PNL-6886

$\overline{\mathrm{UC}-11,41}$

\title{
Hanford Site Ground-Water Monitoring for January through June 1988
}

J. C. Evans

R. W. Bryce

D. R. Sherwood

May 1989

Prepared for the U.S. Department of Energy under Contract DE-ACO6-76RLO 1830

Pacific Northwest Laboratory

Operated for the U.S. Department of Energy

by Battelle Memorial Institute 


\title{
DISCLAIMER
}

This program was prepared as an account of work sponsored by an agency of the United States Government. Neither the United States Government nor any agency thereof, nor Battelle Memorial Institute, nor any of their employees, makes any warranty, express or implied, or assumes any legal liability or responsibility for the accuracy, completeness, or usetulness of any information, apparatus, product, or process disclosed, or represents that its use would not infringe privately owned rights. Reference herein to any specific commerical product, process, or service by trade name, trademark, manufacturer, or otherwise, does not necessarily constitute or imply its endorsement, recommendation, or favoring by the United States Government or any agency thereof, or Battelle Memorial Institute. The views and opinions of authors expressed herein do not necessarily state or refiect those of the United States Government or any agency thereof.

\section{PACIFIC NORTHWEST LABORATORY operated by \\ BATTELLE MEMORIAL. INSTITUTE for the \\ UNITED STATES DEPARTMENT OF ENERGY \\ under Contract DE-ACO6-76RLO 1830}

\author{
Printed in the United States of America \\ Available from \\ National Technical Information Service \\ United States Department of Commerce: \\ 5285 Por Royal Road \\ Springfiedd, Virginia 22161 \\ NTIS Price Codes \\ Microfiche A01 \\ Printed Copy
}

$\begin{array}{cc}\text { Pages } & \begin{array}{c}\text { Price } \\ \text { Codes } \\ 001-025\end{array} \\ 026-050 & \text { A02 } \\ 051-075 & \text { A03 } \\ 076-100 & \text { A04 } \\ 101-125 & \text { A05 } \\ 126-150 & \text { A06 } \\ 151-175 & \text { A07 } \\ 176-200 & \text { A0B } \\ 201-225 & \text { A09 } \\ 226-250 & \text { A10 } \\ 251-275 & \text { A11 } \\ 276-300 & \text { A12 } \\ & \text { A13 }\end{array}$


PNL -6886

UC- 11,41

HANFORD SITE GROUND-WATER MONITORING

FOR JANUARY THROUGH JUNE 1988

J. C. Evans

R. H. Bryce

D. R. Sherwood

May 1989

Prepared for

the U.S. Department of Energy

under Contract DE-ACO6-76RLO 1830

Pacific Northwest Laboratory

Richland, Washington 99352 



\section{SUMMARY}

The Pacific Northwest Laboratory monitors ground-water quality at the Hanford Site for the U.S. Department of Energy to assess the impact of Site operations on the environment. Work undertaken between January and June 1988 included monitoring ground-water elevations across the Site, and monitoring hazardous chemicals and radionuclides in ground water.

Water levels continued to rise in areas receiving increased recharge (e.g., beneath B Pond) and decline in areas where the release of water to disposal facilities has been terminated (e.g., U Pond). The major areas of ground-water contamination defined by monitoring activities are 1) carbon tetrachloride in the 200-West Area; 2) cyanide in and north of the 200-East and 200-West Areas; 3) hexavalcit chromium contamination in the 100-B, $100-\mathrm{D}, 100-\mathrm{F}, 100-\mathrm{H}, 100-\mathrm{K}$, and 200-West Areas; 4) chlorinated hydrocarbons in the vicinity of the Solid Waste Landfill and 300 Area; 5) uranium in the $100-F, 100-H, 200-W e s t$, and 300 Areas; and 6) tritium and nitrate across the Site.

In addition, several new analytical initiatives were undertaken during this period. These include cyanide speciation in the BY Cribs plume, inductively coupled argon plasma/mass spectrometry (ICP/MS) measurements on a broad selection of samples from the 100,200,300, and 600 Areas, and high sensitivity gas chromatography measurements performed at the Solid Waste Landfil1-Nonradioactive Dangerous Waste Landfil1. 



\section{ACKNOWLEDGMENTS}

The authors wish to thank A. G. Law and J. A. Serkowski (Westinghouse Hanford Company) for carefully reviewing this report, D. J. Bates for preparation of tables and report review, R. E. Lundgren for technical editing, and the Sigma $V$ team for word processing. 



\section{CONTENTS}

SUMMARY. . . . . . . . . . . . . . . . . . . .

ACKNOWLEDGMENTS. . . . . . . . . . . . . . . . . . . . V v

1.0 INTRODUCTION . . . . . . . . . . . . . . . . . . . . . 1.1

2.0 WATER-LEVEL ELEVATION MONITORING . . . . . . . . . . . . . 2.1

2.1 DATA COLLECTION . . . . . . . . . . . . . . . 2.1

2.2 RESULTS . . . . . . . . . . . . . . . . . . 2.2

3.0 RADIOLOGICAL AND CHEMICAL GROUND-WATER MONITORING . . . . . . . 3.1

3.1 DATA COLLECTION . . . . . . . . . . . . . . . . . 3.1

3.1.1 Facility-Specific Monitoring . . . . . . . . 3.1

3.1.2 Operational Monitoring........... 3.2

3.1.3 Site-Wide Monitoring . . . . . . . . . . . 3.2

3.1.4 Sample Collection January Through June 1988 . . . 3.5

3.1.5 Monitoring Well Design............ 3.5

3.1.6 Sampling Methods . . . . . . . . . . . . 3.6

3.2 RESULTS . . . . . . . . . . . . . . . . . . . 3.9

3.2.1 Background Concentrations . . . . . . . . 3.10

3.2.2 Cyanide .................. 3.10

3.2.3 Hexavalent Chromium ........... 3.12

3.2.4 Volatile Organic Compounds Across the Site.... . 3.13

3.2.5 Nitrate Across the Site . . . . . . . . 3.17

3.2.6 Tritium Across the Site . . . . . . . . . 3.20

3.2.7 Gross Alpha Activity and Uranium . . . . . . . . . 3.25

3.2.8 Gross Beta Activity . . . . . . . . . . . 3.25

3.2.9 Carbon-14 . . . . . . . . . . . . . . . . . 3.28

3.2.10 Cobalt-60 .................. . . 3.28 
3.2.11 Nicke1-63 ................ . . . 3.29

3.2.12 Strontium-90.............. . . 3.29

3.2 .13 Technetium-99 . . . . . . . . . . . 3.31

3.2.14 Ruthenium-106 ............ 3.33

3.2.15 Iodine-129................ 3.34

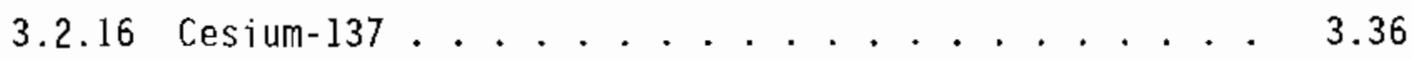

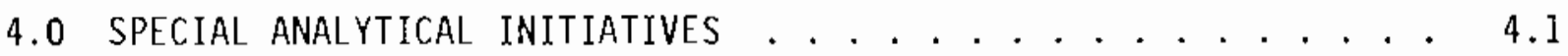

4.1 CYANIDE SPECIATION . . . . . . . . . . . . . 4.1

4.2 INDUCTIVELY COUPLED ARGON PLASMA/MASS SPECTROMETRY
SEMIQUANTITATIVE ANALYSIS

4.3 GAS CHROMATOGRAPHY-VOLATILE ORGANIC ANALYSIS
MEASUREMENTS AT THE SOLID WASTE LANDFILL . . . . . . . . . 4.6

4.3.1 We11 699-24-33 ............ . . 4.7

4.3 .2 We11 699-24-34B ............ . . . 4.7

4.3.3 Pacific Northwest Laboratory Special
Sampling Study at the SWL . . . . . . . 4.9

4.3 .4 Conclusions ................ . 4.11

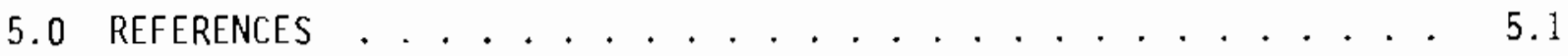

APPENDIX A - WATER LEVELS MEASURED DURING JUNE $1988 \ldots \ldots$. . . . . . A.1

APPENDIX B - MAPS OF OPERATIONAL AND FACILITY-SPECIFIC
MONITORING WELL NETWORKS . . . . . . . B. . . . .

$\begin{aligned} \text { APPENDIX } c & \text { MAXIMUM CONTAMINANT LEVELS AND DERIVED } \\ & \text { CONCENTRATION GUIDELINES ............. . . . . . . . . }\end{aligned}$

$\begin{aligned} \text { APPENDIX D } & \text { DATA OBTAINED BY SPECIAL INDUCTIVELY COUPLED } \\ & \text { PLASMA/MASS SPECTROMETRY METHOD . . . . . . . . . D. . . }\end{aligned}$

APPENDIX E - DATA LISTINGS . . . . . . . . . . . . E.1

PLATE 1 - Hanford Site Water Table Map, June 1988 . . . . . back pocket 


\section{FIGURES}

1.1 Hanford Site Location Map. . . . . . . . . . . . 1.2

3.1 Location of Hanford Site Ground-Water Monitoring Wells . . . . 3.7

3.2 Maximum Chromium Concentrations in Unconfined Aquifer Wells, January 1, 1987, to June $30,1988 \ldots . . . . .3 .13$

3.3 Maximum Carbon Tetrachloride Concentrations in 200-West Area Unconfined Aquifer Wells Between January 1,1987 , and June $30,1988 \ldots \ldots . \ldots . . \ldots 3.15$

3.4 Maximum Nitrate Concentrations in Unconfined Aquifer Wel1s, January 1,1987 , to June $30,1988 \ldots . . . . .3 .18$

3.5 Tritium Concentrations from 1984 to June 1988

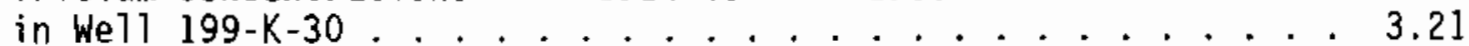

3.6 Tritium Concentrations Through Time in Well 699-S19-E13. . . . . . ............. 3.24

3.7 Tritium Concentrations Through Time in Well 699-S3-E12 ... 3.24

3.8 Maximum Uranium Concentrations in Unconfined Aquifer We1ls, January 1,1987 , to June $30,1988 \ldots . . \ldots 3.26$

3.9 Maximum Gross Beta Concentrations in Unconfined Aquifer Wel1s, January 1 , 1987, to June $30,1988 \ldots . . . . .3 .27$

3.10 Maximum Strontium-90 Concentrations in Unconfined Aquifer We11s, January 1,1987 , to June $30,1988 \ldots \ldots .30$

3.11 Maximum Technetium-99 Concentrations in Unconfined Aquifer Wells, January 1 , 1987, to June $30,1988 \ldots . . . .31$

3.12 Maximum Iodine-129 Concentrations in Unconfined Aquifer We11s, January 1,1987 , to June $30,1988 \ldots . . . .35$

B.1 Location of 100-B Area Ground-Water Monitoring Wells Sampled During January Through June 1988 . . . . . . . . . B.3

B.2 Location of 100-D Area Ground-Water Monitoring We 11s Sampled During January Through June 1988 . . . . . . . . . B.5

B.3 Location of 100-F Area Ground-Water Monitoring We1ls Sampled During January Through June 1988 . . . . . . . . B.7

B.4 Location of 100-H Area Ground-Water Monitoring Welis Sampled During Janaury Through June 1988 
B.5 Location of 100-K Area Ground-Water Monitoring Wells

Sampled During Janaury Through June 1988.......... B.11

B.6 Location of 100-N Area Ground-Water Monitoring Wells

Sampled During January Through June 1988 ........... B.13

B.7 Location of 200-East Area Ground-Water Monitoring Wells

Sampled During January Through June 1988 ......... . B.15

B.8 Location of 200-West Ground-Water Monitoring We1ls

Sampled During January Through June 1988 .......... B.17

B.9 Location of 300-Area Ground-Water Monitoring Wells

Sampled During January Through June 1988 ......... . B.19

B.10 Location of 400 Area Ground-Water Monitoring Wells

Sampled During Janauary Through June 1988. . . . . . . . . . B.21 


\section{$\underline{\text { TABLES }}$}

1.1 Waste Disposal Facilities with Ongoing Sampling Projects . . . 1.3

3.1 Major Chemical Ground-Water Contaminants and Their Link to Site Operations. . . . . . . . . . . . . 3.5

3.2 Estimated Background Levels for Selected Constituents in Hanford Ground Water. . . . . . . . . . . . . . . . 3.11

4.1 Cyanide Speciation in 600 Area Wells . . . . . . . . . . . 4.2

4.2 Method Detection Limits for Semiquantitative Analys is by ICP/MS .................. . 4.4

4.3 Chlorinated Hydrocarbon Contaminants in We11 699-24-33................. 4.8

4.4 Ch1orinated Hydrocarbon Contaminants in We1? $699-24-34 \mathrm{~B}$

$4.51,1,1$-Trichloroethane Results . . . . . . . . . . 4.13

$4.61,1,2$-Trichloroethene Results . . . . . . . . . . 4.14

$4.71,1,2,2$-Tetrachloroethene Results . . . . . . . . . . . 4.15

4.8 Chloroform Results.................... 4.16

4.9 Carbon Tetrachloride Results.............. 4.17

4.10 Chlorinated Hydrocarbons Collected by Sequential Purging of Well 699-24-35 . . . . . . . . . . . . . . 4.18

A.1 Hanford Water Level Measurements in the Unconfined Aquifer, June 1988 . . . . . . . . . . . . . . . . . A.3

C.1 Radiological Maximum Contaminant Levels ............ c.l

C.2 Chemical Maximum Contaminant Levels . . . . . . . . . . . c.2

C.3 Proposed Derived Concentration Guides . . . . . . . . . . . . c.3

D.1 ICP-MS SEMIQUANT Analysis of Hanford Ground-Water Samples . . . 0.2

E.I Key to Constituent and Constituent Group Names Used in Tables E.2, E.3, E.4, E.5, E.6, and E.7 ......... E.3

E.2 Constituents Analyzed for in Ground-Water Samples from Site-wide Chemical Monitoring Wells 
E.3 Results for Site-Wide Chemical Monitoring Wells . . . . . . E.19

E.4 Analytical Plans for Compliance Monitoring Networks . . . . . . E.180

E.5 Results for Compliance Monitoring Wells . . . . . . . . . . E.187

E.6 Constituents Analyzed for in Ground-Water Samples from

Site-Wide Radiological Monitoring Wells Outside the

Site-Wide Chemical or Compliance Monitoring Networks . . . . . E.313

E.7 Results for Site-Wide Radiological Monitoring Wells

Outside the Site-Wide Chemical or Compliance

Monitoring Networks . . . . . . . . . . . . . . . E.321 


\subsection{INTRODUCTION}

The Pacific Northwest Laboratory (PNL)(a) monitors the distribution of radionuclides and other hazardous materials in the ground water at the Hanford Site (Figure 1.1) for the U.S. Department of Energy (DOE). This Site-Wide Ground-Water Monitoring Project also evaluates the impact of past and present Site operations on the environment. The specific objectives of the project are to 1) determine the distribution of radionuclides and nitrate ion to define the extent of impacted ground water, 2) relate the distribution of these constituents to Site operations, 3) establish background concentrations for naturally occurring regulated hazardous materials, and 4) identify in the ground water those hazardous chemicals that resulted from Site operations. The Hanford Site-Wide Ground-Water Monitoring Project involves the measurement of water-table elevations, collection and analysis of groundwater samples, computer modeling of contaminant transport, and use of geochemical models to meet these objectives.

The Hanford Site monitoring well network is designed to meet the intent of DOE Orders 5480.1 and 5484.1, which are applicable to environmental monitoring. DOE Order 5480.1, Chapter XI, lists the permissible concentrations of radionuclides applicable to the discharge of liquid effluent in controlled and uncontrolled areas. This order also requires that the environment be monitored sufficiently to ensure that the radiation doses to which the public is exposed are as low as reasonably achievable. DOE Order 5480.1, Chapter XII, requires that DOE cooperate with the U.S. Environmental Protection Agency (EPA) and state, interstate, and local agencies in the prevention, control, and abatement of environmental pollution. Annual reports (e.g., Jaquish and Mitchell 1988) are published by PNL to document all surface and subsurface monitoring activities at the site, as well as measured and calculated doses to the public.

(a) The Pacific Northwest Laboratory is operated for the U.S. Department of Energy by Battelle Memorial Institute under Contract DE-ACO6-76RLO 1830. 


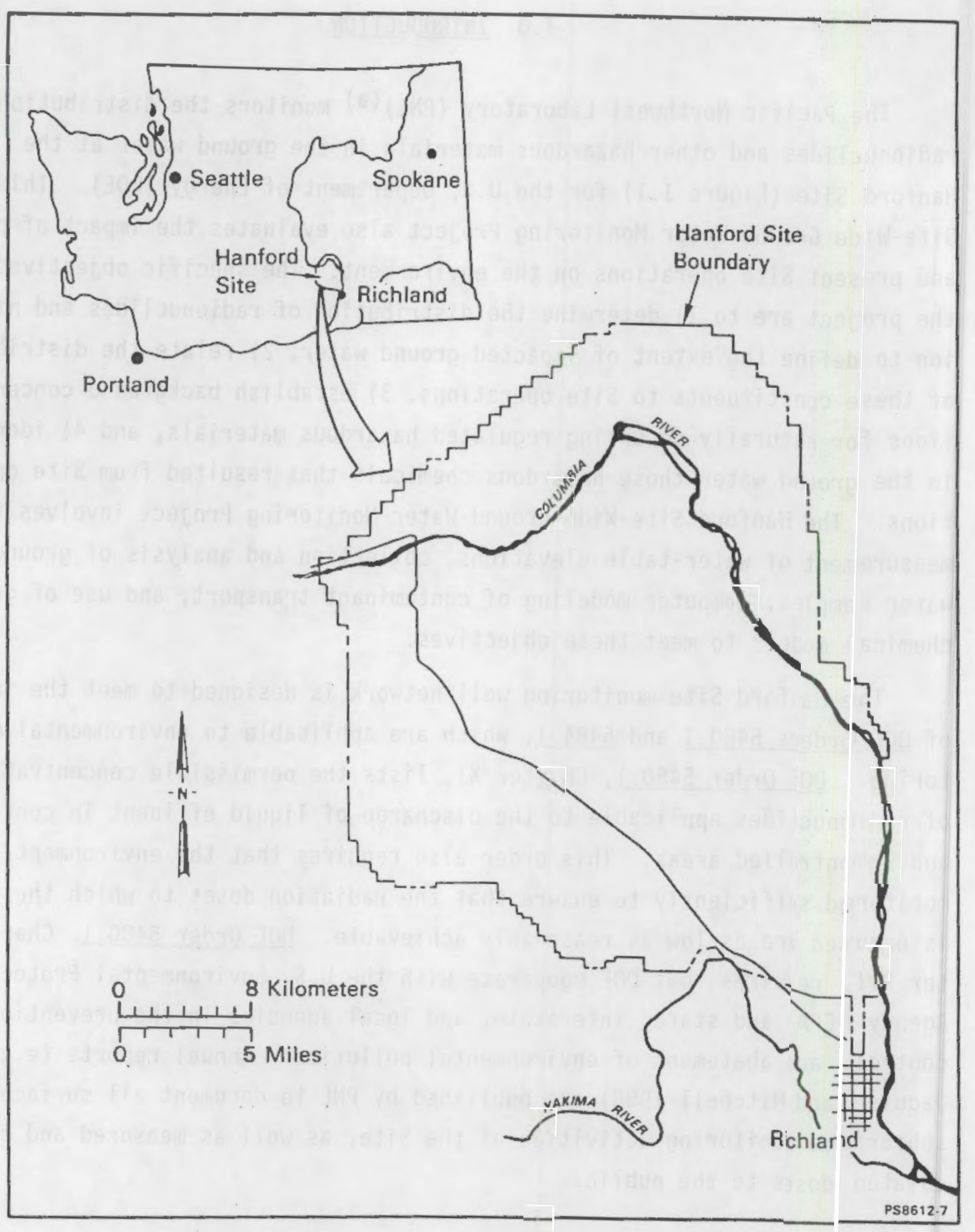

FIGURE 1.1. Hanford Site Location Map 
In addition to the Hanford Site-Wide Ground-Water Monitoring Project, two ground-water monitoring activities are being conducted by PNL at the Hanford Site for Westinghouse Hanford Company. Ground-water samples are collected by PNL for operational monitoring in and around the 200 Areas for compliance with DOE orders and for facility-specific monitoring for compliance with the Resource Conservation and Recovery Act (RCRA) (at 40 CFR 265) and Washington Administrative Code (WAC) 173-303 and -304. The facility-specific activities include sampling programs at facitities 1 isted in Table 1.1. The results of some of these activities are discussed briefly in this report and will be reported in more detail elsewhere. The compliance monitoring results (primarily for chemicals) are valuable in determining the total impact of Site operations on ground water and, therefore, are used by the Hanford Site-Wide Ground-Water Monitoring Project to meet its objectives.

\author{
TABLE 1.1. Waste Disposal Facilities with Ongoing \\ Sampling Projects \\ 183-H Solar Evaporation Basins in the 100-H Area \\ 1301-N/1325-N Liquid Waste Disposal Facilities \\ and 1324-N/NA Ponds in the 100-N Area \\ Transportable Grout Facility \\ 300 Area Process Trenches \\ Solid Waste Landfill (SWL) and Nonradioactive \\ Dangerous Waste Landfili (NRDW') \\ 200 Area Low-Level Burial Grounds \\ $216-\mathrm{A}-10 \mathrm{Crib}$ \\ 216-A-29 Ditch \\ 216-A-36B Crib \\ 216-B-3 Pond \\ 2101-M Pond
}


This semiannual report provides brief discussions and detailed data listings of results for ground-water monitoring at the Hanford Site during January through June 1988. This report presents and discusses the watertable elevation map (Plate 1, in back pocket) for the Hanford Site for June 1988 and results of ground-water sampling and analysis. The following contaminants are discussed in detail because of their high concentrations or widespread distribution: 1) carbon tetrachloride in the 200-West Areas; 2) cyanide in and north of the 200-East and the 200-West Areas; 3) hexavalent chromium contamination in the 100-B, 100-D, 100-F, 100-H, 100-K, and the 200-West Areas; 4) chlorinated hydrocarbons in the vicinity of the Central Landfil1 and 300 Area; 5) uranium in the 100-F, 100-H, 200-West, and 300 Areas; 6) nitrate across the Site; and 7) tritiun across the Site. The implications of several other ohservations are also discussed briefly. To adequately represent Site conditions, the figures in this report show data for an 18-month period (January 1, 1987 to June 30, 1988). The text itself only summarizes data from January 1 to June $30,1.988$.

More detailed discussions of the hydrology and geology of the Site, operational activities, and sampling, analysis, and distributions of average constituent concentrations during 1988 will be included in the environmental monitoring annual report by PNL (to be published in 1989). In addition, Westinghouse Hanford Company (e.g., Serkowski et al. 1988) reports radiological monitoring results for the 200 Areas and some of the surrounding 600 Area. Also RCRA monitoring results are documented in quarterly reports (e.g., PNL 1988a). 


\subsection{WATER-LEVEL ELEVATION MONITORING}

Water levels in selected wells open to the unconfined aquifer on the Hanford Site are measured in June and December of each year to determine the configuration of the water table and to assess the impact of waste disposal on that configuration. Water-level elevations for December 1987 were presented by Evans et al. (1988). Historical water-level data and an evaluation of past water-level changes are presented by Zimmerman et a]. (1986). Water-level elevations for this report were measured in approximately 240 wells open to the unconfined aquifer during a 2-week period in June 1988.

Water-table maps can be used to infer directions of ground-water flow, with ground water moving from regions of higher water levels to regions of lower water levels at right angles to contours in systems with isotropic hydraulic conductivities. Water-table configurations can be used to identify recharge and discharge areas, and to determine the potential for water movement between adjacent ground-water and surface-water bodies.

\subsection{DATA COLLECTION}

Water levels are measured in wells and piezometers following written procedures. Most monitoring welis at the Hanford Site used for water-level measurement are 6 or 8 in. (15 or $20 \mathrm{~cm}$ ) in diameter and are constructed of steel casing. Several small-diameter [2-in. (5-cm)] piezometers and some Targer diameter wells are also used. Monitoring wells used to measure water levels for the unconfined aquifer are completed with well screens or perforated casing generally open to the upper 10 to $20 \mathrm{ft}(3$ to $6 \mathrm{~m}$ ) of the aquifer. This type of completion allows measurements to be representative of the water-table elevation. Well locations for water-level monitoring are shown in Plate 1 (found in back pocket of this document).

A written procedure adapted from the U.S. Geological Survey (USGS 1977) was followed to measure water levels in wells. A chalked steel tape was used to measure the depth to water below the top of the well casing. These measurements were repeated to confirm the initial value. If the second measurement differed by more than $0.05 \mathrm{ft}$ from the first, the measurement was 
repeated until two measurements within the proper tolerance were obtained. Measurements were compared to previous water levels to further reduce human error. Water-level elevations for each well were determined by subtracting the depth to water from the surveyed measuring-point elevation on the well casing. All water levels are measured within a 2-week period to reduce the effects on the water-table map of time-variant water-level changes.

\subsection{RESULTS}

Water levels measured during June 1988 are 1 isted in Appendix A. The water-table map constructed by contouring these water-level elevations is presented in Plate 1 . The following are important features of the watertable map:

- Water-table elevations generally decrease from west to east across the Hanford Site south of Gable Mountain and Gable Butte.

- Water-table elevations decrease northward through the gap between Umtanum Ridge and Gable Butte and between Gable Butte and Gable Mountain.

- Ground-water mounds are present beneath B Pond and the location of the decommissioned U Pond.

- The water level in the Yakima River is of higher elevation than that of the water table in the adjacent region of the Hanford Site.

Elevated water levels in the western region of the Hanford Site may be attributable to ground-water recharge in Cold Creek and Dry Creek valleys and on the adjacent ridges (i.e., Yakima, Umtanum, and Ratt]esnake ridges). Recharge is most likely caused by rain and snow at the higher elevations and by irrigation of agricultural land at the lower elevations. Water-table elevation generally decreases toward the Columbia River at the eastern edge of the Hanford Site and north of the Gable Mountain-Gable Butte anticline, implying discharge of unconfined ground water to the Columbia River along the Hanford reach.

Ground-water mounds beneath B Pond and the location of the decommissioned $U$ Pond have been caused by process cooling water and other liquid wastes recharging the ground water at those locations. A summary of waste discharges to disposal facilities in the 200 Areas is presented by Serkowski 
et a1. (1988). The U Pond was decommissioned in 1984, and the elevation of the ground-water mound at that location continues to decline. A decline of one-half foot $(15 \mathrm{~cm}$ ) was observed between December 1987 and June 1988 (well 299-W18-15). Waste disposal at other facilities in the 200-West Area also influences the elevation of the water table as demonstrated by a water-level increase of approximately $2 \mathrm{ft}(61 \mathrm{~cm})$ in well 299-W23-4 and water-level decines of 1 to $2 \mathrm{ft}(30$ to $61 \mathrm{~cm})$ in we17s north of the Plutonium Finishing Plant.

The elevation of the ground-water mound beneath $B$ Pond, which receives process cooling water from the plutonium-uranium extraction (PUREX) Plant, has increased approximately $1 \mathrm{ft}(30 \mathrm{~cm}$ ) since December 1987 (as reflected in water levels in well 699-43-42). We11 699-39-39 showed a temporary increase in water level of $7 \mathrm{ft}(2.13 \mathrm{~m})$ in June. While the cause of this increase is unknown, it is most likely a result of changes in disposal practices at B Pond. The water level in well 699-39-39 returned to its pre-June elevation in July.

A water-table elevation lower than the Yakima River in the region between the Horn Rapids Dam and the City of Richland implies that the ground water is being recharged by the Yakima River. Water levels measured in this region also indicate that ground water recharged by the Yakima River flows to the east beneath the Hanford Site and discharges to the Columbia River. 



\subsection{RADIOLOGICAL AND CHEMICAL GROUND-WATER MONITORING}

\subsection{DATA COLLECTION}

The network of wells used for the Hanford Site-Wide Ground-Water Monitoring Project is a combination of several networks that have been designed for facility-specific, operational, and Site-wide monitoring activities. The basis for selecting wells, sampling frequencies, and constituents to be analyzed is different for each of these projects based on the project objectives.

\subsubsection{Facility-Specific Monitoring}

We11 networks have been established for Westinghouse Hanford Company around specific waste disposal facilities to comply with RCRA requirements. Facility-specific activities include sampling programs at the 183-H Solar Evaporation Basins in the 100-H Area, 1301-N/1325-N Liquid Waste Disposal Facilities and 1324-N/NA Ponds in the 100-N Area, Transportable Grout Facility, 300 Area Process Trenches, Low Level Burial Grounds, 216-A-10 Crib, 216-A-29 Ditch, 216-A-36B Crib, 216-B-3 Pond, 2101-M Pond, Solid Waste Landfill (SWL), and the Nonradioactive Dangerous Waste Landfill (NRDWL).

The requirements for monitoring well design and location, constituents to be sampled for, and sampling frequencies are specified in RCRA regulations (40 CFR 265) and by Washington Administration Code (WAC 173-303 and -304). Ground-water monitoring systems at each site must consist of at least one monitoring well hydraulically upgradient and at least three monitoring wells downgradient of the facility. The location, depth, and number of wells included in the network must ensure that results obtained to evaluate the migration of contaminants to the uppermost aquifer are statistically significant. The RCRA regulations require that ground water be sampled and analyzed for 1) drinking water parameters, 2) parameters that establish ground-water quality, and 3) parameters used as indicators of ground-water contamination. Samples are also analyzed for contaminants known to have been disposed of at the facility being monitored. The frequency of sampling for each parameter is also specified in the RCRA regulations, based on the status of permitting of the faci]ity (e.g., interim status, permitted status). 
Quarterly reports (i.e., PNL 1988a) document monitoring networks and analytical plans for these RCRA sites. Two such reports have been published that cover the periods January 1 to March 31, 1988 (PNL 1988a) and Apri7 1 to June 30, 1988 (PNL 1988b).

\subsubsection{Qperational Monitoring}

Operational monitoring near waste facilitie: in the 200 Areas is conducted for Westinghouse Hanford Company to allow them to evaluate the performance of waste disposal and storage sites and assess the impact of specific sites on ground water (Serkowski et a1. 1988). Well location, monitoring frequency, and constituents for which samples are analyzed are selected to meet this objective. The sampling network design, analytical plans, and results are presented each year in an annual report (e.g., Serkowski et a1. 1988). Changes in the monitoring network are described in that report. The planned network and schedule for calendar year 1988 are listed in Appendix B of Serkowski et al. (1988).

\subsubsection{Site-Wide Monitoring}

The purpose of the Site-Wide Ground-Water Monitoring Project is to monitor the distribution of radionuclides and other hazardous materials in ground water at the Hanford Site. The selection of wells, constituents for which samples are collected and analyzed, and sampling frequency are based on knowledge of waste disposal practices and inventories, regulatory requirements, contaminant mobility, and the site hydrogeology. The Site-wide monitoring network consists of a network for radiological monitoring and a network for chemical monitoring.

\subsubsection{Radiological Monitoring}

The radiological monitoring network was developed to monitor the extent of contamination, identify new instances of contaminant release to the ground water, and sample for selected radionuclides the.t may contribute to radiation dose. Welis and constituents near operational and facility-specific networks were selected to complement monitoring under these programs. For example, some wells in the 200 Areas monitored by Westinghouse Hanford Company to 
evaluate facility operation are sampled for additional constituents to meet the objectives of site-wide monitoring.

The primary analyses done on samples collected for radiological analysis are for tritium and gross alpha, gross beta, gamma scans, and beta counting for radiochemical separates (i.e., strontium-90, technetium-99). The maximum extent of radionuclide contamination in the ground water beneath the Hanford Site is defined using tritium because nearly all radioactive waste disposed of at Hanford contains tritium. Tritium exists as part of the water molecule and as such moves with the ground water unretarded by chemical and physical interaction with dissolved constituents and aquifer materials. Tritium was also concentrated in certain large-volume wastes, such as reactor coolant in the 100 Areas and process condensates in the 200 Areas.

Gross alpha and gross beta analyses, and gamma scans are used to identify potential new releases of radionuclides in a cost-effective manner at certain locations. These techniques are used to survey wells throughout the Site for a wide variety of alpha-, beta-, and gamma-emitting radionuclides. If results of these survey techniques indicate it is warranted, samples are collected and analyzed for individual radionuclides. Subsequent analyses are chosen on the basis of radionuctide inventories, radionuclide mobilities, and potential dose concerns.

Gross alpha concentrations above background may indicate the sample contains uranium or plutonium. Uranium is an alpha-emitting radionuclide that is mobile in the Hanford ground water. It is commonly the radionuclide responsible for elevated gross alpha concentrations. Uranium is also a potential concern in terms of its dose to man. Plutonium is another alpha emitter that could contribute to gross alpha activity. Past monitoring for plutonium suggests that it is immobile in the ground water and hence is monitored in only a few wells near facilities suspected of receiving plutonium. These wells are all within the 200 Areas.

Elevated gross beta concentrations are more difficult to associate with a single radionuclide because of the potentially large number of betaemitting radionuclides discharged in Hanford liquid wastes. Strontium-90 has been a common contributor to elevated gross beta concentrations in ground 
water. Strontium-90 is monitored in ground-water samples collected throughout the Hanford Site, with emphasis on the operating areas. Another beta emitter of potential dose concern that is relatively mobile in the ground water is technetium-99. Technetium-99 is not efficiently detected using gross beta analysis because it is a low-energy beta emitter. Few technetium-99 measurements have been made in the past because of the costly analytical method; however, a less costly technique was developed in 1987 . Using this new technique, technetium-99 concentrations were determined for ground-water samples collected from 190 wells in many areas during the period covered by this report. The purpose of this greatly expanded analysis program was to gather baseline data and to investigate elevated gross beta concentrations previously observed. Three other beta emitters were also selectively analyzed in a limited number of wells during this same period. These are carbon-14, nickel-63, and iodine-129. Isolated measurements of these radionuclides were conducted to further assess elevated gross beta measurements previously observed and to investigate selected wells near possible sources of these radionuclides.

\subsubsection{Chemical Monitoring}

A subset of both the PNL Site-wide and the Westinghouse Hanford Company operational radiological monitoring networks is used for site-wide chemical sampling by PNL. Chemical sampling wells were selected primarily for their proximity to known active and inactive chemical disposal areas in the 100 , 200, and 600 Areas, and on the basis of the compiled waste inventories (DOE 1986).

Nitrate is monitored in most of the wells sampled. Nitrate, which is mobile in ground water, was present in many of the waste streams disposed of to the ground and, like tritium, can be used to help define the extent of contamination in Hanford aquifers. Extensive historical records also exist for nitrate. Other chemicals related to Site operations are listed in Table 3.1. Ground-water samples collected from wells in areas where these chemicals have been discharged are analyzed for the appropriate contaminant(s). 
IABLE 3.1. Major Chemical Ground-Water Contaminants and Their Link to Site Operations

\begin{tabular}{llll}
\multicolumn{1}{c}{ Type of Facility } & Area & & Constituents \\
\cline { 1 - 1 } Reactor Operations & 100 & $\mathrm{Cr}^{6+}$ & \\
Irradiated Fuel Processing & 200 & $\mathrm{NO}_{3}^{-}, \mathrm{CN}^{-}$, Uranium \\
Plutonium Purification & 200 & $\mathrm{CCl}_{4}$ and other chlorinated hydrocarbons \\
Fuel Fabrication & 300 & Uranium, $\mathrm{Cr}^{6+}$
\end{tabular}

\subsubsection{Sample Collection January Through June 1988}

The 1988 PNL Site-wide radiological sampling network consisted of 294 wells that were monitored quarterly or monthly. An additional 110 wells were monitored semiannually. In the 200 Areas and surrounding 600 Area, PNL sampled 161 wells in Westinghouse Hanford Company's operational monitoring network.

During the first quarter of 1988, 114 wells were monitored for chemical constituents; 103 wells were sampled during the second quarter. Chemical data are available for an additional 93 facility-specific wells.

The Site-wide monitoring network for 1988 is shown in Figure 3.1. Detailed maps of operational and facility-specific monitoring well networks for the 100-B, 100-D, 100-F, 100-H, 100-K, 100-N, 200-East, 200-West, 300, and 400 Areas are included in Appendix B.

\subsubsection{Monitoring We11 Design}

Most monitoring wells at the Hanford Site are 6 or $8 \mathrm{in.}(15$ or $20 \mathrm{~cm}$ ) in diameter and are constructed of steel casing. Several smali-diameter [2-in. $(5-\mathrm{cm})]$ piezometers are sampled for radionuclides only. Monitoring wells for the unconfined aquifer are completed with well screens or perforated casing in the upper 10 to $20 \mathrm{ft}(3$ to $6 \mathrm{~m})$ of the aquifer. Completion at the water table allows samples to be collected near the top of the aquifer where maximum concentrations for some radionuclides were measured at a few locations on the Hanford Site (Eddy, Myers, and Raymond 1978). Confined aquifer monitoring wells have screens, perforated casing, or an open hole 
within the monitored horizon. Only wells containing submersible pumps were chosen for chemical sampling to allow sufficient purging of wells before sampling.

\subsubsection{Sampling Methods}

Samples were collected using documented sampling procedures that follow formal, established guidelines (EPA 1986). Wells fitted with submersibie pumps were sampled after pumping for a sufficient time (at least 20 min) to allow temperature, $\mathrm{pH}$, and specific conductivity to stabilize. The purging process ensured that any stagnant water in the well was removed, allowing collection of a sample that was representative of the ground water near the well. A stainless steel sampling tee was then connected to the pump discharge line. One side of the tee consisted of a $3 / 16-\mathrm{in} .(0.476-\mathrm{cm})$ critical orifice discharging to a $1 / 4-i n .(0.635-\mathrm{cm})$ reflon ${ }^{\circledR}(a)$ sampling line. Excess water was discharged through a ball valve on the other branch of the tee. This arrangement allowed the pump discharge to be throttled back sufficiently to provide a continuous water column, while providing some pressure relief to avoid damage to the header pipe. Samples for volatile organic analyses were taken with zero head space and sealed immediately with a septum-sealed cap. When sampling for filtered trace metals, a disposable, 0.45 -micron pore-sized filter pack was connected to the Teflon ${ }^{\circledR}$ samp 7 ing 1 ine. The filter was purged with $0.13 \mathrm{gat}(500 \mathrm{~mL})$ of well water, then a sample was collected in the appropriate sample bittle. Trace metal samples and some radiochemical samples were preserved by acidification at the time of collection. All samples were placed on ice in ice chests immediately after sampling and were transferred the same day or early the next morning to the analytical subcontractor, United States Testing Company, Inc. (UST), Richland, Washington, for immediate analys is of species with short holding times (e.g., for nitrate and volatile organic analyses). Samples were stored at $39^{\circ} \mathrm{F}\left(4^{\circ} \mathrm{C}\right)$ from the time of sampling until they were analyzed. All samples were tracked using chain-of-custody procedures from sampling through

(a) Teflon is the registered tradename for a fluorocarbon resin product of E. I. du Pont de Nemours and Company, Wilmington, Delaware. 


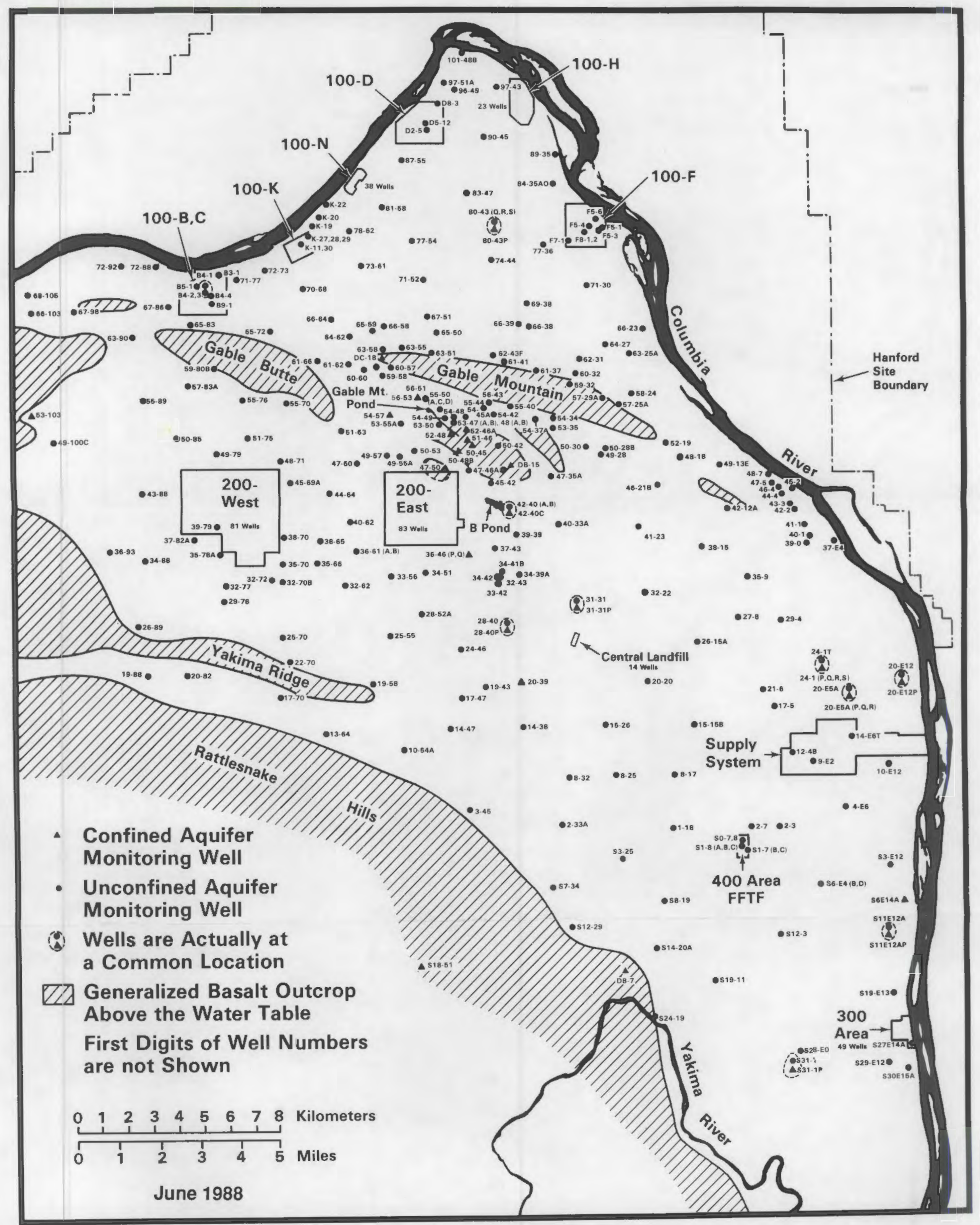

FIGURE 3.1. Location of Hanford Site Ground-Water Monitoring Wells 

analysis and disposal. Procedures for analyzing samples have been described elsewhere (Jaquish and Mitchell 1988, Appendix C).

\subsection{RESULTS}

Results of the Site-wide, operational, and facility-specific groundwater monitoring projects are discussed in this section. Information on contaminants can be found in past environmental monitoring reports by PNL and the operating contractor (previously Rockwell Hanford Operations; now Westinghouse Hanford Company). The most recent reports are Jaquish and Mitchell (1988), Evans et a1. (1988), and Serkowski et al. (1988). Evans et a1. (1988) discussed in detail the following contaminants in Hanford Site ground water: 1) carbon tetrachloride in the 200-West Area, 2) cyanide in and north of the 200-East and 200-West Areas, 3) hexavalent chromium in the 100 and 200-West Areas, 4) chlorinated hydrocarbons near the Centra7 Landfill and 300 Area, 5) nitrate across the Site, and 6) tritium across the Site. This report will update that information based on data that became available during January through June 1988.

In addition to the above-mentioned constituents, this report discusses more recent findings. Such findings clearly show cyanide and chromium contamination in the 200-West Area. Chromium contamination is now also evident in several parts of the extended 100 Area environs and in several wells in the 200-East Area. In addition to the areas previously discussed, trichloroethene has recently been observed at levels of concern in an area directly west of the 100-F Area and in a we1l directly north of Gable Mountain. Other observations of chemical and radiological contaminants are briefly discussed. Results for technetium-99 include the greatly expanded technetium survey carried out during the period covered by this report. This report also includes analysis results of approximately half of the annual iodine-129 samples. These samples were analyzed by a newly implemented direct-counting method with detection sensitivity ranging down to the maximum contaminant level (MCL) of $1 \mathrm{pCi} / \mathrm{L}$. Samples collected from eleven wells for iodine-129 analysis were measured by a more sensitive technique (mass spectrometry). 
Results are discussed relative to the EPA maximum contaminant level (MCL), to the DOE derived concentration guide (DCG) appropriate for each constituent (Appendix C), and to background concentrations. The MCLs for radionuclides are more restrictive than the DCGs because the MCLS are based on an annual dose to the affected organ of $4 \mathrm{mrem} / \mathrm{yr}$, while the DCGs are based on an effective dose of $100 \mathrm{mrem} / \mathrm{yr}$. The DCGs are only relevant to radionuclides. Radionuclide concentrations in Hanford Site ground water are administered by DOE Order 5480.1 .

\subsubsection{Background Concentrations}

Background concentrations of contaminants have been estimated from ground-water samples collected in areas not affected by Hanford operations. Because the effect of Hanford operations is not in all cases obvious, some judgement was needed for the selection of wells to be used. Only 600 Area wells were used because all of the operating areas are clearly affected to some extent. Wells in the region of the 200-East Area cyanide/technetium plume were also excluded because those wells clearly exhibit anomalous chemical and radiological effects. In a few other cases, wells were excluded if they showed evidence of other anomalous effects. For example, well 699-83-47 has repeatedly shown elevated levels of chromium. Although the origin of the material is unknown, that well can be assumed to be affected by Site operations. Estimates of background concentrations for selected constituents are 1isted in Table 3.2. Data used for the calculation of backgrounds as presented in Table 3.2 were taken from two sources. Data listings for the period covered by this report (January to June 1988) were the primary source of data with forty-five to sixty 600 Area wells selected as typical. Data obtained by inductively coupled plasma/mass spectrometry (ICP/MS) on thirty 600 Area wells were used to supplement that information in cases where lower detection limits or additional elemental information could be used effectively. The ICP/MS work and a complete listing of the data are described later in this report.

\section{2 .2 Cyanide}

Sampling for chemicals in March 1987 included several 600 Area wells directly north of the 200-East Area. Cyanide was detected in two we11s, 
TABLE 3.2. Estimated Background Levels for Selected Constituents in Hanford Ground Water

\begin{tabular}{|c|c|c|}
\hline Constituent & $\begin{array}{l}\text { Detection } \\
\text { Limit a) } \\
\end{array}$ & $\begin{array}{c}\text { Background } \\
\text { Concentration (a) }\end{array}$ \\
\hline Aluminum & $2^{(b)}$ & $<2$ (b) \\
\hline Ammonia & 50 & $<50$ \\
\hline Arsenic & $0.2^{(b)}$ & $3.9 \pm 2.4(b)$ \\
\hline Barium & 6 & $42 \pm 29$ \\
\hline Beryllium & 0.3 (b) & $<0.3(b)$ \\
\hline Bismuth & 0,02 (b) & $<0.02$ (b) \\
\hline Boron & 50 (b) & $<50$ (b) \\
\hline Cadmium & $0.2(b)$ & $<0.2$ (b) \\
\hline Calcium & 50 & $40,400 \pm 10,300$ \\
\hline Chloride & 500 & $10,300 \pm 6,500$ \\
\hline Chromium & 2 (b) & $4.9+2.0(b)$ \\
\hline Copper & 1 (b) & $<1(b)$ \\
\hline Cyanide & 10 & $<10$ \\
\hline Fluoride & 500 & $370+100$ \\
\hline Lead & 0.5 (b) & $<0.5(b)$ \\
\hline Magnesium & 10 & $11,800 \pm 3,400$ \\
\hline Manganese & 5 & $7 \pm 5$ \\
\hline Mercury & 0,1 & $<0,1$ \\
\hline Nickel & $4(b)$ & $<4($ b) \\
\hline Phosphate & 1000 & $<1000$ \\
\hline Potassium & 100 & $4,950 \pm 1,240$ \\
\hline Selenium & $2^{(b)}$ & $<2(b)$ \\
\hline Silver & 10 & $<10$ \\
\hline Sodium & 10 & $18,260 \pm 10,150$ \\
\hline Strontium & 20 & $236 \pm 102$ \\
\hline Sulfate & 500 & $34,300 \pm 16,900$ \\
\hline Uranium & $0.5(\mathrm{c})$ & $1.7 \pm 0.8(\mathrm{c})$ \\
\hline Vanadium & 5 & $17 \pm 9$ \\
\hline Zinc & 5 & $6 \pm 2$ \\
\hline Alkal inity & - & $123,000 \pm 21,000$ \\
\hline & $\cdots$ & $7.64 \pm 0.16$ \\
\hline $\begin{array}{l}\text { Total Organic Carbon } \\
\text { Conductivity }\end{array}$ & 200 (d) & $\begin{array}{l}586 \pm 347 \\
380 \pm 82(d)\end{array}$ \\
\hline & & \\
\hline Gross Alpha & $0,5(c)$ & $2.5 \pm 1,4(\mathrm{c})$ \\
\hline $\begin{array}{l}\text { Gross Beta } \\
\text { Radium }\end{array}$ & $\begin{array}{l}4(c) \\
0.2(c)\end{array}$ & $19 \pm 12^{(\mathrm{C})}$ \\
\hline Kadium & & \\
\hline
\end{tabular}

(a) Units in ppb unless otherwise noted.

(b) Based on ICP/MS data.

(c) Units in $\mathrm{pC} i / \mathrm{L}$.

(d) Units in $\mu \mathrm{mho} / \mathrm{cm}$. 
699-49-57 and 699-50-53. More extensive sampling during the third quarter (April to June) confirmed those preliminary results. A third well, 299-E33-5, inside the 200-East Area was also found to be contaminated with cyanide. The presence of cyanide was accompanied by corresponding increases in cobalt-60 and gross beta activity in the ground water over a 1-year period. Although cobalt-60 is normally relatively immobile in the Hanford subsurface environment, it appears to be chemically complexed and mobilized by cyanide. Samples taken through June 1988 show a maximum cyanide concentration of $1690 \mathrm{ppb}$ in wel1 699-50-53 with lesser amounts present in four other wells in or near the north side of the 200-East Area. In addition to cyanide, a relatively high level of technetium-99 $(28,000 \mathrm{pCi} / \mathrm{L})$ was also detected in well 699-50-53. The majority of the gross beta activity observed in that well is likely technetium-99. The cyanide is thus associated with a well-defined but relatively narrow plume that has only recently appeared in that area. The extent of the contamination is currently limited to about $1 \mathrm{mi}^{2}\left(2.5 \mathrm{~km}^{2}\right)$; however, the exact extent of the plume is difficult to determine because there are few wells in the area. No fixed MCL or other standard for cyanide currently exists. The detection limit for cyanide is typically 5 to $10 \mu \mathrm{g} / \mathrm{L}$ using EPA's standard analytical method (SW-846 Method 9010) (EPA 1982).

Low-level cyanide contamination has also been detected in four widely spaced wells in the 200-West Area (299-W12-1, 299-W14-2, 299-W15-8, and 299-W18-7). Cyanide was detected three times in we11 699-38-70, located immediately to the east of the 200-West Area, but was not observed in the May 1988 sample. Low levels of cyanide have also been found in well 699-44-64, which is located between the 200-West and 200-East Areas. The immediate significance of these observations has not been fully assessed. Solutions containing ferrocyanide, which have been disposed of in the 200-West and 200-East Areas are likely to be the source.

\subsubsection{Hexavalent Chromium}

Chromium contamination levels remained essentially constant in the wells sampled in the 100-B, 100-H, 100-F, 200-East, 200-West, and 600 Areas. Chromium contamination observed for the first time during the last two 
quarters in wells throughout the northern portion of the 600 Area was confirmed during this reporting period. Figure 3.2 shows the Site-wide distribution of dissolved chromium in ground water. Diamonds shown on the plot represent measurements below the detection limit of $10 \mu \mathrm{g} / \mathrm{L}$ for the inductively coupled plasma/mass spectrometry method.

\subsubsection{Volatile Organic Compounds Across the Site}

\subsubsection{Carbon Tetrachloride in the 200-West Area}

Carbon tetrachloride contamination has been found beneath much of the 200-West Area. The contamination is believed to be from waste disposal

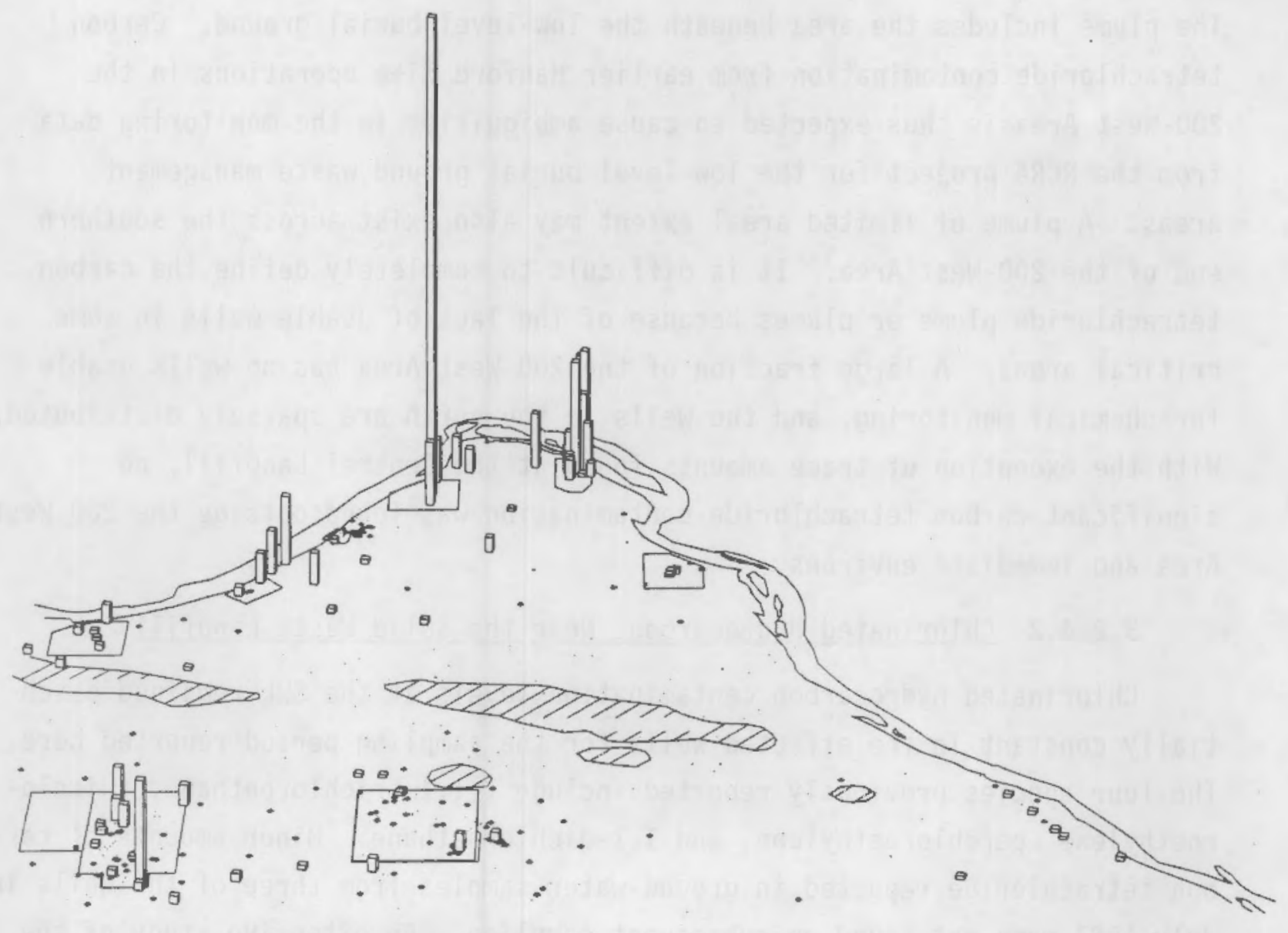

Diamonds Indicate Below Detection

FIGURE 3.2. Maximum Chromium Concentrations in Unconfined Aquifer Wells, January 1, 1987, to June 30, 1988 
operations associated with the Plutonium Finishing Plant (PFP) before 1973. Maximum concentrations detected in each well during the period from January 1987 to June 1988 are shown in Figure 3.3. The MCL for carbon tetrachloride is $5 \mathrm{ppb}$. The detection 7 imit of the gas chromatography/mass spectrometry method used for analysis is also $5 \mathrm{ppb}$. A maximum concentration of $5550 \mathrm{ppb}$ (well 299-W15-11, Figure B.8) was found near the PFP in March 1988 (location of the PFP is also shown in Figure B.8). The concentration of carbon tetrachloride in well 299-W15-11 has generally tended to increase, approximately doubling over a 1-year period since it was first observed. The plot shows high concentrations of carbon tetrachloride near T Plant and the PFP. The plume includes the area beneath the low-level burial ground. Carbon tetrachloride contamination from earlier Hanford Site operations in the 200-West Area is thus expected to cause ambiguities in the monitoring data from the RCRA project for the low-level burial ground waste management areas. A plume of limited areal extent may also exist across the southern end of the 200-West Area. It is difficult to completely define the carbon tetrachloride plume or plumes because of the lack of usable wells in some critical areas. A large fraction of the 200-West Area has no wells usable for chemical monitoring, and the wells to the north are sparsely distributed. With the exception of trace amounts found at the Central Landfi11, no significant carbon tetrachloride contamination was found outside the 200 -West Area and immediate environs.

\subsubsection{Chlorinated Hydrocarbons Near the Sol id Waste Landfill}

Chlorinated hydrocarbon contamination levels at the SWL remained essentially constant in the affected wells for the sampling period reported here. The four species previously reported include 1,1,1-trichloroethane, trichloroethylene, perchloroethylene, and 1,1-dichloroethane. Minor amounts of carbon tetrachloride reported in ground-water samples from three of the wells in July 1987 were not found on subsequent sampling. An extensive study of the chlorinated hydrocarbon contamination at the SWL was carried out by PNL using a highly sensitive gas chromatography method. The results of that study confirmed the earlier work by United States Testing Company, Incorporated (UST). Details of the PNL study are discussed in Section 4.3. The origin of the contamination is believed to be steam stripper waste containing 


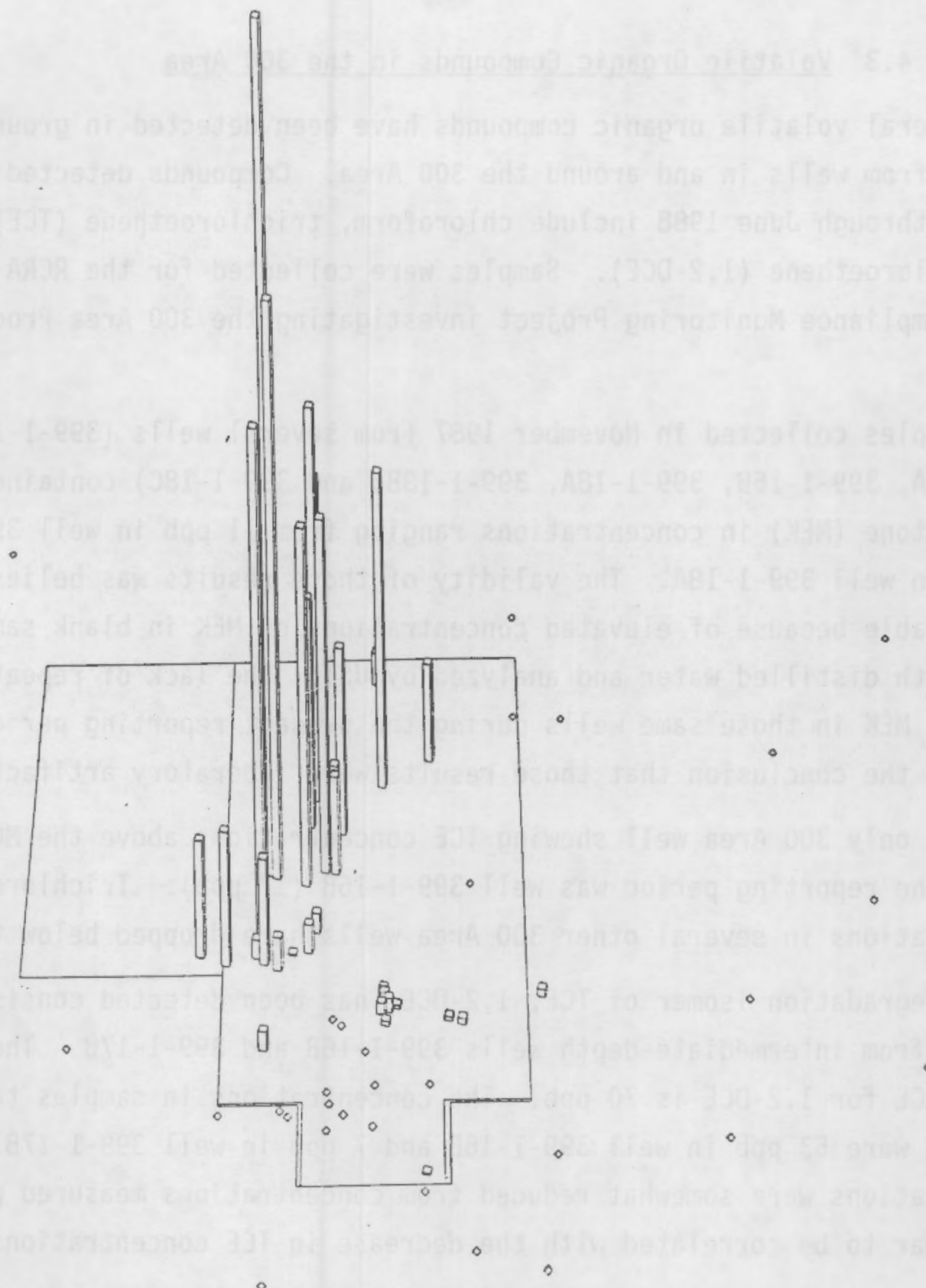

\section{Diamonds Indicate Below Detection}

FIGURE 3.3. Maximum Carbon Tetrachloride Concentrations in 200-West Area Unconfined Aquifer Wells Between January 1, 1987, and June 30, 1988

degreasing solvents originating from motor pool maintenance operations in the 1100 Area and disposed of in the SWL. 


\subsubsection{Volatile Organic Compounds in the 300 Area}

Several volatile organic compounds have been detected in ground-water samples from wells in and around the 300 Area. Compounds detected during January through June 1988 include chloroform, trichloroethene (TCE), and 1,2-dichloroethene (1,2-DCE). Samples were collected for the RCRA GroundWater Compliance Monitoring Project investigating the 300 Area Process Trenches.

Samples collected in November 1987 from several wells (399-1-13, 399-1-16A, 399-1-16B, 399-1-18A, 399-1-18B, and 399-1-18C) contained methy ethyl ketone (MEK) in concentrations ranging from $11 \mathrm{ppb}$ in well 399-1-16A to $45 \mathrm{ppb}$ in well 399-1-18A. The validity of those results was believed to be questionable because of elevated concentrations of MEK in blank samples prepared with distilled water and analyzed by UST. The lack of repeat occurrence of MEK in those same wells during the present reporting period further supports the conclusion that those results were laboratory artifacts.

The only 300 Area well showing TCE concentrations above the MCL (5 ppb) during the reporting period was well 399-1-16B (17 ppb). Trichloroethene concentrations in several other 300 Area wells have dropped below the MCL.

A degradation isomer of TCE, 1,2-DCE, has been detected consistently in samples from intermediate-depth wells 399-1-16B and 399-1-17B. The recommended MCL for 1,2-DCE is $70 \mathrm{ppb}$. The concentrations in samples taken in May 1988 were $53 \mathrm{ppb}$ in well 399-1-16B and $7 \mathrm{ppb}$ in wel1 399-1-17B. These concentrations were somewhat reduced from concentrations measured previously and appear to be correlated with the decrease in TCE concentrations.

The source of the organic compounds detected in 300 Area wells is unclear. With the exception of chloroform, which is normally only detected in wells adjacent to or downgradient from the process trenches, other organic compounds have been detected in wells upgradient from the process trenches (e.g., we11s 399-8-2, 699-S19-E13, and we11s in the 399-1-18 cluster) in addition to wells downgradient from the trenches. The widespread occurrence of the compounds suggests that sources other than, or in addition to, the process trenches may be involved. 


\subsubsection{Trichloroethene in 600 Area}

Two wells west of the 100-F Area (699-77-36 [35 ppb] and 199-F7-1 [14 ppb]) show the presence of TCE at concentrations greater than the MCL $(5 \mathrm{ppb})$. The source of the contamination is unclear but may be associated with the White Bluffs Pickling Acid Crib.

We11 699-71-52 shows persistent traces of TCE close to the MCL. The well is located in an isolated region of the 600 Area and no source of TCE contamination is evident.

\subsubsection{Nitrate Across the Site}

Nitrate is associated with process condensates, decontamination waste streams, and other process 7 iquid wastes discharged to the ground at Hanford (PNL 1988a). The extent of nitrate contamination in the unconfined aquifer reflects the extensive use of nitrogen-bearing compounds (e.g., nitric acid, sodium nitrate, sodium nitrite, and ammonia) in chemical reprocessing, production, and decontamination operations.

Nitrate is analyzed for in most wells in all monitoring networks and was measured at concentrations greater than the MCL ( $45 \mathrm{ppm}$ as nitrate ion) in wells in all operational areas except for the 100-B and 400 Areas. A plot of the maximum nitrate concentrations in ground-water samples taken from January 1987 through June 1988 is presented in Figure 3.4.

\subsubsection{Nitrate in the 100 Areas}

Nitric acid used in reactor decontamination activities represents the major source of nitrate ion in the unconfined aquifer beneath the 100 Areas. Nitrate concentrations in the 100-B Area dropped below the MCL during this reporting period. In well 199-B3-1, the nitrate concentration decreased from 55.6 to $28.5 \mathrm{ppm}$ from July 1987 to January 1988. In the 100-D Area, wells 199-D2-5 and 199-D5-12 remained above the MCL with nitrate concentrations of 80.3 and $73.8 \mathrm{ppm}$, respectively. Wells 199-F5-4, 199-F7-1, 199-F8-1, and 199-F8-2 al1 exceed the MCL in the 100-F Area with the maximum concentration found in well 199-F8-1 of $244 \mathrm{ppm}$. In the 100-H Area, seven of the sampled wells contained nitrate concentrations over the MCL (199-H3-1, 199-H4-3, 199-H4-4, 199-H4-9, 199-H4-12A, 199-H4-12B, and 199-H4-17). Well 199-H4-3 


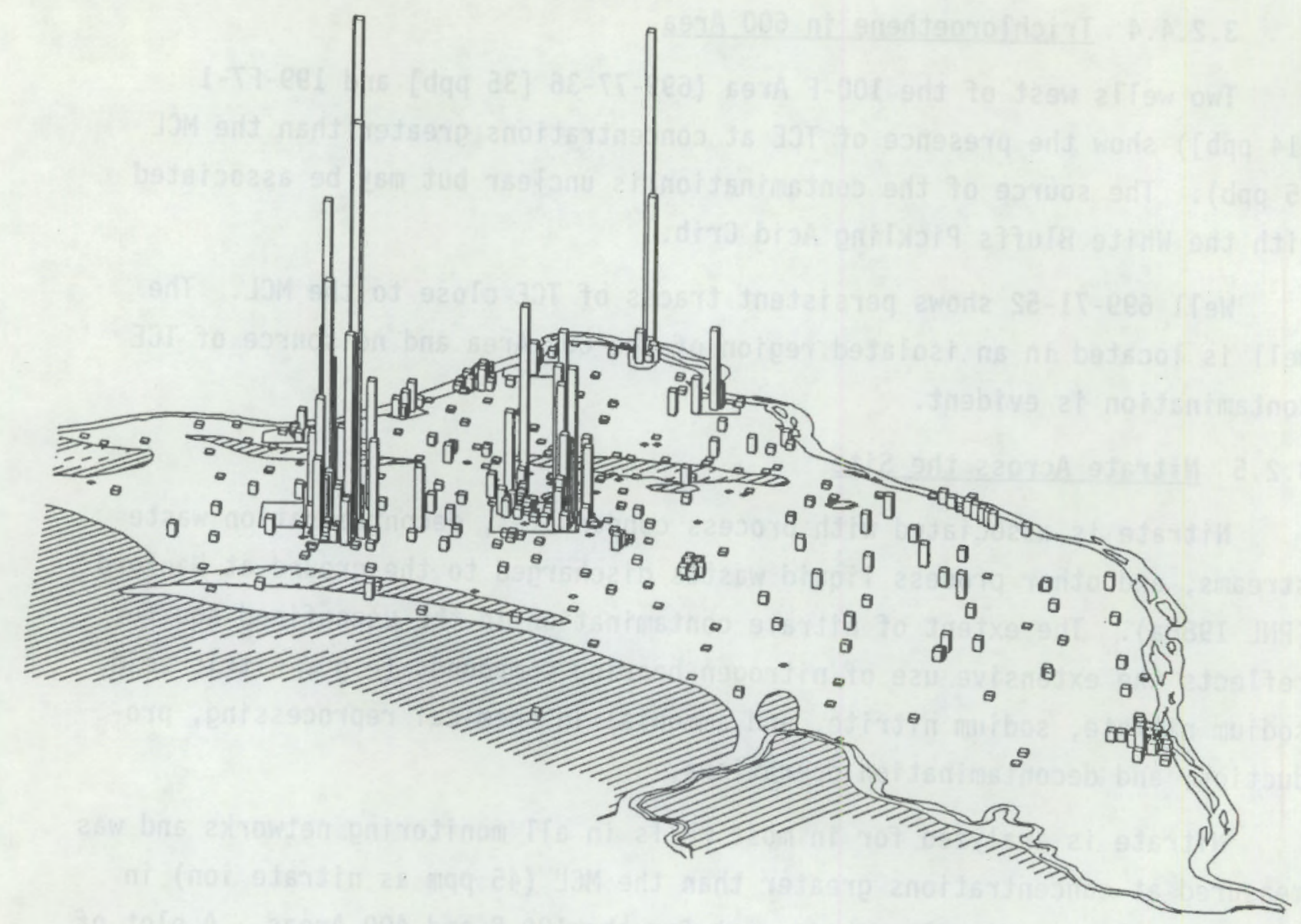

D1amonal Indsate below contractual detection linst of 2500 ppb

FIGURE 3.4. Maximum Nitrate Concentrations in Unconfined Aquifer Wells, January 1, 1987, to June 30, 1988

near the 183-H Solar Evaporation Basins exhibited the maximum nitrate concentration for a 11100 Area wells with a concentration of $663 \mathrm{ppm}$. In the 100-K Area, wel1s 199-K-11, 199-K-19, and 199-K-30 had nitrate concentrations greater than the MCL; concentrations ranged from $41.5 \mathrm{ppm}$ (slightly below the MCL) in well 199-K-19 in June 1988 to a high of $86.8 \mathrm{ppm}$ in that same well during March. During the January to June 1988 reporting period, the only wells detected with nitrate concentrations above the MCL in the 100-N Area were we11s 199-N-32, 199-N-39, 199-N-42, and 199-N-49. 


\subsubsection{Nitrate in the 200-East Area}

Twenty-two wells in the 200-East Area were found to contain nitrate concentrations greater than the MCL. Nineteen of these wells are located in the southeastern portion of 200-East Area near the PUREX Plant. The other three wells with nitrate concentrations above the MCL are located north and west of B Plant. Wells 299-E17-1 and 299-E24-1 contained the highest concentrations of nitrate measured in the 200-East Area, 308 and $334 \mathrm{ppm}$, respectively. Each of these wells is located adjacent to a process condensate liquid disposal facility, which is the likely source of nitrate in these wells.

\subsubsection{Nitrate in the 200-West Area}

Forty-three wells throughout the 200-West Area contained nitrate concentrations in excess of the MCL. The highest nitrate level detected in this area and Site-wide was 2810 ppm in we11 299-W18-17. This well is located near Z Plant between the 216-Z-1 and the 216-Z-19 1iquid disposal facilities. Few other wells in this immediate area contain nitrate concentraiions in excess of the MCL. Past trend plot records for this well indicate that all previous nitrate measurements were below the detection limit. This value is considered suspect. (a) Wells with high nitrate concentrations are located primarily west of T Plant, near U Plant, and around the REDOX Plant. Maximum nitrate concentrations in these locations are 356 ppm (299-W10-9), 1400 ppm (299-W19-19), and 147 ppm (299-W23-10), respectively.

\subsubsection{Nitrate in 0ther Areas}

Elevated nitrate concentrations exist in areas downgradient from reprocessing facilities in the 200 Areas. Nitrate plumes extend from the southeast corner of the 200-East Area to the Columbia River, north from the 200-East Area toward the gap between Gable Mountain and Gable Butte, and east of the 200 -West Area. The largest of these plumes is located between the 200-East Area and the Columbia River. Wells in this portion of the Site exceeded the MCL for nitrate on an intermittent basis except for well

(a) Resampling of this well subsequent to the reporting period detected no nitrate. 
699-38-15, which remained only slightly above the MCL at $55.5 \mathrm{ppm}$ nitrate. Nitrate concentrations north of the 200-East Area are significantly above the MCL with a maximum concentration of $559 \mathrm{ppm}$ in well 699-50-53. The only other wells in this region with nitrate concentrations in excess of the MCL are 699-49-55A and 699-49-57. Four wells east of the 200-West Area contain nitrate concentrations in excess of the MCL, wells 699-38-65, 699-38-70, 699-40-62, and 699-44-64. Nitrate concentrations in these wells range from $45.8 \mathrm{ppm}$ in well 699-44-64 to $239 \mathrm{ppm}$ in well 699-38-70. Finally, one isolated well (699-77-36) near the 100-F Area also contains a nitrate concentration of $67.1 \mathrm{ppm}$, which exceeds the MCL.

\subsubsection{Iritium Across the Site}

Tritium concentrations greater than the $\mathrm{MCL}$ of $20,000 \mathrm{pCi} / \mathrm{L}$ were detected in all areas except 100-F, 100- $\mathrm{H}$, and 300 Areas. Concentrations of tritium in excess of the DCG were found only in the 200-East and 200-West Areas.

\subsubsection{Tritium in the 100 Areas}

Twenty-five wells in the 100 Areas contained tritium concentrations in excess of the MCL. Wells 199-B4-1, 199-D2-5, and 199-K-30 were the only 100 Area monitoring wells outside of the 100-N Area with tritium concentrations greater than the MCL. Tritium concentrations in well 199-B4-1 had dropped from 49,700 pCi/L in January 1988 to $21,000 \mathrm{pCi} / \mathrm{L}$ in early April, while tritium concentrations in well 199-D2-5 remained nearly constant at $33,000 \mathrm{pCi} / \mathrm{L}$. Wel1 199-K-30 continued to exhibit the highest tritium concentration of any 100 Area well, with concentrations in excess of $1,200,000 \mathrm{pCi} / \mathrm{L}$. Figure 3.5 illustrates the trend in tritium concentrations for well 199-K-30 since early 1984.

The remaining 22 wells with tritium concentrations greater than the MCL are located in the 100-N Area adjacent to the 1301-N and 1325-N Liquid Waste Disposal Facilities. Wells in 100-N Area downgradient from these facilities continue to show the influence of reactor cooling water and fuel storage basin effluent disposal even after reactor operations have ceased. The maximum tritium concentration measured in $100-\mathrm{N}$ Area we17s was $152,000 \mathrm{pCi} / \mathrm{L}$ in well 199-N-49. Tritium concentrations in the 100-N Area are expected to 


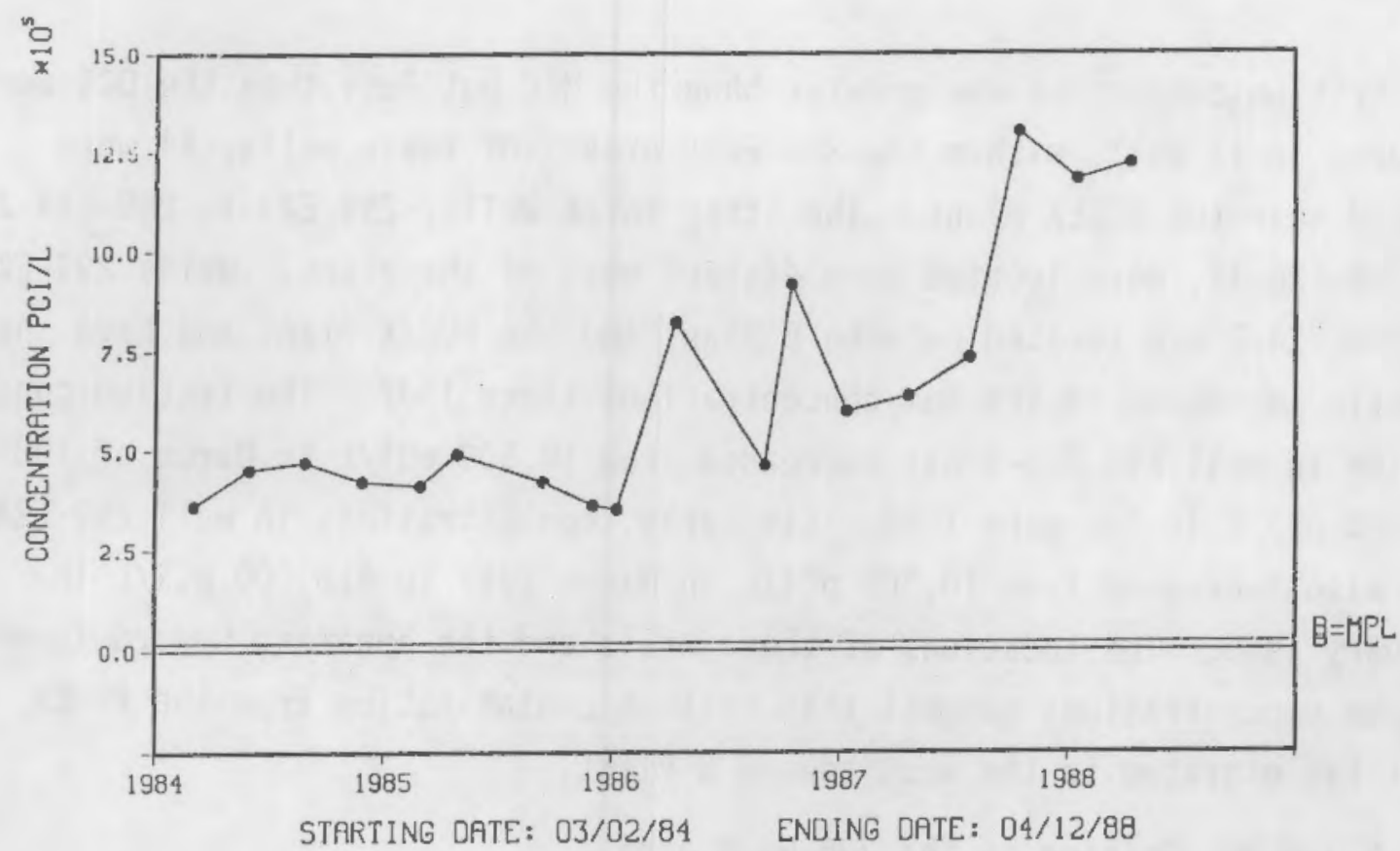

FIGURE 3.5. Tritium Concentrations from 1984 to June 1988 in Well 199-K-30

decrease because the $\mathbb{N}$ Reactor has been placed in cold standby. These future decreases are indicated by comparing data from wells close to the liquid waste disposal facilities with that from more distant downgradient wells. The average tritium concentrations for four wells near the $1325-\mathrm{N}$ Liquid Waste Disposal Facility (199-N-27, 199-N-32, 199-N-36, 199-N-45) were approximately $69,000 \mathrm{pCi} / \mathrm{L}$. Average tritium concentrations in more distant wells (199-N-49, 199-N-50) remained at nearly $134,000 \mathrm{pCi} / \mathrm{L}$ from January to June 1988. These data suggest that the concentrations of tritium and/or discharge volumes of tritiated liquids have decreased.

\subsubsection{Tritium in the 200-East Area}

The highest concentrations of tritium onsite continue to be found in the 200-East Area near the PUREX Plant. Twenty-one wells in this portion of the 200-East Area contain tritium concentrations in excess of the MCL. Nine of these wells had tritium concentrations in excess of the DCG during the Reporting Period. We 71 s 299-E17-1 and 299-E24-11 contained tritium concentrations slightly greater than $8,000,000 \mathrm{pCi} / \mathrm{L}$. The remaining seven wells with tritium concentrations greater than the DCG were 299-E17-5, 299-E17-8, 299-E17-9, 299-E17-13, 299-E24-1, 299-E24-2, and 299-E24-12. 
Tritium concentrations greater than the MCL but less than the DCG were measured in 17 wells within the 200-East Area. Of these wells, 14 were located near the PUREX Plant. The other three we11s, 299-E23-1, 299-E24-7, and 299-E28-12, were located some distant west of the Plant. Wells 299-E23-1 and 299-E24-7 are located between B Plant and the PUREX Plant and have shown dramatic increases in tritium concentrations since 1987. The tritium concentration in we11 299-E23-1 has increased from $10,500 \mathrm{pCi} / \mathrm{L}$ in March of 1987 to $135,000 \mathrm{pCi} / \mathrm{L}$ in February 1988. Similarly, concentrations in well 299-E24-7 have also increased from $10,300 \mathrm{pCi} / \mathrm{L}$ in March 1987 to $418,000 \mathrm{pCi} / \mathrm{L}$ in February 1988. The locations of these wells and the apparent upward trend in tritium concentrations suggest that tritium contamination from the PUREX Plant has migrated to the west toward B Plant.

\subsubsection{Tritium in the 200-West Area}

The concentrations of tritium in 200-West Area wells have remained fairly consistent with previous results. Nineteen wells continue to show tritium concentrations in excess of the MCL. Well 299-W22-9 continued to show the highest concentrations of tritium in the 200-West Area at 7,560,000 pCi/L. Well 299-W23-4 also contained tritium concentrations in excess of the DCG during the February 1988 sampling. Subsequently, tritium concentrations in we11 299-W23-4 dropped from 5,450,000 pCi/L in February to $1,550,000 \mathrm{pCi} / \mathrm{L}$ in June 1988. Previous tritium results for well 299-W23-4 were all below the MCL of $20,000 \mathrm{pCi} / \mathrm{L}$. Of the 17 wells with tritium less than the DCG but greater than the MCL, ten wells (299-W19-2, 299-W21-1, 299-W22-7, 299-W22-10, 299-W22-12, 299-W22-20, 299-W22-26, 299-W23-2, 299-W23-9, and 299-W23-10) were located in the southern portion of the 200-West Area and adjacent to the REDOX Plant.

Several wells located in the northern part of the 200-West Area also continued to show tritium concentrations in excess of the $\mathrm{MCL}$. Wells 299-W6-1, 299-W10-1, 299-W10-3, 299-W10-4, 299-W10-9, 299-W14-2, and 299-W15-4 all contain tritium concentrations greater than the MCL. Tritium concentrations in these northern wel1s range from $54,800 \mathrm{pCi} / \mathrm{L}$ in we1] 299-W10-1 to $155,000 \mathrm{pCi} / \mathrm{L}$ in we11 299-W15-4. Liquid waste sites interspersed between these wells were associated with the early operations of 
T Plant. Tritium concentrations in the northern portion of the 200-West Area are expected to decrease as radioactive decay continues to deplete the tritium sources from these early operations.

\subsubsection{Tritium in Other Areas}

Tritium concentrations in excess of the MCL exist in several locations adjacent to operational areas. We11 699-87-55 lies in an area downgradient from the 100-N Area and contained an average tritium concentration of $52,000 \mathrm{pCi} / \mathrm{L}$ for the reporting period. No other wells north of Gable Mountain and Gable Butte and outside of the 100 Areas contain tritium in excess of the MCL.

Tritium plumes also exist in portions of the Site between the 200-West and 200-East Areas and in the portion of the Hanford Site between the 200-East Area and the Columbia River. The tritium plume between the 200-West and 200-East Areas has remained relatively constant over the reporting period. Wells 699-35-66 and 699-35-70 continue to show the highest tritium concentrations in the plume with tritium concentrations of $1,200,000 \mathrm{pCi} / \mathrm{L}$. We11s 699-32-70B, 699-32-72, 699-38-65, and 699-40-62 a7so were found to contain tritium concentrations in excess of the MCL. Tritium concentrations in these weils range from $77,000 \mathrm{pCi} / \mathrm{L}$ in we11 699-40-62 to nearly $400,000 \mathrm{pCi} / \mathrm{L}$ in $699-38-65$.

Tritium concentrations greater than the MCL extend from the 200-East Area 211 the way to the Columbia River. Maximum tritium concentrations near the river were found in wells 699-39-0, 699-40-1, 699-41-1, 699-42-2, and 699-43-3. Tritium concentrations in these wells ranged from 200,000 to $250,000 \mathrm{pCi} / \mathrm{L}$ during the reporting period. The maximum tritium concentrations detected in this piume were found in well 699-38-15 at 489,000 pCi/L.

The width of the tritium plume between the 200-East Area and the river continues to expand to the south. Expansion of the plume to the south is illustrated in Figure 3.6 (699-S19-E13) and Figure 3.7 (699-S3-E12). These two wells exhibit similar trends that suggest that tritium concentrations will continue to increase in that portion of the Site immediately north of the 300 Area. Concentrations of tritium is excess of the MCL were found in 


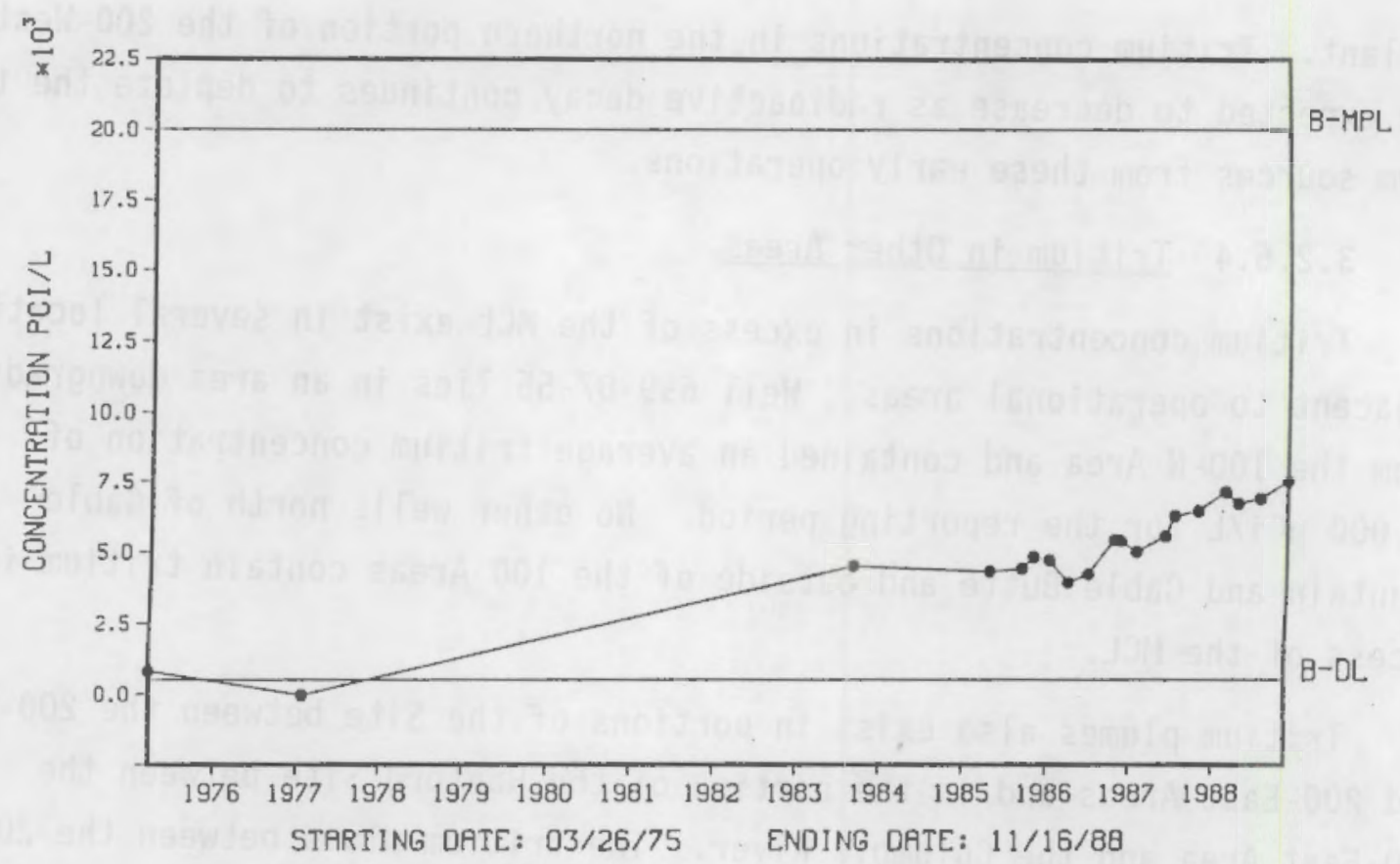

FIGURE 3.6. Tritium Concentrations Through Time in Well 699-S19-E13

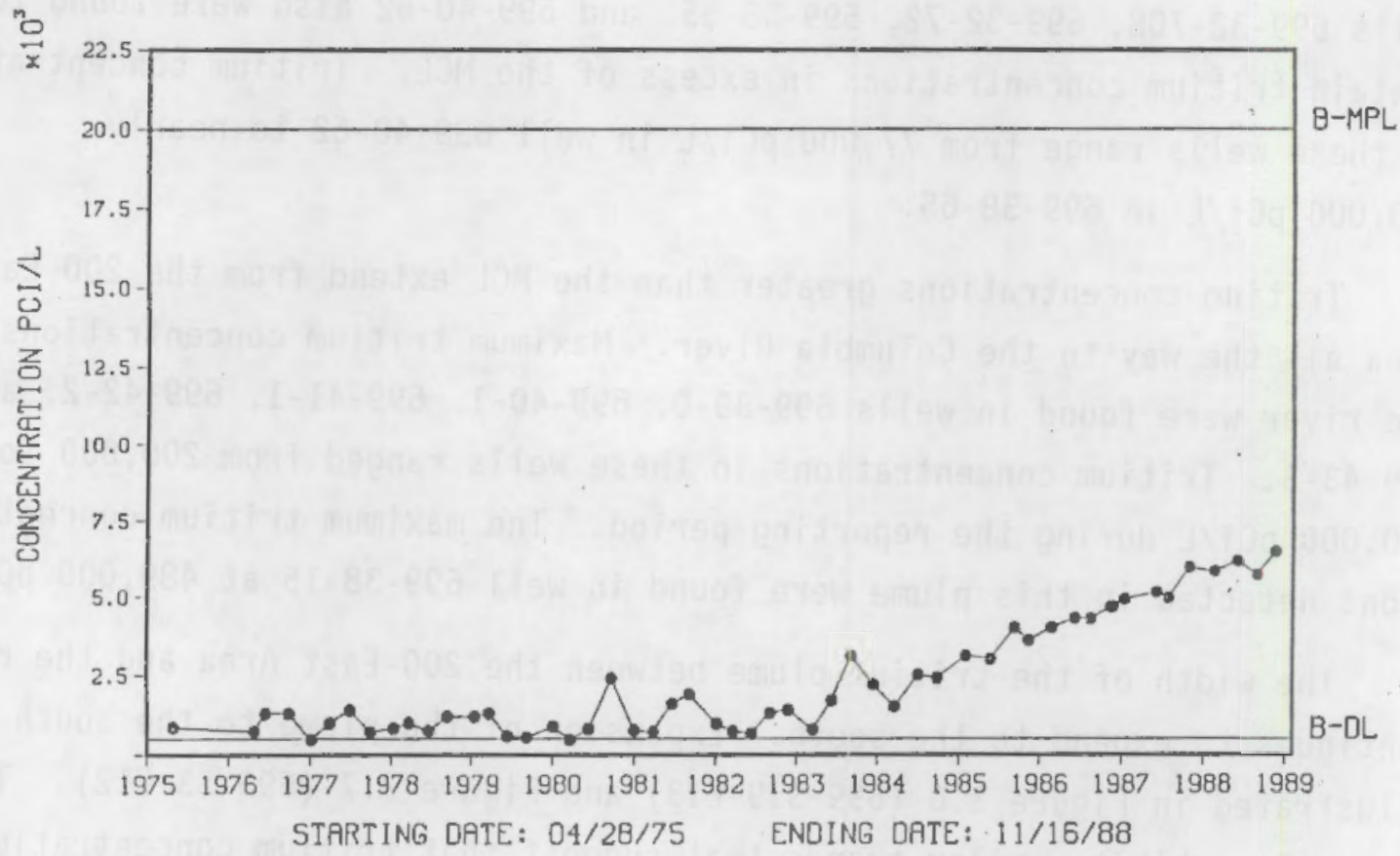

FIGURE 3.7. Tritium Concentrations Through Time in Well 699-S3-E12 
wel1 699-S6-E4B, which is approximately 2 miles inland from well 699-S3-E12. If the present trend continues, wells 699-S3-E12 and 699-S19-E13 will likely exceed the MCL within 5 years.

\subsubsection{Gross Alpha Activity and Uranium}

Alpha activities in Hanford ground water can be attributed to the presence of isotopes of uranium and plutonium discharged to liquid waste disposal facilities. Other alpha-emitting radionuclides, such as lead, radium, polonium, americium, and neptunium, have not been detected in Hanford ground water. Based on past ground-water analyses, the costly nature of alphaemitting radionuclide-specific analytical techniques, and the limited mobi1ity of these radionuclides in the subsurface environment, further analyses of these other alpha emitters has been discontinued. Instead, gross a7pha activity is used as an indicator, and when alpha concentrations exceed an established level further investigations are undertaken to identify which alpha-emitting radionuclide may be responsible. In nearly every instance, the alpha emitter of interest is uranium. One exception to this finding is the presence of plutonium-239,240 in we11 299-E28-23. In the January and May 1988 sampling, well 299-E28-23 had plutonium-239,240 concentrations of 9.3 and $11.1 \mathrm{pCi} / \mathrm{L}$. These results are consistent with past results and represent about one-third of the MCL for plutonium-239,240 (30 pCi/L). Concentrations of plutonium-239,240 in this well are below the contractual detection limit of $17 \mathrm{pCi} / \mathrm{L}$ but still are quantifiable by the present analytical method.

In a11 other we11s, uranium is responsible for the gross alpha activity. Only wells in the 200-West Area contained uranium in excess of the MCL of 600 pCi/L. Wells 299-W19-3, 299-W19-11, 299-W19-16, and 299-W19-18 are the only wells with uranium concentrations in excess of the MCL. Figure 3.8 illustrates the distribution of uranium across the Site.

\subsubsection{Gross Beta Activity}

Gross beta concentrations greater than the MCL of $50 \mathrm{pCi} / \mathrm{L}$ were detected in wells from all operational areas except the $100-\mathrm{K}$ and 400 Areas (Figure 3.9). Although gross beta concentrations greater than the MCL were widespread, the highest concentrations were found in wells near several waste disposal facilities in the 100-N, 200-East, and 200-West Areas and in the 


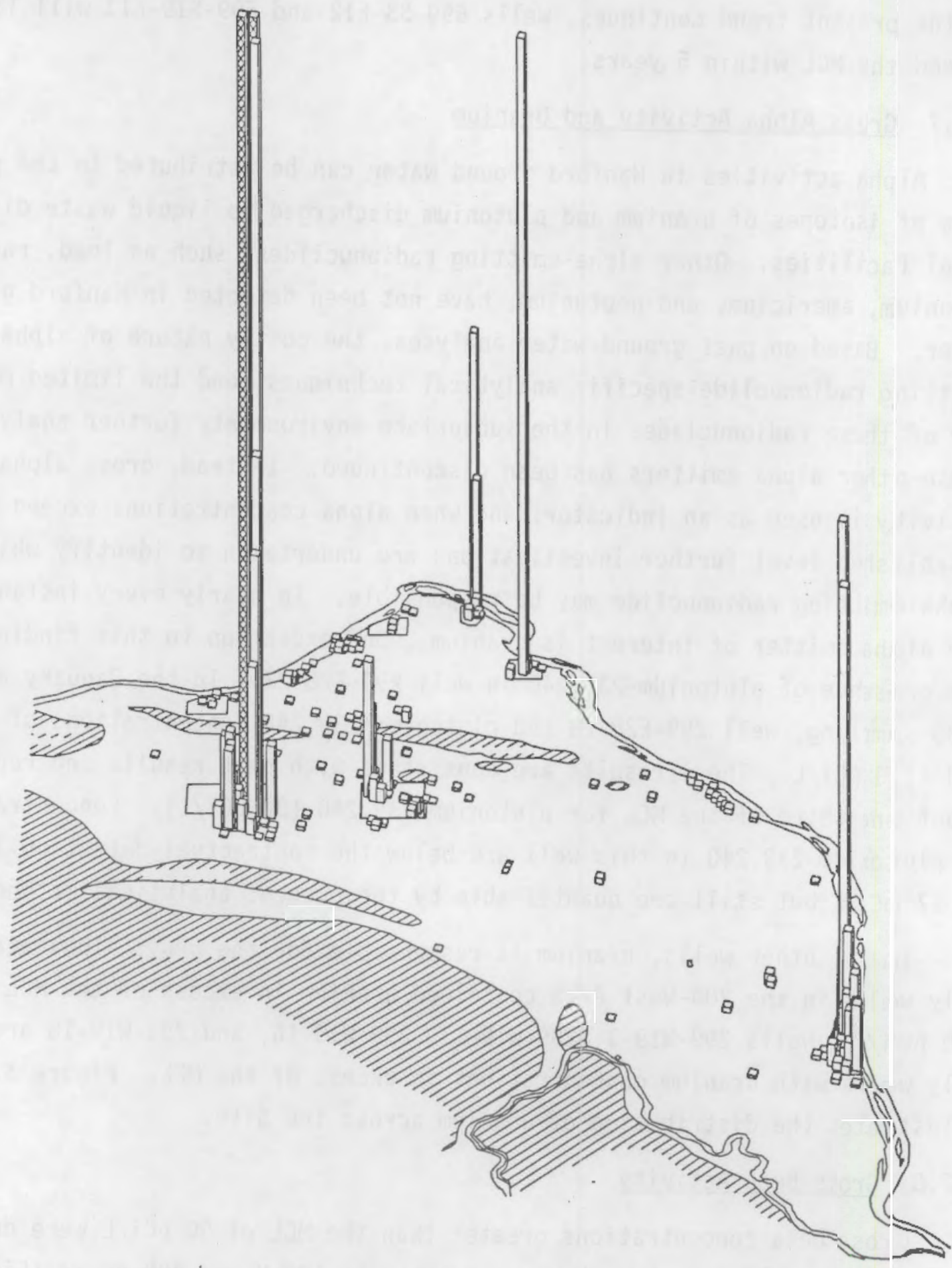

Diamonds indicate values less than analytical error

FIGURE 3.8. Maximum Uranium Concentrations in Unconfined Aquifer Wells, January 1, 1987, to June 30, 1988 


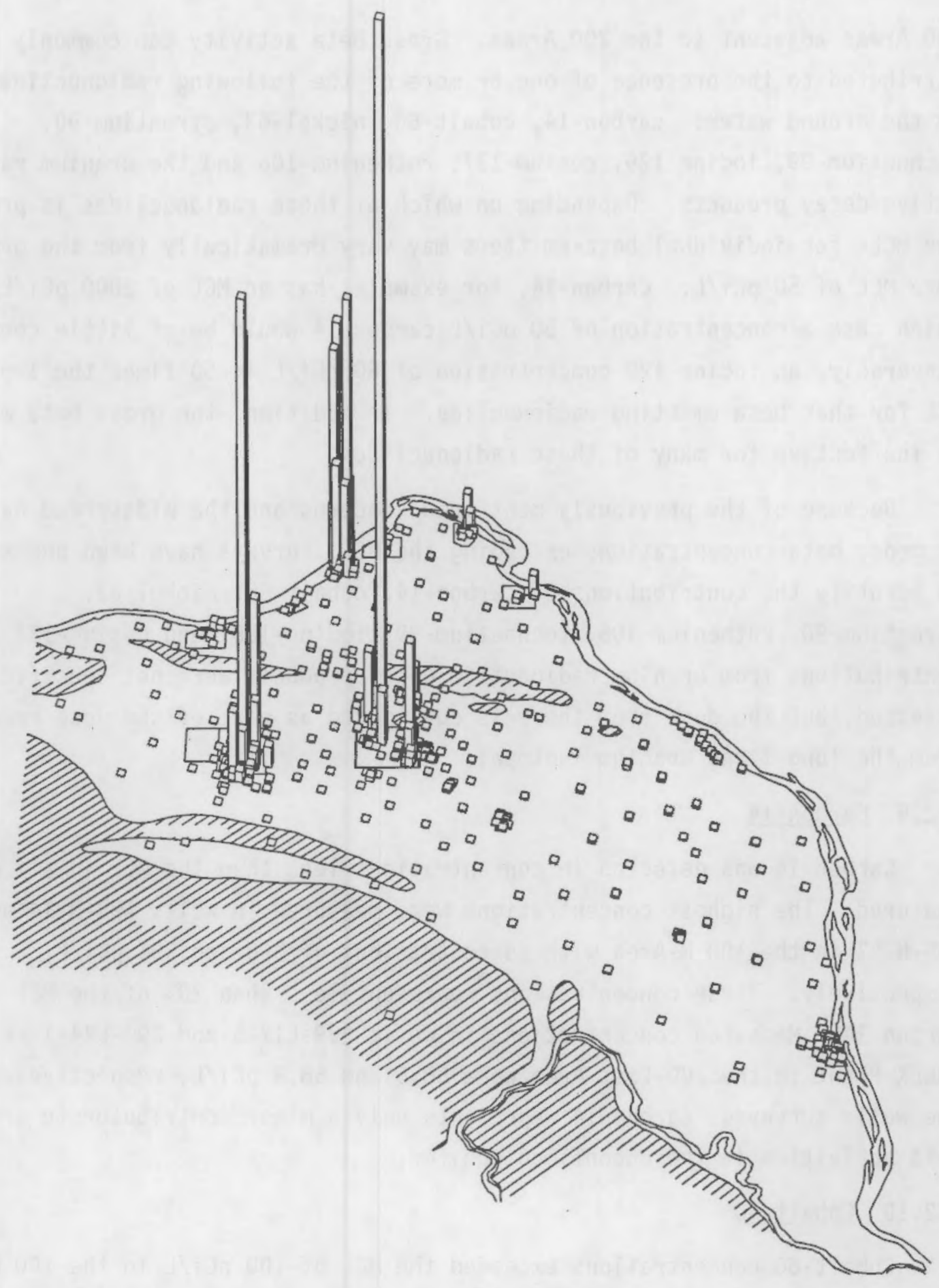

FIGURE 3.9. Maximum Gross Beta Concentrations in Unconfined Aquifer Wells, January 1, 1987, to June 30, 1988 
600 Areas adjacent to the 200 Areas. Gross beta activity can commonly be attributed to the presence of one or more of the following radionuclides in the ground water: carbon-14, cobalt-60, nicke1-63, strontium-90, technetium-99, iodine-129, cesium-137, ruthenium-106 and the uranium radioactive decay products. Depending on which of these radionuclides is present, the MCLs for individual beta-emitters may vary dramatically from the gross beta MCL of $50 \mathrm{pCi} / \mathrm{L}$. Carbon-14, for example, has an MCL of $2000 \mathrm{pCi} / \mathrm{L}$, in which case a concentration of $50 \mathrm{pCi} / \mathrm{L}$ carbon-14 would be of $1 \mathrm{ittle}$ concern. Conversely, an iodine-129 concentration of $50 \mathrm{pCi} / \mathrm{L}$ is 50 times the $1-\mathrm{pCi} / \mathrm{L}$ MCL for that beta-emitting radionuclide. In addition, the gross beta method is ineffective for many of these radionuclides.

Because of the previously mentioned concerns and the widespread nature of gross beta concentrations exceeding the MCL, surveys have been undertaken to identify the contributions of carbon-14, cobalt-60, nickel-63, strontium-90, ruthenium-106, technetium-99, iodine-129, and cesium-137. Contributions from uranium radioactive decay products were not specifically assessed, but the dose from these is considered as part of the dose received from the long-lived uranium isotope.

\subsubsection{Carbon-14}

Carbon-14 was detected in concentrations less than the MCL in all wells measured. The highest concentrations were measured in wells $199-\mathrm{N}-14$ and 199-N-33 in the $100 \mathrm{~N}$-Area with concentrations of 390 and $394 \mathrm{pCi} / \mathrm{L}$, respectively. These concentrations represent less than $20 \%$ of the $\mathrm{MCL}$ for carbon-14. Measured concentrations in we11s 299-E17-5 and 299-E24-1 near PUREX Plant in the 200-East Area were 58.5 and $58.8 \mathrm{pCi} / \mathrm{L}$, respectively. $0 f$ the wells surveyed, carbon-14 represents only a minor contributor to gross beta activities in the unconfined aquifer.

\subsubsection{Cobalt-60}

Cobalt-60 concentrations exceeded the MCL of $100 \mathrm{pCi} / \mathrm{L}$ in the $100-\mathrm{N}$ Area and in an isolated section of the Site north of the 200-East Area. Wells 199-N-32 and 199-N-39 in the 100-N Area had cobalt-60 concentrations of 168 and $196 \mathrm{pCi} / \mathrm{L}$, respectively. In the region north of the 200-East Area, well 699-50-53 contained $469 \mathrm{pCi} / \mathrm{L}$ of cobalt-60, which was the highest cobalt-60 
concentration measured during the survey. Outside of these two locations, only well 299-E17-5 in the southeast corner of the 200-East Area exhibited cobalt-60 concentrations greater than the detection limit. Cobalt-60, 1ike carbon-14, appears to only account for a minor contribution to gross beta activity in the Hanford ground waters.

\subsubsection{Nickel-63}

Nickel-63 was found in only one well at concentrations exceeding the detection limit of $10 \mathrm{pCi} / \mathrm{L}$. Well 199-N-33 in the 100-N Area contained a nickel-63 concentration of $10.71 \mathrm{pCi} / \mathrm{L}$, which is less than the MCL of $50 \mathrm{pCi} / \mathrm{L}$.

\subsubsection{Strontium-90}

Strontium-90 concentrations in excess of the $8 \mathrm{pCi} / \mathrm{L} \mathrm{MCL}$ were detected in all areas except the 300 and 400 Areas. Peak concentrations of strontium-90 in the 100-N and 200-East Areas also exceeded the DCG of $1000 \mathrm{pCi} / \mathrm{L}$. In the 100-N Area, four wells remained in excess of the DCG (199-N-2, 199-N-3, 199-N-14, and 199-N-45), while well 199-N-39, which was previously over the DCG, dropped from $10,400 \mathrm{pCi} / \mathrm{L}$ in July of 1987 to less than $900 \mathrm{pC} i / \mathrm{L}$ during this reporting period. Strontium-90 concentrations were also found to exceed the DCG in wells 299-E28-23 (6150 pCi/L) and 299-E28-25 (6270 pCi/L) in the northern portion of the 200-East Area. These wells were placed in proximity to the 216-B-5 reverse we11, which discharged liquid waste directly into the unconfined aquifer. Other nearby wells, 299-E28-7 and 299-E28-24, exhibit much lower concentrations of strontium-90 at 60.9 and $172 \mathrm{pCi} / \mathrm{L}$, respectively. These data suggest that the extent of strontium-90 migration from the 216-B-5 reverse well is 7 imited.

The Site-wide distribution of maximum strontium-90 concentrations for wells sampled during the gross beta survey period of January 1987 to June 1988 is presented in Figure 3.10. This figure suggests that in some instances strontium-90 represents a major contributor to Site-wide gross beta levels. In the 100 Areas and particularly in 100-N Area, strontium-90 and gross beta exhibit similar trends with gross beta values consistently in excess of the strontium- 90 concentrations. The difference in the values is 


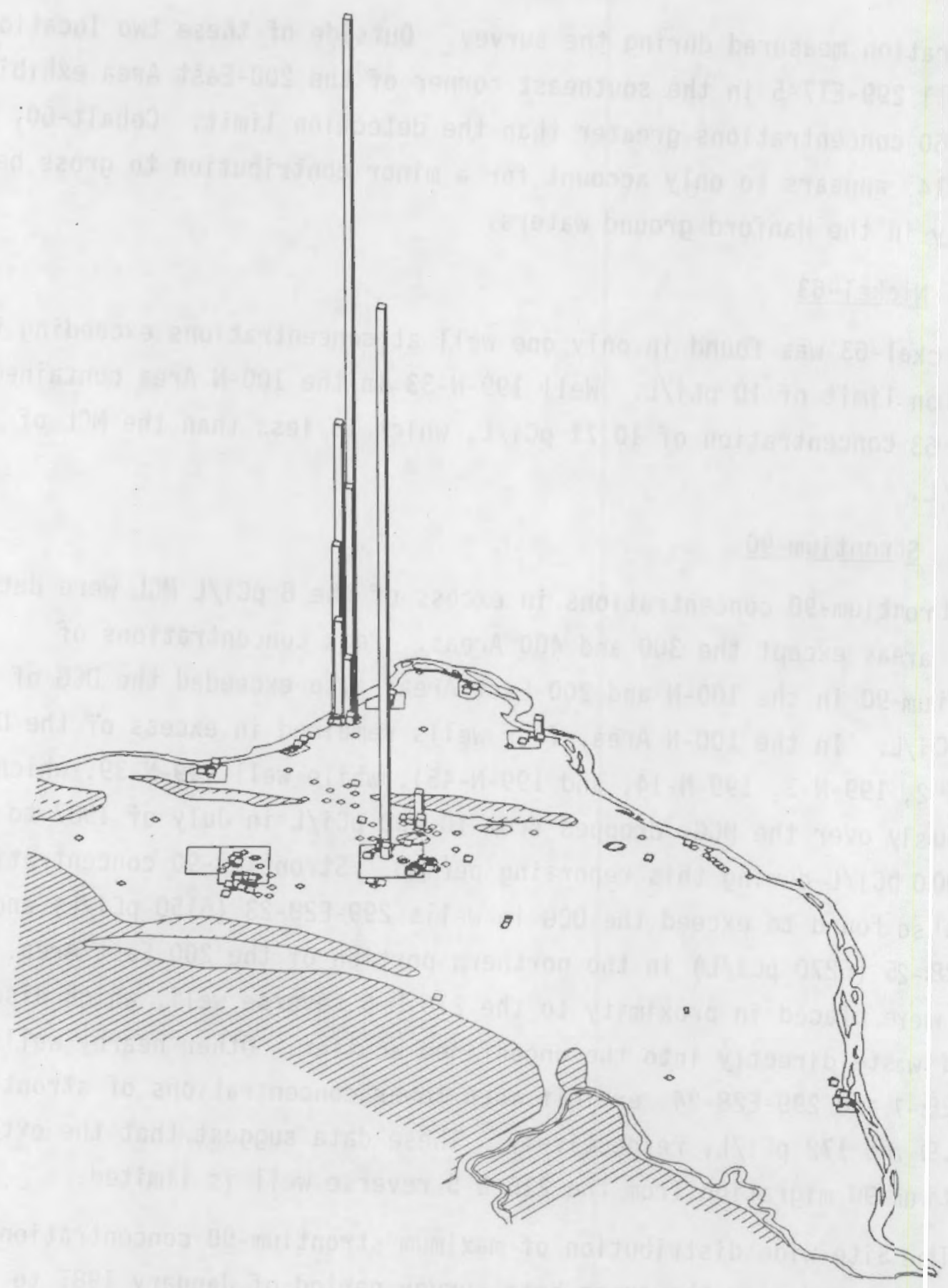

\section{Diamonds Indicate Below Detection}

FIGURE 3.10. Maximum Strontium-90 Concentrations in Unconfined Aquifer Wells, January 1, 1987, to June 30, 1988 
likely a result of the presence of other beta-emitters as well as differences in the efficiencies of the two methods for detecting strontium-90.

\subsubsection{Technetium-99}

Detectable concentrations of technetium- 99 were found in wells across the Site (Figure 3.11). Concentrations greater than the MCL $(900 \mathrm{pCi} / \mathrm{L})$ were measured in the ground water in the $100-\mathrm{H}$ Area and in the portions of the 200-East Area, 200-West Area, and in the 600 Area adjacent to the 200 Areas. No wells contained concentrations greater than the DCG $(100,00 \mathrm{pCi} / \mathrm{L})$. Concentrations of technetium-99 less than the MCL were measured in wells in the $100-\mathrm{B}, 100-\mathrm{K}, 100-\mathrm{N}, 300$, and 600 Areas.

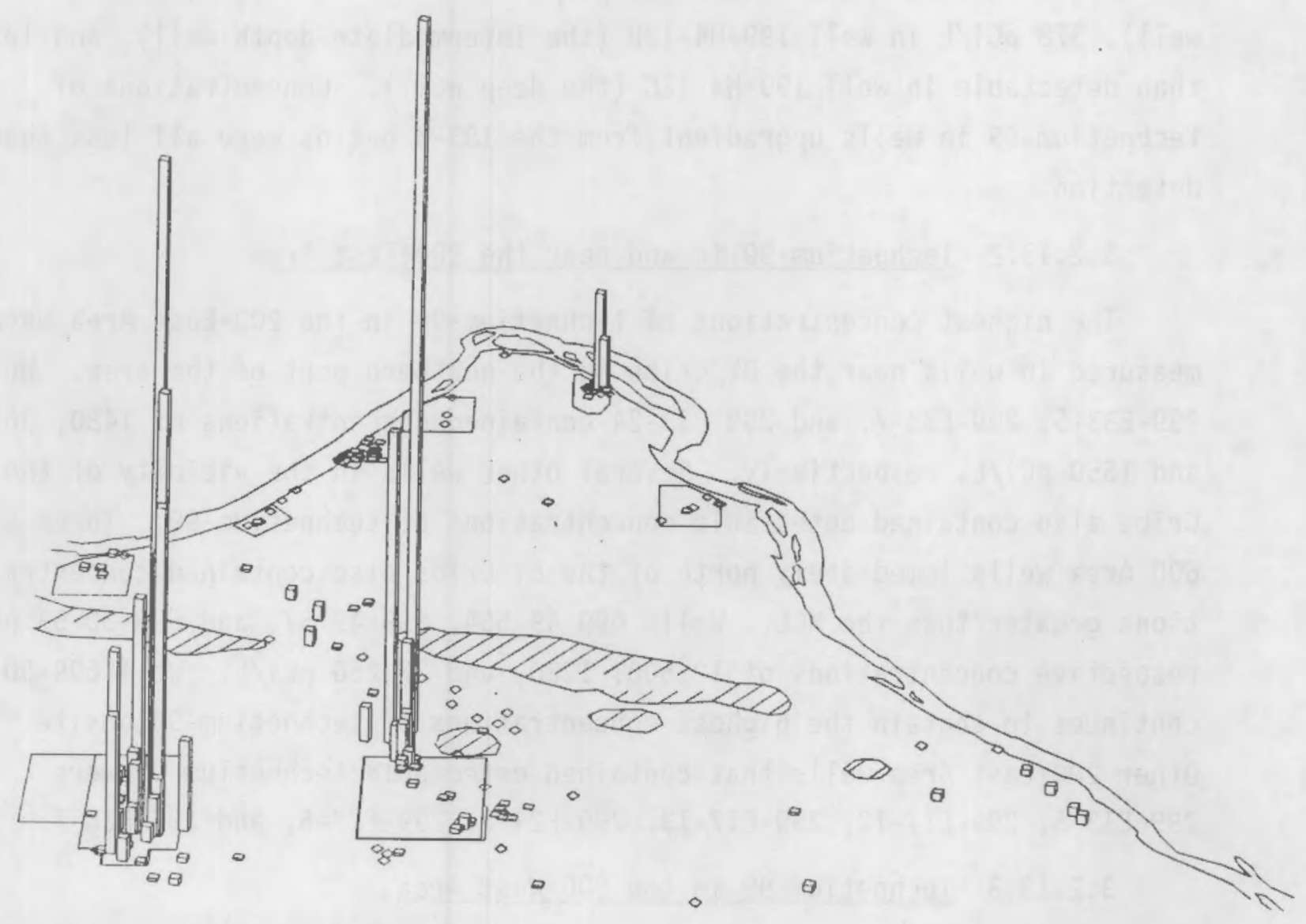

Diamonds Indicate Below Detection

FIGURE 3.11. Maximum Technetium-99 Concentrations in Unconfined Aquifer Wells, January 1, 1987, to June 30, 1988 


\subsubsection{Technetium-99 in the 100-H Area}

A well-defined plume of technetium-99 in the 100-H Area extends from the 183-H Solar Evaporation Basins to the Columbia River. Average concentrations in wells within the plume ranged from $2260 \mathrm{pCi} / \mathrm{L}$ in well 199-H4-3 adjacent to the 183-H basins to $20 \mathrm{pCi} / \mathrm{L}$ in well 199-H4-11 southeast of the basins near the Columbia River. Based on the concentrations detected in the 199-H4-12 well cluster, detectable quantities of technetium-99 extend into the intermediate portion of the unconfined aquifer but are not present in deeper portions of the aquifer. The average concentrations measured in samples from wells in this cluster were $491 \mathrm{pCi} / \mathrm{L}$ in well 199-H4-12A (the shallow we11), $378 \mathrm{pCi} / \mathrm{L}$ in we11 199-H4-12B (the intermediate-depth wel1), and less than detectable in well 199-H4-12C (the deep well). Concentrations of technetium-99 in wells upgradient from the 183-H basins were all less than detection.

\subsubsection{Technetium-99 in and near the 200-East Area}

The highest concentrations of technetium-99 in the 200-East Area were measured in wells near the BY Cribs in the northern part of the area. Wells 299-E33-5, 299-E33-7, and 299-E33-24 contained concentrations of 1480, 3630, and $1550 \mathrm{pCi} / \mathrm{L}$, respectively. Several other wells in the vicinity of the BY Cribs also contained detectable concentrations of technetium-99. Three 600 Area wells immediately north of the BY Cribs also contained concentrations greater than the MCL. Wel1s 699-49-55A, 699-49-57, and 699-50-53 had respective concentrations of $12,500,1220$, and $27,250 \mathrm{pCi} / \mathrm{L}$. Well 699-50-53 continues to contain the highest concentrations of technetium-99 onsite. Other 200-East Area wells that contained detectable technetium-99 were 299-E13-5, 299-E17-12, 299-E17-13, 299-E24-8, 299-E27-5, and 299-E28-7.

\subsubsection{Technetium-99 in the 200-West Area}

Wells located in an area around U Plant adjacent to numerous waste disposal facilities that have received waste from U Plant operations contained the highest concentrations of technetium-99 in the 200-West Area. The highest concentrations are in wells that surround the inactive 216-U-1 and 216-U-2 Cribs, and the 216-U-17 Crib, which has never been used. Well 699-38-70, which is in the 600 Area immediately east of U Plant, contained an 
average concentration of $3238 \mathrm{pCi} / \mathrm{L}$. Wells located in the southwest corner of the 200-West Area also contained concentrations greater than the MCL. Detectable concentrations of technetium-99 were also measured in well 299-W18-3 near Z Plant and in several wells located in the northern part of the 200-West Area near T Plant.

\subsubsection{Technetium-99 in the 600 Area}

Several wells located in the 600 Area between the 200-East Area and the Columbia River to the east also contained technetium-99. The highest concentrations detected were in several wells adjacent to the river (wells 699-40-1, 699-41-1, 699-42-12A, 699-43-3, and 699-46-4) and in two wells (699-27-8 and 699-32-22) located near the center of the plume emanating from the 200-East Area. Several 600 Area wells located between Gable Mountain and Gable Butte also contained detectable technetium-99. Of particular interest was the detection of technetium-99 in wells $699-50-48 \mathrm{~B}(16.5 \mathrm{pCi} / \mathrm{L})$ and 699-54-57 (71.7 pCi/L) north of the 200-East Area. These wells are believed to be completed in a confined aquifer.

\subsubsection{Ruthenium-106}

Ruthenium-106 concentrations above the detection 1 imit were found in only three wells all in the 200-East Area. Wells 299-E17-2, 299-E17-5, and 299-E24-12 contained maximum ruthenium-106 concentrations of 235,352 , and $547 \mathrm{\rho Ci} / \mathrm{L}$, respectively. The detection 1 imit for ruthenium-106 is $172.5 \mathrm{pCi} / \mathrm{L}$, which is nearly six times the $\mathrm{MCL}$ of $30 \mathrm{pCi} / \mathrm{L}$. This relatively high detection limit lends some uncertainty to the contribution of ruthenium106 to gross beta concentrations in Hanford ground waters. Concentrations of ruthenium-106 less than the analytical detection limit may be present in wells that exhibit slightly elevated gross beta and no identified betaemitting radionuclide. For example, well 299-E24-1 was found to contain $66.8 \mathrm{pC} / \mathrm{L}$ gross beta during the June 1988 sampling with no identified betaemitter. This concentration exceeds the MCL for gross beta of $50 \mathrm{pCi} / \mathrm{L}$ and would not have been detected by the present ruthenium-106 measurement technique. Although some uncertainty does exist with the contribution of ruthenium-106, it does not appear to represent a long-term concern with respect to potential dose. The relatively short half-life of ruthenium-106 
(358 days) suggests that it will likely undergo radioactive decay before reaching the accessible environment. This conclusion is supported by the fact that the only wells with detectable ruthenium-106 concentrations are close to active operating facilities near the PUREX Plant.

\section{2 .15 Iodine-129}

The presence of iodine-129 in ground water is significant primarily because of its relatively long half-life (16 million years) and its potential for accumulation in the environment as a result of long-term releases from nuclear fuel reprocessing facilities (Soldat 1976). On the Hanford Site, the main contributor of iodine-129 to the ground water has been liquid discharges to cribs in the 200 Areas. Samples from wells in the 100-N, 200-East, 200-West, 400, and 600 Areas were analyzed for iodine-129. The only wells that contained iodine-129 in concentrations greater than the MCL of $1 \mathrm{pCi} / \mathrm{L}$ (the detection limit for iodine-129 is also $1 \mathrm{pCi} / \mathrm{L}$ using the direct counting method) were confined to the 200 Areas or within plumes emanating from waste disposal facilities within the 200 Areas (Figure 3.12). Wells 299-E17-5 and 299-E24-1 in the 200-East Area near the PUREX Plant contained iodine-129 concentrations of 15.6 and $26.6 \mathrm{pCi} / \mathrm{L}$, respectively. Within the tritium plume that extends east of the 200-East Area to the Columbia River (Jaquish and Mitche11 1988) we11s 699-15-26, 699-20-20, 699-24-33, 699-26-15A, 699-27-8, 699-32-22, 699-32-43, and 699-41-23 had iodine-129 concentrations of $1.6,1.5,2.7,1.9,2.3,1.5,6.7$, and $6.3 \mathrm{pCi} / \mathrm{L}$, respective $1 y$. These wells roughly delineate the extent of the migration of iodine-129 to the east and south from the 200-East Area in concentrations exceeding the MCL. A number of other 600 Area wells surrounding these wells contained concentrations of iodine-129 less than the detection 1imit. The only 200-West Area well that was analyzed for iodine-129 was 299-W19-3, which contained $15.5 \mathrm{pCi} / \mathrm{L}$. Five 600 Area wells located east and south of the 200 -West Area also contained concentrations of iodine- 129 exceeding the MCL. These wells were 699-32-70B, 699-35-66, 699-35-70, 699-38-65, and 699-38-70 with respective concentrations of $3.3,6.6,87.8,3.5$, and $1.4 \mathrm{pCi} / \mathrm{L}$. Well 699-35-70 has historically contained the highest iodine-129 concentration on Site. 


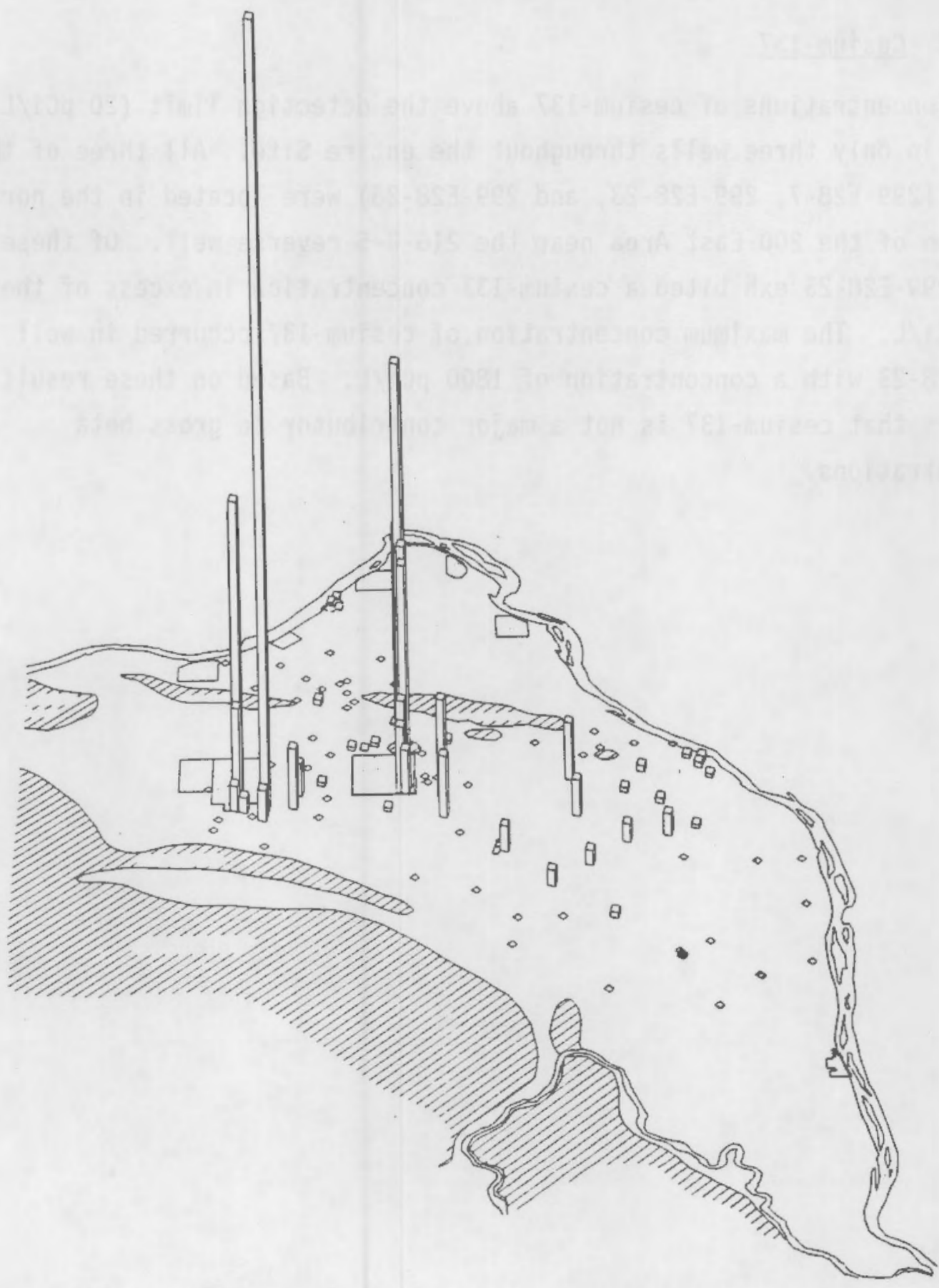

\section{Diamonds Indicate Below Detection}

FIGURE 3.12. Maximum Iodine-129 Concentrations in Unconfined
Aquifer Wells, January 1,1987 , to June 30,1988 


\subsubsection{Cesium-137}

Concentrations of cesium-137 above the detection limit $(20 \mathrm{pCi} / \mathrm{L})$ were found in only three wells throughout the entire Site. All three of these wells (299-E28-7, 299-E28-23, and 299-E28-25) were located in the northern portion of the 200-East Area near the 216-B-5 reverse wel1. Of these wells, only 299-E28-23 exhibited a cesium-137 concentration in excess of the MCL of $200 \mathrm{pCi} / \mathrm{L}$. The maximum concentration of cesium-137 occurred in well 299-E28-23 with a concentration of $1800 \mathrm{pCi} / \mathrm{L}$. Based on these results, it appears that cesium-137 is not a major contributor to gross beta concentrations. 


\subsection{SPECIAL ANALYTICAL INITIATIVES}

Several new analytical initiatives were undertaken during the year. These special studies were performed entirely in-house at PNL. The purpose of these studies was to provide additional information not available through the routine analytical methods employed by UST. Three special studies will be discussed here: 1) cyanide speciation in the BY Cribs plume; 2) inductively coupled argon plasma/mass spectrometry (ICP/MS) measurements on a broad selection of samples from the 100,200, 300, and 600 Areas; and 3) high sensitivity gas chromatography measurements performed at the Solid Waste Landfi11-Nonradioactive Dangerous Waste Landfi11.

\subsection{CYANIDE SPECIATION}

The cyanide contamination found north of the 200-East Area is believed to originate from nickel ferrocyanide scavenging waste disposed of in the BY Cribs approximately 30 years ago. The chemical analysis method employed by UST (SW-846 Method 9010, EPA 1982) provides information only on total cyanide. Because the waste was originally present as the complexed and thus relatively nontoxic form, determination of the chemical form currently found in the ground water is of interest. Toward that end, PNL initiated a 7 imited analytical development effort aimed at providing an improved method for speciation of the cyanide. The work was performed by Dr. K. Poole of the PNL Chemical and Material Sciences Center. Two methods were chosen, ion exclusion chromatography (MPIC) and ion chromatography using a pulsed amperometric detector (PAD). The PAD method is selective for free cyanide while the MPIC method is selective for the complexed form $\left(\mathrm{Fe}[\mathrm{CN}]_{6}^{4-}\right)$. The three wells selected for the special study were 699-49-55A, 699-49-57, and 699-50-53. Those wells had consistently exhibited the highest levels of total cyanide on the Site. The samples were collected as splits at the well as part of the routine sampling of those wells. The samples were collected during February and March 1988. The analytical results are presented in Table 4.1. The results are expressed as $\mu \mathrm{g} / \mathrm{L}$ of cyanide. It appears that approximately one-third of the cyanide is present in the free or uncomplexed form. The remainder may be present as the less toxic ferrocyanide complex. 
IABLE 4.1. Cyanide Speciation in 600 Area Wells

$\begin{array}{lcc}\text { We11 } & \text { Free } \mathrm{CN}^{-} & \begin{array}{c}\text { Total } \\ \text { Cyanide (UST) }\end{array} \\ 699-49-55 A & 53 & 141 \\ 699-49-57 & 24 & 62 \\ 699-50-53 & 160 & 422\end{array}$

Both forms would be expected to have high environmental mobility, and both forms are expected to be capable of forming mobile complexes with cobalt. The results of the MPIC method were not considered of adequate reliability to report at this time.

\subsection{INDUCTIVELY COUPLED ARGON PLASMA/MASS SPECTROMETRY SEMIQUANTIJATIVE. ANALYSIS}

Samples from 140 wells were collected for [CP/MS analysis. Samples were collected in the March through August 1987 timeirame. All samples were filtered and acidified in the field. The samples had all been previously analyzed by UST for some of the elements of interest. Samples were analyzed as received. Analytical work was performed by Dr. M. Smith and R. Lucke of the PNL Chemical Sciences Department. The instrument used was a VG PlasmaQuad Inductively Coupled Argon Plasma-Mass Spectrometer. The plasma source was operated at standard conditions (1300 watts forward power); samples were introduced into the plasma with a peristaltic pump feeding a Meinhard nebulizer. The instrument was operated in the SEMIQUANT mode with most of the periodic table surveyed. Dwe 11 time was $0.25 \mathrm{sec}$ for each mass peak selected in the 7ibrary. A calibration standarcl called a tune solution containing known concentrations of magnesium, iridium, and lead was run between every 10 samples. The tune solution was used to check instrument stability and normalize the internal library of elemental response factors. Blanks consisting of high purity water containirg $1 \%$ U1trex nitric acid were run every 10 samples as well. A series of instrument-specific Saha correction factors was used to provide a final renormalization of instrument response factors after processing. Semiquantitative data obtained in this fashion should be accurate to within a factor of 2. A final correction was 
applied to the data when UST data on the same samples were available. Method detection 1 imits (MDLs) were calculated from the average blank data. The MDLs were computed as the blank value plus twice the standard deviation in the blank. Method detection limits computed in that manner can be regarded as conservative estimates of the method sensitivity. In some cases, the MDLs were adjusted upward even further to eliminate spurious data. The method is extremely sensitive for most of the elements studied. A list of computed MDLs is presented in Table 4.2. Table 4.2 contains information on 62 trace elements. In addition, technetium-99 has a long enough half-1ife to be measured by this method with usable sensitivity. Uranium-235 and uranium-238 can be quantified independently providing useful isotopic ratio information.

A complete risting of the ICP/MS information can be found in Appendix D. Highlights of the results are discussed below.

A large number of elements were either below detection or present at marginally detectable levels. These elements include beryllium, cobalt, germanium, niobium, rhodium, palladium, indium, tin, silver, tellurium, cesium, hafnium, tantaium, osmium, iridium, platinum, gold, mercury, thallium, and bismuth. The lack of detection of bismuth at the 20 parts-pertrillion level of sensitivity is particularly significant because of the extensive use and environinental release of bismuth phosphate during the earliest stages of Hanford operations. A previous search for bismuth by graphite furnace atomic absorption at a detection level of $5 \mu \mathrm{g} / \mathrm{L}$ was uniformly negative. This work extends the level of sensitivity by more than two decades. Heavy rare earth elements (Eu-Lu) were not detected but a few samples did contain lighter rare earths, particularly La, Cc, and Nd (in we11s 199-H3-2C, 399-1-9, 399-1-19, and 699-83-47). Wel1s containing detectable rare earth elements also showed detectable thorium.

High chromium levels were observed in numerous wells at levels similar to those reported by UST.

Bromine levels throughout the Site are extremely variable ranging from a high of $297 \mu \mathrm{g} / \mathrm{L}$ in the 200 -west Area to less than the $7-\mu \mathrm{g} / \mathrm{L} \mathrm{MDL}$ for many of the wells. The high and variable levels of bromine found put constraints 
TABLE 4.2. Method Detection Ljmits (MDLs) for Semiquantitative Analysis by ICP/MS (a)

\begin{tabular}{|c|c|c|c|}
\hline Element & $\mathrm{MDL}$ & Element & MDL \\
\hline Lithium (Li) & 80 & $\operatorname{Tin}\langle\mathrm{S} n\rangle$ & 0.05 \\
\hline Beryllium (Be) & 0.3 & Antimony (Sb) & 0.03 \\
\hline Boron (B) & 50 & Iodine (I) & 0.5 \\
\hline Aluminum (A1) & 2 & Tellurium (Te) & 0.3 \\
\hline Calcium (Ca) & 400 & Cerium (Ce) & 0.2 \\
\hline Scandium (SC) & 0.3 & Praseodymium (Pr) & 0.02 \\
\hline Vanadium (V) & 0.5 & Neollymi um (Nd) & 0.04 \\
\hline Chromium (Cr) & 2 & Samarium (Sm) & 0.04 \\
\hline Manganese (Mn) & 1 & Europium (Eu) & 0.02 \\
\hline Iron $(\mathrm{Fe})$ & 160 & Gadolinium (Gd) & 0.05 \\
\hline Cobalt (Co) & 0.1 & Terbium (Tb) & 0.02 \\
\hline Nickel (Ni) & 4 & Dysprosium (Dy) & 0.02 \\
\hline Copper (Cu) & 1 & Holnium (Ho) & 0.02 \\
\hline Zinc (Zn) & 9 & Erbium (Er) & 0.05 \\
\hline Gallium (Ga) & 0.05 & Thul ium (Tm) & 0.02 \\
\hline Germanium (Ge) & 0.3 & Ytterbium (Yb) & 0.06 \\
\hline Arsenic (As) & 0.2 & Lutetium (Lu) & 0.02 \\
\hline SeTenium (Se) & 2 & Hafrium (Hf) & 0.03 \\
\hline Bromine (Br) & 7 & Tantalum (Ta) & 0.01 \\
\hline Rubidtum (Rb) & 0.03 & Tunçsten (W) & 0.2 \\
\hline Strontium (Sr) & 0.4 & Rher ium (Re) & 0.03 \\
\hline Yttrium $(Y)$ & 0.01 & Osmium (Os) & 0.04 \\
\hline Zirconium $(\mathrm{Zr})$ & 0.15 & Platinum (Pt) & 0.04 \\
\hline Niobium (Nb) & 0.02 & Gold $(A u)$ & 0.03 \\
\hline Morybdenum (Mo) & 0.03 & Mercury (Hg) & l \\
\hline Technet i um-99 (Tc) & $300 \mathrm{pCi} / \mathrm{L}$ & Thallium (Ti) & 0.02 \\
\hline Ruthenium (Ru) & 0.06 & Lead $(\mathrm{Pb})$ & 0.5 \\
\hline Rhodium (Rh) & 0.01 & Bismuth (Bi) & 0.02 \\
\hline Palladium (Pd) & 0.06 & Thorium (Th) & 0.01 \\
\hline Silver $(A g)$ & 0.08 & Uranium-235 (U) & 0.8 \\
\hline Cadmium (Cd) & 0.2 & Uranium-238 (U) & 0.20 \\
\hline Indium (In) & 0.1 & & \\
\hline
\end{tabular}

(a) Units in $\mu \mathrm{kg} / \mathrm{L}$ unless otherwise noted.

on the use of bromide as a ground-water tracer. The origin of the bromine is not apparent but does not appear to be related to interaquifer mixing in most cases.

Detectable zirconium was found in several wells in the 300 Area (399-1-6, 399-1-11, 399-1-13, and 399-1-19). Zirconium was detected in both the April and March 1987 samples from well 399-1-19 at approximately 20 times 
the MDL. The zirconium is likely to have been introduced by the fuel fabrications operation. The levels found even at the highest point are quite low $(2.9 \mu \mathrm{g} / \mathrm{L})$. We11 699-83-47 was also found to contain detectable minor amounts of zirconium.

Ruthenium-101 was detected only in wells 699-49-57 and 699-50-53. Although the levels are extremely low $(0.11 \mathrm{\mu g} / \mathrm{L})$, this observation is of some interest because of the evidence for a cyanide-containing plume from the BY Cribs also found in those same wells. Well 699-50-53 has one of the highest technetium-99 ground-water concentrations on the Site. Because the fission yield of ruthenium-101 is approximately half that of technetium-99, the detected ruthenium is likely a stable-element fission product. This conclusion could be easily verified by an isotopic analysis of the other ruthenium isotopes because their fission yields are quite different than their natural abundances. Other anomalous ground-water effects observed in well 699-50-53 include elevated levels of calcium, iron, cobalt, selenium, bromine, strontium, yttrium, cadmium, and rhenium. The rhenium observation is particularly interesting because rhenium is frequentiy used as a chemical analog for technetium and thus may have been present in the BY Crib waste stream.

Well 699-83-47 also shows a large number of anomalies in the trace element chemistry of the ground water. Elements with elevated levels relative to the rest of the data set include aluminum, chromium, iron, cobalt, yttrium, zirconium, niobium, lanthanum, cerium, praseodymium, neodymium, samarium, dysprosium, lead, and thorium. That well is located near the site of the White Bluffs Pickling Acid Crib. In addition to elements contributed to the soil column from operation of the facility, drainage of sulfuric acid from the crib may have mobilized low levels of some elements from the host rock (rare earths for example).

The ICP/MS method appears to work quite well for detection of technetium-99 at levels 3 times lower than the DWS $(900 \mathrm{pCi} / L)$. Although relatively few technetium-99 measurements were available for comparison during the period of interest, the levels measured by ICP/MS compare well with those determined by radiochemistry where comparison is possible. In 
addition, technetium-99 contamination was found in several wells not previously known to contain that contaminant (199-H4-10, 199-H4-11, 299-Wl2-1, and $299-W-23-3)$.

The ICP/MS method appears to be a very good technique for measuring uranium isotopic ratios. The uranium-235/uranium-238 ratio provides a useful signature of the source material for a uranium plume. Fuel fabrication waste is expected to be enriched in uranium-235, while uranium that has undergone a high degree of irradiation during plutonium production may be depleted in uranium-235 as a result of fission burnup. Prior measurements by direct counting have shown that uranium found in the 2010-West Area ground water is somewhat depleted while uranium from the 100-H Area is enriched. The ICP/MS measurements discussed here produced a similar result with the ground-water samples from the north end of the 300 Area also showing 30 to $40 \%$ relative enrichment. That ratio is consistent with the expected enrichment for fuel fabrications waste. Samples from wells located south of the South Process Pond, however, showed isotopic abundances consistent with natural abundance. This suggests that there may be two separate plumes present in the 300 Area. The natural abundance material may have originated from the earliest fuel fabrications waste disposal operation in the $30 \mathrm{C}$ Area (i.e., the South Process Pond).

\subsection{GAS CHROMATOGRAPHY-VOLATILE ORGANIC ANALYSIS MEASUREMENTS AT THE SOLID WASTE LANDFILL}

We11 699-24-33 is 1ocated approximately $500 \mathrm{ft}$ from the east fenceline of the SWL. That welT was sampled for volatile organic constituents in January of 1986 as part of the 90 well Hazardous Materials Monitoring Project. 1,1,1 trichloroethane (TCA) was found in all three triplicate samples analyzed $(27,22$, and $30 \mu \mathrm{g} / \mathrm{L})$. Funding limitations on the project at that time prevented further followup of that observation. In 1987, the Hazardous Materials Monitoring Project was combined with the Site-Wide Ground-Water Monitoring Project and given a greatly expanded scope. Resampling of wel1 699-24-33 in March 1987 confirmed the presence of contamination in that area. The central Landfill was considered as the most likely source. A request was made at that time to sample the NRDW and the new 
completed SWL wells for chlorinated hydrocarbons. Those samples taken in May and June of 1987 clearly showed the presence of chlorinated hydrocarbons downgradient of a liquid waste disposal trench in the SWL. Species detected included TCA, TCE, perchloroethylene (PCE), and 1,2 dich7oroethane (DCA). Proportions of the individual species were similar to those found in nearby wel1 699-24-33, monitored for the Hanford Site-Wide Ground-Water Monitoring Project. Continued monitoring of those wells on nine separate samplings has clearly confirmed those results. Seven sets of samples have been collected to date through the routine ground-water sampling program. Those samples were analyzed at UST by the routine gas chromatography/mass spectrometry (GC/MS) method. Four of those sample sets included splits made at the well and sent to PNL for quality assurance/quality control purposes. The PNL method employs electron capture gas chromatography, which is at least 100 times more sensitive than the GC/MS method used by UST. In addition to the routine sampling, a special study was undertaken by PNL in June 1988 to conclusively establish the existence of the contaminant plume and verify that the sampling methods used for routine sampling produce samples representative of ground-water conditions. Details of that study are discussed below.

\section{3 .1 We11 699-24-33}

We11 699-24-33 has been sampled nine times since early 1986. A summary of the results is given in Table 4.3. On7y TCA was reported by UST in 1986 because it was not their policy at that time to report results below the contractual detection $1 \mathrm{imit}$ of $10 \mu \mathrm{g} / \mathrm{L}$. Subsequent analyses are reported relative to the quantifiable detection limit for the instrument, which is estimated to be $2 \mu \mathrm{g} / \mathrm{L}$ for the GC/MS method. The contamination leveis have been remarkably constant over a period of 2 years.

\section{3 .2 Wel1 699-24-34B}

The highest chlorinated hydrocarbon levels have been found in well 699-24-34B. A summary of the measurements conducted to date on that well are included in Table 4.4. Only the three most abundant species, TCA, PCE, and TCE, are reported here. Chloroform, DCA, and carbon tetrachloride have also been detected in that we 71 and in several others. Only TCE is present at levels of potential regulatory concern. The MCL for TCE is $5 \mu \mathrm{g} / \mathrm{L}$. Three 
TABLE 4.3. Chlorinated Hydrocarbon Contaminants in We11 699-24-33 ( $\mu \mathrm{g} / \mathrm{L})$

\begin{tabular}{|c|c|c|c|c|c|c|}
\hline \multirow[b]{2}{*}{ Date } & \multicolumn{2}{|c|}{$1,1,1-\mathrm{TCA}$ (a) } & \multicolumn{2}{|c|}{$P C E^{(b)}$} & \multicolumn{2}{|c|}{$\operatorname{TCE}(\mathrm{c})$} \\
\hline & $\mathrm{GC} / \mathrm{MS}(\mathrm{d})$ & $\mathrm{GC}(\mathrm{e})$ & $\underline{G C / M S}$ & $\underline{G C}$ & $\underline{G C / M S}$ & $\underline{G C}$ \\
\hline $1 / 23 / 86$ & 21 & $N A(f)$ & $<10$ & NA & $<10$ & NA \\
\hline $1 / 23 / 86$ & 23 & NA & $<10$ & NA & $<10$ & NA \\
\hline $1 / 23 / 86$ & 30 & NA & $<10$ & NA & $<10$ & NA \\
\hline $3 / 23 / 87$ & 21 & NA & 3 & NA & 4 & NA \\
\hline $5 / 14 / 87$ & 17 & NA & 3 & NA & 3 & NA \\
\hline $8 / 25 / 87$ & 18 & NA & 3 & NA & 3 & NA \\
\hline $11 / 10 / 87$ & 23 & NA & 3 & NA & 3 & NA \\
\hline $2 / 03 / 88$ & 23 & NA & 3 & NA & 3 & NA \\
\hline $4 / 28 / 88$ & 19 & NA & 3 & NA & 3 & NA \\
\hline $5 / 29 / 88$ & NA & 26 & NA & 3.4 & NA & 4.0 \\
\hline $6 / 29 / 88$ & NA & 27 & NA & 3.8 & NA & 4.3 \\
\hline $7 / 30 / 88$ & 20 & 23 & 4 & 3.1 & 3 & 3.9 \\
\hline
\end{tabular}

\footnotetext{
(a) 1,1,1-TCA = 1,1,1-trichioroethane.

(b) $\mathrm{PCE}=$ perchloroethene.

(c) TCE = trichloroethene.

(d) $\mathrm{GC} / \mathrm{MS}$ = analysis by gas chromatography/mass spectrometry method conducted by PNL. Quantifiable detection limit is estimated to be $0.02 \mu \mathrm{g} / \mathrm{L}$ for all three species.

(e) $\mathrm{GC}=$ analys is by gas chromatography method conducted by UST. Quantifiable detection limit is estimated to be $2 \mu \mathrm{g} / \mathrm{L}$ for all three species.

(f) $\quad N A=$ not applicable.
}

other wells at the SWL were also found to have levels of TCE s7ightly above the MCL. We11 699-24-34B has been included in the PNL quality assurance/ quality control program this year. Several split measurements by both GC/MS (UST) and GC (PNL) are reported in Table 4.4. Agreement is excellent in all cases, although the PNL measurements in general have better precision because of the much greater sensitivity of the GC method. Measurements from UST below $10 \mu \mathrm{g} / \mathrm{L}$ are only reported to one significalt figure because these levels are close to the detection limit. 
IABLE 4.4. Chlorinated Hydrocarbon Contaminants in We11 699-24-34B ( $\mu \mathrm{g} / \mathrm{L})$

\begin{tabular}{|c|c|c|c|c|c|c|}
\hline \multirow[b]{2}{*}{ Date } & \multicolumn{2}{|c|}{$1,1,1-\operatorname{TCA}(a)$} & \multicolumn{2}{|c|}{ PCE (b) } & \multicolumn{2}{|c|}{ TCE (c) } \\
\hline & $\mathrm{GC} / \mathrm{MS}(\mathrm{d})$ & $\underline{G C}(\mathrm{e})$ & $\underline{G C / M S}$ & $\underline{G C}$ & $\underline{G C / M S}$ & $\underline{G C}$ \\
\hline $5 / 20 / 87$ & 56 & $N A(f)$ & 7 & NA & 10 & NA \\
\hline $6 / 18 / 87$ & 40 & NA & 6 & NA & 8 & NA \\
\hline $7 / 28 / 87$ & 46 & NA & 5 & NA & 8 & NA \\
\hline $11 / 15 / 87$ & 64 & 60 & 8 & NA & 8 & NA \\
\hline $11 / 15 / 87$ & 61 & NA & 8 & NA & 8 & NA \\
\hline $1 / 18 / 88$ & 58 & 47 & 8 & 6.9 & 8 & NA \\
\hline $4 / 22 / 88$ & 41 & 55 & 8 & 8.5 & 6 & NA \\
\hline $4 / 22 / 88$ & 41 & 50 & 8 & 9.0 & 6 & NA \\
\hline $6 / 27 / 88$ & NA & 51 & NA & 9.6 & NA & 8.7 \\
\hline $6 / 27 / 88$ & NA & 50 & NA & 9.5 & NA & 8.6 \\
\hline $7 / 7 / 88$ & NA & 51 & NA & 9.6 & NA & 8.7 \\
\hline $7 / 7 / 88$ & NA & 50 & NA & 9.5 & NA & 8.6 \\
\hline $7 / 27 / 88$ & 34 & 36 & 7 & 7.6 & 6 & 5.7 \\
\hline $7 / 27 / 88$ & 35 & NA & 7 & NA & 5 & NA \\
\hline
\end{tabular}

\footnotetext{
(a) 1,1,1-TCA = 1,1,1-trichloroethane.

(b) $\mathrm{PCE}=$ perchloroethene.

(c) TCE = trichioroethene.

(d) GC/MS = analysis by gas chromatography/mass spectrometry method conducted by PNL. Quantifiable detection limit is estimated to be $0.02 \mu \mathrm{g} / \mathrm{L}$ for all three species.

(e) $G C=$ analys is by gas chromatography method conducted by UST. Quantifiable detection 1 imit is estimated to be $2 \mu \mathrm{g} / \mathrm{L}$ for al1 three species.

(f) $\mathrm{NA}=$ not applicable.
}

\subsubsection{Pacific Northwest Laboratory Special Sampling Study at the SWL}

Pacific Northwest Laboratory personnel conducted a series of sampling and analysis tests at the SWL from June 27 to JuTy 11, 1988. The purpose of the study was to 1) compare sampling methods employing centrifugal pumps, bladder pumps, and Teflon bailers; 2) obtain a full set of carefully prepared samples for high sensitivity GC analysis; 3 ) perform careful $\mathrm{pH}$ measurements on all SWL wells with a flow through $\mathrm{pH}$ cell; and 4) test soil-gas analys is techniques and equipment. Ali objectives were satisfactorily met. The pH 
and soil-gas results will be discussed in detail elsewhere. B1adder pumps were added to two of the wells (699-24-34B and 699-24-35); however, the pump in well 699-24-35 did not perform satisfactorily and was not used. To facilitate rapid measurement of samples, the PNL mobile GC laboratory was moved to the SWL site. The laboratory is mounted inside a $30-\mathrm{ft}$ motor home. A portable $15-\mathrm{kW}$ generator was also set up at the site to provide power.

Volatile organic analysis (VOA) samples were collected in standard 40-mL VOA bottles, transferred to the motor home, and analyzed immediately. The wells were purged for time periods ranging from 20 to 40 min before collecting samples. A teeing system containing two throttling valves and a critical orifice was used to provide a low flow path for VoA samples. Samples from the centrifugal pumps were collected through a 1/4-in. Teflon tube at a flow rate of approximately 1 Titer per minute. Bailer samples were taken with 1-L Tefion bailers lowered into the wells with a winch and nylon fishing 1ine. Unused dedicated bailers were used in the study. Approximately $20 \mathrm{ft}$ of fishing line was discarded after each use to avoid cross contamination.

Samples were analyzed with a Hewlett-Packard Model $5880 \mathrm{~A}$ gas chromatograph. The GC was equipped with two identical J\&W DB-624 $30 \mathrm{~m} \times 0.53 \mathrm{~mm}$ fused silica capillary columns. The DB-624 columns were coated with a cross-linked and bonded stationary phase composed of cyanopropyl, phenyl, dimethylsiloxane. The two columns were teed together at the inlet and were routed to separate electron capture (ECD) and flame ionization (FID) detectors. Sample introduction was via a Tekmar Model LSC-3 purge and trap unit. The LSC-3 contained a Tenax sorption trap. Samples were thermally desorbed from the Tenax trap and transferred to the colums through a heated transfer 1ine. The LSC-3 was modified by adding a pneumatic valve actuator to permit. full automation of the purge and trap cycle by tie GC run table. The HP 5880A was equipped with two separate integrators to simultaneously integrate data from both detectors. Analytical meas srements were performed in accordance with the guidelines set forth in EPA (1982), methods 8010 (Halogenated Volatile Organics) and 8015 (Nonhalogenated Organics).

Analytical results for five species of interest in nine wells are presented in Tables 4.5 to 4.9 . Table 4.6 is of particular interest because it 
provides rather conclusive evidence for the presence of TCE at levels somewhat above the regulatory limit; levels greater than the $5-\mu \mathrm{g} / \mathrm{L}$ limit were seen 25 times in four different wells in this study. Note that the regulatory limit is about 300 times the measured system blank.

In addition to the measurements reported above, a time-dependent sampling study was performed at the upgradient we11, 699-24-35. The purpose of that test was to determine if purging volume has any effect on the representativeness of the results. The well was first sampled with a bailer without purge (although it had been purged 4 days earlier). The well was then sequentially purged with the centrifugal pump at a flow rate of $5 \mathrm{gpm}$ for 10-min intervais. Samples were taken for VOA at the end of each 10-min interval, the pump turned off, and bailer samples taken; the pump was then restarted immediately. Times were in all cases measured from the appearance of water at the pump discharge line. The sampling continued for $1 \mathrm{~h}$. Each 10-min interval corresponded to about 3.5 well bore volumes. The analytical results for 4 species are shown in Table 4.10 .

\subsubsection{Conclusions}

The sampling program over the past 2 years has generated a Targe amount of chlorinated hydrocarbon data. While these data have not yet been subjected to a rigorous statistical review, a number of conclusions appear to be definitive:

1. Widespread, low-level chlorinated hydrocarbon contamination is detectable throughout the extended landfill area. The concentrations found in well 699-24-33 suggest that the contamination has been present at least since early 1986 and has undergone little change since then. The relative concentrations of contaminants in that well are similar to those in other wells closer to the landfill area.

2. Contaminants detected include chloroform, TCA, TCE, PCE, DCA, and carbon tetrachloride. Chloroform is commonly found in chlorinetreated water. It may also be a decomposition product of TCA; TCA is the most abundant species. It is commonly used as a degreaser solvent. The highest level found is only about $1 / 4$ of the drinking water standard but couid be higher closer to the source (i.e., inside the SWL). Trichloroethylene is present in four of the wells at levels slightly above the DWS. The relative concentrations of TCA, TCE, PCE, and DCA are similar in all wells tested including the Hanford Site-Wide Ground-Water Monitoring Project well and the 
upgradient wel]. In contrast, carbon tetrachloride is relatively constant in all wells tested, although some increase was observed near the NRDW. The carbon tetrachloride data are difficult to understand without further study.

3. The three sampling methods tested (centrifugal pump, bladder pump, and bailer) produced identical results in all cases. The bladder pump was judged to be unsatisfactory for routine use because of the high instantaneous flows produced during each pulse; however, the results showed little sensitivity to such effects.

4. Purge volume had little effect on results for the centrifugal pump. The bailer samples did show a small increase after the first 40 min of purge. The reason for the difference is unclear. During routine sampling the wells are purged for 20 min before sampling. 
IABLE 4.5. 1,1,1-Trichloroethane Results $(\mu \mathrm{g} / \mathrm{L})$

\begin{tabular}{|c|c|c|c|c|}
\hline Wel1 Number & $\begin{array}{c}\text { Date } \\
\text { Collected } \\
\end{array}$ & $\begin{array}{l}\text { Centrifugal } \\
\text { Pump } \\
\end{array}$ & $\begin{array}{l}\text { Bladder } \\
\text { Pump }\end{array}$ & Bailer \\
\hline $\begin{array}{l}699-25-34 C \\
699-25-34 C\end{array}$ & $\begin{array}{l}6 / 29 / 88 \\
6 / 29 / 88\end{array}$ & $\begin{array}{l}5.9 \\
5.9\end{array}$ & $\begin{array}{l}\mathrm{NA}(\mathrm{a}) \\
\mathrm{NA}\end{array}$ & $\begin{array}{l}6.1 \\
6.1\end{array}$ \\
\hline $\begin{array}{l}699-24-34 C \\
699-24-34 C\end{array}$ & $\begin{array}{l}6 / 27 / 88 \\
6 / 27 / 88\end{array}$ & $\begin{array}{l}33 \\
33\end{array}$ & $\begin{array}{l}\text { NA } \\
\text { NA }\end{array}$ & $\begin{array}{l}35 \\
34\end{array}$ \\
\hline $\begin{array}{l}699-24-34 B \\
699-24-34 B \\
699-24-34 B \\
699-24-34 B \\
699-24-34 B\end{array}$ & $\begin{array}{l}6 / 27 / 88 \\
6 / 27 / 88 \\
7 / 07 / 88 \\
7 / 07 / 88 \\
7 / 07 / 88\end{array}$ & $\begin{array}{l}51 \\
50 \\
52 \\
53 \\
52(b)\end{array}$ & $\begin{array}{l}48 \\
47 \\
49 \\
53 \\
50(b)\end{array}$ & $\begin{array}{l}\text { NA } \\
56 \\
52 \\
50 \\
48(b)\end{array}$ \\
\hline $\begin{array}{l}699-24-34 A \\
699-24-34 A\end{array}$ & $\begin{array}{l}6 / 27 / 88 \\
6 / 27 / 88\end{array}$ & $\begin{array}{l}40 \\
\text { NA }\end{array}$ & $\begin{array}{l}\text { NA } \\
\text { NA }\end{array}$ & $\begin{array}{l}43 \\
44\end{array}$ \\
\hline $\begin{array}{l}699-23-34 \\
699-23-34\end{array}$ & $\begin{array}{l}6 / 29 / 88 \\
6 / 29 / 88\end{array}$ & $\begin{array}{l}49 \\
50\end{array}$ & $\begin{array}{l}\text { NA } \\
\text { NA }\end{array}$ & $\begin{array}{l}46 \\
49\end{array}$ \\
\hline $\begin{array}{l}699-24-33 \\
699-24-33\end{array}$ & $\begin{array}{l}6 / 29 / 88 \\
6 / 29 / 88\end{array}$ & $\begin{array}{l}26 \\
27\end{array}$ & $\begin{array}{l}\text { NA } \\
\text { NA }\end{array}$ & $\begin{array}{l}24 \\
26\end{array}$ \\
\hline $\begin{array}{l}699-24-35 \\
699-24-35\end{array}$ & $\begin{array}{l}7 / 11 / 88 \\
7 / 11 / 88\end{array}$ & $\begin{array}{l}4.1 \\
4.1\end{array}$ & $\begin{array}{l}\text { NA } \\
\text { NA }\end{array}$ & $\begin{array}{l}4.3 \\
4.1\end{array}$ \\
\hline $\begin{array}{l}699-25-34 B \\
699-25-34 B\end{array}$ & $\begin{array}{l}7 / 11 / 88 \\
7 / 11 / 88\end{array}$ & $\begin{array}{l}3.6 \\
3.5\end{array}$ & $\begin{array}{l}\text { NA } \\
\text { NA }\end{array}$ & $\begin{array}{l}\text { NA } \\
\text { NA }\end{array}$ \\
\hline $\begin{array}{l}699-26-33 \\
699-26-33\end{array}$ & $\begin{array}{l}7 / 11 / 88 \\
7 / 11 / 88\end{array}$ & $\begin{array}{l}0.85 \\
0.85\end{array}$ & $\begin{array}{l}\text { NA } \\
\text { NA }\end{array}$ & $\begin{array}{l}\text { NA } \\
\text { NA }\end{array}$ \\
\hline System Blank & & 0.014 & & \\
\hline
\end{tabular}

(a) $N A=$ not applicable.

(b) 5-mL glass sampling syringe filled at well. 
TABLE 4.6. 1,1,2-Trichloroethene Results $(\mu \mathrm{g} / \mathrm{L})$

\begin{tabular}{|c|c|c|c|c|}
\hline We11 Number & $\begin{array}{c}\text { Date } \\
\text { Collected }\end{array}$ & $\begin{array}{l}\text { Centrifugal } \\
-\quad \text { Pump } \\
\end{array}$ & $\begin{array}{l}\text { Bl adder } \\
\text { Pump }\end{array}$ & Bajler \\
\hline $\begin{array}{l}699-25-34 C \\
699-25-34 C\end{array}$ & $\begin{array}{l}6 / 29 / 88 \\
6 / 29 / 88\end{array}$ & $\begin{array}{l}1.05 \\
1.05\end{array}$ & $\begin{array}{l}\mathrm{NA}(\mathrm{a}) \\
\mathrm{NA}\end{array}$ & $\begin{array}{l}1.04 \\
1.07\end{array}$ \\
\hline $\begin{array}{l}699-24-34 C \\
699-24-34 C\end{array}$ & $\begin{array}{l}6 / 27 / 88 \\
6 / 27 / 88\end{array}$ & $\begin{array}{l}5.3 \\
5.4\end{array}$ & $\begin{array}{l}\text { NA } \\
\text { NA }\end{array}$ & $\begin{array}{l}5.7 \\
5.6\end{array}$ \\
\hline $\begin{array}{l}699-24-34 B \\
699-24-34 B \\
699-24-34 B \\
699-24-34 B \\
699-24-34 B\end{array}$ & $\begin{array}{l}6 / 27 / 88 \\
6 / 27 / 88 \\
7 / 07 / 88 \\
7 / 07 / 88 \\
7 / 07 / 88\end{array}$ & $\begin{array}{l}8.7 \\
8.6 \\
8.1 \\
8.1 \\
8.2(b)\end{array}$ & $\begin{array}{l}8.2 \\
8.1 \\
8.8 \\
8.2 \\
7.8(b)\end{array}$ & $\begin{array}{l}\text { NA } \\
9.4 \\
8.1 \\
8.0 \\
7.6(b)\end{array}$ \\
\hline $\begin{array}{l}699-24-34 A \\
699-24-34 A\end{array}$ & $\begin{array}{l}6 / 27 / 88 \\
6 / 27 / 88\end{array}$ & ${ }_{\mathrm{NA}}^{7}$ & $\begin{array}{l}\text { NA } \\
\text { NA }\end{array}$ & $\begin{array}{l}8.4 \\
8.6\end{array}$ \\
\hline $\begin{array}{l}699-23-34 \\
699-23-34\end{array}$ & $\begin{array}{l}6 / 29 / 88 \\
6 / 29 / 88\end{array}$ & $\begin{array}{l}8.8 \\
9.0\end{array}$ & $\begin{array}{l}\text { NA } \\
\text { NA }\end{array}$ & $\begin{array}{l}8.3 \\
9.0\end{array}$ \\
\hline $\begin{array}{l}699-24-33 \\
699-24-33\end{array}$ & $\begin{array}{l}6 / 29 / 88 \\
6 / 29 / 88\end{array}$ & $\begin{array}{l}4.0 \\
4.3\end{array}$ & $\begin{array}{l}\text { NA } \\
\text { NA }\end{array}$ & $\begin{array}{l}3.6 \\
4.0\end{array}$ \\
\hline $\begin{array}{l}699-24-35 \\
699-24-35\end{array}$ & $\begin{array}{l}7 / 11 / 88 \\
7 / 11 / 88\end{array}$ & $\begin{array}{l}0.54 \\
0.55\end{array}$ & $\begin{array}{l}\text { NA } \\
\text { NA }\end{array}$ & $\begin{array}{l}0.59 \\
0.57\end{array}$ \\
\hline $\begin{array}{l}699-25-34 B \\
699-25-34 B\end{array}$ & $\begin{array}{l}7 / 11 / 88 \\
7 / 11 / 88\end{array}$ & $\begin{array}{l}0.65 \\
0.63\end{array}$ & $\begin{array}{l}\text { NA } \\
\text { NA }\end{array}$ & $\begin{array}{l}\text { NA } \\
\text { NA }\end{array}$ \\
\hline $\begin{array}{l}699-26-33 \\
699-26-33\end{array}$ & $\begin{array}{l}7 / 11 / 88 \\
7 / 11 / 88\end{array}$ & $\begin{array}{l}0.21 \\
0.21\end{array}$ & $\begin{array}{l}\text { NA } \\
\text { NA }\end{array}$ & $\begin{array}{l}\text { NA } \\
\text { NA }\end{array}$ \\
\hline System B] ank & & 0.017 & & \\
\hline
\end{tabular}

(a) $N A=$ not applicable.

(b) 5-mL glass sampling syringe filled at wel?. 
TABLE 4.7. 1,1,2,2-Tetrach1oroethene Results $(\mu \mathrm{g} / \mathrm{L})$

\begin{tabular}{|c|c|c|c|c|}
\hline We 11 Number & $\begin{array}{c}\text { Date } \\
\text { Collected }\end{array}$ & $\begin{array}{l}\text { Centrifugal } \\
\text { Pump }\end{array}$ & $\begin{array}{l}\text { Bladder } \\
\text { Pump } \\
\end{array}$ & Bailer \\
\hline $\begin{array}{l}699-25-34 C \\
699-25-34 C\end{array}$ & $\begin{array}{l}6 / 29 / 88 \\
6 / 29 / 88\end{array}$ & $\begin{array}{l}0.70 \\
0.70\end{array}$ & $\begin{array}{l}\text { NA }(a) \\
\text { NA }\end{array}$ & $\begin{array}{l}0.70 \\
0.75\end{array}$ \\
\hline $\begin{array}{l}699-24-34 C \\
699-24-34 C\end{array}$ & $\begin{array}{l}6 / 27 / 88 \\
6 / 27 / 88\end{array}$ & $\begin{array}{l}5.6 \\
5.7\end{array}$ & $\begin{array}{l}N A \\
N A\end{array}$ & $\begin{array}{l}6.2 \\
5.9\end{array}$ \\
\hline $\begin{array}{l}699-24-34 B \\
699-24-34 B \\
699-24-34 B \\
699-24-34 B \\
699-24-34 B\end{array}$ & $\begin{array}{l}6 / 27 / 88 \\
6 / 27 / 88 \\
7 / 07 / 88 \\
7 / 07 / 88 \\
7 / 07 / 88\end{array}$ & $\begin{array}{l}9.6 \\
9.5 \\
8.6 \\
8.6 \\
8.6 \text { (b) }\end{array}$ & $\begin{array}{l}9.0 \\
8.8 \\
8.0 \\
8.6 \\
8.0(b)\end{array}$ & $\begin{array}{l}N A \\
10.0 \\
8.6 \\
8.6 \\
7.6(b)\end{array}$ \\
\hline $\begin{array}{l}699-24-34 A \\
699-24-34 A\end{array}$ & $\begin{array}{l}6 / 27 / 88 \\
6 / 27 / 88\end{array}$ & 7.0 & $\begin{array}{l}\text { NA } \\
\text { NA }\end{array}$ & $\begin{array}{l}7.2 \\
7.4\end{array}$ \\
\hline $\begin{array}{l}699-23-34 \\
699-23-34\end{array}$ & $\begin{array}{l}6 / 29 / 88 \\
6 / 29 / 88\end{array}$ & $\begin{array}{l}7.8 \\
8.2\end{array}$ & $\begin{array}{l}\text { NA } \\
\text { NA }\end{array}$ & $\begin{array}{l}7.4 \\
8.0\end{array}$ \\
\hline $\begin{array}{l}699-24-33 \\
699-24-33\end{array}$ & $\begin{array}{l}6 / 29 / 88 \\
6 / 29 / 88\end{array}$ & $\begin{array}{l}3.4 \\
3.8\end{array}$ & $\begin{array}{l}\text { NA } \\
\text { NA }\end{array}$ & $\begin{array}{l}3.3 \\
3.6\end{array}$ \\
\hline $\begin{array}{l}699-24-35 \\
699-24-35\end{array}$ & $\begin{array}{l}7 / 11 / 88 \\
7 / 11 / 88\end{array}$ & $\begin{array}{l}0.54 \\
0.55\end{array}$ & $\begin{array}{l}\text { NA } \\
\text { NA }\end{array}$ & $\begin{array}{l}0.58 \\
0.55\end{array}$ \\
\hline $\begin{array}{l}699-25-34 B \\
699-25-34 B\end{array}$ & $\begin{array}{l}7 / 11 / 88 \\
7 / 11 / 88\end{array}$ & $\begin{array}{l}0.55 \\
0.51\end{array}$ & $\begin{array}{l}\mathrm{NA} \\
\mathrm{NA}\end{array}$ & $\begin{array}{l}\text { NA } \\
\text { NA }\end{array}$ \\
\hline $\begin{array}{l}699-26-33 \\
699-26-33\end{array}$ & $\begin{array}{l}7 / 11 / 88 \\
7 / 11 / 88\end{array}$ & $\begin{array}{l}0.092 \\
0.090\end{array}$ & $\begin{array}{l}\text { NA } \\
\text { NA }\end{array}$ & $\begin{array}{l}\text { NA } \\
\text { NA }\end{array}$ \\
\hline System Blank & & 0.003 & & \\
\hline
\end{tabular}

(a) $\mathrm{NA}=$ not applicable.

(b) 5 -mL glass sampling syringe filled at well. 
IABLE 4.8. Chloroform Results ( $\mu \mathrm{g} / \mathrm{L})$

\begin{tabular}{|c|c|c|c|c|}
\hline We11 Number & $\begin{array}{c}\text { Date } \\
\text { collected }\end{array}$ & $\begin{array}{c}\text { Centrifuga } \\
\quad \text { Pump } \\
\end{array}$ & $\begin{array}{l}\text { Bladder } \\
\text { Pump }\end{array}$ & Bailer \\
\hline $\begin{array}{l}699-25-34 C \\
699-25-34 C\end{array}$ & $\begin{array}{l}6 / 29 / 88 \\
6 / 29 / 88\end{array}$ & $\begin{array}{l}0.33 \\
0.34\end{array}$ & $\begin{array}{l}\text { NA (a) } \\
N A\end{array}$ & $\begin{array}{l}0.34 \\
0.35\end{array}$ \\
\hline $\begin{array}{l}699-24-34 C \\
699-24-34 C\end{array}$ & $\begin{array}{l}6 / 27 / 88 \\
6 / 27 / 88\end{array}$ & $\begin{array}{l}0.99 \\
0.88\end{array}$ & $\begin{array}{l}\text { NA } \\
\text { NA }\end{array}$ & $\begin{array}{l}1.00 \\
0.98\end{array}$ \\
\hline $\begin{array}{l}699-24-34 \mathrm{~B} \\
699-24-34 \mathrm{~B} \\
699-24-34 \mathrm{~B} \\
699-24-34 \mathrm{~B} \\
699-24-34 \mathrm{~B}\end{array}$ & $\begin{array}{l}6 / 27 / 88 \\
6 / 27 / 88 \\
7 / 7 / 88 \\
7 / 7 / 88 \\
7 / 7 / 88\end{array}$ & $\begin{array}{l}1.29 \\
1.33 \\
1.59 \\
1.52 \\
1.46(b)\end{array}$ & $\begin{array}{l}1.33 \\
1.28 \\
1.40 \\
1.46 \\
1.17(b)\end{array}$ & $\begin{array}{l}\text { NA } \\
2.06 \\
1.44 \\
1.51 \text { (b) } \\
1.47 \text { b }\end{array}$ \\
\hline $\begin{array}{l}699-24-34 A \\
699-24-34 A\end{array}$ & $\begin{array}{l}6 / 27 / 88 \\
6 / 27 / 88\end{array}$ & $\begin{array}{l}1.12 \\
1.02\end{array}$ & $\begin{array}{l}\text { NA } \\
\text { NA }\end{array}$ & $\begin{array}{l}1.10 \\
1.19\end{array}$ \\
\hline $\begin{array}{l}699-23-34 \\
699-23-34\end{array}$ & $\begin{array}{l}6 / 29 / 88 \\
6 / 29 / 88\end{array}$ & $\begin{array}{l}1.40 \\
1.35\end{array}$ & $\begin{array}{l}\text { NA } \\
\text { NA }\end{array}$ & $\begin{array}{l}1.48 \\
1.58\end{array}$ \\
\hline $\begin{array}{l}699-24-33 \\
699-24-33\end{array}$ & $\begin{array}{l}6 / 29 / 88 \\
6 / 29 / 88\end{array}$ & $\begin{array}{l}0.80 \\
0.82\end{array}$ & $\begin{array}{l}\text { NA } \\
\text { NA }\end{array}$ & $\begin{array}{l}0.60 \\
0.92\end{array}$ \\
\hline $\begin{array}{l}699-24-35 \\
699-24-35\end{array}$ & $\begin{array}{l}7 / 11 / 88 \\
6 / 11 / 88\end{array}$ & $\begin{array}{l}0.15 \\
0.15\end{array}$ & $\begin{array}{l}\text { NA } \\
\text { NA }\end{array}$ & $\begin{array}{l}0.15 \\
0.14\end{array}$ \\
\hline $\begin{array}{l}699-25-34 B \\
699-25-34 B\end{array}$ & $\begin{array}{l}7 / 11 / 88 \\
7 / 11 / 88\end{array}$ & $\begin{array}{l}0.34 \\
0.33\end{array}$ & $\begin{array}{l}\text { NA } \\
\text { NA }\end{array}$ & $\begin{array}{l}\text { NA } \\
\text { NA }\end{array}$ \\
\hline $\begin{array}{l}699-26-33 \\
699-26-33\end{array}$ & $\begin{array}{l}7 / 11 / 88 \\
7 / 11 / 88\end{array}$ & $\begin{array}{l}0.17 \\
0.18\end{array}$ & $\begin{array}{l}\text { NA } \\
\text { NA }\end{array}$ & $\begin{array}{l}\text { NA } \\
\text { NA }\end{array}$ \\
\hline System Blank & 0.04 & & & \\
\hline
\end{tabular}

(a) $\mathrm{NA}=$ not applicable.

(b) 5-mL glass sampling syringe filled at well. 
IABLE 4.9. Carbon Tetrachloride Results $(\mu \mathrm{g} / \mathrm{L})$

\begin{tabular}{|c|c|c|c|c|}
\hline We11 Number & $\begin{array}{l}\text { Date } \\
\text { Collected }\end{array}$ & $\begin{array}{l}\text { Centrifugal } \\
\text { Pump } \\
\end{array}$ & $\begin{array}{l}\text { Bladder } \\
\text { Pump } \\
\end{array}$ & Bailer \\
\hline $\begin{array}{l}699-25-34 C \\
699-25-34 C\end{array}$ & $\begin{array}{l}6 / 29 / 88 \\
6 / 29 / 88\end{array}$ & $\begin{array}{l}0.72 \\
0.70\end{array}$ & $\begin{array}{l}\text { NA }(a) \\
\text { NA }\end{array}$ & $\begin{array}{l}0.66 \\
0.66\end{array}$ \\
\hline $\begin{array}{l}699-24-34 c \\
699-24-34 c\end{array}$ & $\begin{array}{l}6 / 27 / 88 \\
6 / 27 / 88\end{array}$ & $\begin{array}{l}0.33 \\
0.27\end{array}$ & $\begin{array}{l}\text { NA } \\
\text { NA }\end{array}$ & $\begin{array}{l}0.27 \\
0.30\end{array}$ \\
\hline $\begin{array}{l}699-24-34 B \\
699-24-34 B \\
699-24-34 B \\
699-24-34 B \\
699-24-34 B\end{array}$ & $\begin{array}{l}6 / 27 / 88 \\
6 / 27 / 88 \\
7 / 7 / 88 \\
7 / 7 / 88 \\
7 / 7 / 88\end{array}$ & $\begin{array}{l}0.30 \\
0.26 \\
0.28 \\
0.28 \\
0.30 \text { (b) }\end{array}$ & $\begin{array}{l}0.26 \\
0.23 \\
0.32 \\
0.29 \\
0.29 \text { (b) }\end{array}$ & $\begin{array}{l}0.37 \\
0.29 \\
0.27 \\
0.27 \\
0.27 \text { (b) }\end{array}$ \\
\hline $\begin{array}{l}699-24-34 A \\
699-24-34 A\end{array}$ & $\begin{array}{l}6 / 27 / 88 \\
6 / 27 / 88\end{array}$ & $\begin{array}{l}0.26 \\
0.23\end{array}$ & $\begin{array}{l}\text { NA } \\
\text { NA }\end{array}$ & $\begin{array}{l}0.25 \\
0.26\end{array}$ \\
\hline $\begin{array}{l}699-23-34 \\
699-23-34\end{array}$ & $\begin{array}{l}6 / 29 / 88 \\
6 / 29 / 88\end{array}$ & $\begin{array}{l}0.28 \\
0.31\end{array}$ & $\begin{array}{l}\text { NA } \\
\text { NA }\end{array}$ & $\begin{array}{l}0.26 \\
0.30\end{array}$ \\
\hline $\begin{array}{l}699-24-33 \\
699-24-33\end{array}$ & $\begin{array}{l}6 / 29 / 88 \\
6 / 29 / 88\end{array}$ & $\begin{array}{l}0.41 \\
0.43\end{array}$ & $\begin{array}{l}\text { NA } \\
\text { NA }\end{array}$ & $\begin{array}{l}0.43 \\
0.40\end{array}$ \\
\hline $\begin{array}{l}699-24-35 \\
699-24-35\end{array}$ & $\begin{array}{l}7 / 11 / 88 \\
7 / 11 / 88\end{array}$ & $\begin{array}{l}0.30 \\
0.31\end{array}$ & $\begin{array}{l}\text { NA } \\
\text { NA }\end{array}$ & $\begin{array}{l}0.31 \\
0.31\end{array}$ \\
\hline $\begin{array}{l}699-25-34 \mathrm{~B} \\
699-25-34 \mathrm{~B}\end{array}$ & $\begin{array}{l}7 / 11 / 88 \\
7 / 11 / 88\end{array}$ & $\begin{array}{l}0.82 \\
0.80\end{array}$ & $\begin{array}{l}\text { NA } \\
\text { NA }\end{array}$ & $\begin{array}{l}\text { NA } \\
\text { NA }\end{array}$ \\
\hline $\begin{array}{l}699-26-33 \\
699-26-33\end{array}$ & $\begin{array}{l}7 / 11 / 88 \\
7 / 11 / 88\end{array}$ & $\begin{array}{l}0.43 \\
0.43\end{array}$ & $\begin{array}{l}\text { NA } \\
\text { NA }\end{array}$ & $\begin{array}{l}\text { NA } \\
\text { NA }\end{array}$ \\
\hline System Blank & & $<0.003$ & & \\
\hline
\end{tabular}

(a) $N A=$ not applicable.

(b) 5-mL glass sampling syringe filled at well. 

TABLE 4.10. Chlorinated Hydrocarbons $\{0\}$ lected by Sequential

\begin{tabular}{|c|c|c|c|c|c|c|c|c|}
\hline \multirow{2}{*}{$\begin{array}{c}\text { Purge } \\
\text { Time, min }\end{array}$} & \multicolumn{2}{|c|}{ TCA (b) } & \multicolumn{2}{|c|}{$\operatorname{TCE}(\mathrm{C})$} & \multicolumn{2}{|c|}{$\operatorname{PCE}(d)$} & \multicolumn{2}{|c|}{$\mathrm{CCl}_{4}(\mathrm{e})$} \\
\hline & Pump & Bailer & Pump & Bailer & Fump & Bajler & Pump & Bailer \\
\hline 0 & $N A(f)$ & 3.51 & NA & 0.45 & NA & 0.42 & NA & 0.31 \\
\hline 0 & & 3.50 & NA & 0.45 & NiA & 0.42 & NA & 0.31 \\
\hline 10 & 3.94 & 3.85 & 0.53 & 0.52 & C.51 & 0.51 & 0.32 & 0.30 \\
\hline 10 & 3.95 & 3.97 & 0.53 & 0.52 & C.52 & 0.50 & 0.32 & 0.31 \\
\hline 20 & $\begin{array}{l}4.03 \\
4.18\end{array}$ & 4.12 & 0.54 & $\begin{array}{l}0.55 \\
0.53\end{array}$ & C. 51 & 0.54 & $\begin{array}{l}0.30 \\
0.32\end{array}$ & $\begin{array}{r}0.30 \\
0.39\end{array}$ \\
\hline 30 & 09 & 42 & 054 & 05 & 54 & 058 & 030 & 031 \\
\hline 30 & 4.11 & 4.13 & 0.55 & 0.57 & 0.55 & 0.55 & 0.31 & 0.31 \\
\hline 40 & 4.07 & 4.18 & 0.54 & 0.55 & 0.53 & 0.53 & 0.31 & 0.29 \\
\hline 40 & 4.13 & 4.07 & 0.55 & 0.54 & 0.53 & 0.53 & 0.32 & 0.29 \\
\hline 50 & 4.18 & 5.24 & 0.55 & 0.73 & 0.52 & 0.74 & 0.30 & 0.30 \\
\hline 50 & 4.16 & 5.41 & 0.55 & 0.77 & 0.54 & 0.80 & 0.31 & 0.28 \\
\hline 60 & 4.15 & 5.52 & 0.55 & 0.77 & 0.53 & 0.79 & 0.32 & 0.29 \\
\hline 60 & 4.04 & 5.46 & 0.55 & 0.76 & 0.53 & 0.81 & 0.31 & 0.29 \\
\hline
\end{tabular}

\footnotetext{
(a) A11 results in $\mu \mathrm{g} / \mathrm{L}$ unless otherwise noted.

(b) TCA = trichloroethane.

(c) TCE = trichloroethene.

(d) $P C E=$ perch7oroethene.

(e) $\mathrm{CCl}_{4}=$ carbon tetrach]oride.

(f) $N A=$ not applicable.
} 


\subsection{REFERENCES}

40 CFR 265. 1987. U.S. Envirommental Protection Agency, "Interim Status Standards for Owners and 0perators of Hazardous Waste Treatment, Storage, and Disposal Facilities." U.S. Code of Federal Regulations.

DOE. 1981a. "Environmental Protection, Safety, and Health Protection Program for OOE Operations." DOE Order 5480.1, U.S. Department of Energy, Washington, D.C.

D0E. 1981b. "Environmental Protection, Safety, and Hea7th Protection Information Reporting Requirements." DoE Order 5484.1, U.S. Department of Energy Washington, D.C.

Eddy, P. A., D. A. Myers, and J. R. Raymond. 1978. Vertical Contamination in the Unconfined Groundwater at the Hanford Site, Washington. PNL-2724, Pacific Northwest Laboratory, Richland, Washington.

EPA. 1982. Test Methods for Evaluating Solid Waste: Physical/Chemical Methods. 2nd ed., SW-846, Washington, D.C.

EPA. 1986. Ground-Water Monitoring Technical Enforcement Guidance Document. OWSER 9950.1, U.S. Environmenta1 Protection Agency, Washington, D.C.

Evans, J. C., D. I. Dennison, R. W. Bryce, P. J. Mitche11, D. R. Sherwood, K. M. Krupka, N. W. Hinman, E. A. Jacobson, and M. D. Freshley. 1988. Hanford Site Ground-Water Monitoring for July Through December 1987. PNL-6315-2, Pacific Northwest Laboratory, Richland, Washington.

Jaquish, R. E., and P. J. Mitche11, eds. 1988. Environmental Monitoring at Hanford for 1987. PNL-6464, Pacific Northwest Laboratory, Richland, Washington.

PNL. 1988a. Ground-Water Monitoring Compliance Projects for Hanford Site Facilities: Progress Report for the Period January 1 to March 31, 1988.

PNL-6581, Pacific Northwest Laboratory, Richiand, Washington.

PNL. 1988b. Ground-Water Monitoring Compliance Projects for Hanford Site Facilities: Progress Report for the Period April 1 to June 30, 1988.

PNL-6675, Pacific Northwest Laboratory, Richland, Washington.

Resource Conservation and Recovery Act of 1976 (RCRA). 1976. Public Law 94-580, October 21, 1976, 90 Stat. 2795, Title 42.

Serkowski, J. A., A. G. Law, J. J. Ammerman, and A. L. Schatz. 1988. Results of Ground-Water Monitoring for Radionuclides in the Separations Area 1987. WHC-FP-0152, Westinghouse Hanford Company, Richland, Washington.

Soldat, J. K. 1976. "Radiation Doses from Iodine-129 in the Environment." Health Physics 30:61-70. 
Stenner, R. D., K. H. Cramer, K. A. Higley, S. J. Jette, D. A. Lamar, T. J. McLaughl in, D. R. Sherwood, N. C. VanHouten. 1988. Evaluation Method and Results. Volume 1 of Hazard Ranking System Evaluation of CERCLA Inactive Waste Sites at Hanford. PNL-6456, Pacific Northwest Laboratory, Richland, Washington.

USGS. 1977. National Handbook of Recommended Methods for Water-Data Acquisition. Office of Water Data Coordination, U.S. Geological Survey, Reston, Virginia.

WAC 173-303. 1986. Washington State Department of Ecology, "Dangerous Waste Regulations." Washington Administrative Code, Chapter 173-303, 01 ympia, Washington.

WAC 173-304. 1986. Washington State Department of Ecology, "Minimum Functional Standards for Solid Waste Handling." Washington Administrative Code, Chapter 173-304, 0lympia, Washington.

Zimmerman, D. A., A. E. Reisenauer, G. D. Black, and M. A. Young. 1986. Hanford Site Water Table Changes 1950 Through 1980-Data Observations and Evaluation. PNL-5506, Prepared for Rockwell Hanford Operations by Pacific Northwest Laboratory, Richland, Washington. 


\section{APPENDIX A}

WATER LEVELS MEASURED DURING JUNE 1988 


\section{APPENDIX A}

\section{WATER LEVELS MEASURED DURING JUNE 1988}

This appendix contains water levels measured in unconfined aquifer wells in June 1988.

A. 1 

IABLE A.1. Hanford Water Level Measurements in the Unconfined Aquifer, June 1988

\begin{tabular}{|c|c|c|c|c|}
\hline We11 Number & Date & $\begin{array}{l}\text { Elevation } \\
\text { of Casing, } \\
\text { ft Above MSL }\end{array}$ & $\begin{array}{l}\text { Depth to } \\
\text { water. ft }\end{array}$ & $\begin{array}{c}\text { Water Table } \\
\text { Elevation, } \\
\text { ft Above MSL }\end{array}$ \\
\hline $199-\mathrm{B} 04-01$ & $06 / 16 / 88$ & 461.80 & 63.11 & 398.69 \\
\hline $199-\mathrm{D} 02-05$ & $06 / 14 / 88$ & 460.87 & 74.32 & 386.55 \\
\hline $199-F 05-01$ & $06 / 14 / 88$ & 406.56 & 36.36 & 370.20 \\
\hline $199-\mathrm{H} 03-01$ & $06 / 14 / 88$ & 421.98 & 45.74 & 376.24 \\
\hline $199-\mathrm{K} 00-11$ & $06 / 16 / 88$ & 467.65 & 73.86 & 393.79 \\
\hline $299-\mathrm{E} 13-10$ & $06 / 22 / 88$ & 737.70 & 333.36 & 404.34 \\
\hline $299-E 13-12$ & $06 / 22 / 88$ & 733.49 & 327.69 & 405.80 \\
\hline $299-E 13-14$ & $06 / 22 / 88$ & 745.37 & 339.62 & 405.75 \\
\hline $299-E 17-05$ & $06 / 10 / 88$ & 718.65 & 314.17 & 404.48 \\
\hline $299-E 17-08$ & $06 / 10 / 88$ & 718.73 & 313.74 & 404.99 \\
\hline $299-E 23-01$ & $06 / 10 / 88$ & 709.65 & 304.27 & 405.38 \\
\hline $299-E 23-02(0)$ & $06 / 10 / 88$ & 721.26 & 315.56 & 405.70 \\
\hline $299-E 24-02$ & $06 / 10 / 88$ & 717.19 & 312.68 & 404.51 \\
\hline $299-E 24-04$ & $06 / 10 / 88$ & 697.00 & 291.50 & 405.50 \\
\hline $299-\mathrm{E} 25-04$ & $06 / 30 / 88$ & 659.39 & 252.41 & 406.98 \\
\hline $299-E 25-11$ & $06 / 21 / 88$ & 681.28 & 275.68 & 405.60 \\
\hline $299-\mathrm{E} 25-19$ & $06 / 21 / 88$ & 677.20 & 272.03 & 405.17 \\
\hline $299-E 25-21$ & $06 / 21 / 88$ & 677.27 & 271.32 & 405.95 \\
\hline $299-E 25-25$ & $06 / 21 / 88$ & 669.42 & 261.84 & 407.58 \\
\hline $299-E 25-26$ & $06 / 22 / 88$ & 668.51 & 262.18 & 406.37 \\
\hline $299-\mathrm{E} 25-27$ & $06 / 21 / 88$ & 676.13 & 269.61 & 406.52 \\
\hline $299-\mathrm{E} 26-01$ & $06 / 09 / 88$ & 617.25 & 211.68 & 406.17 \\
\hline $299-E 26-04$ & $06 / 21 / 88$ & 645.82 & 239.52 & 406.30 \\
\hline $299-E 27-01$ & $06 / 10 / 88$ & 681.05 & 275.55 & 405.50 \\
\hline $299-\mathrm{E} 28-07$ & $06 / 13 / 88$ & 685.91 & 281.36 & 404.55 \\
\hline $299-E 28-12$ & $06 / 13 / 88$ & 708.60 & 303.08 & 405.52 \\
\hline $299-E 28-17$ & $06 / 10 / 88$ & 708.56 & 302.35 & 406.21 \\
\hline $299-\mathrm{E} 28-18$ & $06 / 13 / 88$ & 692.58 & 287.50 & 405.08 \\
\hline $299-\mathrm{E} 32-01$ & $06 / 09 / 88$ & 656.17 & 251.11 & 405.06 \\
\hline $299-E 33-07$ & $06 / 21 / 88$ & 626.58 & 221.68 & 404.90 \\
\hline $299-E 33-08$ & $06 / 09 / 88$ & 651.03 & 245.69 & 405.34 \\
\hline $299-E 33-14$ & $06 / 09 / 88$ & 622.12 & 216.80 & 405.32 \\
\hline $299-E 33-17$ & $06 / 21 / 88$ & 631.65 & 226.44 & 405.21 \\
\hline $299-E 33-21$ & $06 / 21 / 88$ & 668.40 & 263.08 & 405.32 \\
\hline $299-\mathrm{E} 34-01$ & $06 / 09 / 88$ & 629.42 & 224.00 & 405.42 \\
\hline $299-E 34-05$ & $06 / 09 / 88$ & 590.79 & 184.86 & 405.93 \\
\hline $299-E 34-06$ & $06 / 09 / 88$ & 579.83 & 192.69 & 405.14 \\
\hline $299-W 10-02$ & $06 / 14 / 88$ & 674.33 & 207.12 & 467.21 \\
\hline $299-W 10-05$ & $06 / 14 / 88$ & 672.31 & 204.46 & 467.85 \\
\hline $299-W 10-08$ & $06 / 21 / 88$ & 680.33 & 213.99 & 466.34 \\
\hline $299-W 11-07$ & $06 / 22 / 88$ & 709.11 & 243.48 & 465.63 \\
\hline $299-W 11-09$ & $06 / 13 / 88$ & 722.94 & 261.55 & 461.39 \\
\hline $299-W 11-10$ & $06 / 13 / 88$ & 728.89 & 270.18 & 458.71 \\
\hline $299-W 11-12$ & $06 / 14 / 88$ & 679.58 & 212.38 & 467.20 \\
\hline $299-W 11-13$ & $06 / 14 / 88$ & 692.27 & 223.92 & 468.35 \\
\hline $299-W 11-23$ & $06 / 21 / 88$ & 685.86 & 219.66 & 466.20 \\
\hline $299-W 12-01$ & $06 / 13 / 88$ & 726.46 & 273.25 & 453.21 \\
\hline
\end{tabular}


IABLE A.1. (contd)

\begin{tabular}{lcc} 
Well Number & Date \\
\cline { 1 - 1 } $299-$ W14-01 & $06 / 14 / 88$ \\
$299-W 15-02$ & $06 / 22 / 88$ \\
$299-W 15-04$ & $06 / 14 / 88$ \\
$299-W 15-05$ & $06 / 16 / 88$ \\
$299-W 15-10$ & $06 / 22 / 88$ \\
$299-W 18-15$ & $06 / 21 / 88$ \\
$299-W 19-01$ & $06 / 15 / 88$ \\
$299-W 19-02$ & $06 / 16 / 88$ \\
$299-W 19-03$ & $06 / 21 / 88$ \\
$299-W 19-04$ & $06 / 15 / 88$ \\
$299-W 19-13$ & $06 / 15 / 88$ \\
$299-W 19-14$ & $06 / 15 / 88$ \\
$299-W 19-15$ & $06 / 15 / 88$ \\
$299-W 19-19$ & $06 / 21 / 88$ \\
$299-W 21-01$ & $06 / 16 / 88$ \\
$299-W 22-07$ & $06 / 16 / 88$ \\
$299-W 22-17$ & $06 / 21 / 88$ \\
$299-W 22-22$ & $06 / 16 / 88$ \\
$299-W 23-04$ & $06 / 20 / 88$ \\
$299-W 23-11$ & $06 / 09 / 88$ \\
$399-08-01$ & $06 / 03 / 88$ \\
$699-02-03$ & $06 / 03 / 88$ \\
$699-03-45$ & $06 / 17 / 88$ \\
$699-08-17$ & $06 / 07 / 88$ \\
$699-08-25$ & $06 / 20 / 88$ \\
$699-09-E 02$ & $06 / 03 / 88$ \\
$699-10-54$ & $06 / 17 / 88$ \\
$699-10-E 12$ & $06 / 03 / 88$ \\
$699-11-45 A$ & $06 / 17 / 88$ \\
$699-14-38$ & $06 / 20 / 88$ \\
$699-14-47$ & $06 / 17 / 88$ \\
$699-14-E 6 T$ & $06 / 03 / 88$ \\
$699-15-15 A$ & $06 / 09 / 88$ \\
$699-15-26$ & $06 / 20 / 88$ \\
$699-17-05$ & $06 / 09 / 88$ \\
$699-17-70$ & $06 / 17 / 88$ \\
$699-19-43$ & $06 / 17 / 88$ \\
$699-20-20$ & $06 / 02 / 88$ \\
$699-20-39$ & $06 / 02 / 88$ \\
$699-20-E 12$ & $06 / 09 / 88$ \\
$699-21-17$ & $06 / 09 / 88$ \\
$699-24-01 T$ & $06 / 09 / 88$ \\
$699-24-33$ & $06 / 02 / 88$ \\
$699-25-55$ & $06 / 14 / 88$ \\
$699-25-70$ & $06 / 09 / 88$ \\
$699-26-15$ & $06 / 30 / 88$ \\
$699-27-08$ & $06 / 09 / 88$
\end{tabular}

\section{Elevation}

of Casing, ft Above MSL

668.83

693.51

662.00

670.95

676.00

660.96

674.04

694.04

695.12

715.52

695.08

693.21

693.28

694.90

699.26

697.41

672.09

690.38

662.82

664.14

394.87

477.14

504.54

522.44

509.30

418.09

516.40

430.86

578.58

514.89

587.23

458.38

547.14

523.83

433.13

563.18

551.58

505.58

539.98

437.25

527.31

475.54

524.21

676.55

629.56

442.64

465.67
Depth to

Water, $\mathrm{ft}$

199.82

225.42

191.04

199.74

206.88

189.24

199.72

231.07

227.43

252.90

227.84

225.77

225.19

232.97

241.59

224.77

204.82

227.93

192.12

195.38

51.36

87.28

93.23

123.62

108.95

46.20

103.52

73.63

167.33

110.83

175.68

95.12

148.24

121.63

44.94

88.94

146.51

103.17

135.39

79.74

128.98

99.12

119.96

262.80

180.12

43.15

70.62
Water Table

Elevation,

ft Above MSl.

468.96

468.09

470.96

471.21

469.12

471.72

474.32

462.97

467.69

462.62

467.24

467.44

468.09

461.93

457.67

462.64

467.27

462.45

470.70

468.76

343.51

389.86

411.30

398.82

400.35

371.89

412.88

357.23

411.25

404.06

411.55

363.26

398.90

402.20

388.25

474.24

405.07

402.41

404.59

357.51

398.33

376.42

404.25

413.75

449.44

399.49

395.05 
TABLE A.1. (contd)

$\begin{array}{lc}\text { Wel1 Number } & \text { Date } \\ 699-28-40 & 06 / 02 / 88 \\ 699-28-52 A & 06 / 14 / 88 \\ 699-29-78 & 06 / 09 / 88 \\ 699-31-31 & 06 / 02 / 88 \\ 699-32-22 & 06 / 13 / 88 \\ 699-32-43 & 06 / 10 / 88 \\ 699-32-62 & 06 / 09 / 88 \\ 699-32-70 \mathrm{~B} & 06 / 09 / 88 \\ 699-32-72 & 06 / 09 / 88 \\ 699-32-77 & 06 / 09 / 88 \\ 699-33-42 & 06 / 10 / 88 \\ 699-33-56 & 06 / 16 / 88 \\ 699-34-39 \mathrm{~A} & 06 / 02 / 88 \\ 699-34-41 & 06 / 10 / 88 \\ 699-34-42 & 06 / 10 / 88 \\ 699-34-51 & 06 / 14 / 88 \\ 699-34-88 & 06 / 07 / 88 \\ 699-35-09 & 06 / 09 / 88 \\ 699-35-66 & 06 / 08 / 88 \\ 699-35-70 & 06 / 08 / 88 \\ 699-35-78 \mathrm{~A} & 06 / 09 / 88 \\ 699-36-61 \mathrm{~A} & 06 / 09 / 88 \\ 699-36-93 & 06 / 12 / 88 \\ 699-37-43 & 06 / 10 / 88 \\ 699-37-82 \mathrm{~A} & 06 / 16 / 88 \\ 699-38-65 & 06 / 09 / 88 \\ 699-38-70 & 06 / 08 / 88 \\ 699-39-39 & 06 / 15 / 88 \\ 699-39-79 & 06 / 20 / 88 \\ 699-40-01 & 06 / 10 / 88 \\ 699-40-33 \mathrm{~A} & 06 / 13 / 88 \\ 699-40-62 \mathrm{~A} & 06 / 07 / 88 \\ 699-41-23 & 06 / 13 / 88 \\ 699-42-12 \mathrm{~A} & 06 / 10 / 88 \\ 699-42-40 \mathrm{~B} & 06 / 13 / 88 \\ 699-43-104 & 06 / 17 / 88 \\ 699-43-42 & 06 / 14 / 88 \\ 699-43-89 & 06 / 07 / 88 \\ 699-44-64 & 06 / 09 / 88 \\ 699-45-42 & 06 / 14 / 88 \\ 699-45-69 & 06 / 07 / 88 \\ 699-46-21 & 06 / 13 / 88 \\ 699-47-35 \mathrm{~B} & 06 / 13 / 88 \\ 699-47-46 & 06 / 14 / 88 \\ 699-47-60 & 06 / 15 / 88 \\ 699-48-07 & 06 / 08 / 88 \\ 699-48-71 & 06 / 15 / 88\end{array}$

\section{Elevation \\ of Casing,}

ft Above MSL

559.44

684.67

647.05

529.32

517.55

516.62

707.09

666.61

668.16

653.74

516.00

717.03

537.07

570.89

540.20

736.76

632.82

499.83

725.65

693.72

660.65

748.11

644.77

690.17

636.75

753.33

710.67

536.65

673.52

438.71

518.05

747.78

466.50

514.27

546.46

766.07

564.48

644.15

725.60

577.33

725.46

522.02

476.65

580.14

649.84

384.72

688.15
Depth to

Water, ft

154.59

278.18

181.64

125.01

115.44

111.53

277.67

213.74

212.77

189.64

110.82

310.76

132.09

165.67

135.00

331.38

161.49

113.45

285.68

241.00

191.81

338.89

171.42

284.52

167.47

322.45

256.01

116.32

203.91

75.30

106.89

430.40

67.50

138.02

121.98

262.83

143.63

176.46

317.46

158.37

275.00

130.54

63.04

173.71

245.32

26.23

240.77
Water Table

Elevation,

ft Above MSL

404.85

406.49

465.41

404.31

402.11

405.09

429.42

452.87

455.39

464.10

405.18

406.27

404.98

405.22

405.20

405.73

470.85

386.60

439.97

452.72

468.84

409.22

473.35

406.06

469.28

430.88

454.66

420.33

469.61

363.41

411.16

407.38

399.00

376.25

424.48

503.24

420.85

467.69

408.14

418.96

449.54

391.48

413.61

406.43

404.52

358.49

447.38 
TABLE A.1. (contc)

\begin{tabular}{lc} 
Well Number & Date \\
\cline { 1 - 1 } $699-49-13 \mathrm{~F}$ & $06 / 08 / 88$ \\
$699-49-28$ & $06 / 08 / 8 B$ \\
$699-49-55 \mathrm{~A}$ & $06 / 16 / 88$ \\
$699-49-57$ & $06 / 16 / 88$ \\
$699-49-79$ & $06 / 16 / 88$ \\
$699-50-28 \mathrm{~B}$ & $06 / 08 / 88$ \\
$699-50-30$ & $06 / 08 / 88$ \\
$699-50-42$ & $06 / 10 / 88$ \\
$699-50-53$ & $06 / 16 / 88$ \\
$699-50-85$ & $06 / 16 / 88$ \\
$699-51-63$ & $06 / 17 / 88$ \\
$699-51-75$ & $06 / 17 / 88$ \\
$699-52-19$ & $06 / 08 / 88$ \\
$699-53-35$ & $06 / 08 / 88$ \\
$699-53-47 \mathrm{~A}$ & $06 / 10 / 88$ \\
$699-53-47 \mathrm{~B}$ & $06 / 20 / 88$ \\
$699-53-48 \mathrm{~A}$ & $06 / 20 / 88$ \\
$699-53-48 \mathrm{~B}$ & $06 / 20 / 88$ \\
$699-53-55 \mathrm{~B}$ & $06 / 20 / 88$ \\
$699-53-55 \mathrm{C}$ & $06 / 20 / 88$ \\
$699-54-19$ & $06 / 08 / 88$ \\
$699-54-48$ & $06 / 20 / 88$ \\
$699-55-21$ & $06 / 08 / 88$ \\
$699-55-40$ & $06 / 17 / 88$ \\
$699-55-50 \mathrm{C}$ & $06 / 20 / 88$ \\
$699-55-70$ & $06 / 15 / 88$ \\
$699-55-76$ & $06 / 17 / 88$ \\
$699-55-89$ & $06 / 06 / 88$ \\
$699-55-95$ & $06 / 07 / 88$ \\
$699-57-25 \mathrm{~A}$ & $06 / 08 / 88$ \\
$699-57-29 \mathrm{~B}$ & $06 / 10 / 88$ \\
$699-57-83$ & $06 / 15 / 88$ \\
$699-58-24$ & $06 / 08 / 88$ \\
$699-59-32$ & $06 / 10 / 88$ \\
$699-59-58$ & $06 / 06 / 88$ \\
$699-59-80 \mathrm{~B}$ & $06 / 15 / 88$ \\
$699-60-32$ & $06 / 10 / 88$ \\
$699-60-60$ & $06 / 06 / 88$ \\
$699-61-37$ & $06 / 10 / 88$ \\
$699-61-41$ & $06 / 10 / 88$ \\
$699-61-62$ & $06 / 06 / 88$ \\
$699-61-66$ & $06 / 06 / 88$ \\
$699-62-31$ & $06 / 10 / 88$ \\
$699-62-43 \mathrm{~A}$ & $06 / 10 / 88$ \\
$699-63-25 \mathrm{~A}$ & $06 / 13 / 88$ \\
$699-63-51$ & $06 / 10 / 88$ \\
$699-63-58$ & $06 / 06 / 88$
\end{tabular}

Elevation

of Casing,

ft Above MSL

412.72

535.40

530.14

55.00

688.59

537.30

528.84

466.84

556.30

739.35

571.84

641.51

411.08

530.99

438.28

438.58

44.00

442.71

576.16

576.08

383.60

457.02

395.96

543.13

444.43

569.03

583.24

617.43

777.05

414.57

416.18

577.96

418.80

424.29

497.77

583.25

424.30

512.03

442.94

428.92

497.51

522.18

434.12

432.30

396.15

424.54

491.90
Depth to

Water, $f t$

51.41

141.39

125.16

148.00

230.82

143.75

135.05

55.36

151.67

282.32

165.33

190.75

49.56

134.05

31.84

32.14

37.79

37.84

172.56

172.23

21.99

53.23

35.59

133.24

40.65

136.16

138.20

162.44

310.79

50.60

54.45

144.70

57.19

62.27

94.83

155.64

63.47

108.44

60.73

32.49

94.00

119.69

72.34

35.52

33.87

23.86

89.80
Water Table

Elevation,

ft Above MS

361.31

394.01

404.98

404.81

457.77

393.55

393.79

411.48

404.63

457.03

406.68

450.76

361.52

396.94

406.44

406.44

405.06

404.87

403.60

403.85

361.61

403.79

360.37

409.89

403.78

432.87

445.04

454.99

466.26

363.97

361.73

433.26

361.61

362.02

402.94

427.61

361.83

403.59

382.21

396.43

403.51

402.49

361.78

396.78

361.28

400.68

402.10 
IABLE A. 1. (contd)

\begin{tabular}{lc} 
We1l Number & Date \\
\hline $699-63-90$ & $06 / 06 / 88$ \\
$699-64-27$ & $06 / 13 / 88$ \\
$699-64-62$ & $06 / 16 / 88$ \\
$699-65-50$ & $06 / 10 / 88$ \\
$699-65-59 A$ & $06 / 14 / 88$ \\
$699-65-72$ & $06 / 06 / 88$ \\
$699-65-83$ & $06 / 06 / 88$ \\
$699-65-95$ & $06 / 16 / 88$ \\
$699-66-103$ & $06 / 16 / 88$ \\
$699-66-23$ & $06 / 13 / 88$ \\
$699-66-38$ & $06 / 13 / 88$ \\
$699-66-39$ & $06 / 13 / 88$ \\
$699-66-58$ & $06 / 14 / 88$ \\
$699-66-64$ & $06 / 16 / 88$ \\
$699-66-91$ & $06 / 16 / 88$ \\
$699-67-51$ & $06 / 20 / 88$ \\
$699-67-86$ & $06 / 16 / 88$ \\
$699-67-98$ & $06 / 16 / 88$ \\
$699-68-105$ & $06 / 16 / 88$ \\
$699-69-38$ & $06 / 13 / 88$ \\
$699-69-45(0)$ & $06 / 13 / 88$ \\
$699-70-23$ & $06 / 13 / 88$ \\
$699-70-68$ & $06 / 16 / 88$ \\
$699-71-30$ & $06 / 13 / 88$ \\
$699-71-52$ & $06 / 10 / 88$ \\
$699-71-77$ & $06 / 16 / 88$ \\
$699-72-73$ & $06 / 16 / 88$ \\
$699-72-88$ & $06 / 16 / 88$ \\
$699-72-92$ & $06 / 06 / 88$ \\
$699-74-44$ & $06 / 13 / 88$ \\
$699-74-48$ & $06 / 13 / 88$ \\
$699-77-36$ & $06 / 13 / 88$ \\
$699-77-54$ & $06 / 14 / 88$ \\
$699-78-62$ & $06 / 14 / 88$ \\
$699-81-38$ & $06 / 13 / 88$ \\
$699-82-45 A$ & $06 / 04 / 88$ \\
$699-83-47$ & $06 / 14 / 88$ \\
$699-86-42$ & $06 / 14 / 88$ \\
$699-87-55$ & $06 / 14 / 88$ \\
$699-89-35$ & $06 / 14 / 88$ \\
$699-90-45$ & $06 / 14 / 88$ \\
$699-91-37$ & $06 / 14 / 88$ \\
$699-92-49$ & $06 / 14 / 88$ \\
$699-97-43$ & $06 / 14 / 88$ \\
$699-503-25$ & $06 / 07 / 88$ \\
$699-503-E 12$ & $06 / 03 / 88$ \\
$699-506-E 04 D$ & $06 / 03 / 88$
\end{tabular}

\section{Elevation \\ of Casing,} ft Above MSL

509.73

414.29

500.25

467.06

506.96

540.28

485.63

452.26

463.01

389.01

436.20

453.70

503.33

505.92

467.75

524.59

472.39

455.47

451.85

422.93

487.18

391.71

526.21

400.68

523.04

472.28

482.57

437.37

452.22

445.18

487.18

412.28

480.59

469.88

406.47

413.73

435.27

409.92

458.63

397.46

422.15

422.93

432.00

421.81

523.50

397.90

430.47
Depth to

Water, ft

111.65

52.56

98.50

66.77

105.31

140.83

87.18

53.49

65.76

26.96

33.02

47.25

101.82

104.91

68.19

123.82

74.01

57.37

56.21

21.40

87.15

29.07

126.38

29.92

122.87

75.74

84.81

36.88

52.58

48.03

88.13

35.71

82.22

73.50

26.97

24.40

45.88

25.00

70.30

26.43

37.32

49.50

48.42

43.06

124.15

43.74

58.44
Water Table

Elevation,

ft Above MSL

398.08

361.73

401.75

400.69

401.65

399.45

398.45

398.77

397.25

362.05

403.18

406.45

401.51

401.01

399.56

400.77

398.38

398.10

395.64

401.53

400.03

362.64

399.83

370.76

400.17

396.97

397.76

400.49

399.64

397.15

399.05

376.56

398.37

396.38

379.50

389.33

389.39

384.92

388.33

371.03

384.83

373.43

383.58

378.75

399.35

354.16

372.03 
TABLE A.1. (contd)

$\begin{array}{lc}\text { Well Number } & \text { Date } \\ \text { 699-S06-E14A } & 06 / 03 / 88 \\ 699-\mathrm{S} 07-34 & 06 / 17 / 88 \\ 699-\mathrm{S} 08-19 & 06 / 07 / 88 \\ 699-\mathrm{S} 12-03 & 06 / 03 / 88 \\ 699-\mathrm{S} 12-29 & 06 / 17 / 88 \\ 699-\mathrm{S} 14-20 & 06 / 07 / 88 \\ 699-\mathrm{S} 18-\mathrm{E} 02 \mathrm{~A} & 06 / 03 / 88 \\ 699-\mathrm{S} 19-\mathrm{E} 13 & 06 / 03 / 88 \\ 699-\mathrm{S} 29-\mathrm{E} 12 & 06 / 02 / 88 \\ 699-\mathrm{S} 30-\mathrm{E} 15 \mathrm{~A} & 06 / 07 / 88 \\ 699-\mathrm{S} 31-01 & 06 / 02 / 88\end{array}$

$\begin{array}{ll}\text { Elevation } & \\ \text { of Casing, } & \text { Depth to } \\ \mathrm{ft} \text { Above MSL } & \text { Water, } \mathrm{ft}\end{array}$

378.29

527.12

503.81

435.52

487.68

492.74

434.85

394.55

387.97

400.39

460.11
27.97

119.59

107.73

55.53

83.03

92.02

75.58

49.63

42.86

57.29

81.74
Water Table?

Elevation,

ft Above MSL

350.32

407.53

396.08

379.99

404.65

400.72

359.27

344.92

345.11

343.10

378.37 
APPENDIX B

MAPS OF OPERATIONAL AND FACILITY-SPECIFIC MONITORING WELL NETWORKS 


\section{APPENDIX B}

MAPS OF OPERATIONAL AND FACILITY-SPECIFIC MONITORING WELL NETWORKS

Figures B.1 through B.10 (foldouts) are maps of the operational and facility-specific monitoring well networks.

B. 1 



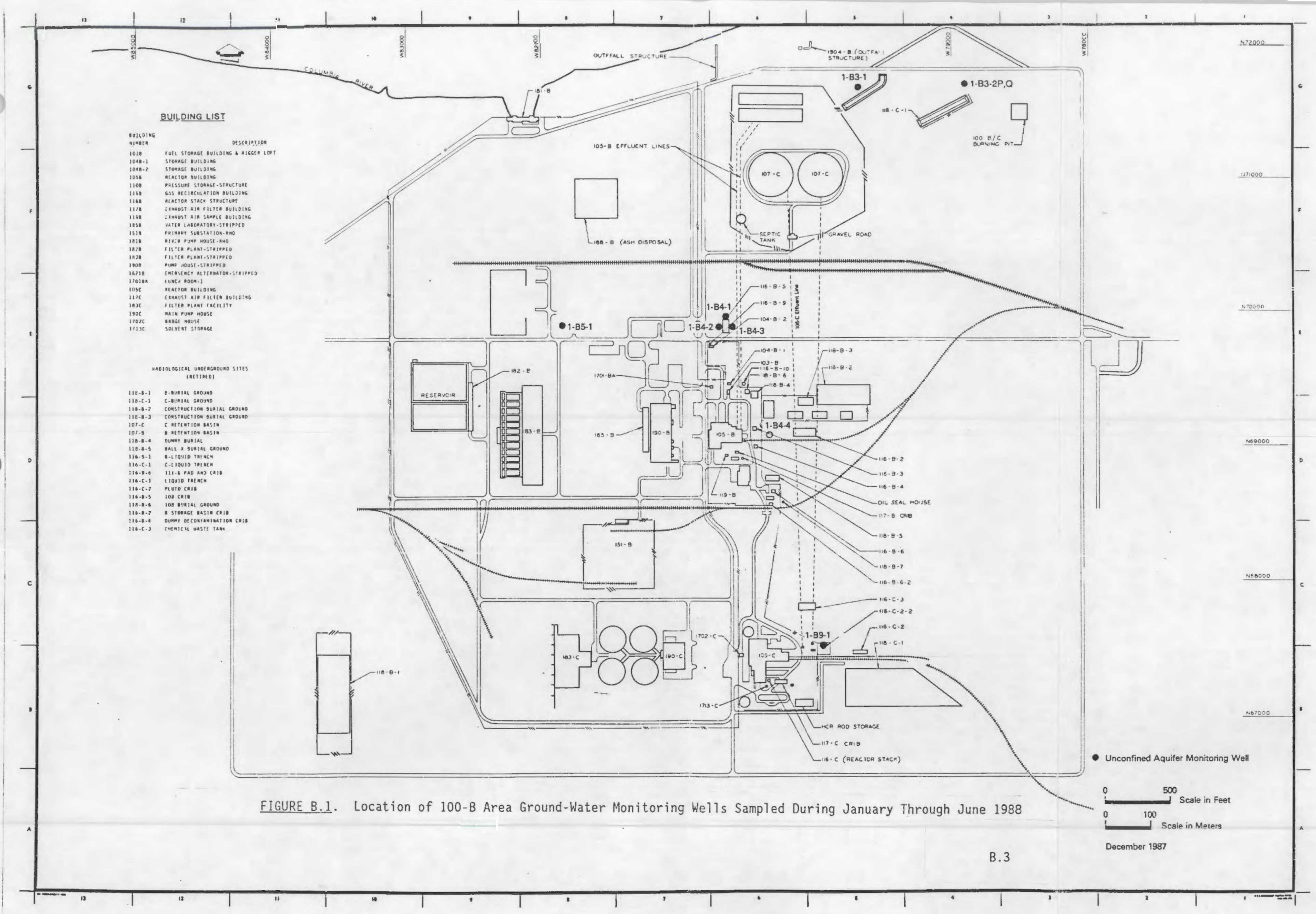





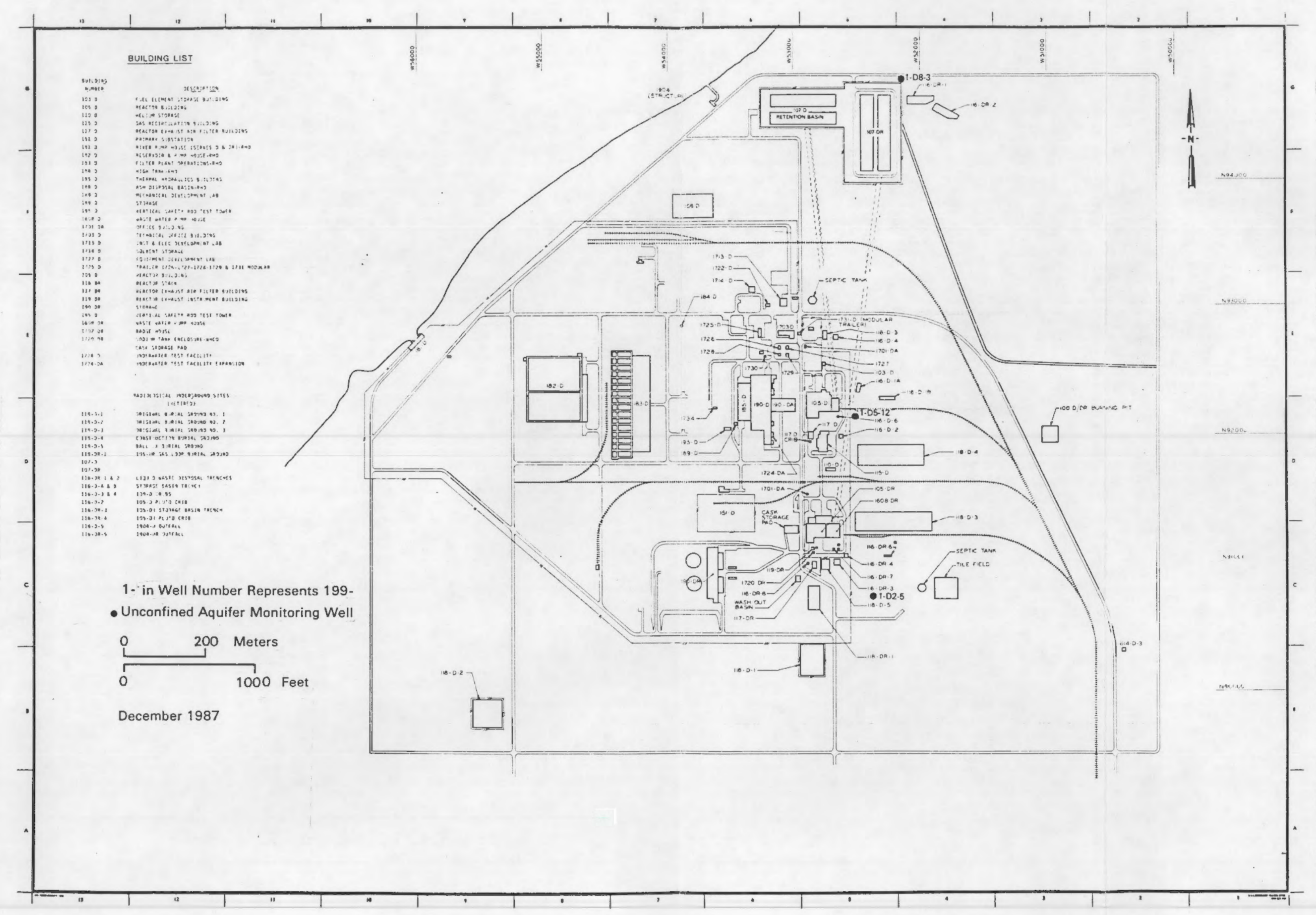

FIGURE B.2. Location of 100-D Area Ground-Water Monitoring Wells Sampled During January Through June 1988 



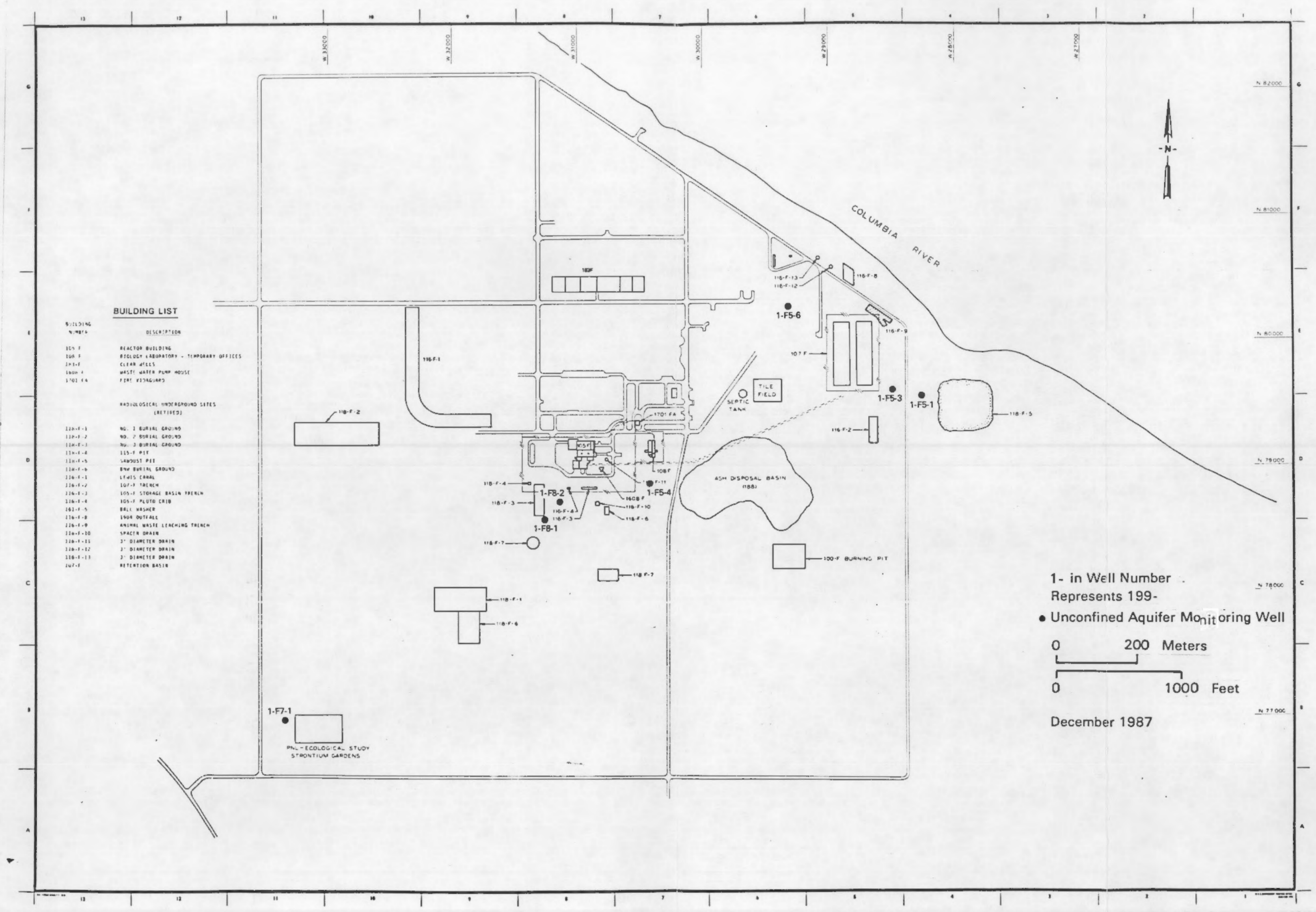

FIGURE B.3. Location of 100-F Area Ground-Water Monitoring Wells Sampled During January Through June 1988 



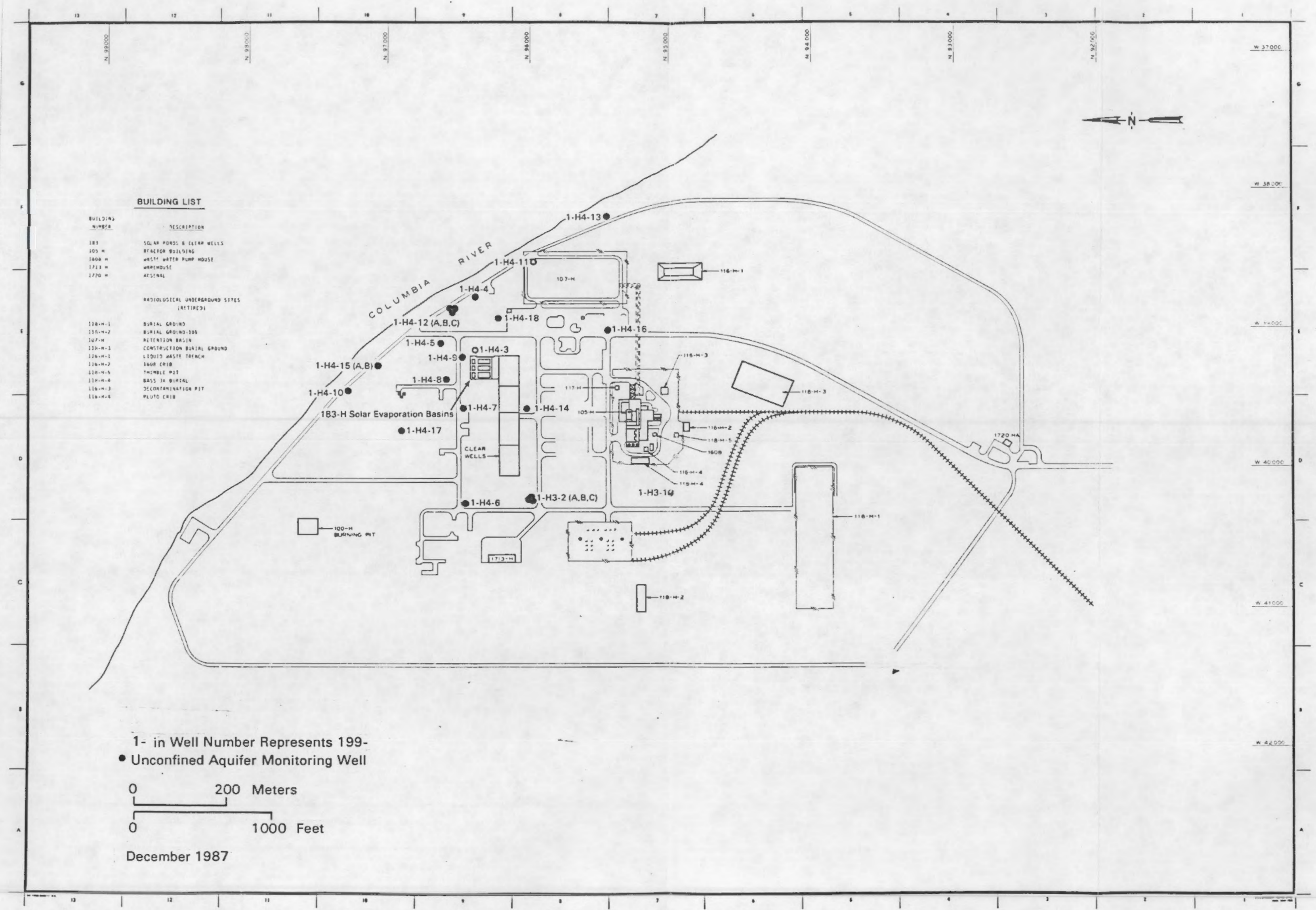

FIGURE B.4. Location of 100-H Area Ground-Water Monitoring Wells Sampled During January Through June 1988 



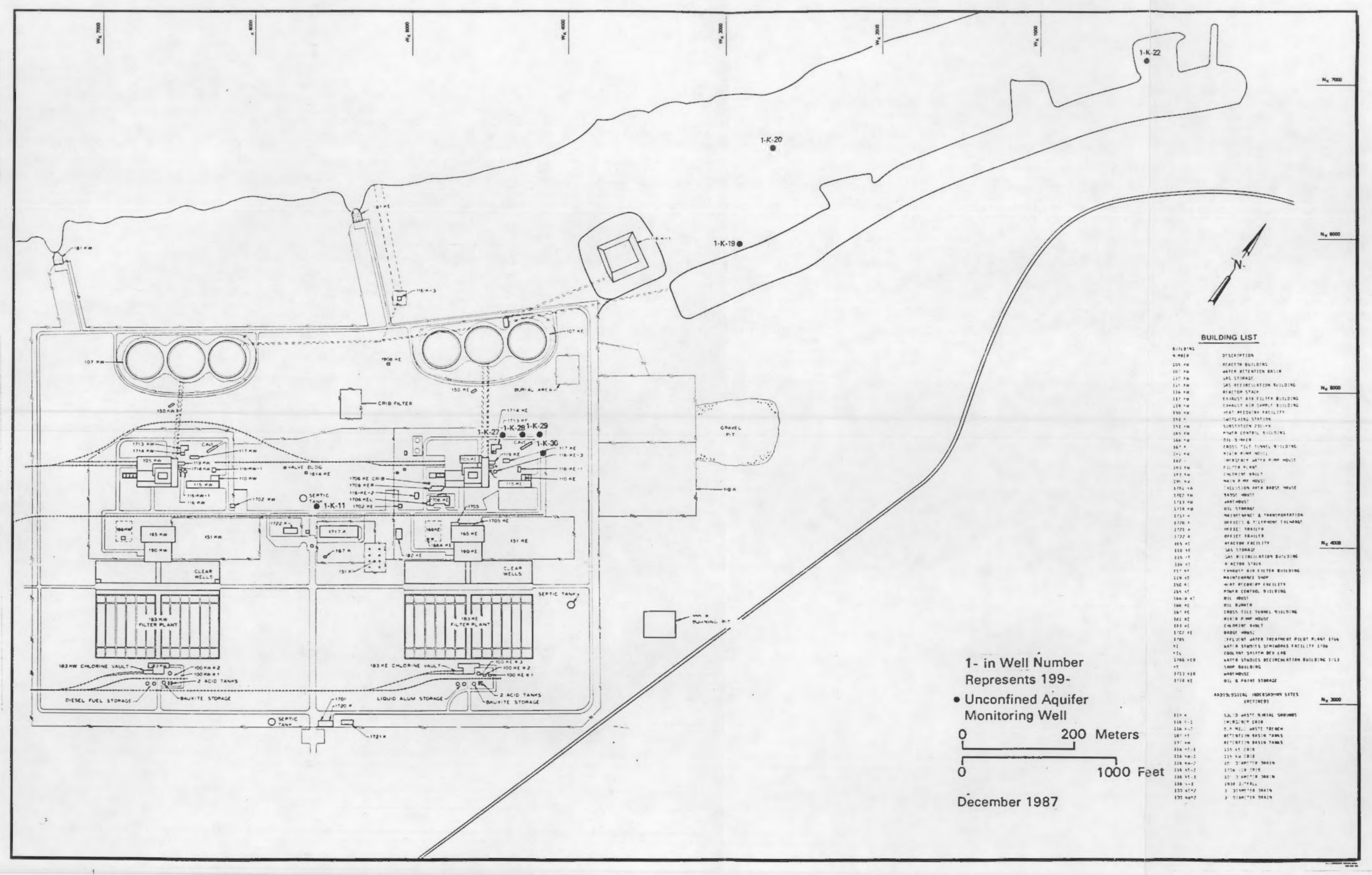

FIGURE B.5. Location of 100-K Area Ground-Water Monitoring Wells Sampled During January Through June 1988 



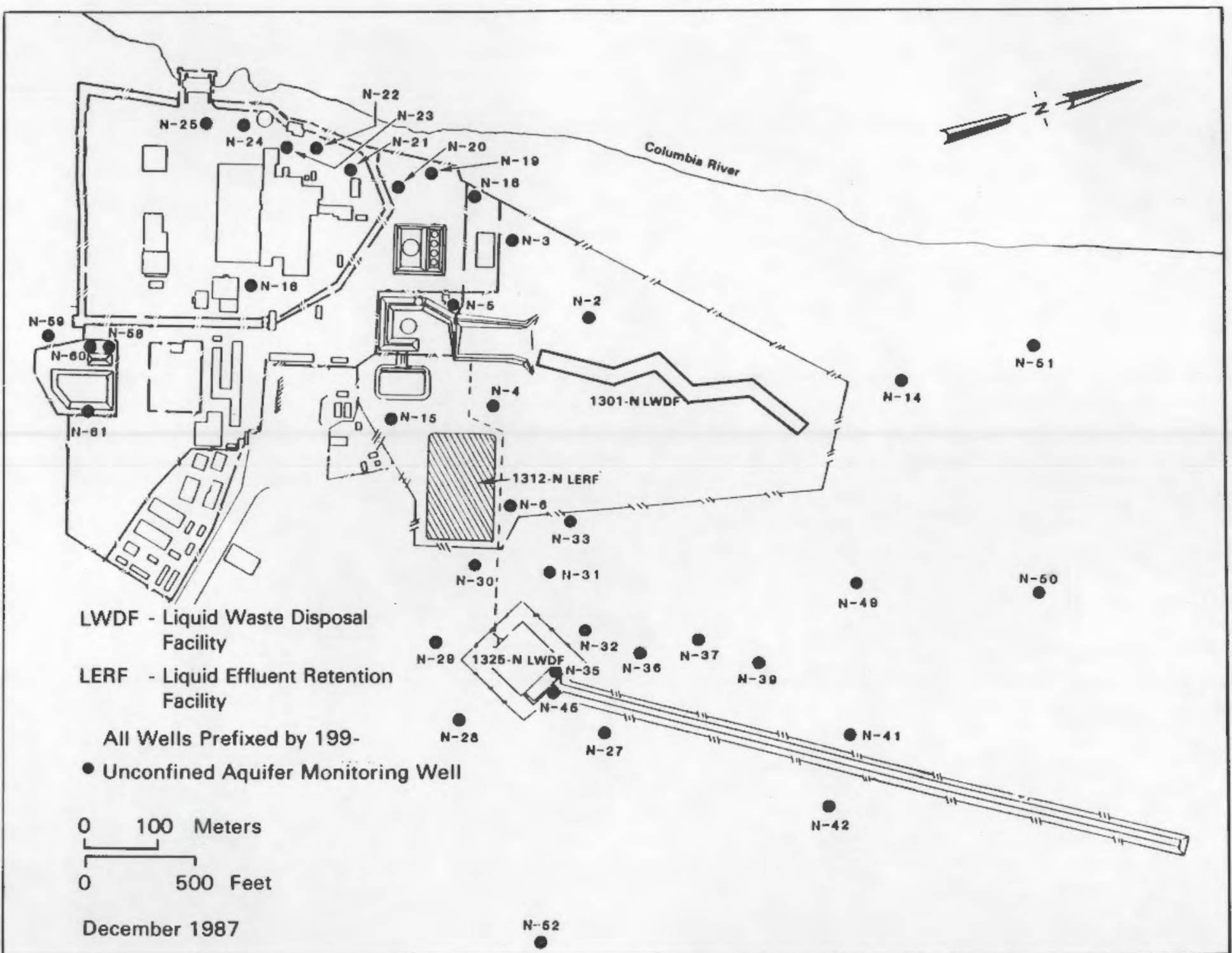

FIGURE B.6. Location of 100-N Area Ground-Water Monitoring Wells Sampled During January Through June 1988 



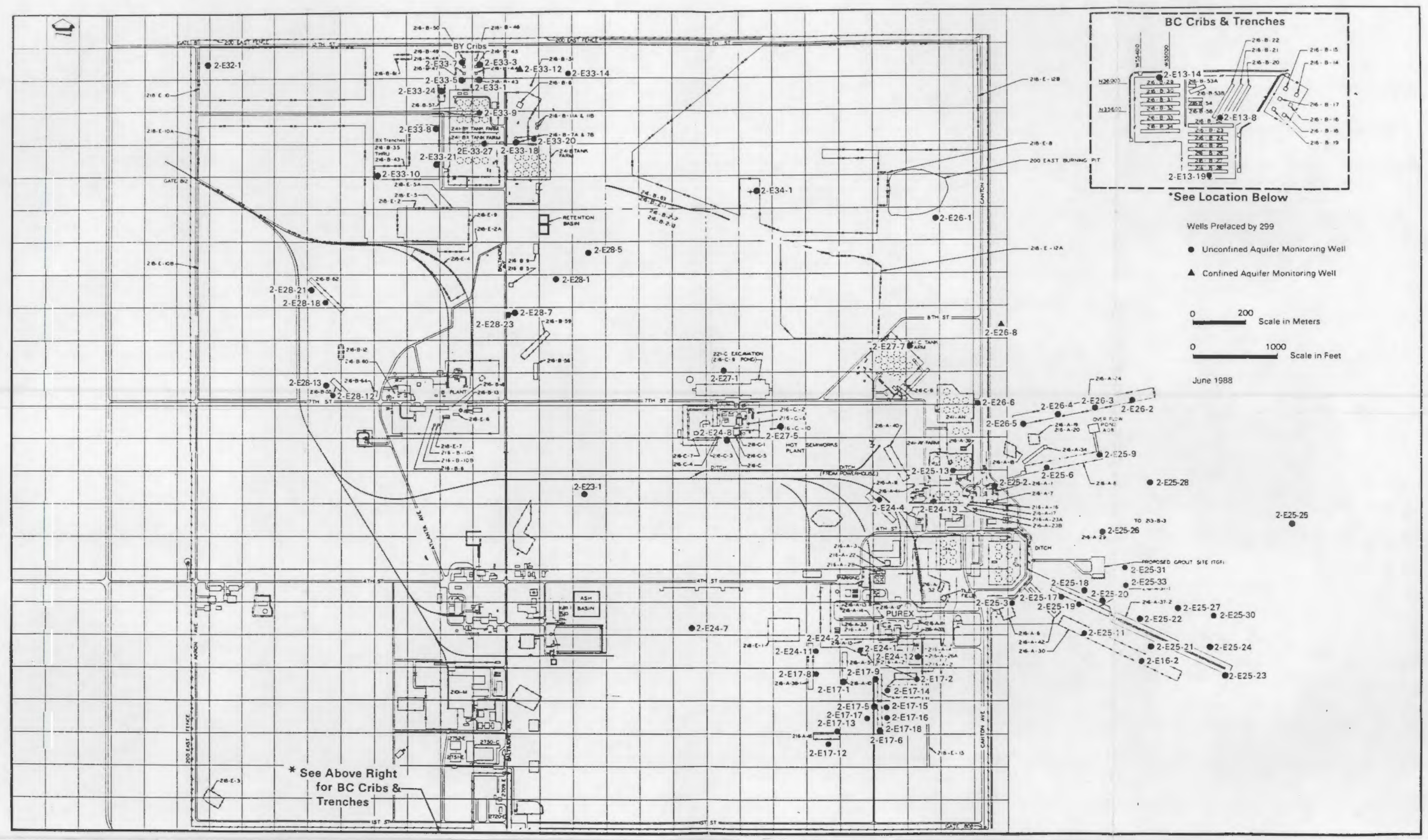

FIGURE B.7. Location of 200-East Area Ground-Water Monitoring Wells Sampled During January Through June 1988 


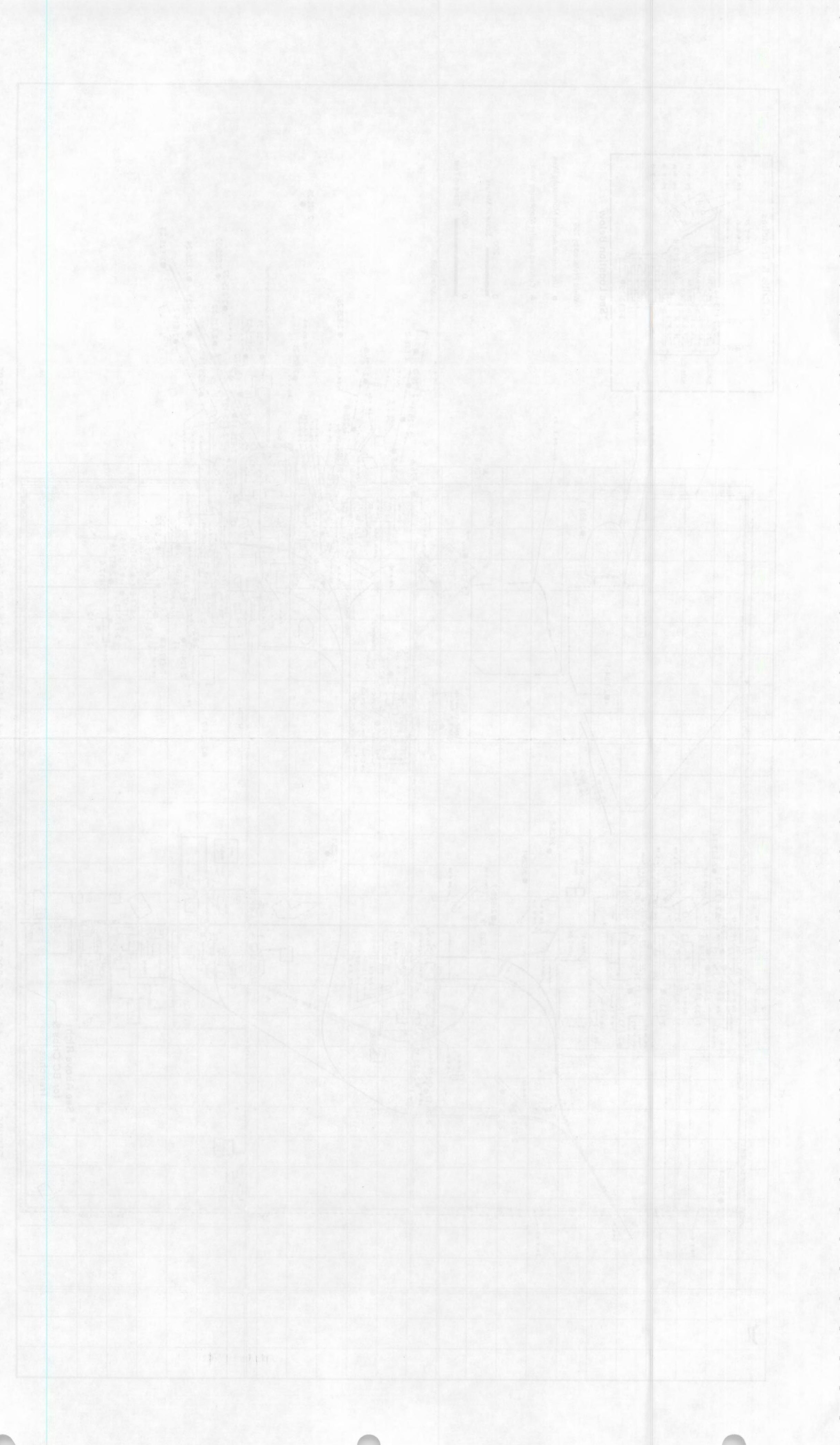




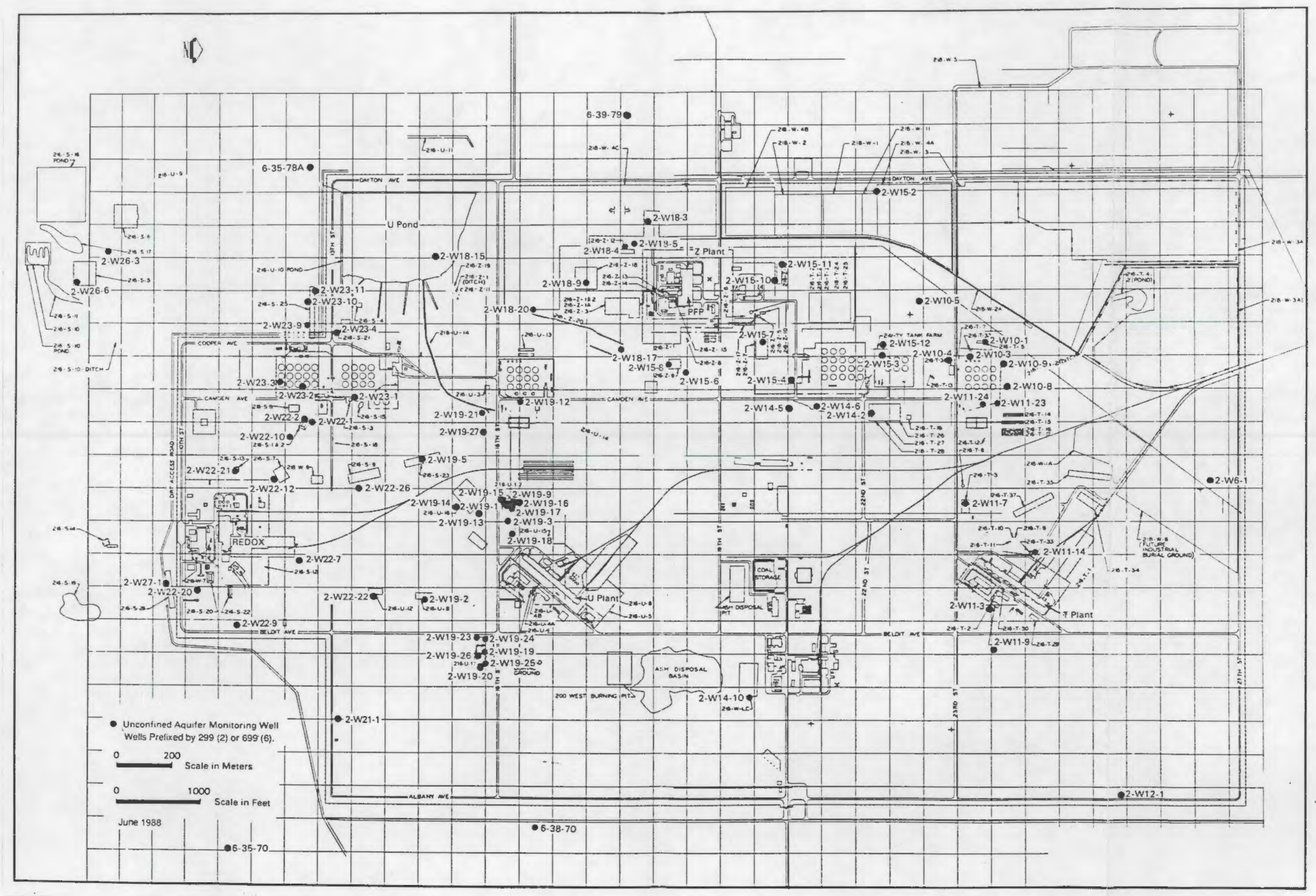

FIGURE B.8. Location of 200-West Ground-Water Monitoring Wells Sampled During January Through June 1988 



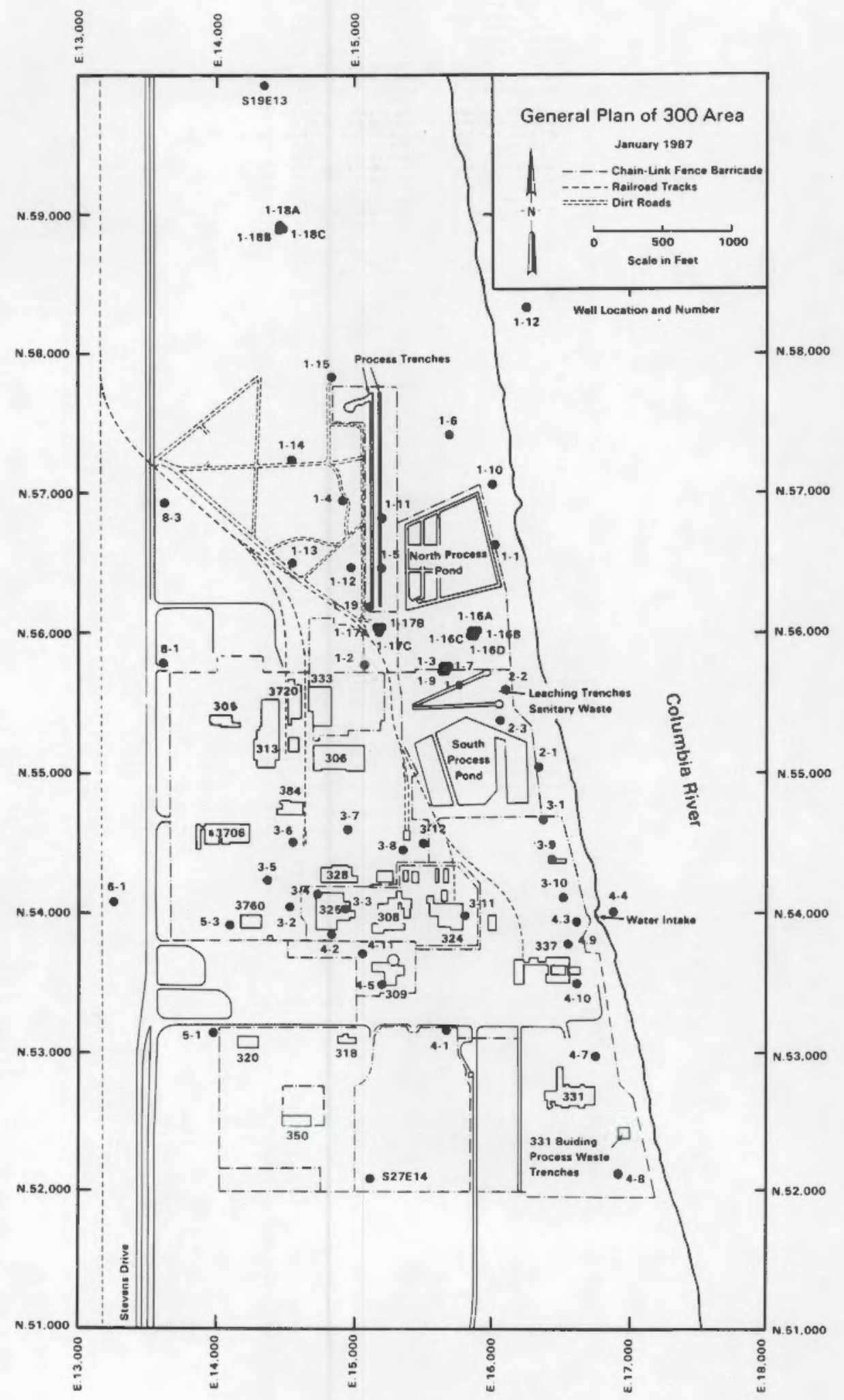

FIGURE B.9. Location of 300-Area Ground-Water Monitoring Wells Sampled During January Through June 1988 



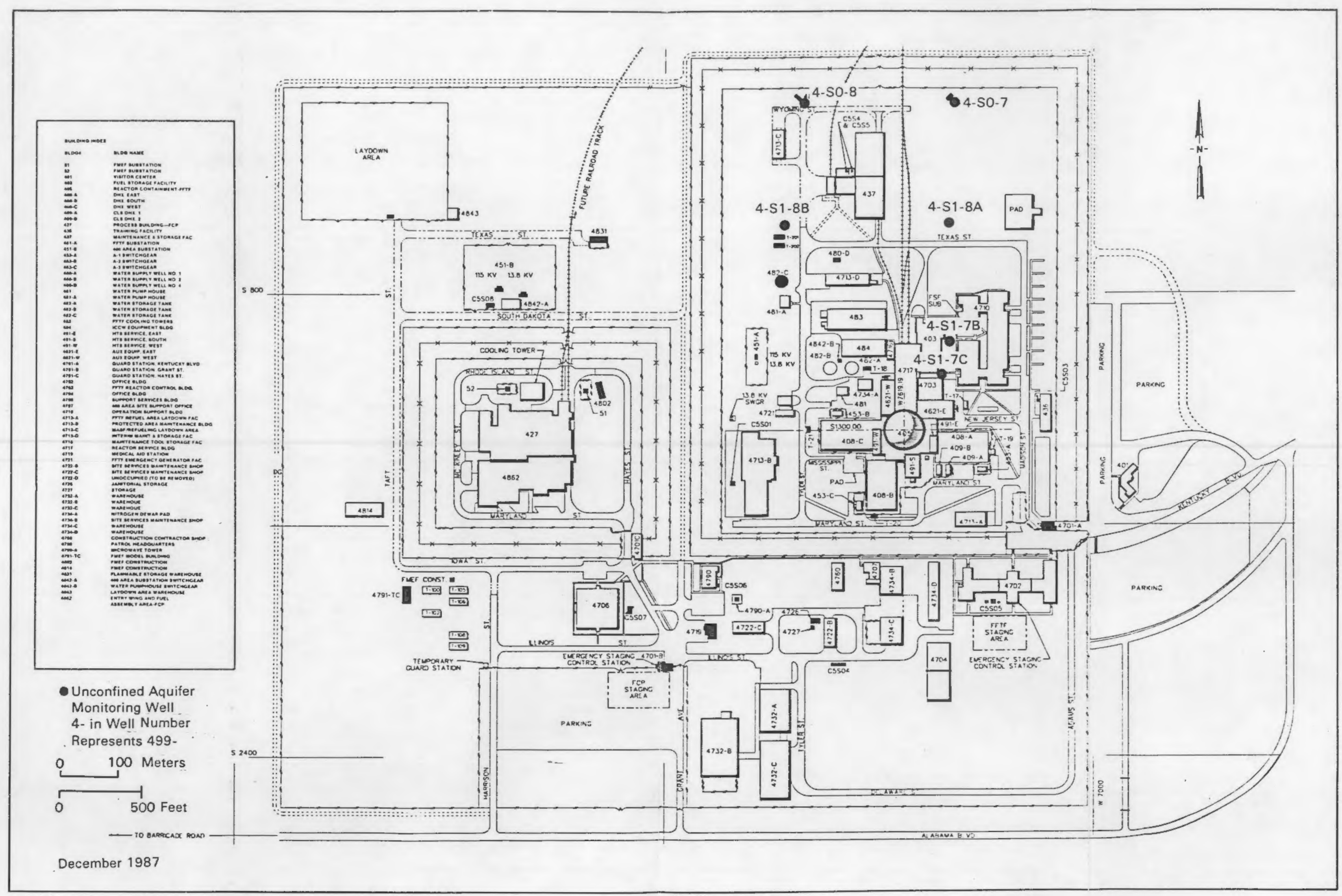

FIGURE B.10. Location of 400 Area Ground-Water Monitoring Wells Sampled During January Through June 1988 

APPENDIX C

MAXIMUM CONTAMINANT LEVELS AND

DERIVED CONCENTRATION GUIDELINES 


\section{APPENDIX C}

\section{MAXIMUM CONTAMINANT LEVELS AND \\ DERIVED CONCENTRATION GUIDEL INES}

TABLE C.1. Radiological Maximum Contaminant Levels [from U.S. Environmenta) Protection Agency (OFR 1986) and State of Washington Public Water Supplies (WDSHS 1983)]

Contaminant

Gross alpha (excluding uranium)

Combined radium-226 and radium-228

Radium-226 (State of Washington only) Gross beta and gamma radioactivity

from manmade radionuclides

\section{Limit}

\section{$15 \mathrm{pCi} / \mathrm{L}$}

$5 \mathrm{pCi} / \mathrm{L}$

$3 \mathrm{pCi} / \mathrm{L}$

Annual average concentration shall not produce an annual dose from manmade radionuclides equivalent to the total body or any internal organ dose greater than 4 mrem/yr. If two or more radionuclides are present, the sutn of their annual dose equivalent shall not exceed 4 mrem $/ y r$.

Compliance may be assumed if annual average concentrations for gross beta activity, tritium, and strontium-90 are less than $50 \mathrm{pCi} / \mathrm{L}, 20,000 \mathrm{pCi} / \mathrm{L}$, and $8 \mathrm{pCi} / \mathrm{L}$, respectively. It should be noted that these "screening levels" are conservatively calculated and not directiy equivalent to an annual dose of 4 inrem. 
The following list provides the annual average concentrations that yield an annual dose of 4 millirem to the indicated orzan assuming a 2-L daily intake. Data are taken from EPA (1976).

\begin{tabular}{|c|c|c|}
\hline Radionuclide & Critical Organ & $\begin{array}{c}\text { Concentration, } \\
\mathrm{pC} \mathrm{C} / \mathrm{L}\end{array}$ \\
\hline Antimony-125 & GI (LLI) & 300 \\
\hline Carbon-14 & Fat & 2,000 \\
\hline Cesium-137 & Whole body & 200 \\
\hline Cobalt-60 & GI (LLI) & 100 \\
\hline Iodine-129 & Thyroid & 1 \\
\hline Nicke]-63 & Bone & 50 \\
\hline Ruthen i um- 103 & GI (LLI) & 200 \\
\hline Ruthen i um- 106 & GI (LLI) & 30 \\
\hline Stront jum-90 & Bone marrow & 8 \\
\hline Technetium-99 & GI (LLI) & 900 \\
\hline
\end{tabular}

TABLE C.2. Chemical Maximum Contaminant Levels [from U.S. Environmenta] Protection Agency (OFR 1986) and state of Washington Public Water Supplies (WDSHS 1983)]

\begin{tabular}{|c|c|c|}
\hline Chemical Constituent & Concen & atrati \\
\hline Arsenic & 50 & $p p b^{(a)}$ \\
\hline Barium & 1 & $\mathrm{ppm}$ \\
\hline Cadmi um & 10 & $\mathrm{ppb}$ \\
\hline Carbon tetrachloride & 5 & $\mathrm{ppb}$ \\
\hline $\mathrm{Chloroform}$ & 100 & $\mathrm{ppb}$ \\
\hline Chromium & 50 & $\mathrm{ppb}$ \\
\hline Copper & 1.3 & $\mathrm{ppm}$ \\
\hline Fluorine & 2 & $\mathrm{ppm}$ \\
\hline Lead & 50 & $\mathrm{ppb}$ \\
\hline Mercury & 2 & $\mathrm{ppb}$ \\
\hline Nitrate ion & 45 & $\mathrm{ppm}$ \\
\hline Selenium & 10 & $\mathrm{ppb}_{(\mathrm{h})}$ \\
\hline $\begin{array}{l}\text { Trans-1,2- } \\
\text { Dichloroethene }\end{array}$ & 70 & $\mathrm{ppb}^{(\mathrm{D})}$ \\
\hline Trichloroethene & 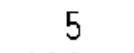 & $\mathrm{ppb}$ \\
\hline $1,1,1$-Trichioroethane & 200 & $\mathrm{ppb}$ \\
\hline
\end{tabular}

(a) $\mathrm{ppb}=\mathrm{ppm} / 1000$.

(b) Proposed recommended maximum contaminant level. 
IABLE_C.3. Proposed Derived Concentration Guides (a)

\begin{tabular}{l}
\multicolumn{1}{c}{ Radionuclide } \\
\hline Americium-241 \\
Ant imony-125 \\
Cesium-137 \\
Cobalt-60 \\
Iodine-129 \\
Nickel-63 \\
Plutonium-238 \\
PTutonium-239,240 \\
Radium-226 \\
Ruthenium-103 \\
Ruthenium-106 \\
Strontium-89 \\
Strontium-90 \\
Technet ium-99 \\
Tritium \\
Uranium-234 \\
Uranium-235 \\
Uranium-236 \\
Uranium-238
\end{tabular}

$\begin{gathered}\text { Concentration, } \\ \mathrm{pCi} i / \mathrm{L}\end{gathered}$
30
60,000
3,000
5,000
500
300,000
400
300
100
50,000
6,000
20,000
1,000
100,000
$2,000,000$
500
600
500
600

(a) Concentrations of radionuclides in water that could be continuously consumed and not exceed an effective dose equivalent of $100 \mathrm{mrem} / \mathrm{yr}$. Consumption is assumed to be $730 \mathrm{~L}$ of drinking water per year. 


\section{REFERENCES}

40 CFR Parts 100 to 149. 1986. U.S. Environmental Protection Agency, "Protection of Environment." U.S. Code of Federal Regulations.

EPA. 1976. National Interim Primary Drinking Water Requlations.

EPA-570/9-76-003, U.S. Environmental Protection f.gency, Office of Water Supply, Washington, D.C.

U.S. Department of Energy (DOE). 1981. "Envirormental Protection, Safety, and Health Protection Program for DOE Operations." DOE Order 5480.1A

Washington State Department of Social and Health Services (WDSHS).

August 1983 (Revised). "Public Water Supplies". Washington Administration Code, Chapter 248-54, 0lympia, Washington. 
APPENDIX D

DATA OBTAINED BY SPECIAL INDUCTIVELY COUPLED

PLASMA/MASS SPECTROMETRY METHOD 


\section{APPENDIX D}

\section{DATA OBTAINED BY SPECIAL INDUCT IVELY COUPLED PLASMA/MASS SPECTROMETRY METHOD}

This appendix table is data from the inductively coupled plasma/mass spectrometry method (ICP/MS) SEMIQUANT analysis of Hanford ground-water samples.

0.1 
TABLE D.1. ICP-MS SEMIQUANT Analys is of Hanford Ground-Water Samples

\begin{tabular}{|c|c|c|c|c|c|c|c|c|c|c|c|c|c|c|c|c|}
\hline & \multicolumn{16}{|c|}{ (nll concentrations exaressed da l: arograms per liter) } \\
\hline & & Li & $\mathrm{Be}$ & B & Al & $\mathrm{C}_{\mathrm{a}}$ & Sc & $\mathrm{v}$ & $\mathrm{C}_{\mathrm{r}}$ & Mn & $\mathrm{Fe}$ & co & $\mathrm{Ni}$ & $\mathrm{Cu}$ & $2 n$ & Ga \\
\hline hell & collected & 7 & 9 & 10 & 27 & 44 & 45 & 51 & 52 & 55 & 57 & 59 & 60 & 63 & 66 & 69 \\
\hline $1-83-1$ & MAY & ND & ND & No & ND & 43160 & 0.9 & 2.3 & 60 & ND & ND & ND & ND & ND & ND & 0.27 \\
\hline $1-84-1$ & MARCH & & & & 3 & & 0.9 & 7.5 & 13 & ND & ND & ND & ND & ND & ND & 0.23 \\
\hline $1-84-4$ & APRIL & ND & ND & ND & 3 & 50385 & 2.4 & 8.8 & 13 & 11 & ND & ND & NO & ND & NO & 0.18 \\
\hline $1-85-1$ & JUNE & ND & ND & ND & ND & 41330 & 1.8 & 6.7 & 31 & ND & ND & ND & ND & ND & ND & 0.29 \\
\hline $1-89-1$ & MARCH & & & & ND & & 0.8 & 10.4 & 13 & 4 & ND & ND & NO & NO & 10 & ND \\
\hline 1.02 .5 & APR IL & 87 & ND & ND & ND & 75734 & 1.5 & 11.4 & 216 & ND & 208 & ND & ND & NO & ND & 0.83 \\
\hline $1.05-12$ & APRIL & 97 & ND & ND & 3 & 114710 & 2.6 & 13.4 & 2263 & ND & 224 & ND & ND & ND & ND & 0.80 \\
\hline $1-F 5-1$ & APRIL & ND & ND & ND & 3 & 50257 & 9.5 & 1.4 & 3 & ND & ND & ND & ND & ND & ND & 0.34 \\
\hline $1-F 5-3$ & MAY & ND & ND & ND & ND & 27589 & 1.0 & 0.9 & 6 & 2 & ND & ND & ND & ND & ND & 0.20 \\
\hline $1-\mathrm{H} 3-1$ & MARCH & & & & ND & & 0.6 & 7.8 & 61 & ND & RD & ND & ND & ND & ND & 0.13 \\
\hline $1-\mathrm{H} 3-2 \mathrm{~A}$ & JULY & ND & ND & NO & 4 & 52761 & 1.6 & 7.8 & 57 & ND & ND & ND & ND & ND & ND & 0.20 \\
\hline $1-H 3-2 B$ & AUGUST & ND & ND & ND & 6 & 42176 & 1.1 & 4.6 & 35 & 59 & ND & ND & ND & ND & ND & 0.30 \\
\hline $1-43-2 C$ & MAY & 99 & ND & ND & 7 & 20627 & 1.5 & 14.9 & 3 & 34 & ND & ND & ND & 7 & 54 & 0.19 \\
\hline $1-H_{3}-2 \mathrm{C}$ & JUNE & ND & ND & ND & 3 & 22160 & 1.8 & 12.7 & ND & 32 & ND & ND & ND & NO & 11 & 0.22 \\
\hline $1-H 3-2 C$ & MARCH & & & & & & ND & 7.8 & ND & 44 & ND & ND & ND & 4 & ND & 0.06 \\
\hline $1-1+4-3$ & JULY & ND & ND & ND & 16 & 12056 & 1.8 & 17.5 & 403 & 2 & ND & 0.2 & 24 & 4 & ND & 0.22 \\
\hline $1-\mathrm{H}_{4}-3$ & MAY & ND & ND & NO & 10 & 20875 & 2.3 & 13.9 & 407 & 2 & ND & 0.2 & 34 & 4 & NO & 0.25 \\
\hline 1-H4-3 & MARCH & & & & 4 & & 0.8 & 7.5 & 475 & 5 & ND & 0.5 & 67 & 22 & ND & 0.32 \\
\hline i-H4-3 & AUGUST & ND & ND & ND & 6 & 26016 & 1.4 & 7.4 & 278 & 3 & ND & 0.4 & 43 & 11 & ND & 0.46 \\
\hline $1-H 4-4$ & JULY & & ND & ND & 4 & 39285 & 1.9 & 6.3 & 216 & ND & ND & ND & 13 & ND & 38 & 0.30 \\
\hline 1- $\mathrm{H}_{4}-4$ & MAY & 187 & ND & ND & ND & 25419 & 0.8 & 4.0 & 195 & ND & ND & ND & 15 & ND & 36 & 0.32 \\
\hline $7-H_{4}-4$ & JUNE & & ND & ND & 6 & 28956 & 1.5 & 7.3 & 33 & ND & ND & ND & ND & ND & 7 & 0.25 \\
\hline $1-\mathrm{H} 4 \cdot 4$ & AUGUST & ND & ND & ND & ND & 38729 & 1.4 & 5.0 & 317 & ND & ND & ND & 25 & ND & 51 & 0.59 \\
\hline $1-H 4-5$ & MARCH & & & & ND & & 0.7 & 10.4 & 251 & ND & ND & ND & ND & ND & 61 & 0.28 \\
\hline
\end{tabular}




\section{TABLE D.1. (contd)}

(All concentrations expressed as micrograns per liter)

\begin{tabular}{|c|c|c|c|c|c|c|c|c|c|c|c|c|c|c|c|c|}
\hline & & $L \mathfrak{i}$ & Be & B & A! & $\mathrm{Ca}$ & Sc & v & $\mathrm{Cr}$ & $\mathrm{Mn}$ & $\mathrm{Fe}$ & Co & $\mathrm{Ni}$ & Cu & $2 n$ & $\mathrm{Ga}$ \\
\hline Well & collected & 7 & 9 & 10 & 27 & 44 & 45 & 51 & 52 & 5.5 & 57 & 59 & 60 & 63 & 66 & 69 \\
\hline $2-E 17-9$ & APRIL & 100 & ND & ND & ND & 63645 & 2.5 & 26.9 & 3 & 5 & ND & ND & ND & ND & ND & 0.49 \\
\hline 2-E24-12 & APR IL & 88 & ND & ND & NO & 53148 & 2.7 & 22.4 & ND & 2 & ND & ND & ND & ND & ND & 0.36 \\
\hline $2-E 24-7$ & JUNE & NO & ND & ND & 3 & 30087 & 2.6 & 23.2 & ND & 4 & ND & ND & ND & ND & ND & 0.25 \\
\hline $2-E 24-8$ & AUGUST & ND & ND & ND & ND & 34010 & 2.3 & 24.6 & 6 & 4 & ND & NO & ND & ND & ND & 0.37 \\
\hline $2-E 25-18$ & APRIL & ND & ND & ND & ND & 26898 & 2.1 & 31.9 & ND & 8 & ND & ND & No & ND & ND & 0.13 \\
\hline $2-E 25-20$ & MARCH & & & & ND & & ND & 2.4 & ND & ND & ND & ND & ND & ND & ND & 0.11 \\
\hline $2-E 25-21$ & APRIL & 115 & ND & ND & 4 & 30162 & 3.8 & 82.7 & 3 & 11 & ND & ND & ND & ND & ND & 0.22 \\
\hline $2-E 25-27$ & JUNE & ND & ND & & 4 & 21007 & 1.9 & 41.1 & ND & ND & ND & ND & ND & ND & ND & 0.16 \\
\hline $2-E 25-28$ & JUNE & $N D$ & ND & ND & 3 & 20298 & 2.0 & 45.3 & 3 & ND & ND & ND & ND & ND & 83 & 0.19 \\
\hline 2-E25-7 & JUNE & ND & ND & NO & 3 & 28958 & 2.6 & 37.4 & 3 & 2 & ND & ND & ND & ND & ND & 0.23 \\
\hline $2-E 26-1$ & JUNE & ND & ND & ND & 4 & 34688 & 1.8 & 14.4 & ND & 3 & ND & ND & ND & ND & ND & 0.22 \\
\hline $2-E 26-3$ & JUNE & ND & ND & ND & 4 & 26001 & 2.2 & 41.0 & 3 & ND & ND & ND & ND & ND & ND & 0.16 \\
\hline $2-E 27-5$ & MARCH & & & & ND & & ND & 4.5 & ND & ND & ND & ND & ND & ND & ND & 0.11 \\
\hline $2-E 28-17$ & JUNE & ND & ND & 152 & 15 & 44857 & 1.6 & 10.7 & 5 & 2 & ND & ND & ND & ND & ND & 0.32 \\
\hline $2-E 2 B-7$ & JUNE & ND & ND & ND & $\mathrm{ND}$ & 25463 & 1.7 & 20.2 & 3 & ND & ND & ND & ND & ND & ND & 0.31 \\
\hline $2-E 32-1$ & AUGUST & ND & ND & ND & ND & 27241 & 1.6 & 20.3 & 5 & ND & ND & ND & ND & ND & ND & 0.31 \\
\hline $2-E 33-1$ & MAY & ND & ND & ND & ND & 21049 & 1.5 & 25.3 & 7 & NO & ND & ND & ND & ND & ND & 0.17 \\
\hline $2-E 33-18$ & MAY & ND & ND & ND & ND & 22770 & 1.5 & 18.8 & 3 & 2 & ND & ND & ND & ND & ND & 0.23 \\
\hline $2-E 33-2$ & JUNE & ND & NO & ND & ND & 20282 & 1.2 & 21.0 & 7 & NO & ND & ND & ND & ND & ND & 0.14 \\
\hline $2-E 33-21$ & AUGUST & ND & ND & ND & ND & 23664 & 1.5 & 17.7 & 4 & 2 & ND & ND & ND & ND & ND & 0.23 \\
\hline $2-E 33-24$ & AUGUST & ND & ND & ND & ND & 21864 & 1.3 & 15.4 & 4 & ND & ND & ND & ND & ND & ND & 0.20 \\
\hline $2-E 33-3$ & AUGUST & ND & ND & ND & ND & 30928 & 2.0 & 29.1 & 14 & ND & ND & ND & ND & ND & ND & 0.21 \\
\hline $2-E 33-5$ & AUGUST & ND & ND & ND & ND & 34452 & 2.2 & 26.2 & 10 & 2 & ND & ND & ND & ND & ND & 0.32 \\
\hline $2-E 34-1$ & JULY & ND & ND & ND & NO & 62698 & 1.7 & 12.2 & NO & 12 & ND & ND & ND & ND & 16 & 0.22 \\
\hline $2-46-1$ & JUNE & ND & ND & NO & ND & 61481 & 1.9 & 14.1 & 54 & 12 & 176 & ND & NO & ND & ND & 0.53 \\
\hline $2-610-4$ & MARCH & & & & NO & & ND & 3.8 & 27 & ND & ND & ND & ND & ND & ND & 0.06 \\
\hline $2-W 10-9$ & MAY & No & $\mathrm{NC}$ & ND & No & 36705 & 1.9 & 56.3 & 147 & NO & ND & ND & ND & ND & ND & 0.58 \\
\hline $2-412-1$ & JUNE & ND & ND & ND & ND & 77635 & 1.9 & 18.1 & 68 & ND & 240 & NO & ND & ND & ND & 0.61 \\
\hline $2 \cdot \operatorname{lin} 4-5$ & MARCH & & & & 80 & & 0.4 & 19.3 & ND & 7 & ND & NO & ND & ND & ND & ND \\
\hline $2 \cdot-15-14$ & MARCH & & & & ND & & ND & 6.6 & ND & $B$ & NO & ND & ND & ND & ND & ND \\
\hline $2-418-15$ & JULY & NO & ND & ND & ND & 23875 & 1.9 & 19.4 & 3 & 2 & ND & ND & NO & ND & ND & 0.16 \\
\hline
\end{tabular}




\section{TABLE D.1. (contd)}

(All concentrations expressed as micrograms per (iter)

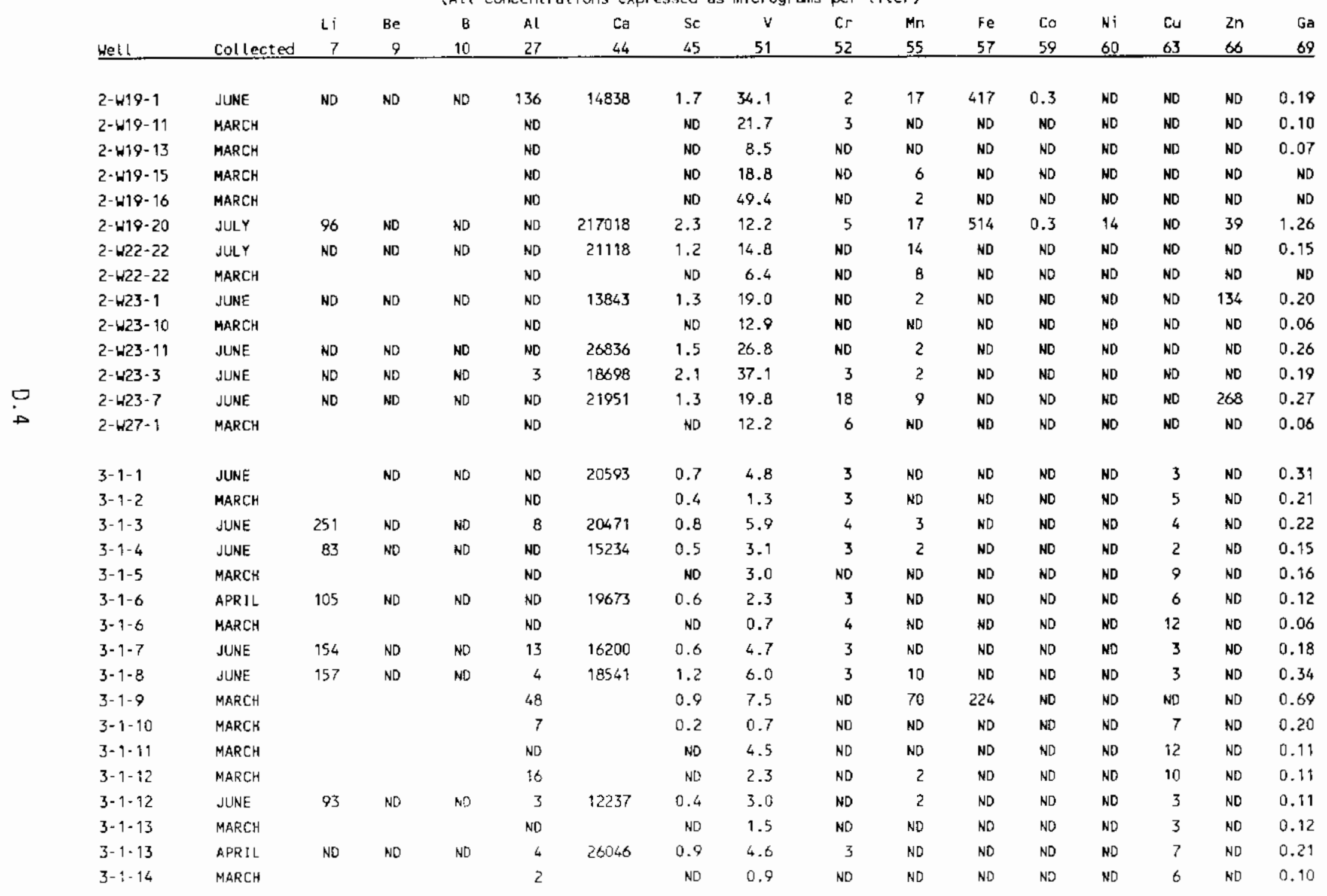




\section{TABLE D.1. (contd)}

(All concentrations expressed as micrograms per liter)

\begin{tabular}{|c|c|c|c|c|c|c|c|c|c|c|c|c|c|c|c|c|}
\hline & & Li & $6 e$ & B & Al & $\mathrm{Ca}$ & Se & $v$ & $\mathrm{Cr}$ & Mn & $\mathrm{Fe}$ & Co & $\mathrm{Ni}$ & $\mathrm{Cu}$ & $\mathrm{Zn}$ & $\mathrm{Ga}$ \\
\hline Hell & Collected & 7 & 9 & 10 & 27 & 44 & 45 & 51 & 52 & 55 & 57 & 59 & 60 & 63 & 60 & 69 \\
\hline $3-1-15$ & MARCH & & & & NO & & 0.7 & 2.2 & NO & 35 & ND & ND & ND & ND & ND & 0.26 \\
\hline $3-1-15$ & APR IL & ND & ND & ND & 8 & 45522 & 1.2 & 6.4 & 3 & No & NO & ND & ND & ND & ND & 0.32 \\
\hline $3-1-16 A$ & JUNE & & ND & ND & 4 & 21570 & 1.0 & 5.1 & 4 & ND & NO & ND & ND & 4 & 11 & 0.33 \\
\hline $3-1-168$ & JUNE & 199 & ND & 52 & ND & 14335 & 9.4 & 2.0 & ND & 79 & NO & NO & ND & NO & ND & 0.41 \\
\hline $3-1-16 c$ & MARCH & & & & ND & & 0.7 & 9.2 & ND & 37 & ND & ND & ND & ND & ND & 0.26 \\
\hline $3-1-16 C$ & APRIL & 257 & ND & 58 & ND & 9636 & 1.6 & 2.2 & ND & 38 & ND & NO & ND & ND & ND & 0.56 \\
\hline $3 \cdot 1-17 A$ & MARCH & & & & 58 & & ND & 8.9 & ND & 2 & ND & ND & ND & 8 & ND & 0.14 \\
\hline $3-1-17 B$ & MARCH & & & & NO & & 0.6 & 6.4 & ND & 50 & ND & ND & ND & ND & ND & 0.24 \\
\hline $3-1 \cdot 17 c$ & JUNE & 221 & ND & ND & ND & 9022 & 2.7 & 1.1 & ND & 24 & ND & ND & ND & ND & ND & 0.68 \\
\hline $3-1-18 A$ & MARCH & & & & 6 & & 0.4 & 7.8 & ND & ND & ND & ND & ND & ND & ND & 0.18 \\
\hline $3-1-188$ & MARCH & & & & ND & & 0.6 & 6.4 & NO & 31 & ND & NO & ND & ND & ND & 0.10 \\
\hline $3-1-18 C$ & APR IL & 227 & ND & NO & 9 & 13190 & 2.6 & 1.6 & NO & 48 & ND & ND & ND & ND & ND & 0.39 \\
\hline $3.1-19$ & APRIL & 264 & ND & NO & 332 & 12472 & 0.5 & 5.5 & 6 & 39 & 1255 & ND & ND & 9 & ND & 0.22 \\
\hline $3-1-19$ & MARCH & & & & & & & 2.3 & & & 1351 & NO & ND & 9 & ND & 0.17 \\
\hline $3-2-1$ & JUNE & & NO & ND & NO & $1987 \mathrm{C}$ & 0.7 & 3.7 & ND & ND & ND & ND & ND & 2 & ND & 0.35 \\
\hline $3-3-10$ & APRIL & & NO & 77 & 3 & 34035 & 1.4 & 2.4 & 3 & 19 & ND & 0.2 & ND & ND & ND & 0.37 \\
\hline $3-3-10$ & MARCH & & & & ND & & 0.4 & 3.1 & ND & 19 & 434 & ND & ND & ND & ND & 0.30 \\
\hline $3-3-7$ & MARCH & & & & ND & & 0.4 & 2.0 & ND & ND & NO & NO & NO & ND & ND & 0.17 \\
\hline $3-4-1$ & JUNE & ND & ND & ND & 4 & 36017 & 1.6 & 9.4 & 5 & ND & ND & NO & ND & ND & ND & 0.35 \\
\hline $3-4-11$ & MARCH & & & & NO & & 0.5 & 1.3 & NO & NO & ND & ND & ND & ND & ND & 0.19 \\
\hline $3-4-7$ & JUNE & ND & NO & 82 & ND & 32572 & 1.0 & 6.0 & 3 & NO & NO & ND & ND & ND & ND & 0.32 \\
\hline $3-8-2$ & MARCH & & & & ND & & 0.6 & 1.2 & ND & ND & NO & ND & ND & ND & ND & 0.32 \\
\hline $6-20-20$ & MARCH & & & & ND & & 0.6 & 1.9 & ND & 3 & ND & NO & ND & ND & ND & 0.39 \\
\hline $6-20-39$ & JUNE & ND & ND & NO & NO & 26533 & 1.1 & 5.2 & ND & 14 & ND & ND & ND & ND & ND & 0.25 \\
\hline $6-20-E 5 A$ & JUNE & NO & $\mathrm{ND}$ & NO & ND & 30110 & 1.2 & 13.5 & 6 & 2 & ND & ND & NO & ND & ND & 0.44 \\
\hline $6-23-34$ & JUNE & 150 & ND & ND & NO & 76107 & 2.3 & 14.7 & 6 & 2 & 256 & ND & ND & ND & 40 & 0.71 \\
\hline $6-24-33$ & MAY & 105 & ND & ND & ND & 50067 & 1.7 & 15.9 & 5 & ND & $\mathrm{ND}$ & NO & ND & ND & ND & 0.41 \\
\hline $6-24-34 A$ & JUNE & 143 & NO & NO & ND & 69089 & 2.4 & 16.2 & 6 & 4 & 240 & ND & ND & NO & 52 & 0.57 \\
\hline $6 \cdot 24 \cdot 348$ & JUNE & 127 & ND & ND & ND & 81610 & 2.9 & 15.0 & 7 & 7 & NO & ND & ND & NO & 44 & 0.43 \\
\hline $6-24-34 C$ & JULY & 89 & ND & NO & NO & 69398 & 2.6 & 13.5 & 6 & 5 & ND & ND & ND & ND & 34 & 0.37 \\
\hline $6-24 \cdot 35$ & JULY & NO & ND & ND & NO & 41934 & 2.0 & 16.3 & 7 & ND & ND & ND & ND & ND & 11 & 0.35 \\
\hline $6-25 \cdot 34$ & JULY $Y$ & ND & ND & No & ND & 36774 & 2.1 & 20.1 & 5 & ND & ND & ND & NO & ND & ND & 0.25 \\
\hline
\end{tabular}




\section{TABLE. D.1. (contd)}

(Alt concentrations expressed as micrograms per (iter)

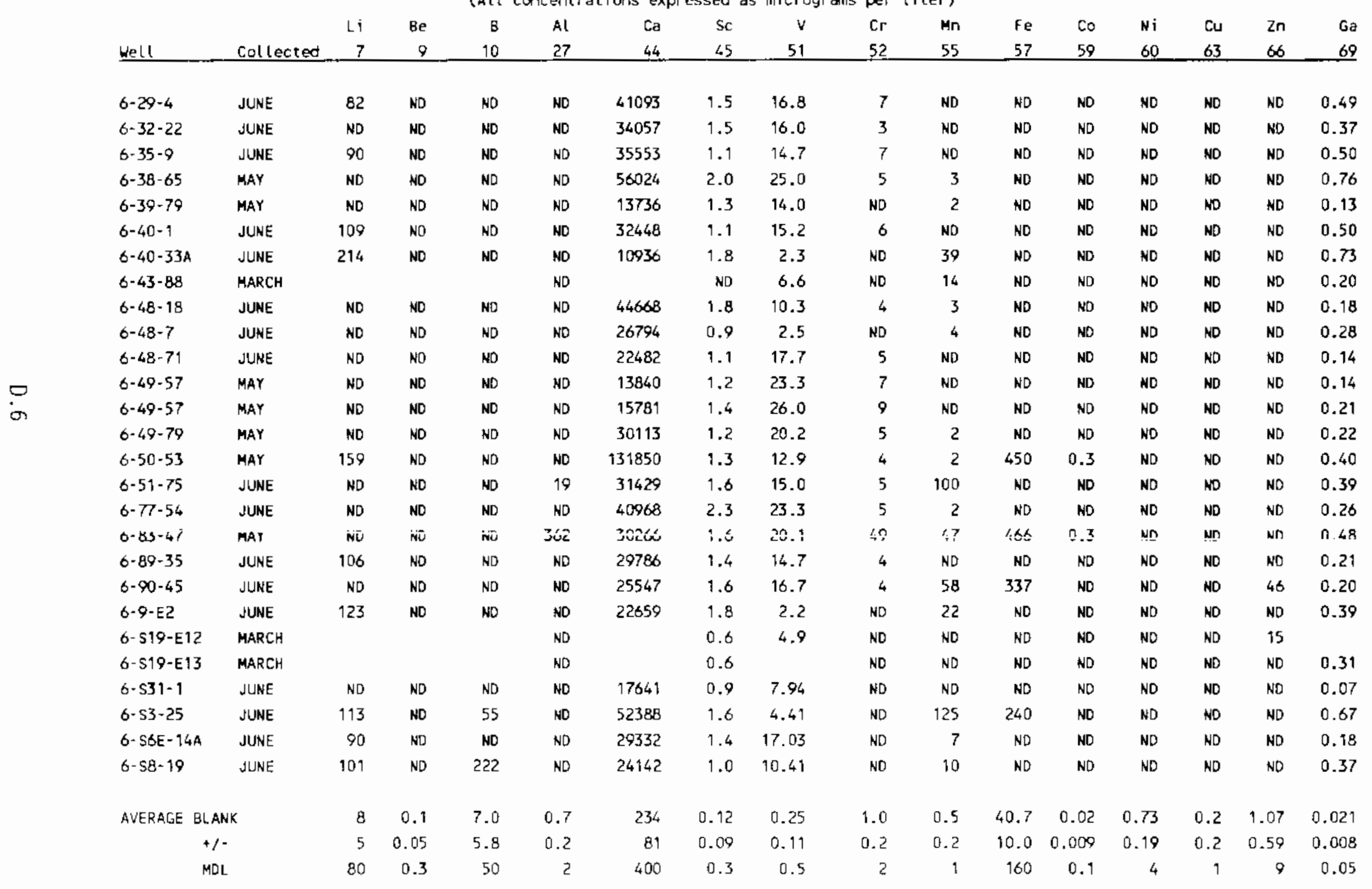


TABLE D.1. (contd)

(All concentrations expressed as micrograms per liter)

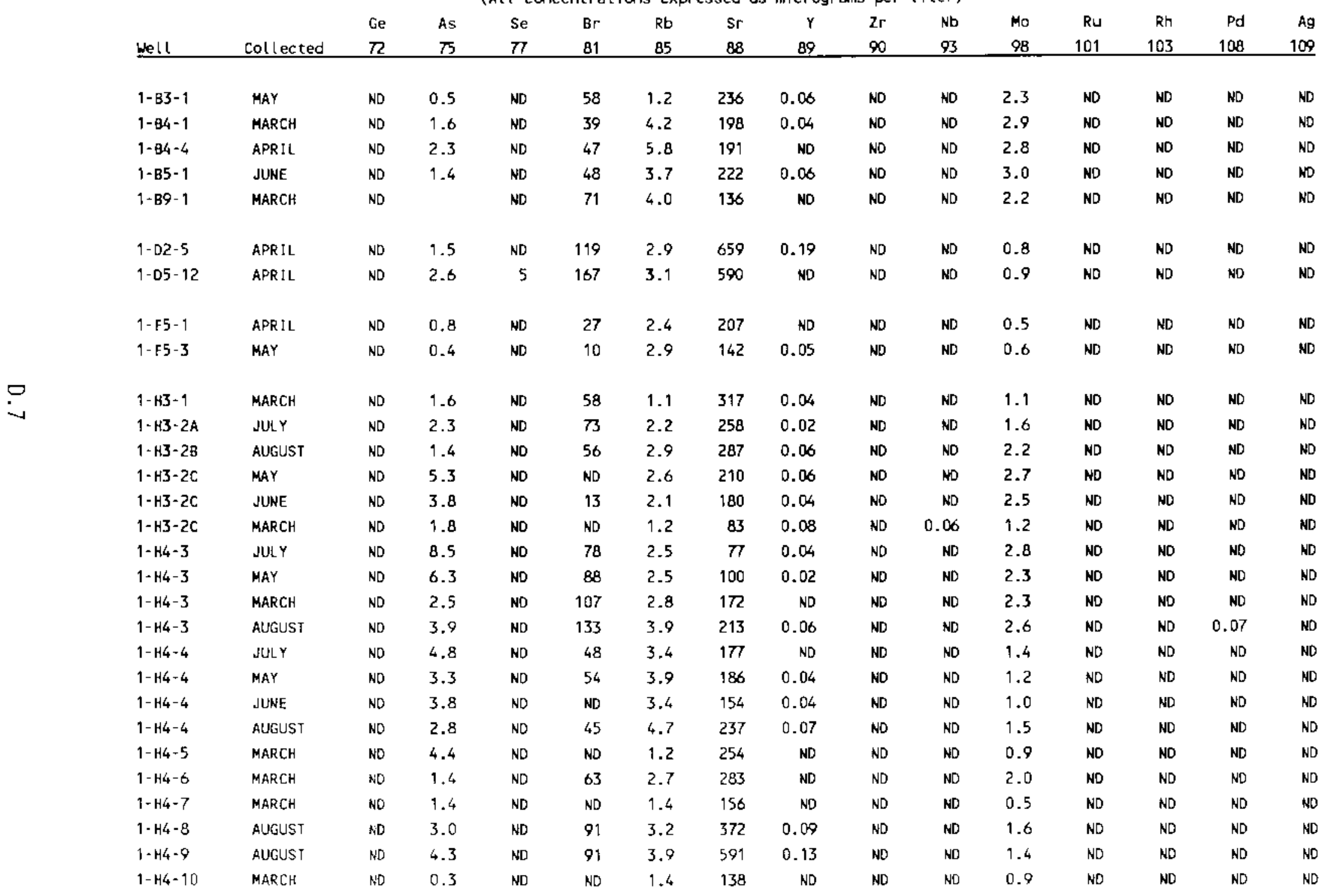


TABLE D.1. (contd)

(All concentrations expressed as micrograms per liter)

\begin{tabular}{|c|c|c|c|c|c|c|c|c|c|c|c|c|c|c|c|}
\hline tell 1 & Collected & $\begin{array}{l}\mathrm{Ge} \\
72\end{array}$ & $\begin{array}{l}\text { As } \\
75\end{array}$ & $\begin{array}{l}\text { se } \\
\pi\end{array}$ & $\begin{array}{l}\mathrm{Br} \\
81\end{array}$ & $\begin{array}{l}R b \\
85\end{array}$ & $\begin{array}{l}\mathrm{Sr} \\
88\end{array}$ & $\begin{array}{r}Y \\
89 \\
\end{array}$ & $\begin{array}{l}\mathrm{Zr} \\
90 \\
\end{array}$ & $\begin{array}{l}\text { Nb } \\
93 \\
\end{array}$ & $\begin{array}{l}\text { Mo } \\
98\end{array}$ & $\begin{array}{r}\text { Ru } \\
101 \\
\end{array}$ & $\begin{array}{r}\text { Rh } \\
103 \\
\end{array}$ & $\begin{array}{r}\text { Pd } \\
108 \\
\end{array}$ & $\begin{array}{r}\mathrm{Ag} \\
109 \\
\end{array}$ \\
\hline $1-\mathrm{H} 4-11$ & MARCH & ND & 0.5 & ND & ND & 1.5 & 112 & ND & ND & ND & 0.4 & ND & ND & ND & KD \\
\hline $1-H 4-12 A$ & JUL $\gamma$ & ND & 3.1 & ND & 55 & 5.1 & 368 & 0.12 & ND & ND & 1.1 & ND & ND & ND & ND \\
\hline $1-H 4-12 B$ & JULY & ND & 1.8 & ND & ND & 2.6 & 308 & 0.10 & ND & ND & 1.1 & ND & ND & ND & ND \\
\hline $1-\mathrm{H}_{4}-12 \mathrm{C}$ & AUGUST & ND & 4.4 & ND & ND & 1.4 & 169 & 0.03 & ND & ND & 0.2 & ND & NO & ND & ND \\
\hline $1-H_{4}-13$ & AUGUST & ND & 1.6 & ND & 59 & 3.8 & 215 & 0.09 & ND & ND & 1.2 & ND & ND & ND & ND \\
\hline $1-\mathrm{H}_{4}-14$ & AUGUST & NO & 2.6 & ND & 41 & 1.5 & 180 & 0.04 & ND & ND & 0.8 & ND & ND & ND & ND \\
\hline $1-144-15 \mathrm{~A}$ & AUGUST & ND & 2.3 & $\mathrm{ND}$ & 45 & 3.2 & 270 & 0.08 & ND & ND & 1.0 & ND & ND & ND & ND \\
\hline $1-H 4-15 B$ & AUGUST & ND & 1.8 & ND & ND & 2.6 & 198 & 0.05 & $N D$ & ND & 0.7 & ND & ND & ND & ND \\
\hline $1-\mathrm{H} 4-16$ & JULY & ND & 2.3 & ND & ND & 2.7 & 221 & ND & ND & ND & 1.1 & ND & ND & ND & ND \\
\hline $1-144-17$ & AUGUST & ND & 0.5 & ND & 56 & 4.8 & 351 & 0.07 & 0.33 & ND & 3.4 & ND & ND & ND & ND \\
\hline $1-H 4-18$ & AUGUST & ND & 2.2 & ND & ND & 1.6 & 214 & 0.08 & ND & ND & 0.8 & ND & ND & ND & ND \\
\hline $1-k-11$ & APRIL & ND & 6.9 & ND & 67 & 1.0 & 215 & 0.03 & ND & ND & 2.0 & NO & NO & ND & ND \\
\hline $1-k-20$ & MARCH & ND & 1.0 & ND & ND & 0.3 & 119 & 0.02 & ND & ND & 0.5 & ND & ND & ND & ND \\
\hline$i-k-22$ & APR IL & ND & 0.8 & ND & ND & 0.7 & 202 & NO & ND & ND & 1.1 & ND & ND & ND & ND \\
\hline $1-k-27$ & APR IL & ND & 1.4 & ND & ND & 0.3 & 186 & 0.07 & 0.64 & ND & 0.9 & ND & ND & NO & ND \\
\hline $1-k-28$ & APRIL & NO & 2.4 & ND & 58 & 0.4 & 295 & ND & ND & ND & 0.9 & ND & ND & ND & ND \\
\hline $1-x-20$ & $A P R I I$ & Nก & 3.5 & ND & 37 & 0.3 & 176 & ND & ND & ND & 0.9 & ND & ND & ND & ND \\
\hline $1-k-30$ & APRIL & ND & 2.7 & ND & 74 & 0.4 & 249 & ND & ND & ND & 1.0 & ND & ND & ND & ND \\
\hline $1-N-14$ & MARCH & ND & 0.6 & ND & ND & 0.5 & 83 & ND & ND & ND & 0.4 & ND & ND & ND & ND \\
\hline $1-\mathrm{N}-29$ & MARCH & ND & 6.3 & ND & NO & 1.5 & 51 & ND & ND & ND & 0.4 & ND & ND & ND & ND \\
\hline Z-E $13-5$ & MARCH & NO & 0.7 & ND & ND & 0.1 & 76 & ND & ND & ND & 1.8 & ND & ND & ND & ND \\
\hline $2 \cdot E \cdot 17-1$ & APRIL & ND & 3.8 & ND & ND & 1.4 & 489 & ND & ND & ND & 2.5 & ND & ND & ND & ND \\
\hline $2-E 17-5$ & APRIL & $N D$ & 5.0 & ND & ND & 1.2 & 244 & ND & ND & ND & 2.7 & ND & ND & ND & ND \\
\hline $2-E 17-6$ & APR IL & ND & 1.0 & ND & ND & 8.8 & 229 & 0.07 & ND & ND & 4.9 & ND & NO & ND & ND \\
\hline $2-E 17 \cdot 9$ & AFRIL & ND & 10.0 & ND & ND & 0.7 & 321 & ND & NC & ND & 3.9 & ND & $N D$ & $\mathrm{NO}$ & ND \\
\hline $2-E 24-12$ & AORIL & ND & 5.8 & ND & ND & 0.8 & 250 & 0.02 & NO & ND & 3.2 & ND & ND & ND & NO \\
\hline $2-E \geq 4-7$ & JUNE & ND & 5.1 & ND & ND & 1.2 & 134 & 0.02 & ND & NO & 3.1 & ND & ND & ND & NO \\
\hline $2-E 24-8$ & AUGUST & NO & 7.0 & 3 & ND & 1.0 & 198 & 0.07 & ND & ND & 2.8 & ND & ND & ND & NO \\
\hline $2-E 25-18$ & APRIL & $\$ D$ & 8.1 & $\mathrm{NC}$ & ND & 0.7 & 118 & 0.03 & NO & ND & 2.9 & ND & ND & NO & No \\
\hline
\end{tabular}


TABLE D.1. (contd)

(All concentrations expressed as micrograms per liter)

\begin{tabular}{|c|c|c|c|c|c|c|c|c|c|c|c|c|c|c|c|}
\hline & & Ge & As & Se & $\mathrm{B} \Gamma$ & $\mathrm{Rb}$ & $\mathrm{S} r$ & $Y$ & $2 r$ & Nb & Mo & $R \omega$ & $\mathrm{Rh}$ & $P d$ & $\mathrm{Ag}$ \\
\hline We!! & Collected & 72 & 75 & 77 & 81 & 85 & 88 & 89 & 90 & 93. & 98 & 101 & 103 & 108 & 109 \\
\hline $2-E 25-20$ & MARCH & $\mathrm{ND}$ & 2.8 & ND & ND & 0.8 & 138 & ND & ND & ND & 6.3 & ND & NO & ND & ND \\
\hline 2-E25-21 & APRIL & ND & 12.0 & ND & ND & 1.2 & 147 & ND & ND & ND & 11.5 & ND & ND & ND & ND \\
\hline $2-E 25-27$ & JUME & ND & 6.2 & ND & ND & 0.8 & 135 & 0.04 & ND & ND & 6.4 & ND & ND & NO & ND \\
\hline $2-E 25-28$ & JUNE & NO & 9.8 & ND & ND & 1.9 & 124 & 0.07 & ND & ND & 1.3 & ND & ND & NO & ND \\
\hline $2-E 25-7$ & JUNE & ND & 11.2 & ND & ND & 0.6 & 117 & 0.05 & ND & ND & 1.4 & ND & ND & NO & ND \\
\hline $2-E 26-1$ & JUNE & ND & 3.6 & ND & ND & 2.5 & 157 & 0.02 & ND & ND & 2.6 & ND & ND & ND & ND \\
\hline $2-\varepsilon 26-3$ & JUNE & ND & 8.8 & ND & ND & 1.1 & 122 & 0.09 & ND & ND & 1.0 & ND & ND & ND & ND \\
\hline 2-E27-5 & MARCH & ND & 1.9 & ND & ND & 0.3 & 54 & ND & ND & NO & 0.7 & ND & ND & No & ND \\
\hline $2-E 28-17$ & JUNE & ND & 2.5 & 3 & 73 & 1.9 & 270 & 0.07 & ND & NO & 9.6 & ND & ND & ND & ND \\
\hline $2-E 28-7$ & JUNE & ND & 3.8 & NO & ND & 1.0 & 153 & 0.05 & ND & NO & 3.2 & ND & ND & NO & ND \\
\hline $2-E 32-1$ & AUGUST & NO & 5.0 & NO & ND & 1.2 & 154 & 0.03 & NO & ND & 3.0 & ND & ND & NO & ND \\
\hline $2-E 33-1$ & MAY & NO & 7.5 & ND & ND & 0.9 & 129 & 0.05 & ND & ND & 3.0 & NO & ND & NO & ND \\
\hline $2-E 33-18$ & MAY & ND & 6.2 & ND & ND & 0.9 & 153 & 0.04 & ND & ND & 2.6 & ND & ND & ND & ND \\
\hline $2-E 33-2$ & JUNE & ND & 8.2 & ND & ND & 1.0 & 130 & 0.03 & ND & ND & 2.7 & HD & NO & ND & ND \\
\hline $2-E 33-21$ & AUGUST & ND & 6.6 & ND & ND & 1.0 & 154 & 0.02 & ND & ND & 2.2 & ND & ND & ND & ND \\
\hline $2-E 33-24$ & AUGUST & ND & 5.5 & ND & ND & 0.7 & 137 & 0.03 & ND & ND & 2.3 & ND & ND & ND & ND \\
\hline $2-E 33-3$ & AUGUST & ND & 11.3 & ND & ND & 1.0 & 198 & 0.06 & ND & NO & 3.5 & ND & ND & ND & ND \\
\hline $2-E 33-5$ & AUGUST & ND & 8.0 & ND & ND & 1.2 & 196 & 0.07 & NO & ND & 3.2 & ND & ND & NO & ND \\
\hline $2-E 34-1$ & JULY & ND & 2.7 & 4 & 68 & 3.3 & 300 & ND & ND & ND & 3.8 & ND & ND & ND & ND \\
\hline $2-W 6-1$ & JUNE & ND & 0.7 & NO & 82 & 3.7 & 307 & 0.09 & ND & ND & 1.7 & ND & ND & ND & ND \\
\hline $2-W 10-4$ & MARCH & ND & 2.3 & ND & 48 & 0.7 & 47 & ND & ND & ND & 2.6 & ND & ND & ND & ND \\
\hline $2-w 10-9$ & MAY & ND & 19.1 & ND & 218 & 3.8 & 256 & 0.07 & ND & NO & 5.3 & ND & ND & ND & ND \\
\hline $2-w 12-1$ & JUNE & ND & 1.2 & ND & 297 & 2.9 & 566 & 0.16 & ND & ND & 2.5 & AD & ND & ND & ND \\
\hline $2-w 14-5$ & MARCH & ND & 1.9 & ND & 167 & 1.9 & 85 & ND & NO & ND & 3.5 & ND & ND & ND & $\mathrm{ND}$ \\
\hline $2-W 15-14$ & MARCH & ND & 0.7 & ND & ND & 1.9 & 51 & ND & ND & ND & 2.0 & ND & ND & ND & ND \\
\hline $2-w 18-15$ & JULY & ND & 9.3 & ND & ND & 1.8 & 95 & ND & ND & NO & 1.0 & ND & ND & ND & ND \\
\hline $2 \cdot w 19-1$ & JUNE & ND & 6.5 & ND & ND & 1.7 & 72 & 0.06 & ND & ND & 6.1 & ND & ND & ND & ND \\
\hline $2-\omega 19-11$ & MARCH & ND & 1.1 & ND & NO & 2.0 & 122 & ND & ND & ND & 1.8 & ND & ND & ND & ND \\
\hline 2-W19-13 & MARCH & $\mathrm{ND}$ & 1.5 & ND & ND & 1.3 & 91 & ND & ND & ND & 1.9 & ND & ND & ND & ND \\
\hline 2- -19-15 & MARCH & $\mathrm{ND}$ & 0.7 & $\mathrm{ND}$ & 39 & 1.4 & 118 & ND & NO & ND & 1.3 & ND & $\mathrm{ND}$ & ND & $\mathrm{ND}$ \\
\hline
\end{tabular}


TABLE D.1. (contd)

(All concentrations expressed as micrograms per liter)

\begin{tabular}{|c|c|c|c|c|c|c|c|c|c|c|c|c|c|c|c|}
\hline & & Ge & AS & Se & $\mathrm{Br}$ & Rb & $\mathrm{Sr}$ & $Y$ & $\mathrm{Zr}$ & $\mathrm{Nb}$ & Mo & Ru & $\mathrm{Rh}$ & $P d$ & $\mathrm{Ag}$ \\
\hline Hell & Collected & 72 & 75 & $\pi$ & 81 & 85 & 88 & 89 & 90 & 93 & 98 & 101 & 103 & 108 & 100 \\
\hline $2-\$ 19-16$ & MARCH & NO & 0.7 & ND & ND & 0.8 & 66 & ND & ND & ND & 1.6 & ND & NO & ND & ND \\
\hline $2-\omega 19-20$ & JULY & ND & 1.3 & ND & 101 & 3.7 & 1023 & ND & NO & ND & 1.3 & ND & ND & ND & ND \\
\hline $2-w 22-22$ & JULY & ND & 1.2 & ND & NO & 2.5 & 139 & 0.04 & NO & ND & 5.5 & ND & NO & ND & ND \\
\hline $2-122-22$ & MARCH & ND & 0.7 & ND & NO & 0.8 & 51 & ND & ND & ND & 2.0 & ND & ND & ND & ND \\
\hline $2-423-1$ & JUNE & NO & 3.1 & ND & NO & 1.6 & 84 & 0.02 & ND & ND & 6.7 & ND & ND & ND & ND \\
\hline $2-w 23-10$ & MARCH & NO & 1.1 & ND & NO & 1.1 & 114 & NO & ND & ND & 0.6 & ND & ND & ND & ND \\
\hline $2-423-11$ & JUNE & ND & 8.2 & NO & NO & 1.8 & 135 & 0.03 & ND & NO & 1.8 & NO & ND & ND & ND \\
\hline $2-623-3$ & JUNE & ND & 4.1 & NO & ND & 1.9 & 94 & 0.02 & ND & ND & 9.6 & ND & ND & ND & ND \\
\hline $2-w 23-7$ & JUNE & ND & 2.8 & 3 & 74 & 1.1 & 143 & 0.03 & ND & ND & 4.4 & NO & ND & ND & NO \\
\hline $2-w 27-1$ & MARCH & ND & 0.5 & 9 & 43 & 0.1 & 119 & No & ND & ND & 1.2 & ND & NO & ND & ND \\
\hline 3-1-1 & JUNE & ND & 1.1 & ND & 36 & 2.4 & 105 & 0.08 & ND & NO & 1.1 & ND & ND & ND & NO \\
\hline $3-1-2$ & MARCH & ND & 0.7 & ND & ND & 2.5 & 98 & 0.09 & ND & ND & 1.0 & ND & ND & NO & NO \\
\hline $3 \cdot 1 \cdot 3$ & JUNE & NO & 1.3 & NO & 57 & 3.3 & 103 & 0.07 & ND & ND & 2.2 & ND & ND & NO & NO \\
\hline $3 \cdot 1 \cdot 4$ & JUNE & ND & 0.5 & ND & ND & 1.8 & 83 & 0.06 & NO & $\mathrm{ND}$ & 0.8 & ND & ND & ND & ND \\
\hline $3-1-5$ & MARCH & ND & 0.3 & ND & No & 0.9 & 62 & 0.08 & ND & ND & 0.7 & ND & ND & ND & ND \\
\hline $3 \cdot 1.6$ & APRIL & ND & 0.7 & NO & 39 & 1.5 & 87 & 0.17 & 0.26 & No & 0.6 & ND & ND & ND & ND \\
\hline $3.1-6$ & MARCH & ND & 0.3 & ND & ND & 0.9 & 60 & 0.07 & ND & ND & 0.7 & $\mathrm{ND}$ & NO & ND & $\mathrm{N}$ \\
\hline 3.1 .7 & JUNE & NO & 1.0 & ND & ND & 2.6 & 91 & 0.08 & ND & ND & 1.3 & ND & ND & ND & ND \\
\hline $3-1-8$ & JUNE & ND & 3.0 & $\mathrm{ND}$ & 51 & 5.7 & 114 & 0.09 & ND & ND & 7.5 & ND & ND & ND & ND \\
\hline $3-1-9$ & MARCH & ND & ND & ND & NO & 3.0 & 82 & 0.05 & ND & ND & 3.3 & ND & ND & ND & ND \\
\hline $3-1-10$ & MARCH & ND & 0.4 & ND & ND & 1.4 & 65 & 0.21 & ND & ND & 0.7 & ND & ND & ND & ND \\
\hline $3 \cdot 1 \cdot 11$ & MARCH & NO & 0.4 & ND & ND & 0.7 & 53 & 0.06 & 0.23 & ND & 0.6 & ND & ND & ND & ND \\
\hline $3-1-12$ & MARCH & ND & 0.4 & NDD & ND & 1.0 & 56 & 0.21 & NO & ND & 0.8 & ND & ND & $N D$ & NO \\
\hline $3-1-12$ & JUNE & ND & 0.8 & ND & ND & 1.8 & 76 & 0.05 & 0.18 & ND & 0.8 & NO & NO & ND & NO \\
\hline $3-1-13$ & MARCH & ND & 0.7 & ND & ND & 1.0 & 68 & 0.07 & $N D$ & ND & 0.8 & ND & ND & ND & ND \\
\hline $3-1-13$ & APR $1 L$ & ND & 1.3 & ND & 40 & 1.6 & 104 & 0.11 & ND & ND & 1.2 & $\mathrm{ND}$ & ND & NO & ND \\
\hline $3-1-14$ & MARCH & ND & 0.3 & ND & ND & 0.7 & 54 & 0.31 & ND & ND & 0.7 & ND & $\mathrm{ND}$ & ND & ND \\
\hline $3 \cdot 1-15$ & MARCH & ND & $N D$ & ND & ND & 2.7 & 51 & ND & ND & ND & 2.3 & ND & ND & ND & ND \\
\hline $3 \cdot 1 \cdot 15$ & APR IL & ND & 2.1 & ND & 71 & 2.3 & 188 & 0.02 & ND & No & 2.6 & ND & ND & ND & ND \\
\hline $3-1-16 A$ & JUNE & ND & 1.3 & ND & 36 & 2.8 & 113 & 0.09 & NO & No & 1.3 & ND & ND & ND & NO \\
\hline $3-i-16 B$ & JUNE & ND & 1.4 & ND & 38 & 6.2 & 125 & 0.04 & NO & ND & 5.6 & NO & ND & ND & NO \\
\hline
\end{tabular}


TABLE D.1. (contd)

(All concentrations expressed as micrograns per (iter)

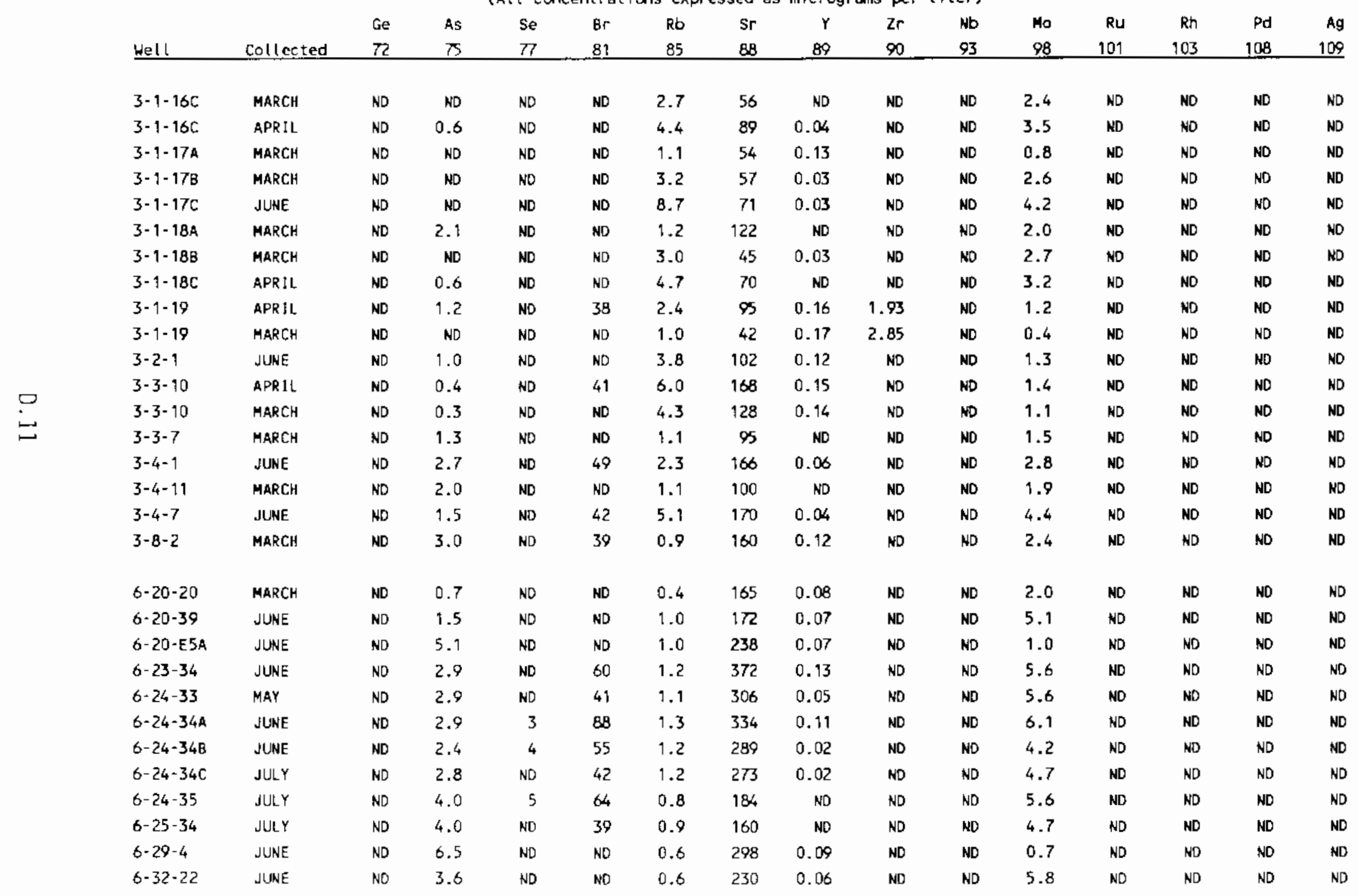


TABLE D.1. (contd)

(All concentrations expressed as micrograns per liter)

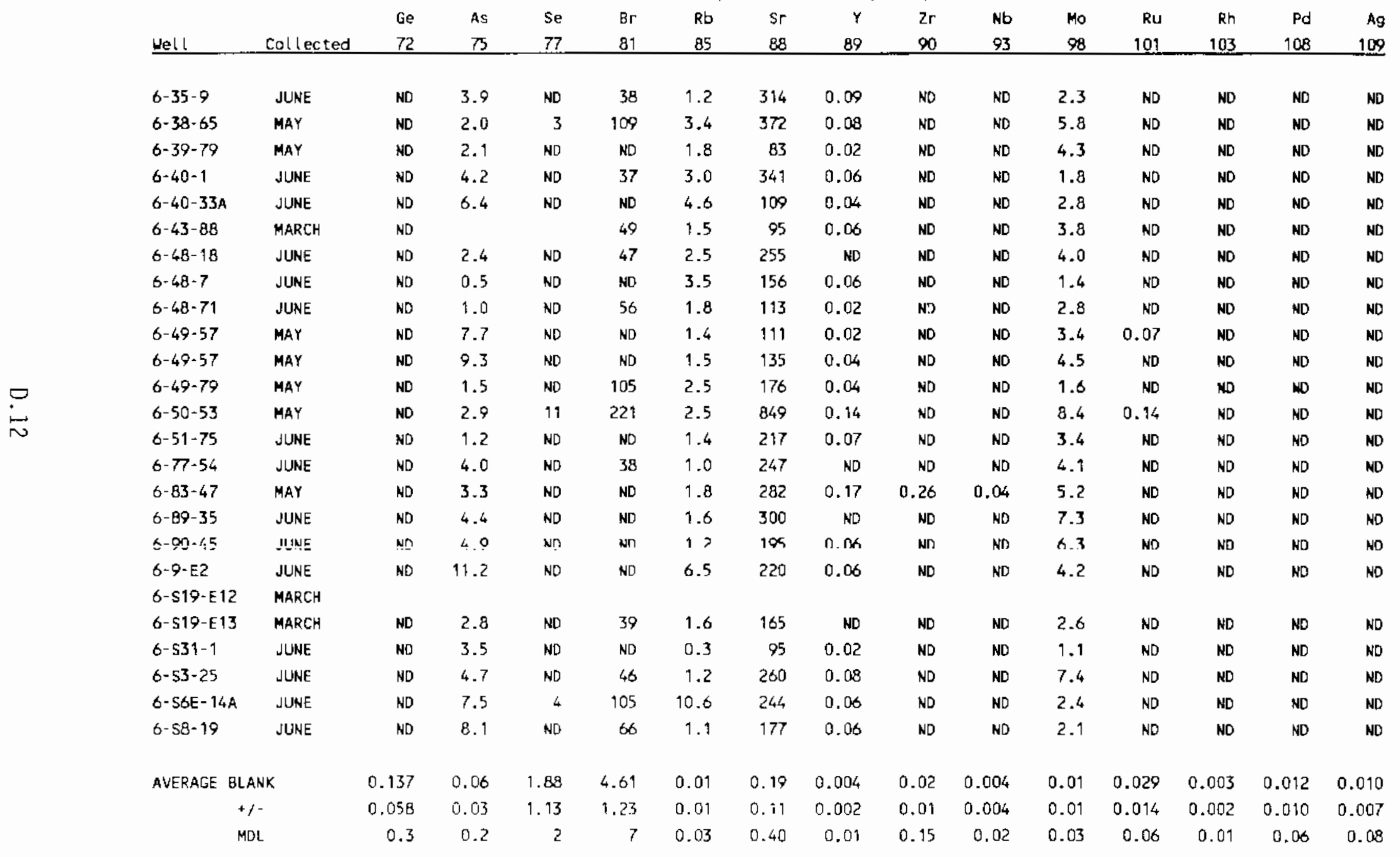


TABLE D.1. (contd)

(All concentrations expressed as micrograms per liter)

\begin{tabular}{|c|c|c|c|c|c|c|c|c|c|c|c|c|c|c|c|c|}
\hline & & $\begin{array}{r}\mathrm{Li} \\
7\end{array}$ & Be & 8 & Al & $\mathrm{Ca}$ & Sc & $V$ & $\mathrm{Cr}$ & $\mathrm{Mn}$ & $\mathrm{Fe}$ & $\begin{array}{l}\text { Co } \\
50\end{array}$ & $\begin{array}{l}\mathrm{Ni} \\
60\end{array}$ & $\mathrm{Cu}$ & $2 n$ & $\begin{array}{l}\text { Ga } \\
60\end{array}$ \\
\hline ell & collected & & $y$ & Fu & 27 & 44 & 45 & & 52 & 35 & & & & & & 09 \\
\hline $1-\mathrm{H} 4-6$ & MARCH & & & & ND & & 0.7 & & 53 & 76 & ND & 0.2 & ND & ND & 145 & 0.29 \\
\hline $1-\mathrm{H} 4-7$ & MARCH & & & & 11 & & 0.5 & 7.8 & 95 & ND & ND & NO & ND & ND & ND & 0.07 \\
\hline $1 \cdot H_{4}-8$ & AUGUST & ND & ND & 68 & 7 & 52872 & 1.4 & 6.6 & 93 & 2 & ND & ND & ND & ND & ND & 0.43 \\
\hline $1-H 4-9$ & AUGUST & ND & ND & 54 & 6 & 85813 & 1.3 & 5.3 & 106 & ND & 241 & NO & ND & NO & ND & 0.61 \\
\hline $1-H 4-10$ & MARCH & & & & ND & & ND & 7.8 & 66 & ND & ND & 0.2 & ND & ND & ND & 0.12 \\
\hline $1-H 4-11$ & MARCH & & & & 4 & & ND & 9.9 & 40 & ND & ND & ND & ND & ND & ND & 0.14 \\
\hline $1 \cdot H 4-12 A$ & JULY $Y$ & ND & ND & ND & 4 & 68612 & 1.8 & 7.0 & 267 & ND & 208 & ND & ND & ND & ND & 0.61 \\
\hline $1-\mathrm{H}_{4}-12 \mathrm{~B}$ & JULY & ND & ND & ND & 3 & 53323 & 1.3 & 4.8 & 203 & ND & ND & ND & ND & ND & ND & 0.83 \\
\hline $1-\mathrm{H}_{4}-12 \mathrm{C}$ & AUGUST & ND & ND & ND & 3 & 21157 & 1.7 & 13.9 & $12 \uparrow$ & 10 & ND & 0.2 & 24 & ND & ND & ND \\
\hline $1-H 4-13$ & AUGUST & ND & ND & ND & 6 & 41301 & 1.6 & 4.6 & 30 & ND & NO & ND & ND & ND & ND & 0.26 \\
\hline $1-\mathrm{H}_{4}-14$ & AUGUST & ND & ND & ND & 4 & 30382 & 1.0 & 5.1 & 215 & ND & ND & ND & ND & ND & ND & 0.17 \\
\hline $1-H 4-15 A$ & AUGUST & 45 & ND & ND & ND & 36407 & 1.5 & 6.8 & 125 & ND & ND & ND & MD & ND & ND & 0.66 \\
\hline $1-H 4-158$ & ALUGUST & ND & ND & ND & ND & 30494 & 1.1 & 6.1 & 102 & 2 & ND & ND & ND & ND & 15 & 0.60 \\
\hline $1-144-16$ & JULY & ND & ND & ND & 5 & 51277 & 1.7 & 5.5 & 265 & 7 & ND & ND & ND & ND & ND & 0.25 \\
\hline $1-H 4-17$ & AUGUST & ND & ND & ND & 106 & 58766 & 1.3 & 2.8 & 31 & 18 & 256 & 0.3 & 23 & ND & ND & 0.71 \\
\hline $1-H 4-18$ & AUGUST & ND & ND & ND & 5 & 40077 & 1.1 & 4.1 & 4 & ND & ND & ND & ND & ND & ND & D.22 \\
\hline $1-k-11$ & APRIL & ND & ND & ND & ND & 42639 & 1.7 & 18.9 & 32 & ND & ND & 0.4 & 17 & ND & ND & 0.22 \\
\hline $1-k-20$ & MARCH & & & & ND & & 0.4 & 7.5 & 100 & $\mathrm{ND}$ & ND & ND & ND & ND & ND & ND \\
\hline $1-k-22$ & APRIL & ND & ND & ND & ND & 43671 & 1.1 & 3.9 & 207 & 2 & ND & 0.2 & ND & ND & 290 & 0.18 \\
\hline $1-k-27$ & APRIL & ND & ND & ND & 3 & 33765 & 1.1 & 7.4 & 4 & 2 & ND & ND & ND & ND & ND & 0.21 \\
\hline $1-k-28$ & APRIL & ND & ND & ND & ND & 66400 & 2.0 & 11.3 & 5 & 2 & ND & ND & ND & ND & ND & 0.30 \\
\hline $1-x-29$ & APRIL & ND & ND & ND & 3 & 41920 & 1.9 & 12.4 & 3 & 7 & ND & ND & ND & ND & ND & 0.19 \\
\hline $1-k-30$ & APRIL & ND & ND & ND & 3 & 53999 & 1.9 & 9.6 & 4 & ND & ND & ND & ND & ND & ND & 0.20 \\
\hline $1-N=14$ & MARCH & & & & ND & & ND & 6.6 & ND & ND & ND & NO & ND & ND & ND & 0.09 \\
\hline $1-N-29$ & MARCH & & & & ND & & ND & 5.6 & ND & ND & ND & ND & ND & ND & ND & $\mathrm{ND}$ \\
\hline $2 \cdot \operatorname{E13-5}$ & MARCH & & & & ND & & ND & 5.2 & ND & ND & ND & ND & ND & ND & ND & 0.06 \\
\hline $2-E 17-1$ & APRIL & 157 & ND & ND & ND & 99552 & 2.6 & 19.3 & ND & 2 & 192 & 0.2 & ND & NO & 17 & 0.64 \\
\hline $2-E 17 \cdot 5$ & APRIL & 96 & ND & ND & 3 & 50489 & 2.8 & 23.2 & ND & ND & ND & ND & ND & ND & ND & 0.29 \\
\hline $2-E 17-6$ & APR IL & 159 & ND & ND & ND & 28153 & 2.6 & 0.6 & ND & 81 & 256 & ND & ND & ND & ND & 0.59 \\
\hline
\end{tabular}




\section{IABLE D.1. (contd)}

(All concentrations expressed as micrograns per liter)

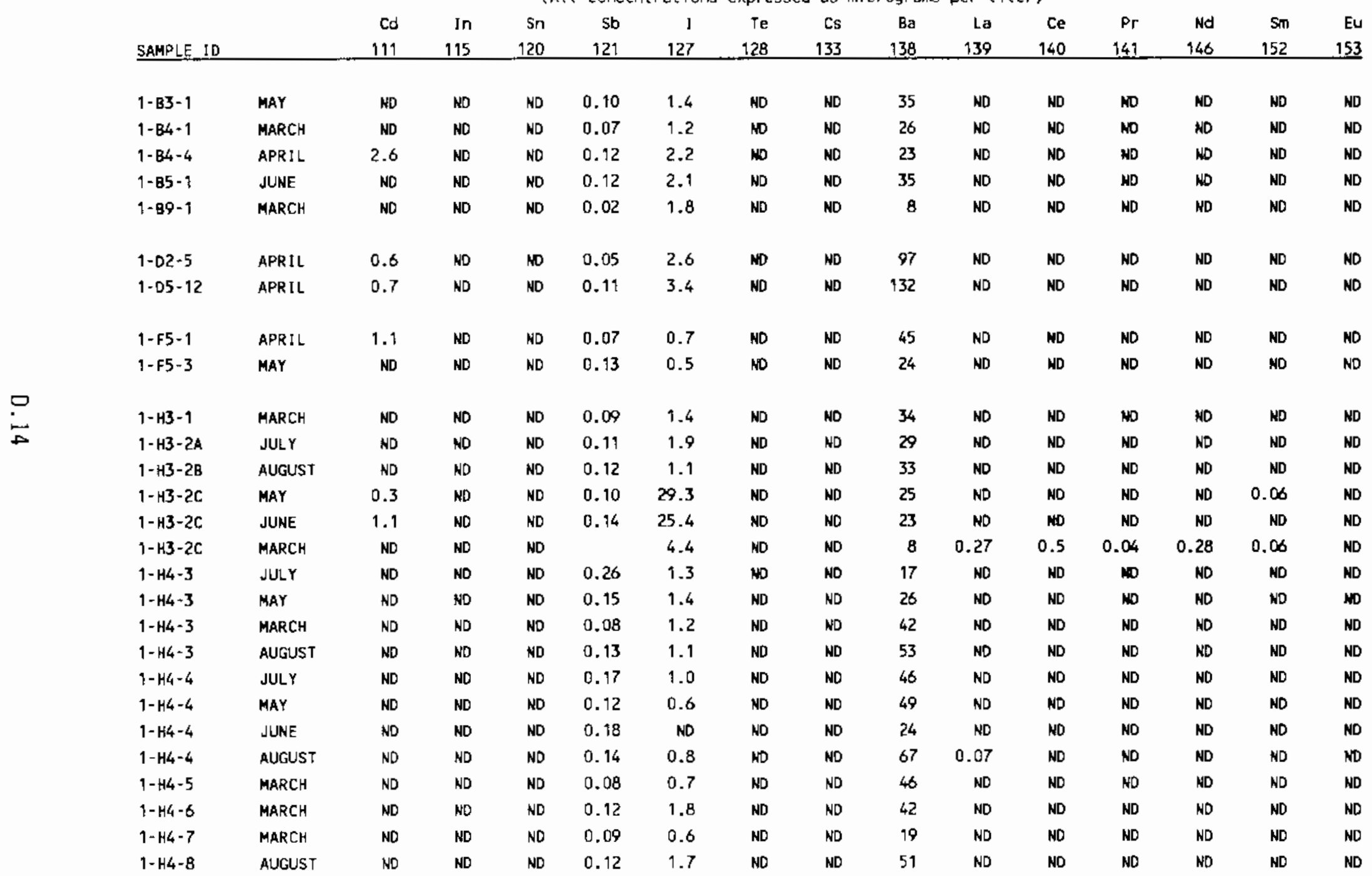


TABLE D.1. (contd)

(All concentrations expressed as micrograns per liter)

\begin{tabular}{|c|c|c|c|c|c|c|c|c|c|c|c|c|c|c|c|}
\hline SAMPLE IO & & $\begin{array}{r}\text { cd } \\
111 \\
\end{array}$ & $\begin{array}{r}\text { In } \\
115\end{array}$ & $\begin{array}{r}\text { Sn } \\
120 \\
\end{array}$ & $\begin{array}{r}\text { Sb } \\
121\end{array}$ & $\begin{array}{r}1 \\
127 \\
\end{array}$ & $\begin{array}{r}\mathrm{Te} \\
128 \\
\end{array}$ & $\begin{array}{r}\mathrm{Cs} \\
133\end{array}$ & $\begin{array}{r}8 a \\
138 \\
\end{array}$ & $\begin{array}{r}48 \\
139 \\
\end{array}$ & $\begin{array}{r}\mathrm{Ce} \\
140 \\
\end{array}$ & $\begin{array}{r}\mathrm{Pr} \\
141 \\
\end{array}$ & $\begin{array}{r}\text { Nd } \\
146\end{array}$ & $\begin{array}{r}5 \mathrm{sin} \\
152 \\
\end{array}$ & $\begin{array}{r}\text { Eu } \\
153 \\
\end{array}$ \\
\hline $1-1+4-9$ & AUGUST & ND & ND & ND & 0.12 & 1.3 & ND & ND & 92 & 0.06 & ND & ND & HD & ND & ND \\
\hline $1-\mathrm{R} 4-10$ & MARCH & ND & ND & ND & NO & 0.5 & ND & ND & 45 & NO & ND & ND & ND & $M$ & NO \\
\hline $1-H 4-11$ & MARCH & ND & ND & ND & 0.07 & ND & ND & NO & 18 & ND & ND & ND & ND & ND & W \\
\hline $9-H 4-12 A$ & JULY & ND & ND & ND & 0.12 & 1.1 & ND & NO & 65 & ND & ND & ND & NDD & NO & ND \\
\hline $1-H 4-128$ & JULY & ND & ND & $\mathrm{ND}$ & 0.11 & 0.8 & ND & ND & 92 & 0.09 & ND & ND & NO & 10 & ND \\
\hline $1-H_{4}-12 \mathrm{C}$ & AUGUST & NO & ND & ND & 0.04 & 0.8 & ND & NO & 5 & ND & ND & NO & ND & ND & NO \\
\hline $1-H 4-13$ & AUGUST & ND & ND & ND & 0.10 & ND & ND & NO & 25 & ND & ND & NO & ND & ND & ND \\
\hline $1-1,14-14$ & AUGUST & ND & ND & ND & 0.09 & ND & ND & ND & 18 & ND & no & NO & ND & ND & ND \\
\hline $1-\mathrm{H} 4-15 A$ & AUGUST & ND & ND & ND & 0.08 & 1.1 & ND & $N D$ & 83 & ND & ND & ND & ND & ND & ND \\
\hline $4-H 4-158$ & AUGUST & ND & ND & ND & 0.05 & 0.6 & ND & NO & 81 & ND & ND & $N D$ & ND & ND & ND \\
\hline $9-H_{4}-16$ & JULY & NO & ND & ND & 0.10 & 0.9 & ND & ND & 35 & ND & MD & ND & NO & ND & ND \\
\hline $1-H 4-17$ & AUGUST & ND & ND & ND & 0.22 & 1.4 & ND & ND & $n$ & 0.07 & ND & ND & NO & ND & ND \\
\hline $1-H 4-18$ & AUGUST & ND & ND & ND & 0.12 & 0.6 & ND & NO & 23 & ND & ND & $N D$ & ND & ND & $D$ \\
\hline $1-k-11$ & APR IL & 0.8 & ND & ND & 0.13 & 3.4 & ND & ND & 34 & ND & ND & MD & ND & NO & No \\
\hline $1-k-20$ & MARCH & ND & ND & ND & 0.09 & ND & ND & ND & 10 & ND & ND & NO & $W$ & No & $\mathbf{N D}$ \\
\hline $1-k-22$ & APRIL & 1.1 & NO & ND & 0.11 & 0.8 & ND & ND & 19 & ND & $\mathrm{mo}$ & ND & NO & ND & ND \\
\hline $1-k-27$ & APRIL & 3.1 & NO & ND & 0.11 & 0.6 & ND & ND & 23 & ND & ND & ND & ND & NO & WD \\
\hline $1-k-28$ & APR IL & ND & YND & ND & 0.08 & 1.8 & no & ND & 38 & ND & ND & ND & $\omega$ & NO & ND \\
\hline $1-k-29$ & APRIL & ND & ND & ND & 0.11 & 0.9 & ND & ND & 20 & ND & ND & NO & NO & $W$ & ND \\
\hline $1-k-30$ & APRIL & ND & ND & ND & 0.09 & 1.4 & ND & ND & 27 & ND & ND & NO & ND & ND & ND \\
\hline $1-N-14$ & MARCH & ND & ND & ND & 0.08 & ND & ND & NO & 11 & ND & NO & ND & ND & ND & KD \\
\hline $1-N-29$ & MARCH & ND & ND & ND & 0.15 & ND & NO & ND & 6 & ND & ND & ND & ND & ND & $N$ \\
\hline 2-E13-5 & MARCH & ND & ND & ND & 0.03 & 1.4 & ND & ND & 21 & ND & ND & ND & ND & NO & ND \\
\hline 2-E17-1 & APRIL & 0.6 & ND & ND & 0.09 & 1.2 & ND & ND & 88 & $R D$ & ND & ND & ND & ND & ND \\
\hline $2-E 17-5$ & APR IL & 0.6 & ND & ND & 0.11 & 1.5 & ND & NO & 45 & ND & ND & ND & ND & ND & ND \\
\hline $2-E 17-6$ & APRIL & $\mathrm{ND}$ & ND & ND & ND & 6.7 & ND & NO & 73 & 0.07 & ND & ND & ND & ND & NO \\
\hline $2-E 17-9$ & APRIL & ND & ND & ND & 0.10 & 1.4 & ND & ND & 67 & ND & ND & ND & ND & ND & ND \\
\hline $2-E 24-12$ & APRIL & ND & ND & ND & 0.08 & 1.2 & ND & ND & 48 & ND & ND & ND & ND & ND & NO \\
\hline $2-E 24-7$ & JUNE & 0.4 & ND & ND & 0.76 & 3.1 & ND & ND & 35 & ND & ND & ND & ND & ND & NO \\
\hline
\end{tabular}




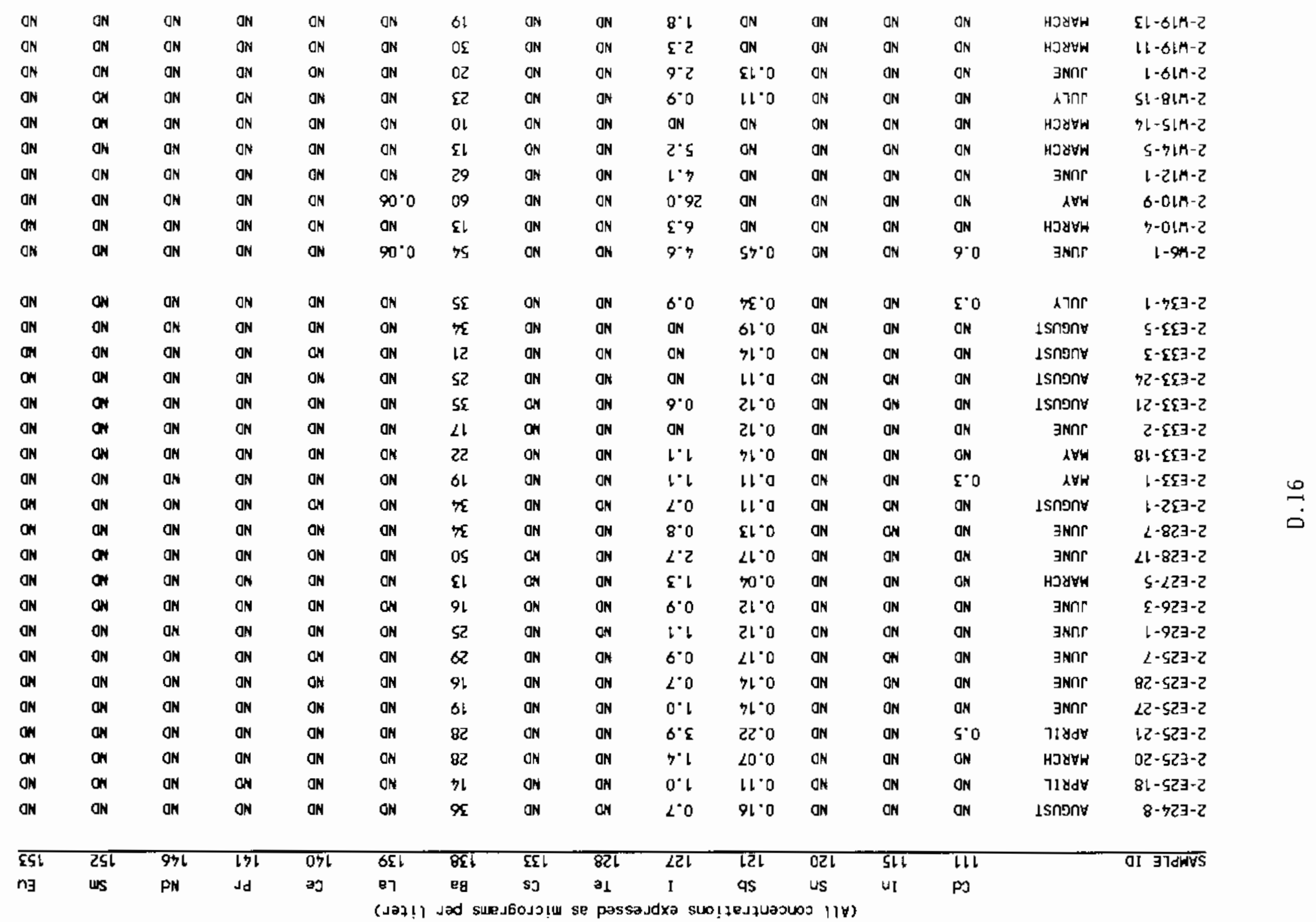

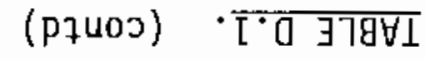




\section{TABLE D.1. (contd)}

(All concentrations expressed as micrograms per liter)

\begin{tabular}{|c|c|c|c|c|c|c|c|c|c|c|c|c|c|c|c|}
\hline SAMPLE 10 & & $\begin{array}{r}\text { Cd } \\
111 \\
\end{array}$ & $\begin{array}{r}\text { In } \\
115 \\
\end{array}$ & $\begin{array}{r}\text { Sn } \\
120 \\
\end{array}$ & $\begin{array}{r}\text { Sb } \\
121 \\
\end{array}$ & $\begin{array}{r}1 \\
127 \\
\end{array}$ & $\begin{array}{r}\text { Ye } \\
128 \\
\end{array}$ & $\begin{array}{r}\text { Cs } \\
133 \\
\end{array}$ & $\begin{array}{r}80 \\
138 \\
\end{array}$ & $\begin{array}{r}\text { La } \\
139 \\
\end{array}$ & $\begin{array}{r}\mathrm{Ce} \\
140 \\
\end{array}$ & $\begin{array}{r}\mathrm{Pr} \\
141 \\
\end{array}$ & $\begin{array}{r}\text { Wd } \\
146 \\
\end{array}$ & $\begin{array}{r}5 \pi \\
152 \\
\end{array}$ & $\begin{array}{r}\text { Eu } \\
153 \\
\end{array}$ \\
\hline $2-\$ 19-15$ & MARCH & ND & ND & ND & NO & 0.3 & ND & ND & 22 & NO & 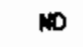 & $N D$ & ND & ND & No \\
\hline $2+w 19 \cdot 16$ & MARCH & ND & MD & $N D$ & ND & ND & No & ND & 18 & NO & ND & ND & ND & ND & ND \\
\hline $2-w 19+20$ & JULYY & 0.3 & ND & W & ND & 4.0 & ND & $D$ & 195 & ND & ND & ND & NO & NO & ND \\
\hline $2-422-22$ & JULY & 0.8 & ND & ND & ND & 10.1 & NO & ND & 23 & NO & $N$ & wo & ND & ND & No \\
\hline $2-422-22$ & MARCH & ND & ND & ND & NO & ND & ND & NO & 10 & ND & $N$ & in & ND & ND & ND \\
\hline 2-w23-1 & JUNE & NO & ND & ND & 0.06 & 0.8 & ND & NO & 23 & WD & 10 & ND & ND & ND & ND \\
\hline $2-x_{23}-10$ & MARCH & ND & ND & ND & ND & ND & ND & NO & 34 & ND & ND & ND & NO & ND & MD \\
\hline $2-v_{23}-11$ & JUNE & ND & ND & ND & 0.06 & 0.6 & ND & ND & 24 & ND & NO & ND & NO & ND & No \\
\hline $2-423-3$ & JUNE & ND & ND & ND & 0.06 & 0.9 & ND & ND & 20 & ND & No & ND & ND & ND & No \\
\hline $2-w 23-7$ & JUNE & 0.3 & ND & ND & 0.04 & 0.8 & ND & $N D$ & 33 & ND & NO & NO & $M$ & ND & No \\
\hline $2-w 27-1$ & MARCH & ND & ND & ND & ND & ND & No & NO & 19 & ND & $M$ & 10 & $N D$ & ND & ND \\
\hline $3 \cdot 1 \cdot 1$ & JUNE & ND & ND & ND & D. 13 & ND & ND & NO & 31 & MD & NO & ND & No & ND & ND \\
\hline $3-1 \cdot 2$ & MARCH & ND & ND & $M$ & 0.11 & ND & ND & ND & 21 & ND & ND & ND & ND & ND & No \\
\hline $3-1-3$ & JUNE & ND & ND & ND & 0.19 & 0.6 & ND & ND & 19 & ND & ND & 10 & $N D$ & MD & No \\
\hline $3-1-4$ & JUNE & ND & NO & NO & 0.18 & ND & ND & ND & 16 & NO & No & 10 & ND & ND & $M 0$ \\
\hline $3-1-5$ & MARCH & ND & NO & ND & 0.12 & ND & ND & ND & 13 & No & $M$ & ND & ND & ND & MD \\
\hline $3-1-6$ & APR IL & ND & NO & ND & 0.13 & ND & NO & ND & 18 & ND & WD & ND & ND & ND & ND \\
\hline $3-1-6$ & MARCH & ND & NO & ND & 0.08 & ND & NO & ND & 12 & NO & ND & no & No & ND & No \\
\hline $3-1-7$ & JUNE & ND & NO & ND & 0.15 & ND & NO & ND & 19 & ND & ND & WD & ND & ND & ND \\
\hline $3-1-8$ & JUNE & ND & ND & ND & 0.28 & 1.2 & ND & ND & 26 & ND & ND & ND & NO & ND & NO \\
\hline $3-1-9$ & MARCH & ND & ND & ND & 0.07 & 63.8 & ND & ND & 93 & 0.11 & 0.24 & 0.04 & 0.18 & 0.05 & 10 \\
\hline $3-1-10$ & MARCH & ND & ND & $N D$ & 0.07 & 1.5 & ND & NO & 19 & ND & ND & ND & ND & ND & ND \\
\hline $3-1-11$ & MARCH & ND & ND & ND & 0.09 & ND & ND & ND & 10 & ND & ND & ND & ND & ND & ND \\
\hline $3-1-12$ & MARCH & ND & ND & ND & 0.09 & ND & ND & ND & 13 & ND & ND & ND & ND & ND & 10 \\
\hline $3-1-12$ & JUNE & ND & ND & NO & 0.18 & ND & ND & ND & 14 & ND & מא & ND & ND & ND & ND \\
\hline $3-1-13$ & MARCH & ND & ND & ND & 0.07 & ND & ND & ND & 16 & ND & ND & ND & NO & ND & ND \\
\hline $3-1-13$ & APRIL & ND & ND & ND & 0.09 & 1.0 & ND & ND & 23 & ND & ND & ND & NO & ND & NO \\
\hline $3-9-14$ & MARCH & ND & ND & ND & 0.06 & ND & ND & ND & 12 & ND & ND & ND & ND & ND & NO \\
\hline $3-1-15$ & MARCH & ND & ND & ND & ND & 27.8 & ND & ND & 45 & ND & ND & ND & ND & ND & ND \\
\hline $3-1-15$ & APRIL & ND & ND & $N D$ & 0.08 & 2.2 & ND & ND & 43 & NO & ND & ND & ND & ND & ND \\
\hline $3-1-16 A$ & JUNE & ND & ND & ND & 0.11 & ND & ND & ND & 29 & 0.06 & ND & ND & ND & ND & ND \\
\hline
\end{tabular}




\section{IABLE D.1. (contd)}

(All concentrations expressed as microgrems per liter)

\begin{tabular}{|c|c|c|c|c|c|c|c|c|c|c|c|c|c|c|c|}
\hline SAMPLE ID & & $\begin{array}{r}\text { Cd } \\
111 \\
\end{array}$ & $\begin{array}{r}1 n \\
115 \\
\end{array}$ & $\begin{array}{r}\text { Sn } \\
120 \\
\end{array}$ & $\begin{array}{r}\text { Sb } \\
121\end{array}$ & $\begin{array}{r}1 \\
127 \\
\end{array}$ & $\begin{array}{r}\mathrm{Te} \\
128 \\
\end{array}$ & $\begin{array}{r}\text { Cs } \\
133 \\
\end{array}$ & $\begin{array}{r}\mathrm{Ba} \\
138 \\
\end{array}$ & $\begin{array}{r}\text { ta } \\
139 \\
\end{array}$ & $\begin{array}{r}\text { Ce } \\
140 \\
\end{array}$ & $\begin{array}{r}\mathrm{Pr} \\
143 \\
\end{array}$ & $\begin{array}{r}\text { Nd } \\
146 \\
\end{array}$ & $\begin{array}{r}\text { Sm } \\
152 \\
\end{array}$ & $\begin{array}{r}\text { Eu } \\
153 \\
\end{array}$ \\
\hline $3-1-168$ & JUNE & NO & NO & ND & ND & 29.3 & ND & NO & 55 & ND & no & no & ND & 0.06 & ND \\
\hline $3-1+16 c$ & MARCH & ND & NO & ND & NO & 28.8 & ND & ND & 46 & ND & KD & ND & ND & ND & ND \\
\hline $3-1-16 C$ & APR IL & ND & ND & ND & ND & 25.4 & ND & ND & 61 & 0.07 & ND & ND & ND & ND & NO \\
\hline $3-1-17 A$ & MARCH & ND & ND & ND & ND & NO & ND & ND & 14 & ND & ND & ND & No & ND & ND \\
\hline $3-1-17 B$ & MARCH & NO & ND & ND & ND & 13.8 & ND & ND & 42 & ND & ND & ND & ND & ND & no \\
\hline $3-1-17 c$ & JUNE & NO & ND & ND & ND & 19.7 & ND & ND & 82 & 0.09 & ND & ND & $N D$ & ND & 10 \\
\hline $3-1-18 A$ & MARCH & ND & ND & ND & ND & 1.4 & ND & ND & 30 & ND & ND & ND & ND & ND & no \\
\hline $3-1-18 B$ & MARCH & ND & ND & ND & ND & 28.5 & ND & ND & 27 & ND & ND & ND & ND & ND & no \\
\hline $3-1-18 \mathrm{C}$ & APRIL & ND & ND & ND & ND & 16.9 & NO & ND & 60 & ND & ND & ND & ND & ND & w \\
\hline $3 \cdot 1-19$ & APRIL & ND & ND & ND & 0.15 & 1.2 & NO & ND & 21 & ND & ND & ND & ND & NO & no \\
\hline $3-1-19$ & MARCK & ND & ND & NO & ND & ND & NO & ND & 16 & 0.23 & 0.42 & 0.06 & 0.23 & 0.06 & no \\
\hline $3-2-1$ & JUNE & ND & ND & ND & 0.15 & ND & NO & ND & 43 & NO & ND & no & ND & No & ND \\
\hline $3-3-10$ & APRIL & ND & ND & ND & 0.11 & 0.8 & ND & ND & 51 & ND & $m$ & no & ND & ND & HD \\
\hline $3-3-10$ & MARCH & ND & ND & ND & ND & 0.6 & NO & ND & 40 & ND & ND & ND & ND & ND & ND \\
\hline $3-3-7$ & MARCH & ND & ND & ND & 0.07 & 1.3 & NO & ND & 23 & NO & ND & ND & ND & ND & ND \\
\hline $3-4-1$ & JUNE & ND & ND & ND & 0.12 & 1.8 & ND & ND & 38 & ND & ND & ND & No & ND & ND \\
\hline $3-4-11$ & MARCH & ND & ND & ND & 0.06 & 1.2 & ND & ND & 25 & ND & ND & ND & ND & NO & ND \\
\hline $3+4-7$ & JUNE & HD & ND & ND & 0.13 & 1.7 & ND & ND & 40 & ND & ND & ND & ND & ND & ND \\
\hline $3-8-2$ & MARCH & ND & ND & ND & 0.07 & 1.2 & ND & NO & 35 & ND & No & ND & ND & ND & no \\
\hline $6 \cdot 20-20$ & MARCH & ND & ND & ND & 0.11 & 1.7 & ND & ND & 54 & ND & ND & No & ND & ND & ND \\
\hline $6-20-39$ & JUNE & ND & ND & ND & 0.05 & 2.7 & ND & ND & 27 & ND & ND & ND & ND & ND & ND \\
\hline $6-20-E 5 A$ & JUNE & ND & ND & ND & 0.08 & 0.9 & ND & ND & 55 & ND & ND & NO & ND & ND & ND \\
\hline $6-23-34$ & JUNE & 0.9 & ND & ND & 0.08 & 3.3 & ND & ND & 82 & 0.09 & ND & NO & No & ND & ND \\
\hline $6-24-33$ & MAY & 0.3 & ND & ND & 0.06 & 3.0 & ND & ND & 82 & ND & ND & ND & ND & ND & ND \\
\hline $6-24-34 A$ & JUNE & 0.4 & NO & ND & 0.10 & 6.3 & ND & ND & 68 & 0.07 & ND & ND & ND & NO & ND \\
\hline $6-24-348$ & JUNE & 0.3 & ND & NO & 0.08 & 3.8 & ND & ND & 72 & ND & ND & ND & ND & ND & ND \\
\hline $6-24-34 \mathrm{C}$ & JULY & ND & ND & ND & 0.09 & 3.4 & ND & NO & 54 & ND & ND & AD & NO & ND & ND \\
\hline $6-24-35$ & JULYY & 1.5 & ND & ND & 0.11 & 3.4 & ND & ND & 44 & ND & ND & No & ND & ND & ND \\
\hline $6-25-34$ & JULY & ND & ND & ND & 0.10 & 2.8 & ND & ND & 33 & ND & ND & ND & ND & No & ND \\
\hline $6-29-4$ & JUNE & ND & ND & ND & 0.14 & 1.4 & ND & ND & 71 & 0.05 & ND & ND & ND & ND & ND \\
\hline $6-32-22$ & JUNE & ND & $\mathrm{ND}$ & ND & 0.12 & 3.0 & ND & ND & 37 & 0.05 & ND & ND & ND & ND & ND \\
\hline
\end{tabular}


TABLE D.1. (contd)

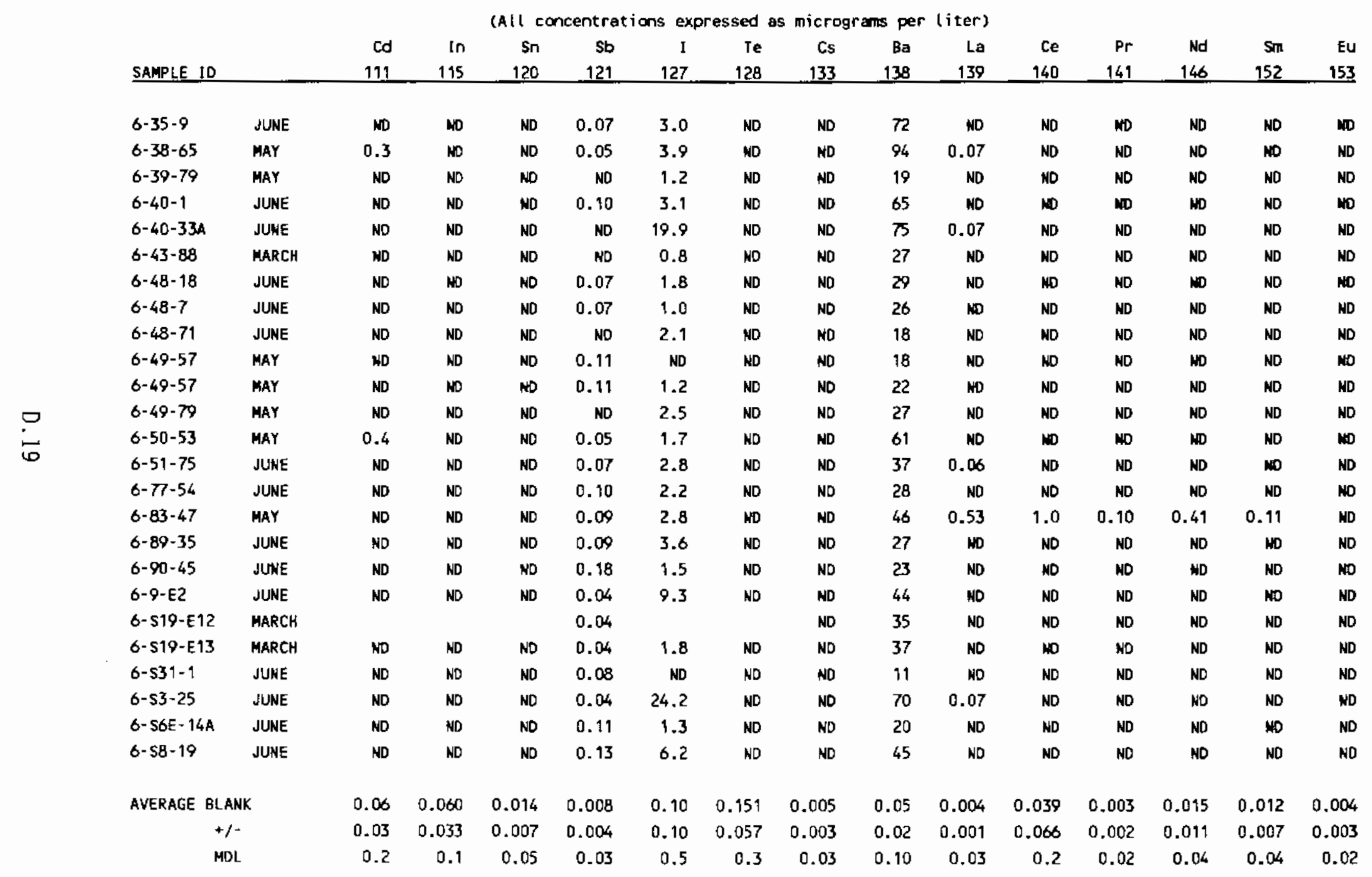


TABLE D.1. (contd)

(All concentrations expressed as micrograms per (iter)

\begin{tabular}{|c|c|c|c|c|c|c|c|c|c|c|c|c|c|c|c|}
\hline SAMPLE ID & & $\begin{array}{r}\text { Gd } \\
158\end{array}$ & $\begin{array}{r}\text { Tb } \\
159 \\
\end{array}$ & $\begin{array}{r}\text { Dy } \\
163\end{array}$ & $\begin{array}{r}\text { Ho } \\
165 \\
\end{array}$ & $\begin{array}{r}\text { Er } \\
166 \\
\end{array}$ & $\begin{array}{r}\text { Im } \\
16 \theta\end{array}$ & $\begin{array}{r}Y b \\
172 \\
\end{array}$ & $\begin{array}{r}\text { Lu } \\
175 \\
\end{array}$ & $\begin{array}{r}H f \\
178\end{array}$ & $\begin{array}{r}\mathrm{Ta} \\
181 \\
\end{array}$ & $\begin{array}{r}\omega \\
182 \\
\end{array}$ & $\begin{array}{r}\mathrm{Re} \\
187 \\
\end{array}$ & $\begin{array}{r}0 s \\
189 \\
\end{array}$ & $\begin{array}{r}\text { Ir } \\
193 \\
\end{array}$ \\
\hline $1-B 3-1$ & MAY & ND & ND & NO & ND & ND & ND & NO & ND & ND & ND & ND & ND & ND & ND \\
\hline $9+B 4-1$ & MARCH & ND & ND & ND & ND & NO & NO & ND & ND & ND & ND & ND & ND & ND & ND \\
\hline $1-B 4-4$ & APR IL & ND & ND & ND & HO & NO & ND & ND & ND & ND & ND & ND & ND & ND & ND \\
\hline $1-B 5-1$ & JUNE & ND & ND & ND & ND & ND & ND & ND & $N D$ & $M D$ & ND & ND & ND & ND & $\$ 0$ \\
\hline $1-B 9-1$ & MARCH & ND & ND & NO & ND & ND & ND & ND & ND & NO & ND & ND & NO & ND & ND \\
\hline $1-02-5$ & APRIL & ND & ND & ND & ND & ND & ND & ND & NO & ND & ND & ND & ND & ND & ND \\
\hline $1-05 \cdot 12$ & APRIL & ND & ND & ND & ND & ND & $M D$ & ND & ND & ND & ND & 0.3 & no & ND & ND \\
\hline $1-F 5-1$ & APRIL & ND & NO & ND & ND & No & ND & ND & NO & ND & ND & ND & ND & ND & ND \\
\hline $1-F 5-3$ & MAY & ND & ND & ND & ND & No & ND & ND & ND & ND & ND & ND & ND & ND & 10 \\
\hline $1-\mathrm{H3}-1$ & MARCH & ND & NO & ND & NO & ND & ND & ND & ND & ND & ND & ND & ND & ND & ND \\
\hline $1-H 3-2 A$ & JULY & ND & NO & ND & NO & ND & ND & ND & ND & ND & ND & ND & ND & no & ND \\
\hline $1-H 3-2 B$ & AUGUST & ND & NO & ND & ND & ND & ND & ND & ND & MO & ND & ND & ND & NO & ND \\
\hline $1-H 3-2 C$ & MAY & ND & ND & ND & ND & ND & ND & ND & 10 & ND & ND & 0.4 & ND & 0.09 & ND \\
\hline $1-H 3-2 C$ & JUNE & MD & NO & ND & ND & ND & ND & NO & NO & ND & ND & 0.4 & ND & ND & ND \\
\hline$i-H 3-2 C$ & MARCH & ND & NO & ND & ND & ND & ND & 0.07 & ND & ND & ND & NO & Nn & ND & Mn \\
\hline $1-44-3$ & JULY & ND & NO & ND & ND & ND & ND & NO & ND & ND & ND & 0.3 & ND & ND & ND \\
\hline $1-H 4-3$ & MAY & ND & NO & ND & ND & ND & ND & ND & ND & ND & ND & ND & ND & ND & ND \\
\hline $1-H 4-3$ & MARCH & NO & ND & ND & ND & ND & ND & ND & ND & ND & HD & ND & ND & ND & ND \\
\hline $1-H 4-3$ & AUGUST & ND & ND & ND & ND & ND & ND & 0.10 & ND & ND & ND & ND & ND & ND & ND \\
\hline $1-H 4-4$ & JULY & ND & ND & ND & ND & ND & ND & ND & ND & NO & ND & ND & ND & ND & ND \\
\hline $1-H 4-4$ & MAY & ND & ND & ND & NO & ND & No & ND & NO & ND & ND & ND & ND & ND & ND \\
\hline 1-H4-4 & JUNE & ND & ND & ND & ND & ND & No & NO & ND & ND & ND & ND & ND & ND & ND \\
\hline 1-H4-4 & AUGUST & ND & ND & ND & ND & ND & ND & ND & ND & ND & ND & ND & ND & ND & ND \\
\hline $9-\mathrm{H} 4-5$ & MARCH & ND & ND & NO & ND & ND & ND & NO & NO & ND & ND & ND & ND & ND & ND \\
\hline $1-1+14-6$ & MARCH & ND & ND & ND & ND & ND & ND & ND & ND & ND & NO & ND & ND & ND & ND \\
\hline $1-\mathrm{H} 4-7$ & MARCH & ND & ND & ND & NO & ND & ND & ND & ND & ND & ND & ND & ND & ND & ND \\
\hline $9-H 4-8$ & AUGUST & ND & ND & ND & ND & No & ND & ND & ND & ND & ND & ND & ND & 0.06 & ND \\
\hline $1-H 4 \cdot 9$ & AUGUST & NO & ND & ND & ND & NO & ND & ND & ND & ND & ND & NO & ND & ND & ND \\
\hline $1-H 4-10$ & MARCH & ND & ND & ND & ND & ND & ND & ND & ND & ND & ND & ND & ND & ND & ND \\
\hline
\end{tabular}


TABLE D.1. (contd)

(All concentrations expressed as micrograms per liter)

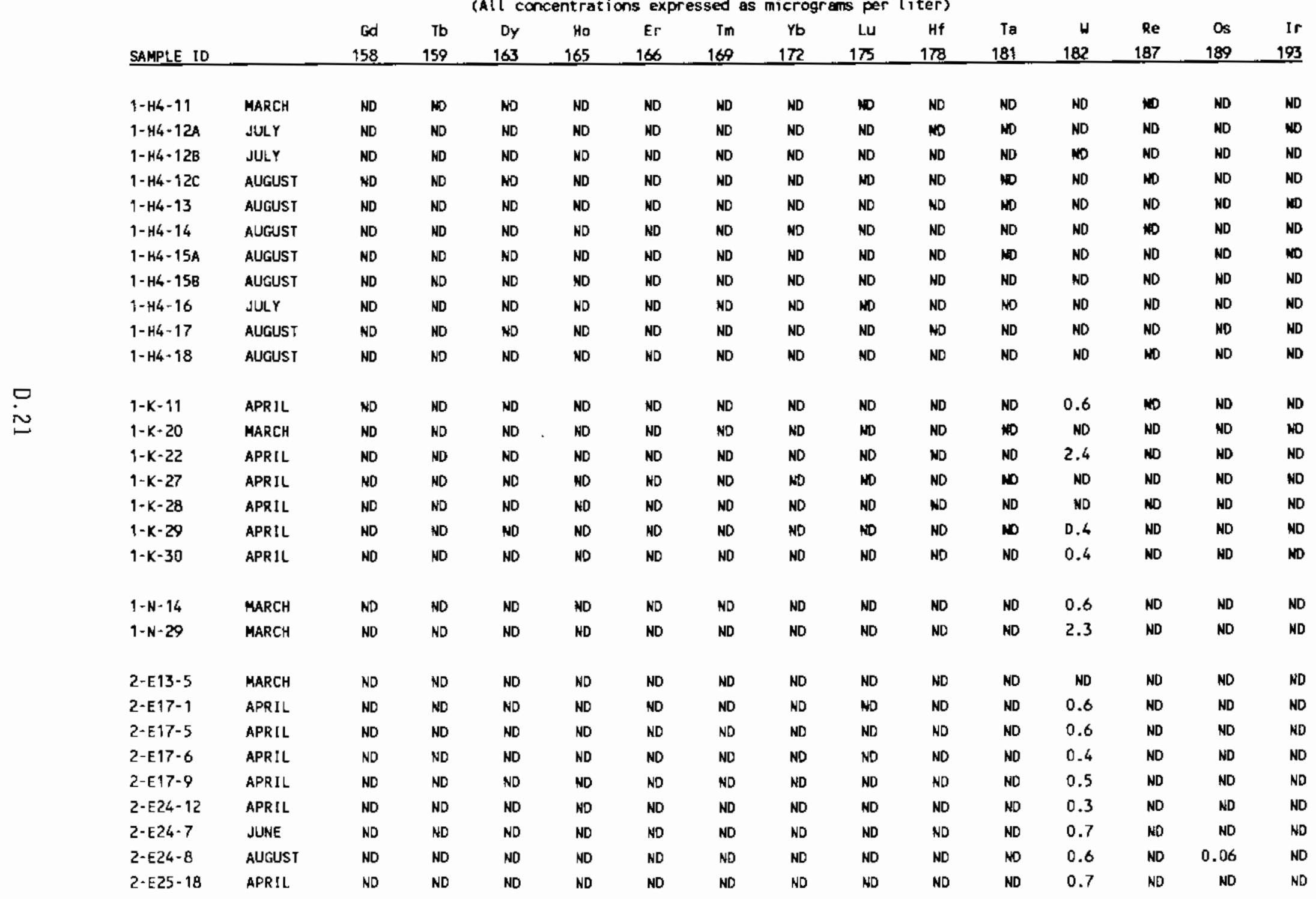


TABLE D.1. (contd)

(Al! concentrations expressed as micrograms per liter)

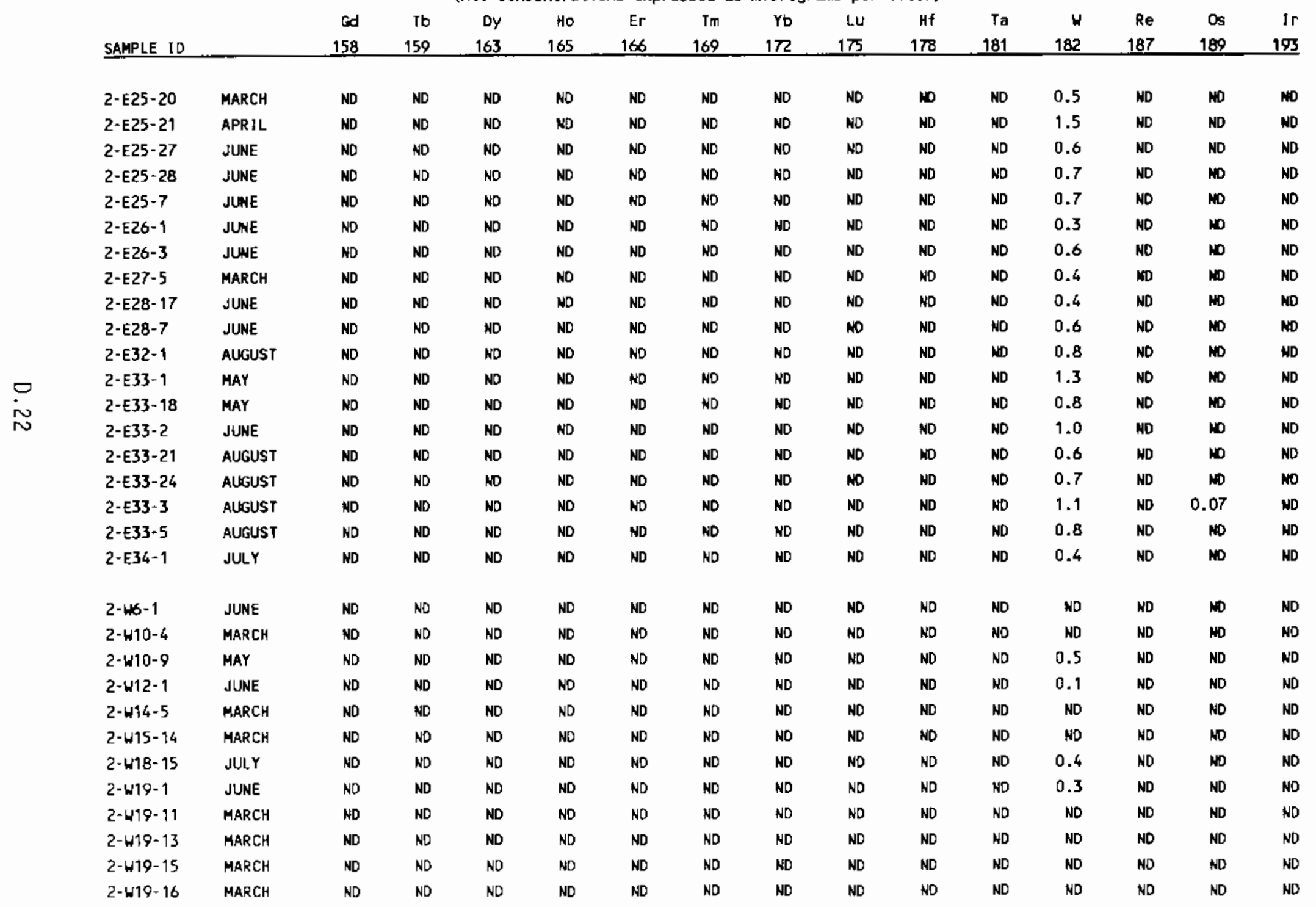




\section{IABLE D.1. (contd)}

(All concentrations expressed as microgrens per liter)

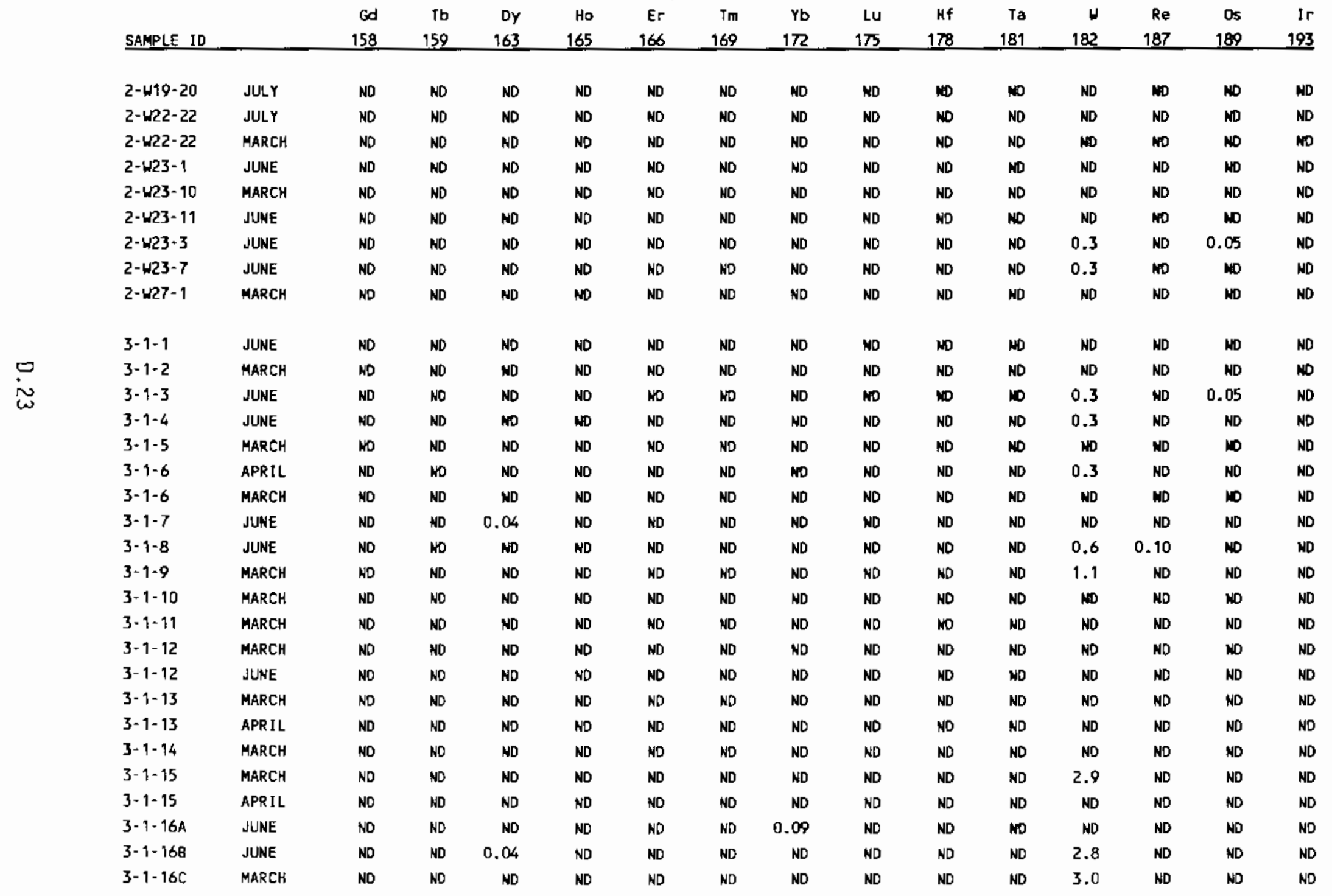


TABLE D.. . (contd)

(All concentrations expressed as micrograms per (iter)

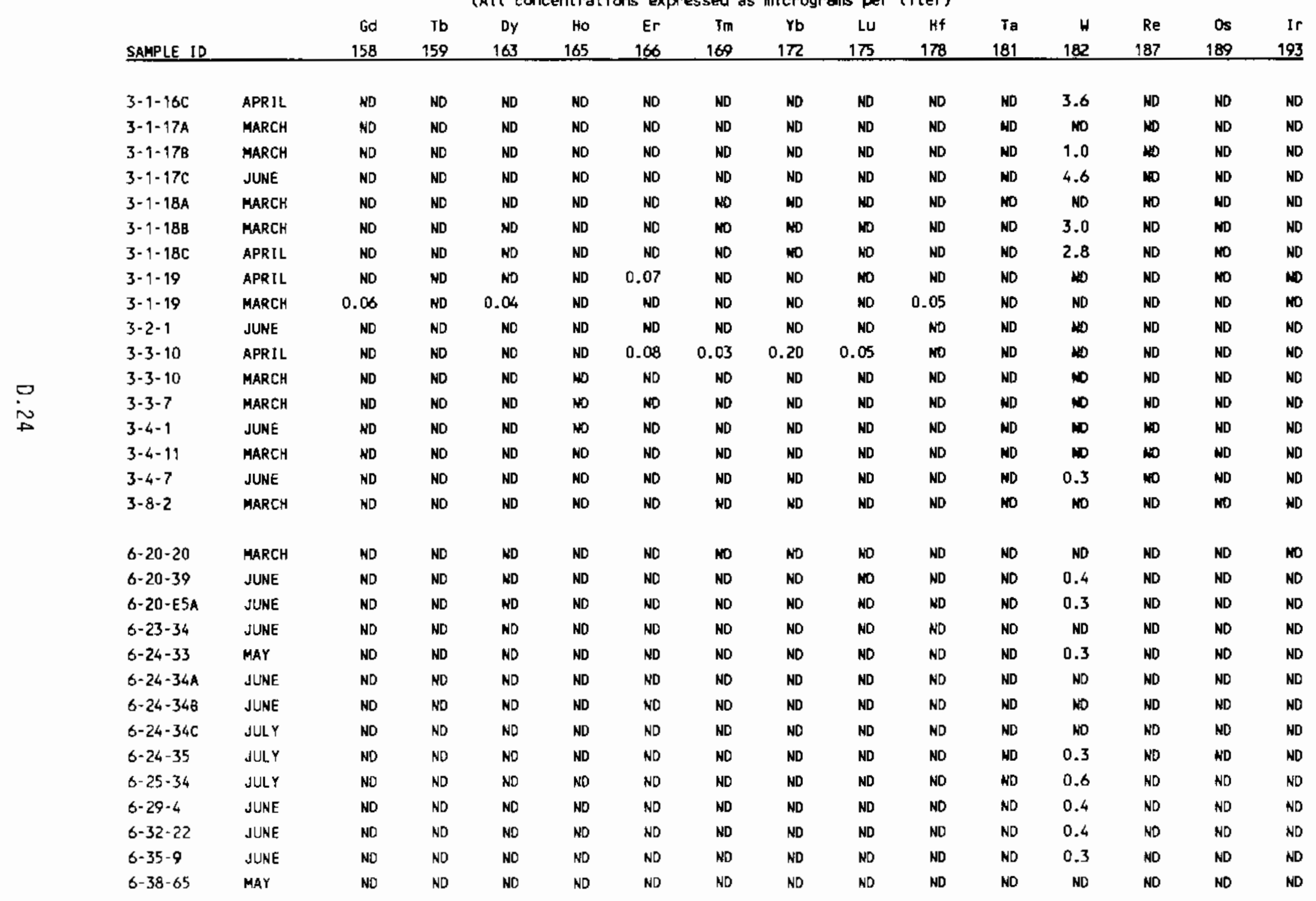




\section{TABLE D.1. (contd)}

(All concentrations expressed as micrograns per $(i \mathrm{ter})$

\begin{tabular}{|c|c|c|c|c|c|c|c|c|c|c|c|c|c|c|c|}
\hline SAMPLE ID & & $\begin{array}{r}\text { Gd } \\
158 \\
\end{array}$ & $\begin{array}{r}\mathrm{Tb} \\
159 \\
\end{array}$ & $\begin{array}{r}0 y \\
163 \\
\end{array}$ & $\begin{array}{r}\text { Ho } \\
165 \\
\end{array}$ & $\begin{array}{r}E r \\
186 \\
\end{array}$ & $\begin{array}{r}\text { Tm } \\
169 \\
\end{array}$ & $\begin{array}{r}10 \\
172 \\
\end{array}$ & $\begin{array}{r}14 \\
175 \\
\end{array}$ & $\begin{array}{r}H f \\
178 \\
\end{array}$ & $\begin{array}{r}\text { Ta } \\
189 \\
\end{array}$ & $\begin{array}{r}W \\
182 \\
\end{array}$ & $\begin{array}{r}\text { Re } \\
187 \\
\end{array}$ & $\begin{array}{r}\text { Os } \\
189 \\
\end{array}$ & $\begin{array}{r}15 \\
193 \\
\end{array}$ \\
\hline $6-39-79$ & MAY & ND & ND & ND & ND & ND & ND & ND & ND & ND & ND & 0.5 & ND & 0.05 & ND \\
\hline $6-40-1$ & JUNE & ND & No & ND & ND & ND & ND & ND & ND & ND & ND & ND & ND & NO & 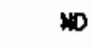 \\
\hline $6-40-33 A$ & JUNE & No & ND & ND & ND & ND & No & ND & ND & ND & ND & 1.4 & ND & ND & ND \\
\hline $6-43-88$ & MARCH & ND & ND & ND & ND & ND & ND & ND & ND & ND & MD & ND & ND & ND & ND \\
\hline $6-48-18$ & JUNE & NB & ND & ND & ND & ND & M & NO & ND & ND & NO & MD & ND & ND & $\$ 0$ \\
\hline $6-48-7$ & JUNE & ND & ND & ND & No & ND & ND & ND & ND & ND & ND & ND & ND & No & ND \\
\hline $6-48-79$ & JUNE & ND & ND & ND & ND & ND & M & ND & ND & ND & ND & 0.3 & No & No & MD \\
\hline $6-49-57$ & MAY & ND & ND & ND & ND & ND & ND & ND & ND & $N D$ & ND & 1.0 & ND & ND & ND \\
\hline $6-49-57$ & MAY & ND & ND & ND & ND & ND & ND & NO & ND & ND & ND & 0.9 & ND & ND & ND \\
\hline $6.49-79$ & MAY & ND & ND & MD & ND & ND & $\omega$ & NO & ND & ND & ND & ND & $\infty$ & ND & ND \\
\hline $6-50-53$ & MAY & ND & ND & ND & ND & NO & ND & $m$ & NO & ND & ND & 0.3 & 0.12 & NO & ND \\
\hline $6-51-75$ & JUNE & ND & ND & ND & ND & NO & ND & ND & ND & ND & ND & 0.9 & ND & ND & ND \\
\hline $6-77-54$ & JUNE & ND & ND & No & WD & ND & ND & ND & ND & ND & ND & 0.5 & ND & ND & ND \\
\hline $6-83-47$ & MAY & No & ND & 0.06 & ND & ND & ND & ND & ND & ND & ND & 0.8 & ND & ND & ND \\
\hline $6-89-35$ & \UNE & ND & ND & ND & ND & ND & No & ND & ND & ND & ND & ND & ND & ND & ND \\
\hline $6-90-45$ & JUNE & ND & ND & No & ND & ND & ND & No & ND & ND & ND & 0.5 & ND & NO & ND \\
\hline O-9-E2 & JUNE & ND & ND & ND & ND & ND & ND & ND & ND & ND & ND & 0.6 & ND & ND & NO \\
\hline $6-519-E 12$ & MARCH & ND & ND & ND & ND & ND & ND & ND & ND & ND & ND & NO & ND & ND & $M$ \\
\hline $6-519-E 13$ & MARCH & ND & ND & MD & ND & ND & ND & ND & ND & ND & ND & ND & 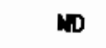 & ND & ND \\
\hline $6-531=1$ & JUNE & ND & ND & ND & ND & ND & No & ND & NO & ND & ND & ND & ND & ND & NO \\
\hline $6-53-25$ & JUNE & ND & ND & ND & ND & ND & ND & M & ND & ND & ND & 1.0 & ND & ND & No \\
\hline $6-56 E-14 A$ & JUNE & NO & ND & ND & ND & ND & ND & ND & NO & ND & NO & 0.8 & ND & ND & ND \\
\hline $6-58-19$ & JUNE & ND & ND & ND & ND & ND & ND & ND & ND & ND & ND & 2.1 & ND & ND & ND \\
\hline \multicolumn{2}{|c|}{ AVERAGE BLANK } & 0.008 & 0.002 & 0.009 & 0.002 & 0.008 & 0.002 & 0.011 & 0.002 & 0.012 & 0.002 & 0.019 & 0.005 & 0.016 & 0.005 \\
\hline \multicolumn{2}{|c|}{$+1-$} & 0.006 & 0.001 & 0.007 & 0.001 & 0.005 & 0.002 & 0.007 & 0.001 & 0.005 & 0.001 & 0.011 & 0.003 & 0.010 & 0.004 \\
\hline \multicolumn{2}{|c|}{$\mathrm{MDL}$} & 0.05 & 0.02 & 0.02 & 0.02 & 0.05 & 0.02 & 0.06 & 0.02 & 0.03 & 0.01 & 0.2 & 0.03 & 0.04 & 0.02 \\
\hline
\end{tabular}




\section{TABLE D.1. (contd)}

(All concentrations expressed as micrograms per liter)

\begin{tabular}{|c|c|c|c|c|c|c|c|c|c|c|c|c|}
\hline SAMPLE ID & & $\begin{array}{r}\rho t \\
195 \\
\end{array}$ & $\begin{array}{r}\mathrm{AU} \\
197 \\
\end{array}$ & $\begin{array}{r}\mathrm{Hg} \\
202 \\
\end{array}$ & $\begin{array}{r}\mathrm{Tl} \\
205 \\
\end{array}$ & $\begin{array}{r}\mathrm{Pb} \\
208 \\
\end{array}$ & $\begin{array}{r}\mathrm{Bi} \\
209 \\
\end{array}$ & $\begin{array}{r}\text { Th } \\
232 \\
\end{array}$ & $\begin{array}{r}U \\
235 \\
\end{array}$ & $\begin{array}{r}U \\
238 \\
\end{array}$ & U235/U238 & $\begin{array}{l}\text { Tc } \\
g 9\left(\mathrm{pC}^{2} \mathrm{~L} / \mathrm{L}\right)\end{array}$ \\
\hline $1-B 3-1$ & MAY & ND & ND & ND & ND & 0.9 & ND & ND & 2.8 & 2.7 & & ND \\
\hline $1-84-1$ & MARCH & ND & ND & ND & ND & No & ND & ND & & 1.5 & & \\
\hline $1-84-4$ & APRIL & ND & ND & ND & NO & ND & ND & ND & 1.2 & 1.1 & & ND \\
\hline $1-85-1$ & JUNE & ND & No & ND & ND & 0.6 & No & NO & 1.3 & 1.5 & & NO \\
\hline $1-89 \cdot 1$ & MARCH & ND & No & NO & ND & ND & No & ND & & 0.7 & & \\
\hline $1-D 2-5$ & APRIL & ND & NO & ND & ND & 1.4 & ND & ND & 2.5 & 2.6 & & ND \\
\hline $1-05-12$ & APRIL & ND & NO & ND & ND & ND & ND & ND & 2.1 & 2.7 & & ND \\
\hline $1-F 5-1$ & APRIL & ND & ND & ND & ND & ND & ND & ND & 1.5 & 1.0 & & ND \\
\hline $1-F 5-3$ & MAY & ND & ND & ND & ND & ND & ND & ND & 0.5 & 0.7 & & ND \\
\hline $1-\mathrm{H} 3-1$ & MARCH & ND & 10 & ND & ND & NO & ND & ND & & 5.8 & & \\
\hline $4-H 3-2 A$ & JULY & ND & ND & ND & ND & no & ND & ND & 2.9 & 3.3 & & ND \\
\hline $1-H 3-2 B$ & AUGUST & ND & ND & ND & ND & ND & ND & ND & 2.7 & 2.8 & & ND \\
\hline $1-H 3-2 C$ & MAY & ND & ND & ND & ND & ND & ND & ND & 0.5 & 1.6 & & ND \\
\hline $1-H_{3}-2 C$ & JUNE & ND & ND & ND & ND & ND & ND & NO & 1.3 & 1.5 & & ND \\
\hline $1-\mathrm{H3}-2 \mathrm{C}$ & MARCH & ND & Nn & Nn & NO & MD & ND & 0.22 & & 0.9 & & \\
\hline $1-\mathrm{H}_{4}-3$ & JULY & ND & ND & ND & ND & NO & ND & ND & 206.9 & 148.6 & 1.39 & 1486 \\
\hline $1-\mathrm{H} 4-3$ & MAY & ND & ND & ND & ND & NO & ND & ND & 205.5 & 156.5 & 1.31 & 1982 \\
\hline $1-\mathrm{H} 4-3$ & MARCH & ND & NO & ND & ND & NO & ND & ND & & 230.9 & & \\
\hline $1-1+4-3$ & AUGUST & NO & ND & ND & ND & ND & ND & ND & 330.5 & 248.4 & 1.33 & 4078 \\
\hline $1-\mathrm{H}_{4}-4$ & JULY & ND & ND & ND & NO & ND & ND & ND & 60.9 & 46.3 & 1.31 & 849 \\
\hline 1-H4-4 & MAY & ND & ND & ND & NO & ND & NO & ND & 87.6 & 68.0 & 1.29 & 956 \\
\hline 1- $\mathrm{H4}-4$ & JUNE & ND & ND & ND & ND & ND & ND & ND & 6.7 & 4.4 & & ND \\
\hline $1-144-4$ & AUGUST & ND & ND & ND & ND & ND & ND & ND & 152.7 & 109.3 & 1.40 & 1998 \\
\hline $1-\mathrm{H}_{4}-5$ & MARCH & ND & ND & ND & NO & ND & ND & ND & & 2.5 & & \\
\hline $1-H 4-6$ & MARCH & NO & ND & ND & ND & NO & ND & ND & & 3.8 & & \\
\hline $1-34-7$ & MARCH & ND & ND & ND & ND & ND & ND & ND & & 2.0 & & \\
\hline $1-\mathrm{H}_{4}-8$ & AUGUST & ND & ND & ND & ND & ND & ND & ND & 4.3 & 4.1 & & ND \\
\hline $1-H 4-9$ & AUGUST & ND & ND & ND & ND & ND & ND & ND & 14.5 & 11.7 & 1.24 & 919 \\
\hline $1-\mathrm{H}_{4}-10$ & MARCH & ND & ND & NO & ND & ND & ND & ND & & 1.9 & & \\
\hline
\end{tabular}


TABLE D.1. (contd)

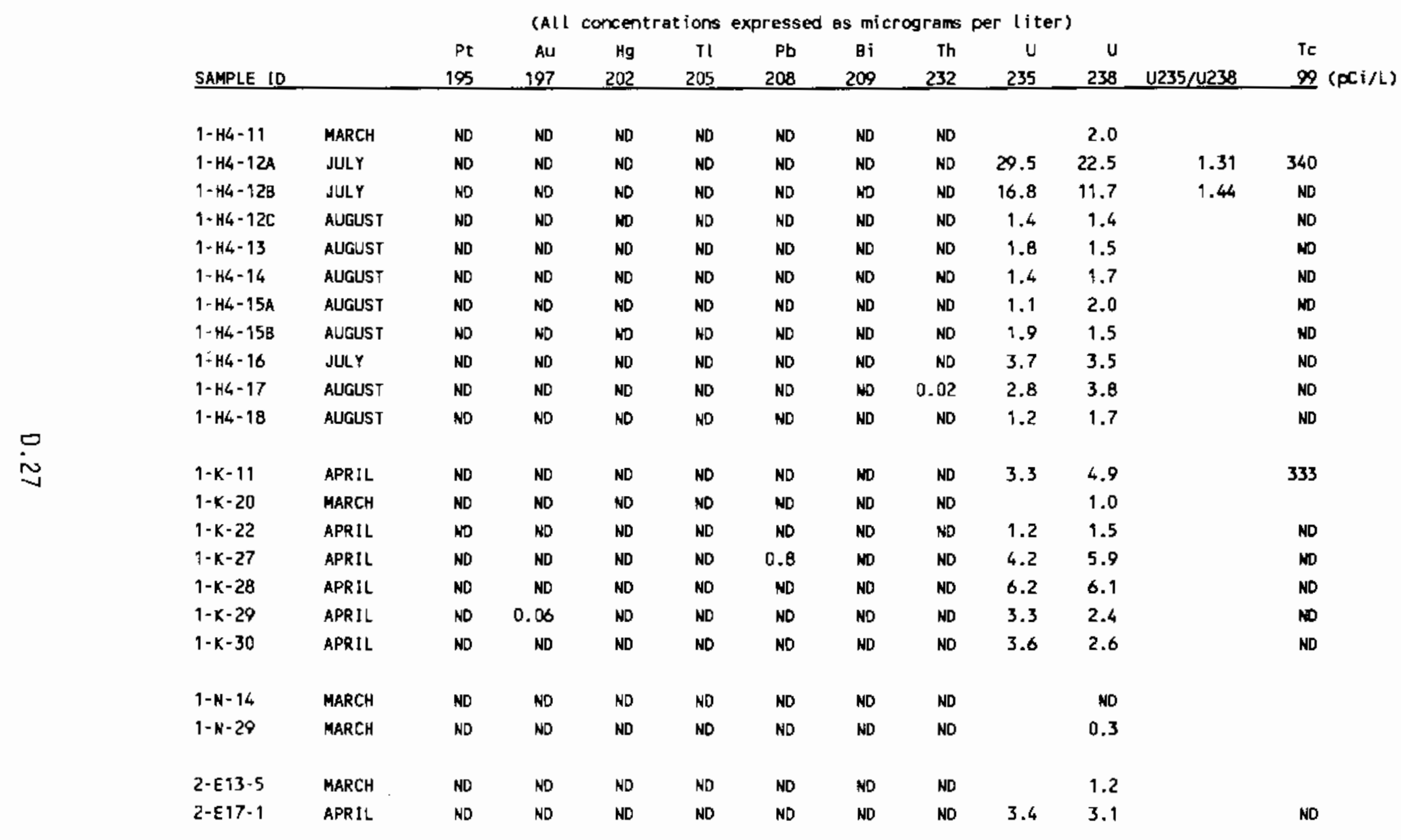


IABLE D.1. (contd)

(All concentrations expressed as micrograms per (iter)

\begin{tabular}{|c|c|c|c|c|c|c|c|c|c|c|c|c|}
\hline SAMPLE ID & & $\begin{array}{r}\text { Pt } \\
195 \\
\end{array}$ & $\begin{array}{r}\text { Au } \\
197 \\
\end{array}$ & $\begin{array}{r}\mathrm{Hg} \\
202 \\
\end{array}$ & $\begin{array}{r}11 \\
205 \\
\end{array}$ & $\begin{array}{r}P b \\
208 \\
\end{array}$ & $\begin{array}{r}8 i \\
209 \\
\end{array}$ & $\begin{array}{r}\text { in } \\
232 \\
\end{array}$ & $\begin{array}{r}U \\
235 \\
\end{array}$ & $\begin{array}{r}u \\
238 \\
\end{array}$ & $\mathrm{U} 235 / \mathrm{U} 238$ & $\begin{array}{l}\text { Tc } \\
99(p c i / L)\end{array}$ \\
\hline $2-E 17-5$ & APRIL & No & ND & NO & ND & No & ND & ND & 8.8 & 8.3 & & 394 \\
\hline 2-E17-6 & APRIL & ND & NO & ND & ND & NO & ND & ND & ND & NO & & No \\
\hline $2-E 17-9$ & APRIL & ND & NO & ND & NO & ND & NO & ND & 4.4 & 3.7 & & ND \\
\hline $2-E 24-12$ & APRIL & ND & ND & ND & ND & ND & ND & ND & 28.1 & 29.8 & 0.94 & ND \\
\hline 2-E24-7 & JUWE & ND & ND & NO & ND & ND & ND & ND & 5.9 & 7.0 & & ND \\
\hline 2-E24-8 & AUGUST & ND & NO & ND & ND & ND & ND & ND & 1.8 & 2.0 & & NO \\
\hline $2-E 25-18$ & APRIL & NO & ND & No & NO & ND & ND & No & 1.0 & 1.2 & & ND \\
\hline $2-E 25-20$ & MARCH & ND & ND & ND & NO & ND & ND & ND & & 1.1 & & \\
\hline 2-E25-21 & APRIL & ND & ND & ND & ND & ND & ND & ND & 1.5 & $1 . B$ & & $\mathbf{N D}$ \\
\hline $2-E 25-27$ & JUNE & ND & NO & ND & ND & ND & ND & ND & 2.0 & 1.7 & & ND \\
\hline $2-E 25-28$ & JUNE & ND & No & ND & ND & No & ND & NO & 1.7 & 1.1 & & ND \\
\hline $2-E 25-7$ & JUNE & ND & ND & ND & NO & ND & ND & ND & 1.8 & 1.4 & & ND \\
\hline 2-E26- 1 & JUWE & ND & ND & NO & ND & no & $\mathrm{ND}$ & ND & 1.4 & $1 . B$ & & No \\
\hline 2-E26-3 & JUWE & ND & No & No & ND & ND & ND & ND & 1.2 & 1.3 & & ND \\
\hline 2-E27-5 & MARCH & MD & NO & ND & NO & ND & ND & No & & 1.1 & & \\
\hline $2-E 28-17$ & JUNE & ND & ND & ND & No & ND & NO & ND & 10.9 & 13.9 & 0.78 & ND \\
\hline $2-E 28-7$ & JUNE & ND & ND & ND & ND & 0.6 & MD & ND & 3.6 & 3.6 & & 302 \\
\hline $2-E 32-1$ & AUGUST & ND & Nn & ND & ND & ND & ND & ND & 4.4 & 3.8 & & ND \\
\hline 2-E33-1 & MAY & ND & No & ND & ND & NO & ND & ND & 1.8 & 1.7 & & 304 \\
\hline $2-E 33-18$ & MAY & ND & ND & ND & ND & ND & NO & ND & 1.7 & 2.2 & & HD \\
\hline $2-E 33-2$ & JUNE & ND & ND & ND & NO & ND & ND & ND & 1.1 & 1.3 & & ND \\
\hline $2-E 33-21$ & AUGUST & ND & ND & ND & ND & ND & ND & ND & 2.5 & 2.1 & & \\
\hline $2-E 33-24$ & ALIGUST & ND & ND & NO & ND & ND & ND & ND & 2.5 & 2.1 & & 1220 \\
\hline $2-\varepsilon 33-3$ & AUGUST & ND & No & ND & ND & ND & ND & ND & 1.7 & 1.9 & & 1557 \\
\hline 2-E33-5 & AUGUST & ND & ND & ND & No & ND & ND & ND & 2.0 & 2.0 & & 1633 \\
\hline
\end{tabular}




\section{TABLE D.1. (contd)}

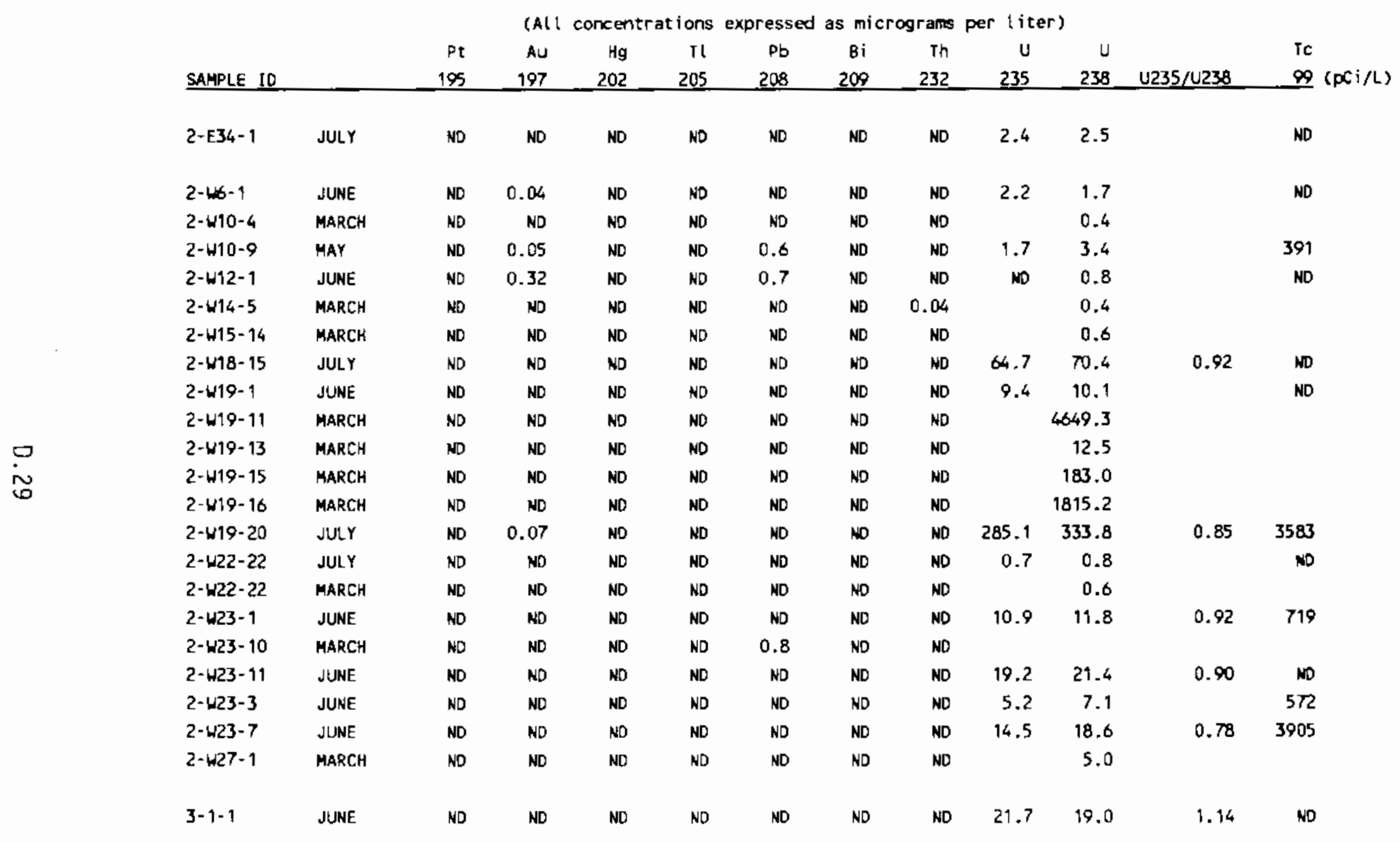


TABLE D.1. (contd)

\begin{tabular}{|c|c|c|c|c|c|c|c|c|c|c|c|c|c|}
\hline & & & & (Al & incent & ions & oresse & is mic & xgrams & Der lit & & & \\
\hline & & & $\mathrm{Pt}$ & AL & $\mathrm{Hg}$ & $T 1$ & $\mathrm{~Pb}$ & $\mathrm{Bi}$ & Th & U & U & & TC \\
\hline & SAMPLE ID & & 195 & 197 & 202 & 205 & 208 & 209 & 232 & 235 & 238 & U235/U238 & $\mathscr{\phi}(\mathrm{pC} \mathrm{i} / \mathrm{L})$ \\
\hline & $3-1-2$ & MARCH & ND & ND & ND & ND & ND & ND & ND & 11.0 & 11.1 & 0.99 & \\
\hline & $3-1-3$ & JUNE & ND & ND & ND & ND & ND & ND & ND & 49.4 & 36.9 & 1.34 & $m$ \\
\hline & $3-1-4$ & JUWE & ND & ND & ND & NO & ND & ND & ND & 33.3 & 24.5 & 1.36 & \\
\hline & $3-1-5$ & MARCH & ND & ND & ND & ND & ND & ND & ND & 150.9 & 121.7 & 1.24 & \\
\hline & $3-1-6$ & APR IL & ND & ND & ND & ND & ND & ND & ND & 31.9 & 22.6 & 1.41 & ND \\
\hline & $3-1-6$ & MARCH & ND & NO & ND & ND & ND & ND & ND & 17.0 & 14.3 & 1.19 & \\
\hline & $3-1-7$ & JUWE & NO & ND & ND & ND & ND & ND & ND & 43.8 & 33.8 & 1.30 & $M D$ \\
\hline & $3-1-8$ & JUNE & ND & ND & ND & ND & ND & ND & ND & 28.9 & 24.2 & 1.19 & ND \\
\hline & $3-1-9$ & MARCH & ND & ND & ND & ND & ND & ND & 0.05 & & ND & & \\
\hline & $3-1-10$ & MARCH & ND & ND & ND & ND & ND & ND & ND & 14.5 & 13.9 & 1.05 & \\
\hline & $3-1-11$ & MARCH & ND & ND & ND & ND & NO & ND & ND & 138.3 & 115.0 & 1.20 & \\
\hline$\square$ & $3-1-12$ & MARCH & NO & ND & ND & ND & ND & ND & ND & 74.0 & 55.8 & 1.33 & \\
\hline e & $3-1-12$ & JUNE & ND & ND & ND & ND & ND & ND & ND & 46.2 & 33.9 & 1.36 & ND \\
\hline & $3-1-13$ & MARCH & NO & ND & ND & AD & ND & NO & ND & B.7 & 7.0 & 1.23 & \\
\hline & $3-1-13$ & APR It & NO & ND & ND & No & ND & NO & NO & 20.6 & 16.5 & 1.24 & ND \\
\hline & $3-1-16$ & MARCH & ND & ND & ND & No & ND & ND & NO & 29.5 & 23.3 & 1.27 & \\
\hline & $3-1-15$ & MARCH & NO & ND & NO & ND & ND & ND & ND & 3.1 & 2.4 & & \\
\hline & $3-1-15$ & APRIL & ND & ND & ND & ND & ND & ND & ND & 26.7 & 21.2 & 1.25 & \\
\hline & $3-1-16 A$ & JUNE & NO & ND & ND & ND & ND & NO & No & 17.9 & 15.2 & 1.18 & ND \\
\hline & $3-1-16 B$ & JUWE & ND & ND & ND & ND & ND & ND & ND & 2.7 & 2.1 & & \\
\hline & $3-1-16 C$ & MARCH & ND & ND & ND & ND & ND & ND & ND & 2.2 & 2.4 & & \\
\hline & $3-1-16 c$ & APRIL & ND & NO & ND & ND & ND & ND & ND & 3.4 & 2.7 & & \\
\hline & $3-1-17 \mathrm{~A}$ & MARCH & ND & ND & ND & NO & ND & ND & ND & 97.6 & 80.7 & 1.21 & \\
\hline & $3-1-17 \mathrm{~B}$ & MARCH & ND & ND & ND & ND & ND & ND & ND & ND & ND & & \\
\hline & $3-1-17 c$ & JUNE & NO & ND & ND & ND & 0.7 & ND & ND & ND & ND & & ND \\
\hline
\end{tabular}


TABLE D.1. (contd)

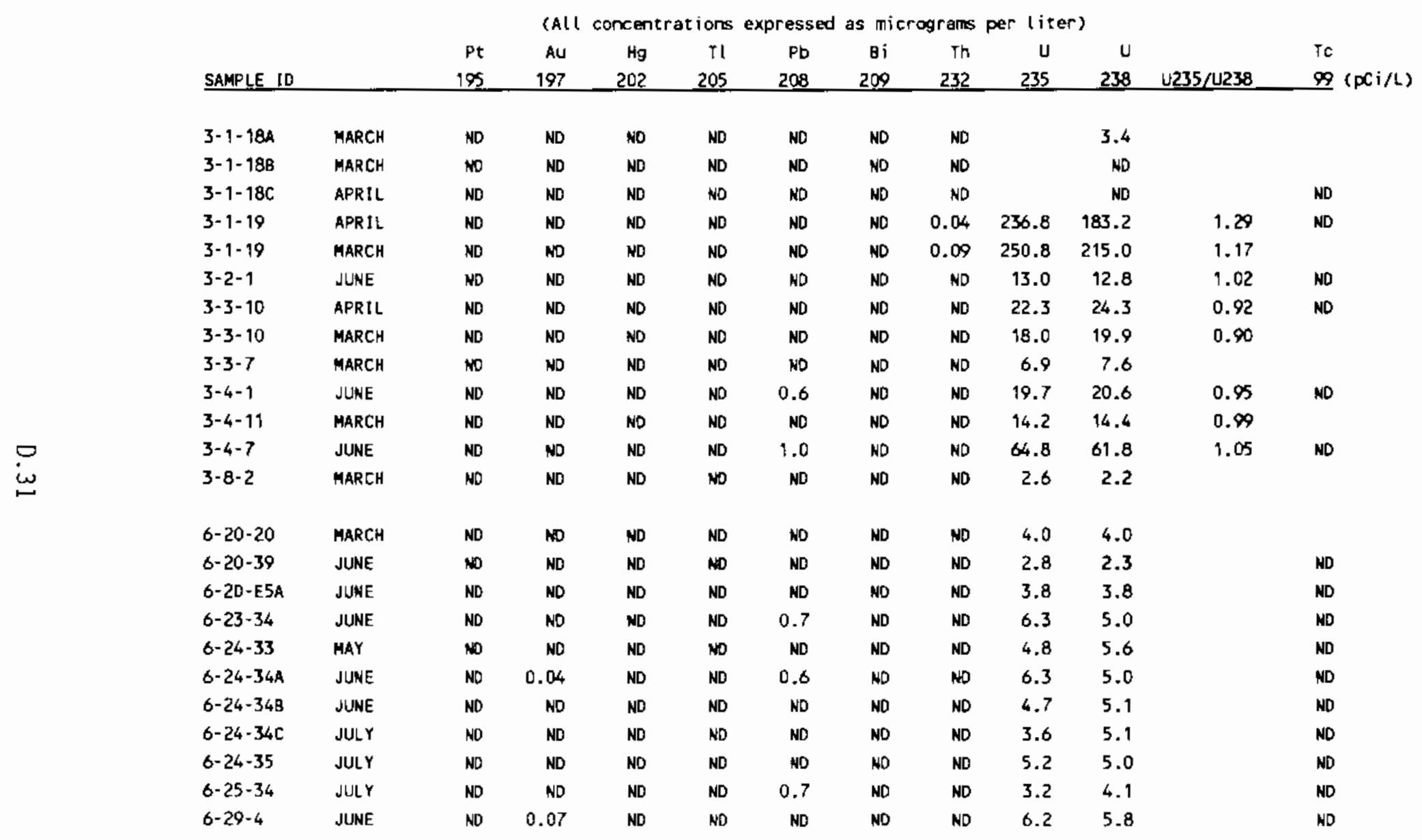




$$
\text { HWIII }
$$


IABLE D.1. (contd)

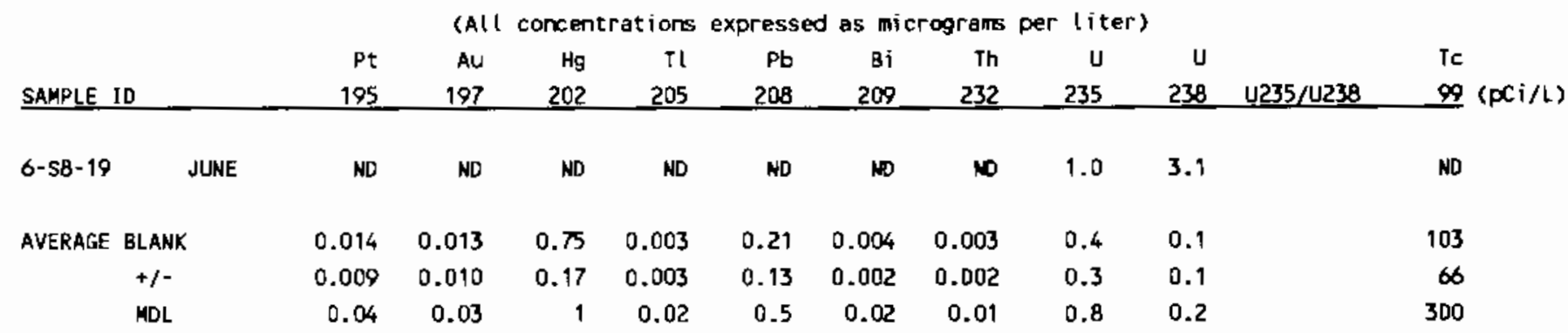

$\dot{\omega}_{\omega}$ 

APPENDIX E

DATA LISTINGS 


\section{APPENDIX E}

\section{DATA LISTINGS}

This appendix contains tables 1 isting the constituents for which samples taken during the period July through December 1987 had been analyzed and reported by United States Testing Company, Inc. (UST). Table E.l is a key to the constituent and constituent group names used throughout the remaining tables. Some constituents appear more than once in the list in different forms (e.g., NITRATE and HNITRATE are both nitrate ion, but HNITRATE has a higher detection 1 imit). The constituents $U$ and $U$-CHEM are both total uranium, but in units of $\mathrm{pCi} / \mathrm{L}$ and $\mathrm{ppb}(\mathrm{a})$, respectively. The constituent names CHROMUM and FCHROMI represent chromium analyses done on unfiltered and filtered samples, respectively.

Each monitoring well sampled on the Hanford Site from July through December 1987 was placed into one of three groups: 1) Site-wide chemical monitoring wells (Tables E.2 and E.3), 2) compliance monitoring wells (Tables E.4 and E.5), and 3) additional Site-wide radiological and nitrate monitoring wells that were not in either of the other two networks (Tables E.6 and E.7).

Most wells were sampled once during each quarter. Some exceptions included a few wells that were sampled monthly, wells that were dropped from the chemical network after three sampling periods, and wells that were sampled semiannually for radiological constituents. Compliance monitoring in the $100-H$ and 300 Areas is conducted monthly and quarterly, respectively.

Tables E.3, E.5, and E.7 contain all chemical results (above detection levels) and radiochemical results (for which the result is at least as large as the counting error). Blank spaces in the tables or constituents missing

(a) The conversion from ppb uranium to $\mathrm{pC} i / \mathrm{L}$ uranium is approximately 0.699 for natural uranium. Other conversion factors may be calculated where the isotopic composition has been measured [e.g., Law, Serkowski, and Schatz (1987) report a conversion factor of 0.679 for their data]. 
from the first column indicate that the results did not meet these criteria. The letters "NR" in the tables indicate that the analysis was not requested or the results had not yet been reported by the laboratory at the time of writing.

Analyses were performed by UST following approved procedures (EPA 1982) or other standard methods. Specific conductance, $\mathrm{pH}$, and temperature were measured in the field at the time of collection, in accordance with documented procedures. The analytical procedures have been described elsewhere (Jaquish and Mitche11 1988, Appendix C).

The following symbols are used in Tables E.3 and E.7:

* - Value exceeds primary drinking water standard.

\# - Value exceeds proposed primary drinking water standard.

+ - Value exceeds screening level for further investigation.

0 - Detection limit was not available for comparison.

NR - Analysis not requested ro not yet reponted.

Values in ( ) are counting errors for radionuclides.

Water standard (S) in parentheses are proposed. 
TABLE E.1. Key to Constituent and Constituent Group Names Used in Tables E.2, E.3, E.4, E.5, E.6, and E.7

Individual Constituents

\begin{tabular}{|c|c|c|}
\hline Name in Tables & Units & Full Name \\
\hline 12PRDOL & $\mathrm{ppb}$ & 12-Propanediol \\
\hline ACETONE & $\mathrm{ppb}$ & Acetone \\
\hline ALKALIN & $\mathrm{ppb}$ & Total alkalinity, as $\mathrm{CaCO} 3$ \\
\hline ALPHA & $\mathrm{pCi} / \mathrm{L}$ & Gross alpha \\
\hline AMMONIU & $\mathrm{ppb}$ & Ammonium ion \\
\hline BETA & $\mathrm{pCi} / \mathrm{L}$ & Gross beta \\
\hline$c-14$ & $\mathrm{pCi} / \mathrm{L}$ & Carbon-14 \\
\hline CITRUSR & $\mathrm{ppb}$ & Citrus red \\
\hline COLIFRM & $\operatorname{MPN}(a)$ & Coliform bacteria \\
\hline CONDFLD & $\mu$ mho & Specific conductance, field \\
\hline CONDLAB & who & Specific conductance, laboratory \\
\hline CYANIDE & $\mathrm{ppb}$ & Cyanide \\
\hline DIOXIN & $\mathrm{ppb}$ & Dioxin \\
\hline ETHYGLY & $\mathrm{ppb}$ & Ethylene glycol \\
\hline HNITRAT & $\mathrm{ppb}$ & Nitrate, high $\mathrm{DL}(\mathrm{b})$ \\
\hline I-129DW & $\mathrm{pCi} / \mathrm{L}$ & Iodine-129 (for drinking water regs) \\
\hline LFLUORD & $\mathrm{ppb}$ & Fluoride, low DL \\
\hline LHYDRAZ & $\mathrm{ppb}$ & Hydrazine, low DL \\
\hline LPHENOL & $\mathrm{ppb}$ & Phenol, low DL \\
\hline$N I-63$ & $\mathrm{pCi} / \mathrm{L}$ & Nickel-63 \\
\hline PERCHLO & $\mathrm{ppb}$ & Perchlorate \\
\hline PH-LAB & & $\mathrm{pH}$, laboratory \\
\hline PHFIELD & & $\mathrm{pH}$, field \\
\hline RADIUM & $\mathrm{pCi} / \mathrm{L}$ & Total radium \\
\hline SR-90 & $\mathrm{PCi} / \mathrm{L}$ & Strontium-90 \\
\hline STYRENE & $\mathrm{ppb}$ & Styrene \\
\hline SULFIDE & $\mathrm{ppb}$ & Sulfide \\
\hline $\mathrm{TC}$ & $\mathrm{ppb}$ & Tota? carbon \\
\hline TC -99 & $\mathrm{pCi} / \mathrm{L}$ & Technetium-99 \\
\hline TDS & $\mathrm{ppb}$ & Total dissolved solids \\
\hline TOC & $\mathrm{ppb}$ & Total organic carbon \\
\hline TOX & $\mathrm{ppb}$ & Total organic halogen \\
\hline TOXLDL & $\mathrm{ppb}$ & Total organic halogens, low DL \\
\hline TRITIUM & $\mathrm{pCi} / \mathrm{L}$ & Tritjum $(H-3)$ \\
\hline U & $\mathrm{pCi} / \mathrm{L}$ & Uranium \\
\hline U-CHEM & $\mu \mathrm{g} / \mathrm{L}(\mathrm{C})$ & Urnaium, chemical \\
\hline UNKNOWN & $\mathrm{ppb}$ & Unknown \\
\hline
\end{tabular}

\footnotetext{
(a) MPN is most probable number.

(b) DL is detection limit.

(c) $\mu \mathrm{g} / \mathrm{L}$ equals $\mathrm{ppb}$.
} 
TABLEE.1. (contd)

Group: ANIONS = Ion Chromatography

Name in Tables Units

Fu11 Name

CHOLORID

FLUORID

NITRATE

PHOSPHA

ppb

$\mathrm{ppb}$

$\mathrm{ppb}$

$\mathrm{PPb}$

SULFATE

$\mathrm{ppb}$

Chloride

Fluoride

Nitrate

Phosphate

Sulfate

Group: ANIONSE = Ion Chromatography, enhanced list

Name in Tables Units

Full Name

BROMIDE $\quad$ ppb $\quad$ Bromide

NITRITE $\quad \mathrm{ppb} \quad$ Nitrite

Group: DIRAQIN = Direct Aqueous Injection

Name in Tables Units

Full Name

ACRYIDE

ALLYLAL

CHLACET

CHLPROP

ETHCARB

ETHCYAN

HYDRAZI

ISOBUTY

PARALDE

PROPYLA

PROPYNO $\mathrm{ppb}$

$\mathrm{ppb}$

$\mathrm{ppb}$

$\mathrm{ppb}$

$p p b$

$\mathrm{ppb}$

$\mathrm{ppb}$

$\mathrm{ppb}$

$\mathrm{ppb}$

$\mathrm{ppb}$

$\mathrm{ppb}$

Acrylamide

Allyl alcohol

Chloroacetaldehyde

3-Chloropropionitrile

Ethyl carbamate

Ethyl cyanide

Hydrazine

Isobutyl alcoho

Paraldehyde

n-Propylamine

2-Propyn-1-01

Group: GAMMA = Gamma Scan

Name in Tables Units

Ful1 Name

Co- 60

CS- 137

$\mathrm{pCi} / \mathrm{L}$

$\mathrm{pCi} / \mathrm{L}$

$\mathrm{pCi} / \mathrm{L}$

$\mathrm{RU}-106$

$\mathrm{pC} \mathrm{i} / \mathrm{L}$

Cesium-137

Ruthenium- 106

Anthimony- 125

Group: HERB $=$ Herbicides

Name in Tables Units

Fu11 Name

$2,4,5 T P$

$\mathrm{ppb}$

$2,4-D$

$\mathrm{ppb}$

$2,4,5$-TP silvex

2,4-D [2,4-Dichlorophenoxyacetic acid]

E.4 
TABLE E.1. (contd)

Group: HERBE $=$ Herbicides, enhanced list

Name in Tables Units

Full Name

2,4,5-T $\quad$ ppb 2,4,5-T

Group: $\quad$ ICPMT = ICP Metals, unfiltered

Name in Tables Units

Full Name

$\begin{array}{lll}\text { ALUMNUM } & \mathrm{ppb} & \text { Aluminum } \\ \text { BARIUM } & \mathrm{ppb} & \text { Barium } \\ \text { CAMIUM } & \mathrm{ppb} & \text { Cadmium } \\ \text { CALCIUM } & \mathrm{ppb} & \text { Calcium } \\ \text { CHROMUM } & \mathrm{ppb} & \text { Chromium } \\ \text { COPPER } & \mathrm{ppb} & \text { Copper } \\ \text { IRON } & \mathrm{ppb} & \text { Iron } \\ \text { MAGNES } & \mathrm{ppb} & \text { Magnesium } \\ \text { MANGESE } & \mathrm{ppb} & \text { Manganese } \\ \text { NICKEL } & \mathrm{ppb} & \text { Nickel } \\ \text { POTASUM } & \mathrm{ppb} & \text { Potassium } \\ \text { SILVER } & \mathrm{ppb} & \text { Silver } \\ \text { SODIUM } & \mathrm{ppb} & \text { Sodium } \\ \text { VANADUM } & \mathrm{ppb} & \text { Vanadium } \\ \text { ZINC } & \mathrm{ppb} & \text { Zinc }\end{array}$

Group: ICPMTE = ICP Metals, unfiltered, enhanced list

Name in Tables Units Full Name

ANTIONY

BERYLUM

STRONUM

$\mathrm{ppb} \quad$ Antimony

ppb Beryllium

$\mathrm{ppb} \quad$ Strontium

Group: ICPMTF $=$ ICP Metals, filtered

Name in Tables Units Ful1 Name

FALUMIN $\quad \mathrm{ppb} \quad$ Aluminum, filtered

FBARIUM $\quad \mathrm{ppb} \quad$ Barium, filtered

FCAOMIU $\quad \mathrm{ppb}$

FCALCIU $\quad \mathrm{ppb}$

FCHROMI $\mathrm{ppb}$

FCOPPER $\quad \mathrm{pPb}$

FIRON $\quad \mathrm{ppb}$

FMAGNES $\quad \mathrm{ppb}$

FMANGAN $\quad \mathrm{ppb}$

FNICKEL $\quad \mathrm{ppb}$

FPOTASS $\quad \mathrm{ppb}$ Cadmium, fi7tered Calcium, filtered Chromium, filtered Copper, filtered Iron, filtered Magnesium, filtered Manganese, filtered Nicke?, filtered Potassium, filtered 


\section{TABLE E.1. (contd)}

Group: ICPMTF = ICP Metals, filtered (contd)

Name in Tables Units

Fu11 Name

$\begin{array}{lll}\text { FSILVER } & \mathrm{ppb} & \text { Silver, filtered } \\ \text { FSODIUM } & \mathrm{ppb} & \text { Sodium, filtered } \\ \text { FVANADI } & \mathrm{ppb} & \text { Vanadium, filtered } \\ \text { FZINC } & \mathrm{ppb} & \text { Zinc, filtered }\end{array}$

Group: ICPMTFE $=$ ICP Metals, filtered, enhanced list

Name in Tables Units

Full Name

$\begin{array}{lll}\text { FANTIMO } & \mathrm{ppb} & \text { Antimony, filtered } \\ \text { FBERYLL } & \mathrm{ppb} & \text { Beryllium, filtered } \\ \text { FSTRONT } & \mathrm{ppb} & \text { Strontium, filtered }\end{array}$

Group: $\quad$ OMET $=$ Other metals

Name in Tables Units

Full Name

$\begin{array}{lll}\text { ARSENIC } & \mathrm{ppb} & \text { Arsenic } \\ \text { LEADGF } & \mathrm{ppb} & \text { Lead (graphite - urnace) } \\ \text { MERCURY } & \mathrm{ppb} & \text { Mercury } \\ \text { SELENUM } & \mathrm{ppb} & \text { Selenium }\end{array}$

Group: OMETE = Other metals, enhanced 1 ist

Name in Tables Units Fu11 Name

THALIUM

$\mathrm{ppb}$

ThaTlium

Group: OMETF $=$ Other metals, filtered

Name in Tabies Units Fu11 Name

FARSENI $\quad$ ppb Arsenic, filtered

FLEAD $\quad$ ppb Lead, filtered

FMERCUR $\quad$ ppb Mercury, filtered

FSELENI $\quad \mathrm{ppb} \quad$ Selenium, filtered

Group: OMETFE $=$ Other metals, filtered, enhanced list

Name in Tables Units Ful1 Name

FTHALLI

ppb Thallium, filtered 
TABLE E.1. (contd)

Group: $\quad$ PCBS $=$ Polychlorinated biphenyls

Name in Tables Units

Full Name

AR1016 $\quad$ ppb $\quad$ Arochlor 1016

ARI221 $\quad \mathrm{ppb} \quad$ Arochlor 1221

AR1232 $\quad \mathrm{ppb} \quad$ Arochlor 1232

AR1242 $\quad \mathrm{ppb} \quad$ Arochlor 1242

AR1248 $\quad \mathrm{ppb} \quad$ Arochlor 1248

AR1254 $\quad \mathrm{ppb} \quad$ Arochior 1254

AR1260 $\quad \mathrm{ppb} \quad$ Arochlor 1260

Group: $\quad$ PEST $=$ Pesticides

Name in Tables Units

Full Name

$\begin{array}{lll}\text { ENDRIN } & p p b & \text { Endrin } \\ \text { METHLOR } & p p b & \text { Methoxychior } \\ \text { TOXAENE } & p p b & \text { Toxaphene } \\ \text { a-BHC } & p p b & \text { Lindane, a7pha-BHC } \\ \text { b-BHC } & p p b & \text { Lindane, beta-BHC } \\ \text { d-BHC } & p p b & \text { Lindane, delta-BHC } \\ \text { g-BHC } & p p b & \text { Lindane, gamma-BHC }\end{array}$

Group: PESTE = Pesticides, enhanced 1ist

Name in Tables Units

Full Name

$\begin{array}{lll}\text { ALDRIN } & \mathrm{ppb} & \text { Aldrin } \\ \text { CHLLATE } & \mathrm{ppb} & \text { Chlorobenzilate } \\ \text { CHLOANE } & \mathrm{ppb} & \text { Chlordane } \\ \text { DOD } & \mathrm{ppb} & \text { DOD } \\ \text { DDE } & \mathrm{ppb} & \text { DDE } \\ \text { ODT } & \mathrm{ppb} & \text { DDT } \\ \text { DIELRIN } & \mathrm{ppb} & \text { Dieldrin } \\ \text { ENDO1 } & \mathrm{ppb} & \text { Endosuifan I (alpha) } \\ \text { ENDO2 } & \mathrm{ppb} & \text { Endosulfan II (beta) } \\ \text { HEPTIDE } & \mathrm{ppb} & \text { Heptachior epoxide } \\ \text { HEPTLOR } & \mathrm{ppb} & \text { Heptachlor }\end{array}$


IABLE E.1. (contd)

Group: PHOSPST $=$ Phosphorus pesticides

$\begin{array}{llll}\text { Name in Tables } & \text { Units } & & \\ \text { CARBPHT } & & & \\ \text { DIMETH0 } & \mathrm{ppb} & & \text { Carbophenothion } \\ \text { DISULF0 } & \mathrm{ppb} & & \text { Dimethoate } \\ \text { METHPAR } & \mathrm{ppb} & & \text { Misulfotony Name } \\ \text { PARATHI } & \mathrm{ppb} & & \text { Parathion } \\ \text { TETEPHY } & \mathrm{ppb} & & \text { Tetraethylpyrophosphate }\end{array}$

Group: PU-ISO = Plutonium I sotopes

Name in Tables Units Full Name

$\begin{array}{lll}\text { PU-238 } & \mathrm{PC} \mathrm{i} / \mathrm{L} & \text { Plutonium-238 } \\ \mathrm{PU} 39-40 & \mathrm{pC} i / \mathrm{L} & \text { Plutonium-239,240 }\end{array}$

Group: SEMvOL - Semivolatile Organics

Name in TabTes Units Fu?1 Name

$\begin{array}{lll}\text { 12-dben } & \mathrm{ppb} & 1,2-\text { Dichlorobenzene } \\ \text { 1234TE } & \mathrm{ppb} & 1,2,3,4 \text {-Tetrachlorobenzene } \\ \text { 1235TE } & \mathrm{ppb} & 1,2,3,5-\text { Tetrachlorobenzene } \\ \text { 123TRI } & \mathrm{ppb} & 1,2,3-\text { Trichlorobenzene } \\ \text { 13-dben } & \mathrm{ppb} & 1,3-\text { Dichlorobenzene } \\ \text { 135TRI } & \mathrm{ppb} & 1,3,5-\text { Trichlorobenzene } \\ \text { 14-dben } & \mathrm{ppb} & 1,4-\text { Dichlorobenzene } \\ \text { HEXACHL } & \mathrm{ppb} & \text { Hexachlorophene } \\ \text { HEXCBEN } & \mathrm{ppb} & \text { Hexachlorobenzene } \\ \text { KEROSEN } & \mathrm{ppb} & \text { Kerosene } \\ \text { NAPHTHA } & \mathrm{ppb} & \text { Naphthalene } \\ \text { PENTCHB } & \mathrm{ppb} & \text { Pentachlorobenzene } \\ \text { PHENOL } & \mathrm{ppb} & \text { Phenol } \\ \text { TETRCHB } & \mathrm{ppb} & 1,2,4,5 \text {-Tetrachlorobenzene } \\ \text { TRIBUPH } & \mathrm{ppb} & \text { Tributylphospho'ic acid } \\ \text { TRICHLB } & \mathrm{ppb} & 1,2,4-\text { Trichlorolsenzene }\end{array}$

Group: SEMvOLE $=$ Semivolatile Organics, enhanced list

\begin{tabular}{|c|c|c|}
\hline Name in Tables & Units & Fuil Name \\
\hline 1-napha & $\mathrm{ppb}$ & 1-Naphthyl amine \\
\hline 2-napha & $\mathrm{ppb}$ & 2-Naphthylamine \\
\hline $24-d c h p$ & $\mathrm{ppb}$ & 2,4 -0ichlorophenol \\
\hline 24 -dint & $\mathrm{ppb}$ & 2,4-Dinitrotoluene \\
\hline $245-\operatorname{trp}$ & ppb & $2,4,5$-Trichloropheno? \\
\hline $246-\operatorname{trp}$ & ppb & $2,4,6$-Trichiorophenol \\
\hline
\end{tabular}


IABLE E.1. (contd)

Group: SEMVOLE $=$ Semivolatile Organics, enhanced list (contd)

\begin{tabular}{|c|c|c|}
\hline Name in Tables & Units & Ful1 Name \\
\hline $26-d c h p$ & $\mathrm{ppb}$ & 2,6-Dich1 orophenol \\
\hline 26-dint & $\mathrm{ppb}$ & 2,6-Dinitrotoluene \\
\hline ACEFENE & $\mathrm{ppb}$ & 2-Acetyl aminofluorene \\
\hline ACETOPH & $\mathrm{ppb}$ & Acetophenone \\
\hline AMI ISOX & $\mathrm{ppb}$ & 5-(Aminomethyl)-3-isoxazolol \\
\hline AMINOYL & $\mathrm{ppb}$ & 4-Aminobiphenyi \\
\hline AMITROL & $\mathrm{ppb}$ & Amitrole \\
\hline ANILINE & $\mathrm{ppb}$ & Aniline \\
\hline ARAMITE & $\mathrm{ppb}$ & Aramite \\
\hline AURAMIN & $\mathrm{ppb}$ & Auramine \\
\hline BENDICM & $\mathrm{ppb}$ & Benzene, dichloromethy1 \\
\hline BENDINE & $\mathrm{ppb}$ & Benzidine \\
\hline BENTHOL & $\mathrm{ppb}$ & Benzenethiol \\
\hline BENZAAN & $\mathrm{ppb}$ & Benz [a] anthracene \\
\hline BENZBFL & $\mathrm{ppb}$ & Benzo[b] fluoranthene \\
\hline BENZCAC & $\mathrm{ppb}$ & Benz $[c]$ acridine \\
\hline BENZCHL & $\mathrm{ppb}$ & Benzyl chloride \\
\hline BENZJJL & $\mathrm{ppb}$ & Benzo $[j]$ 'fluoranthene \\
\hline BENZOPY & $\mathrm{ppb}$ & Benzo[a]pyrene \\
\hline BIS2CHE & $\mathrm{ppb}$ & Bis $(2$-chloroethyl) ether \\
\hline BIS2CHM & $\mathrm{ppb}$ & Bis (2-chloroethoxy) methane \\
\hline BIS2EPH & $\mathrm{ppb}$ & Bis (2-ethylhexyl) phthalate \\
\hline BIS2ETH & $\mathrm{ppb}$ & Bis (2-chloroisopropyl) ether \\
\hline BROPHEN & $\mathrm{ppb}$ & 4-Bromophenyl phenyl ether \\
\hline BUTBENP & $\mathrm{ppb}$ & Butyl benzyl phthalate \\
\hline BUTDINP & $\mathrm{ppb}$ & 2-sec-Buty1-4,6-dinitrophenol (DNBP) \\
\hline CHALETH & $\mathrm{ppb}$ & Chloroalkyl ethers \\
\hline CHLANIL & $\mathrm{ppb}$ & p-Chloroaniline \\
\hline CHLCRES & $\mathrm{ppb}$ & $p$-chloro-m-cresol \\
\hline CHLEPOX & $\mathrm{ppb}$ & 1-Ch1oro-2,3-epoxypropane \\
\hline CHLNAPH & $\mathrm{ppb}$ & 2-Chloronaphthalene \\
\hline CHLNAPZ & $\mathrm{ppb}$ & Chlornaphaz ine \\
\hline CHLPHEN & $\mathrm{ppb}$ & 2-Ch7orophenol \\
\hline CHRYSEN & $\mathrm{ppb}$ & Chrysene \\
\hline CRESOLS & $\mathrm{ppb}$ & Cresols \\
\hline CYCHDIN & $\mathrm{ppb}$ & 2-Cyclohexy7-4,6-dinitrophenol \\
\hline DIBAEPY & $\mathrm{ppb}$ & Dibenzo[a,e]pyrene \\
\hline DIBAHAC & $\mathrm{ppb}$ & Dibenzo[a,h]acridine \\
\hline DIBAHAN & $\mathrm{ppb}$ & Dibenz $[a, h]$ anthracene \\
\hline DIBAHPY & $\mathrm{ppb}$ & Dibenzo $[a, h]$ pyrene \\
\hline DIBAIPY & $\mathrm{ppb}$ & Dibenzo[a, $i]$ pyrene \\
\hline DIBAJAC & $\mathrm{ppb}$ & Dibenz $[a, j]$ acridine \\
\hline OIBCGCA & $\mathrm{ppb}$ & $7 \mathrm{H}$-Dibenzo[c,g]carbazole \\
\hline DIBPHTH & $\mathrm{ppb}$ & Di-n-butyl phthalate \\
\hline DICHBEN & $\mathrm{ppb}$ & $3,3^{\prime}$-Dichlorobenzidine \\
\hline
\end{tabular}


TABLE E.1. (contd)

Group: SEMVOLE = Semivolatile Organics, enhanced 7ist (contd)

\begin{tabular}{|c|c|c|}
\hline Name in Tables & Units & Fu1l Name \\
\hline DIEPHTH & $\mathrm{ppb}$ & Diethyl phthalate \\
\hline DIHYSAF & $\mathrm{ppb}$ & Dihydrosafrole \\
\hline DIMBENZ & $\mathrm{ppb}$ & 7,12-Dimethyibenz [a] anthracene \\
\hline DIMEAMB & $\mathrm{ppb}$ & p-Dimethyl aminoazobenzene \\
\hline DIMETHB & $\mathrm{ppb}$ & $3,3^{\prime}$-Dimethoxybenzidine \\
\hline DIMEYLB & $\mathrm{ppb}$ & $3,3^{\prime}$-Dimethy benzidine \\
\hline DIMPHAM & $\mathrm{ppb}$ & alpha, alpha-Dimethylphenethyl amine \\
\hline DIMPHEN & $\mathrm{ppb}$ & 2,4-Dimethy]pheno \\
\hline DIMPHTH & $\mathrm{ppb}$ & Dimethyl phthalate \\
\hline DINBENZ & $\mathrm{ppb}$ & Dinitrobenzene \\
\hline DINCRES & $\mathrm{ppb}$ & 4,6-Dinitro-0-cresol and salts \\
\hline DINPHEN & $\mathrm{ppb}$ & 2,4-Dinitrophenol \\
\hline DIOPHTH & $\mathrm{ppb}$ & Di-n-octyl phthialate \\
\hline DIPHAMI & $\mathrm{ppb}$ & Diphenylamine \\
\hline DIPHHYD & $\mathrm{ppb}$ & 1,2-Diphenylhydrazine \\
\hline DIPRNIT & $\mathrm{ppb}$ & Di-n-propylnitrosamine \\
\hline ETHMETS & $\mathrm{ppb}$ & Ethyl methanesulfonate \\
\hline ETHMINE & $\mathrm{ppb}$ & Ethyleneimine \\
\hline FLUORAN & $\mathrm{ppb}$ & Fluoranthene \\
\hline HEXAENE & $\mathrm{ppb}$ & Hexachloropropene \\
\hline HEXCBUT & $\mathrm{ppb}$ & Hexachlorobutadiene \\
\hline HEXCCYC & $\mathrm{ppb}$ & Hexachlorocyclopentadiene \\
\hline HEXCETH & $\mathrm{ppb}$ & Hexachloroethane \\
\hline INDENOP & $\mathrm{ppb}$ & Indeno $(1,2,3$-cd pyrene \\
\hline ISOSOLE & $\mathrm{ppb}$ & Isosafrole \\
\hline MALHYDR & $\mathrm{ppb}$ & Majeic hydrazide \\
\hline MALOILE & $\mathrm{ppb}$ & Malononitrile \\
\hline MELPHAL & $\mathrm{ppb}$ & MelphaTan \\
\hline METACTO & $\mathrm{ppb}$ & 2-Methyllactonitrile \\
\hline METAZIR & $\mathrm{ppb}$ & 2-Methylaziridine \\
\hline METBISC & $\mathrm{ppb}$ & $4,4^{\prime}$-Methylenebis (2-chloroaniline) \\
\hline METCHAN & $\mathrm{ppb}$ & 3-Methylcholanthrene \\
\hline METHAPY & $\mathrm{ppb}$ & Methapyrilene \\
\hline METHIOU & $\mathrm{ppb}$ & Methyl thiouraci: \\
\hline METHNYL & $\mathrm{ppb}$ & Metholonyl \\
\hline METMSUL & $\mathrm{ppb}$ & Methyl methanesulfonate \\
\hline METPROP & $\mathrm{ppb}$ & 2-Methyl-2-(methylthio) propionaldehyde- \\
\hline NAPHQUI & $\mathrm{ppb}$ & 1,4-Naphthoquinone \\
\hline NICOTIN & $\mathrm{ppb}$ & Nicotinic acid \\
\hline NITBENZ & $\mathrm{ppb}$ & 4-Nitrobenzene \\
\hline NITPHEN & $\mathrm{ppb}$ & p-Nitroaniline \\
\hline NITRANI & $\mathrm{ppb}$ & p-Nitroaniline \\
\hline NITRPYR & $\mathrm{ppb}$ & Nitrosopyrrolidine \\
\hline NITRTOL & $\mathrm{ppb}$ & 5-Nitro-o-toluidine \\
\hline NNIBUTY & $\mathrm{ppb}$ & $N$-nitrosodi-n-butylamine \\
\hline
\end{tabular}


TABLE E.1. (contd)

Group: SEMVOLE $=$ Semivolatile Organics, enhanced 1 ist (contd)

\begin{tabular}{|c|c|c|}
\hline Name in Tables & Units & Full Name \\
\hline NNIDIEA & $\mathrm{ppb}$ & N-nitrosodiethanol amine \\
\hline NNIDIEY & $\mathrm{ppb}$ & $\mathrm{N}$-nitrosodimethylamine \\
\hline NNIDIME & $\mathrm{ppb}$ & $\mathrm{N}$-nitrosodimethylamine \\
\hline NNIMETH & $\mathrm{ppb}$ & $\mathrm{N}$-nitrosomethylethylamine \\
\hline NNIMORP & $\mathrm{ppb}$ & N-nitrosomorphol ine \\
\hline NNINICO & $\mathrm{ppb}$ & $\mathrm{N}$-nitrosonornicotine \\
\hline NNIPIPE & $\mathrm{ppb}$ & N-nitrosopiperidine \\
\hline NNIURET & $\mathrm{ppb}$ & $\mathrm{N}$-nitroso-n-methylurethane \\
\hline NNIVINY & $\mathrm{ppb}$ & $\mathrm{N}$-nitrosomethylvinylamine \\
\hline OTOLHYD & $\mathrm{ppb}$ & o-Toluidine hydrochloride \\
\hline PBENZQU & $\mathrm{ppb}$ & p-Benzoquinone \\
\hline PENTCHN & $\mathrm{ppb}$ & Pentachloronitrobenzene \\
\hline PENTCHP & $\mathrm{ppb}$ & Pentachlorophenol \\
\hline PHENINE & $\mathrm{ppb}$ & Phenylenediamine \\
\hline PHENTIN & $\mathrm{ppb}$ & Phenacet in \\
\hline PHTHEST & $\mathrm{ppb}$ & Phthalic acid esters \\
\hline PICOLIN & $\mathrm{ppb}$ & 2-Picoline \\
\hline PRONIDE & $\mathrm{ppb}$ & Pronamide \\
\hline RESERPI & $\mathrm{ppb}$ & Reserpine \\
\hline RESORCI & $\mathrm{ppb}$ & Resorcinot \\
\hline SAFROL & $\mathrm{ppb}$ & Safrol \\
\hline STRYCHN & $\mathrm{ppb}$ & Strychnine \\
\hline SYMTRIN & $\mathrm{ppb}$ & Sym-trinitrobenzene \\
\hline TETRCHP & $\mathrm{ppb}$ & $2,3,4,6$-Tetrachlorophenol \\
\hline THIONOX & $\mathrm{ppb}$ & Thiofanox \\
\hline THIURAM & $\mathrm{ppb}$ & Thiuram \\
\hline TOLUDIA & $\mathrm{ppb}$ & Toluenediamine \\
\hline TRIPHOS & $\mathrm{ppb}$ & $0,0,0$-triethyl phosphorothioate \\
\hline TRISPHO & $\mathrm{ppb}$ & Tris (2,3-dibromopropyl) phosphate \\
\hline WARFRIN & $\mathrm{ppb}$ & Warfarin \\
\hline
\end{tabular}

Name in Tables Units

Fu17 Name

ACETREA

CHLOREA

DIETROL

ETHYREA

NAPHREA

PHENREA

THIOURA $\mathrm{ppb}$

$\mathrm{ppb}$

$\mathrm{ppb}$

$\mathrm{ppb}$

$\mathrm{ppb}$

$\mathrm{ppb}$

$\mathrm{ppb}$
1-Acetyl-2-thiourea

1-(0-Chiorophenyl) thiourea

0iethylstilbesterol

Ethyl enethiourea

1-Naphthyl-2-thiourea

$\mathrm{N}$-phenyl thiourea

Thiourea 
TABLE E.1. (contd)

Group: U-ISO $=$ Uranium Isotopes

Name in Tables Units

Full Name

$\mathrm{U}-234$

$\mathrm{U}-235$

$\mathrm{pCi} / \mathrm{L}$

$\mathrm{pCi} / \mathrm{L}$

$\mathrm{pCi} / \mathrm{L}$

- 234

Uranium-235

$\mathrm{U}-238$

Uranium-238

Group: $\quad$ VOLORG $=$ Volatile Organics

Name in Tables Units

Full Name

$\begin{array}{lll}1,1,1-T & \mathrm{ppb} & 1,1,1-\text { Trich]oroethane } \\ 1,1,2-T & \mathrm{ppb} & 1,1,2-\text { Trichloroethane } \\ \text { CHLFORM } & \mathrm{ppb} & \text { Chloroform [Trichloromethane] } \\ \text { HEXONE } & \mathrm{ppb} & \text { Hexone } \\ \text { M-XYLE } & \mathrm{ppb} & \text { Xylene-m } \\ \text { METHONE } & \mathrm{ppb} & \text { Methyl ethyl ketone } \\ \text { METHYCH } & \mathrm{ppb} & \text { Methylene chloride } \\ \text { OPXYLE } & \mathrm{ppb} & \text { Xylene-0, } \\ \text { PERCENE } & \mathrm{ppb} & \text { Perchloroethylene [Tetrachloroethene] } \\ \text { TETRANE } & \mathrm{ppb} & \text { Tetrachloromethane [Carbon Tetrachloride] } \\ \text { TRICENE } & \mathrm{ppb} & \text { Trichloroethylene [1,1,2-Trichloroethene] }\end{array}$

Group: VOLORGE $=$ Volatile Organics, enhanced list

Name in Tables Units Ful1 Name

1,1-DIC

$1,2-D I C$

$1112-\mathrm{tc}$

$1122-\mathrm{tc}$

$123-\operatorname{trp}$

ACETILE

ACROLIN

ACRYILE

BENZENE

BISTHER

BROMONE

BROMORM

CARBIDE

CHLBENZ

CHLTHER

CHMTHER

CROTONA

DIBRCHL

DIBRETH

DIBRMET

DIBUTEN ppb 1,1-Dichloroethane

$\mathrm{ppb}$ 1,2-Dichloroethane

$\mathrm{ppb} \quad 1,1,1,2$-Tetrachlorethane

ppb 1,1,2,2-Tetrachlorethane

ppb 1,2,3-Trichlorooropane

$\mathrm{ppb} \quad$ Acetonitrile

$\mathrm{ppb} \quad$ Acrolein

ppb Acrylonitrile

ppb Benzene

$\mathrm{ppb}$

$\mathrm{ppb}$

$\mathrm{ppb}$

$\mathrm{ppb}$

$\mathrm{ppb}$

$\mathrm{ppb}$

$\mathrm{ppb}$

$\mathrm{ppb}$

$\mathrm{ppb}$

$\mathrm{ppb}$

$\mathrm{ppb}$

$\mathrm{ppb}$
Bis (chloromethyl) ether

Bromoacetone

Bromoform [Tribromomethane]

Carbon disulfid?

Chlorobenzene

2-Chloroethyl vinyl ether

Chloromethyl methyl ether

Crotonaldehyde

1,2-Dibromo-3-c17oropropane

1,2-Dibromoetha e

Dibromomethane

1,4-Dichloro-2-sutene 
TABLEE.1. (contd)

Group: VOLORGE $=$ Volatile Organics, enhanced 7 ist (contd)

\begin{tabular}{|c|c|c|}
\hline Name in Tables & Units & Full Name \\
\hline DICDIFM & $\mathrm{ppb}$ & Dichlorodifluoromethane \\
\hline DICETHY & $\mathrm{ppb}$ & 1,1-Dichloroethylene \\
\hline DICPANE & $\mathrm{ppb}$ & 1,2-Dichloropropane \\
\hline DICPENE & $\mathrm{ppb}$ & 1,3-Dichloropropene \\
\hline DIETHY & $\mathrm{ppb}$ & Diethylarsine \\
\hline DIOXANE & $\mathrm{ppb}$ & DIOXANE \\
\hline ETHMETH & $\mathrm{ppb}$ & Ethyl methacrylate \\
\hline ETHOXID & $\mathrm{ppb}$ & Ethylene oxide \\
\hline FORMALN & $\mathrm{ppb}$ & Formal in \\
\hline HYDRSUL & $\mathrm{ppb}$ & Hydrogen sulfide \\
\hline IDDOMET & $\mathrm{ppb}$ & Iodomethane \\
\hline METACRY & $\mathrm{ppb}$ & Methyl methacrylate \\
\hline METHACR & $\mathrm{ppb}$ & Methacrylonitrile \\
\hline METHBRO & $\mathrm{ppb}$ & Methyl bromide \\
\hline METHCHL & $\mathrm{ppb}$ & Methyl chloride [Chloromethane] \\
\hline METHTHI & $\mathrm{ppb}$ & Methanethiol \\
\hline NNDIEHY & $\mathrm{ppb}$ & $\mathrm{N}, \mathrm{N}$-diethylhydrazine \\
\hline PENTACH & $\mathrm{ppb}$ & Pentachloroethane \\
\hline PYRIDIN & $\mathrm{ppb}$ & Pyridine \\
\hline - TDLUENE & $\mathrm{ppb}$ & Toluene \\
\hline TRANDCE & $\mathrm{ppb}$ & trans-1,2-Dichioroethene \\
\hline TRCMEOL & $\mathrm{ppb}$ & Trichloromethanethiol \\
\hline TRCMFLM & $\mathrm{ppb}$ & Trichloromonofluoromethane \\
\hline TRCPANE & $\mathrm{ppb}$ & Trichloropropane \\
\hline VINYIDE & $\mathrm{ppb}$ & Vinyl chloride \\
\hline
\end{tabular}


TABLE E.2. Constituents Analyzed for in Ground-Water Samples from Site-Wide Chemical Monitoring Wells
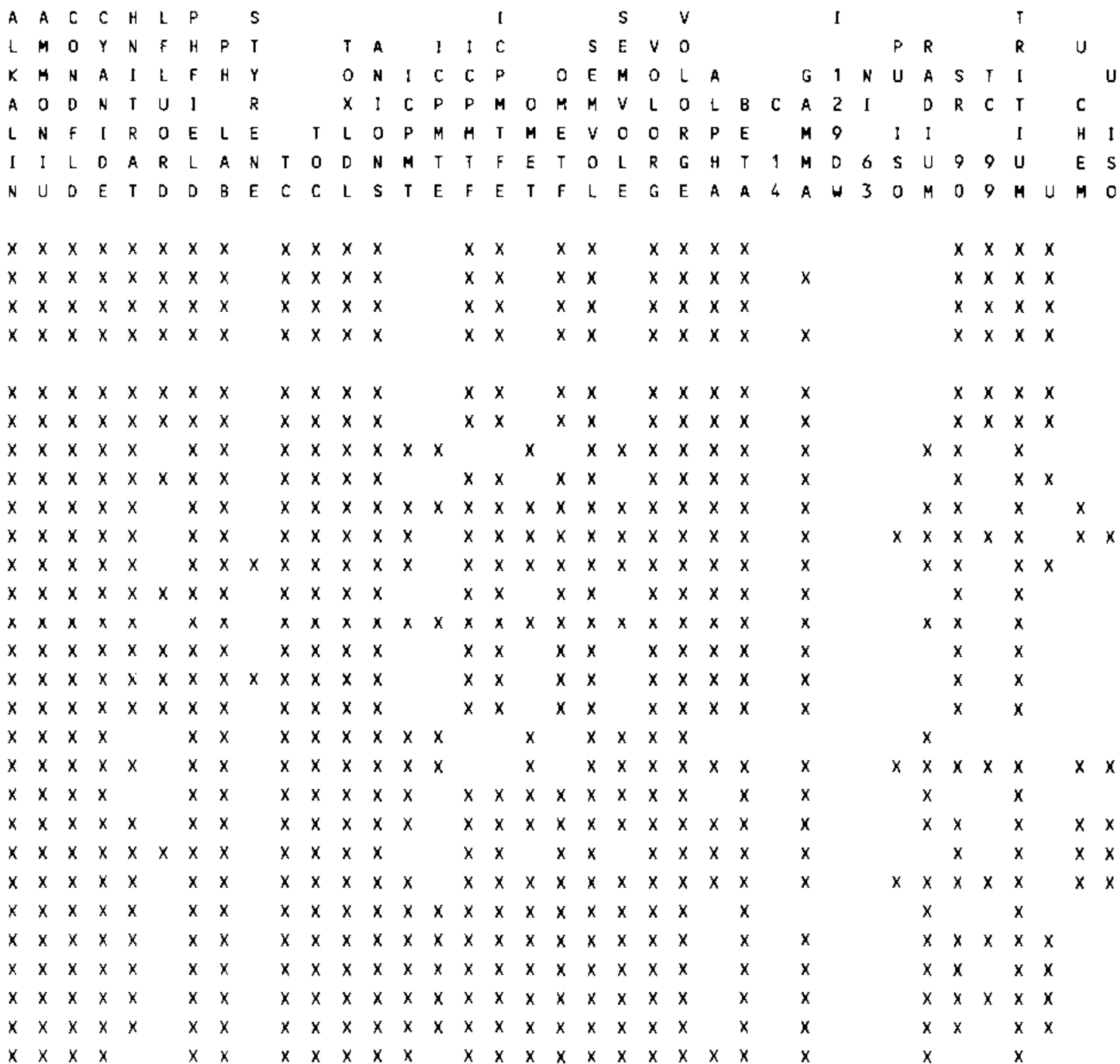


\section{IABLE E.2. (contd)}

\begin{tabular}{|c|c|c|c|c|c|c|c|c|c|c|c|c|c|c|c|c|c|c|c|c|c|c|c|c|c|c|c|c|c|c|c|c|}
\hline$E$ & A & A & C & $c$ & H L & L $\quad P$ & & $s$ & & & & & & & $i$ & & & $\mathrm{~s}$ & S & $v$ & & & & & I & & & & & $T$ & & \\
\hline L & $L$ & $H$ & 0 & $Y$ & N F & $\mathrm{F} \mathrm{H}$ & $P$ & $T$ & & $\mathbf{T}$ & A & & 1 & ! & $c$ & & & $S E$ & $E V$ & 0 & & & & & & $P$ & $R$ & & & $\mathbf{R}$ & & u \\
\hline L & $k$ & $M$ & $\mathbf{N}$ & A & i 1 & L $F$ & $H$ & $Y$ & & 0 & $\mathbf{N}$ & I & C & C & $P$ & & 0 & $E M$ & 40 & $\mathrm{~L}$ & A & & & G & $1 \mathrm{~N}$ & $\mathrm{~N} U$ & $A$ & $\mathbf{S}$ & $T$ & 1 & & \\
\hline N & A & 0 & D & N & $T$ & $U$ I & & $R$ & & $x$ & 1 & $c$ & $P$ & $P$ & $M$ & 0 & $M$ & $M V$ & $V L$ & 0 & L & B & c & A & 21 & 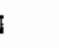 & D & $R$ & $c$ & $T$ & & C \\
\hline A & L & $\mathrm{N}$ & $F$ & $\mathrm{I}$ & $R \quad O$ & $O E$ & L & $\varepsilon$ & $T$ & $L$ & 0 & $P$ & $M$ & $M$ & $\tau$ & $M$ & E & $\vee 0$ & 00 & $R$ & $P$ & $E$ & & M & 9 & 1 & I & & & 1 & & $H$ \\
\hline M & I & 1 & $L$ & 0 & A $R$ & $R \quad L$ & A & N $T$ & 0 & 0 & $\mathrm{~N}$ & M & $T$ & $T$ & $F$ & $E$ & T & o L & $1 \quad R$ & $\mathbf{G}$ & H & $t$ & 1 & $M$ & 06 & 65 & U & 9 & 9 & $\mathrm{U}$ & & $E$ \\
\hline$E$ & $\mathbf{N}$ & $U$ & $D$ & $E$ & $\begin{array}{ll}T & 0\end{array}$ & 00 & B & $E \quad C$ & $c$ & $L$ & $s$ & $T$ & $E$ & $F$ & $E$ & $T$ & $F$ & L. $E$ & $E \quad G$ & $E$ & A & A & 4 & A & $\checkmark 3$ & 30 & $M$ & 0 & 9 & $M$ & $\downarrow$ & M \\
\hline $2-W 10-4$ & $x$ & $x$ & $x$ & $x$ & $x$ & $x$ & $x$ & $x$ & $x$ & $x$ & $x$ & $x$ & $x$ & $x$ & $x$ & $x$ & $x$ & $x \quad x$ & $x \quad x$ & $x$ & & $x$ & & $x$ & & & $x$ & $x$ & & $x$ & $x$ & \\
\hline $2-410-9$ & $x$ & $x$ & $x$ & $x$ & $x$ & $x$ & $x$ & $x$ & $x$ & $x$ & $x$ & $x$ & $x$ & $x$ & $x$ & $x$ & $x$ & $x x$ & $x \quad x$ & $x$ & $x$ & $x$ & & $x$ & & & $x$ & $x$ & & $x$ & $x$ & \\
\hline 2-W11-7 & $x$ & $x$ & $x$ & $x$ & & $x$ & $x$ & $x$ & $x$ & $x$ & $x$ & $x$ & $x$ & & & $x$ & & $x \quad x$ & $x \quad x$ & $x$ & $x$ & $x$ & & & & & $x$ & & & & & \\
\hline $2-w 11-14$ & $x$ & $x$ & $x$ & $x$ & & $x$ & $x$ & $x$ & $x$ & $x$ & $x$ & $x$ & $x$ & & & $x$ & & $x \quad x$ & $x \quad x$ & $x$ & $x$ & $x$ & & & & & $x$ & & & & & \\
\hline $2-414-2$ & $x$ & $x$ & $x$ & $x$ & $x$ & $x$ & $x$ & $x$ & $x$ & $x$ & $x$ & $x$ & $x$ & $x$ & $x$ & $x$ & $x$ & $x x$ & $x \quad x$ & $x$ & $x$ & $x$ & & $x$ & & & $x$ & $x$ & $x$ & $x$ & $x$ & \\
\hline $2-414-5$ & $x$ & $x$ & $x$ & $x$ & $x$ & $x$ & $x$ & $x$ & $x$ & $x$ & $x$ & $x$ & $x$ & $x$ & $x$ & $x$ & $x$ & $x \quad x$ & $x \times$ & $x$ & & $x$ & & $x$ & & & $x$ & $x$ & & $x$ & $x$ & \\
\hline $2-214-6$ & $x$ & $x$ & $x$ & $x$ & $x \quad x$ & $x \quad x$ & $x$ & $x$ & $x$ & $x$ & $x$ & & & $x$ & $x$ & & $x$ & $x$ & $x$ & $x$ & $x$ & $x$ & & $x$ & & & & $x$ & & $x$ & $x$ & \\
\hline $2-414-10$ & $x$ & $x$ & $x$ & $x$ & $x$ & $x$ & $x$ & $x$ & $x$ & $x$ & $x$ & $x$ & & $x$ & $x$ & $x$ & $x$ & $x \quad x$ & $x \quad x$ & $x$ & $x$ & $x$ & & $x$ & & & $x$ & $x$ & & $x$ & & \\
\hline$-415-4$ & $x$ & $x$ & $x$ & $x$ & $x$ & $x$ & $x$ & $x$ & $x$ & $x$ & $x$ & $x$ & $x$ & $x$ & $x$ & $x$ & $\mathrm{x}$ & $x \quad x$ & $x \quad x$ & $x$ & & $x$ & & & & & $x$ & $x$ & $x$ & $x$ & $x$ & \\
\hline $2-\$ 15-7$ & $x$ & $x$ & $x$ & $x$ & $x$ & $x$ & $x$ & $x$ & $x$ & $x$ & $x$ & $x$ & $x$ & $x$ & $x$ & $x$ & $x$ & $x x$ & $x \quad x$ & $x$ & $x$ & $x$ & & $x$ & & & $x$ & $x$ & & $x$ & & \\
\hline $2.415-8$ & $x$ & $x$ & $x$ & $x$ & & $x$ & $x$ & $x$ & $x$ & $x$ & $x$ & $x$ & $x$ & & & $x$ & & $x \quad x$ & $x \quad x$ & $x$ & $x$ & $x$ & & & & & $x$ & & & & & \\
\hline$-415-10$ & $x$ & $x$ & $x$ & $x$ & $x$ & $x$ & $x$ & $x$ & $x$ & $x$ & $x$ & $x$ & $x$ & $x$ & $x$ & $x$ & $x$ & $x \quad x$ & $x \quad x$ & $x$ & $x$ & $x$ & & & & & $x$ & & & $x$ & & \\
\hline $2-415-11$ & $x$ & $x$ & $x$ & $x$ & $x$ & $x$ & $x$ & $x$ & $x$ & $x$ & $x$ & $x$ & $x$ & $x$ & $x$ & $x$ & $x$ & $x \quad x$ & $x \times$ & $x$ & $x$ & $x$ & & $x$ & & & $x$ & $x$ & & $x$ & & \\
\hline $2-415-12$ & $x$ & $x$ & $x$ & $x$ & & $x$ & $x$ & $x$ & $x$ & $x$ & $x$ & $x$ & $x$ & & & $x$ & & $x \quad x$ & $x \quad x$ & $x$ & & & & & & & $x$ & & & & & \\
\hline$-w 18-4$ & $x$ & $x$ & $x$ & $x$ & & $x$ & $x$ & $x$ & $x$ & $x$ & $x$ & $x$ & $x$ & & & $x$ & & $x \quad x$ & $x \quad x$ & $x$ & & & & & & & $x$ & & & & & \\
\hline$-418-5$ & $x$ & $x$ & $x$ & $x$ & & $x$ & $x$ & $x$ & $x$ & $x$ & $x$ & $x$ & $x$ & $x$ & $x$ & $x$ & $x$ & $x \quad x$ & $x \quad x$ & $x$ & $x$ & $x$ & & & & & $x$ & & & & & \\
\hline$-418-9$ & $x$ & $x$ & $x$ & $x$ & $x$ & $x$ & $x$ & $x$ & $x$ & $x$ & $x$ & $x$ & $x$ & & & $x$ & & $x \quad x$ & $x \quad x$ & $x$ & $x$ & $x$ & & & & & $x$ & & & & & \\
\hline $2-118-15$ & $x$ & $x$ & $x$ & $x$ & $x$ & $x$ & $x$ & $x$ & $x$ & $x$ & $x$ & $x$ & & $x$ & $x$ & $x$ & $x$ & $x \quad x$ & $x \quad x$ & $x$ & $x$ & $x$ & & $x$ & & $x$ & $x$ & & & $x$ & & $x$ \\
\hline$-418-17$ & $x$ & $x$ & $x$ & $x$ & $x$ & $x$ & $x$ & $x$ & $x$ & $x$ & $x$ & $x$ & $x$ & & & $x$ & & $x x$ & $x \quad x$ & $x$ & $x$ & $x$ & & $x$ & & & $x$ & & & $x$ & & \\
\hline$-619-3$ & $x$ & $x$ & $x$ & $x$ & $x$ & $x$ & $x$ & $x$ & $x$ & $x$ & $x$ & $x$ & $x$ & $n$ & $x$ & $x$ & x & $x \quad x$ & $x \quad x$ & $x$ & $x$ & $x$ & $x$ & $x$ & $x$ & & $x$ & $x$ & $x$ & $x$ & & $x$ \\
\hline $2-419-9$ & $x$ & $x$ & $x$ & $x$ & $x \quad x$ & $x \quad x$ & $x$ & $x$ & $x$ & $x$ & $x$ & & & $x$ & $x$ & & $x$ & $x$ & $x$ & $x$ & $x$ & $x$ & $x$ & $x$ & & & & $x$ & $x$ & $x$ & & $x$ \\
\hline $2-419-11$ & $x$ & $x$ & $x$ & $x$ & $x$ & $x$ & $x$ & $x$ & $x$ & $x$ & $x$ & $x$ & $x$ & $x$ & $x$ & $x$ & $\mathrm{x}$ & $x x$ & $x \quad x$ & $x$ & $x$ & $x$ & $x$ & $x$ & & & $x$ & $x$ & $x$ & $x$ & & $x$ \\
\hline $2-419-13$ & $x$ & $x$ & $x$ & $x$ & $x$ & $x$ & $x$ & $x$ & $x$ & $x$ & $x$ & $x$ & $x$ & $x$ & $x$ & $x$ & $x$ & $x \quad x$ & $x \quad x$ & $x$ & $x$ & $x$ & & $x$ & & & $x$ & $x$ & & $x$ & & $x$ \\
\hline -W19-15 & $x$ & $x$ & $x$ & $x$ & $x \quad x$ & $x \quad x$ & $x$ & $x$ & $x$ & $x$ & $x$ & & & $x$ & $x$ & & $x$ & $x$ & $x$ & $x$ & $x$ & $x$ & $x$ & $x$ & & & & $x$ & $x$ & $x$ & & $x$ \\
\hline $2-419-16$ & $x$ & $x$ & $x$ & $x$ & $x \quad x$ & $x \quad x$ & $x$ & $x$ & $x$ & $x$ & $x$ & & & $x$ & $x$ & & $x$ & $x$ & $x$ & $x$ & $x$ & $x$ & $x$ & $x$ & & & & $x$ & $x$ & $x$ & & $x$ \\
\hline $2 \cdot 419-18$ & $x$ & $x$ & $x$ & $x$ & $x$ & $x$ & $x$ & $x$ & $x$ & $x$ & $x$ & $x$ & & $x$ & $x$ & $x$ & $x$ & $x \quad x$ & $x \quad x$ & $x$ & $x$ & $x$ & & $x$ & & & $x$ & $x$ & $x$ & $x$ & & $x$ \\
\hline $2 \cdot 419-20$ & $x$ & $x$ & $x$ & $x$ & $x$ & $x$ & $x$ & $x$ & $x$ & $x$ & $x$ & $x$ & & $x$ & $x$ & $x$ & $x$ & $x \quad x$ & $x \quad x$ & $x$ & $x$ & $x$ & & $x$ & & & $x$ & $x$ & $x$ & $x$ & & $x$ \\
\hline $2-419-21$ & $x$ & $x$ & $x$ & $x$ & $x$ & $x$ & $x$ & $x$ & $x$ & $x$ & $x$ & $x$ & & $x$ & $x$ & $x$ & $x$ & $x \quad x$ & $x \quad x$ & $x$ & $x$ & $x$ & & $x$ & & $x$ & $x$ & $x$ & & $x$ & & $x$ \\
\hline $2 \cdot 419-24$ & $x$ & $x$ & $x$ & $x$ & $x$ & $x$ & $x$ & $x$ & $x$ & $x$ & $x$ & $x$ & & $x$ & $x$ & $x$ & $x$ & $x x$ & $x \quad x$ & $x$ & $x$ & $x$ & & $x$ & & $x$ & $x$ & $x$ & $x$ & $x$ & & $x$ \\
\hline $2-422-1$ & $x$ & $x$ & $x$ & $x$ & $x$ & $x$ & $x$ & $x$ & $x$ & $x$ & $x$ & $x$ & $x$ & $x$ & $x$ & $x$ & $x$ & $x \quad x$ & $x \quad x$ & $x$ & $x$ & $x$ & & $x$ & & & $x$ & $x$ & & $x$ & $x$ & \\
\hline $2-422-20$ & $x$ & $x$ & $x$ & $x$ & $x$ & $x$ & $x$ & $x$ & $x$ & $x$ & $x$ & $x$ & $x$ & $x$ & $x$ & $x$ & $x$ & $x \quad x$ & $x \times$ & $x$ & & $x$ & & $x$ & & & $x$ & $x$ & & $x$ & $x$ & \\
\hline
\end{tabular}




\section{TABLE E.2. (contd)}

\begin{tabular}{|c|c|c|c|c|c|c|c|c|c|c|c|c|c|c|c|c|c|c|c|c|c|c|c|c|c|c|c|c|c|c|c|c|c|c|c|}
\hline $\mathbf{E}$ & $A$ & $A$ & $\mathrm{c}$ & $\mathrm{C}$ & $H$ & $\mathrm{~L}$ & $P$ & & $s$ & & & & & & & I & & & & $s$ & & $v$ & & & & & 1 & & & & & & $T$ & & \\
\hline L & $L$ & $M$ & 0 & $Y$ & $\mathrm{~N}$ & $F$ & $\mathrm{H}$ & $P$ & $T$ & & $T$ & $A$ & & 1 & 1 & $c$ & & & $s$ & $E$ & $v$ & 0 & & & & & & & $P$ & $R$ & & & $\mathbf{R}$ & & $u$ \\
\hline$L$ & $k$ & $M$ & $\mathrm{~N}$ & A & 1 & L & $F$ & $H$ & $Y$ & & 0 & $N$ & I & C & C & $P$ & & 0 & $E$ & M & 0 & L & A & & & G & 1 & $\mathrm{~N}$ & $\mathrm{U}$ & A & $s$ & $T$ & 1 & & \\
\hline $\mathbf{N}$ & $A$ & 0 & $D$ & $\mathrm{~N}$ & $\mathbf{T}$ & $\mathrm{u}$ & I & & $R$ & & $x$ & I & $\mathrm{C}$ & $\mathrm{P}$ & $P$ & $M$ & 0 & $M$ & $M$ & $v$ & L & 0 & $L$ & B & $\mathrm{c}$ & A & 2 & I & & D & $R$ & C & $T$ & & c \\
\hline A & $L$ & $\mathbf{N}$ & $F$ & I & $R$ & 0 & $E$ & $L$ & $E$ & $T$ & L & 0 & $P$ & $M$ & $M$ & $T$ & M & $E$ & $v$ & 0 & 0 & $R$ & $P$ & $E$ & & $M$ & 9 & & 1 & I & & & $I$ & & $H$ \\
\hline M & 1 & 1 & L & D & $A$ & $R$ & L & A & $N \quad T$ & 0 & $D$ & $N$ & $M$ & 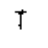 & $\Upsilon$ & $F$ & $E$ & $T$ & 0 & L & $R$ & G & $\mathrm{H}$ & $T$ & $\imath$ & $M$ & D & 6 & $\mathrm{~s}$ & $\mathrm{u}$ & 9 & 9 & U & & $E$ \\
\hline$E$ & $\mathbf{N}$ & U & D & $E$ & $T$ & $D$ & D & B & E C & $c$ & L & $s$ & $\mathrm{~T}$ & $\mathrm{E}$ & $F$ & $E$ & $T$ & $F$ & L & $E$ & $G$ & $E$ & A & A & 4 & A & $W$ & 3 & 0 & M & 0 & 9 & $M$ & U & M \\
\hline $2-+22-22$ & $x$ & & $x$ & $x$ & $x$ & & $x$ & $x$ & $\mathrm{x}$ & $x$ & $x$ & $x$ & $x$ & & $x$ & $x$ & $x$ & $x$ & $x$ & $x$ & $x$ & $x$ & $x$ & $x$ & & $x$ & & & & $x$ & $x$ & & $x$ & & $x$ \\
\hline $2-w 23-10$ & $x$ & $x$ & $x$ & $x$ & $x$ & $x$ & $x$ & $x$ & $\mathrm{x}$ & $x$ & $x$ & $x$ & & & $x$ & $x$ & & $x$ & $x$ & $x$ & $x$ & $x$ & $x$ & $x$ & & $x$ & & & & & $x$ & $x$ & $x$ & & $x$ \\
\hline $2-427-1$ & $x$ & $x$ & $x$ & $x$ & $x$ & & $x$ & $x$ & $x$ & $x$ & $x$ & $x$ & $x$ & & $x$ & $x$ & $x$ & $x$ & $x$ & $x$ & $x$ & $x$ & $x$ & $x$ & & $x$ & & & $x$ & $x$ & $x$ & & $x$ & & $x$ \\
\hline $4-51-7 C$ & $x$ & $x$ & $x$ & $x$ & $x$ & $x$ & $x$ & $x$ & $\mathrm{x}$ & $x$ & $x$ & $x$ & & & $x$ & $x$ & & $x$ & $x$ & & $x$ & $x$ & $x$ & $x$ & & & $x$ & & & & & & $x$ & & \\
\hline $4-S 1-8 A$ & $x$ & $x$ & $x$ & $x$ & $x$ & $x$ & $x$ & $x$ & $\mathrm{x}$ & $x$ & $x$ & $x$ & & & $x$ & $x$ & & $x$ & $x$ & & $x$ & $x$ & $x$ & $x$ & & & $x$ & & & & & & $x$ & & \\
\hline $4-51-88$ & $x$ & $x$ & $x$ & $x$ & $x$ & $x$ & $x$ & $x$ & $\mathrm{x}$ & $x$ & $\mathrm{x}$ & $x$ & & & $x$ & $x$ & & $x$ & $x$ & & $x$ & $x$ & $x$ & $x$ & & & $x$ & & & & & & $x$ & & \\
\hline $6-53+E 12$ & $x$ & $x$ & $x$ & $x$ & $x$ & $\mathrm{x}$ & $x$ & $x$ & $x$ & $x$ & $x$ & $x$ & & & $x$ & $x$ & & $x$ & $x$ & & $x$ & $x$ & $x$ & $x$ & & & $x$ & & & & & & $x$ & & \\
\hline $6-10-E 12$ & $x$ & $x$ & $x$ & $x$ & $x$ & $x$ & $x$ & $x$ & $x$ & $x$ & $x$ & $x$ & & & $x$ & $x$ & & $x$ & $x$ & & $x$ & $x$ & $x$ & $x$ & & & $x$ & & & & & & $x$ & & \\
\hline $6-12-4 B$ & $x$ & $x$ & $x$ & $x$ & & $x$ & $x$ & $x$ & $x$ & $x$ & $x$ & $x$ & & & $x$ & $x$ & & $x$ & $x$ & & $x$ & $x$ & $x$ & $x$ & & & & & & & & & & & \\
\hline $6-14-38$ & $x$ & $x$ & $x$ & $x$ & $x$ & $x$ & $x$ & $x$ & $x$ & $x$ & $x$ & $x$ & & & $x$ & $x$ & & $x$ & $x$ & & $x$ & $x$ & $x$ & $x$ & & & $x$ & & & & & & $x$ & & \\
\hline $6-19-43$ & $x$ & $x$ & $x$ & $x$ & $x$ & $x$ & $x$ & $x$ & $x$ & $x$ & $x$ & $x$ & & & $x$ & $x$ & & $x$ & $x$ & & $x$ & $x$ & $x$ & $x$ & & & & & & & & & $x$ & & \\
\hline $6-20-39$ & $x$ & $x$ & $x$ & $x$ & $x$ & $x$ & $x$ & $x$ & $x$ & $x$ & $x$ & $x$ & & & $x$ & $x$ & & $x$ & $x$ & & $x$ & $x$ & $x$ & $x$ & & $x$ & & & & & & $x$ & $x$ & & \\
\hline $6-24-33$ & $x$ & $x$ & $x$ & $x$ & $x$ & $x$ & $x$ & $x$ & $x$ & $x$ & $x$ & $x$ & & & $x$ & $x$ & & $x$ & $x$ & & $x$ & $x$ & $x$ & $x$ & & & $x$ & & & & & & $x$ & & \\
\hline $6-24-46$ & $x$ & $x$ & $x$ & $x$ & $x$ & $x$ & $x$ & $x$ & $x$ & $x$ & $x$ & $x$ & & & $x$ & $x$ & & $x$ & $x$ & & $x$ & $x$ & $x$ & $x$ & & & & & & & & & $x$ & & \\
\hline $6-29-78$ & $x$ & $x$ & $x$ & $x$ & $x$ & $x$ & $x$ & $x$ & $x$ & $x$ & $x$ & $x$ & & & $x$ & $x$ & & $x$ & $x$ & & $x$ & $x$ & $x$ & $x$ & & & $x$ & & & & & & $x$ & & \\
\hline $6-32-70 B$ & $x$ & $x$ & $x$ & $x$ & $x$ & $x$ & $x$ & $x$ & $x$ & $x$ & $x$ & $x$ & & & $x$ & $x$ & & $x$ & $x$ & & $x$ & $x$ & $x$ & $x$ & & & $x$ & & & & $x$ & $x$ & $x$ & $x$ & \\
\hline $6-32-72$ & $x$ & $x$ & $x$ & $x$ & $x$ & $x$ & $x$ & $x$ & $x$ & $x$ & $x$ & $x$ & & & $x$ & $x$ & & $x$ & $x$ & & $x$ & $x$ & $x$ & $x$ & & $x$ & $x$ & & & & $x$ & $x$ & $x$ & $x$ & \\
\hline $6-32-77$ & $x$ & $x$ & $x$ & $x$ & $x$ & $x$ & $x$ & $x$ & $\mathrm{x}$ & $x$ & $\mathrm{x}$ & $x$ & & & $x$ & $x$ & & $x$ & $x$ & & $x$ & $x$ & $x$ & $x$ & & $x$ & $x$ & & & & & & $x$ & & \\
\hline $6-35-66$ & $x$ & $x$ & $x$ & $x$ & $x$ & $x$ & $x$ & $x$ & $\mathrm{x}$ & $x$ & $x$ & $x$ & & & $x$ & $x$ & & $x$ & $x$ & & $x$ & $x$ & $x$ & $x$ & & & $x$ & & & & $x$ & $x$ & $x$ & $x$ & \\
\hline $6-36-61 A$ & $x$ & $x$ & $x$ & $x$ & $x$ & $x$ & $x$ & $x$ & $x$ & $x$ & $x$ & $x$ & & & $x$ & $x$ & & $x$ & $x$ & & $x$ & $x$ & $x$ & $x$ & & & & & & & & & & & \\
\hline $6-37-E 4$ & $x$ & $x$ & $x$ & $x$ & $x$ & $x$ & $x$ & $x$ & $x$ & $x$ & $x$ & $x$ & & & $x$ & $x$ & & $x$ & $x$ & & $x$ & $x$ & $x$ & $x$ & & & & & & & & & $x$ & & \\
\hline $6-37-43$ & $x$ & $x$ & $x$ & $x$ & $x$ & $x$ & $x$ & $x$ & $\mathrm{x}$ & $x$ & $x$ & $x$ & & & $x$ & $x$ & & $x$ & $x$ & & $x$ & $x$ & $x$ & $x$ & $x$ & $x$ & & & & & & $x$ & $x$ & & \\
\hline $6-38-70$ & $x$ & $x$ & $x$ & $x$ & $x$ & $x$ & $x$ & $x$ & $x$ & $x$ & $x$ & $x$ & & & $x$ & $x$ & & $x$ & $x$ & & $x$ & $x$ & $x$ & $x$ & $x$ & & $x$ & $x$ & & & $x$ & $x$ & $x$ & & $x$ \\
\hline $6-39-39$ & $x$ & $x$ & $x$ & $x$ & $x$ & $x$ & $x$ & $x$ & $x$ & $x$ & $x$ & $x$ & & & $x$ & $x$ & & $x$ & $x$ & & $x$ & $x$ & $x$ & $x$ & & $x$ & $x$ & & & & & & $x$ & & \\
\hline $6-39-79$ & $x$ & $x$ & $x$ & $x$ & $x$ & $x$ & $x$ & $x$ & $x$ & $x$ & $x$ & $x$ & & & $x$ & $x$ & & $x$ & $x$ & & $x$ & $x$ & $x$ & $x$ & & $x$ & $x$ & & & & & & $x$ & & \\
\hline $6-40-62$ & $x$ & $x$ & $x$ & $x$ & $x$ & $x$ & $x$ & $x$ & $x$ & $x$ & $x$ & $x$ & & & $x$ & $x$ & & $x$ & $x$ & & $x$ & $x$ & $x$ & $x$ & & & $x$ & & & & & & $x$ & & \\
\hline $6-41-1$ & $x$ & $x$ & $x$ & $x$ & $x$ & $x$ & $x$ & $x$ & $x$ & $x$ & $x$ & $x$ & & & $x$ & $x$ & & $x$ & $x$ & & $x$ & $x$ & $x$ & $x$ & & $x$ & $x$ & & & & $x$ & $x$ & & $x$ & \\
\hline
\end{tabular}




\section{TABLEE.2. (contd)}

\begin{tabular}{|c|c|c|c|c|c|c|c|c|c|c|c|c|c|c|c|c|c|c|c|c|c|c|c|c|c|c|c|c|c|c|c|c|c|c|c|}
\hline 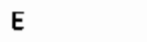 & A & $A$ & $c$ & C & $H$ & L & $P$ & & s & & & & & & & l & & & $\$$ & & $v$ & & & & & 1 & & & & & & $T$ & & & \\
\hline$L$ & $\mathbf{L}$ & M & 0 & $Y$ & $\mathbf{N}$ & $F$ & $H$ & $P$ & $T$ & & $T$ & A & & $t$ & I & C & & $\mathbf{s}$ & $E$ & $v$ & 0 & & & & & & & $P$ & $\mathbf{R}$ & & & $\mathbf{R}$ & & U & \\
\hline L & $k$ & $\mathbf{M}$ & $\mathbf{N}$ & A & 1 & $L$ & $F$ & $\mathbf{H}$ & $Y$ & & 0 & $\mathbf{N}$ & I & C & c & $p$ & 0 & $E$ & $M$ & 0 & L & A & & & G & 1 & $N$ & u & A & $\mathbf{s}$ & $T$ & I & & & $v$ \\
\hline$N$ & $A$ & 0 & $D$ & $N$ & $T$ & $v$ & 1 & & $\mathbf{R}$ & & $x$ & I & $c$ & $P$ & $P$ & M O & $M$ & $M$ & $v$ & L & 0 & L & B & c & A & 2 & I & & D & $\mathbf{R}$ & $C$ & $T$ & & c & \\
\hline A & $\mathrm{L}$ & $\mathbf{N}$ & $\mathbf{F}$ & I & $R$ & 0 & $E$ & $L$ & $E$ & $T$ & $\mathrm{~L}$ & 0 & $\mathrm{P}$ & $M$ & M & $T H$ & $E$ & $v$ & 0 & 0 & $\mathbf{R}$ & $P$ & E & & $M$ & 9 & & $!$ & I & & & 1 & & H & I \\
\hline$M$ & 1 & 1 & L & D & A & $R$ & $L$ & A & N $\quad T$ & 0 & D & $\mathrm{N}$ & $\mathbf{M}$ & $T$ & $T$ & $F \quad E$ & $T$ & 0 & L & $\mathbf{R}$ & G & H & $T$ & 1 & $\mathbf{k}$ & D & 6 & s & U & 9 & 9 & $u$ & & $E$ & $\mathbf{s}$ \\
\hline E & $\mathbf{N}$ & $u$ & $D$ & $\mathrm{E}$ & $T$ & $D$ & $D$ & $B$ & E C & $c$ & $\mathrm{~L}$ & $\mathbf{s}$ & $T$ & $E$ & $\mathbf{F}$ & E T & $\mathbf{F}$ & $\mathrm{L}$ & $E$ & G & $E$ & A & A & 4 & A & $W$ & 3 & 0 & M & 0 & 9 & H & U & $M$ & 0 \\
\hline $6-42-2$ & $x$ & $x$ & $x$ & $x$ & $x$ & $x$ & $x$ & $x$ & $x$ & $x$ & $x$ & $x$ & & & $x$ & $x$ & $x$ & $x$ & & $x$ & $x$ & $x$ & $x$ & & & & & & & & & $x$ & & & \\
\hline $6-42-40 A$ & $x$ & $x$ & $x$ & $x$ & $x$ & & $x$ & $x$ & $x$ & $x$ & $x$ & $x$ & $x$ & & $x$ & $x \quad x$ & $x$ & $x$ & $x$ & $x$ & $x$ & $x$ & $x$ & & $x$ & & & $x$ & $x$ & $x$ & & $x$ & & $x$ & $x$ \\
\hline $6-43-3$ & $x$ & $x$ & $x$ & $x$ & $x$ & $x$ & $x$ & $x$ & $x$ & $x$ & $x$ & $x$ & & & $x$ & $x$ & $x$ & $x$ & & $x$ & $x$ & $x$ & $x$ & & $x$ & $x$ & & & & & $x$ & $x$ & & & \\
\hline $6.44-64$ & $x$ & $x$ & $x$ & $x$ & $x$ & $x$ & $x$ & $x$ & $x$ & $x$ & $x$ & $x$ & & & $x$ & $x$ & $x$ & $x$ & & $x$ & $x$ & $x$ & $x$ & & & $x$ & & & & & & $x$ & & & \\
\hline $6-45-2$ & $x$ & $x$ & $x$ & $x$ & $x$ & $x$ & $x$ & $x$ & $x$ & $x$ & $x$ & $x$ & & & $x$ & $x$ & $x$ & $x$ & & $x$ & $x$ & $x$ & $x$ & & $x$ & $x$ & & & & & & $x$ & & & \\
\hline $6-45-42$ & $x$ & $x$ & $x$ & $x$ & $x$ & $x$ & $x$ & $x$ & $x$ & $x$ & $x$ & $x$ & $x$ & & $x$ & $x \quad x$ & $x$ & $x$ & $x$ & $x$ & $x$ & $x$ & $x$ & & $x$ & & & $x$ & $x$ & $x$ & & $x$ & & $x$ & $x$ \\
\hline $6-46-4$ & $x$ & $x$ & $x$ & $x$ & $x$ & $x$ & $x$ & $x$ & $x$ & $x$ & $x$ & $x$ & & & $x$ & $x$ & $x$ & $x$ & & $x$ & $x$ & $x$ & $x$ & & & $x$ & & & & & $x$ & $x$ & & & \\
\hline $6.47-5$ & $x$ & $x$ & $x$ & $x$ & $x$ & $x$ & $x$ & $x$ & $x$ & $x$ & $x$ & $x$ & & & $x$ & $x$ & $x$ & $x$ & & $x$ & $x$ & $x$ & $x$ & & $x$ & & & & & $x$ & & $x$ & $x$ & & \\
\hline $6-47-46 \mathrm{~A}$ & $x$ & $x$ & $x$ & $x$ & $x$ & $x$ & $x$ & $x$ & $x$ & $x$ & $x$ & $x$ & & & $x$ & $x$ & $x$ & $x$ & & $x$ & $x$ & $x$ & $x$ & & $x$ & $x$ & & & & & & $x$ & $x$ & & \\
\hline $6-47-50$ & $x$ & $x$ & $x$ & $x$ & $x$ & $x$ & $x$ & $x$ & $x$ & $x$ & $x$ & $x$ & & & $x$ & $x$ & $x$ & $x$ & & $x$ & $x$ & $x$ & $x$ & $x$ & $x$ & & & & & $x$ & $x$ & $x$ & & & \\
\hline $6-47-60$ & $x$ & $x$ & $x$ & $x$ & $x$ & $x$ & $x$ & $x$ & $x$ & $x$ & $x$ & $x$ & & & $x$ & $x$ & $x$ & $x$ & & $x$ & $x$ & $x$ & $x$ & & $x$ & $x$ & & & & & & $x$ & $x$ & & \\
\hline $6-49-55 A$ & $x$ & $x$ & $x$ & $x$ & $x$ & $x$ & $x$ & $x$ & $x$ & $x$ & $x$ & $x$ & & & $x$ & $x$ & $x$ & $x$ & & $x$ & $x$ & $x$ & $x$ & & $x$ & $x$ & & & & $x$ & $x$ & $x$ & $x$ & & \\
\hline $6-49-57$ & $x$ & $x$ & $x$ & $x$ & $x$ & $x$ & $x$ & $x$ & $x$ & $x$ & $x$ & $x$ & & & $x$ & $x$ & $x$ & $x$ & & $x$ & $x$ & $x$ & $x$ & $x$ & $x$ & $x$ & & & & $x$ & $x$ & $x$ & $x$ & & \\
\hline $6-49-79$ & $x$ & $x$ & $x$ & $x$ & $x$ & $x$ & $x$ & $x$ & $x$ & $x$ & $x$ & $x$ & & & $x$ & $x$ & $x$ & $x$ & & $x$ & $x$ & $x$ & $x$ & & & & & & & & & $x$ & & & \\
\hline $6.50-53$ & $x$ & $x$ & $x$ & $x$ & $x$ & $x$ & $x$ & $x$ & $x$ & $x$ & $x$ & $x$ & & & $x$ & $x$ & $x$ & $x$ & & $x$ & $x$ & $x$ & $x$ & $x$ & $x$ & $x$ & & & & $x$ & $x$ & $x$ & & & \\
\hline $6-50-85$ & $x$ & $x$ & $x$ & $x$ & $x$ & $x$ & $x$ & $x$ & $x$ & $x$ & $x$ & $x$ & & & $x$ & $x$ & $x$ & $x$ & & $x$ & $x$ & $x$ & $x$ & & & & & & & & & $x$ & & & \\
\hline $6-53-47 A$ & $x$ & $x$ & $x$ & $x$ & & & $x$ & $x$ & $x$ & $x$ & $x$ & $x$ & $x$ & & $x$ & $x \quad x$ & $x$ & $x$ & $x$ & $x$ & $x$ & $x$ & $x$ & & $x$ & & & & $x$ & $x$ & & & & & \\
\hline $6-53-48 B$ & & & $x$ & & & & $x$ & & & & & & & & & & & & & & & $x$ & $x$ & & $x$ & & & & & $x$ & & & & & \\
\hline $6-55-50 \mathrm{C}$ & $x$ & $x$ & $x$ & $x$ & $x$ & $x$ & $x$ & $x$ & $x$ & $x$ & $x$ & $x$ & & & $x$ & $x$ & $x$ & $x$ & & $x$ & $x$ & $x$ & $x$ & & $x$ & $x$ & & & & $x$ & & $x$ & $x$ & & \\
\hline $6-55-76$ & $x$ & $x$ & $x$ & $x$ & $x$ & $x$ & $x$ & $x$ & $x$ & $x$ & $x$ & $x$ & & & $x$ & $x$ & $x$ & $x$ & & $x$ & $x$ & $x$ & $x$ & & & & & & & & & & & & \\
\hline $6-65-72$ & $x$ & $x$ & $x$ & $x$ & $x$ & $x$ & $x$ & $x$ & $x$ & $x$ & $x$ & $x$ & & & $x$ & $x$ & $x$ & $x$ & & $x$ & $x$ & $x$ & $x$ & & & $x$ & & & & & & $x$ & $x$ & & \\
\hline $6.65-83$ & $x$ & $x$ & $x$ & $x$ & $x$ & $x$ & $x$ & $x$ & $x$ & $x$ & $x$ & $x$ & & & $x$ & $x$ & $x$ & $x$ & & $x$ & $x$ & $x$ & $x$ & & & & & & & & & $x$ & & & \\
\hline $6-67-86$ & $x$ & $x$ & $x$ & $x$ & $x$ & $x$ & $x$ & $x$ & $x$ & $x$ & $x$ & $x$ & & & $x$ & $x$ & $x$ & $x$ & & $x$ & $x$ & $x$ & $x$ & & & & & & & & & $x$ & & & \\
\hline $6-70-68$ & $x$ & $x$ & $x$ & $x$ & $x$ & $x$ & $x$ & $x$ & $x$ & $x$ & $x$ & $x$ & & & $x$ & $x$ & $x$ & $x$ & & $x$ & $x$ & $x$ & $x$ & & $x$ & $x$ & & & & $x$ & $x$ & $x$ & $x$ & & \\
\hline $6 \cdot 71-30$ & $x$ & $x$ & $x$ & $x$ & $x$ & $x$ & $x$ & $x$ & $x$ & $x$ & $x$ & $x$ & & & $x$ & $x$ & $x$ & $x$ & & $x$ & $x$ & $x$ & $x$ & & & & & & & & & $x$ & & & \\
\hline $6-71-52$ & $x$ & $x$ & $x$ & $x$ & $x$ & $x$ & $x$ & $x$ & $x$ & $x$ & $x$ & $x$ & & & $x$ & $x$ & $x$ & $x$ & & $x$ & $x$ & $x$ & $x$ & & & $x$ & & & & & & $x$ & $x$ & & \\
\hline $6-71-77$ & $x$ & $x$ & $x$ & $x$ & $x$ & $x$ & $x$ & $x$ & $x$ & $x$ & $x$ & $x$ & & & $x$ & $x$ & $x$ & $x$ & & $x$ & $x$ & $x$ & $x$ & & & $x$ & & & & & & $x$ & $x$ & & \\
\hline $6-72-73$ & $x$ & $x$ & $x$ & $x$ & $x$ & $x$ & $x$ & $x$ & $x$ & $x$ & $x$ & $x$ & & & $x$ & $x$ & $x$ & $x$ & & $x$ & $x$ & $x$ & $x$ & & & & & & & & & $x$ & & & \\
\hline $6-73-61$ & $x$ & $x$ & $x$ & $x$ & $x$ & $x$ & $x$ & $x$ & $x$ & $x$ & $x$ & $x$ & & & $x$ & $x$ & $x$ & $x$ & & $x$ & $x$ & $x$ & $x$ & & & $x$ & & & & & & $x$ & $x$ & & \\
\hline $6-74-44$ & $x$ & $x$ & $x$ & $x$ & $x$ & $x$ & $x$ & $x$ & $x$ & $x$ & $x$ & $x$ & & & $x$ & $x$ & $x$ & $x$ & & $x$ & $x$ & $x$ & $x$ & & & & & & & & & $x$ & & & \\
\hline $6-77-36$ & $x$ & $x$ & $x$ & $x$ & $x$ & $x$ & $x$ & $x$ & $x$ & $x$ & $x$ & $x$ & & & $x$ & $x$ & $x$ & $x$ & & $x$ & $x$ & $x$ & $x$ & & & & & & & & & $x$ & & & \\
\hline
\end{tabular}


TABLE E.2. (contd)

\begin{tabular}{|c|c|c|c|c|c|c|c|c|c|c|c|c|c|c|c|c|c|c|c|c|c|c|c|c|c|c|c|c|c|c|c|c|c|c|c|c|}
\hline$E$ & $A$ & $A$ & $\mathrm{C}$ & $\mathrm{C}$ & $H$ & $\mathrm{~L}$ & $P$ & & $\mathbf{s}$ & & & & & & & & 1 & & & & $s$ & & $v$ & & & & & 1 & & & & & & $T$ & & \\
\hline L. & $\mathrm{L}$ & $M$ & 0 & $Y$ & $\mathbf{N}$ & $F$ & $H$ & $P$ & $T$ & & & $T$ & A & & 1 & 1 & $c$ & & & $\mathrm{~s}$ & $\mathrm{E}$ & $v$ & 0 & & & & & & & $P$ & $\mathbf{R}$ & & & $R$ & & u \\
\hline L. & $\mathrm{K}$ & $M$ & $\mathbf{N}$ & A & I & $\mathrm{L}$ & $\mathrm{F}$ & H & $Y$ & & & 0 & $\mathbf{N}$ & 1 & $\mathrm{C}$ & $c$ & $P$ & & 0 & $E$ & $M$ & 0 & $\mathrm{~L}$ & A & & & G & 1 & $\mathbf{N}$ & $u$ & A & 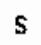 & $\mathrm{T}$ & I & & \\
\hline N & $A$ & 0 & $D$ & $\mathbf{N}$ & $T$ & $\mathrm{u}$ & $\mathrm{I}$ & & $R$ & & & $x$ & I & $\mathrm{C}$ & $p$ & $\rho$ & $M$ & 0 & $\mathbf{M}$ & $M$ & $v$ & $L$ & 0 & L & B & $c$ & $A$ & 2 & l & & D & $\mathbf{R}$ & C & $T$ & & C \\
\hline A & L & $\mathrm{N}$ & $F$ & 1 & $R$ & 0 & $\mathrm{E}$ & $\mathrm{L}$ & $E$ & & $T$ & $\mathrm{~L}$ & 0 & $\mathbf{P}$ & $M$ & $M$ & $T$ & $M$ & $E$ & $v$ & 0 & 0 & $R$ & $P$ & $E$ & & $M$ & 9 & & I & I & & & $\mathrm{I}$ & & $\mathrm{H}$ \\
\hline M & 1 & 1 & $L$ & $D$ & A & $R$ & $\mathrm{~L}$ & $A$ & $N$ & $T$ & 0 & $\mathrm{D}$ & $N$ & $M$ & $T$ & $\mathbf{T}$ & $F$ & $\mathrm{E}$ & $\mathbf{T}$ & 0 & $L$ & $\mathbf{R}$ & G & $\mathrm{H}$ & $\mathbf{T}$ & 1 & $M$ & $D$ & 6 & $\mathbf{s}$ & $\mathrm{u}$ & 9 & 9 & $\mathrm{u}$ & & $\mathrm{E}$ \\
\hline$E$ & $\mathbf{N}$ & $\mathrm{u}$ & $D$ & $E$ & $T$ & $D$ & $D$ & B & $\varepsilon$ & $c$ & $c$ & $\mathrm{~L}$ & s & $T$ & $\mathrm{E}$ & $F$ & $E$ & $\mathrm{~T}$ & $\mathrm{~F}$ & $\mathbf{L}$ & $\mathrm{E}$ & $G$ & $\mathrm{E}$ & A & $A$ & 4 & A & $\boldsymbol{u}$ & 3 & 0 & M & 0 & 9 & $M$ & U & M \\
\hline $6-78-62$ & $x$ & $x$ & $x$ & $x$ & $x$ & $x$ & $x$ & $x$ & & $x$ & $x$ & $x$ & $x$ & & & $x$ & $x$ & & $x$ & $x$ & & $x$ & $x$ & $x$ & $x$ & & & & & & & & & & & \\
\hline $6-83-47$ & $x$ & $x$ & $x$ & $x$ & & $x$ & $x$ & $x$ & & $x$ & $x$ & $x$ & $x$ & & & $x$ & $x$ & & $x$ & $x$ & & $x$ & $x$ & $x$ & $x$ & & & & & & & $x$ & $x$ & $x$ & & \\
\hline $6-96-49$ & $x$ & $x$ & $x$ & $x$ & $x$ & $x$ & $x$ & $x$ & & $x$ & $x$ & $x$ & $x$ & & & $x$ & $x$ & & $x$ & $x$ & & $x$ & $x$ & $x$ & $x$ & $x$ & & & $x$ & & & & & $x$ & $x$ & \\
\hline $6-97-43$ & $x$ & $x$ & $x$ & $x$ & $x$ & $x$ & $x$ & $x$ & & $x$ & $x$ & $x$ & $x$ & & & $x$ & $x$ & & $x$ & $x$ & & $x$ & $x$ & $x$ & $x$ & & & & & & & & & $x$ & & \\
\hline $6.97-51 \mathrm{~A}$ & $x$ & $x$ & $x$ & $x$ & $x$ & $x$ & $x$ & $x$ & & $x$ & $x$ & $x$ & $x$ & & & $x$ & $x$ & & $x$ & $x$ & & $x$ & $x$ & $x$ & $x$ & $x$ & & & $x$ & & & & & $x$ & $x$ & \\
\hline $6-101-488$ & $x$ & $x$ & $x$ & $x$ & $x$ & $x$ & $x$ & $x$ & & $x$ & $x$ & $x$ & $x$ & & & $x$ & $x$ & & $x$ & $x$ & & $x$ & $x$ & $x$ & $x$ & & & & & & & $x$ & $x$ & $x$ & & \\
\hline
\end{tabular}


IABLE E.3. Results for Site-Wide Chemical Monitoring Wells

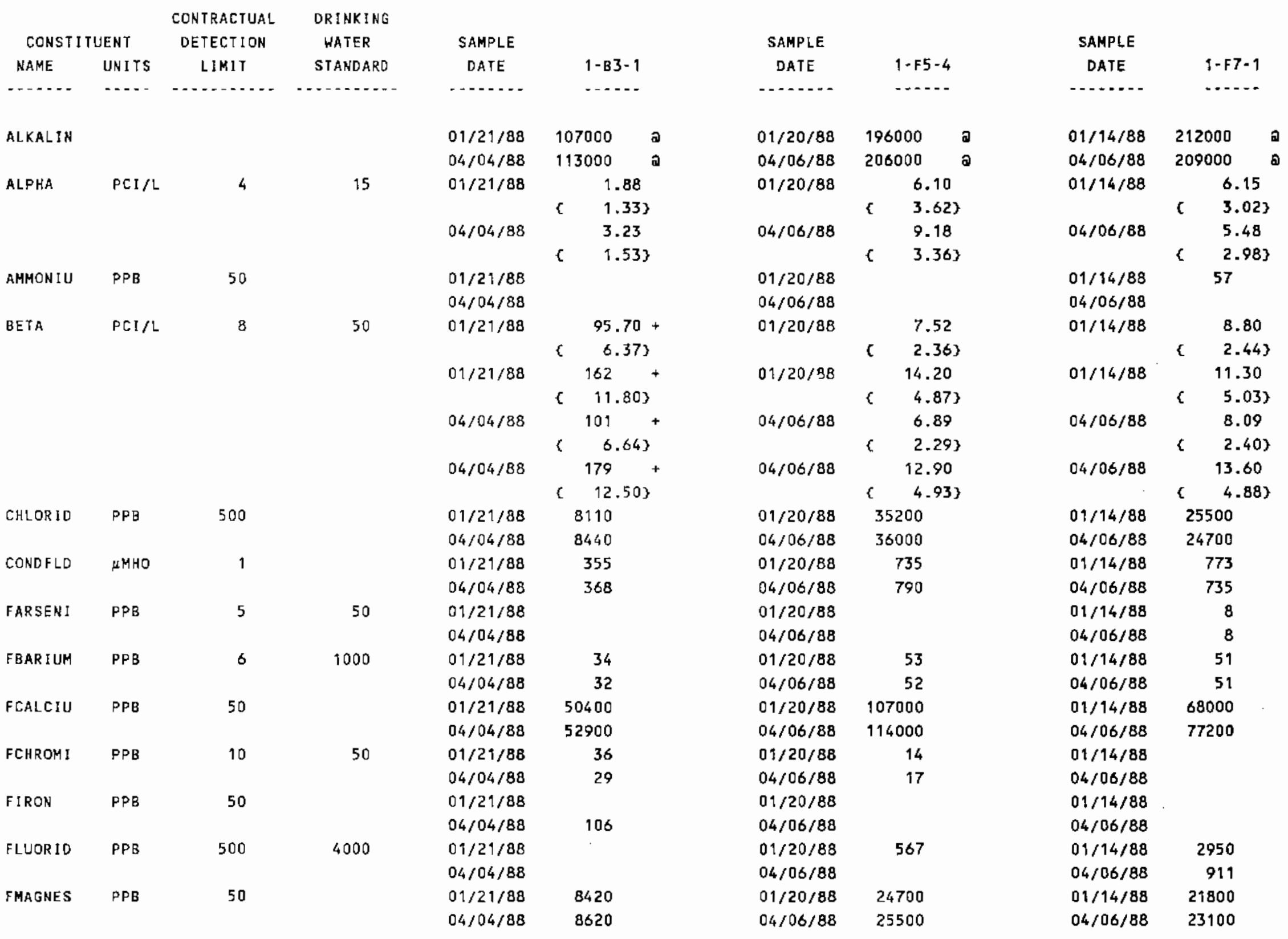


TABLE E.3. (contd)

\begin{tabular}{|c|c|c|c|c|c|c|c|c|c|}
\hline \multicolumn{2}{|c|}{ CONSTI TUENT } & $\begin{array}{l}\text { CONTRACTUAL } \\
\text { DETECTION }\end{array}$ & $\begin{array}{l}\text { DRINKING } \\
\text { WATER }\end{array}$ & SAMPLE & & SAMPLE & & SAMPLE & \\
\hline NAME & UNITS & LIMIT & STANDARD & DATE & $1-B 3-1$ & DATE & $9-F 5-4$ & DATE & $1-F 7-1$ \\
\hline - - . - - & $\cdots-$ & - & $-\ldots \ldots \ldots$ & $\cdots$ & $\ldots \ldots$ & $\cdots$ & $\cdots$ & - - & $\cdots$ \\
\hline \multirow[t]{2}{*}{ FMANGAM } & PPB & 5 & & $01 / 21 / 88$ & & $01 / 20 / 88$ & & $01 / 14 / 88$ & \\
\hline & & & & $04 / 04 / 88$ & 7 & $04 / 06 / 88$ & & $04 / 06 / 88$ & \\
\hline \multirow[t]{2}{*}{ FPOTASS } & $\mathrm{PPB}$ & 100 & & $01 / 21 / 88$ & 2040 & $09 / 20 / 88$ & 6180 & $01 / 14 / 88$ & 7890 \\
\hline & & & & $04 / 04 / 88$ & 2160 & $04 / 06 / 88$ & 6250 & $04 / 06 / 88$ & 7760 \\
\hline \multirow[t]{2}{*}{ FSODIUM } & PPB & 200 & & $01 / 21 / 88$ & 10600 & $01 / 20 / 88$ & 22500 & $01 / 14 / 88$ & 60900 \\
\hline & & & & $04 / 04 / 88$ & 10700 & $04 / 06 / 88$ & 22300 & $04 / 06 / 88$ & 61500 \\
\hline \multirow{2}{*}{ FSTRONT } & PPB & 10 & & $01 / 21 / 88$ & 236 & $01 / 20 / 88$ & 736 & $01 / 14 / 88$ & 484 \\
\hline & & & & $04 / 04 / 88$ & 241 & $04 / 06 / 88$ & 761 & $04 / 06 / 88$ & 502 \\
\hline \multirow[t]{2}{*}{ FVANADI } & PPB & 5 & & $01 / 21 / 88$ & & $01 / 20 / 88$ & 5 & $01 / 14 / 88$ & 14 \\
\hline & & & & $04 / 04 / 88$ & 5 & $04 / 06 / 88$ & 6 & $04 / 06 / 88$ & 16 \\
\hline \multirow[t]{2}{*}{ FZINC } & $\mathrm{PPB}$ & 5 & & $01 / 21 / 88$ & & $01 / 20 / 88$ & & $01 / 14 / 88$ & 18 \\
\hline & & & & $04 / 04 / 88$ & & $04 / 06 / 88$ & & $04 / 06 / 88$ & 14 \\
\hline \multirow[t]{2}{*}{ HNITRAT } & PPB & 2500 & 45000 & $01 / 21 / 88$ & 25000 & $01 / 20 / 88$ & 65800 & $01 / 14 / 88$ & 93200 \\
\hline & & & & $04 / 04 / 88$ & 27200 & $04 / 06 / 88$ & 69100 & $04 / 06 / 88$ & 87800 \\
\hline
\end{tabular}




\begin{tabular}{|c|c|c|c|c|c|c|c|}
\hline \multirow{2}{*}{\multicolumn{2}{|c|}{ CONST I TUENT }} & CONTRACTUAL & DRINKING & & & \multicolumn{2}{|c|}{ TABLE } \\
\hline & & DETECTION & HATER & SAMPLE & & \multirow{2}{*}{\multicolumn{2}{|c|}{$1-B 3-1$}} \\
\hline NAME & UNITS & LIMIT & STANDARD & DATE & & & \\
\hline - & $\cdots-$ & - & 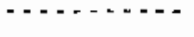 & $\cdots$ & & \multicolumn{2}{|c|}{$\ldots$} \\
\hline \multirow[t]{2}{*}{ LFLUORD } & PPB & 50 & 4000 & $01 / 21 / 88$ & & \multicolumn{2}{|l|}{166} \\
\hline & & & & $04 / 04 / 88$ & & \multicolumn{2}{|l|}{178} \\
\hline \multirow[t]{2}{*}{ NITRATE } & $P P B$ & 500 & 45000 & $01 / 21 / 88$ & \multicolumn{3}{|c|}{28500} \\
\hline & & & & $04 / 04 / 88$ & \multicolumn{3}{|c|}{26800} \\
\hline \multirow[t]{2}{*}{$P H-L A B$} & & 0.01 & & $01 / 21 / 88$ & & \multicolumn{2}{|c|}{7.85} \\
\hline & & & & $04 / 04 / 88$ & & \multicolumn{2}{|c|}{8.03} \\
\hline \multirow[t]{2}{*}{ PHFIELD } & & 0.10 & & $01 / 21 / 88$ & & & \\
\hline & & & & $04 / 04 / 88$ & & \multicolumn{2}{|c|}{8.20} \\
\hline \multirow[t]{4}{*}{ SR 90} & $\mathrm{PCI} / \mathrm{L}$ & 5 & 8 & $01 / 21 / 88$ & \multicolumn{3}{|c|}{50 * } \\
\hline & & & & & 〔 & \multicolumn{2}{|c|}{3.623} \\
\hline & & & & $04 / 04 / 88$ & \multicolumn{3}{|c|}{$57.50 *$} \\
\hline & & & & & C & \multicolumn{2}{|c|}{3.923} \\
\hline \multirow[t]{2}{*}{ SULFATE } & PPB & 500 & & $01 / 21 / 88$ & \multicolumn{3}{|c|}{38600} \\
\hline & & & & $04 / 04 / 88$ & \multicolumn{3}{|c|}{41600} \\
\hline \multirow[t]{2}{*}{ TC } & PPB & 2000 & & $01 / 21 / 88$ & \multicolumn{3}{|c|}{27000} \\
\hline & & & & $04 / 04 / 88$ & \multicolumn{3}{|c|}{25100} \\
\hline \multirow[t]{2}{*}{ TC-99 } & $\mathrm{PCI} / \mathrm{L}$ & 15 & 900 & $01 / 21 / 88$ & \multicolumn{3}{|c|}{118} \\
\hline & & & & & $\zeta$ & 2.2 & \\
\hline \multirow[t]{2}{*}{ TOXLOL } & PPB & 10 & & $01 / 21 / 88$ & & & \\
\hline & & & & $04 / 04 / 88$ & & & \\
\hline TRICENE & PPB & 10 & 5 & $01 / 21 / 88$ & & & \\
\hline & & & & $04 / 04 / 88$ & & & \\
\hline TR I I I UM & $\mathrm{PCl} / \mathrm{L}$ & 500 & 20000 & $01 / 21 / 88$ & & 3670 & \\
\hline & & & & & c & 253 & 3 \\
\hline & & & & $04 / 04 / 88$ & & 3620 & \\
\hline & & & & & $\zeta$ & 296 & 3 \\
\hline $\mathrm{u}$ & $\mathrm{PCI} / \mathrm{L}$ & 0.50 & 600 & $01 / 21 / 88$ & & 2.3 & \\
\hline & & & & $04 / 04 / 88$ & & 2.2 & \\
\hline
\end{tabular}

\begin{tabular}{|c|c|c|}
\hline \multicolumn{3}{|l|}{ SAKPLE } \\
\hline DATE & \multicolumn{2}{|l|}{$1-F 5-4$} \\
\hline 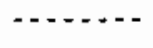 & \multicolumn{2}{|l|}{ - } \\
\hline $01 / 20 / 88$ & 160 & \\
\hline $04 / 06 / 88$ & 151 & \\
\hline $01 / 20 / 88$ & 66200 & 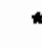 \\
\hline $04 / 06 / 88$ & 68800 & 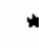 \\
\hline $01 / 20 / 88$ & \multicolumn{2}{|l|}{7.59} \\
\hline $04 / 06 / 88$ & \multicolumn{2}{|c|}{7.90} \\
\hline $01 / 20 / 88$ & \multicolumn{2}{|c|}{7.60} \\
\hline $04 / 06 / 88$ & \multirow{2}{*}{\multicolumn{2}{|c|}{7.90}} \\
\hline $01 / 20 / 88$ & & \\
\hline $04 / 06 / 88$ & & \\
\hline $01 / 20 / 88$ & \multicolumn{2}{|l|}{106000} \\
\hline $04 / 06 / 88$ & \multicolumn{2}{|l|}{108000} \\
\hline $01 / 20 / 88$ & \multicolumn{2}{|l|}{50600} \\
\hline $04 / 06 / 88$ & \multirow{2}{*}{\multicolumn{2}{|c|}{47500}} \\
\hline $01 / 20 / 88$ & & \\
\hline \multicolumn{3}{|l|}{$01 / 20 / 88$} \\
\hline \multicolumn{3}{|l|}{$04 / 06 / 88$} \\
\hline \multicolumn{3}{|l|}{$01 / 20 / 88$} \\
\hline \multicolumn{3}{|l|}{$04 / 06 / 88$} \\
\hline \multirow[t]{2}{*}{$01 / 20 / 88$} & \multicolumn{2}{|l|}{13500} \\
\hline & C 479 & 3 \\
\hline \multirow[t]{2}{*}{$04 / 06 / 88$} & \multicolumn{2}{|l|}{11500} \\
\hline & C 428 & 3 \\
\hline $01 / 20 / 88$ & \multicolumn{2}{|c|}{6.17} \\
\hline $04 / 06 / 88$ & 7.60 & \\
\hline
\end{tabular}

\begin{tabular}{|c|c|c|c|}
\hline \multicolumn{4}{|l|}{ SAMPLE } \\
\hline DATE & \multicolumn{3}{|c|}{$1-F 7-1$} \\
\hline$\cdots$ & \multicolumn{3}{|c|}{$\cdots$} \\
\hline $01 / 14 / 88$ & & 653 & \\
\hline $04 / 06 / 88$ & & 588 & \\
\hline $01 / 74 / 88$ & & 86600 & * \\
\hline $04 / 06 / 88$ & & 87200 & * \\
\hline $01 / 14 / 88$ & \multicolumn{3}{|c|}{7.60} \\
\hline $04 / 06 / 88$ & \multicolumn{3}{|c|}{7.66} \\
\hline $01 / 14 / 88$ & \multicolumn{3}{|c|}{6.20} \\
\hline $04 / 06 / 88$ & \multirow{2}{*}{\multicolumn{3}{|c|}{7.90}} \\
\hline $01 / 14 / 88$ & & & \\
\hline $04 / 06 / 88$ & & & \\
\hline $01 / 14 / 88$ & \multicolumn{3}{|c|}{71900} \\
\hline $04 / 06 / 88$ & \multicolumn{3}{|c|}{75000} \\
\hline $03 / 14 / 88$ & \multicolumn{3}{|c|}{52000} \\
\hline $04 / 06 / 88$ & \multicolumn{3}{|c|}{49400} \\
\hline \multicolumn{4}{|l|}{$01 / 14 / 88$} \\
\hline $01 / 14 / 88$ & \multicolumn{3}{|c|}{26} \\
\hline $04 / 06 / 88$ & \multicolumn{3}{|c|}{24} \\
\hline $01 / 14 / 88$ & \multicolumn{3}{|c|}{14} \\
\hline $04 / 06 / 88$ & \multicolumn{3}{|c|}{13} \\
\hline $01 / 14 / 88$ & \multicolumn{3}{|c|}{565} \\
\hline & c & 211 & 3 \\
\hline \multirow[t]{2}{*}{$04 / 06 / 88$} & \multicolumn{3}{|c|}{637} \\
\hline & C & 211 & 3 \\
\hline $01 / 14 / 88$ & \multicolumn{3}{|c|}{5.08} \\
\hline $04 / 06 / 88$ & \multicolumn{3}{|c|}{6.59} \\
\hline
\end{tabular}


TABLE E.3. (contd)

\begin{tabular}{|c|c|c|c|}
\hline \multicolumn{2}{|c|}{ CONSTITUENT } & $\begin{array}{l}\text { CONTRACTUAL } \\
\text { DETECTION }\end{array}$ & $\begin{array}{l}\text { DRINKING } \\
\text { WATER }\end{array}$ \\
\hline NAME & UN ITS & LIMIT & STANDARD \\
\hline - - - - & $-\ldots$ & - & - \\
\hline
\end{tabular}

\section{ALKALIN}

ALPMA

$$
P C I / L
$$

4

15

AMMONIU

PPB

50

BETA

$$
P C 1 / L
$$$$
8
$$

N

$\begin{array}{lll} & & \\ \text { CHLORID } & \text { PPB } & 500 \\ \text { CO-60 } & \text { PCI L } & 22.50 \\ \text { CONDFLD } & \text { HMHO } & 1 \\ \text { FBARIUM } & \text { PPB } & 6 \\ \text { FCALCIU } & \text { PPB } & 50 \\ \text { FCHROMI } & \text { PPB } & 10 \\ \text { FIRON } & \text { PPB } & 50\end{array}$

SAHPLE

DATE $1-\mathrm{N}-28$

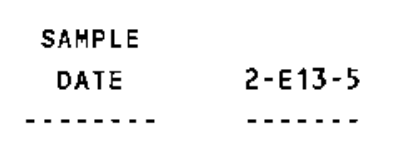

$\begin{array}{lcc}01 / 27 / 88 & 161000 \\ 04 / 19 / 88 & 165000 \\ 01 / 27 / 88 & 1.47 \\ & < & 1.15\} \\ 04 / 19 / 88 & & 2.86 \\ & \& & 1.52\} \\ 01 / 27 / 88 & & \end{array}$

$03 / 16 / 88$

$03 / 16 / 88$

$04 / 27 / 88$

$04 / 27 / 88$

$04 / 27 / 88$

$01 / 22 / 88$

$03 / 16 / 88$

$04 / 27 / 88$

$04 / 27 / 88$

$03 / 16 / 88$

$04 / 27 / 88$

$01 / 22 / 88$

$04 / 27 / 88$

$03 / 16 / 88$

$04 / 27 / 88$

$03 / 16 / 88$

$04 / 27 / 88$

$03 / 16 / 88$

$04 / 27 / 88$

$03 / 16 / 88$

$04 / 27 / 88$

$03 / 16 / 88$
82900 a

69200 ล

2.96

$\{\quad 1.42\}$

1.22
< $\quad 0.893$

1250

1290

1260

1600

1700

1700

$228+$

\{ 9.30$\}$

$\begin{gathered}351 \\ 16.703\end{gathered}+$

285

( 10.70 )

( $316.503+$

1550

1400

100

c 20.103

70.80
$18.80\}$

208

184

21
17

34100

23200

95
$04 / 19 / 88$

SAMPLE

DATE

2-E13-14

-...-. - -.....

$01 / 27 / 88 \quad 117000$

$04 / 19 / 88 \quad 139000 \quad$ a

$01 / 27 / 88$

$04 / 19 / 88 \quad 1.58$

$01 / 27 / 88$

$04 / 19 / 88$

NR

NR

NR

NR

NR

$01 / 27 / 88$

6.52

$01 / 27 / 88$

3.68 )

( 1.97

$04 / 19 / 88$

$04 / 19 / 88$

$01 / 27 / 88$

$04 / 19 / 88$

$01 / 27 / 88$

9.88

C 4.12

7.98
$\{\quad 2.13\}$

8640

7310

$01 / 27 / 88$

$04 / 27 / 88$

$04 / 19 / 88$

$04 / 19 / 88$

$01 / 27 / 88$

$04 / 19 / 88$

$04 / 29 / 88$

NR

NR

$01 / 27 / 88$

422

$4 / 19 / 88$

530

$01 / 27 / 88$

55
56

47100

$01 / 27 / 88$

45400

$1 / 27 / 88$

$04 / 19 / 88$

$01 / 27 / 88$
$01 / 27 / 88$

$04 / 19 / 88$

$01 / 27 / 88$

$04 / 19 / 88$

$01 / 27 / 88$

$04 / 19 / 88$

$01 / 27 / 88$

$04 / 19 / 88$

$01 / 27 / 88$
397

473

42

42000

37400

28

41 
TABLE E.3. (contd)

\begin{tabular}{|c|c|c|c|c|c|c|c|c|c|}
\hline \multicolumn{2}{|c|}{ CONST I TUEN T } & $\begin{array}{l}\text { CONTRACTUAL } \\
\text { DETECTION }\end{array}$ & $\begin{array}{l}\text { DRINKING } \\
\text { WATER }\end{array}$ & SAMPLE & \multicolumn{3}{|c|}{ SAMPLE } & \multicolumn{2}{|l|}{ SAMPLE } \\
\hline NAME & UNITS & LIMIT & STANDARD & DATE & $1-N-28$ & DATE & $2-E 13-5$ & DATE & 2-E13-14 \\
\hline \multirow[t]{2}{*}{$\ldots$} & $\cdots$ & 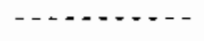 & - n. & - & - . . - & - & …... & - & $\cdots$ \\
\hline & & & & $04 / 27 / 88$ & & $04 / 19 / 88$ & & $04 / 19 / 88$ & \\
\hline \multirow[t]{2}{*}{ FLUORID } & $\mathrm{PPB}$ & 500 & 4000 & $03 / 16 / 88$ & & $01 / 27 / 88$ & 572 & $01 / 27 / 88$ & \\
\hline & & & & $04 / 27 / 88$ & & $04 / 19 / 88$ & & $04 / 19 / 88$ & \\
\hline \multirow[t]{2}{*}{ FMAGNES } & $\mathrm{PPB}$ & 50 & & $03 / 16 / 88$ & 6200 & $01 / 27 / 88$ & 15100 & $01 / 27 / 88$ & 15500 \\
\hline & & & & $04 / 27 / 88$ & 4360 & $04 / 19 / 88$ & 15000 & $04 / 19 / 88$ & 14100 \\
\hline \multirow[t]{2}{*}{ FPOTASS } & PPB & 100 & & $03 / 16 / 88$ & 4110 & $01 / 27 / 88$ & 5910 & $01 / 27 / 88$ & 5730 \\
\hline & & & & $04 / 27 / 88$ & 3430 & $04 / 19 / 88$ & 6190 & $04 / 19 / 88$ & 5670 \\
\hline \multirow[t]{2}{*}{ FSODIUM } & PPB & 200 & & $03 / 16 / 88$ & 2730 & $01 / 27 / 88$ & 19600 & $01 / 27 / 88$ & 20900 \\
\hline & & & & $04 / 27 / 88$ & 2290 & $04 / 19 / 88$ & 19000 & $04 / 19 / 88$ & 18900 \\
\hline \multirow[t]{2}{*}{ FSTRONT } & PPB & 10 & & $03 / 16 / 88$ & 177 & $01 / 27 / 88$ & 218 & $01 / 27 / 88$ & 220 \\
\hline & & & & $04 / 27 / 88$ & 119 & $04 / 19 / 88$ & 223 & $04 / 19 / 88$ & 203 \\
\hline \multirow[t]{2}{*}{ FVANADI } & $\mathrm{PPB}$ & 5 & & $03 / 16 / 88$ & 16 & $01 / 27 / \varepsilon 8$ & 17 & $01 / 27 / 88$ & 9 \\
\hline & & & & $04 / 27 / 88$ & 14 & $04 / 19 / 88$ & 15 & $04 / 19 / 8 B$ & 14 \\
\hline
\end{tabular}


TABLE E.3. (contd)

\begin{tabular}{|c|c|c|c|c|c|c|c|c|c|c|}
\hline \multicolumn{2}{|c|}{ CONSTITUENT } & $\begin{array}{l}\text { CONTRACTUAL } \\
\text { DETECTION }\end{array}$ & DRINKING & SAMPLE & \multicolumn{3}{|r|}{ SAMPLE } & \multicolumn{2}{|r|}{ SAMPLE } & \multirow[b]{2}{*}{$2-E 13-14$} \\
\hline WAME & UNITS & LIMIT & STANDARD & DATE & $1-N-$ & & DATE & 2-E13-5 & DATE & \\
\hline ….... & $\cdots$ & . . . n & 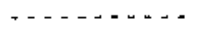 & $\ldots . .$. & $\cdots$ & & 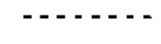 & - n...... & . & . \\
\hline \multirow[t]{2}{*}{ FZINC } & PPB & 5 & & $03 / 16 / 88$ & & & $01 / 27 / 88$ & 12 & $01 / 27 / 88$ & \\
\hline & & & & $04 / 27 / 88$ & & & $04 / 19 / 88$ & 12 & $04 / 19 / 88$ & \\
\hline \multirow[t]{2}{*}{ HNITRAT } & PPB & 2500 & 45000 & $01 / 22 / 88$ & 12000 & & $01 / 27 / 88$ & 10800 & $01 / 29 / 88$ & 12900 \\
\hline & & & & $04 / 27 / 8 B$ & 11300 & & & $\mathrm{NR}$ & & NR \\
\hline \multirow[t]{2}{*}{ LFLUORD } & PP3 & 50 & 4000 & $03 / 16 / 8 B$ & 85 & & $01 / 27 / 88$ & 406 & $01 / 27 / 88$ & 377 \\
\hline & & & & $04 / 27 / 88$ & 83 & & $04 / 19 / 88$ & 423 & $04 / 19 / 88$ & 371 \\
\hline \multirow[t]{4}{*}{ METHONE } & $P P B$ & 10 & & $03 / 96 / 88$ & & & $01 / 27 / 88$ & 16 & $01 / 27 / 88$ & \\
\hline & & & & $03 / 16 / 8 B$ & & & $04 / 19 / 88$ & & $04 / 19 / 88$ & \\
\hline & & & & $03 / 16 / 88$ & & & & NR & & NR \\
\hline & & & & $04 / 27 / 88$ & & & & NR & & NR \\
\hline \multirow[t]{2}{*}{ NITRATE } & PPB & 500 & 45000 & $03 / 16 / 88$ & 27200 & & $01 / 27 / 38$ & 12200 & $01 / 27 / 88$ & 13800 \\
\hline & & & & $04 / 27 / 88$ & 12000 & & $04 / 19 / 88$ & 11800 & $04 / 19 / 88$ & 14200 \\
\hline \multirow[t]{2}{*}{ PH-LAB } & & 0.01 & & $03 / 16 / 88$ & 8. & & $01 / 27 / 88$ & 7.82 & $01 / 27 / 88$ & 7.99 \\
\hline & & & & $04 / 27 / 88$ & 8. & & $04 / 19 / 88$ & 8.10 & $04 / 19 / 88$ & 8.20 \\
\hline \multirow[t]{2}{*}{ PHFIELD } & & 0.10 & & $03 / 16 / 88$ & 8. & & $01 / 27 / 88$ & 7.80 & $01 / 27 / 88$ & 8 \\
\hline & & & & $04 / 27 / 88$ & 8. & & $04 / 19 / 88$ & 7.50 & $04 / 19 / 88$ & 7.70 \\
\hline \multirow[t]{2}{*}{ SB 125} & $\mathrm{PCI} / \mathrm{L}$ & & & $01 / 22 / 88$ & 143 & a & & NR & & $N R$ \\
\hline & & & & & \& 30. & & & & & \\
\hline \multirow[t]{4}{*}{ SR 90} & $\mathrm{PCI} / \mathrm{L}$ & 5 & 8 & $01 / 22 / 88$ & 49 & * & $01 / 27 / 88$ & 3.90 & $01 / 29 / 88$ & 0.96 \\
\hline & & & & & 3. & & & 1.263 & & 0.853 \\
\hline & & & & $04 / 27 / 88$ & 103 & * & & NR & & NR \\
\hline & & & & & 5. & & & & & \\
\hline \multirow[t]{2}{*}{ SULFATE } & PPB & 500 & & $03 / 16 / 88$ & 13300 & & $01 / 27 / 88$ & 48400 & $01 / 27 / 88$ & 77000 \\
\hline & & & & $04 / 27 / 88$ & 10200 & & $04 / 19 / 88$ & 42500 & $04 / 19 / 88$ & 39700 \\
\hline \multirow[t]{2}{*}{ TC } & PPB & 2000 & & $03 / 16 / 88$ & 19700 & & $01 / 27 / 88$ & 40000 & $01 / 27 / 88$ & 28900 \\
\hline & & & & $04 / 27 / 88$ & 15700 & & $04 / 19 / 88$ & 37400 & $04 / 19 / 88$ & 31600 \\
\hline \multirow[t]{2}{*}{$T C-99$} & $\mathrm{PCI} / \mathrm{L}$ & 15 & 900 & $01 / 22 / 88$ & 17. & & $01 / 27 / 88$ & 67.70 & $01 / 29 / 88$ & \\
\hline & & & & & 1 & & & 1.843 & & \\
\hline \multirow[t]{4}{*}{ TRITIUM } & $\mathrm{PCI} / \mathrm{L}$ & 500 & 20000 & $01 / 22 / 88$ & 72700 & * & $01 / 27 / 88$ & & $01 / 29 / 88$ & \\
\hline & & & & & c 882 & 3 & & & & \\
\hline & & & & $04 / 27 / 88$ & 48200 & * & $04 / 19 / 88$ & & & NR \\
\hline & & & & & c 834 & 3 & & & & \\
\hline \multirow[t]{2}{*}{$u$} & $\mathrm{PCI} / \mathrm{L}$ & 0.50 & 600 & $01 / 22 / 88$ & 0. & & $01 / 27 / 88$ & 1.61 & $01 / 29 / 88$ & 1.78 \\
\hline & & & & $04 / 27 / 88$ & 3. & & & $\mathrm{NR}$ & & $\mathrm{NR}$ \\
\hline
\end{tabular}




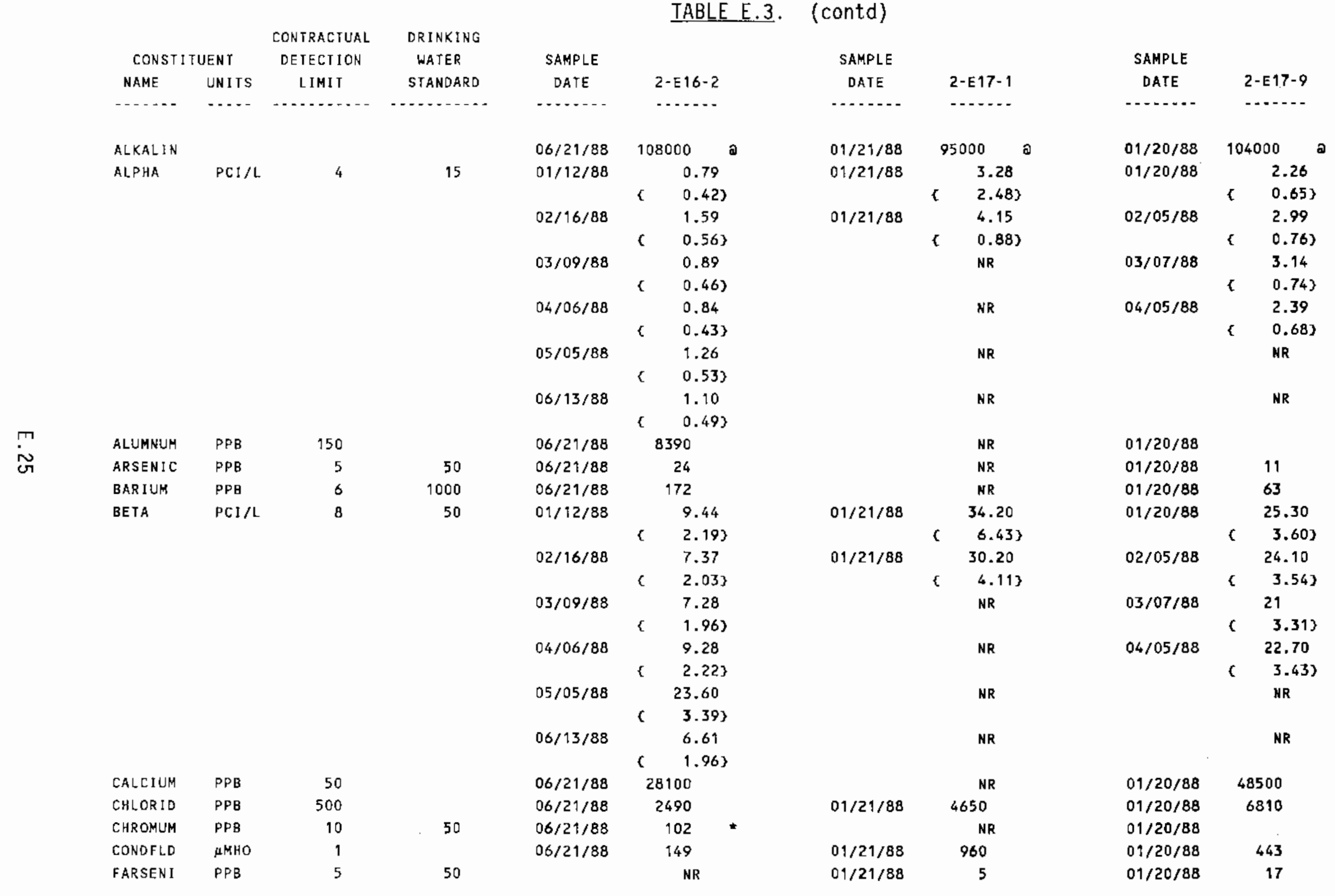


IABLE E. 3. (contd)

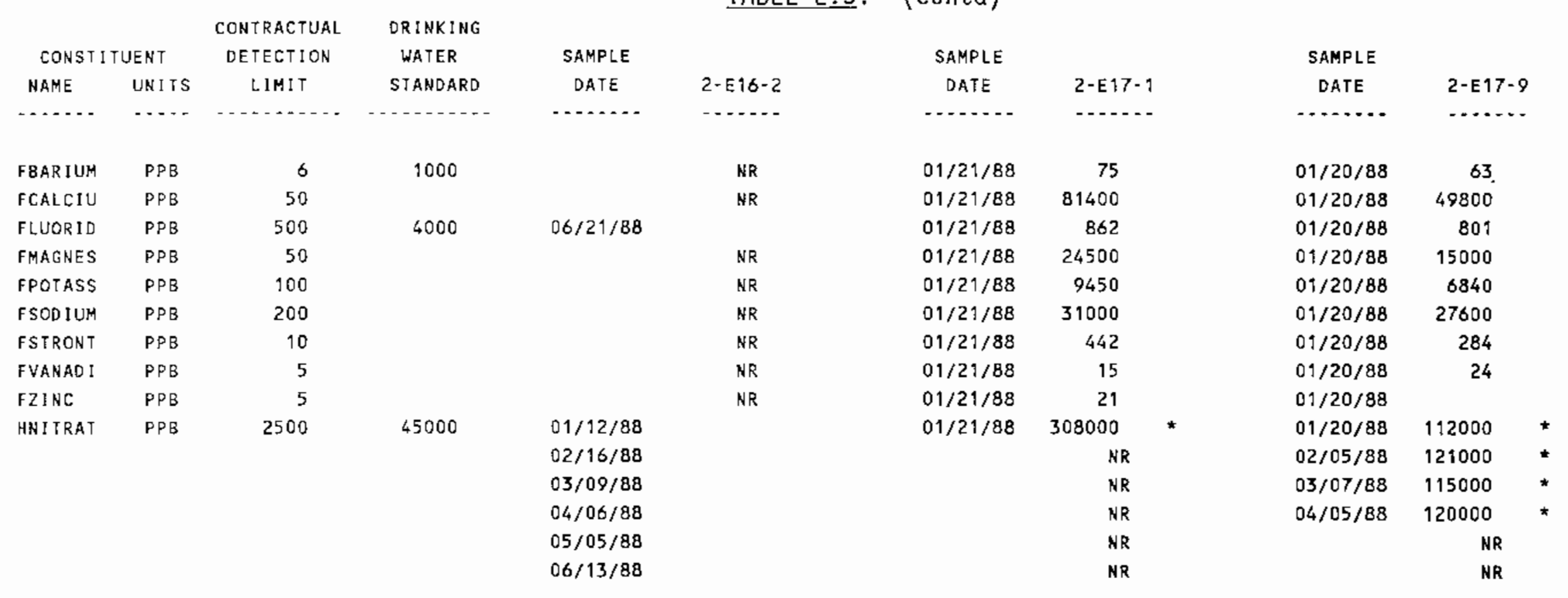


TABLE E.3. (contd)

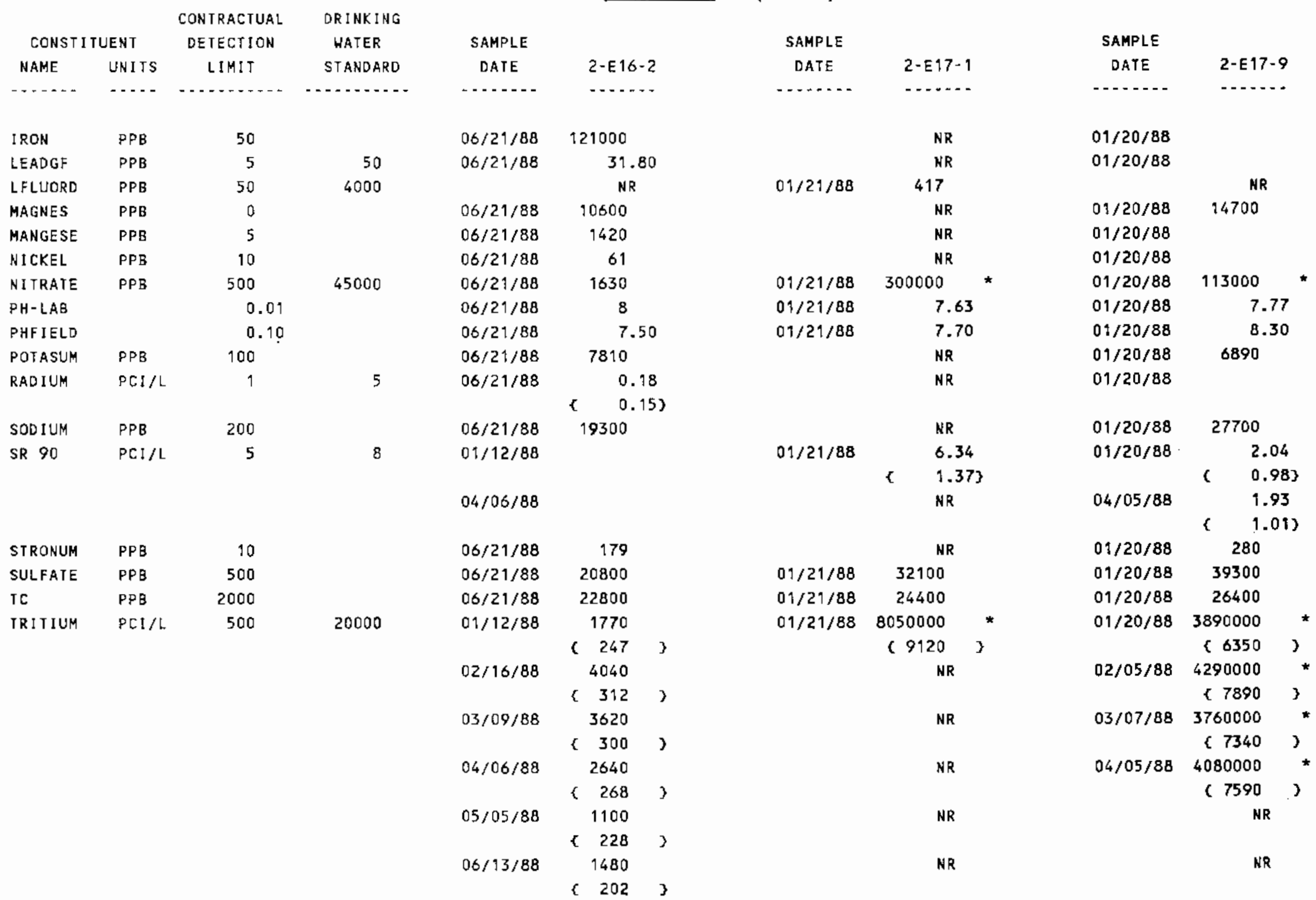


TABLE E.3. (contd)

\begin{tabular}{|c|c|c|c|c|c|c|c|c|c|}
\hline NAME & UNITS & LIMIT & STANDARD & DATE & $2-E 16-2$ & DATE & 2-E17-1 & DATE & $2 \cdot 817-9$ \\
\hline n & $\cdots$ & , n n & 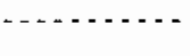 & - & $\cdots$ & - & 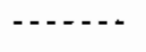 & $\ldots \ldots$ & ........ \\
\hline u & $\mathrm{PCI} / \mathrm{L}$ & 0.50 & 600 & & NR & $01 / 21 / 88$ & 2.25 & & NR \\
\hline \multirow[t]{4}{*}{$U$-CHEM } & UG/L & 0.73 & & & NR & & NR & $01 / 20 / 88$ & 3.78 \\
\hline & & & & & NR & & NR & $02 / 05 / 88$ & 3.74 \\
\hline & & & & & NR & & NR & $03 / 07 / 88$ & 3.36 \\
\hline & & & & & NR & & NR & $04 / 05 / 88$ & 3.95 \\
\hline VANADUM & PPB & 5 & & $06 / 21 / 88$ & 261 & & $\mathrm{NR}$ & $01 / 20 / 88$ & 23 \\
\hline $21 N C$ & PPB & 5 & & $06 / 21 / 88$ & 76 & & NR & $01 / 20 / 88$ & \\
\hline
\end{tabular}




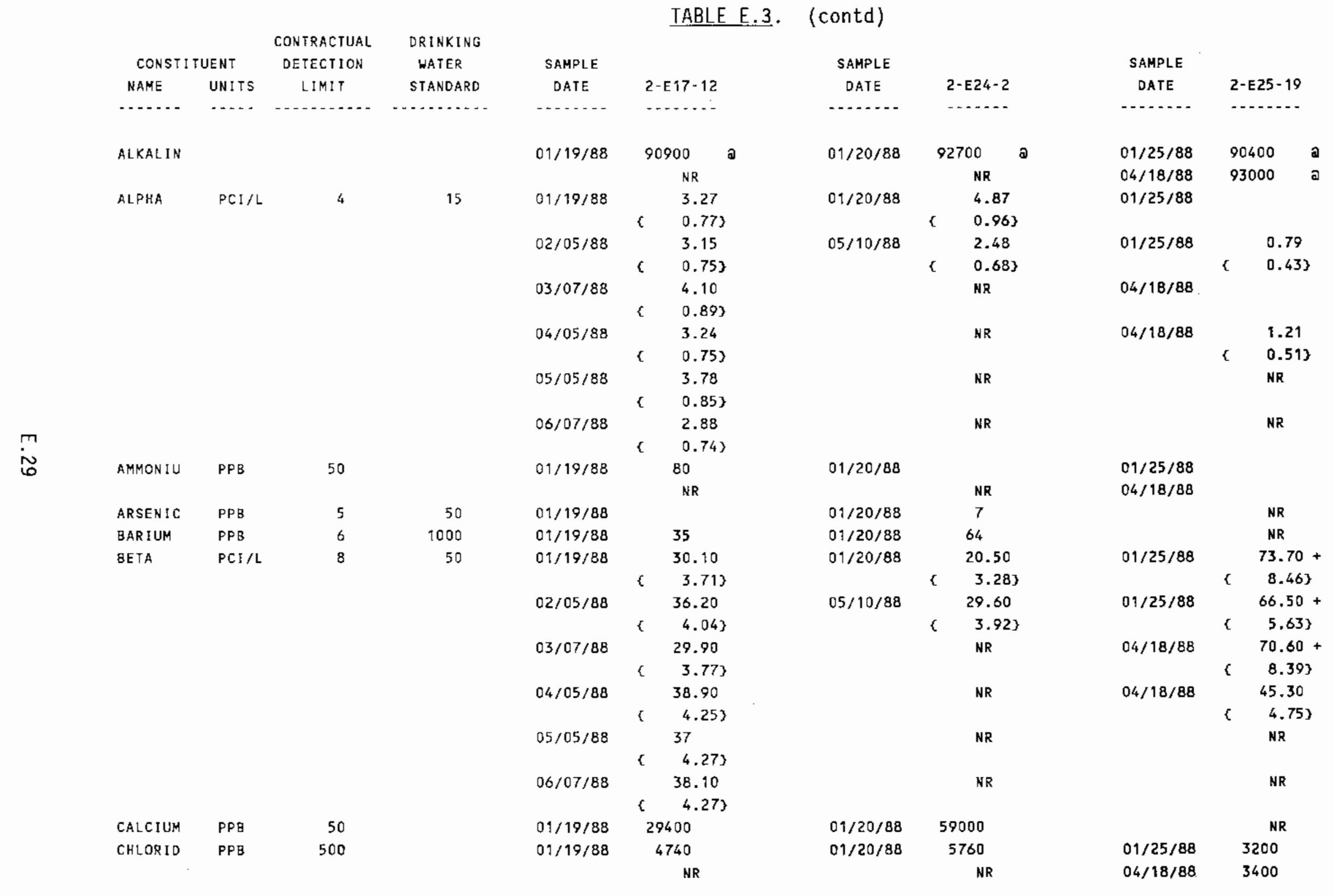


TABLE E.3. (contd)

\begin{tabular}{|c|c|c|c|c|c|c|c|c|c|}
\hline \multirow{2}{*}{\multicolumn{2}{|c|}{ CONST I TUENT }} & CONTRACTUAL & DRINKING & & & \multirow{2}{*}{\multicolumn{2}{|c|}{ SAMPLE }} & \multirow[b]{2}{*}{ SAMPLE } & \multirow[b]{3}{*}{ 2-E25-19 } \\
\hline & & DETECTION & HATER & SAMPLE & \multirow[b]{2}{*}{$2-E 17-12$} & & & & \\
\hline NAME & UN ITS & LIMIT & STANDARO & DATE & & DATE & $2-E 24-2$ & DATE & \\
\hline - n..... & .... & ……... & 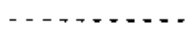 & $\ldots$ & $\ldots \ldots$ & .......... & ........ & - & - - - - \\
\hline \multirow[t]{8}{*}{$c 0-60$} & $P C I / L$ & 22.50 & 100 & $01 / 19 / 88$ & & $01 / 20 / 88$ & & $01 / 25 / 88$ & 5.65 \\
\hline & & & & & & & & & $5.05 \mathrm{~J}$ \\
\hline & & & & $02 / 05 / 88$ & & $05 / 10 / 88$ & & $04 / 18 / 88$ & \\
\hline & & & & $03 / 07 / 88$ & & & NR & & NR \\
\hline & & & & $04 / 05 / 88$ & 5.35 & & NR & & NR \\
\hline & & & & & 4.783 & & & & \\
\hline & & & & $05 / 05 / 88$ & & & NR & & NR \\
\hline & & & & $06 / 07 / 88$ & & & NR & & NR \\
\hline \multirow[t]{2}{*}{ CONDFLD } & НMHO & 1 & & $01 / 19 / 88$ & 297 & $01 / 20 / 88$ & 497 & $01 / 25 / 88$ & 460 \\
\hline & & & & & NR & & NR & $04 / 18 / 88$ & 559 \\
\hline COPPER & PPB & 10 & $(1300.0)$ & $01 / 19 / 88$ & 20 & $01 / 20 / 83$ & & & NR \\
\hline \multirow[t]{4}{*}{ CS 137} & $\mathrm{PCI} / \mathrm{L}$ & 20 & 200 & $01 / 19 / 88$ & 9.13 & $01 / 20 / 88$ & & $01 / 25 / 88$ & \\
\hline & & & & & 6.233 & & & & \\
\hline & & & & $02 / 05 / 88$ & & $05 / 10 / 88$ & & $04 / 18 / 88$ & \\
\hline & & & & $03 / 07 / 88$ & & & NR & & NR \\
\hline
\end{tabular}


IABLE E.3. (contd)

\begin{tabular}{|c|c|c|c|c|c|c|c|c|c|}
\hline \multicolumn{2}{|c|}{ CONSTITUENT } & $\begin{array}{l}\text { CONTRACTUAL } \\
\text { DETECIION }\end{array}$ & $\begin{array}{l}\text { DRINKING } \\
\text { WATER }\end{array}$ & SAMPLE & \multirow{2}{*}{$2-E 17-12$} & \multirow{2}{*}{$\begin{array}{l}\text { SAMPLE } \\
\text { DATE }\end{array}$} & \multirow[b]{2}{*}{$2-E 24-2$} & SAMPLE & \multirow[b]{2}{*}{$2-E 25-19$} \\
\hline NAME & UNITS & L. IMIT & STANDARD & DATE & & & & DATE & \\
\hline$\cdots$ & $\cdots$ & - & - & $\cdots$ & $\ldots$ & $\ldots$ & $\cdots$ & $\ldots \ldots$ & $-\ldots$ \\
\hline \multirow[t]{3}{*}{ CS- 137} & $\mathrm{PCI} / \mathrm{L}$ & 20 & 200 & $04 / 05 / 88$ & & & NR & & NR \\
\hline & & & & $05 / 05 / 88$ & & & NR & & NR \\
\hline & & & & $06 / 07 / 88$ & & & NR & & NR \\
\hline \multirow[t]{2}{*}{ FARSEN I } & $\mathrm{PPB}$ & 5 & 50 & $01 / 19 / 88$ & & $01 / 20 / 88$ & 12 & $01 / 25 / 88$ & 7 \\
\hline & & & & & NR & & NR & $04 / 18 / 88$ & 8 \\
\hline \multirow[t]{2}{*}{ FBARIUM } & $P P B$ & 6 & 1000 & $01 / 19 / 88$ & 36 & $01 / 20 / 88$ & 64 & $01 / 25 / 88$ & 66 \\
\hline & & & & & NR & & NR & $04 / 18 / 88$ & 72 \\
\hline \multirow[t]{2}{*}{ FCALCIU } & PPB & 50 & & $01 / 19 / 88$ & 27000 & $01 / 20 / 88$ & 64300 & $01 / 25 / 88$ & 46600 \\
\hline & & & & & NR & & NR & $04 / 18 / 88$ & 53100 \\
\hline \multirow[t]{2}{*}{ FLUORID } & $P P B$ & 500 & 4000 & $01 / 19 / 88$ & 707 & $01 / 20 / 88$ & 702 & $01 / 25 / 88$ & 687 \\
\hline & & & & & NR & & NR & $04 / 18 / 88$ & \\
\hline \multirow[t]{2}{*}{ FMAGNES } & PPB & 50 & & $01 / 19 / 88$ & 8240 & $01 / 20 / 88$ & 18800 & $01 / 25 / 88$ & 12400 \\
\hline & & & & & NR & & NR & $04 / 18 / 88$ & 13400 \\
\hline \multirow[t]{2}{*}{ FMERCUR } & PPB & 0.10 & 2 & $01 / 19 / 88$ & & $01 / 20 / 88$ & 0.11 & $01 / 25 / 88$ & \\
\hline & & & & & NR & & NR & $04 / 18 / 88$ & \\
\hline \multirow[t]{2}{*}{ FPOTASS } & PPB & 100 & & $01 / 19 / 88$ & 5470 & $01 / 20 / 88$ & 7010 & $01 / 25 / 88$ & 9700 \\
\hline & & & & & NR & & NR & $04 / 18 / 88$ & 9240 \\
\hline \multirow[t]{2}{*}{ F SOD IUM } & PPB & 200 & & $01 / 19 / 88$ & 22700 & $01 / 20 / 88$ & 21000 & $01 / 25 / 88$ & 35600 \\
\hline & & & & & NR & & NR & $04 / 18 / 88$ & 34800 \\
\hline \multirow[t]{2}{*}{ FSTRONT } & $P P B$ & 10 & & $01 / 19 / 88$ & 131 & $01 / 20 / 88$ & 312 & $01 / 25 / 88$ & 248 \\
\hline & & & & & $\mathrm{NR}$ & & NR & $04 / 18 / 88$ & 278 \\
\hline \multirow[t]{2}{*}{ FУANADI } & PPB & 5 & & $01 / 19 / 88$ & 20 & $01 / 20 / 88$ & 23 & $01 / 25 / 88$ & 41 \\
\hline & & & & & $N R$ & & NR & $04 / 18 / 88$ & 42 \\
\hline \multirow[t]{2}{*}{ FZINC } & PPB & 5 & & $01 / 19 / 88$ & 9 & $01 / 20 / 88$ & 36 & $01 / 25 / 88$ & 28 \\
\hline & & & & & NR & & NR & $04 / 18 / 88$ & 28 \\
\hline \multirow[t]{6}{*}{ HN I TRAT } & PPB & 2500 & 45000 & $01 / 19 / 88$ & 40600 & $01 / 20 / 88$ & 176000 & $01 / 25 / 88$ & 161000 \\
\hline & & & & $02 / 05 / 88$ & 43900 & $05 / 10 / 88$ & 139000 & $04 / 18 / 88$ & 168000 \\
\hline & & & & $03 / 07 / 88$ & 52700 & $\star$ & NR & & NR \\
\hline & & & & $04 / 05 / 88$ & 56600 & * & NR & & HR \\
\hline & & & & $05 / 05 / 88$ & 54700 & $\star$ & NR & & NR \\
\hline & & & & $06 / 07 / 88$ & 55600 & * & $N R$ & & NR \\
\hline I RON & PPB & 50 & & $01 / 19 / 88$ & 3770 & $01 / 20 / 88$ & 151 & & NR \\
\hline LEADGF & PPB & 5 & 50 & $01 / 19 / 88$ & 6 & $01 / 20 / 88$ & & & NR \\
\hline
\end{tabular}


IABLE E.3. (contd)

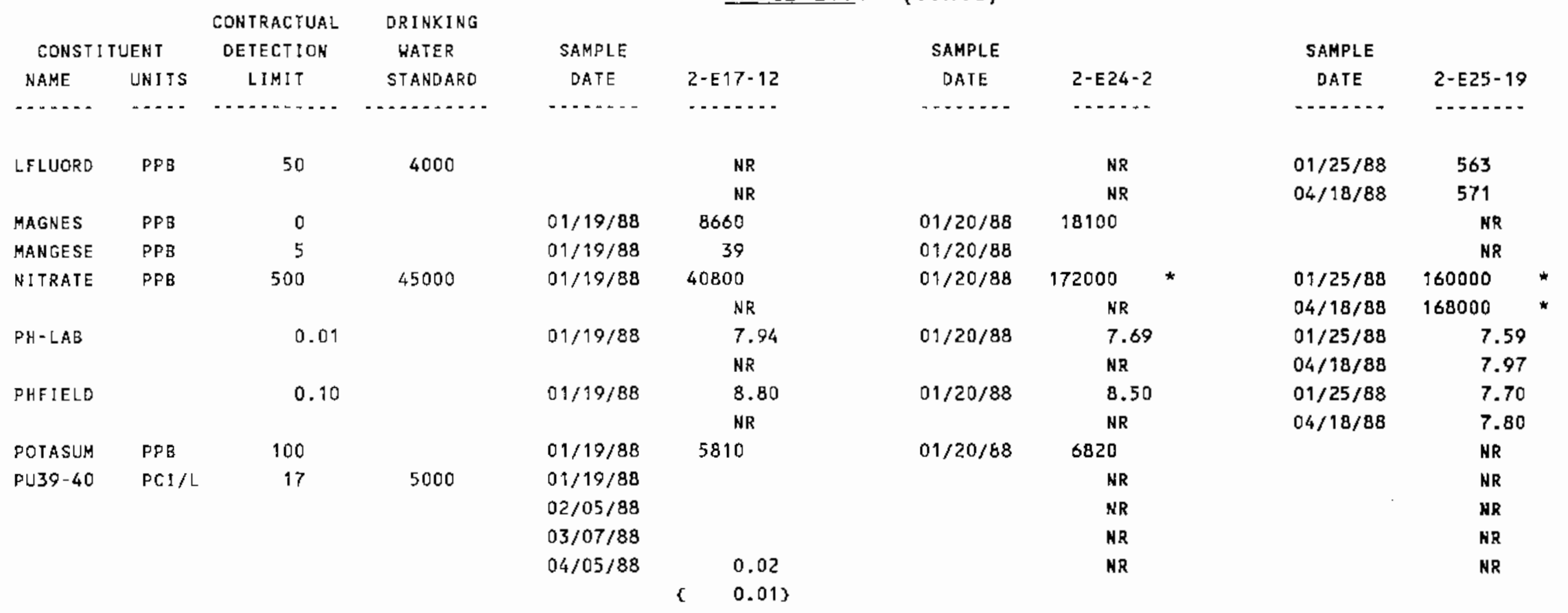


TABLE E.3. (contd)

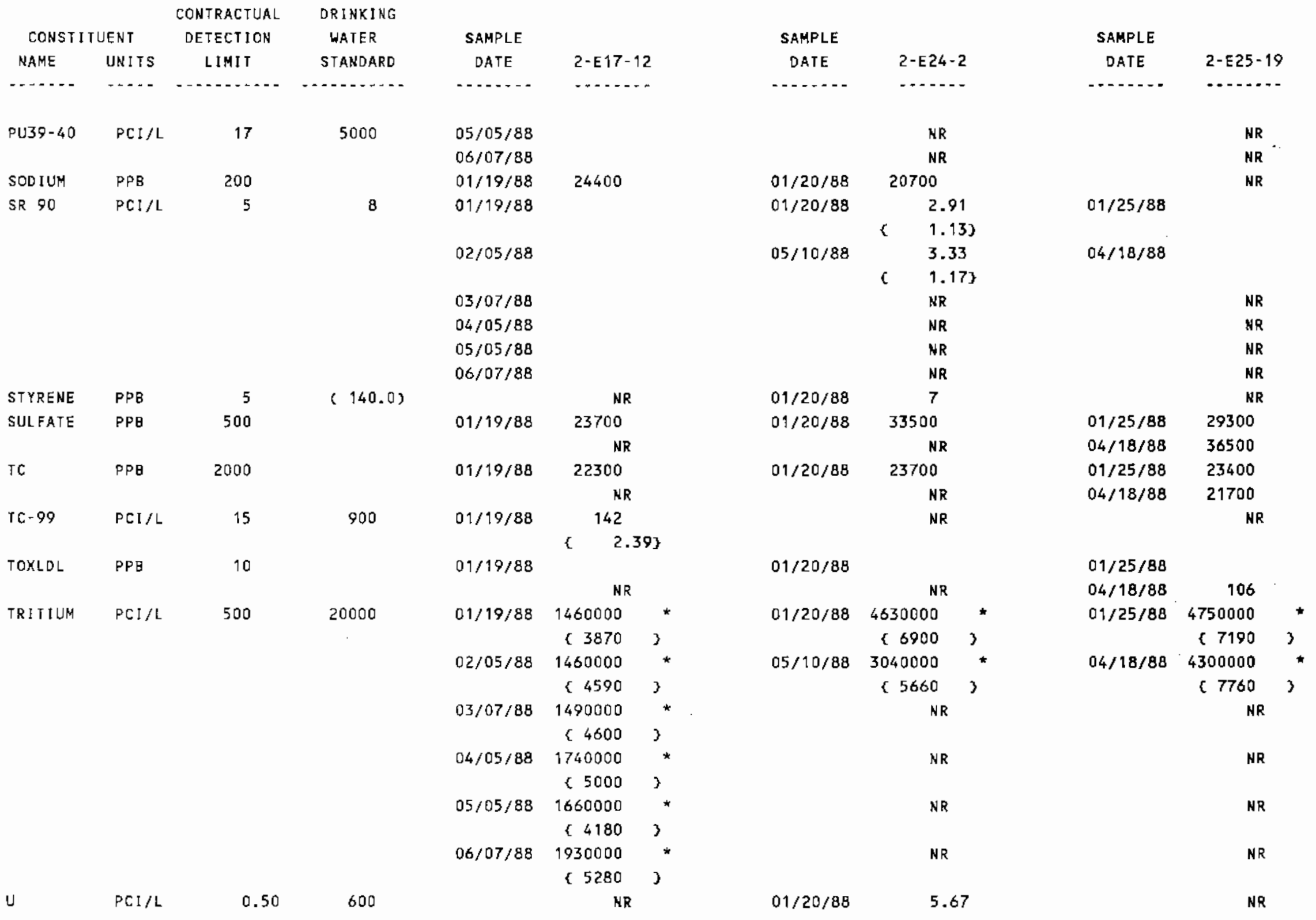


TABLE E.3. (contd)

\begin{tabular}{|c|c|c|c|c|c|c|c|c|c|}
\hline \multirow{3}{*}{\multicolumn{2}{|c|}{ CONSTI I TUENT }} & \multirow{3}{*}{$\begin{array}{l}\text { CONTRACTUAL } \\
\text { DETECTION }\end{array}$} & \multirow{2}{*}{ DRINKING } & & \multirow{3}{*}{ SAMPLE } & \multirow{4}{*}{$2 \cdot E 25-19$} \\
\hline & & & & & \multirow[b]{3}{*}{$2-\mathrm{E} 17-12$} & \multirow{2}{*}{\multicolumn{2}{|c|}{ SAMPLE }} & & \\
\hline & & & WATER & SAMPLE & & & & & \\
\hline NAME & UNITS & LIMIT & STANDARD & DATE & & DATE & $2-E 24-2$ & DATE & \\
\hline , n....... & $\cdots$ & - & - nen & $\ldots \ldots$ & 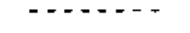 & $\cdots+\ldots$ & $\cdots++\cdots$ & 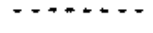 & $\ldots$ \\
\hline \multirow[t]{12}{*}{$U-234$} & PCI/L & 0.10 & 32 & $01 / 19 / 88$ & 1.58 & & NR & & NR \\
\hline & & & & & 0.113 & & & & \\
\hline & & & & $02 / 05 / 88$ & 1.66 & & NR & & NR \\
\hline & & & & & $0.12\}$ & & & & \\
\hline & & & & $03 / 07 / 88$ & 1.63 & & NR & & NR \\
\hline & & & & & 0.123 & & & & \\
\hline & & & & $04 / 05 / 88$ & 1.83 & & NR & & NR \\
\hline & & & & & 0.133 & & & & \\
\hline & & & & $05 / 05 / 88$ & 1.90 & & NR & & NR \\
\hline & & & & & 0.133 & & & & \\
\hline & & & & $06 / 07 / 88$ & 1.92 & & NR & & NR \\
\hline & & & & & 0.163 & & & & \\
\hline \multirow[t]{4}{*}{ U-235 } & $P[I / L$ & 0.10 & 32 & $01 / 19 / 88$ & 0.06 & & NR & & NR \\
\hline & & & & & 0.023 & & & & \\
\hline & & & & $02 / 05 / 88$ & 0.08 & & NR & & NR \\
\hline & & & & & $0.03\}$ & & & & \\
\hline
\end{tabular}




\begin{tabular}{|c|c|c|c|c|c|c|c|c|c|}
\hline \multicolumn{2}{|c|}{ CONST I TUENT } & $\begin{array}{l}\text { CONTRACTUAL } \\
\text { DETECTIION }\end{array}$ & $\begin{array}{l}\text { DRINKING } \\
\text { WATER }\end{array}$ & SAMPLE & & \multicolumn{2}{|l|}{ SAMPLE } & \multirow{2}{*}{$\begin{array}{l}\text { SAMPLE } \\
\text { DAJE }\end{array}$} & \\
\hline NAME & UNITS & LIMIT & STANDARD & DATE & $2-\mathrm{E} 17-12$ & DATE & $2-E 24-2$ & & $2-E 25-19$ \\
\hline - ....... & $\ldots$ & n.t.n. & - & $\ldots$ & - . L L & - - & $\cdots$ & $\cdots$ & 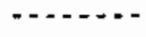 \\
\hline \multirow[t]{8}{*}{$u-235$} & $\mathrm{PCl} / \mathrm{L}$ & 0.10 & 32 & $03 / 07 / 88$ & 0.06 & & NR & & NR \\
\hline & & & & & 0.023 & & & & \\
\hline & & & & $04 / 05 / 88$ & 0.07 & & NR & & NR \\
\hline & & & & & 0.023 & & & & \\
\hline & & & & $05 / 05 / 88$ & 0.07 & & NR & & NR \\
\hline & & & & & 0.033 & & & & \\
\hline & & & & $06 / 07 / 88$ & 0.10 & & NR & & NR \\
\hline & & & & & 0.043 & & & & \\
\hline \multirow[t]{12}{*}{$u-238$} & $P C I / L$ & 0.10 & 4.80 & $01 / 19 / 88$ & 1.33 & & NR & & NR \\
\hline & & & & & 0.103 & & & & \\
\hline & & & & $02 / 05 / 88$ & 1.60 & & NR & & NR \\
\hline & & & & & 0.123 & & & & \\
\hline & & & & $03 / 07 / 88$ & 1.58 & & NR & & MR \\
\hline & & & & & 0.123 & & & & \\
\hline & & & & $04 / 05 / 88$ & 1.68 & & NR & & NR \\
\hline & & & & & 0.123 & & & & \\
\hline & & & & $05 / 05 / 88$ & 1.67 & & NR & & NR \\
\hline & & & & & 0.123 & & & & \\
\hline & & & & $06 / 07 / 88$ & 1.76 & & NR & & NR \\
\hline & & & & & 0.153 & & & & \\
\hline \multirow[t]{6}{*}{ U-CHEM } & $U G / L$ & 0.73 & & $01 / 19 / 88$ & 3.22 & & NR & & $\$ R$ \\
\hline & & & & $02 / 05 / 88$ & 3.40 & & NR & & NR \\
\hline & & & & $03 / 07 / 88$ & 3.90 & & NR & & HR \\
\hline & & & & $04 / 05 / 88$ & 4.18 & & NR & & NR \\
\hline & & & & $05 / 05 / 88$ & 4.38 & & NR & & NR \\
\hline & & & & $06 / 07 / 88$ & 4.52 & & NR & & NR \\
\hline VANADUM & PPB & 5 & & $01 / 19 / 88$ & 29 & $01 / 20 / 88$ & 21 & & NR \\
\hline IINC & PPB & 5 & & $01 / 19 / 88$ & 27 & $01 / 20 / 88$ & 46 & & N8 \\
\hline
\end{tabular}


TABLE E. 3. (contd)

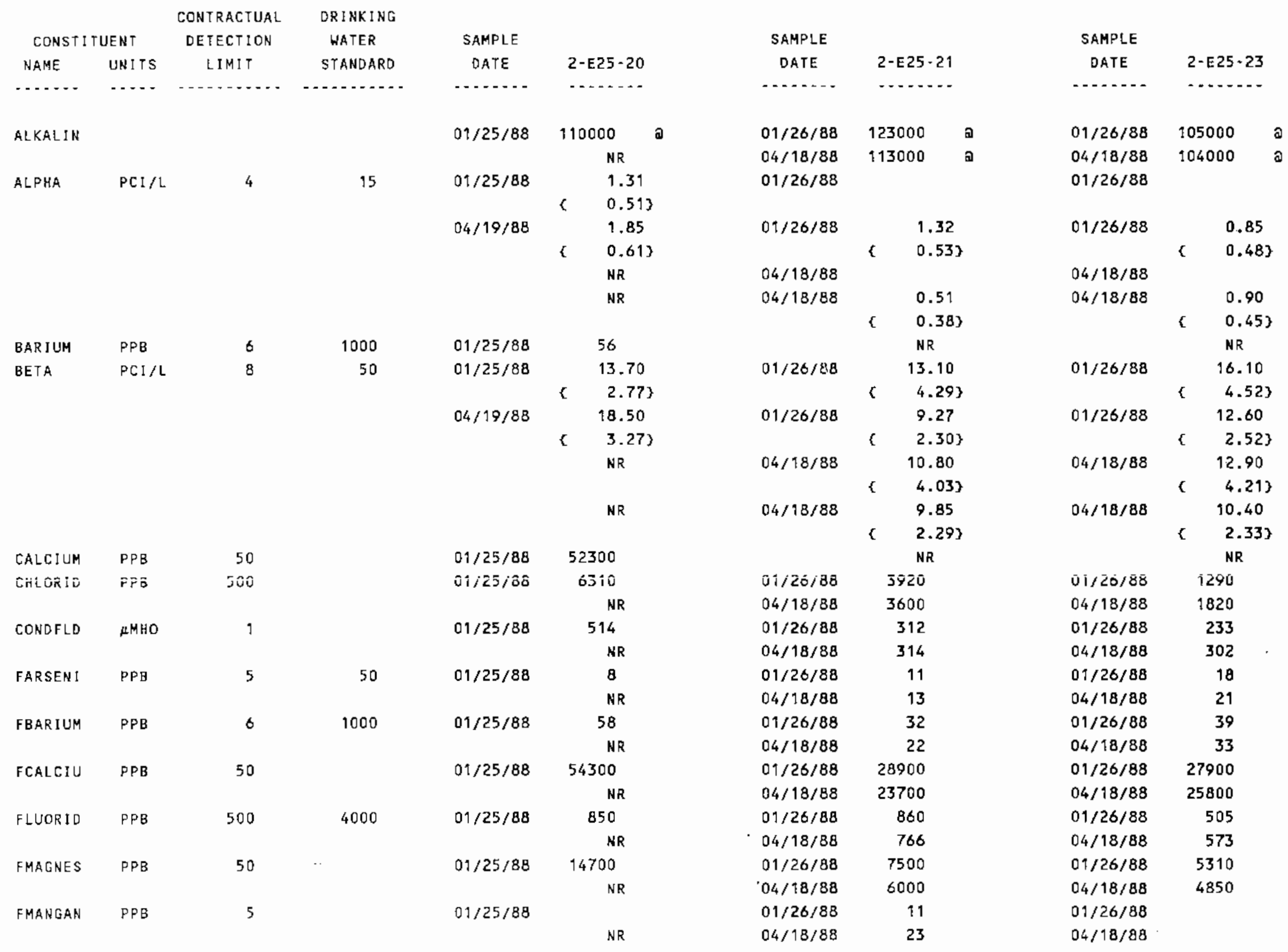


TABLE E. 3. (contd)

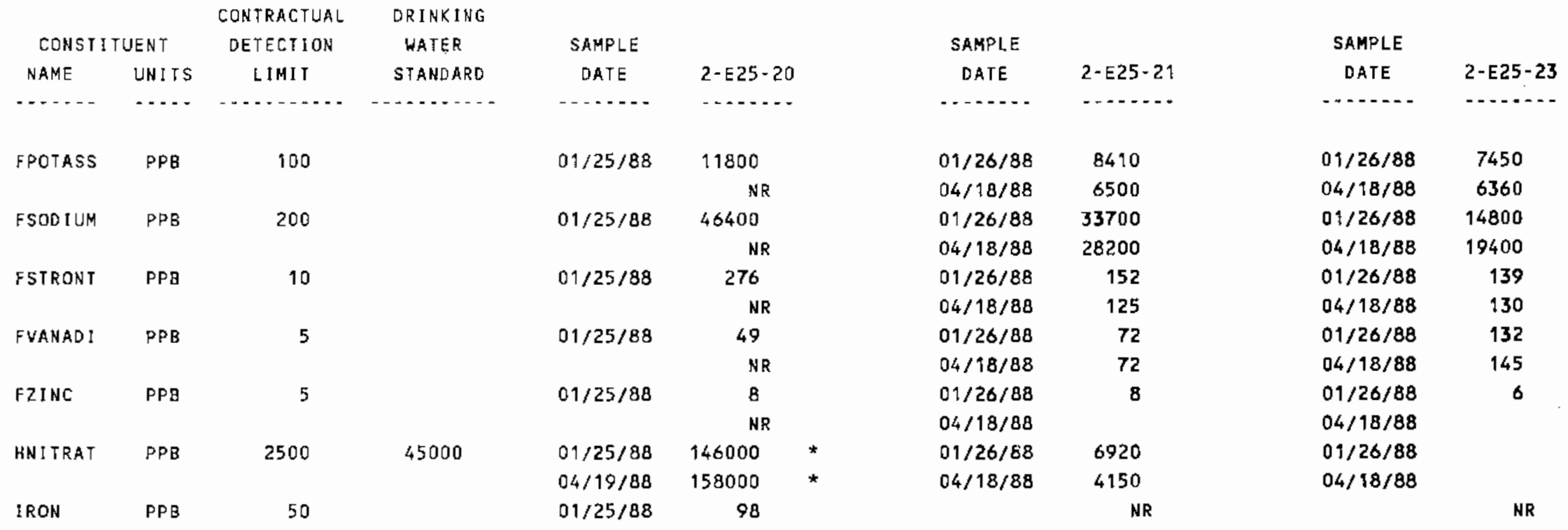


TABLE E.3. (contd)

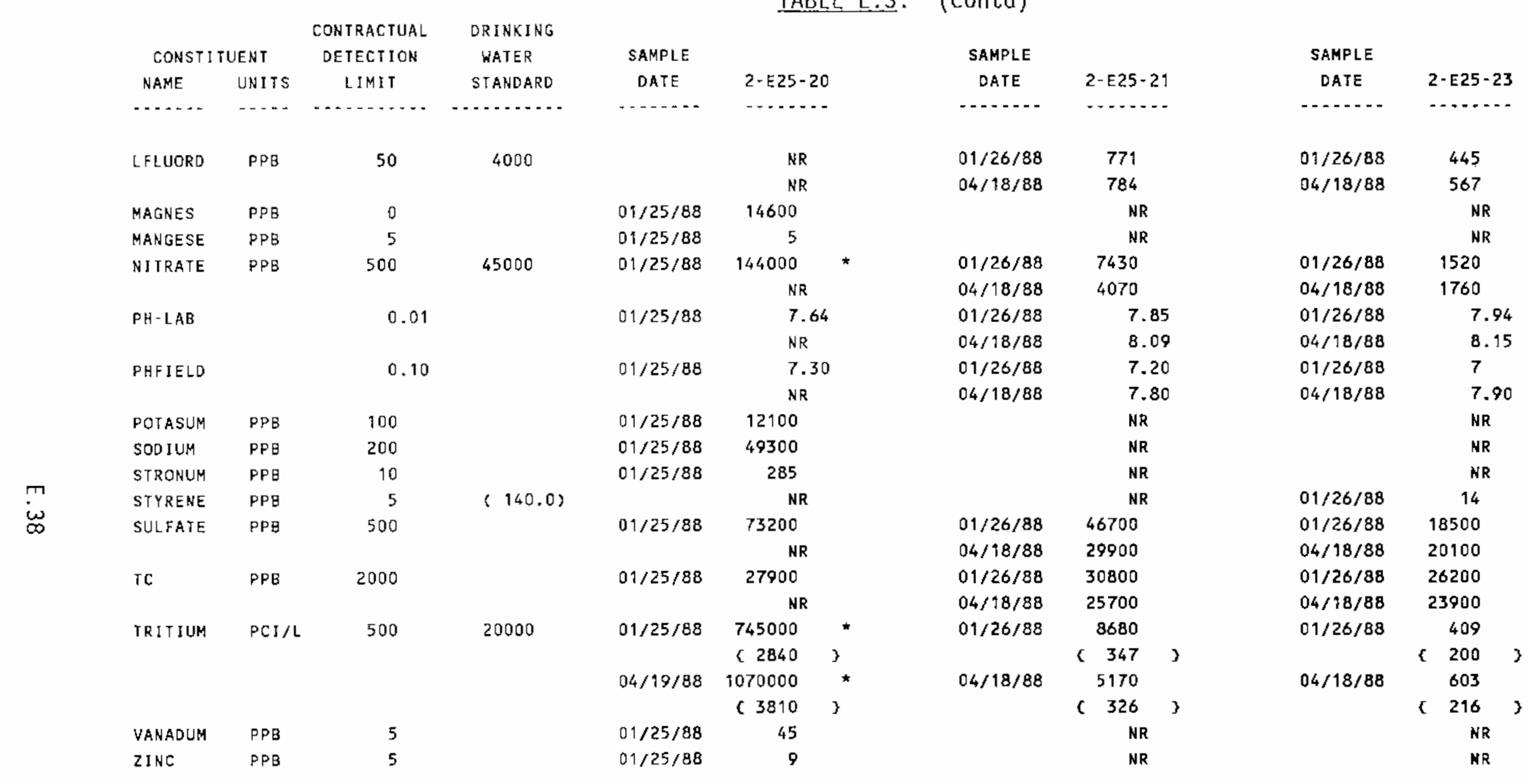


IABLE E.3. (contd)

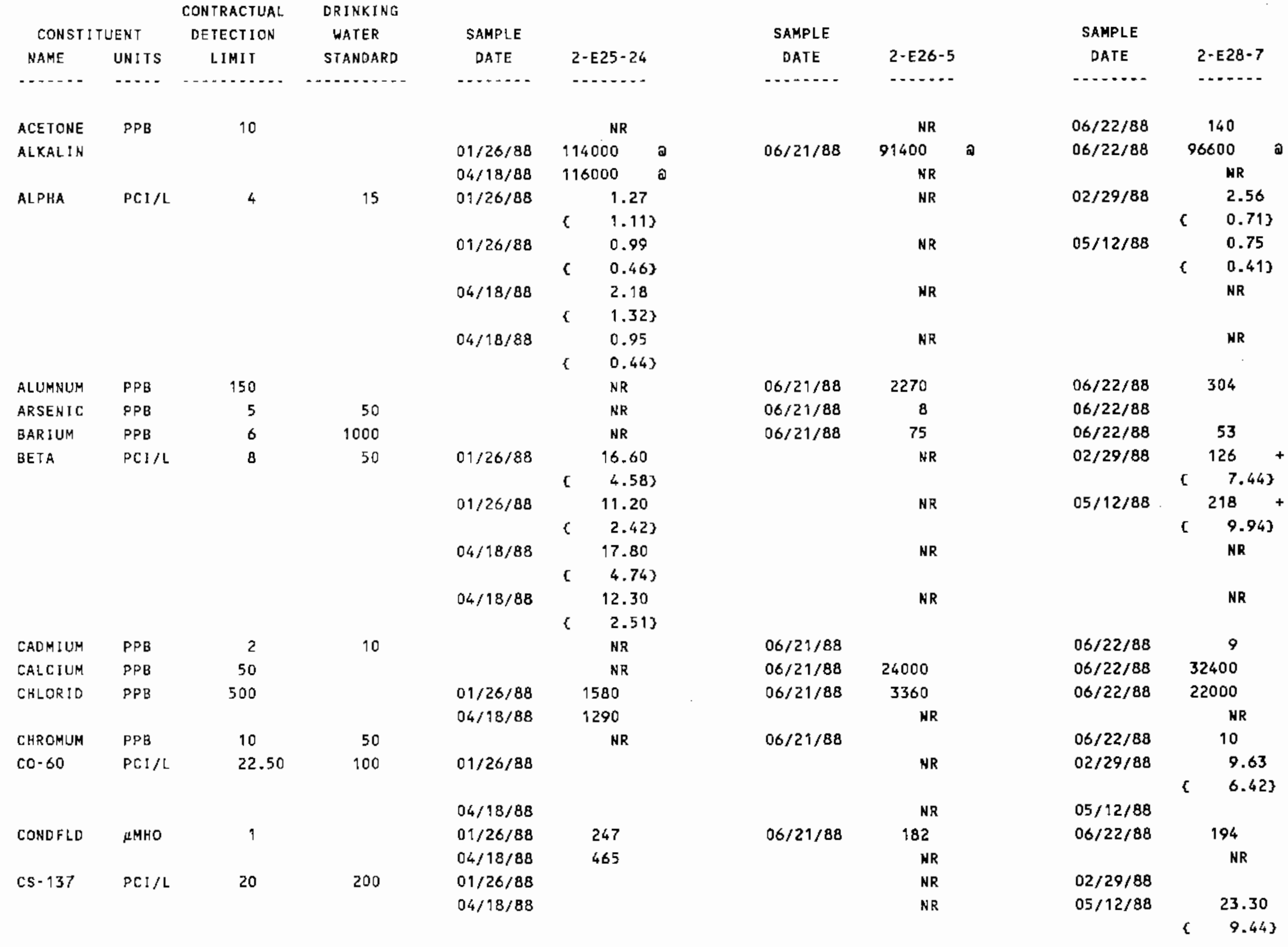


TABLE E.3. (contd)

\begin{tabular}{|c|c|c|c|c|c|c|c|c|c|}
\hline \multicolumn{2}{|c|}{ CONST I TUENT } & $\begin{array}{l}\text { CONTRACTUAL } \\
\text { DETECTION }\end{array}$ & $\begin{array}{l}\text { DR INKING } \\
\text { HATER }\end{array}$ & \multicolumn{2}{|l|}{ SAMPLE } & \multicolumn{2}{|l|}{ SAMPLE } & SAMPLE & \multirow[b]{2}{*}{$2-E 28-7$} \\
\hline NAME & UNITS & LIMIT & STANDARD & DATE & $2-E 25-24$ & DATE & $2-E 26-5$ & DATE & \\
\hline -...... & … & $\cdots$ & 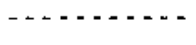 & n....... & …..... & $\cdots$ & ........ & n......... & ….... \\
\hline \multirow[t]{2}{*}{ FARSENI } & PPB & 5 & 50 & $01 / 26 / 88$ & 15 & & NR & & NR \\
\hline & & & & $04 / 18 / 88$ & 15 & & NR & & HR \\
\hline \multirow[t]{2}{*}{ FBARIUM } & PPB & 6 & 1000 & $01 / 26 / 88$ & 32 & & NR & & NR \\
\hline & & & & $04 / 18 / 88$ & 32 & & NR & & MR \\
\hline \multirow[t]{2}{*}{ FCALCIU } & $P P B$ & 50 & & $01 / 26 / 88$ & 25500 & & NR & & NR \\
\hline & & & & $04 / 18 / 88$ & 29600 & & NR & & NR \\
\hline \multirow[t]{2}{*}{ FLUORID } & PPB & 500 & 4000 & $01 / 26 / 88$ & 759 & $06 / 21 / 88$ & & $06 / 22 / 88$ & \\
\hline & & & & $04 / 18 / 88$ & 762 & & NR & & FR \\
\hline \multirow[t]{2}{*}{ FMAGNES } & $P P B$ & 50 & & $01 / 26 / 88$ & 5400 & & NR & & NR \\
\hline & & & & $04 / 18 / 88$ & 6120 & & NR & & NR \\
\hline \multirow[t]{2}{*}{ FPOTASS } & PPB & 100 & & $01 / 26 / 88$ & 8610 & & NR & & NR \\
\hline & & & & $04 / 18 / 88$ & 8110 & & NR & & NR \\
\hline FSODIUM & PPB & 200 & & $01 / 26 / 88$ & 25800 & & NR & & NR \\
\hline
\end{tabular}




\begin{tabular}{|c|c|c|c|c|c|c|c|c|c|}
\hline \multicolumn{2}{|c|}{ CONSTITUENT } & $\begin{array}{l}\text { CONTRACTUAL } \\
\text { DETECTION }\end{array}$ & $\begin{array}{l}\text { DRINKING } \\
\text { WATER }\end{array}$ & SAMPLE & & SAMPLE & & SAMPLE & \\
\hline NAME & UNITS & LIMIT & STANDARD & DATE & $2-E 25-24$ & DATE & 2-E26-5 & DATE & $2-E 28-7$ \\
\hline (n...... & $\cdots-$ & , n & n.t. & ......... & - n. & .......... & ….... & -........ & -...... \\
\hline FSODIUM & PPB & 200 & & $04 / 18 / 88$ & 20600 & & NR & & NR \\
\hline \multirow[t]{2}{*}{ FSTRONT } & PPQ & 10 & & $01 / 26 / 88$ & 141 & & NR & & MR \\
\hline & & & & $04 / 18 / 88$ & 160 & & NR & & NR \\
\hline \multirow[t]{2}{*}{ FVANAD I } & PPB & 5 & & $01 / 26 / 88$ & 126 & & NR & & NR \\
\hline & & & & $04 / 18 / 88$ & 128 & & NR & & NR \\
\hline \multirow[t]{2}{*}{ HNI TRAT } & PPB & 2500 & 45000 & $01 / 26 / 88$ & 3070 & & NR & $02 / 29 / 88$ & 8090 \\
\hline & & & & $04 / 18 / 88$ & & & NR & & SR \\
\hline IRON & $P P B$ & 50 & & & NR & $06 / 21 / 88$ & 67800 & $06 / 22 / 88$ & 15800 \\
\hline LEADGF & PPB & 5 & 50 & & NR & $06 / 21 / 88$ & 17 & $06 / 22 / 88$ & \\
\hline \multirow[t]{2}{*}{ LFLUORD } & PPB & 50 & 4000 & $01 / 26 / 88$ & $7 \uparrow 4$ & & NR & & NR \\
\hline & & & & $04 / 18 / 88$ & 714 & & NR & & NR \\
\hline MAGNES & PPB & 0 & & & NR & $06 / 21 / 88$ & 7630 & $06 / 22 / 88$ & 10300 \\
\hline MANGESE & PPB & 5 & & & NR & $06 / 21 / 88$ & 1090 & $06 / 22 / 88$ & 259 \\
\hline \multirow[t]{2}{*}{ NITRATE } & PPB & 500 & 45000 & $01 / 26 / 88$ & 3050 & $06 / 21 / 88$ & 1490 & $06 / 22 / 88$ & 8290 \\
\hline & & & & $04 / 18 / 88$ & 1640 & & NR & & NR \\
\hline \multirow[t]{2}{*}{$P H-L A B$} & & 0.01 & & $01 / 26 / 88$ & 7.70 & $06 / 21 / 88$ & 7.90 & $06 / 22 / 88$ & 7.90 \\
\hline & & & & $04 / 18 / 88$ & 7.96 & & NR & & HR \\
\hline \multirow[t]{2}{*}{ PHFIELD } & & 0.10 & & $01 / 26 / 88$ & 6.50 & $06 / 21 / 88$ & 7.90 & $06 / 22 / 88$ & 7.40 \\
\hline & & & & $04 / 18 / 88$ & 7.90 & & NR & & NR \\
\hline POTASUM & PPB & 100 & & & NR & $06 / 21 / 88$ & 4870 & $06 / 22 / 88$ & 6370 \\
\hline \multirow[t]{2}{*}{ RADIUM } & $\mathrm{PCI} / \mathrm{L}$ & 1 & 5 & & NR & $06 / 21 / 88$ & 0.26 & $06 / 22 / 88$ & 0.17 \\
\hline & & & & & & & 0.193 & & 0.143 \\
\hline SODIUM & $P P B$ & 200 & & & NR & $06 / 21 / 88$ & 11000 & $06 / 22 / 88$ & 24700 \\
\hline \multirow[t]{3}{*}{ SR 90} & $\mathrm{PCI} / \mathrm{L}$ & 5 & 8 & $01 / 26 / 88$ & & & NR & $02 / 29 / 88$ & $60.90 \star$ \\
\hline & & & & & & & & & 3.973 \\
\hline & & & & $04 / 18 / 88$ & & & NR & $05 / 12 / 88$ & 105 * \\
\hline & & & & & & & & & 5.473 \\
\hline SULFATE & PPB & 10 & & & $N R$ & $06 / 21 / 88$ & 139 & $06 / 22 / 88$ & 166 \\
\hline \multirow[t]{2}{*}{ SULFATE } & PPB & 500 & & $01 / 26 / 88$ & 30700 & $06 / 21 / 88$ & 12900 & $06 / 22 / 88$ & 40500 \\
\hline & & & & $04 / 18 / 88$ & 28200 & & NR & & NR \\
\hline \multirow[t]{2}{*}{ TC } & PPB & 2000 & & $01 / 26 / 88$ & 29500 & $06 / 21 / 88$ & 23400 & $06 / 22 / 88$ & 24200 \\
\hline & & & & $04 / 18 / 88$ & 26800 & & NR & & NR \\
\hline \multirow[t]{2}{*}{$T C .99$} & $\mathrm{PCI} / \mathrm{L}$ & 15 & 900 & & NR & & NR & $02 / 29 / 88$ & 136 \\
\hline & & & & & & & & & f 2.34$\}$ \\
\hline
\end{tabular}


TABLE E. 3. (contd)

\begin{tabular}{|c|c|c|c|c|c|c|c|c|c|c|c|}
\hline \multicolumn{2}{|c|}{ CONSTITUENT } & $\begin{array}{l}\text { CONTRACTUAL } \\
\text { DETECTION }\end{array}$ & $\begin{array}{l}\text { DRINKING } \\
\text { WATER }\end{array}$ & SAMPLE & & & SAMPLE & & SAMPLE & & \\
\hline \multirow{2}{*}{$\begin{array}{l}\text { NAME } \\
\ldots \ldots\end{array}$} & UNITS & LIMIT & STANDARD & DATE & \multicolumn{2}{|c|}{$2-E 25-24$} & DATE & $2-E 26-5$ & DATE & \multicolumn{2}{|r|}{$2-E 2 B-7$} \\
\hline & - . - - & - n. & 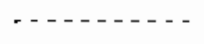 & - & - - - - & & $\cdots$ & - n & - - - - - & & - \\
\hline \multirow[t]{2}{*}{ TOLUENE } & PPB & 10 & $(2000.0)$ & $01 / 26 / 88$ & & & $06 / 21 / 88$ & & $06 / 22 / 88$ & & 20 \\
\hline & & & & $04 / 18 / 88$ & & & & NR & & & NR \\
\hline \multirow[t]{2}{*}{ rOXLDL } & $\mathrm{PPB}$ & 10 & & $01 / 26 / 88$ & & & $06 / 21 / 88$ & & $06 / 22 / 88$ & & 21.50 \\
\hline & & & & $04 / 18 / 88$ & & & & NR & & & NR \\
\hline \multirow[t]{4}{*}{ TRITIUM } & $\mathrm{PCI} / \mathrm{L}$ & 500 & 20000 & $01 / 26 / 88$ & 1040 & & & NR & $02 / 29 / 88$ & & 7280 \\
\hline & & & & & \& 220 & 3 & & & & 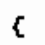 & 377 \\
\hline & & & & $04 / 18 / 88$ & 564 & & & NR & & & NR \\
\hline & & & & & C 214 & 3 & & & & & \\
\hline \multirow[t]{2}{*}{$u-234$} & $P C 1 / L$ & 0.10 & 32 & & NR & & & NR & $02 / 29 / 88$ & & 0.73 \\
\hline & & & & & & & & & & c & 0.083 \\
\hline \multirow[t]{2}{*}{$U-235$} & $\mathrm{PCI} / \mathrm{L}$ & 0.10 & 32 & & $\mathrm{NR}$ & & & NR & $02 / 29 / 88$ & & 0.03 \\
\hline & & & & & & & & & & 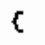 & 0.023 \\
\hline \multirow[t]{2}{*}{$u-238$} & $\mathrm{PCI} / \mathrm{L}$ & 0.10 & 4.80 & & NR & & & NR & $02 / 29 / 88$ & & 0.61 \\
\hline & & & & & & & & & & $\varepsilon$ & 0.073 \\
\hline U-CHEM & $U G / L$ & 0.73 & & & NR & & & NR & $02 / 29 / 88$ & & 1.70 \\
\hline
\end{tabular}




\section{IABLE E.3. (contd)}

\begin{tabular}{lccc}
\multicolumn{2}{c}{ CONSTITUENT } & $\begin{array}{c}\text { CONTRACTUAL } \\
\text { DETECTION }\end{array}$ & $\begin{array}{c}\text { DRINKING } \\
\text { WATER }\end{array}$ \\
NAME & UNITS & LIMIT & STANDARD \\
\hdashline & $\ldots \ldots .-$ & & \\
U-CKEM & UG $/ L$ & 0.73 & \\
VANADUM & PPB & 5 & \\
ZINC & PPB & 5 &
\end{tabular}

SAMPLE

$2-E 25-24$

......

NR

NR

NR

$$
\begin{aligned}
& \text { SAMPLE } \\
& \text { DATE } \\
& \ldots \ldots \ldots-\ldots 26-5
\end{aligned}
$$

$06 / 21 / 88$

$06 / 21 / 88$
NR
128

57
SAMPLE

DATE

- . - . . .

2-E28-7

$05 / 12 / 88$

$06 / 22 / 88$

$06 / 22 / 88$
36 


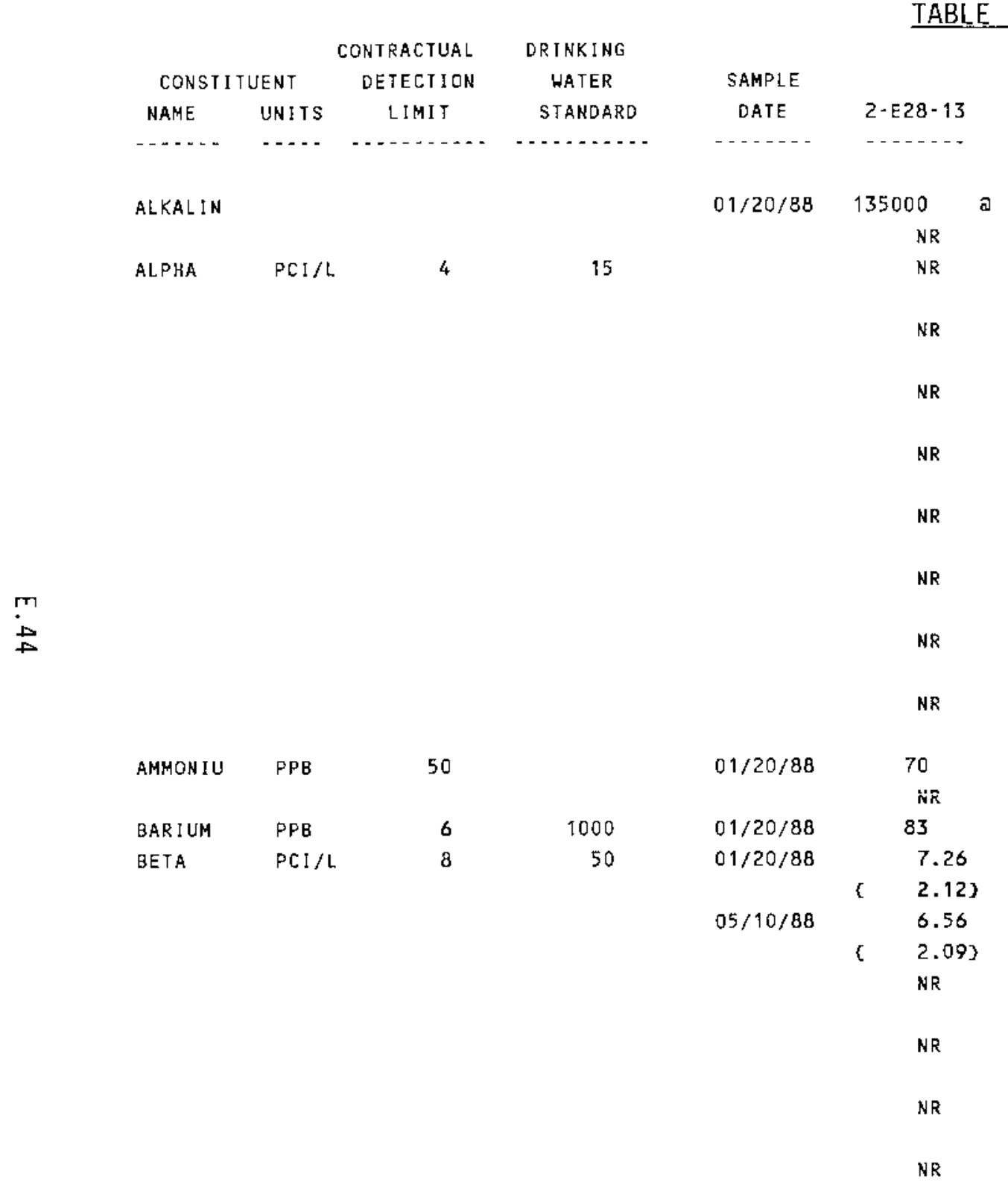

TABLE E.3. (contd)

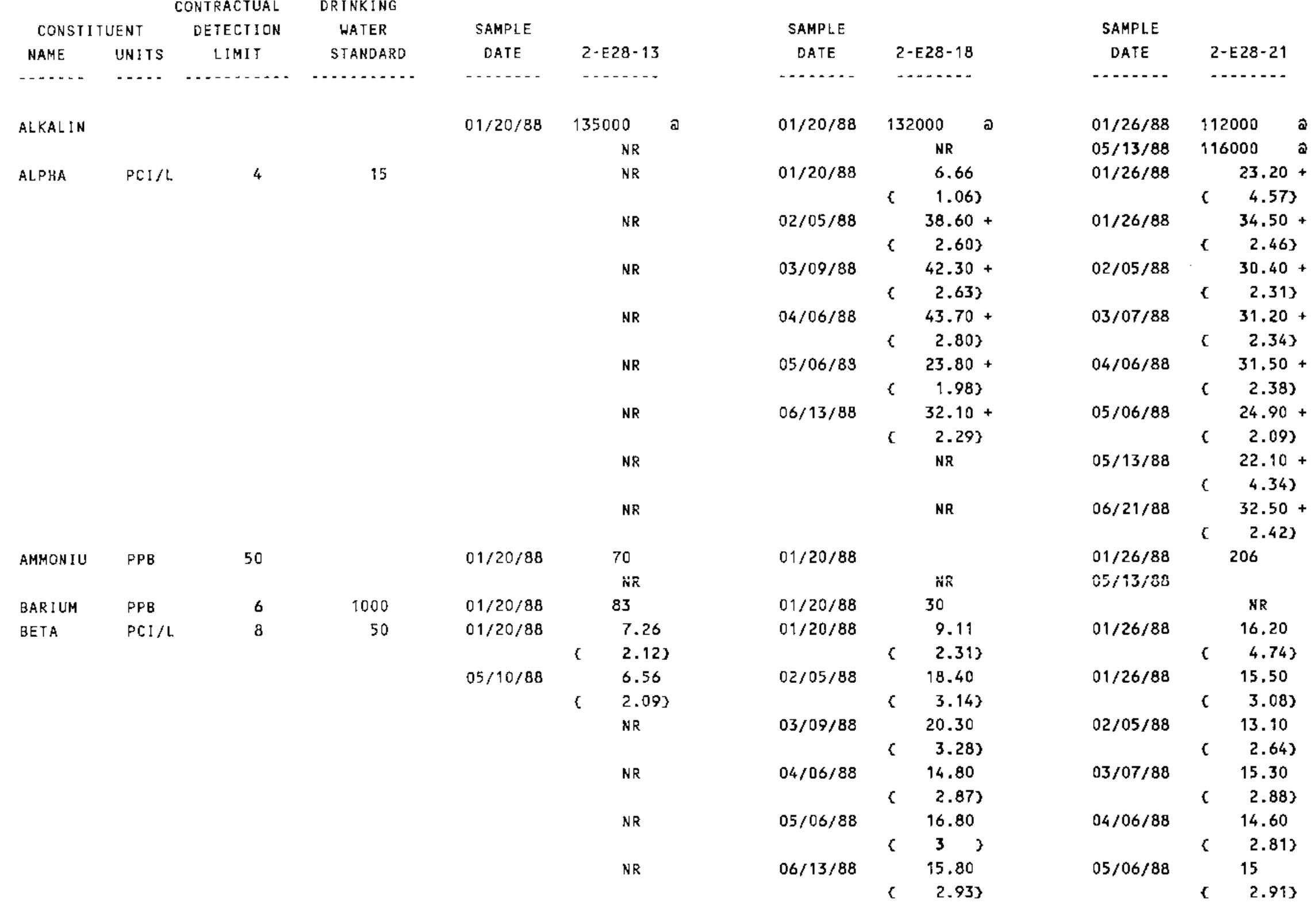




\begin{tabular}{|c|c|c|c|}
\hline \multirow{2}{*}{\multicolumn{2}{|c|}{ CONSTITUENT }} & \multirow{2}{*}{$\begin{array}{r}\text { CONTRACTUAL } \\
\text { DETECTION }\end{array}$} & \multirow{2}{*}{$\begin{array}{l}\text { DRINKING } \\
\text { HATER }\end{array}$} \\
\hline & & & \\
\hline NAME & UNITS & LIMIT & STANOARD \\
\hline & & $\cdots$ & \\
\hline
\end{tabular}

$\begin{array}{llrl} & & & \\ \text { CALCIUM } & \text { PPB } & 50 & \\ \text { CHLORID } & \text { PPB } & 500 & \\ \text { CO-60 } & \text { PCI/L } & 22.50 & \\ & & & \\ & & & \\ \text { CONDFLO } & & & \end{array}$

IABLE E.3. (contd)

\begin{tabular}{|c|c|c|c|c|c|}
\hline $\begin{array}{l}\text { SAMPLE } \\
\text { DATE }\end{array}$ & 2-E28-13 & $\begin{array}{l}\text { SAMPLE } \\
\text { DATE }\end{array}$ & 2-E28-18 & $\begin{array}{l}\text { SAMPLE } \\
\text { DATE }\end{array}$ & $2-E 28-21$ \\
\hline & NR & & NR & $05 / 13 / 88$ & 20.20 \\
\hline & & & & & $5.14 \mathrm{~J}$ \\
\hline & NR & & NR & $06 / 21 / 88$ & $\begin{array}{ll} & 13.80 \\
c \quad 2.76)\end{array}$ \\
\hline $01 / 20 / 88$ & 49300 & $01 / 20 / 88$ & 50700 & & NR \\
\hline $01 / 20 / 88$ & 7500 & $01 / 20 / 88$ & 8000 & $09 / 26 / 88$ & 12000 \\
\hline & NR & & NR & $05 / 13 / 88$ & 10380 \\
\hline $01 / 20 / 88$ & & $01 / 20 / 88$ & & $01 / 26 / 88$ & \\
\hline $05 / 10 / 88$ & & $02 / 05 / 88$ & & $02 / 05 / 88$ & \\
\hline & NR & $03 / 09 / 88$ & & $03 / 07 / 88$ & \\
\hline & NR & $04 / 06 / 88$ & 5.65 & $04 / 06 / 88$ & \\
\hline & & & 5.053 & & \\
\hline & NR & $05 / 06 / 88$ & & $05 / 06 / 88$ & \\
\hline & NR & $06 / 13 / 88$ & & $06 / 21 / 88$ & \\
\hline $01 / 20 / 88$ & 415 & $01 / 20 / 88$ & 274 & $01 / 26 / 88$ & 406 \\
\hline
\end{tabular}


TABLE E.3. (contd)

\begin{tabular}{|c|c|c|c|c|c|c|c|c|c|}
\hline \multicolumn{2}{|c|}{ CONST I TUENT } & CONTRACTUAL & DRINKING & SAMPIE & \multicolumn{3}{|c|}{ SAMPLE } & \multicolumn{2}{|l|}{ SAMPLE } \\
\hline NAME & UNITS & LIMIT & STANDARD & DATE & $2-E 28-13$ & DATE & $2-E 28-18$ & DATE & $2-\varepsilon 28-21$ \\
\hline$\ldots .$. & $\ldots$ & $\ldots$ & - & $\ldots$ & $\ldots . .$. & $\ldots . .$. & . . . . . & . & . \\
\hline CONDFLD & MMHO & 1 & & & NR & & $N R$ & $05 / 13 / 88$ & 467 \\
\hline \multirow[t]{2}{*}{ FARSEN I } & PPB & 5 & 50 & $01 / 20 / 88$ & & $01 / 20 / 88$ & 6 & $01 / 26 / 88$ & \\
\hline & & & & & NR & & $\mathrm{NR}$ & $05 / 13 / 88$ & \\
\hline \multirow[t]{2}{*}{ FBARIUM } & PPB & 6 & 1000 & $01 / 20 / 88$ & 77 & $01 / 20 / 88$ & 31 & $01 / 26 / 88$ & 47 \\
\hline & & & & & NR & & NR & $05 / 13 / 88$ & 46 \\
\hline \multirow[t]{2}{*}{ FCALCIU } & $\mathrm{PPB}$ & 50 & & $01 / 20 / 88$ & 45300 & $01 / 20 / 88$ & 46300 & $01 / 26 / 88$ & 68000 \\
\hline & & & & & NR & & NR & $05 / 13 / 88$ & 59100 \\
\hline \multirow[t]{2}{*}{ FLUORID } & PPB & 500 & 4000 & $01 / 20 / 88$ & 724 & $01 / 20 / 88$ & 670 & $01 / 26 / 88$ & 645 \\
\hline & & & & & $N R$ & & NR & $05 / 13 / 88$ & 670 \\
\hline \multirow[t]{2}{*}{ FMAGNES } & $P P B$ & 50 & & $01 / 20 / 88$ & 14100 & $01 / 20 / 88$ & 13100 & $01 / 26 / 88$ & 15600 \\
\hline & & & & & NR & & $N R$ & $05 / 13 / 88$ & 15500 \\
\hline \multirow[t]{2}{*}{ FPOTASS } & PPB & 100 & & $01 / 20 / 88$ & 6430 & $01 / 20 / 88$ & 6550 & $01 / 26 / 88$ & 5200 \\
\hline & & & & & NR & & NR & $05 / 13 / 88$ & 5220 \\
\hline \multirow[t]{2}{*}{ FSODIUM } & PPS & 200 & & $01 / 20 / 88$ & 26800 & $01 / 20 / 88$ & 25800 & $01 / 26 / 88$ & 23700 \\
\hline & & & & & NR & & NR & $05 / 13 / 88$ & 25500 \\
\hline \multirow[t]{2}{*}{ FSTRONT } & $P P B$ & 10 & & $01 / 20 / 88$ & 227 & $01 / 20 / 88$ & 212 & $01 / 26 / 88$ & 260 \\
\hline & & & & & NR & & NR & $05 / 13 / 88$ & 246 \\
\hline \multirow[t]{2}{*}{ FVANADI } & PPB & 5 & & $01 / 20 / 88$ & 16 & $01 / 20 / 88$ & 17 & $01 / 26 / 88$ & 16 \\
\hline & & & & & NR & & NR & $05 / 13 / 88$ & 15 \\
\hline \multirow[t]{2}{*}{ FZINC } & FPB & 5 & & $01 / 20 / 38$ & 8 & $01 / 20 / 80$ & & $01 / 26 / 88$ & 5 \\
\hline & & & & & $N R$ & & NR & $05 / 13 / 88$ & \\
\hline \multirow[t]{6}{*}{ HN I TRAT } & $\mathrm{PPB}$ & 2500 & 45000 & & NR & $01 / 20 / 88$ & 23300 & $01 / 26 / 88$ & 37500 \\
\hline & & & & & NR & $02 / 05 / 88$ & 35400 & $02 / 05 / 88$ & 34500 \\
\hline & & & & & NR & $03 / 09 / 88$ & 38000 & $03 / 07 / 88$ & 38400 \\
\hline & & & & & NR & $04 / 06 / 88$ & 38900 & $04 / 06 / 88$ & 40500 \\
\hline & & & & & NR & $05 / 06 / 88$ & 34100 & $05 / 06 / 88$ & 34700 \\
\hline & & & & & NR & $06 / 13 / 88$ & 38600 & $06 / 21 / 88$ & 35700 \\
\hline$I R O N$ & PPB & 50 & & $01 / 20 / 88$ & 260 & $01 / 20 / 88$ & 94 & & NR \\
\hline LEADGF & $P P B$ & 5 & 50 & $01 / 20 / 88$ & & $01 / 20 / 88$ & 6 & & SR \\
\hline \multirow[t]{2}{*}{ LFLUORD } & $\mathrm{PPB}$ & 50 & 4000 & & NR & & NR & $01 / 26 / 88$ & 467. \\
\hline & & & & & NR & & NR & $05 / 13 / 88$ & 490 \\
\hline MAGNES & PPB & 0 & & $01 / 20 / 88$ & 14800 & $01 / 20 / 88$ & 13800 & & $N R$ \\
\hline MANGESE & PPB & 5 & & $01 / 20 / 88$ & 24 & $01 / 20 / 88$ & 5 & & $N R$ \\
\hline
\end{tabular}


TABLE E.3. (contd)

\begin{tabular}{|c|c|c|c|c|c|c|c|c|c|}
\hline \multicolumn{2}{|c|}{ CONST : TUENT } & $\begin{array}{l}\text { CONTRACTUAL } \\
\text { DETECTION }\end{array}$ & $\begin{array}{l}\text { DRINKING } \\
\text { WATER }\end{array}$ & SAMPLE & & SAMPLE & & SAMPLE & \\
\hline NAME & UNITS & LIMIT & STANDARO & DATE & $2-E 28-13$ & DATE & $2-E 28-18$ & DATE & $2-E 2 B-21$ \\
\hline$\ldots$. & - - . & $\cdots$ & $-\ldots$ & $\ldots$ & $\ldots$ & . & $\ldots \ldots$ & - & $\ldots$ \\
\hline \multirow[t]{2}{*}{ NITRATE } & PPB & 500 & 45000 & $01 / 20 / 88$ & 48700 & $01 / 20 / 88$ & 26800 & $01 / 26 / 88$ & 34900 \\
\hline & & & & & NR & & NR & $05 / 13 / 88$ & 36000 \\
\hline \multirow[t]{2}{*}{$P H-L A B$} & & 0.01 & & $01 / 20 / 88$ & 7.65 & $01 / 20 / 88$ & 7.81 & $01 / 26 / 88$ & 7.57 \\
\hline & & & & & NR & & NR & $05 / 13 / 88$ & 7.80 \\
\hline \multirow[t]{2}{*}{ PHFIELD } & & 0.10 & . & $01 / 20 / 88$ & 8.20 & $01 / 20 / 88$ & 8.50 & $01 / 26 / 88$ & 7 \\
\hline & & & & & NR & & NR & $05 / 13 / 88$ & 7.50 \\
\hline POIASUM & PPQ & 100 & & $01 / 20 / 88$ & 6700 & $01 / 20 / 88$ & 6980 & & NR \\
\hline SODIUH & PPQ & 200 & & $01 / 20 / 88$ & 27700 & $01 / 20 / 88$ & 27400 & & NR \\
\hline \multirow[t]{2}{*}{ SULFATE } & PPB & 500 & & $01 / 20 / 88$ & 48000 & $01 / 20 / 88$ & 65200 & $01 / 26 / 88$ & 116000 \\
\hline & & & & & NR & & NR & $05 / 13 / 88$ & 104000 \\
\hline \multirow[t]{2}{*}{$T C$} & $P P B$ & 2000 & & $01 / 20 / 88$ & 34100 & $01 / 20 / 8 \varepsilon$ & 32400 & $09 / 26 / 88$ & 28700 \\
\hline & & & & & NR & & NR & $05 / 13 / 88$ & 14700 \\
\hline \multirow[t]{2}{*}{ TOXLDL } & $\mathrm{PPB}$ & 10 & & $01 / 20 / 88$ & 23.20 & $01 / 20 / 88$ & & $01 / 26 / 88$ & 20.40 \\
\hline & & & & & NR & & NR & $05 / 13 / 88$ & \\
\hline \multirow[t]{2}{*}{ TRITILM } & $\mathrm{PCl} / \mathrm{L}$ & 500 & 20000 & $01 / 20 / 88$ & 7470 & $01 / 20 / 88$ & 8410 & $01 / 26 / 88$ & 9590 \\
\hline & & & & & \& 322 & \} & ( $\quad 337$ & & c 362 \\
\hline
\end{tabular}


TABLE E.3. (contd)

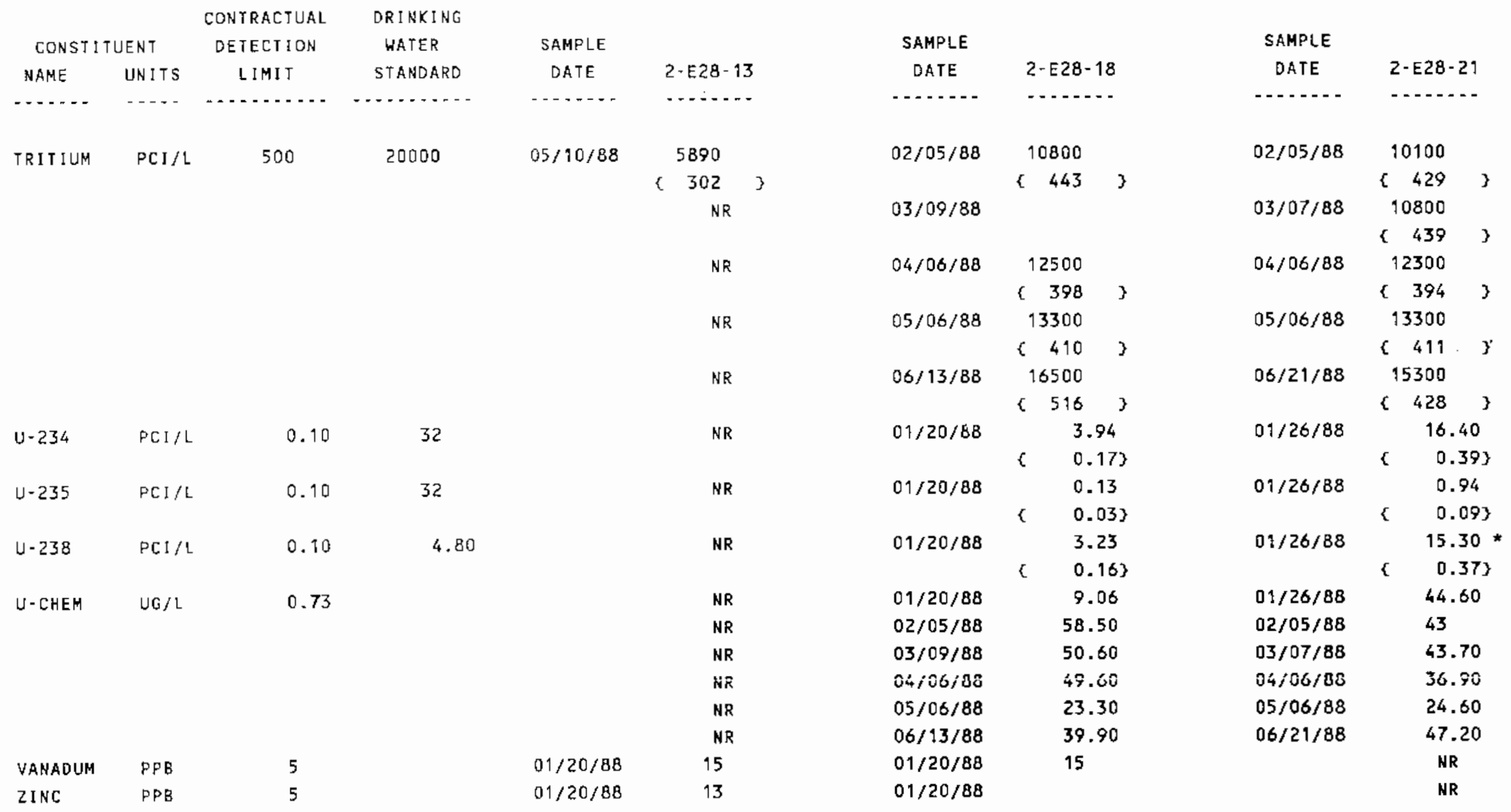


TABLE E. 3. (contd)

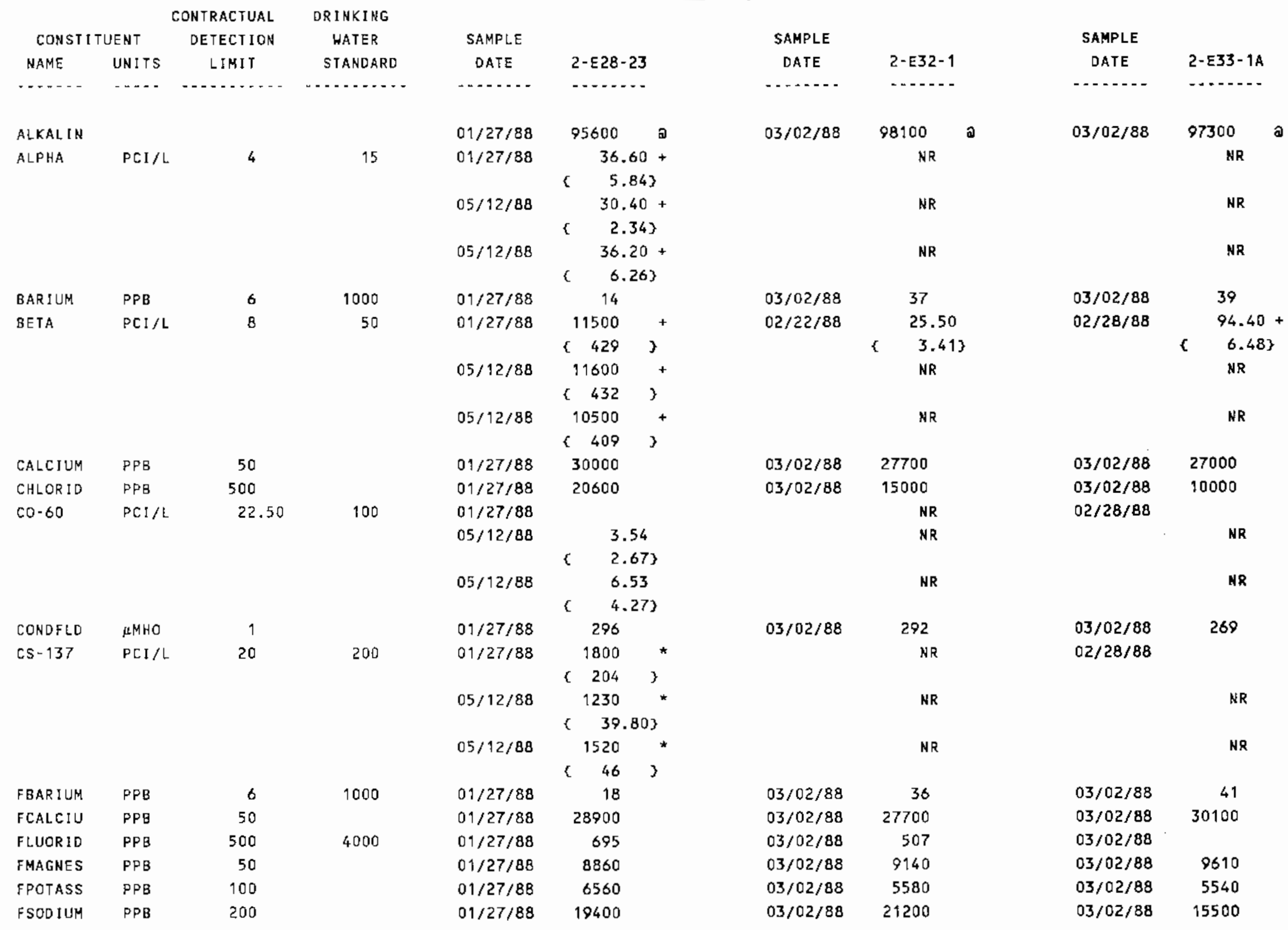


IABLE E.3. (contd)

\begin{tabular}{|c|c|c|c|c|c|c|c|}
\hline \multicolumn{2}{|c|}{ CONSTI I UENT } & $\begin{array}{l}\text { CONTRACTUAL } \\
\text { DETECTIDN }\end{array}$ & & SAMPLE & \multicolumn{2}{|r|}{ SAMPLE } & \multirow[b]{2}{*}{$2-E 32-1$} \\
\hline NAME & UNITS & LIMIT & STANDARD & DATE & $2-E 28-23$ & DATE & \\
\hline -....... & $\ldots$. & $\ldots$ & n. & . n........ & .......... & - n....... & n....... \\
\hline FSTRONT & PPB & 10 & & $01 / 27 / 88$ & 133 & $03 / 02 / 88$ & 144 \\
\hline FVANAD I & PPB & 5 & & $01 / 27 / 88$ & 19 & $03 / 02 / 88$ & 18 \\
\hline FZINC & PPB & 5 & & $01 / 27 / 88$ & & $03 / 02 / 88$ & 5 \\
\hline \multirow[t]{2}{*}{ HNITRAT } & $\mathrm{PPB}$ & 2500 & 45000 & $01 / 27 / 88$ & 8920 & $02 / 22 / 88$ & 8600 \\
\hline & & & & $05 / 12 / 88$ & 9480 & & NR \\
\hline IRON & $\mathrm{PPB}$ & 50 & & $01 / 27 / 88$ & 73 & $03 / 02 / 88$ & 57 \\
\hline MAGNES & PPB & 0 & & $01 / 27 / 88$ & 9380 & $03 / 02 / 88$ & 9180 \\
\hline NITRATE & PPB & 500 & 45000 & $01 / 27 / 88$ & 9580 & $03 / 02 / 88$ & 10200 \\
\hline$P H-L A B$ & & 0.01 & & $01 / 27 / 88$ & 7.92 & $03 / 02 / 88$ & 7.86 \\
\hline PHFIELD & & 0.10 & & $01 / 27 / 88$ & 7.80 & $03 / 02 / 88$ & 8.40 \\
\hline POTASUM & $P P B$ & 100 & & $01 / 27 / 88$ & 7150 & $03 / 02 / 38$ & 5510 \\
\hline \multirow[t]{3}{*}{$P U-238$} & $\mathrm{PCl} / \mathrm{L}$ & 17 & 5000 & $01 / 27 / 88$ & 0.04 & & NR \\
\hline & & & & & $0.02\}$ & & \\
\hline & & & & $05 / 12 / 88$ & 0.07 & & NR \\
\hline
\end{tabular}

SAMPLE

DATE

$2-E 33 \div 1 A$

-.......

$03 / 02 / 88$

$03 / 02 / 88$

$03 / 02 / 88$

$02 / 28 / 88$

46300

$03 / 02 / 88$

$03 / 02 / 88$

$03 / 02 / 88$

$03 / 02 / 88$

$03 / 02 / 88$

$03 / 02 / 88$

8.40

5120

NR

NR 


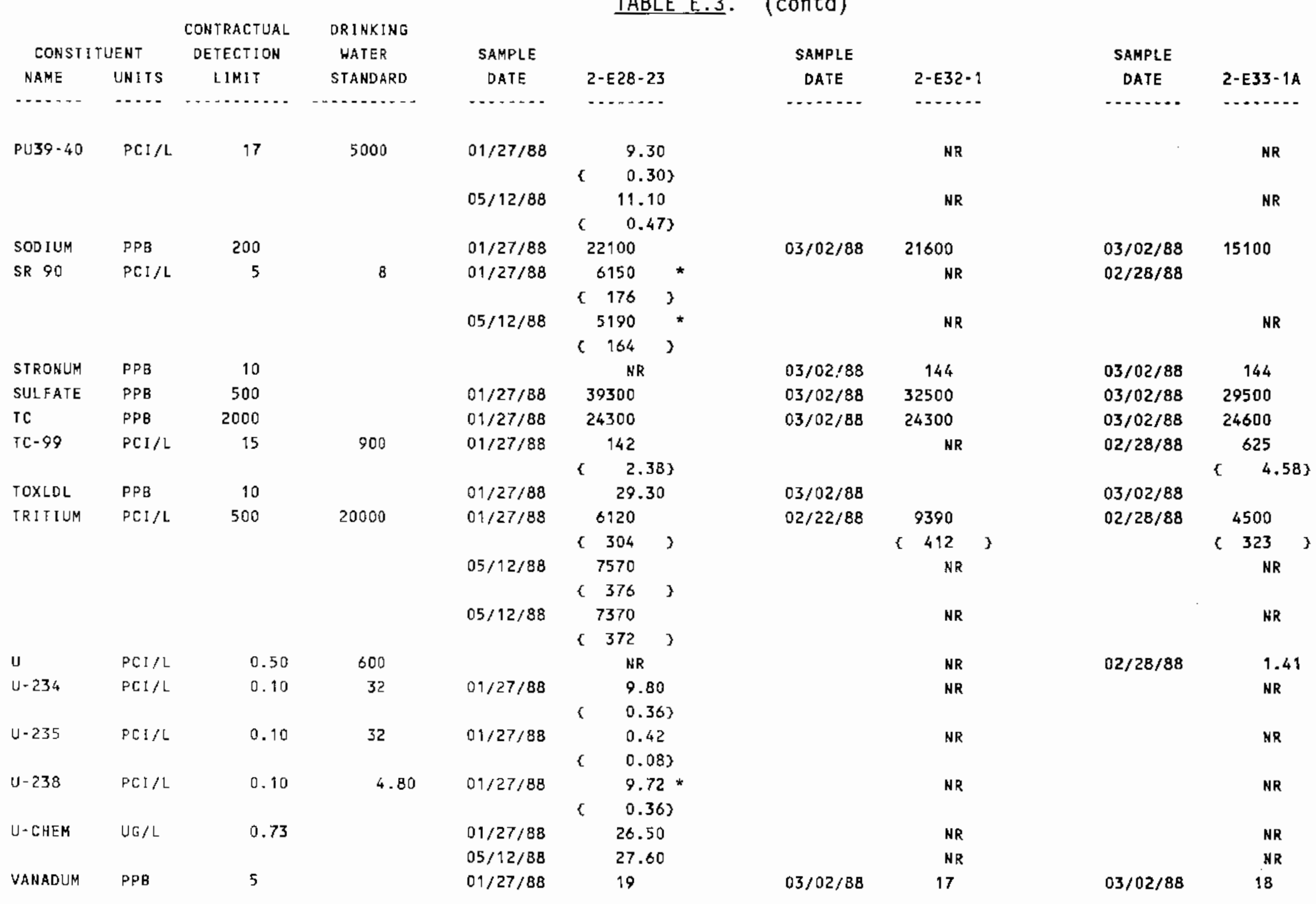


TABLE E.3. (contd)

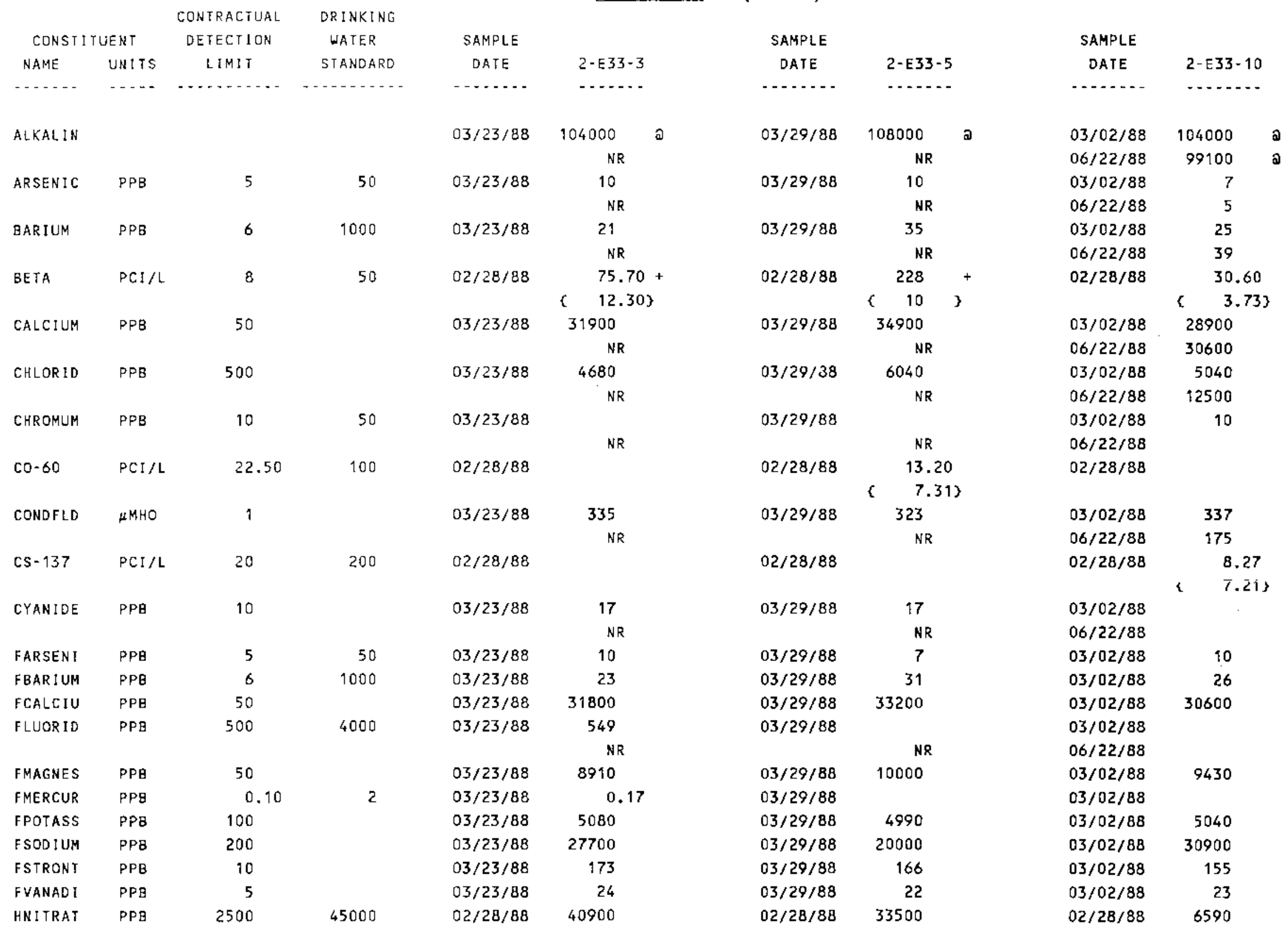


IABLE E.3. (contd)

\begin{tabular}{|c|c|c|c|c|c|c|c|c|c|}
\hline \multicolumn{2}{|c|}{ CONSTI TUENT } & $\begin{array}{l}\text { CONTRACTUAL } \\
\text { DETECTION }\end{array}$ & $\begin{array}{l}\text { DRINKING } \\
\text { WATER }\end{array}$ & SAMPLE & & SAMPLE & & SAMPLE & \\
\hline NAME & UNITS & LIMIT & STANDARD & DATE & $2-E 33-3$ & DATE & $2-E 33-5$ & DATE & $2-E 33-10$ \\
\hline$\ldots . .$. & $\ldots$ & $\cdots$ & n. & $\ldots$ & ….... & $\ldots$ & -........ & . & $\ldots$ \\
\hline \multirow[t]{2}{*}{ IRON } & PPB & 50 & & $03 / 23 / 88$ & & $03 / 29 / 88$ & 73 & $03 / 02 / 88$ & \\
\hline & & & & & NR & & NR & $06 / 22 / 88$ & 148 \\
\hline \multirow[t]{2}{*}{ MAGNES } & $P P B$ & 0 & & $03 / 23 / 88$ & 8760 & $03 / 29 / 88$ & 10500 & $03 / 02 / 88$ & 8720 \\
\hline & & & & & $N R$ & & NR & $06 / 22 / 88$ & 9480 \\
\hline \multirow[t]{2}{*}{ MERCURY } & $\mathrm{PPB}$ & 0.10 & 2 & $03 / 23 / 88$ & 0.25 & $03 / 29 / 88$ & & $03 / 02 / 88$ & \\
\hline & & & & & NR & & NR & $06 / 22 / 88$ & \\
\hline \multirow[t]{2}{*}{ NITRATE } & $P P B$ & 500 & 45000 & $03 / 23 / 88$ & 44600 & $03 / 29 / 88$ & 30900 & $03 / 02 / 88$ & 44700 \\
\hline & & & & & $\mathrm{NR}$ & & NR & $06 / 22 / 88$ & 7140 \\
\hline \multirow[t]{2}{*}{$P H-L A B$} & & 0.01 & & $03 / 23 / 88$ & 8.11 & $03 / 29 / 88$ & 8.14 & $03 / 02 / 88$ & 7.75 \\
\hline & & & & & NR & & NR & $06 / 22 / 88$ & 8 \\
\hline \multirow[t]{2}{*}{ PHF IELD } & & 0.10 & & $03 / 23 / 88$ & 8.10 & $03 / 29 / 88$ & 7.20 & $03 / 02 / 88$ & 8.50 \\
\hline & & & & & NR & & NR & $06 / 22 / 88$ & 7.80 \\
\hline \multirow[t]{2}{*}{ POTASUM } & PPB & 100 & & $03 / 23 / 88$ & 4870 & $03 / 29 / 88$ & 5210 & $03 / 02 / 88$ & 4420 \\
\hline & & & & & NR & & NR & $06 / 22 / 88$ & $5450^{-}$ \\
\hline
\end{tabular}


TABLE E.3. (contd)

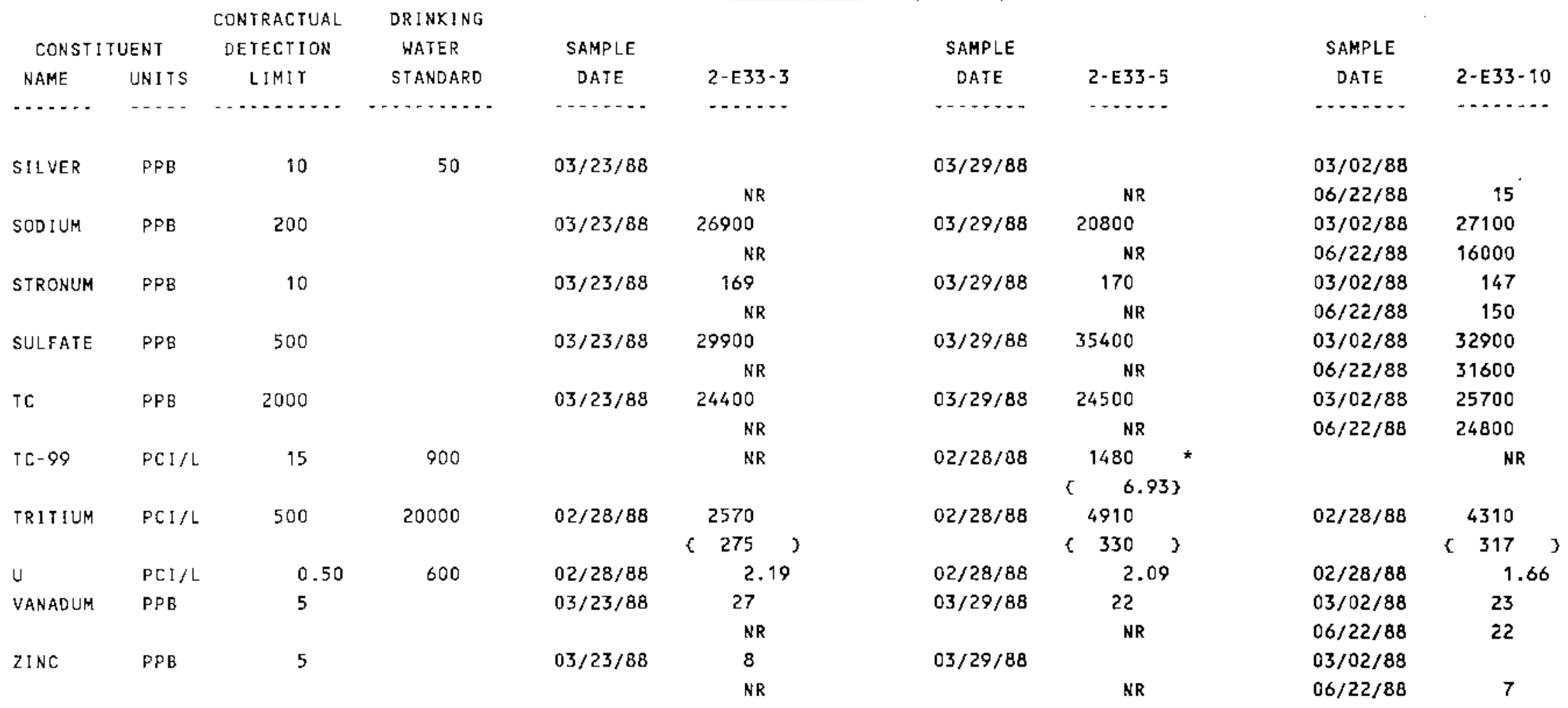


IABLE_E.3. (contd)

\begin{tabular}{|c|c|c|c|c|c|c|c|c|c|c|c|}
\hline \multicolumn{2}{|c|}{ CONST I TUENT } & $\begin{array}{l}\text { CONTRACTUAL } \\
\text { DETECTION }\end{array}$ & $\begin{array}{l}\text { DRINKING } \\
\text { WATER }\end{array}$ & SAMPLE & & SAMPLE & & & SAMPLE & \multirow{2}{*}{\multicolumn{2}{|c|}{$2-410-9$}} \\
\hline NAME & UNITS & LIMIT & STANDARD & DATE & $2-E 34-1$ & DATE & \multicolumn{2}{|l|}{$2-H 10-4$} & DATE & & \\
\hline 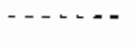 & $\cdots$ & - & 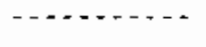 & $\cdots$ & $\ldots \ldots$ & $\cdots$ & \multicolumn{2}{|l|}{ 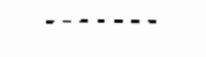 } & $\cdots$ & $\cdots$ & \\
\hline ALKALIN & & & & $01 / 27 / 88$ & 89100 & $03 / 04 / 88$ & 157000 & a & $03 / 21 / 88$ & 169000 & อ \\
\hline \multirow[t]{4}{*}{ ALPHA } & $\mathrm{PCI} / \mathrm{L}$ & 4 & 15 & $01 / 27 / 88$ & 1.55 & & NR & & $02 / 28 / 88$ & 3.93 & \\
\hline & & & & & $0.55\}$ & & & & & 0.843 & \\
\hline & & & & $06 / 03 / 88$ & 1.87 & & $N R$ & & & NR & \\
\hline & & & & & 0.603 & & & & & & \\
\hline ARSENIC & PPB & 5 & 50 & $01 / 27 / 88$ & & $03 / 04 / 88$ & 8 & & $03 / 21 / 88$ & 16 & \\
\hline BARIUH & PPB & 6 & 1000 & $01 / 27 / 88$ & 28 & $03 / 04 / 88$ & 34 & & $03 / 21 / 88$ & 60 & \\
\hline \multirow[t]{4}{*}{ BETA } & PCI/L & 8 & 50 & $01 / 27 / 88$ & 8.91 & $02 / 25 / 88$ & 79.90 & + & $02 / 28 / 88$ & 49.40 & \\
\hline & & & & & 2.323 & & 6.453 & & & 5.35 & \\
\hline & & & & $06 / 03 / 88$ & 9.05 & & NR & & & NR & \\
\hline & & & & & 2.373 & & & & & & \\
\hline BIS2EPH & $P P B$ & 10 & & $01 / 27 / 88$ & 10 & $03 / 04 / 88$ & & & $03 / 21 / 88$ & & \\
\hline CALCIUM & PPB & 50 & & $01 / 27 / 88$ & 58000 & $03 / 04 / 88$ & 23600 & & $03 / 21 / 88$ & 43500 & \\
\hline CHLFORH & PPB & 10 & 100 & $01 / 27 / 88$ & & $03 / 04 / 88$ & 20 & & $03 / 21 / 88$ & 16 & \\
\hline CHLORID & PPB & 500 & & $01 / 27 / 88$ & 16600 & $03 / 04 / 88$ & 16900 & & $03 / 21 / 88$ & 21700 & \\
\hline CHROMUM & PPB & 10 & 50 & $01 / 27 / 88$ & & $03 / 04 / 88$ & 66 & $\star$ & $03 / 21 / 88$ & 157 & * \\
\hline \multirow[t]{3}{*}{$c 0-60$} & PCI / & 22.50 & 100 & $01 / 27 / 88$ & & $02 / 25 / 88$ & 6.43 & & $02 / 28 / 88$ & & \\
\hline & & & & & & & 5.253 & & & & \\
\hline & & & & $06 / 03 / 88$ & & & NR & & & NR & \\
\hline CONDFLD & „MМHO & 1 & & $01 / 27 / 88$ & 464 & $03 / 04 / 88$ & 648 & & $03 / 29 / 88$ & 1055 & \\
\hline FARSEN ] & PPB & 5 & 50 & $01 / 27 / 88$ & & $03 / 04 / 88$ & 6 & & $03 / 21 / 88$ & 16 & \\
\hline FBARIUM & PPB & 6 & 1000 & $01 / 27 / 88$ & 27 & $03 / 04 / 88$ & 35 & & $03 / 21 / 88$ & 59 & \\
\hline FCALCIU & PPB & 50 & & $01 / 27 / 88$ & 61900 & $03 / 04 / 88$ & 23700 & & $03 / 21 / 88$ & 45500 & \\
\hline FCHROMI & PPB & 10 & 50 & $01 / 27 / 88$ & & $03 / 04 / 88$ & 65 & $*$ & $03 / 21 / 88$ & 152 & $\star$ \\
\hline FIRON & $\mathrm{PPB}$ & 50 & & $01 / 27 / 88$ & 50 & $03 / 04 / 88$ & & & $03 / 21 / 88$ & & \\
\hline FLUOR ID & $\mathrm{PPB}$ & 500 & 4000 & $01 / 27 / 88$ & 724 & $03 / 04 / 88$ & 3170 & & $03 / 21 / 88$ & 5050 & $\star$ \\
\hline FMAGNES & PPB & 50 & & $01 / 27 / 88$ & 16200 & $03 / 04 / 88$ & 7340 & & $03 / 21 / 88$ & 15300 & \\
\hline FMANGAN & PPB & 5 & & $01 / 27 / 88$ & 22 & $03 / 04 / 88$ & & & $03 / 21 / 88$ & & \\
\hline FPOTASS & PPB & 100 & & $01 / 27 / 88$ & 8130 & $03 / 04 / 88$ & 2950 & & $03 / 21 / 88$ & 4890 & \\
\hline FSELEN I & $P P B$ & 5 & 10 & $01 / 27 / 88$ & 7 & $03 / 04 / 88$ & & & $03 / 21 / 88$ & & \\
\hline FSODIUH & PPB & 200 & & $01 / 27 / 88$ & 27100 & $03 / 04 / 88$ & 149000 & & $03 / 21 / 88$ & 181000 & \\
\hline FSTRONT & PPB & 10 & & $01 / 27 / 88$ & 283 & $03 / 04 / 88$ & 126 & & $03 / 21 / 88$ & 249 & \\
\hline FVANADI & PPB & 5 & & $01 / 27 / 88$ & 7 & $03 / 04 / 88$ & 52 & & $03 / 21 / 88$ & 67 & \\
\hline HNI TRAT & PPB & 2500 & 45000 & & NR & $02 / 25 / 88$ & 209000 & $\star$ & $02 / 28 / 88$ & 396000 & * \\
\hline
\end{tabular}


TABLE E.3. (contd)

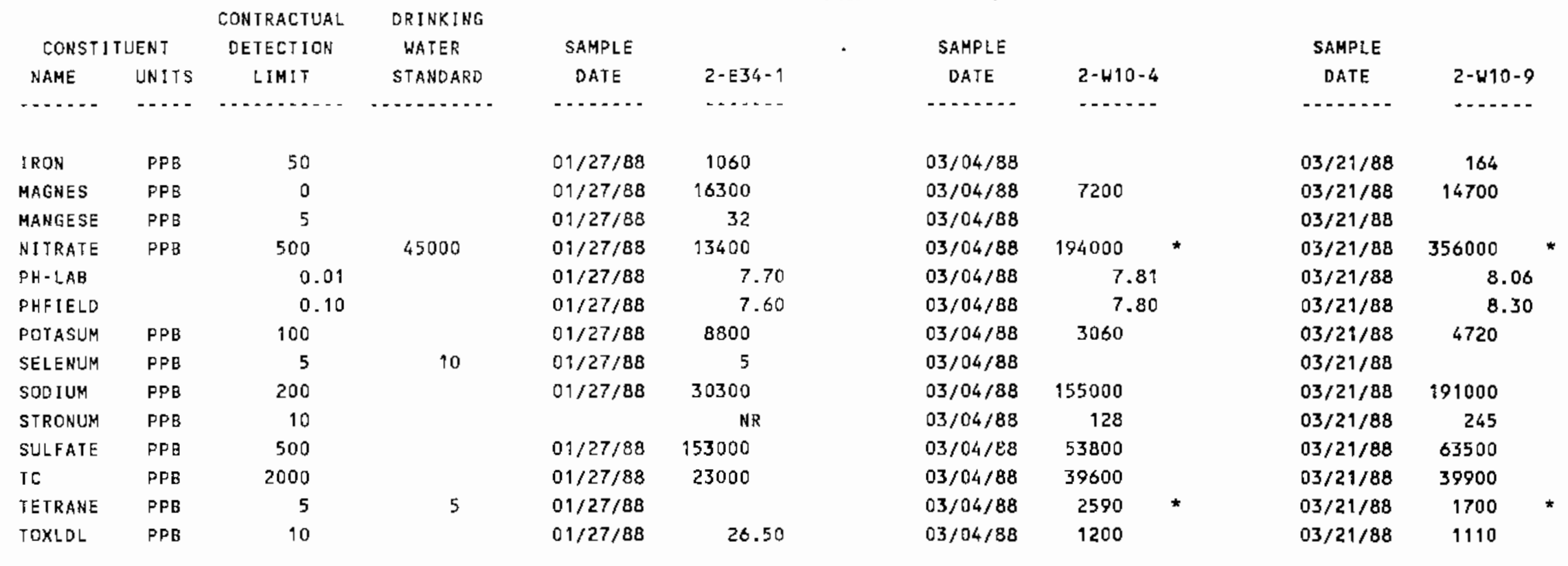


TABLE E. 3 . (contd)

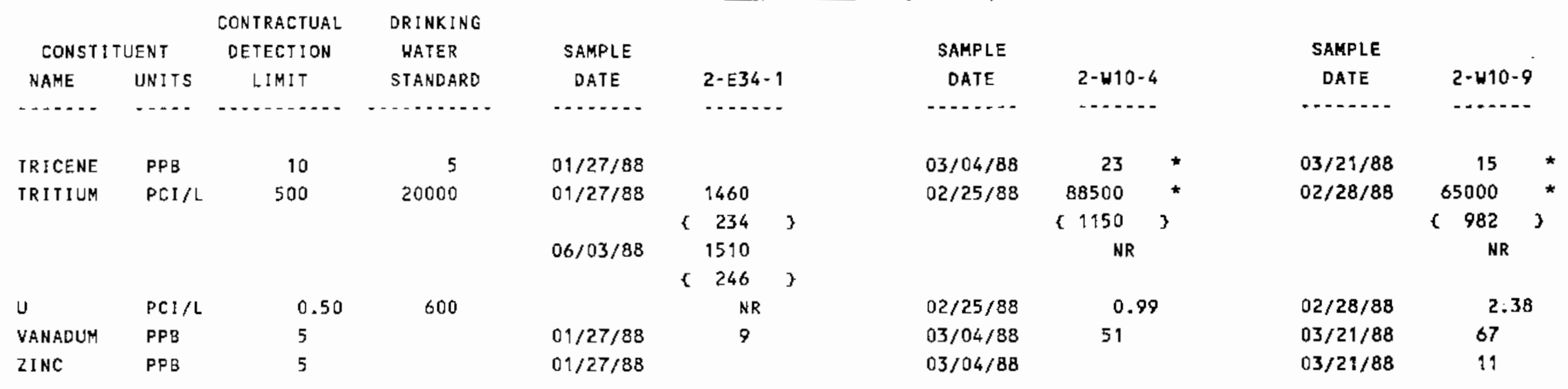


TABLEE.3. (contd)

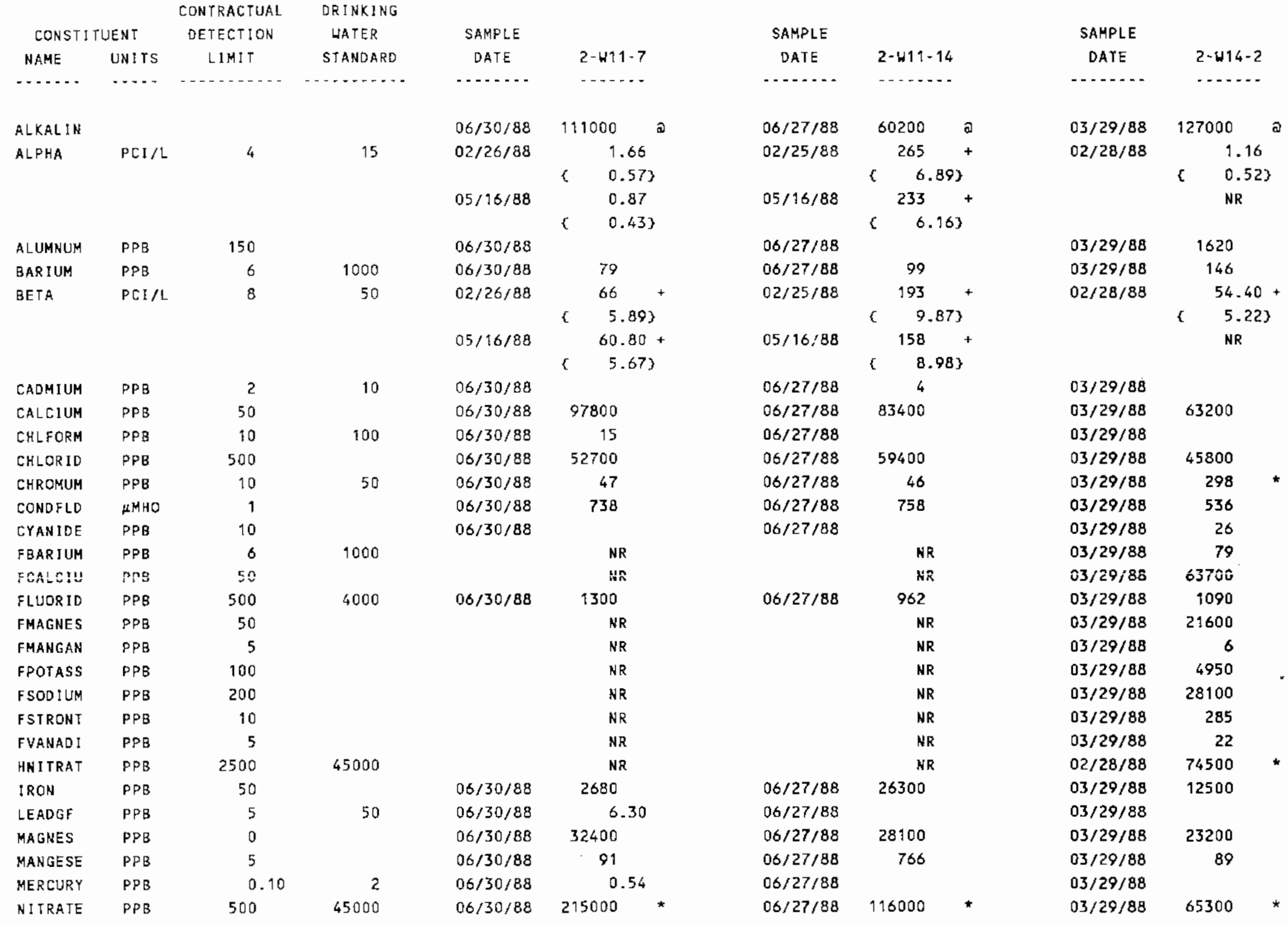


IABLE E.3. (contd)

\begin{tabular}{|c|c|c|c|c|c|c|c|c|c|}
\hline \multicolumn{2}{|c|}{ CONSTITUENT } & $\begin{array}{l}\text { CONTRACTUAL } \\
\text { DETECTION }\end{array}$ & $\begin{array}{l}\text { DRINKING } \\
\text { WATER }\end{array}$ & SAMPLE & \multicolumn{3}{|c|}{ SAMPLE } & \multicolumn{2}{|l|}{ SAMPLE } \\
\hline NAME & UNITS & LIMIT & STANDARD & DATE & $2-H 11-7$ & DATE & $2-H 11-14$ & DATE & $2-414-2$ \\
\hline$\ldots$ & $\cdots$ & 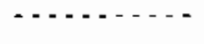 & 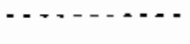 & ........... & ....... & $\ldots+\ldots$ & - & $\ldots$ & $\cdots$ \\
\hline$P H-L A B$ & & 0.01 & & $06 / 30 / 88$ & 7.90 & $06 / 27 / 88$ & 7.80 & $03 / 29 / 88$ & 7.92 \\
\hline PHFIELD & & 0.10 & & $06 / 30 / 88$ & 8.10 & $06 / 27 / 88$ & 7.80 & $03 / 29 / 88$ & 7.40 \\
\hline POTASUM & PPB & 100 & & $06 / 30 / 88$ & 5840 & $06 / 27 / 88$ & 5420 & $03 / 29 / 88$ & 5140 \\
\hline \multirow[t]{2}{*}{ RADIUM } & $\mathrm{PCI} / \mathrm{L}$ & 1 & 5 & $06 / 30 / 88$ & & $06 / 27 / 88$ & 0.88 & $03 / 29 / 88$ & \\
\hline & & & & & & & 0.323 & & \\
\hline SELENUM & PPB & 5 & 10 & $06 / 30 / 88$ & & $06 / 27 / 88$ & 7.10 & $03 / 29 / 88$ & \\
\hline SODIUM & $P P B$ & 200 & & $06 / 30 / 88$ & 27400 & $06 / 27 / 88$ & 36500 & $03 / 29 / 88$ & 28700 \\
\hline STRONUH & PPB & 10 & & $06 / 30 / 88$ & 436 & $06 / 27 / 88$ & 431 & $03 / 29 / 88$ & 293 \\
\hline SULFATE & PPB & 500 & & $06 / 30 / 88$ & 46300 & $06 / 27 / 88$ & 64900 & $03 / 29 / 88$ & 51000 \\
\hline TC & PPB & 2000 & & $06 / 30 / 88$ & 30200 & $06 / 27 / 88$ & 28600 & $03 / 29 / 88$ & 29100 \\
\hline \multirow[t]{2}{*}{ TC- 99} & $\mathrm{PCI} / \mathrm{L}$ & 15 & 900 & & $\mathrm{NR}$ & & NR & $02 / 28 / 88$ & 351 \\
\hline & & & & & & & & & ( $\quad 3.511$ \\
\hline TE TRANE & PPB & 5 & 5 & $06 / 30 / 88$ & 2080 & $06 / 27 / 88$ & 650 & $03 / 29 / 88$ & 1050 \\
\hline TOXLDL & PPB & 10 & & $06 / 30 / 88$ & & $06 / 27 / 88$ & 148 & $03 / 29 / 88$ & 788 \\
\hline
\end{tabular}


IABLE E.3. (contd)

\begin{tabular}{|c|c|c|c|c|c|c|c|c|c|c|}
\hline \multirow{2}{*}{\multicolumn{2}{|c|}{ CONST I TUENT }} & CONTRACTUAL & DRINKING & & & & & & \multirow{3}{*}{\multicolumn{2}{|c|}{$2-W 14-2$}} \\
\hline & & DETECTION & HATER & SAMPLE & & SAMPLE & & SAMPLE & & \\
\hline NAME & UNITS & LIM $1 \mathrm{~T}$ & STANDARD & DATE & $2-H 11-7$ & DATE & $2-H 11-14$ & DATE & & \\
\hline$\ldots \ldots$ & - . - & $-\ldots \ldots$ & $\cdots$ & - & - - - - - & $\ldots$ & . & - & $\ldots$ & \\
\hline TRICENE & $P P B$ & 10 & 5 & $06 / 30 / 88$ & & $06 / 27 / 88$ & & $03 / 29 / 88$ & 11 & * \\
\hline \multirow[t]{2}{*}{ TRITIUM } & $P C I / L$ & 500 & 20000 & & NR & & NR & $02 / 28 / 88$ & 80300 & * \\
\hline & & & & & & & & & $<1100$ & 3 \\
\hline $\mathrm{U}$ & $\mathrm{PCI} / \mathrm{L}$ & 0.50 & 600 & & NR & & $N R$ & $02 / 28 / 88$ & \multicolumn{2}{|c|}{1.07} \\
\hline VANADUM & PPB & 5 & & $06 / 30 / 88$ & 23 & $06 / 27 / 88$ & 57 & $03 / 29 / 88$ & \multicolumn{2}{|l|}{45} \\
\hline ZINC & $P P B$ & 5 & & $06 / 30 / 88$ & 36 & $06 / 27 / 88$ & 41 & $03 / 29 / 88$ & \multicolumn{2}{|l|}{15} \\
\hline
\end{tabular}




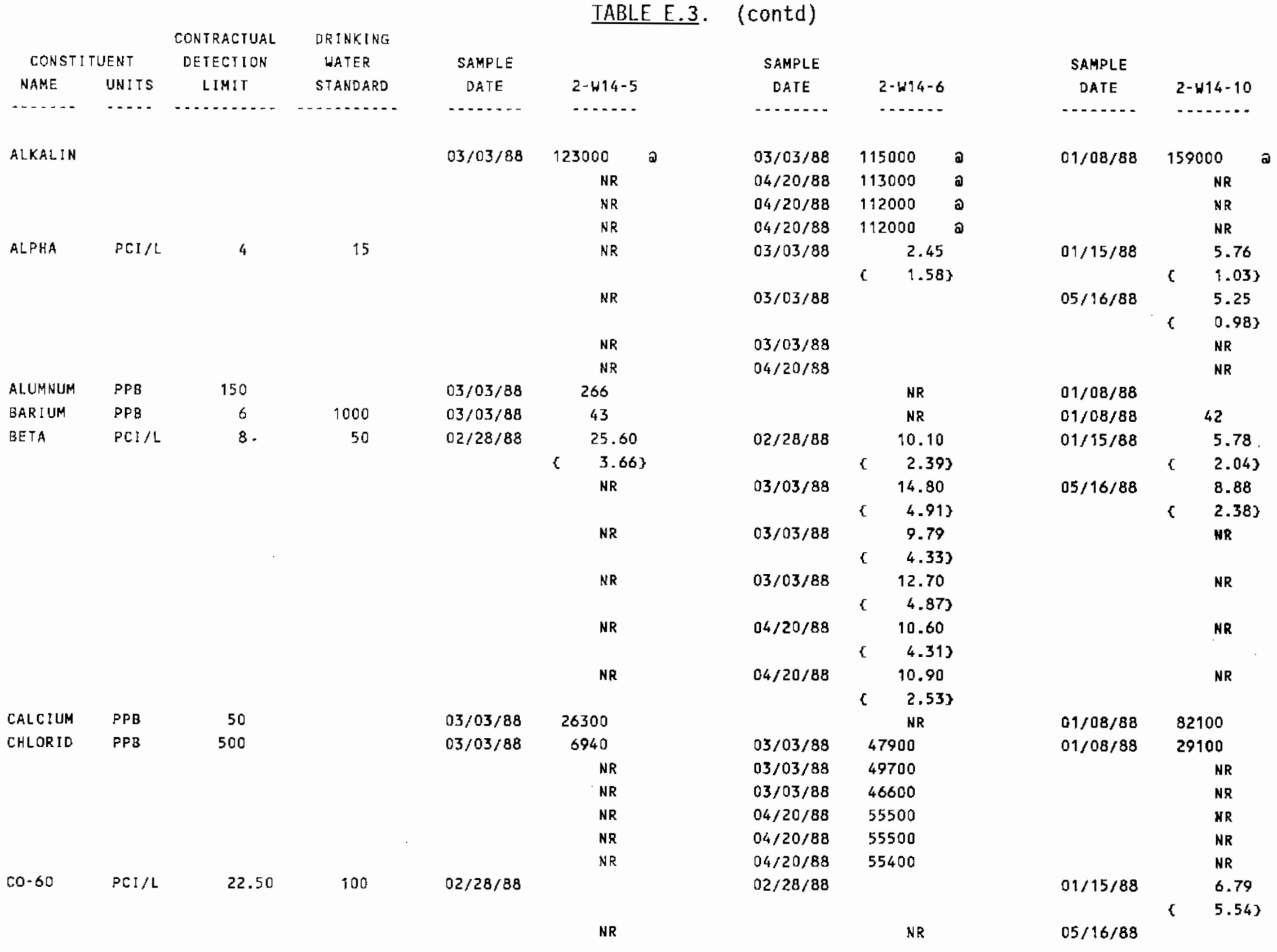


TABLE E.3. (contd)

\begin{tabular}{|c|c|c|c|}
\hline \multirow{2}{*}{\multicolumn{2}{|c|}{ CONST I TUENT }} & CONTRACTUAL & DRINKING \\
\hline & & DETECTION & WATER \\
\hline NAME & UNITS & LIMIT & STANDARD \\
\hline$\cdots$ & $\cdots$ & - & - \\
\hline
\end{tabular}

$\begin{array}{llcc}\text { CONDFLD } & \text { HMHO } & 1 & \\ & & & \\ \text { COPPER } & \text { PPB } & 10 & (1300.0) \\ \text { CS-137 } & \text { PCI/L } & 20 & 200 \\ & & & \end{array}$

FBARIUM PPB

6

1000

FCALCIU

PPB 
TABLEE.3. (contd)

\begin{tabular}{|c|c|c|c|c|c|c|c|c|c|}
\hline \multicolumn{2}{|c|}{ CONSTI TUENT } & $\begin{array}{l}\text { CONTRACIUAL } \\
\text { DETECTION }\end{array}$ & $\begin{array}{l}\text { DRINXING } \\
\text { WATER }\end{array}$ & SAMPLE & & SAMPLE & & SAMPLE & \\
\hline NAME & UNITS & LIMIT & STANDARD & DATE & $2-W 14-5$ & DATE & $2-W 14-6$ & DATE & $2-w 14-90$ \\
\hline$\cdots . .$. & ..... & n.t. & . & $\ldots . .$. & …… & $\ldots$ & -....... & - - - - - & - \\
\hline \multirow[t]{4}{*}{ FIRON } & PPB & 50 & & $03 / 03 / 88$ & & $03 / 03 / 88$ & 165 & $01 / 08 / 88$ & \\
\hline & & & & & NR & $03 / 03 / 88$ & 487 & & NR \\
\hline & & & & & $\mathrm{NR}$ & $03 / 03 / 88$ & & & NR \\
\hline & & & & & $N R$ & $04 / 20 / 88$ & & & NR \\
\hline \multirow[t]{6}{*}{ FLUORID } & $P P B$ & 500 & 4000 & $03 / 03 / 88$ & 2260 & $03 / 03 / 88$ & 3310 & $01 / 08 / 88$ & 640 \\
\hline & & & & & NR & $03 / 03 / 88$ & 3300 & & NR \\
\hline & & & & & NR & $03 / 03 / 88$ & 3320 & & NR \\
\hline & & & & & NR & $04 / 20 / 88$ & 2520 & & NR \\
\hline & & & & & NR & $04 / 20 / 88$ & 2470 & & NR \\
\hline & & & & & NR & $04 / 20 / 88$ & 2550 & & NR \\
\hline \multirow[t]{4}{*}{ FMAGNES } & PPB & 50 & & $03 / 03 / 88$ & 8960 & $03 / 03,88$ & 13700 & $01 / 08 / 88$ & 25100 \\
\hline & & & & & NR & $03 / 03 / 88$ & 13100 & & NR \\
\hline & & & & & NR & $03 / 03 / 88$ & 12900 & & NR \\
\hline & & & & & NR & $04 / 20 / 88$ & 13600 & & NR \\
\hline \multirow[t]{4}{*}{ FMANGAN } & $P P B$ & 5 & & $03 / 03 / 88$ & & $03 / 03 / 88$ & 6 & $01 / 08 / 88$ & \\
\hline & & & & & NR & $03 / 03 / 88$ & 11 & & NR \\
\hline & & & & & NR & $03 / 03 / 88$ & & & NR \\
\hline & & & & & NR & $04 / 20 / 88$ & & & NR \\
\hline \multirow[t]{4}{*}{ FPOTASS } & PPB & 100 & & $03 / 03 / 88$ & 4370 & $03 / 03 / 88$ & 5020 & $01 / 08 / 88$ & 5370 \\
\hline & & & & & NR & $03 / 03 / 88$ & 4760 & & NR \\
\hline & & & & & NR & $03 / 03 / 88$ & 4690 & & NR \\
\hline & & & & & NR & $04 / 20 / 88$ & 5060 & & NR \\
\hline \multirow[t]{4}{*}{ FSOD I UM } & PPB & 200 & & $03 / 03 / 88$ & 90900 & $03 / 03 / 88$ & 48600 & $01 / 08 / 88$ & 20900 \\
\hline & & & & & NR & $03 / 03 / 88$ & 45700 & & NR \\
\hline & & & & & NR & $03 / 03 / 88$ & 45000 & & NR \\
\hline & & & & & NR & $04 / 20 / 88$ & 38600 & & NR \\
\hline \multirow[t]{4}{*}{ FSTRONT } & $\mathrm{PPB}$ & 10 & & $03 / 03 / 88$ & 120 & $03 / 03 / 88$ & 171 & $01 / 08 / 88$ & 342 \\
\hline & & & & & NR & $03 / 03 / 88$ & 165 & & NR \\
\hline & & & & & NR & $03 / 03 / 88$ & 162 & & NR \\
\hline & & & & & NR & $04 / 20 / 88$ & 175 & & NR \\
\hline \multirow[t]{4}{*}{ FVANADI } & PPB & 5 & & $03 / 03 / 88$ & 88 & $03 / 03 / 88$ & 35 & $01 / 08 / 88$ & 15 \\
\hline & & & & & NR & $03 / 03 / 88$ & 36 & & NR \\
\hline & & & & & NR & $03 / 03 / 88$ & 34 & & NR \\
\hline & & & & & NR & $04 / 20 / 88$ & 32 & & NR \\
\hline
\end{tabular}


TABLE E.3. (contd)

\begin{tabular}{|c|c|c|c|c|c|c|c|c|c|}
\hline \multirow{2}{*}{\multicolumn{2}{|c|}{ CONSTITUENT }} & CONTRACTUAL & DRINKING & & & \multirow{2}{*}{\multicolumn{2}{|c|}{ SAMPLE }} & \multirow{2}{*}{\multicolumn{2}{|c|}{ SAMPLE }} \\
\hline & & DETECTION & WATER & SAMPLE & & & & & \\
\hline NAME & UNITS & LIMIT & STANDARD & DATE & $2-W 14-5$ & DATE & $2-w 14-6$ & DATE & $2-H 14-10$ \\
\hline$\ldots \ldots$ & $\cdots$ & - - - - - - & - & $\cdots$ & $\ldots$ & - - - - - & $-\cdots$ & $\ldots$ & $\cdots$ \\
\hline \multirow[t]{4}{*}{ FZINC } & $\mathrm{PPB}$ & 5 & & $03 / 03 / 88$ & & $03 / 03 / 88$ & & $01 / 08 / 88$ & 14 \\
\hline & & & & & NR & $03 / 03 / 88$ & & & NR \\
\hline & & & & & $N R$ & $03 / 03 / 88$ & & & NR \\
\hline & & & & & $N R$ & $04 / 20 / 88$ & & & NR \\
\hline \multirow[t]{2}{*}{ HNI IRAT } & PPB & 2500 & 45000 & $02 / 28 / 88$ & 41400 & $02 / 28 / 88$ & 15600 & $01 / 15 / 88$ & 84300 \\
\hline & & & & & NR & $04 / 20 / 88$ & 14800 & $05 / 16 / 88$ & 82500 \\
\hline IRON & $\mathrm{PPB}$ & 50 & & $03 / 03 / 88$ & 1240 & & NR & $01 / 08 / 88$ & 836 \\
\hline \multirow[t]{6}{*}{ LFLUORD } & $P P B$ & 50 & 4000 & & NR & $03 / 03 / 88$ & 3590 & & NR \\
\hline & & & & & $N R$ & $03 / 03 / 88$ & 3620 & & NR \\
\hline & & & & & NR & $03 / 03 / 88$ & 3620 & & NR \\
\hline & & & & & NR & $04 / 20 / 98$ & 2480 & & NR \\
\hline & & & & & NR & $04 / 20 / 88$ & 2630 & & NR \\
\hline & & & & & NR & $04 / 20 / 88$ & 2630 & & NR \\
\hline MAGNES & $P P B$ & 0 & & $03 / 03 / 88$ & 8740 & & NR & $01 / 08 / 88$ & 26100 \\
\hline
\end{tabular}


TABLE E.3. (contd)

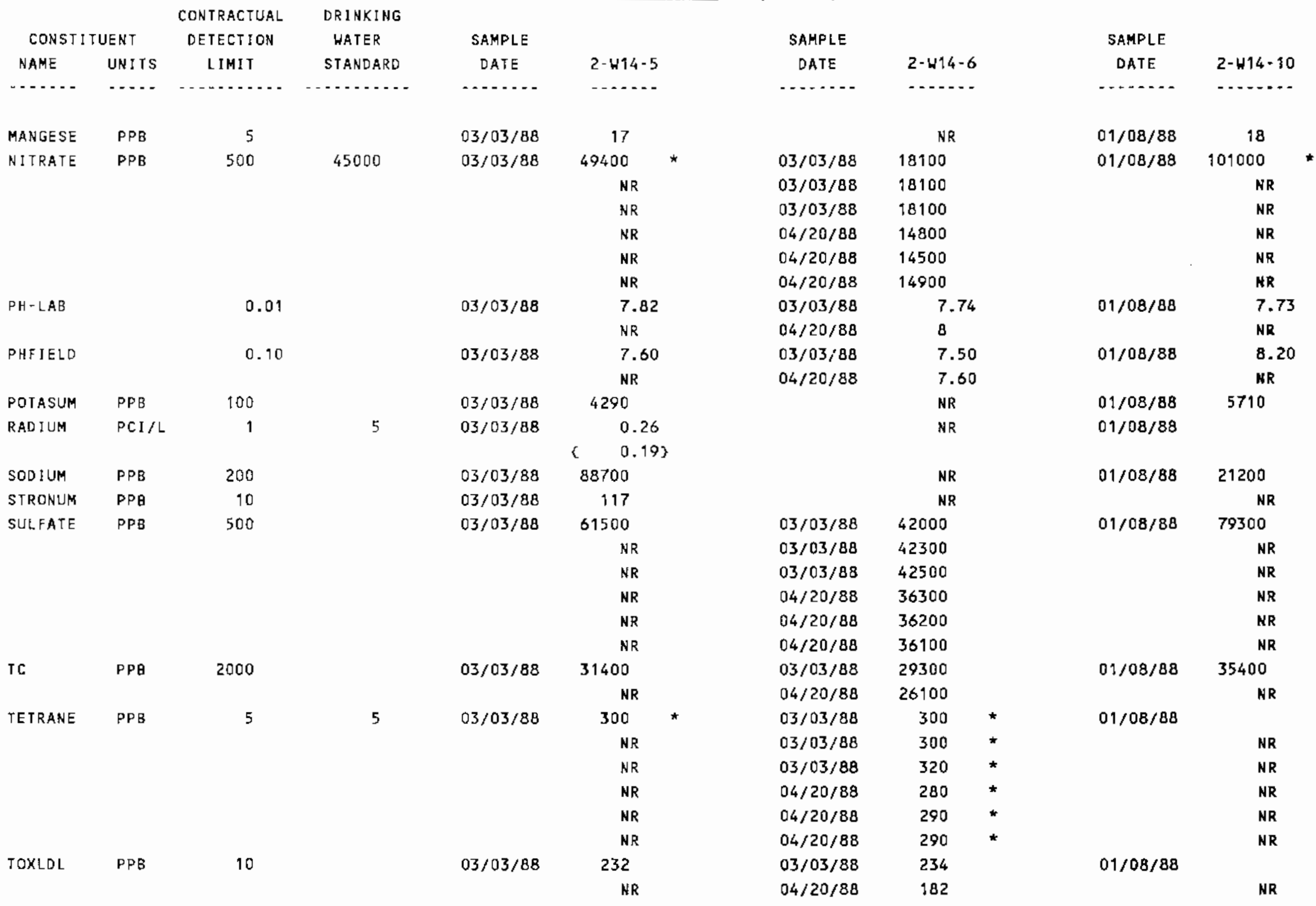


TABLE E.3. (contd)

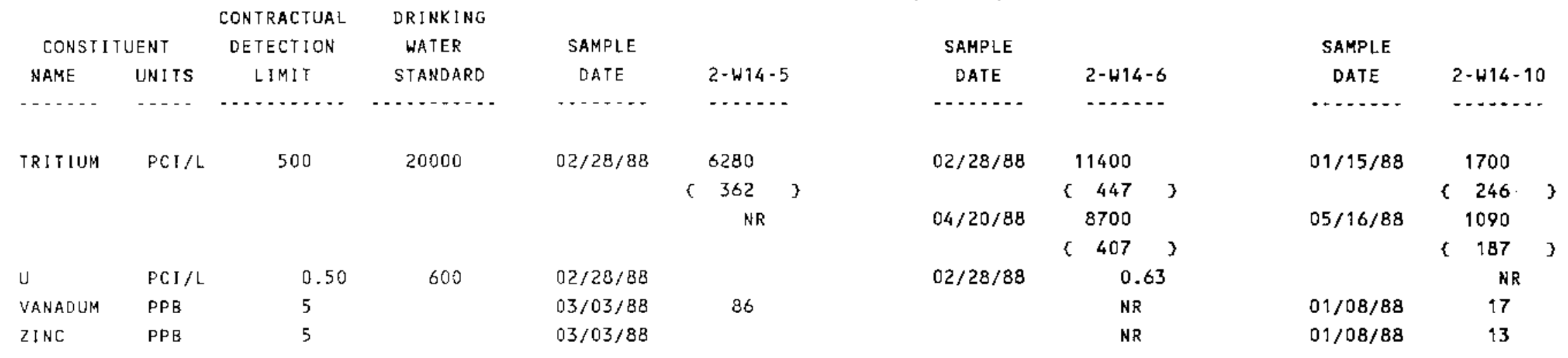




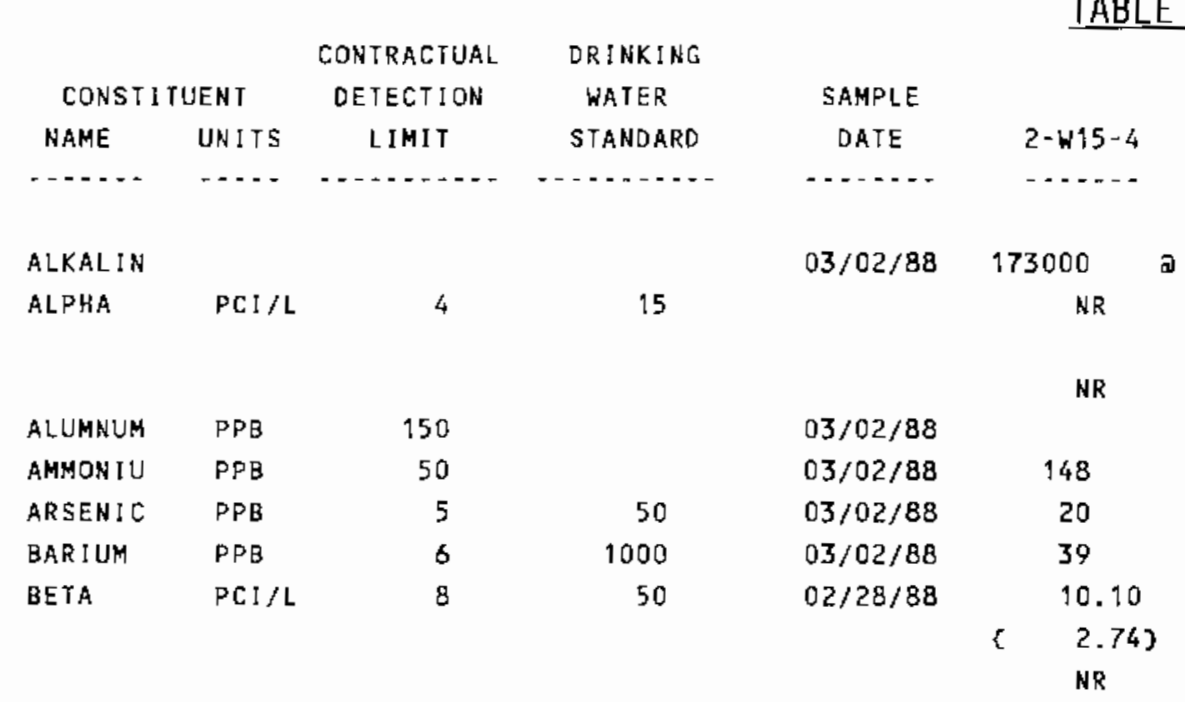

\begin{tabular}{|c|c|c|c|c|c|}
\hline CALCIUM & $\mathrm{PPB}$ & 50 & & $03 / 02 / 88$ & 22800 \\
\hline CHLFORH & PPB & 10 & 100 & $03 / 02 / 88$ & 16 \\
\hline CHLORID & $\mathrm{PPB}$ & 500 & & $03 / 02 / 88$ & 15600 \\
\hline CHROMUM & PPB & 10 & 50 & $03 / 02 / 88$ & \\
\hline$c 0-60$ & $\mathrm{PCl} / \mathrm{L}$ & 22.50 & 100 & & NR \\
\hline CONDFLD & AMHO & 1 & & $03 / 02 / 88$ & 1635 \\
\hline COPPER & PPB & 10 & $(1300.0)$ & $03 / 02 / 88$ & \\
\hline CS -137 & $P C I / L$ & 20 & 200 & & NR \\
\hline CYANIDE & PPB & 10 & & $03 / 02 / 88$ & \\
\hline FARSENI & PPB & 5 & 50 & $03 / 02 / 88$ & 24 \\
\hline FBARIUM & PPB & 6 & 1000 & $03 / 02 / 88$ & 39 \\
\hline FCALCIU & $\mathrm{PPB}$ & 50 & & $03 / 02 / 88$ & 24500 \\
\hline FLUORID & PPB & 500 & 4000 & $03 / 02 / 88$ & 12800 \\
\hline FMAGNES & PPB & 50 & & $03 / 02 / 88$ & 7050 \\
\hline FMANGAN & PPB & 5 & & $03 / 02 / 88$ & 10 \\
\hline FPOTASS & PPB & 100 & & $03 / 02 / 88$ & 5950 \\
\hline FSODIUM & PPB & 200 & & $03 / 02 / 88$ & 269000 \\
\hline FSTRONT & $P P B$ & 10 & & $03 / 02 / 88$ & 122 \\
\hline FVANADI & PPB & 5 & & $03 / 02 / 88$ & 269 \\
\hline HNITRAT & PPB & 2500 & 45000 & $02 / 28 / 88$ & 399000 \\
\hline
\end{tabular}

TABLE E. 3. (contd)

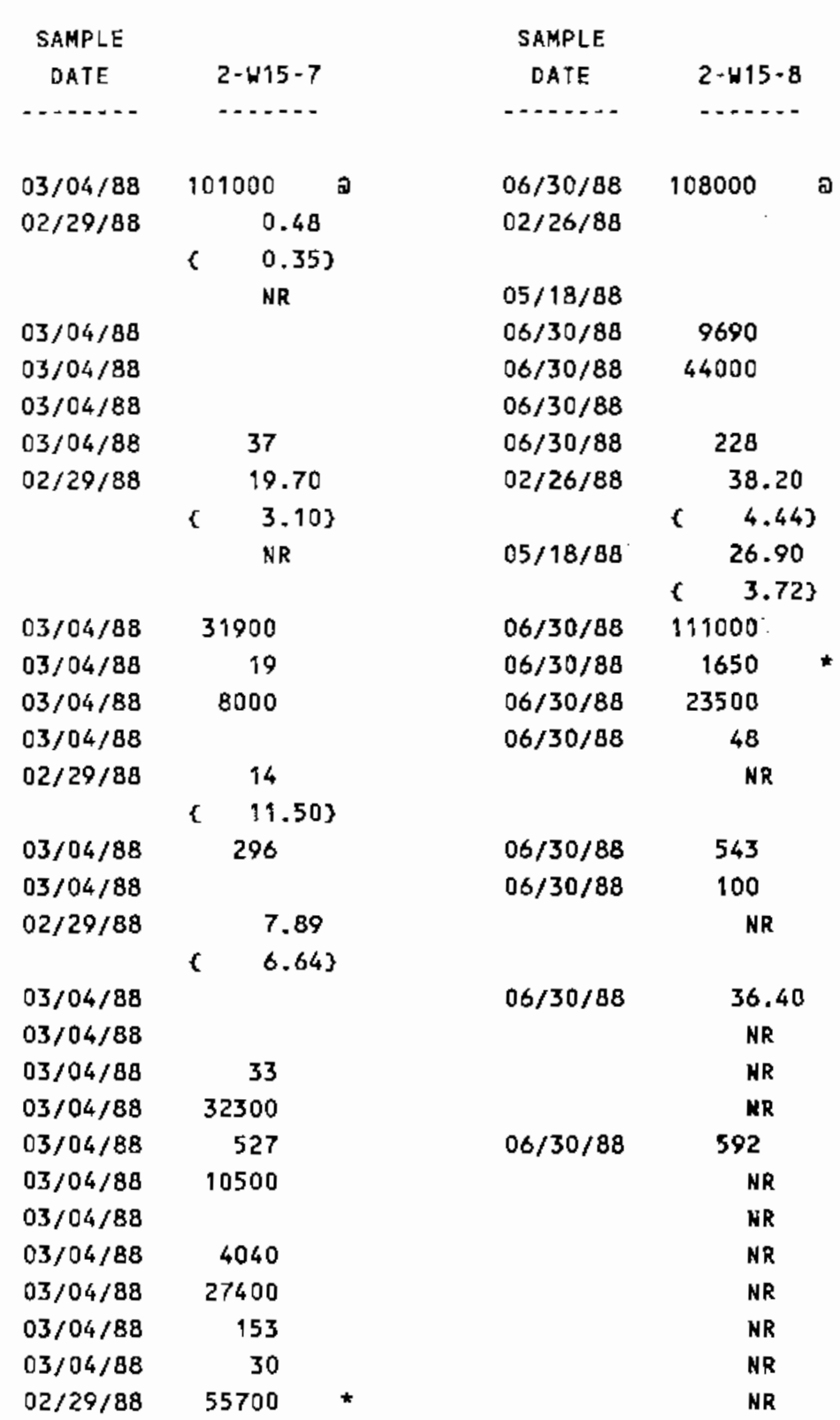


TABLE E.3. (contd)

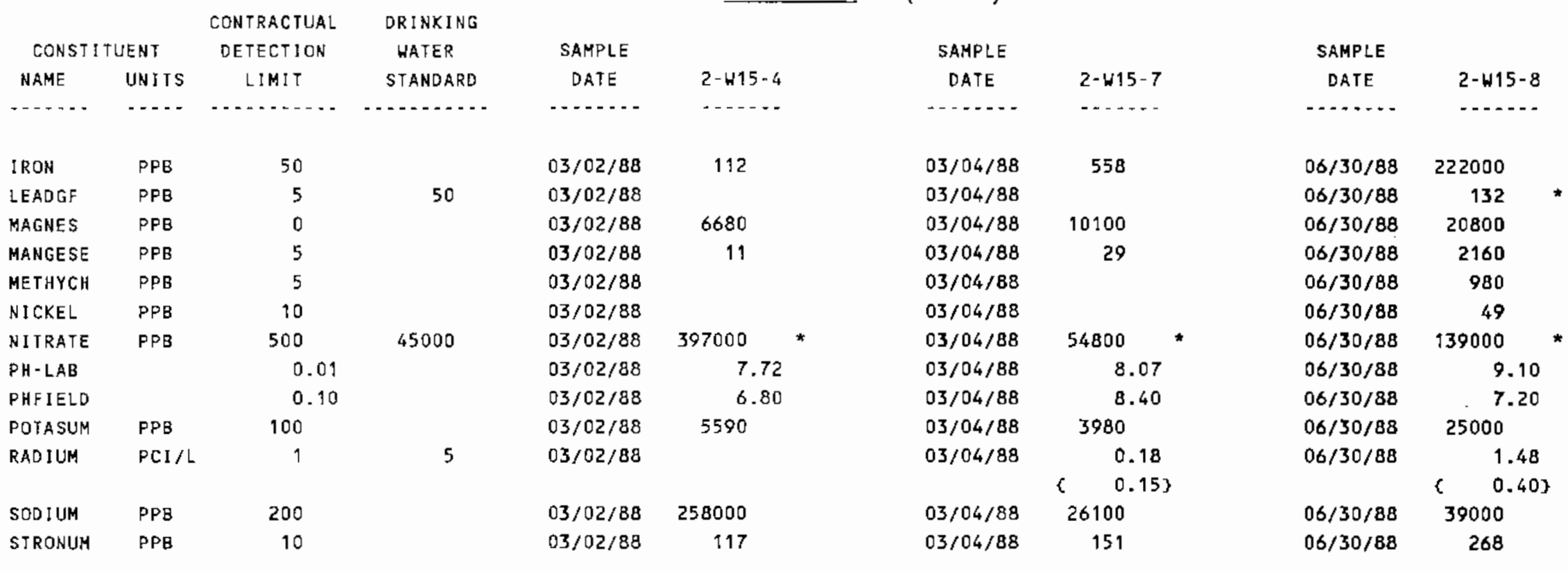


TABLE E. 3 . (contd)

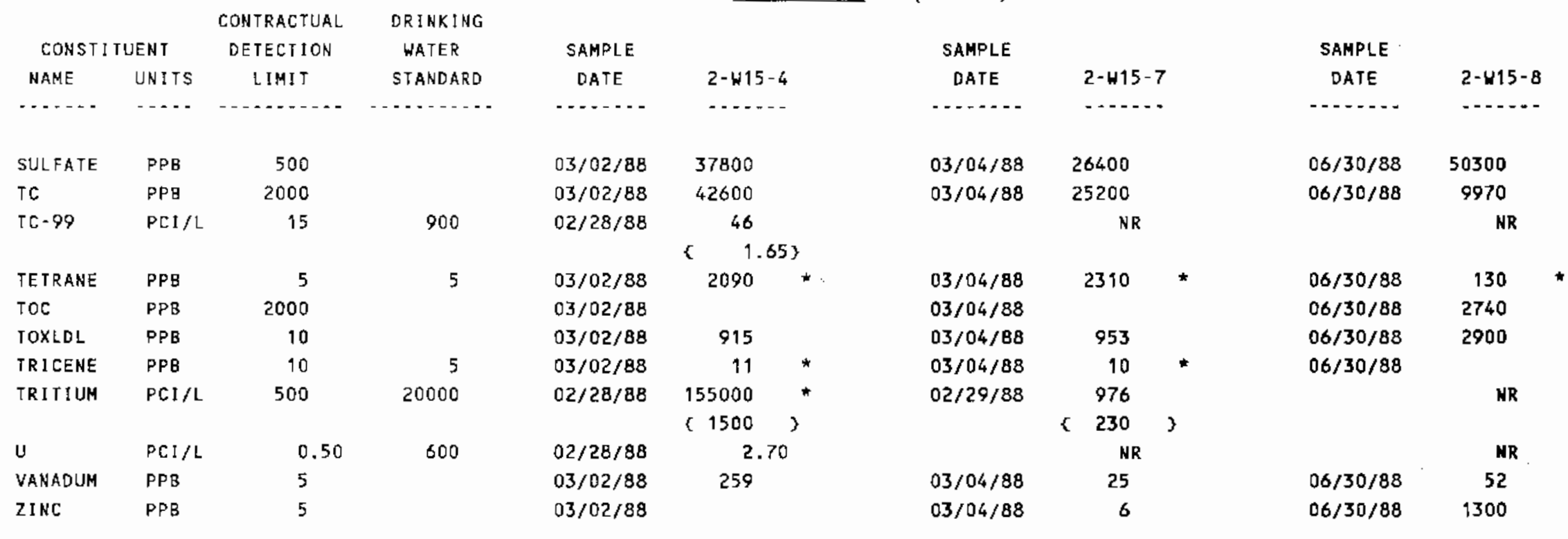


IABLE E. 3 . (contd)

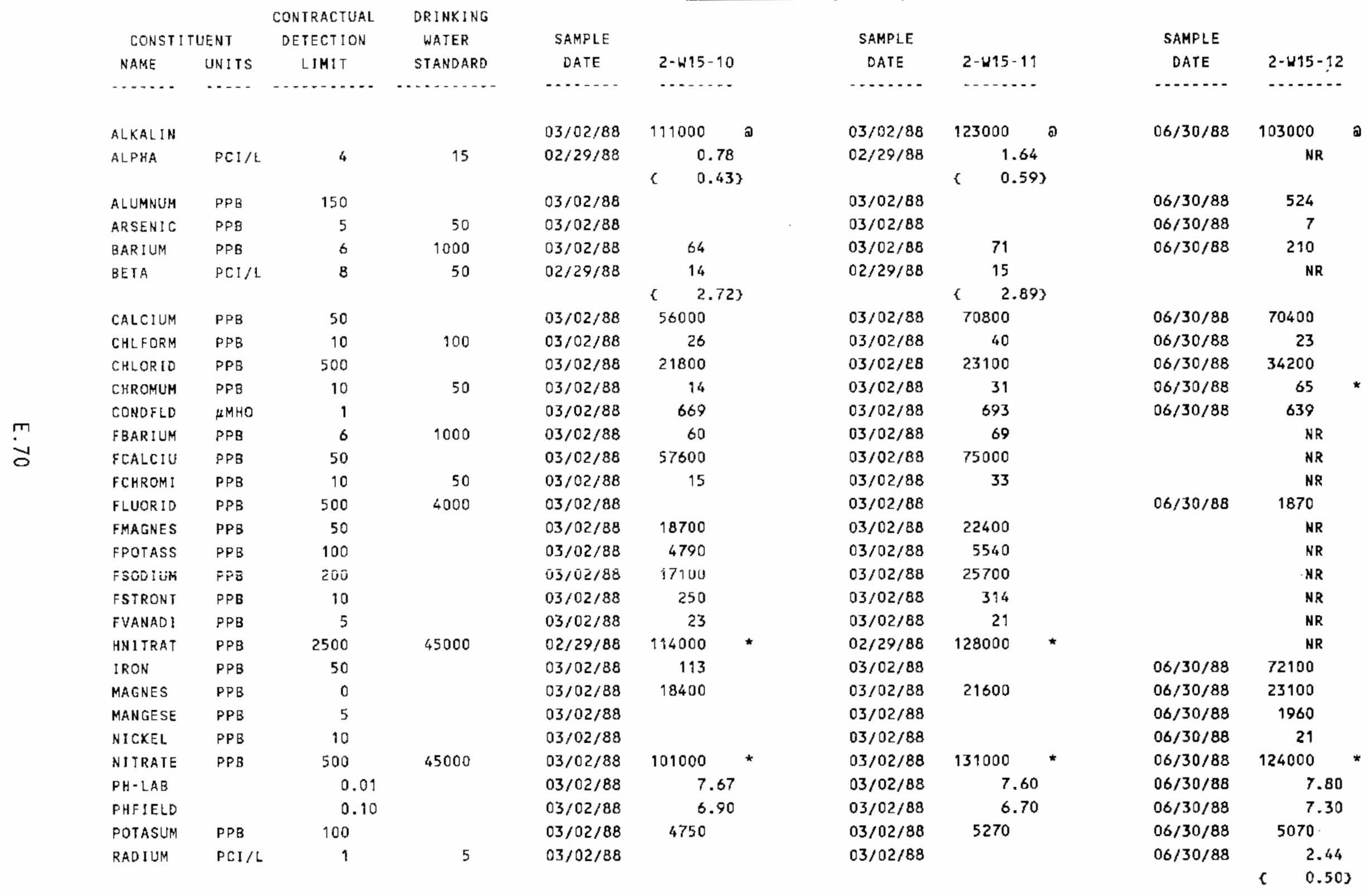


TABLE E.3. (contd)

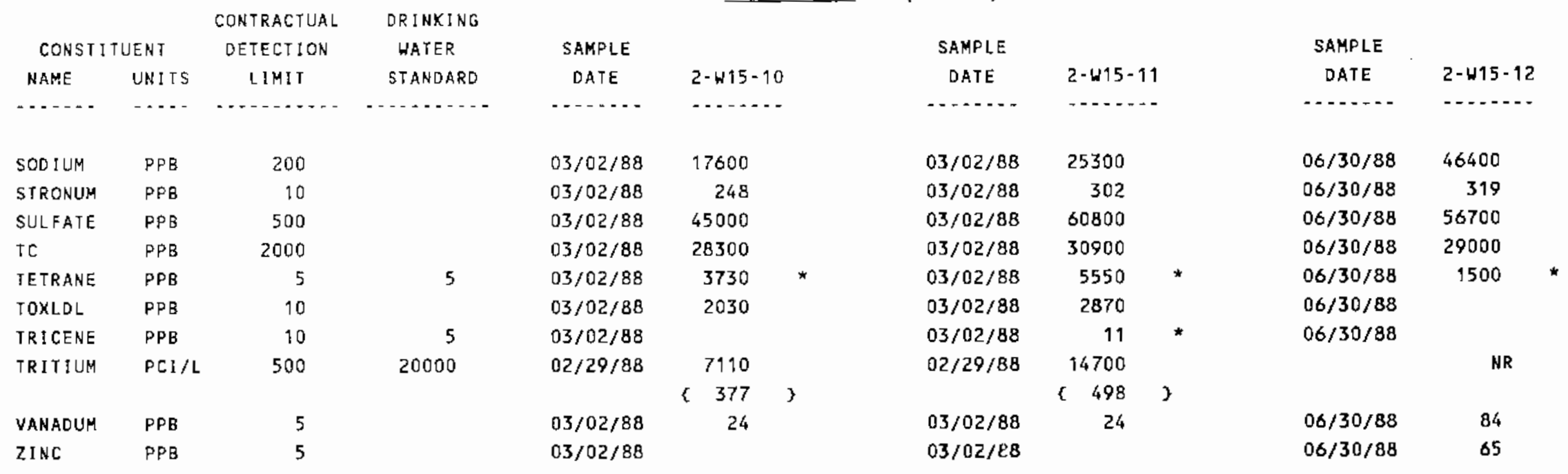


TABLE E.3. (contd)

\begin{tabular}{|c|c|c|c|c|c|c|c|c|c|c|}
\hline \multicolumn{2}{|c|}{ CONSTITUENT } & $\begin{array}{l}\text { CONTRACTUAL } \\
\text { OETECTION }\end{array}$ & $\begin{array}{l}\text { DRINKING } \\
\text { WATER }\end{array}$ & SAMPLE & \multirow{2}{*}{\multicolumn{2}{|c|}{$2-W 18-4$}} & \multicolumn{2}{|l|}{ SAMPLE } & SAMPLE & \multirow[b]{2}{*}{$2-W 18-9$} \\
\hline NAME & UNITS & LIMIT & STANDARD & DATE & & & DATE & $2-418-5$ & DATE & \\
\hline …... & $\cdots$ & , n............ & $\cdots$ & $\ldots$ & $\cdots$ & & $\cdots$ & n....... & ........... & ........ \\
\hline ALKALIN & & & & $06 / 27 / 88$ & 109000 & a & $03 / 17 / 88$ & 107000 & $06 / 27 / 88$ & 67700 \\
\hline \multirow[t]{3}{*}{ ALPHA } & $P C I / L$ & 4 & 15 & & NR & & $02 / 22 / 88$ & 1.02 & $02 / 22 / 88$ & \\
\hline & & & & & & & & 0.493 & & \\
\hline & & & & & NR & & & NR & $05 / 18 / 88$ & \\
\hline ALUMNUM & $P P B$ & 150 & & $06 / 27 / 88$ & 555 & & $03 / 17 / 88$ & & $06 / 27 / 88$ & 839 \\
\hline AMMONIU & PPB & 50 & & $06 / 27 / 88$ & 15000 & & $03 / 17 / 88$ & 420 & $06 / 27 / 88$ & 58 \\
\hline BARIUM & PPB & 6 & 1000 & $06 / 27 / 88$ & 34 & & $03 / 17 / 88$ & 75 & $06 / 27 / 88$ & 28 \\
\hline \multirow[t]{4}{*}{ BETA } & $\mathrm{PCI} / \mathrm{L}$ & 8 & 50 & & NR & & $02 / 22 / 88$ & 6.95 & $02 / 22 / 88$ & \\
\hline & & & & & & & & $2.26)$ & & \\
\hline & & & & & NR & & & $\mathrm{NR}$ & $05 / 18 / 88$ & 4.54 \\
\hline & & & & & & & & & & 1.873 \\
\hline CADHIUM & PPB & 2 & 10 & $06 / 27 / 88$ & 3 & & $03 / 17 / 88$ & & $06 / 27 / 88$ & 5 \\
\hline CALCIUM & PPB & 50 & & $06 / 27 / 88$ & 17200 & & $03 / 17 / 88$ & 63300 & $06 / 27 / 88$ & 18300 \\
\hline CHLFORH & $\mathrm{PPB}$ & 10 & 100 & $06 / 27 / 88$ & 510 & * & $03 / 17 / 88$ & 28 & $06 / 27 / 88$ & 19 \\
\hline CHLORID & $\mathrm{PPB}$ & 500 & & $06 / 27 / 88$ & 9580 & & $03 / 17 / 88$ & 4970 & $06 / 27 / 88$ & 4410 \\
\hline CHROMUM & PPB & 10 & 50 & $06 / 27 / 88$ & 22 & & $03 / 17 / 88$ & 21 & $06 / 27 / 88$ & 28 \\
\hline CONDFLD & «MHO & 1 & & $06 / 27 / 88$ & 347 & & $03 / 17 / 88$ & 760 & $06 / 27 / 88$ & 179 \\
\hline COPPER & $P P B$ & 10 & $(1300.0)$ & $06 / 27 / 88$ & & & $03 / 17 / 88$ & & $06 / 27 / 88$ & 14 \\
\hline FBARIUM & PPB & 6 & 1000 & & NR & & $03 / 17 / 88$ & 69 & & NR \\
\hline ICALGIU & FPB & 50 & & & NR & & $03 / 17 / 88$ & 64300 & & NR \\
\hline FCHROMI & PPB & 10 & 50 & & $N R$ & & $03 / 17 / 88$ & 14 & & NR \\
\hline F COPPER & PPB & 10 & $(1300.0)$ & & NR & & $03 / 17 / 88$ & 10 & & HR \\
\hline FIRON & PPB & 50 & & & NR & & $03 / 17 / 88$ & 54 & & NR \\
\hline FLUORID & $\mathrm{PPB}$ & 500 & 4000 & $06 / 27 / 88$ & & & $03 / 17 / 88$ & 622 & $06 / 27 / 88$ & \\
\hline FMAGNES & PPB & 50 & & & NR & & $03 / 17 / 88$ & 18600 & & MR \\
\hline FPOTASS & PPB & 100 & & & $\mathrm{NR}$ & & $03 / 17 / 88$ & 4820 & & NR \\
\hline FSODIUM & PPB & 200 & & & NR & & $03 / 17 / 88$ & 51100 & & $\mathrm{NR}$ \\
\hline FSTRONT & PPB & 10 & & & NR & & $03 / 17 / 88$ & 243 & & NR \\
\hline FVANADI & PPB & 5 & & & $N R$ & & $03 / 17 / 88$ & 25 & & NR \\
\hline FZINC & $\mathrm{PPB}$ & 5 & & & $\mathrm{NR}$ & & $03 / 17 / 88$ & 6 & & NR \\
\hline \multirow[t]{2}{*}{ HNITRAT } & $\mathrm{PPB}$ & 2500 & 45000 & & NR & & & NR & $02 / 22 / 88$ & 8080 \\
\hline & & & & & NR & & & NR & $05 / 18 / 88$ & 3670 \\
\hline IRON & PPB & 50 & & $06 / 27 / 88$ & 7350 & & $03 / 17 / 88$ & 1970 & $06 / 27 / 88$ & 27300 \\
\hline LEADGF & $P P B$ & 5 & 50 & $06 / 27 / 88$ & 32.60 & & $03 / 17 / 88$ & & $06 / 27 / 88$ & 18.90 \\
\hline
\end{tabular}


IABLE E.3. (contd)

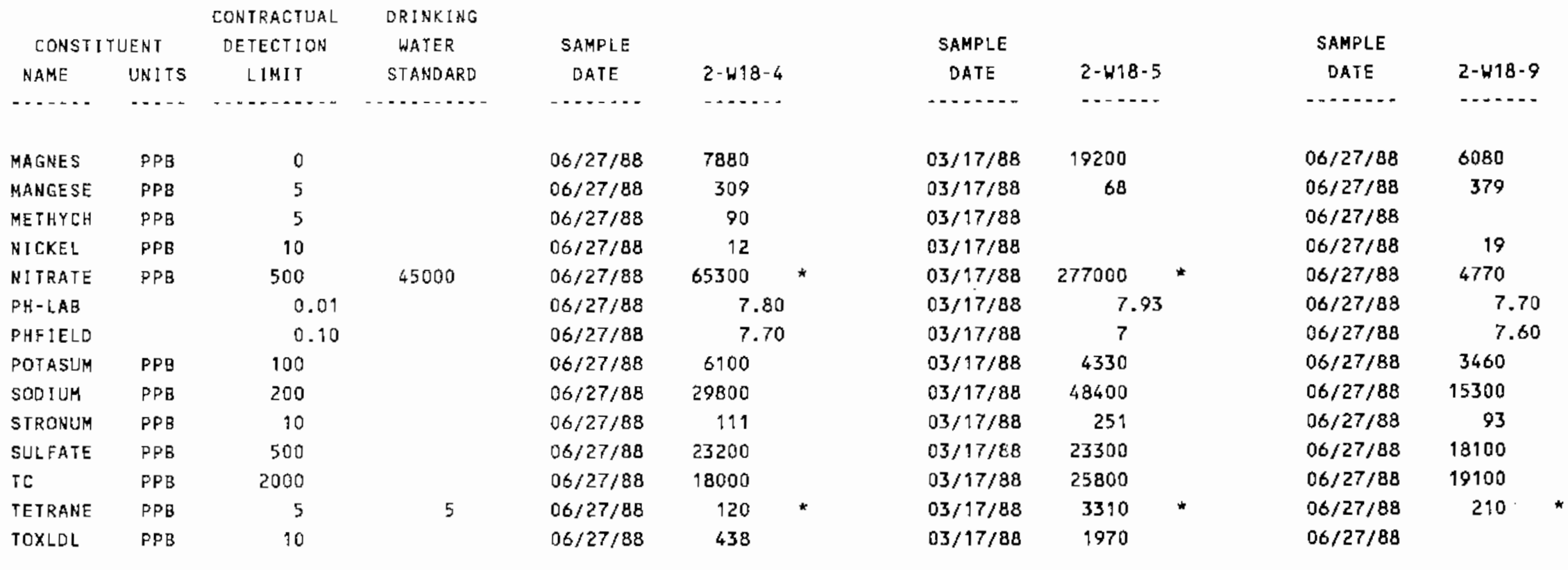


TABLE E.3. (contd)

\begin{tabular}{|c|c|c|c|c|c|c|c|c|c|}
\hline \multicolumn{2}{|c|}{ CONSTI TUENT } & $\begin{array}{l}\text { CONTRACTUAL } \\
\text { DETECTIONN }\end{array}$ & $\begin{array}{l}\text { DRINKING } \\
\text { WATER }\end{array}$ & SAMPLE & & SAMPLE & & SAMPLE & \\
\hline NAME & UN! TS & LIMIT & STANDARD & DATE & $2 \cdot w 18-4$ & DATE & $2-618-5$ & DATE & $2-418-9$ \\
\hline - . . . & $\cdots$ & 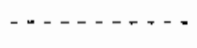 & 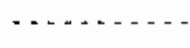 & $\ldots$ & ...... & $\cdots$ & $\ldots$ & - - - - & ....... \\
\hline VANADUM & PPB & 5 & & $06 / 27 / 88$ & 10 & $03 / 17 / 88$ & 32 & $06 / 27 / 88$ & 34 \\
\hline ZINC & $\mathrm{PPB}$ & 5 & & $06 / 27 / 88$ & 5610 & $03 / 17 / 88$ & 9 & $06 / 27 / 88$ & 7380 \\
\hline
\end{tabular}


TABLE E.3. (contd)

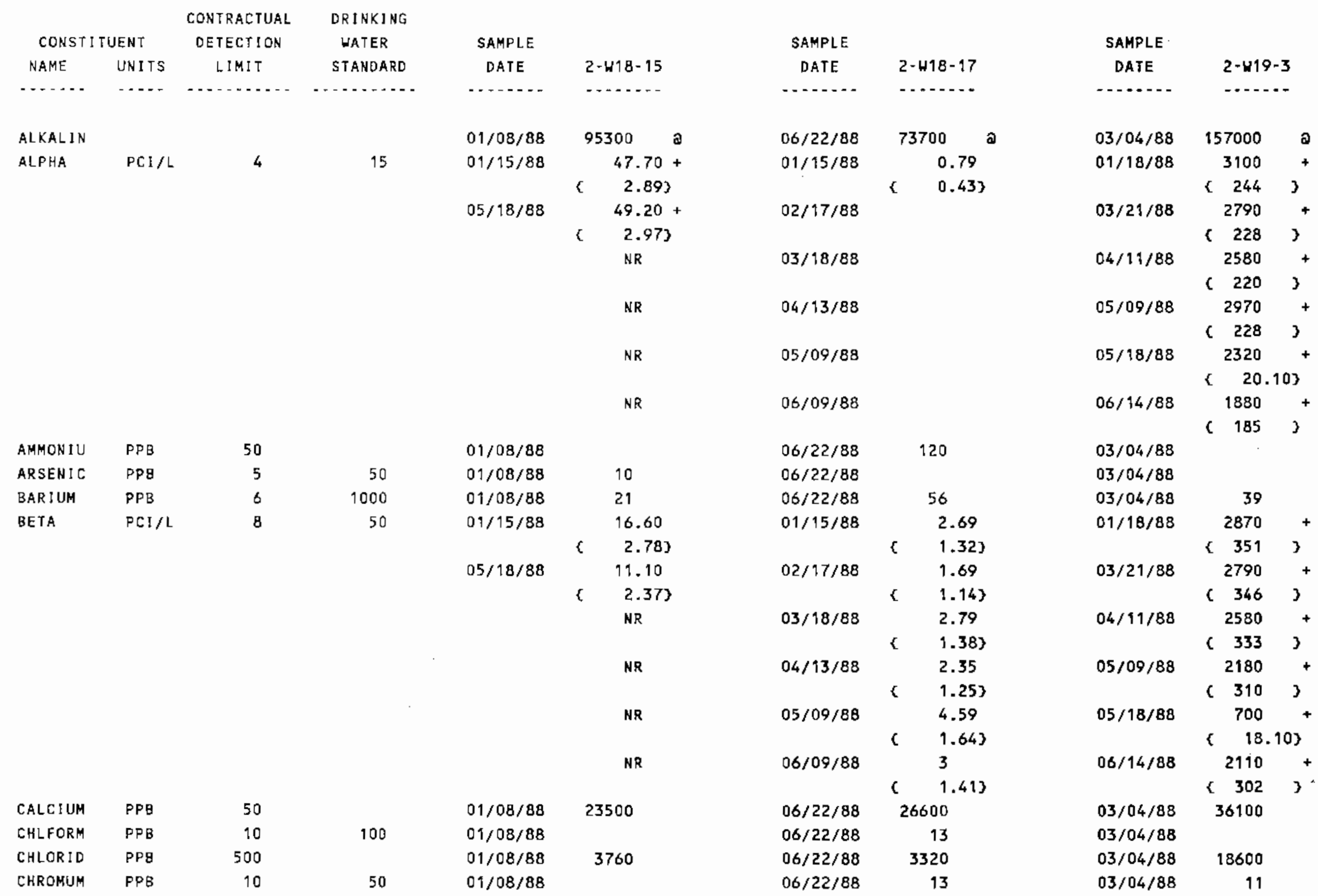


TABLE E.3. (contd)

\begin{tabular}{|c|c|c|c|c|c|c|c|c|c|}
\hline \multicolumn{2}{|c|}{ CONST I TUENT } & $\begin{array}{c}\text { CONTRACTUAL } \\
\text { DETECTION }\end{array}$ & $\begin{array}{l}\text { DRINKING } \\
\text { WATER }\end{array}$ & SAMPLE & \multirow[b]{2}{*}{$2-H \uparrow B-15$} & \multicolumn{2}{|l|}{ SAMPLE } & \multicolumn{2}{|l|}{ SAMPLE } \\
\hline NAME & UNITS & LIMIT & STAMDARD & DATE & & DATE & 2-W18-17 & DATE & $2-499-3$ \\
\hline$\cdots$ & $\cdots$ & $\cdots \cdots$ & - - - - - - - - - & $\ldots$ & $\cdots$ & $-\ldots$ & 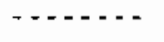 & $\ldots$ & $-\cdots$ \\
\hline \multirow[t]{7}{*}{$\mathrm{co-}-60$} & $\mathrm{PCl} / \mathrm{L}$ & 22.50 & 100 & $01 / 15 / 88$ & & $01 / 15 / 88$ & & $01 / 18 / 88$ & \\
\hline & & & & $05 / 18 / 88$ & 6.83 & $04 / 13 / 88$ & & $03 / 21 / 88$ & \\
\hline & & & & & $5.57\}$ & & & & \\
\hline & & & & & NR & & NR & $04 / 11 / 88$ & \\
\hline & & & & & NR & & NR & $05 / 09 / 88$ & \\
\hline & & & & & NR & & NR & $05 / 18 / 88$ & \\
\hline & & & & & NR & & NR & $06 / 14 / 88$ & \\
\hline COND FLD & HMHO & 1 & & $01 / 08 / 88$ & & $06 / 22 / 88$ & 183 & $03 / 04 / 88$ & 403 \\
\hline FARSEN I & PPB & 5 & 50 & $01 / 08 / 88$ & 12 & & NR & $03 / 04 / 88$ & \\
\hline FBAR IUM & PPB & 6 & 1000 & $01 / 08 / 88$ & 20 & & NR & $03 / 04 / 88$ & 38 \\
\hline FCALCIU & PPB & 50 & & $01 / 08 / 88$ & 21000 & & NR & $03 / 04 / 88$ & 33600 \\
\hline FCHROHI & $P P B$ & 10 & 50 & $01 / 08 / 88$ & & & $N R$ & $03 / 04 / 88$ & 10 \\
\hline FLUORID & PPB & 500 & 4000 & $01 / 08 / 88$ & & $06 / 22 / 88$ & & $03 / 04 / 88$ & 524 \\
\hline FMAGNES & PPB & 50 & & $01 / 08 / 88$ & 8070 & & NR & $03 / 04 / 88$ & 10100 \\
\hline FPOTASS & PPB & 100 & & $01 / 08 / 88$ & 3400 & & NR & $03 / 04 / 88$ & 3930 \\
\hline F SOD IUM & PPB & 200 & & $01 / 08 / 88$ & 11300 & & NR & $03 / 04 / 88$ & 62900 \\
\hline
\end{tabular}


TABLE E.3. (contd)

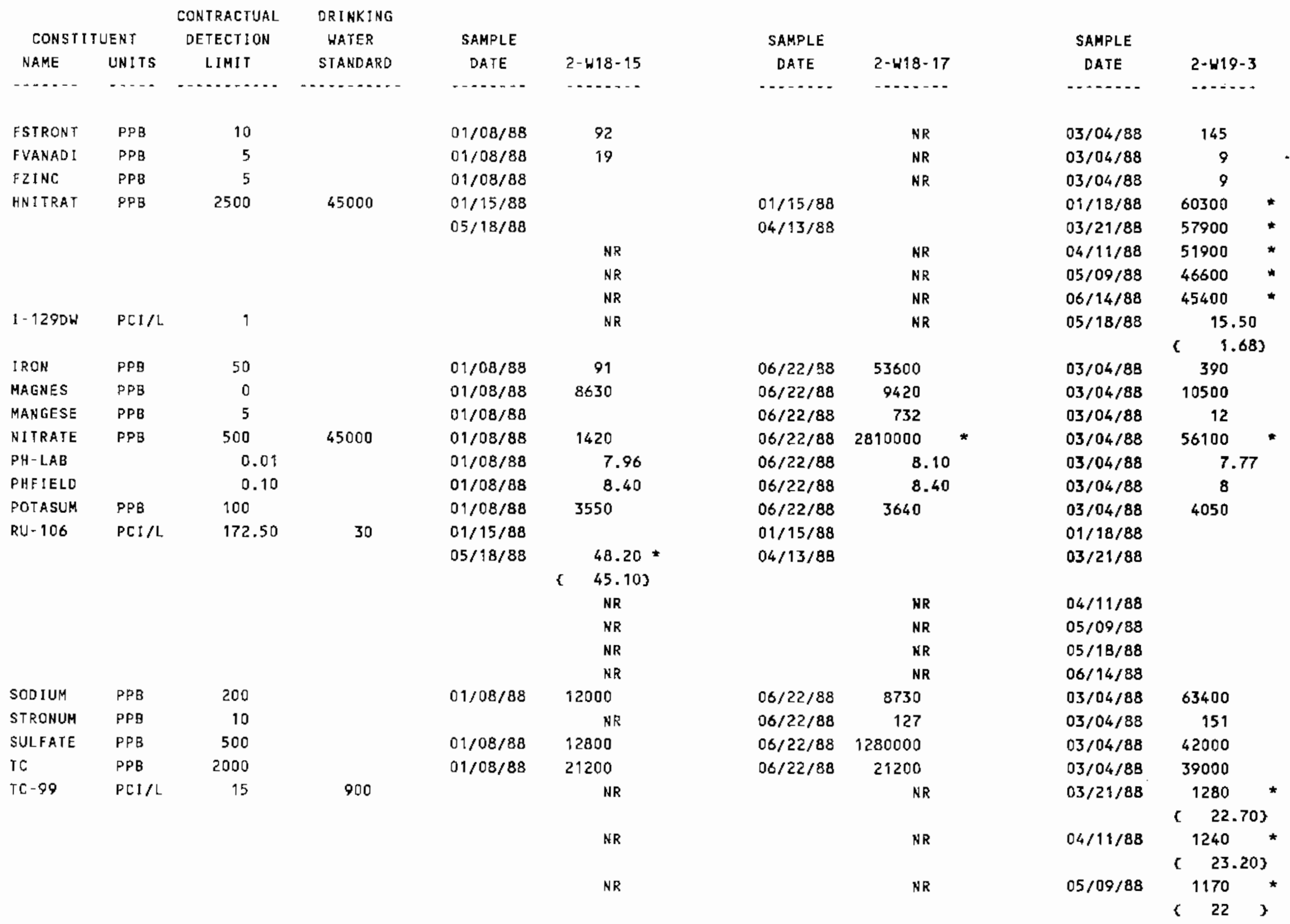


TABLE E.3. (contd)

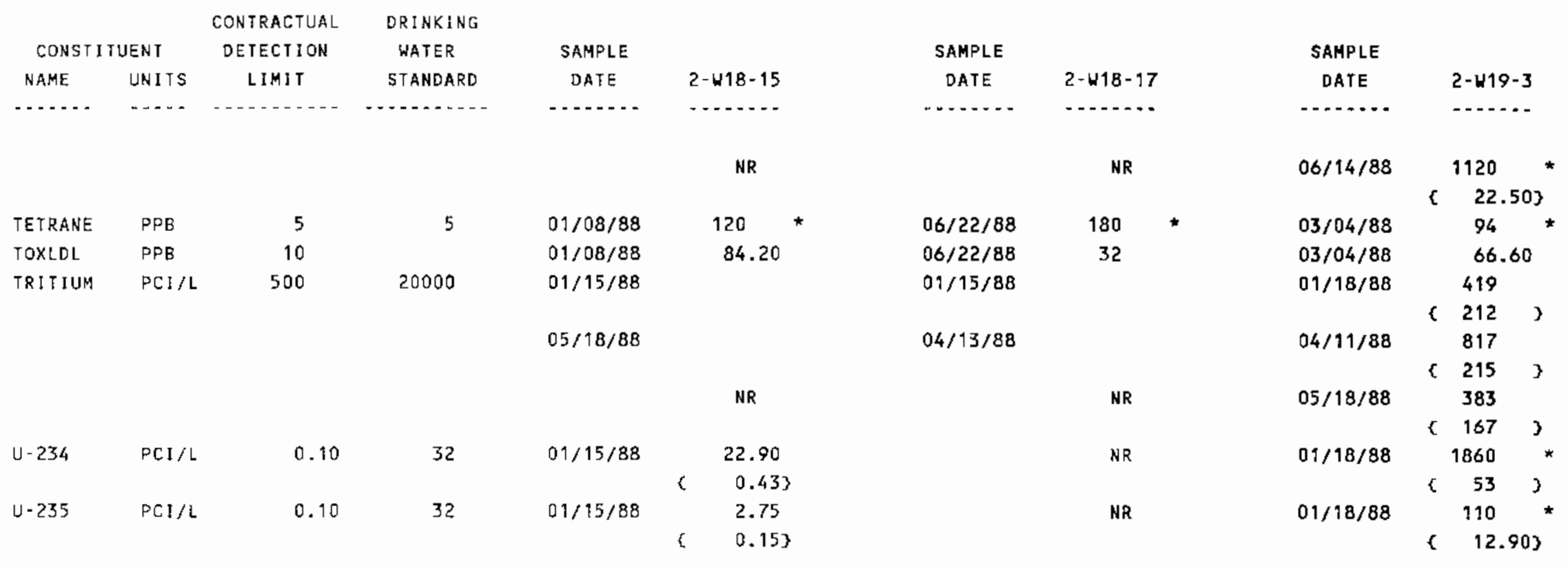


TABLE E.3. (contd)

\begin{tabular}{|c|c|c|c|c|c|c|c|c|c|}
\hline \multicolumn{2}{|c|}{ CONST I TUENT } & $\begin{array}{c}\text { CONTRACTUAL } \\
\text { DETECTION }\end{array}$ & $\begin{array}{l}\text { DRINKING } \\
\text { WATER }\end{array}$ & SAMPLE & \multirow[b]{2}{*}{$2-W 18-15$} & \multirow{2}{*}{$\begin{array}{c}\text { SAMPLE } \\
\text { DATE }\end{array}$} & \multicolumn{3}{|c|}{ SAMPLE } \\
\hline NAME & UNIIS & LIMIT & STANDARD & DATE & & & $2-W 18-17$ & DATE & $2 \cdot 419 \cdot 3$ \\
\hline$\ldots . .$. & $\cdots$ & $\cdots$ & $\ldots$ & $\ldots$ & - & ........ & ......... & 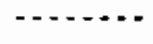 & ........ \\
\hline \multirow[t]{2}{*}{$U-238$} & $\mathrm{PCl} / \mathrm{L}$ & 0.10 & 4.80 & $01 / 15 / 88$ & $24.50 *$ & & $\mathrm{NR}$ & $01 / 18 / 88$ & 1910 \\
\hline & & & & & 0.443 & & & & $(53.60)$ \\
\hline \multirow[t]{5}{*}{$U$-CHEM } & $U G / L$ & 0.73 & & $01 / 15 / 88$ & 56.50 & & NR & $01 / 18 / 88$ & 5150 \\
\hline & & & & $05 / 18 / 88$ & 63.20 & & NR & $03 / 21 / B B$ & 3630 \\
\hline & & & & & NR & & NR & $04 / 11 / 88$ & 3930 \\
\hline & & & & & NR & & KR & $05 / 09 / 88$ & 2750 \\
\hline & & & & & NR & & NR & $06 / 14 / 88$ & 3270 \\
\hline VANADUM & PPB & 5 & & $01 / 08 / 88$ & 21 & $06 / 22 / 88$ & 100 & $03 / 04 / 88$ & 16 \\
\hline ZINC & PPB & 5 & & $01 / 08 / 88$ & & $06 / 22 / 88$ & 19 & $03 / 04 / 88$ & 18 \\
\hline
\end{tabular}


TABLE E.3. (contd)

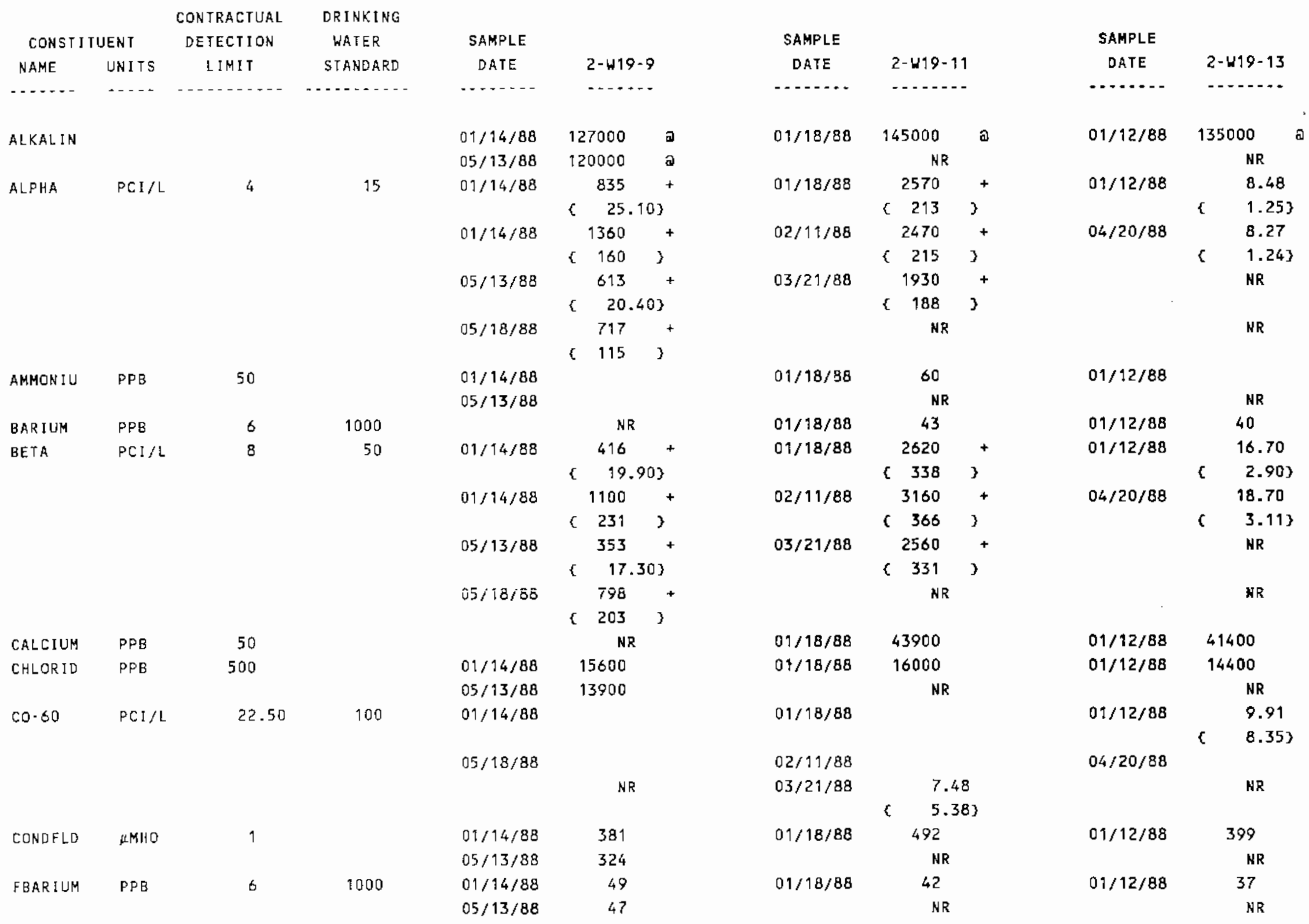


TABLE E.3. (contd)

\begin{tabular}{|c|c|c|c|c|c|c|c|c|c|}
\hline \multicolumn{2}{|c|}{ CONSTI TUENT } & $\begin{array}{l}\text { CONTRACTUAL } \\
\text { DETECTION }\end{array}$ & $\begin{array}{l}\text { DRINKING } \\
\text { WATER }\end{array}$ & SAMPLE & & \multicolumn{2}{|l|}{ SAMPLE } & \multicolumn{2}{|l|}{ SAMPLE } \\
\hline NAME & UNITS & LIMIT & STANDARD & DATE & $2-\$ 19-9$ & DATE & $2-w 19-11$ & DATE & $2-419 \cdot 13$ \\
\hline - n...... & ..... & n............. & - & .......... & ….... & .......... & ........ & - & - n. \\
\hline \multirow[t]{2}{*}{ FCALCIU } & PPB & 50 & & $01 / 14 / 88$ & 33200 & $01 / 18 / 88$ & 45200 & $01 / 12 / 88$ & 40800 \\
\hline & & & & $05 / 13 / 88$ & 31600 & & NR & & NR \\
\hline \multirow[t]{2}{*}{ FLUORID } & $P P B$ & 500 & 4000 & $01 / 14 / 88$ & 756 & $01 / 18 / 88$ & 667 & $01 / 12 / 88$ & 830 \\
\hline & & & & $05 / 13 / 88$ & 750 & & NR & & NR \\
\hline \multirow[t]{2}{*}{ FMAGNES } & PPB & 50 & & $01 / 14 / 88$ & 10500 & $01 / 18 / 88$ & 13600 & $01 / 12 / 88$ & 12900 \\
\hline & & & & $05 / 13 / 88$ & 9920 & & NR & & NR \\
\hline \multirow[t]{2}{*}{ FMANGAN } & PPB & 5 & & $01 / 14 / 88$ & & $01 / 18 / 88$ & & $01 / 12 / 88$ & \\
\hline & & & & $05 / 13 / 88$ & 8 & & NR & & NR \\
\hline \multirow[t]{2}{*}{ FPOTASS } & PPB & 100 & & $01 / 14 / 88$ & 3820 & $01 / 18 / 88$ & 4410 & $01 / 12 / 88$ & 4600 \\
\hline & & & & $05 / 13 / 88$ & 3650 & & NR & & HR \\
\hline \multirow[t]{2}{*}{ FSODIUM } & PPB & 200 & & $01 / 14 / 88$ & 35000 & $01 / 18 / 88$ & 48100 & $01 / 12 / 88$ & 26400 \\
\hline & & & & $05 / 13 / 88$ & 30900 & & NR & & KR \\
\hline \multirow[t]{2}{*}{ FSTRONT } & PPB & 10 & & $01 / 14 / 88$ & 148 & $01 / 18 / 88$ & 188 & $01 / 12 / 88$ & 182 \\
\hline & & & & $05 / 13 / 88$ & 133 & & NR & & NR \\
\hline FVANAD I & PPB & 5 & & $01 / 14 / 88$ & 16 & $01 / 18 / 88$ & 12 & $01 / 12 / 88$ & 26 \\
\hline
\end{tabular}


TABLE E.3. (contd)

\begin{tabular}{|c|c|c|c|c|c|c|c|c|c|}
\hline CONSTI & TUENT & $\begin{array}{l}\text { CONTRACTUAL } \\
\text { DETECTION }\end{array}$ & $\begin{array}{l}\text { DRINKING } \\
\text { WATER }\end{array}$ & SAMPLE & & SAMPLE & & SAMPLE & \\
\hline NAME & UNITS & LIMIT & STANDARD & DATE & $2-$ W19-9 & DATE & $2-W 19-11$ & DATE & $2-W 19-13$ \\
\hline - n..... & $\cdots$ & $\cdots$ & $-\ldots \ldots$ & 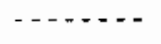 & - - n & ......... & -........ & $\ldots . .$. & ......... \\
\hline FVANADI & $\mathrm{PPB}$ & 5 & & $05 / 13 / 88$ & 22 & & NR & & NR \\
\hline FZINC & $\mathrm{PPB}$ & 5 & & $01 / 14 / 88$ & 35 & $01 / 18 / 88$ & 9 & $01 / 12 / 88$ & 7 \\
\hline & & & & $05 / 13 / 88$ & 44 & & NR & & NR \\
\hline HNITRAT & $P P B$ & 2500 & 45000 & $01 / 14 / 88$ & 36000 & $01 / 18 / 88$ & 92100 & $01 / 12 / 88$ & 18000 \\
\hline & & & & $05 / 18 / 88$ & 18400 & $02 / 11 / 88$ & 87400 & $04 / 20 / 88$ & 20000 \\
\hline & & & & & NR & $03 / 21 / 88$ & 86200 & * & NR \\
\hline IRON & PPB & 50 & & & $\mathrm{NR}$ & $01 / 18 / 88$ & 316 & $01 / 12 / 88$ & \\
\hline LFLUORD & PPB & 50 & 4000 & $01 / 14 / 88$ & 574 & & NR & & NR \\
\hline & & & & $05 / 13 / 88$ & 604 & & NR & & NR \\
\hline MAGNES & PPB & 0 & & & NR & $01 / 18 / 88$ & 13800 & $01 / 12 / 88$ & 12800 \\
\hline NITRATE & PPB & 500 & 45000 & $01 / 14 / 88$ & 34400 & $01 / 18 / 88$ & 93100 & $01 / 12 / 88$ & 21000 \\
\hline & & & & $05 / 13 / 88$ & 19800 & & NR & & NR \\
\hline$P H-L A B$ & & 0.01 & & $01 / 14 / 88$ & 7.76 & $01 / 18 / 88$ & 7.87 & $01 / 12 / 88$ & 7.68 \\
\hline & & & & $05 / 13 / 88$ & 8.10 & & NR & & NR \\
\hline PHFIELD & & 0.10 & & $01 / 14 / 88$ & 8.50 & $01 / 18 / 88$ & 7.50 & $01 / 12 / 88$ & 7.80 \\
\hline & & & & $05 / 13 / 88$ & 7.90 & & NR & & NR \\
\hline POTASUM & PPB & 100 & & & NR & $01 / 18 / 88$ & 4970 & $01 / 12 / 88$ & 4400 \\
\hline SODIUM & PPB & 200 & & & NR & $01 / 18 / 88$ & 53000 & $01 / 12 / 88$ & 25600 \\
\hline STRONUM & PPB & 10 & & & NR & $01 / 18 / 88$ & 194 & $01 / 12 / 88$ & 179 \\
\hline SULtATE & fro & 500 & & 0ิ1/i4/80 & Żं & $01 / 18 / 88$ & 38400 & $01 / 12 / 88$ & 38800 \\
\hline & & & & $05 / 13 / 88$ & 27100 & & NR & & NR \\
\hline IC & PPB & 2000 & & $01 / 14 / 88$ & 29200 & $01 / 18 / 88$ & 33400 & $01 / 12 / 88$ & 31300 \\
\hline & & & & $05 / 13 / 88$ & 27600 & & NR & & NR \\
\hline$T C-99$ & $\mathrm{PCI/L}$ & 15 & 900 & $01 / 14 / 88$ & 1020 & $01 / 18 / 88$ & 355 & & NR \\
\hline & & & & & $(21.10\}$ & & 3.543 & & \\
\hline & & & & $05 / 18 / 88$ & 348 & $02 / 11 / 88$ & 2870 & * & NR \\
\hline & & & & & $\{15.10\}$ & & \& 32 & 3 & \\
\hline & & & & & NR & $03 / 21 / 88$ & 2080 & * & NR \\
\hline & & & & & & & $\{\quad 28.40\}$ & & \\
\hline TETRANE & $P P B$ & 5 & 5 & $01 / 14 / 88$ & 99 & $01 / 18 / 88$ & 115 & $01 / 12 / 88$ & 33 \\
\hline & & & & $05 / 13 / 8 B$ & 110 & * & NR & & $\mathrm{NR}$ \\
\hline TOXLDL & $\mathrm{PPB}$ & 10 & & $01 / 14 / 88$ & 81.10 & $01 / 18 / 88$ & 110 & $01 / 12 / 88$ & \\
\hline & & & & $05 / 13 / 88$ & 41.20 & & NR & & NR \\
\hline
\end{tabular}


IABLE E.3. (contd)

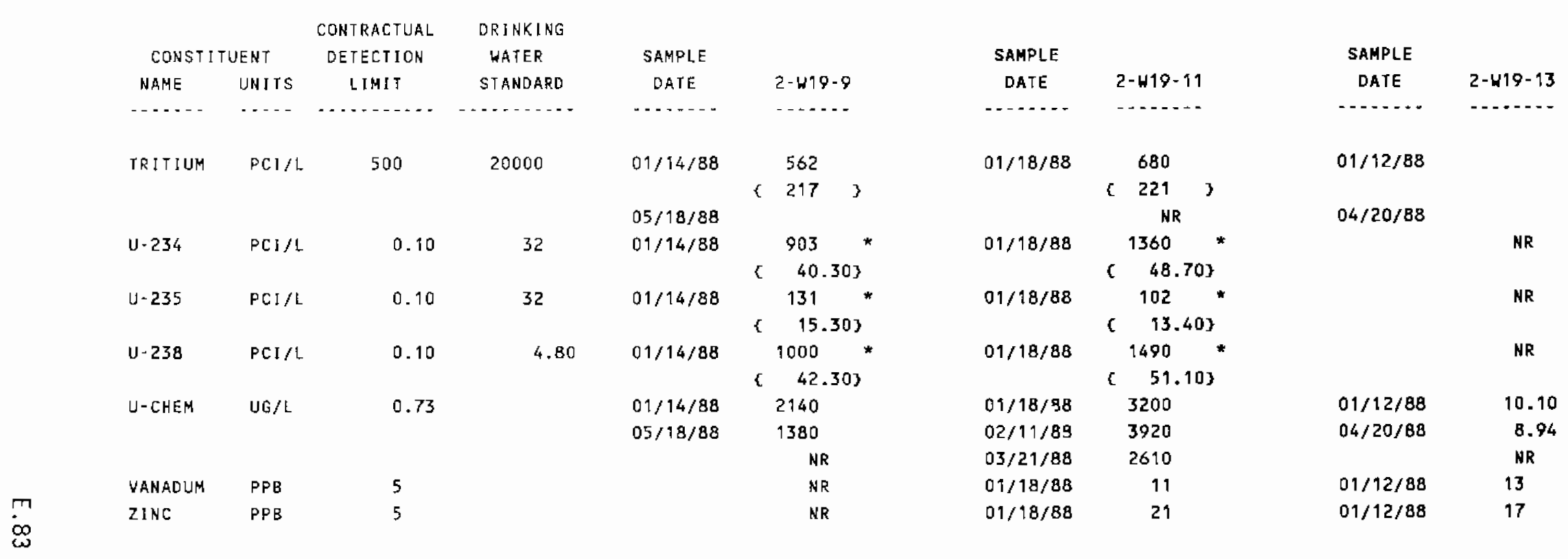


IABLE E.3. (contd)

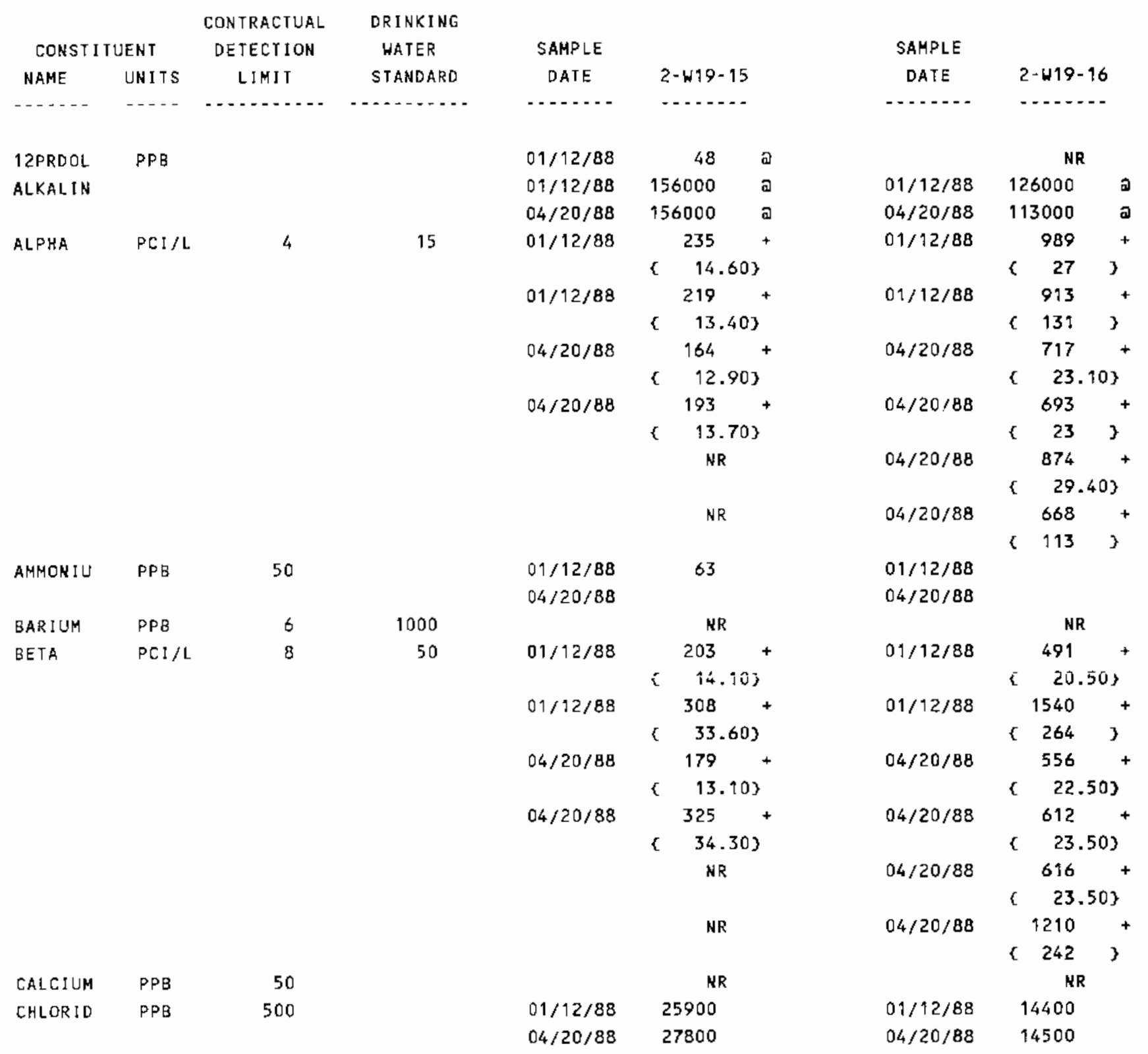

SAMPLE

DATE

2-พ19-18

-...- - -

$01 / 14 / 88 \quad 189000$ a

$01 / 14 / 88$

$02 / 10 / 88$

$03 / 18 / 88$

$04 / 11 / 88$

$05 / 06 / 88$

$06 / 09 / 88$

$01 / 14 / 88$

$01 / 14 / 88$

$01 / 14 / 88$

$01 / 14 / 88$

$02 / 10 / 88$

$03 / 18 / 88$

$04 / 11 / 88$

$05 / 06 / 88$

$06 / 09 / 88$

$01 / 14 / 88$

$01 / 14 / 88$ 
IABLE E.3. (contd)

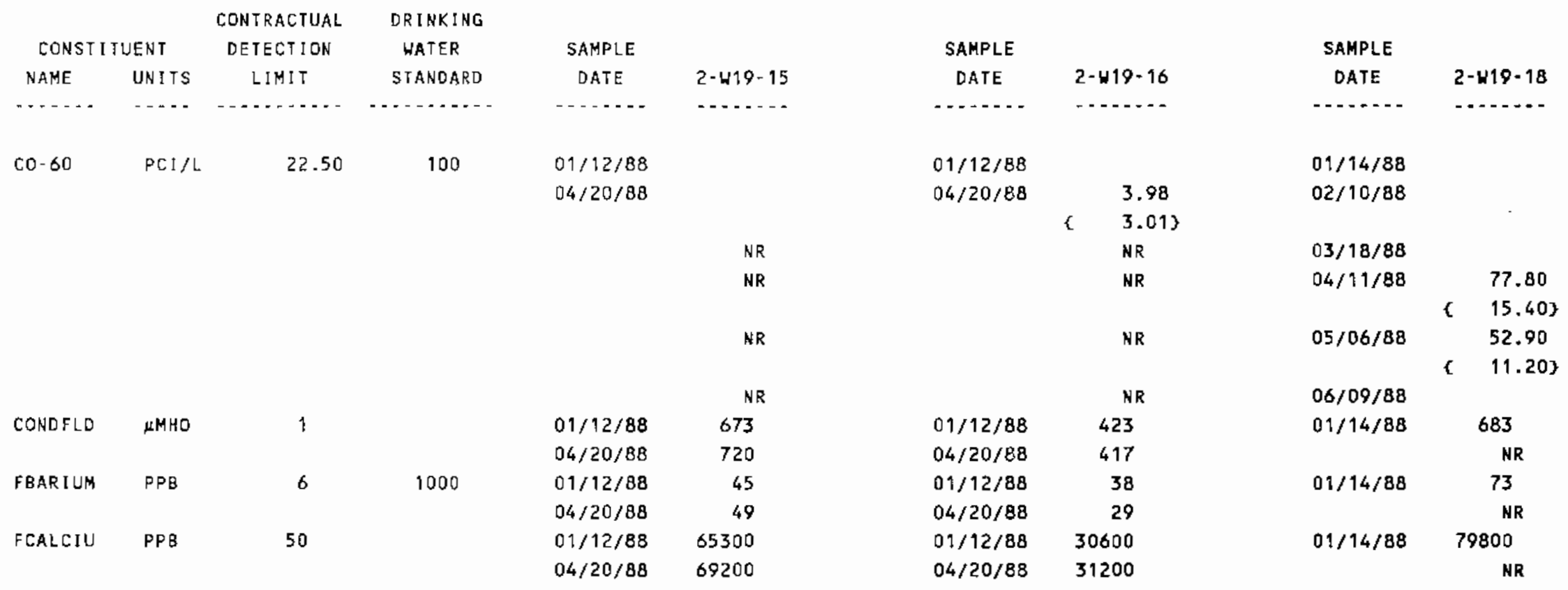


IABLE E.3. (contd)

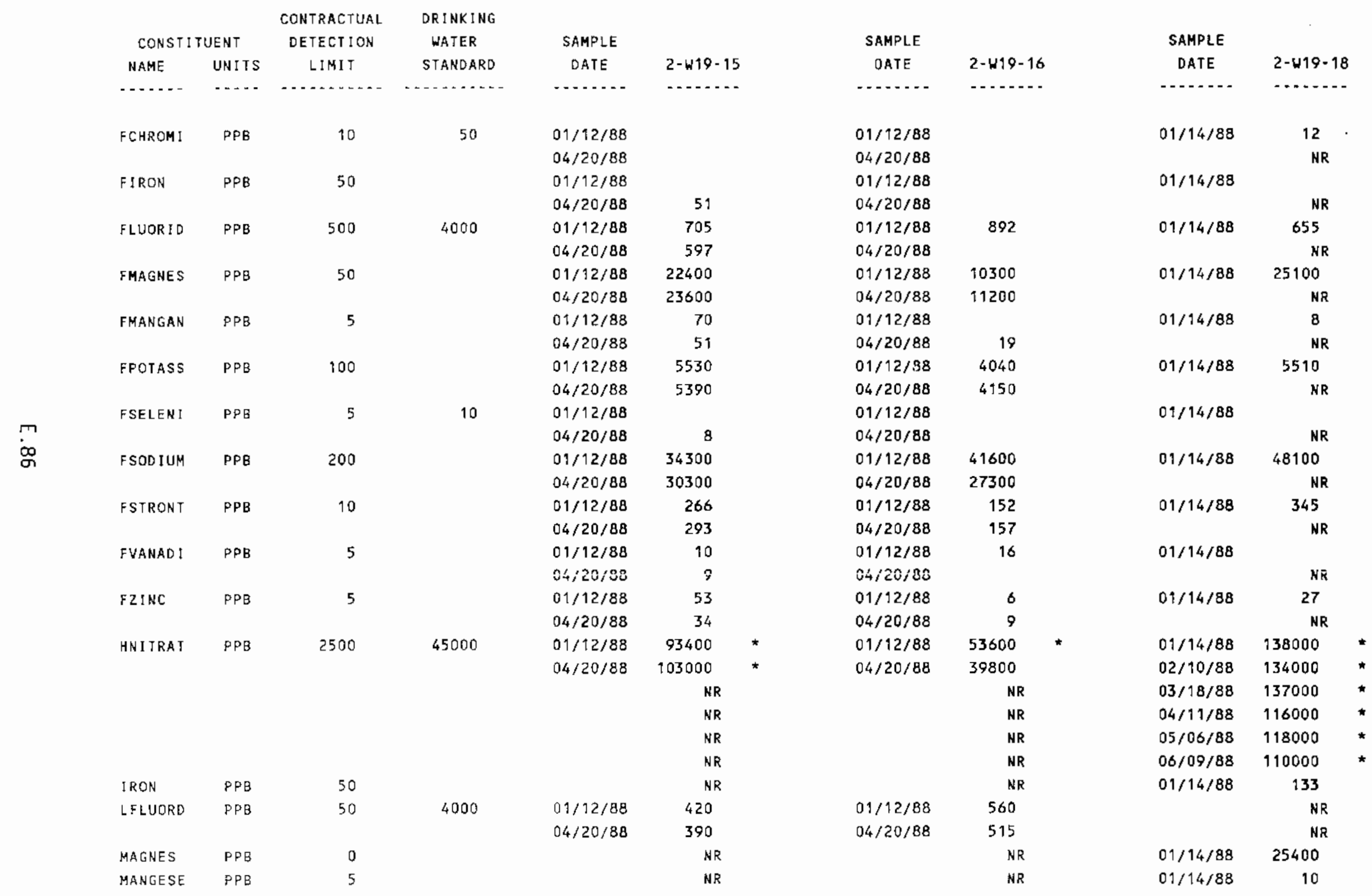


IABLE E.3. (contd)

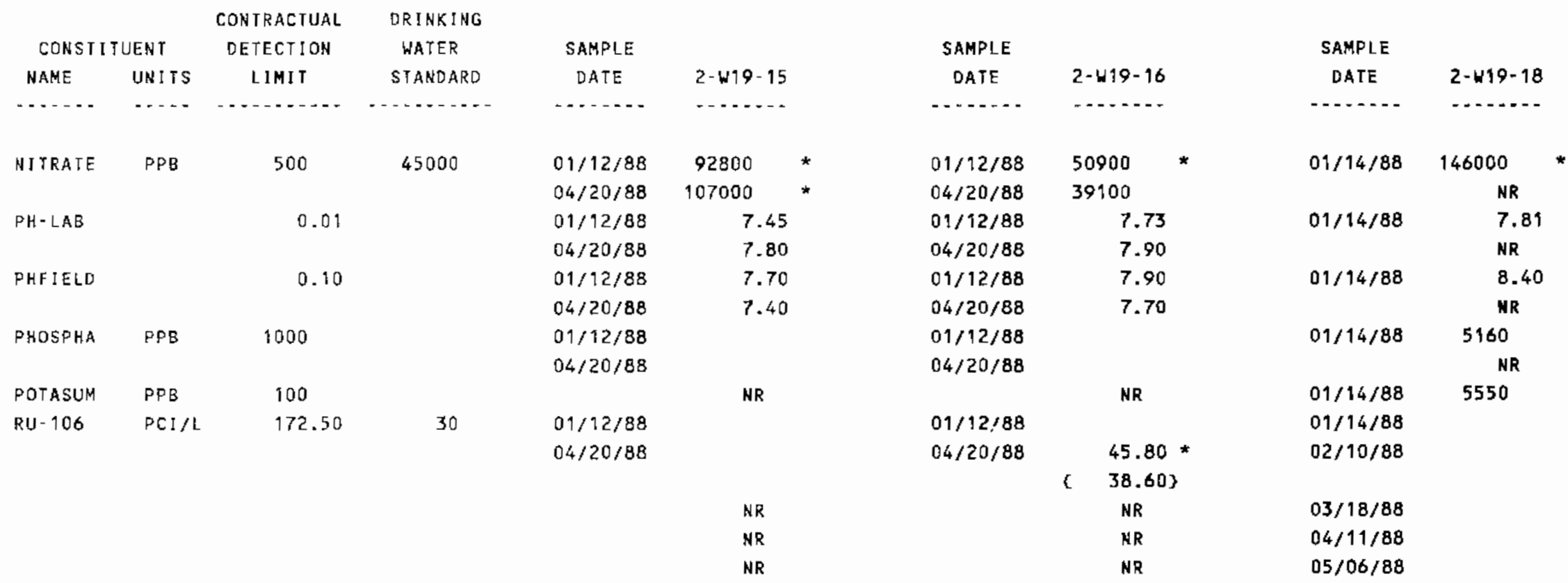


TABLE E. 3. (contd)

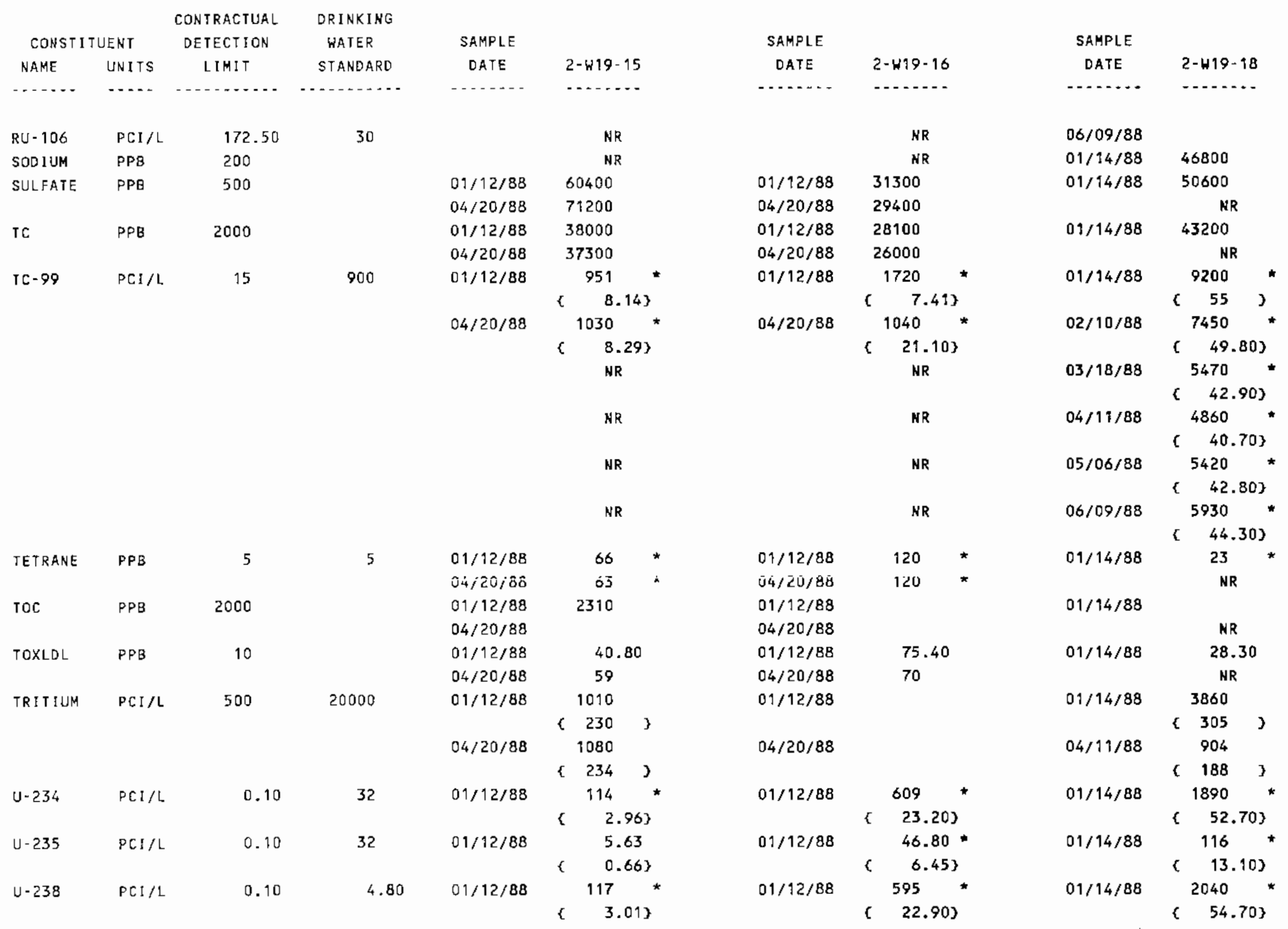


IABLE E.3. (contd)

\begin{tabular}{|c|c|c|c|}
\hline \multirow{2}{*}{\multicolumn{2}{|c|}{ CONSTITUENT }} & CONTRACTUAL & DRINKING \\
\hline & & DETECTION & WATER \\
\hline NAME & UNITS & LIMIT & STANDARD \\
\hline-- & $\cdots$ & $\cdots$ & $\cdots$ \\
\hline
\end{tabular}

\begin{tabular}{|c|c|c|c|}
\hline SAMPLE & & SAMPLE & \\
\hline DATE & $2-419-15$ & DATE & $2-W 19-16$ \\
\hline - & 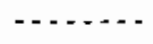 & - & - - - - - - \\
\hline
\end{tabular}

SAMPLE

DATE

2- $419-18$

U-CHEM UG/L $\quad 0.73$

$01 / 12 / 88$

336

$04 / 20 / 88$

219

NR

$01 / 12 / 88 \quad 1540$

$04 / 20 / 88$

694

NR

NR

NR

NR

ZINC

PPB

5

....... .......

$01 / 14 / 88 \quad 5760$

$02 / 10 / 88 \quad 5480$

$03 / 18 / 88 \quad 4690$

$04 / 11 / 88 \quad 4410$

$05 / 06 / 88 \quad 3410$

$06 / 09 / 88 \quad 3470$

$01 / 14 / 88$ 
TABLE E.3. (contd)

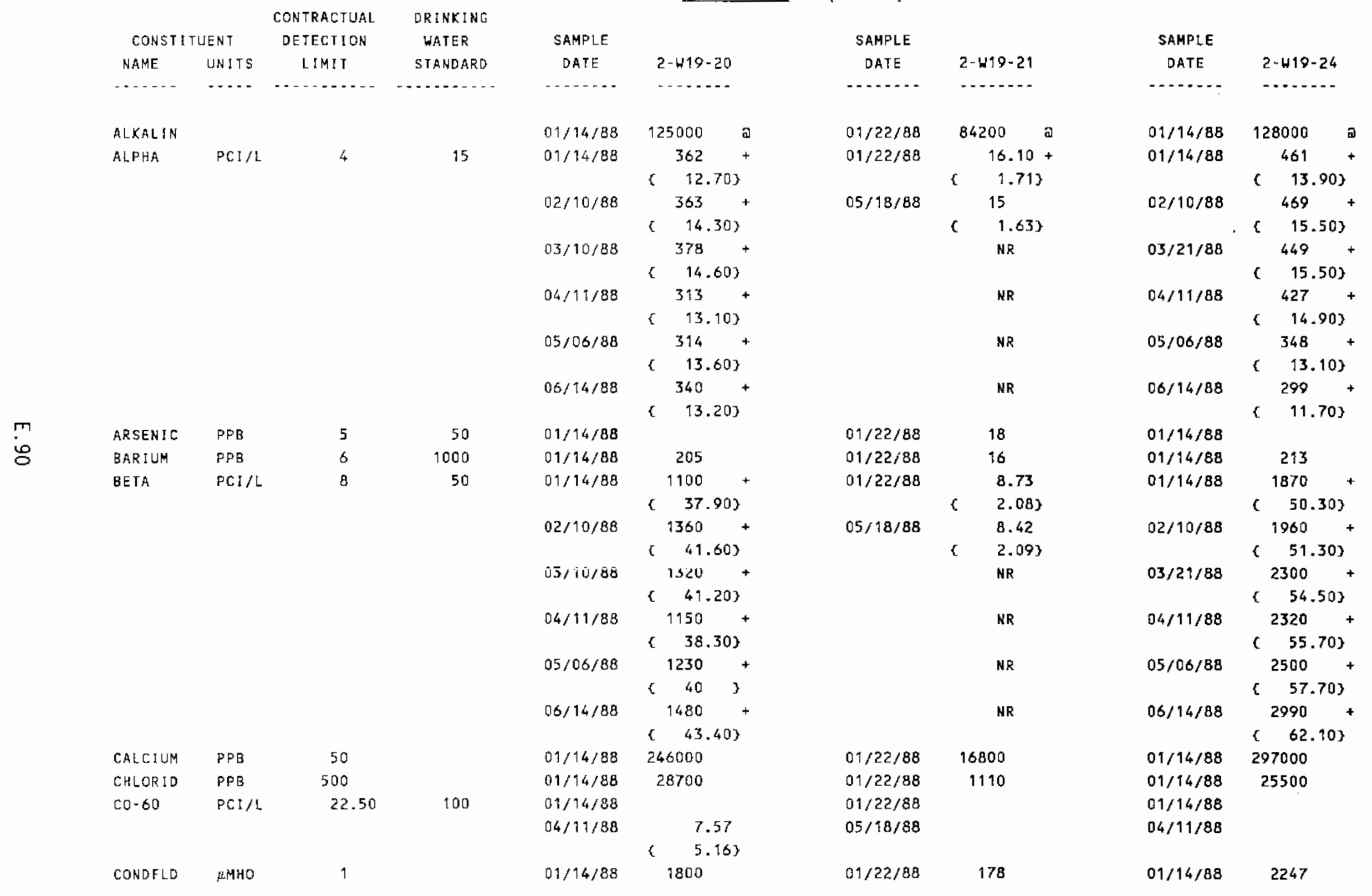


IABLE E.3. (contd)

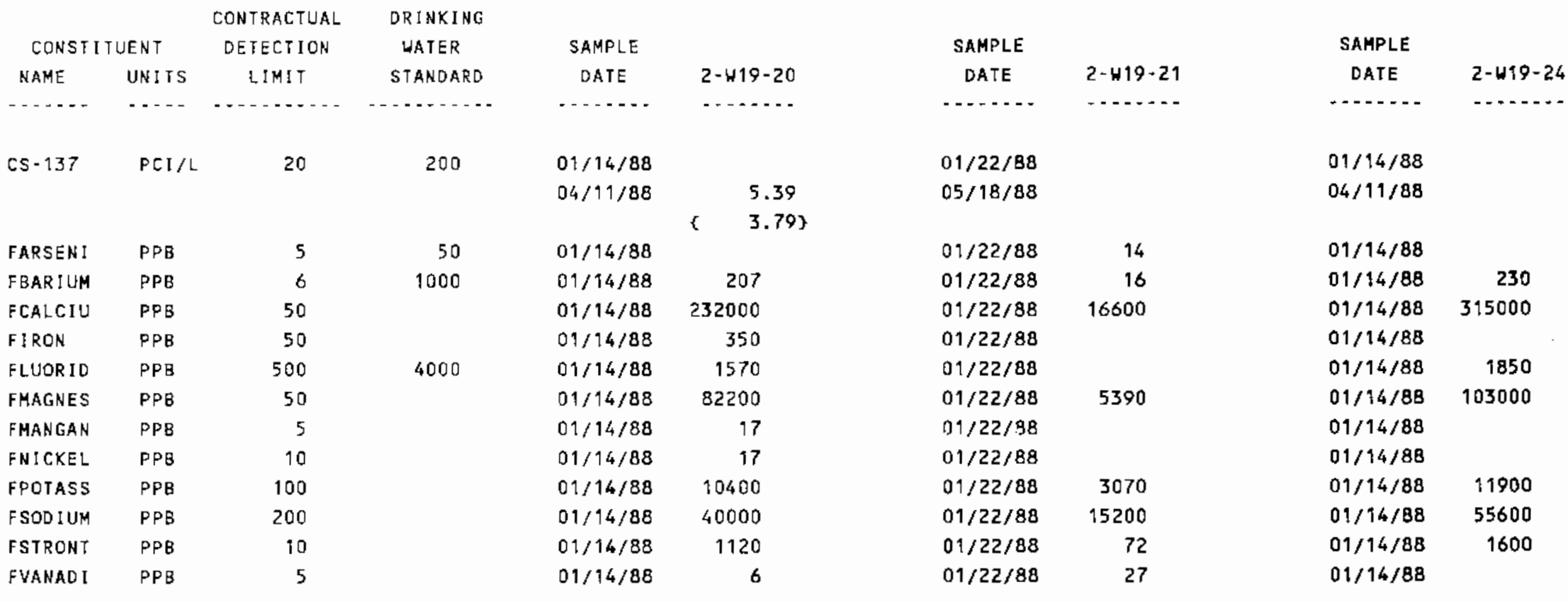


IABLE E.3. (contd)

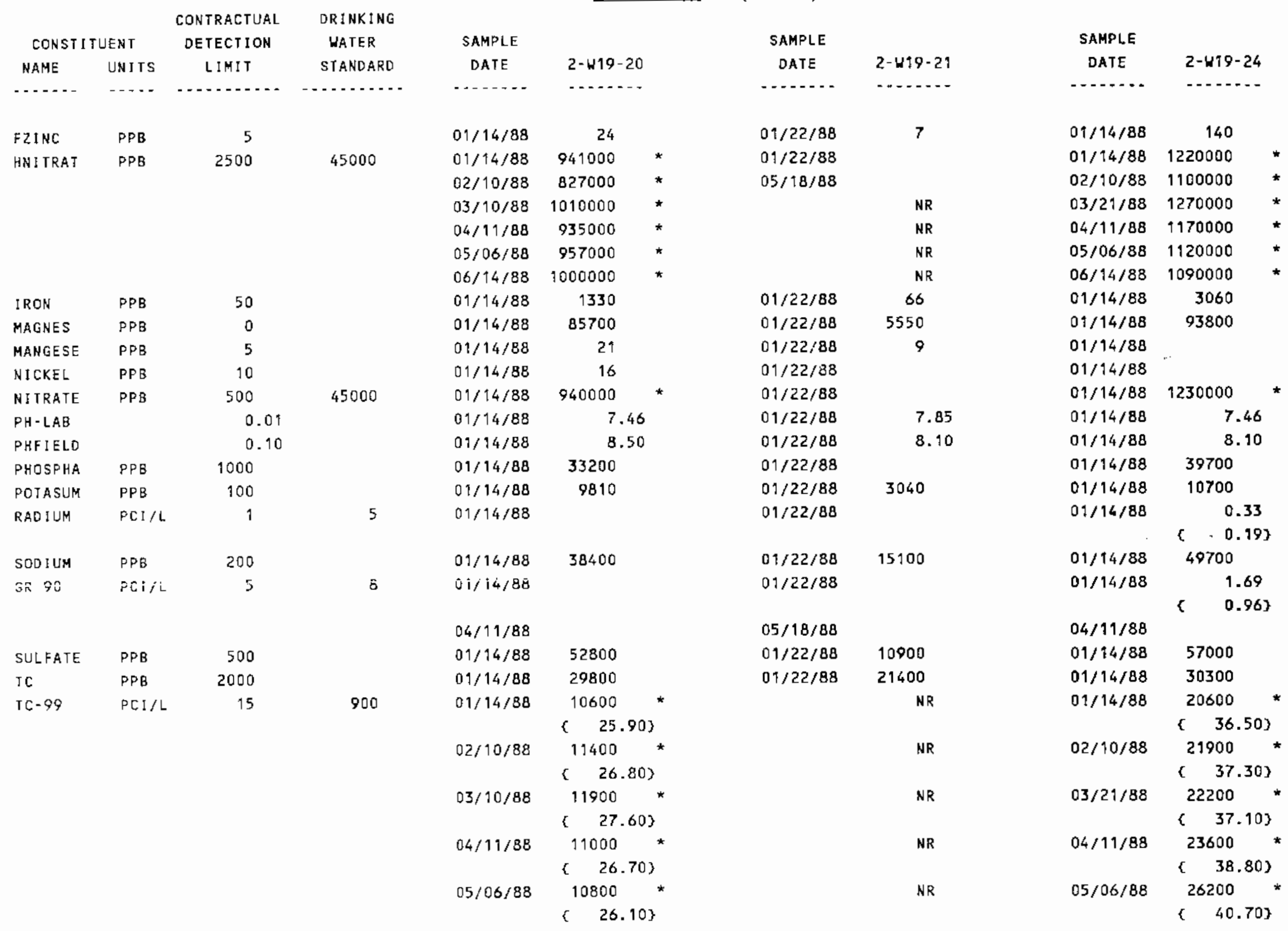


TABLE E.3. (contd)

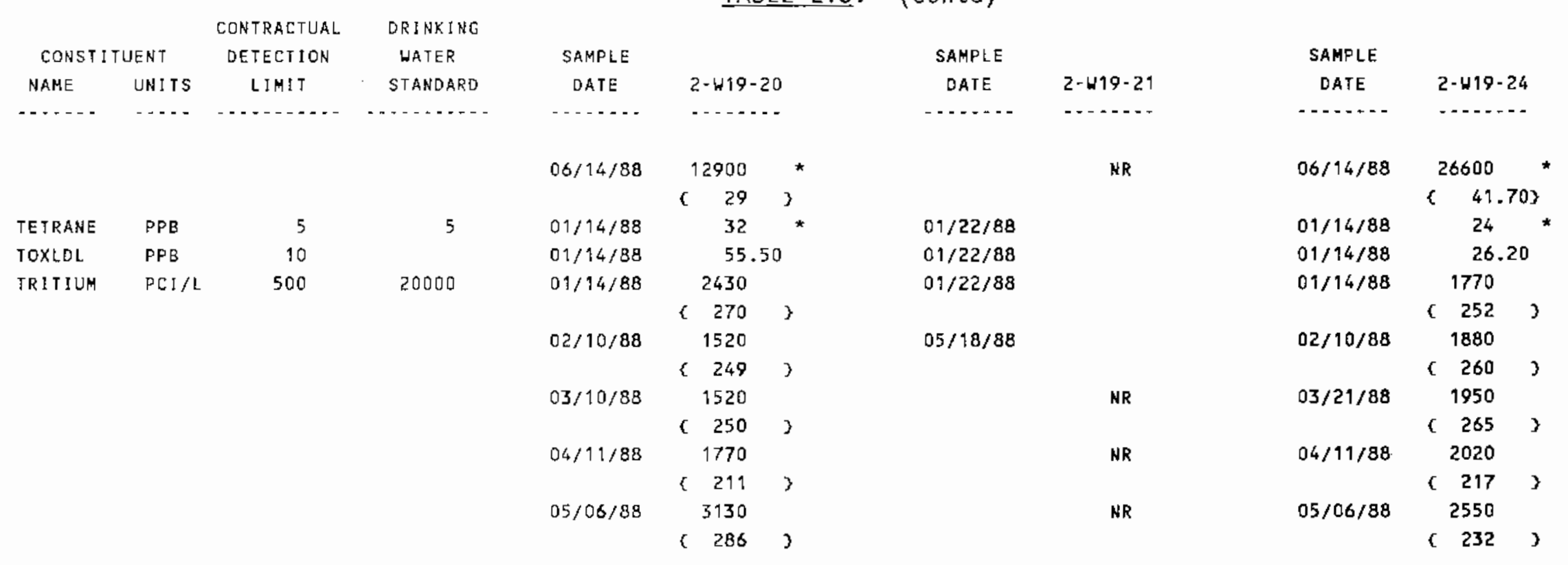


TABLE E.3. (contd)

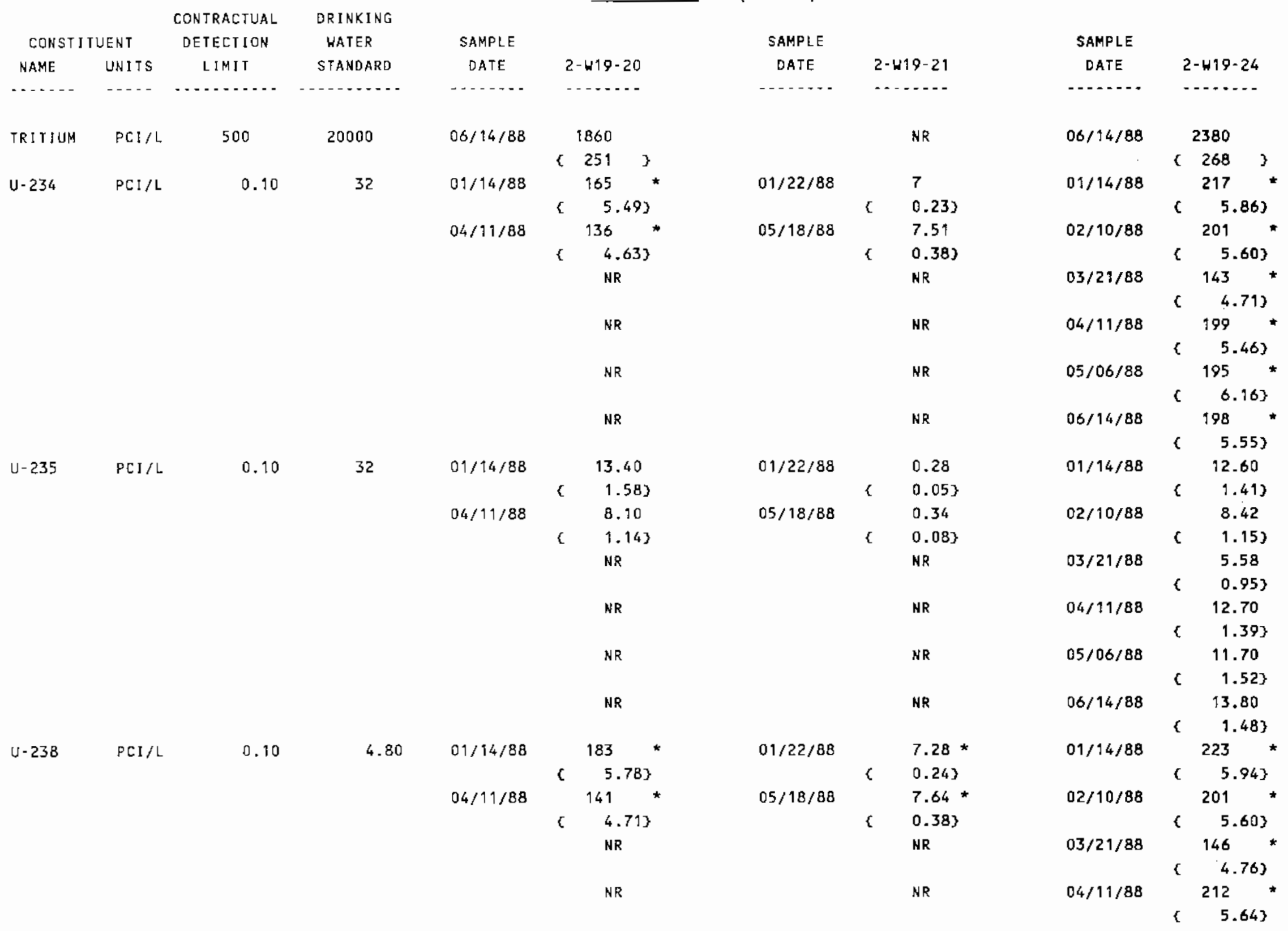


TABLE.E.3. (contd)

\begin{tabular}{|c|c|c|c|c|c|c|c|c|c|}
\hline \multicolumn{2}{|c|}{ CONSTI TUENT } & $\begin{array}{l}\text { CONTRACTUAL } \\
\text { DETECTION }\end{array}$ & $\begin{array}{l}\text { DRINKING } \\
\text { WATER }\end{array}$ & SAMPLE & & SAMPLE & & SAMPLE & \multirow[b]{2}{*}{ 2-W19-24 } \\
\hline NAME & UNITS & LIMIT & STANDARD & DATE & $2-419-20$ & DATE & $2-w 19-21$ & DATE & \\
\hline \multirow{5}{*}{........ } & $\cdots$ & n. & $\ldots$ & $\ldots$ & ........... & ......... & $\ldots$ & -......... & - \\
\hline & & & & & NR & & NR & $05 / 06 / 88$ & 206 \\
\hline & & & & & & & & & $6.33)$ \\
\hline & & & & & NR & & NR & $06 / 14 / 88$ & 209 \\
\hline & & & & & & & & & $5.70\}$ \\
\hline \multirow[t]{6}{*}{$U$ - CHEM } & UG/L & 0.73 & & $01 / 14 / 88$ & 494 & $01 / 22 / 88$ & 21.40 & $01 / 14 / 8 B$ & 592 \\
\hline & & & & $02 / 10 / 88$ & 491 & $05 / 18 / 88$ & 14.60 & $02 / 10 / 88$ & 613 \\
\hline & & & & $03 / 10 / 88$ & 429 & & NR & $03 / 21 / 88$ & 488 \\
\hline & & & & $04 / 11 / 88$ & 449 & & NR & $04 / 19 / 88$ & 575 \\
\hline & & & & $05 / 06 / 88$ & 389 & & NR & $05 / 06 / 8 B$ & 452 \\
\hline & & & & $06 / 14 / 88$ & 542 & & NR & $06 / 14 / 88$ & 537 \\
\hline VANADUM & PPB & 5 & & $01 / 14 / 88$ & & $01 / 22 / 38$ & 27 & $01 / 14 / 8 B$ & \\
\hline ZINC & PPB & 5 & & $01 / 94 / 88$ & 43 & $01 / 22 / 88$ & 11 & $01 / 14 / 88$ & 213 \\
\hline
\end{tabular}


TABLE E.3. (contd)

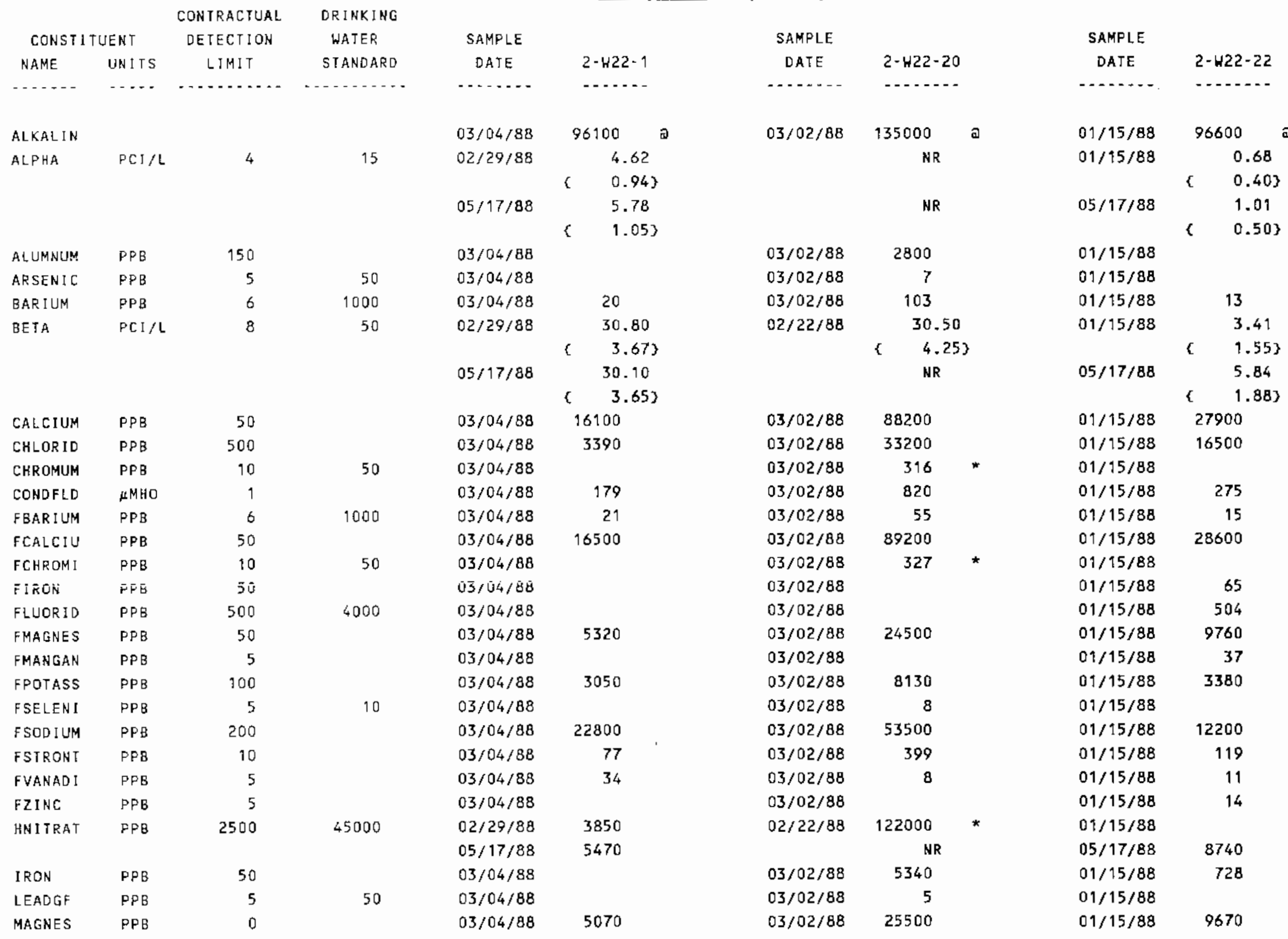


IABLE E.3. (contd)

\begin{tabular}{|c|c|c|c|c|c|c|c|c|c|}
\hline \multicolumn{2}{|c|}{ CONSTITUENT } & $\begin{array}{l}\text { CONTRACTUAL } \\
\text { DETECTION }\end{array}$ & $\begin{array}{l}\text { DRINKING } \\
\text { WATER }\end{array}$ & SAMPLE & & SAMPLE & & \multicolumn{2}{|l|}{ SAMPLE } \\
\hline NAME & UNITS & LIMIT & STANDARD & DATE & $2-H 22-1$ & DATE & $2-W 22-20$ & DATE & $2-w 22-22$ \\
\hline ...... & $\ldots$ & - n. & 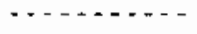 & $\ldots$ & n..... & . & n....... & -- & - \\
\hline MANGESE & PPB & 5 & & $03 / 04 / 88$ & & $03 / 02 / 88$ & 260 & $01 / 15 / 88$ & 56 \\
\hline NICKEL & $P P B$ & 10 & & $03 / 04 / 88$ & & $03 / 02 / 88$ & 12 & $01 / 15 / 88$ & \\
\hline NITRATE & $\mathrm{PPB}$ & 500 & 45000 & $03 / 04 / 88$ & 3830 & $03 / 02 / 88$ & 124000 & $01 / 15 / 88$ & 2810 \\
\hline$P H-L A B$ & & 0.01 & & $03 / 04 / 88$ & 7.95 & $03 / 02 / 88$ & 7.48 & $01 / 15 / 88$ & 7.95 \\
\hline PHFIELD & & 0.10 & & $03 / 04 / 88$ & 8.30 & $03 / 02 / 88$ & 6.80 & $01 / 15 / 88$ & 8.20 \\
\hline POTASUM & $\mathrm{PPB}$ & 100 & & $03 / 04 / 88$ & 2870 & $03 / 02 / 88$ & 8150 & $01 / 15 / 88$ & 3600 \\
\hline \multirow[t]{2}{*}{ RADIUM } & $\mathrm{PCI} / \mathrm{L}$ & 1 & 5 & $03 / 04 / 88$ & & $03 / 02 / 88$ & 1.53 & $01 / 15 / 88$ & \\
\hline & & & & & & & 0.393 & & \\
\hline SELENUM & PPB & 5 & 10 & $03 / 04 / 88$ & & $03 / 02 / 88$ & 14 & $01 / 15 / 88$ & \\
\hline SODIUM & $P P B$ & 200 & & $03 / 04 / 88$ & 20900 & $03 / 02 / 88$ & 54600 & $01 / 15 / 88$ & 13100 \\
\hline \multirow[t]{4}{*}{ SR 90} & $\mathrm{PCI} / \mathrm{L}$ & 5 & 8 & $02 / 29 / 88$ & 7.44 & $02 / 22 / 38$ & & $01 / 15 / 88$ & \\
\hline & & & & & $1.57\}$ & & & & \\
\hline & & & & $05 / 17 / 88$ & 7.96 & & NR & $05 / 17 / 88$ & \\
\hline & & & & & 1.663 & & & & \\
\hline
\end{tabular}


TABLE E.3. (contd)

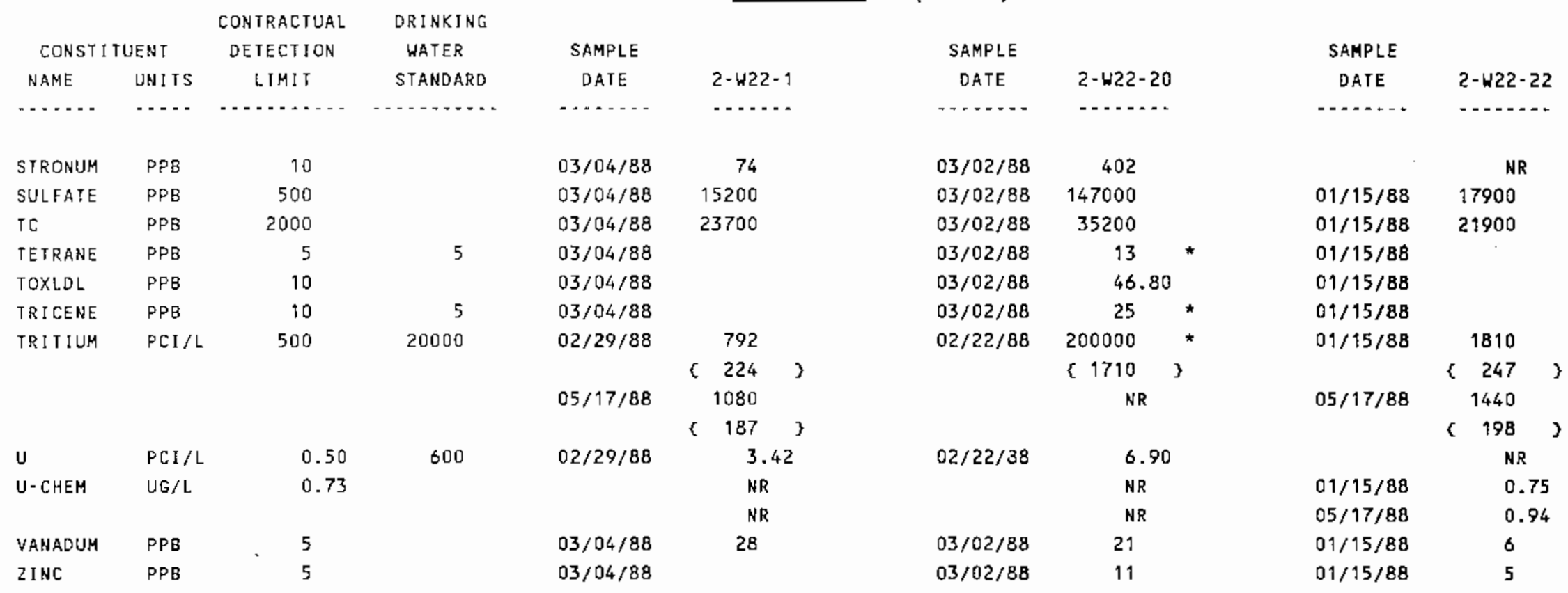


TABLE E.3. (contd)

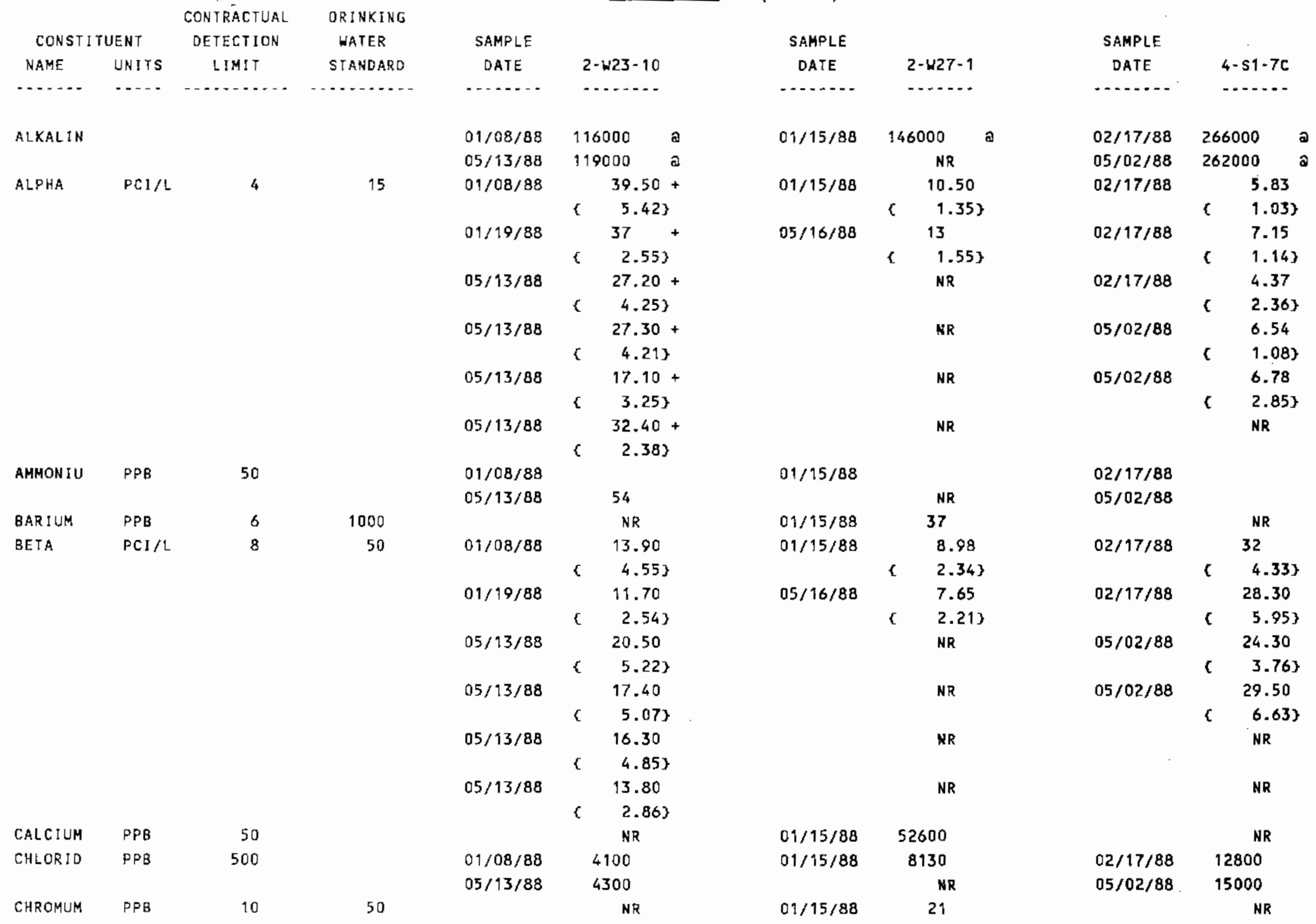


TABLE E.3. (contd)

\begin{tabular}{|c|c|c|c|c|c|c|c|c|c|}
\hline \multirow{2}{*}{\multicolumn{2}{|c|}{ CONSTITUENT }} & \multirow{3}{*}{$\begin{array}{l}\text { CONTRACTUAL } \\
\text { DETECTION } \\
\text { LIMIT }\end{array}$} & \multirow{3}{*}{$\begin{array}{l}\text { DRINKING } \\
\text { WATER } \\
\text { STANDARD }\end{array}$} & \multirow{3}{*}{$\begin{array}{l}\text { SAMPLE } \\
\text { DATE }\end{array}$} & \multirow[b]{3}{*}{$2-\$ 23-10$} & \multirow[b]{2}{*}{ SAMPLE } & \multirow{2}{*}{\multicolumn{3}{|c|}{ SAMPLE }} \\
\hline & & & & & & & & & \\
\hline NAME & UNITS & & & & & DATE & $2-W 27-1$ & DATE & $4-51-7 c$ \\
\hline$\cdots \cdots$ & $\cdots-$ & , n.......... & - N & $\cdots$ & - & -....... & - . . & ........ & $\ldots$ \\
\hline \multirow[t]{2}{*}{ CONDFLD } & सMHO & 1 & & $01 / 08 / 88$ & & $01 / 15 / 88$ & 479 & $02 / 17 / 88$ & 486 \\
\hline & & & & $05 / 13 / 88$ & 408 & & NR & $05 / 02 / 88$ & 763 \\
\hline \multirow[t]{2}{*}{ FBARIUM } & PPB & 6 & 1000 & $01 / 08 / 88$ & 57 & $01 / 15 / 88$ & 34 & $02 / 17 / 88$ & 70 \\
\hline & & & & $05 / 13 / 88$ & 54 & & NR & $05 / 02 / 88$ & 68 \\
\hline \multirow[t]{2}{*}{ FCADMIU } & PPB & 2 & 10 & $01 / 08 / 88$ & & $01 / 15 / 88$ & 2 & $02 / 17 / 88$ & \\
\hline & & & & $05 / 13 / 88$ & & & NR & $05 / 02 / 88$ & \\
\hline \multirow[t]{2}{*}{ FCALCIU } & PPB & 50 & & $01 / 08 / 88$ & 52200 & $01 / 15 / 88$ & 52300 & $02 / 17 / 88$ & 103000 \\
\hline & & & & $05 / 13 / 88$ & 46000 & & NR & $05 / 02 / 88$ & 105000 \\
\hline \multirow[t]{2}{*}{ FCHROMI } & PPB & 10 & 50 & $01 / 08 / 88$ & & $01 / 15 / 88$ & 24 & $02 / 17 / 88$ & \\
\hline & & & & $05 / 13 / 88$ & & & NR & $05 / 02 / 88$ & \\
\hline \multirow[t]{2}{*}{ FIRON } & PPB & 50 & & $01 / 08 / 88$ & & $01 / 15 / 88$ & & $02 / 17 / 88$ & 92 \\
\hline & & & & $05 / 13 / 88$ & & & NR & $05 / 02 / 88$ & 151 \\
\hline \multirow[t]{2}{*}{ FLUORID } & PPB & 500 & 4000 & $01 / 08 / 88$ & & $01 / 15 / 88$ & & $02 / 17 / 88$ & \\
\hline & & & & $05 / 13 / 88$ & & & NR & $05 / 02 / 88$ & 537 \\
\hline FMAGNES & PPB & 50 & & $01 / 08 / 88$ & 17700 & $01 / 15 / 88$ & 13200 & $02 / 17 / 88$ & 24800 \\
\hline
\end{tabular}


IABLE E.3. (contd)

\begin{tabular}{|c|c|c|c|c|c|c|c|c|c|}
\hline \multicolumn{2}{|c|}{ CONSTITUENT } & $\begin{array}{l}\text { CONTRACTUAL } \\
\text { DETECTION }\end{array}$ & $\begin{array}{l}\text { DRINKING } \\
\text { HATER }\end{array}$ & SAMPLE & \multirow{2}{*}{\multicolumn{2}{|c|}{$\begin{array}{l}\text { SAMPLE } \\
\text { DATE }\end{array}$}} & \multicolumn{3}{|c|}{ SAMPLE } \\
\hline NAME & UNITS & LIMIT & STANDARD & DATE & & & $2-w 27-1$ & DATE & $4-\$ 1-7 c$ \\
\hline$\cdots$ & $\cdots$ & - & - & - & , & $\ldots \ldots$ & - . n & - - & $\cdots$ \\
\hline FMAGNES & $\mathrm{PPB}$ & 50 & & $05 / 13 / 88$ & 15800 & & NR & $05 / 02 / 88$ & 25900 \\
\hline \multirow[t]{2}{*}{ FMANGAN } & $\mathrm{PPB}$ & 5 & & $01 / 08 / 8 B$ & & $01 / 15 / 88$ & & $02 / 17 / 88$ & \\
\hline & & & & $05 / 13 / 88$ & & & NR & $05 / 02 / 88$ & 12 \\
\hline \multirow[t]{2}{*}{ FPOTASS } & PPB & 100 & & $01 / 08 / 88$ & 4900 & $01 / 15 / 88$ & 5820 & $02 / 17 / 88$ & 8800 \\
\hline & & & & $05 / 13 / 88$ & 4230 & & NR & $05 / 02 / 88$ & 9030 \\
\hline \multirow[t]{2}{*}{ FSELEN I } & PPB & 5 & 10 & $01 / 08 / 88$ & & $01 / 15 / 88$ & 24 & $02 / 17 / 88$ & \\
\hline & & & & $05 / 13 / 88$ & & & NR & $05 / 02 / 88$ & \\
\hline \multirow[t]{2}{*}{ FSODIUM } & PPB & 200 & & $01 / 08 / 88$ & 22900 & $01 / 15 / 88$ & 33800 & $02 / 17 / 88$ & 27200 \\
\hline & & & & $05 / 13 / 88$ & 19600 & & NR & $05 / 02 / 88$ & 28000 \\
\hline \multirow[t]{2}{*}{ FSTRONT } & PPB & 10 & & $01 / 08 / 88$ & 217 & $01 / 15 / 88$ & 252 & $02 / 17 / 88$ & 442 \\
\hline & & & & $05 / 13 / 88$ & 190 & & NR & $05 / 02 / 88$ & 435 \\
\hline \multirow[t]{2}{*}{ FVANADI } & PPB & 5 & & $01 / 08 / 88$ & 21 & $01 / 15 / 88$ & 18 & $02 / 17 / 88$ & 6 \\
\hline & & & & $05 / 13 / 88$ & 23 & & NR & $05 / 02 / 88$ & \\
\hline \multirow[t]{2}{*}{ FZINC } & $P P B$ & 5 & & $01 / 08 / 88$ & & $01 / 15 / 88$ & & $02 / 17 / 88$ & 27 \\
\hline & & & & $05 / 13 / 88$ & & & NR & $05 / 02 / 88$ & 48 \\
\hline \multirow[t]{2}{*}{ HNITRAT } & $P P B$ & 2500 & 45000 & $01 / 19 / 88$ & 103000 & $01 / 15 / 88$ & 61100 & $02 / 17 / 88$ & 24900 \\
\hline & & & & $05 / 13 / 88$ & 116000 & $05 / 16 / 88$ & 49300 & $05 / 02 / 88$ & 25800 \\
\hline IRON & PPB & 50 & & & NR & $01 / 15 / 88$ & 106 & & KR \\
\hline \multirow[t]{2}{*}{ LFLUORD } & PPB & 50 & 4000 & $01 / 08 / 88$ & 240 & & $N R$ & $02 / 17 / 88$ & 191 \\
\hline & & & & $05 / 13 / 88$ & 253 & & NR & $05 / 02 / 88$ & 179 \\
\hline MAGNES & PPB & 0 & & & NR & $01 / 15 / 88$ & 13400 & & NR \\
\hline \multirow[t]{2}{*}{ NITRATE } & PPB & 500 & 45000 & $01 / 08 / 88$ & 147000 & $01 / 15 / 8 B$ & 60000 & $02 / 17 / 88$ & 27900 \\
\hline & & & & $05 / 13 / 88$ & 121000 & * & NR & $05 / 02 / 88$ & 27400 \\
\hline NNIDIME & $\mathrm{PPB}$ & 10 & & $01 / 08 / 88$ & 27 & $01 / 15 / 8 B$ & & & NR \\
\hline \multirow[t]{2}{*}{ PH-LAB } & & 0.01 & & $01 / 08 / 88$ & 7.66 & $01 / 15 / 88$ & 7.89 & $02 / 17 / 88$ & 7.17 \\
\hline & & & & $05 / 13 / 88$ & 8.10 & & MR & $05 / 02 / 88$ & 7.70 \\
\hline \multirow[t]{2}{*}{ PHFIELD } & & 0.10 & & $01 / 08 / 88$ & 8.20 & $01 / 15 / 88$ & 8.30 & $02 / 17 / 88$ & 7.30 \\
\hline & & & & $05 / 13 / 88$ & 7.80 & & NR & $05 / 02 / 88$ & 7.30 \\
\hline POTASUM & PPB & 100 & & & NR & $01 / 15 / 88$ & 6460 & & NR \\
\hline SELENUM & PPB & 5 & 10 & & NR & $01 / 15 / 88$ & 21 & * & NR \\
\hline SODIUM & $P P B$ & 200 & & & NR & $01 / 15 / 88$ & 38000 & & NR \\
\hline \multirow[t]{2}{*}{ SR 90} & $\mathrm{PCI} / \mathrm{L}$ & 5 & 8 & $01 / 19 / 88$ & & $01 / 15 / 88$ & 0.88 & & NR \\
\hline & & & & & & & 0.813 & & \\
\hline
\end{tabular}


TABLE E.3. (contd)

\begin{tabular}{|c|c|c|c|c|c|}
\hline \multicolumn{2}{|c|}{ CONSTI TUENT } & $\begin{array}{l}\text { CONTRACTUAL } \\
\text { DETECTION }\end{array}$ & $\begin{array}{l}\text { ORINKING } \\
\text { WATER }\end{array}$ & SAMPLE & \multirow[b]{2}{*}{$2-w 23-10$} \\
\hline NAME & UNITS & LIMIT & STANDARD & DATE & \\
\hline$\cdots$ & $\cdots$ & 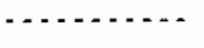 & . . . . . . . . & $\ldots$ & . \\
\hline \multirow[t]{2}{*}{ SULFATE } & $P P B$ & 500 & & $01 / 08 / 88$ & 13100 \\
\hline & & & & $05 / 13 / 88$ & 13500 \\
\hline \multirow[t]{2}{*}{ TC } & $P P B$ & 2000 & & $01 / 08 / 88$ & 26300 \\
\hline & & & & $05 / 13 / 88$ & 27600 \\
\hline \multirow[t]{5}{*}{ TRITIUM } & $\mathrm{PCI} / \mathrm{L}$ & 500 & 20000 & $01 / 19 / 88$ & 579000 \\
\hline & & & & & $\{2440$ \\
\hline & & & & $05 / 13 / 88$ & 687000 \\
\hline & & & & & < 2710 \\
\hline & & & & & $\mathrm{NR}$ \\
\hline \multirow[t]{4}{*}{$U-234$} & $P C: / L$ & 0.10 & 32 & $01 / 19 / 88$ & 18.80 \\
\hline & & & & & 0.413 \\
\hline & & & & $05 / 13 / 8 B$ & 22.30 \\
\hline & & & & & 1.413 \\
\hline \multirow{2}{*}{ U-235 } & $\mathrm{PCI} / \mathrm{L}$ & 0.10 & 32 & $01 / 19 / 88$ & 0.81 \\
\hline & & & & & 0.093 \\
\hline
\end{tabular}

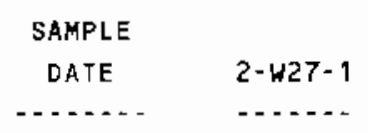

$01 / 15 / 88 \quad 53100$

$01 / 15 / 88 \quad 32900$

$01 / 15 / 88 \quad$ NR

[ 265 )

5760

\{ 297$\}$

NR

$01 / 15 / e 8$

$05 / 16 / 88$

$01 / 15 / 88$
5.02

$\{0.20\}$

5.09

( 0.21$\}$

0.16

$\{\quad 0.04\}$

\section{SAMPLE \\ DATE \\ $4-51-7 C$ \\ -...... -....}

$02 / 17 / 88 \quad 72300$

$05 / 02 / 88 \quad 68000$

$02 / 17 / 88 \quad 70300$

$05 / 02 / 88 \quad 70700$

$02 / 17 / 88 \quad 76100$

( 1050$\}$

$02 / 17 / 88 \quad 75000$ *

$05 / 02 / 88 \quad \begin{array}{r}3040 \\ 75300\end{array}$

( 895$)$

NR

NR 
TABLE E.3. (contd)

\begin{tabular}{|c|c|c|c|c|c|c|c|c|c|}
\hline \multicolumn{2}{|c|}{ CONSTITUENT } & $\begin{array}{l}\text { CONTRACTUAL } \\
\text { DETECTION }\end{array}$ & $\begin{array}{l}\text { DRINKING } \\
\text { WATER }\end{array}$ & SAMPLE & & SAMPLE & & SAMPLE & \\
\hline \multirow{2}{*}{ NAME } & UNITS & LIMIT & STANDARD & DATE & $2 \cdot \omega 23 \cdot 10$ & DATE & $2-W 27-1$ & DATE & $4-51-7 c$ \\
\hline & $\ldots$ & $\cdots$. & $\cdots$ & -........ & n...... & ......... & ........ & n...... & ......... \\
\hline \multirow[t]{2}{*}{$U-235$} & $\mathrm{PCI} / \mathrm{L}$ & 0.10 & 32 & $05 / 13 / 88$ & 0.95 & $05 / 16 / 88$ & 0.19 & & NR \\
\hline & & & & & $0.30\}$ & & 0.043 & & \\
\hline \multirow[t]{4}{*}{$u-238$} & $\mathrm{PCI} / \mathrm{L}$ & 0.10 & 4.80 & $01 / 19 / 88$ & $18.80 *$ & $01 / 15 / 88$ & 4.47 & & NR \\
\hline & & & & & 0.413 & & 0.193 & & \\
\hline & & & & $05 / 13 / 88$ & $22.80 *$ & $05 / 16 / 88$ & 4.63 & & NR \\
\hline & & & & & 1.423 & & 0.203 & & \\
\hline \multirow[t]{2}{*}{ U-CHEM } & $U G / L$ & 0.73 & & $01 / 19 / 88$ & 61.60 & $01 / 15 / 88$ & 11.20 & & NR \\
\hline & & & & $05 / 13 / 88$ & 53.30 & $05 / 16 / 88$ & 14 & & NR \\
\hline VANADUM & $\mathrm{PPB}$ & 5 & & & NR & $01 / 15 / 88$ & 16 & & $\mathrm{NR}$ \\
\hline
\end{tabular}


TABLE E.3. (contd)

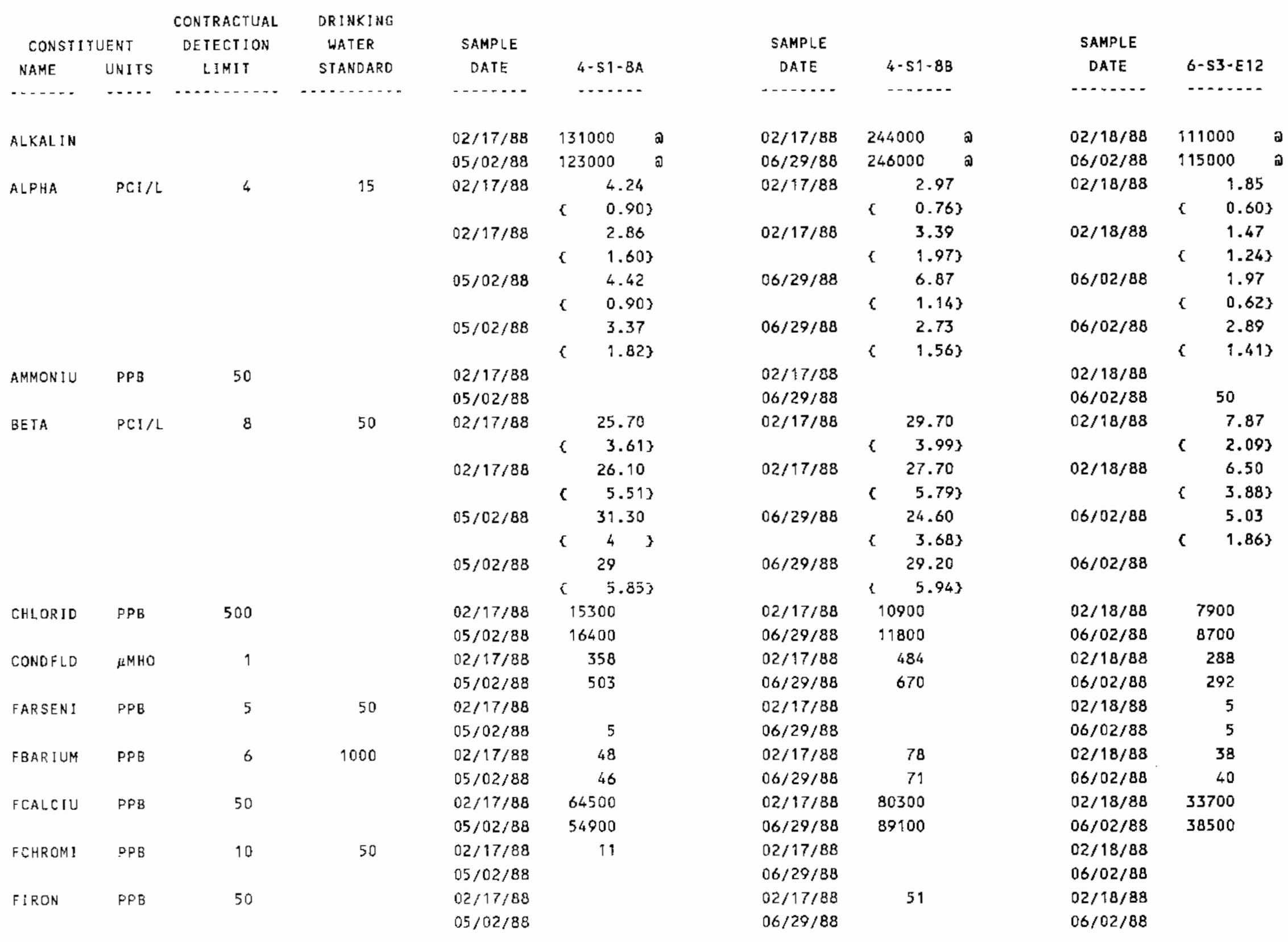


IABLE E.3. (contd)

\begin{tabular}{|c|c|c|c|c|c|c|c|c|c|}
\hline \multicolumn{2}{|c|}{ CONSTI I TUEN T } & $\begin{array}{l}\text { CONTRACTUAL } \\
\text { DETECTION }\end{array}$ & $\begin{array}{l}\text { DRINKING } \\
\text { HATER }\end{array}$ & \multirow{2}{*}{$\begin{array}{l}\text { SAMPLE } \\
\text { DATE }\end{array}$} & \multicolumn{3}{|c|}{ SAMPLE } & \multicolumn{2}{|l|}{ SAMPLE } \\
\hline NAME & UNITS & LIMIT & STANDARD & & $4-51-8 A$ & DATE & $4-\$ 1-88$ & DATE & $6-S 3-E 12$ \\
\hline ........ & $\cdots$ & 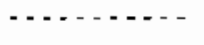 & $\cdots+\ldots+\ldots$ & ......... & …… & n........ & n...... & -....... & . \\
\hline \multirow[t]{2}{*}{ FLUORID } & РPB & 500 & 4000 & $02 / 17 / 88$ & & $02 / 17 / 88$ & & $02 / 18 / 88$ & \\
\hline & & & & $05 / 02 / 88$ & 523 & $06 / 29 / 88$ & & $06 / 02 / 88$ & \\
\hline \multirow[t]{2}{*}{ FMAGNES } & PPB & 50 & & $02 / 17 / 8 B$ & 15200 & $02 / 17 / 88$ & 21500 & $02 / 18 / 88$ & 9680 \\
\hline & & & & $05 / 02 / 88$ & 14200 & $06 / 29 / 88$ & 23300 & $06 / 02 / 88$ & 11100 \\
\hline \multirow[t]{2}{*}{ FMANGAN } & PPB & 5 & & $02 / 17 / 88$ & & $02 / 17 / 88$ & & $02 / 18 / 88$ & \\
\hline & & & & $05 / 02 / 88$ & & $06 / 29 / 88$ & & $06 / 02 / 88$ & 5 \\
\hline \multirow[t]{2}{*}{ FPOTASS } & PPB & 100 & & $02 / 17 / 88$ & 7050 & $02 / 17 / 88$ & 7890 & $02 / 18 / 88$ & 4200 \\
\hline & & & & $05 / 02 / 88$ & 6550 & $06 / 29 / 88$ & 8380 & $06 / 02 / 88$ & 4890 \\
\hline \multirow[t]{2}{*}{ FSODIUM } & $\mathrm{PPB}$ & 200 & & $02 / 17 / 88$ & 26200 & $02 / 17 / 88$ & 25300 & $02 / 18 / 88$ & 13000 \\
\hline & & & & $05 / 02 / 88$ & 25100 & $06 / 29 / 88$ & 26000 & $06 / 02 / 88$ & 15100 \\
\hline \multirow[t]{2}{*}{ FSTRONT } & PPB & 10 & & $02 / 17 / 88$ & 283 & $02 / 17 / \varepsilon 8$ & 190 & $02 / 18 / 88$ & 201 \\
\hline & & & & $05 / 02 / 88$ & 257 & $06 / 29 / 88$ & 403 & $06 / 02 / 88$ & 221 \\
\hline \multirow[t]{2}{*}{ FYANADI } & $P P B$ & 5 & & $02 / 17 / 88$ & 13 & $02 / 17 / 88$ & 8 & $02 / 18 / 88$ & 20 \\
\hline & & & & $05 / 02 / B B$ & 10 & $06 / 29 / 88$ & & $06 / 02 / 88$ & 18 \\
\hline
\end{tabular}


TABLE E.3. (contd)

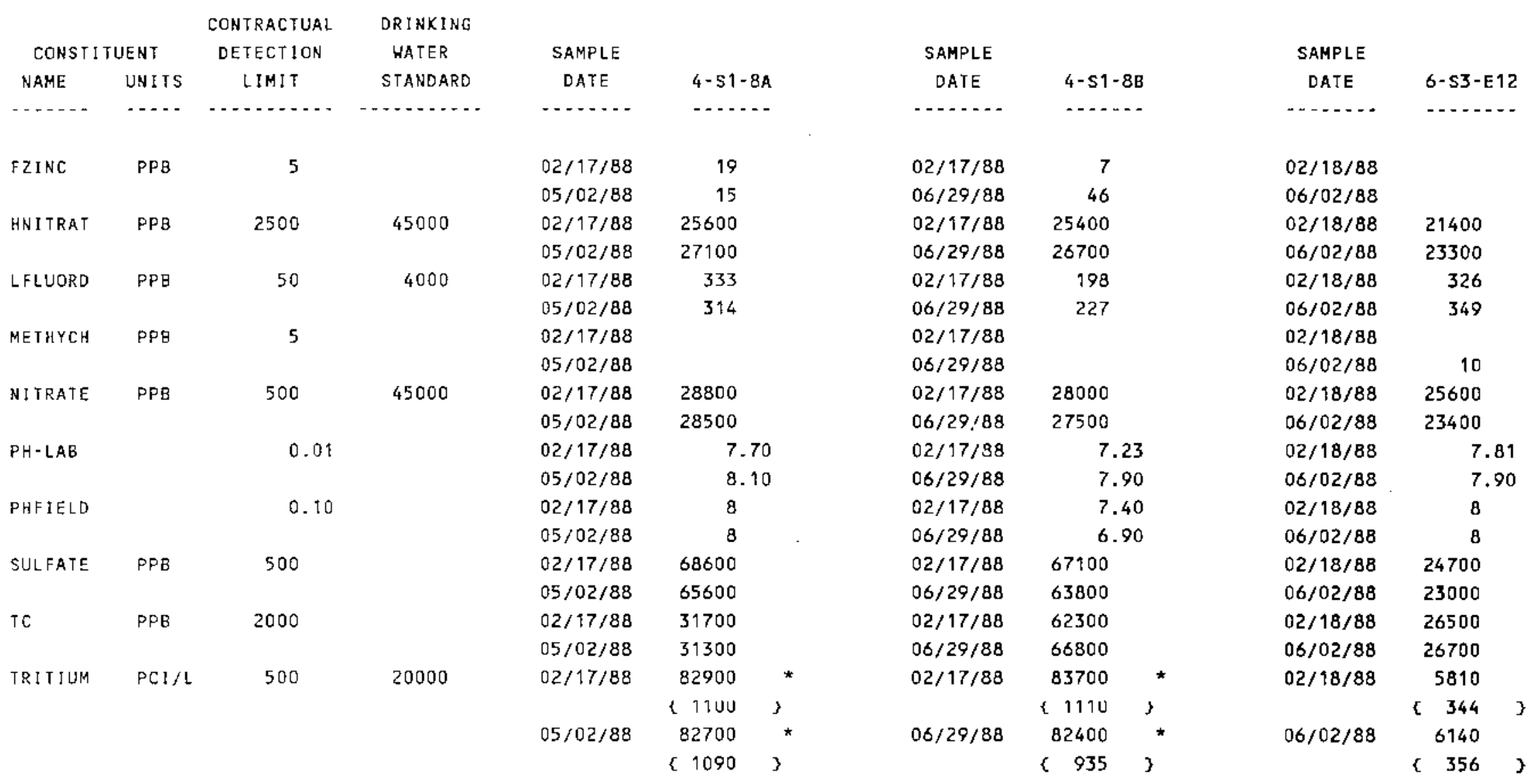


TABLE E.3. (contd)

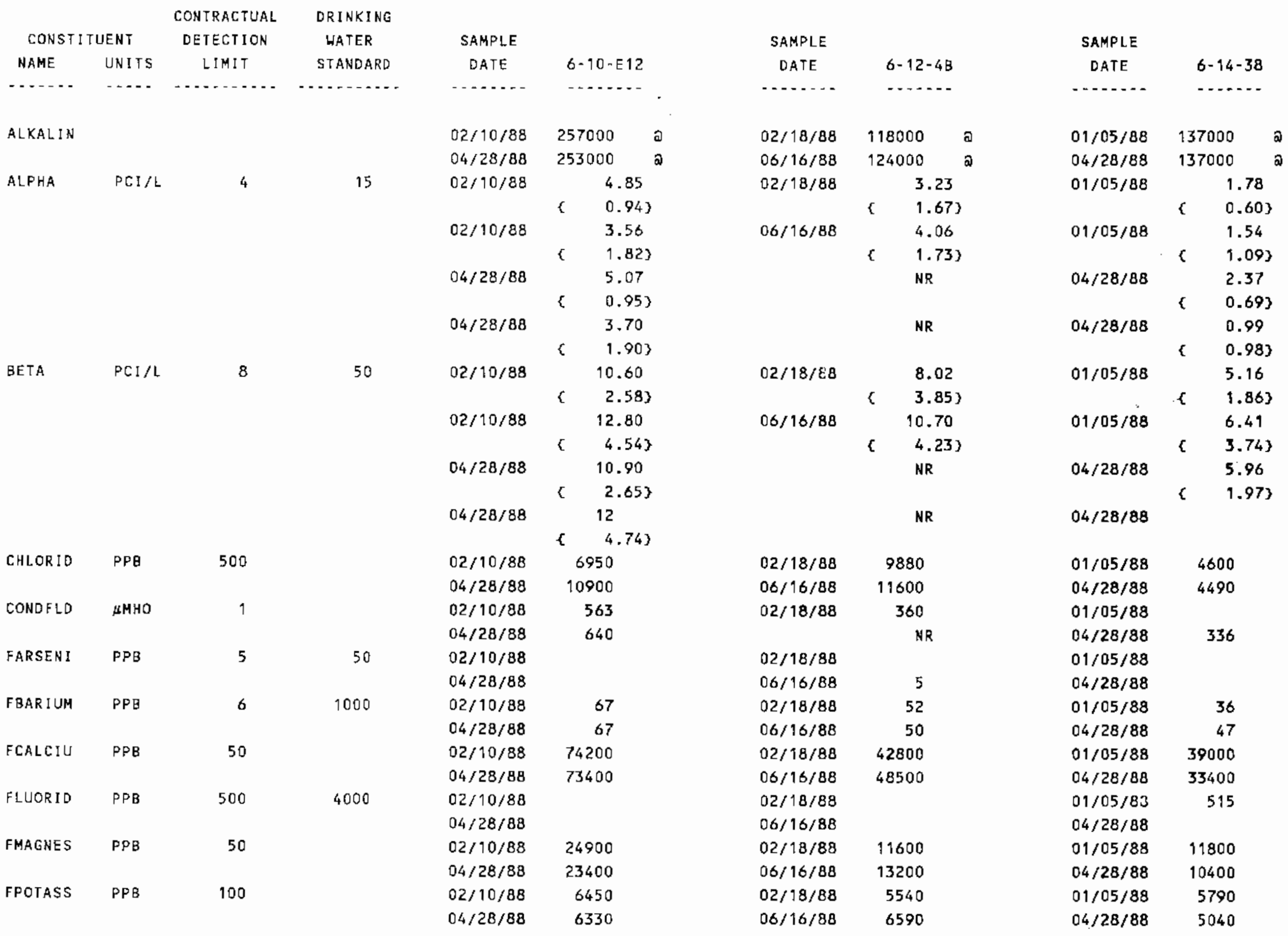


IABLE E.3. (contd)

\begin{tabular}{|c|c|c|c|c|c|c|c|c|c|}
\hline \multicolumn{2}{|c|}{ CONST I TUENT } & $\begin{array}{l}\text { CONTRACTUAL } \\
\text { DETECTION }\end{array}$ & $\begin{array}{l}\text { ORINKING } \\
\text { WATER }\end{array}$ & SAMPLE & & SAMPLE & & SAMPLE & \\
\hline NAME & UNITS & LIMIT & STANDARD & DATE & $6-10-E 12$ & DATE & $6-12-4 B$ & DATE & $6-14-38$ \\
\hline$-\ldots .$. & .... & - n. & - n & $\ldots$ & $\ldots$ & …...... & ....... & - n...... & - n. \\
\hline FSODIUM & $P P B$ & 200 & & $02 / 10 / 88$ & 18400 & $02 / 18 / 88$ & 16000 & $01 / 05 / 88$ & 17100 \\
\hline & & & & $04 / 28 / 88$ & 18300 & $06 / 16 / 88$ & 18300 & $04 / 28 / 88$ & 15000 \\
\hline FSIRDNT & $P P B$ & 10 & & $02 / 10 / 88$ & 520 & $02 / 18 / 88$ & 230 & $01 / 05 / 88$ & 192 \\
\hline & & & & $04 / 28 / 88$ & 500 & $06 / 16 / 88$ & 255 & $04 / 28 / 88$ & 171 \\
\hline FVANAD I & $\mathrm{PPB}$ & 5 & & $02 / 10 / 88$ & 15 & $02 / 18 / 88$ & 9 & $01 / 05 / 88$ & \\
\hline & & & & $04 / 28 / 88$ & 10 & $06 / 16 / 88$ & 10 & $04 / 28 / 88$ & \\
\hline FZINC & $P P B$ & 5 & & $02 / 10 / 88$ & & $02 / 18 / 88$ & & $01 / 05 / 88$ & \\
\hline & & & & $04 / 28 / 88$ & & $06 / 16 / 88$ & 6 & $04 / 28 / 88$ & \\
\hline HNITRAT & $P P B$ & 2500 & 45000 & $02 / 10 / 88$ & 19100 & & NR & $01 / 05 / 88$ & 3530 \\
\hline & & & & $04 / 28 / 88$ & 21300 & & NR & $04 / 28 / 88$ & 3660 \\
\hline LFLUORD & PPB & 50 & 4000 & $02 / 10 / 88$ & 201 & $02 / 18 / 38$ & 283 & $01 / 05 / 88$ & 415 \\
\hline & & & & $04 / 28 / 88$ & 201 & $06 / 16 / 88$ & 306 & $04 / 28 / 88$ & 349 \\
\hline NITRATE & PPB & 500 & 45000 & $02 / 10 / 88$ & 22200 & $02 / 18 / 88$ & 27400 & $01 / 05 / 88$ & 3590 \\
\hline & & & & $04 / 28 / 88$ & 21300 & $06 / 16 / 88$ & 26700 & $04 / 28 / 88$ & 3290 \\
\hline
\end{tabular}


IABLE E.3. (contd)

\begin{tabular}{|c|c|c|c|c|c|c|c|c|c|}
\hline \multicolumn{2}{|c|}{ CONST 1 TUENT } & $\begin{array}{l}\text { CONTRACTUAL } \\
\text { DETECTION }\end{array}$ & $\begin{array}{l}\text { DRINKING } \\
\text { WATER }\end{array}$ & SAMPLE & & SAMPLE & & SAMPLE & \\
\hline NAME & UNITS & LIHIT & STANDARD & DATE & $6-10-E_{12}$ & DATE & $6-12-48$ & DATE & $6-14 \cdot 38$ \\
\hline ...... & $-\cdots$ & . & (n. & $\ldots$ & $\ldots$ & ......... & $\cdots$ & ......... & -....... \\
\hline \multirow[t]{2}{*}{$P H-L A B$} & & 0.01 & & $02 / 10 / 88$ & 7.43 & $02 / 18 / 88$ & 7.56 & $01 / 05 / 88$ & 7.74 \\
\hline & & & & $04 / 28 / 88$ & 7.60 & $06 / 16 / 88$ & 7.90 & $04 / 28 / 88$ & 8 \\
\hline \multirow[t]{2}{*}{ PHEIELD } & & 0.10 & & $02 / 10 / 88$ & 7.80 & $02 / 18 / 88$ & 7.80 & $01 / 05 / 88$ & \\
\hline & & & & $04 / 28 / 88$ & 7.40 & & NR & $04 / 28 / 88$ & 7.40 \\
\hline \multirow[t]{2}{*}{ SULFATE } & $P P B$ & 500 & & $02 / 10 / 88$ & 29200 & $02 / 18 / 88$ & 44300 & $01 / 05 / 88$ & 23500 \\
\hline & & & & $04 / 28 / 88$ & 27900 & $06 / 16 / 88$ & 44600 & $04 / 28 / 88$ & 21800 \\
\hline \multirow[t]{2}{*}{ TC } & $\mathrm{PPB}$ & 2000 & & $02 / 10 / 88$ & 59900 & $02 / 18 / 88$ & 28700 & $01 / 05 / 88$ & 30800 \\
\hline & & & & $04 / 28 / 88$ & 63900 & $06 / 16 / 88$ & 28100 & $04 / 28 / 88$ & 32300 \\
\hline \multirow[t]{4}{*}{ TRITIUM } & $\mathrm{PCI} / \mathrm{L}$ & 500 & 20000 & $02 / 10 / 88$ & 17000 & & NR & $01 / 05 / 88$ & \\
\hline & & & & & c 527 & & & & \\
\hline & & & & $04 / 28 / 88$ & 17500 & & NR & $04 / 28 / 88$ & \\
\hline & & & & & $\{533\}$ & & & & \\
\hline
\end{tabular}


TABLE E.3. (contd)

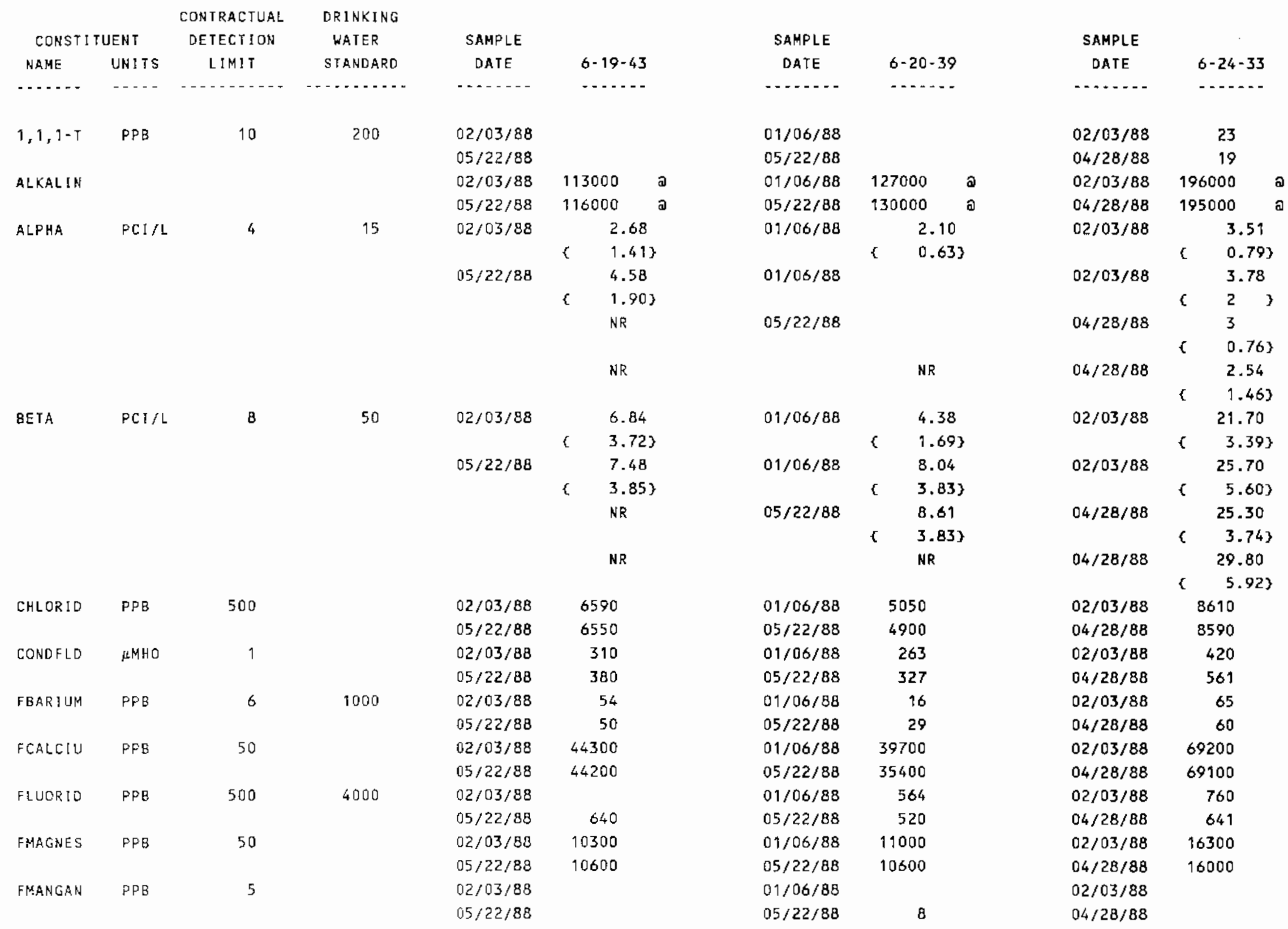


TABLE E.3. (contd)

\begin{tabular}{|c|c|c|c|c|c|c|c|c|c|}
\hline \multirow{2}{*}{\multicolumn{2}{|c|}{ CONST I TUENT }} & CONTRACTUAL & DRINKING & & & & & \multirow{2}{*}{\multicolumn{2}{|c|}{ SAMPLE }} \\
\hline & & DETECTION & WATER & SAMPLE & \multicolumn{3}{|c|}{ SAMPLE } & & \\
\hline NAME & UNITS & LIMIT & STANDARD & DATE & $6-19-43$ & DATE & $6-20-39$ & DATE & $6-24-33$ \\
\hline$\cdots \cdots$ & $-\cdots$ & - & - & 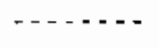 & $\cdots$ & $\ldots \ldots$ & $\ldots$ & $\ldots$ & $\cdots$ \\
\hline \multirow[t]{2}{*}{ FPOTASS } & $P P B$ & 100 & & $02 / 03 / 88$ & 5240 & $01 / 06 / 88$ & 5160 & $02 / 03 / 88$ & 7330 \\
\hline & & & & $05 / 22 / 88$ & 5730 & $05 / 22 / 88$ & 4920 & $04 / 28 / 88$ & 7400 \\
\hline \multirow[t]{2}{*}{ FSODIUM } & $\mathrm{PPB}$ & 200 & & $02 / 03 / 88$ & 17400 & $01 / 06 / 88$ & 16300 & $02 / 03 / 88$ & 23400 \\
\hline & & & & $05 / 22 / 88$ & 18400 & $05 / 22 / 88$ & 14900 & $04 / 28 / 88$ & 23700 \\
\hline \multirow[t]{2}{*}{ FSTRONT } & PPS & 10 & & $02 / 03 / 88$ & 203 & $01 / 06 / 88$ & 194 & $02 / 03 / 88$ & 278 \\
\hline & & & & $05 / 22 / 88$ & 206 & $05 / 22 / 88$ & 186 & $04 / 28 / 88$ & 274 \\
\hline \multirow[t]{2}{*}{ FVANADI } & $\mathrm{PPB}$ & 5 & & $02 / 03 / 88$ & 9 & $01 / 06 / 88$ & & $02 / 03 / 88$ & 17 \\
\hline & & & & $05 / 22 / 88$ & 8 & $05 / 22 / 88$ & 7 & $04 / 28 / 88$ & 15 \\
\hline \multirow[t]{2}{*}{ FZINE } & PPB & 5 & & $02 / 03 / 88$ & 183 & $01 / 06 / 88$ & & $02 / 03 / 88$ & \\
\hline & & & & $05 / 22 / 88$ & $23 i$ & $05 / 22 / 88$ & & $04 / 28 / 88$ & \\
\hline \multirow[t]{2}{*}{ HNITRAT } & $\mathrm{PPB}$ & 2500 & 45000 & $02 / 03 / 88$ & 9140 & $01 / 06 / 88$ & 5540 & $02 / 03 / 88$ & 24100 \\
\hline & & & & & NR & & NR & $04 / 28 / 88$ & 27800 \\
\hline \multirow[t]{2}{*}{$I-1290 W$} & $P C I / L$ & 1 & & & NR & & NR & $04 / 28 / 88$ & 2.71 \\
\hline & & & & & & & & & 0.433 \\
\hline
\end{tabular}


IABLE E.3. (contd)

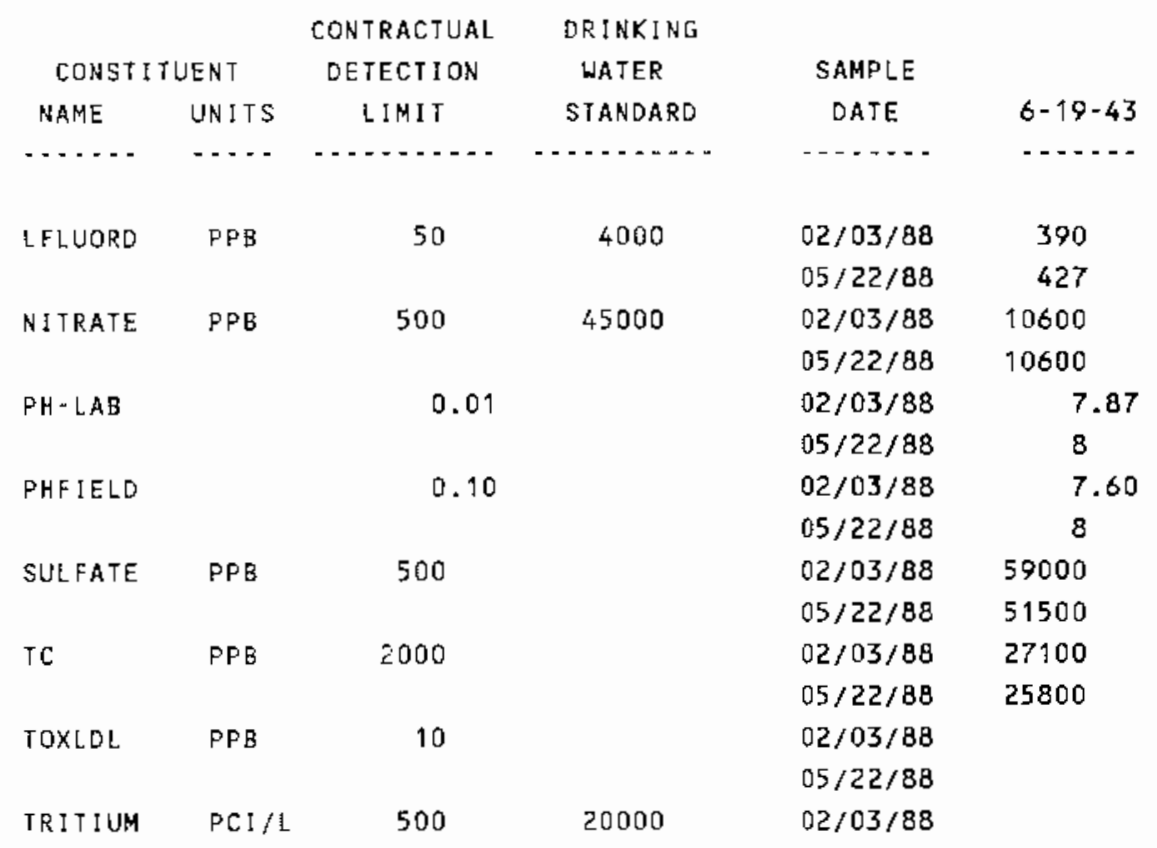

$\begin{array}{lc}\begin{array}{c}\text { SAMPLE } \\ \text { DATE }\end{array} & 6-20-39 \\ \ldots \ldots \ldots & \ldots \ldots \ldots \\ & \\ 01 / 06 / 88 & 400 \\ 05 / 22 / 88 & 418 \\ 01 / 06 / 88 & 5590 \\ 05 / 22 / 88 & 4820 \\ 01 / 06 / 88 & 7.80 \\ 05 / 22 / 88 & 8 \\ 01 / 06 / 88 & 7.80 \\ 05 / 22 / 88 & 8.10 \\ 01 / 06 / 88 & 27500 \\ 05 / 22 / 88 & 27000 \\ 01 / 06 / 38 & 28400 \\ 05 / 22 / 88 & 28800 \\ 01 / 06 / 88 & \\ 05 / 22 / 88 & \\ 01 / 06 / 88 & \end{array}$

NR
SAMPLE

DATE $6-24-33$

$\begin{array}{lc}02 / 03 / 88 & 428 \\ 04 / 28 / 88 & 425 \\ 02 / 03 / 88 & 28200 \\ 04 / 28 / 88 & 29200 \\ 02 / 03 / 88 & 7.52 \\ 04 / 28 / 88 & 7.60 \\ 02 / 03 / 88 & 6.90 \\ 04 / 28 / 88 & 6.80 \\ 02 / 03 / 88 & 48300 \\ 04 / 28 / 88 & 41900 \\ 02 / 03 / 88 & 49800 \\ 04 / 28 / 88 & 50300 \\ 02 / 03 / 88 & 25 \\ 04 / 28 / 88 & 21 \\ 02 / 03 / 88 & 127000 \\ & \{1380 \\ 04 / 28 / 88 & 149000\end{array}$ *


IABLE E.3. (contd)

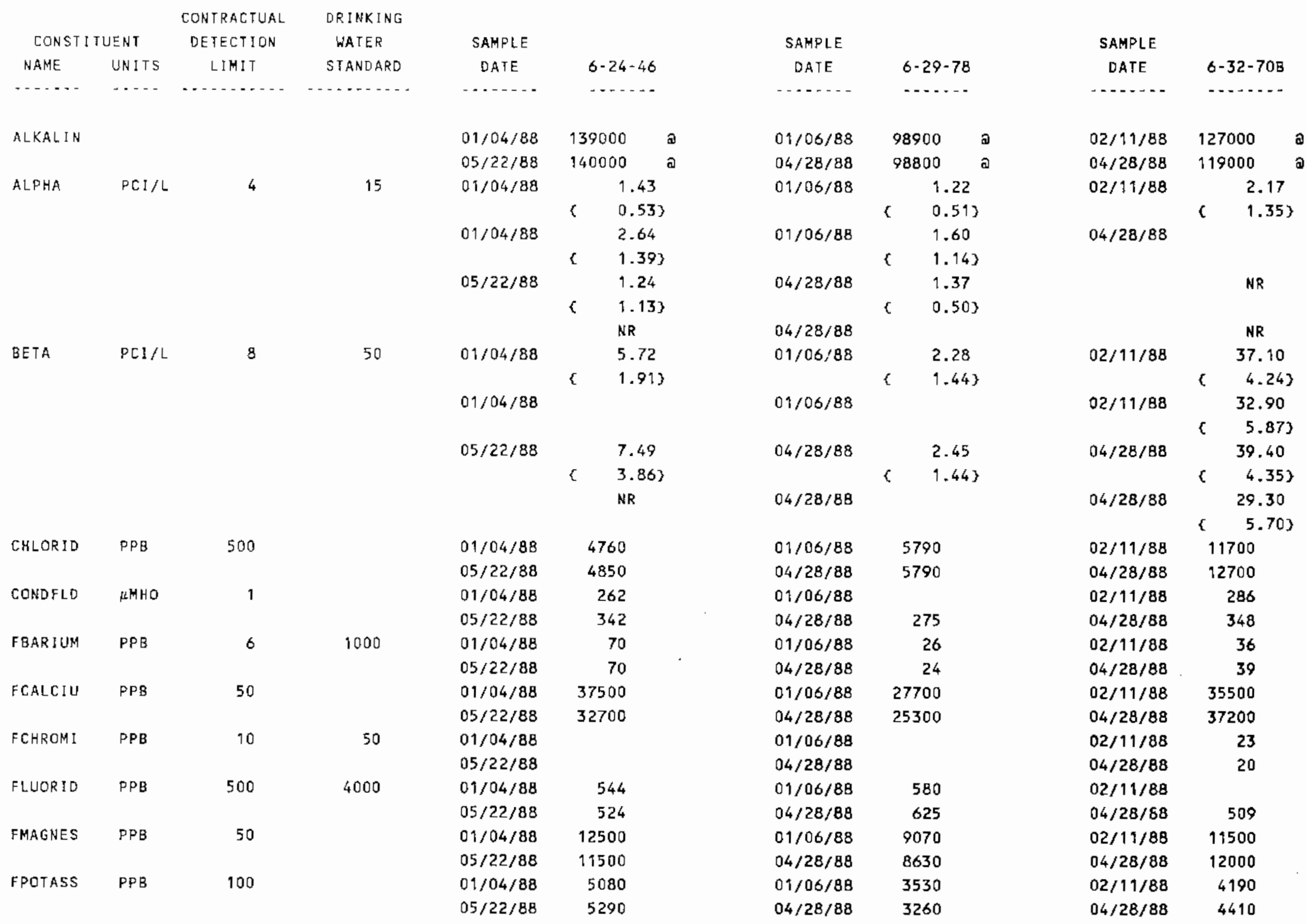


TABLE E.3. (contd)

\begin{tabular}{|c|c|c|c|}
\hline \multirow{2}{*}{\multicolumn{2}{|c|}{ CONST I TUENT }} & CONTRACTUAL & DRINKING \\
\hline & & DETECIION & WATER \\
\hline NAME & UN ITS & LIMIT & STANDARD \\
\hline 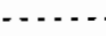 & $\cdots$ & 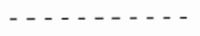 & $\cdots$ \\
\hline
\end{tabular}

SAMPLE$$
\text { DATE }
$$

- - - D...

$\begin{array}{llr}\text { FSODIUM } & \text { PPB } & 200 \\ \text { FSTRONT } & \text { PPB } & 10 \\ \text { FVANADI } & \text { PPB } & 5 \\ \text { FZINC } & \text { PPB } & 5\end{array}$

HNITRAT PPB $\quad 2500 \quad 45000$

$1-1290 \mathrm{~W} \quad \mathrm{PCI} / \mathrm{L} \quad 1$

LFLUORD PPB $\quad 50$

NITRATE PPB $\quad 500$
$01 / 04 / 88$

$05 / 22 / 88$

$01 / 04 / 88$

$05 / 22 / 88$

$01 / 04 / 88$

$05 / 22 / 88$

$01 / 04 / 88$

$05 / 22 / 88$

$01 / 04 / 88$

$01 / 04 / 88$

$05 / 22 / 88$

$01 / 04 / 88$
SAMPLE

6-24-46

…...-

\section{0}

14800

166

152

8

6

8900

NR

NR

465

459
DATE

-......

$6-29-78$

$01 / 06 / 88$

$04 / 28 / 88$

$01 / 06 / 88$

$04 / 28 / 88$

$01 / 06 / 88$

$04 / 28 / 88$

$01 / 06 / 88$

$04 / 28 / 88$

$01 / 06 / 88$

$04 / 28 / 88$

$04 / 28 / 38$

$01 / 06 / 88$

$04 / 28 / 88$

$01 / 06 / 88$
SAMPLE

DATE

$6-32-70 B$

-......

-.-...

$02 / 11 / 88 \quad 18400$

$04 / 28 / 88 \quad 19500$

$02 / 11 / 88 \quad 156$

$04 / 28 / 88 \quad 159$

$02 / 11 / 88 \quad 28$

$04 / 28 / 88 \quad 30$

$02 / 91 / 88$

$04 / 28 / 88 \quad 11$

$02 / 11 / 88 \quad 13200$

$04 / 28 / 88 \quad 17400$

$04 / 28 / 88 \quad 3.25$

$02 / 19 / 88 \quad 403$

$04 / 28 / 88 \quad 393$

$02 / 11 / 88 \quad 18800$ 
IABLE E.3. (contd)

\begin{tabular}{|c|c|c|c|c|c|c|c|c|c|}
\hline \multicolumn{2}{|c|}{ CONSIITUENT } & $\begin{array}{l}\text { CONTRACTUAL } \\
\text { DETECTIDN }\end{array}$ & $\begin{array}{l}\text { DRINKING } \\
\text { WATER }\end{array}$ & SAMPLEE & & SAMPLE & & SAMPLE & \\
\hline NAME & UNITS & LIMIT & STANDARD & DATE & $6-24-46$ & DATE & $6-29-78$ & DATE & $6+32-70 \mathrm{~B}$ \\
\hline ….... & $\cdots$ & 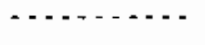 & …...... & ........... & ........ & .......... & ….... & $\ldots$ & . \\
\hline NITRATE & PPB & 500 & 45000 & $05 / 22 / 88$ & 8310 & $04 / 28 / 88$ & 7410 & $04 / 28 / 88$ & 18100 \\
\hline \multirow[t]{2}{*}{$P H-L A B$} & & 0.01 & & $01 / 04 / 88$ & 7.85 & $01 / 06 / 88$ & 7.73 & $02 / 11 / 88$ & 7.86 \\
\hline & & & & $05 / 22 / 88$ & 7.90 & $04 / 28 / 88$ & 8 & $04 / 28 / 88$ & 7.90 \\
\hline \multirow[t]{2}{*}{ PHFIELD } & & 0,10 & & $01 / 04 / 88$ & 6.10 & $01 / 06 / 88$ & 8.10 & $02 / 11 / 88$ & 6.90 \\
\hline & & & & $05 / 22 / 88$ & 8 & $04 / 28 / 88$ & 7.80 & $04 / 28 / 88$ & 7 \\
\hline \multirow[t]{2}{*}{ SULFATE } & PPB & 500 & & $01 / 04 / 88$ & 28400 & $01 / 06 / 88$ & 21900 & $02 / 11 / 88$ & 23900 \\
\hline & & & & $05 / 22 / 88$ & 26700 & $04 / 28 / 88$ & 20400 & $04 / 28 / 88$ & 22300 \\
\hline \multirow[t]{2}{*}{ TC } & $P P B$ & 2000 & & $01 / 04 / 88$ & 30900 & $01 / 06 / 88$ & 22700 & $02 / 11 / 88$ & 30700 \\
\hline & & & & $05 / 22 / 88$ & 31300 & $04 / 28 / 88$ & 24700 & $04 / 28 / 88$ & 30600 \\
\hline \multirow[t]{2}{*}{ TC-99 } & $\mathrm{PCI} / \mathrm{L}$ & 15 & 900 & & NR & & NR & $02 / 11 / 88$ & 199 \\
\hline & & & & & & & & & ( $\quad 2.72)$ \\
\hline \multirow[t]{4}{*}{ TRITIUM } & PCI/L & 500 & 20000 & $01 / 04 / 88$ & & $01 / 06 / 88$ & & $02 / 11 / 88$ & 269000 \\
\hline & & & & & & & & & \& 1980 \\
\hline & & & & & NR & $04 / 28 / 88$ & & $04 / 28 / 88$ & 261000 \\
\hline & & & & & & & & & $(1930)$ \\
\hline \multirow[t]{2}{*}{$u$} & $\mathrm{PCI} / \mathrm{L}$ & 0.50 & 600 & & NR & & NR & $02 / 11 / 88$ & 1.74 \\
\hline & & & & & $N R$ & & NR & $04 / 28 / 88$ & 1.08 \\
\hline
\end{tabular}


TABLE E.3. (contd)

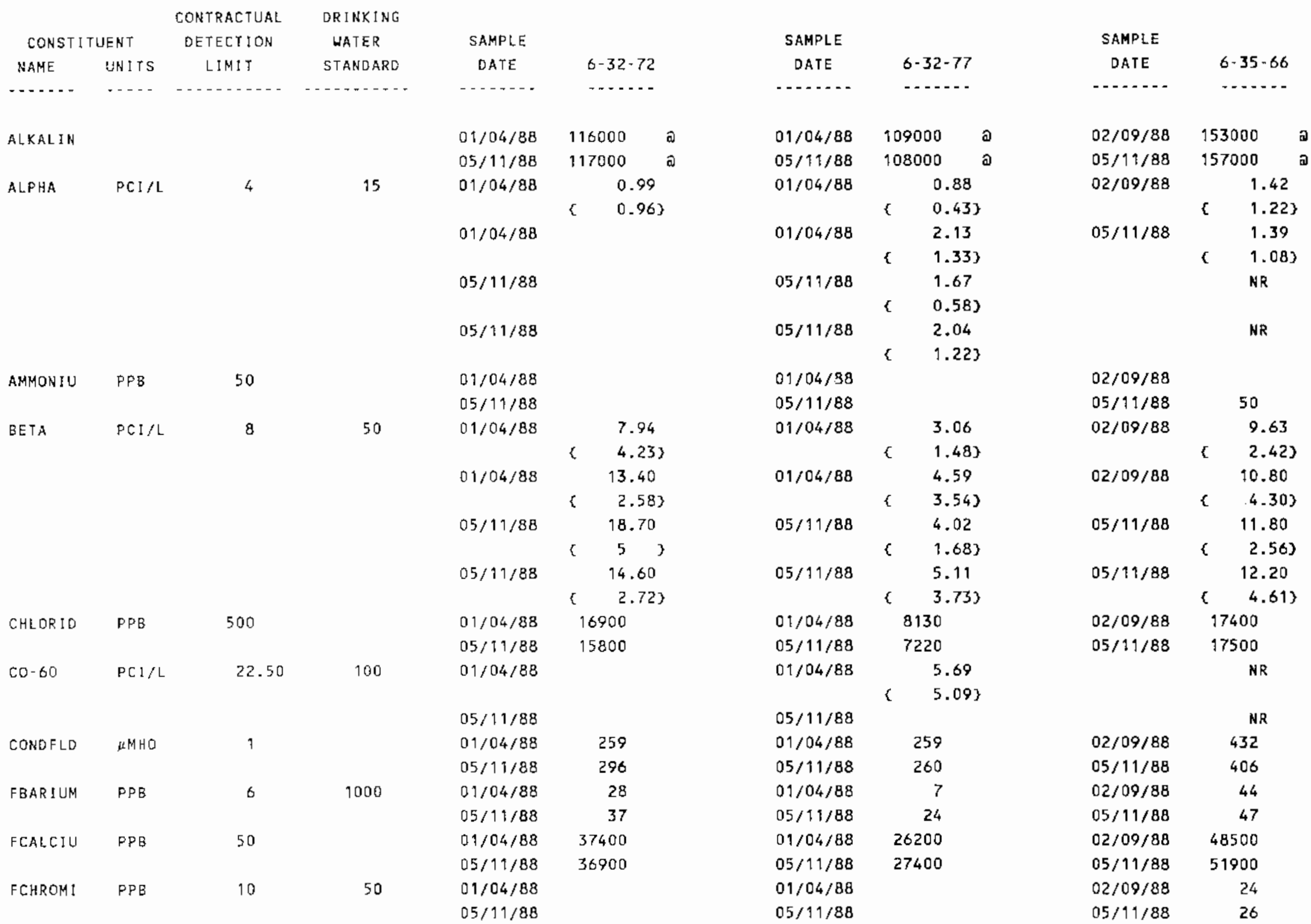


IABLE E. 3. (contd)

\begin{tabular}{|c|c|c|c|c|c|c|c|c|c|}
\hline \multirow{2}{*}{\multicolumn{2}{|c|}{ CONSTITUENT }} & \multirow{3}{*}{$\begin{array}{c}\text { CONTRACTUAL } \\
\text { DETECTION } \\
\text { LIMII }\end{array}$} & \multirow{3}{*}{$\begin{array}{l}\text { DRINKING } \\
\text { WATER } \\
\text { STANDARD }\end{array}$} & \multirow[b]{2}{*}{ SAMPLE } & \multirow{2}{*}{\multicolumn{3}{|c|}{ SAMPLE }} & \multirow[b]{2}{*}{ SAMPLE } & \multirow[b]{3}{*}{$6-35-66$} \\
\hline & & & & & & & & & \\
\hline NAME & UNITS & & & DATE & $6 \cdot 32-72$ & DATE & $6-32-77$ & DATE & \\
\hline$\cdots \ldots$ & $\cdots$ & , n. & $\ldots \ldots \ldots$ & $\ldots$ & - . . - & - . . & …... & $\cdots$ & - . n. \\
\hline \multirow[t]{2}{*}{ FLUORIO } & $P P B$ & 500 & 4000 & $01 / 04 / 88$ & 560 & $01 / 04 / 88$ & 676 & $02 / 09 / 88$ & 521 \\
\hline & & & & $05 / 11 / 88$ & 548 & $05 / 11 / 88$ & 620 & $05 / 11 / 88$ & \\
\hline \multirow[t]{2}{*}{ FMAGNES } & PPB & 50 & & $01 / 04 / 88$ & 12800 & $01 / 04 / 88$ & 8060 & $02 / 09 / 88$ & 14200 \\
\hline & & & & $05 / 11 / 88$ & 12500 & $05 / 11 / 88$ & 7890 & $05 / 11 / 88$ & 14200 \\
\hline \multirow[t]{2}{*}{ FMANGAN } & PPB & 5 & & $01 / 04 / 88$ & 14 & $01 / 04 / 88$ & & $02 / 09 / 88$ & \\
\hline & & & & $05 / 11 / 88$ & 10 & $05 / 11 / 88$ & & $05 / 11 / 88$ & \\
\hline \multirow[t]{2}{*}{ FPOTASS } & PPB & 100 & & $01 / 04 / 88$ & 3660 & $01 / 04 / 88$ & 3420 & $02 / 09 / 88$ & 5680 \\
\hline & & & & $05 / 11 / 88$ & 3990 & $05 / 11 / 8 B$ & 3070 & $05 / 11 / 88$ & 5250 \\
\hline \multirow[t]{2}{*}{ FSOD IUM } & PPB & 200 & & $01 / 04 / 88$ & 14300 & $01 / 04 / 88$ & 19800 & $02 / 09 / 88$ & 21800 \\
\hline & & & & $05 / 11 / 88$ & 15300 & $05 / 11 / 88$ & 17700 & $05 / 11 / 88$ & 20200 \\
\hline \multirow[t]{2}{*}{ FSTRONT } & PPB & 10 & & $01 / 04 / 88$ & 143 & $01 / 04 / 38$ & 107 & $02 / 09 / 88$ & 211 \\
\hline & & & & $05 / 11 / 88$ & 141 & $05 / 11 / 88$ & 112 & $05 / 11 / 88$ & 207 \\
\hline \multirow[t]{2}{*}{ FVANADI } & $P P B$ & 5 & & $01 / 04 / 88$ & 25 & $01 / 04 / 88$ & & $02 / 09 / 88$ & 31 \\
\hline & & & & $05 / 11 / 88$ & 26 & $05 / 11 / 88$ & 39 & $05 / 11 / 88$ & 30 \\
\hline FZINC & $P P B$ & 5 & & $01 / 04 / 88$ & & $01 / 04 / 88$ & & $02 / 09 / 88$ & 8 \\
\hline
\end{tabular}


TABLE E.3. (contd)

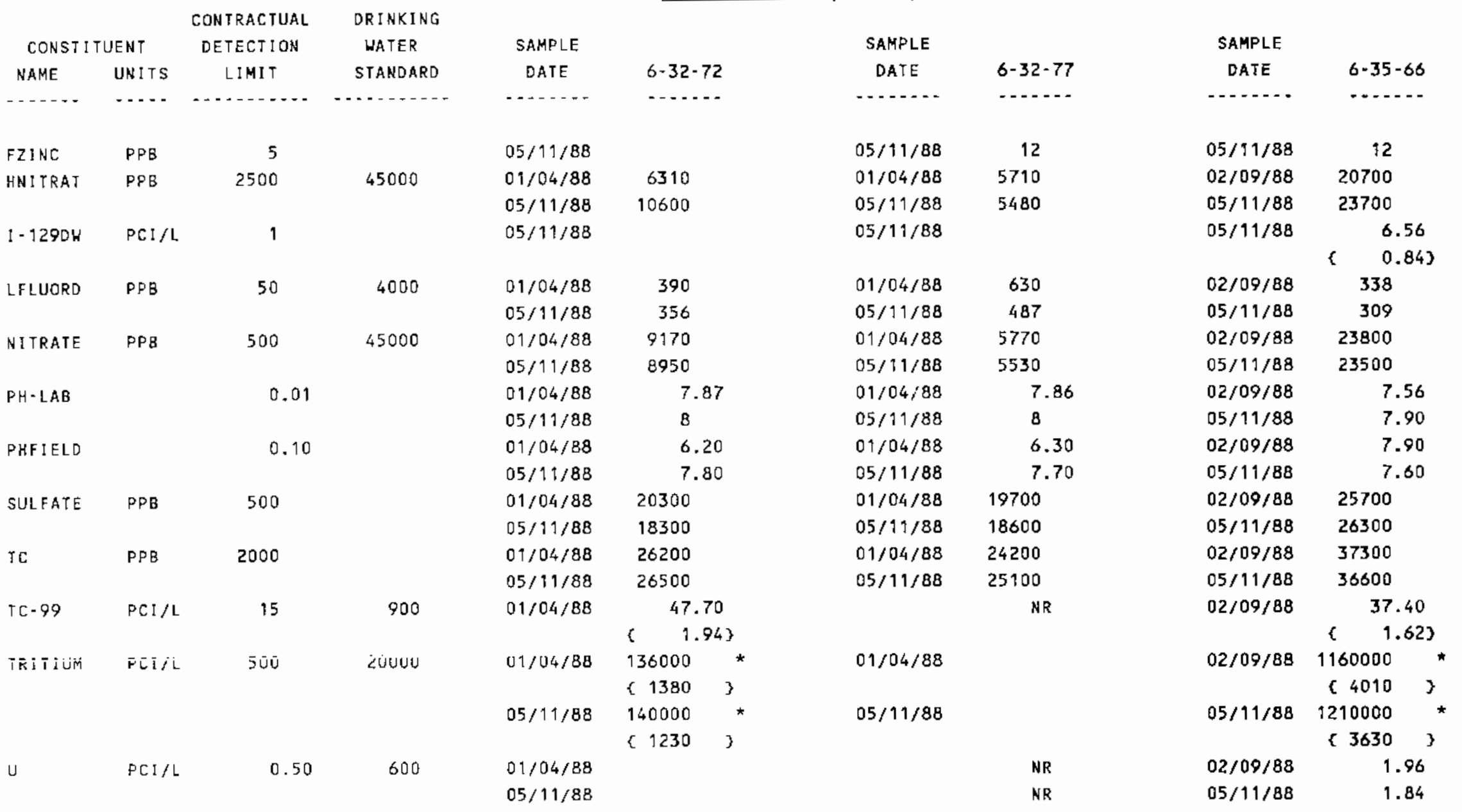


TABLE E.3. (contd)

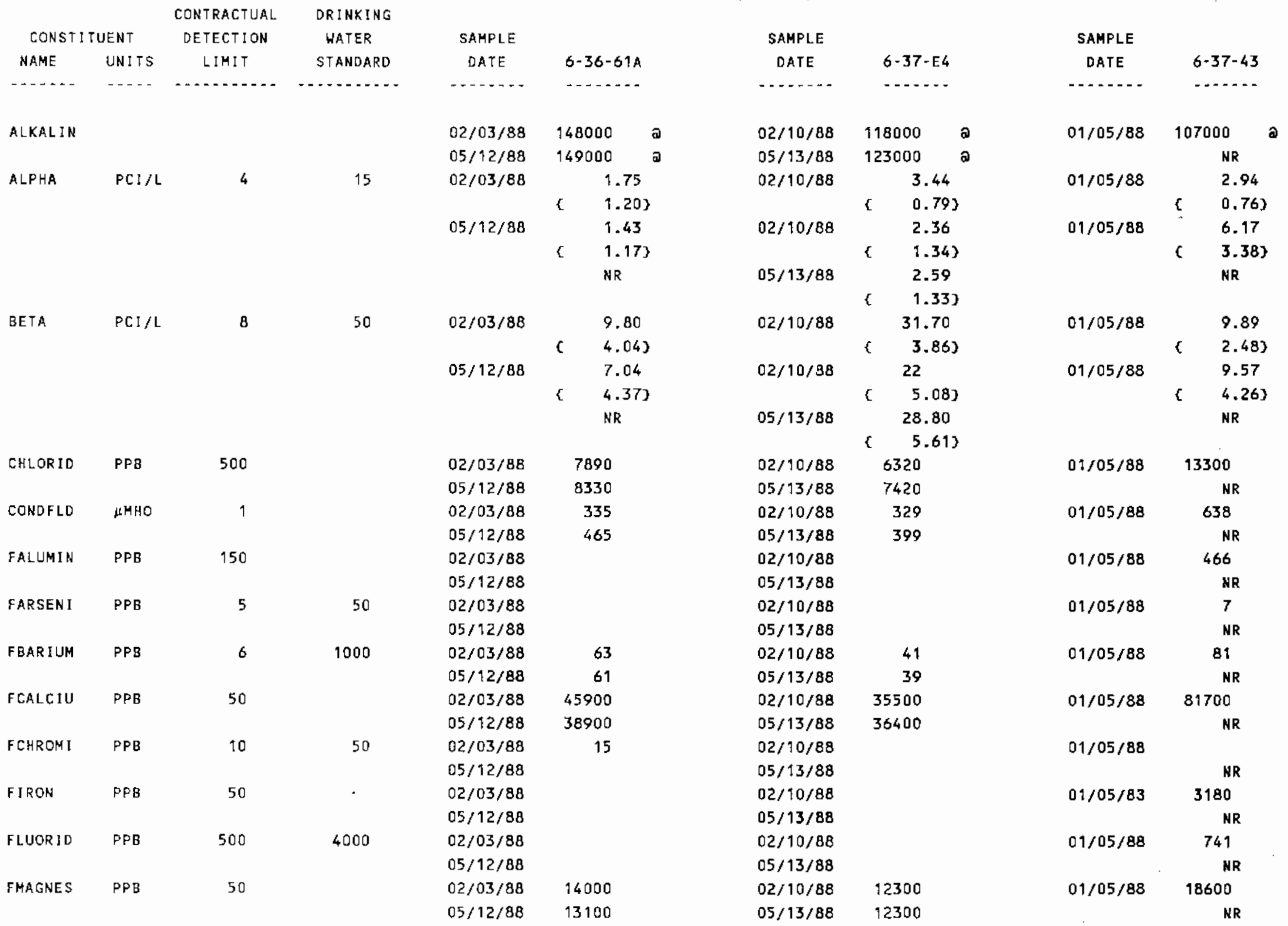


TABLE E.3. (contd)

\begin{tabular}{|c|c|c|c|c|c|c|c|c|c|}
\hline \multicolumn{2}{|c|}{ CONST I TUENT } & $\begin{array}{l}\text { CONTRACTUAL } \\
\text { DETECTION }\end{array}$ & $\begin{array}{l}\text { DRINKING } \\
\text { WATER }\end{array}$ & SAMPLE & & SAMPLE & & SAMPLE & \\
\hline \multirow{2}{*}{ NAME } & UNITS & LIMIT & STANDARD & DATE & $6-36-61 \mathrm{~A}$ & DATE & $6-37-E 4$ & DATE & $6-37-43$ \\
\hline & $\cdots$ & ...... & מ & ......... & ......... & - n & - n. & - $\ldots \ldots$ & - - - \\
\hline \multirow[t]{2}{*}{ FMANGAN } & PPB & 5 & & $02 / 03 / 8 B$ & & $02 / 10 / 88$ & & $01 / 05 / 88$ & 30 \\
\hline & & & & $05 / 12 / 88$ & & $05 / 13 / 88$ & & & NR \\
\hline \multirow[t]{2}{*}{ FPOTASS } & $\mathrm{PPB}$ & 100 & & $02 / 03 / 88$ & 5210 & $02 / 10 / 88$ & 5270 & $01 / 05 / 88$ & 8520 \\
\hline & & & & $05 / 12 / 88$ & 4980 & $05 / 13 / 88$ & 5100 & & NR \\
\hline \multirow[t]{2}{*}{ FSELEN I } & PPB & 5 & 10 & $02 / 03 / 88$ & 5 & $02 / 10 / 88$ & & $01 / 05 / 88$ & \\
\hline & & & & $05 / 12 / 88$ & & $05 / 13 / 88$ & & & NR \\
\hline \multirow[t]{2}{*}{ F SOD I UM } & PPB & 200 & & $02 / 03 / 88$ & 17200 & $02 / 10 / 88$ & 16400 & $01 / 05 / 88$ & 54700 \\
\hline & & & & $05 / 12 / 88$ & 17900 & $05 / 13 / 88$ & 16500 & & NR \\
\hline \multirow[t]{2}{*}{ FSTRONT } & PPB & 10 & & $02 / 03 / 88$ & 190 & $02 / 10 / 88$ & 287 & $01 / 05 / 88$ & 404 \\
\hline & & & & $05 / 12 / 88$ & 173 & $05 / 13.8 B$ & 284 & & NR \\
\hline \multirow[t]{2}{*}{ FVANADI } & PPB & 5 & & $02 / 03 / 88$ & 25 & $02 / 10 / 38$ & 13 & $01 / 05 / 88$ & \\
\hline & & & & $05 / 12 / 88$ & 26 & $05 / 13 / 88$ & 15 & & NR \\
\hline \multirow[t]{2}{*}{ FZINC } & PPB & 5 & & $02 / 03 / 88$ & & $02 / 10 / 88$ & & $01 / 05 / 88$ & 165 \\
\hline & & & & $05 / 12 / 88$ & & $05 / 13 / 88$ & & & NR \\
\hline
\end{tabular}


TABLE E.3. (contd)

\begin{tabular}{|c|c|c|c|c|c|}
\hline \multirow{3}{*}{\multicolumn{2}{|c|}{ CONSTI IUENT }} & \multirow{3}{*}{$\begin{array}{c}\text { CONTRACTUAL } \\
\text { DETECTION } \\
\text { LIMIT }\end{array}$} & \multirow{2}{*}{$\begin{array}{l}\text { DRINKING } \\
\text { WATER }\end{array}$} & \multirow[b]{2}{*}{ SAMPLE } & \multirow[b]{3}{*}{$6.36-61 A$} \\
\hline & & & & & \\
\hline & UNITS & & STANDARD & DATE & \\
\hline$\cdots+$ & $\cdots$ & $---1, \ldots$ & - & - & - \\
\hline \multirow[t]{2}{*}{ HNI TRAT } & PPB & 2500 & 45000 & $02 / 03 / 88$ & 18300 \\
\hline & & & & $05 / 12 / 88$ & 20200 \\
\hline \multirow[t]{2}{*}{ LFLUORD } & PPB & 50 & 4000 & $02 / 03 / 88$ & 363 \\
\hline & & & & $05 / 12 / 88$ & 321 \\
\hline \multirow[t]{2}{*}{ NITRATE } & PPB & 500 & 45000 & $02 / 03 / 88$ & 20900 \\
\hline & & & & $05 / 12 / 88$ & 20800 \\
\hline \multirow[t]{2}{*}{$P H-L A B$} & & 0.01 & & $02 / 03 / 88$ & 7.66 \\
\hline & & & & $05 / 12 / 88$ & 7.90 \\
\hline \multirow[t]{2}{*}{ PHFIELD } & & 0.10 & & $02 / 03 / 88$ & 7.30 \\
\hline & & & & $05 / 12 / 88$ & 7.30 \\
\hline \multirow[t]{2}{*}{ SULFATE } & $P P B$ & 500 & & $02 / 03 / 88$ & 30000 \\
\hline & & & & $05 / 12 / 88$ & 30800 \\
\hline \multirow[t]{2}{*}{ TC } & PPB & 2000 & & $02 / 03 / 88$ & 35100 \\
\hline & & & & $05 / 12 / 88$ & 34700 \\
\hline TRITIUM & $\mathrm{PCI} / \mathrm{L}$ & 500 & 20000 & & $N R$ \\
\hline
\end{tabular}

$\begin{array}{cc}\text { SAMPLE } & \\ \text { DATE } & 6-37-E 4 \\ \ldots \ldots \ldots & -\ldots-\ldots \\ & \\ 02 / 10 / 88 & 25900 \\ & \text { NR } \\ 02 / 10 / 88 & 307 \\ 05 / 13 / 88 & 321 \\ 02 / 10 / 88 & 27700 \\ 05 / 13 / 88 & 28400 \\ 02 / 10 / 88 & 7.76 \\ 05 / 13 / 88 & 8 \\ 02 / 10 / 88 & 8.10 \\ 05 / 13 / 88 & 7.80 \\ 02 / 10 / 88 & 27100 \\ 05 / 13 / 88 & 27600 \\ 02 / 10 / 88 & 28300 \\ 05 / 13 / 88 & 27800 \\ 02 / 10 / 88 & 51100 \\ & 6873\end{array}$

$\begin{array}{cc}\text { SAMPLE } & \\ \text { DATE } & 6-37-43 \\ 01 / 05 / 88 & 11900 \\ & \text { HR } \\ 01 / 05 / 88 & 460 \\ & \text { HR } \\ 01 / 05 / 88 & 12300 \\ & \text { NR } \\ 01 / 05 / 88 & 7.83 \\ & \text { HR } \\ 01 / 05 / 88 & 8 \\ & \text { NR } \\ 01 / 05 / 88 & 248000 \\ & \text { NR } \\ 01 / 05 / 88 & 24200 \\ & \text { NR } \\ 01 / 05 / 88 & 58300 \\ & 910\end{array}$


IABLE E.3. (contd)

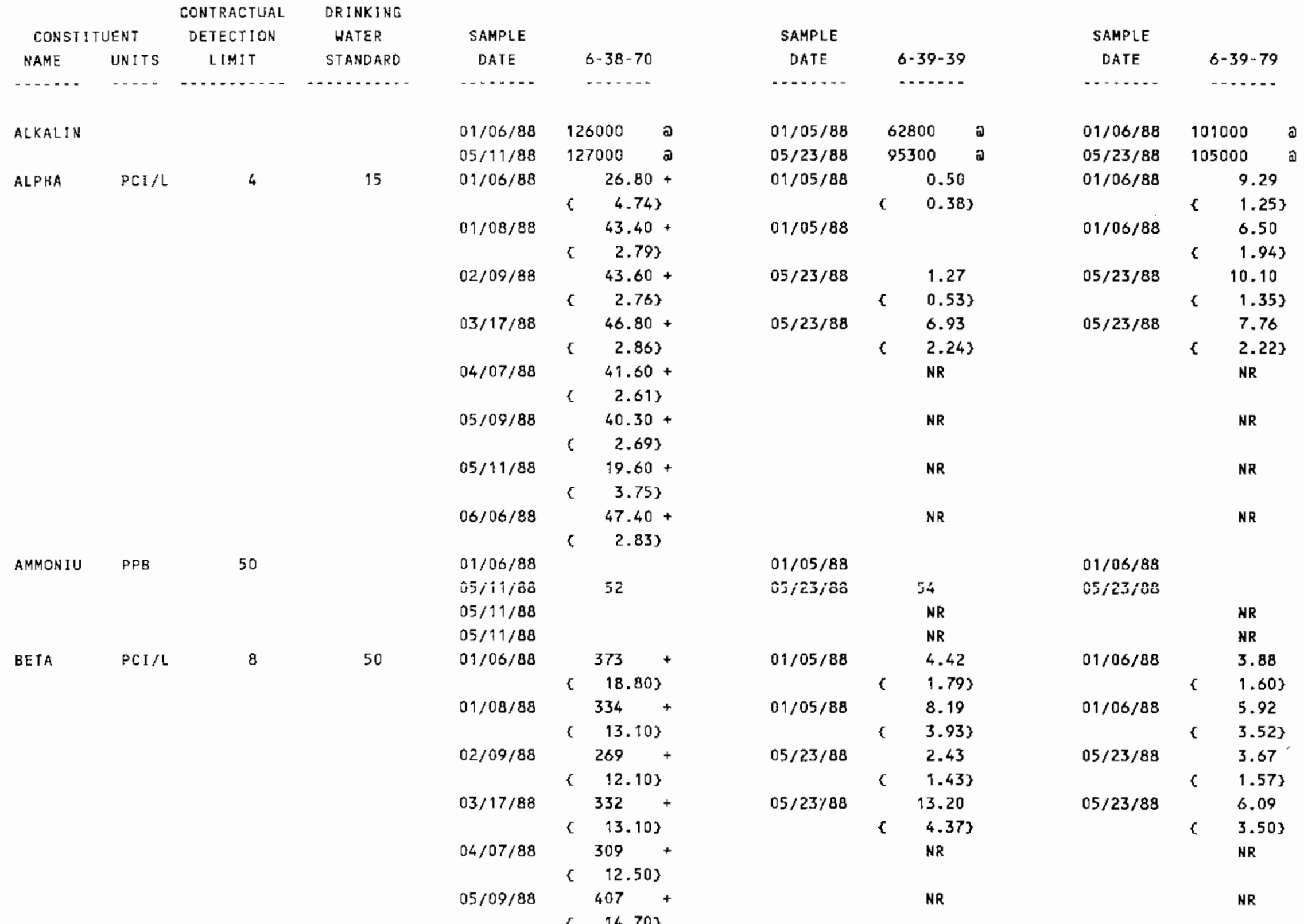


TABLE E.3. (contd)

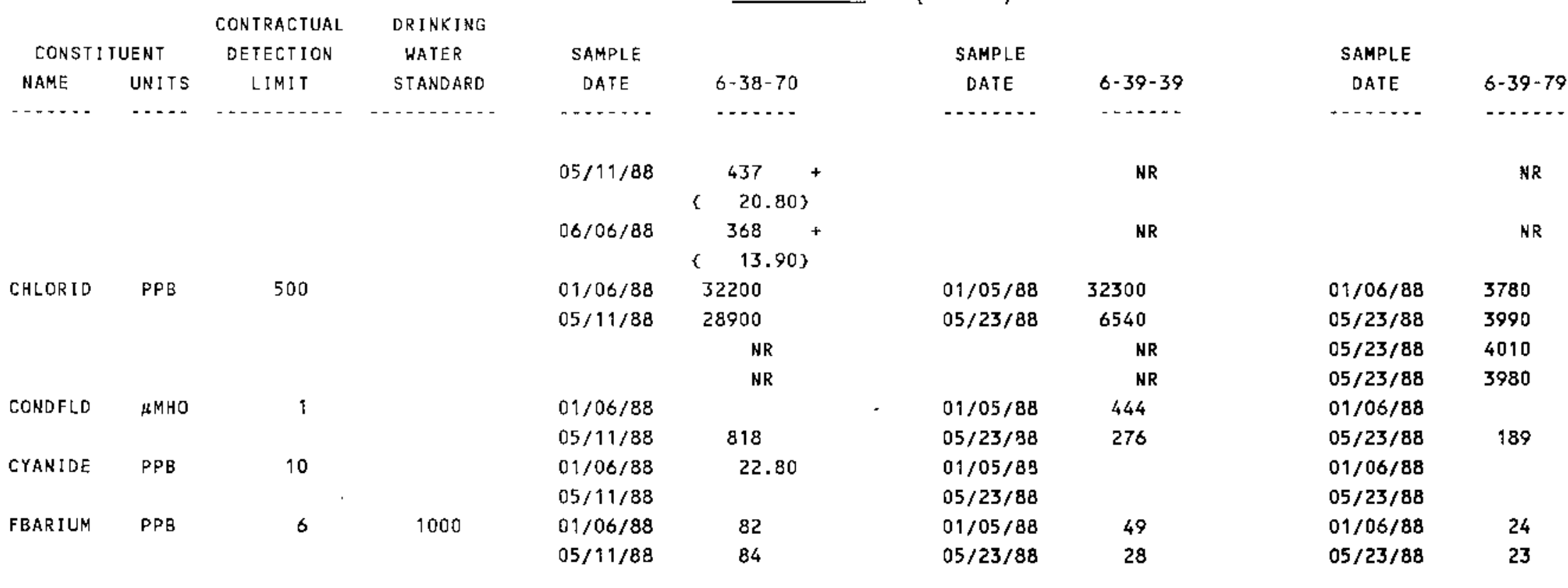




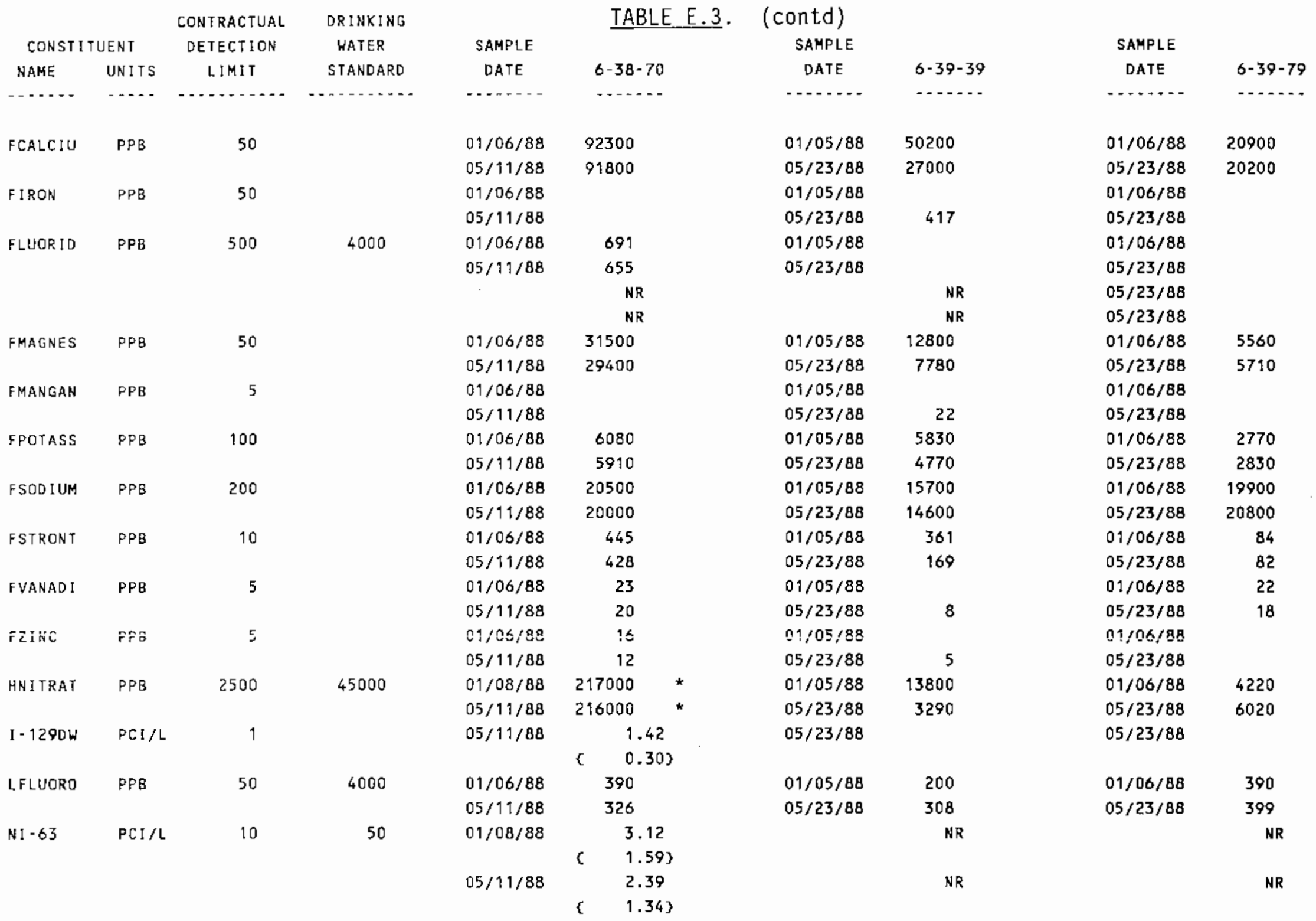


TABLE E.3. (contd)

\begin{tabular}{|c|c|c|c|c|c|c|c|c|c|}
\hline \multicolumn{2}{|c|}{ CONSTI TUENT } & $\begin{array}{l}\text { CONTRACIUAL } \\
\text { DETECTION }\end{array}$ & $\begin{array}{l}\text { DRINKING } \\
\text { HATER }\end{array}$ & SAMPLE & \multicolumn{3}{|c|}{ SAMPLE } & \multicolumn{2}{|l|}{ SAMPLE } \\
\hline NAME & UNITS & LIMIT & STANDARD & DATE & $6-38-70$ & DATE & $6-39-39$ & OATE & $6 \cdot 39-79$ \\
\hline - n...... & $\ldots .$. & 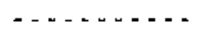 & - & - & $\ldots$ & -...... & 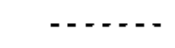 & $\ldots \ldots$ & ......- \\
\hline \multirow[t]{4}{*}{ NITRATE } & PPB & 500 & 45000 & $01 / 06 / 88$ & 239000 & $01 / 05 / 88$ & 15200 & $01 / 06 / 88$ & 4000 \\
\hline & & & & $05 / 11 / 88$ & 220000 & $05 / 23 / 88$ & 2690 & $05 / 23 / 88$ & 6220 \\
\hline & & & & & NR & & NR & $05 / 23 / 88$ & 6170 \\
\hline & & & & & NR & & NR & $05 / 23 / 88$ & 6360 \\
\hline \multirow[t]{2}{*}{$P H-\angle A B$} & & 0.01 & & $01 / 06 / 88$ & 7.51 & $01 / 05 / 88$ & 7.90 & $01 / 06 / 88$ & $\begin{array}{r}8.13 \\
+\quad 8\end{array}$ \\
\hline & & & & $05 / 11 / 88$ & 7.80 & $05 / 23 / 88$ & 8.20 & $05 / 23 / 88$ & 8.30 \\
\hline \multirow[t]{2}{*}{ PHFIELD } & & 0.10 & & $01 / 06 / 88$ & 7.90 & $01 / 05 / 88$ & 8.20 & $01 / 06 / 88$ & 8.60 \\
\hline & & & & $05 / 11 / 88$ & 7.80 & $05 / 23 / 88$ & 8.20 & $05 / 23 / 88$ & 8.10 \\
\hline \multirow[t]{4}{*}{ PHOSPHA } & PPB & 1000 & & $01 / 06 / 88$ & 9190 & $01 / 05 / 88$ & & $01 / 06 / 88$ & \\
\hline & & & & $05 / 11 / 88$ & & $05 / 23 / 88$ & & $05 / 23 / 88$ & \\
\hline & & & & & NR & & NR & $05 / 23 / 88$ & \\
\hline & & & & & NR & & NR & $05 / 23 / 88$ & \\
\hline \multirow[t]{4}{*}{ SULFATE } & PPB & 500 & & $01 / 06 / 88$ & 48400 & $01 / 05 / 88$ & 110000 & $09 / 06 / 88$ & 15300 \\
\hline & & & & $05 / 11 / 88$ & 46000 & $05 / 23 / 88$ & 28100 & $05 / 23 / 88$ & 96300 \\
\hline & & & & & NR & & NR & $05 / 23 / 88$ & 16200 \\
\hline & & & & & NR & & NR & $05 / 23 / 88$ & 16300 \\
\hline
\end{tabular}


TABLE E.3. (contd)

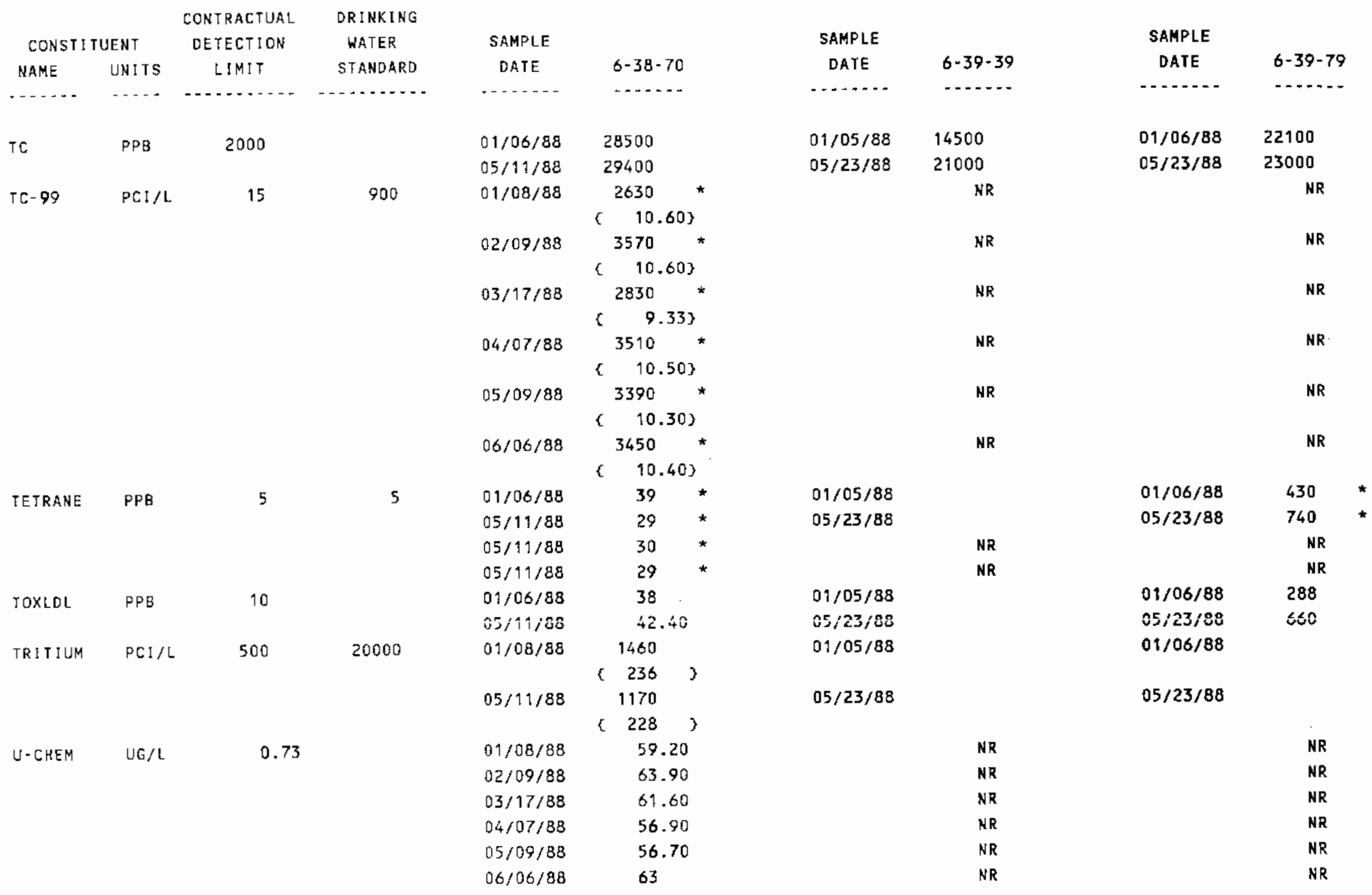




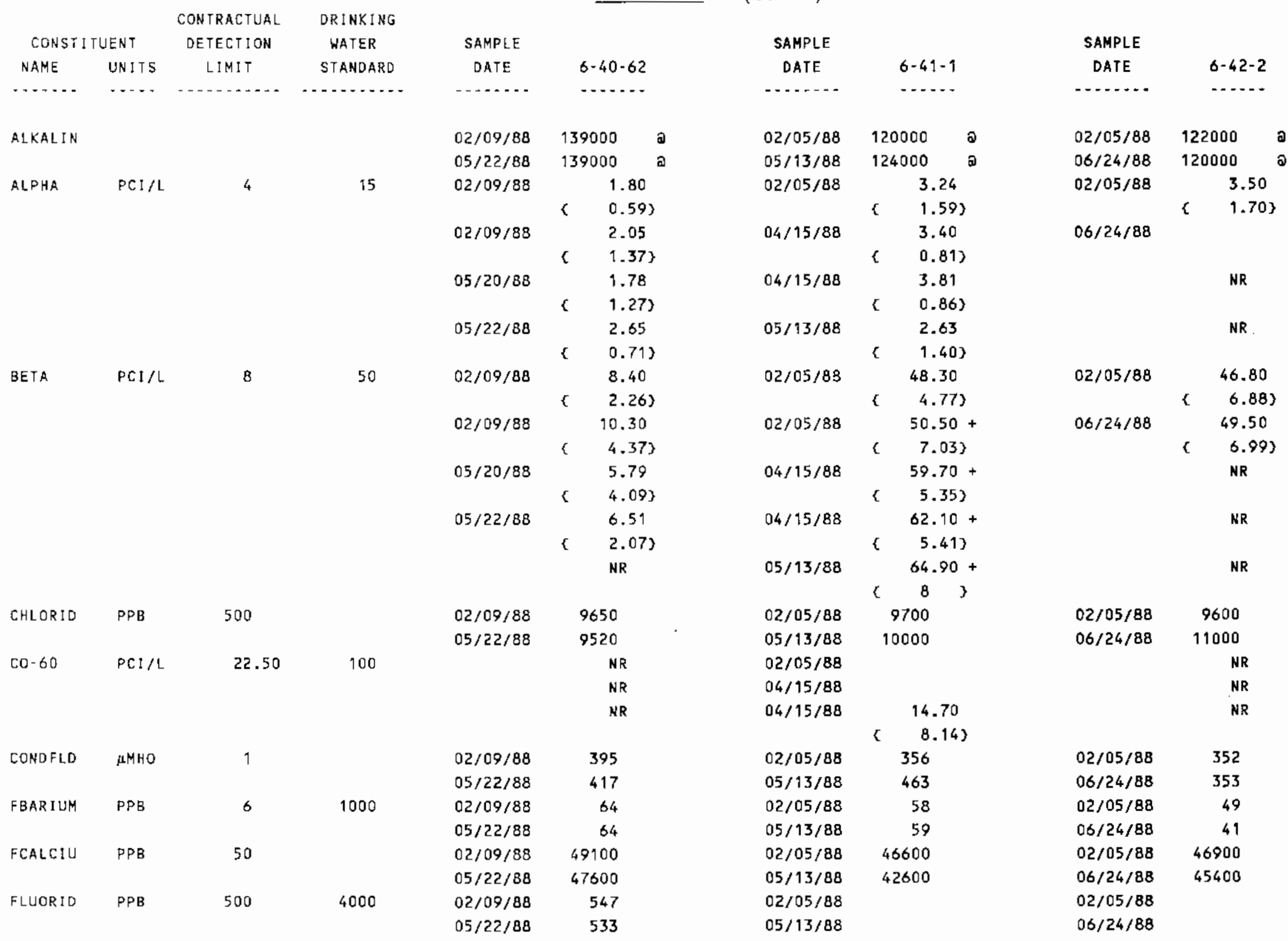


TABLE E.3. (contd)

\begin{tabular}{|c|c|c|c|c|c|c|c|c|c|}
\hline \multicolumn{2}{|c|}{ CONSTI TUENT } & $\begin{array}{l}\text { CONTRACTUAL } \\
\text { DETECTION }\end{array}$ & $\begin{array}{l}\text { DRINKING } \\
\text { WATER }\end{array}$ & SAMPLE & & \multicolumn{2}{|l|}{ SAMPLE } & \multicolumn{2}{|l|}{ SAMPLE } \\
\hline NAME & UNITS & LIMIT & STANDARD & DATE & $6-40-62$ & DATE & $6-41-1$ & DATE & $6-42-2$ \\
\hline n..... & ..... & .......... & $\ldots \ldots$ & - - - & ........ & ......... & ....... & ........ & - \\
\hline \multirow[t]{2}{*}{ FMAGNES } & $\mathrm{PPB}$ & 50 & & $02 / 09 / 8 B$ & 16700 & $02 / 05 / 88$ & 13000 & $02 / 05 / 88$ & 13300 \\
\hline & & & & $05 / 22 / 88$ & 16300 & $05 / 13 / 88$ & 12600 & $06 / 24 / 88$ & 13000 \\
\hline \multirow[t]{2}{*}{ FPOTASS } & $\mathrm{PPB}$ & 100 & & $02 / 09 / 88$ & 5590 & $02 / 05 / 88$ & 5230 & $02 / 05 / 88$ & 5380 \\
\hline & & & & $05 / 22 / 88$ & 5420 & $05 / 13 / 88$ & 5330 & $06 / 24 / 88$ & 5610 \\
\hline \multirow{2}{*}{ FSODIUM } & PPB & 200 & & $02 / 09 / 88$ & 19100 & $02 / 05 / 88$ & 17700 & $02 / 05 / 88$ & 19600 \\
\hline & & & & $05 / 22 / 88$ & 18400 & $05 / 13 / 88$ & 18800 & $06 / 24 / 88$ & 19200 \\
\hline \multirow[t]{2}{*}{ FSTRONT } & $\mathrm{PPB}$ & 10 & & $02 / 09 / 88$ & 217 & $02 / 05 / 88$ & 307 & $02 / 05 / 88$ & 315 \\
\hline & & & & $05 / 22 / 88$ & 207 & $05 / 13 / 88$ & 306 & $06 / 24 / 88$ & 316 \\
\hline \multirow[t]{2}{*}{ FVANADI } & PPB & 5 & & $02 / 09 / 88$ & 27 & $02 / 05 / 88$ & 16 & $02 / 05 / 88$ & 16 \\
\hline & & & & $05 / 22 / 88$ & 26 & $05 / 13 / 88$ & 16 & $06 / 24 / 88$ & 19 \\
\hline \multirow[t]{2}{*}{ FZINC } & PPB & 5 & & $02 / 09 / 88$ & & $02 / 05 / 38$ & & $02 / 05 / 88$ & \\
\hline & & & & $05 / 22 / 88$ & & $05 / 13 / 88$ & & $06 / 24 / 88$ & 6 \\
\hline \multirow[t]{2}{*}{ HNITRAT } & PPB & 2500 & 45000 & $02 / 09 / 88$ & 41100 & $02 / 05 / 88$ & 36100 & $02 / 05 / 88$ & 35200 \\
\hline & & & & $05 / 22 / 88$ & 45700 & $04 / 15 / 88$ & 38700 & & NR \\
\hline
\end{tabular}




\begin{tabular}{|c|c|c|c|c|c|c|}
\hline & & CONTRACTUAL & DRINKING & & & \\
\hline CONST & UENT & DETECTION & WATER & SAMPLE & & \\
\hline NAME & UNITS & LIMIT & STANDARD & DATE & $6-40-6$ & \\
\hline$\cdots \cdots$ & - - - & $\cdots$ & $\ldots$ & $\cdots$ & $\cdots \cdots$ & \\
\hline $1-1290 W$ & $\mathrm{PCI} / \mathrm{L}$ & 1 & & $05 / 22 / 88$ & 0.2 & \\
\hline & & & & & 0.2 & \\
\hline LFLUORD & $\mathrm{PPB}$ & 50 & 4000 & $02 / 09 / 88$ & 334 & \\
\hline & & & & $05 / 22 / 88$ & 352 & \\
\hline NITRATE & PPB & 500 & 45000 & $02 / 09 / 88$ & 41100 & \\
\hline & & & & $05 / 22 / 88$ & 46500 & * \\
\hline$P H-L A B$ & & 0.01 & & $02 / 09 / 88$ & 7.5 & \\
\hline & & & & $05 / 22 / 88$ & 7.9 & \\
\hline PHFIELD & & 0.10 & & $02 / 09 / 88$ & 7.9 & \\
\hline & & & & $05 / 22 / 88$ & 7.4 & \\
\hline SULFATE & $P P B$ & 500 & & $02 / 09 / 88$ & 39700 & \\
\hline & & & & $05 / 22 / 88$ & 41800 & \\
\hline TC & PPB & 2000 & & $02 / 09 / 8 \mathrm{~B}$ & 33000 & \\
\hline & & & & $05 / 22 / 88$ & 31600 & \\
\hline TC-98 & $\mathrm{PCI} / \mathrm{L}$ & 15 & 900 & & NR & \\
\hline TRITIUM & $\mathrm{PCI} / \mathrm{L}$ & 500 & 20000 & $02 / 09 / 88$ & 76600 & * \\
\hline & & & & & \& 1060 & 3 \\
\hline & & & & $05 / 22 / 88$ & 78800 & * \\
\hline & & & & & \{ 1080 & 3 \\
\hline & & & & & NR & \\
\hline & & & & & NR & \\
\hline U & $P[J / L$ & 0.50 & 600 & & $N R$ & \\
\hline & & & & & NR & \\
\hline & & & & & NR & \\
\hline UNKNOWN & $P P B$ & 0 & & & NR & \\
\hline & & & & & NR & \\
\hline
\end{tabular}

\begin{tabular}{|c|c|c|}
\hline \multicolumn{3}{|l|}{ SAMPLE } \\
\hline DATE & \multicolumn{2}{|c|}{$6-41-1$} \\
\hline$\ldots$ & \multicolumn{2}{|c|}{ - . - - } \\
\hline $04 / 15 / 88$ & \multicolumn{2}{|c|}{0.28} \\
\hline & \multicolumn{2}{|c|}{0.243} \\
\hline $02 / 05 / 88$ & \multicolumn{2}{|c|}{324} \\
\hline $05 / 13 / 88$ & \multicolumn{2}{|l|}{366} \\
\hline $02 / 05 / 88$ & \multicolumn{2}{|l|}{39800} \\
\hline $05 / 13 / 88$ & \multicolumn{2}{|l|}{38700} \\
\hline $02 / 05 / 88$ & \multicolumn{2}{|c|}{7.63} \\
\hline $05 / 13 / 88$ & \multicolumn{2}{|c|}{7.90} \\
\hline $02 / 05 / 88$ & \multicolumn{2}{|c|}{7.70} \\
\hline $05 / 13,88$ & \multicolumn{2}{|c|}{7.80} \\
\hline $02 / 05 / 38$ & \multicolumn{2}{|l|}{38400} \\
\hline $05 / 13 / 88$ & \multicolumn{2}{|l|}{37600} \\
\hline $02 / 05 / 88$ & \multicolumn{2}{|l|}{28800} \\
\hline $05 / 13 / 88$ & \multicolumn{2}{|l|}{28500} \\
\hline \multirow[t]{2}{*}{$02 / 05 / 88$} & \multicolumn{2}{|l|}{340} \\
\hline & \multicolumn{2}{|c|}{3.443} \\
\hline \multirow[t]{2}{*}{$02 / 05 / 88$} & \multicolumn{2}{|l|}{236000} \\
\hline & \multicolumn{2}{|l|}{ ₹ 1850} \\
\hline \multirow[t]{2}{*}{$02 / 05 / 88$} & \multicolumn{2}{|l|}{235000} \\
\hline & \multicolumn{2}{|l|}{$<1870$} \\
\hline \multirow[t]{2}{*}{$04 / 15 / 88$} & \multicolumn{2}{|l|}{241000} \\
\hline & \multicolumn{2}{|l|}{ c 1850} \\
\hline \multirow[t]{2}{*}{$04 / 15 / 88$} & \multicolumn{2}{|l|}{235000} \\
\hline & $<1820$ & 3 \\
\hline $02 / 05 / 88$ & \multicolumn{2}{|c|}{3.24} \\
\hline $04 / 15 / 88$ & & 52 \\
\hline $04 / 15 / 88$ & & 86 \\
\hline $05 / 13 / 88$ & 14 & \\
\hline $05 / 13 / 88$ & 11 & \\
\hline
\end{tabular}

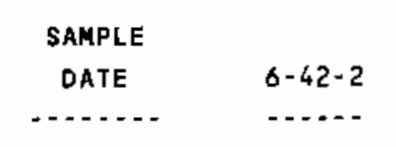

\begin{tabular}{|c|c|c|}
\hline \multicolumn{3}{|c|}{ NR } \\
\hline $02 / 05 / 88$ & 330 & \\
\hline $06 / 24 / 88$ & 348 & \\
\hline $02 / 05 / 88$ & 38700 & \\
\hline $06 / 24 / 88$ & 38000 & \\
\hline $02 / 05 / 88$ & 7.67 & \\
\hline $06 / 24 / 88$ & 7.90 & \\
\hline $02 / 05 / 88$ & 7.60 & \\
\hline $06 / 24 / 88$ & 7.30 & \\
\hline $02 / 05 / 88$ & 37500 & \\
\hline $06 / 24 / 88$ & 37100 & \\
\hline $02 / 05 / 88$ & 29000 & \\
\hline \multirow{2}{*}{$06 / 24 / 88$} & 30300 & \\
\hline & $N R$ & \\
\hline \multirow[t]{10}{*}{$02 / 05 / 88$} & 219000 & $\star$ \\
\hline & \& 1790 & 3 \\
\hline & NR & \\
\hline & NR & \\
\hline & NR & \\
\hline & NR & \\
\hline & NR & \\
\hline & NR & \\
\hline & $N R$ & \\
\hline & NR & \\
\hline
\end{tabular}


TABLE E. 3 . (contd)

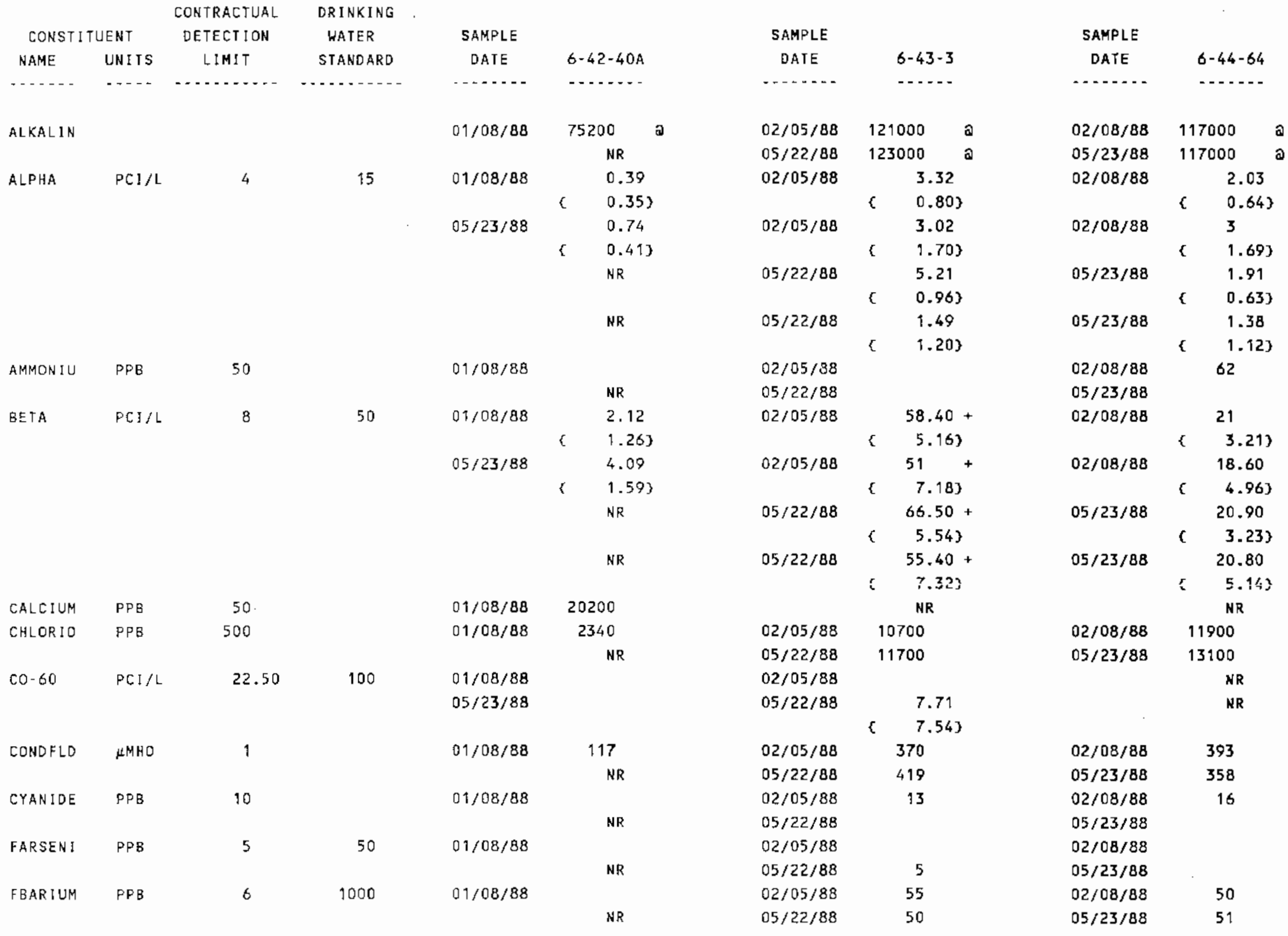


TABLE E.3. (contd)

\begin{tabular}{|c|c|c|c|c|c|c|c|c|c|}
\hline \multicolumn{2}{|c|}{ CONSTI IUENT } & $\begin{array}{l}\text { CONTRACTUAL } \\
\text { DETECTION }\end{array}$ & $\begin{array}{l}\text { DRINKING } \\
\text { WATER }\end{array}$ & SAMPLE & & SAMPLE & & SAMPLE & \\
\hline NAME & UNITS & LIMIT & STANDARD & DATE & $6-42-40 \mathrm{~A}$ & DATE & $6-43-3$ & DATE & $6-44.64$ \\
\hline …… & $\cdots$ & - & n. & - & $\cdots$ & $\ldots$ & $-\ldots$ & 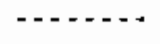 & ........ \\
\hline \multirow{2}{*}{ FCALCIU } & $P P B$ & 50 & & $01 / 08 / 88$ & 21300 & $02 / 05 / 88$ & 44100 & $02 / 08 / 88$ & 42700 \\
\hline & & & & & NR & $05 / 22 / 88$ & 44500 & $05 / 23 / 88$ & 45400 \\
\hline \multirow[t]{2}{*}{ FIRON } & PPB & 50 & & $01 / 08 / 88$ & 171 & $02 / 05 / 88$ & & $02 / 08 / 88$ & \\
\hline & & & & & $\mathrm{NR}$ & $05 / 22 / 88$ & & $05 / 23 / 88$ & \\
\hline \multirow[t]{2}{*}{ FLUORID } & PPB & 500 & 4000 & $01 / 08 / 88$ & & $02 / 05 / 88$ & & $02 / 08 / 88$ & \\
\hline & & & & & NR & $05 / 22 / 88$ & & $05 / 23 / 88$ & 745 \\
\hline \multirow[t]{2}{*}{ FMAGNES } & PPB & 50 & & $01 / 08 / 88$ & 5630 & $02 / 05 / 88$ & 13400 & $02 / 08 / 88$ & 16000 \\
\hline & & & & & NR & $05 / 22 / 88$ & 13300 & $05 / 23 / 88$ & 16800 \\
\hline \multirow{2}{*}{ FMANGAN } & PPB & 5 & & $01 / 08 / 88$ & 11 & $02 / 05 / 88$ & & $02 / 08 / 88$ & \\
\hline & & & & & NR & $05 / 22 / 88$ & & $05 / 23 / 88$ & \\
\hline \multirow[t]{2}{*}{ FPOTASS } & PPB & 100 & & $01 / 08 / 88$ & 2800 & $02 / 05 / 38$ & 4900 & $02 / 08 / 88$ & 4950 \\
\hline & & & & & $N R$ & $05 / 22 / 88$ & 5050 & $05 / 23 / 88$ & 5120 \\
\hline \multirow[t]{2}{*}{ FSODIUM } & $P P B$ & 200 & & $01 / 08 / 88$ & 4460 & $02 / 05 / 88$ & 19600 & $02 / 08 / 88$ & 14900 \\
\hline & & & & & NR & $05 / 22 / 88$ & 19100 & $05 / 23 / 88$ & 15500 \\
\hline
\end{tabular}


IABLE E.3. (contd)

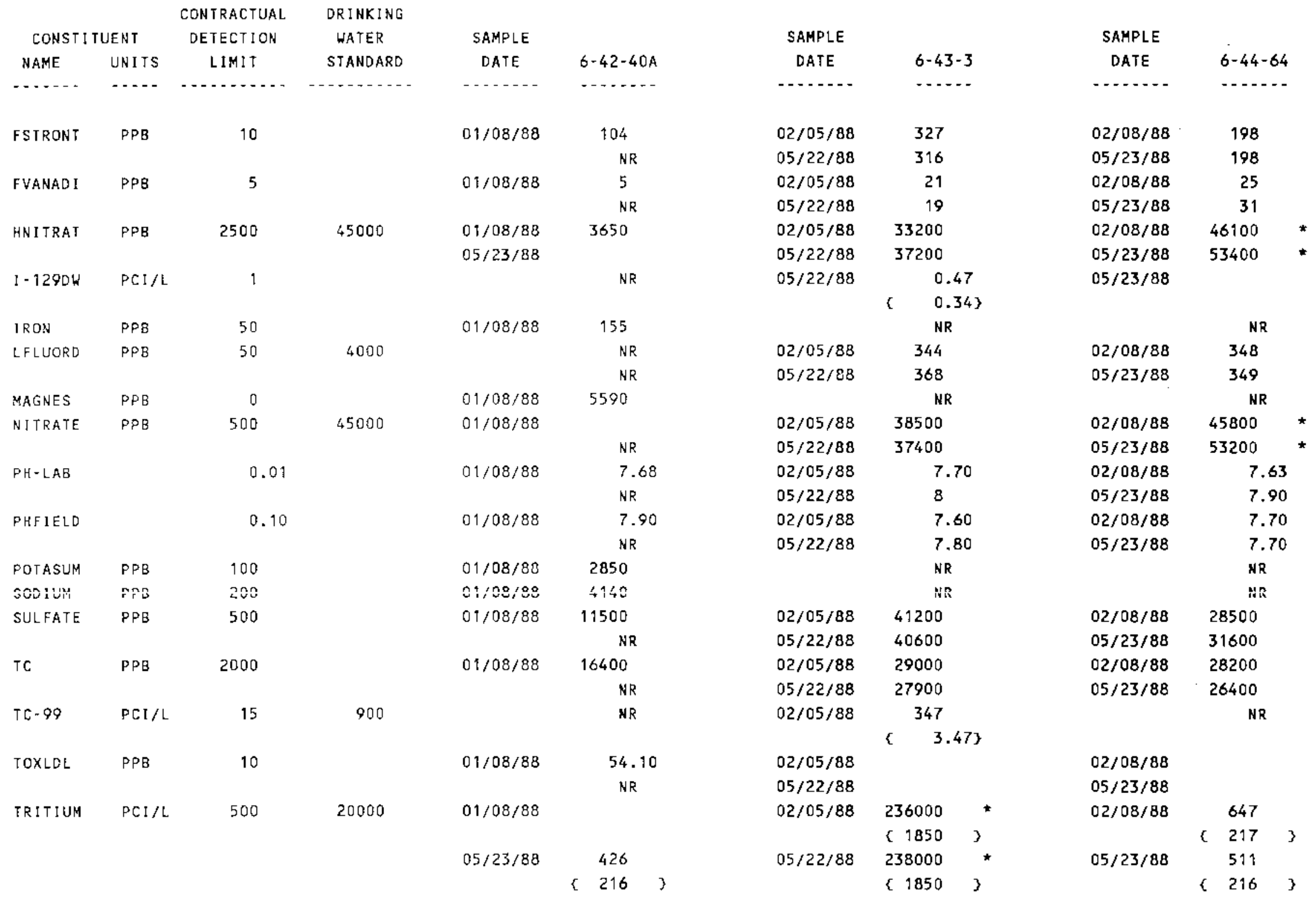


TABLE E.3. (contd)

\begin{tabular}{|c|c|c|c|c|c|c|c|c|c|}
\hline \multicolumn{2}{|c|}{ CONSTITUENT } & $\begin{array}{l}\text { CONTRACTUAL } \\
\text { OETECTION }\end{array}$ & $\begin{array}{l}\text { DRINKING } \\
\text { WATER }\end{array}$ & SAMPLE & \multirow[b]{2}{*}{$6-42-40 \mathrm{~A}$} & \multicolumn{2}{|l|}{ SAMPLE } & \multicolumn{2}{|l|}{ SAMPLE } \\
\hline NAME & UNITS & LIMIT & STANDARD & DATE & & DATE & $6-43-3$ & DATE & $6-44-64$ \\
\hline ….... & $\cdots$ & 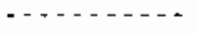 & n............... & - . - . & $\cdots \ldots$ & . & ....... & $\cdots$ & ..... \\
\hline \multirow[t]{4}{*}{$v-234$} & $\mathrm{PCI} / \mathrm{L}$ & 0.10 & 32 & $01 / 08 / 88$ & 0.47 & & NR & & NR \\
\hline & & & & & $0.07\}$ & & & & \\
\hline & & & & $05 / 23 / 88$ & 0.47 & & $\mathrm{NR}$ & & NR \\
\hline & & & & & 0.073 & & & & \\
\hline \multirow[t]{3}{*}{ U. 235} & $\mathrm{PCI} / \mathrm{L}$ & 0.10 & 32 & $01 / 08 / 88$ & 0.06 & & NR & & NR \\
\hline & & & & & 0.033 & & & & \\
\hline & & & & $05 / 23 / 88$ & & & NR & & $\mathrm{NR}$ \\
\hline \multirow[t]{4}{*}{ U. 238} & $P C I / L$ & 0.10 & 4.80 & $01 / 08 / 88$ & 0.44 & & NR & & NR \\
\hline & & & & & 0.073 & & & & \\
\hline & & & & $05 / 23 / 88$ & 0.33 & & NR & & NR \\
\hline & & & & & $0.06\}$ & & & & \\
\hline \multirow[t]{2}{*}{ U-CHEM } & $U G / L$ & 0.73 & & $01 / 08 / 88$ & 0.81 & & NR & & NR \\
\hline & & & & $05 / 23 / 88$ & 0.83 & & NR & & NR \\
\hline
\end{tabular}


TABLE E.3. (contd)

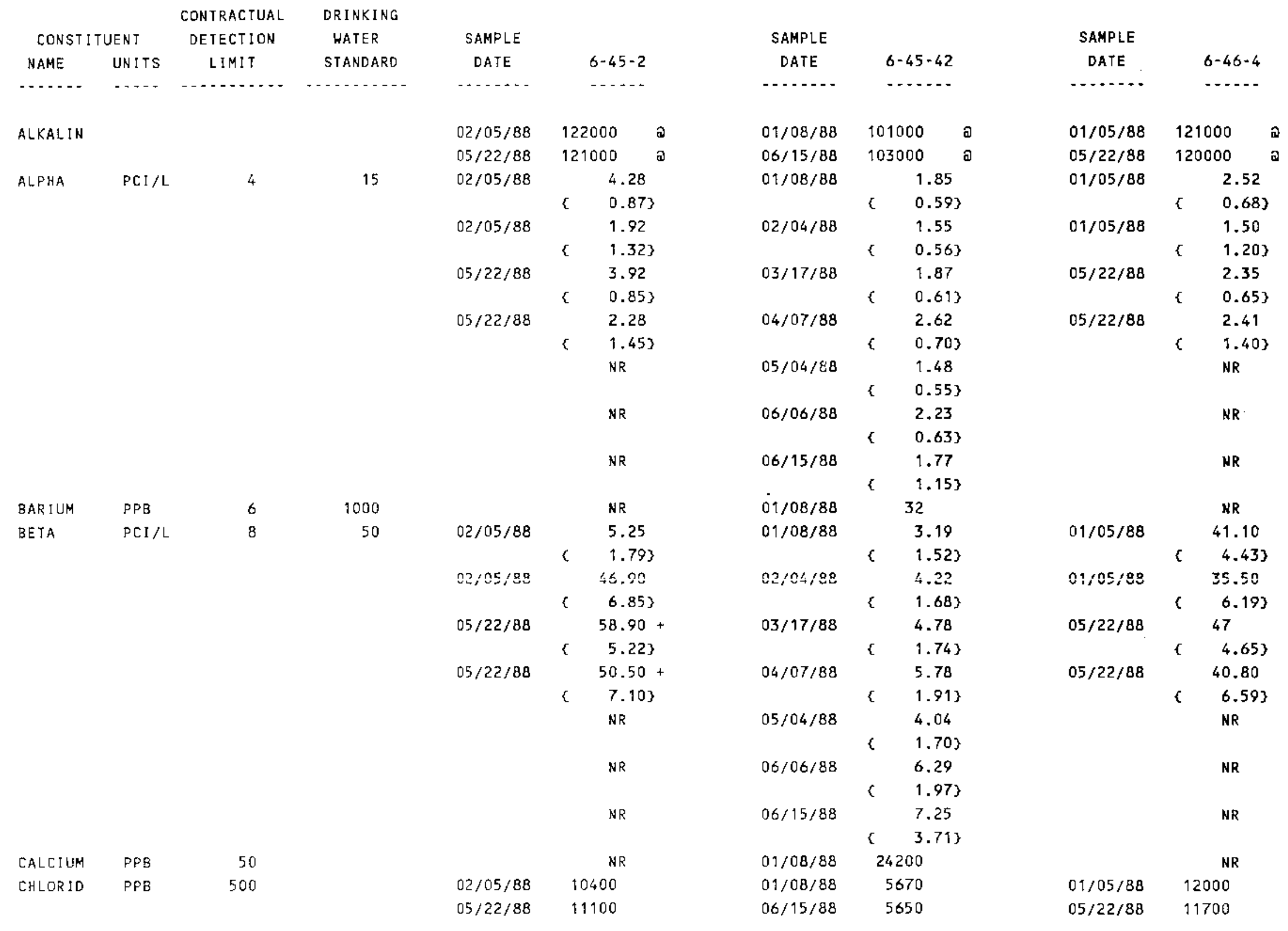




\begin{tabular}{|c|c|c|c|}
\hline \multirow{2}{*}{\multicolumn{2}{|c|}{ CONSII TUENT }} & CONTRACTUAL & ORINKING \\
\hline & & DETECTION & WATER \\
\hline NAME & UNITS & LIMII & STANDARD \\
\hline & $\cdots$ & 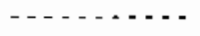 & $-\ldots$ \\
\hline
\end{tabular}

$\cos 60$

$\mathrm{PCI} / \mathrm{L}$

22.50

100

CONDFLD AMHO

CYANIDE PPE

10

FARSENI PPB

FBARIUM PPB

TABLE E.3. (contd)

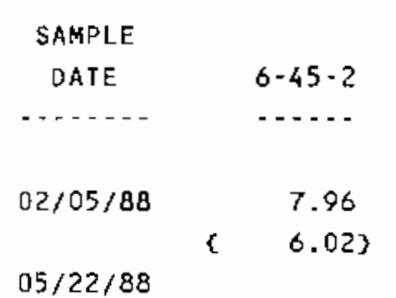

$02 / 05 / 88$

$05 / 22 / 88$

$02 / 05 / 88$

$05 / 22 / 88$

$02 / 05 / 88$

$05 / 22 / 88$

$02 / 05 / 88$

SAMPLE
DATE
$\ldots \ldots . .6$

$01 / 08 / 88$

$02 / 04 / 88$

$03 / 17 / 88$

$04 / 07 / 88$

$05 / 04 / 88$

$06 / 06 / 88$

$01 / 08 / 88$

$01 / 08 / 88$

$06 / 15 / 88$

$01 / 08 / 88$

$06 / 15 / 88$

$01 / 08 / 88$
SAMPLE

DATE

6-46-4

....... -...-

NR

WR

NR

NR

NR

NR

$01 / 05 / 88$

$05 / 22 / 88$

$01 / 05 / 88$

$05 / 22 / 88$

$01 / 05 / 88$

$05 / 22 / 88$

$09 / 05 / 88$
344

387

5

5 


\begin{tabular}{|c|c|c|c|c|c|c|c|c|c|}
\hline \multirow{2}{*}{\multicolumn{2}{|c|}{ CONSTITUENT }} & CONTRACTUAL & DRINKING & & & \multirow{2}{*}{\multicolumn{2}{|c|}{ SAMPLE }} & \multirow{2}{*}{\multicolumn{2}{|c|}{ SAMPLE }} \\
\hline & & DETECTION & WATER & SAMPLE & & & & & \\
\hline NAHE & UNITS & LIMIT & STANDARD & DATE & $6-45-2$ & DATE & $6-45-42$ & DATE & $6-46-4$ \\
\hline$-\cdots$ & $\cdots$ & - - - - - - - - - - - & 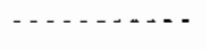 & $\cdots \cdots$ & $\ldots \ldots$ & $\cdots$ & $\ldots$ & $-\ldots$ & $\ldots \ldots$ \\
\hline FBARIUM & PPB & 6 & 1000 & $05 / 22 / 88$ & 35 & $06 / 15 / 88$ & 35 & $05 / 22 / 88$ & 35 \\
\hline \multirow[t]{2}{*}{ FCALCIU } & PPB & 50 & & $02 / 05 / 88$ & 42200 & $01 / 08 / 88$ & 26300 & $01 / 05 / 88$ & 43300 \\
\hline & & & & $05 / 22 / 88$ & 43700 & $06 / 15 / 88$ & 26900 & $05 / 22 / 88$ & 39300 \\
\hline \multirow[t]{2}{*}{ FIRON } & PPB & 50 & & $02 / 05 / 88$ & & $01 / 08 / 88$ & 60 & $01 / 05 / 88$ & \\
\hline & & & & $05 / 22 / 88$ & & $06 / 15 / 88$ & 53 & $05 / 22 / 88$ & \\
\hline \multirow[t]{2}{*}{ FLUORID } & PPB & 500 & 4000 & $02 / 05 / 88$ & & $01 / 08 / 88$ & 667 & $01 / 05 / 88$ & 546 \\
\hline & & & & $05 / 22 / 88$ & 591 & $06 / 15 / 88$ & 1200 & $05 / 22 / 88$ & 607 \\
\hline \multirow[t]{2}{*}{ FMAGNES } & PPB & 50 & & $02 / 05 / 88$ & 11900 & $01 / 08 / 88$ & 10500 & $01 / 05 / 88$ & 12700 \\
\hline & & & & $05 / 22 / 88$ & 12300 & $06 / 15 / 88$ & 10800 & $05 / 22 / 88$ & 12300 \\
\hline \multirow[t]{2}{*}{ FMANGAN } & PPB & 5 & & $02 / 05 / 88$ & & $01 / 08 / 88$ & 10 & $01 / 05 / 88$ & \\
\hline & & & & $05 / 22 / 88$ & & $06 / 15 / 88$ & 7 & $05 / 22 / 88$ & \\
\hline \multirow[t]{2}{*}{ FPOTASS } & PPB & 100 & & $02 / 05 / 88$ & 4880 & $01 / 08 / 88$ & 4320 & $01 / 05 / 88$ & 4980 \\
\hline & & & & $05 / 22 / 88$ & 5200 & $06 / 15 / 88$ & 3920 & $05 / 22 / 88$ & 4920 \\
\hline \multirow[t]{2}{*}{ FSODIUM } & PPB & 200 & & $02 / 05 / 88$ & 19200 & $01 / 08 / 88$ & 18400 & $01 / 05 / 88$ & 22200 \\
\hline & & & & $05 / 22 / 88$ & 19400 & $06 / 15 / 88$ & 17300 & $05 / 22 / 88$ & 21700 \\
\hline \multirow[t]{2}{*}{ FSIRDNT } & $\mathrm{PPB}$ & 10 & & $02 / 05 / 88$ & 276 & $01 / 08 / 88$ & 192 & $01 / 05 / 88$ & 305 \\
\hline & & & & $05 / 22 / 88$ & 282 & $06 / 15 / 88$ & 196 & $05 / 22 / 88$ & 295 \\
\hline \multirow[t]{2}{*}{ FVANAD 1} & PPB & 5 & & $02 / 05 / 88$ & 18 & $01 / 08 / 88$ & 30 & $01 / 05 / 88$ & \\
\hline & & & & $05 / 22 / 88$ & 21 & $06 / 15 / 88$ & 34 & $05 / 22 / 88$ & 19 \\
\hline \multirow[t]{2}{*}{ rzita } & SPS & 5 & & $02 / 05 / 88$ & & $09 / 08 / 89$ & & $01 / 05 / 8 \mathrm{8}$ & \\
\hline & & & & $05 / 22 / 88$ & & $06 / 15 / 88$ & & $05 / 22 / 88$ & 6 \\
\hline \multirow[t]{6}{*}{ HN I TRAT } & $P P B$ & 2500 & 45000 & $02 / 05 / 88$ & 32300 & $01 / 08 / 88$ & 6910 & $01 / 05 / 88$ & 29300 \\
\hline & & & & $05 / 22 / 88$ & 35200 & $02 / 04 / 88$ & 6140 & $05 / 22 / 88$ & 27400 \\
\hline & & & & & NR & $03 / 17 / 88$ & 5210 & & NR \\
\hline & & & & & $N R$ & $04 / 07 / 88$ & 5960 & & NR \\
\hline & & & & & NR & $05 / 04 / 88$ & 6610 & & NR \\
\hline & & & & & $N R$ & $06 / 06 / 88$ & 7730 & & NR \\
\hline \multirow[t]{2}{*}{$l-1290 \mathrm{~W}$} & $\mathrm{PCS} / \mathrm{L}$ & 1 & & $05 / 22 / 88$ & 0.39 & & NR & $05 / 22 / 88$ & 0.26 \\
\hline & & & & & 0.313 & & & & 0.213 \\
\hline I RON & PPB & 50 & & & NR & $01 / 08 / 88$ & 428 & & NR \\
\hline \multirow[t]{2}{*}{ LFLUORD } & PPB & 50 & 4000 & $02 / 05 / 88$ & 365 & $06 / 15 / 88$ & 488 & $01 / 05 / 88$ & 435 \\
\hline & & & & $05 / 22 / 88$ & 402 & & NR & $05 / 22 / 88$ & 410 \\
\hline MAGNES & $\mathrm{PPB}$ & 0 & & & $\mathrm{NR}$ & $01 / 08 / 88$ & 10100 & & NR \\
\hline
\end{tabular}


TABLE E.3. (contd)

\begin{tabular}{|c|c|c|c|c|c|c|c|c|c|}
\hline \multirow{2}{*}{\multicolumn{2}{|c|}{ CONSTITUENT }} & CONTRACTUAL & DRINKING & & & & & & \\
\hline & & DETECTION & WATER & SAMPLE & \multirow[b]{2}{*}{$6-45-2$} & SAMPLE & & SAMPLE & \\
\hline NAME & UNITS & LIMIT & STANDARD & DATE & & DATE & $6-45-42$ & DATE & \\
\hline$\ldots$ & $\cdots$ & $-\cdots$ & $\cdots$ & - - - - - - & $\ldots$ & - & $\cdots$ & 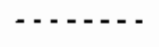 & $\begin{array}{l}6-46-4 \\
-\ldots \ldots\end{array}$ \\
\hline \multirow[t]{2}{*}{ NITRATE } & \multirow[t]{2}{*}{$P P B$} & \multirow[t]{2}{*}{500} & \multirow[t]{2}{*}{45000} & $02 / 05 / 88$ & 35200 & $01 / 08 / 88$ & 8860 & $01 / 05 / 88$ & 30000 \\
\hline & & & & $05 / 22 / 88$ & 35500 & $06 / 15 / 88$ & 7270 & $05 / 22 / 88$ & 29100 \\
\hline \multirow[t]{2}{*}{$P H-L A B$} & \multirow{2}{*}{\multicolumn{3}{|c|}{0.01}} & $02 / 05 / 88$ & 7.62 & $01 / 08 / 88$ & 7.74 & $01 / 05 / 88$ & 7.83 \\
\hline & & & & $05 / 22 / 88$ & 7.80 & $06 / 15 / 88$ & 7.90 & $05 / 22 / 88$ & 7.90 \\
\hline \multirow[t]{2}{*}{ PHFIELD } & \multirow{2}{*}{\multicolumn{3}{|c|}{0.10}} & $02 / 05 / 88$ & 7.60 & $01 / 08 / 88$ & 7.50 & $01 / 05 / 88$ & 7.60 \\
\hline & & & & $05 / 22 / 88$ & 7.40 & & NR & $05 / 22 / 88$ & 7.50 \\
\hline POTASUM & PPB & 100 & & & NR & $01 / 08 / 88$ & 4420 & & KR \\
\hline SODIUM & PPB & 200 & & & NR & $01 / 08 / 88$ & 18800 & & NR \\
\hline \multirow[t]{2}{*}{ SULFATE } & PPB & 500 & & $02 / 05 / 88$ & 39100 & $01 / 08 / 88$ & 35200 & $01 / 05 / 88$ & 48400 \\
\hline & & & & $05 / 22 / 88$ & 39500 & $06 / 15 / 98$ & 34200 & $05 / 22 / 88$ & 43200 \\
\hline JC & $P P B$ & 2000 & & $02 / 05 / 88$ & 29100 & $01 / 08 / 88$ & 22600 & $01 / 05 / 88$ & 27200 \\
\hline & & & & $05 / 22 / 88$ & 28100 & $06 / 15 / 88$ & 23300 & $05 / 22 / 88$ & 27300 \\
\hline$T C-99$ & $P C 1 / L$ & 15 & 900 & & NR & & NR & $01 / 05 / 88$ & 210 \\
\hline & & & & & & & & & 3.183 \\
\hline TRITIUM & $P C 1 / L$ & 500 & 20000 & $02 / 05 / 83$ & 202000 & $01 / 08 / 88$ & 52300 & $01 / 05 / 88$ & 154000 \\
\hline & & & & & $\{1720$ & & \{ 867 & & < 1470 \\
\hline
\end{tabular}


TABLE E.3. (contd)

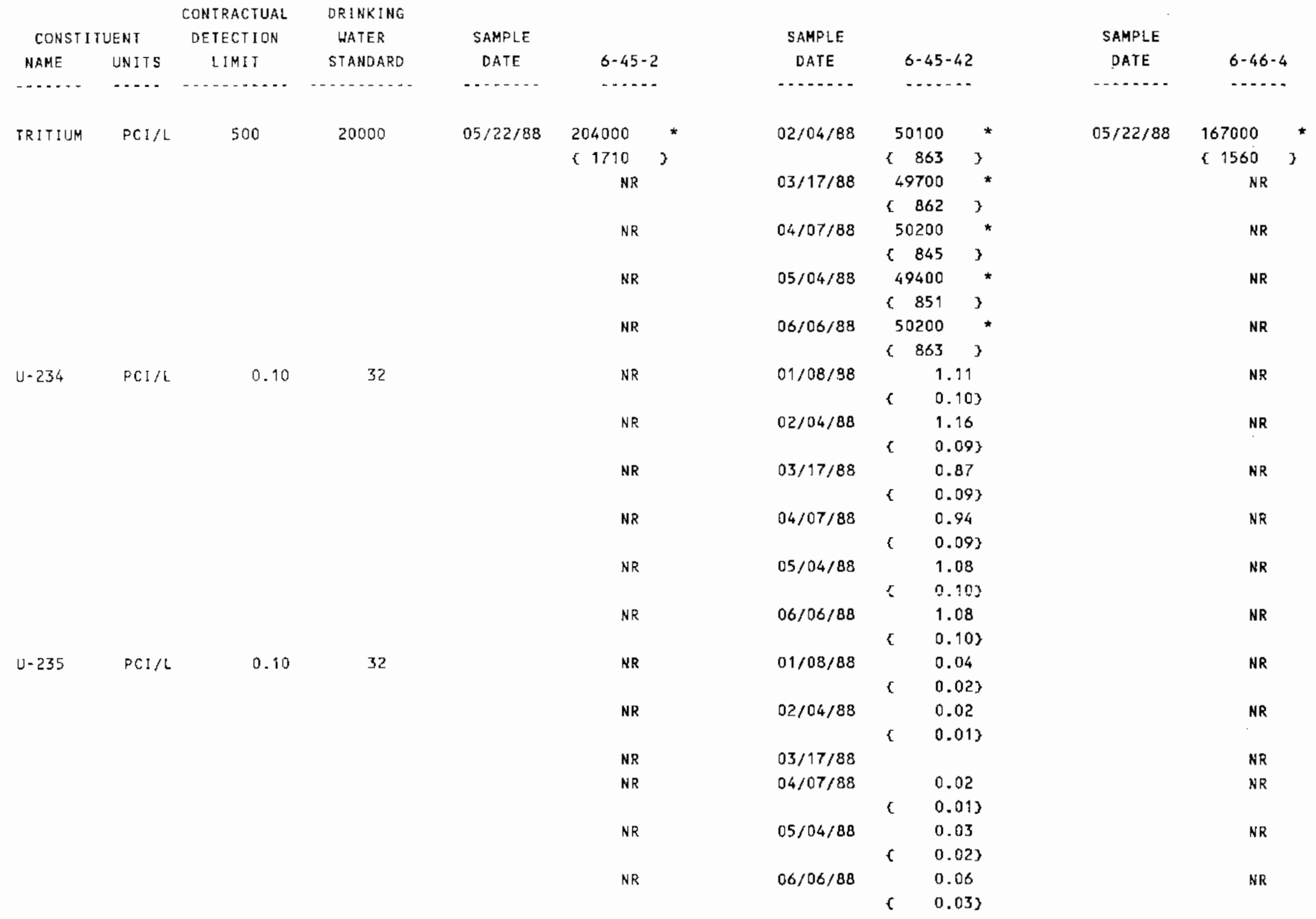


TABLE E.3. (contd)

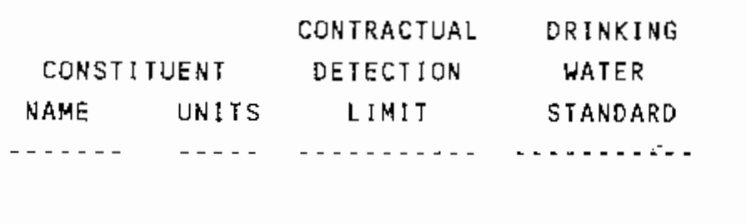

$\begin{array}{ll}\text { SAMPLE } & \\ \text { DATE } & 6-45-2 \\ \ldots \ldots-\ldots & \cdots . . .\end{array}$

SAMPLE
DATE
$\ldots \ldots-2-45-42$

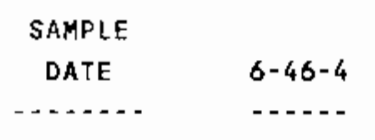

$\begin{array}{ll}\mathrm{U}-23 \mathrm{~PB} & 0.10 \quad 4.80 \\ \end{array}$

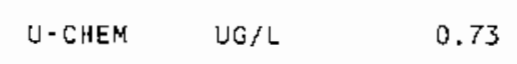

$\begin{array}{lll}U \text {-CHEM } & \text { UG } / L & 0.73 \\ \text { VANADUM } & \text { PPB } & 5\end{array}$

NR

NR

NR

NR

NR

NR

NR

NR

NR

-..... -....

\begin{tabular}{|c|c|c|}
\hline \multirow[t]{2}{*}{$01 / 08 / 88$} & & 0.86 \\
\hline & $c$ & $0.09\}$ \\
\hline \multirow[t]{2}{*}{$02 / 04 / 88$} & & 0.87 \\
\hline & $c$ & 0.083 \\
\hline \multirow[t]{2}{*}{$03 / 17 / 88$} & & 0.57 \\
\hline & $c$ & 0.073 \\
\hline \multirow[t]{2}{*}{$04 / 07 / 8 B$} & & 0.79 \\
\hline & $\mathfrak{c}$ & 0.083 \\
\hline \multirow[t]{2}{*}{$05 / 04 / 88$} & & 0.82 \\
\hline & $\kappa$ & 0.093 \\
\hline \multirow[t]{2}{*}{$06 / 06 / 83$} & & 0.89 \\
\hline & 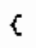 & $0.09\}$ \\
\hline $01 / 08 / 88$ & & 2.17 \\
\hline $02 / 04 / 88$ & & 2.32 \\
\hline $03 / 17 / 88$ & & 1.92 \\
\hline $04 / 07 / 8 B$ & & 1.88 \\
\hline
\end{tabular}

NR

NR

NR

NR

SAMPLE

DATE

$6-45-42$

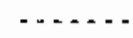

SAMPLE

DATE 6-46-4

$05 / 04 / 88$

$06 / 06 / 88$

$01 / 08 / 88$ 
IABLE E. 3. (contd)

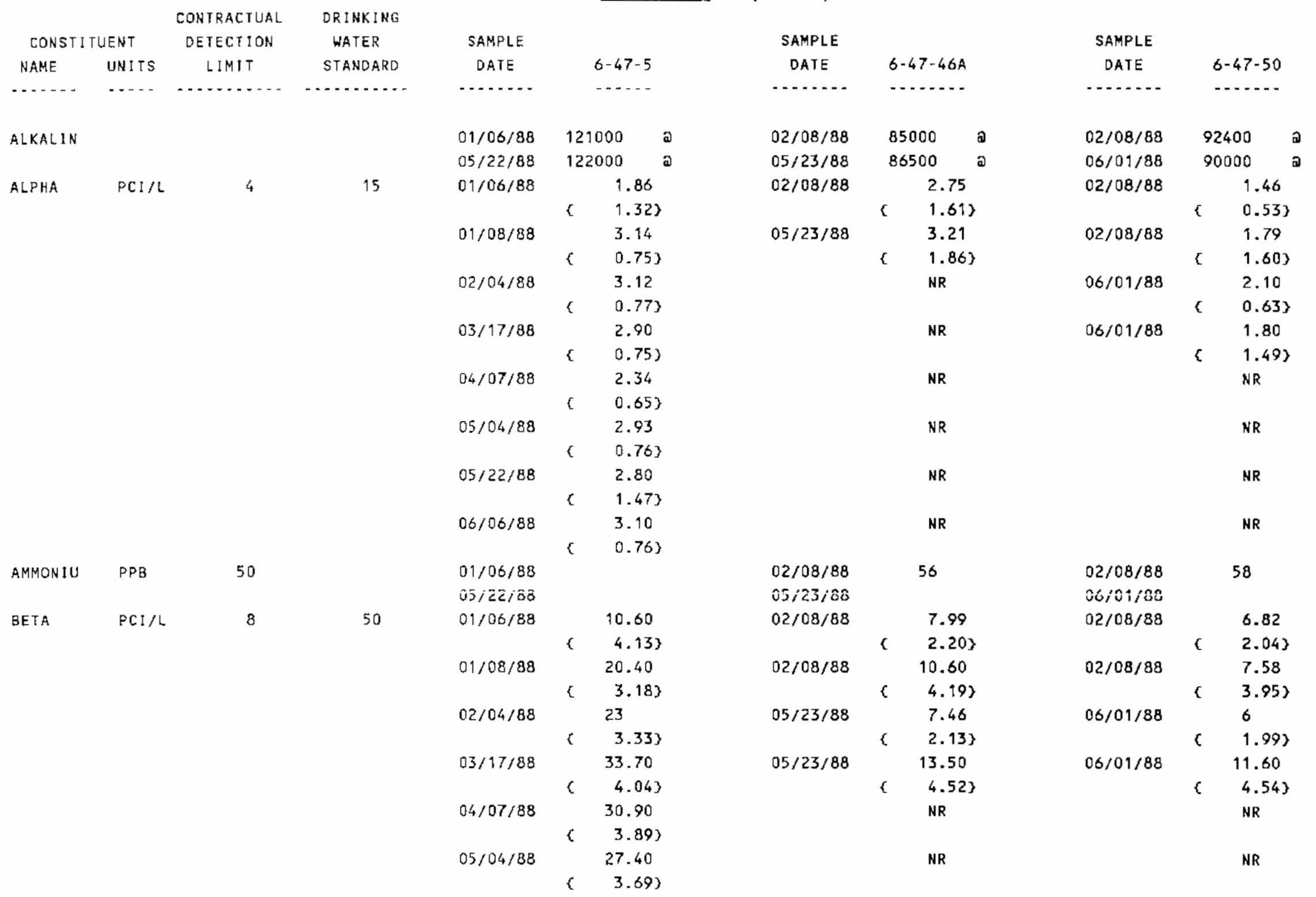


TABLE E.3. (contd)

\begin{tabular}{|c|c|c|c|c|c|c|c|c|c|}
\hline \multicolumn{2}{|c|}{ CONSTI IUENT } & $\begin{array}{l}\text { CONTRACTUAL } \\
\text { DETECTION }\end{array}$ & $\begin{array}{l}\text { DRINKING } \\
\text { WATER }\end{array}$ & SAMPLE & \multirow[b]{2}{*}{$6-47-5$} & \multicolumn{2}{|l|}{ SAMPLE } & \multicolumn{2}{|l|}{ SAMPLE } \\
\hline NAME & UNITS & LIMIT & STANDARD & DATE & & DATE & $6-47-46 A$ & DATE & $6-47-50$ \\
\hline \multirow[t]{5}{*}{ ….... } & ..... & ..... & - & $\ldots \ldots$ & ...... & . & .......... & ......... & $\ldots$ \\
\hline & & & & $05 / 22 / 88$ & 22.10 & & NR & & NR \\
\hline & & & & & 5.423 & & & & \\
\hline & & & & $06 / 06 / 88$ & 23.60 & & NR & & NR \\
\hline & & & & & 3.523 & & & & \\
\hline \multirow{2}{*}{ CHLORID } & PPB & 500 & & $01 / 06 / 88$ & 11600 & $02 / 08 / 88$ & 20900 & $02 / 08 / 88$ & 28900 \\
\hline & & & & $05 / 22 / 88$ & 10600 & $05 / 23 / 88$ & 21000 & $06 / 01 / 88$ & 29500 \\
\hline \multirow[t]{4}{*}{$c 0-60$} & $\mathrm{PCI} / \mathrm{L}$ & 22.50 & 100 & $01 / 08 / 88$ & 8.71 & $02 / 08 / 88$ & & $02 / 08 / 88$ & \\
\hline & & & & & 83 & & & & \\
\hline & & & & $04 / 07 / 88$ & 8.48 & $05 / 23 / 88$ & & $06 / 01 / 88$ & \\
\hline & & & & & 5.013 & & & & \\
\hline \multirow[t]{2}{*}{ CONDFLD } & AMHO & 1 & & $01 / 06 / 88$ & 323 & $02 / 08 / 88$ & 386 & $02 / 08 / 88$ & 400 \\
\hline & & & & $05 / 22 / 88$ & 345 & $05 / 23 / 88$ & 378 & $06 / 01 / 88$ & 481 \\
\hline \multirow[t]{2}{*}{ FBARIUM } & PPB & 6 & 1000 & $01 / 06 / 88$ & 27 & $02 / 08 / 88$ & 53 & $02 / 08 / 88$ & 74 \\
\hline & & & & $05 / 22 / 88$ & 30 & $05 / 23 / 88$ & 50 & $06 / 01 / 88$ & 67 \\
\hline \multirow[t]{2}{*}{ FCALCIU } & PPB & 50 & & $01 / 06 / 88$ & 44100 & $02 / 08 / 88$ & 45400 & $02 / 08 / 88$ & 55200 \\
\hline & & & & $05 / 22 / 88$ & 44900 & $05 / 23 / 88$ & 44600 & $06 / 01 / 88$ & 48700 \\
\hline
\end{tabular}


TABLE E. 3 . (contd)

\begin{tabular}{|c|c|c|c|c|c|c|c|c|c|}
\hline & & CONTRACTUAL & DRINKING & & & & & & \\
\hline CONSTI & UENT & DETECTION & WATER & SAMPLE & & SAMPLE & & SAMPLE & \\
\hline RAME & UNITS & LIM1T & STANDARD & DATF & $6-47 \cdot 5$ & DATE & $6-47-46 \mathrm{~A}$ & DATE & $6-47-50$ \\
\hline …..... & $\cdots$ & n............. & . & $\ldots \ldots$ & $\cdots$ & $\ldots$ & ......... & $\ldots$ & ...... \\
\hline FIRON & PPB & 50 & . & $01 / 06 / 88$ & & $02 / 08 / 88$ & & $02 / 08 / 88$ & 262 \\
\hline & & & & $05 / 22 / 8 B$ & & $05 / 23 / 88$ & & $06 / 01 / 88$ & 203 \\
\hline FLUORID & $P P B$ & 500 & 4000 & $01 / 06 / 88$ & 592 & $02 / 08 / 88$ & 602 & $02 / 08 / 88$ & 648 \\
\hline & & & & $05 / 22 / 88$ & 517 & $05 / 23 / 88$ & 668 & $06 / 01 / 88$ & \\
\hline FMAGNES & PPB & 50 & & $01 / 06 / 88$ & 9750 & $02 / 08 / 88$ & 15000 & $02 / 08 / 88$ & 16200 \\
\hline & & & & $05 / 22 / 88$ & 10200 & $05 / 23 / 88$ & 14900 & $06 / 01 / 88$ & 14800 \\
\hline FMANGAN & $\mathrm{PPB}$ & 5 & & $01 / 06 / 88$ & & $02 / 08 / 88$ & & $02 / 08 / 88$ & 14 \\
\hline & & & & $05 / 22 / 88$ & & $05 / 23 / 88$ & & $06 / 01 / 88$ & 12 \\
\hline FPOTASS & $P P B$ & 100 & & $01 / 06 / 88$ & 4540 & $02 / 08 / 88$ & 8070 & $02 / 08 / 88$ & 7220 \\
\hline & & & & $05 / 22 / 88$ & 4910 & $05 / 23 / 88$ & 7640 & $06 / 01 / 88$ & 6910 \\
\hline FSELEN I & PPB & 5 & 10 & $01 / 06 / 83$ & & $02 / 08 / 28$ & 7 & $02 / 08 / 88$ & \\
\hline & & & & $05 / 22 / 88$ & & $05 / 23 / 88$ & & $06 / 01 / 88$ & \\
\hline FSODIUM & $P P B$ & 200 & & $01 / 06 / 88$ & 21800 & $02 / 08 / 88$ & 23000 & $02 / 08 / 88$ & 21400 \\
\hline & & & & $05 / 22 / 88$ & 22100 & $05 / 23 / 88$ & 21800 & $06 / 01 / 88$ & 20400 \\
\hline FSTRONT & PPB & 10 & & $01 / 06 / 88$ & 218 & $02 / 08 / 88$ & 318 & $02 / 08 / 8 B$ & 285 \\
\hline & & & & $05 / 22 / 88$ & 229 & $05 / 23 / 88$ & 300 & $06 / 01 / 88$ & 254 \\
\hline FVANAD I & $P P B$ & 5 & & $01 / 06 / 88$ & 16 & $02 / 08 / 88$ & 20 & $02 / 08 / 88$ & 8 \\
\hline & & & & $05 / 22 / 88$ & 15 & $05 / 23 / 88$ & 21 & $06 / 01 / 88$ & 9 \\
\hline FZINC & PPB & 5 & & $01 / 06 / 88$ & & $02 / 08 / 88$ & & $02 / 08 / 88$ & \\
\hline & & & & $\bar{U} \bar{S} / \bar{C} \bar{C} / \bar{O} \overline{0}$ & & $05 / 23 / 00$ & 5 & $0 \leq / 01 / 88$ & \\
\hline HNI TRAT & $P P B$ & 2500 & 45000 & $01 / 08 / 88$ & 22400 & $02 / 08 / 88$ & 12000 & $02 / 08 / 88$ & 6790 \\
\hline & & & & $02 / 04 / 88$ & 22900 & $05 / 23 / 88$ & 13400 & $02 / 08 / 88$ & 7310 \\
\hline & & & & $03 / 17 / 88$ & 33200 & & NR & $06 / 01 / 88$ & 7420 \\
\hline & & & & $04 / 07 / 88$ & 31300 & & NR & & $N R$ \\
\hline & & & & $05 / 04 / 88$ & 36000 & & NR & & NR \\
\hline & & & & $06 / 06 / 88$ & 25200 & & NR & & NR \\
\hline LFLUORD & PPB & 50 & 4000 & $01 / 06 / 88$ & 415 & $02 / 08 / 88$ & 409 & $02 / 08 / 88$ & 469 \\
\hline & & & & $05 / 22 / 88$ & 415 & $05 / 23 / 88$ & 443 & $06 / 01 / 88$ & 518 \\
\hline NITRATE & $P P B$ & 500 & 45000 & $01 / 06 / 88$ & 27500 & $02 / 08 / 88$ & 12600 & $02 / 08 / 88$ & 7470 \\
\hline & & & & $05 / 22 / 88$ & 26000 & $05 / 23 / 88$ & 13600 & $06 / 01 / 88$ & 8120 \\
\hline$P H-L A B$ & & 0.01 & & $01 / 06 / 88$ & 7.58 & $02 / 08 / 88$ & 7.73 & $02 / 08 / 88$ & 7.58 \\
\hline & & & & $05 / 22 / 88$ & 7.70 & $05 / 23 / 88$ & 7.90 & $06 / 01 / 88$ & 7.80 \\
\hline PHF JELD & & 0.10 & & $01 / 06 / 88$ & 7.70 & $02 / 08 / 88$ & 7.90 & $02 / 08 / 88$ & 7.90 \\
\hline & & & & $05 / 22 / 88$ & 7.60 & $05 / 23 / 88$ & 7.80 & $06 / 01 / 88$ & 8.40 \\
\hline
\end{tabular}




\begin{tabular}{|c|c|c|c|}
\hline \multirow{2}{*}{\multicolumn{2}{|c|}{ CONSTI TUENT }} & CONTRACTUAL & ORINKING \\
\hline & & DETECTION & WATER \\
\hline NAME & UNITS & LIMIT & STANDARD \\
\hline$\ldots$ & $-\ldots$ & - & $-\ldots$ \\
\hline
\end{tabular}

$\begin{array}{lrrr}\text { SR } 90 & \text { PCI/L } & 5 & 8 \\ \text { SULFATE } & \text { PPB } & 500 & \\ \text { TC } & \text { PPB } & 2000 & \\ \text { TRITIUM } & \text { PCI/L } & 500 & 20000 \\ & & & \end{array}$

$\underset{⿱ 亠 䒑}{m}$

\begin{tabular}{|c|c|c|c|c|c|}
\hline & & CONTRACTUAL & DRINKING & & \\
\hline CONSTI & JENT & DETECTION & HATER & SAMPLE & \\
\hline NAME & UNITS & LIMIT & STANDARD & DATE & $6-47-5$ \\
\hline$\ldots \ldots$ & - - - - & $\ldots \ldots$ & $\ldots$ & $\ldots \ldots$ & $\ldots$. \\
\hline TRITIUM & $\mathrm{PCl} / \mathrm{L}$ & 500 & 20000 & $04 / 07 / 88$ & 218000 \\
\hline & & & & & $<1530$ \\
\hline & & & & $05 / 04 / 88$ & 187000 \\
\hline & & & & & ( 1410 \\
\hline & & & & $06 / 06 / 88$ & 172000 \\
\hline & & & & & < 1570 \\
\hline u & PCI $/ L$ & 0.50 & 600 & $01 / 08 / 88$ & 2.09 \\
\hline & & & & $04 / 07 / 88$ & 1.53 \\
\hline
\end{tabular}

TABLE E.3. (contd)

\begin{tabular}{|c|c|c|}
\hline \multicolumn{3}{|l|}{ SAMPLE } \\
\hline DATE & \multicolumn{2}{|c|}{$6-47-5$} \\
\hline 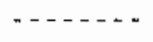 & \multicolumn{2}{|c|}{$\ldots$} \\
\hline $01 / 08 / 88$ & \multicolumn{2}{|c|}{1.22} \\
\hline & \multicolumn{2}{|c|}{0.853} \\
\hline $04 / 07 / 88$ & & \\
\hline $01 / 06 / 88$ & \multicolumn{2}{|c|}{49100} \\
\hline $05 / 22 / 8 B$ & \multicolumn{2}{|c|}{45600} \\
\hline $01 / 06 / 88$ & \multicolumn{2}{|c|}{27700} \\
\hline $05 / 22 / 88$ & \multicolumn{2}{|c|}{28000} \\
\hline \multirow[t]{2}{*}{$01 / 08 / 88$} & \multicolumn{2}{|c|}{139000} \\
\hline & \multicolumn{2}{|l|}{ c 1390} \\
\hline \multirow[t]{2}{*}{$02 / 04 / 80$} & \multicolumn{2}{|l|}{160000} \\
\hline & \multicolumn{2}{|c|}{ < 1530} \\
\hline \multirow[t]{2}{*}{$03 / 17 / 88$} & \multicolumn{2}{|c|}{221000} \\
\hline & \& 1780 & $j$ \\
\hline
\end{tabular}

\begin{tabular}{|c|c|}
\hline SAMPLE & \\
\hline DATE & $6-47-46 A$ \\
\hline - - & \\
\hline
\end{tabular}

SAMPLE
DATE
$\ldots \ldots-47-50$

\begin{tabular}{lr} 
& NR \\
& NR \\
$02 / 08 / 88$ & 89800 \\
$05 / 23 / 88$ & 95100 \\
$02 / 08 / 88$ & 20400 \\
$05 / 23 / 88$ & 19100 \\
$02 / 08 / 88$ & \\
$05 / 23 / 88$ & \\
& \\
\hline
\end{tabular}

$02 / 08 / 88$

$06 / 01 / 88$

0.92

$02 / 08 / 88 \quad 95100$

$06 / 01 / 88 \quad 98200$

$02 / 08 / 88 \quad 22900$

$06 / 01 / 88 \quad 24800$

$02 / 08 / 88$

$02 / 08 / 88$

$06 / 01 / 88$

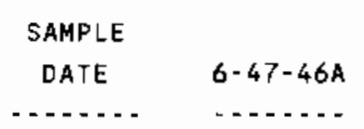

NR

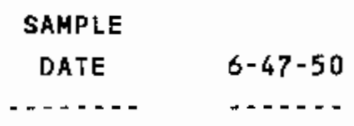

NR

NR

NR

NR

NR 
TABLE E.3. (contd)

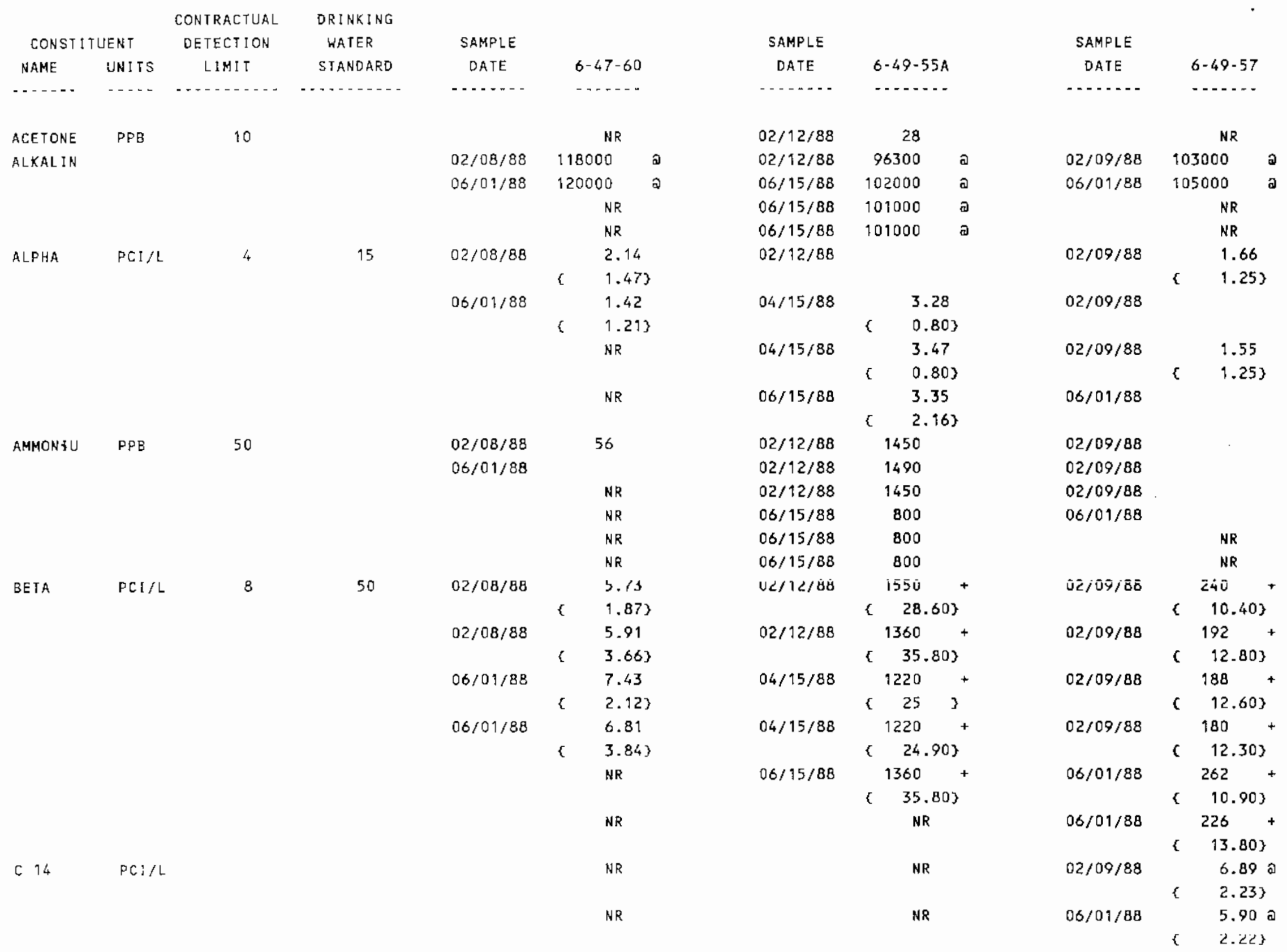


TABLE E. 3. (contd)

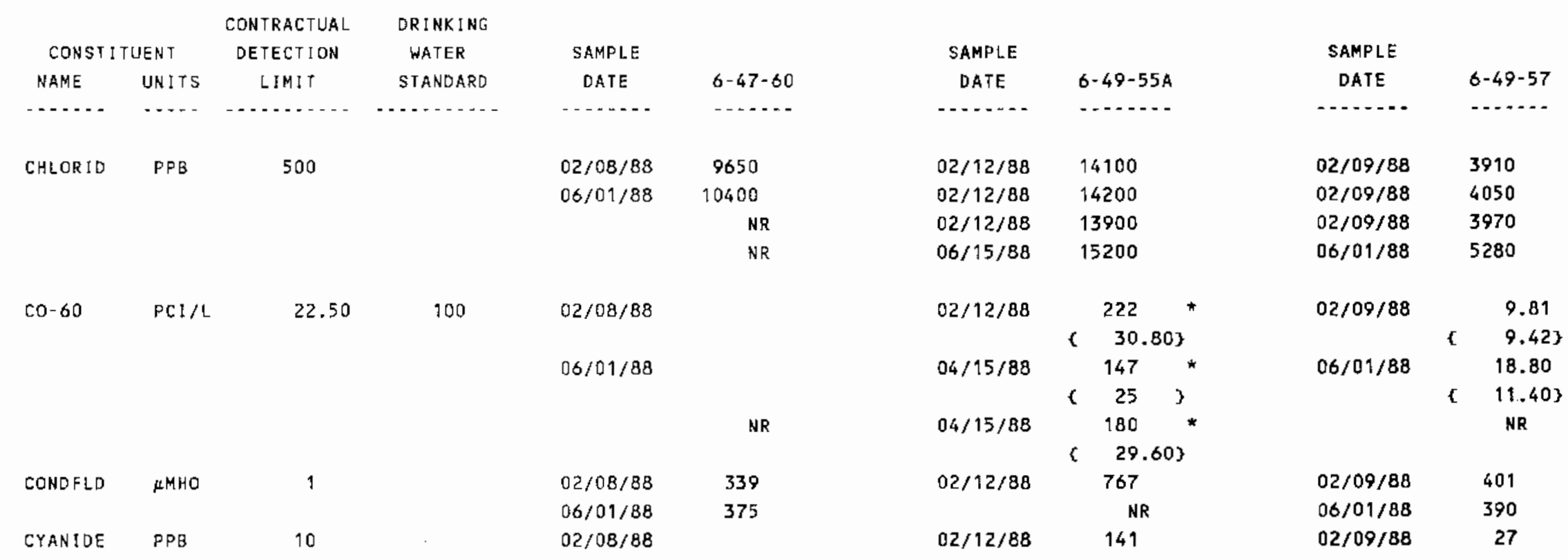


TABLE E.3. (contd)

\begin{tabular}{|c|c|c|c|c|c|c|c|c|c|}
\hline \multicolumn{2}{|c|}{ CONSTI TUENT } & $\begin{array}{l}\text { CONTRACTUAL } \\
\text { DETECTION }\end{array}$ & $\begin{array}{l}\text { DRINKING } \\
\text { HATER }\end{array}$ & SAMPLE & & SAMPLE & & SAMPLE & \\
\hline NAME & UNITS & LIMIT & STANDARD & DATE & $6-47-60$ & DATE & $6-49-55 A$ & DATE & $6-49-57$ \\
\hline$-\ldots .$. & $\cdots$ & $\cdots$ & - & $-\ldots$ & 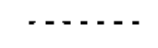 & $\cdots$ & - & 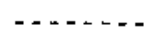 & $-\ldots$ \\
\hline \multirow[t]{5}{*}{ CYANIDE } & PPB & 10 & & $06 / 01 / 88$ & & $02 / 12 / 88$ & 247 & $02 / 09 / 88$ & 21 \\
\hline & & & & & $N R$ & $02 / 12 / 88$ & 106 & $02 / 09 / 88$ & 21 \\
\hline & & & & & $N R$ & $06 / 15 / 88$ & 104 & $06 / 01 / 88$ & 28 \\
\hline & & & & & $N R$ & $06 / 15 / 88$ & 77 & $06 / 01 / 88$ & 25 \\
\hline & & & & & NR & $06 / 15 / 88$ & & $06 / 01 / 88$ & 27 \\
\hline \multirow[t]{6}{*}{ FARSEN I } & PPB & 5 & 50 & $02 / 08 / 88$ & & $02 / 12 / 88$ & & $02 / 09 / 88$ & 9 \\
\hline & & & & $06 / 01 / 88$ & 7 & $06 / 15 / 88$ & 5 & $02 / 09 / 88$ & 10 \\
\hline & & & & & $\mathrm{NR}$ & $06 / 15 / 88$ & & $02 / 09 / 88$ & 9 \\
\hline & & & & & NR & $06 / 15 / 88$ & & $06 / 01 / 88$ & 10 \\
\hline & & & & & MR & & NR & $06 / 01 / 88$ & 9 \\
\hline & & & & & NR & & NR & $06 / 01 / 88$ & 9 \\
\hline \multirow[t]{6}{*}{ FBARIUM } & $P P B$ & 6 & 1000 & $02 / 08 / 88$ & 34 & $02 / 12 / 88$ & 39 & $02 / 09 / 88$ & 20 \\
\hline & & & & $06 / 01 / 88$ & 37 & $06 / 15 / 88$ & 45 & $02 / 09 / 88$ & 21 \\
\hline & & & & & NR & $06 / 15 / 88$ & 43 & $02 / 09 / 88$ & 21 \\
\hline & & & & & NR & $06 / 15 / 88$ & 43 & $06 / 01 / 88$ & 24 \\
\hline & & & & & NR & & NR & $06 / 01 / 88$ & 25 \\
\hline & & & & & NR & & NR & $06 / 01 / 88$ & 24 \\
\hline \multirow[t]{6}{*}{ FCALCIU } & PPB & 50 & & $02 / 08 / 88$ & 37300 & $02 / 12 / 88$ & 99200 & $02 / 09 / 88$ & 22100 \\
\hline & & & & $06 / 01 / 88$ & 36400 & $06 / 15 / 88$ & 94800 & $02 / 09 / 88$ & 23600 \\
\hline & & & & & NR & $06 / 15,80$ & 93900 & $02 / 09 ; 66$ & 24500 \\
\hline & & & & & $N R$ & $06 / 15 / 88$ & 91100 & $06 / 01 / 88$ & 24100 \\
\hline & & & & & NR & & NR & $06 / 01 / 88$ & 26200 \\
\hline & & & & & NR & & $N R$ & $06 / 01 / 88$ & 25600 \\
\hline \multirow[t]{6}{*}{ FCHROMI } & PPB & 10 & 50 & $02 / 08 / 88$ & & $02 / 12 / 88$ & & $02 / 09 / 88$ & 11 \\
\hline & & & & $06 / 01 / 88$ & & $06 / 15 / 88$ & & $02 / 09 / 88$ & 19 \\
\hline & & & & & NR & $06 / 15 / 88$ & & $02 / 09 / 88$ & 11 \\
\hline & & & & & $N R$ & $06 / 15 / 88$ & & $06 / 01 / 88$ & \\
\hline & & & & & NR & & NR & $06 / 01 / 88$ & \\
\hline & & & & & NR & & NR & $06 / 01 / 88$ & \\
\hline \multirow[t]{6}{*}{ FIRON } & PPB & 50 & & $02 / 08 / 88$ & & $02 / 12 / 88$ & 234 & $02 / 09 / 88$ & \\
\hline & & & & $06 / 01 / 88$ & & $06 / 15 / 88$ & 100 & $02 / 09 / 88$ & \\
\hline & & & & & NR & $06 / 15 / 88$ & 121 & $02 / 09 / 88$ & \\
\hline & & & & & NR & $06 / 15 / 88$ & 103 & $06 / 01 / 88$ & \\
\hline & & & & & NR & & NR & $06 / 01 / 88$ & \\
\hline & & & & & $\mathrm{NR}$ & & NR & $06 / 01 / 88$ & \\
\hline
\end{tabular}


TABLE E.3. (contd)

\begin{tabular}{|c|c|c|c|c|c|c|c|c|c|}
\hline \multirow{2}{*}{\multicolumn{2}{|c|}{ CONSTITUENT }} & CONTRACTUAL & DRINKING & & & & & & \\
\hline & & DETECTION & WATER & SAMPLE & & SAMPLE & & SAMPLE & \\
\hline NAME & UNITS & LIMIT & STANDARD & DATE & $6-47-60$ & DATE & $6-49-55 A$ & DATE & $6-49-57$ \\
\hline$\cdots$ & $\cdots$ & - - - - - - - - & $\ldots \ldots \ldots$ & $\ldots$ & - - - - - & $\cdots \ldots$ & $\ldots \ldots$ & - & $\ldots \ldots$ \\
\hline \multirow[t]{4}{*}{ FLUORIO } & PPB & 500 & 4000 & $02 / 08 / 88$ & 744 & $02 / 12 / 88$ & & $02 / 09 / 88$ & 1010 \\
\hline & & & & $06 / 01 / 88$ & 579 & $02 / 12 / 88$ & & $02 / 09 / 88$ & 1010 \\
\hline & & & & & NR & $02 / 12 / 88$ & & $02 / 09 / 88$ & 1010 \\
\hline & & & & & NR & $06 / 15 / 88$ & & $06 / 01 / 88$ & 878 \\
\hline \multirow[t]{6}{*}{ FMAGNES } & PPB & 50 & & $02 / 08 / 88$ & 11700 & $02 / 12 / 88$ & 26000 & $02 / 09 / 88$ & 7100 \\
\hline & & & & $06 / 01 / 88$ & 11900 & $06 / 15 / 88$ & 26800 & $02 / 09 / 88$ & 7350 \\
\hline & & & & & NR & $06 / 15 / 88$ & 26300 & $02 / 09 / 88$ & 7680 \\
\hline & & & & & NR & $06 / 15 / 88$ & 25600 & $06 / 01 / 88$ & 7700 \\
\hline & & & & & NR & & NR & $06 / 01 / 88$ & 8390 \\
\hline & & & & & NR & & NR & $06 / 01 / 88$ & 8080 \\
\hline \multirow[t]{4}{*}{ FMANGAN } & PPB & 5 & & $02 / 08 / 88$ & & $02 / 12 / 86$ & 25 & $02 / 09 / 88$ & \\
\hline & & & & $06 / 01 / 88$ & & $06 / 15 / 88$ & 21 & $02 / 09 / 88$ & \\
\hline & & & & & NR & $06 / 15 / 88$ & 21 & $02 / 09 / 88$ & \\
\hline & & & & & NR & $06 / 15 / 88$ & 20 & $06 / 01 / 88$ & \\
\hline
\end{tabular}


TABLE E.3. (contd)

\begin{tabular}{|c|c|c|c|c|c|c|c|c|c|}
\hline & & CONTRACTUAL & DRINKING & & & & & & \\
\hline CONSTI & JENT & DETECTION & WATER & SAMPLE & & SAMPLE & & SAMPLE & \\
\hline NAME & UNITS & LIMIT & STANDARD & DATE & $6-47-60$ & DATE & $6-49-55 A$ & DATE & $6-49-57$ \\
\hline$+\ldots$ & $\cdots$ & $\cdots$ & $\cdots$ & $\cdots$ & $\cdots \cdots$ & $\cdots$ & - & 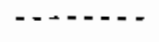 & $-\cdots-\cdots$ \\
\hline FMANGAN & PPB & 5 & & & NR & & NR & $06 / 01 / 88$ & \\
\hline & & & & & NR & & NR & $06 / 01 / 88$ & \\
\hline FPOTASS & PP8 & 100 & & $02 / 08 / 88$ & 5540 & $02 / 12 / 88$ & $13 \% 00$ & $02 / 09 / 88$ & 5540 \\
\hline & & & & $06 / 01 / 88$ & 6140 & $06 / 15 / 88$ & 12000 & $02 / 09 / 88$ & 5760 \\
\hline & & & & & NR & $06 / 15 / 88$ & 11900 & $02 / 09 / 88$ & 5990 \\
\hline & & & & & NR & $06 / 15 / 88$ & 12200 & $06 / 01 / 88$ & 5960 \\
\hline & & & & & NR & & NR & $06 / 01 / 88$ & 6210 \\
\hline & & & & & NR & & NR & $06 / 01 / 88$ & 5840 \\
\hline FSELENI & $P P B$ & 5 & 10 & $02 / 08 / 88$ & & $02 / 12 / 88$ & 6 & $02 / 09 / 88$ & \\
\hline & & & & $06 / 01 / 88$ & & $06 / 15 / 98$ & & $02 / 09 / 88$ & \\
\hline & & & & & NR & $06 / 15 / 88$ & & $02 / 09 / 8 \mathrm{~B}$ & \\
\hline & & & & & NR & $06 / 15 / 88$ & & $06 / 01 / 88$ & \\
\hline & & & & & NR & & NR & $06 / 01 / 88$ & \\
\hline & & & & & NR & & NR & $06 / 01 / 88$ & \\
\hline FSODIUM & PPB & 200 & & $02 / 08 / 88$ & 24100 & $02 / 12 / 88$ & 47700 & $02 / 09 / 88$ & 43900 \\
\hline & & & & $06 / 01 / 88$ & 24900 & $06 / 15 / 88$ & 48200 & $02 / 09 / 88$ & 45200 \\
\hline & & & & & NR & $06 / 15 / 88$ & 46700 & $02 / 09 / 88$ & 45300 \\
\hline & & & & & NR & $06 / 15 / 8 B$ & 46000 & $06 / 01 / 88$ & 41200 \\
\hline & & & & & NR & & NR & $06 / 09 / 88$ & 45000 \\
\hline & & & & & NR & & AN $\pi$ & $06 / 01 / 88$ & 44800 \\
\hline FSTRONT & $P P B$ & 10 & & $02 / 08 / 88$ & 196 & $02 / 12 / 88$ & 487 & $02 / 09 / 88$ & 126 \\
\hline & & & & $06 / 01 / 88$ & 204 & $06 / 15 / 88$ & 443 & $02 / 09 / 88$ & 128 \\
\hline & & & & & NR & $06 / 15 / 8 B$ & 440 & $02 / 09 / 88$ & 930 \\
\hline & & & & & $N R$ & $06 / 15 / 88$ & 436 & $06 / 01 / 88$ & 130 \\
\hline & & & & & $N R$ & & $N R$ & $06 / 01 / 88$ & 139 \\
\hline & & & & & $N R$ & & NR & $06 / 01 / 88$ & 133 \\
\hline FVANADI & PPB & 5 & & $02 / 08 / 88$ & 17 & $02 / 12 / 88$ & 8 & $02 / 09 / 88$ & 33 \\
\hline & & & & $06 / 01 / 8 B$ & 18 & $06 / 15 / 88$ & 9 & $02 / 09 / 88$ & 29 \\
\hline & & & & & NR & $06 / 15 / 88$ & 9 & $02 / 09 / 88$ & 32 \\
\hline & & & & & NR & $06 / 15 / 88$ & 9 & $06 / 01 / 88$ & 28 \\
\hline & & & & & $N R$ & & NR & $06 / 01 / 88$ & 28 \\
\hline & & & & & NR & & NR & $06 / 01 / 88$ & 27 \\
\hline
\end{tabular}


IABLE E.3. (contd)

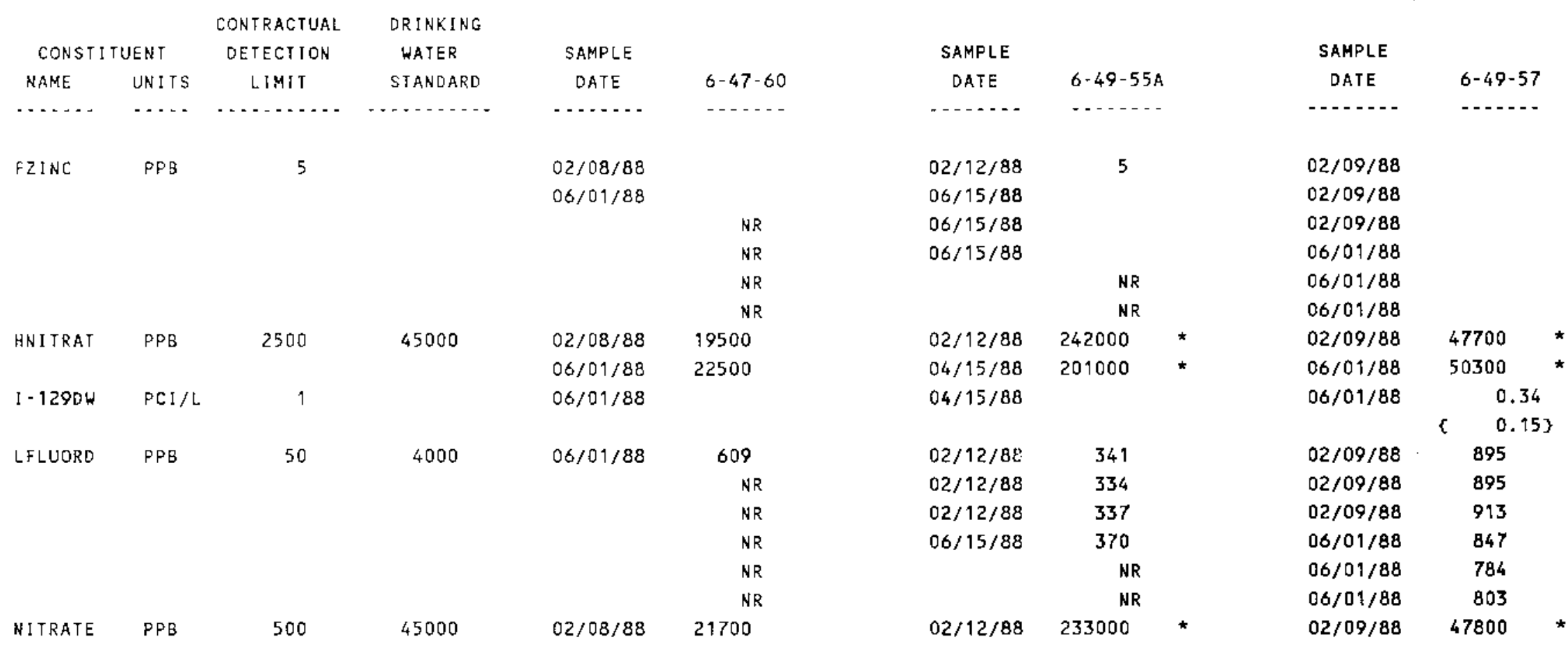


TABLE E.3. (contd)

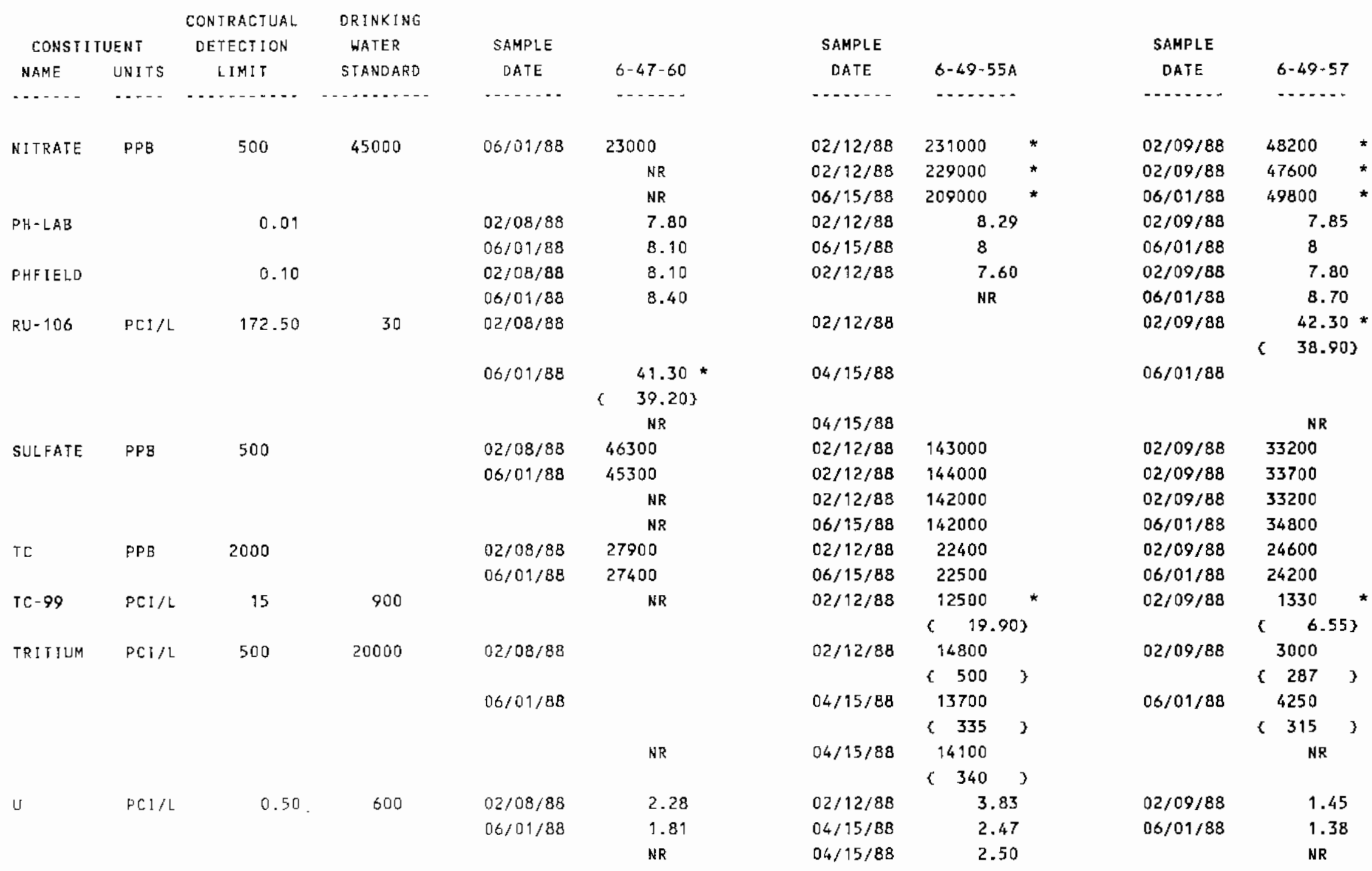


IABLE E. 3. (contd)

\begin{tabular}{|c|c|c|c|c|c|c|c|c|c|c|c|c|}
\hline \multicolumn{2}{|c|}{ CONSTI TUENT } & $\begin{array}{l}\text { CONTRACTUAL } \\
\text { DETECTION }\end{array}$ & $\begin{array}{l}\text { DRINKING } \\
\text { WATER }\end{array}$ & SAMPLE & \multirow{2}{*}{\multicolumn{2}{|c|}{$6-49-79$}} & \multirow{2}{*}{$\begin{array}{l}\text { SAMPLE } \\
\text { DATE }\end{array}$} & \multirow{2}{*}{\multicolumn{2}{|c|}{$6-50-53$}} & SAMPLE & \multirow{2}{*}{\multicolumn{2}{|c|}{$6-50-85$}} \\
\hline NAME & UNITS & LIMIT & STANDARD & DATE & & & & & & DATE & & \\
\hline 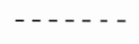 & $\cdots$ & - & - & $\ldots$ & & - . . - & , & $\cdots$ & & - & & $\ldots$ \\
\hline \multirow[t]{4}{*}{ ALKAL IN } & & & & $02 / 09 / 88$ & & 00 & $03 / 06 / 88$ & 68500 & a & $03 / 23 / 88$ & & 00 \\
\hline & & & & $06 / 07 / 88$ & & 00 & $06 / 06 / 88$ & 68500 & a & $06 / 06 / 88$ & & 00 \\
\hline & & & & & & NR & $06 / 06 / 88$ & 67700 & a & & & NR \\
\hline & & & & & & NR & $06 / 06 / 88$ & 68000 & a & & & NR \\
\hline \multirow[t]{18}{*}{ ALPHA } & $\mathrm{PCI} / \mathrm{L}$ & 4 & 15 & $02 / 09 / 88$ & & 0.74 & $03 / 06 / 88$ & & & $02 / 19 / 88$ & & 0.54 \\
\hline & & & & & c & $0.43\}$ & & $\varepsilon$ & & & c & $0.37)$ \\
\hline & & & & $02 / 09 / 88$ & & & $03 / 06 / 88$ & & & $03 / 23 / 88$ & & \\
\hline & & & & & & & & $c$ & & & & \\
\hline & & & & $06 / 07 / 88$ & & 1.01 & $03 / 06 / 88$ & & & $06 / 06 / 88$ & & 0.65 \\
\hline & & & & & $\mathfrak{c}$ & $0.48\}$ & & & & & ( & $0.38)$ \\
\hline & & & & $06 / 07 / 88$ & & 1.68 & $03 / 06 / 82$ & & & $06 / 06 / 88$ & & $9: 09$ \\
\hline & & & & & $c$ & 1.343 & & $\varepsilon$ & & & C & 0.953 \\
\hline & & & & & & NR & $04 / 15 / 88$ & & & & & NR \\
\hline & & & & & & & & c & & & & \\
\hline & & & & & & NR & $04 / 15 / 88$ & & & & & NR \\
\hline & & & & & & & & $c$ & & & & \\
\hline & & & & & & NR & $06 / 06 / 88$ & & & & & NR \\
\hline & & & & & & & & c & & & & \\
\hline & & & & & & NR & $06 / 06 / 88$ & & & & & MR \\
\hline & & & & & & & & c & & & & \\
\hline & & & & & & NR & $06 / 06 / 88$ & & & & & NR \\
\hline & & & & & & & & \& & & & & \\
\hline \multirow[t]{2}{*}{ AMMONIU } & $\mathrm{PPB}$ & 50 & & $02 / 09 / 88$ & & & $03 / 06 / 88$ & 82 & & $03 / 23 / 88$ & & \\
\hline & & & & $06 / 07 / 88$ & & & $06 / 06 / 88$ & 55 & & $06 / 06 / 88$ & & \\
\hline \multirow[t]{9}{*}{ BETA } & $\mathrm{PCI} / \mathrm{L}$ & 8 & 50 & $02 / 09 / 88$ & & 3.93 & $03 / 06 / 88$ & 2350 & + & $02 / 19 / 88$ & & 4.86 \\
\hline & & & & & c & 1.703 & & C $3 E$ & 3 & & c & $1.78)$ \\
\hline & & & & $02 / 09 / 88$ & & & $03 / 06 / 88$ & 2560 & + & $03 / 23 / 88$ & & 4.94 \\
\hline & & & & & & & & c 53 & & & $\varepsilon$ & 3.493 \\
\hline & & & & $06 / 07 / 88$ & & 4.72 & $03 / 06 / 88$ & 2430 & + & $06 / 06 / 88$ & & 3.44 \\
\hline & & & & & \{ & 1.843 & & $\{51$ & & & $\varepsilon$ & 1.663 \\
\hline & & & & $06 / 07 / 88$ & & 6.85 & $03 / 06 / 88$ & 2570 & + & $06 / 06 / 88$ & & 4.22 \\
\hline & & & & & \{ & 3.863 & & $\{54$ & 3 & & $\varepsilon$ & 3.773 \\
\hline & & & & & & NR & $04 / 15 / 88$ & 2260 & + & & & NR \\
\hline
\end{tabular}


IABLE E.3. (contd)

\begin{tabular}{|c|c|c|c|c|c|c|c|c|c|}
\hline \multicolumn{2}{|c|}{ CONST I TUENT } & $\begin{array}{l}\text { CONTRACTUAL } \\
\text { DETECTION }\end{array}$ & $\begin{array}{l}\text { DRINKING } \\
\text { WATER }\end{array}$ & SAMPLE & & SAMPLE & & SAMPLE & \\
\hline \multirow{11}{*}{ NAME } & UNITS & LIMIT & STANDARO & DATE & $6-49-79$ & DATE & $6-50-53$ & DATE & $6-50-85$ \\
\hline & $\cdots$ & $\cdots$ & . & $\cdots$ & n. & $\ldots$ & $\cdots$ & $\ldots$ & - . . \\
\hline & & & & & & & $\{\quad 38.20\}$ & & \\
\hline & & & & & NR & $04 / 15 / 88$ & 2030 & & NR \\
\hline & & & & & & & $\{34.80\}$ & & \\
\hline & & & & & NR & $06 / 06 / 88$ & 3020 & & NR \\
\hline & & & & & & & $\{\quad 59.80\}$ & & \\
\hline & & & & & NR & $06 / 06 / 88$ & $2980+$ & & NR \\
\hline & & & & & & & $\{\quad 58.80\}$ & & \\
\hline & & & & & NR & $06 / 06 / 88$ & 2630 & & NR \\
\hline & & & & & & & $(53.90\}$ & & \\
\hline \multirow[t]{6}{*}{ CHLORID } & $P P B$ & 500 & & $02 / 09 / 88$ & 10000 & $03 / 06 / 88$ & 38200 & $03 / 23 / 8 B$ & 9330 \\
\hline & & & & $06 / 07 / 88$ & 9960 & $03 / 06 / 88$ & 37300 & $06 / 06 / 88$ & 9960 \\
\hline & & & & & NR & $03 / 06 / 88$ & 35500 & & NR \\
\hline & & & & & NR & $06 / 06 / 88$ & 36800 & & NR \\
\hline & & & & & NR & $06 / 06 / 88$ & 36300 & & NR \\
\hline & & & & & NR & $06 / 06 / 88$ & 33900 & & NR \\
\hline
\end{tabular}


TABLEE. .3. (contd)

\begin{tabular}{|c|c|c|c|c|c|c|c|c|c|}
\hline CONST: & TUENT & $\begin{array}{l}\text { CONTRACTUAL } \\
\text { DETECTION }\end{array}$ & $\begin{array}{l}\text { DRINKING } \\
\text { HATER }\end{array}$ & SAMPLE & & SAMPLE & & SAMPLE & \\
\hline NAME & UNITS & LIMIT & STANDARD & DATE & $6-49-79$ & DATE & $6-50-53$ & DATE & $6-50-85$ \\
\hline 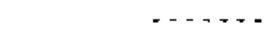 & $\cdots$ & 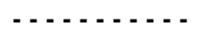 & - n. & - & - n. & - - - & ........ & - . . . & - \\
\hline & & & & & & & $\{\quad 38.40\}$ & & \\
\hline & & & & & $\mathrm{NR}$ & $04 / 15 / 88$ & $469 *$ & & $N R$ \\
\hline & & & & & & & $\{\quad 48.20\}$ & & \\
\hline & & & & & NR & $04 / 15 / 88$ & 460 & & NR \\
\hline & & & & & & & $\{\quad 48.40\}$ & & \\
\hline CONDFLD & «MНО & 1 & & $02 / 09 / 88$ & 385 & $03 / 06 / 88$ & 1295 & $03 / 23 / 88$ & 316 \\
\hline & & & & $06 / 07 / 88$ & 386 & $06 / 06 / 88$ & 1588 & $06 / 06 / 88$ & 304 \\
\hline CYANIDE & $P P B$ & 10 & & $02 / 09 / 88$ & & $03 / 06 / 88$ & 422 & $03 / 23 / 88$ & \\
\hline & & & & $06 / 07 / 88$ & 11 & $03 / 06 / 88$ & 827 & $06 / 06 / 88$ & \\
\hline & & & & & NR & $03 / 06 / 83$ & 489 & & NR \\
\hline & & & & & NR & $06 / 06 / 88$ & 1690 & & NR \\
\hline$\pi$ & & & & & NR & $06 / 06 / 88$ & 1300 & & NR \\
\hline 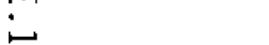 & & & & & NR & $06 / 06 / 88$ & 1360 & & NR \\
\hline & & & & $06 / 07 / 88$ & 29 & $03 / 06 / 88$ & 73 & $06 / 06 / 88$ & 30 \\
\hline & & & & & $\mathrm{NR}$ & $03 / 06 / 88$ & 77 & & NR \\
\hline & & & & & NR & $06 / 06 / 88$ & 76 & & $N R$ \\
\hline & & & & & NR & $06 / 06 / 88$ & 74 & & $N R$ \\
\hline & & & & & NR & $06 / 06 / 88$ & 74 & & NR \\
\hline FCALCIU & $\mathrm{PPB}$ & 50 & & $02 / 09 / 88$ & 44300 & $03 / 06 / 88$ & 222000 & $03 / 23 / 88$ & 39600 \\
\hline & & & & $06 / 07 / 88$ & 43500 & $03 / 06 / 88$ & 244000 & $06 / 06 / 88$ & 38400 \\
\hline & & & & & $\mathrm{NR}$ & $03 / 06 / 88$ & 238000 & & NR \\
\hline & & & & & $\mathrm{NR}$ & $06 / 06 / 88$ & 244000 & & NR \\
\hline & & & & & $\mathrm{NR}$ & $06 / 06 / 88$ & 242000 & & NR \\
\hline & & & & & NR & $06 / 06 / 88$ & 245000 & & NR \\
\hline F1RON & PPB & 50 & & $02 / 09 / 88$ & & $03 / 06 / 88$ & 265 & $03 / 23 / 88$ & \\
\hline & & & & $06 / 07 / 88$ & & $03 / 06 / 88$ & 276 & $06 / 06 / 88$ & \\
\hline & & & & & NR & $03 / 06 / 88$ & 286 & & NR \\
\hline & & & & & $N R$ & $06 / 06 / 88$ & 318 & & NR \\
\hline & & & & & $N R$ & $06 / 06 / 88$ & 305 & & NR \\
\hline & & & & & NR & $06 / 06 / 88$ & 307 & & NR \\
\hline
\end{tabular}


IABLE E. 3. (contd)

\begin{tabular}{|c|c|c|c|c|c|c|c|c|c|}
\hline \multicolumn{2}{|c|}{ CONSTIIUENT } & \multirow{2}{*}{$\begin{array}{l}\text { CONTRACTUAL } \\
\text { DETECTION } \\
\text { S.IMIT }\end{array}$} & \multirow{2}{*}{$\begin{array}{l}\text { DRINKING } \\
\text { WATER } \\
\text { STANDARO }\end{array}$} & \multirow{2}{*}{$\begin{array}{c}\text { SAMPLE } \\
\text { DATE }\end{array}$} & \multirow[b]{2}{*}{$6-49-79$} & \multirow{2}{*}{$\begin{array}{l}\text { SAMPLE } \\
\text { DATEE }\end{array}$} & \multirow[b]{2}{*}{$6-50-53$} & \multirow{2}{*}{$\begin{array}{l}\text { SAMPLE } \\
\text { DATE }\end{array}$} & \multirow[b]{2}{*}{$6-50-85$} \\
\hline NAME & UNITS & & & & & & & & \\
\hline \multirow[t]{5}{*}{ FLUORID } & PPB & 500 & 4000 & $02 / 09 / 88$ & & $03 / 06 / 88$ & 567 & $03 / 23 / 88$ & \\
\hline & & & & $06 / 07 / 88$ & & $03 / 06 / 88$ & 564 & $06 / 06 / 88$ & \\
\hline & & & & & NR & $\begin{array}{l}03 / 06 / 88 \\
06 / 06 / 88\end{array}$ & & & NR \\
\hline & & & & & $\begin{array}{l}\text { NR } \\
\text { NR }\end{array}$ & $\begin{array}{l}06 / 06 / 88 \\
06 / 06 / 88\end{array}$ & $\begin{array}{l}1020 \\
1000\end{array}$ & & $\begin{array}{l}\text { NR } \\
\text { NR }\end{array}$ \\
\hline & & & & & NR & $06 / 08 / 88$ & 1000 & & NR \\
\hline \multirow[t]{5}{*}{ FMAGNES } & POB & 50 & & $02 / 09 / 88$ & 14300 & $03 / 06 / 88$ & 63000 & $03 / 23 / 88$ & 13400 \\
\hline & & & & $06 / 07 / 88$ & 14000 & $03 / 06 / 88$ & 70300 & $06 / 06 / 88$ & 13100 \\
\hline & & & & & NR & $03 / 06 / 88$ & 68600 & & NR \\
\hline & & & & & NR & $06 / 06 / 88$ & 69000 & & NR \\
\hline & & & & & NR & $06 / 08 / 89$ & 67400 & & NR \\
\hline & & & & & NR & $06 / 06 / 88$ & 68500 & & NR \\
\hline \multirow[t]{4}{*}{ FPOTASS } & PPB & 100 & & $02 / 09 / 88$ & 3700 & $03 / 06 / 88$ & 14100 & $03 / 23 / 88$ & 3990 \\
\hline & & & & $06 / 07 / 88$ & 3480 & $03 / 06 / 88$ & 15400 & $06 / 06 / 88$ & 3690 \\
\hline & & & & & NR & $03 / 06 / 88$ & 15200 & & NR \\
\hline & & & & & NR & $06 / 06 / 88$ & 14300 & & NR \\
\hline
\end{tabular}


TABLE_E.3. (contd)

\begin{tabular}{|c|c|c|c|c|c|c|c|c|c|}
\hline CONST: & FUENT & $\begin{array}{l}\text { CONTRACTUAL } \\
\text { DETECTION }\end{array}$ & $\begin{array}{l}\text { DRINKING } \\
\text { WATER }\end{array}$ & SAMPLE & & SAMPLE & & SAMPLE & \\
\hline NAME & UNITS & & STANDARD & DATE & $6.49-79$ & DATE & $6-50-53$ & DATE & $6-50-85$ \\
\hline FPOTASS & PPB & 100 & & & NR & $06 / 06 / 88$ & 13500 & & NR \\
\hline & & & & & NR & $06 / 06 / 88$ & 14000 & & NR \\
\hline FSELEN I & PPB & 5 & 10 & $02 / 09 / 88$ & & $03 / 06 / 88$ & 19 & $03 / 23 / 88$ & \\
\hline & & & & $06 / 07 / 88$ & & $03 / 06 / 88$ & 19 & $06 / 06 / 88$ & \\
\hline & & & & & NR & $03 / 06 / 88$ & 19 & * & NR \\
\hline & & & & & NR & $06 / 06 / 88$ & 27 & * & NR \\
\hline & & & & & NR & $06 / 06 / 88$ & 23 & * & NR \\
\hline & & & & & NR & $06 / 06 / 88$ & 25 & * & NR \\
\hline FSODIUM & $P P B$ & 200 & & $02 / 09 / 88$ & 8550 & $03 / 06 / 88$ & 60200 & $03 / 23 / 88$ & 10300 \\
\hline & & & & $06 / 07 / 88$ & 8690 & $03 / 06 / 88$ & 65900 & $06 / 06 / 88$ & 10300 \\
\hline & & & & & NR & $03 / 06 / 88$ & 65100 & & NR \\
\hline & & & & & NR & $06 / 06 / 88$ & 62800 & & NR \\
\hline$m$ & & & & & NR & $06 / 06 / 88$ & 59000 & & NR \\
\hline & & & & & NR & $06 / 06 / 88$ & 61300 & & NR \\
\hline FSTRONT & PPB & 10 & & $02 / 09 / 88$ & 175 & $03 / 06 / 88$ & 960 & $03 / 23 / 88$ & 142 \\
\hline & & & & $06 / 07 / 88$ & 168 & $03 / 06 / 88$ & 995 & $06 / 06 / 88$ & 135 \\
\hline & & & & & NR & $03 / 06 / 88$ & 1050 & & NR \\
\hline & & & & & NR & $06 / 06 / 88$ & 971 & & NR \\
\hline & & & & & NR & $06 / 06 / 88$ & 944 & & NR \\
\hline & & & & & NR & $06 / 06 / 88$ & 961 & & $\mathrm{NR}$ \\
\hline FVANAD I & PPB & 5 & & $02 / 09 / 88$ & 27 & $03 / 06 / 88$ & 5 & $03 / 23 / 88$ & 24 \\
\hline & & & & $06 / 07 / 88$ & 26 & $03 / 06 / 88$ & 5 & $06 / 06 / 88$ & 27 \\
\hline & & & & & NR & $03 / 06 / 88$ & 7 & & NR \\
\hline & & & & & NR & $06 / 06 / 88$ & & & $\mathrm{NR}$ \\
\hline & & & & & NR & $06 / 06 / 88$ & & & NR \\
\hline & & & & & NR & $06 / 06 / 88$ & & & NR \\
\hline FZINC & $P P B$ & 5 & & $02 / 09 / 88$ & 6 & $03 / 06 / 88$ & 6 & $03 / 23 / 88$ & \\
\hline & & & & $06 / 07 / 8 B$ & 27 & $03 / 06 / 88$ & 7 & $06 / 06 / 88$ & \\
\hline & & & & & NR & $03 / 06 / 88$ & 7 & & NR \\
\hline & & & & & NR & $06 / 06 / 88$ & 8 & & NR \\
\hline & & & & & NR & $06 / 06 / 88$ & 7 & & NR \\
\hline & & & & & NR & $06 / 06 / 88$ & 7 & & NR \\
\hline
\end{tabular}


TABLEE.3. (contd)

\begin{tabular}{|c|c|c|c|c|c|c|c|c|c|c|}
\hline & CONST I TUENT & $\begin{array}{l}\text { CONTRACTUAL } \\
\text { DETECTION }\end{array}$ & $\begin{array}{l}\text { DRINKING } \\
\text { WATER }\end{array}$ & SAMPLE & & SAMPLE & & & SAMPLE & \\
\hline - . . . & .... & - & - & $\cdots \cdots$ & ....... & $\ldots \ldots$ & \multicolumn{2}{|c|}{ n....... } & - & - . . . . \\
\hline HNITRAT & & & & $06 / 07 / 88$ & 38400 & $04 / 15 / 88$ & 506000 & * & $06 / 06 / 88$ & 22800 \\
\hline \multirow[t]{2}{*}{ I - $1290 \mathrm{H}$} & PCI/L & 1 & & & NR & $04 / 15 / 88$ & \multicolumn{2}{|c|}{0.27} & & $\mathrm{NR}$ \\
\hline & & & & & & & \multicolumn{2}{|c|}{0.21} & & \\
\hline \multirow{4}{*}{ LFLUORO } & & & & & NR & $03 / 06 / 88$ & \multicolumn{2}{|l|}{257} & & NR \\
\hline & & & & & $N R$ & $06 / 06 / 88$ & \multicolumn{2}{|l|}{273} & & NR \\
\hline & & & & & $\mathrm{NR}$ & $06 / 06 / 88$ & \multicolumn{2}{|l|}{278} & & NR \\
\hline & & & & & NR & $06 / 06 / 88$ & \multicolumn{2}{|l|}{279} & & NR \\
\hline \multirow[t]{5}{*}{ NITRATE } & PPB & 500 & 45000 & $02 / 09 / 88$ & 38100 & $03 / 06 / 83$ & \multirow{2}{*}{\multicolumn{2}{|c|}{$\begin{array}{l}538000 \\
533000\end{array}$}} & $03 / 23 / 88$ & 24900 \\
\hline & & & & $06 / 07 / 88$ & 40100 & $03 / 06 / 88$ & & & $06 / 06 / 88$ & 24600 \\
\hline & & & & & NR & $03 / 06 / 88$ & \multicolumn{2}{|l|}{509000} & & NR \\
\hline & & & & & NR & $06 / 06 / 88$ & \multicolumn{2}{|l|}{557000} & & NR \\
\hline & & & & & NR & $06 / 06 / 88$ & 554000 & * & & NR \\
\hline \multicolumn{2}{|l|}{$P H-\angle A B$} & \multicolumn{2}{|l|}{0.01} & $02 / 09 / 88$ & 7.75 & $03 / 06 / 88$ & \multicolumn{2}{|c|}{7.73} & $03 / 23 / 88$ & 8.10 \\
\hline
\end{tabular}


TABLE E. 3 . (contd)

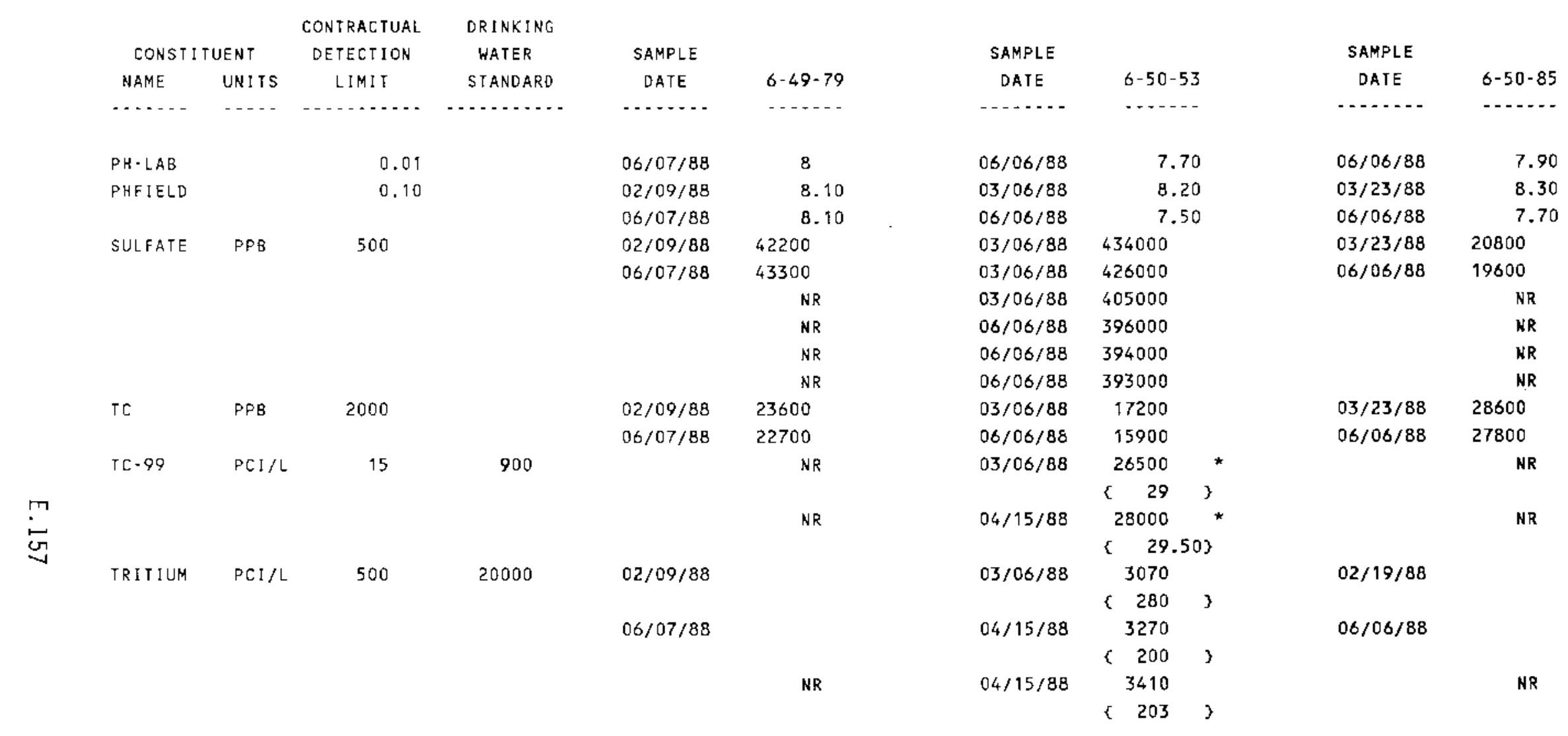


TABLE E.3. (contd)

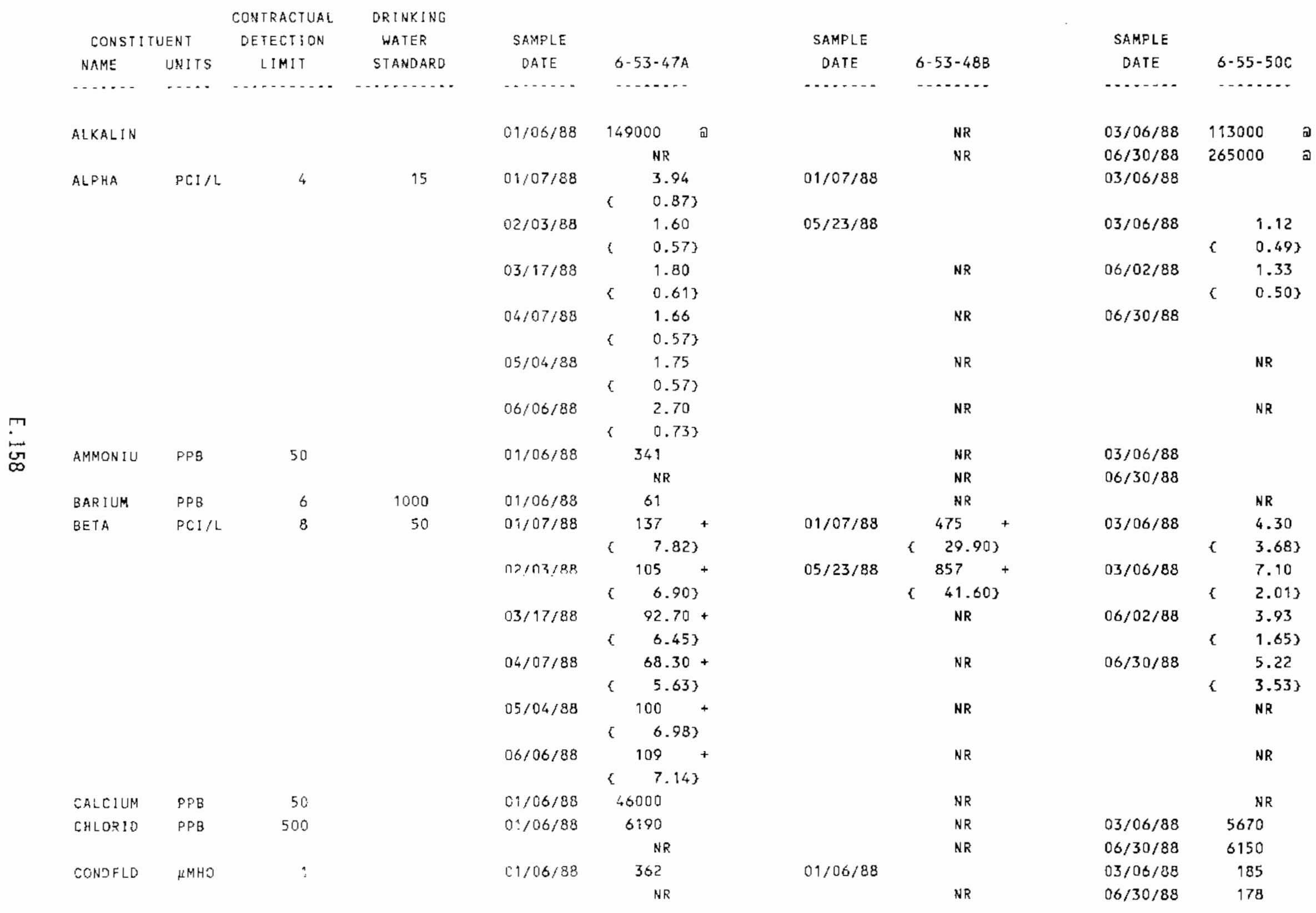


IABLE E.3. (contd)

\begin{tabular}{|c|c|c|c|c|c|c|c|c|c|}
\hline \multicolumn{2}{|c|}{ CONSTITUENT } & $\begin{array}{l}\text { CONTRACTUAL } \\
\text { DETECTION }\end{array}$ & $\begin{array}{l}\text { DRINKING } \\
\text { WATER }\end{array}$ & SAMPLE & & SAMPLE & & SAMPLE & \\
\hline NAME & UNITS & LIMIT & STANDARD & DATE & $6-53-47 A$ & DATE & $6-53-48 B$ & DATE & $6.55 .50 \mathrm{C}$ \\
\hline ….... & $\cdots$ & 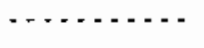 & (n........... & $\cdots$ & $\ldots \ldots$ & -........ & ........... & - & $\ldots+\ldots$ \\
\hline CS. 137 & $\mathrm{PCI} / \mathrm{L}$ & 20 & 200 & $01 / 07 / 88$ & & $01 / 07 / 88$ & & $03 / 06 / 88$ & \\
\hline & & & & $02 / 03 / 88$ & & $05 / 23 / 88$ & & $06 / 02 / 88$ & \\
\hline & & & & $03 / 17 / 88$ & & & NR & & NR \\
\hline & & & & $04 / 07 / 88$ & 4.89 & & NR & & NR \\
\hline & & & & & $4.63\}$ & & & & \\
\hline & & & & $05 / 04 / 88$ & & & NR & & NR \\
\hline & & & & $06 / 06 / 88$ & & & NR & & NR \\
\hline FBARIUM & PPB & 6 & 1000 & $01 / 06 / 88$ & 63 & & NR & $03 / 06 / 88$ & 12 \\
\hline & & & & & NR & & NR & $06 / 30 / 88$ & 12 \\
\hline FCALCIU & PPB & 50 & & $01 / 06 / 88$ & 49000 & & NR & $03 / 06 / 88$ & 32400 \\
\hline & & & & & NR & & NR & $06 / 30 / 88$ & 33400 \\
\hline FMAGNES & PPB & 50 & & $01 / 06 / 88$ & 14100 & & NR & $03 / 06 / 88$ & 9720 \\
\hline & & & & & NR & & NR & $06 / 30 / 88$ & 9620 \\
\hline EMANGAN & $\mathrm{FPB}$ & 5 & & $01 / 08 / 88$ & 77 & & NR & $03 / 06 / 88$ & \\
\hline
\end{tabular}


TABLE E. 3. (contd)

\begin{tabular}{|c|c|c|c|c|c|c|c|c|c|}
\hline & & CONTRACTUAL & $\begin{array}{l}\text { DRINKING } \\
\text { WATER }\end{array}$ & & & & & & \\
\hline const 1 & LENT & DETECTION & WATER & SAMPLE & & SAMPLE & & SAMPLE & \\
\hline NAME & UNITS & LIMIT & STANDARD & DATE & $6-53-47 A$ & DATE & $6-53-48 B$ & DATE & $6-55-50 \mathrm{C}$ \\
\hline - . . . & $\cdots$ & $\ldots \ldots$ & $\ldots$ & $\cdots \cdots$ & $-\ldots$ & $\cdots$ & $-\cdots$ & $\cdots$ & $\cdots-\cdots$ \\
\hline FMANGAN & $\mathrm{PPB}$ & 5 & & & $M R$ & & NR & $06 / 30 / 88$ & \\
\hline FPOTASS & PPB & 100 & & $01 / 06 / 88$ & 4360 & & NR & $03 / 06 / 88$ & 4580 \\
\hline & & & & & NR & & NR & $06 / 30 / 88$ & 4230 \\
\hline F SOOIUM & PPB & 200 & & $01 / 06 / 88$ & 7100 & & NR & $03 / 06 / 88$ & 5650 \\
\hline & & & & & NR & & NR & $06 / 30 / 88$ & 4920 \\
\hline FSTRONT & $\mathrm{PPB}$ & 10 & & $01 / 06 / 88$ & 291 & & $\mathrm{NR}$ & $03 / 06 / 88$ & 146 \\
\hline & & & & & NR & & NR & $06 / 30 / 88$ & 138 \\
\hline FVANADI & PPB & 5 & & $01 / 06 / 88$ & & & NR & $03 / 06 / 88$ & 6 \\
\hline & & & & & NR & & NR & $06 / 30 / 88$ & 9 \\
\hline FZINC & PPB & 5 & & $01 / 06 / 88$ & 12 & & NR & $03 / 06 / 88$ & \\
\hline & & & & & NR & & NR & $06 / 30 / 88$ & \\
\hline IRON & $\mathrm{PPB}$ & 50 & & $01 / 06 / 88$ & 553 & & $N R$ & & NR \\
\hline LFLUORD & $P P B$ & 50 & 4000 & & NR & & NR & $03 / 06 / 88$ & 184 \\
\hline i- & & & & & NR & & $\mathrm{NR}$ & $06 / 30 / 88$ & 187 \\
\hline MAGNES & PPB & 0 & & $01 / 06 / 88$ & 13600 & & $N R$ & & NR \\
\hline MANGESE & $\mathrm{PPB}$ & 5 & & $01 / 06 / 88$ & 60 & & NR & & NR \\
\hline NITRAIE & PPB & 500 & 45000 & $01 / 06 / 88$ & 5690 & & NR & $03 / 06 / 88$ & 1540 \\
\hline & & & & & NR & & NR & $06 / 30 / 88$ & 1530 \\
\hline$P H-L A B$ & & 0.01 & & $01 / 06 / 88$ & 7.59 & & NR & $03 / 06 / 88$ & 8.16 \\
\hline & & & & & NR & & NR & $06 / 30 / 88$ & 8.20 \\
\hline PHFIELD & & 0.10 & & $01 / 06 / 88$ & 7.20 & $01 / 06 / 88$ & & $03 / 06 / 88$ & 8.80 \\
\hline & & & & & $N R$ & & NR & $06 / 30 / 8 B$ & 7.50 \\
\hline POTASUM & PPB & 100 & & $01 / 06 / 88$ & 4500 & & NR & & NR \\
\hline$R U-106$ & $P C I / L$ & 172.50 & 30 & $01 / 07 / 88$ & & $01 / 07 / 88$ & & $03 / 06 / 88$ & \\
\hline & & & & $02 / 03 / 88$ & & $05 / 23 / 88$ & & $06 / 02 / 88$ & $50.70 *$ \\
\hline & & & & & & & & & 43.603 \\
\hline & & & & $03 / 17 / 88$ & & & $\mathrm{NR}$ & & NR \\
\hline & & & & $04 / 07 / 88$ & & & NR & & NR \\
\hline & & & & $05 / 04 / 88$ & & & NR & & $\mathrm{NR}$ \\
\hline & & & & $06 / 06 / 83$ & & & NR & & NR \\
\hline SODILM & $\mathrm{PPB}$ & 200 & & $01 / 06 / 88$ & 7040 & & NR & & NR \\
\hline
\end{tabular}


IABLE E. 3. (contd)

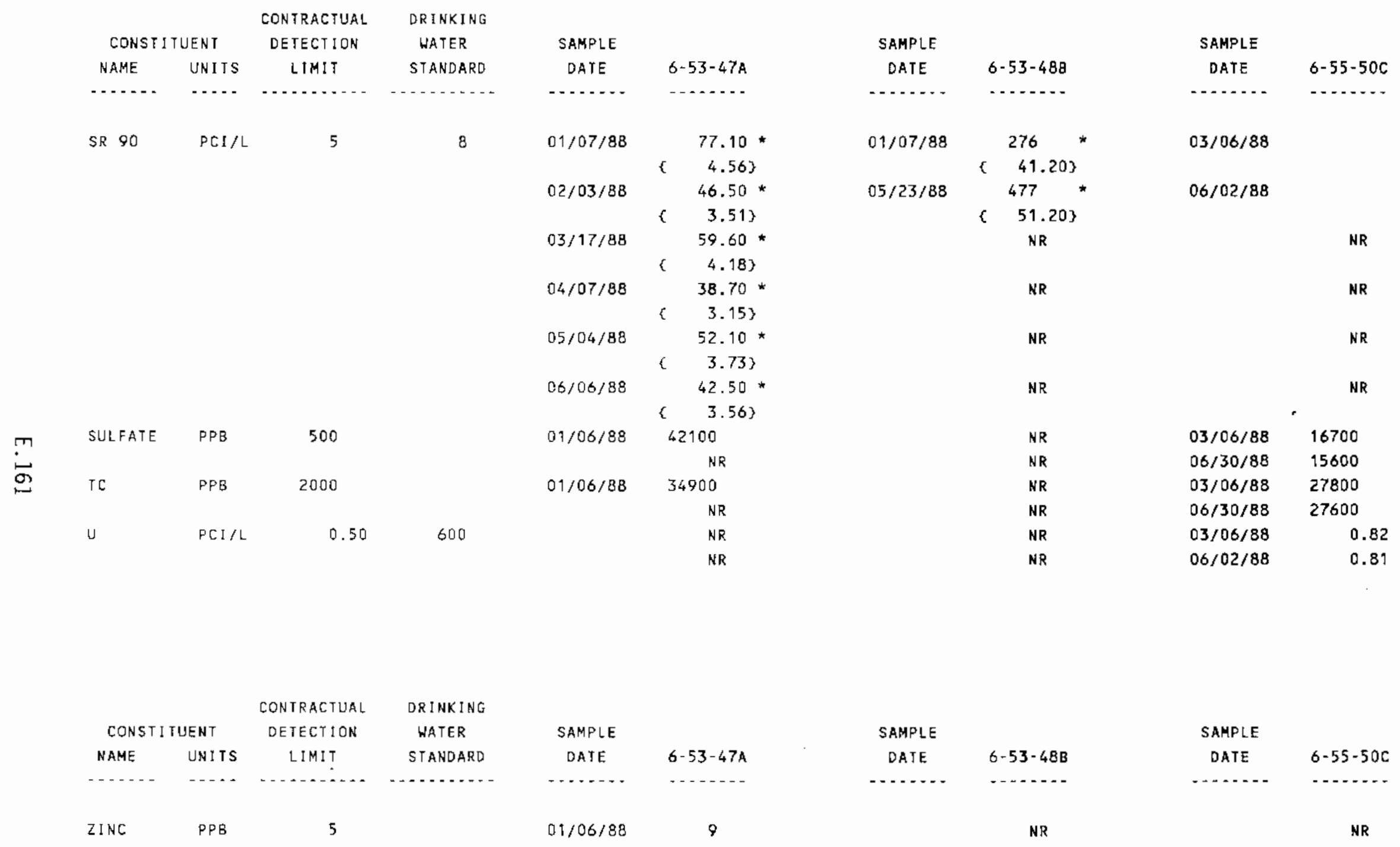


TABLE E.3. (contd)

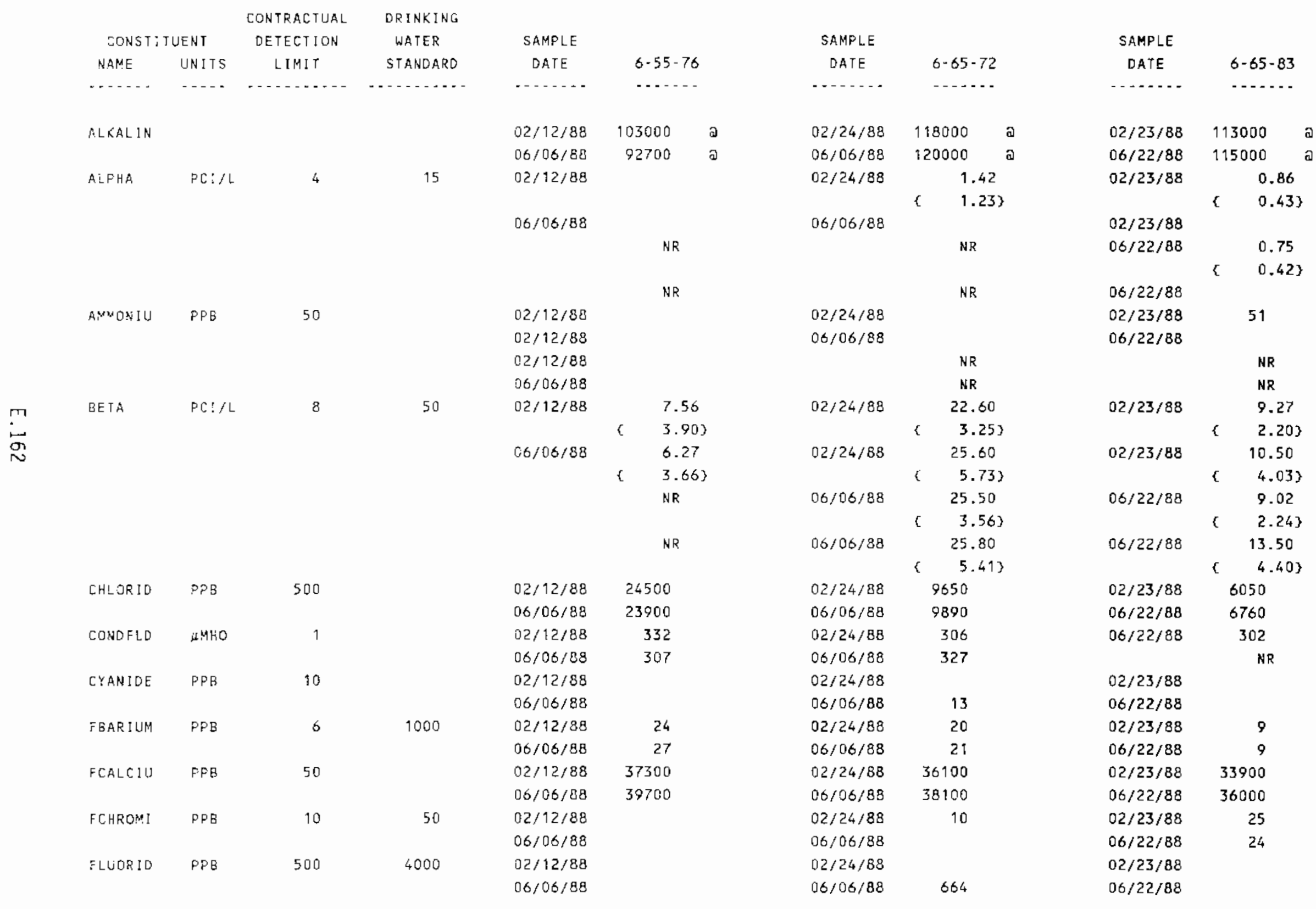


TABLEE. E. 3. (contd)

\begin{tabular}{|c|c|c|c|c|c|c|c|c|c|}
\hline \multicolumn{2}{|c|}{ CONST 1 TUENT } & $\begin{array}{l}\text { CONTRACTUAL } \\
\text { DETECTION }\end{array}$ & $\begin{array}{l}\text { DRINKING } \\
\text { WATER }\end{array}$ & SAMPLE & & SAMPLE & & SAMPLE & \\
\hline \multirow[t]{2}{*}{ NAME } & UNITS & LIMIT & STANDARD & DATE & $6-55-76$ & DATE & $6-65-72$ & DATE & $6-65-83$ \\
\hline & $\ldots$ & - & $--\ldots \ldots \ldots$ & - & $\cdots \cdots$ & $\cdots$ & - . . . . & .......... & ….... \\
\hline \multirow[t]{2}{*}{ FMAGNES } & $P P B$ & 50 & & $02 / 12 / 88$ & 15000 & $02 / 24 / 88$ & 11000 & $02 / 23 / 88$ & 10300 \\
\hline & & & & $06 / 06 / 88$ & 16400 & $06 / 06 / 88$ & 11700 & $06 / 22 / 88$ & 10900 \\
\hline \multirow[t]{2}{*}{ FMANGAN } & PPB & 5 & & $02 / 12 / 88$ & 20 & $02 / 24 / 88$ & & $02 / 23 / 88$ & \\
\hline & & & & $06 / 06 / 88$ & 30 & $06 / 06 / 88$ & & $06 / 22 / 88$ & \\
\hline \multirow[t]{2}{*}{ FPOTASS } & PPB & 100 & & $02 / 12 / 88$ & 4500 & $02 / 24 / 88$ & 6070 & $02 / 23 / 88$ & 7140 \\
\hline & & & & $06 / 06 / 88$ & 4670 & $06 / 06 / 88$ & 6440 & $06 / 22 / 88$ & 7880 \\
\hline \multirow[t]{2}{*}{ FSODIUM } & $P P B$ & 200 & & $02 / 12 / 88$ & 9270 & $02 / 24 / 88$ & 23300 & $02 / 23 / 88$ & 15100 \\
\hline & & & & $06 / 06 / 88$ & 10100 & $06 / 06 / 88$ & 26000 & $06 / 22 / 88$ & 16400 \\
\hline \multirow[t]{2}{*}{ FSTRONT } & PPB & 10 & & $02 / 12 / 88$ & 187 & $02 / 24 / 88$ & 168 & $02 / 23 / 88$ & 138 \\
\hline & & & & $06 / 06 / 88$ & 192 & $06 / 06 / 88$ & 177 & $06 / 22 / 88$ & 146 \\
\hline \multirow[t]{2}{*}{ FVANAD 1} & PPB & 5 & & $02 / 12 / 88$ & 6 & $02 / 24 / 83$ & 20 & $02 / 23 / 88$ & 29 \\
\hline & & & & $06 / 06 / 88$ & 7 & $06 / 06 / 88$ & 18 & $06 / 22 / 88$ & 29 \\
\hline \multirow[t]{2}{*}{ FZINC } & PPB & 5 & & $02 / 12 / 88$ & & $02 / 24 / 88$ & & $02 / 23 / 88$ & \\
\hline & & & & $06 / 06 / 88$ & & $06 / 06 / 88$ & & $06 / 22 / 88$ & 6 \\
\hline
\end{tabular}


TABLE.E.3. (contd)

\begin{tabular}{|c|c|c|c|c|c|c|c|c|c|}
\hline & CONST ITUENT & $\begin{array}{l}\text { CONTRACTUAL } \\
\text { DETECTION }\end{array}$ & $\begin{array}{l}\text { DRINKING } \\
\text { HATER }\end{array}$ & SAMPLE & & SAMPLE & & SAMPLE & \\
\hline NAME: & UNITS & LIMIT & STANDARD & DATE & $6.55-76$ & DATE & $6-65-72$ & DATE & $6-65-83$ \\
\hline$\ldots \ldots$ & $\cdots$ & $\ldots$ & $\ldots$ & $\cdots$ & …… & - . . . . & …... & ........ & $\cdots$ \\
\hline HNITRAT & PPB & 2500 & 45000 & $02 / 12 / 88$ & 6240 & $02 / 24 / 88$ & 19800 & $02 / 23 / 88$ & 4200 \\
\hline & & & & $06 / 06 / 88$ & 3360 & $06 / 06 / 88$ & 20400 & $06 / 22 / 88$ & $5 \uparrow 10$ \\
\hline LFLUORD & $\mathrm{PPB}$ & 50 & 4000 & $02 / 12 / 88$ & 184 & $02 / 24 / 8 B$ & 577 & $02 / 23 / 88$ & 339 \\
\hline & & & & $06 / 06 / 88$ & 218 & $06 / 06 / 88$ & 637 & $06 / 22 / 88$ & 353 \\
\hline NITRATE & $P P B$ & 500 & 45000 & $02 / 12 / 8 B$ & 5980 & $02 / 24 / 88$ & 24400 & $02 / 23 / 88$ & 4910 \\
\hline & & & & $06 / 06 / 88$ & 4370 & $06 / 06 / 88$ & 21300 & $06 / 22 / 88$ & 3890 \\
\hline$P H-L A B$ & & 0.01 & & $02 / 12 / 88$ & 7.94 & $02 / 24 / 88$ & 7.62 & $02 / 23 / 88$ & 7.75 \\
\hline & & & & $06 / 06 / 88$ & 7.90 & $06 / 06 / 88$ & 7.70 & $06 / 22 / 88$ & 7.90 \\
\hline PRFIELD & & 0.10 & & $02 / 12 / 88$ & 7.50 & $02 / 24 / 88$ & 8.20 & $06 / 22 / 88$ & 7.60 \\
\hline & & & & $06 / 06 / 88$ & 7.70 & $06 / 06 / 88$ & 7.50 & & NR \\
\hline SULFATE & PPB & 500 & & $02 / 12 / 88$ & 47300 & $02 / 24 / 88$ & 42200 & $02 / 23 / 88$ & 40100 \\
\hline & & & & $06 / 06 / 88$ & 43000 & $06 / 06 / 88$ & 38800 & $06 / 22 / 88$ & 39200 \\
\hline TC & PPE & 2000 & & $02 / 12 / 88$ & 23600 & $02 / 24 / 88$ & 28500 & $02 / 23 / 88$ & 27100 \\
\hline & & & & $06 / 06 / 88$ & 21600 & $06 / 06 / 88$ & 28200 & $06 / 22 / 88$ & 27900 \\
\hline FRITIUM & $P C I / L$ & 500 & 20000 & & NR & $02 / 24 / 88$ & 3090 & $02 / 23 / 88$ & 1000 \\
\hline & & & & & & & f 286 & & < 228 \\
\hline & & & & & NR & $06 / 06 / 88$ & 3230 & $06 / 22 / 88$ & 943 \\
\hline & & & & & & & $\{293\}$ & & $6 \quad 221$ \\
\hline$v$ & $P C I / L$ & 0.50 & 600 & & NR & $02 / 24 / 8 B$ & 1.71 & & NR \\
\hline & & & & & NR & $06 / 06 / 88$ & 1.62 & & NR \\
\hline
\end{tabular}


TABLE E.3. (contd)

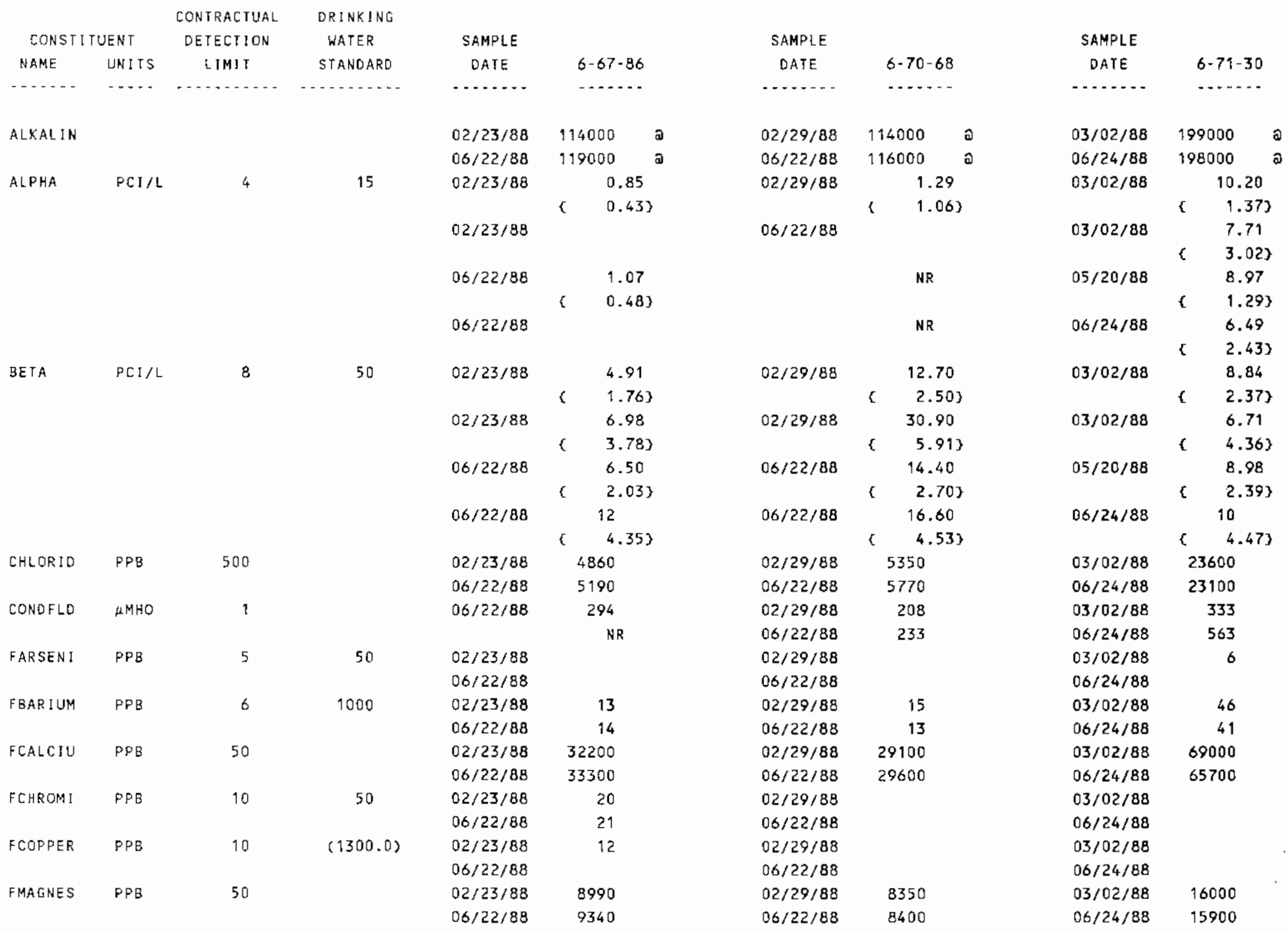


TABLEE.3. (contd)

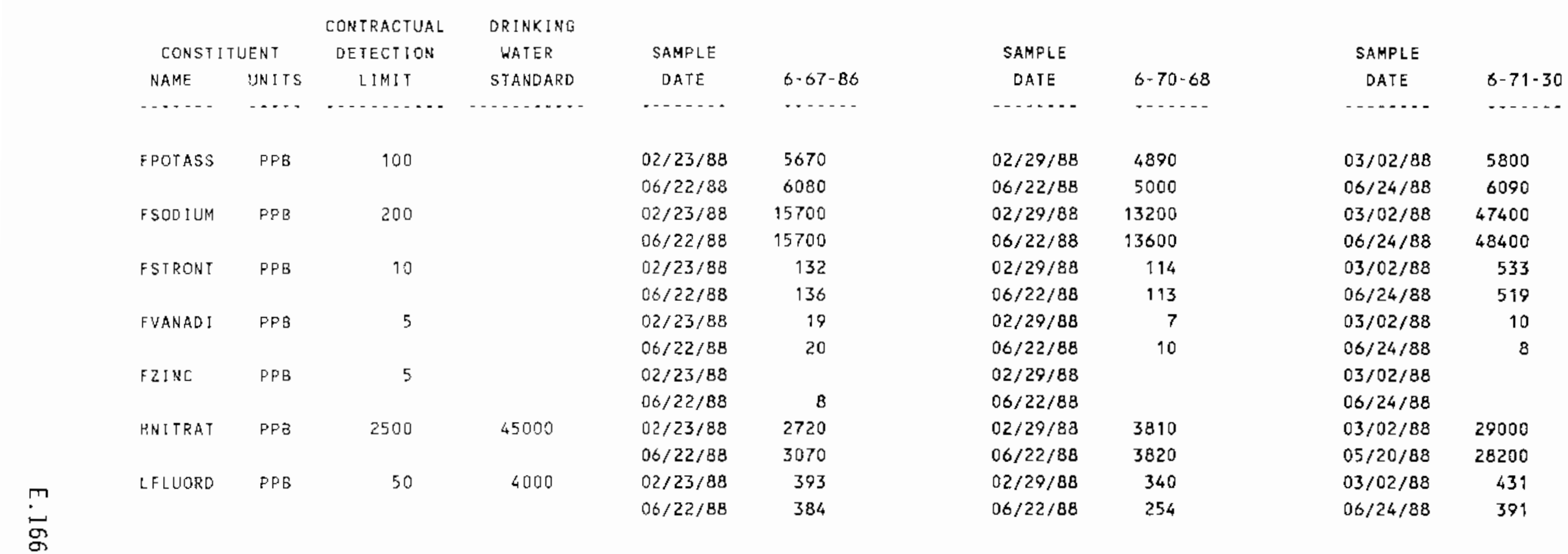


IABLE E.3. (contd)

\begin{tabular}{|c|c|c|c|c|c|c|c|c|c|}
\hline & CONSTI TUENT & $\begin{array}{l}\text { CONTRACIUAL } \\
\text { DETECTION }\end{array}$ & $\begin{array}{l}\text { DRINKING } \\
\text { WATER }\end{array}$ & SAMPLE & & \multirow{2}{*}{$\begin{array}{c}\text { SAMPLE } \\
\text { DATE }\end{array}$} & \multicolumn{3}{|c|}{ SAMPLE } \\
\hline NAME & UNITS & LIMIT & STANDARD & DATE & $6-67.86$ & & $6-70-68$ & DATE & $6-79-30$ \\
\hline$\ldots$ & .... & . & n.t.n. & $\ldots$ & $\ldots$ & -....... & - n..... & - n. & $\ldots . .$. \\
\hline NI TRATE & PPB & 500 & 45000 & $02 / 23 / 88$ & 3000 & $02 / 29 / 88$ & 3780 & $03 / 02 / 88$ & 29800 \\
\hline & & & & $06 / 22 / 88$ & 3000 & $06 / 22 / 88$ & 3720 & $06 / 24 / 88$ & 29700 \\
\hline$P H-L A B$ & & 0.01 & & $02 / 23 / 88$ & 7.49 & $02 / 29 / 88$ & 7.66 & $03 / 02 / 88$ & 7.61 \\
\hline & & & & $06 / 22 / 88$ & 7.80 & $06 / 22 / 88$ & 7.90 & $06 / 24 / 88$ & 7.90 \\
\hline PHF IELD & & 0.10 & & $06 / 22 / 88$ & 7.50 & $02 / 29 / 88$ & 8.60 & $03 / 02 / 88$ & 7.90 \\
\hline & & & & & NR & $06 / 22 / 88$ & 7.50 & $06 / 24 / 88$ & 7.50 \\
\hline SULFATE & PPB & 500 & & $02 / 23 / 88$ & 37800 & $02 / 29 / 88$ & 19500 & $03 / 02 / 88$ & 82100 \\
\hline & & & & $06 / 22 / 88$ & 37300 & $06 / 22 / 88$ & 17900 & $06 / 24 / 88$ & 75000 \\
\hline IC & PPB & 2000 & & $02 / 23 / 88$ & 27900 & $02 / 29 / 88$ & 28700 & $03 / 02 / 88$ & 50600 \\
\hline & & & & $06 / 22 / 88$ & 28500 & $06 / 22 / 38$ & 28100 & $06 / 24 / 88$ & 49700 \\
\hline 10.99 & $\mathrm{PCI} / \mathrm{L}$ & 15 & 900 & & NR & $02 / 29 / 83$ & 59 & & NR \\
\hline & & & & & & & 1.793 & & \\
\hline IRITIUM & $P C I / L$ & 500 & 20000 & $02 / 23 / 88$ & 872 & $02 / 29 / 88$ & 1850 & $03 / 02 / 88$ & \\
\hline d & & & & & c 225 & 3 & $(256)$ & & \\
\hline & & & & $06 / 22 / 88$ & 1110 & $06 / 22 / 88$ & 1790 & $05 / 20 / 88$ & \\
\hline & & & & & c 225 & 3 & ( 245 & & \\
\hline$u$ & $\mathrm{PCI} / \mathrm{L}$ & 0.50 & 600 & & NR & $02 / 29 / 88$ & 1.26 & & NR \\
\hline & & & & & NR & $06 / 22 / 88$ & 0.63 & & NR \\
\hline
\end{tabular}


TABLE E.3. (contd)

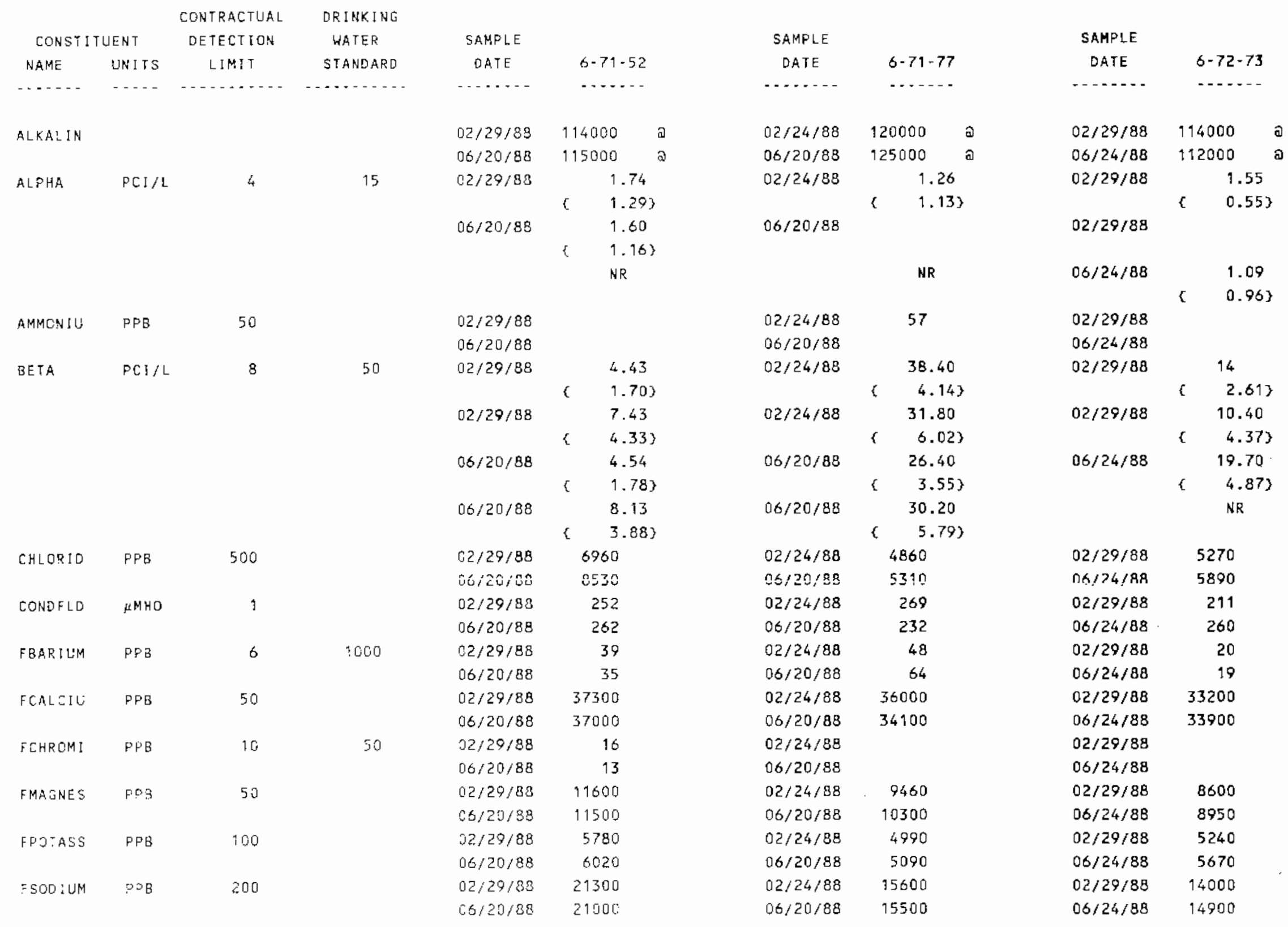


TABLE E.3. (contd)

\begin{tabular}{|c|c|c|c|}
\hline \multirow{2}{*}{\multicolumn{2}{|c|}{ CONST I TUENT }} & CONTRACTUAL & DRINKING \\
\hline & & DETECTION & WATER \\
\hline NAME & UNITS & LIMIT & STANDARD \\
\hline$\ldots$ & $\ldots$ & - & - . . . . . . . \\
\hline
\end{tabular}

\begin{tabular}{|c|c|c|c|c|c|}
\hline \multicolumn{2}{|c|}{ CONST I TUENT } & DETECTION & WATER & \multicolumn{2}{|l|}{ SAMPLE } \\
\hline NAME & UNITS & LIMIT & STANDARD & DATE & $6-71-52$ \\
\hline - - - - - & $\cdots-$ & - & $-\ldots$ & $\cdots$ & $\ldots$ \\
\hline \multirow[t]{2}{*}{ FSTRONT } & PPB & 10 & & $02 / 29 / 88$ & 227 \\
\hline & & & & $06 / 20 / 88$ & 234 \\
\hline \multirow[t]{2}{*}{ FVANADI } & PPB & 5 & & $02 / 29 / 88$ & 17 \\
\hline & & & & $06 / 20 / 88$ & 98 \\
\hline \multirow[t]{2}{*}{ FZINC } & PPB & 5 & & $02 / 29 / 88$ & \\
\hline & & & & $06 / 20 / 88$ & \\
\hline \multirow[t]{2}{*}{ HN I TRAT } & $P P B$ & 2500 & 45000 & $02 / 29 / 88$ & 7160 \\
\hline & & & & $06 / 20 / 88$ & 7120 \\
\hline \multirow[t]{2}{*}{ LFLUORD } & PPB & 50 & 4000 & $02 / 29 / 88$ & 382 \\
\hline & & & & $06 / 20 / 88$ & 373 \\
\hline \multirow[t]{2}{*}{ NITRATE } & $P P B$ & 500 & 45000 & $02 / 29 / 88$ & 7720 \\
\hline & & & & $06 / 20 / 88$ & 7260 \\
\hline \multirow[t]{2}{*}{$P H-L A B$} & \multirow{2}{*}{\multicolumn{3}{|c|}{0.01}} & $02 / 29 / 88$ & 7.75 \\
\hline & & & & $06 / 20 / 88$ & 7.90 \\
\hline
\end{tabular}

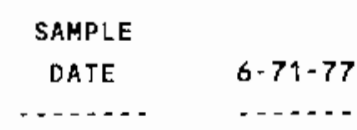

$\begin{array}{lr}02 / 24 / 88 & 186 \\ 06 / 20 / 88 & 220 \\ 02 / 24 / 88 & 19 \\ 06 / 20 / 88 & 29 \\ 02 / 24 / 88 & \\ 06 / 20 / 88 & \\ 02 / 24 / 88 & 8350 \\ 06 / 20 / 88 & 9260 \\ 02 / 24 / 88 & 323 \\ 06 / 20 / 88 & 346 \\ 02 / 24 / 83 & 10200 \\ 06 / 20 / 88 & 11400 \\ 02 / 24 / 88 & 7.67 \\ 06 / 20 / 88 & 7.80\end{array}$

$02 / 29 / 8$

$06 / 24 / 88$

$02 / 29 / 88$

$06 / 24 / 88$

$02 / 29 / 88$

$06 / 24 / 88$

$02 / 29 / 88$

$02 / 29 / 88$

$06 / 24 / 88$

$02 / 29 / 88$

$06 / 24 / 88$

$02 / 29 / 88$

$06 / 24 / 88$
$6-72-73$

\section{7}

131

15

15

5

6
4140

NR

340

342

4030

4080

7.56

7.90

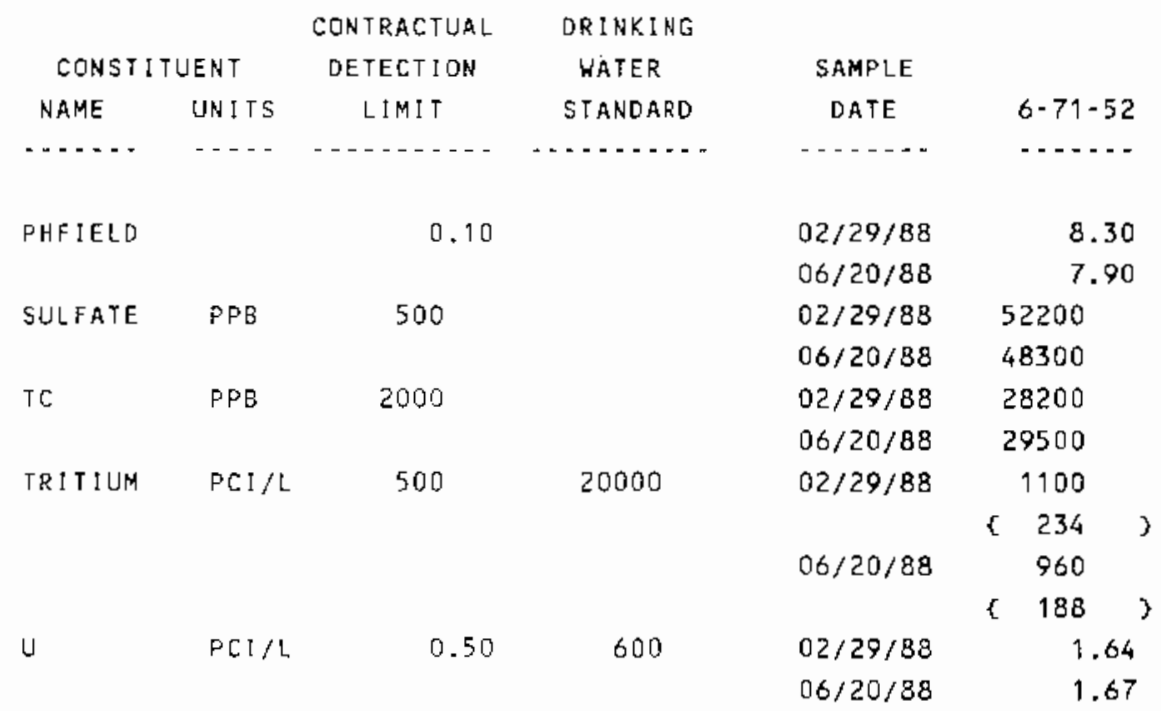

\begin{tabular}{lc}
$\begin{array}{c}\text { SAMPLE } \\
\text { DATE }\end{array}$ & $6-71-77$ \\
$\ldots \ldots \ldots$ & $\ldots \ldots .$. \\
& \\
$02 / 24 / 88$ & 8.20 \\
$06 / 20 / 88$ & 7.50 \\
$02 / 24 / 88$ & 28200 \\
$06 / 20 / 88$ & 27200 \\
$02 / 24 / 88$ & 29100 \\
$06 / 20 / 88$ & 32200 \\
$02 / 24 / 88$ & 4320 \\
& $\{314$ \\
$06 / 20 / 88$ & 2520 \\
\multicolumn{2}{c}{228} \\
$02 / 24 / 88$ & 1.43 \\
$06 / 20 / 88$ & 1.50
\end{tabular}

$\begin{array}{cc}\text { SAMPLE } & \\ \text { DATE } & 6-72-73 \\ \ldots \ldots \ldots & \cdots \\ & \\ 02 / 29 / 88 & 8.40 \\ 06 / 24 / 88 & 7.50 \\ 02 / 29 / 88 & 20100 \\ 06 / 24 / 88 & 18100 \\ 02 / 29 / 88 & 29100 \\ 06 / 24 / 88 & 29400 \\ 02 / 29 / 88 & 1670 \\ & 252 \\ & \text { NR } \\ & \text { NR } \\ & \text { NR }\end{array}$


TABLEE.3. (contd)

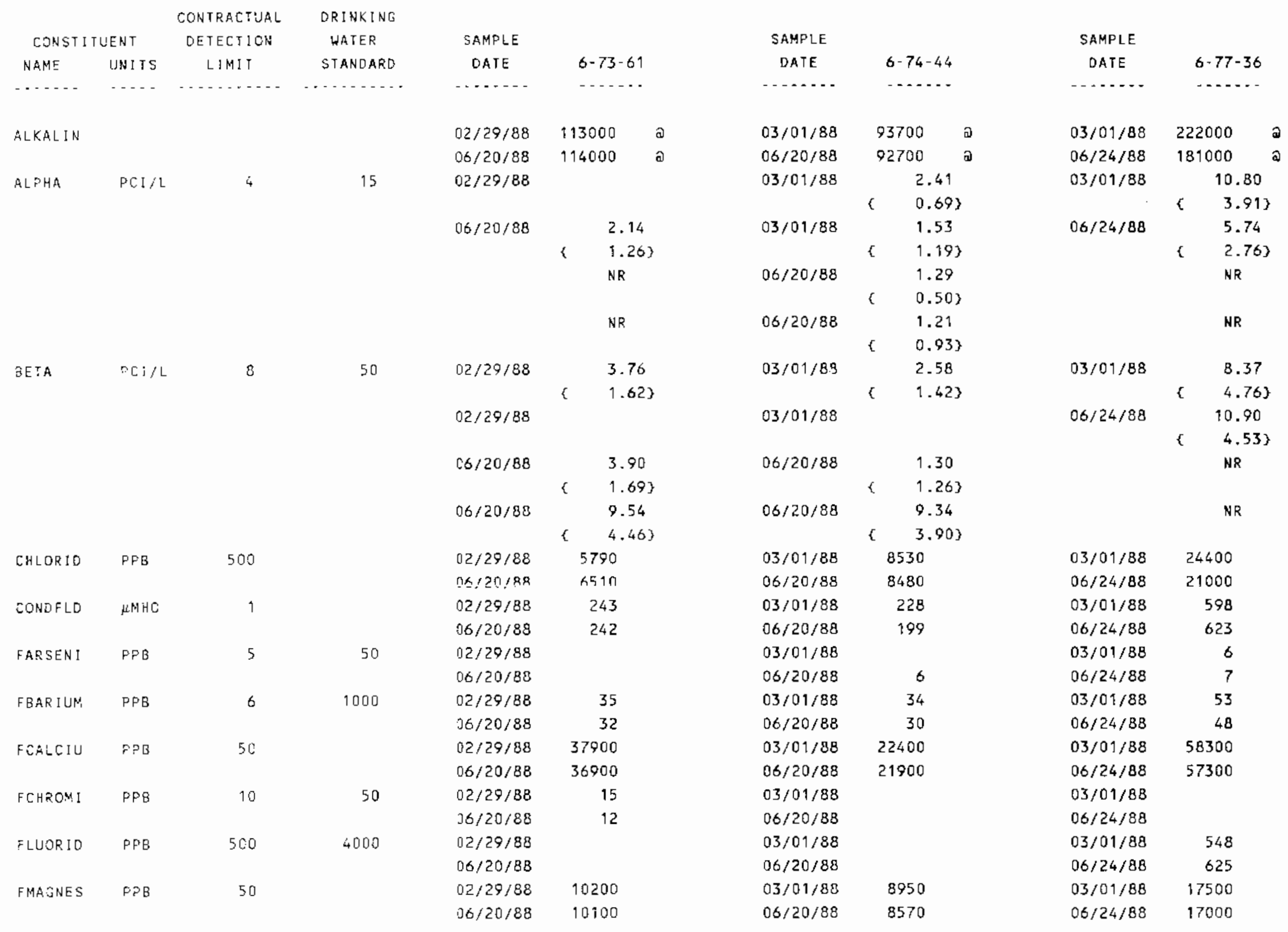


TABLE E.3. (contd)

\begin{tabular}{|c|c|c|c|c|c|c|c|c|c|}
\hline \multicolumn{2}{|c|}{ CONSTI I FUENT } & CONIRACIUAL & DRINKING & CAMPIE & & \multicolumn{2}{|l|}{ SAMPLE } & \multicolumn{2}{|l|}{ SAMPLE } \\
\hline NAME & UNITS & LIMIT & STANDARD & DATE & $6-73-61$ & DATE & $6-74.44$ & DATE & $6-77-36$ \\
\hline$\cdots \cdots$ & $\cdots$ & - $\ldots \ldots \ldots$ & - & $\ldots$ & n..... & - & $-\ldots \ldots$ & $\ldots$ & +...en- \\
\hline \multirow[t]{2}{*}{ FMANGAN } & $\mathrm{PPB}$ & 5 & & $02 / 29 / 88$ & 9 & $03 / 01 / 88$ & & $03 / 01 / 88$ & \\
\hline & & & & $06 / 20 / 88$ & 6 & $06 / 20 / 88$ & & $06 / 24 / 88$ & \\
\hline \multirow[t]{2}{*}{ FPOTASS } & $P P B$ & 100 & & $02 / 29 / 88$ & 4820 & $03 / 01 / 88$ & 2870 & $03 / 01 / 88$ & 5860 \\
\hline & & & & $06 / 20 / 88$ & 5000 & $06 / 20 / 88$ & 2750 & $06 / 24 / 88$ & 5800 \\
\hline \multirow[t]{2}{*}{ F SOOIUM } & $P P B$ & 200 & & $02 / 29 / 88$ & 17400 & $03 / 01 / 88$ & 24900 & $03 / 01 / 88$ & 69900 \\
\hline & & & & $06 / 20 / 88$ & 16900 & $06 / 20 / 8 B$ & 22900 & $06 / 24 / 88$ & 67100 \\
\hline \multirow[t]{2}{*}{ FSTRONT } & $P P B$ & 10 & & $02 / 29 / 88$ & 207 & $03 / 01 / 88$ & 239 & $03 / 01 / 88$ & 507 \\
\hline & & & & $06 / 20 / 88$ & 209 & $06 / 20 / 88$ & 233 & $06 / 24 / 88$ & 481 \\
\hline \multirow[t]{2}{*}{ FVANAOI } & PPB & 5 & & $02 / 29 / 88$ & 11 & $03 / 01 / 88$ & 8 & $03 / 01 / 88$ & 14. \\
\hline & & & & $06 / 20 / 88$ & 14 & $06 / 20 / 88$ & 14 & $06 / 24 / 88$ & 18 \\
\hline \multirow[t]{2}{*}{ FZINC } & PPB & 5 & - & $02 / 29 / 88$ & 148 & $03 / 01 / 88$ & & $03 / 01 / 88$ & 8 \\
\hline & & & & $06 / 20 / 88$ & 152 & $06 / 20 / 88$ & & $06 / 24 / 88$ & \\
\hline \multirow[t]{2}{*}{ HNIYRAT } & $P P B$ & 2500 & 45000 & $02 / 29 / 88$ & 9160 & $03 / 01 / 88$ & 6190 & $03 / 01 / 88$ & 66700 \\
\hline & & & & $06 / 20 / 88$ & 8950 & $06 / 20 / 88$ & 5820 & & NR \\
\hline
\end{tabular}


TABLE E.3. (contd)

\begin{tabular}{|c|c|c|c|c|c|c|c|c|c|c|}
\hline \multirow{2}{*}{\multicolumn{2}{|c|}{ CONST: TUEN? }} & CONTRACTUAL & DRISKING & & \multirow{2}{*}{\multicolumn{3}{|c|}{ SAMPLE }} & \multirow{2}{*}{\multicolumn{3}{|c|}{ SAMPLE }} \\
\hline & & DETECTICN & WATER & SAMPLE & & & & & & \\
\hline NAME & ISNITS & LIMIT & SIANDARD & DATE & $6-73-61$ & DATE & $6-74-44$ & DATE & $6-77-36$ & \\
\hline$-\ldots$ & $\cdots$ & - . - - - - - & $\cdots$ & $\cdots$ & $-\ldots$ & $\cdots \cdots$ & $-\cdots$ & $\ldots$ & $\cdots$ & \\
\hline \multirow[t]{2}{*}{ LFLUORD } & $\mathrm{PPB}$ & 50 & 4000 & $02 / 29 / 88$ & 295 & $03 / 01 / 88$ & 313 & $03 / 01 / 88$ & 610 & \\
\hline & & & & $06 / 20 / 88$ & 308 & $06 / 20 / 88$ & 306 & $06 / 24 / 88$ & 594 & \\
\hline \multirow[t]{4}{*}{ METHYCH } & PPB & 5 & & $02 / 29 / 88$ & & $03 / 01 / 88$ & & $03 / 01 / 88$ & & \\
\hline & & & & $06 / 20 / 88$ & 67 & $06 / 20 / 88$ & & $06 / 24 / 88$ & & \\
\hline & & & & & $N R$ & & NR & $06 / 24 / 88$ & & \\
\hline & & & & & NR & & $\mathrm{NR}$ & $06 / 24 / 88$ & & \\
\hline \multirow[t]{2}{*}{ NITRATE } & $\mathrm{PPB}$ & 500 & 45000 & $02 / 29 / 88$ & 9740 & $03 / 01 / 88$ & 7840 & $03 / 01 / 88$ & 67100 & * \\
\hline & & & & $06 / 20 / 88$ & 9550 & $06 / 20 / 88$ & 6010 & $06 / 24 / 88$ & 57800 & * \\
\hline \multirow[t]{2}{*}{$P H-L A B$} & & 0.01 & & $02 / 29 / 88$ & 7.63 & $03 / 01 / 88$ & 8.09 & $03 / 01 / 88$ & 7.53 & \\
\hline & & & & $06 / 20 / 88$ & 7.80 & $06 / 20 / 88$ & 8.20 & $06 / 24 / 88$ & 7.80 & \\
\hline \multirow[t]{2}{*}{ FHF:ELD } & & $0 .: 0$ & & $02 / 29 / 88$ & 8.30 & $03 / 01 / 83$ & 9 & $03 / 01 / 88$ & 8.40 & \\
\hline & & & & $06 / 20 / 88$ & 7.70 & $06 / 20 / 88$ & 8.40 & $06 / 24 / 88$ & 7.40 & \\
\hline \multirow[t]{2}{*}{ SULFA?E } & PP5 & 500 & & $02 / 29 / 88$ & 40900 & $03 / 01 / 88$ & 41100 & $03 / 01 / 88$ & 63100 & \\
\hline & & & & $06 / 20 / 88$ & 40800 & $06 / 20 / 88$ & 34100 & $06 / 24 / 88$ & 52700 & \\
\hline \multirow[t]{2}{*}{ TC } & $\mathrm{PPB}$ & 2000 & & $02 / 29 / 88$ & 27800 & $03 / 01 / 88$ & 22900 & $03 / 01 / 88$ & 56000 & \\
\hline & & & & $06 / 20 / 88$ & 29700 & $06 / 20 / 88$ & 23200 & $06 / 24 / 88$ & 57000 & \\
\hline \multirow[t]{2}{*}{ TOXLDL } & PPB & 10 & & $02 / 29 / 88$ & & $03 / 01 / 88$ & & $03 / 01 / 88$ & 30.20 & \\
\hline & & & & $06 / 20 / 88$ & 26.90 & $06 / 20 / 88$ & & $06 / 24 / 88$ & 29.90 & \\
\hline \multirow[t]{4}{*}{ TRICENE } & PPG & 10 & 5 & $02 / 29 / 88$ & & $03 / 01 / 88$ & & $03 / 01 / 88$ & 32 & * \\
\hline & & & & $06 / 70 / 88$ & & $06 / 20 / 88$ & & $06 / 24 / 88$ & 35 & * \\
\hline & & & & & NR & & NR & $06 / 24 / 88$ & 35 & * \\
\hline & & & & & $\mathrm{NR}$ & & NR & $06 / 24 / 88$ & 35 & * \\
\hline \multirow[t]{2}{*}{ 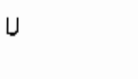 } & $\mathrm{PCI} / \mathrm{L}$ & 0.50 & 600 & $02 / 29 / 88$ & 1.39 & & $N R$ & & NR & \\
\hline & & & & $06 / 20 / 8 B$ & 1.51 & & NR & & $N R$ & \\
\hline
\end{tabular}


TABLE E.3. (contd)

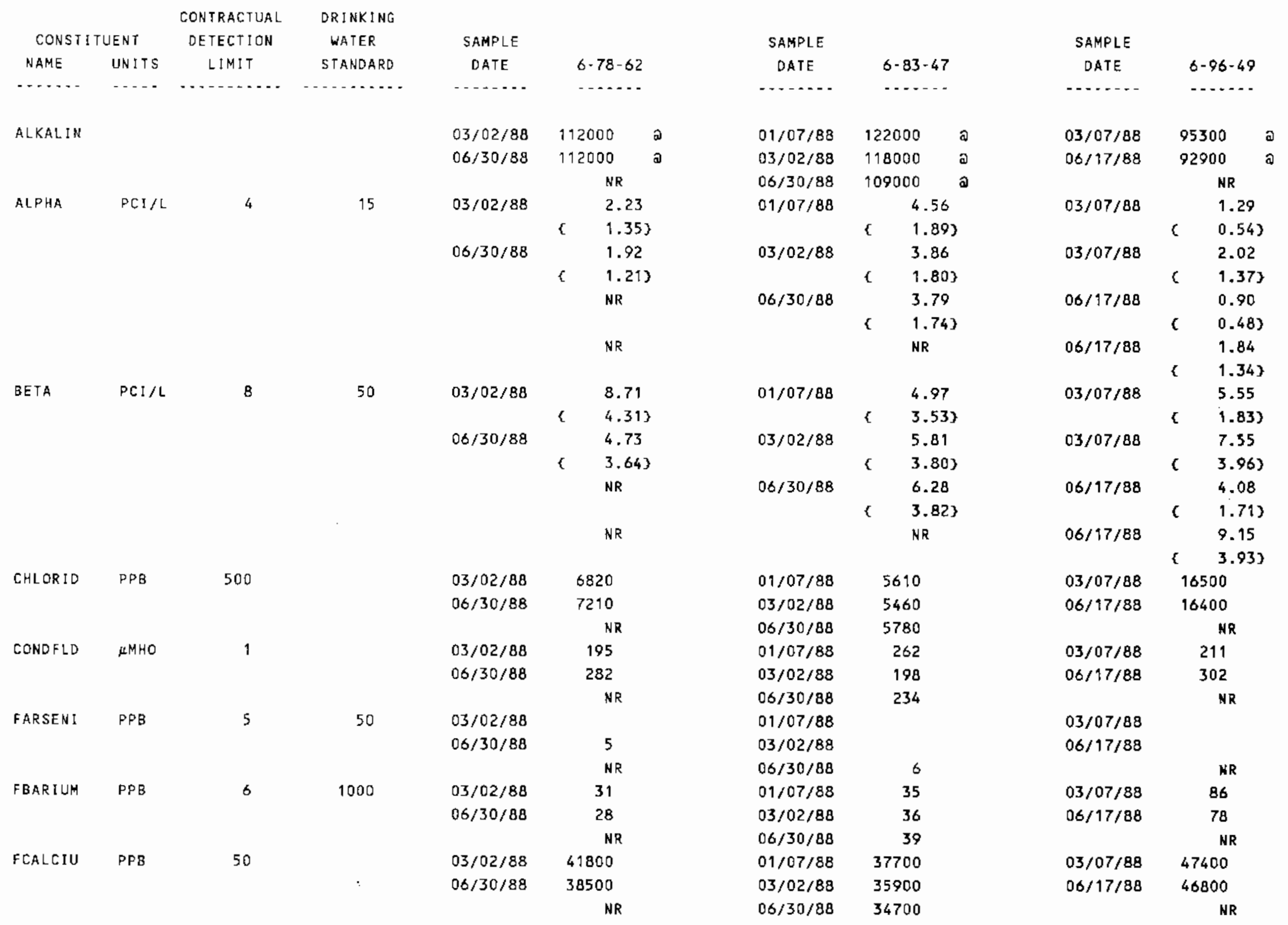


TABLE E.3. (contd)

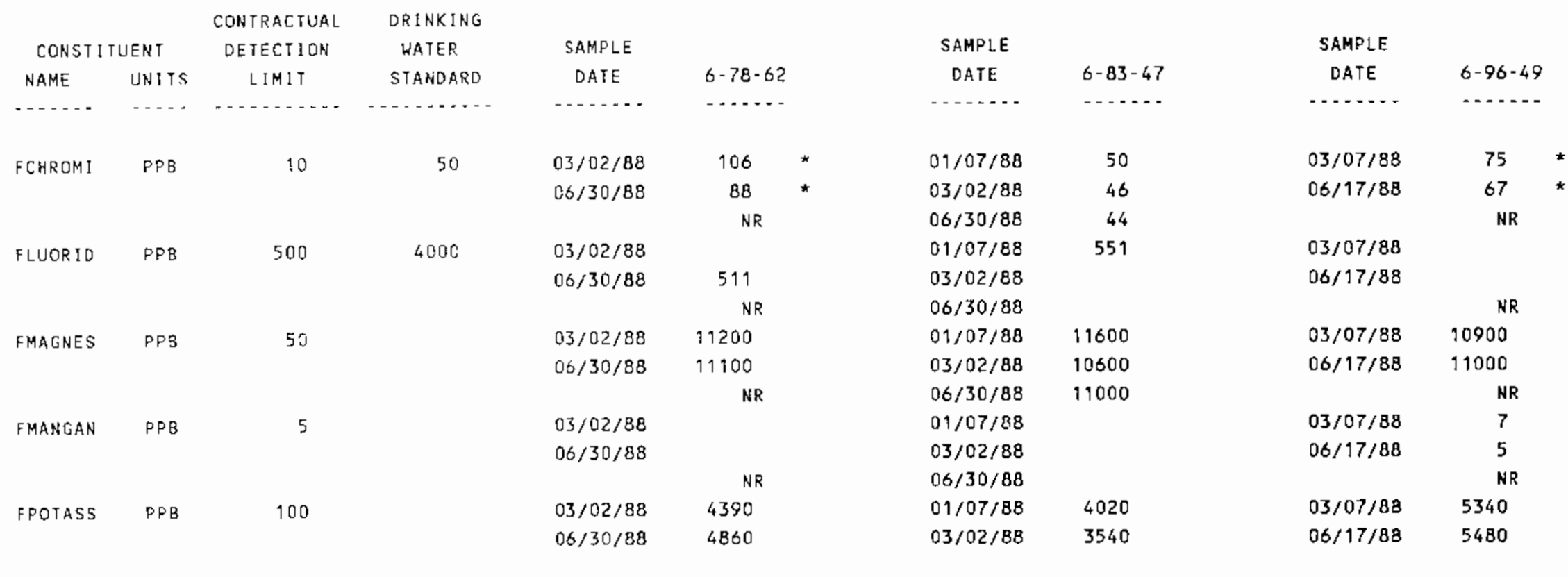


TABLE E.3. (contd)

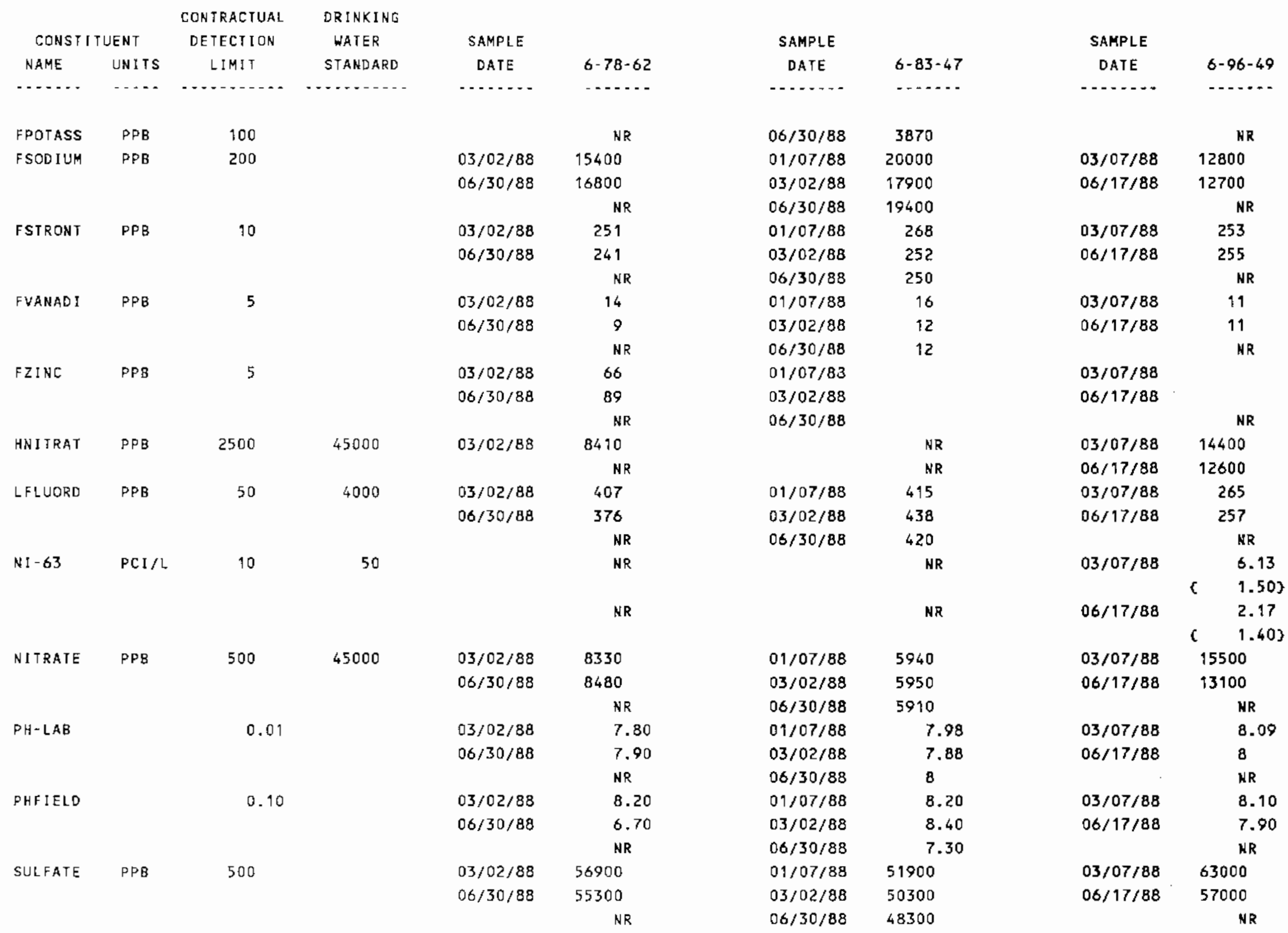


IABLE E.3. (contd)

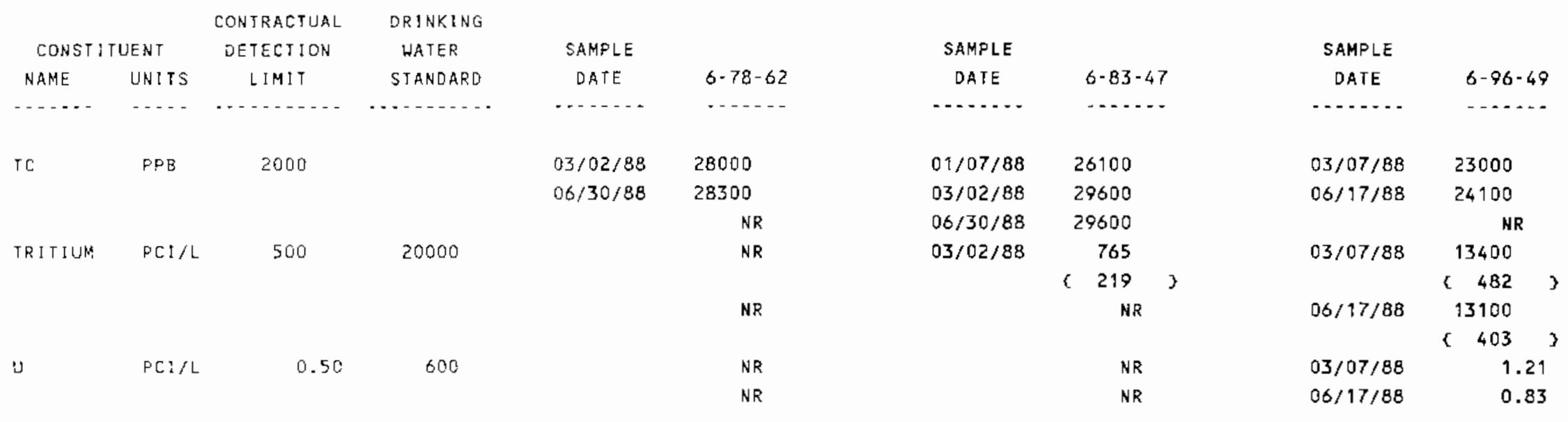


TABLE E.3. (contd)

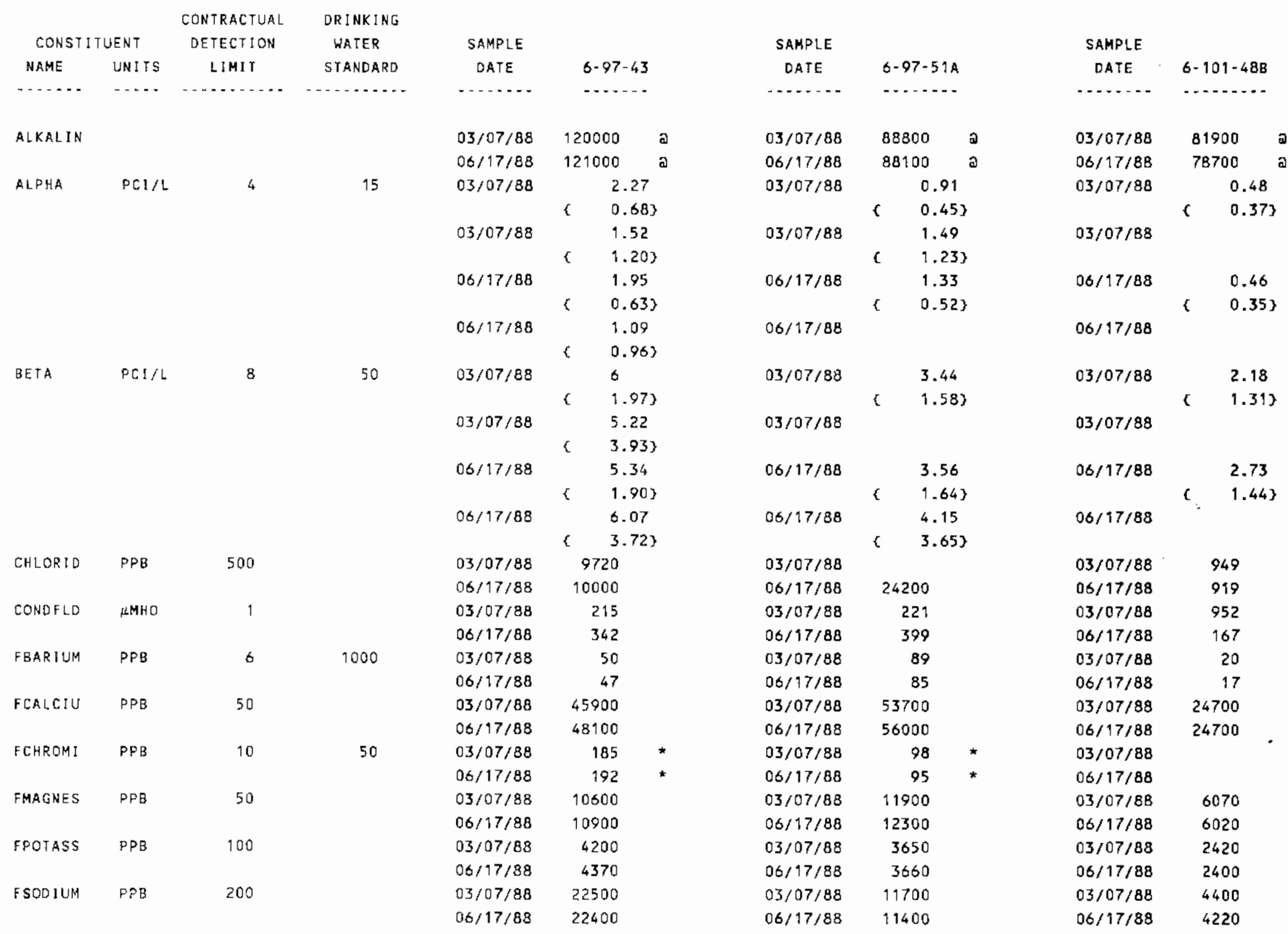


TABLE E.3. (contd)

\begin{tabular}{|c|c|c|c|c|c|c|c|c|c|}
\hline \multirow{2}{*}{\multicolumn{2}{|c|}{ CONS T I TUEN T }} & CCLTRACTUAL & JR:NK: INE & & \multirow{2}{*}{\multicolumn{3}{|c|}{ SAMPLE }} & \multirow{2}{*}{\multicolumn{2}{|c|}{ SAMPLE }} \\
\hline & & CETECT:OX & WA :E'R & SAMPLE & & & & & \\
\hline NAME & :N]IS & LiM1? & $S$ : ANDARU & DATE & 6.97 .43 & DATE & $6.97-51 \mathrm{~A}$ & DATE & $6-101-48 B$ \\
\hline - . - . & $\cdots$ & - & - - - - - - - - - & - - - - - - & $\cdots$ & $\cdots \ldots$ & 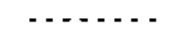 & $\cdots$ & .......... \\
\hline \multirow[t]{2}{*}{ FSTRONT } & FPS & 10 & & $03 / 07 / 88$ & 223 & $03 / 07 / 88$ & 244 & $03 / 07 / 88$ & 111 \\
\hline & & & & $05 / 17 / 88$ & 230 & $06 / 17 / 88$ & 251 & $06 / 17 / 88$ & 112 \\
\hline \multirow[t]{2}{*}{ FUANAEI } & $P O B$ & 5 & & $03 / 07 / 88$ & & $03 / 07 / 88$ & & $03 / 07 / 88$ & 10 \\
\hline & & & & $06 / 17 / 88$ & & $06 / 17 / 88$ & & $06 / 17 / 88$ & 12 \\
\hline \multirow[t]{2}{*}{$F Z: N C$} & $P P B$ & 5 & & $03 / 07 / 88$ & & $03 / 07 / 88$ & 6 & $03 / 07 / 88$ & \\
\hline & & & & $06 / 17 / 88$ & & $06 / 17 / 88$ & 6 & $06 / 17 / 88$ & \\
\hline \multirow[t]{2}{*}{ HNITRAT } & $F \circ B$ & 2500 & $450 \mathrm{co}$ & $03 / 07 / 88$ & 20000 & $03 / 07 / 88$ & 20700 & $03 / 07 / 88$ & \\
\hline & & & & $06 / 17 / 88$ & 18500 & $06 / 17 / 88$ & 19900 & $06 / 17 / 88$ & \\
\hline \multirow[t]{2}{*}{ LFL:OORL } & $\rho \rho B$ & 50 & 4000 & $03 / 07 / 88$ & 291 & $03 / 07 / 88$ & 271 & $03 / 07 / 88$ & 161 \\
\hline & & & & $06 / 17 / 88$ & 283 & $06 / 17 / 88$ & 262 & $06 / 17 / 88$ & 157 \\
\hline \multirow[t]{3}{*}{$9 i 63$} & $F=1 /-$ & 10 & $5 \mathrm{C}$ & & NR & $03 / 07 / 88$ & 5.86 & & $N R$ \\
\hline & & & & & & & 1.523 & & \\
\hline & & & & & NR & $06 / 17 / 88$ & & & NR \\
\hline$\because I-R A T E$ & $F B$ & 50 & $\operatorname{sicc}$ & $03 / 07 / 88$ & 21300 & $03 / 07 / 88$ & 22200 & $03 / 07 / 88$ & 917 \\
\hline
\end{tabular}


TABLE E.3. (contd)

\begin{tabular}{|c|c|c|c|c|c|c|c|c|c|c|}
\hline \multicolumn{2}{|c|}{ CONSI I TUENT } & $\begin{array}{l}\text { CONTRACTUAL } \\
\text { DETECIION }\end{array}$ & $\begin{array}{l}\text { DRINKING } \\
\text { WATER }\end{array}$ & SAMPLE & \multirow{2}{*}{\multicolumn{2}{|c|}{$6.97-43$}} & SAMPLE & & \$AMPLE & \\
\hline NAME & UNITS & LIMIT & STANDARD & DATE & & & DATE & $6-97.51 \mathrm{~A}$ & DATE & $6-101-48 B$ \\
\hline$\ldots$ & $\ldots$. & . & - & $\cdots \ldots$ & \multicolumn{2}{|c|}{$\ldots$} & $\ldots$ & $\ldots$ & $\cdots$ & $\cdots$ \\
\hline NITRATE & $P P B$ & 500 & 45000 & $06 / 17 / 88$ & \multicolumn{2}{|l|}{19000} & $06 / 17 / 88$ & 20800 & $06 / 17 / 88$ & 1230 \\
\hline \multirow[t]{2}{*}{$P H-L A B$} & & \multirow[t]{2}{*}{0.01} & & $03 / 07 / 88$ & \multicolumn{2}{|c|}{7.88} & $03 / 07 / 88$ & 7.61 & $03 / 07 / 88$ & 7.85 \\
\hline & & & & $06 / 17 / 88$ & \multicolumn{2}{|c|}{7.90} & $06 / 17 / 88$ & 7.60 & $06 / 17 / 88$ & 7.80 \\
\hline \multirow[t]{2}{*}{ PHFIELD } & & \multirow[t]{2}{*}{0.10} & & $03 / 07 / 88$ & \multicolumn{2}{|c|}{7.70} & $03 / 07 / 88$ & 7.40 & $03 / 07 / 88$ & 8 \\
\hline & & & & $06 / 17 / 88$ & \multicolumn{2}{|c|}{7.50} & $06 / 17 / 88$ & 7.30 & $06 / 17 / 88$ & 7.70 \\
\hline \multirow[t]{2}{*}{ SULFATE } & PPB & 500 & & $03 / 07 / 88$ & \multicolumn{2}{|l|}{62600} & $03 / 07 / 88$ & 80900 & $03 / 07 / 88$ & 12200 \\
\hline & & & & $06 / 17 / 88$ & \multicolumn{2}{|l|}{57800} & $06 / 17 / 88$ & 74400 & $06 / 17 / 88$ & 11000 \\
\hline \multirow[t]{2}{*}{ TC } & $\mathrm{PPB}$ & 2000 & & $03 / 07 / 88$ & \multicolumn{2}{|l|}{29800} & $03 / 07 / 88$ & 22400 & $03 / 07 / 88$ & 20100 \\
\hline & & & & $06 / 17 / 88$ & \multicolumn{2}{|l|}{30800} & $06 / 17 / 88$ & 24000 & $06 / 17 / 88$ & 21900 \\
\hline \multirow[t]{4}{*}{ TRITIUM } & $\mathrm{PCI} / \mathrm{L}$ & 500 & 20000 & $03 / 07 / 88$ & \multicolumn{2}{|l|}{10100} & $03 / 07 / 88$ & 15000 & $03 / 07 / 88$ & \\
\hline & & & & & $<429$ & 3 & & $<507$ & & \\
\hline & & & & $06 / 17 / 88$ & \multicolumn{2}{|l|}{9250} & $06 / 17 / 88$ & 14200 & $06 / 17 / 88$ & \\
\hline & & & & & < 349 & ) & & \& 417$\}$ & & \\
\hline \multirow[t]{2}{*}{$u$} & $\mathrm{PCI} / \mathrm{L}$ & 0.50 & 600 & & NR & & $03 / 07 / 88$ & 1.30 & & NR \\
\hline & & & & & NR & & $06 / 17 / 88$ & 1.33 & & NR \\
\hline
\end{tabular}

- VALUE EXCEEDS PRIMARY DRINKING WATER STANDARD.

* - Value exceEds pRoposed primary dRINKINg hater standard.

+ - value exceeds screening level for further investigation.

a - DETECTION LIMIT WAS NOT AVAILABLE FOR COMPARISON

NR - ANALYSIS NOT REQUESTEO OR NOT YET REPORTED

VALUES IN \{\} ARE COUNTING ERRORS FOR RADIONUCLIDES

WATER STANDARD(S) IN PARENTHESES ARE PROPOSED ONLY

JOb DN_ANA (queue SY\$BATCH_Y, entry 1575) completed 
TABLE E.4. Analytical Plans for Compliance Monitoring Networks

$\operatorname{comp}=1301 \cdot \mathrm{N}$

E AACCCCC EHLLLPP $\$$

LMIOOOYOINFH PEHPU

KMTLNNAIHILYHRFHL

AORIDONDYTUDECI F

LNUFFLIXGRORNHELI

NURMDBENYTDZLDDBECSCXLSENBETEFETEFESTETLEEGEAA4AH3MO9MUM
$\begin{array}{llr}P & S & V \\ H & S & V\end{array}$

$\begin{array}{ll}T & T \\ R & R\end{array}$

POEMTOLA GINAST I

2 I DRCT

\begin{tabular}{|c|c|c|c|c|}
\hline $1-\mathbf{N}-\mathbf{Z}$ & $x$ & $x x$ & $x$ & $\times x$ \\
\hline $1-N-3$ & $x$ & $\times x$ & & $x \times$ \\
\hline $1-N-4$ & $x$ & $x \times$ & $x$ & $x \times$ \\
\hline $1-N=14$ & $x$ & $x x$ & $x$ & $x \times$ \\
\hline $1-N-49$ & $x$ & $x \times$ & $x$ & $x x$ \\
\hline
\end{tabular}

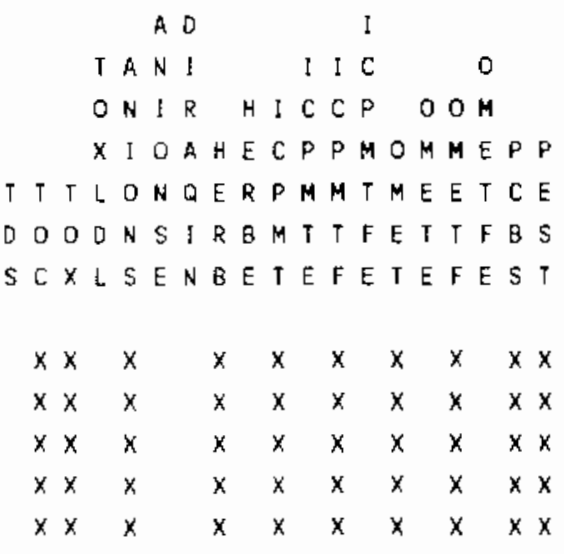

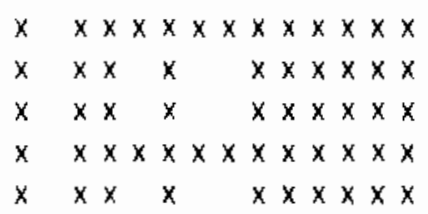

$\operatorname{COMP}=1324-\mathrm{N}$

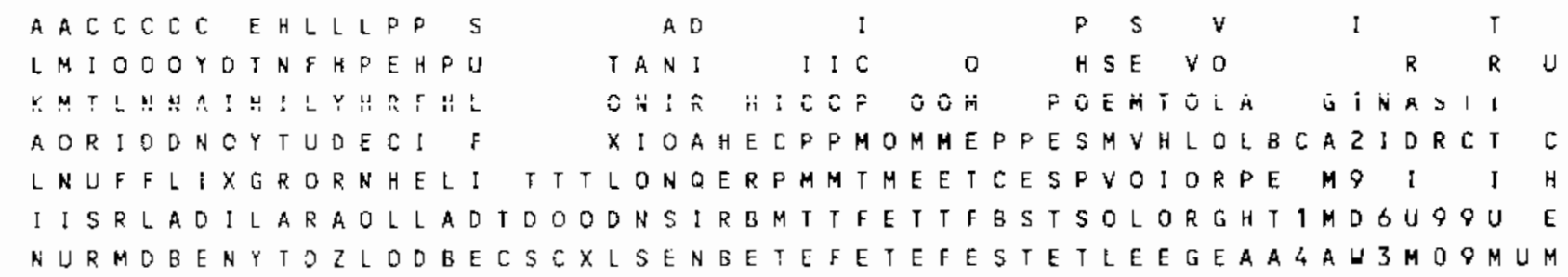

\begin{tabular}{|c|c|c|c|c|c|c|c|c|c|c|c|c|c|c|}
\hline $3-N-58$ & $x$ & $x \times x$ & $x$ & $x x$ & $x$ & $x$ & $x$ & $x$ & $x$ & $x$ & $x$ & $x$ & $x$ & $x \times$ \\
\hline $1-N-59$ & & $x \times x$ & $x$ & $x \times$ & $x$ & $x$ & $x$ & $x$ & $x$ & $x$ & $x$ & $x$ & $x$ & $x x$ \\
\hline $1-N-60$ & & $x \times x$ & $x$ & $x \times$ & $x$ & $x$ & $x$ & $x$ & $x$ & $x$ & $x$ & $x$ & $x$ & $x \times$ \\
\hline $1-N-61$ & & $x \times x$ & $x$ & $x \times$ & $x$ & $x$ & $x$ & $x$ & $x$ & $x$ & $x$ & $x$ & $x$ & $x \times$ \\
\hline
\end{tabular}


TABLE E.4. (contd)

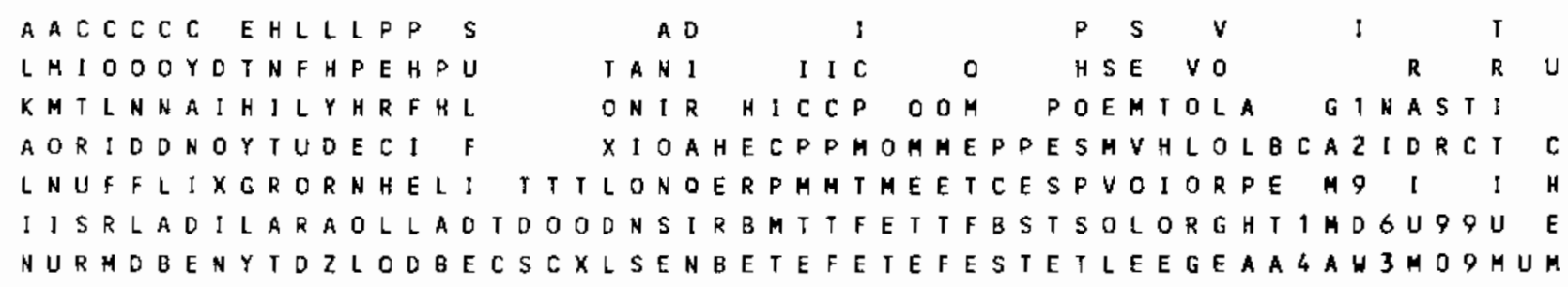

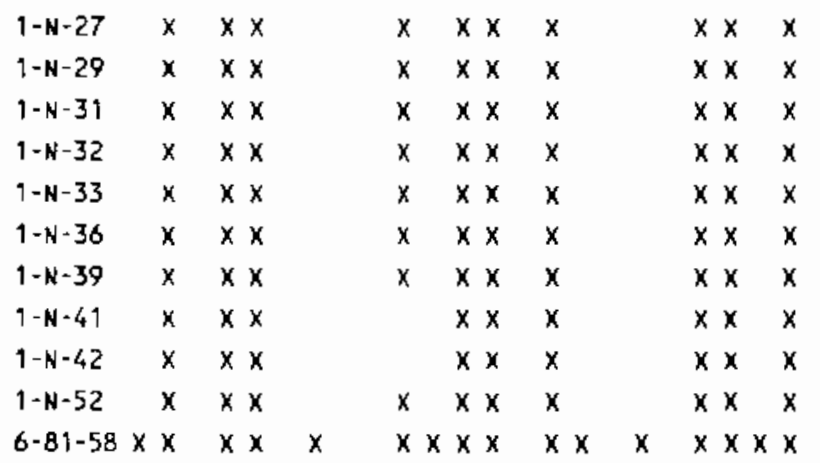

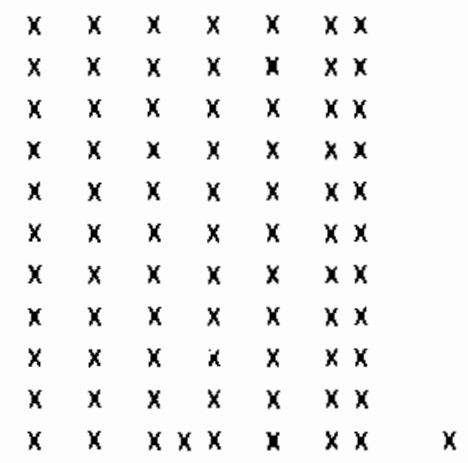




\title{
TABLE E.4. (contd)
}

$\operatorname{COMP}=183-\mathrm{H}$

\begin{abstract}
A ACCCCC EHLLLPP S LMIDOOYDTNFHPEHPU KMTL LNAIHILYYHRFHL A D $T A N 1$ $\begin{array}{lll}P & S\end{array}$ I ONIR HICCP OOM POEMTOLA $\begin{array}{lll}R & R & \\ R & & \end{array}$ AORIDDNOYTUDECI F XI OAHECPPMOMMEPPESMVHLOLBCAZIDRCT C LNUFFLIXGRORNHELI TTTLONQERPMMTMEETCESPVOIORPE M 9 I I H I ISRLAOILARAOLLADTDOOONSIRBMTTFETTFBSTSOLORGHTIMD $6 U 99 U$ U NURMDBENYTDZLODBECSCXLSENBETEFETEFESTETLEEGEAAGAH3MO9MUM
\end{abstract}

\begin{tabular}{|c|c|c|c|}
\hline $\begin{array}{l}1-H 3-1 \\
1-H^{3}-2 A\end{array}$ & $\begin{array}{l}x \times \\
\times x\end{array}$ & & $\begin{array}{l}x \quad x \\
x \quad x\end{array}$ \\
\hline $1-H 3-26$ & $x x$ & & $x x$ \\
\hline $1-H 3-2 C$ & $x x$ & & $x \times$ \\
\hline $1-\mathrm{H}_{4}-3$ & & $x$ & $x \times \times$ \\
\hline $1-\mathrm{H}_{4}-4$ & $x x$ & & $x \times$ \\
\hline $1-\mathrm{H}_{4}-5$ & $x>$ & & $x \times$ \\
\hline $1-\mathrm{H}_{4}-6$ & $x x$ & & $x \times$ \\
\hline $1-\mathrm{H}_{4}-7$ & $x x$ & & $x \times$ \\
\hline $1-\mathrm{H}_{4}-8$ & $x x$ & $x$ & $x \times$ \\
\hline 1-H4-9 & $x y$ & & $x \times$ \\
\hline $1-H_{4}-10$ & $x>$ & & $x \times$ \\
\hline $1-4 t:-1 !$ & $x:$ & & $x x$ \\
\hline $1-H_{4}-12 A$ & $x>$ & & $x \times$ \\
\hline $1-H_{4}-128$ & $x x$ & & $x \times$ \\
\hline $1-44-12 C$ & $x y$ & & $x x$ \\
\hline $1 \cdot H 4-13$ & $x y$ & & $x \times$ \\
\hline $1-1.44-14$ & $x$ & & $x \times$ \\
\hline $1 \cdot-44-15 \mathrm{~A}$ & $x$ & & $x \times$ \\
\hline $1-44-159$ & $x$ & & $x \times$ \\
\hline$?-4 \dot{4}-16$ & . & & $x \times$ \\
\hline$i-H 4 \cdot 17$ & r & & $x \times$ \\
\hline$H H_{1}-\cdots$ & $x$ & & $x \times x$ \\
\hline
\end{tabular}

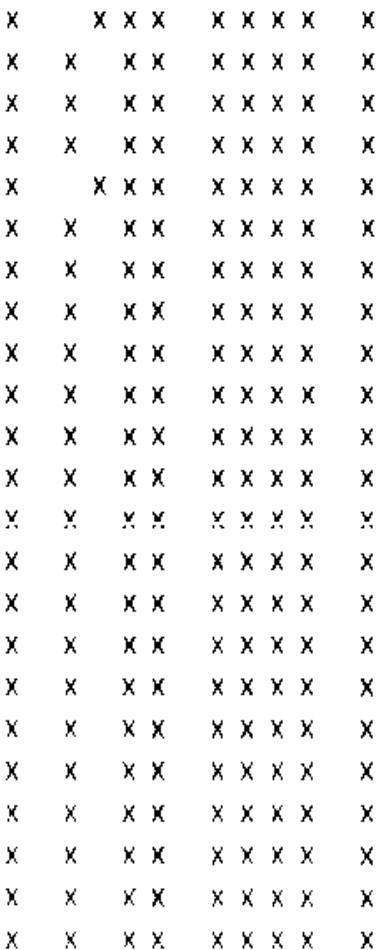

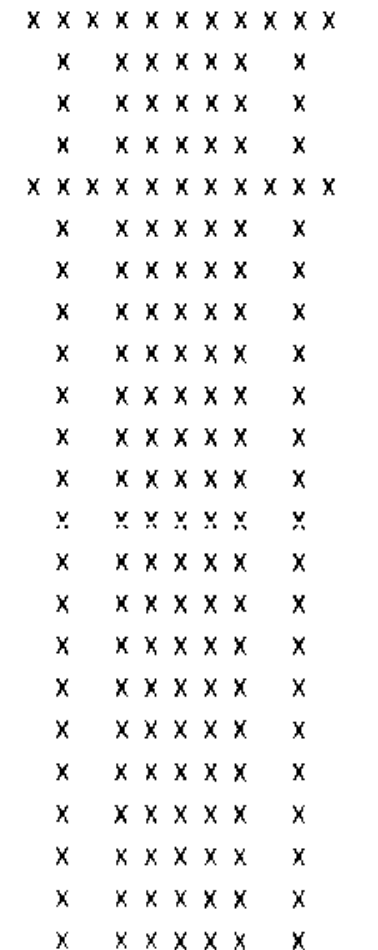

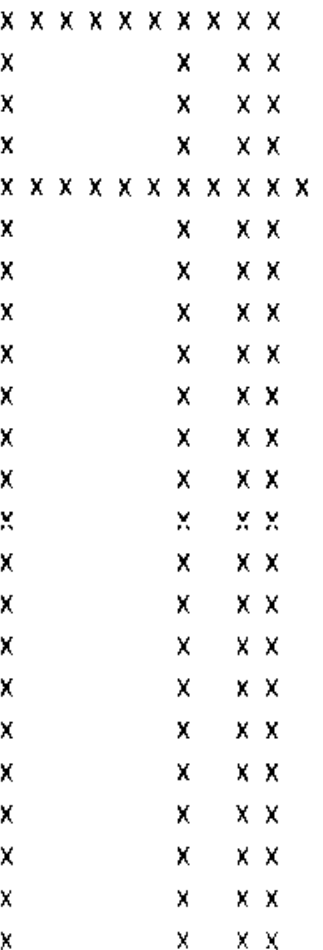
$x \quad x \quad x$ $x \times x$ $x \quad x \quad x$ $x \times \times \times \times x$ $x \times x \times$ 
IABLE E.4. (contd)

$C O M P=2 E-A 36 B$

AACCCCC EHLLLPP S
LMIOOOYDTNFHPEHPU TAN

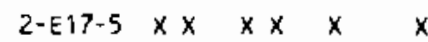

2-E17-6 $\times \times \times \times \times \times \times \times$

2-E17-14 $X \quad X \times$

2-E17-15 $X \times X$

2-E17-16 $X X X$

2-E17-17 $x \quad x \times$

2-E17-18 $x \times x$

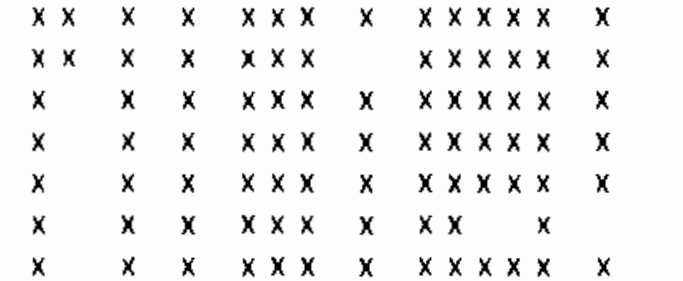

$\begin{array}{lllll}x \times & \times \times \times \times \times \times \times & x \times & x & x \\ x & \times \times \times \times \times & x & \\ x & x \times \times \times & x & \\ x & x \times x \times & x & \\ x & x \times x \times & x & \\ x & x \times \times x & x \times x & \end{array}$

\section{$\operatorname{COMP}=2 E-G T F$}
A ACCCCC EHLLLPP S
A D
1
TA N I
I I C
ONIR HICCP OOM
P S
HSE VO
L MIOOOOYYOT N F H PE H P U

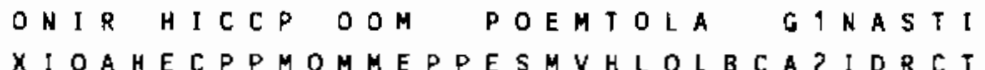
AORIDDNOYTUDECI F
XIOAHECPPMOMMEPPESMVHLOLBCAZIDRCT C
LNUFFLIXGRORNHELI TTTLONOERPMMTHEETCESPVOIORPE M 9 I I H
IISRLADILARAOLLADTDOOONSIRBMTTFETTFBSTSOLORGHTIMDOU O OU E

NURMDBENYTDZLODBECSCXLSENBETEFETEFESTETLEEGEAA4AU3MOGMU H

$\begin{array}{lllll}2-E 25-18 & \times & \times & \times & \times \\ 2-E 25-22 & \times \times & \times & \times & \times \\ 2-E 25-25 & \times \times \times \times & \times \times \times \\ 2-E 25-26 & \times & \times & \\ 2-E 25-27 & \times & \times & \\ 2-E 25-28 & \times & \times & \\ 2-E 25-30 & \times \times & \times & \times \times \times \\ 2-E 25-31 & \times \times \times \times & \times \times \times \\ 2-E 25-33 & \times \times \times \times & \times \times \times\end{array}$

$x \times \times \times \times \times \times \times \times \times \times \times \times \times \times \times \times \times \times \times \times \times \times \times \times \times \times$

$x \times x$

$x \times$

$x \times$

$x \times$

$x \times$

$x \times x$

$\times \quad x \times$ 
TABLE E.4. (contd)

COMP $=300$ APT

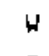

AACCCCC EHLLLPPS

LHIOOOYDTNFHPEHPU KMTLNNAIHILYHRFHL AORIDDNOYTUDECI F LNUFFLIXGRORNHELI IISRLADILARAOLLADTDOODNSIRBMTTFETTFBSTSOLORGHT1MD6U99U E NURMOBENYTDZLODBECSCXLSENBETEFETEFESTETLEEGEAA4AW $3 M O 9 M U M$

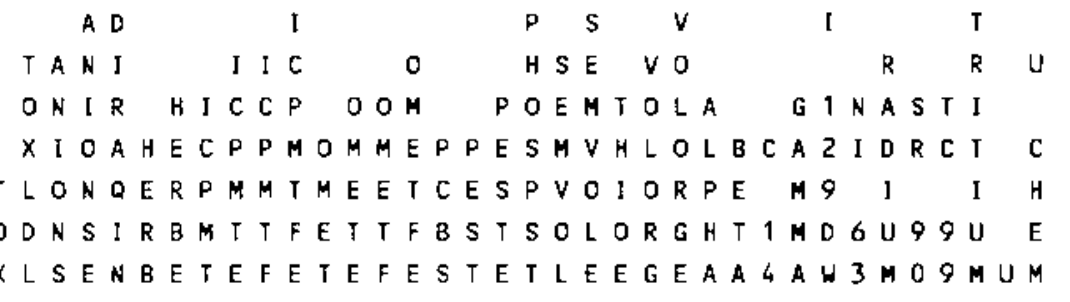

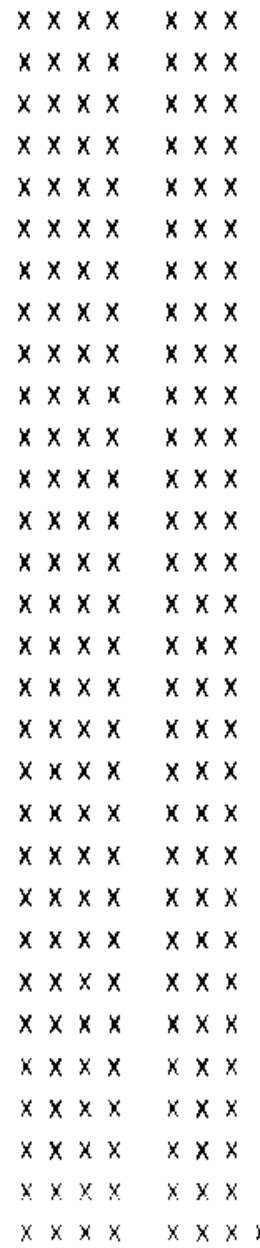

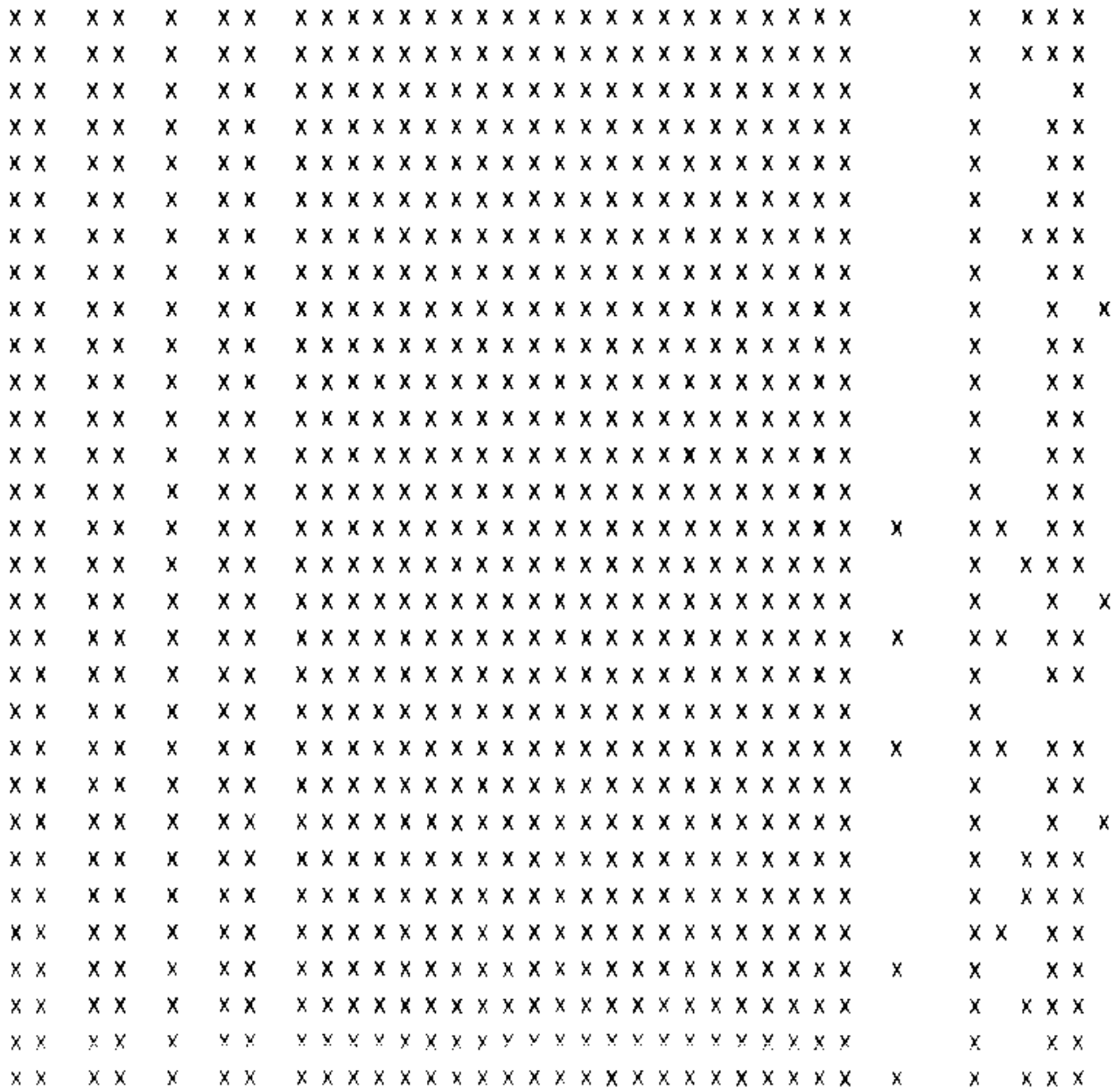


TABLE E.4. (contd)

COMP $=300$ APT

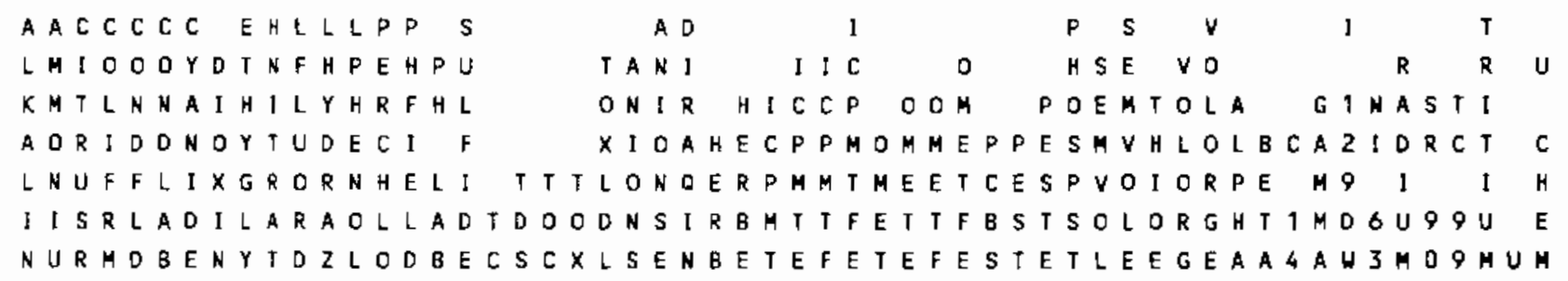

NURMOBENYTDZLODBECS CXLSENBETEFETEFESTET LEEGEAA4AU3MDOMUM

\begin{tabular}{|c|c|c|c|c|c|c|c|c|c|c|}
\hline $3-8-2$ & $\times \times \times \times$ & $x \times \times$ & $x \times$ & $x \times$ & $x$ & $x \times$ & $\times \times \times \times \times \times \times \times \times \times \times \times \times \times \times \times \times \times \times \times \times \times$ & & $x$ & $x$ \\
\hline $3-8-3$ & $x \times \times \times$ & $x \times \times \times$ & $\times x$ & $x \times$ & $x$ & $x \times$ & $x \times \times \times \times \times \times \times \times \times \times \times \times \times \times \times \times \times \times \times \times \times$ & $x$ & $x$ & $x \times)$ \\
\hline$-\$ 30-E 15 A$ & $x \times \times \times$ & $x$ & $x \times$ & $x \times$ & $x$ & $\times x$ & $\times \times \times \times \times \times \times \times \times \times \times$ & & $x$ & $x)$ \\
\hline$-\$ 19-E 13$ & $x \times \times \times$ & $\times \times \times$ & $x \times$ & $x \times$ & $x$ & $x \times$ & $\times \times \times \times \times \times \times \times \times \times x$ & $x$ & $x$ & \\
\hline
\end{tabular}

$\operatorname{COMP}=6-\mathrm{HROH}$

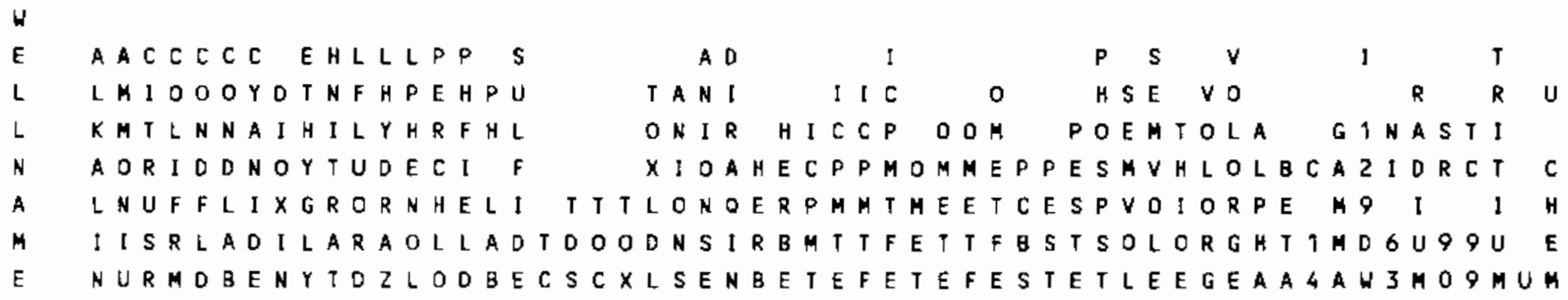

\begin{tabular}{|c|c|c|c|c|c|c|c|c|c|}
\hline $6-25 \cdot 33 \mathrm{~A}$ & $x \times x$ & $x$ & $x$ & $x \times$ & $x \times x$ & $x \times x$ & $x \times \times x \times$ & $x$ & $x \times x \times$ \\
\hline $6-25-34 \mathrm{~A}$ & $x \times x$ & $x$ & $x$ & $x \times$ & $x \times x$ & $x \times x$ & $x \times \times \times \times$ & $x$ & $x \times \times \times$ \\
\hline $6-25 \cdot 34 \mathrm{~B}$ & $x \times x$ & $x$ & $x$ & $x \times$ & $x \times x$ & $x \times x$ & $x \times \times \times x$ & $x$ & $x \times x \times$ \\
\hline $6 \cdot 26-33$ & $x \times x$ & $x$ & $x$ & $x \times$ & $x \times x$ & $x \times x$ & $x \times \times \times \times$ & $x$ & $x \times x \times$ \\
\hline $6-26-34$ & $x \times x$ & $x$ & $x$ & $x \times$ & $x \times x$ & $x \times x$ & $x \times \times \times x$ & $x$ & $x \times \times \times$ \\
\hline $6-26-35 A$ & $x \times x$ & $x$ & $x$ & $x \times$ & $x \times x$ & $x \times x$ & $x \times x \times x$ & $x$ & $x \times x \times$ \\
\hline $6-26-35 c$ & $x \times x$ & $x$ & $x$ & $x \times$ & $x \times x$ & $x \times x$ & $x \times \times \times x$ & $x$ & $x \times \times x$ \\
\hline
\end{tabular}


TABLE E.4. (contd)

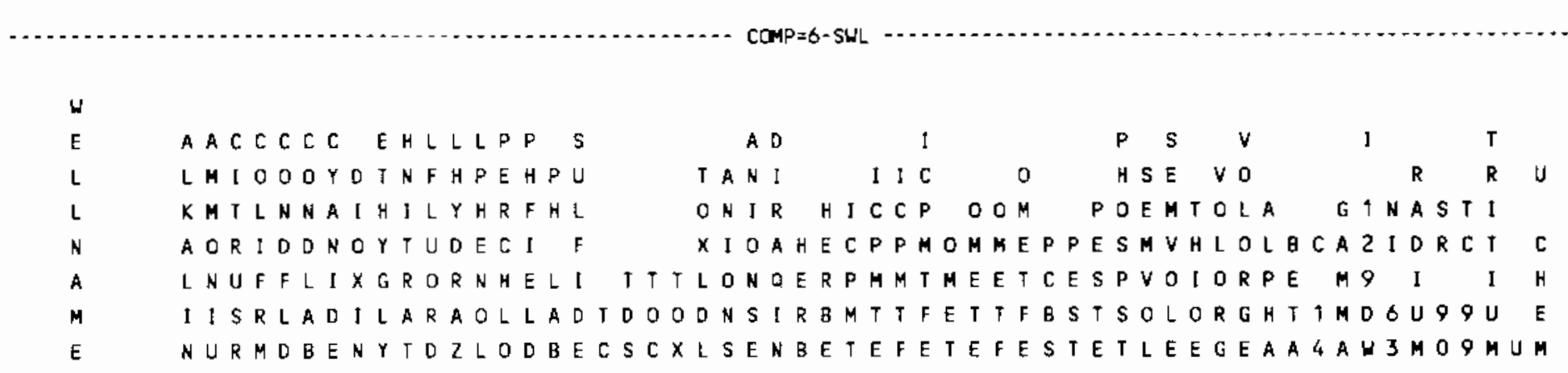

\begin{tabular}{|c|c|c|c|c|c|c|c|c|c|c|c|c|c|}
\hline $6-23-34$ & $x \times$ & $x \times x$ & $x$ & $x$ & $x \times$ & $x \times x$ & $x \times x$ & $x$ & $x \times x \times x$ & $x$ & $x$ & $x \times x \times$ & $x$ \\
\hline $6-24-34 A$ & $x \times$ & $x \times x$ & $x$ & $x$ & $x \times$ & $x \times x$ & $x \times x$ & $x$ & $x \times \times \times \times$ & $x$ & $x$ & $x \times x \times$ & $x$ \\
\hline $6-24-34 B$ & $x \times$ & $x \times x$ & $x$ & $x$ & $x \times$ & $x \times x$ & $x \times x$ & $x$ & $x \times \times \times x$ & $x$ & $x$ & $x \times x \times$ & $x$ \\
\hline $6-24-34 \mathrm{C}$ & $x \times$ & $x \times x$ & $x$ & $x$ & $x \times$ & $x \times x$ & $x \times x$ & $x$ & $x \times \times \times \times$ & $x$ & $x$ & $x \times x \times$ & $x$ \\
\hline $6-24-35$ & $x \times$ & $x \times x$ & $x$ & $x$ & $x \times$ & $x \times x$ & $x \times x$ & $x$ & $x \times x \times x$ & $x$ & $x$ & $\times \times \times \times$ & $x$ \\
\hline $6-25-34 \mathrm{C}$ & $x \times$ & $x \times x$ & $x$ & $x$ & $\times x$ & $x \times x$ & $x \times x$ & $x$ & $x \times \times \times \times$ & $x$ & $x$ & $x \times \times \times$ & $x$ \\
\hline
\end{tabular}


TABLE E.5. Results for Compliance Monitoring Wells

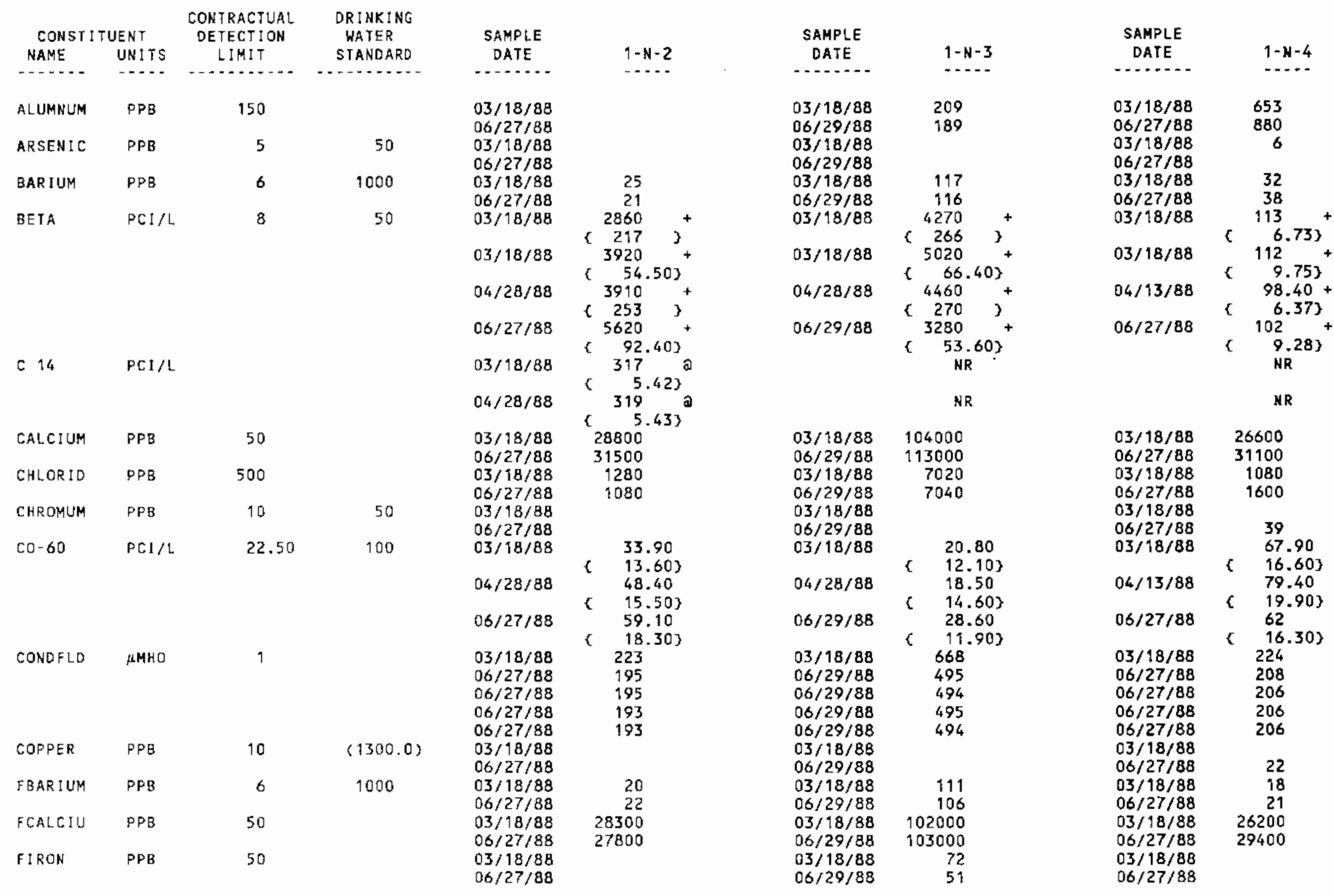


TABLE E.5. (contd)

\begin{tabular}{|c|c|c|c|c|c|c|c|c|c|c|}
\hline & CONSII & JENT & $\begin{array}{l}\text { CONTRACTUAL } \\
\text { DETECTION }\end{array}$ & $\begin{array}{l}\text { DRINKING } \\
\text { WATER }\end{array}$ & SAMPLE & & SAMPLE & & SAMPLE & \\
\hline & NAME & UNITS & L IMIT & STANDARD & DATE & $1-N-2$ & DATE & $1-N-3$ & DATE & $1-N-4$ \\
\hline & 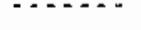 & -..... & & 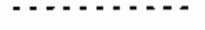 & $\cdots$ & & 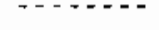 & & - nen & $-\cdots$ \\
\hline & FLUORID & PPB & 500 & 4000 & $\begin{array}{l}03 / 18 / 88 \\
06 / 27 / 88\end{array}$ & & $\begin{array}{l}03 / 18 / 88 \\
06 / 29 / 88\end{array}$ & 1610 & $\begin{array}{l}03 / 18 / 88 \\
06 / 27 / 88\end{array}$ & \\
\hline & FMAGNES & PPB & 50 & & $\begin{array}{l}03 / 18 / 88 \\
06 / 27 / 88\end{array}$ & $\begin{array}{l}4880 \\
4870\end{array}$ & $\begin{array}{l}03 / 18 / 88 \\
06 / 29 / 88\end{array}$ & $\begin{array}{l}16400 \\
16700\end{array}$ & $\begin{array}{l}03 / 18 / 88 \\
06 / 27 / 88\end{array}$ & $\begin{array}{l}4480 \\
5110\end{array}$ \\
\hline & FMANGAN & PPB & 5 & & $03 / 18 / 88$ & & $03 / 18 / 88$ & 10 & $03 / 18 / 88$ & 8 \\
\hline & FMANGAN & PPB & 5 & & $06 / 27 / 88$ & & $06 / 29 / 88$ & 19 & $06 / 27 / 88$ & 12 \\
\hline & FPOTASS & PPB & 100 & & $03 / 18 / 88$ & 1630 & $03 / 18 / 88$ & 3190 & $03 / 18 / 88$ & 2620 \\
\hline & & & & & $06 / 27 / 88$ & 1730 & $06 / 29 / 88$ & 3510 & $06 / 27 / 88$ & 2920 \\
\hline & FSODIUM & PPB & 200 & & $\begin{array}{l}03 / 18 / 88 \\
06 / 27 / 88\end{array}$ & $\begin{array}{l}2710 \\
3030\end{array}$ & $\begin{array}{l}03 / 18 / 88 \\
06 / 29 / 88\end{array}$ & $\begin{array}{l}13000 \\
15700\end{array}$ & $\begin{array}{l}03 / 18 / 88 \\
06 / 27 / 88\end{array}$ & $\begin{array}{l}3620 \\
4650\end{array}$ \\
\hline & FVANAD I & PPB & 5 & & $03 / 18 / 88$ & & $03 / 18 / 88$ & & $03 / 98 / 88$ & 6 \\
\hline & & & & & $06 / 27 / 88$ & 7 & $06 / 29 / 88$ & & $06 / 27 / 88$ & 9 \\
\hline & FZINC & PPB & 5 & & $03 / 98 / 88$ & 6 & $03 / 18 / 88$ & & $03 / 18 / 88$ & 11 \\
\hline & & & & & $06 / 27 / 88$ & 6 & $06 / 29 / 88$ & & $06 / 27 / 88$ & 9 \\
\hline & HNITRAT & PPB & 2500 & 45000 & $\begin{array}{l}03 / 18 / 88 \\
04 / 28 / 88\end{array}$ & $\begin{array}{l}38100 \\
34500\end{array}$ & & $\begin{array}{l}\text { NR } \\
\text { NR }\end{array}$ & $\begin{array}{l}03 / 18 / 88 \\
04 / 13 / 88\end{array}$ & 29900 \\
\hline & I $-1290 \mathrm{~W}$ & $P C I / L$ & 1 & & $04 / 28 / 88$ & 0.22 & & NR & & NR \\
\hline & & & & & & $0.24)$ & & & & \\
\hline- & IRON & PPB & 50 & & $03 / 18 / 88$ & 119 & $03 / 18 / 88$ & 2160 & $03 / 18 / 88$ & 5010 \\
\hline & MAGNES & PPB & 0 & & $03 / 18 / 88$ & 5010 & $03 / 18 / 88$ & 16600 & $03 / 18 / 88$ & 4670 \\
\hline & & & & & $06 / 27 / 88$ & 5090 & $06 / 29 / 88$ & 17400 & $06 / 27 / 88$ & 5360 \\
\hline & MANGESE & PPB & 5 & & $03 / 18 / 88$ & 6 & $03 / 18 / 88$ & 91 & $03 / 18 / 88$ & 120 \\
\hline & NILKEL & PPB & 10 & & $03 / 18 / 88$ & & $03 / 18 / 88$ & & $03 / 18 / 88$ & \\
\hline & & & & & $06 / 27 / 88$ & & $06 / 29 / 88$ & & $06 / 27 / 88$ & $\begin{array}{r}112 \\
712\end{array}$ \\
\hline & NITRATE & PPB & 500 & 45000 & $\begin{array}{l}03 / 18 / 88 \\
06 / 27 / 88\end{array}$ & $\begin{array}{l}38700 \\
33000\end{array}$ & $\begin{array}{l}03 / 18 / 88 \\
06 / 29 / 88\end{array}$ & $\begin{array}{l}28400 \\
25100\end{array}$ & $\begin{array}{l}03 / 18 / 88 \\
06 / 27 / 88\end{array}$ & $\begin{array}{l}32000 \\
26800\end{array}$ \\
\hline & PHFIELO & & 0.10 & & $03 / 18 / 88$ & 8 & $03 / 18 / 88$ & 7.70 & $03 / 18 / 88$ & 5.70 \\
\hline & & & & & $06 / 27 / 88$ & 7.10 & $06 / 29 / 88$ & 7.10 & $06 / 27 / 88$ & 7.90 \\
\hline & & & & & $06 / 27 / 88$ & 7.30 & $06 / 29 / 88$ & 7.20 & $06 / 27 / 88$ & 7.80 \\
\hline & & & & & $06 / 27 / 88$ & 7.30 & $06 / 29 / 88$ & 7.20 & $06 / 27 / 88$ & 7.80 \\
\hline & POTASIM & & 100 & & $00 / 27 / 88$ & 7.40 & $06 / 29 / 88$ & 7.20 & $06 / 27 / 88$ & 7.80 \\
\hline & PUTASUM & $P P B$ & 100 & & $\begin{array}{l}03 / 18 / 88 \\
06 / 27 / 88\end{array}$ & $\begin{array}{l}1690 \\
1820\end{array}$ & $\begin{array}{l}03 / 18 / 88 \\
06 / 29 / 88\end{array}$ & $\begin{array}{l}3250 \\
3620\end{array}$ & $\begin{array}{l}03 / 18 / 88 \\
06 / 27 / 88\end{array}$ & $\begin{array}{l}2670 \\
3050\end{array}$ \\
\hline & $R U=106$ & $P C 1 / L$ & 172.50 & 30 & $03 / 18 / 88$ & $76.70 *$ & $03 / 18 / 88$ & & $03 / 18 / 88$ & \\
\hline & & & & & $04 / 28 / 88$ & & $04 / 28 / 88$ & & $04 / 13 / 88$ & \\
\hline & & & & & $06 / 27 / 88$ & $\left\{\begin{array}{l}95.90 * \\
60.10\}\end{array}\right.$ & $06 / 29 / 88$ & & $06 / 27 / 88$ & \\
\hline & SODIUM & PPB & 200 & & $03 / 18 / 88$ & 2750 & $03 / 18 / 88$ & 13000 & $03 / 18 / 88$ & 3530 \\
\hline & & & & & $06 / 27 / 88$ & 3100 & $06 / 29 / 88$ & 15700 & $06 / 27 / 88$ & 4220 \\
\hline & SR 90 & $\mathrm{PCI} / \mathrm{L}$ & 5 & 8 & $03 / 18 / 88$ & 1640 & $03 / 18 / 88$ & 2290 & $03 / 18 / 88$ & 12 \\
\hline & & & & & & < 144 & & 240 & & 1.88 \\
\hline
\end{tabular}


TABLE E.5. (contd)

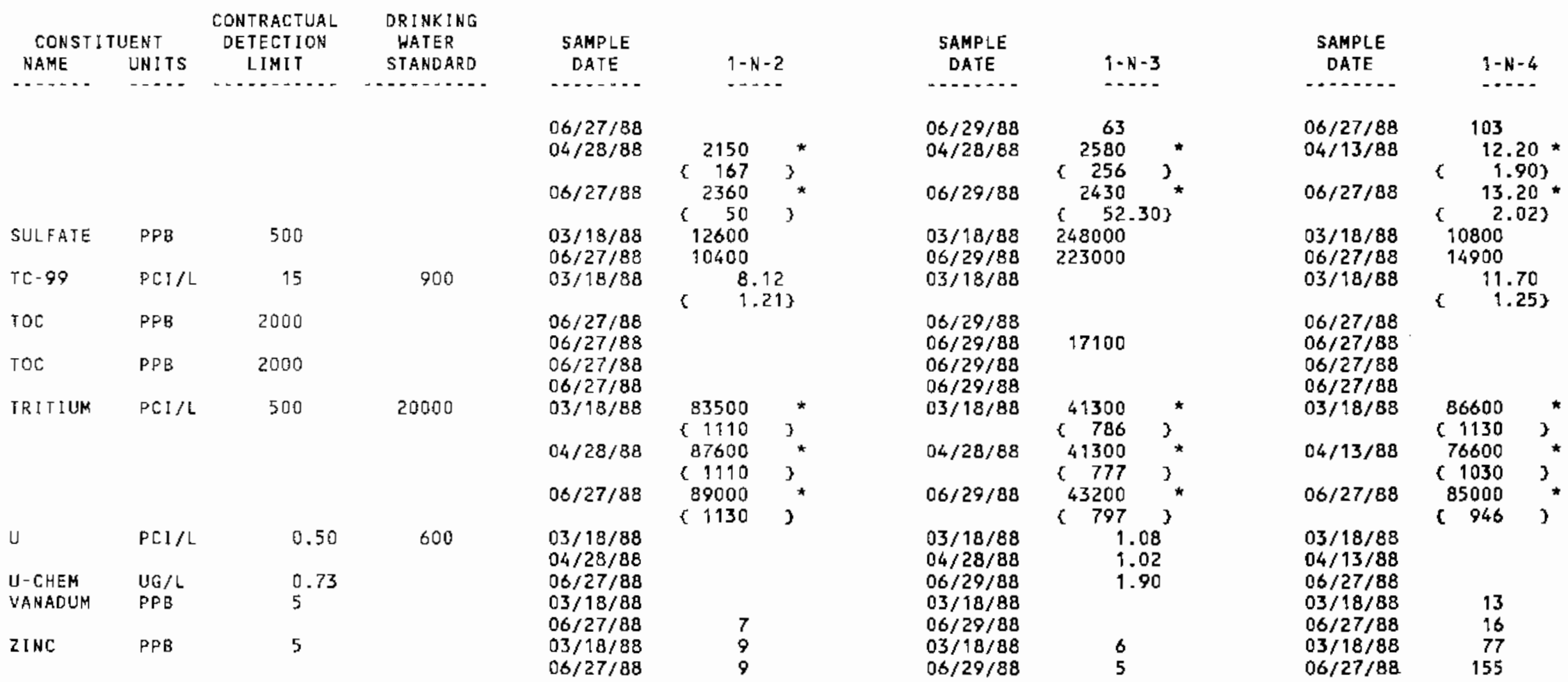


TABLE E.5. (contd)

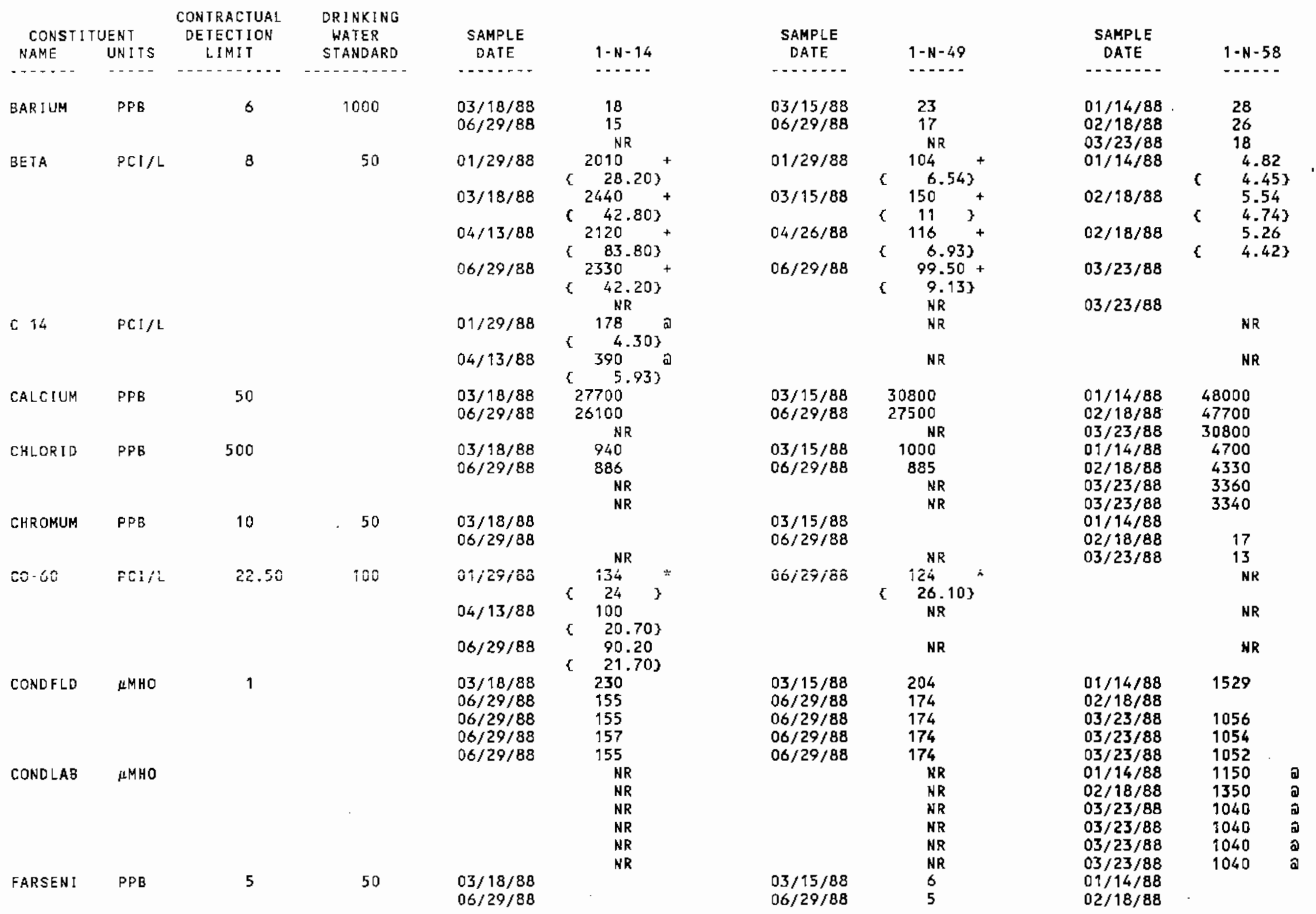


TABLE E.5. (contd)

\begin{tabular}{|c|c|c|c|c|c|c|c|c|c|}
\hline \multicolumn{2}{|c|}{ CONSTITUENT } & $\begin{array}{l}\text { CONTRACTURL } \\
\text { DETECTION }\end{array}$ & DRINKING & SAMDIE & & & & & \\
\hline NAME & UNITS & LIMIT & STANDARO & $\begin{array}{l}\text { SAMPLE } \\
\text { DATE }\end{array}$ & $1-N-14$ & $\begin{array}{l}\text { SAMPLE } \\
\text { DATE }\end{array}$ & $1-k-49$ & $\begin{array}{l}\text { SAAPLE } \\
\text { DATE }\end{array}$ & $1-N-58$ \\
\hline \multirow{3}{*}{$-\ldots \ldots$} & $\cdots$ & $\ldots$ & $\ldots \ldots \ldots$ & $\ldots$ & $\ldots . .$. & $\ldots$ & $\cdots$ & $\ldots$ & $\ldots \ldots$ \\
\hline & & & & & NR & & NR & $02 / 18 / 88$ & \\
\hline & & & & & NR & & NR & $03 / 23 / 88$ & \\
\hline FARSEN! & $P P B$ & 5 & 50 & & NR & & NR & $03 / 23 / 88$ & \\
\hline \multirow[t]{4}{*}{ FBAR I UM } & PPB & 6 & 1000 & $\begin{array}{l}03 / 18 / 88 \\
06 / 29 / 88\end{array}$ & $\begin{array}{l}15 \\
17\end{array}$ & $\begin{array}{l}03 / 15 / 88 \\
06 / 29 / 88\end{array}$ & $\begin{array}{l}20 \\
22\end{array}$ & $\begin{array}{l}01 / 14 / 88 \\
02 / 18 / 88\end{array}$ & $\begin{array}{l}29 \\
26\end{array}$ \\
\hline & & & & & NR & & NR & $02 / 18 / 88$ & 26 \\
\hline & & & & & NR & & NR & $03 / 23 / 88$ & 18 \\
\hline & & & & & NR & & NR & $03 / 23 / 88$ & \\
\hline \multirow[t]{4}{*}{ FCALCIU } & PPB & 50 & & $\begin{array}{l}03 / 18 / 88 \\
06 / 29 / 88\end{array}$ & $\begin{array}{l}29800 \\
25300\end{array}$ & $\begin{array}{l}03 / 15 / 88 \\
06 / 29 / 88\end{array}$ & $\begin{array}{l}30700 \\
30900\end{array}$ & $\begin{array}{l}01 / 14 / 88 \\
02 / 18 / 88\end{array}$ & $\begin{array}{l}49500 \\
48700\end{array}$ \\
\hline & & & & & NR & & NR & $02 / 18 / 88$ & 47600 \\
\hline & & & & & NR & & NR & $03 / 23 / 88$ & 29100 \\
\hline & & & & & NR & & NR & $03 / 23 / 88$ & 32100 \\
\hline \multirow[t]{4}{*}{ FCHROMI } & $\mathrm{PPB}$ & 10 & 50 & $\begin{array}{l}03 / 18 / 88 \\
06 / 29 / 88\end{array}$ & & $\begin{array}{l}03 / 15 / 88 \\
06 / 29 / 88\end{array}$ & & $\begin{array}{l}01 / 14 / 88 \\
02 / 18 / 88\end{array}$ & \\
\hline & & & & & NR & & HR & $02 / 18 / 88$ & 14 \\
\hline & & & & & NR & & NR & $03 / 23 / 88$ & \\
\hline & & & & & NR & & NR & $03 / 23 / 88$ & \\
\hline \multirow[t]{4}{*}{ FIRON } & $P P B$ & 50 & & $\begin{array}{l}03 / 18 / 88 \\
06 / 29 / 88\end{array}$ & & $\begin{array}{l}03 / 15 / 88 \\
06 / 29 / 88\end{array}$ & & $\begin{array}{l}01 / 14 / 88 \\
02 / 18 / 88\end{array}$ & \\
\hline & & & & & NR & & NR & $02 / 18 / 88$ & 61 \\
\hline & & & & & NR & & NR & $03 / 23 / 88$ & \\
\hline & & & & & $\mathrm{NR}$ & & NR & $03 / 23 / 88$ & \\
\hline \multirow[t]{2}{*}{ FLUORID } & PPB & 500 & 4000 & $\begin{array}{l}03 / 18 / 88 \\
06 / 29 / 88\end{array}$ & & $\begin{array}{l}03 / 15 / 88 \\
06 / 29 / 88\end{array}$ & & $\begin{array}{l}01 / 14 / 88 \\
02 / 18 / 88\end{array}$ & 1280 \\
\hline & & & & & $\begin{array}{l}\text { NR } \\
\text { NR }\end{array}$ & & $N R$ & $03 / 23 / 88$ & 1050 \\
\hline \multirow{5}{*}{ FMAGNES } & & & & & & & NR & $03 / 23 / 88$ & 1090 \\
\hline & PPB & 50 & & $\begin{array}{l}03 / 18 / 88 \\
06 / 29 / 88\end{array}$ & $\begin{array}{l}4820 \\
4110\end{array}$ & $\begin{array}{l}03 / 15 / 88 \\
06 / 29 / 88\end{array}$ & $\begin{array}{l}6140 \\
6280\end{array}$ & $\begin{array}{l}01 / 14 / 88 \\
02 / 18 / 88\end{array}$ & $\begin{array}{l}9280 \\
9330\end{array}$ \\
\hline & & & & & NR & & NR & $02 / 18 / 88$ & 9180 \\
\hline & & & & & NR & & NR & $03 / 23 / 88$ & 5650 \\
\hline & & & & & $N R$ & & NR & $03 / 23 / 88$ & 6110 \\
\hline \multirow[t]{3}{*}{ FNICKEL } & PPB & 10 & & $\begin{array}{l}03 / 18 / 88 \\
06 / 29 / 88\end{array}$ & & $\begin{array}{l}03 / 15 / 88 \\
06 / 29 / 88\end{array}$ & & $\begin{array}{l}01 / 14 / 88 \\
02 / 18 / 88\end{array}$ & \\
\hline & & & & & NR & & NR & $02 / 18 / 88$ & 10 \\
\hline & & & & & $\begin{array}{l}\text { NR } \\
\text { NR }\end{array}$ & & NR & $03 / 23 / 88$ & \\
\hline \multirow[t]{4}{*}{ FPOTASS } & PPB & 100 & & $03 / 18 / 88$ & 1690 & & NR & $03 / 23 / 88$ & \\
\hline & & & & $06 / 29 / 88$ & $\begin{array}{l}1690 \\
1580\end{array}$ & $\begin{array}{l}03 / 15 / 88 \\
06 / 29 / 88\end{array}$ & $\begin{array}{l}2910 \\
3180\end{array}$ & $\begin{array}{l}01 / 14 / 88 \\
02 / 18 / 88\end{array}$ & $\begin{array}{l}3760 \\
3260\end{array}$ \\
\hline & & & & & $\begin{array}{l}\text { NR } \\
\text { NR }\end{array}$ & & $\begin{array}{l}\text { NR } \\
\text { NR }\end{array}$ & $\begin{array}{l}02 / 18 / 88 \\
03 / 23 / 88\end{array}$ & $\begin{array}{l}3130 \\
2410\end{array}$ \\
\hline & & & & & NR & & $\mathrm{HR}$ & $03 / 23 / 88$ & 2590 \\
\hline FSODIUM & PPB & 200 & & $\begin{array}{l}03 / 18 / 88 \\
06 / 29 / 88\end{array}$ & $\begin{array}{l}3550 \\
3120\end{array}$ & $\begin{array}{l}03 / 15 / 88 \\
06 / 29 / 88\end{array}$ & $\begin{array}{l}4320 \\
4460\end{array}$ & $\begin{array}{l}01 / 14 / 88 \\
02 / 18 / 88\end{array}$ & $\begin{array}{l}285000 \\
250000\end{array}$ \\
\hline
\end{tabular}


TABLE E.5. (contd)

\begin{tabular}{|c|c|c|c|c|c|}
\hline \multicolumn{2}{|c|}{ CONST I TUENT } & $\begin{array}{l}\text { CONTRACTUAL } \\
\text { DETECTION }\end{array}$ & $\begin{array}{l}\text { DRINKING } \\
\text { WATER }\end{array}$ & $\begin{array}{l}\text { SAMPLE } \\
\text { DAIE }\end{array}$ & \\
\hline NAME & UN I TS & LIMIT & STANDARD & DATE & $1-N-14$ \\
\hline & & & & & $\begin{array}{l}N R \\
N R\end{array}$ \\
\hline FVANADI & PPB & 5 & & $\begin{array}{l}03 / 18 / 88 \\
06 / 29 / 88\end{array}$ & $\begin{array}{l}6 \\
6 \\
\text { NR } \\
\text { NR }\end{array}$ \\
\hline FYANAD 1 & PPB & 5 & & & NR \\
\hline FZINC & PPB & 5 & & $\begin{array}{l}03 / 18 / 88 \\
06 / 29 / 88\end{array}$ & $\begin{array}{l}12 \\
11\end{array}$ \\
\hline & & & & & $\begin{array}{l}\text { NR } \\
\text { NR }\end{array}$ \\
\hline & & & & & NR \\
\hline HNITRAT & $P P B$ & 2500 & 45000 & $\begin{array}{l}01 / 29 / 88 \\
04 / 13 / 88\end{array}$ & $\begin{array}{l}38600 \\
35100\end{array}$ \\
\hline $1-1290 \mathrm{~W}$ & $P C I / L$ & 1 & & $04 / 13 / 88$ & $\begin{array}{ll} & 0.20 \\
\{\quad 0.17\}\end{array}$ \\
\hline IRON & PPB & 50 & & $\begin{array}{l}03 / 18 / 88 \\
06 / 29 / 88\end{array}$ & $\begin{array}{c}650 \\
55 \\
N R\end{array}$ \\
\hline LEADGF & PPB & 5 & 50 & $\begin{array}{l}03 / 18 / 88 \\
06 / 29 / 88\end{array}$ & \\
\hline MAGNES & PPB & 0 & & $\begin{array}{l}03 / 18 / 88 \\
06 / 29 / 88\end{array}$ & $\begin{array}{l}\text { NR } \\
4500 \\
4360\end{array}$ \\
\hline MANGESE & PPB & 5 & & $\begin{array}{l}03 / 18 / 88 \\
06 / 29 / 88\end{array}$ & $\underset{i B}{N R}$ \\
\hline$N I-63$ & $\mathrm{PCl} / \mathrm{L}$ & 10 & 50 & $01 / 29 / 88$ & $\begin{array}{l}\text { NR } \\
6.38 \\
\{.82\}\end{array}$ \\
\hline HICKEL & PPB & 10 & & $\begin{array}{l}04 / 13 / 88 \\
03 / 18 / 88 \\
06 / 29 / 88\end{array}$ & \\
\hline NITRATE & PPB & 500 & 45000 & $\begin{array}{l}03 / 18 / 88 \\
06 / 29 / 88\end{array}$ & $\begin{array}{l}\quad \text { NR } \\
39700 \\
34900\end{array}$ \\
\hline & & & & & NR \\
\hline$P H-L A B$ & & 0.01 & & & $\begin{array}{l}\text { NR } \\
\text { NR } \\
\text { NR } \\
\text { NR } \\
\text { NR } \\
\text { NR }\end{array}$ \\
\hline
\end{tabular}

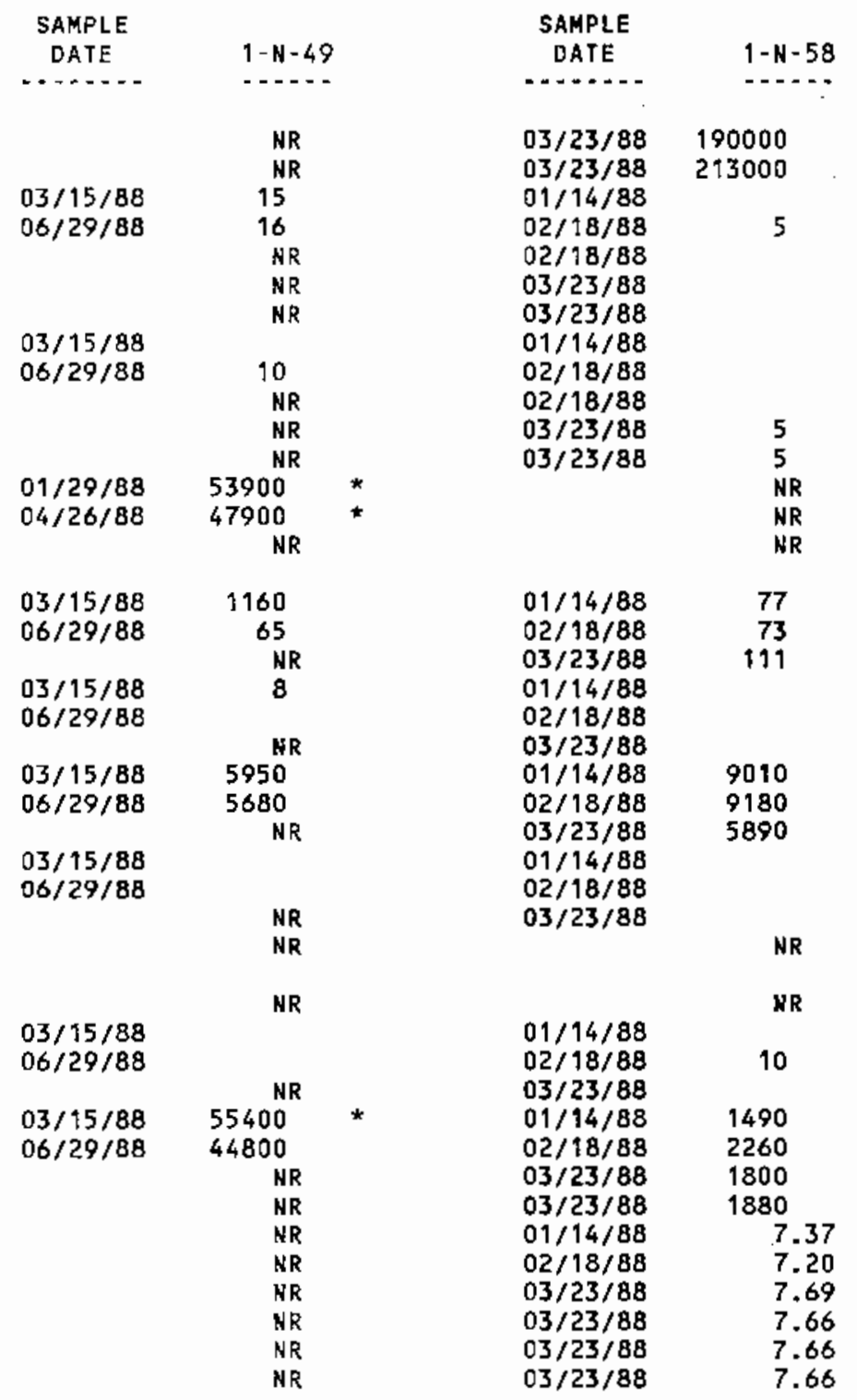


TABLE E.5. (contd)

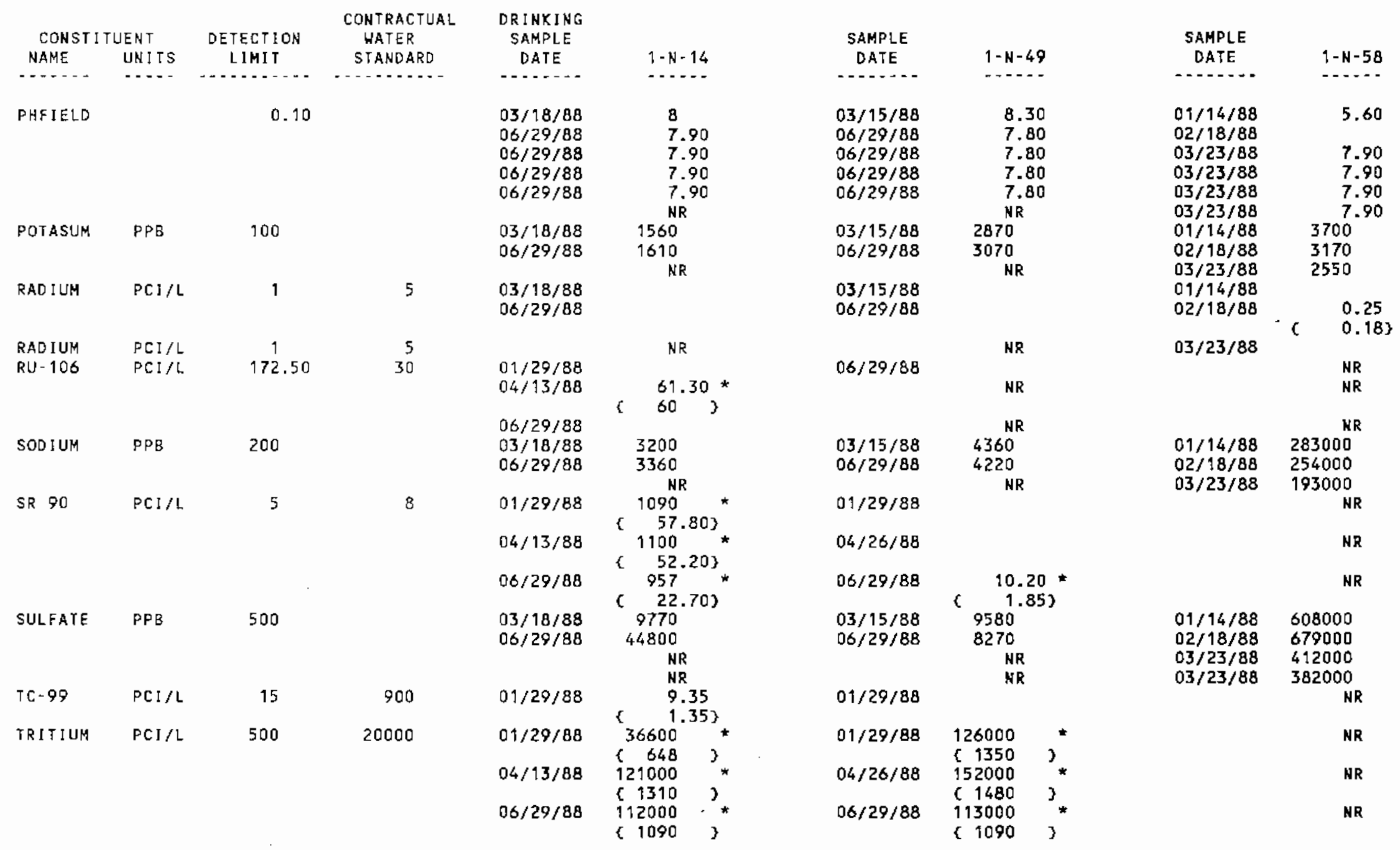


TABLE E.5. (contd)

\begin{tabular}{|c|c|c|c|c|c|}
\hline \multicolumn{2}{|c|}{ CONSTITUENT } & DETECTION & $\begin{array}{l}\text { CONTRACTUAL } \\
\text { WATER }\end{array}$ & $\begin{array}{l}\text { DR INKING } \\
\text { SAMPLE }\end{array}$ & \\
\hline NAME & UNITS & LINIT & STANDARD & DATE & $1-\mathrm{N}-14$ \\
\hline & & & & & \\
\hline $\mathrm{u}$ & $\mathrm{PCI} / \mathrm{L}$ & 0.50 & 600 & $\begin{array}{l}01 / 29 / 88 \\
04 / 13 / 88\end{array}$ & \\
\hline VANADUM & PPB & 5 & & $\begin{array}{l}03 / 18 / 88 \\
06 / 29 / 88\end{array}$ & $\begin{array}{l}7 \\
6 \\
\text { NR }\end{array}$ \\
\hline ZINC & PPB & 5 & & $\begin{array}{l}03 / 18 / 88 \\
06 / 29 / 88\end{array}$ & $\frac{38}{7}$ \\
\hline
\end{tabular}

$\begin{array}{lcc}\text { SAMPLE } & \\ \text { DATE } & 1-\mathrm{N}-49 \\ & \cdots & \cdots \\ & 01 / 29 / 88 & 0.65 \\ 7 & 04 / 26 / 88 & \\ 6 & 03 / 15 / 88 & 16 \\ \text { NR } & 06 / 29 / 88 & 10 \\ 38 & & \text { NR } \\ 7 & 03 / 15 / 88 & 17 \\ \text { NR } & 06 / 29 / 88 & 6 \\ & & \text { NR }\end{array}$

SAMPLE

$\begin{array}{ll}\text { DATE } & 1-N-58 \\ & \end{array}$

$01 / 14 / 88$
$02 / 18 / 88$
$03 / 23 / 88$
$01 / 14 / 88$
$02 / 18 / 88$
$03 / 23 / 88$ 
TABLE E.5. (contd)

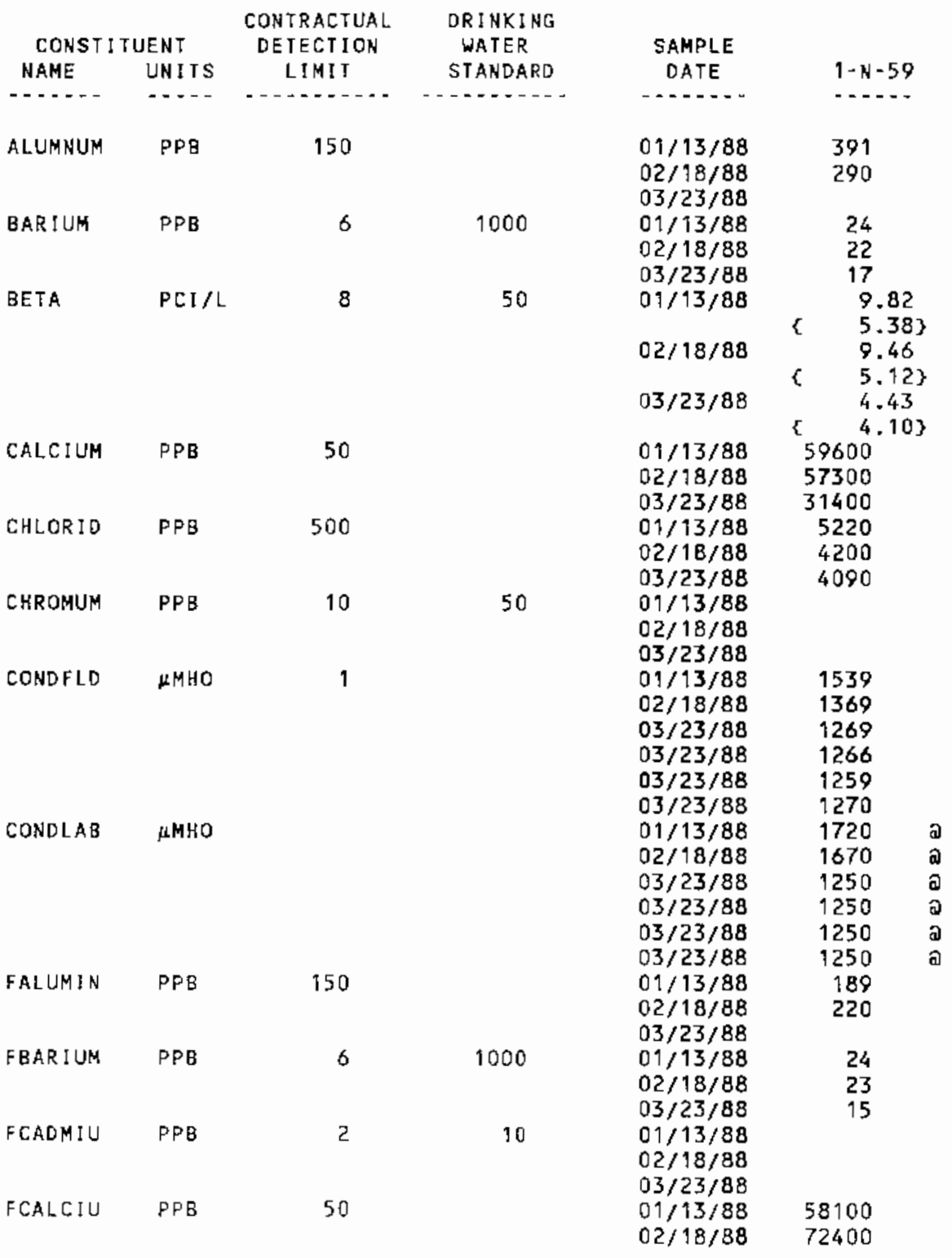

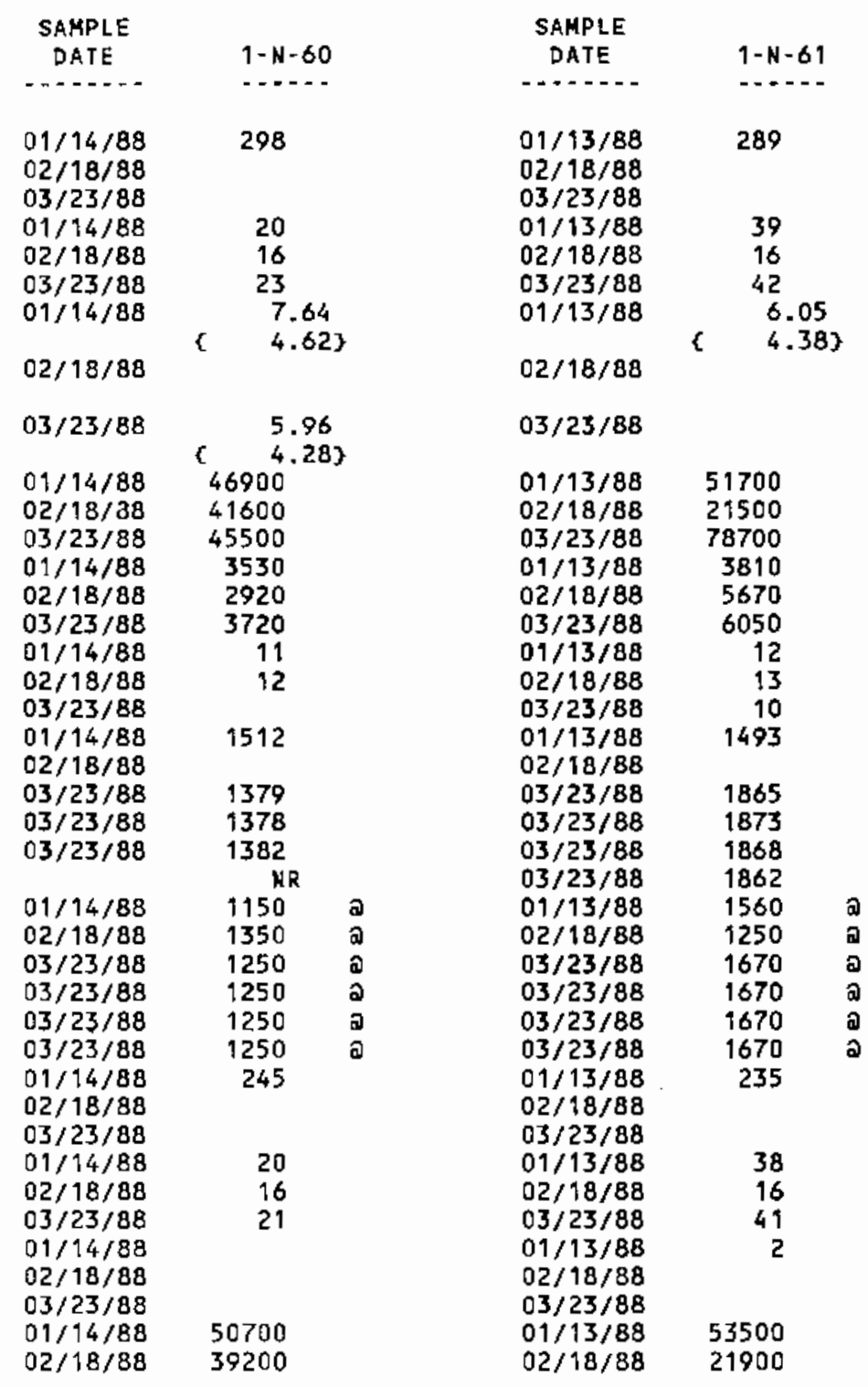


IABLE E.5. (contd)

\begin{tabular}{|c|c|c|c|c|c|c|c|c|c|}
\hline \multicolumn{2}{|c|}{ CONST I TUENT } & $\begin{array}{l}\text { CONTRACIUAL } \\
\text { DETECTION }\end{array}$ & $\begin{array}{l}\text { DRINKING } \\
\text { HATER }\end{array}$ & SAMPLE & & SAMPLE & & SAMPLE & \\
\hline NAME & UNITS & LIMIT & STANDARD & DATE & $1-N-59$ & DATE & $1-N-60$ & DATE & $9-N-61$ \\
\hline$\cdots+-\infty$ & $\cdots$ & 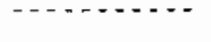 & - n- & $\cdots$ & $\cdots+\infty$ & $\cdots$ & n...... & 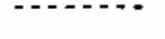 & - - \\
\hline FIRON & $P P B$ & 50 & & $\begin{array}{l}03 / 23 / 88 \\
01 / 13 / 88 \\
02 / 18 / 88 \\
03 / 23 / 88\end{array}$ & 35100 & $\begin{array}{l}03 / 23 / 88 \\
01 / 14 / 88 \\
02 / 18 / 88 \\
03 / 23 / 88\end{array}$ & 47500 & $\begin{array}{l}03 / 23 / 88 \\
01 / 13 / 88 \\
02 / 18 / 88 \\
03 / 23 / 88\end{array}$ & $\begin{array}{c}75800 \\
68\end{array}$ \\
\hline FLUORID & PPA & 500 & 4000 & $\begin{array}{l}01 / 13 / 88 \\
02 / 18 / 88 \\
03 / 23 / 88\end{array}$ & $\begin{array}{r}1460 \\
590 \\
1030\end{array}$ & $\begin{array}{l}01 / 14 / 88 \\
02 / 18 / 88 \\
03 / 23 / 88\end{array}$ & $\begin{array}{l}1300 \\
1110\end{array}$ & $\begin{array}{l}01 / 13 / 88 \\
02 / 18 / 88 \\
03 / 23 / 88\end{array}$ & $\begin{array}{l}1480 \\
1560\end{array}$ \\
\hline FMAGNES & PPB & 50 & & $\begin{array}{l}01 / 13 / 88 \\
02 / 18 / 88 \\
03 / 23 / 88\end{array}$ & $\begin{array}{r}13300 \\
13600 \\
7370\end{array}$ & $\begin{array}{l}01 / 14 / 88 \\
02 / 18 / 88 \\
03 / 23 / 88\end{array}$ & $\begin{array}{r}12300 \\
8680 \\
9080\end{array}$ & $\begin{array}{l}01 / 13 / 88 \\
02 / 18 / 88 \\
03 / 23 / 88\end{array}$ & $\begin{array}{r}13200 \\
5250 \\
15700\end{array}$ \\
\hline FMANGAN & PPB & 5 & & $\begin{array}{l}01 / 13 / 88 \\
02 / 18 / 88 \\
03 / 23 / 88\end{array}$ & $\begin{array}{r}216 \\
172 \\
42\end{array}$ & $\begin{array}{l}01 / 14 / 88 \\
02 / 18 / 88 \\
03 / 23 / 88\end{array}$ & $\begin{array}{r}230 \\
95\end{array}$ & $\begin{array}{l}01 / 13 / 88 \\
02 / 18 / 88 \\
03 / 23 / 88\end{array}$ & $\begin{array}{r}116 \\
29 \\
62\end{array}$ \\
\hline FNI CKE L & PPB & 10 & & $\begin{array}{l}01 / 13 / 88 \\
02 / 18 / 88 \\
03 / 23 / 88\end{array}$ & $\begin{array}{l}10 \\
12\end{array}$ & $\begin{array}{l}01 / 14 / 88 \\
02 / 18 / 88 \\
03 / 23 / 88\end{array}$ & 11 & $\begin{array}{l}01 / 13 / 88 \\
02 / 18 / 88 \\
03 / 23 / 88\end{array}$ & \\
\hline FPOTASS & PPB & 100 & & $\begin{array}{l}01 / 13 / 88 \\
02 / 18 / 88 \\
03 / 23 / 88\end{array}$ & $\begin{array}{l}4010 \\
4080 \\
3340\end{array}$ & $\begin{array}{l}01 / 14 / 88 \\
02 / 18 / 88 \\
03 / 23 / 88\end{array}$ & $\begin{array}{l}3450 \\
2840 \\
4360\end{array}$ & $\begin{array}{l}01 / 13 / 88 \\
02 / 18 / 88 \\
03 / 23 / 88\end{array}$ & $\begin{array}{l}2510 \\
1590 \\
2400\end{array}$ \\
\hline FSOD IUM & $P P B$ & 200 & & $\begin{array}{l}01 / 13 / 88 \\
02 / 18 / 88 \\
03 / 23 / 88\end{array}$ & $\begin{array}{l}315000 \\
361000 \\
237000\end{array}$ & $\begin{array}{l}01 / 14 / 88 \\
02 / 18 / 88 \\
03 / 23 / 88\end{array}$ & $\begin{array}{l}272000 \\
253000 \\
256000\end{array}$ & $\begin{array}{l}01 / 13 / 88 \\
02 / 18 / 88 \\
03 / 23 / 88\end{array}$ & $\begin{array}{l}280000 \\
273000 \\
322000\end{array}$ \\
\hline FVANAD I & PPB & 5 & & $\begin{array}{l}01 / 13 / 88 \\
02 / 18 / 88 \\
03 / 23 / 88\end{array}$ & & $\begin{array}{l}01 / 14 / 88 \\
02 / 18 / 88 \\
03 / 23 / 88\end{array}$ & . & $\begin{array}{l}01 / 13 / 88 \\
02 / 18 / 88 \\
03 / 23 / 88\end{array}$ & \\
\hline FZINC & PPB & 5 & & $\begin{array}{l}01 / 13 / 88 \\
02 / 18 / 88 \\
03 / 23 / 88\end{array}$ & $\begin{array}{r}24 \\
18 \\
8\end{array}$ & $\begin{array}{l}01 / 14 / 88 \\
02 / 18 / 88 \\
03 / 23 / 88\end{array}$ & $\begin{array}{r}11 \\
5\end{array}$ & $\begin{array}{l}01 / 13 / 88 \\
02 / 18 / 88 \\
03 / 23 / 88\end{array}$ & $\begin{array}{r}18 \\
6 \\
9\end{array}$ \\
\hline IRON & PPB & 50 & & $\begin{array}{l}01 / 13 / 88 \\
02 / 18 / 88 \\
03 / 23 / 88\end{array}$ & 57 & $\begin{array}{l}01 / 14 / 88 \\
02 / 18 / 88 \\
03 / 23 / 88\end{array}$ & $\begin{array}{l}92 \\
82\end{array}$ & $\begin{array}{l}01 / 13 / 88 \\
02 / 18 / 88 \\
03 / 23 / 88\end{array}$ & $\begin{array}{r}208 \\
84 \\
195\end{array}$ \\
\hline MAGNES & $\mathrm{PPB}$ & 0 & & $\begin{array}{l}01 / 13 / 88 \\
02 / 18 / 88 \\
03 / 23 / 88\end{array}$ & $\begin{array}{r}13600 \\
12800 \\
6660\end{array}$ & $\begin{array}{l}01 / 14 / 88 \\
02 / 18 / 88 \\
03 / 23 / 88\end{array}$ & $\begin{array}{r}11700 \\
9020 \\
8680\end{array}$ & $\begin{array}{l}01 / 13 / 88 \\
02 / 18 / 88 \\
03 / 23 / 88\end{array}$ & $\begin{array}{r}13200 \\
5090 \\
16000\end{array}$ \\
\hline HANGESE & PPB & 5 & & $\begin{array}{l}01 / 13 / 88 \\
02 / 18 / 88 \\
03 / 23 / 88\end{array}$ & $\begin{array}{r}196 \\
167 \\
43\end{array}$ & $\begin{array}{l}01 / 14 / 88 \\
02 / 18 / 88 \\
03 / 23 / 88\end{array}$ & $\begin{array}{l}213 \\
101\end{array}$ & $\begin{array}{l}01 / 13 / 88 \\
02 / 18 / 88 \\
03 / 23 / 88\end{array}$ & $\begin{array}{l}97 \\
29 \\
64\end{array}$ \\
\hline NI CKEL & PPB & 10 & & $\begin{array}{l}01 / 13 / 88 \\
02 / 18 / 88 \\
03 / 23 / 88\end{array}$ & 11 & $\begin{array}{l}01 / 14 / 88 \\
02 / 18 / 88 \\
03 / 23 / 88\end{array}$ & $\begin{array}{l}11 \\
10\end{array}$ & $\begin{array}{l}01 / 13 / 88 \\
02 / 18 / 88 \\
03 / 23 / 88\end{array}$ & \\
\hline NITRATE & $\mathrm{PPB}$ & 500 & 45000 & $\begin{array}{l}01 / 13 / 88 \\
02 / 18 / 88 \\
03 / 23 / 88\end{array}$ & $\begin{array}{l}1890 \\
2210 \\
2120\end{array}$ & $\begin{array}{l}01 / 14 / 88 \\
02 / 18 / 88 \\
03 / 23 / 88\end{array}$ & $\begin{array}{l}1570 \\
2120 \\
1960\end{array}$ & $\begin{array}{l}01 / 13 / 88 \\
02 / 18 / 88 \\
03 / 23 / 88\end{array}$ & $\begin{array}{l}1610 \\
2770 \\
2340\end{array}$ \\
\hline
\end{tabular}


TABLEE E.5. (contd)

\begin{tabular}{|c|c|c|c|c|c|c|c|c|c|}
\hline \multicolumn{2}{|c|}{ CONSTI I TUENT } & $\begin{array}{l}\text { CONTRACTUAL } \\
\text { DETECTION }\end{array}$ & $\begin{array}{l}\text { DRINKING } \\
\text { WATER }\end{array}$ & SAMPLE & & SAMPLE & & SAMPLE & \\
\hline NAME & UNI IS & LIMIT & STANDARD & DATE & $1-N-59$ & DATE & $1-N-60$ & DATE & $1-N-61$ \\
\hline 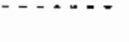 & $\cdots$ & $\ldots$ & , n & $\cdots \cdots$ & $\cdots \cdots$ & $\cdots$ & $\cdots-\cdots$ & $\cdots$ & $\cdots$ \\
\hline \multirow[t]{5}{*}{$P H-L A B$} & & 0.01 & & $01 / 13 / 88$ & 6.59 & $01 / 14 / 88$ & 5.87 & $01 / 13 / 88$ & 5.54 \\
\hline & & & & $\begin{array}{l}02 / 18 / 88 \\
03 / 23 / 88\end{array}$ & $\begin{array}{l}6.07 \\
6.64\end{array}$ & $\begin{array}{l}02 / 18 / 88 \\
03 / 23 / 88\end{array}$ & $\begin{array}{l}5.95 \\
7.77\end{array}$ & $02 / 18 / 88$ & 64 \\
\hline & & & & $03 / 23 / 88$ & 6.71 & $03 / 23 / 88$ & 7.73 & $\begin{array}{l}03 / 23 / 88 \\
03 / 23 / 88\end{array}$ & $\begin{array}{l}6.41 \\
6.41\end{array}$ \\
\hline & & & & $03 / 23 / 88$ & 6.69 & $03 / 23 / 88$ & 7.68 & $03 / 23 / 88$ & 6.48 \\
\hline & & & & $03 / 23 / 88$ & 6.71 & $03 / 23 / 88$ & 7.74 & $03 / 23 / 88$ & 6.64 \\
\hline \multirow[t]{2}{*}{ PHFIELD } & & 0.10 & & $\begin{array}{l}01 / 13 / 88 \\
02 / 18 / 88\end{array}$ & 5.60 & $\begin{array}{l}01 / 14 / 88 \\
02 / 18 / 88\end{array}$ & 3.70 & $\begin{array}{l}01 / 13 / 88 \\
02 / 18 / 8 B\end{array}$ & 5.10 \\
\hline & & & & $03 / 23 / 88$ & 6.60 & $03 / 23 / 88$ & 8.20 & $03 / 23 / 88$ & 6.40 \\
\hline \multirow[t]{3}{*}{ PHF IELO } & & 0.10 & & $03 / 23 / 88$ & 6.60 & $03 / 23 / 88$ & 8.10 & $03 / 23 / 88$ & 6.40 \\
\hline & & & & $03 / 23 / 83$ & 6.60 & $03 / 23 / 88$ & 8.10 & $03 / 23 / 88$ & 6.40 \\
\hline & & & & $03 / 23 / 88$ & 6.60 & $03 / 23 / 88$ & 8.10 & $03 / 23 / 88$ & 6.40 \\
\hline \multirow[t]{2}{*}{ POTASUM } & PPB & 100 & & $01 / 13 / 88$ & 4320 & $01 / 14 / 88$ & 3370 & $01 / 13 / 88$ & 2580 \\
\hline & & & & $02 / 18 / 88$ & 3790 & $02 / 18 / 88$ & 2920 & $02 / 18 / 88$ & 1530 \\
\hline \multirow[t]{4}{*}{ RAD!UM } & PC!/L, & $\hat{j}$ & 5 & $\begin{array}{l}03 / 23 / 88 \\
01 / 13 / 88\end{array}$ & 3020 & $\begin{array}{l}03 / 23 / 68 \\
01 / 14 / 88\end{array}$ & 4250 & $\begin{array}{l}03 / 23 / 88 \\
01 / 13 / 88\end{array}$ & 2430 \\
\hline & & & & $02 / 18 / 88$ & 0.32 & $02 / 18 / 88$ & 0.29 & $02 / 18 / 88$ & \\
\hline & & & & & 0.173 & & 0.173 & & \\
\hline & & & & $05 / 23 / 88$ & & $03 / 23 / 88$ & & $03 / 23 / 88$ & $\begin{array}{l}0.16 \\
0.153\end{array}$ \\
\hline \multirow[t]{3}{*}{ SODIUM } & PPB & 200 & & $01 / 13 / 88$ & 307000 & $01 / 14 / 88$ & 267000 & $01 / 13 / 88$ & 295000 \\
\hline & & & & $02 / \uparrow 8 / 88$ & 351000 & $02 / 18 / 88$ & 271000 & $02 / 18 / 88$ & 251000 \\
\hline & & & & $03 / 23 / 88$ & 234000 & $03 / 23 / 88$ & 247000 & $03 / 23 / 88$ & 347000 \\
\hline \multirow{2}{*}{ SULSATE } & $P P B$ & 500 & & $\begin{array}{l}01 / 13 / 88 \\
02 / 18 / 88\end{array}$ & $\begin{array}{l}833000 \\
904000\end{array}$ & $\begin{array}{l}01 / 14 / 88 \\
07 / 18 / 88\end{array}$ & $\begin{array}{l}681000 \\
736000\end{array}$ & $01 / 13 / 88$ & 792000 \\
\hline & & & & & 534000 & $03 / 23 / 88$ & $\begin{array}{l}736000 \\
519000\end{array}$ & $02 / 18 / 88$ & $\begin{array}{l}669000 \\
910000\end{array}$ \\
\hline \multirow[t]{3}{*}{ VANADU: } & $P P B$ & $\overline{5}$ & & $01 / 13 / 88$ & & $01 / 14 / 88$ & & $\begin{array}{l}03 / 23 / 88 \\
01 / 13 / 88\end{array}$ & 870000 \\
\hline & & & & $02 / 18 / 88$ & & $02 / 18 / 88$ & & $02 / 18 / 8 B$ & \\
\hline & & & & $03 / 23 / 88$ & & $03 / 23 / 88$ & 6 & $03 / 23 / 88$ & \\
\hline \multirow[t]{3}{*}{ ZINC } & $\mathrm{PPB}$ & 5 & & $01 / 13 / 88$ & 10 & $01 / 14 / 88$ & 11 & $01 / 13 / 88$ & \\
\hline & & & & $02 / 18 / 88$ & 19 & $02 / 18 / 88$ & 6 & $02 / 18 / 88$ & 6 \\
\hline & & & & $03 / 23 / 88$ & 7 & $03 / 23 / 88$ & 28 & $03 / 23 / 88$ & 10 \\
\hline
\end{tabular}


IABLE E.5. (contd)

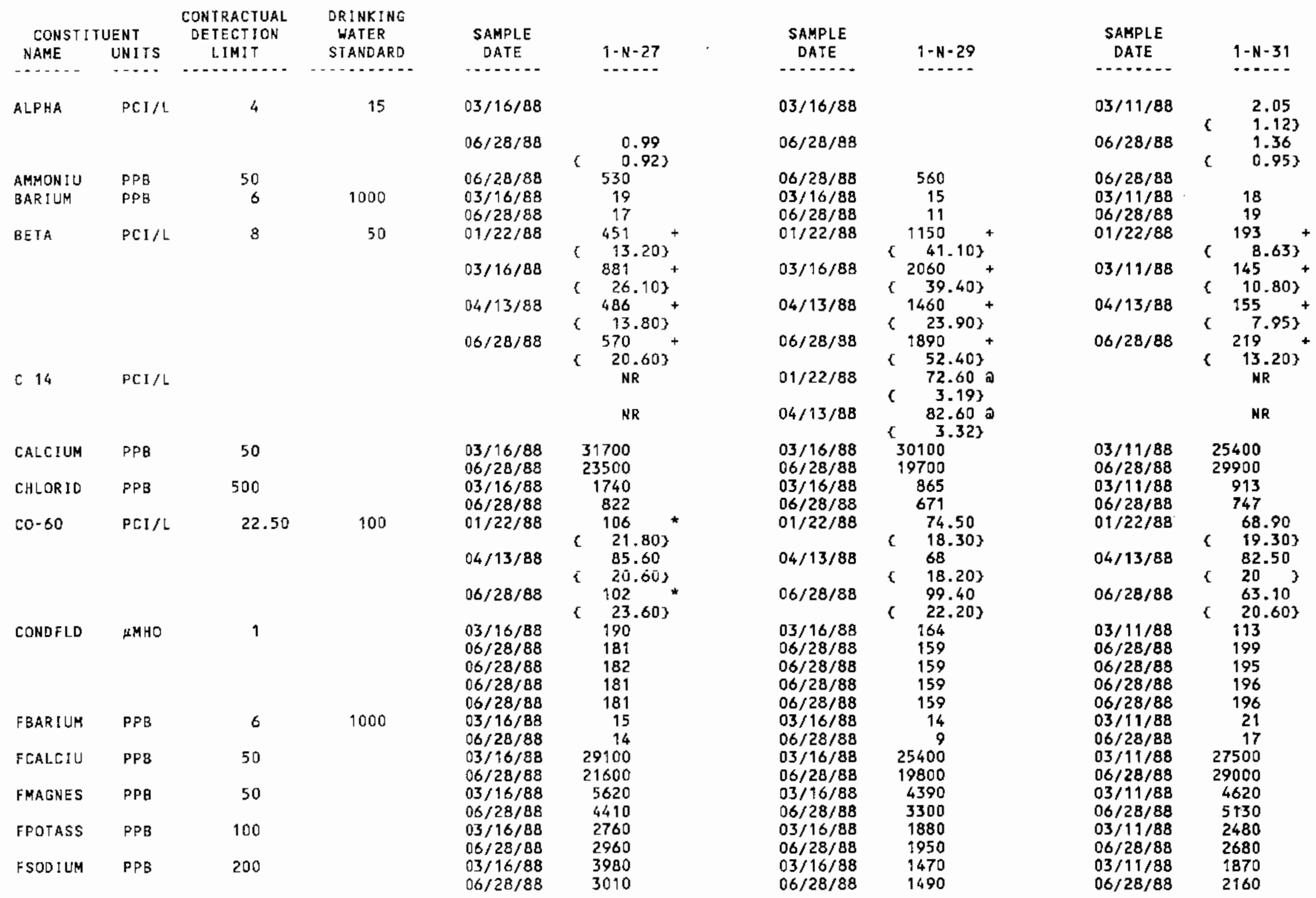


IABLE E.5. (contd)

\begin{tabular}{|c|c|c|c|c|c|}
\hline \multicolumn{2}{|c|}{ COHS : TUEN I } & $\begin{array}{c}\text { CONTRACTSA! } \\
\text { DETECIION }\end{array}$ & $\begin{array}{l}\text { DRINKINA } \\
\text { WATER }\end{array}$ & SAMPLE & \\
\hline MAM:: & $\therefore N I T S$ & I IMIT & STANOARD & DATE & $1-N-27$ \\
\hline - - - . - & $\cdots$ & $\ldots \ldots$ & $\ldots \ldots$ & $-\ldots \ldots$ & $\ldots$ \\
\hline FVANAYI & $P F B$ & 5 & & $03 / 16 / 88$ & 6 \\
\hline & & & & $06 / 28 / 88$ & 12 \\
\hline HETRAR & $F P 3$ & 2500 & 45000 & $0: 1 / 22 / 88$ & 27700 \\
\hline & & & & $04 / 13 / 88$ & 17700 \\
\hline ! ROM & $F ? 5$ & 50 & & $03 / 16 / 88$ & 70 \\
\hline MhonES & $P P B$ & 0 & & $\begin{array}{l}06 / 28 / 38 \\
03 / 16 / 88 \\
06 / 28 / 88\end{array}$ & $\begin{array}{l}5880 \\
4600\end{array}$ \\
\hline$S I-53$ & $\mathrm{PCJ} / \mathrm{L}$ & 10 & 50 & & NR \\
\hline & & & & & NR \\
\hline NITRATE & PPB & 500 & 45000 & $\begin{array}{l}03 / 16 / 88 \\
06 / 28 / 88\end{array}$ & $\begin{array}{l}20600 \\
16700\end{array}$ \\
\hline PHFIELD & & 0.10 & & $\begin{array}{l}03 / 16 / 88 \\
06 / 28 / 88\end{array}$ & $\begin{array}{l}8.60 \\
8.20\end{array}$ \\
\hline & & & . & $\begin{array}{l}06 / 28 / 88 \\
06 / 28 / 88\end{array}$ & $\begin{array}{l}8.30 \\
8.30\end{array}$ \\
\hline & & & & $06 / 28 / 88$ & 8.30 \\
\hline PCTASUM & PFB & 100 & & $03 / 16 / 88$ & 2760 \\
\hline & & & & $06 / 28 / 88$ & 3120 \\
\hline RAD I LM & POI/L & 1 & 5 & $03 / 16 / 88$ & 0.20 \\
\hline & & & & $06 / 28 / 88$ & $0.16\}$ \\
\hline$R U-10 t$ & $D C I / L$ & 172.50 & 30 & $01 / 22 / 88$ & 127 \\
\hline & & & & $04 / 13 / 88$ & $\left\{\begin{array}{l}83.30\} \\
60.60\end{array}\right.$ \\
\hline & & & & & $50.70\}$ \\
\hline \$B 125 & $\mathrm{PCI} / \mathrm{L}$ & & & & $N R$ \\
\hline & & & & & NR \\
\hline SODIUM & PPB & 200 & & $\begin{array}{l}03 / 16 / 88 \\
06 / 28 / 88\end{array}$ & $\begin{array}{l}4080 \\
3150\end{array}$ \\
\hline$S R 90$ & $\mathrm{PCI} / \mathrm{L}$ & 5 & 8 & $01 / 22 / 88$ & 149 \\
\hline & & & & $04 / 13 / 88$ & $\left\{2^{6.02\}}\right.$ * \\
\hline & & & & $06 / 28 / 88$ & $\left\{8^{8.74\}} *\right.$ \\
\hline SULFATE & pos & 500 & & & c 7.343 \\
\hline SULा AाL & & 300 & & $\begin{array}{l}03 / 16 / 88 \\
06 / 28 / 88\end{array}$ & $\begin{array}{r}15700 \\
8170\end{array}$ \\
\hline 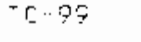 & PCI/L & 15 & 900 & $01 / 22 / 88$ & 19.20 \\
\hline & & & & & 1.363 \\
\hline
\end{tabular}

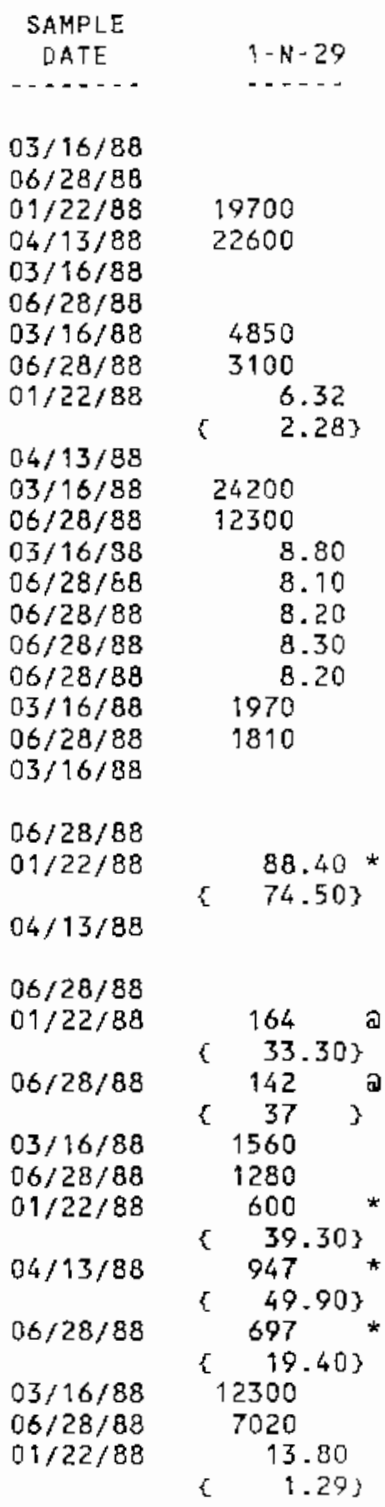

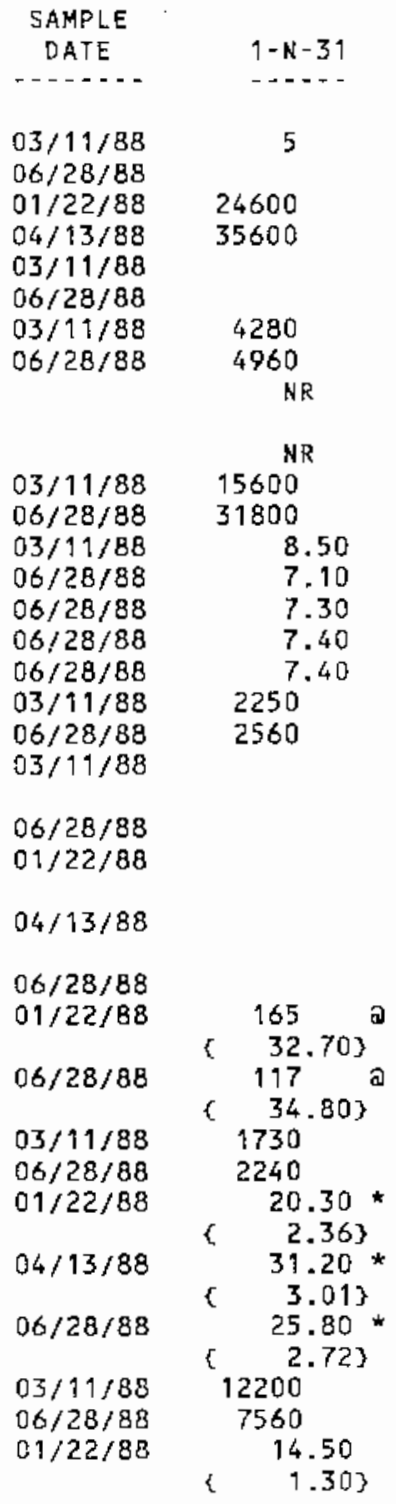


TABLE E.5. (contd)

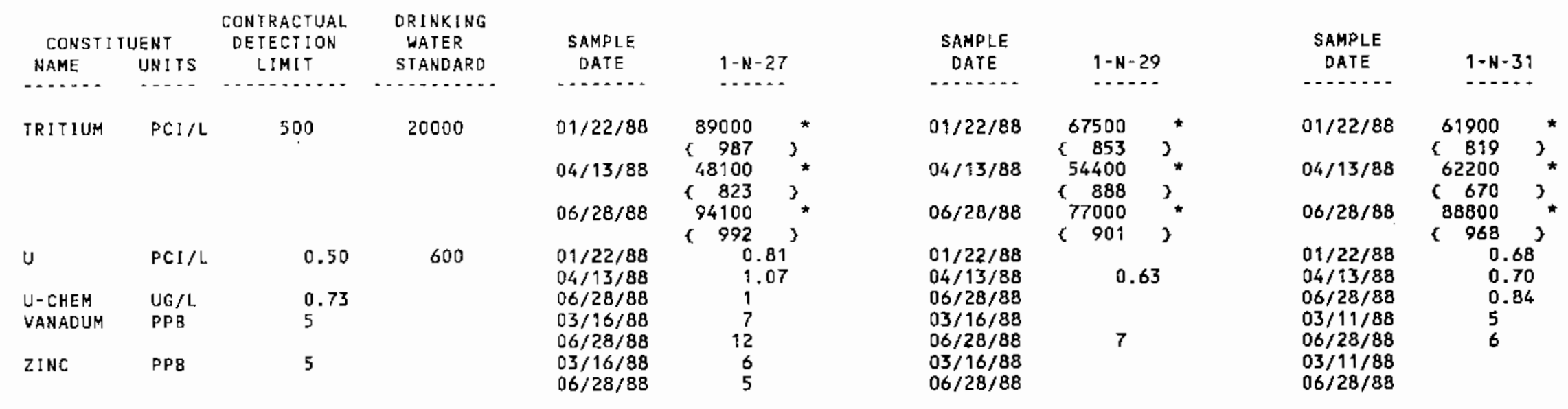


TABLE E.. . (contd)

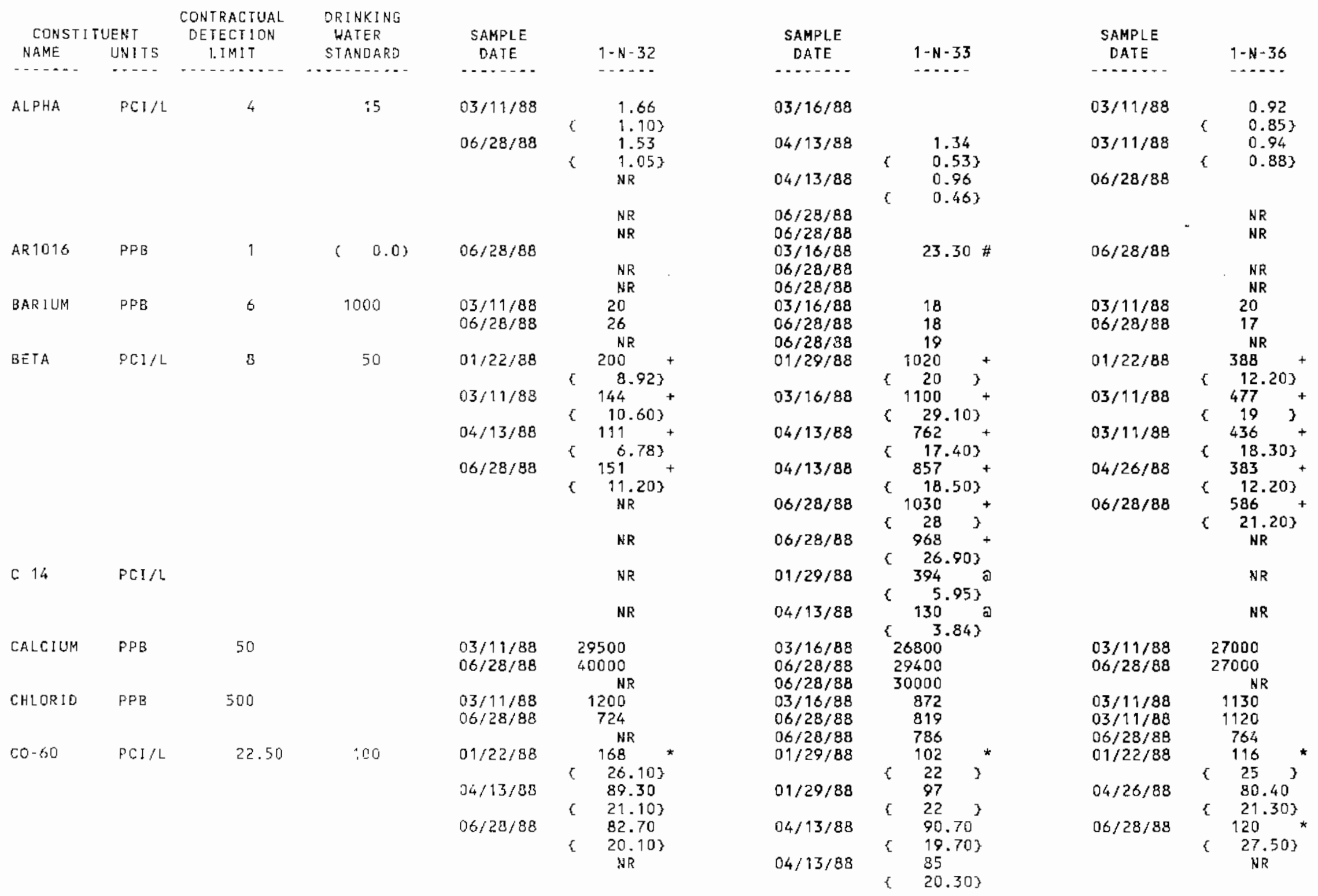


TABLE E. 5 . (contd)

\begin{tabular}{|c|c|c|c|c|c|c|c|c|c|}
\hline \multicolumn{2}{|c|}{ CONSTITUENT } & $\begin{array}{l}\text { CONTRACTUAL } \\
\text { DETECTION }\end{array}$ & $\begin{array}{l}\text { DRINKING } \\
\text { WATER }\end{array}$ & SAMPLE & & SAMPLE & & SAMPLE & \\
\hline NAME & UNITS & LIMIT & STANDARD & DATE & $1-\mathrm{N}-32$ & DATE & $1-N-33$ & DATE & $1-N-36$ \\
\hline$\ldots$ & $\cdots$ & 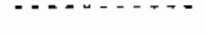 & n. & n & - & $\cdots$ & $\cdots+\infty$ & 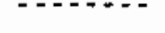 & $\cdots+1$ \\
\hline & & & & & $N R$ & $06 / 28 / 88$ & $\begin{array}{l}66.30 \\
16.803\end{array}$ & & NR \\
\hline & & & & & NR & $06 / 28 / 88$ & $\begin{array}{ll} & 72.30 \\
\{\quad 20.10\}\end{array}$ & & NR \\
\hline COLIFRM & MPN & 2 & 1 & $\begin{array}{l}03 / 91 / 88 \\
06 / 28 / 88\end{array}$ & $5.10 *$ & $\begin{array}{l}03 / 16 / 88 \\
06 / 28 / 88\end{array}$ & & $\begin{array}{l}03 / 11 / 88 \\
06 / 28 / 88\end{array}$ & \\
\hline CONDFLD & $\not M H O$ & 1 & & $\begin{array}{l}03 / 11 / 88 \\
06 / 28 / 88 \\
06 / 28 / 88 \\
06 / 28 / 88 \\
06 / 28 / 88\end{array}$ & $\begin{array}{l}136 \\
252 \\
193 \\
245 \\
248\end{array}$ & $\begin{array}{l}03 / 16 / 88 \\
06 / 28 / 88 \\
06 / 28 / 88 \\
06 / 28 / 88 \\
06 / 28 / 88\end{array}$ & $\begin{array}{l}176 \\
187 \\
188 \\
188 \\
188\end{array}$ & $\begin{array}{l}03 / 11 / 88 \\
06 / 28 / 88 \\
06 / 28 / 88 \\
06 / 28 / 88 \\
06 / 28 / 88\end{array}$ & $\begin{array}{l}105 \\
164 \\
165 \\
164 \\
164\end{array}$ \\
\hline FARSEN I & PPB & 5 & 50 & $\begin{array}{l}03 / 11 / 88 \\
06 / 28 / 88\end{array}$ & $\begin{array}{l}5 \\
\text { NR }\end{array}$ & $\begin{array}{l}03 / 16 / 88 \\
06 / 28 / 88 \\
06 / 28 / 38\end{array}$ & 5 & $\begin{array}{l}03 / 11 / 88 \\
03 / 11 / 88 \\
06 / 28 / 88\end{array}$ & \\
\hline FBARIUM & PPB & 6 & 1000 & $\begin{array}{l}03 / 11 / 88 \\
06 / 28 / 88\end{array}$ & $\begin{array}{l}21 \\
24 \\
\text { NR }\end{array}$ & $\begin{array}{l}03 / 16 / 88 \\
06 / 28 / 88 \\
06 / 28 / 88\end{array}$ & $\begin{array}{l}77 \\
16 \\
16\end{array}$ & $\begin{array}{l}03 / 11 / 88 \\
03 / 11 / 88 \\
06 / 28 / 88\end{array}$ & $\begin{array}{l}20 \\
20 \\
14\end{array}$ \\
\hline FCALCIU & PPB & 58 & & $\begin{array}{l}03 / 11 / 88 \\
06 / 28 / 88\end{array}$ & $\begin{array}{r}28400 \\
37000 \\
\text { NR }\end{array}$ & $\begin{array}{l}03 / 16 / 88 \\
06 / 28 / 88 \\
06 / 28 / 88\end{array}$ & $\begin{array}{l}25400 \\
27800 \\
28800\end{array}$ & $\begin{array}{l}03 / 11 / 88 \\
03 / 11 / 88 \\
06 / 28 / 88\end{array}$ & $\begin{array}{l}25600 \\
25300 \\
24500\end{array}$ \\
\hline FMAGNES & PPB & 50 & & $\begin{array}{l}03 / 11 / 88 \\
06 / 28 / 88\end{array}$ & $\begin{array}{c}5620 \\
7810 \\
\text { NR }\end{array}$ & $\begin{array}{l}03 / 16 / 88 \\
06 / 28 / 88 \\
06 / 28 / 88\end{array}$ & $\begin{array}{l}4220 \\
4800 \\
4980\end{array}$ & $\begin{array}{l}03 / 11 / 88 \\
03 / 11 / 88 \\
06 / 28 / 88\end{array}$ & $\begin{array}{l}4860 \\
4780 \\
4700\end{array}$ \\
\hline FPOTASS & PPB & 100 & & $\begin{array}{l}03 / 11 / 88 \\
06 / 28 / 88\end{array}$ & $\begin{array}{r}3260 \\
3490 \\
\text { NR }\end{array}$ & $\begin{array}{l}03 / 16 / 88 \\
06 / 28 / 88 \\
06 / 28 / 88\end{array}$ & $\begin{array}{l}2240 \\
2570 \\
2640\end{array}$ & $\begin{array}{l}03 / 11 / 88 \\
03 / 11 / 88 \\
06 / 28 / 88\end{array}$ & $\begin{array}{l}2190 \\
2140 \\
2030\end{array}$ \\
\hline rSOD : & fFE & żôv & & $\begin{array}{l}0 \overline{3} / \mathrm{i} i / 8 \overline{8} \\
06 / 28 / 88\end{array}$ & $\begin{array}{r}2960 \\
3350 \\
\text { NR }\end{array}$ & $\begin{array}{l}03 / 16 / 88 \\
06 / 28 / 88 \\
06 / 28 / 88\end{array}$ & $\begin{array}{l}2580 \\
2940 \\
2750\end{array}$ & $\begin{array}{l}03 / 11 / 88 \\
03 / 11 / 88 \\
06 / 28 / 88\end{array}$ & $\begin{array}{l}2400 \\
2340 \\
2270\end{array}$ \\
\hline FVANAD I & PPB & 5 & & $\begin{array}{l}03 / 11 / 88 \\
06 / 28 / 88\end{array}$ & $\begin{array}{l}12 \\
10 \\
\text { NR }\end{array}$ & $\begin{array}{l}03 / 16 / 88 \\
06 / 28 / 88 \\
06 / 28 / 88\end{array}$ & $\begin{array}{l}8 \\
9 \\
9\end{array}$ & $\begin{array}{l}03 / 11 / 88 \\
03 / 11 / 88 \\
06 / 28 / 88\end{array}$ & $\begin{array}{l}7 \\
7 \\
8\end{array}$ \\
\hline FZINC & PPB & 5 & & $\begin{array}{l}03 / 11 / 88 \\
06 / 28 / 88\end{array}$ & $\begin{array}{l}5 \\
\mathrm{HR}\end{array}$ & $\begin{array}{l}03 / 16 / 88 \\
06 / 28 / 88 \\
06 / 28 / 88\end{array}$ & 6 & $\begin{array}{l}03 / 11 / 88 \\
03 / 11 / 88 \\
06 / 28 / 88\end{array}$ & 6 \\
\hline HN I TRAT & $P P B$ & 2500 & 45000 & $\begin{array}{l}01 / 22 / 88 \\
04 / 13 / 88\end{array}$ & $\begin{array}{l}43900 \\
48400\end{array}$ & $\begin{array}{l}01 / 29 / 88 \\
04 / 13 / 88\end{array}$ & $\begin{array}{l}26400 \\
28600\end{array}$ & $\begin{array}{l}01 / 22 / 88 \\
04 / 26 / 88\end{array}$ & $\begin{array}{l}26900 \\
18000\end{array}$ \\
\hline I RON & $P P B$ & 50 & & $\begin{array}{l}03 / 11 / 88 \\
06 / 28 / 88\end{array}$ & & $\begin{array}{l}03 / 16 / 88 \\
06 / 28 / 88\end{array}$ & 98 & $\begin{array}{l}03 / 11 / 88 \\
06 / 28 / 88\end{array}$ & \\
\hline & & & & & NR & $06 / 28 / 88$ & 73 & & NR \\
\hline MAGNES & PPB & 0 & & $\begin{array}{l}03 / 11 / 88 \\
06 / 28 / 88\end{array}$ & $\begin{array}{l}5580 \\
7980\end{array}$ & $\begin{array}{l}03 / 16 / 88 \\
06 / 28 / 88\end{array}$ & $\begin{array}{l}4510 \\
4760\end{array}$ & $\begin{array}{l}03 / 11 / 88 \\
06 / 28 / 88\end{array}$ & $\begin{array}{l}5030 \\
4870\end{array}$ \\
\hline & & & & & NR & $06 / 28 / 88$ & 4820 & & NR \\
\hline$N 1-63$ & $\mathrm{PCI} / \mathrm{L}$ & 10 & 50 & & NR & $01 / 29 / 88$ & $\begin{array}{c}10.70 \\
\{\quad 3.17\}\end{array}$ & & NR \\
\hline
\end{tabular}


TABLE E.5. (contd)

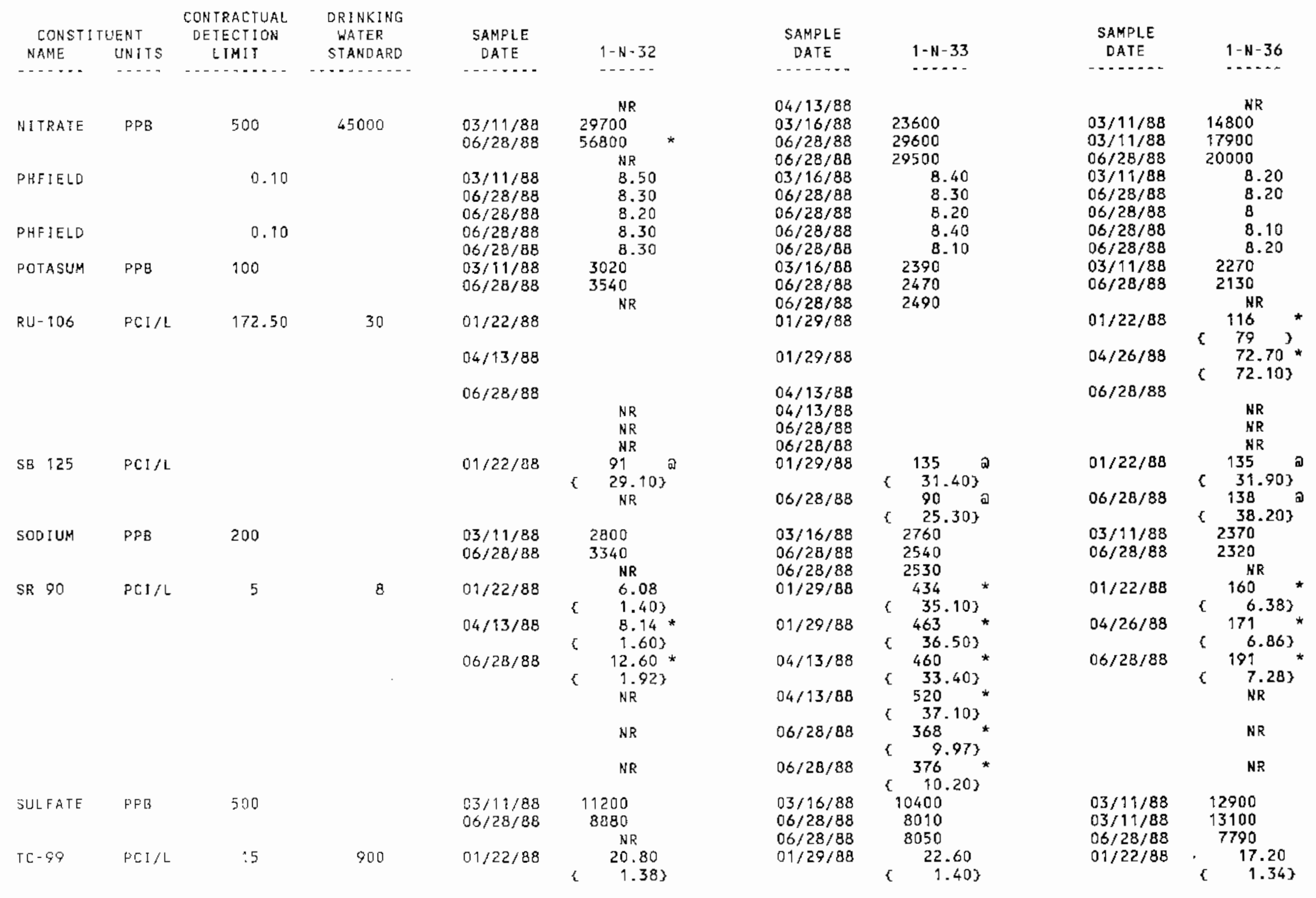


TABLE E.5. (contd)

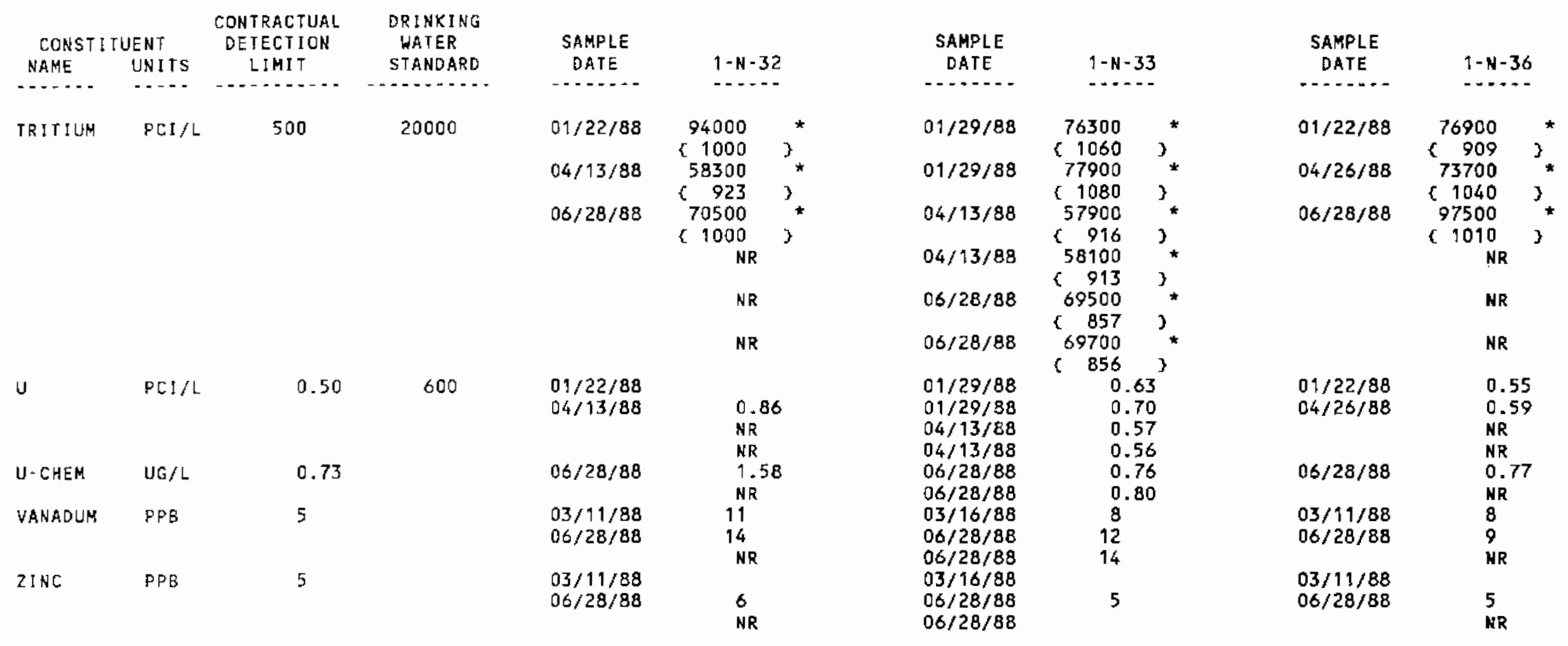


TABLE E.5. (contd)

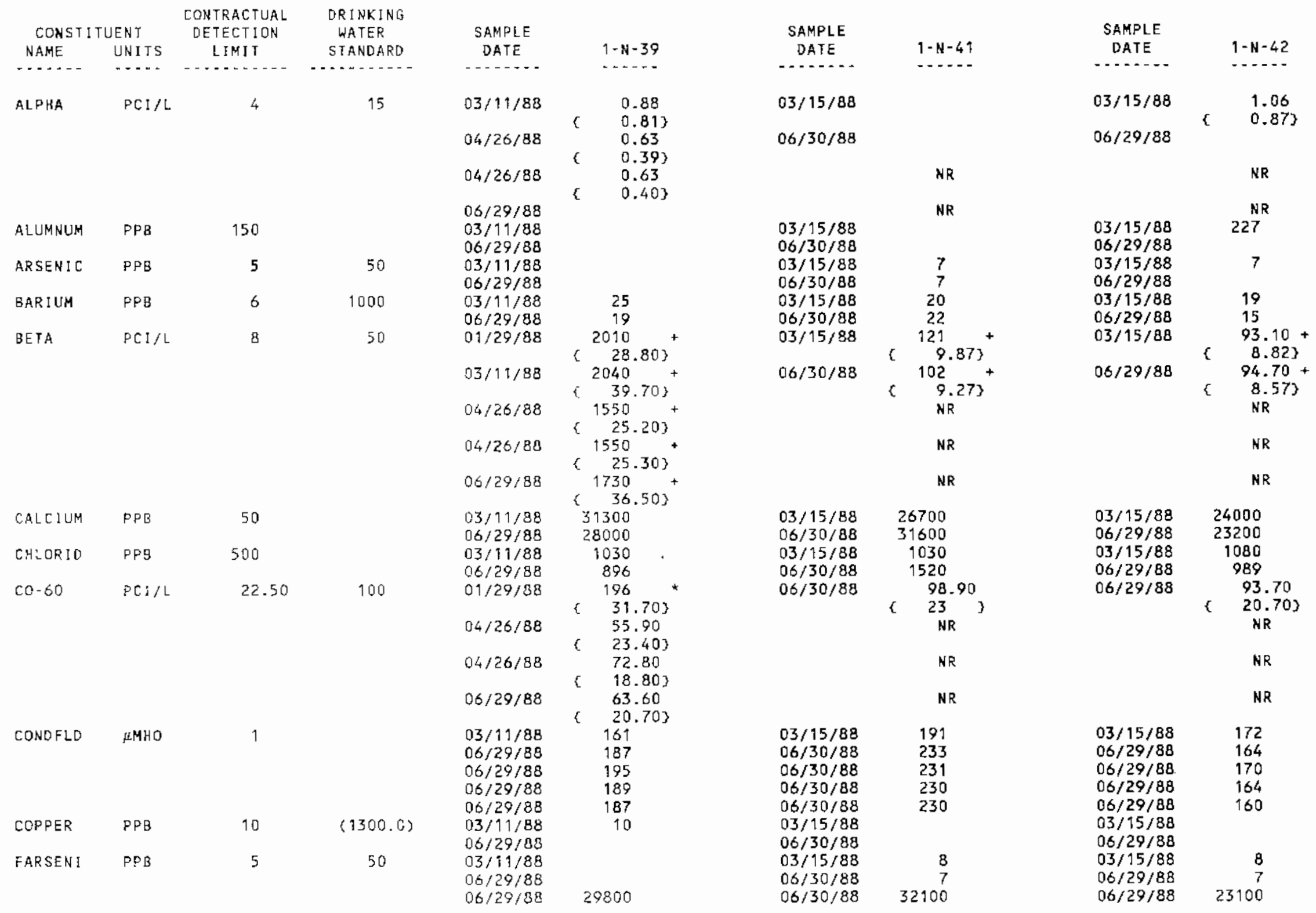


TABLE E.5. (contd)

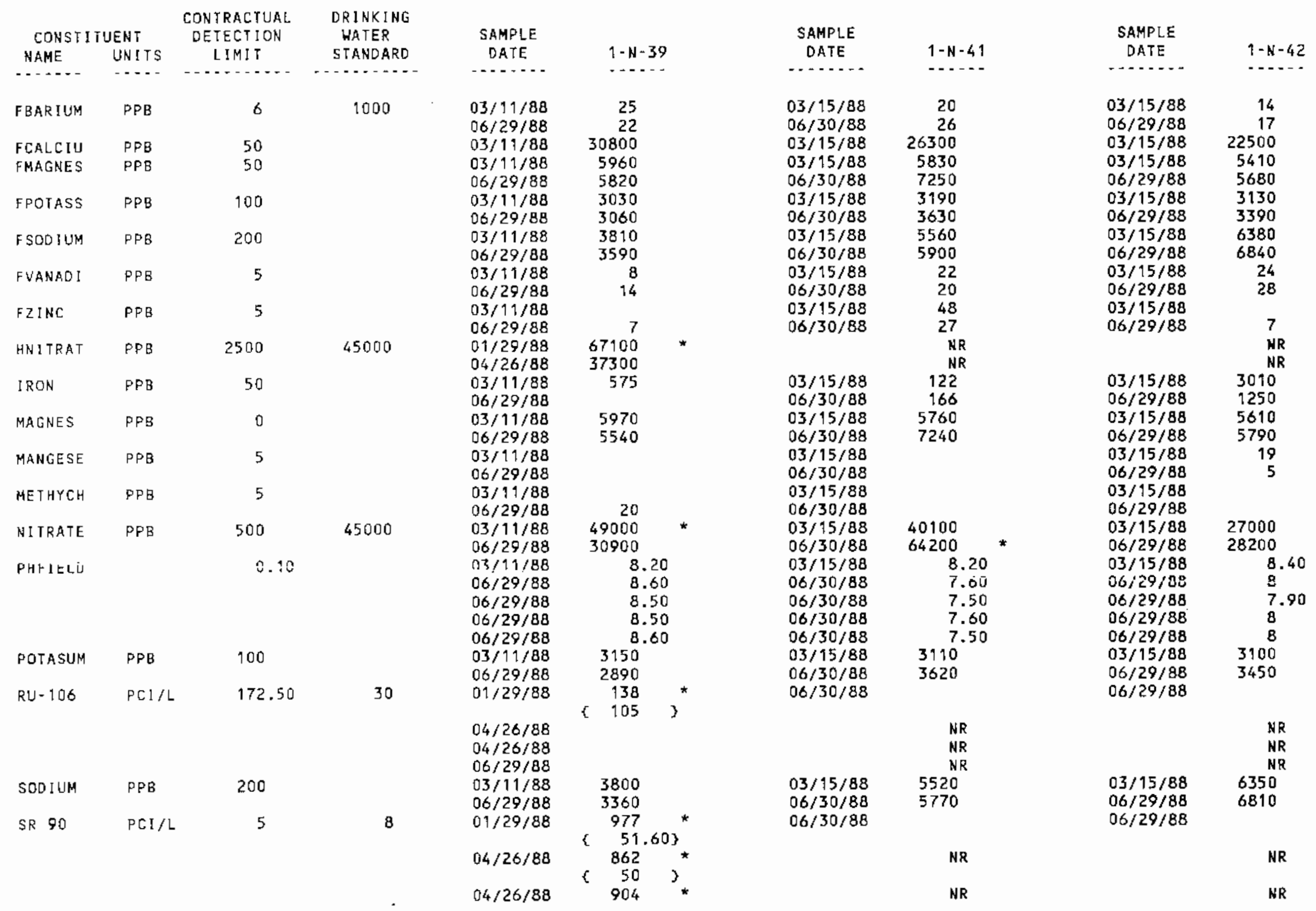


TABLE E.5. (contd)

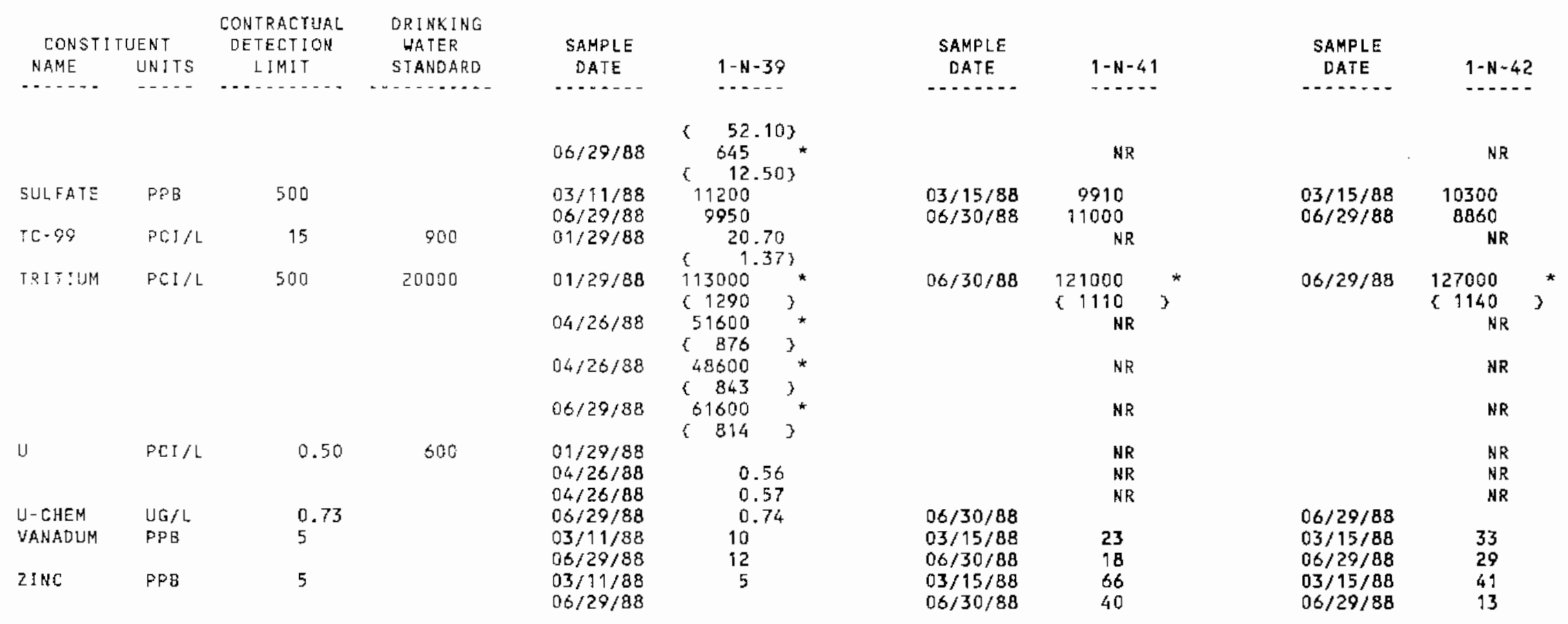


TABLE E.5. (contd)

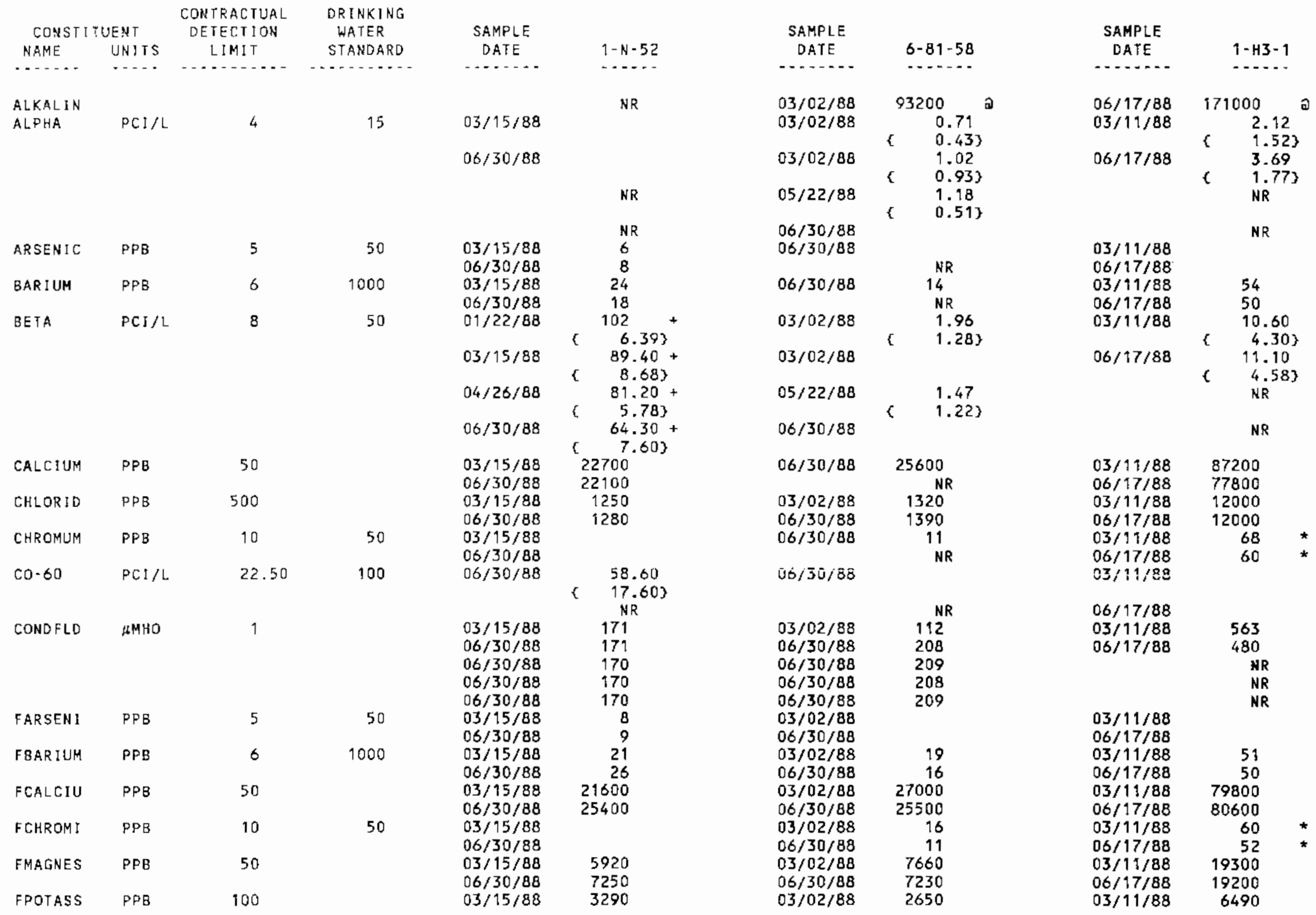


IABLE E.5. (contd)

\begin{tabular}{|c|c|c|c|c|c|}
\hline \multicolumn{2}{|c|}{ CONSI 1 TUENT } & $\begin{array}{l}\text { COETRACTUAL } \\
\text { DETECTION }\end{array}$ & $\begin{array}{l}\text { CRINKING } \\
\text { WATER }\end{array}$ & SAMPLE & \\
\hline NAME & UNITS & LIMIT & STANDARO & $\begin{array}{l}\text { SAMPLE } \\
\text { DATE }\end{array}$ & $1-N-52$ \\
\hline$-\cdots$ & $-\cdots$ & - - - - - - - & $\cdots$ & $-\ldots$ & $\cdots$ \\
\hline FSOOIUM & $P P B$ & 200 & & $\begin{array}{l}06 / 30 / 88 \\
03 / 15 / 88 \\
06 / 30 / 88\end{array}$ & $\begin{array}{l}4140 \\
5820 \\
7100\end{array}$ \\
\hline FSTRONT & PPB & 10 & & & $\begin{array}{l}\text { NR } \\
\text { NR }\end{array}$ \\
\hline FVANADI & PPB & 5 & & $\begin{array}{l}03 / 15 / 88 \\
06 / 30 / 88\end{array}$ & $\begin{array}{l}26 \\
30\end{array}$ \\
\hline FZINC & PPB & 5 & & $\begin{array}{l}03 / 15 / 88 \\
06 / 30 / 88\end{array}$ & 6 \\
\hline HNITRAT & PPB & 2500 & 45000 & $\begin{array}{l}01 / 22 / 88 \\
04 / 26 / 88\end{array}$ & $\begin{array}{l}24500 \\
21700\end{array}$ \\
\hline IRON & PPB & 50 & & $\begin{array}{l}03 / 15 / 88 \\
06 / 30 / 88\end{array}$ & \\
\hline LFLUORD & $P P B$ & 50 & 4000 & & NR \\
\hline MAGNES & PPB & 0 & & $\begin{array}{l}03 / 15 / 88 \\
06 / 30 / 88\end{array}$ & $\begin{array}{l}6020 \\
6400\end{array}$ \\
\hline MANGESE & PPB & 5 & & $\begin{array}{l}03 / 15 / 88 \\
06 / 30 / 88\end{array}$ & \\
\hline N1TRATE & PPB & 500 & 45000 & $\begin{array}{l}03 / 15 / 88 \\
06 / 30 / 88\end{array}$ & $\begin{array}{l}23700 \\
25800\end{array}$ \\
\hline$P H-L A B$ & & 0.01 & & & $\begin{array}{l}\text { NR } \\
\text { NR }\end{array}$ \\
\hline PHF IELD & & 0.10 & & $\begin{array}{l}03 / 15 / 88 \\
06 / 30 / 88 \\
06 / 30 / 88 \\
06 / 30 / 88 \\
06 / 30 / 88\end{array}$ & $\begin{array}{l}8.40 \\
7.80 \\
7.90 \\
7.80 \\
7.90\end{array}$ \\
\hline POTASUM & PPB & 100 & & $\begin{array}{l}03 / 15 / 88 \\
06 / 30 / 88\end{array}$ & $\begin{array}{l}3280 \\
3420\end{array}$ \\
\hline RADIUM & $\mathrm{PCI} / \mathrm{L}$ & 1 & 5 & $03 / 15 / 8 \mathrm{~d}$ & \\
\hline & & & & $06 / 30 / 88$ & $\begin{array}{l}0.16 \\
0.15\end{array}$ \\
\hline SOD IUM & PPB & 200 & & $\begin{array}{l}03 / 15 / 88 \\
06 / 30 / 88\end{array}$ & $\begin{array}{l}5850 \\
6000\end{array}$ \\
\hline STRONUM & PPB & 10 & & & $\begin{array}{l}\text { NR } \\
\text { NR }\end{array}$ \\
\hline SULFAIE & PPB & 500 & & $\begin{array}{l}03 / \uparrow 5 / 88 \\
06 / 30 / 88\end{array}$ & $\begin{array}{l}11200 \\
11600\end{array}$ \\
\hline TC & PPB & 2000 & & & $\begin{array}{l}N R \\
N R\end{array}$ \\
\hline TC- 99 & $\mathrm{PCI} / \mathrm{L}$ & 15 & 900 & $01 / 22 / 88$ & $\begin{array}{c}13.70 \\
1.293 \\
N R\end{array}$ \\
\hline
\end{tabular}

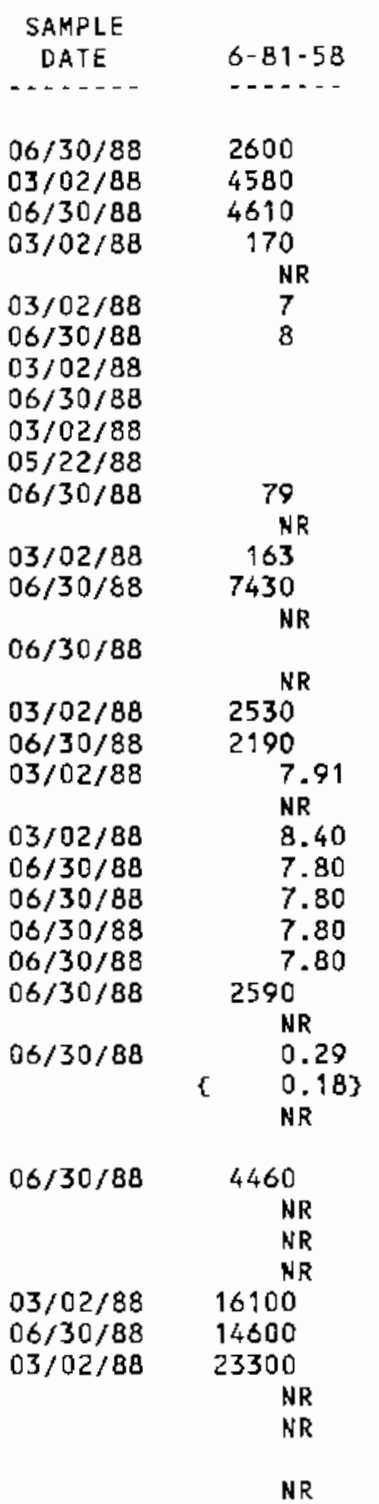

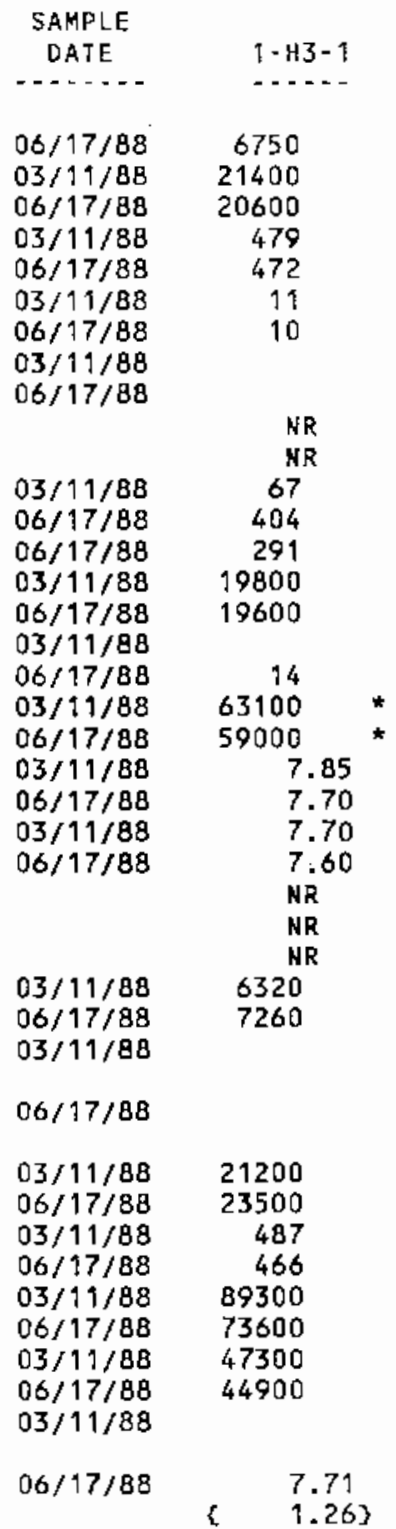


TABLE E.5. (contd)

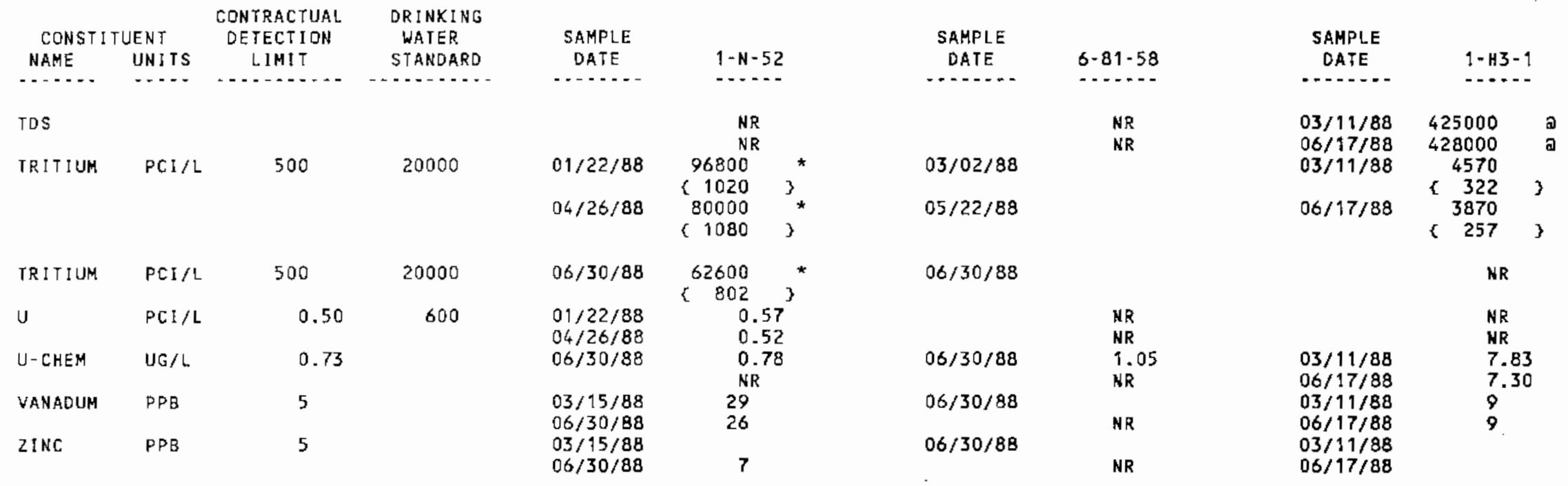


TABLE E.5. (contd)

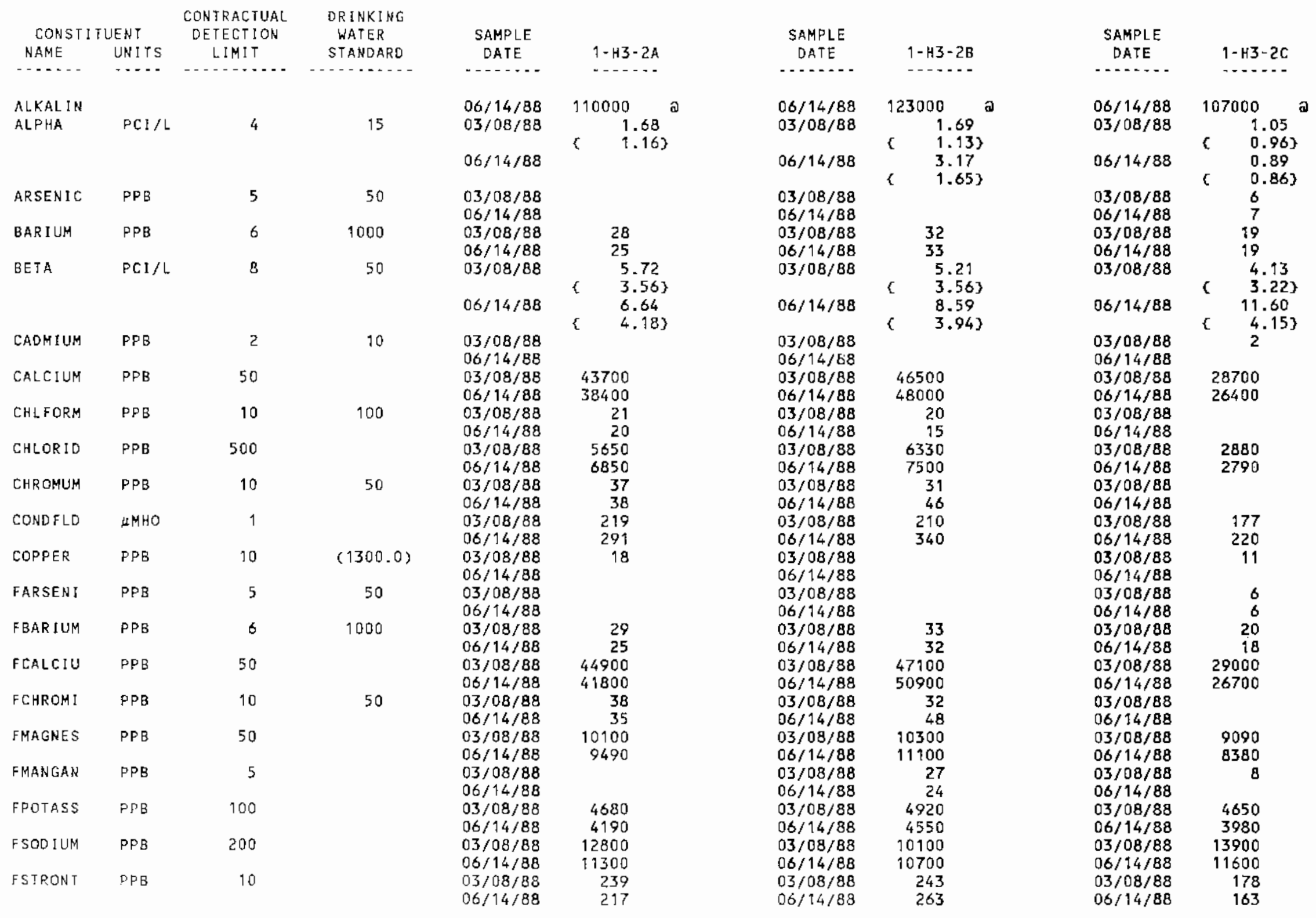


TABLE E. 5 . (contd)

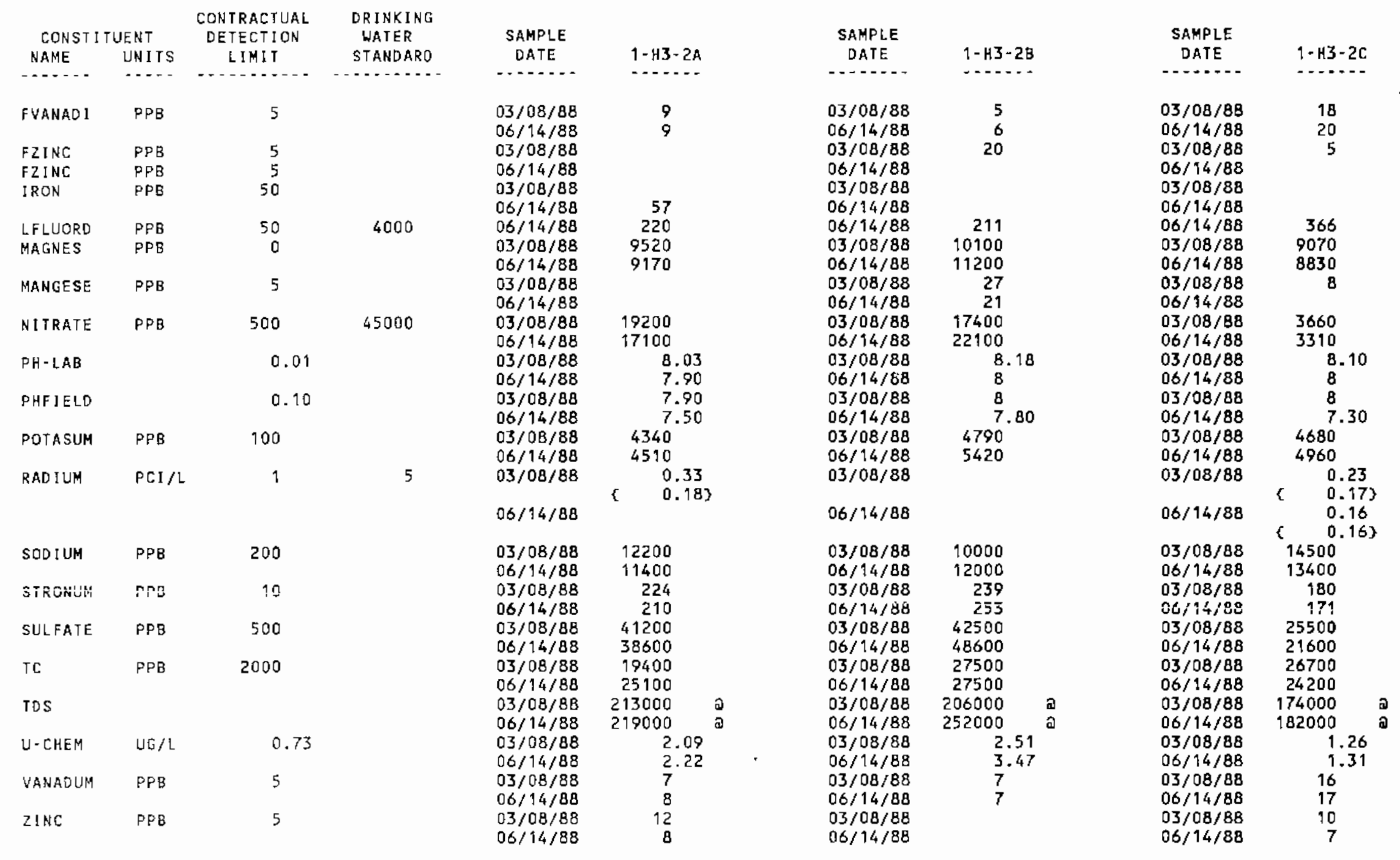


IABLE E.5. (contd)

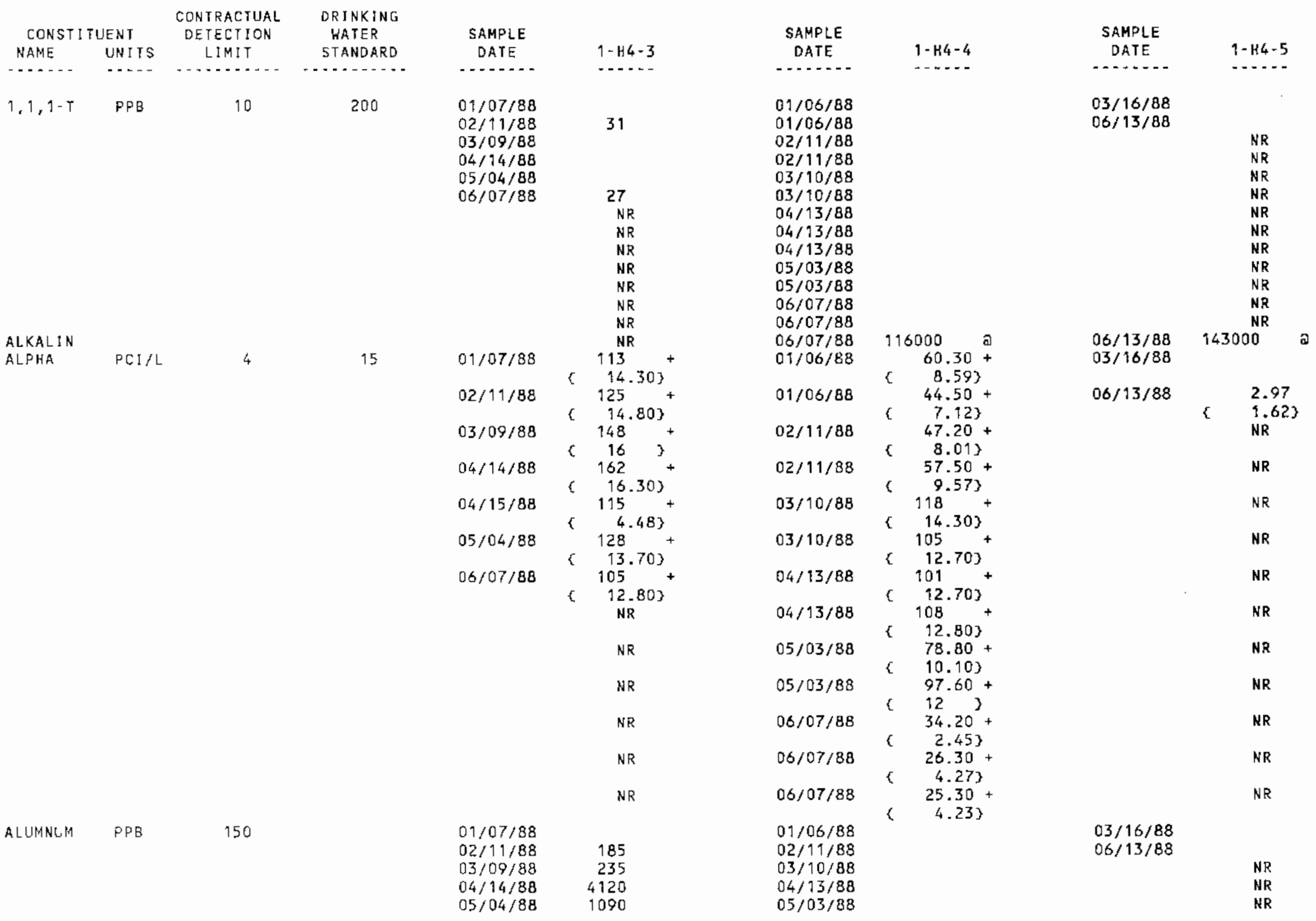


TABLE E. 5. (contd)

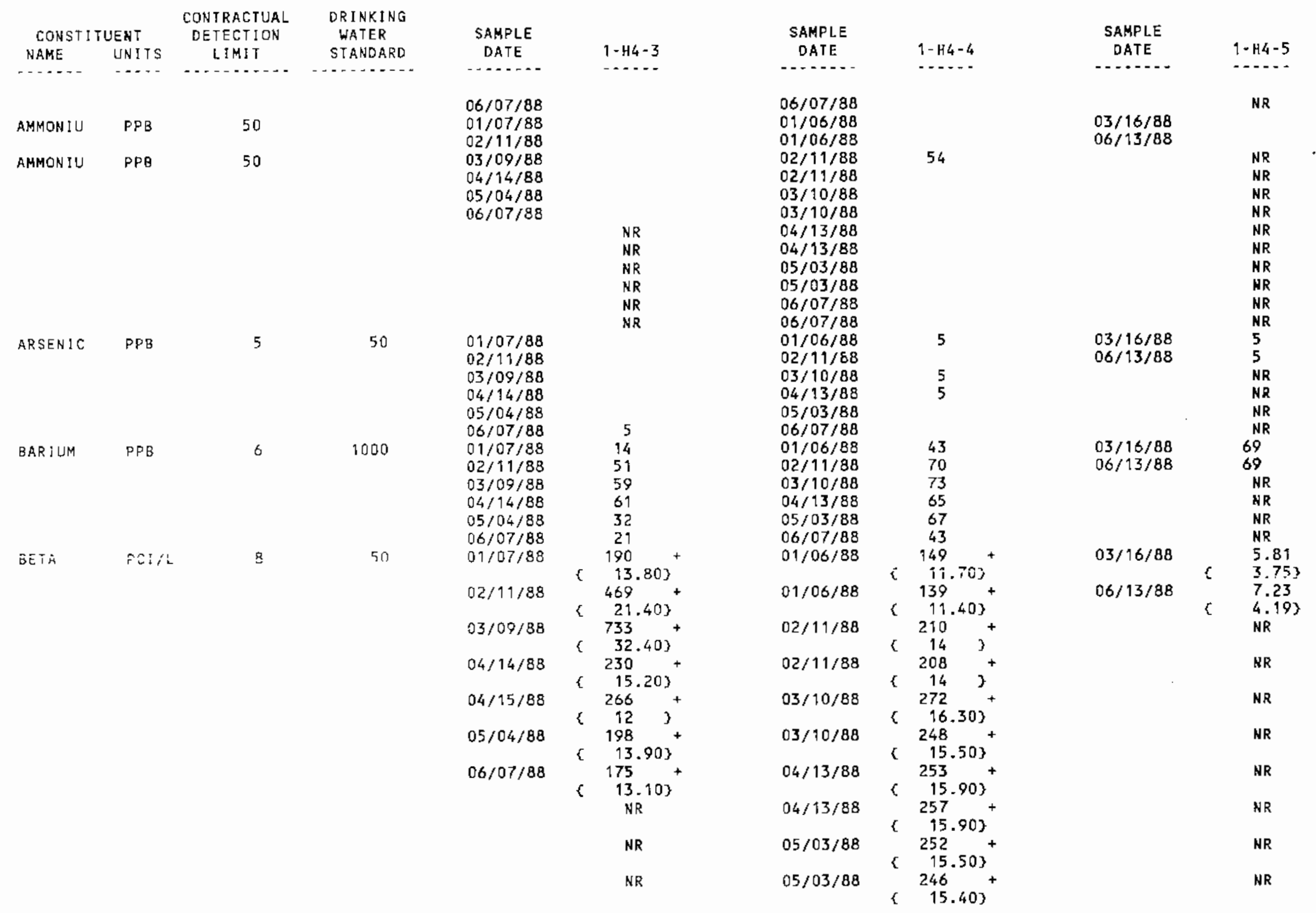


TABLEE.5. (contd)

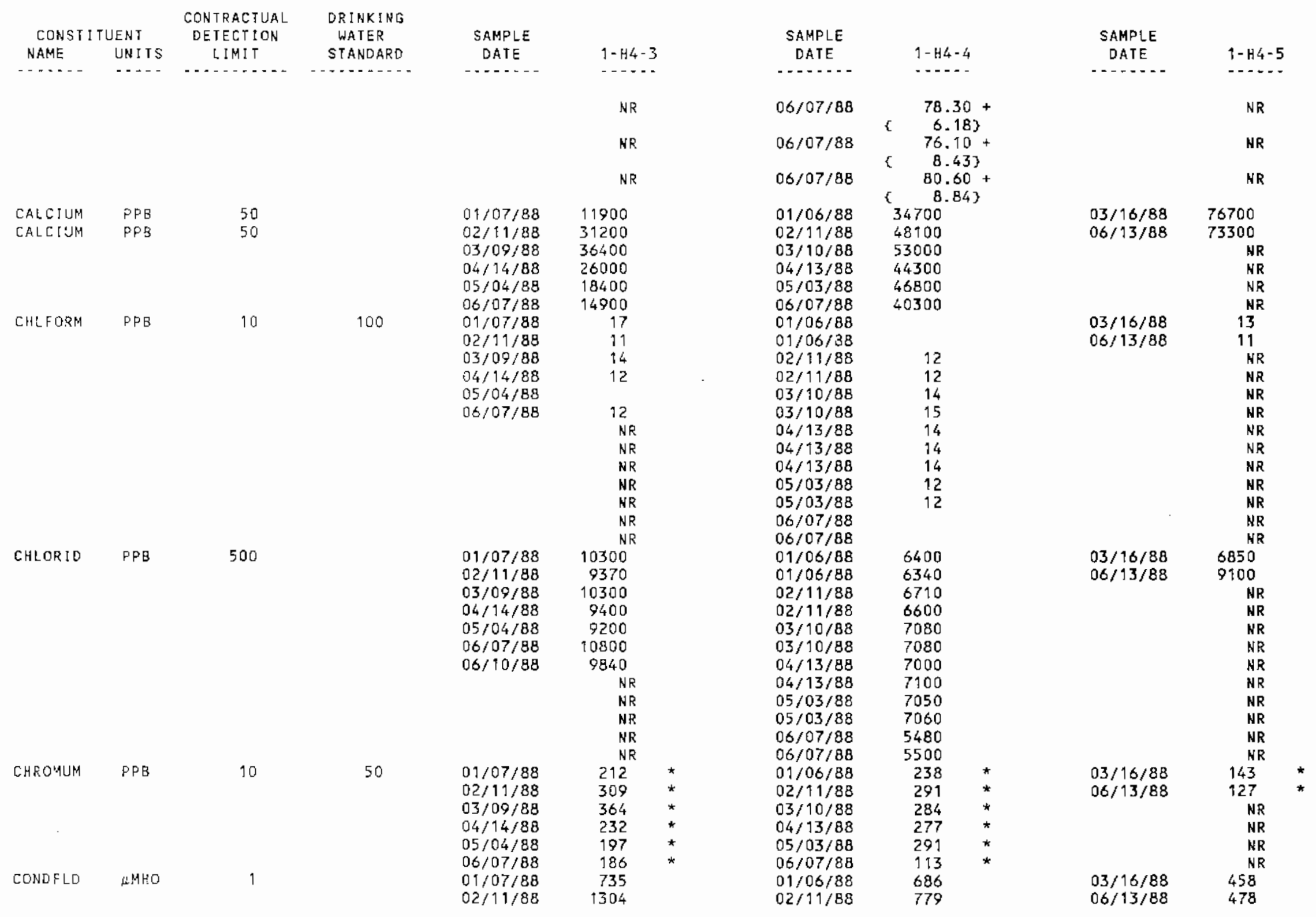


TABLE_E.5. (contd)

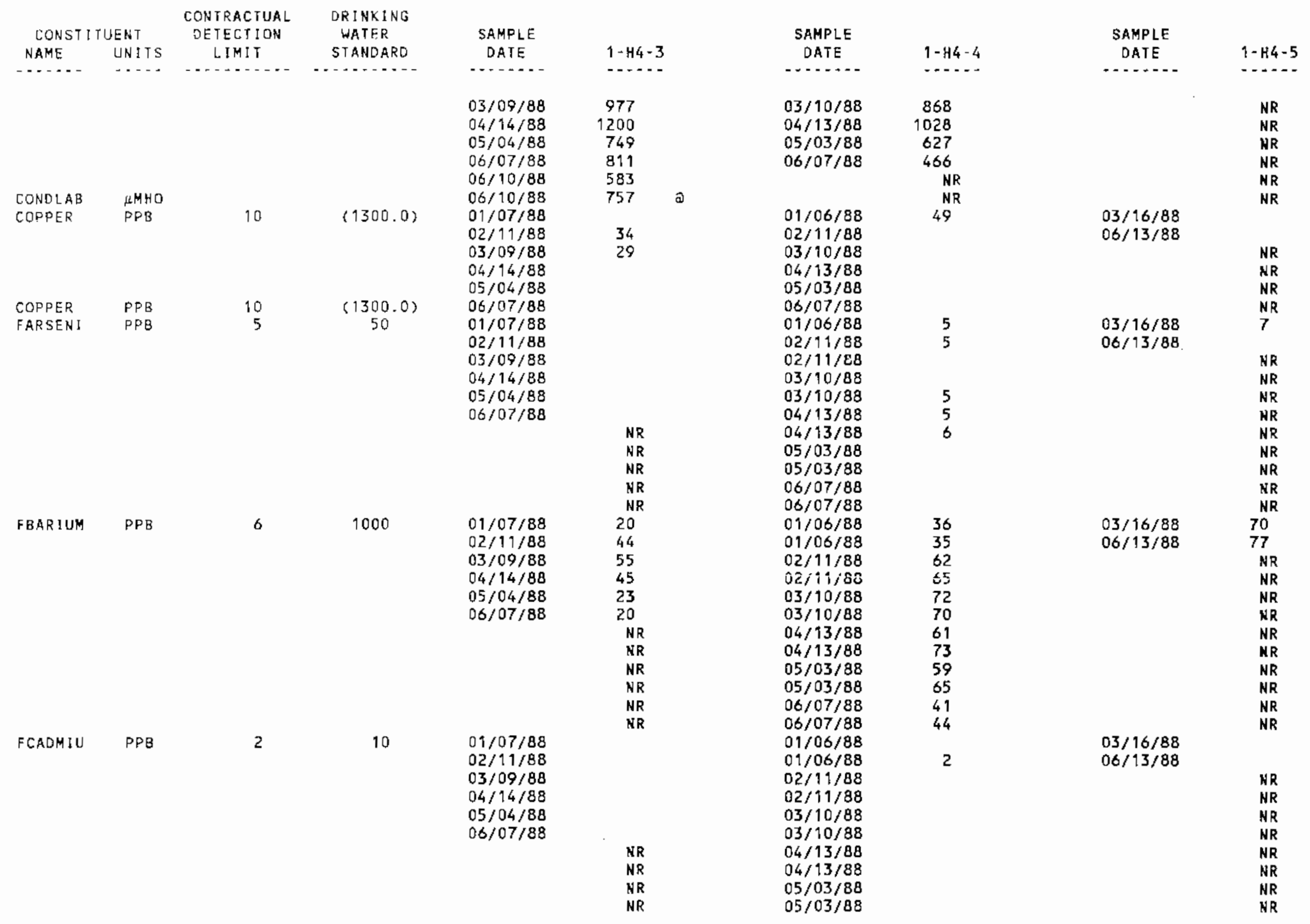


TABLE E. 5. (contd)

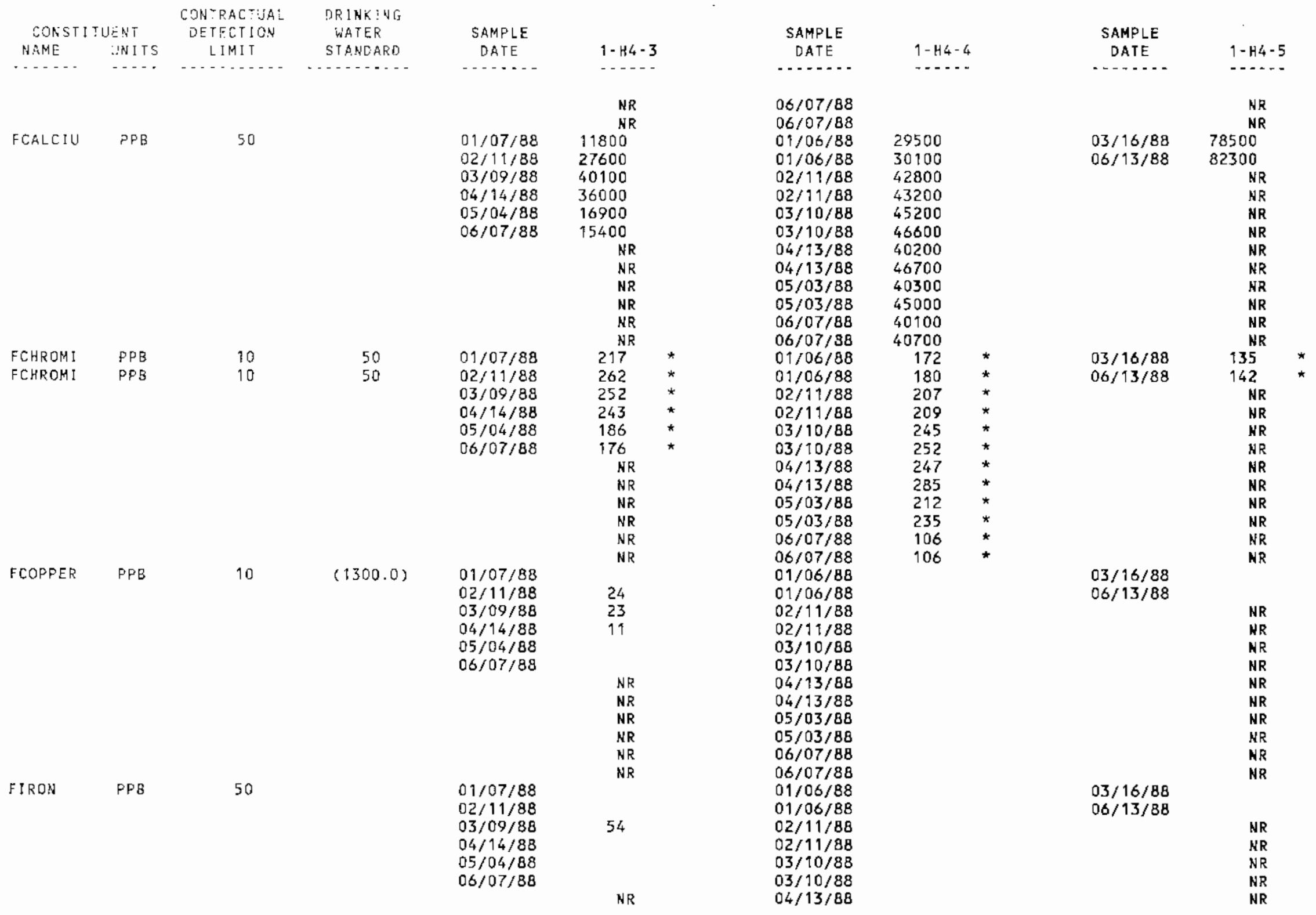


TABLE E.5. (contd)

\begin{tabular}{|c|c|c|c|c|c|c|c|c|c|}
\hline CONSTI & UENT & $\begin{array}{l}\text { CONTRACTUAL } \\
\text { DETECTION }\end{array}$ & $\begin{array}{l}\text { DR INKING } \\
\text { WATER }\end{array}$ & SAMPLE & & SAMPLE & & SAMPLE & \\
\hline NAME & UNITS & LIMIT & STANDARD & DATE & $1-44-3$ & DATE & $1-\mathrm{H} 4-4$ & DATE & $1-H_{4}-5$ \\
\hline$\cdots$ & $\cdots \cdots$ & - & - & $\cdots$ & $\cdots$ & n......... & ....... & - n. & $\cdots$ \\
\hline & & & & & NR & $04 / 13 / 88$ & & & NR \\
\hline & & & & & NR & $05 / 03 / 88$ & & & NR \\
\hline & & & & & NR & $05 / 03 / 88$ & & & NR \\
\hline & & & & & NR & $06 / 07 / 88$ & & & NR \\
\hline & & & & & NR & $06 / 07 / 88$ & & & NR \\
\hline FLUOR ID & PPB & 500 & 4000 & $\begin{array}{l}01 / 07 / 88 \\
02 / 11 / 88\end{array}$ & 939 & $\begin{array}{l}01 / 06 / 88 \\
01 / 06 / 88\end{array}$ & $\begin{array}{l}715 \\
702\end{array}$ & $\begin{array}{l}03 / 16 / 88 \\
06 / 13 / 88\end{array}$ & \\
\hline & & & & $03 / 09 / 88$ & & $02 / 11 / 88$ & & & NR \\
\hline & & & & $04 / 14 / 88$ & 904 & $02 / 11 / 88$ & & & NR \\
\hline & & & & $05 / 04 / 88$ & 743 & $03 / 10 / 88$ & 1300 & & HR \\
\hline & & & & $06 / 07 / 88$ & 756 & $03 / 10 / 88$ & 1310 & & NR \\
\hline & & & & $06 / 10 / 88$ & 947 & $04 / 13 / 88$ & 741 & & NR \\
\hline & & & & & NR & $04 / 13 / 88$ & 825 & & NR \\
\hline & & & & & NR & $05 / 03 / 88$ & 693 & & NR \\
\hline & $\cdot$ & & & & NR & $05 / 03 / 88$ & 688 & & NR \\
\hline & & & & & NR & $06 / 07 / 88$ & & & NR \\
\hline & & & & & NR & $06 / 07 / 88$ & & & , NR \\
\hline FMAGNES & PPB & 50 & & $\begin{array}{l}01 / 07 / 88 \\
02 / 11 / 88\end{array}$ & $\begin{array}{l}1960 \\
4820\end{array}$ & $\begin{array}{l}01 / 06 / 88 \\
01 / 06 / 88\end{array}$ & $\begin{array}{l}4250 \\
4430\end{array}$ & $\begin{array}{l}03 / 16 / 88 \\
06 / 13 / 88\end{array}$ & $\begin{array}{l}12800 \\
14200\end{array}$ \\
\hline FMAGNES & $P P B$ & 50 & & $03 / 09 / 88$ & 6690 & $02 / 11 / 88$ & 7010 & & NR \\
\hline & & & & $04 / 14 / 88$ & 5830 & $02 / 11 / 88$ & 7040 & & NR \\
\hline & & & & $05 / 04 / 88$ & 2910 & $03 / 10 / 88$ & 7120 & & NR \\
\hline & & & & $06 / 07 / 88$ & 2640 & $03 / 10 / 88$ & 7240 & & NR \\
\hline & & & & & $N R$ & $04 / 13 / 88$ & 6150 & & NR \\
\hline & & & & & NR & $04 / 13 / 88$ & 6800 & & NR \\
\hline & & & & & NR & $05 / 03 / 88$ & 6160 & & NR \\
\hline & & & & & NR & $05 / 03 / 80$ & 0690 & & NQR \\
\hline & & & & & $\mathrm{NR}$ & $06 / 07 / 88$ & 7130 & & NR \\
\hline & & & & & NR & $06 / 07 / 88$ & 7230 & & NR \\
\hline FMANGAN & PPB & 5 & & $\begin{array}{l}01 / 07 / 88 \\
02 / 11 / 88\end{array}$ & & $\begin{array}{l}01 / 06 / 88 \\
01 / 06 / 88\end{array}$ & & $\begin{array}{l}03 / 16 / 88 \\
06 / 13 / 88\end{array}$ & \\
\hline & & & & $03 / 09 / 88$ & 7 & $02 / 11 / 88$ & & & NR \\
\hline & & & & $04 / 14 / 88$ & & $02 / 19 / 88$ & & & NR \\
\hline & & & & $05 / 04 / 88$ & & $03 / 10 / 88$ & & & NR \\
\hline & & & & $06 / 07 / 88$ & & $03 / 10 / 88$ & & & NR \\
\hline & & & & & $N R$ & $04 / 13 / 88$ & & & NR \\
\hline & & & & & NR & $04 / 13 / 88$ & & & NR \\
\hline & & & & & NR & $05 / 03 / 88$ & & & NR \\
\hline & & & & & NR & $05 / 03 / 88$ & & & NR \\
\hline & & & & & NR & $06 / 07 / 88$ & & & NR \\
\hline & & & & & NR & $06 / 07 / 88$ & & & NR \\
\hline FNICKEL & PPB & 10 & & $01 / 07 / 88$ & & $01 / 06 / 88$ & & $03 / 16 / 88$ & \\
\hline & & & & $02 / 11 / 88$ & 27 & $01 / 06 / 88$ & & $06 / 13 / 88$ & \\
\hline & & & & $03 / 09 / 88$ & 27 & $02 / 11 / 88$ & & & NR \\
\hline & & & & $04 / 14 / 88$ & 23 & $02 / 11 / 88$ & 10 & & NR \\
\hline
\end{tabular}


TABLE_E.5. (contd)

\begin{tabular}{|c|c|c|c|c|c|c|c|c|c|}
\hline \multicolumn{2}{|c|}{ CONSTITUENAT } & \multirow{2}{*}{$\begin{array}{c}\text { CONTRACTUAL } \\
\text { DETECIION } \\
\text { LIMIT }\end{array}$} & \multirow{2}{*}{$\begin{array}{c}\text { DRINKING } \\
\text { WATER } \\
\text { STANDARD }\end{array}$} & \multirow{2}{*}{$\begin{array}{l}\text { SAMPLE } \\
\text { DATE }\end{array}$} & \multirow[b]{2}{*}{$1-H_{4}-3$} & SAMPLE & \multicolumn{3}{|c|}{ SAMPLE } \\
\hline NAME & UNITS & & & & & DATE & $1-H 4-4$ & DATE & $1-\mathrm{H}_{4}-5$ \\
\hline (n) & $\cdots$ & 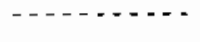 & 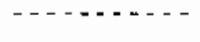 & 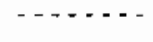 & $\cdots-$ & $\cdots$ & $-\cdots$ & $\ldots$ & $\cdots$ \\
\hline & & & & $05 / 04 / 88$ & & $03 / 10 / 88$ & 13 & & NR \\
\hline & & & & $06 / 07 / 88$ & 13 & $03 / 10 / 88$ & 15 & & NR \\
\hline & & & & & NR & $04 / 13 / 88$ & 13 & & $N R$ \\
\hline & & & & & NR & $04 / 13 / 88$ & 11 & & NR \\
\hline & & & & & NR & $05 / 03 / 88$ & 10 & & NR \\
\hline & & & & & NR & $05 / 03 / 88$ & 11 & & NR \\
\hline & & & & & $\mathrm{NR}$ & $06 / 07 / 88$ & & & NR \\
\hline & & & & & NR & $06 / 07 / 88$ & & & NR \\
\hline FPOTASS & PPB & 100 & & $01 / 07 / 88$ & 3750 & $01 / 06 / 88$ & 4120 & $03 / 16 / 88$ & 5420 \\
\hline & & & & $02 / 11 / 88$ & 5730 & $01 / 06 / 88$ & 4380 & $06 / 13 / 88$ & 5780 \\
\hline & & & & $03 / 09 / 88$ & 6450 & $02 / 11 / 88$ & 5230 & & NR \\
\hline & & & & $04 / 14 / 88$ & 5470 & $02 / 19 / 88$ & 5200 & & NR \\
\hline & & & & $05 / 04 / 88$ & 3940 & $03 / 10 / 88$ & 6030 & & NR \\
\hline & & & & $06 / 07 / 88$ & 3920 & $03 / 10 / 38$ & 6000 & & NR \\
\hline & & & & & NR & $04 / 13 / 88$ & 5630 & & NR \\
\hline & & & & & NR & $04 / 13 / 88$ & 5670 & & NR \\
\hline & & & & & NR & $05 / 03 / 88$ & 5540 & & $\mathrm{NR}$ \\
\hline & & & & & NR & $05 / 03 / 88$ & 6020 & & NR \\
\hline & & & & & NR & $06 / 07 / 88$ & 4020 & & NR \\
\hline & & & & & $N R$ & $06 / 07 / 88$ & 4320 & & NR \\
\hline FSODIUM & $P P B$ & 200 & & $\begin{array}{l}01 / 07 / 88 \\
02 / 11 / 88\end{array}$ & $\begin{array}{l}197000 \\
296000\end{array}$ & $\begin{array}{l}01 / 06 / 88 \\
01 / 06 / 88\end{array}$ & $\begin{array}{l}95900 \\
99200\end{array}$ & $\begin{array}{l}03 / 16 / 88 \\
06 / 13 / 88\end{array}$ & $\begin{array}{l}12900 \\
13500\end{array}$ \\
\hline & & & & $03 / 09 / 88$ & 323000 & $02 / 11 / 88$ & 121000 & & NR \\
\hline FSOD IUM & PPB & 200 & & $04 / 14 / 88$ & 574000 & $02 / 11 / 88$ & 120000 & & NR \\
\hline & & & & $05 / 04 / 88$ & 178000 & $03 / 10 / 88$ & 165000 & & NR \\
\hline & & & & $06 / 07 / 88$ & 194000 & $03 / 10 / 88$ & 168000 & & NR \\
\hline & & & & & NR & $04 / 13 / 88$ & 161000 & & $\mathrm{NR}$ \\
\hline & & & & & NR & $04 / 13 / 88$ & 164000 & & $N R$ \\
\hline & & & & & NR & $05 / 03 / 88$ & 137000 & & NR \\
\hline & & & & & $N R$ & $05 / 03 / 88$ & $\uparrow 46000$ & & NR \\
\hline & & & & & $\mathrm{NR}$ & $06 / 07 / 88$ & 42400 & & NR \\
\hline & & & & & NR & $06 / 07 / 88$ & 41400 & & NR \\
\hline FSTRONT & $\mathrm{PPB}$ & 10 & & $0 \% / 07 / 88$ & 65 & $01 / 06 / 88$ & 142 & $03 / 16 / 88$ & 355 \\
\hline & & & & $02 / 11 / 88$ & 163 & $01 / 06 / 88$ & 147 & $06 / 13 / 88$ & 361 \\
\hline & & & & $03 / 09 / 88$ & 218 & $02 / 11 / 88$ & 231 & & NR \\
\hline & & & & $04 / 14 / 88$ & 193 & $02 / 11 / 88$ & 233 & & NR \\
\hline & & & & $05 / 04 / 88$ & 87 & $03 / 10 / 88$ & 259 & & NR \\
\hline & & & & $06 / 07 / 88$ & 83 & $03 / 10 / 88$ & 260 & & NR \\
\hline & & & & & NR & $04 / 13 / 88$ & 216 & & NR \\
\hline & & & & & NR & $04 / 13 / 88$ & 248 & & NR \\
\hline & & & & & NR & $05 / 03 / 88$ & 211 & & NR \\
\hline & & & & & NR & $05 / 03 / 88$ & 228 & & NR \\
\hline & & & & & NR & $06 / 07 / 88$ & 191 & & $N R$ \\
\hline & & & & & NR & $06 / 07 / 88$ & 194 & & $N R$ \\
\hline FVANADI & PPB & 5 & & $01 / 07 / 88$ & 8 & $01 / 06 / 88$ & 6 & $03 / 16 / 88$ & \\
\hline
\end{tabular}


IABLE E.5. (contd)

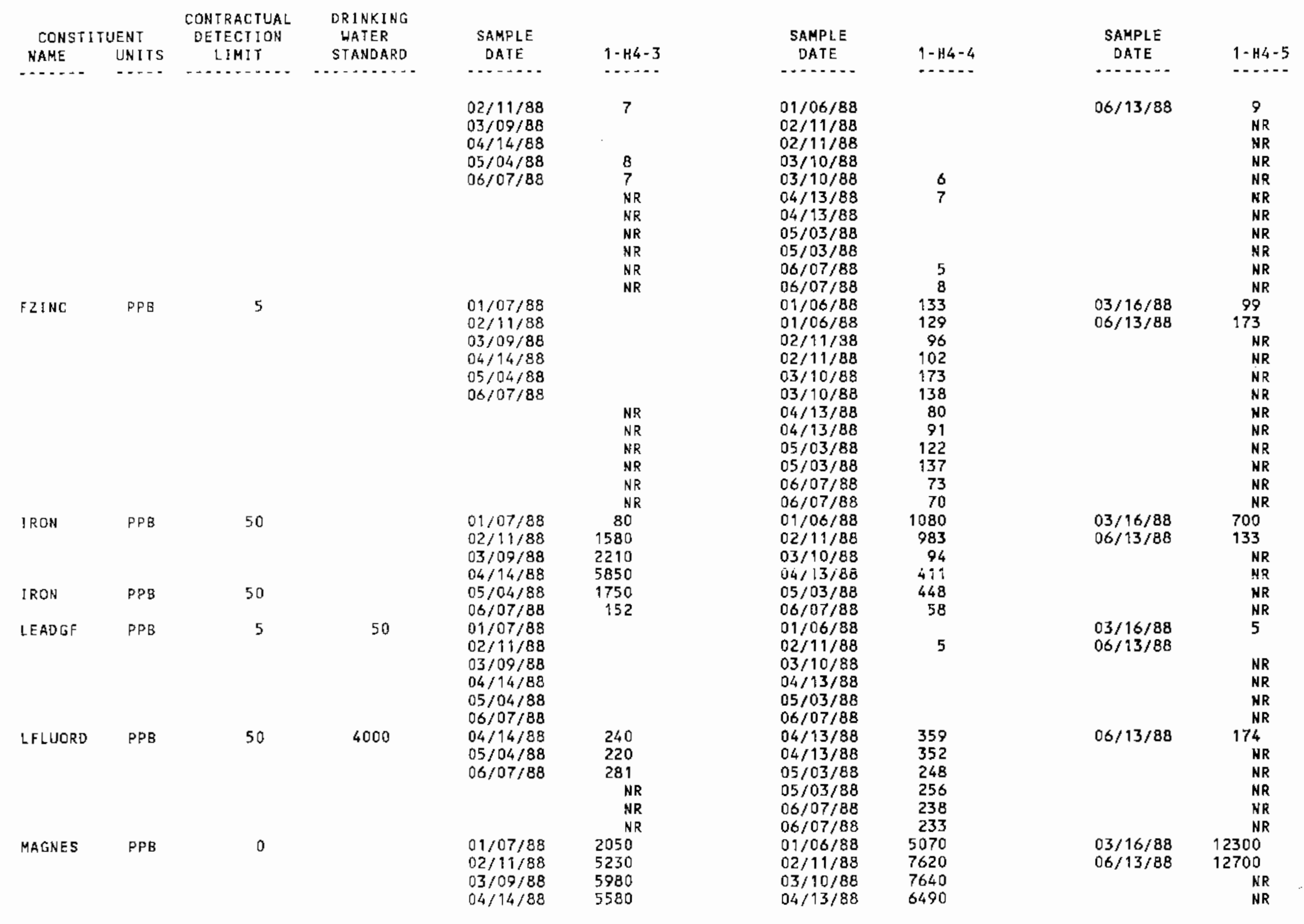


IABLE E.5. (contd)

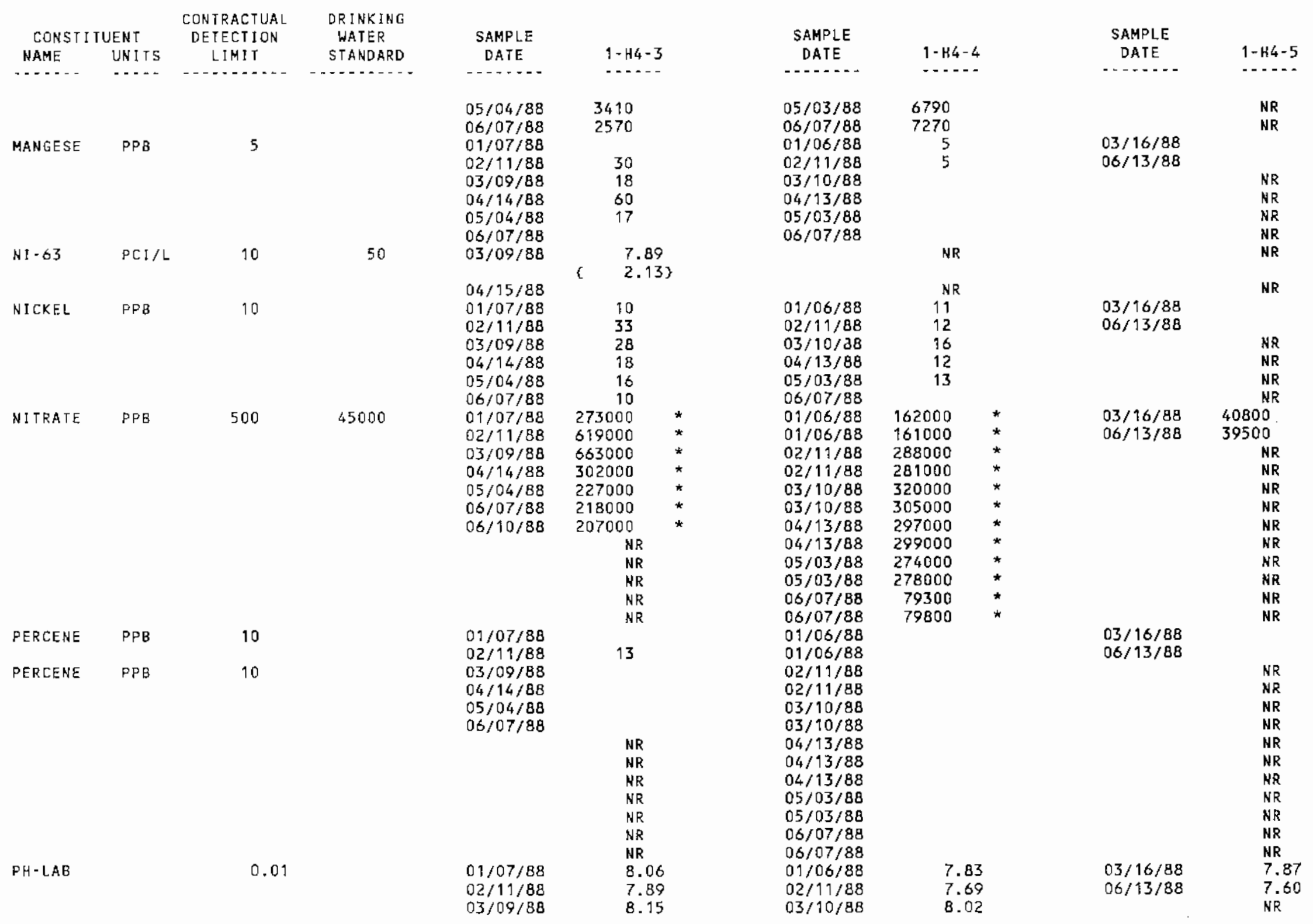


TABLE E.5. (contd)

\begin{tabular}{|c|c|c|c|c|c|c|c|c|c|}
\hline \multicolumn{2}{|c|}{ COHST I TUENT } & $\begin{array}{l}\text { CONTRACTUAL } \\
\text { DETECTION }\end{array}$ & $\begin{array}{l}\text { DRINKING } \\
\text { WATER }\end{array}$ & SAMPLE & & SAMPLE & & SAMPLE & \\
\hline \multirow{5}{*}{$\begin{array}{l}\text { NAME } \\
-\ldots . .\end{array}$} & UNITS & LIMIT & STANDARD & DATE & $1-\mathrm{H}_{4}-3$ & DATE & $1-\mathrm{H} 4-4$ & DATE & $1-\mathrm{H} 4-5$ \\
\hline & $-\ldots$ & & & $\cdots$ & $\cdots$ & $\ldots$ & $\ldots$ & $\cdots$ & $\cdots$ \\
\hline & & & & $04 / 14 / 88$ & 8.20 & $04 / 13 / 88$ & 7.99 & & NR \\
\hline & & & & $05 / 04 / 88$ & 8.10 & $05 / 03 / 88$ & 8 & & HR \\
\hline & & & & $06 / 10 / 88$ & 8.20 & $06 / 07 / 88$ & 8 & & NR \\
\hline \multirow[t]{6}{*}{ PHFIELD } & & 0.10 & & $01 / 07 / 88$ & 8.70 & $01 / 06 / 88$ & 6.80 & $03 / 16 / 88$ & 7.60 \\
\hline & & & & $02 / 11 / 88$ & 7.70 & $02 / 11 / 88$ & 7.70 & $06 / 13 / 88$ & 7.70 \\
\hline & & & & $03 / 09 / 88$ & $\begin{array}{l}8.10 \\
8.40\end{array}$ & $03 / 10 / 88$ & 8 8 & & MR \\
\hline & & & & $05 / 04 / 88$ & 8.30 & $05 / 03 / 88$ & 7.90 & & NR \\
\hline & & & & $06 / 07 / 88$ & 8.30 & $06 / 07 / 88$ & 8 & & NR \\
\hline & & & & $06 / 10 / 88$ & 8.60 & & NR & & HR \\
\hline \multirow[t]{11}{*}{ PHOSPHA } & $P P B$ & 1000 & & $01 / 07 / 88$ & 13100 & $01 / 06 / 88$ & 7350 & $03 / 16 / 88$ & \\
\hline & & & & $\begin{array}{l}02 / 11 / 88 \\
03 / 09 / 88\end{array}$ & & $\begin{array}{l}01 / 06 / 88 \\
02 / 11 / 88\end{array}$ & 8670 & $06 / 13 / 88$ & NR \\
\hline & & & & $04 / 14 / 88$ & & $02 / 11 / 98$ & & & NR \\
\hline & & & & $05 / 04 / 88$ & & $03 / 10 / 88$ & & & NR \\
\hline & & & & $06 / 07 / 88$ & & $03 / 10 / 88$ & & & NR \\
\hline & & & & $D 6 / 10 / 88$ & & $04 / 13 / 88$ & & & NR \\
\hline & & & & & NR & $04 / 13 / 88$ & & & NR \\
\hline & & & & & NR & $05 / 03 / 88$ & & & NR \\
\hline & & & & & NR & $05 / 03 / 88$ & & & NR \\
\hline & & & & & NR & $06 / 07 / 88$ & & & NR \\
\hline & & & & & NR & $06 / 07 / 88$ & & & NR \\
\hline \multirow[t]{6}{*}{ POTASUM } & $P P B$ & 100 & & $01 / 07 / 88$ & 3740 & $01 / 06 / 88$ & 3960 & $03 / 16 / 88$ & 4440 \\
\hline & & & & $02 / 11 / 88$ & 5690 & $02 / 11 / 88$ & 5330 & $06 / 13 / 88$ & 5370 \\
\hline & & & & $03 / 09 / 88$ & 6040 & $03 / 10 / 88$ & 5910 & & NR \\
\hline & & & & $04 / 14 / 88$ & 5070 & $04 / 13 / 88$ & 5670 & & NR \\
\hline & & & & $05 / 04 / 8 B$ & $4 \bar{L} \bar{U}$ & $05 / 03 / 38$ & 5750 & & NR \\
\hline & & & & $06 / 07 / 88$ & 4100 & $06 / 07 / 88$ & 4690 & & NR \\
\hline \multirow[t]{6}{*}{ RADIUM } & $\mathrm{PCI} / \mathrm{L}$ & 1 & 5 & $01 / 07 / 88$ & & $01 / 06 / 88$ & & $03 / 16 / 88$ & \\
\hline & & & & $02 / 11 / 88$ & & $02 / 11 / 88$ & 0.16 & $06 / 13 / 88$ & \\
\hline & & & & $03 / 09 / 88$ & & $03 / 10 / 88$ & & & NR \\
\hline & & & & $04 / 14 / 88$ & & $04 / 13 / 88$ & & & NR \\
\hline & & & & $05 / 04 / 88$ & & $05 / 03 / 88$ & & & NR \\
\hline & & & & $06 / 07 / 88$ & & $06 / 07 / 88$ & & & NR \\
\hline \multirow[t]{5}{*}{ SELENUM } & PPB & 5 & 10 & $01 / 07 / 88$ & & $01 / 06 / 88$ & & $03 / 16 / 88$ & \\
\hline & & & & $\begin{array}{l}02 / 11 / 88 \\
03 / 09 / 88\end{array}$ & & $02 / 11 / 88$ & & $06 / 13 / 88$ & NR \\
\hline & & & & $04 / 14 / 88$ & 7 & $04 / 13 / 88$ & & & NR \\
\hline & & & & $05 / 04 / 88$ & & $05 / 03 / 88$ & & & NR \\
\hline & & & & $06 / 07 / 88$ & & $06 / 07 / 88$ & & & NR \\
\hline \multirow[t]{4}{*}{$\$ 00 \mathrm{IUM}$} & PPB & 200 & & $09 / 07 / 88$ & 209000 & $01 / 06 / 88$ & 81400 & $03 / 16 / 88$ & 10800 \\
\hline & & & & $02 / 11 / 88$ & 301000 & $02 / 11 / 88$ & 116000 & $06 / 13 / 88$ & 12200 \\
\hline & & & & $03 / 09 / 88$ & 310000 & $03 / 10 / 88$ & 159000 & & NR \\
\hline & & & & $04 / 14 / 88$ & 209000 & $04 / 13 / 88$ & 157000 & & NR \\
\hline
\end{tabular}


TABLE E.5. (contd)

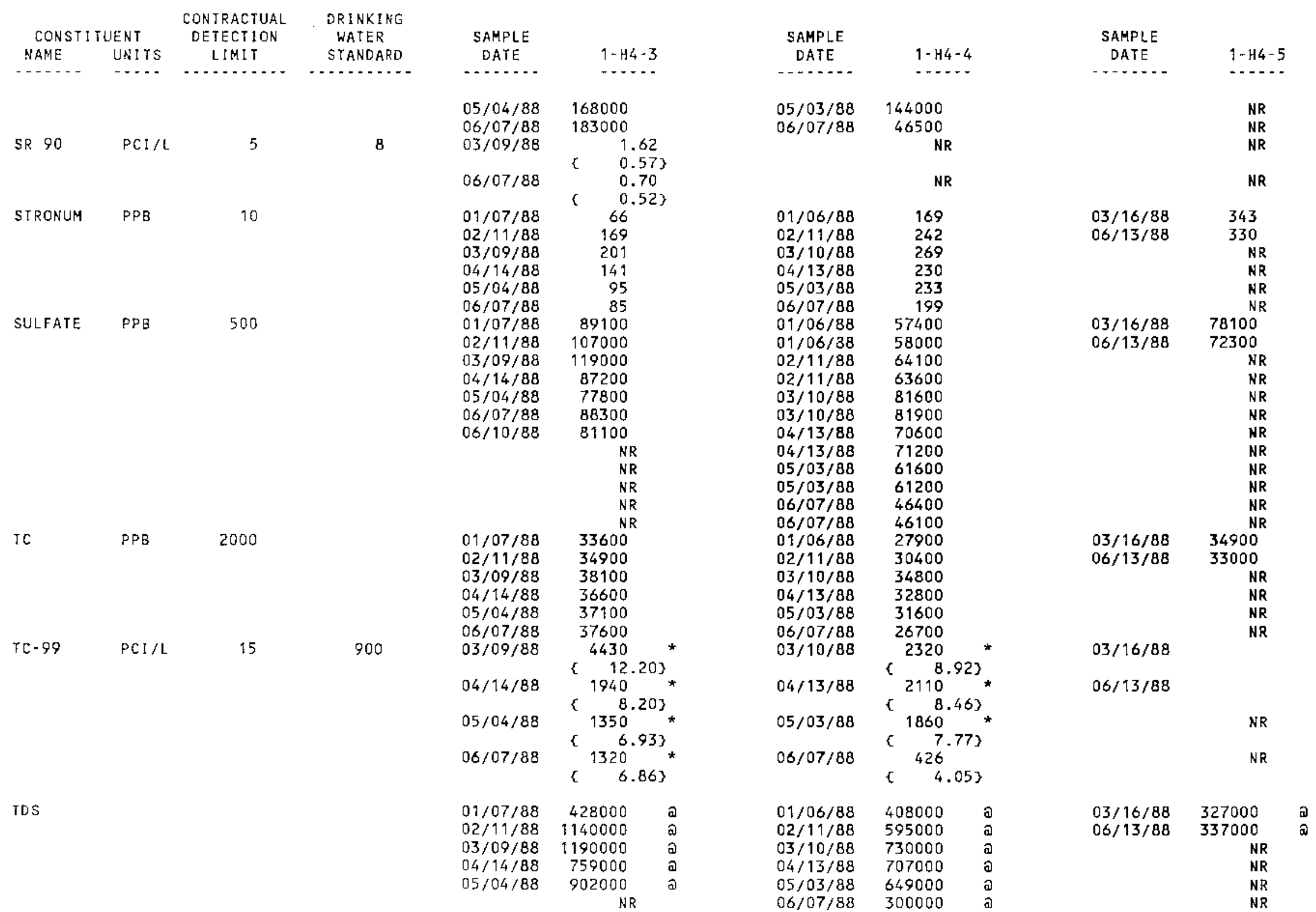


TABLE E.5. (contd)

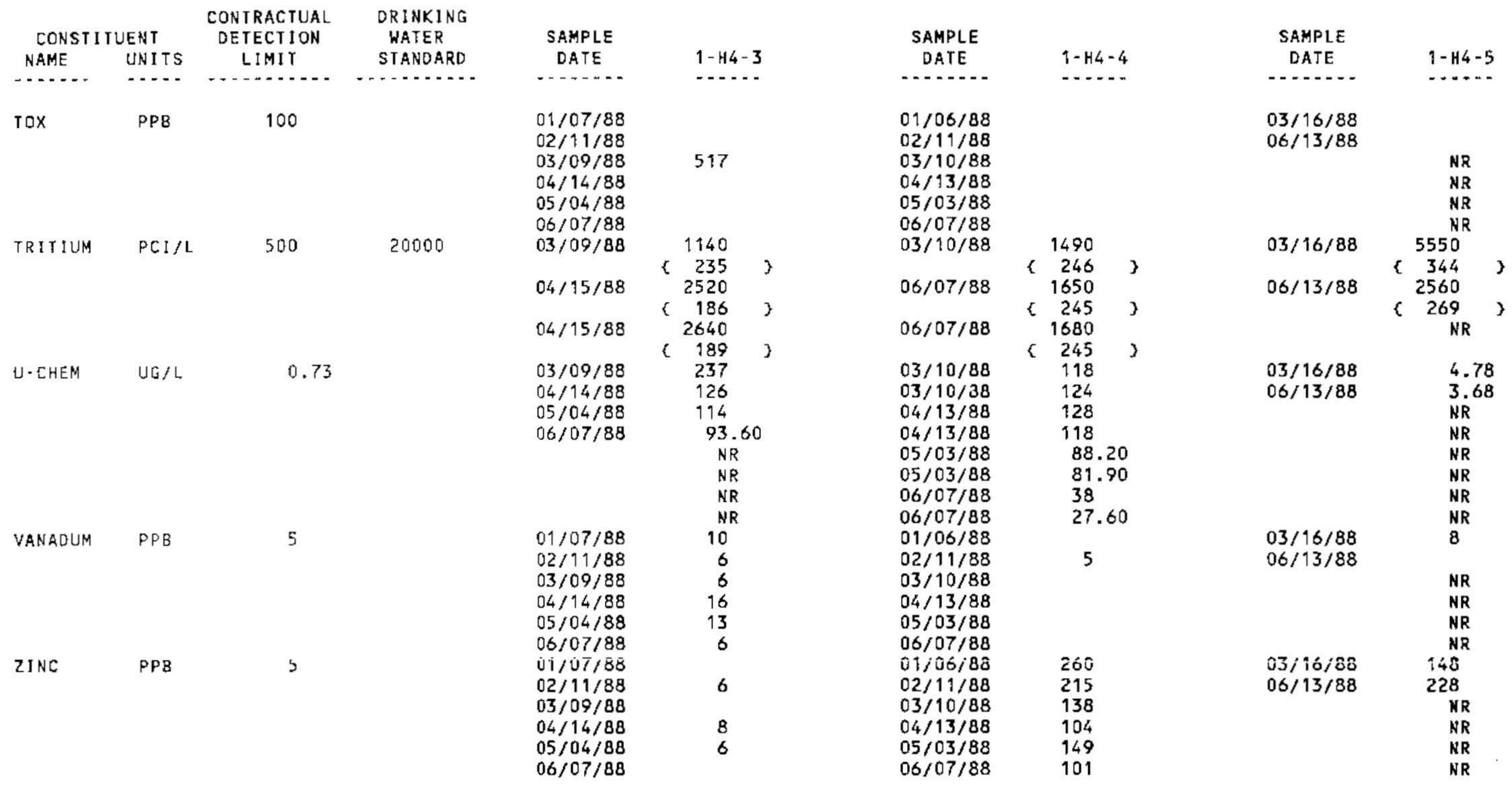


TABLE E.5. (contd)

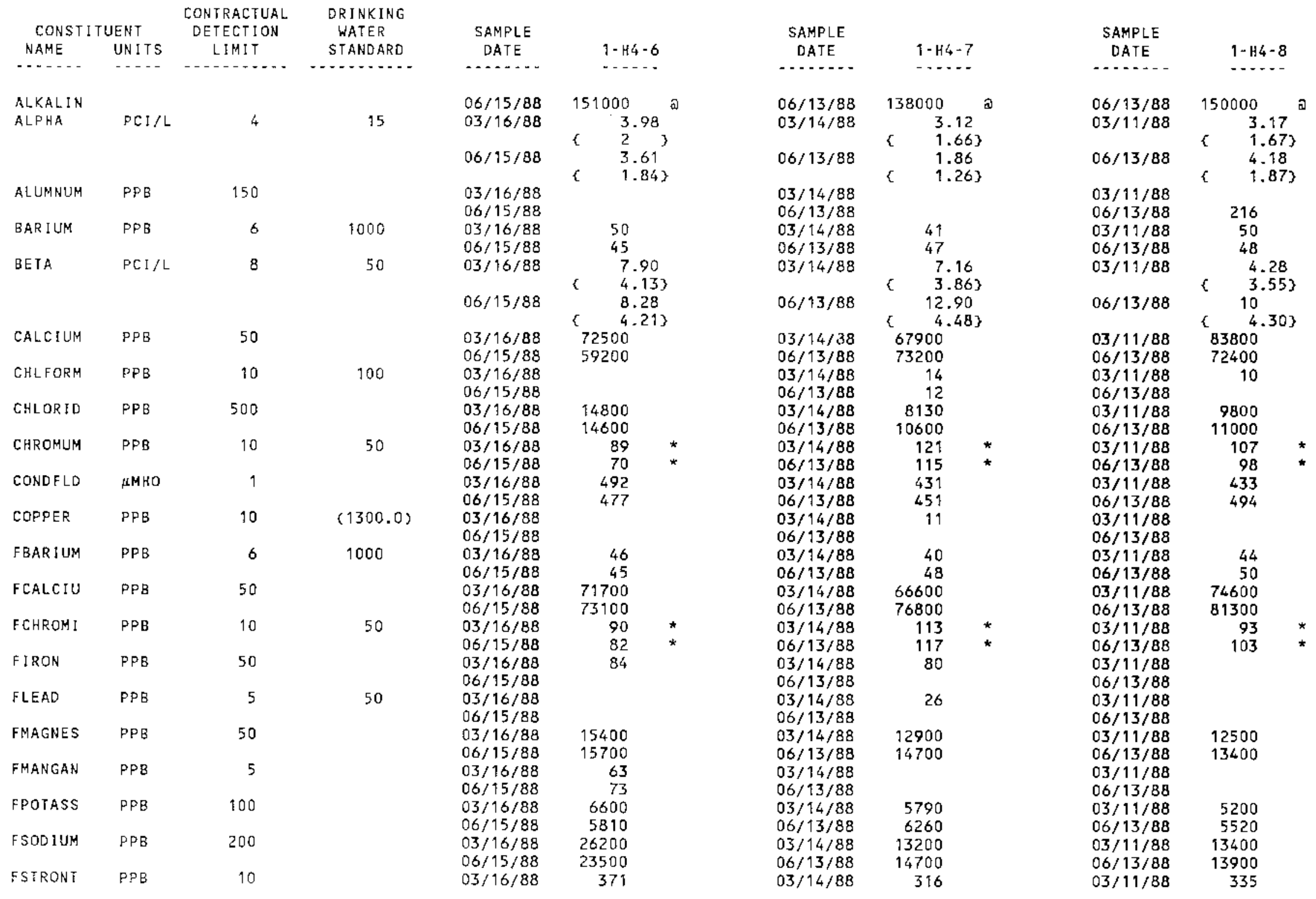


TABLE_E.5. (contd)

\begin{tabular}{|c|c|c|c|c|c|c|}
\hline \multicolumn{2}{|c|}{ CONST I TUENT } & $\begin{array}{l}\text { CONTRACTUAL } \\
\text { DETECTION }\end{array}$ & $\begin{array}{l}\text { DRINKING } \\
\text { WATER }\end{array}$ & SAMPLE & & \\
\hline NAME & UNITS & LIMIT & SIANDARD & DATE & 1-H4- & \\
\hline$-\ldots$. & $\cdots$ & $\cdots$ & - & $\ldots . .$. & $\ldots$. & \\
\hline FVANADI & PPB & 5 & & $\begin{array}{l}06 / 15 / 88 \\
03 / 16 / 88 \\
06 / 15 / 88\end{array}$ & 367 & \\
\hline FZINC & PPB & 5 & & $03 / 16 / 88$ & 171 & \\
\hline $\begin{array}{l}\text { FZINC } \\
\text { IRON }\end{array}$ & $\begin{array}{l}P P B \\
P P B\end{array}$ & $\begin{array}{r}5 \\
50\end{array}$ & & $\begin{array}{l}06 / 15 / 88 \\
03 / 16 / 88 \\
06 / 15 / 88\end{array}$ & $\begin{array}{l}243 \\
234 \\
146\end{array}$ & \\
\hline $\begin{array}{l}\text { LFLUORD } \\
\text { MAGNES }\end{array}$ & $\begin{array}{l}\text { PPB } \\
\text { PPB }\end{array}$ & $\begin{array}{r}50 \\
0\end{array}$ & 4000 & $\begin{array}{l}06 / 15 / 88 \\
03 / 16 / 88 \\
06 / 15 / 88\end{array}$ & $\begin{array}{r}225 \\
15400 \\
14300\end{array}$ & \\
\hline MANGESE & PPB & 5 & & $\begin{array}{l}03 / 16 / 88 \\
06 / 15 / 88\end{array}$ & $\begin{array}{r}102 \\
77\end{array}$ & \\
\hline METHYCH & PPB & 5 & & $\begin{array}{l}03 / 16 / 88 \\
06 / 15 / 88\end{array}$ & 580 & \\
\hline NITRATE & PPB & 500 & 45000 & $\begin{array}{l}03 / 16 / 88 \\
06 / 15 / 88\end{array}$ & $\begin{array}{l}40000 \\
38900\end{array}$ & \\
\hline$P H-L A B$ & & 0.01 & & $\begin{array}{l}03 / 16 / 88 \\
06 / 15 / 88\end{array}$ & $\begin{array}{l}8.0 \\
7.8\end{array}$ & \\
\hline PHFIELD & & 0.10 & & $\begin{array}{l}03 / 16 / 88 \\
06 / 15 / 88\end{array}$ & $\begin{array}{l}8.1 \\
7.4\end{array}$ & \\
\hline POTASUM & PPB & 100 & & $\begin{array}{l}03 / 16 / 88 \\
06 / 15 / 88\end{array}$ & $\begin{array}{l}5890 \\
6530\end{array}$ & \\
\hline RADIUM & $P C I / L$ & 1 & 5 & $03 / 16 / 88$ & & \\
\hline SODIUM & PPB & 200 & & $\begin{array}{l}06 / 15 / 88 \\
03 / 16 / 88 \\
06 / 15 / 38\end{array}$ & $\begin{array}{l}23300 \\
25600\end{array}$ & \\
\hline STRONUM & PPB & 10 & & $\begin{array}{l}03 / 16 / 88 \\
06 / 15 / 88\end{array}$ & $\begin{array}{l}378 \\
337\end{array}$ & \\
\hline SULFATE & PPB & 500 & & $\begin{array}{l}03 / 16 / 88 \\
06 / 15 / 88\end{array}$ & $\begin{array}{l}99400 \\
91600\end{array}$ & \\
\hline TC & $P P B$ & 2000 & & $\begin{array}{l}03 / 16 / 88 \\
06 / 15 / 88\end{array}$ & $\begin{array}{l}34900 \\
33800\end{array}$ & \\
\hline TDS & & & & $\begin{array}{l}03 / 16 / 88 \\
06 / 15 / 88\end{array}$ & $\begin{array}{l}360000 \\
386000\end{array}$ & a \\
\hline tax & PPB & 100 & & $\begin{array}{l}03 / 16 / 88 \\
06 / 15 / 88\end{array}$ & 720 & \\
\hline TRITIUM & PCI/L & 500 & 20000 & $\begin{array}{l}03 / 16 / 88 \\
06 / 15 / 88\end{array}$ & $\begin{array}{r}2320 \\
269 \\
4900 \\
6 \quad 278\end{array}$ & $\begin{array}{l}3 \\
3\end{array}$ \\
\hline
\end{tabular}

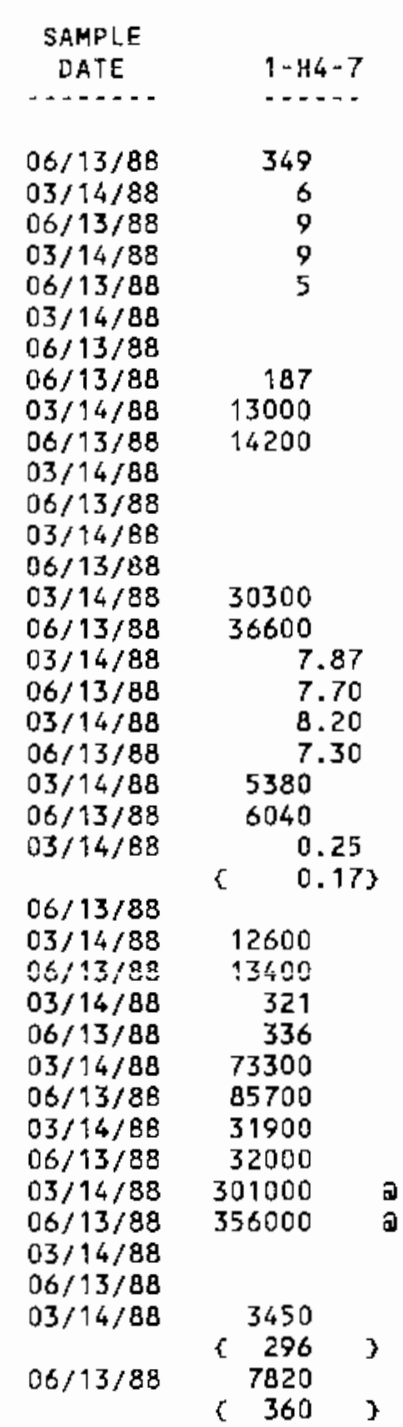

\begin{tabular}{l} 
SAMPLE \\
DATE \\
\hdashline$-1-H 4-8$
\end{tabular}

$06 / 13 / 88 \quad 352$ $\begin{array}{ll}03 / 11 / 88 & 7 \\ 06 / 13 / 88 & 7\end{array}$ $03 / 11 / 8 B \quad 8$

$06 / 13 / 88$ $03 / 11 / 88 \quad 50$ $06 / 13 / 88 \quad 453$ $03 / 11 / 88 \quad 13400$ $03 / 11 / 88$

$06 / 13 / 88.15$

$03 / 11 / 88$

$06 / 13 / 88$

$03 / 11 / 88 \quad 37100$

$06 / 13 / 88 \quad 38400$

$03 / 41 / 88 \quad 7.84$

$03 / 11 / 88-7.70$

$\begin{array}{ll}06 / 13 / 88 & 7.70 \\ 03 / 11 / 88 & 7.50\end{array}$

$03 / 11 / 88 \quad 5240^{\circ}$

$\begin{array}{ll}06 / 13 / 88 & 5420\end{array}$

$03 / 11 / 88$

$06 / 13 / 88$

$03 / 11 / 88 \quad 13700$

$06 / 13 / 8 \mathrm{~B} \quad 12000$

$03 / 11 / 88 \quad 353$

$06 / 13 / 88 \quad 321$

$03 / 11 / 88 \quad 77600$

$03 / 11 / 88$ \% 77600

$\begin{array}{ll}06 / 13 / 88 & 74000 \\ 03 / 11 / 88 & 38700\end{array}$

$\begin{array}{ll}03 / 11 / 88 & 38700 \\ 06 / 13 / 88 & 34200\end{array}$

$03 / 11 / 88 \quad 335000$

$06 / 13 / 88 \quad 331000$

$03 / 11 / 88$

$06 / 13 / 88$

NR 
IABLE E.5. (contd)

\begin{tabular}{|c|c|c|c|c|c|c|c|c|c|}
\hline \multicolumn{2}{|c|}{ CONST I YUENT } & $\begin{array}{c}\text { CCNTRACTUAL } \\
\text { DETECTION }\end{array}$ & $\begin{array}{l}\text { DRINKING } \\
\text { WATER }\end{array}$ & SAMPLE & & SAMPLE & & SAMPLE & \\
\hline NAME & UNI IS & LIMIT & STANDARD & DATE & $1-\mathrm{H}_{4}-6$ & DATE & $1-\mathrm{H}_{4}-7$ & DATE & $1-H 4-8$ \\
\hline$\cdots$ & $\cdots$ & - & $-\ldots \ldots \ldots$ & $\ldots$ & $\ldots-$ & $-\ldots$ & $\ldots$ & - - - - & \\
\hline U-CHEM & UG $/ \mathrm{L}$ & 0.73 & & $\begin{array}{l}03 / 16 / 88 \\
06 / 15 / 88\end{array}$ & $\begin{array}{l}4.43 \\
5.14\end{array}$ & $\begin{array}{l}03 / 14 / 88 \\
06 / 13 / 88\end{array}$ & $\begin{array}{l}5.49 \\
4.22\end{array}$ & $\begin{array}{l}03 / 11 / 88 \\
06 / 13 / 88\end{array}$ & $\begin{array}{l}4 \\
4.91\end{array}$ \\
\hline VANADUM & PPB & 5 & & $\begin{array}{l}03 / 16 / 88 \\
06 / 15 / 88\end{array}$ & 8 & $\begin{array}{l}03 / 14 / 88 \\
06 / 13 / 88\end{array}$ & 9 & $\begin{array}{l}03 / 11 / 88 \\
06 / 13 / 88\end{array}$ & \\
\hline 2 INC & PPB & 5 & & $\begin{array}{l}03 / 16 / 88 \\
06 / 15 / 88\end{array}$ & $\begin{array}{l}177 \\
176\end{array}$ & $\begin{array}{l}03 / 14 / 88 \\
06 / 13 / 88\end{array}$ & $\begin{array}{r}12 \\
9\end{array}$ & $\begin{array}{l}03 / 11 / 88 \\
06 / 13 / 88\end{array}$ & $\begin{array}{l}6 \\
8\end{array}$ \\
\hline
\end{tabular}


TABLE E.5. (contd)

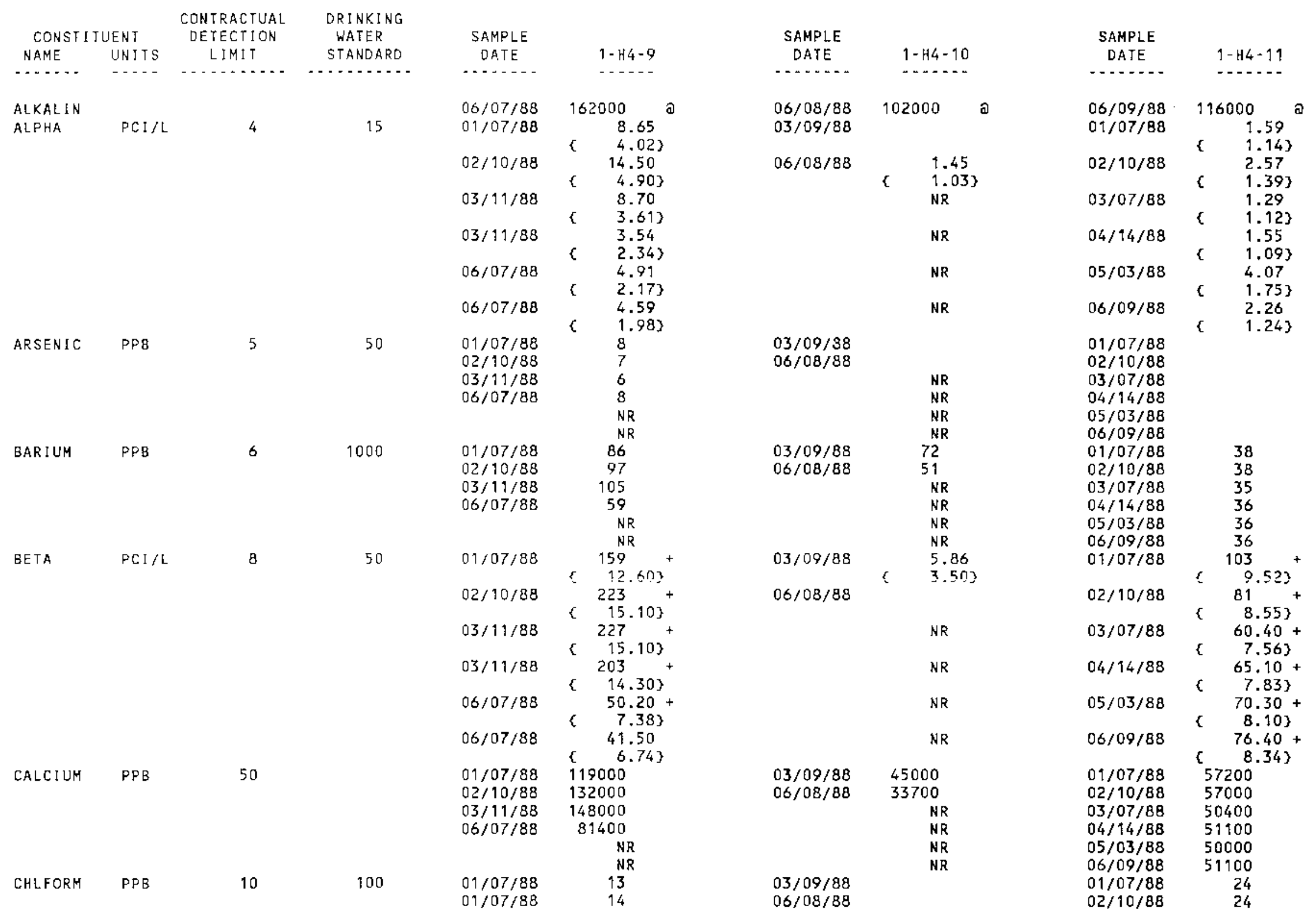


IABLE. F.5. (contd)

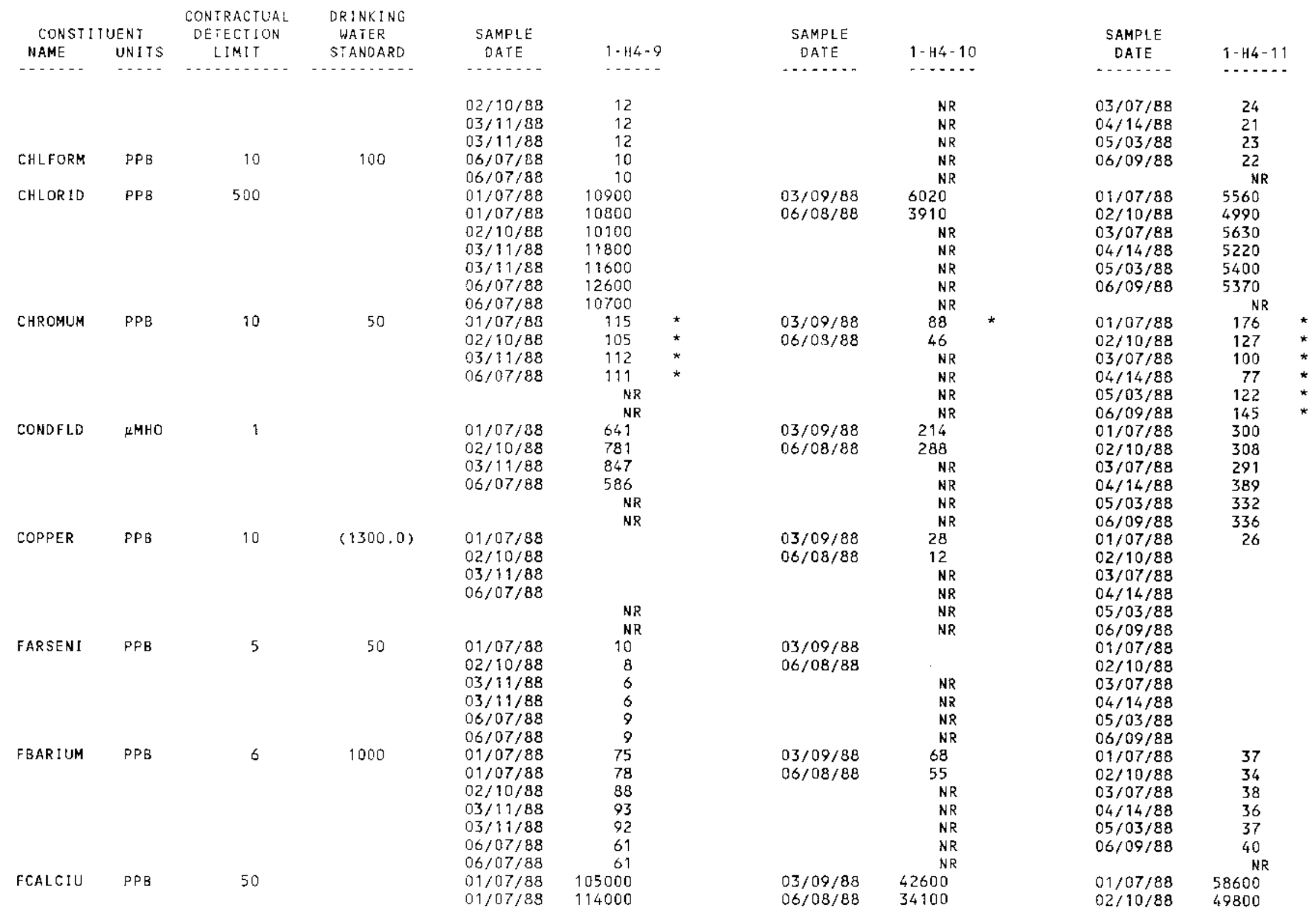


TABLE E. 5. (contd)

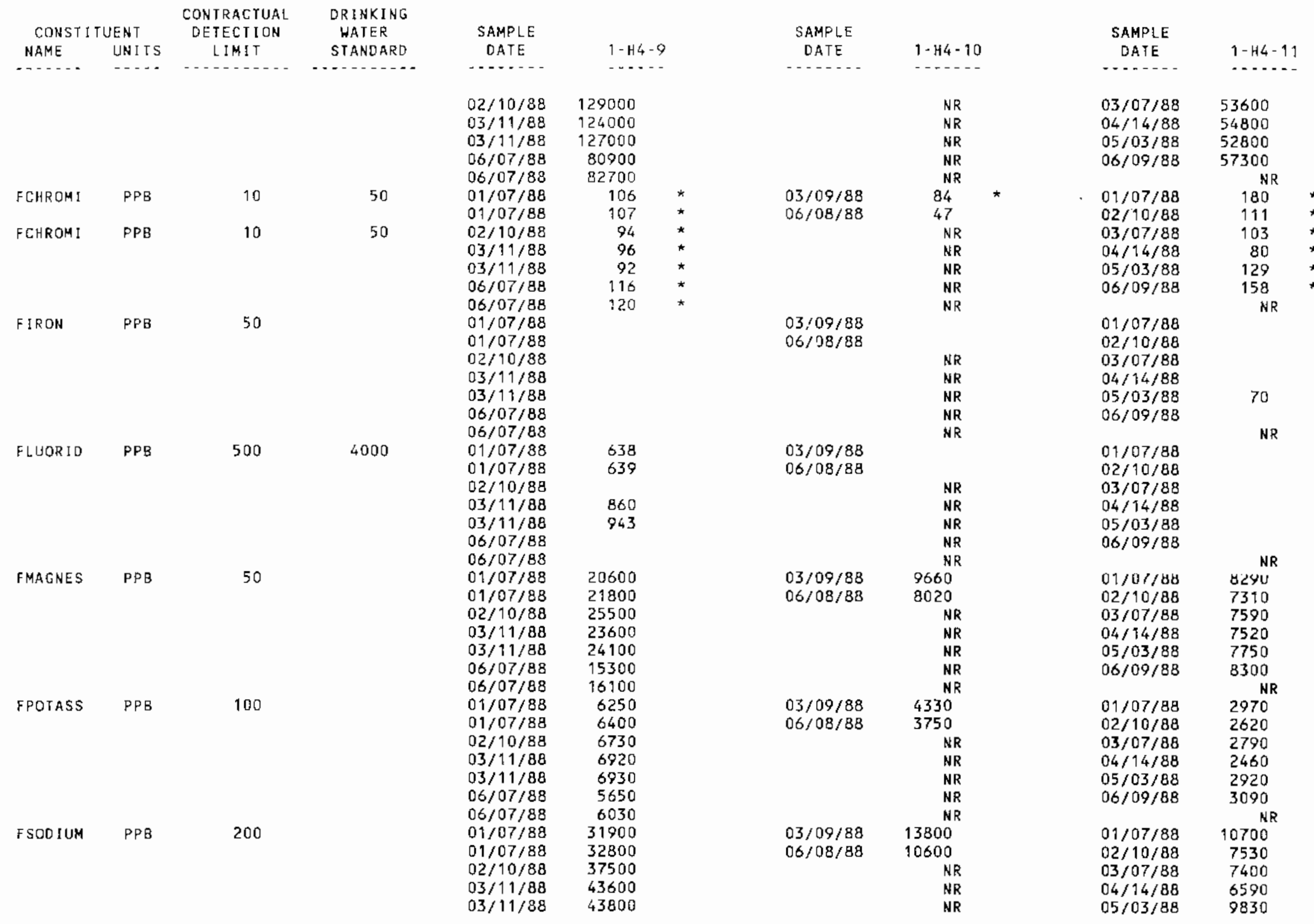


TABLE E.5. (contd)

\begin{tabular}{|c|c|c|c|c|c|c|c|c|c|}
\hline CONSTI & UENT & $\begin{array}{l}\text { CONTRACTUAL } \\
\text { OETECTIDN }\end{array}$ & $\begin{array}{l}\text { DR INK ING } \\
\text { WATER }\end{array}$ & SAMPLE & & SAMPLE & & & \\
\hline NAME & UNITS & LIMIT & STANDARD & DATE & $1-\mathrm{H}_{4}-\mathrm{C}$ & $\begin{array}{l}\text { SAMPLE } \\
\text { DATE }\end{array}$ & $1-\mathrm{H}_{4}-10$ & $\begin{array}{l}\text { SAMPLE } \\
\text { DATE }\end{array}$ & $1-H_{4}-11$ \\
\hline - . . - & -- & n & $\ldots \ldots$ & $\cdots \cdots$ & $-\cdots$ & $\ldots \ldots$ & - - . - & $\cdots$ & $\cdots-\cdots$ \\
\hline & & & & $06 / 07 / 88$ & 25100 & & $\mathrm{NR}$ & $06 / 09 / 88$ & 9610 \\
\hline & & & & $06 / 07 / 88$ & 26600 & & $\mathrm{NR}$ & & NR \\
\hline FSTRONT & $\mathrm{PPB}$ & 10 & & $01 / 07 / 88$ & 495 & $03 / 09 / 88$ & 206 & $01 / 07 / 88$ & 254 \\
\hline & & & & $01 / 07 / 88$ & 500 & $06 / 08 / 88$ & 164 & $02 / 70 / 88$ & 221 \\
\hline & & & & $02 / 10 / 88$ & 572 & & $\mathrm{NR}$ & $03 / 07 / 88$ & 237 \\
\hline & & & & $03 / 11 / 88$ & 576 & & $\mathrm{NR}$ & $04 / 14 / 88$ & 241 \\
\hline & & & & $03 / 11 / 88$ & 572 & & NR & $05 / 03 / 88$ & 231 \\
\hline & & & & $06 / 07 / 88$ & 359 & & NR & $06 / 09 / 88$ & 250 \\
\hline & & & & $06 / 07 / 88$ & 370 & & NR & & NR \\
\hline FVANAD 1 & PPB & 5 & & $01 / 07 / 88$ & 5 & $03 / 09 / 88$ & 7 & $01 / 07 / 88$ & \\
\hline & & & & $01 / 07 / 88$ & 5 & $06 / 08 / 88$ & 9 & $02 / 10 / 88$ & \\
\hline FVANADI & PPB & 5 & & $02 / 10 / 88$ & & & NR & $03 / 07 / 88$ & \\
\hline & & & & $03 / 11 / 88$ & & & NR & $04 / 14 / 88$ & \\
\hline & & & & $03 / 1 \uparrow / 88$ & & & NR & $05 / 03 / 88$ & \\
\hline & & & & $06 / 07 / 88$ & 7 & & NR & $06 / 09 / 88$ & \\
\hline & & & & $06 / 07 / 88$ & 8 & & $\mathrm{NR}$ & & NR \\
\hline FZINC & $P P B$ & 5 & & $\begin{array}{l}01 / 07 / 88 \\
01 / 07 / 88\end{array}$ & & $\begin{array}{l}03 / 09 / 88 \\
06 / 08 / 88\end{array}$ & & $\begin{array}{l}01 / 07 / 88 \\
02 / 10 / 88\end{array}$ & \\
\hline & & & & $02 / 10 / 88$ & & $06 / 08 / 88$ & NR & $03 / 07 / 88$ & 13 \\
\hline & & & & $03 / 11 / 88$ & 10 & & NR & $04 / 14 / 88$ & \\
\hline & & & & $03 / 11 / 38$ & 6 & & NR & $05 / 03 / 88$ & \\
\hline & & & & $06 / 07 / 88$ & & & NR & $06 / 09 / 88$ & \\
\hline & & & & $06 / 07 / 88$ & & & NR & & NR \\
\hline IRON & $\mathrm{PPB}$ & 50 & & $\begin{array}{l}01 / 07 / 88 \\
02 / 10 / 88\end{array}$ & $\begin{array}{l}54 \\
57\end{array}$ & $\begin{array}{l}03 / 09 / 88 \\
06 / 08 / 88\end{array}$ & & $\begin{array}{l}01 / 07 / 88 \\
02 / 10 / 88\end{array}$ & \\
\hline & & & & $03 / 11 / 88$ & & & NR & $03 / 07 / 88$ & \\
\hline & & & & $06 / 07 / 88$ & 95 & & NR & $04 / 14 / 88$ & 151 \\
\hline & & & & & $\mathrm{NR}$ & & NR & $05 / 03 / 88$ & \\
\hline & & & & & NR & & NR & $06 / 09 / 88$ & \\
\hline LFLUORD & PPB & 50 & 4000 & $06 / 07 / 88$ & 255 & $06 / 08 / 88$ & 251 & $04 / 14 / 88$ & 180 \\
\hline & & & & $06 / 07 / 88$ & 257 & & NR & $05 / 03 / 88$ & 144 \\
\hline & & & & & NR & & NR & $06 / 09 / 88$ & 165 \\
\hline MAGNES & $\mathrm{PPB}$ & 0 & & $01 / 07 / 88$ & 23500 & $03 / 09 / 88$ & 10100 & $01 / 07 / 88$ & 8460 \\
\hline & & & & $02 / 10 / 88$ & 25600 & $06 / 08 / 88$ & 7770 & $02 / 10 / 88$ & 8090 \\
\hline & & & & $03 / 11 / 88$ & 26800 & & NR & $03 / 07 / 88$ & 7240 \\
\hline & & & & $06 / 07 / 88$ & 15700 & & NR & $04 / 14 / 88$ & 7080 \\
\hline & & & & & NR & & NR & $05 / 03 / 88$ & 7230 \\
\hline & & & & & NR & & NR & $06 / 09 / 88$ & 7630 \\
\hline NITRATE & PPB & 500 & 45000 & $01 / 07 / 38$ & 206000 & $03 / 09 / 88$ & 17000 & $01 / 07 / 88$ & 50400 \\
\hline & & & & $01 / 07 / 88$ & 206000 & $06 / 08 / 88$ & 9620 & $02 / 10 / 88$ & 26100 \\
\hline & & & & $02 / 10 / 88$ & 131000 & * & NR & $03 / 07 / 88$ & 25800 \\
\hline & & & & $03 / 11 / 88$ & 256000 & * & NR & $04 / 14 / 88$ & 25400 \\
\hline & & & & $03 / 11 / 88$ & 256000 & * & NR & $05 / 03 / 88$ & 22900 \\
\hline & & & & $06 / 07 / 88$ & 63600 & $\star$ & NR & $06 / 09 / 88$ & 29500 \\
\hline & & & & $06 / 07 / 88$ & 62800 & * & NR & & NR \\
\hline
\end{tabular}


TABLE E.5. (contd)

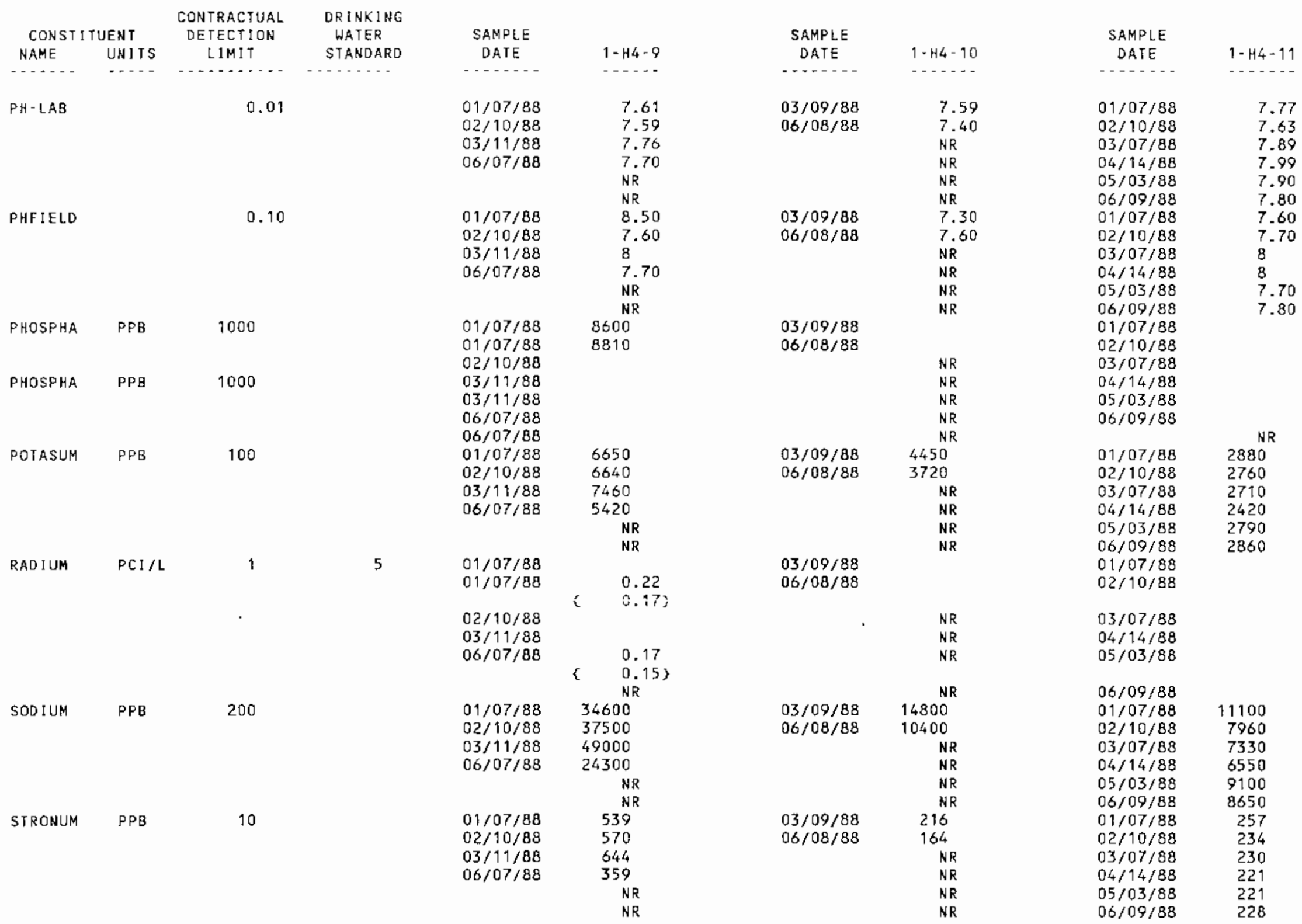


IABLE E.5. (contd)

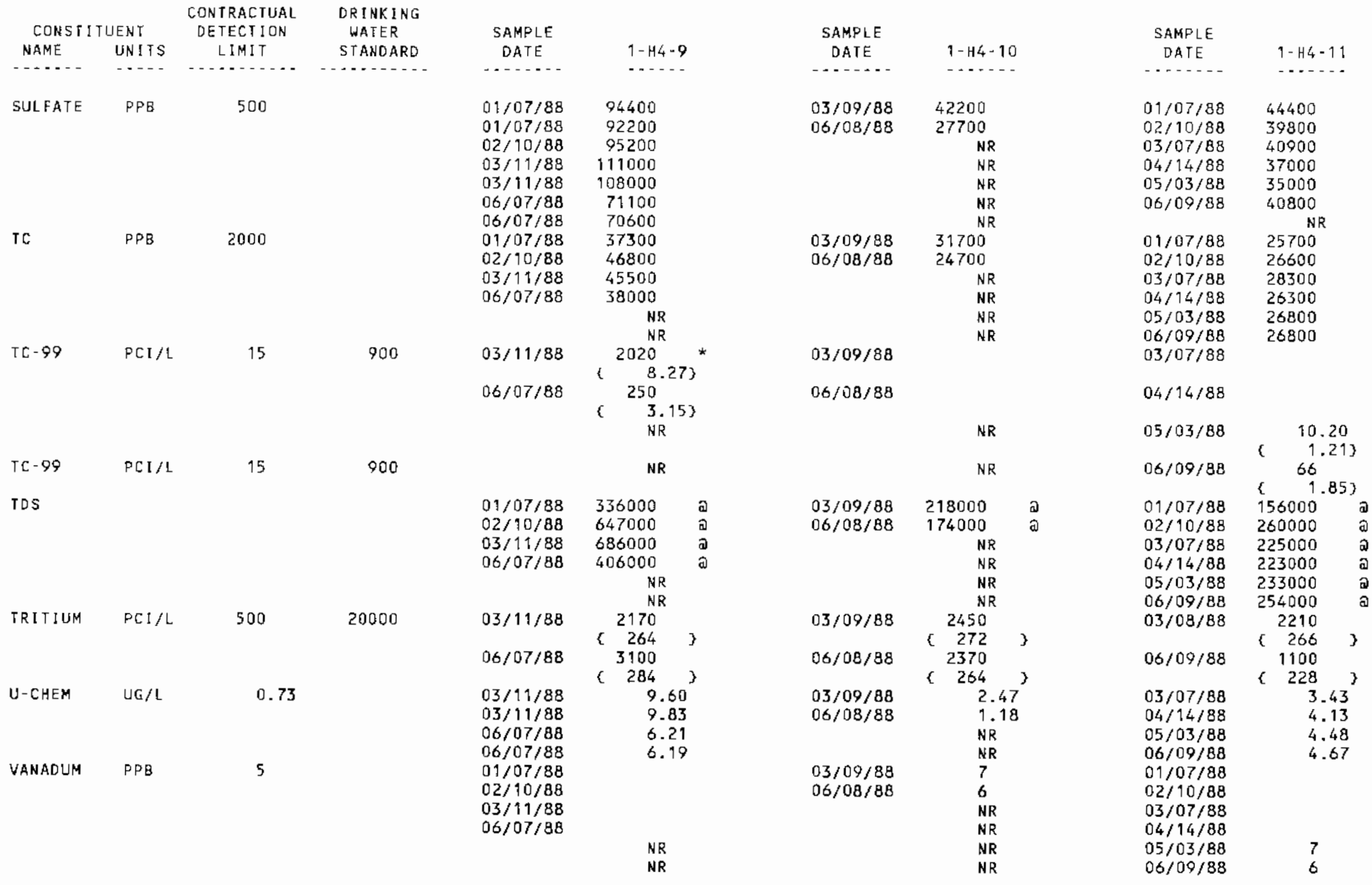


TABLEE.5. (contd)

\begin{tabular}{|c|c|c|c|}
\hline \multirow{2}{*}{\multicolumn{2}{|c|}{ CONST I TUEN T }} & CONTRACTUAL & DRINK ING \\
\hline & & DETECTION & WATER \\
\hline NAME & UNITS & LIMIT & STANDARD \\
\hline - & 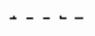 & $\ldots$ & 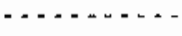 \\
\hline
\end{tabular}

$\begin{array}{cc}\text { SAMPI.E } & \\ \text { DATE } & 1-H 4-9 \\ -\cdots & \cdots \\ 01 / 07 / 88 & 6 \\ 02 / 10 / 88 & \\ 03 / 11 / 88 & 5 \\ 06 / 07 / 88 & \\ & \text { NR } \\ & \text { NR }\end{array}$

$\begin{array}{cc}\text { SAMPLE } & \\ \text { DATE } & 1-\mathrm{H} 4-10 \\ \cdots & \\ 03 / 09 / 88 & 20 \\ 06 / 08 / 88 & 11 \\ & \text { NR } \\ & \text { NR } \\ & \text { NR } \\ & \text { NR }\end{array}$

SAMPLE

DATE

1-H4- 11

ZINC

PPB

5

$01 / 07 / 88$

$02 / 10 / 88$

$03 / 07 / 88$

$04 / 14 / 88$

$05 / 03 / 88$

$06 / 09 / 88$ 
TABLE E.5. (contd)

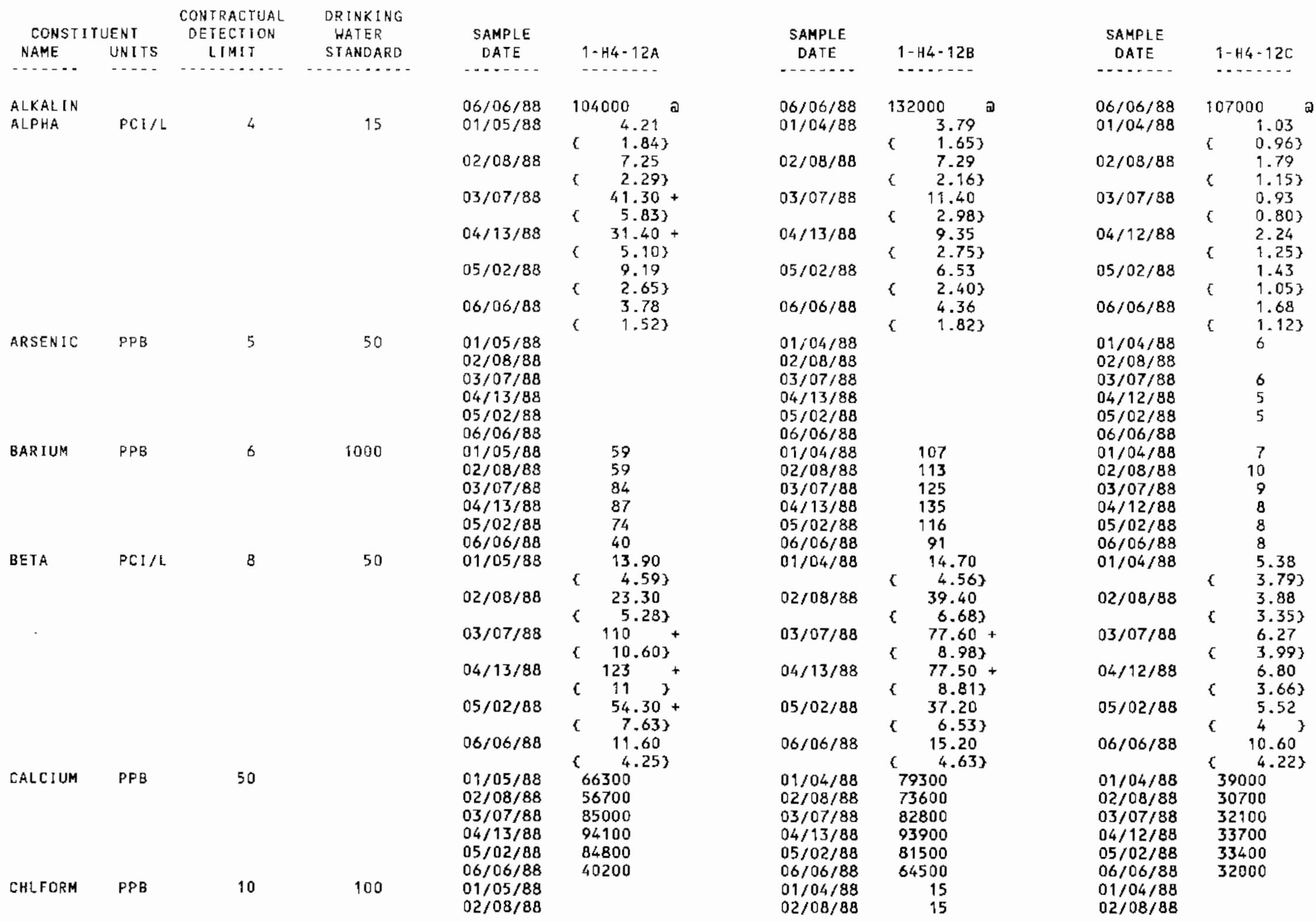


TABLE E.5. (contd)

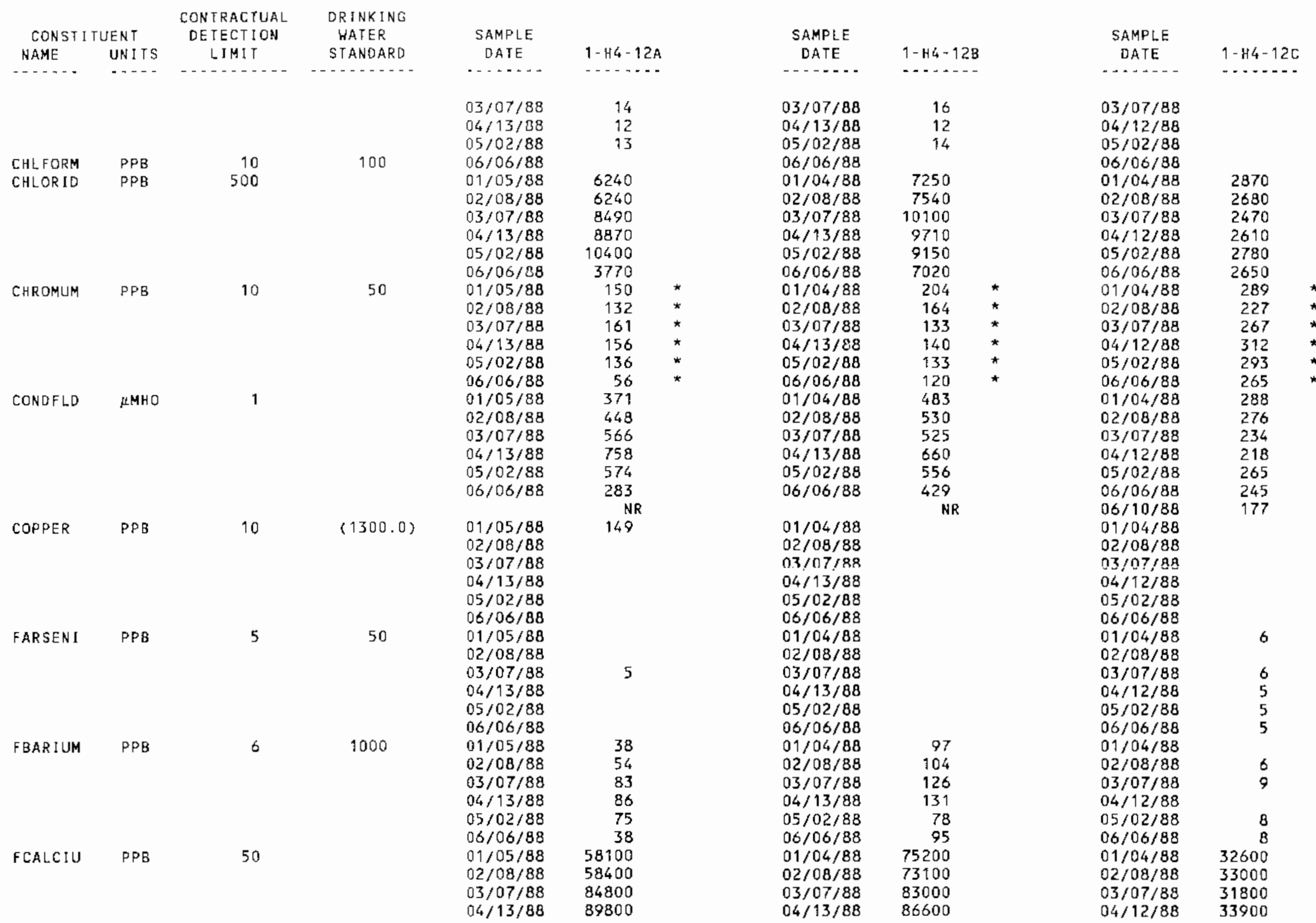


TABLE E.5. (contd)

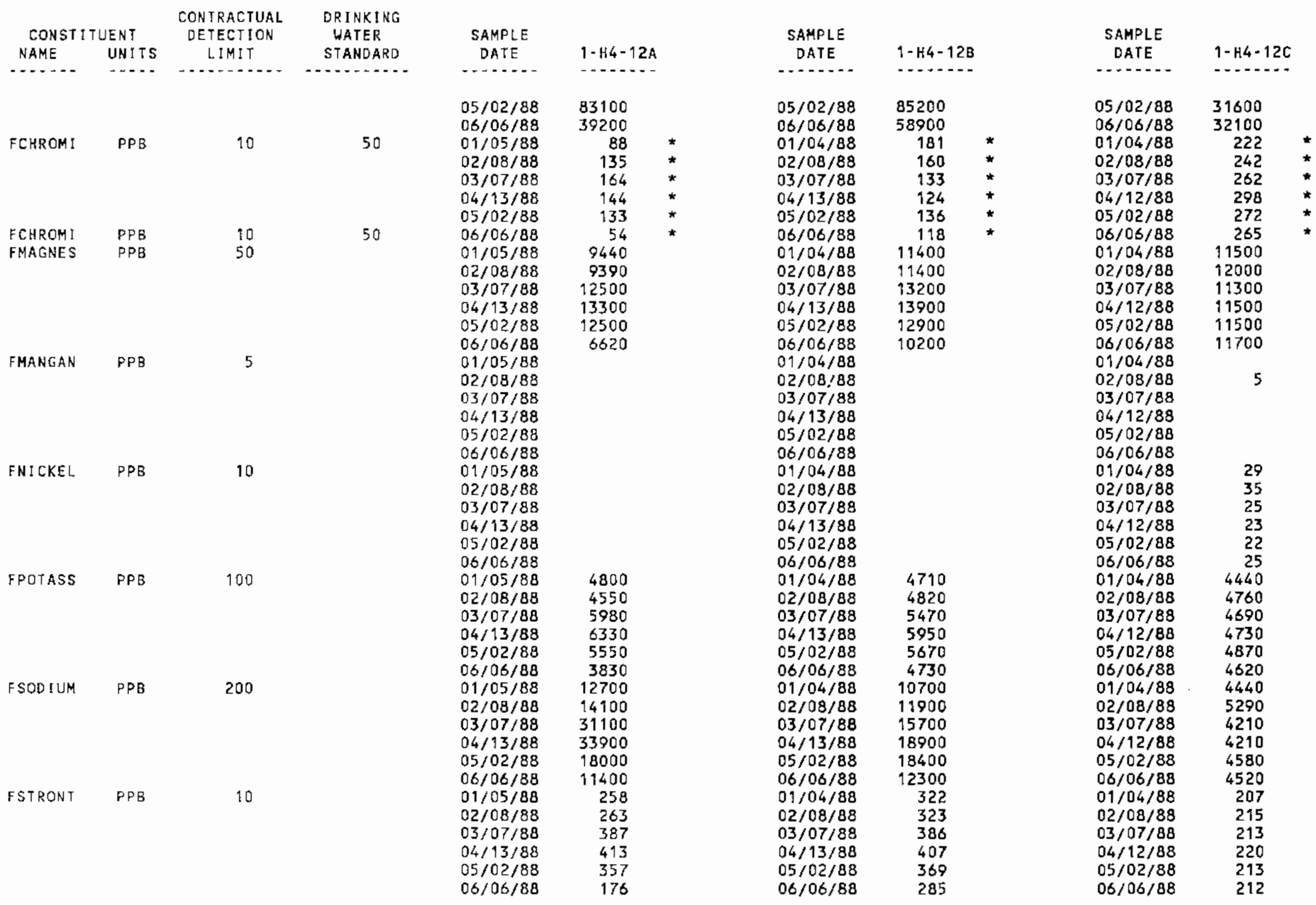


TABLE E.5. (contd)

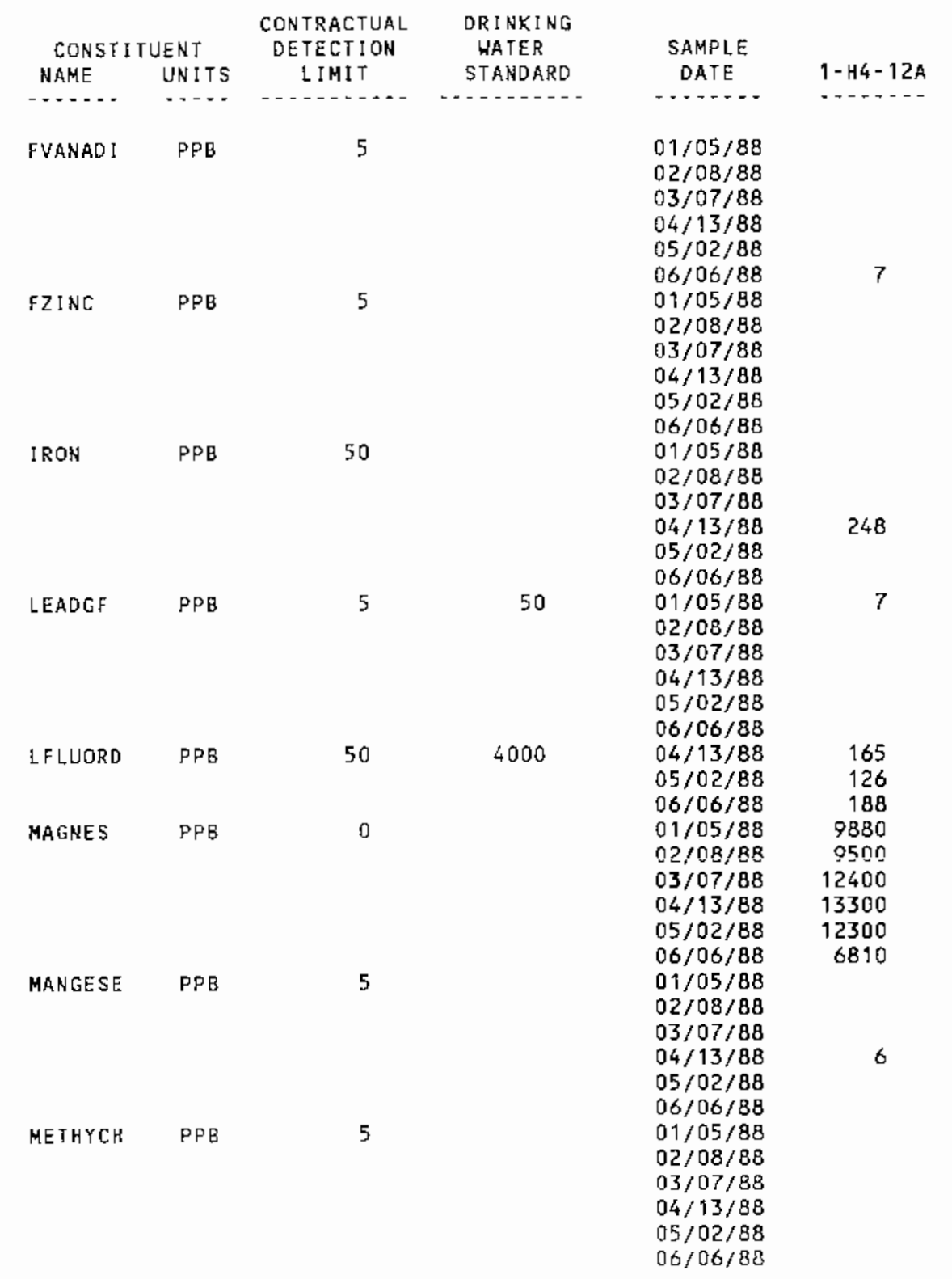

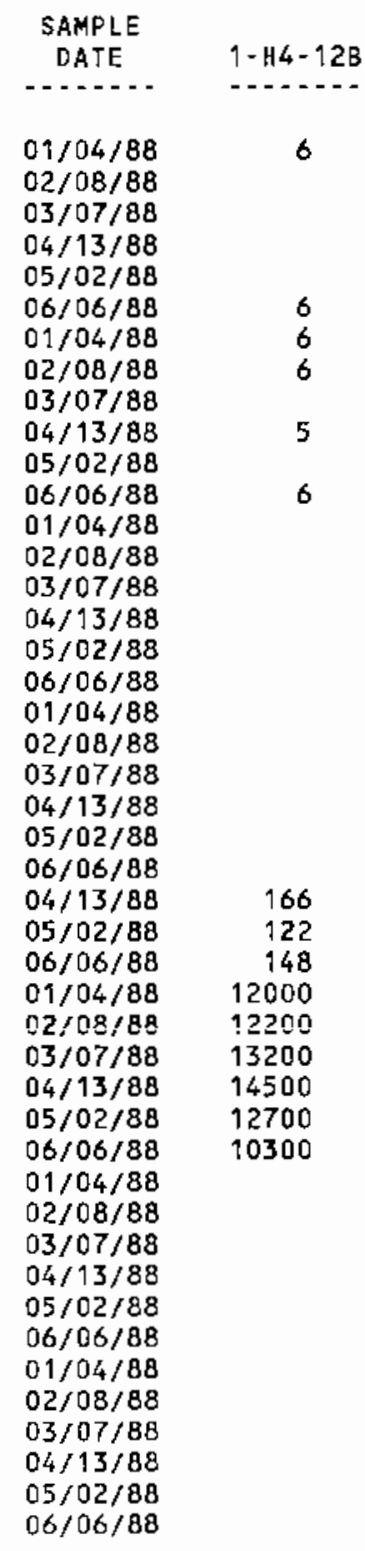

SAMPLE

DATE

$1-\mathrm{H} 4-12 \mathrm{C}$

$01 / 04 / 88 \quad 26$

$02 / 08 / 88$

$03 / 07 / 88 \quad 25$

$04 / 12 / 88 \quad 24$

$\begin{array}{ll}05 / 02 / 88 & 23 \\ 06 / 06 / 88 & 26\end{array}$

$\begin{array}{lr}06 / 06 / 88 & 26 \\ 01 / 04 / 88 & 5\end{array}$

$03 / 07 / 88$

$04 / 12 / 88$

$05 / 02 / 88$

$06 / 06 / 88$

$01 / 04 / 88$

$02 / 08 / 88$

$03 / 07 / 88$

$04 / 12 / 88$

$2 / 88$

$06 / 06 / 88$

$01 / 04 / 88$

$02 / 08 / 88$

$03 / 07 / 88$

$04 / 12 / 88$.

$05 / 02 / 88$

$06 / 06 / 88$

$04 / 12 / 88$

$05 / 02 / 88$

$06 / 06 / 88$

$01 / 04 / 88$

$02 / 08 / 88$

$03 / 07 / 88$

$04 / 12 / 88$

$05 / 02 / 88$

$06 / 06 / 88$

$01 / 04 / 88$

$02 / 08 / 88$

$03 / 07 / 88$

$04 / 12 / 88$

$05 / 02 / 88$

$06 / 06 / 88$

$01 / 04 / 88$

$02 / 08 / 88$

$03 / 07 / 88$

$04 / 12 / 88$

$05 / 02 / 88$

$06 / 06 / 88$ 
TABLE E.5. (contd)

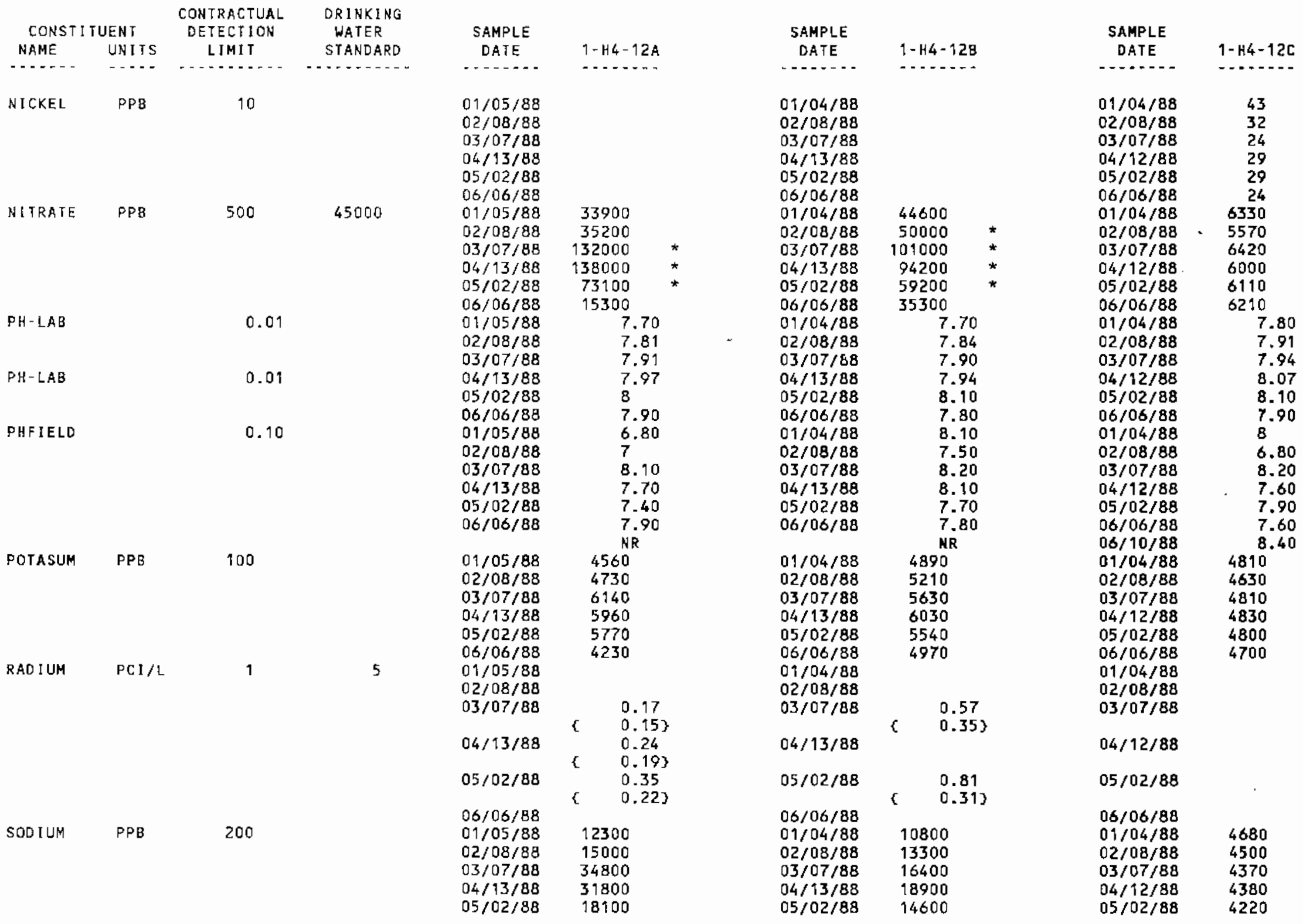


TABLE E.5. (contd)

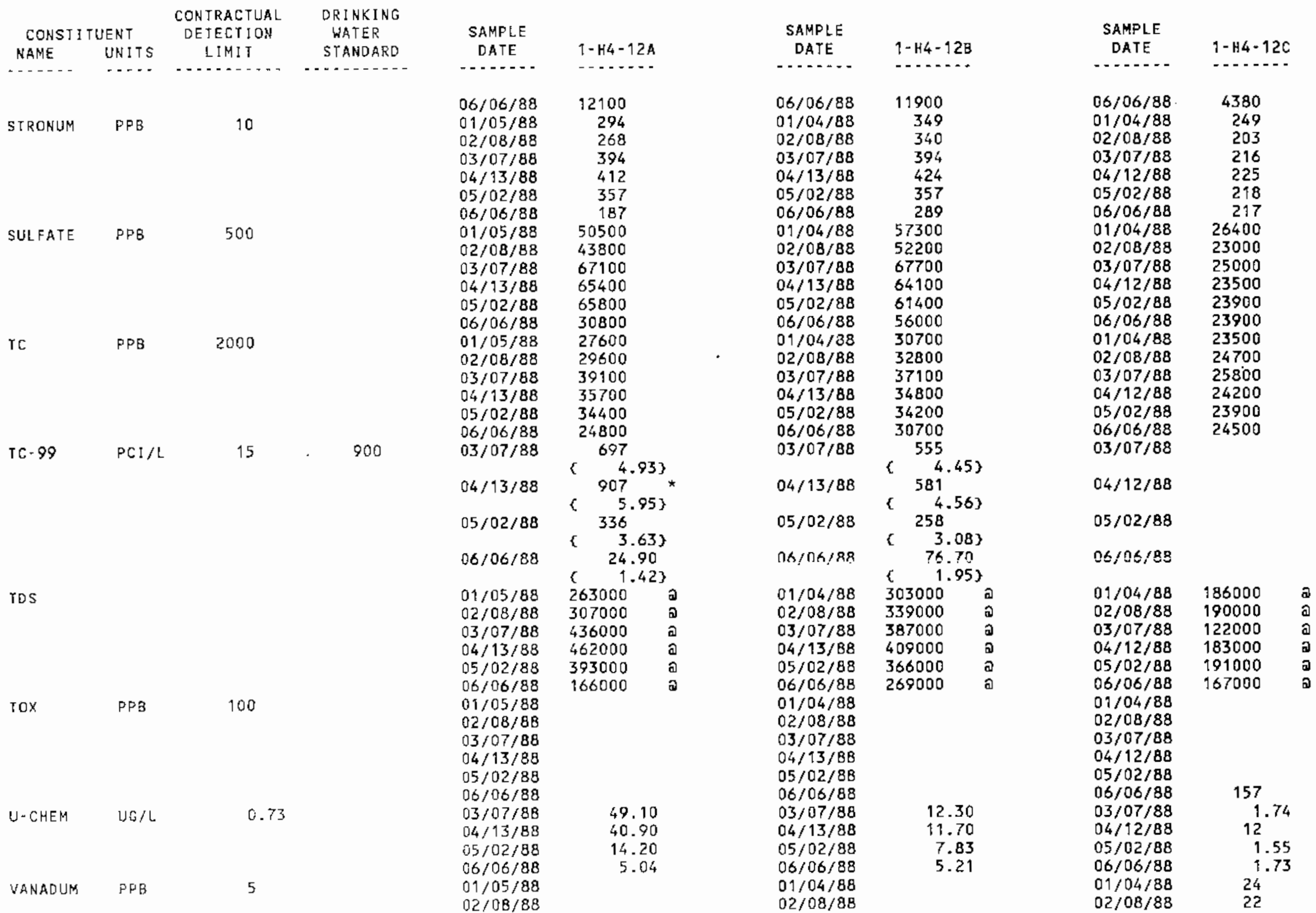


TABLE E.5. (contd)

\begin{tabular}{|c|c|c|c|c|c|}
\hline \multicolumn{2}{|c|}{ CONST I TUENT } & \multirow{2}{*}{$\begin{array}{c}\text { CONTRACTUAL } \\
\text { DETECTION } \\
\text { LIMIT }\end{array}$} & \multirow{2}{*}{$\begin{array}{l}\text { DRINKING } \\
\text { WATER } \\
\text { STANDARD }\end{array}$} & \multirow{2}{*}{$\begin{array}{l}\text { SAMPLE } \\
\text { DATE }\end{array}$} & \multirow[b]{2}{*}{$1-H 4-12 A$} \\
\hline NAME & UNITS & & & & \\
\hline 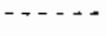 & $\cdots-$ & - & - & $\ldots . .$. & $\cdots$ \\
\hline & & & & $\begin{array}{l}03 / 07 / 88 \\
04 / 13 / 88 \\
05 / 02 / 88\end{array}$ & 6 \\
\hline & & & & $06 / 06 / 88$ & 6 \\
\hline $2 I N C$ & PPB & 5 & & $\begin{array}{l}01 / 05 / 88 \\
02 / 08 / 88 \\
03 / 07 / 88 \\
04 / 13 / 88 \\
05 / 02 / 88 \\
06 / 06 / 88\end{array}$ & 91 \\
\hline
\end{tabular}

$\begin{array}{lr}\text { SAMPLE } \\ \text { DATE } & 1-H 4-12 \\ & \\ 03 / 07 / 88 & 6 \\ 04 / 13 / 88 & \\ 05 / 02 / 88 & \\ 06 / 06 / 88 & 6 \\ 01 / 04 / 88 & 7 \\ 02 / 08 / 88 & 9 \\ 03 / 07 / 88 & 7 \\ 04 / 13 / 88 & 6 \\ 05 / 02 / 88 & 10 \\ 06 / 06 / 88 & 6\end{array}$

SAMPLE

DATE

$03 / 07 / 88$

$04 / 12 / 88$

$05 / 02 / 88$

$06 / 06 / 88$

$01 / 04 / 88$

$01 / 04 / 88$

$02 / 08 / 88$

$03 / 07 / 88$

$04 / 12 / 88$

$05 / 02 / 88$

$06 / 06 / 88$

1- $H 4-12 \mathrm{C}$

-......

25
23
25
25
11
6
13
11
9
5


IABLE E.5. (contd)

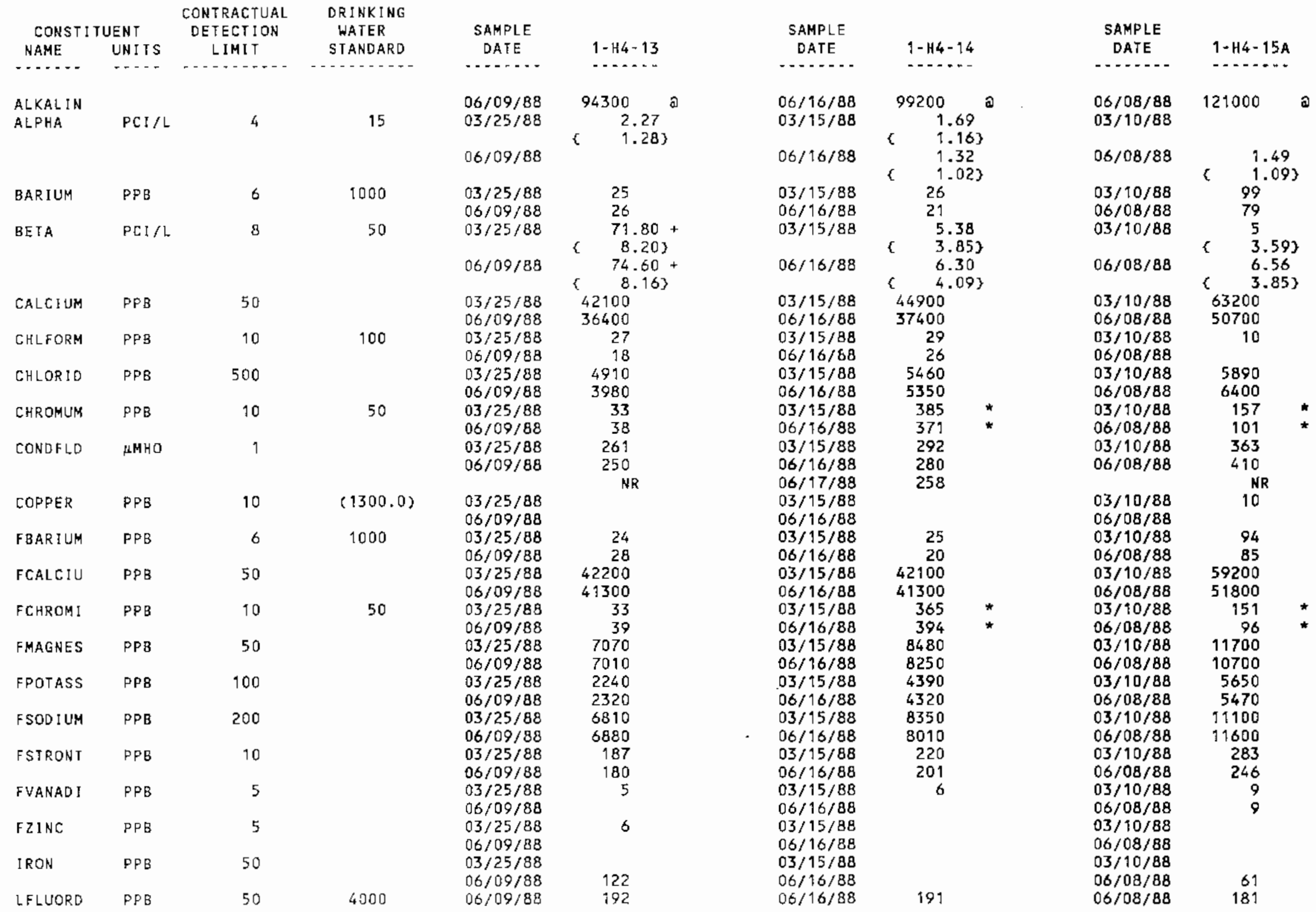


TABLE E.5. (contd)

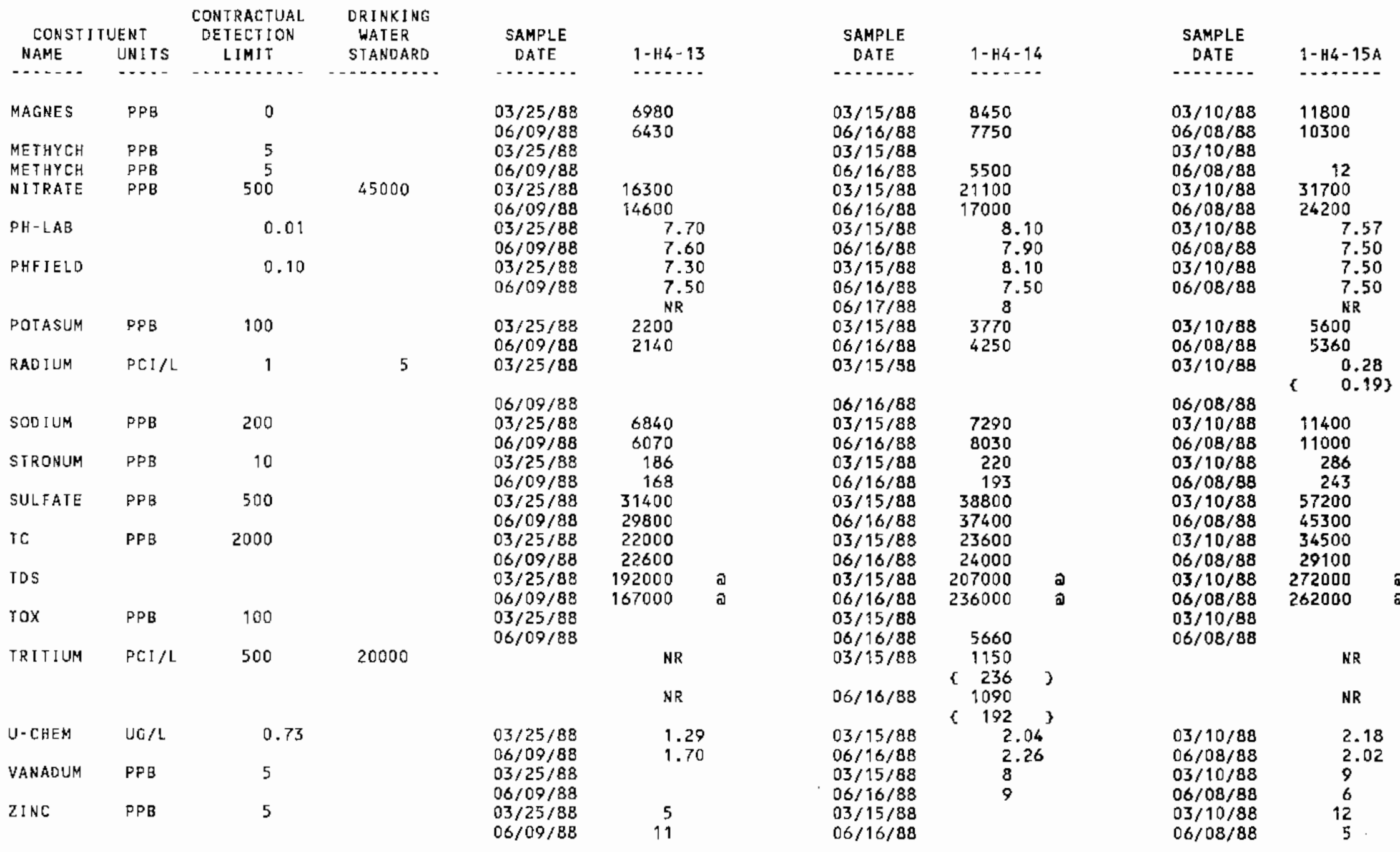


TABLE E.5. (contd)

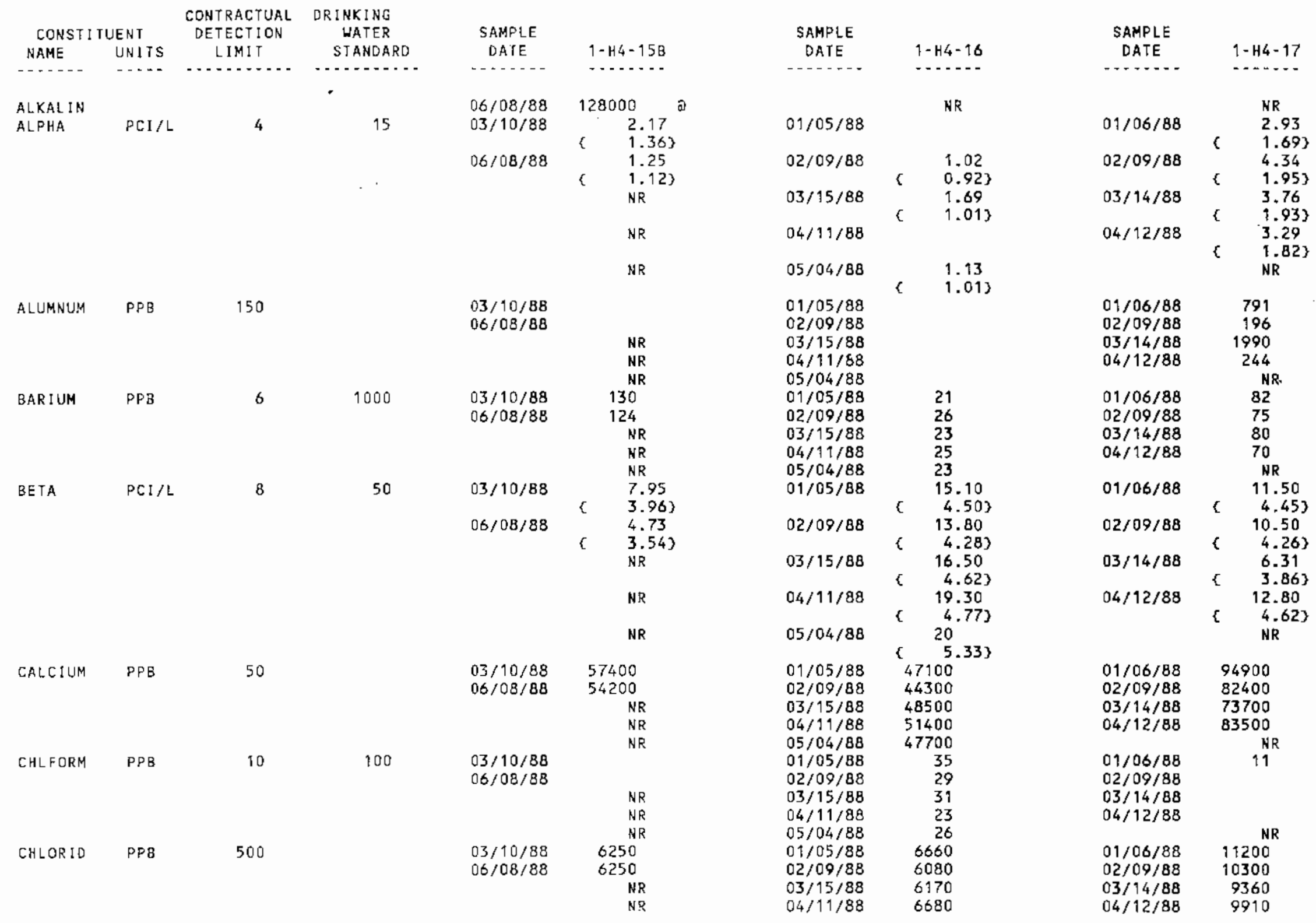


TABLE E. 5. (contd)

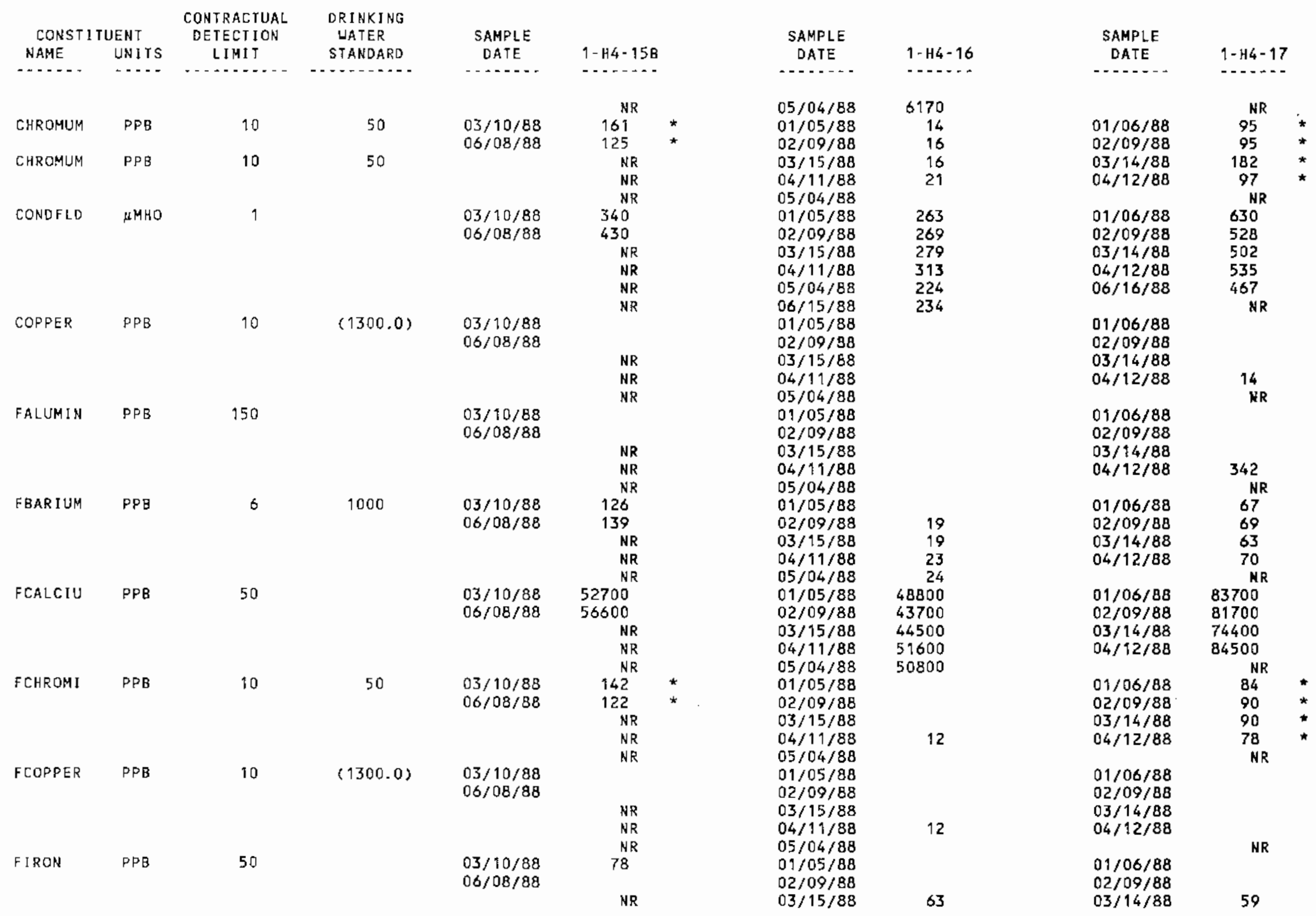


TABLE E.5. (contd)

\begin{tabular}{|c|c|c|c|c|c|c|c|c|c|}
\hline \multicolumn{2}{|c|}{ CONSTI I TUENT } & $\begin{array}{l}\text { CONTRACTUAL } \\
\text { DETECTION }\end{array}$ & $\begin{array}{l}\text { DRINKING } \\
\text { WATER }\end{array}$ & SAMPLE & & SAMPLE & & SAMPLE & \\
\hline NAME & UNITS & LIHIT & STANDARD & DATE & $\mathrm{P}-\mathrm{H4}-15 \mathrm{~B}$ & DATE & $1-H 4-16$ & DATE & $1-144-17$ \\
\hline$-\ldots \ldots$ & $\cdots$ & $\ldots$ & $\cdots$ & $\cdots$ & $\cdots$ & $-\ldots$ & $-\ldots$ & $\ldots$ & -...... \\
\hline & & & & & $\begin{array}{l}\text { NR } \\
\text { NR }\end{array}$ & $\begin{array}{l}04 / 11 / 88 \\
05 / 04 / 88\end{array}$ & & $04 / 12 / 88$ & 657 \\
\hline FLUORID & PPB & 500 & 4000 & $\begin{array}{l}03 / 10 / 88 \\
06 / 08 / 88\end{array}$ & $\begin{array}{l}\text { NR } \\
N R \\
\text { NR }\end{array}$ & $\begin{array}{l}01 / 05 / 88 \\
02 / 09 / 88 \\
03 / 15 / 88 \\
04 / 11 / 88 \\
05 / 04 / 88\end{array}$ & & $\begin{array}{l}01 / 06 / 88 \\
02 / 09 / 88 \\
03 / 14 / 88 \\
04 / 12 / 88\end{array}$ & 501 \\
\hline FMAGNES & PPB & 50 & & $\begin{array}{l}03 / 10 / 88 \\
06 / 08 / 88\end{array}$ & $\begin{array}{r}12200 \\
13200 \\
\text { NR } \\
\text { NR } \\
\text { NR }\end{array}$ & $\begin{array}{l}01 / 05 / 88 \\
02 / 09 / 88 \\
03 / 15 / 88 \\
04 / 11 / 88 \\
05 / 04 / 88\end{array}$ & $\begin{array}{l}5840 \\
5450 \\
5510 \\
6030 \\
6110\end{array}$ & $\begin{array}{l}01 / 06 / 88 \\
02 / 09 / 88 \\
03 / 14 / 88 \\
04 / 12 / 88\end{array}$ & $\begin{array}{l}15400 \\
15100 \\
14100 \\
14900 \\
\text { NR }\end{array}$ \\
\hline FMANGAN & PPB & 5 & & $\begin{array}{l}03 / 10 / 88 \\
06 / 08 / 88\end{array}$ & $\begin{array}{c}19 \\
8 \\
\text { NR }\end{array}$ & $\begin{array}{l}01 / 05 / 88 \\
02 / 09 / 38 \\
03 / 15 / 88\end{array}$ & & $\begin{array}{l}01 / 06 / 88 \\
02 / 09 / 88 \\
03 / 14 / 88\end{array}$ & $\mathrm{NK}$ \\
\hline & & & & & $\begin{array}{l}\text { NR } \\
\text { NR }\end{array}$ & $\begin{array}{l}04 / 19 / 88 \\
05 / 04 / 88\end{array}$ & & $04 / 12 / 88$ & 17 \\
\hline FNICKEL & PPB & 10 & & $\begin{array}{l}03 / 10 / 88 \\
06 / 08 / 88\end{array}$ & $\begin{array}{l}14 \\
20 \\
\text { NR } \\
\text { NR }\end{array}$ & $\begin{array}{l}01 / 05 / 88 \\
02 / 09 / 88 \\
03 / 15 / 88 \\
04 / 11 / 88\end{array}$ & & $\begin{array}{l}01 / 05 / 88 \\
02 / 09 / 88 \\
03 / 14 / 88 \\
04 / 12 / 88\end{array}$ & 14 \\
\hline & & & & & NR & $05 / 04 / 88$ & & & NR \\
\hline FPOTASS & PPB & 100 & & $\begin{array}{l}03 / 10 / 88 \\
06 / 08 / 88\end{array}$ & $\begin{array}{r}5610 \\
6090 \\
\text { NR } \\
\text { NR } \\
\text { NR }\end{array}$ & $\begin{array}{l}01 / 05 / 88 \\
02 / 09 / 88 \\
03 / 15 / 88 \\
04 / 19 / 88 \\
05 / 04 / 88\end{array}$ & $\begin{array}{l}3010 \\
2830 \\
2910 \\
2900 \\
3050\end{array}$ & $\begin{array}{l}01 / 06 / 88 \\
02 / 09 / 88 \\
03 / 14 / 88 \\
04 / 12 / 80\end{array}$ & $\begin{array}{l}6650 \\
6750 \\
6630 \\
6690 \\
\text { NR }\end{array}$ \\
\hline F SODIUM & PPB & 200 & & $\begin{array}{l}03 / 10 / 88 \\
06 / 08 / 88\end{array}$ & $\begin{array}{l}10900 \\
12100\end{array}$ & $\begin{array}{l}01 / 05 / 88 \\
02 / 09 / 88\end{array}$ & $\begin{array}{l}4800 \\
4600\end{array}$ & $\begin{array}{l}01 / 06 / 88 \\
02 / 09 / 88\end{array}$ & $\begin{array}{l}15700 \\
14800\end{array}$ \\
\hline & & & & & $\begin{array}{l}\text { NR } \\
\text { NR }\end{array}$ & $\begin{array}{l}03 / 15 / 88 \\
04 / 11 / 88\end{array}$ & $\begin{array}{l}4710 \\
4610\end{array}$ & $\begin{array}{l}03 / 14 / 88 \\
04 / 12 / 88\end{array}$ & $\begin{array}{l}15400 \\
15400\end{array}$ \\
\hline & & & & & NR & $05 / 04 / 88$ & 4930 & & NR \\
\hline FSTRONT & $P P B$ & 10 & & $\begin{array}{l}03 / 10 / 88 \\
06 / 08 / 88\end{array}$ & $\begin{array}{l}282 \\
287 \\
\text { NR }\end{array}$ & $\begin{array}{l}01 / 05 / 88 \\
02 / 09 / 88 \\
03 / 15 / 88\end{array}$ & $\begin{array}{l}198 \\
196 \\
202\end{array}$ & $\begin{array}{l}01 / 06 / 88 \\
02 / 09 / 88 \\
03 / 14 / 88\end{array}$ & $\begin{array}{l}366 \\
371 \\
344\end{array}$ \\
\hline & & & & & $\begin{array}{l}N R \\
N R\end{array}$ & $\begin{array}{l}04 / 11 / 88 \\
05 / 04 / 88\end{array}$ & $\begin{array}{l}221 \\
214\end{array}$ & $04 / 12 / 88$ & 357 \\
\hline FVANADI & PPB & 5 & & $\begin{array}{l}03 / 10 / 88 \\
06 / 08 / 88\end{array}$ & $\begin{array}{l}13 \\
11\end{array}$ & $\begin{array}{l}01 / 05 / 88 \\
02 / 09 / 88\end{array}$ & & $\begin{array}{l}01 / 06 / 88 \\
02 / 09 / 88\end{array}$ & \\
\hline & & & & & $\begin{array}{l}\text { NR } \\
\text { NR }\end{array}$ & $\begin{array}{l}03 / 15 / 88 \\
04 / 11 / 88\end{array}$ & $\begin{array}{l}5 \\
6\end{array}$ & $\begin{array}{l}03 / 14 / 88 \\
04 / 12 / 88\end{array}$ & \\
\hline & & & & & $\mathrm{NR}$ & $05 / 04 / 88$ & 6 & & NR \\
\hline FZINC & $P P_{B}$ & 5 & & $\begin{array}{l}03 / 10 / 88 \\
06 / 08 / 88\end{array}$ & $\begin{array}{l}22 \\
18 \\
M R\end{array}$ & $\begin{array}{l}01 / 05 / 88 \\
02 / 09 / 88 \\
03 / 15 / 88\end{array}$ & 9 & $\begin{array}{l}01 / 06 / 88 \\
02 / 09 / 88 \\
03 / 44 / 88\end{array}$ & \\
\hline
\end{tabular}


IABLE E.5. (contd)

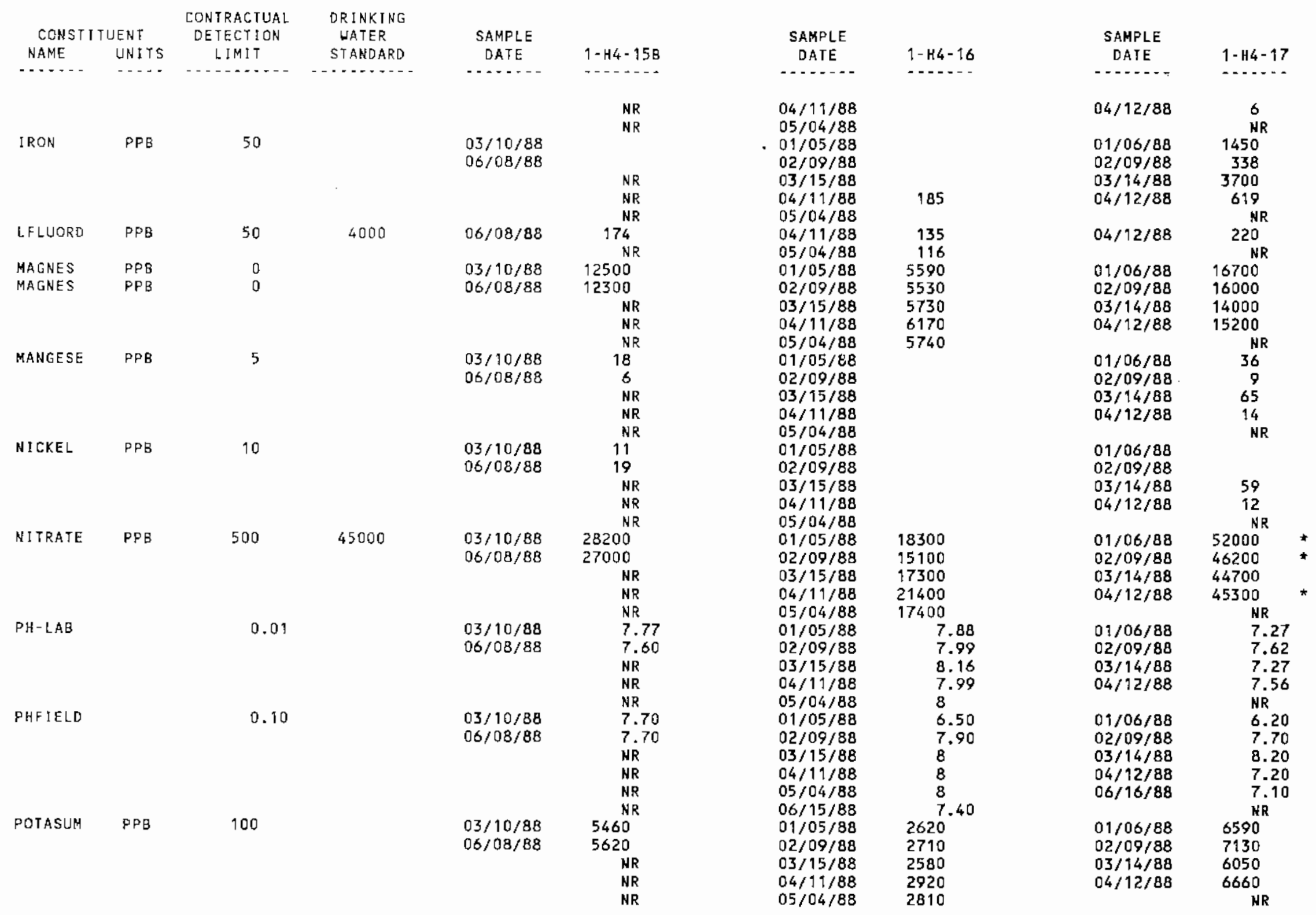


TABLE E.5. (contd)

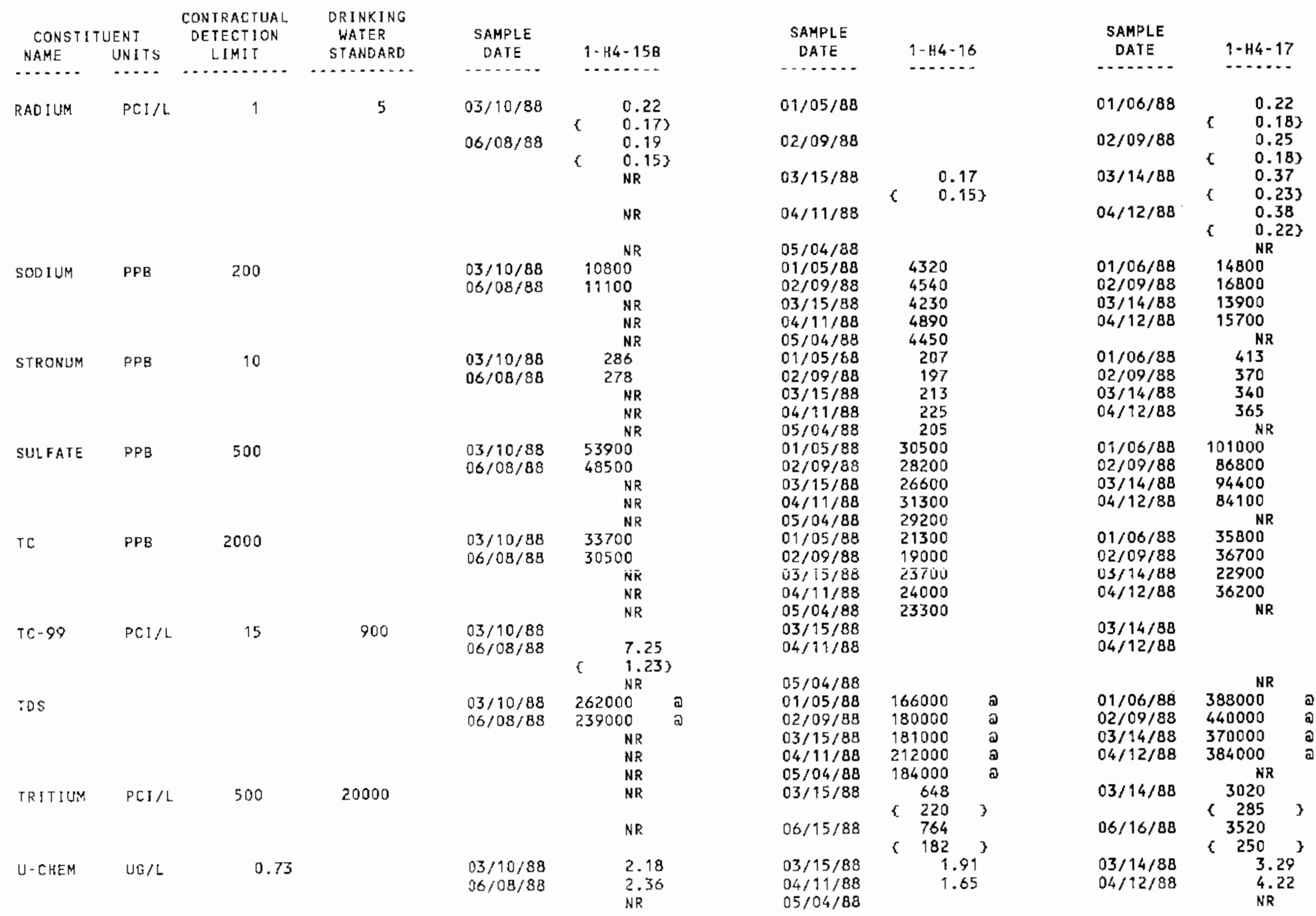


IABLE E.5. (contd)

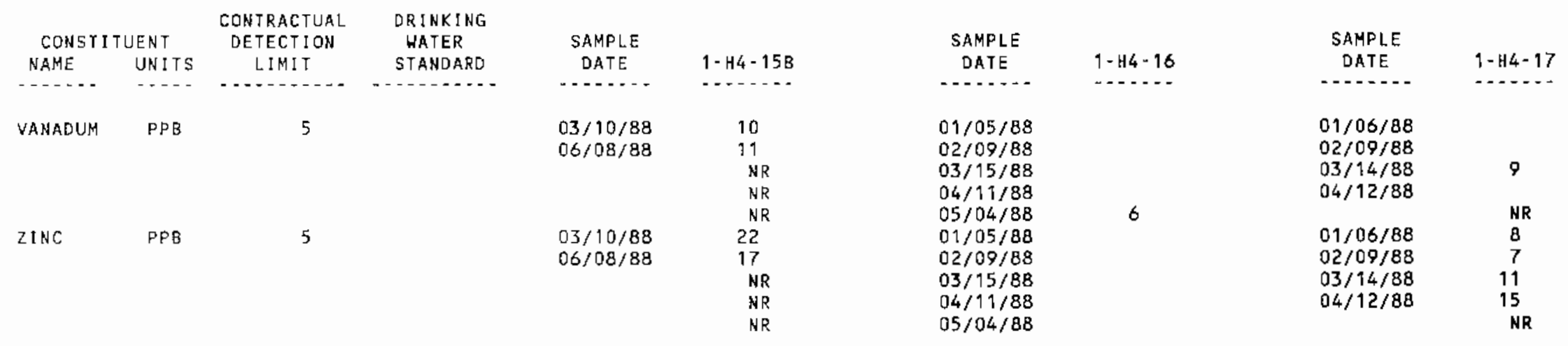


TABLE E.5. (contd)

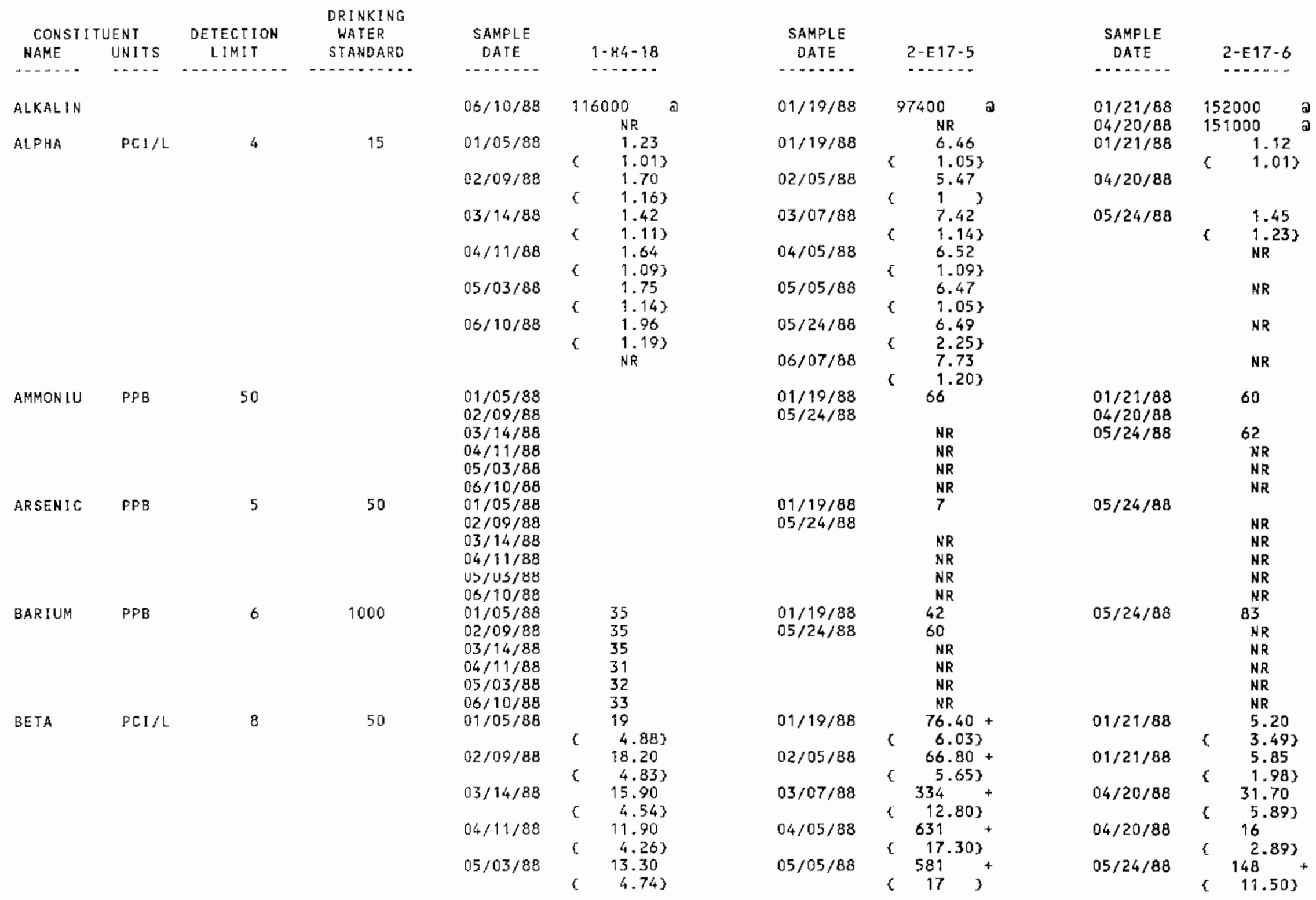


TABLE E.5. (contd)

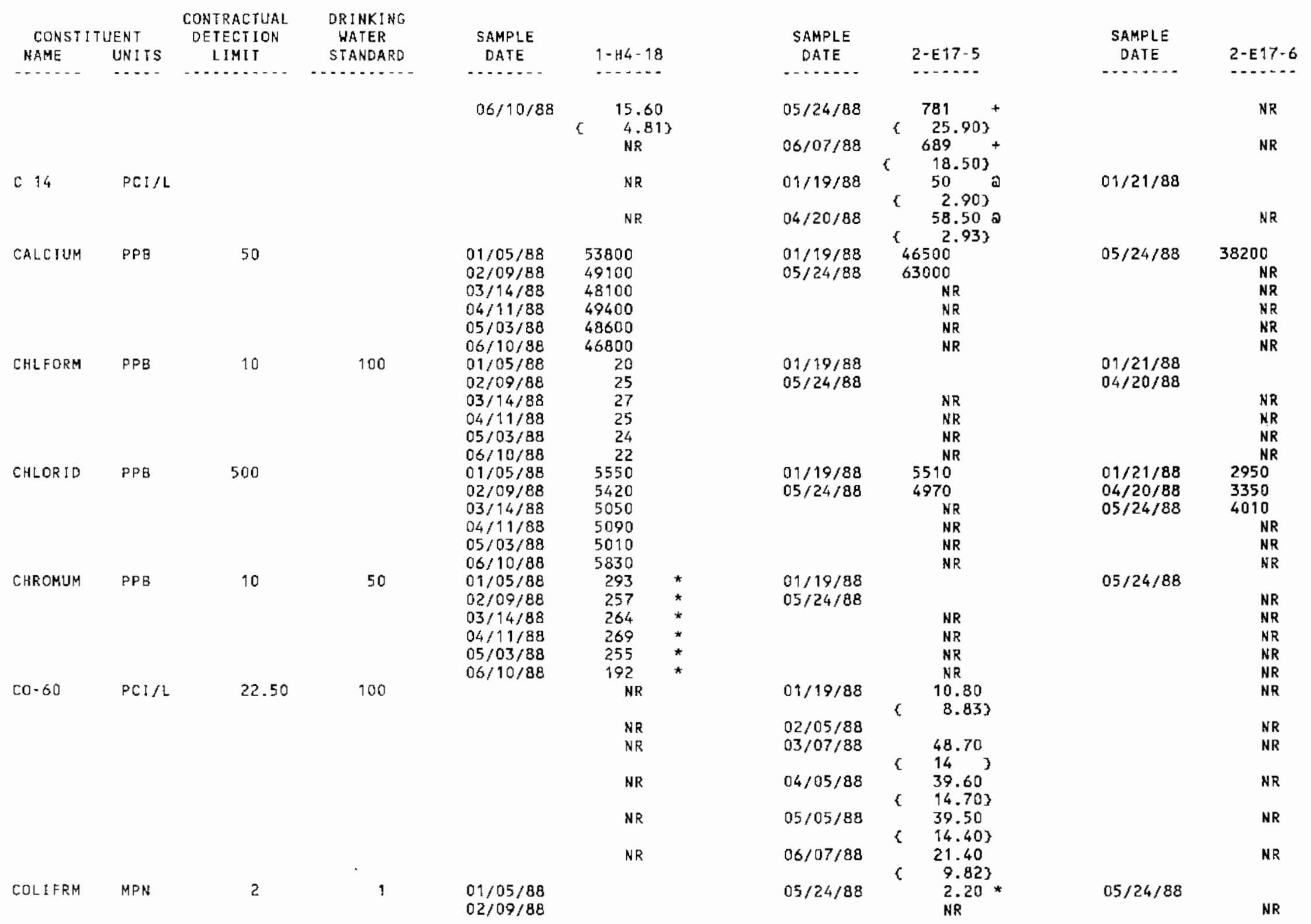


TABLE E.5. (contd)

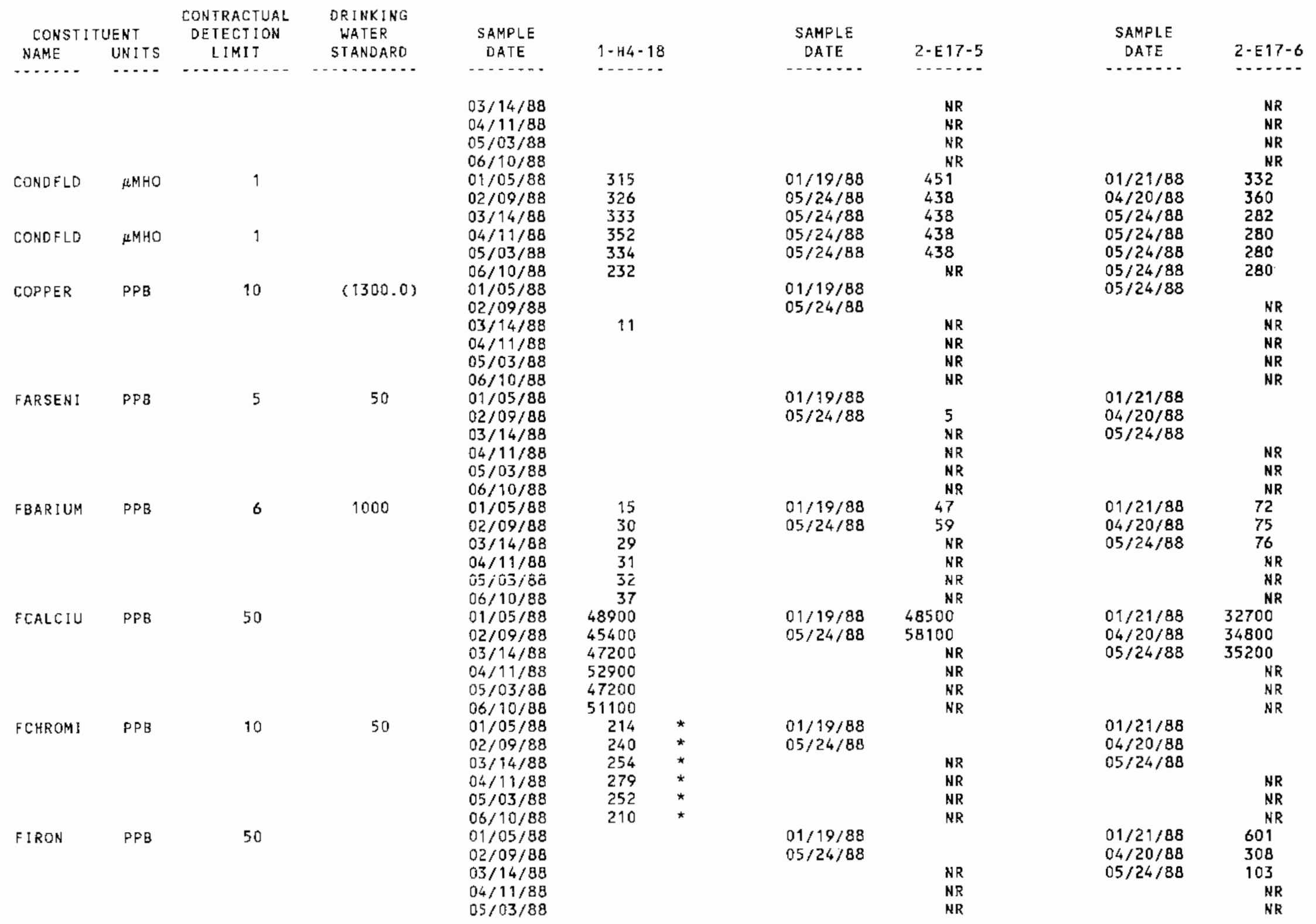


TABLE E. .5 . (contd)

\begin{tabular}{|c|c|c|c|c|c|c|c|c|c|}
\hline \multicolumn{2}{|c|}{ CONSTI IUENT } & $\begin{array}{l}\text { CONTRACTUAL } \\
\text { DETECTIDN }\end{array}$ & $\begin{array}{l}\text { DRINKING } \\
\text { WATER }\end{array}$ & SAMPLE & & SAMPLE & & SAMPLE & \\
\hline NAME & UNITS & LIMIT & STANDARD & DATE & $1-H 4-18$ & DATE & 2-E17-5 & DATE & 2-E $17-6$ \\
\hline \multirow{2}{*}{$\cdots$} & $\cdots$ & , n & - nen & $\cdots$ & 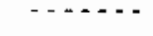 & $\ldots$ & $-\ldots .$. & $\cdots$ & $\ldots$ \\
\hline & & & & $06 / 10 / 88$ & & & NR & & NR \\
\hline \multirow[t]{4}{*}{ FLUORID } & PPB & 500 & 4000 & $\begin{array}{l}01 / 05 / 88 \\
02 / 09 / 88\end{array}$ & & $\begin{array}{l}01 / 19 / 88 \\
05 / 24 / 88\end{array}$ & $\begin{array}{l}666 \\
679\end{array}$ & $\begin{array}{l}01 / 21 / 88 \\
04 / 20 / 88\end{array}$ & 576 \\
\hline & & & & $\begin{array}{l}03 / 14 / 88 \\
04 / 11 / 88\end{array}$ & & & KR & $05 / 24 / 88$ & 563 \\
\hline & & & & $05 / 03 / 88$ & & & NR & & $\begin{array}{l}\text { NR } \\
\text { NR }\end{array}$ \\
\hline & & & & $06 / 10 / 88$ & & & $N_{R}$ & & NR \\
\hline \multirow[t]{3}{*}{ FMAGNES } & $\mathrm{PPB}$ & 50 & & $\begin{array}{l}01 / 05 / 88 \\
02 / 09 / 88\end{array}$ & $\begin{array}{l}8200 \\
8070\end{array}$ & $\begin{array}{l}01 / 19 / 88 \\
05 / 24 / 88\end{array}$ & $\begin{array}{l}14800 \\
18400\end{array}$ & $\begin{array}{l}01 / 21 / 88 \\
04 / 20 / 88\end{array}$ & $\begin{array}{l}12200 \\
12900\end{array}$ \\
\hline & & & & $03 / 14 / 88$ & 8210 & & NR & $05 / 24 / 88$ & 13000 \\
\hline & & & & $04 / 11 / 88$ & 8680 & & NR & & NR \\
\hline \multirow[t]{2}{*}{ FMAGNES } & PPB & 50 & & $05 / 03 / 88$ & 8240 & & NR & & NR \\
\hline & & & & $06 / 10 / 88$ & 8950 & & NR & & NR \\
\hline \multirow[t]{5}{*}{ FMANGAN } & PPB & 5 & & $\begin{array}{l}01 / 05 / 88 \\
02 / 09 / 88\end{array}$ & & $\begin{array}{l}01 / 19 / 88 \\
05 / 24 / 88\end{array}$ & & $\begin{array}{l}01 / 21 / 88 \\
04 / 20 / 88\end{array}$ & $\begin{array}{l}97 \\
77\end{array}$ \\
\hline & & & & $03 / 14 / 88$ & & & NR & $05 / 24 / 88$ & 58 \\
\hline & & & & $04 / 11 / 88$ & & & NR & & NR \\
\hline & & & & $05 / 03 / 88$ & & & NR & & NR \\
\hline & & & & $06 / 10 / 88$ & & & NR & & NR \\
\hline \multirow[t]{5}{*}{ FPOTASS } & PPB & 100 & & $\begin{array}{l}01 / 05 / 88 \\
02 / 09 / 88\end{array}$ & $\begin{array}{l}4080 \\
4040\end{array}$ & $\begin{array}{l}01 / 19 / 88 \\
05 / 24 / 88\end{array}$ & $\begin{array}{l}7140 \\
7660\end{array}$ & $\begin{array}{l}01 / 21 / 88 \\
04 / 20 / 88\end{array}$ & $\begin{array}{l}6600 \\
6870\end{array}$ \\
\hline & & & & $03 / 14 / 88$ & 4030 & & NR & $05 / 24 / 88$ & 6530 \\
\hline & & & & $04 / 11 / 88$ & 4180 & & NR & & HR \\
\hline & & & & $05 / 03 / 88$ & 3990 & & NR & & NR \\
\hline & & & & $06 / 10 / 88$ & 4410 & & HR & & NR \\
\hline \multirow[t]{5}{*}{ FSODIUM } & PPB & 200 & & $\begin{array}{l}01 / 05 / 88 \\
02 / 09 / 88\end{array}$ & $\begin{array}{l}10300 \\
10300\end{array}$ & $\begin{array}{l}01 / 19 / 88 \\
05 / 24 / 88\end{array}$ & $\begin{array}{l}24400 \\
26000\end{array}$ & $\begin{array}{l}01 / 21 / 88 \\
04 / 20 / 88\end{array}$ & 17000 \\
\hline & & & & $03 / 14 / 88$ & 9570 & & NR & $05 / 24 / 88$ & 20100 \\
\hline & & & & $04 / 11 / 88$ & 9460 & & NR & & HR \\
\hline & & & & $05 / 03 / 88$ & 9140 & & NR & & HR \\
\hline & & & & $06 / 10 / 88$ & 10900 & & NR & & NR \\
\hline \multirow[t]{5}{*}{ FSTRONT } & $P P B$ & 10 & & $\begin{array}{l}01 / 05 / 88 \\
02 / 09 / 88\end{array}$ & $\begin{array}{l}212 \\
215\end{array}$ & $\begin{array}{l}01 / 19 / 88 \\
05 / 24 / 88\end{array}$ & $\begin{array}{l}264 \\
323\end{array}$ & $\begin{array}{l}01 / 21 / 88 \\
04 / 20 / 88\end{array}$ & $\begin{array}{l}212 \\
229\end{array}$ \\
\hline & & & & $03 / 14 / 88$ & 223 & & NR & $05 / 24 / 88$ & 217 \\
\hline & & & & $04 / 11 / 88$ & 235 & & NR & & $H R$ \\
\hline & & & & $05 / 03 / 88$ & 217 & & NR & & NR \\
\hline & & & & $06 / 10 / 88$ & 239 & & NR & & NR \\
\hline \multirow[t]{5}{*}{ FVANAD 1} & PPB & 5 & & $01 / 05 / 88$ & & $01 / 19 / 88$ & 16 & $01 / 21 / 88$ & \\
\hline & & & & $03 / 14 / 88$ & $\begin{array}{l}0 \\
7\end{array}$ & $05 / 24$ & ${ }^{20} \mathrm{NR}$ & $\begin{array}{l}04 / 20 / 88 \\
05 / 24 / 88\end{array}$ & \\
\hline & & & & $04 / 11 / 88$ & & & NR & & NR \\
\hline & & & & $05 / 03 / 88$ & & . & NR & & NR \\
\hline & & & & $06 / 10 / 88$ & & & NR & & NR \\
\hline FZINC & PPB & 5 & & $\begin{array}{l}01 / 05 / 88 \\
02 / 09 / 88\end{array}$ & & $\begin{array}{l}01 / 19 / 88 \\
05 / 24 / 88\end{array}$ & & $\begin{array}{l}01 / 21 / 88 \\
04 / 20 / 88\end{array}$ & \\
\hline
\end{tabular}


TABLE E.5. (contd)

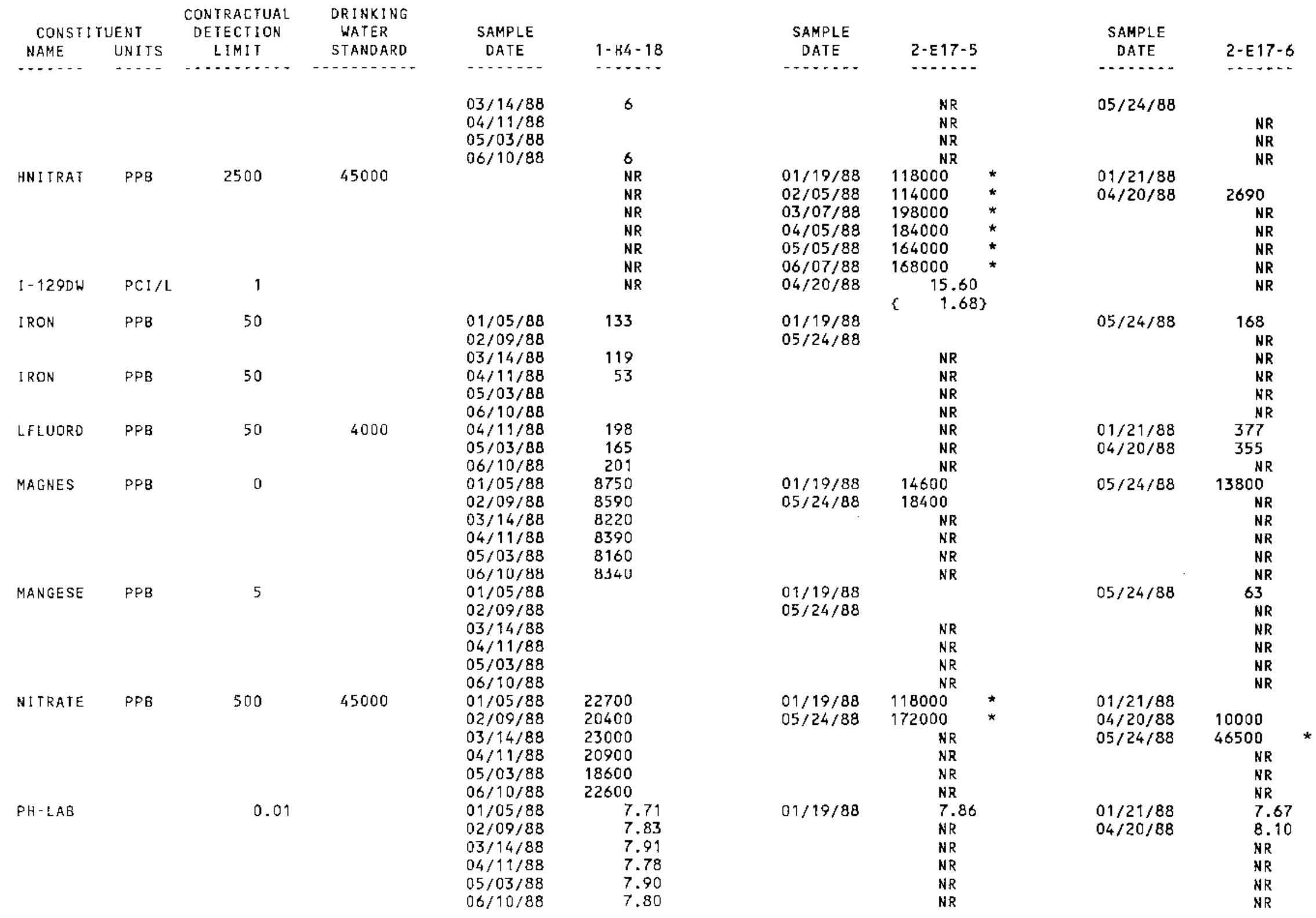


TABLEE.5. (contd)

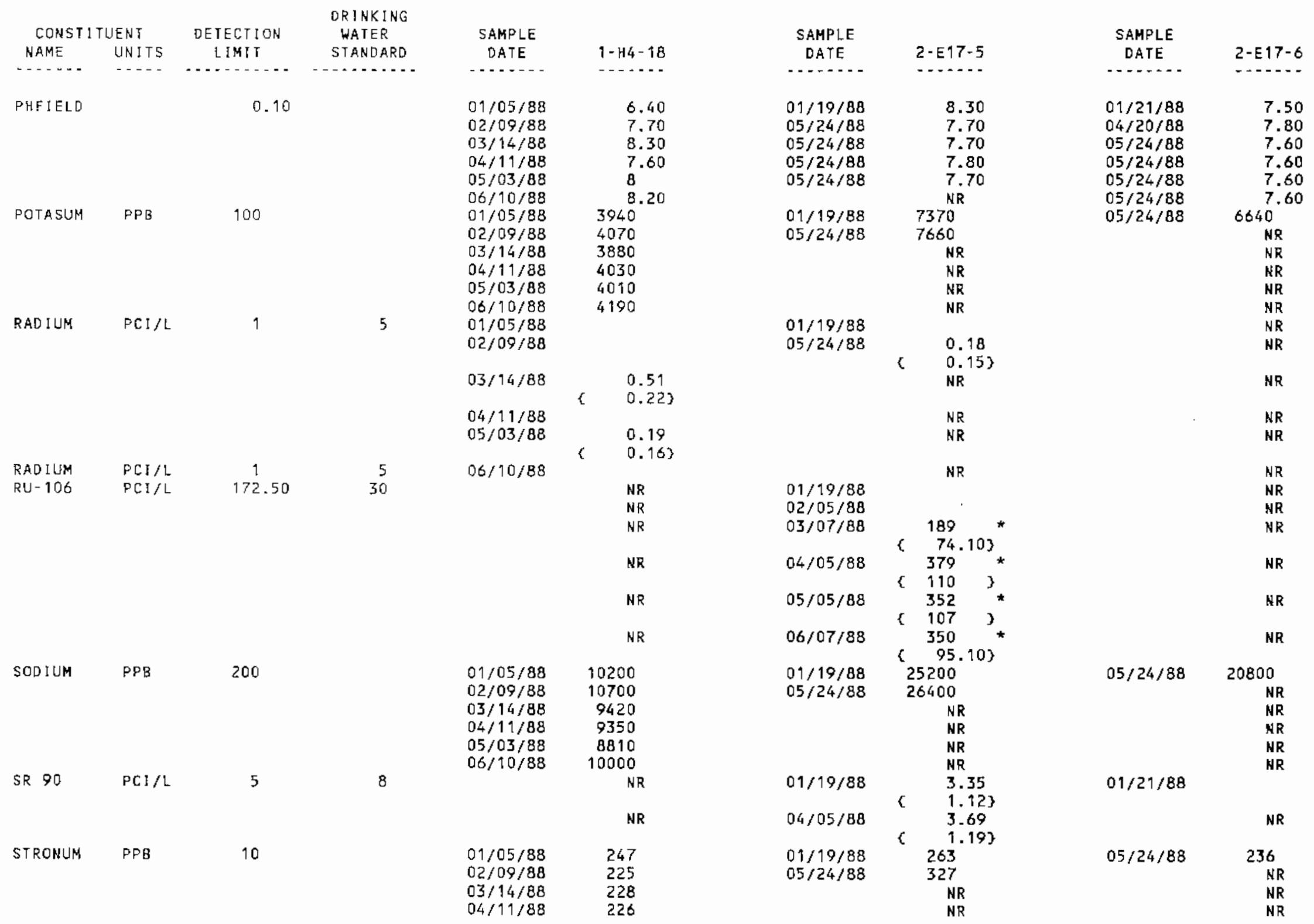


TABLE E.5. (contd)

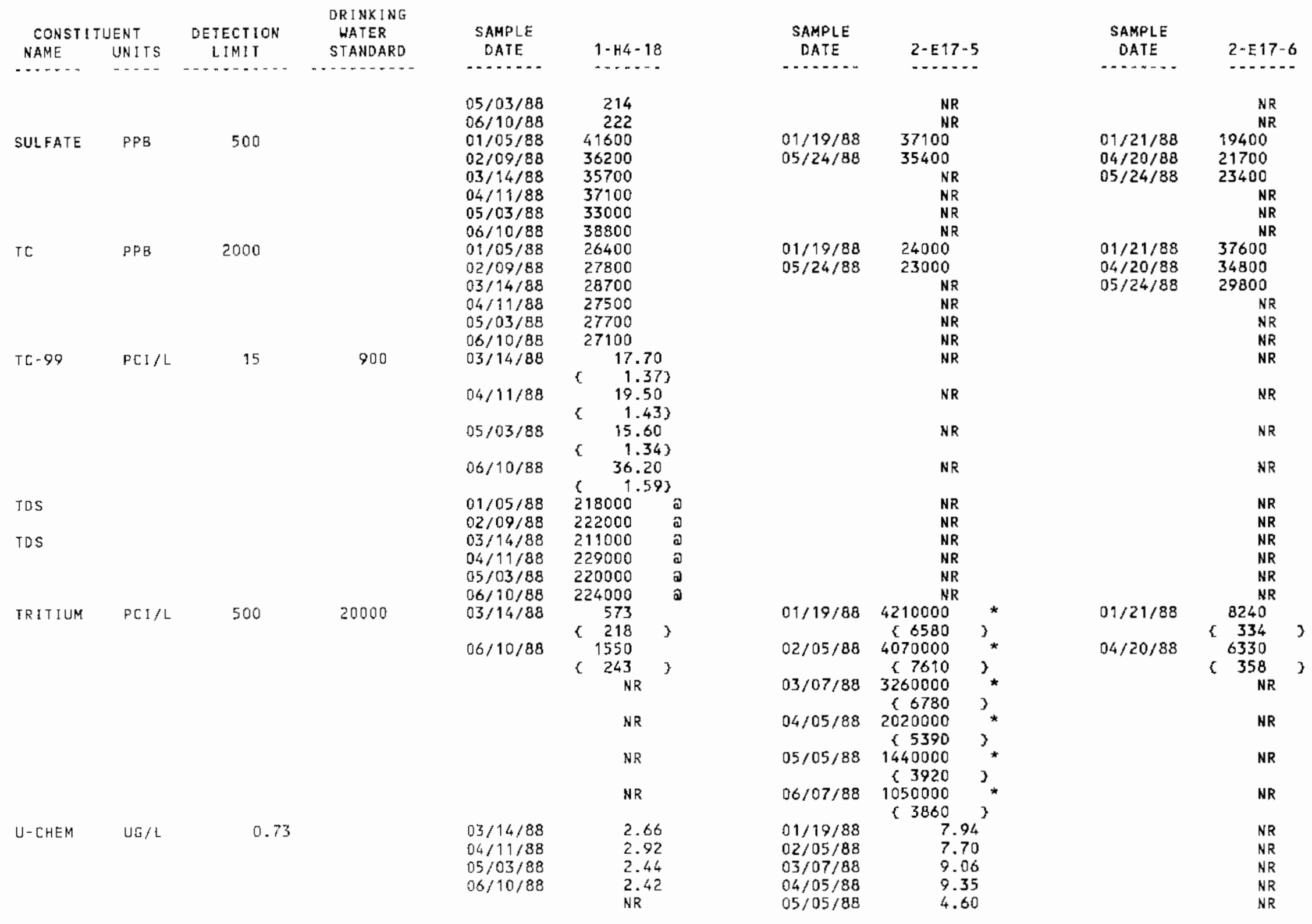


TABLE_E.5. (contd)

\begin{tabular}{|c|c|c|c|c|c|}
\hline \multicolumn{2}{|c|}{ CONST I TUENT } & CONTRACTUAL & $\begin{array}{l}\text { DR JNKIKG } \\
\text { WATER }\end{array}$ & SAMPLE & \\
\hline NAME & UNITS & LIMIT & SIANDARD & DATE & $1-\$ 4-1$ \\
\hline & $\cdots$ & $\ldots \ldots$ & $\cdots$ & & \\
\hline VANAOUM & $P P \beta$ & 5 & & $01 / 05 / 8 R$ & NR \\
\hline & & & & $\begin{array}{l}02 / 09 / 88 \\
03 / 14 / 88 \\
04 / 11 / 88 \\
05 / 03 / 88 \\
06 / 10 / 88\end{array}$ & $\begin{array}{l}5 \\
7\end{array}$ \\
\hline ZINC & $P P B$ & 5 & & $\begin{array}{l}01 / 05 / 8 B \\
02 / 09 / 8 B \\
03 / 14 / 88 \\
04 / 11 / 8 B \\
05 / 03 / 8 B \\
06 / 10 / 88\end{array}$ & $\begin{array}{r}10 \\
9 \\
13 \\
5 \\
7 \\
6\end{array}$ \\
\hline
\end{tabular}

$\begin{array}{lc}\text { SAMPLE } & \\ \text { DATE } & 2-E 17-5 \\ \cdots & \\ 06 / 07 / 88 & 8.34 \\ 01 / 19 / 88 & 17 \\ 05 / 24 / 88 & 19 \\ & \text { NR } \\ & \text { NR } \\ & \text { NR } \\ & \text { NR } \\ 01 / 19 / 88 & \\ 05 / 24 / 88 & \\ & \text { NR } \\ & \text { NR } \\ & \text { NR } \\ & \text { NR }\end{array}$

SAMPLE

DATE

2-E17-6

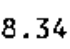

-......

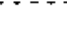

$05 / 24 / 88$

${ }^{N R}$

19

NR

NR

7
NR

7

NR

$05 / 24 / 88$ 
TABLE E.5. (contd)

\begin{tabular}{|c|c|c|c|c|c|c|c|c|c|c|c|c|}
\hline \multicolumn{2}{|c|}{ CONSTITUENT } & $\begin{array}{c}\text { CONTRACTUAL } \\
\text { DETECTION }\end{array}$ & $\begin{array}{l}\text { DRINKING } \\
\text { WATER }\end{array}$ & SAMPLE & \multirow{2}{*}{\multicolumn{2}{|c|}{$2-E 17-14$}} & SAMPLE & \multirow{2}{*}{\multicolumn{2}{|c|}{$2-E 17-15$}} & SAMPLE & \multirow{2}{*}{\multicolumn{2}{|c|}{$2-E 17-16$}} \\
\hline NAME & UNITS & LIMIT & STANDARO & DATE & & & DATE & & & DATE & & \\
\hline$\ldots-$ & $\cdots-$ & $\cdots$ & $\ldots$ & $\cdots \cdots$ & - & & $\ldots \ldots$ & $\cdots-$ & & $\cdots$ & - & \\
\hline ALPHA & $\mathrm{PCl} / \mathrm{L}$ & 4 & 15 & $06 / 08 / 88$ & $\begin{array}{r}14.50 \\
\{\quad 3.72\}\end{array}$ & & $05 / 31 / 88$ & 5 & & $05 / 25 / 88$ & $\begin{array}{l}3.47 \\
1.933\end{array}$ & \\
\hline ALUMNUM & PPB & 150 & & $06 / 08 / 88$ & 1010 & & $05 / 31 / 88$ & & & $05 / 25 / 88$ & & \\
\hline AMMONIU & PPB & 50 & & $06 / 08 / 88$ & 110 & & $05 / 31 / 88$ & & & $05 / 25 / 88$ & & \\
\hline ARSENIC & PPB & 5 & 50 & $06 / 08 / 88$ & 6 & & $05 / 31 / 88$ & 5 & & $05 / 25 / 88$ & 5 & \\
\hline $\begin{array}{l}\text { BARIUM } \\
\text { BETA }\end{array}$ & $\begin{array}{l}\text { PPB } \\
\text { PCl } 1 / 1\end{array}$ & $\begin{array}{l}6 \\
8\end{array}$ & $\begin{array}{r}1000 \\
50\end{array}$ & $\begin{array}{l}06 / 08 / 88 \\
06 / 08 / 88\end{array}$ & $\begin{array}{r}82 \\
781\end{array}$ & + & $\begin{array}{l}05 / 31 / 88 \\
05 / 31 / 88\end{array}$ & $\begin{array}{r}59 \\
2640\end{array}$ & + & $\begin{array}{l}05 / 25 / 88 \\
05 / 25 / 88\end{array}$ & $\begin{array}{r}53 \\
302\end{array}$ & + \\
\hline & & & & & $(\quad 27.40)$ & & & $\{\quad 196$ & 3 & & 16.303 & \\
\hline CADMIUM & PPB & 2 & 10 & $06 / 08 / 88$ & 14 & * & $05 / 31 / 88$ & & & $05 / 25 / 88$ & & \\
\hline CALCIUM & PPB & 50 & & $06 / 08 / 88$ & 77800 & & $05 / 31 / 88$ & 79300 & & $05 / 25 / 88$ & 52400 & \\
\hline CHLORID & PPB & 500 & & $06 / 08 / 88$ & 5030 & & $05 / 31 / 88$ & 4470 & & $05 / 25 / 88$ & 10900 & \\
\hline CHROMUM & PPB & 10 & 50 & $06 / 08 / 88$ & 28 & & $05 / 31 / 88$ & 225 & * & $05 / 25 / 88$ & 223 & $\star$ \\
\hline COL IFRM & MPN & 2 & 1 & $06 / 08 / 88$ & 16 & * & $05 / 31 / 88$ & & & $05 / 25 / 88$ & & \\
\hline CONDFLD & $\angle M H O$ & 1 & & $\begin{array}{l}06 / 08 / 88 \\
06 / 08 / 88 \\
06 / 08 / 88 \\
06 / 08 / 88\end{array}$ & $\begin{array}{l}918 \\
923 \\
926 \\
924\end{array}$ & & $\begin{array}{l}05 / 31 / 88 \\
05 / 31 / 88 \\
05 / 31 / 88 \\
05 / 31 / 88\end{array}$ & $\begin{array}{l}749 \\
749 \\
749 \\
750\end{array}$ & & $\begin{array}{l}05 / 25 / 88 \\
05 / 25 / 88 \\
05 / 25 / 88 \\
05 / 25 / 88\end{array}$ & $\begin{array}{l}500 \\
500 \\
500 \\
499\end{array}$ & \\
\hline COPPER & PPB & 10 & $(1300.0)$ & $06 / 08 / 88$ & 10 & & $05 / 31 / 88$ & & & $05 / 25 / 88$ & & \\
\hline FALUIMIN & PPB & 150 & & $06 / 08 / 88$ & 377 & & $05 / 31 / 88$ & & & $05 / 25 / 88$ & & \\
\hline FARSEN I & PPB & 5 & 50 & $06 / 08 / 88$ & 6 & & $05 / 31 / 88$ & & & $05 / 25 / 88$ & 6 & \\
\hline FBARIUM & $\mathrm{PPB}$ & 6 & 1000 & $06 / 08 / 88$ & 76 & & $05 / 31 / 88$ & 58 & & $05 / 25 / 88$ & 51 & \\
\hline FCADMIU & $\mathrm{PPB}$ & 2 & 10 & $06 / 08 / 88$ & 12 & $\star$ & $05 / 31 / 88$ & & & $05 / 25 / 88$ & & \\
\hline FCALC:U & PPB & 50 & & $06 / 08 / 88$ & 74500 & & $05 / 31 / 88$ & 77500 & & $05 / 25 / 88$ & 49200 & \\
\hline FCHROMI & PPB & 10 & 50 & $06 / 08 / 88$ & 18 & & $05 / 31 / 88$ & 55 & * & $05 / 25 / 88$ & & \\
\hline F I RON & PPB & 50 & & $06 / 08 / 88$ & 2470 & & $05 / 31 / 88$ & 411 & & $05 / 25 / 88$ & & \\
\hline FLEÁ & FFo & 5 & 50 & 06/OR/BR & 8 & & $05 / 31 / 88$ & & & $05 / 25 / 88$ & & \\
\hline FLUORID & PPB & 500 & 4000 & $06 / 08 / 88$ & 509 & & $05 / 31 / 8 B$ & 739 & & $05 / 25 / 88$ & 834 & \\
\hline FMAGNES & PPB & 50 & & $06 / 08 / 88$ & 22800 & & $05 / 31 / 88$ & 18800 & & $05 / 25 / 88$ & 15000 & \\
\hline FMANGAN & PPB & 5 & & $06 / 08 / 88$ & 28 & & $05 / 31 / 88$ & 30 & & $05 / 25 / 88$ & 17. & \\
\hline FN I CKEL & PPB & 10 & & $06 / 08 / 88$ & & & $05 / 31 / 88$ & 49 & & $05 / 25 / 88$ & 19 & \\
\hline FPOTASS & PPB & 100 & & $06 / 08 / 88$ & 7440 & & $05 / 31 / 88$ & 7460 & & $05 / 25 / 88$ & 6810 & \\
\hline FSELEN: & PPB & 5 & 10 & $06 / 08 / 88$ & & & $05 / 31 / 88$ & & & $05 / 25 / 88$ & 6 & \\
\hline FSODIUN & PPB & 200 & & $06 / 08 / 88$ & 28600 & & $05 / 31 / 88$ & 29600 & & $05 / 25 / 88$ & 26100 & \\
\hline FSTRONT & PPB & 10 & & $06 / 08 / 88$ & 395 & & $05 / 31 / 88$ & 360 & & $05 / 25 / 88$ & 251 & \\
\hline FVANAD I & PPB & 5 & & $06 / 08 / 88$ & 19 & & $05 / 31 / 88$ & 14 & & $05 / 25 / 88$ & 17 & \\
\hline FZINC & PPB & 5 & & $06 / 08 / 88$ & 14 & & $05 / 31 / 88$ & 6 & & $05 / 25 / 88$ & & \\
\hline IRON & $P P B$ & 50 & & $06 / 08 / 88$ & 5630 & & $05 / 31 / 88$ & 1080 & & $05 / 25 / 88$ & 1040 & \\
\hline LEADGF & PPB & 5 & 50 & $06 / 08 / 88$ & 13 & & $05 / 31 / 88$ & & & $05 / 25 / 88$ & & \\
\hline MAGNES & PPB & 0 & & $06 / 08 / 88$ & 24500 & & $05 / 31 / 88$ & 19800 & & $05 / 25 / 88$ & 15500 & \\
\hline MANGESE & PPB & 5 & & $06 / 08 / 88$ & 65 & & $05 / 31 / 88$ & 40 & & $05 / 25 / 88$ & 34 & \\
\hline NICKEL & PPB & 10 & & $06 / 08 / 88$ & 19 & & $05 / 3,1 / 88$ & 108 & & $05 / 25 / 88$ & 113 & \\
\hline NITRATE & PPB & 500 & 45000 & $06 / 08 / 88$ & 225000 & * & $05 / 31 / 88$ & 203000 & * & $05 / 25 / 88$ & 74100 & * \\
\hline PHFIELD & & 0.10 & & $\begin{array}{l}06 / 08 / 88 \\
06 / 08 / 88\end{array}$ & $\begin{array}{r}7.90 \\
7.90\end{array}$ & & $\begin{array}{l}05 / 31 / 88 \\
05 / 31 / 88\end{array}$ & 7. & & $\begin{array}{l}05 / 25 / 88 \\
05 / 25 / 88\end{array}$ & $\begin{array}{r}8.20 \\
8.20\end{array}$ & \\
\hline
\end{tabular}


TABLE E.5. (contd)

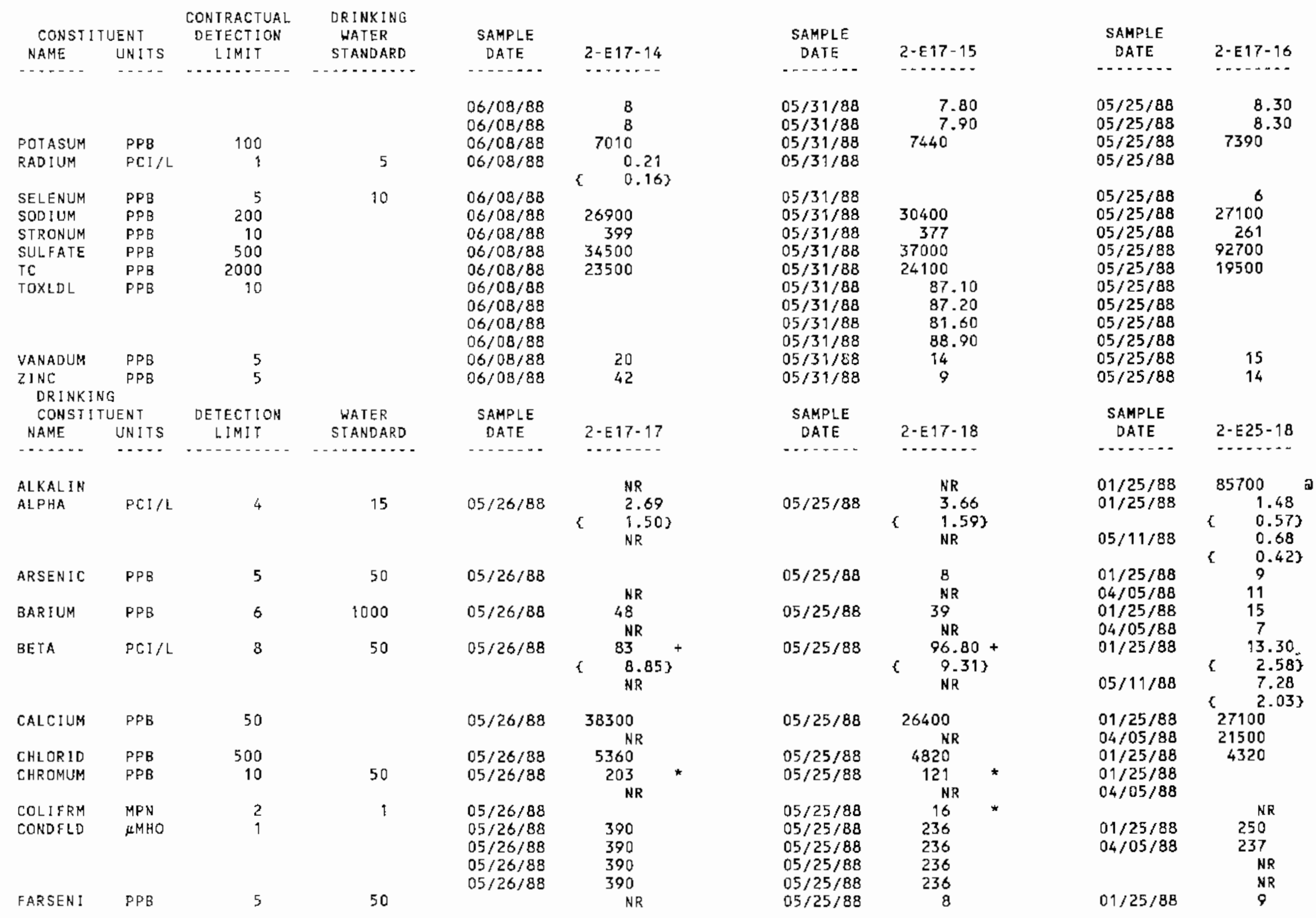


TABLE E.5. (contd)

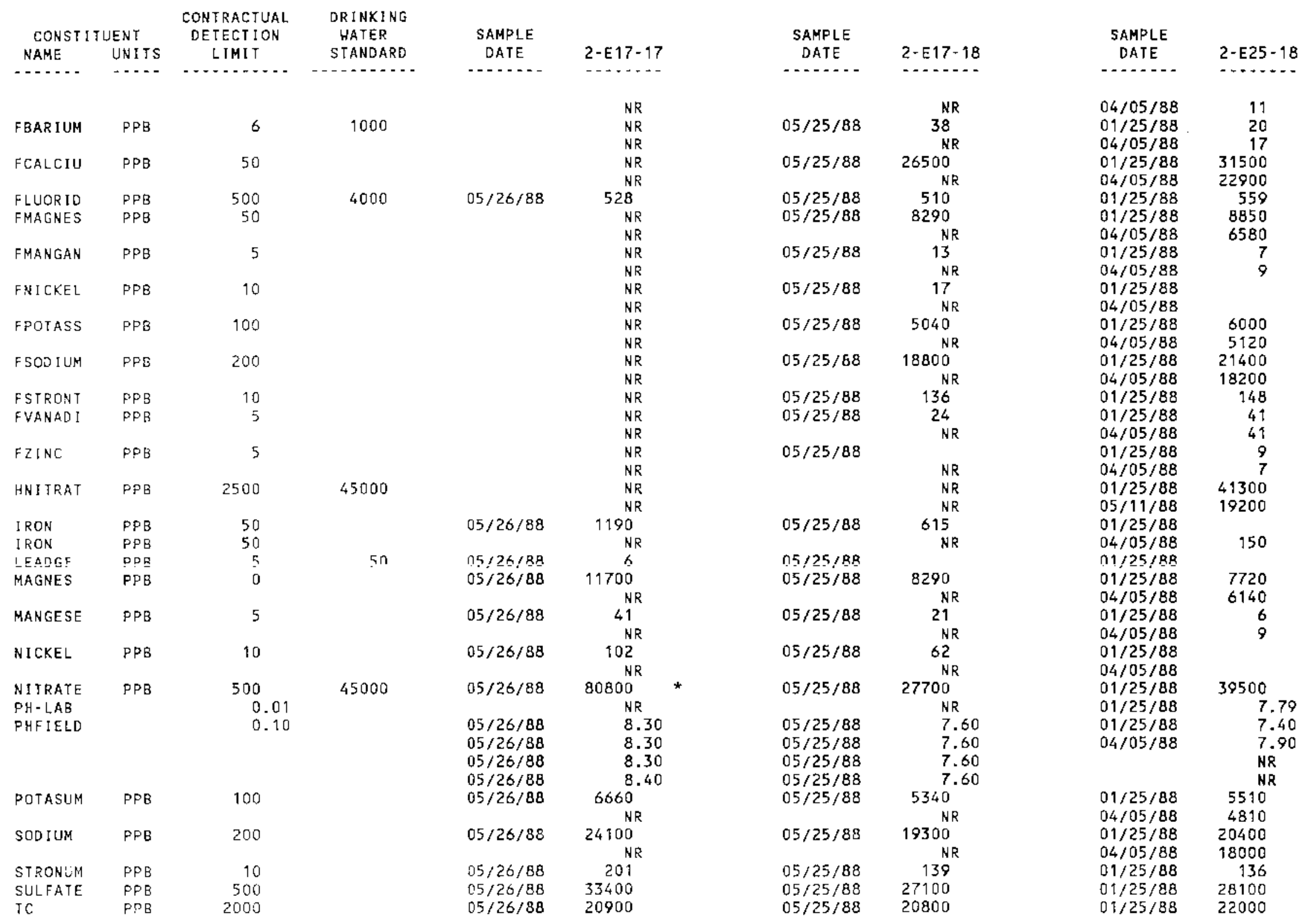


TABLE E.5. (contd)

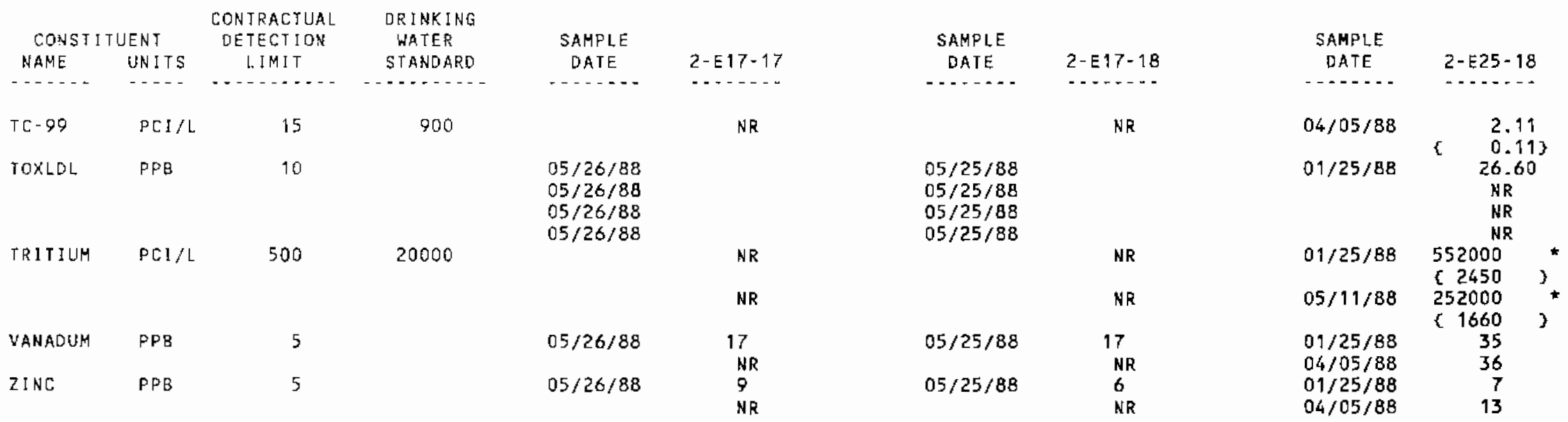


TABLE E.5. (contd)

\begin{tabular}{|c|c|c|c|c|c|c|c|c|c|}
\hline \multicolumn{2}{|c|}{ CONST I IUEN T } & DETECTION & $\begin{array}{l}\text { DRINKING } \\
\text { WATER }\end{array}$ & SAMPLE & \multirow[b]{2}{*}{$2-E 25-22$} & \multicolumn{2}{|l|}{ SAMPLE } & \multicolumn{2}{|l|}{ SAMPLE } \\
\hline NAME & LNITS & LIMIT & STANDARD & DATE & & DATE & $2-E 25-25$ & DATE & $2-E 25-26$ \\
\hline$\cdots-\cdots$ & $\cdots$ & 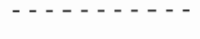 & - & $\ldots$ & - . . . & 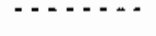 & $\cdots$ & n...... & $-\cdots$ \\
\hline ALKALIN & & & & $01 / 25 / 88$ & 89100 & & NR & & NR \\
\hline ALPHA & $P C I / L$ & 4 & 15 & $01 / 25 / 88$ & 1.23 & $03 / 29 / 88$ & & & $\mathrm{NR}$ \\
\hline & & & & $04 / 19 / 88$ & 0.98 & $03 / 29 / 88$ & & & $\mathrm{NR}$ \\
\hline & & & & & $0.47\}$ & & & & \\
\hline ARSENIC & PPB & 5 & 50 & $\begin{array}{l}01 / 25 / 98 \\
04 / 01 / 88\end{array}$ & $\begin{array}{r}8 \\
10\end{array}$ & $\begin{array}{l}01 / 19 / 88 \\
01 / 19 / 88\end{array}$ & 6 & $03 / 28 / 88$ & $\begin{array}{l}7 \\
\text { NR }\end{array}$ \\
\hline & & & & & NR & $03 / 29 / 88$ & & & NR \\
\hline BARIUM & PPB & 6 & 1000 & $01 / 25 / 88$ & 15 & $01 / 19 / 88$ & 14 & $03 / 28 / 88$ & 13 \\
\hline & & & & $04 / 01 / 88$ & 9 & $01 / 19 / 88$ & 15 & & NR \\
\hline & & & & & $\mathrm{N} R$ & $03 / 29 / 88$ & 20 & & NR \\
\hline BETA & $\mathrm{PCI} / \mathrm{L}$ & 8 & 50 & $01 / 25 / 88$ & 5.76 & $03 / 29 / 88$ & 8.40 & & NR \\
\hline & & & & & $1.81\}$ & & $3.70\}$ & & \\
\hline & & & & $04 / 19 / 88$ & 6.69 & $03 / 29 / 98$ & 5.38 & & NR \\
\hline & & & & & 1.913 & & 3.353 & & \\
\hline CALCIUM & PPB & 50 & & $\begin{array}{l}01 / 25 / 88 \\
04 / 01 / 88\end{array}$ & $\begin{array}{l}23200 \\
20400\end{array}$ & $\begin{array}{l}01 / 19 / 88 \\
01 / 19 / 88\end{array}$ & $\begin{array}{l}16500 \\
16900\end{array}$ & $03 / 28 / 88$ & 20100 \\
\hline & & & & & NR & $03 / 29 / 88$ & 20300 & & NR \\
\hline CHLORID & PPB & 500 & & $01 / 25 / 88$ & 3830 & $01 / 19 / 88$ & 2310 & $03 / 28 / 88$ & 2990 \\
\hline & & & & $04 / 01 / 88$ & 3370 & $03 / 29 / 88$ & 3370 & & NR \\
\hline & & & & & NR & $03 / 29 / 88$ & 3340 & & NR \\
\hline CHROMUM & $P P B$ & 10 & 50 & $01 / 25 / 88$ & & $01 / 19 / 88$ & & $03 / 28 / 88$ & \\
\hline & & & & $04 / 01 / 88$ & & $01 / 19 / 88$ & & & NR \\
\hline & & & & & NR & $03 / 29 / 88$ & 26 & & NR \\
\hline CONDFLD & АMHO & 1 & & $01 / 25 / 88$ & 222 & $01 / 19 / 88$ & 163 & $03 / 28 / 88$ & 185 \\
\hline & & & & $04 / 01 / 88$ & 208 & $01 / 19 / 88$ & 163 & & NR \\
\hline & & & & & NR & $01 / 19 / 8 B$ & 162 & & NR \\
\hline & & & & & NR & $01 / 19 / 88$ & 162 & & NR \\
\hline & & & & & NR & $03 / 29 / 88$ & 124 & & NR \\
\hline & & & & & NR & $03 / 29 / 88$ & 124 & & $\mathrm{NR}$ \\
\hline & & & & & NR & $03 / 29 / 88$ & 124 & & NR \\
\hline & & & & & NR & $03 / 29 / 88$ & 124 & & $N R$ \\
\hline FARSEN I & FPB & 5 & 50 & $01 / 25 / 88$ & 11 & $01 / 19 / 88$ & & $03 / 28 / 88$ & 5 \\
\hline & & & & $04 / 01 / 88$ & 6 & $01 / 19 / 88$ & 6 & & $N R$ \\
\hline & & & & & $N R$ & $03 / 29 / 88$ & & & NR \\
\hline & & & & & NR & $03 / 29 / 88$ & & & NR \\
\hline FBARIUM & PPB & 6 & 1000 & $01 / 25 / 88$ & 20 & $01 / 19 / 88$ & 19 & $03 / 28 / 88$ & 18 \\
\hline & & & & $04 / 01 / 88$ & 20 & $01 / 19 / 88$ & 18 & & NR \\
\hline & & & & & NR & $03 / 29 / 88$ & 16 & & NR \\
\hline & & & & & NR & $03 / 29 / 88$ & 18 & & NR \\
\hline FCALCIU & PPB & 50 & & $01 / 25 / 88$ & 22200 & $01 / 19 / 88$ & 17300 & $03 / 28 / 88$ & 18600 \\
\hline & & & & $04 / 01 / 88$ & 20700 & $01 / 19 / 88$ & 16900 & & NR \\
\hline & & & & & NR & $03 / 29 / 88$ & 21100 & & NR \\
\hline & & & & & NR & $03 / 29 / 8 B$ & 20400 & & $N R$ \\
\hline FCOPPER & PPE & 10 & $(1300.0)$ & $01 / 25 / 88$ & & $01 / 19 / 88$ & & $03 / 28 / 88$ & 17 \\
\hline
\end{tabular}


IABLE E.5. (contd)

\begin{tabular}{|c|c|c|c|c|c|c|c|c|c|}
\hline \multicolumn{2}{|c|}{ CONSTI TUENT } & \multirow{2}{*}{$\begin{array}{l}\text { CONTRACTUAL } \\
\text { DETECTI ON } \\
\text { LIMIT }\end{array}$} & \multirow{2}{*}{$\begin{array}{l}\text { DRINKING } \\
\text { WATER } \\
\text { STANDARO }\end{array}$} & \multirow{2}{*}{$\begin{array}{l}\text { SAMPLE } \\
\text { DATE }\end{array}$} & \multirow[b]{2}{*}{$2-E 25-22$} & SAMPLE & \multirow[b]{2}{*}{ Z-E25-25 } & \multirow{2}{*}{$\begin{array}{l}\text { SAMPLE } \\
\text { DATE }\end{array}$} & \multirow[b]{2}{*}{$2-E 25-26$} \\
\hline NAME & UNITS & & & & & DATE & & & \\
\hline \multirow[t]{4}{*}{$\cdots-\cdots$} & $\cdots$ & $\cdots \cdots$ & 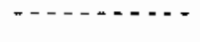 & - & $\cdots$ & $\cdots$ & 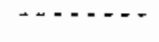 & - - - - - & $--\cdots$ \\
\hline & & & & $04 / 01 / 88$ & & $01 / \uparrow 9 / 88$ & & & NR \\
\hline & & & & & NR & $03 / 29 / 88$ & & & NR \\
\hline & & & & & NR & $03 / 29 / 88$ & & & NR \\
\hline \multirow[t]{3}{*}{ FLUORIO } & PPB & 500 & 4000 & $01 / 25 / 88$ & 507 & $01 / 19 / 88$ & & $03 / 28 / 88$ & \\
\hline & & & & $04 / 01 / 88$ & 527 & $03 / 29 / 88$ & & & NR \\
\hline & & & & & $N R$ & $03 / 29 / 88$ & & & NR \\
\hline \multirow[t]{4}{*}{ FMAGNES } & PPB & 50 & & $01 / 25 / 88$ & 6110 & $01 / 19 / 88$ & 5040 & $03 / 28 / 88$ & 5270 \\
\hline & & & & $04 / 01 / 88$ & 5850 & $01 / 19 / 88$ & 4890 & & NR \\
\hline & & & & & NR & $03 / 29 / 8 \mathrm{~B}$ & 6150 & & NR \\
\hline & & & & & NR & $03 / 29 / 88$ & 5790 & & NR \\
\hline \multirow[t]{4}{*}{ FPOTASS } & PPB & 100 & & $01 / 25 / 88$ & 5530 & $01 / 19 / 88$ & 4450 & $03 / 28 / 88$ & 4060 \\
\hline & & & & $04 / 01 / 88$ & 5350 & $01 / 19 / 88$ & 4330 & & NR \\
\hline & & & & & NR & $03 / 29 / 88$ & 4020 & & NR \\
\hline & & & & & NR & $03 / 29 / 38$ & 3810 & & NR \\
\hline \multirow[t]{4}{*}{ FSODIUA } & $\mathrm{PPB}$ & 200 & & $01 / 25 / 88$ & 17700 & $01 / 19 / 8 B$ & 7320 & $03 / 28 / 88$ & 11000 \\
\hline & & & & $04 / 01 / 88$ & 17600 & $01 / 19 / 88$ & 7290 & & NR \\
\hline & & & & & $N R$ & $03 / 29 / 88$ & 6200 & & NR \\
\hline & & & & & $N R$ & $03 / 29 / 88$ & 5780 & & NR \\
\hline \multirow[t]{4}{*}{ FSTRONT } & PPB & 10 & & $01 / 25 / 88$ & 115 & $01 / 19 / 88$ & 103 & $03 / 28 / 88$ & 105 \\
\hline & & & & & NR & $01 / 19 / 88$ & 101 & & NR \\
\hline & & & & & NR & $03 / 29 / 88$ & 120 & & NR \\
\hline & & & & & NR & $03 / 29 / 88$ & 114 & & NR \\
\hline \multirow[t]{4}{*}{ FVANADI } & $P P B$ & 5 & & $01 / 25 / 88$ & 51 & $01 / 19 / 88$ & 19 & $03 / 28 / 88$ & 34 \\
\hline & & & & $04 / 01 / 88$ & 51 & $01 / 19 / 88$ & 20 & & NR \\
\hline & & & & & NR & $03 / 29 / 88$ & 14 & & NR \\
\hline & & & & & NR & $03 / 29 / 88$ & 8 & & NR \\
\hline \multirow[t]{4}{*}{ FZINC } & PPB & 5 & & $01 / 25 / 88$ & 16 & $01 / 19 / 88$ & 7 & $03 / 28 / 88$ & 13 \\
\hline & & & & $04 / 01 / 88$ & 10 & $01 / 19 / 88$ & $\mathrm{~g}$ & & NR \\
\hline & & & & & NR & $03 / 29 / 88$ & 9 & & $N R$ \\
\hline & & & & & NR & $03 / 29 / 88$ & & & NR \\
\hline \multirow[t]{2}{*}{ HNITRAT } & PPB & 2500 & 45000 & $01 / 25 / 88$ & 5830 & & NR & & NR \\
\hline & & & & $04 / 19 / 88$ & 4430 & & NR & & NR \\
\hline \multirow[t]{3}{*}{ I RON } & $\mathrm{PPB}$ & 50 & & $01 / 25 / 88$ & & $01 / 19 / 88$ & 362 & $03 / 28 / 88$ & 363 \\
\hline & & & & $04 / 01 / 88$ & & $01 / 19 / 88$ & 399 & & NR \\
\hline & & & & & NR & $03 / 29 / 88$ & 385 & & NR \\
\hline \multirow[t]{2}{*}{ MAGNES } & $\mathrm{PPB}$ & 0 & & $01 / 25 / 88$ & 6570 & $01 / 19 / 88$ & 4870 & $03 / 28 / 88$ & 5510 \\
\hline & & & & $04 / 01 / 88$ & 5710 & $01 / 19 / 88$ & 4990 & & NR \\
\hline \multirow{3}{*}{ MANGESE } & $P P B$ & 5 & & $01 / 25 / 88$ & $N R$ & $03 / 29 / 88$ & 5960 & & NR \\
\hline & & & & $04 / 01 / 88$ & & $\begin{array}{l}01 / 19 / 88 \\
01 / 19 / 88\end{array}$ & $\begin{array}{l}6 \\
6\end{array}$ & $03 / 28 / 88$ & NR \\
\hline & & & & & NR & $03 / 29 / 88$ & 9 & & NR \\
\hline NICKEL & PPB & 10 & & $01 / 25 / 88$ & & $01 / 19 / 88$ & & $03 / 28 / 88$ & \\
\hline & & & & $04 / 01 / 88$ & & $01 / 19 / 88$ & & & NR \\
\hline & & & & & $N R$ & $03 / 29 / 88$ & 13 & & NR \\
\hline NITRATE & PPB & 500 & 45000 & $01 / 25 / 88$ & 5880 & $01 / 19 / 88$ & 582 & $03 / 28 / 88$ & 1210 \\
\hline
\end{tabular}


TABLE E.5. (contd)

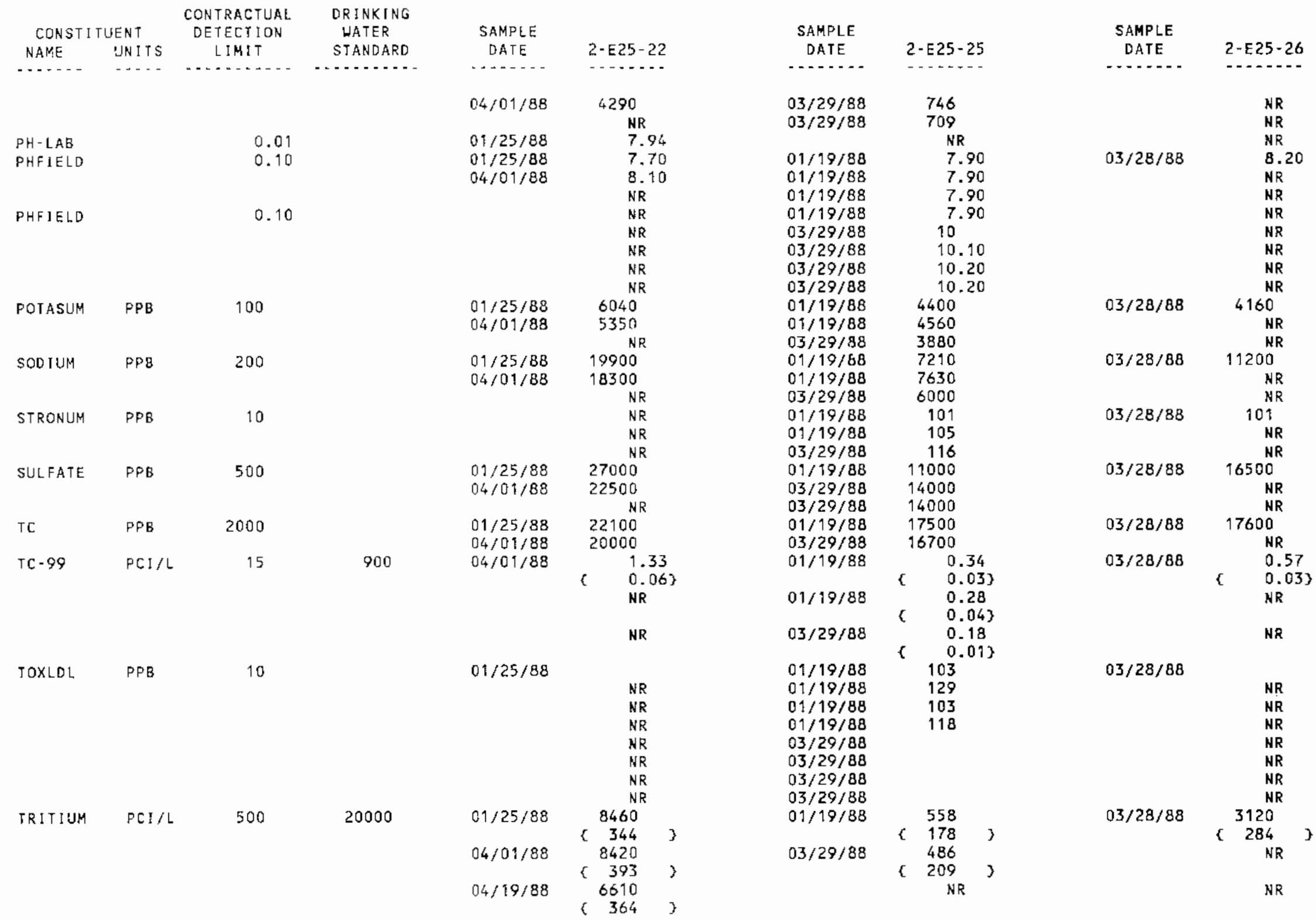


TABLEE.5. (contd)

\begin{tabular}{|c|c|c|c|c|c|c|c|c|c|}
\hline \multicolumn{2}{|c|}{ CONSSITUENT } & $\begin{array}{c}\text { CONTRAETUAL } \\
\text { DETECTION }\end{array}$ & $\begin{array}{l}\text { DR INK ING } \\
\text { WATER }\end{array}$ & SAMPLE & & SAMPLE & & SAMPLE & \\
\hline NAME & UNITS & LIMIT & STANDARD & DATE & $2-E 25-22$ & DATE & $2-E 25-25$ & DATE & $2 \cdot E 25-26$ \\
\hline - - n & $\cdots$ & $\ldots$ & $\ldots$ & $\cdots$ & $\cdots \cdots$ & $\cdots \ldots$ & $\cdots \cdots$ & $\cdots \ldots$ & -1 \\
\hline VANADUM & PPB & 5 & & $\begin{array}{l}01 / 25 / 88 \\
04 / 01 / 88\end{array}$ & $\begin{array}{l}53 \\
48 \\
N R\end{array}$ & $\begin{array}{l}01 / 19 / 88 \\
01 / 19 / 88 \\
03 / 29 / 88\end{array}$ & $\begin{array}{l}20 \\
21 \\
13\end{array}$ & $03 / 28 / 88$ & $\begin{array}{l}43 \\
\text { NR } \\
\text { NR }\end{array}$ \\
\hline $21 \mathrm{NC}$ & PPB & 5 & & $\begin{array}{l}01 / 25 / 88 \\
04 / 01 / 88\end{array}$ & $\begin{array}{l}17 \\
13 \\
\text { NR }\end{array}$ & $\begin{array}{l}01 / 19 / 88 \\
01 / 19 / 88 \\
03 / 29 / 88\end{array}$ & $\begin{array}{r}11 \\
10 \\
6\end{array}$ & $03 / 28 / 88$ & $\begin{array}{l}13 \\
N R \\
N R\end{array}$ \\
\hline
\end{tabular}

$\underset{m}{m}$ 
TABLE E.5. (contd)

\begin{tabular}{|c|c|c|c|c|c|}
\hline \multicolumn{2}{|c|}{ CONSTI TUENT } & $\begin{array}{l}\text { CONTRACTUAL } \\
\text { DETECTION }\end{array}$ & $\begin{array}{l}\text { DRINKING } \\
\text { WATER }\end{array}$ & SAMPLE & \\
\hline NAME & UNITS & LIMIT & STANDARD & DATE & $2-E 25-27$ \\
\hline$-\ldots$ & $\cdots \cdots$ & $\cdots$ & $\ldots+\ldots, \ldots$ & $\cdots . .$. & $\cdots$ \\
\hline ALUMNUM & $P P B$ & 150 & & $03 / 30 / 88$ & \\
\hline ARSEN IC & PPB & 5 & 50 & $03 / 30 / 88$ & $\begin{array}{l}7 \pi \\
? R\end{array}$ \\
\hline BARIUM & PPB & 6 & 1000 & $03 / 30 / 88$ & $\begin{array}{l}16 \\
N R\end{array}$ \\
\hline CALCIUM & PPB & 50 & & $03 / 30 / 88$ & $\begin{array}{r}18700 \\
N R\end{array}$ \\
\hline CHLOR ID & PPB & 500 & & $03 / 30 / 88$ & 3260 \\
\hline CHROMUM & PPB & 10 & 50 & $03 / 30 / 88$ & NR \\
\hline $00-60$ & $\mathrm{PCI} / \mathrm{L}$ & 22.50 & 100 & $03 / 30 / 88$ & \\
\hline CONOFLD & HMHO & 1 & & $03 / 30 / 88$ & 167 \\
\hline CS- 137 & $\mathrm{PCI} / \mathrm{L}$ & 20 & 200 & $03 / 30 / 88$ & \\
\hline FARSEN! & $P P B$ & 5 & 50 & $03 / 30 / 88$ & 7 \\
\hline FBAR IUS & PPB & 6 & 1000 & $03 / 30 / 88$ & 16 \\
\hline FCALCIU & PPE & 50 & & $03 / 30 / 88$ & 19100 \\
\hline FLUORID & PPB & 500 & 4000 & $03 / 30 / 88$ & \\
\hline FMAGNES & PPB & 50 & & $03 / 30 / 88$ & 5680 \\
\hline FPOTASS & $P P B$ & 100 & & $03 / 30 / 88$ & 4240 \\
\hline FSODIUM & PPB & 200 & & $03 / 30 / 88$ & 10900 \\
\hline FSTRONT & $P P B$ & 10 & & $03 / 30 / 88$ & 105 \\
\hline FVANADI & PPB & 5 & & $03 / 30 / 88$ & 35 \\
\hline$F Z I N C$ & $P P B$ & 5 & & $03 / 30 / 88$ & 7 \\
\hline I RON & PFE & 50 & & $03 / 30 / 80$ & $\begin{array}{l}107 \\
\mathrm{NR}\end{array}$ \\
\hline MAGNES & PP3̈ & 0 & & $03 / 30 / 88$ & $\begin{array}{c}5760 \\
N R\end{array}$ \\
\hline MANGESE & PF3 & 5 & & $03 / 30 / 88$ & $\mathrm{NR}$ \\
\hline SIICKEL & PPB & 10 & & $03 / 30 / 88$ & NR \\
\hline $\begin{array}{l}\text { NITRATE } \\
\text { PHFIEID }\end{array}$ & $P P B$ & $\begin{array}{l}500 \\
0.10\end{array}$ & 45000 & $\begin{array}{l}03 / 30 / 88 \\
03 / 30 / 88\end{array}$ & $\begin{array}{c}1520 \\
8.40 \\
N R\end{array}$ \\
\hline PHOSPHA & PPB & 1000 & & $03 / 30 / 88$ & \\
\hline POTASUM & PPB & 100 & & $03 / 30 / 88$ & $\begin{array}{l}4420 \\
\mathrm{NR}\end{array}$ \\
\hline SODIUM & $P P B$ & 200 & - & $03 / 30 / 88$ & $\begin{array}{c}11500 \\
\mathrm{NR}\end{array}$ \\
\hline STRONUM & $P P B$ & 10 & & $63 / 30 / 88$ & $\frac{105}{N R}$ \\
\hline
\end{tabular}

\begin{tabular}{|c|c|}
\hline $\begin{array}{l}\text { SAMPLE } \\
\text { DATE }\end{array}$ & $2-E 25-28$ \\
\hline - & \\
\hline $03 / 31 / 88$ & \\
\hline $03 / 31 / 88$ & $\begin{array}{c}\text { NR } \\
11 \\
\text { ND }\end{array}$ \\
\hline $03 / 31 / 88$ & 20 \\
\hline $03 / 31 / 88$ & $\begin{array}{r}19600 \\
\text { NR }\end{array}$ \\
\hline $\begin{array}{l}03 / 31 / 88 \\
03 / 31 / 88\end{array}$ & 3210 \\
\hline $03 / 31 / 88$ & $A R$ \\
\hline $03 / 31 / 38$ & 174 \\
\hline $03 / 31 / 88$ & \\
\hline $\begin{array}{l}03 / 31 / 88 \\
03 / 31 / 88 \\
03 / 31 / 88 \\
03 / 31 / 88\end{array}$ & $\begin{array}{r}9 \\
19 \\
19700\end{array}$ \\
\hline $\begin{array}{l}03 / 31 / 88 \\
03 / 31 / 88\end{array}$ & $\begin{array}{l}5800 \\
3840\end{array}$ \\
\hline $03 / 31 / 88$ & 11600 \\
\hline $03 / 31 / 88$ & 104 \\
\hline $03 / 31 / 88$ & 43 \\
\hline $03 / 31 / 88$ & 23 \\
\hline $03 / 31 / 88$ & 118 \\
\hline $03 / 31 / 88$ & 6070 \\
\hline $03 / 31 / 88$ & $N R$ \\
\hline $03 / 31 / 88$ & $N R$ \\
\hline & $N R$ \\
\hline $\begin{array}{l}03 / 31 / 88 \\
03 / 31 / 88\end{array}$ & $\begin{array}{c}812 \\
8.60 \\
\text { NR }\end{array}$ \\
\hline $\begin{array}{l}03 / 31 / 88 \\
03 / 31 / 88\end{array}$ & 4050 \\
\hline $03 / 31 / 88$ & $\begin{array}{c}N R \\
12400 \\
N R\end{array}$ \\
\hline $03 / 31 / 88$ & $\begin{array}{c}108 \\
N R\end{array}$ \\
\hline
\end{tabular}

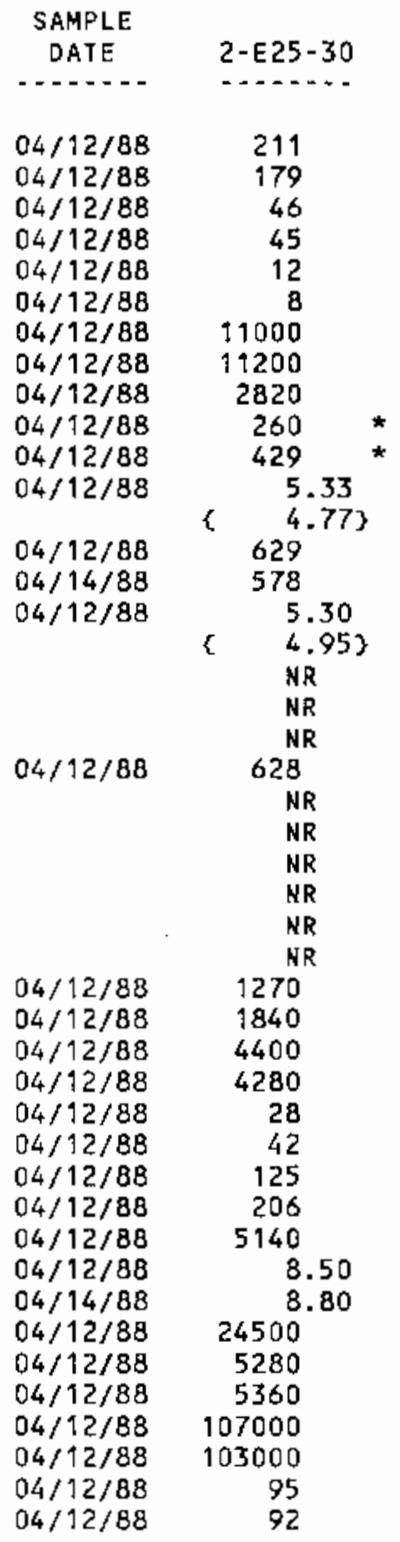


IABLE E.5. (contd)

\begin{tabular}{|c|c|c|c|c|c|}
\hline \multicolumn{2}{|c|}{ CONSTIJUENT } & $\begin{array}{c}\text { CONTRACTUAL } \\
\text { DETECTION }\end{array}$ & $\begin{array}{l}\text { DRINKING } \\
\text { WAIER }\end{array}$ & SAMPLE & \\
\hline NAME & UNITS & LIMIT & STANDARD & DATE & $2-E 25-27$ \\
\hline$\cdots-$ & $-\cdots$ & - & $-\cdots$ & 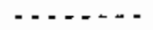 & 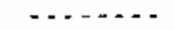 \\
\hline SULFATE & PPB & 500 & & $03 / 30 / 88$ & 17700 \\
\hline TC & PPB & 2000 & & $03 / 30 / 8 B$ & 17000 \\
\hline TC-99 & $\mathrm{PCI} / \mathrm{L}$ & 15 & 900 & $03 / 30 / 88$ & $\begin{array}{ll} & 1.40 \\
0 & 0.04\}\end{array}$ \\
\hline TRITIUM & $\mathrm{PCI} / \mathrm{L}$ & 500 & 20000 & $03 / 30 / 88$ & $\begin{array}{r}3120 \\
\times \quad 284\end{array}$ \\
\hline UNKNOUN & PPB & 0 & & & NR \\
\hline VANADUM & PPB & 5 & & $03 / 30 / 88$ & $\begin{array}{c}34 \\
\text { NR }\end{array}$ \\
\hline ZINC & $\mathrm{PPB}$ & 5 & & $03 / 30 / 88$ & $\stackrel{13}{\mathrm{NR}}$ \\
\hline
\end{tabular}

\begin{tabular}{|c|c|}
\hline $\begin{array}{l}\text { SAMPLE } \\
\text { DATE }\end{array}$ & $2-E 25-28$ \\
\hline$\cdots$ & - - - - - - \\
\hline $\begin{array}{l}03 / 31 / 88 \\
03 / 31 / 88\end{array}$ & $\begin{array}{l}12600 \\
19000\end{array}$ \\
\hline $03 / 31 / 88$ & $\left\{\begin{array}{l}0.62 \\
\{\quad 0.05\}\end{array}\right.$ \\
\hline $03 / 3 \uparrow / 88$ & $\left.\begin{array}{c}1690 \\
245 \\
\text { NR }\end{array}\right]$ \\
\hline $03 / 31 / 88$ & $\begin{array}{l}42 \\
K R\end{array}$ \\
\hline $03 / 39 / 88$ & $\begin{array}{c}52 \\
\text { NR }\end{array}$ \\
\hline
\end{tabular}

SAMPLE

DATE

$04 / 12 / 88$

$04 / 12 / 88$
$04 / 12 / 88$

$04 / 12 / 88$
$04 / 14 / 88$

$04 / 92 / 88$

$04 / 14 / 88$

$04 / 12 / 88$

$04 / 12 / 88$

$04 / 12 / 88$

$04 / 12 / 88$

\section{2-E25-30}

66500

35500

1.48

( 0.11$\}$

\{ 179 )

23

97

104 
TABLE E.5. (contd)

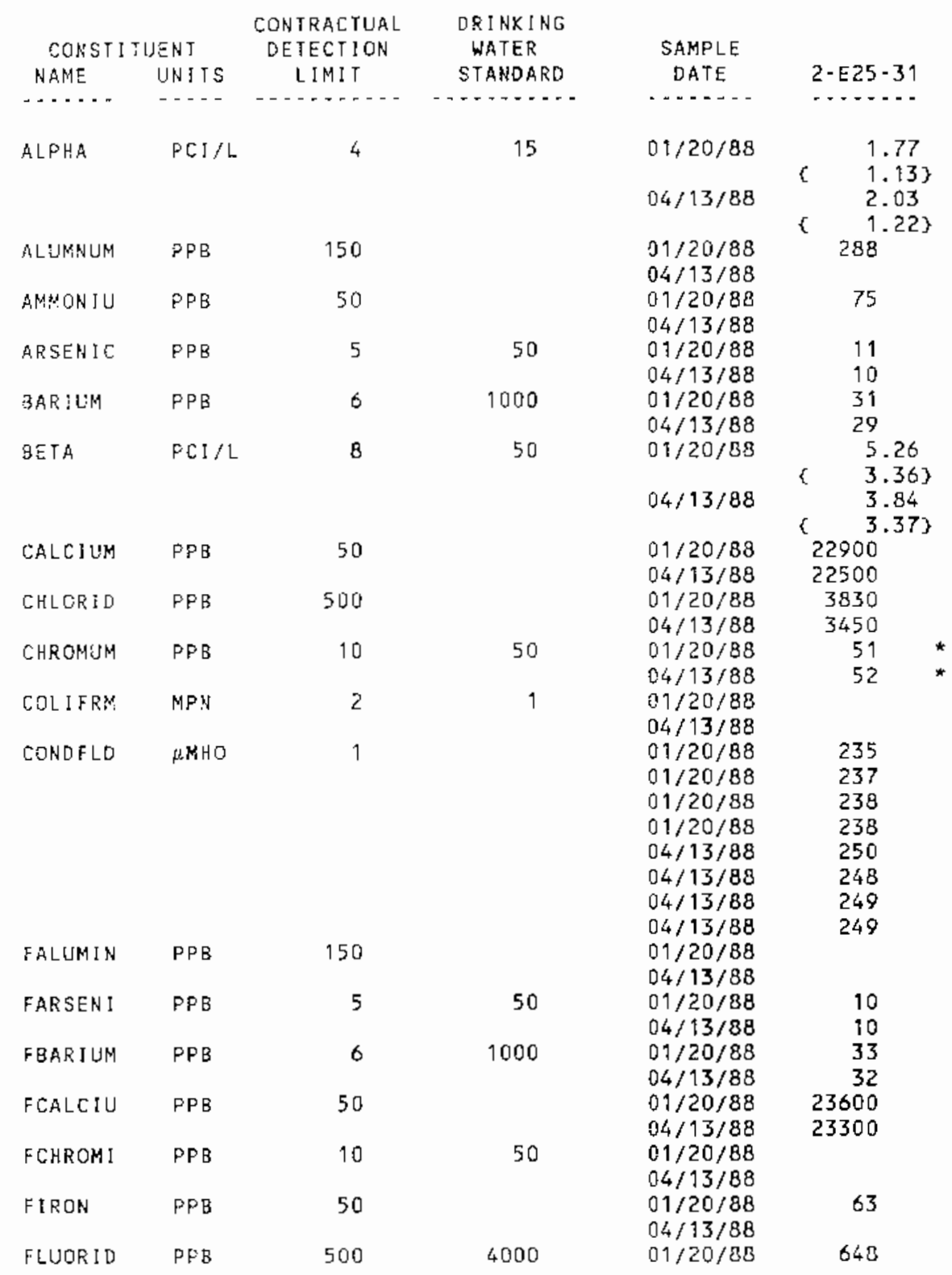

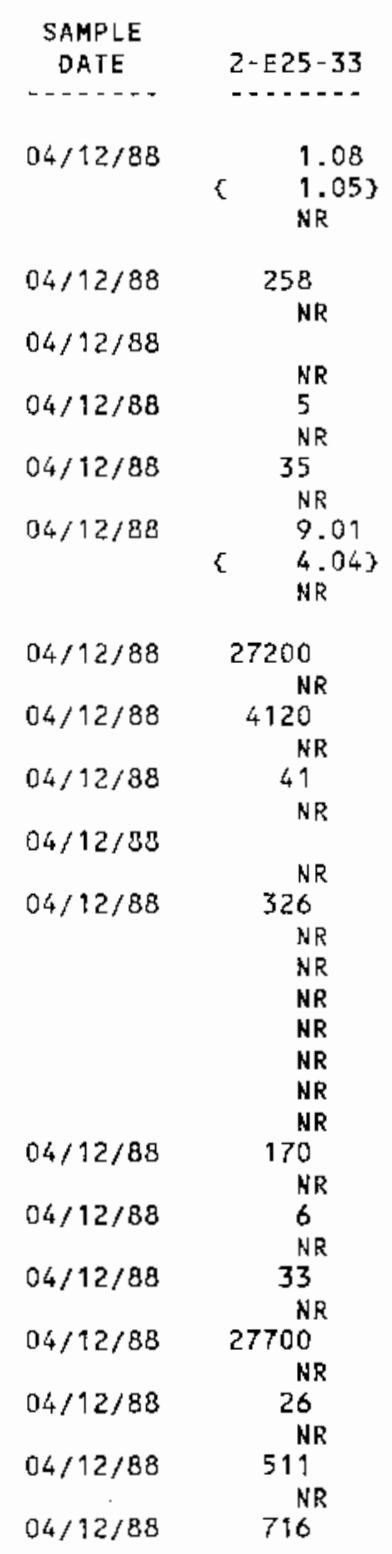

\begin{tabular}{|c|c|}
\hline $\begin{array}{l}\text { SAMPLE } \\
\text { DATE }\end{array}$ & $3-1-1$ \\
\hline 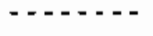 & $\cdots$ \\
\hline $05 / 19 / 88$ & $\begin{array}{l}17.70 \\
3.023 \\
\mathrm{NR}\end{array}$ \\
\hline $05 / 19 / 88$ & \\
\hline $05 / 19 / 88$ & $\begin{array}{l}\text { NR } \\
56 \\
N R\end{array}$ \\
\hline $05 / 19 / 88$ & \\
\hline $05 / 19 / 88$ & $\begin{array}{l}\text { NR } \\
35 \\
\end{array}$ \\
\hline $05 / 19 / 88$ & $\begin{array}{l}\text { NR } \\
8.39 \\
3.713 \\
\text { NR }\end{array}$ \\
\hline $05 / 19 / 88$ & $\begin{array}{r}22700 \\
\text { NR }\end{array}$ \\
\hline $05 / 19 / 88$ & $\begin{array}{c}10800 \\
N R\end{array}$ \\
\hline $05 / 19 / 88$ & NR \\
\hline $05 / 19 / 88$ & \\
\hline $05 / 19 / 88$ & $\begin{array}{c}179 \\
\text { NR } \\
\text { NR } \\
\text { NR } \\
\text { NR } \\
\text { NR } \\
\text { NR } \\
\text { NR }\end{array}$ \\
\hline $05 / 19 / 88$ & \\
\hline $05 / 19 / 88$ & NR \\
\hline $05 / 19 / 88$ & $\begin{array}{l}\text { NR } \\
35 \\
\text { NR }\end{array}$ \\
\hline $05 / 19 / 88$ & $\begin{array}{c}22300 \\
\text { NR }\end{array}$ \\
\hline $05 / 19 / 88$ & $\mathrm{NR}$ \\
\hline $05 / 19 / 88$ & \\
\hline $05 / 19 / 88$ & $\begin{array}{c}N R \\
595\end{array}$ \\
\hline
\end{tabular}


IABLE E.5. (contd)

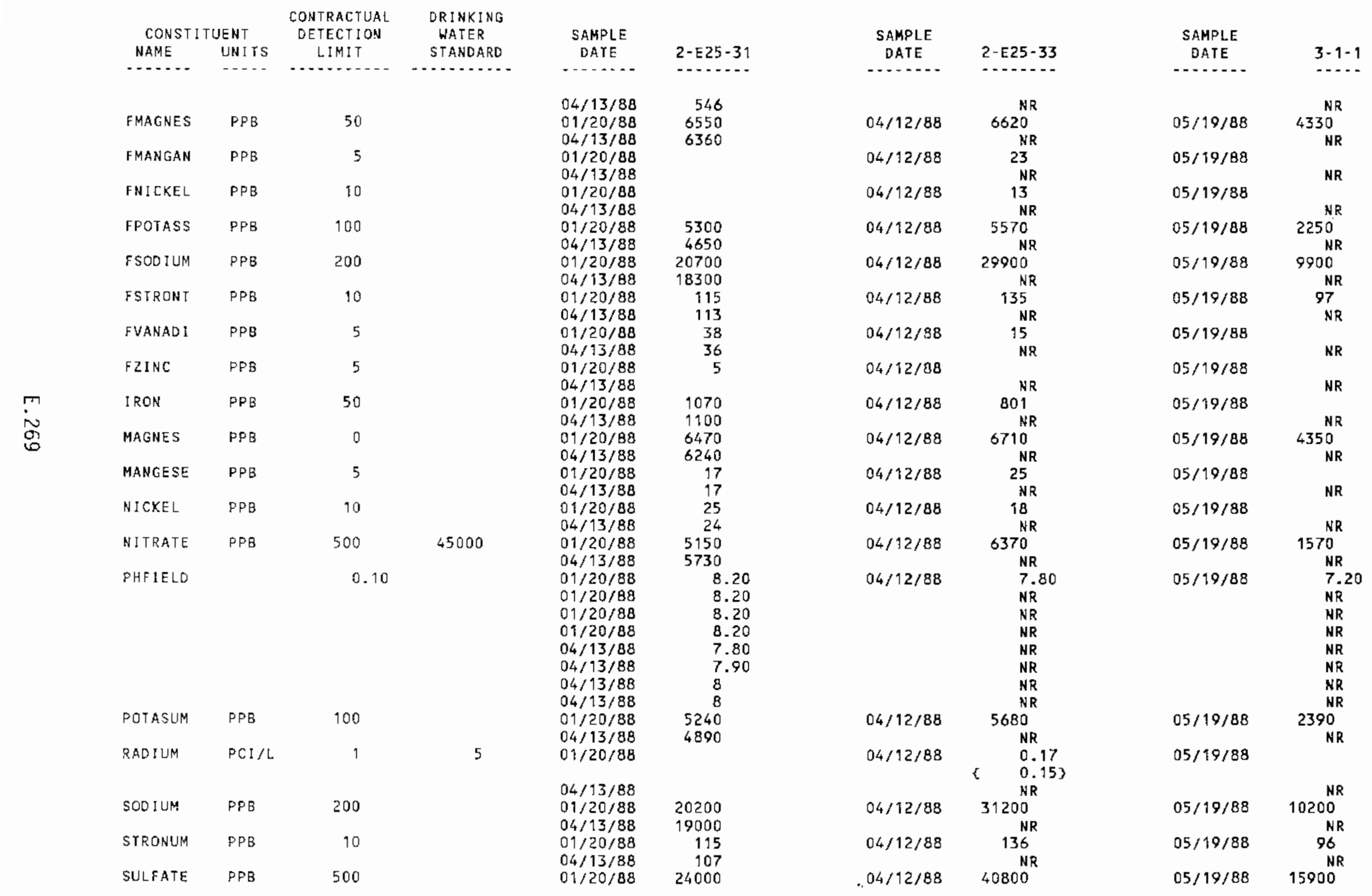


IABLE E.5. (contd)

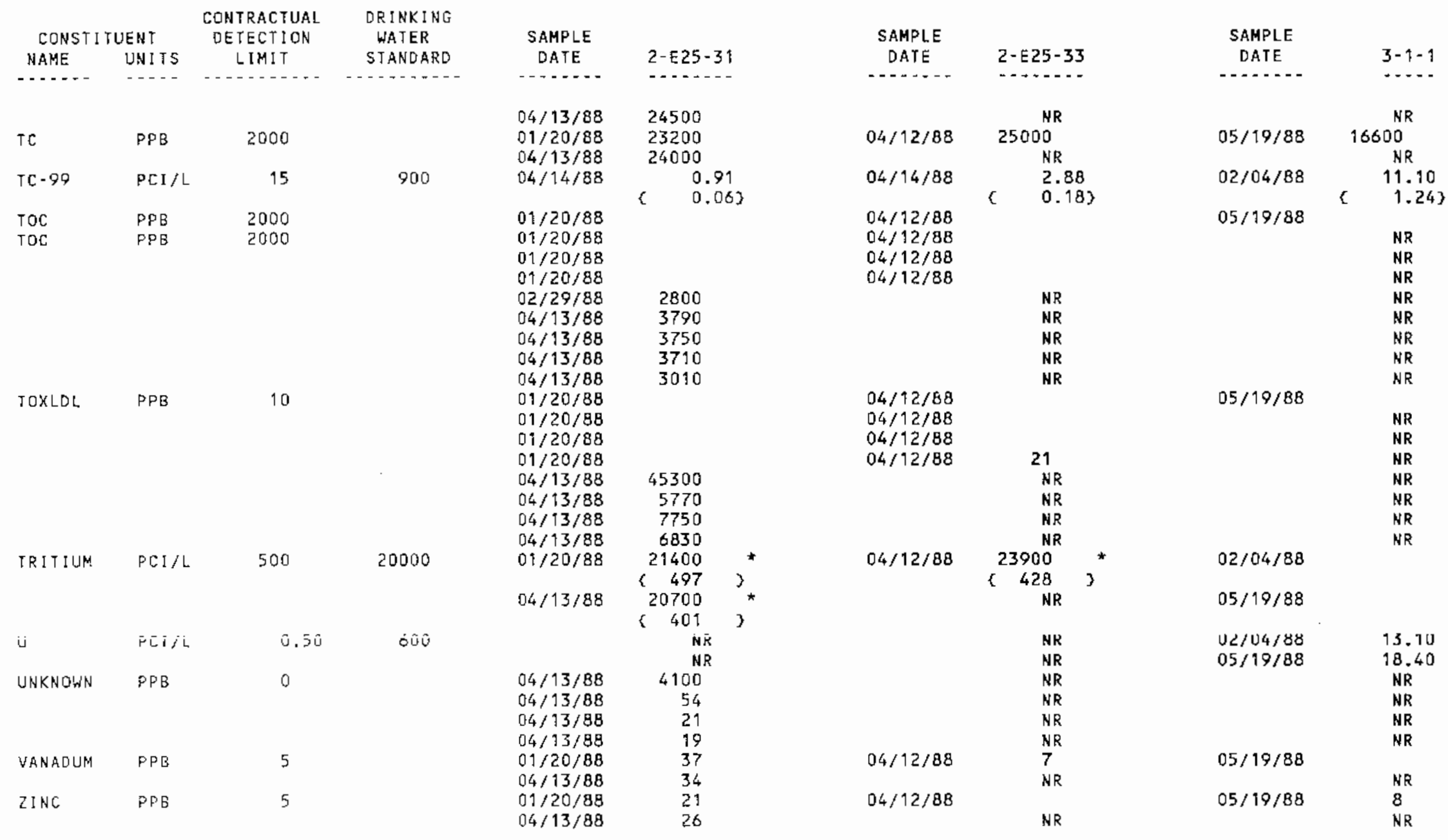


TABLE E.5. (contd)

\begin{tabular}{|c|c|c|c|c|c|c|c|c|c|}
\hline \multicolumn{2}{|c|}{ CONST I TUENT } & $\begin{array}{l}\text { CONTRACTUAL } \\
\text { DETECTION }\end{array}$ & $\begin{array}{l}\text { DRINKING } \\
\text { WATER }\end{array}$ & SAMPLE & & SAMPLE & & SAMPLE & \\
\hline NAME & UNITS & LIMIT & STANDARD & DATE & $3-1-2$ & DATE & $3-1-4$ & DATE & $3-1-6$ \\
\hline$\ldots$ & $\ldots .$. & $\cdots$ & (......... & $\ldots \ldots$ & $\ldots$ & $\ldots$ & $\cdots$ & $\cdots$ & $\cdots$ \\
\hline ALPHA & $\mathrm{PCI} / \mathrm{L}$ & 4 & 15 & $05 / 90 / 88$ & $\begin{array}{c}34.20+ \\
4.423\end{array}$ & $05 / 24 / 88$ & ${ }_{3.17\}^{21}}^{+}$ & $05 / 27 / 88$ & $\begin{array}{c}27.70+ \\
3.71\}\end{array}$ \\
\hline AMMONIU & $P P B$ & 50 & & $05 / 10 / 88$ & 56 & $05 / 24 / 88$ & & $05 / 27 / 88$ & \\
\hline BAR IUM & $P P B$ & 6 & 1000 & $05 / 10 / 88$ & 31 & $05 / 24 / 88$ & 16 & $05 / 27 / 88$ & 20 \\
\hline BETA & PCI/L & 8 & 50 & $05 / 10 / 88$ & $\begin{array}{r}22.90 \\
\{\quad 5.24\}\end{array}$ & $05 / 24 / 88$ & $\left\{\begin{array}{l}12 \\
\{\quad 4.11\}\end{array}\right.$ & $05 / 27 / 88$ & $\begin{array}{c}14.40 \\
4.243\end{array}$ \\
\hline CALCIUM & $P P B$ & 50 & & $05 / 10 / 88$ & 33900 & $05 / 24 / 88$ & 17800 & $05 / 27 / 88$ & 22300 \\
\hline CHLFORM & PPB & 10 & 100 & $05 / 10 / 88$ & & $05 / 24 / 88$ & 22 & $05 / 27 / 88$ & 16 \\
\hline CHLORID & PPB & 500 & & $05 / 10 / 88$ & 14800 & $05 / 24 / 88$ & 4890 & $05 / 27 / 88$ & 6800 \\
\hline COL I FRM & MPN & 2 & 1 & $05 / 10 / 88$ & & $05 / 24 / 88$ & $9.20 *$ & $05 / 27 / 88$ & \\
\hline CONDFLD & $\mu \mathrm{MHO}$ & 1 & & $05 / 10 / 88$ & 239 & $05 / 24 / 88$ & 125 & $05 / 27 / 88$ & 154 \\
\hline FBARIUM & PPB & 6 & $\$ 000$ & $05 / 10 / 88$ & 30 & $05 / 24 / 88$ & 16 & $05 / 27 / 88$ & 20 \\
\hline FCALCIU & PPB & 50 & & $05 / 10 / 88$ & 34200 & $05 / 24 / 88$ & 16900 & $05 / 27 / 8 B$ & 23300 \\
\hline FCOPPER & PPB & 10 & $(1300.0)$ & $05 / 10 / 88$ & & $05 / 24 / 38$ & & $05 / 27 / 88$ & 10 \\
\hline FIRON & PPB & 50 & & $05 / 10 / 88$ & & $05 / 24 / 88$ & 62 & $05 / 27 / 88$ & 81 \\
\hline FMAGNES & $P P B$ & 50 & & $05 / 10 / 88$ & 6820 & $05 / 24 / 88$ & 3650 & $05 / 27 / 88$ & 4960 \\
\hline FPOTASS & PPB & 100 & & $05 / 10 / 88$ & 3370 & $05 / 24 / 88$ & 1650 & $05 / 27 / 88$ & 2000 \\
\hline F SOD I UM & PPB & 200 & & $05 / 10 / 88$ & 11000 & $05 / 24 / 88$ & 7260 & $05 / 27 / 88$ & 7330 \\
\hline FSTRONT & PPB & 10 & & $05 / 10 / 88$ & 136 & $05 / 24 / 88$ & 74 & $05 / 27 / 88$ & 103 \\
\hline FVANAD I & PPB & 5 & & $05 / 10 / 88$ & 7 & $05 / 24 / 88$ & & $05 / 27 / 8 B$ & \\
\hline FZINC & PPB & 5 & & $05 / 10 / 88$ & 5 & $05 / 24 / 88$ & 5 & $05 / 27 / 88$ & 13 \\
\hline I RON & PPB & 50 & & $05 / 10 / 88$ & 51 & $05 / 24 / 88$ & 61 & $05 / 27 / 88$ & 135 \\
\hline MAGNES & PPB & 0 & & $05 / 10 / 88$ & 6680 & $05 / 24 / 88$ & 3810 & $05 / 27 / 88$ & 4760 \\
\hline MET HYCH & PPB & 5 & & $05 / 10 / 88$ & & $05 / 24 / 88$ & 43 & $05 / 27 / 88$ & \\
\hline NITRATE & $\mathrm{PPB}$ & 500 & 45000 & $05 / 10 / 88$ & 6900 & $05 / 24 / 88$ & 761 & $05 / 27 / 88$ & 1810 \\
\hline PHFIELD & & 0.10 & & $05 / 10 / 88$ & 7.20 & $05 / 24 / 88$ & 7.20 & $05 / 27 / 88$ & 6.60 \\
\hline POTASUM & PPB & 100 & & $05 / 10 / 88$ & $3150^{\circ}$ & $05 / 24 / 88$ & 1640 & $05 / 27 / 88$ & 1960 \\
\hline SODIUM & PPB & 200 & & $05 / 10 / 88$ & 10300 & $05 / 24 / 88$ & 7610 & $05 / 27 / 88$ & 7050 \\
\hline STRONUM & PPB & 10 & & $05 / 10 / 88$ & 137 & $05 / 24 / 88$ & 79 & $05 / 27 / 88$ & 103 \\
\hline SULFATE & PPB & 500 & & $05 / 10 / 88$ & 26100 & $05 / 24 / 88$ & 20100 & $05 / 27 / 88$ & 20400 \\
\hline TC & PPB & 2000 & & $05 / 10 / 88$ & 21300 & $05 / 24 / 88$ & 13300 & $05 / 27 / 88$ & 15800 \\
\hline re. 99 & $\mathrm{PCI} / \mathrm{L}$ & 15. & 900 & $02 / 04 / 88$ & $\begin{array}{c}10.90 \\
1.243\end{array}$ & & NR & & NR \\
\hline TOXLDL & PPB & 10 & & $05 / 10 / 88$ & & $05 / 24 / 88$ & 78 & $05 / 27 / 88$ & 25.90 \\
\hline TRITIUM & $P C 1 / L$ & 500 & 20000 & $02 / 04 / 88$ & $\begin{array}{r}575 \\
\{\quad 214\end{array}$ & & $N R$ & $02 / 04 / 88$ & \\
\hline & & & & $05 / 10 / 88$ & 1690 & & NR & $05 / 27 / 88$ & 397 \\
\hline & & & & & $\{244$ & & & & \& 202 \\
\hline U & $\mathrm{PCI} / \mathrm{L}$ & 0.50 & 600 & $02 / 04 / 88$ & 9.74 & $02 / 08 / 88$ & 38.10 & $02 / 04 / 88$ & 19.30 \\
\hline$Z I N C$ & PPB & 5 & & $\begin{array}{l}05 / 10 / 88 \\
05 / 10 / 88\end{array}$ & $\begin{array}{r}29.90 \\
8\end{array}$ & $\begin{array}{l}05 / 24 / 88 \\
05 / 24 / 88\end{array}$ & 18.50 & $05 / 27 / 88$ & 19.50 \\
\hline
\end{tabular}


TABLE E.5. (contd)

\begin{tabular}{|c|c|c|c|c|c|c|c|c|c|}
\hline \multicolumn{2}{|c|}{ CONST I TUENT } & DETECTION & $\begin{array}{l}\text { DRINKING } \\
\text { WATER }\end{array}$ & SAMPLE & & SAMPLE & & SAMPLE & \\
\hline NAME & UNITS & LIMIT & STANDARD & DATE & $3-1-7$ & DATE & $3-1-8$ & DATE & $3-1-9$ \\
\hline$\cdots \cdots$ & $\cdots \cdot-$ & $\ldots$ & $\ldots \ldots$ & $\cdots+---$ & $\cdots \cdot$ & $\cdots$ & $\cdots$ & $\ldots$ & $\cdots$ \\
\hline ALPHA & $\mathrm{PCI} / \mathrm{L}$ & 4 & 15 & $05 / 24 / 88$ & $\begin{array}{c}64.70+ \\
5.843\end{array}$ & $05 / 25 / 88$ & $\begin{array}{c}47.30+ \\
5.30\}\end{array}$ & $05 / 25 / 88$ & \\
\hline AMMON IU & PPB & 50 & & $05 / 24 / 88$ & & $05 / 25 / 88$ & & $05 / 25 / 88$ & 140 \\
\hline BARIUM & PPB & 6 & 1000 & $05 / 24 / 88$ & 24 & $05 / 25 / 88$ & 33 & $05 / 25 / 88$ & 119 \\
\hline BETA & $\mathrm{PCI} / \mathrm{L}$ & 8 & 50 & $05 / 24 / 88$ & $\begin{array}{r}33.80 \\
\{\quad 5.95\}\end{array}$ & $05 / 25 / 88$ & $\begin{array}{r}18.60 \\
<\quad 4.71\}\end{array}$ & $05 / 25 / 88$ & $\begin{array}{ll} & 8.52 \\
\{\quad 4.25\}\end{array}$ \\
\hline CALCIUM & $\mathrm{PPB}$ & 50 & & $05 / 24 / 88$ & 24200 & $05 / 25 / 88$ & 19300 & $05 / 25 / 88$ & 18900 \\
\hline CHLFORM & $\mathrm{PPB}$ & 10 & 100 & $05 / 24 / 88$ & 14 & $05 / 25 / 88$ & & $05 / 25 / 88$ & \\
\hline CHLORID & PPB & 500 & & $05 / 24 / 88$ & 16100 & $05 / 25 / 88$ & 13500 & $05 / 25 / 88$ & 11300 \\
\hline CHROMUM & PPB & 10 & 50 & $05 / 24 / 88$ & & $05 / 25 / 88$ & & $05 / 25 / 88$ & 64 \\
\hline CONDFLD & АMHO & 1 & & $05 / 24 / 88$ & 152 & $05 / 25 / 88$ & 243 & $05 / 25 / 88$ & 343 \\
\hline COPPER & PPB & 10 & $(1300.0)$ & $05 / 24 / 88$ & 14 & $05 / 25 / 88$ & & $05 / 25 / 88$ & \\
\hline FBARIUM & $P P B$ & 6 & 1000 & $05 / 24 / 88$ & 22 & $05 / 25 / 88$ & 31 & $05 / 25 / 88$ & $\$ 15$ \\
\hline FCALCIU & PPB & 50 & & $05 / 24 / 88$ & 23300 & $05 / 25 / 88$ & 18500 & $05 / 25 / 88$ & 18000 \\
\hline FCOPPER & PPB & 10 & $(1300.0)$ & $05 / 24 / 88$ & 10 & $05 / 25 / 88$ & & $05 / 25 / 88$ & \\
\hline FIRON & PPB & 50 & & $05 / 24 / 88$ & 58 & $05 / 25 / 88$ & & $05 / 25 / 88$ & 115 \\
\hline FLUORID & PPB & 500 & 4000 & $05 / 24 / 88$ & 558 & $05 / 25 / 88$ & 611 & $05 / 25 / 88$ & 1300 \\
\hline FMAGNES & PPB & 50 & & $05 / 24 / 88$ & 4710 & $05 / 25 / 88$ & 5830 & $05 / 25 / 88$ & 7550 \\
\hline FMANGAN & PPB & 5 & & $05 / 24 / 88$ & & $05 / 25 / 88$ & 17 & $05 / 25 / 88$ & 62 \\
\hline FPOTASS & PPB & 100 & & $05 / 24 / 88$ & 2280 & $05 / 25 / 88$ & 5070 & $05 / 25 / 88$ & 5860 \\
\hline FSODIUM & PPB & 200 & & $05 / 24 / 88$ & 14700 & $05 / 25 / 88$ & 25600 & $05 / 25 / 88$ & 55200 \\
\hline FSTRONT & $P P B$ & 10 & & $05 / 24 / 88$ & 99 & $05 / 25 / 88$ & 104 & $05 / 25 / 88$ & 114 \\
\hline FZ1NC & PPB & 5 & & $05 / 24 / 88$ & 6 & $05 / 25 / 88$ & & $05 / 25 / 88$ & \\
\hline IRON & PPB & 50 & & $05 / 24 / 88$ & 108 & $05 / 25 / 88$ & 66 & $05 / 25 / 88$ & 544 \\
\hline MAGNES & PPB & 0 & & $05 / 24 / 88$ & 4820 & $05 / 25 / 88$ & 6120 & $05 / 25 / 88$ & 7860 \\
\hline MANGESE & PPB & 5 & & $05 / 24 / 88$ & & $05 / 25 / 88$ & 17 & $05 / 25 / 88$ & 73 \\
\hline NICKEL. & PPB & 10 & & $05 / 24 / 88$ & & $05 / 25 / 88$ & & $05 / 25 / 88$ & 32 \\
\hline NITRATE & PPB & 500 & 45000 & $05 / 24 / 98$ & 2720 & $05 / 25 / 88$ & 1160 & $05 / 25 / 88$ & \\
\hline PHFIELD & & 0.10 & & $05 / 24 / 88$ & 7 & $05 / 25 / 88$ & 7.70 & $05 / 25 / 88$ & 8.20 \\
\hline POTASUM & PPB & 100 & & $05 / 24 / 88$ & 2200 & $05 / 25 / 88$ & 5630 & $05 / 25 / 88$ & 6330 \\
\hline SODIUM & PPB & 200 & & $05 / 24 / 88$ & 14700 & $05 / 25 / 88$ & 27600 & $05 / 25 / 88$ & 59800 \\
\hline STRONUM & $P P B$ & 10 & & $05 / 24 / 88$ & 105 & $05 / 25 / 88$ & 109 & $05 / 25 / 88$ & 121 \\
\hline SULFATE & PPE & 500 & & $05 / 24 / 88$ & 17500 & $05 / 25 / 88$ & 11200 & $05 / 25 / 88$ & 533 \\
\hline$T C$ & PPB & 2000 & & $05 / 24 / 88$ & 17200 & $05 / 25 / 88$ & 22900 & $05 / 25 / 88$ & 40300 \\
\hline TOXLDL & PPB & 10 & & $05 / 24 / 88$ & 27 & $05 / 25 / 88$ & & $05 / 25 / 88$ & \\
\hline$u$ & $\mathrm{PCI} / \mathrm{L}$ & 0.50 & 600 & $\begin{array}{l}02 / 05 / 88 \\
05 / 24 / 88\end{array}$ & $\begin{array}{l}32.80 \\
53.20\end{array}$ & $\begin{array}{l}02 / 05 / 88 \\
05 / 25 / 88\end{array}$ & $\begin{array}{l}11.90 \\
30.90\end{array}$ & $\begin{array}{l}02 / 05 / 88 \\
05 / 25 / 88\end{array}$ & \\
\hline ZINC & PPB & 5 & & $05 / 24 / 88$ & 8 & $05 / 25 / 88$ & & $05 / 25 / 88$ & 6 \\
\hline
\end{tabular}


TABLE E.5. (contd)

\begin{tabular}{|c|c|c|c|c|c|c|c|c|c|}
\hline CONSTI & UENT & DETECTION & $\begin{array}{l}\text { DRINKING } \\
\text { WATER }\end{array}$ & SAMPLE & & SAMPLE & & SAMPLE & \\
\hline NAME & UNITS & LIMIT & STANDARD & DATE & $3-1-10$ & DATE & $3-1-11$ & DATE & $3-1-12$ \\
\hline$\cdots$ & $\cdots$ & $\ldots \ldots$ & $\cdots$ & $\cdots$ & $\ldots$ & $\ldots$ & $\ldots$ & $-\ldots$ & $\cdots$ \\
\hline ALPHA & $\mathrm{PCl} / \mathrm{L}$ & 4 & 15 & $05 / 26 / 88$ & $\begin{array}{c}25.80+ \\
3.647\end{array}$ & $05 / 17 / 88$ & ${ }_{4.453^{4}}^{+}$ & $05 / 17 / 88$ & $\begin{array}{c}87.90+ \\
6.757\end{array}$ \\
\hline AMMONIU & $\mathrm{PPB}$ & 50 & & $05 / 26 / 88$ & & $05 / 17 / 88$ & 66 & $05 / 17 / 88$ & 58 \\
\hline BAR I UM & PPB & 6 & 1000 & $05 / 26 / 88$ & 29 & $05 / 17 / 88$ & 18 & $05 / 17 / 88$ & 20 \\
\hline BETA & $\mathrm{PCI} / \mathrm{L}$ & 8 & 50 & $05 / 26 / 88$ & $\begin{array}{r}11.80 \\
\{\quad 3.99\}\end{array}$ & $05 / 17 / 8 B$ & $\begin{array}{ll} & 9.77 \\
\{\quad 4.26\}\end{array}$ & $05 / 17 / 88$ & $\begin{array}{r}26.40 \\
\{\quad 5.28\}\end{array}$ \\
\hline CALCIUM & PPB & 50 & & $05 / 26 / 88$ & 23700 & $05 / 17 / 88$ & 20600 & $05 / 17 / 88$ & 22900 \\
\hline CHLFORM & PPB & 10 & 100 & $05 / 26 / 88$ & 15 & $01 / 07 / 88$ & 11 & $05 / 17 / 88$ & 17 \\
\hline & & & & & NR & $01 / 13 / 88$ & 12 & & NR \\
\hline & & & & & NR & $01 / 20 / 88$ & 12 & & NR \\
\hline & & & & & NR & $01 / 28 / 88$ & 10 & & NR \\
\hline & & & & & NR & $02 / 05 / 88$ & 13 & & NR \\
\hline & & & & & NR & $02 / 19 / 88$ & 12 & & NR \\
\hline & & & & & NR & $02 / 18 / 88$ & & & NR \\
\hline & & & & & NR & $02 / 26 / 88$ & 11 & & NR \\
\hline & & & & & $N R$ & $03 / 03 / 88$ & 12 & & NR \\
\hline & & & & & NR & $03 / 10 / 88$ & 10 & & NR \\
\hline & & & & & $N R$ & $03 / 17 / 88$ & 12 & & NR \\
\hline & & & & & NR & $03 / 25 / 88$ & 10 & & $\mathrm{NR}$ \\
\hline & & & & & NR & $03 / 31 / 88$ & 12 & & NR \\
\hline & & & & & NR & $04 / 08 / 88$ & 10 & & NR \\
\hline & & & & & NR & $04 / 15 / 88$ & 12 & & NR \\
\hline & & & & & NR & $04 / 21 / 88$ & 14 & & NR \\
\hline & & & & & $N R$ & $04 / 28 / 88$ & 15 & & NR \\
\hline & & & & & NR & $05 / 05 / 88$ & 18 & & NR \\
\hline & & & & & NR & $05 / 12 / 88$ & 17 & & NR \\
\hline & & & & & NR & $05 / 17 / 88$ & 18 & & NR \\
\hline & & & & & NR & $05 / 26 / 88$ & 21 & & NR \\
\hline & & & & & NR & $06 / 02 / 88$ & 21 & & NR \\
\hline & & & & & NR & $06 / 08 / 88$ & 23 & & NR \\
\hline & & & & & NR & $06 / 16 / 88$ & 27 & & NR \\
\hline & & & & & $\mathrm{N} R$ & $06 / 23 / 88$ & 28 & & NR \\
\hline CHLORIO & PPB & 500 & & $05 / 26 / 88$ & 11200 & $01 / 07 / 88$ & 15600 & $05 / 17 / 88$ & 13700 \\
\hline & & & & & NR & $01 / 13 / 88$ & 28000 & & NR \\
\hline & & & & & NR & $01 / 20 / 88$ & 20200 & & NR \\
\hline & & & & & NR & $01 / 28 / 88$ & 35400 & & NR \\
\hline & & & & & NR & $02 / 05 / 88$ & 5070 & & NR \\
\hline & & & & & $N R$ & $02 / 11 / 88$ & 10600 & & NR \\
\hline & & & & & NR & $02 / 18 / 88$ & 3550 & & NR \\
\hline & & & . & & NR & $02 / 26 / 88$ & 26600 & & NR \\
\hline & & & & & $N R$ & $03 / 03 / 88$ & 18800 & & NR \\
\hline & & & & & NR & $03 / 10 / 88$ & 21500 & & $N R$ \\
\hline & & & & & NR & $03 / 17 / 88$ & 9210 & & NR \\
\hline & & & & & NR & $03 / 25 / 88$ & 6140 & & NR \\
\hline
\end{tabular}


TABLE E.5. (contd)

\begin{tabular}{|c|c|c|c|c|c|c|c|c|c|}
\hline \multicolumn{2}{|c|}{ CONST I TUENT } & $\begin{array}{l}\text { CONTRACTUAL } \\
\text { DETECTION }\end{array}$ & $\begin{array}{l}\text { DRINKING } \\
\text { WATER }\end{array}$ & SAMPLE & & SAMPLE & & SAMPLE & \\
\hline NAME & UNITS & LIMIT & STANDARD & DATE & $3-1-10$ & DATE & $3-1-11$ & DATE & $3-1-12$ \\
\hline$\cdots \cdots$ & - . - & 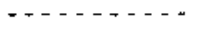 & ............... & $\cdots$ & $\cdots$ & $-\cdots$ & - & $\cdots$ & $-\cdots$ \\
\hline & & & & & NR & $03 / 31 / 88$ & 4000 & & NR \\
\hline & & & & & NR & $04 / 08 / 88$ & 5610 & & NR \\
\hline & & & & & NR & $04 / 95 / 88$ & 4620 & & NR \\
\hline & & & & & NR & $04 / 21 / 88$ & 24400 & & NR \\
\hline CHLORID & PPB & 500 & & & NR & $04 / 28 / 88$ & 5600 & & NR \\
\hline & & & & & NR & $05 / 05 / 88$ & 9180 & & NR \\
\hline & & & & & NR & $05 / 12 / 88$ & 10700 & & NR \\
\hline & & & & & NR & $05 / 17 / 88$ & 5470 & & NR \\
\hline & & & & & NR & $05 / 26 / 88$ & 4720 & & NR \\
\hline & & & & & NR & $06 / 02 / 88$ & 36300 & & NR \\
\hline & & & & & NR & $06 / 08 / 88$ & 4810 & & NR \\
\hline & & & & & $\mathrm{N} R$ & $06 / 16 / 88$ & 5800 & & NR \\
\hline & & & & & NR & $06 / 23 / 88$ & 74800 & & NR \\
\hline CONDFLD & MMHO & 1 & & $05 / 26 / 88$ & 177 & $01 / 07 / 38$ & & $05 / 17 / 8 B$ & 195 \\
\hline & & & & & $N R$ & $01 / 13 / 88$ & 190 & & NR \\
\hline & & & & & $N R$ & $01 / 20 / 88$ & 219 & & NR \\
\hline & & & & & $\mathrm{NR}$ & $01 / 28 / 88$ & 233 & & NR \\
\hline & & & & & $N R$ & $02 / 05 / 88$ & 140 & & NR \\
\hline & & & & & NR & $02 / 11 / 88$ & 134 & & NR \\
\hline & & & & & $\mathrm{NR}$ & $02 / 18 / 88$ & 154 & & NR \\
\hline & & & & & $N R$ & $02 / 26 / 88$ & 180 & & NR \\
\hline & & & & & $M R$ & $03 / 03 / 88$ & 174 & & NR \\
\hline & & & & & NR & $03 / 10 / 88$ & 206 & & NR \\
\hline & & & & & NR & $03 / 97 / 86$ & 173 & & NR \\
\hline & & & & & NR & $03 / 25 / 88$ & 157 & & NR \\
\hline & & & & & NR & $03 / 31 / 88$ & 134 & & NR \\
\hline & & & & & NR & $04 / 08 / 88$ & 184 & & NR \\
\hline & & & & & $\mathrm{NR}$ & $04 / 15 / 88$ & 178 & & NR \\
\hline & & & & & NR & $04 / 21 / 88$ & 293 & & $N R$ \\
\hline & & & & & NR & $04 / 28 / 88$ & 204 & & $N R$ \\
\hline & & & & & NR & $05 / 05 / 88$ & 198 & & NR \\
\hline & & & & & NR & $05 / 12 / 88$ & 189 & & NR \\
\hline & & & & & NR & $05 / 17 / 88$ & 158 & & NR \\
\hline & & & & & NR & $05 / 26 / 88$ & 136 & & NR \\
\hline & & & & & NR & $06 / 02 / 88$ & 226 & & $\mathrm{NR}$ \\
\hline & & & & & NR & $06 / 08 / 88$ & 126 & & NR \\
\hline & & & & & NR & $06 / 16 / 88$ & 142 & & $N R$ \\
\hline & & & & & $\mathrm{NR}$ & $06 / 23 / 88$ & 329 & & NR \\
\hline COPPER & $\mathrm{PPB}$ & 10 & $(1300.0)$ & $05 / 26 / 88$ & & $05 / 17 / 88$ & & $05 / 17 / 88$ & 11 \\
\hline FBARIUM & $\mathrm{PPB}$ & 6 & 1000 & $05 / 26 / 88$ & 28 & $05 / 17 / 88$ & 19 & $05 / 17 / 88$ & 20 \\
\hline FCALCIU & PPB & 50 & & $05 / 26 / 88$ & 22900 & $05 / 17 / 88$ & 21600 & $05 / 17 / 88$ & 23300 \\
\hline FCOPPER & $P P B$ & 10 & $(1300.0)$ & $05 / 26 / 88$ & & $05 / 17 / 88$ & 10 & $05 / 17 / 88$ & 11 \\
\hline FHAGNES & PPB & 50 & & $05 / 26 / 88$ & 4860 & $05 / 17 / 88$ & 4930 & $05 / 17 / 88$ & 5010 \\
\hline FN I CKEL & $\mathrm{PPB}$ & 10 & & $05 / 26 / 88$ & & $05 / 17 / 88$ & & $05 / 17 / 88$ & 15 \\
\hline FPOTASS & PPB & .100 & & $05 / 26 / 88$ & 2190 & $05 / 17 / 88$ & 1640 & $05 / 17 / 88$ & 1990 \\
\hline
\end{tabular}


TABLE E.5. (contd)

\begin{tabular}{|c|c|c|c|c|c|c|c|c|c|}
\hline \multicolumn{2}{|c|}{ CONST I TUENT } & $\begin{array}{l}\text { CONTRACIUAL } \\
\text { DETECTION }\end{array}$ & $\begin{array}{l}\text { DRINKING } \\
\text { WATER }\end{array}$ & SAMPLE & \multirow[b]{2}{*}{$3-1-10$} & \multicolumn{2}{|l|}{ SAMPLE } & SAMPLE & \multirow[b]{2}{*}{$3-1-12$} \\
\hline NAME & UNITS & LIMIT & STANDARO & DATE & & DATE & $3-1-11$ & DATE & \\
\hline$\cdots$ & $\cdots \cdots$ & $\cdots$ & - & $-\cdots$ & $\cdots \cdots$ & $\ldots . . . .$. & ...... & ......... & $\cdots$ \\
\hline FSOD I UM & PPB & 200 & & $05 / 26 / 88$ & 9310 & $05 / 17 / 88$ & 9580 & $05 / 17 / 88$ & .12500 \\
\hline FSTRONT & PPB & 10 & & $05 / 26 / 88$ & 101 & $05 / 17 / 88$ & 102 & $05 / 17 / 88$ & 104 \\
\hline FZINC & PPB & 5 & & $05 / 26 / 88$ & 8 & $05 / 17 / 88$ & & $05 / 17 / 88$ & \\
\hline IRON & PPB & 50 & & $05 / 26 / 88$ & 126 & $05 / 17 / 88$ & & $05 / 17 / 88$ & \\
\hline MAGNES & PPB & 0 & & $05 / 26 / 88$ & 4920 & $05 / 17 / 88$ & 4700 & $05 / 17 / 88$ & $4880^{\circ}$ \\
\hline NITRATE & PPB & 500 & 45000 & $05 / 26 / 88$ & 4410 & $\begin{array}{l}01 / 07 / 88 \\
01 / 13 / 88\end{array}$ & $\begin{array}{l}3590 \\
2290\end{array}$ & $05 / 17 / 88$ & 1910 \\
\hline & & & & & NR & $\begin{array}{l}01 / 75 / 88 \\
01 / 20 / 88\end{array}$ & $\begin{array}{l}2290 \\
2770\end{array}$ & & $\begin{array}{l}\text { NR } \\
\text { KR }\end{array}$ \\
\hline NITRATE & PPB & 500 & 45000 & & NR & $01 / 28 / 88$ & 2400 & & NR \\
\hline & & & & & NR & $02 / 05 / 88$ & 2000 & & NR \\
\hline & & & & & NR & $02 / 11 / 88$ & 3040 & & NR \\
\hline & & & & & NR & $02 / 18 / 88$ & 1880 & & NR \\
\hline & & & & & NR & $02 / 26 / 88$ & 1990 & & NR \\
\hline & & & & & NR & $03 / 03 / 88$ & 2420 & & NR \\
\hline & & & & & MR & $03 / 10 / \varepsilon 8$ & 3840 & & NR \\
\hline & & & & & NR & $03 / 17 / 88$ & 1450 & & NR \\
\hline & & & & & NR & $03 / 25 / 88$ & 1390 & & HR \\
\hline & & & & & NR & $03 / 31 / 88$ & 1970 & & NR \\
\hline & & & & & NR & $04 / 08 / 88$ & 2290 & & NR \\
\hline & & & & & NR & $04 / 15 / 88$ & 2520 & & NR \\
\hline & & & & & NR & $04 / 21 / 88$ & 2080 & & NR \\
\hline & & & & & NR & $04 / 28 / 88$ & 1010 & & NR \\
\hline & & & & & NR & $05 / 05 / 88$ & 1220 & & NR \\
\hline & & & & & NR & $05 / 12 / 88$ & 2480 & & NR \\
\hline & & & & & NR & $05 / 17 / 88$ & 1570 & & NR \\
\hline & & & & & NR & $05 / 26 / 88$ & 1700 & & NR \\
\hline & & & & & NR & $06 / 02 / 88$ & 1520 & & NR \\
\hline & & & & & NR & $06 / 08 / 88$ & 1270 & & NR \\
\hline & & & & & NR & $06 / 16 / 88$ & 2190 & & NR \\
\hline & & & & & NR & $06 / 23 / 88$ & 1630 & & NR \\
\hline PHFIELD & & 0.10 & & $05 / 26 / 88$ & 7.20 & $01 / 07 / 88$ & 8 & $05 / 17 / 88$ & 7.50 \\
\hline & & & & & NR & $01 / 13 / 88$ & 7.40 & & NR \\
\hline & & & & & NR & $01 / 20 / 88$ & 7.50 & & NR \\
\hline & & & & & NR & $01 / 28 / 88$ & 7.40 & & NR \\
\hline & & & & & NR & $02 / 05 / 88$ & 7.30 & & NR \\
\hline & & & & & NR & $02 / 11 / 88$ & 7.90 & & NR \\
\hline & & & & & NR & $02 / 18 / 88$ & 7.70 & & NR \\
\hline & & & & & NR & $02 / 26 / 88$ & 8.10 & & NR \\
\hline & & & & & NR & $03 / 03 / 88$ & 7.90 & & NR \\
\hline & & & & & NR & $03 / 10 / 88$ & 7.70 & & NR \\
\hline & & & & & NR & $03 / 17 / 88$ & 8.10 & & NR \\
\hline & & & & & NR & $03 / 25 / 88$ & 7.70 & & NR \\
\hline & & & & & NR & $03 / 31 / 88$ & 7.60 & & NR \\
\hline & & & & & NR & $04 / 08 / 88$ & 7.40 & & NR \\
\hline & & & & & NR & $04 / 15 / 88$ & 7.70 & & NR \\
\hline
\end{tabular}


TABLE E.5. (contd)

\begin{tabular}{|c|c|c|c|c|c|c|c|c|c|}
\hline const t & UENT & $\begin{array}{l}\text { CONTRACTUAL } \\
\text { DETECTION }\end{array}$ & $\begin{array}{l}\text { DRINKING } \\
\text { WATER }\end{array}$ & SAMPLE & & SAMPLE & & SAMPLE & \\
\hline NAME & UNITS & LIMIT & SIANDARD & DATE & $3-1-10$ & DATE & $3-9-11$ & DATE & $3-1-12$ \\
\hline$\ldots \ldots$ & $\cdots-$ & - & - & - & $\cdots$ & $\ldots \ldots$ & $\ldots .$. & $\cdots \cdots$ & $-\cdots \cdots$ \\
\hline & & & & & $\begin{array}{l}\text { NR } \\
\text { NR } \\
\text { NR } \\
\text { NR } \\
\text { NR } \\
\text { NR } \\
\text { NR } \\
\text { NR } \\
\text { NR } \\
\text { NR }\end{array}$ & $\begin{array}{l}04 / 21 / 88 \\
04 / 28 / 88 \\
05 / 05 / 88 \\
05 / 12 / 88 \\
05 / 17 / 88 \\
05 / 26 / 88 \\
06 / 02 / 88 \\
06 / 08 / 88 \\
06 / 16 / 88 \\
06 / 23 / 88\end{array}$ & $\begin{array}{l}7.40 \\
7.40 \\
7.90 \\
7.20 \\
7.50 \\
7.50 \\
7.30 \\
7.30 \\
7.10 \\
5.80\end{array}$ & & $\begin{array}{l}\text { NR } \\
\text { NR } \\
\text { NR } \\
\text { NR } \\
\text { NR } \\
\text { NR } \\
\text { NR } \\
\text { NR } \\
\text { NR } \\
\text { NR }\end{array}$ \\
\hline POTASUM & $\mathrm{PPB}$ & 100 & & $05 / 26 / 88$ & 2300 & $05 / 17 / 88$ & 1560 & $05 / 17 / 88$ & 1940 \\
\hline RADIUM & $\mathrm{PCI} / \mathrm{L}$ & 1 & 5 & $05 / 26 / 88$ & $\begin{array}{ll} & 0.58 \\
<\quad & 0.203\end{array}$ & $05 / 17 / 88$ & & $05 / 17 / 88$ & \\
\hline SODIUM & PPB & 200 & & $05 / 26 / 88$ & 9490 & $05 / 17 / 38$ & 9220 & $05 / 17 / 88$ & 12900 \\
\hline STRONUM & PPB & 10 & & $05 / 26 / 88$ & 103 & $05 / 17 / 88$ & 95 & $05 / 17 / 88$ & 101 \\
\hline SULFATE & PPB & 500 & & $05 / 26 / 88$ & 18600 & $01 / 07 / 88$ & 15600 & $05 / 17 / 88$ & 19700 \\
\hline & & & & & NR & $01 / 13 / 88$ & 16200 & & NR \\
\hline & & & & & NR & $01 / 20 / 88$ & 15900 & & NR \\
\hline & & & & & NR & $01 / 28 / 88$ & 18100 & & NR \\
\hline & & & & & NR & $02 / 05 / 88$ & 17200 & & NR \\
\hline & & & & & NR & $02 / 11 / 88$ & 17900 & & NR \\
\hline & & & & & NR & $02 / 98 / 88$ & 18500 & & NR \\
\hline & & & & & NR & $02 / 26 / 88$ & 20400 & & NR \\
\hline & & & & & NR & $03 / 03 / 88$ & 17800 & & NR \\
\hline & & & & & NR & $03 / 10 / 88$ & 19300 & & NR \\
\hline & & & & & NR & $03 / 17 / 88$ & 20000 & & NR \\
\hline & & & & & NR & $03 / 25 / 88$ & 15600 & & NR \\
\hline & & & & & NR & $03 / 31 / 88$ & 16700 & & NR \\
\hline & & & & & NR & $04 / 08 / 88$ & 17500 & & NR \\
\hline & & & & & NR & $04 / 15 / 88$ & 17000 & & NR \\
\hline & & & & & NR & $04 / 21 / 88$ & 17500 & & NR \\
\hline & & & & & NR & $04 / 28 / 88$ & 17800 & & NR \\
\hline & & & & & NR & $05 / 05 / 88$ & 18700 & & NR \\
\hline & & & & & NR & $05 / 12 / 88$ & 20700 & & NR \\
\hline & & & & & NR & $05 / 17 / 88$ & 19800 & & NR \\
\hline & & & & & NR & $05 / 26 / 88$ & 19000 & & NR \\
\hline & & & & & NR & $06 / 02 / 88$ & 18700 & & NR \\
\hline & & & & & NR & $06 / 08 / 88$ & 17500 & & NR \\
\hline & & & & & NR & $06 / 16 / 88$ & 17000 & & NR \\
\hline & & & & & NR & $06 / 23 / 88$ & 15600 & & NR \\
\hline TC & PPB & 2000 & & $05 / 26 / 88$ & 16700 & $05 / 17 / 88$ & 16200 & $05 / 17 / 88$ & 17300 \\
\hline TOXLDL & PPB & 10 & & $05 / 26 / 88$ & & $05 / 17 / 88$ & 46.60 & $05 / 97 / 88$ & 41 \\
\hline u & $\mathrm{PCl} / \mathrm{L}$ & 0.50 & 600 & $02 / 03 / 8 B$ & 11.90 & & NR & $02 / 11 / 88$ & 51.40 \\
\hline & & & & $05 / 26 / 88$ & 20 & & NR & $05 / 17 / 88$ & 63.60 \\
\hline U-CHEM & $U G / L$ & 0.73 & & & NR & $01 / 07 / 88$ & 152 & & NR \\
\hline
\end{tabular}


IABLE E.5. (contd)

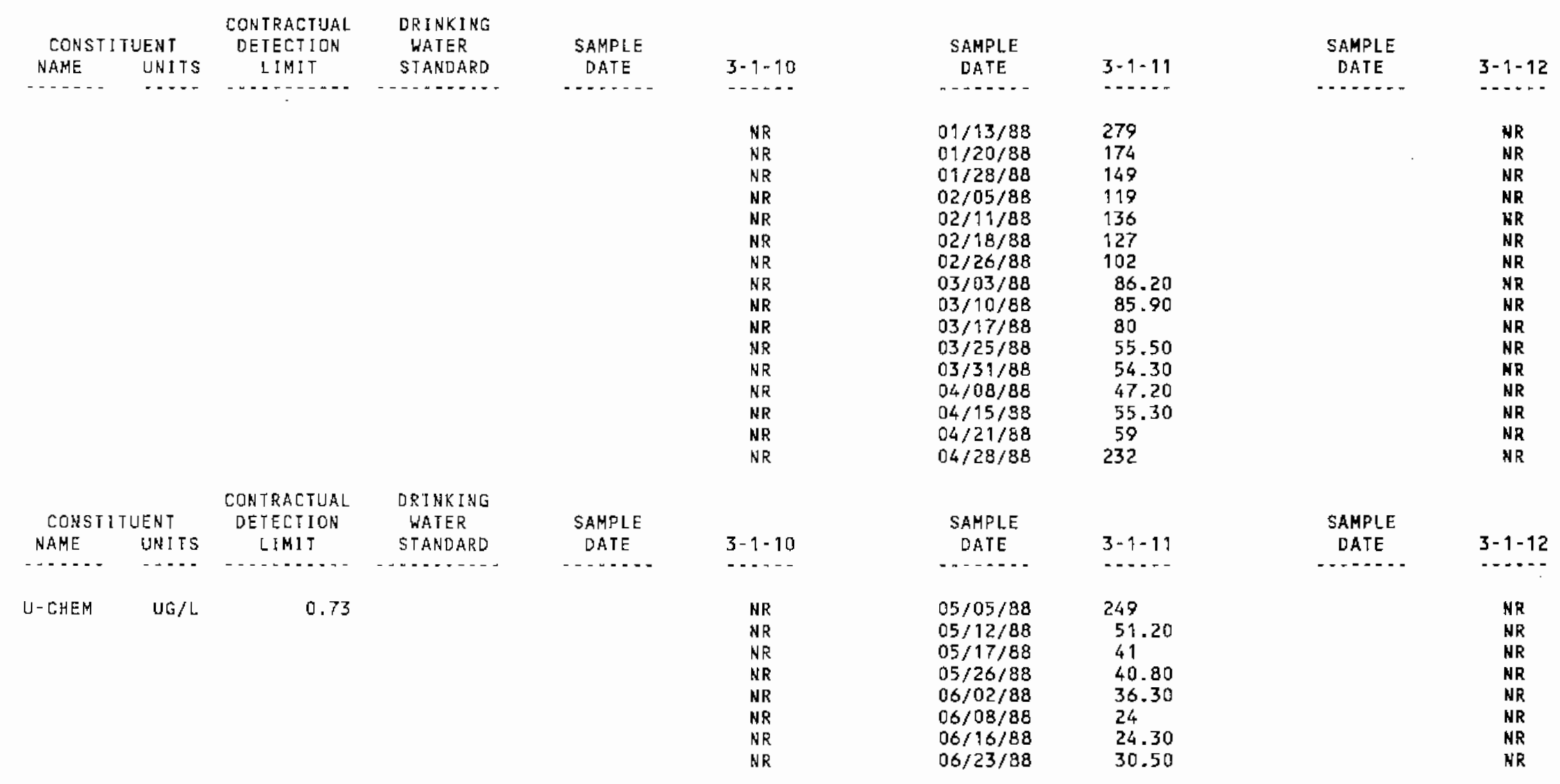


TABLE E.5. (contd)

\begin{tabular}{|c|c|c|c|c|c|c|c|c|c|}
\hline CONSTI & JENT & $\begin{array}{l}\text { CONTRACTUAL } \\
\text { DETECTION }\end{array}$ & $\begin{array}{l}\text { DRINKING } \\
\text { HATER }\end{array}$ & SAMPLE & & SAMPLE & & $\begin{array}{l}\text { SAMPLE } \\
\text { DAIE }\end{array}$ & \\
\hline $\begin{array}{l}\text { NAME } \\
\text { A...... }\end{array}$ & $\begin{array}{l}\text { UNITS } \\
\ldots . . .\end{array}$ & $\begin{array}{c}\text { LIMIT } \\
\ldots\end{array}$ & $\begin{array}{l}\text { SIANDARD } \\
-\end{array}$ & DATE & $\begin{array}{l}3-1-13 \\
-\ldots\end{array}$ & $\begin{array}{c}\text { DATE } \\
-\end{array}$ & $\begin{array}{l}3-1-14 \\
-\cdots-1\end{array}$ & DATE & $\begin{array}{l}3-1-15 \\
---\ldots\end{array}$ \\
\hline ALPHA & $\mathrm{PCI} / \mathrm{L}$ & 4 & 15 & $\begin{array}{l}05 / 27 / 88 \\
05 / 27 / 88\end{array}$ & $\begin{array}{cc} & 18.70+ \\
& 3.11\} \\
& 19.80+ \\
< & 3.31\}\end{array}$ & $05 / 26 / 88$ & $\begin{array}{l}22.30+ \\
3.393 \\
\mathrm{NR}\end{array}$ & $05 / 26 / 88$ & $\begin{array}{l}8.80 \\
2.46\} \\
N R\end{array}$ \\
\hline BARIUM & $P P B$ & 6 & 1000 & $05 / 27 / 88$ & 25 & $05 / 26 / 88$ & 20 & $05 / 26 / 88$ & 40 \\
\hline BETA & $\mathrm{PC} 1 / \mathrm{L}$ & 8 & 50 & $\begin{array}{l}05 / 27 / 88 \\
05 / 27 / 88\end{array}$ & $\left\{\begin{array}{l}11.10 \\
3.95\} \\
\{\quad 9.53 \\
\{\quad 3.90\}\end{array}\right.$ & $05 / 26 / 88$ & $\begin{array}{l}9.38 \\
3.86\} \\
\text { NR }\end{array}$ & $05 / 26 / 88$ & $\begin{array}{l}12.20 \\
4.283 \\
\text { NR }\end{array}$ \\
\hline $\begin{array}{l}\text { CALCIUM } \\
\text { CHLFORM }\end{array}$ & $\begin{array}{l}\text { PPB } \\
\text { PPB }\end{array}$ & $\begin{array}{l}50 \\
10\end{array}$ & 100 & $\begin{array}{l}05 / 27 / 88 \\
05 / 27 / 88\end{array}$ & $\begin{array}{r}28400 \\
13\end{array}$ & $\begin{array}{l}05 / 26 / 88 \\
05 / 26 / 88\end{array}$ & $\begin{array}{r}25600 \\
16\end{array}$ & $\begin{array}{l}05 / 26 / 88 \\
05 / 26 / 88\end{array}$ & 39400 \\
\hline CHLORID & PP8 & 500 & & $\begin{array}{l}05 / 27 / 88 \\
05 / 27 / 88 \\
05 / 27 / 88\end{array}$ & $\begin{array}{r}13 \\
16200 \\
16300\end{array}$ & $05 / 26 / 88$ & $\begin{array}{c}\text { NR } \\
12300_{\text {NR }}\end{array}$ & $05 / 26 / 88$ & $\begin{array}{c}\text { NR } \\
16300^{N R}\end{array}$ \\
\hline COLIFRM & MPN & 2 & 1 & $05 / 27 / 88$ & & $05 / 26 / 88$ & $2.20 *$ & $05 / 26 / 88$ & \\
\hline $\begin{array}{l}\text { CONDFLD } \\
\text { COPPER }\end{array}$ & $\begin{array}{l}\text { HMHO } \\
\mathrm{PPB}\end{array}$ & $\begin{array}{r}1 \\
10\end{array}$ & $(1300.0)$ & $\begin{array}{l}05 / 27 / 88 \\
05 / 27 / 88\end{array}$ & $\begin{array}{r}203 \\
13\end{array}$ & $\begin{array}{l}05 / 26 / 88 \\
05 / 26 / 88\end{array}$ & $\begin{array}{r}155 \\
12\end{array}$ & $\begin{array}{l}05 / 26 / 88 \\
05 / 26 / 88\end{array}$ & 312 \\
\hline FBARIUM & PPB & 6 & 1000 & $\begin{array}{l}05 / 27 / 88 \\
05 / 27 / 88\end{array}$ & $\begin{array}{l}25 \\
24\end{array}$ & $05 / 26 / 88$ & 20 & $05 / 26 / 88$ & $\begin{array}{l}43 \\
N R\end{array}$ \\
\hline FCALCIU & PPB & 50 & & $\begin{array}{l}05 / 27 / 88 \\
05 / 27 / 88\end{array}$ & $\begin{array}{l}28300 \\
25700\end{array}$ & $05 / 26 / 88$ & $\begin{array}{r}24900 \\
N R\end{array}$ & $05 / 26 / 88$ & $\begin{array}{r}41400 \\
N R\end{array}$ \\
\hline FCOPPER & $P P B$ & 10 & $(1300.0)$ & $\begin{array}{l}05 / 27 / 88 \\
05 / 27 / 88\end{array}$ & $\begin{array}{l}13 \\
16\end{array}$ & $05 / 26 / 88$ & $\begin{array}{l}10 \\
N R\end{array}$ & $05 / 26 / 88$ & $N R$ \\
\hline FMAGNES & PPB & 50 & & $\begin{array}{l}05 / 27 / 88 \\
05 / 27 / 88\end{array}$ & $\begin{array}{l}5990 \\
5640\end{array}$ & $05 / 26 / 88$ & 5020 & $05 / 26 / 88$ & 10400 \\
\hline FPOTASS & PPB & 100 & & $\begin{array}{l}05 / 27 / 88 \\
05 / 27 / 88\end{array}$ & $\begin{array}{l}3310 \\
3370\end{array}$ & $05 / 26 / 88$ & 2340 & $05 / 26 / 88$ & $\begin{array}{l}5500 \\
\text { NR }\end{array}$ \\
\hline FSOD I UM & PPB & 200 & & $\begin{array}{l}05 / 27 / 88 \\
05 / 27 / 88\end{array}$ & $\begin{array}{l}9920 \\
9580\end{array}$ & $05 / 26 / 88$ & $\begin{array}{r}8650 \\
\text { NR }\end{array}$ & $05 / 26 / 88$ & $\begin{array}{r}20200 \\
\text { NR }\end{array}$ \\
\hline FSTRONT & $\mathrm{PPB}$ & 10 & & $\begin{array}{l}05 / 27 / 88 \\
05 / 27 / 88\end{array}$ & $\begin{array}{l}113 \\
109\end{array}$ & $05 / 26 / 88$ & $\begin{array}{l}101 \\
\text { NR }\end{array}$ & $05 / 26 / 88$ & $\begin{array}{l}182 \\
N R\end{array}$ \\
\hline FVANAD I & PPB & 5 & & $\begin{array}{l}05 / 27 / 88 \\
05 / 27 / 88\end{array}$ & & $05 / 26 / 88$ & NR & $05 / 26 / 88$ & $\begin{array}{l}9 \\
\text { NR }\end{array}$ \\
\hline $\begin{array}{l}\text { IRON } \\
\text { MAGNES }\end{array}$ & $\begin{array}{l}P P B \\
P P B\end{array}$ & $\begin{array}{r}50 \\
0\end{array}$ & & $\begin{array}{l}05 / 27 / 88 \\
05 / 27 / 88\end{array}$ & $\begin{array}{r}53 \\
6090\end{array}$ & $\begin{array}{l}05 / 26 / 88 \\
05 / 26 / 88\end{array}$ & 5940 & $\begin{array}{l}05 / 26 / 88 \\
05 / 26 / 88\end{array}$ & $\begin{array}{r}55 \\
9870\end{array}$ \\
\hline NITRATE & PPB & 500 & 45000 & $\begin{array}{l}05 / 27 / 88 \\
05 / 27 / 88\end{array}$ & $\begin{array}{l}2690 \\
2750\end{array}$ & $05 / 26 / 88$ & $\begin{array}{r}2000 \\
N R\end{array}$ & $05 / 26 / 88$ & $\begin{array}{r}13300 \\
\text { NR }\end{array}$ \\
\hline PHFIELO & & 0.10 & & $05 / 27 / 88$ & 6.90 & $05 / 26 / 88$ & 7.70 & $05 / 26 / 88$ & 8 \\
\hline POTASUM & PPB & 100 & & $05 / 27 / 88$ & 3500 & $05 / 26 / 88$ & 2530 & $05 / 26 / 88$ & 5560 \\
\hline SOD iUM & PPB & 200 & & $05 / 27 / 88$ & 10300 & $05 / 26 / 88$ & 8810 & $05 / 26 / 88$ & 19800 \\
\hline SIRONUM & PPB & 10 & & $05 / 27 / 88$ & 120 & $05 / 26 / 88$ & 103 & $05 / 26 / 88$ & 178 \\
\hline SULFATE & PPB & 500 & & $\begin{array}{l}05 / 27 / 88 \\
05 / 27 / 88\end{array}$ & $\begin{array}{l}20000 \\
20100\end{array}$ & $05 / 26 / 88$ & $\begin{array}{l}19000 \\
\text { NR }\end{array}$ & $05 / 26 / 88$ & $\begin{array}{r}37300 \\
N R\end{array}$ \\
\hline $\begin{array}{l}\text { TC } \\
\text { TCXLDL. }\end{array}$ & $\begin{array}{l}\text { PPB } \\
\text { PPE }\end{array}$ & $\begin{array}{r}2000 \\
10\end{array}$ & & $\begin{array}{l}05 / 27 / 88 \\
05 / 27 / 88\end{array}$ & $\begin{array}{l}18400 \\
27.20\end{array}$ & $\begin{array}{l}05 / 26 / 88 \\
05 / 26 / 88\end{array}$ & $\begin{array}{l}16600 \\
29.60\end{array}$ & $\begin{array}{l}05 / 26 / 88 \\
05 / 26 / 88\end{array}$ & 25700 \\
\hline
\end{tabular}


TABLE E.5. (contd)

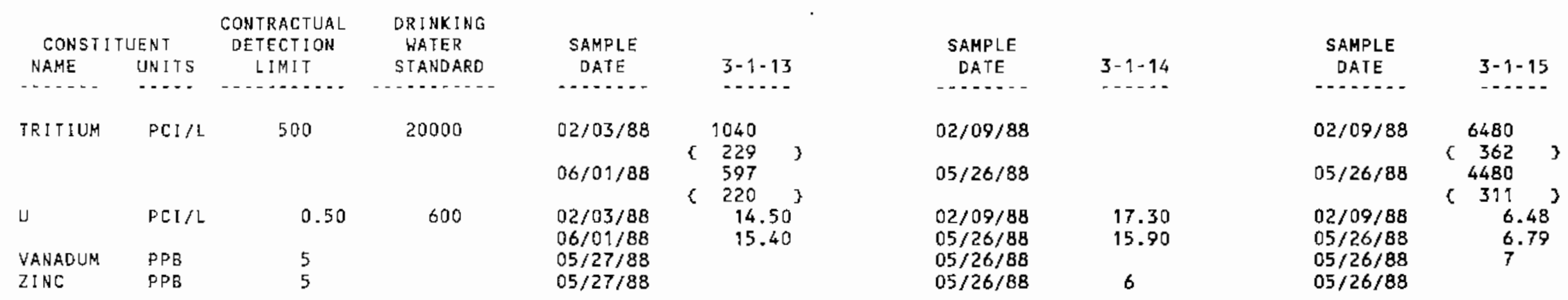


TABLE E. 5. (contd)

\begin{tabular}{|c|c|c|c|c|c|c|c|c|c|}
\hline CONSTI & UENT & $\begin{array}{l}\text { CONTRACTUAL } \\
\text { DETECTION }\end{array}$ & $\begin{array}{l}\text { ORINKING } \\
\text { WATER }\end{array}$ & SAMPLE & & SAMPLE & & SAMPLE & \\
\hline NAME & UNITS & LIMIT & STANOARD & DATE & $3-1-16 \mathrm{~A}$ & DATE & $3-1-16 B$ & DATE & $3-1-16 \mathrm{C}$ \\
\hline$\ldots$ & $\cdots-$ & , n & $\ldots$ & $\cdots$ & $\ldots \ldots$ & - & - n. & n. & $-\ldots, \ldots$ \\
\hline ALPHA & $\mathrm{PCI} / \mathrm{L}$ & 4 & 15 & $\begin{array}{l}05 / 18 / 88 \\
05 / 18 / 88\end{array}$ & $\begin{array}{c}16.50+ \\
2.87\} \\
\left\{\quad 17{ }^{+}\right. \\
\{\quad 3.02\}\end{array}$ & $05 / 18 / 88$ & $\begin{array}{l}1.53 \\
1.123 \\
\text { NR }\end{array}$ & $05 / 18 / 88$ & NR \\
\hline AMMONIU & PPB & 50 & & $\begin{array}{l}05 / 18 / 88 \\
05 / 18 / 88\end{array}$ & & $05 / 18 / 88$ & 125 & $05 / 18 / 88$ & $\begin{array}{l}150 \\
\text { NR }\end{array}$ \\
\hline BARIUM & PPB & 6 & 1000 & $05 / 18 / 88$ & $3 \hat{\imath}$ & $05 / 18 / 88$ & 50 & $05 / 98 / 88$ & 61 \\
\hline BETA & $\mathrm{PCI} / \mathrm{L}$ & 8 & 50 & $\begin{array}{l}05 / 18 / 88 \\
05 / 18 / 88\end{array}$ & $\begin{array}{ll} & 9.72 \\
\{\quad 3.83\} \\
\{\quad 7.64 \\
\{\quad 4.12\}\end{array}$ & $05 / 18 / 88$ & $\begin{array}{l}4.83 \\
3.533 \\
N R\end{array}$ & $05 / 18 / 88$ & $\begin{array}{ll} & 8.69 \\
\mathrm{~K} \quad & 4.12\} \\
& \mathrm{NR}\end{array}$ \\
\hline $\begin{array}{l}\text { CALCIUM } \\
\text { CHLFORM }\end{array}$ & $\begin{array}{l}\text { PPB } \\
\text { PPB }\end{array}$ & $\begin{array}{l}50 \\
10\end{array}$ & 100 & $\begin{array}{l}05 / 18 / 88 \\
05 / 18 / 88\end{array}$ & $\begin{array}{r}23700 \\
10\end{array}$ & $\begin{array}{l}05 / 18 / 88 \\
05 / 18 / 88\end{array}$ & 18200 & $\begin{array}{l}05 / 18 / 88 \\
05 / 18 / 88\end{array}$ & 13100 \\
\hline CHLORID & PPB & 500 & & $\begin{array}{l}05 / 18 / 88 \\
05 / 18 / 88 \\
05 / 18 / 88\end{array}$ & $\begin{array}{r}10 \\
15100 \\
15100\end{array}$ & $05 / 18 / 88$ & $\begin{array}{r}\text { NR } \\
11300^{N R}\end{array}$ & $05 / 18 / 88$ & $\begin{array}{r}\text { NR } \\
10900 \\
\text { NR }\end{array}$ \\
\hline CHROMUM & PPB & 10 & 50 & $05 / 18 / 88$ & & $05 / 18 / 88$ & 11 & $05 / 18 / 88$ & \\
\hline CONDFED & AMHO & 1 & & $05 / 18 / 88$ & 183 & $05 / 18 / 88$ & 303 & $05 / 18 / 88$ & 337 \\
\hline FBAR IUM & $\mathrm{PPB}$ & 6 & 1000 & $\begin{array}{l}05 / 18 / 88 \\
05 / 18 / 88\end{array}$ & $\begin{array}{l}31 \\
32\end{array}$ & $05 / 18 / 88$ & $\begin{array}{l}53 \\
N R\end{array}$ & $05 / 18 / 88$ & $\begin{array}{l}65 \\
\text { NR }\end{array}$ \\
\hline FCADMIU & PPB & 2 & 10 & $\begin{array}{l}05 / 18 / 88 \\
05 / 18 / 88\end{array}$ & & $05 / 18 / 88$ & $\stackrel{2}{N R}^{2}$ & $05 / 18 / 88$ & NR \\
\hline FCALCIU & PPB & 50 & & $\begin{array}{l}05 / 18 / 88 \\
05 / 18 / 88\end{array}$ & $\begin{array}{l}24500 \\
24700\end{array}$ & $05 / 18 / 88$ & $\begin{array}{r}19000 \\
\text { NR }\end{array}$ & $05 / 18 / 88$ & $\begin{array}{r}14900 \\
\text { NR }\end{array}$ \\
\hline FCOPPER & $P P B$ & 10 & $(1300.0)$ & $\begin{array}{l}05 / 18 / 88 \\
05 / 18 / 88\end{array}$ & $\begin{array}{l}10 \\
16\end{array}$ & $05 / 18 / 88$ & NR & $05 / 18 / 88$ & NR \\
\hline rIKON & $\mu \mu b$ & כن & & $\begin{array}{l}0 \overline{5} / 1 \overline{8} / 8 \overline{8} \\
05 / 18 / 88\end{array}$ & & $\overline{0} \bar{S} / \overline{8} \overline{8} / \overline{8} \overline{8}$ & $\begin{array}{l}\overline{8} \dot{4} \\
\text { NR }\end{array}$ & $\overline{0} 5 / 1 \bar{\delta} / \overline{8} \overline{8}$ & $\begin{array}{l}64 \\
\text { NR }\end{array}$ \\
\hline FLUORIO & PPB & 500 & 4000 & $\begin{array}{l}05 / 18 / 88 \\
05 / 18 / 88\end{array}$ & & $05 / 18 / 88$ & $\begin{array}{l}1530 \\
\text { NR }\end{array}$ & $05 / 18 / 88$ & $\begin{array}{l}1900 \\
\text { NR }\end{array}$ \\
\hline FMAGNES & PPB & 50 & & $\begin{array}{l}05 / 18 / 88 \\
05 / 18 / 88\end{array}$ & $\begin{array}{l}4600 \\
4760\end{array}$ & $05 / 18 / 88$ & $\begin{array}{l}6430 \\
\text { NR }\end{array}$ & $05 / 18 / 88$ & $\begin{array}{l}5680 \\
\text { NR }\end{array}$ \\
\hline FMANGAN & $P P B$ & 5 & & $\begin{array}{l}05 / 18 / 88 \\
05 / 18 / 88\end{array}$ & & $05 / 18 / 88$ & $\begin{array}{l}80 \\
\text { NR }\end{array}$ & $05 / 18 / 88$ & $\stackrel{55}{\text { NR }}$ \\
\hline FNICKEL & $P P B$ & 10 & & $\begin{array}{l}05 / 18 / 88 \\
05 / 18 / 88\end{array}$ & $\begin{array}{l}37 \\
39\end{array}$ & $05 / 18 / 88$ & NR & $05 / 18 / 88$ & NR \\
\hline FPOTASS & PPB & 100 & & $\begin{array}{l}05 / 18 / 88 \\
05 / 18 / 88\end{array}$ & $\begin{array}{l}2500 \\
2680\end{array}$ & $05 / 18 / 88$ & $\begin{array}{r}5270 \\
\text { NR }\end{array}$ & $05 / 18 / 88$ & $\begin{array}{l}6910 \\
\text { NR }\end{array}$ \\
\hline FSODIUM & PPB & 200 & & $\begin{array}{l}05 / 18 / 88 \\
05 / 18 / 88\end{array}$ & $\begin{array}{l}12000 \\
12600\end{array}$ & $05 / 18 / 88$ & $\begin{array}{r}53000 \\
\text { NR }\end{array}$ & $05 / 18 / 88$ & $\begin{array}{r}62100 \\
N R\end{array}$ \\
\hline FSTRONT & PPB & 10 & & $\begin{array}{l}05 / 18 / 88 \\
05 / 18 / 88\end{array}$ & $\begin{array}{l}104 \\
108\end{array}$ & $05 / 18 / 88$ & $\begin{array}{c}106 \\
\text { NR }\end{array}$ & $05 / 18 / 88$ & 91 \\
\hline$F Z I N C$ & PPB & 5 & & $\begin{array}{l}05 / 18 / 88 \\
05 / 18 / 88\end{array}$ & $\begin{array}{l}11 \\
12\end{array}$ & $05 / 18 / 88$ & NR & $05 / 18 / 88$ & NR \\
\hline IRON & PPB & 50 & & $05 / 18 / 88$ & & $05 / 18 / 88$ & 114 & $05 / 18 / 88$ & 63 \\
\hline
\end{tabular}


TABLE E.5. (contd)

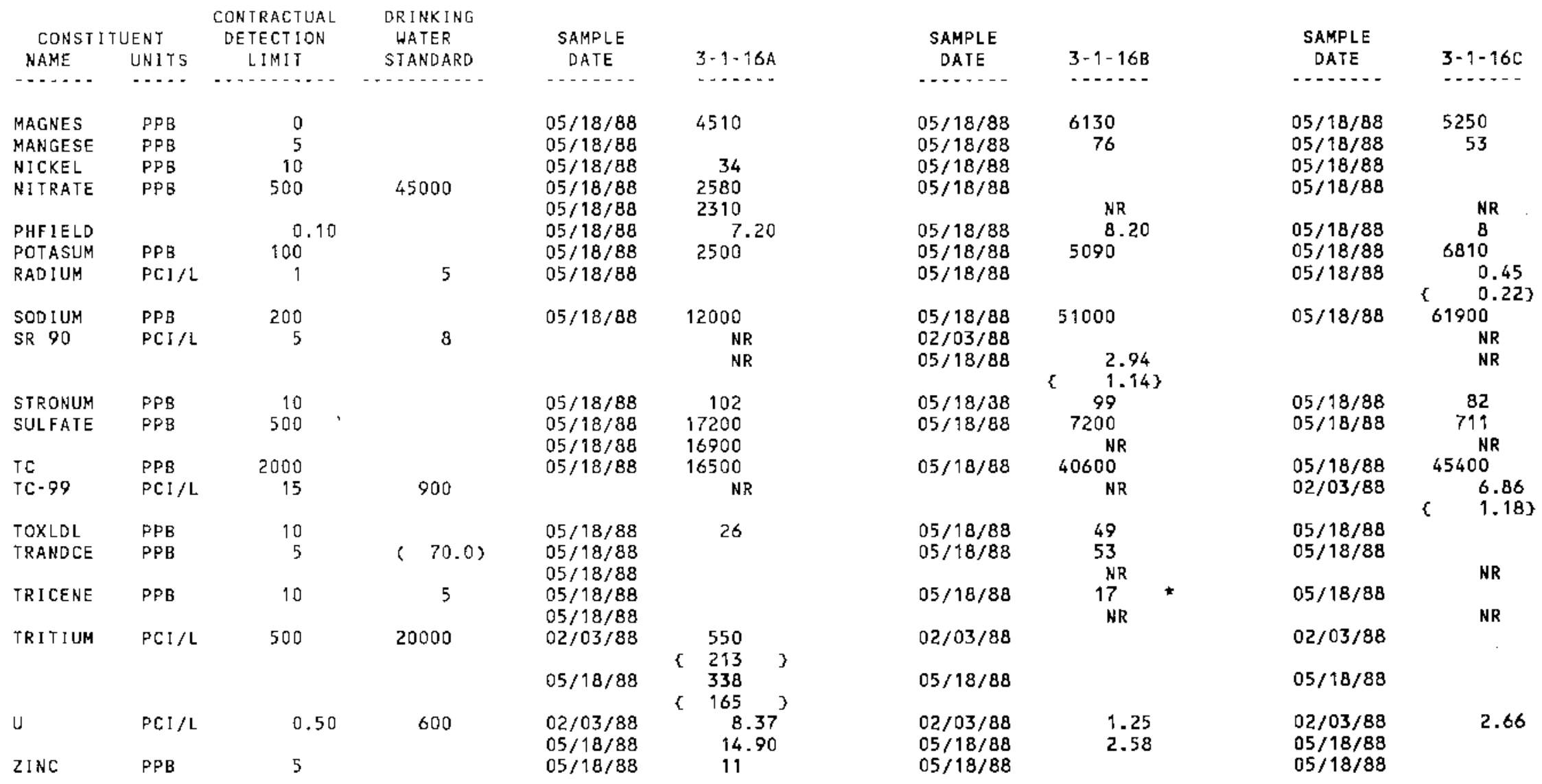


TABLE.E.5. (contd)

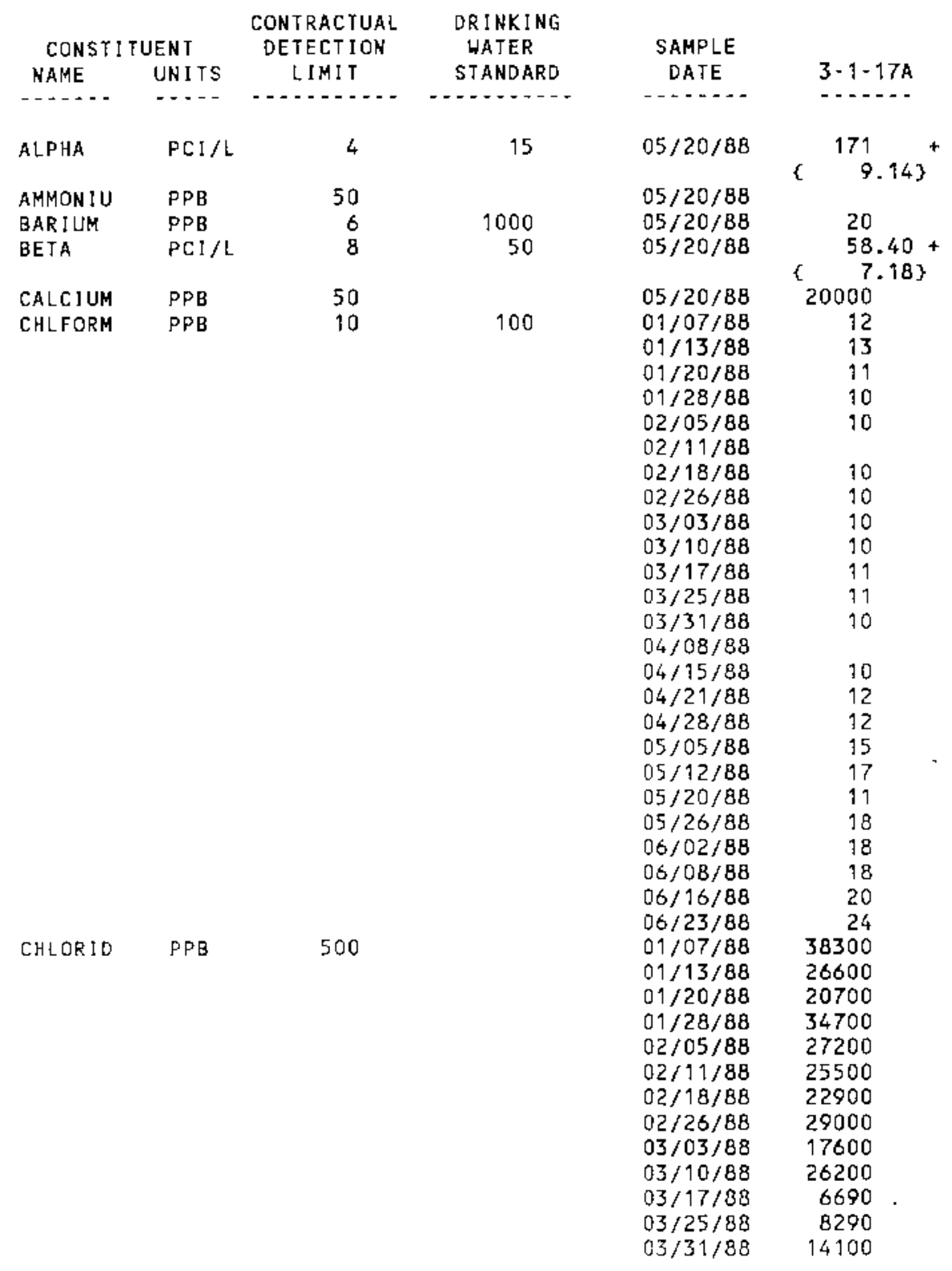

\begin{tabular}{|c|c|c|c|}
\hline $\begin{array}{l}\text { SAMPLE } \\
\text { DATE }\end{array}$ & $3-1-17 B$ & $\begin{array}{l}\text { SAMPLE } \\
\text { DATE }\end{array}$ & $3-1-17 c$ \\
\hline - - - - - - & 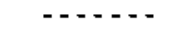 & - & - \\
\hline $05 / 20 / 88$ & & $05 / 20 / 88$ & \\
\hline $\begin{array}{l}05 / 20 / 88 \\
05 / 20 / 88 \\
05 / 20 / 88\end{array}$ & $\begin{array}{l}105 \\
68 \\
11.10 \\
\times \quad 4.113\end{array}$ & $\begin{array}{l}05 / 20 / 88 \\
05 / 20 / 88 \\
05 / 20 / 88\end{array}$ & $\begin{array}{c}155 \\
80 \\
12.60 \\
\{\quad 4.37\}\end{array}$ \\
\hline \multirow[t]{25}{*}{$\begin{array}{l}05 / 20 / 88 \\
05 / 20 / 88\end{array}$} & 18800 & $\begin{array}{l}05 / 20 / 88 \\
05 / 20 / 88\end{array}$ & 10200 \\
\hline & NR & & NR \\
\hline & NR & & NR \\
\hline & NR & & NR \\
\hline & NR & & NR \\
\hline & NR & & NR \\
\hline & NR & & NR \\
\hline & NR & & NR \\
\hline & NR & & NR \\
\hline & NR & & NR \\
\hline & NR & & NR \\
\hline & NR & & NR \\
\hline & NR & & NR \\
\hline & NR & & NR \\
\hline & NR & & NR \\
\hline & NR & & NR \\
\hline & NR & & NR \\
\hline & NR & & NR \\
\hline & NR & & NR \\
\hline & NR & & NR \\
\hline & NR & & NR \\
\hline & NR & & NR \\
\hline & NR & & NR \\
\hline & NR & & NR \\
\hline & NR & & NR \\
\hline \multirow[t]{13}{*}{$05 / 20 / 88$} & 10600 & $05 / 20 / 88$ & 11700 \\
\hline & NR & & NR \\
\hline & NR & & NR \\
\hline & NR & & NR \\
\hline & NR & & NR \\
\hline & NR & & NR \\
\hline & NR & & NR \\
\hline & NR & & $\mathrm{NR}$ \\
\hline & NR & & NR \\
\hline & NR & & NR \\
\hline & NR & & NR \\
\hline & NR & & NR \\
\hline & NR & & NR \\
\hline
\end{tabular}


TABLE E.5. (contd)

\begin{tabular}{|c|c|c|c|c|c|c|c|c|c|}
\hline CONST I & UEN T & $\begin{array}{l}\text { CONTRACTUAL } \\
\text { DETECTION }\end{array}$ & $\begin{array}{l}\text { DRINKING } \\
\text { WATER }\end{array}$ & SAMPLE & & SAMPLE & & SAMPLE & \\
\hline NAME & UNITS & L. JMI T & STANDARD & DATE & $3-1-17 A$ & DATE & $3-1-17 b$ & DATE & $3-1-17 c$ \\
\hline$\cdots \cdots--$ & $-\cdots$ & - - - - - & - - - - - - & - - - - - - & 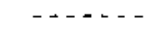 & $\cdots$ & $\cdots \cdots$ & $\cdots$ & $\cdots$ \\
\hline & & & & $04 / 08 / 88$ & 8550 & & NR & & NR \\
\hline & & & & $04 / 15 / 88$ & 9280 & & NR & & NR \\
\hline & & & & $04 / 21 / 88$ & 5230 & & NR & & NR \\
\hline CHLORID & PPB & 500 & & $04 / 28 / 88$ & 14800 & & NR & & NR \\
\hline & & & & $05 / 05 / 88$ & 27800 & & NR & & NR \\
\hline & & & & $05 / 12 / 88$ & 10800 & & NR & & NR \\
\hline & & & & $05 / 20 / 88$ & 6730 & & NR & & NR \\
\hline & & & & $05 / 26 / 88$ & 8030 & & NR & & NR \\
\hline & & & & $06 / 02 / 88$ & 6560 & & NR & & NR \\
\hline & & & & $06 / 08 / 88$ & 10500 & & NR & & NR \\
\hline & & & & $06 / 16 / 88$ & 19500 & & NR & & NR \\
\hline & & & & $06 / 23 / 88$ & 19500 & & NR & & NR \\
\hline $60-60$ & $\mathrm{PCI} / \mathrm{L}$ & 22.50 & 100 & & $\mathrm{NR}$ & $02 / 03 / 88$ & 6.42 & & NR \\
\hline & & & & & NR & $05 / 20 / E B$ & $5.24\}$ & & NR \\
\hline CONDFLD & АМно & 1 & & $01 / 07 / 88$ & & $05 / 20 / 88$ & 370 & $05 / 20 / 88$ & 396 \\
\hline & & & & $01 / 13 / 88$ & 205 & 每 & NR & $03 / 20 / 00$ & NR \\
\hline & & & & $01 / 20 / 88$ & 210 & & NR & & NR \\
\hline & & & & $01 / 28 / 88$ & 260 & & NR & & NR \\
\hline & & & & $02 / 05 / 88$ & 211 & & NR & & NR \\
\hline & & & & $02 / 11 / 88$ & 161 & & NR & & NR \\
\hline & & & & $02 / 18 / 88$ & 205 & & NR & & NR \\
\hline & & & & $02 / 26 / 88$ & 187 & & NR & & NR \\
\hline & & & & $03 / 03 / 88$ & 175 & & NR & & NR \\
\hline & & & & $03 / 10 / 88$ & 183 & & NR & & NR \\
\hline & & & & $03 / 17 / 88$ & 168 & & NR & & NR \\
\hline & & & & $03 / 25 / 88$ & 169 & & NR & & NR \\
\hline & & & & $03 / 31 / 88$ & 166 & & NR & & MR \\
\hline & & & & $04 / 08 / 88$ & 187 & & NR & & NR \\
\hline & & & & $04 / 15 / 88$ & 181 & & NR & & NR \\
\hline & & & & $04 / 21 / 88$ & 231 & & NR & & NR \\
\hline & & & & $04 / 28 / 88$ & 230 & & NR & & NR \\
\hline & & & & $05 / 05 / 88$ & 270 & & NR & & NR \\
\hline & & & & $05 / 12 / 88$ & 239 & & NR & & NR \\
\hline & & & & $05 / 20 / 88$ & 191 & & NR & & NR \\
\hline & & & & $05 / 26 / 88$ & 156 & & NR & & NR \\
\hline & & & & $06 / 02 / 88$ & 159 & & NR & & NR \\
\hline & & & & $06 / 08 / 88$ & 162 & & NR & & HR \\
\hline & & & & $06 / 16 / 88$ & 198 & & NR & & NR \\
\hline & & & & $06 / 23 / 88$ & 165 & & NR & & NR \\
\hline FBARIUM & PPB & 6 & 1000 & $05 / 20 / 88$ & 20 & $05 / 20 / 88$ & 69 & $05 / 20 / 88$ & 80 \\
\hline FCALCIU & PPB & 50 & & $05 / 20 / 88$ & 20000 & $05 / 20 / 88$ & 19200 & $05 / 20 / 88$ & 10900 \\
\hline FIRON & PPB & 50 & & $05 / 20 / 88$ & & $05 / 20 / 88$ & 139 & $05 / 20 / 88$ & 71 \\
\hline FLUOR ID & PPB & 500 & 4000 & $01 / 07 / 88$ & & $05 / 20 / 88$ & 1180 & $05 / 20 / 88$ & 1780 \\
\hline & & & & $01 / 13 / 88$ & 537 & & NR & & NR \\
\hline
\end{tabular}


TABLE E.5. (contd)

\begin{tabular}{|c|c|c|c|c|c|c|c|c|c|}
\hline \multicolumn{2}{|c|}{ CONSTI TUENT } & \multirow{2}{*}{$\begin{array}{c}\text { CONTRACTUAL } \\
\text { DETECTION } \\
\text { LIMIT }\end{array}$} & \multirow{2}{*}{$\begin{array}{l}\text { DRINKING } \\
\text { WATER } \\
\text { STANDARD }\end{array}$} & \multirow{2}{*}{$\begin{array}{l}\text { SAMPLE } \\
\text { DATE }\end{array}$} & \multirow[b]{2}{*}{$3-1-17 \mathrm{~A}$} & SAMPLE & & SAMPLE & \multirow{2}{*}{$3-1-17 c$} \\
\hline NAME & UN I TS & & & & & DATE & $3-1-17 B$ & DATE & \\
\hline , & ........ & - & $\cdots$ & 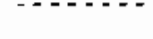 & 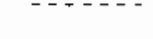 & (n) & $-+-1,--$ & 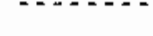 & $\cdots$ \\
\hline & & & & $01 / 20 / 88$ & & & NR & & NR \\
\hline & & & & $01 / 28 / 88$ & 522 & & NR & & NR \\
\hline & & & & $02 / 05 / 88$ & & & NR & & NR \\
\hline & & & & $02 / 11 / 88$ & & & NR & & NR \\
\hline & & & & $02 / 18 / 88$ & & & NR & & NR \\
\hline & & & & $02 / 26 / 88$ & & & NR & & $\mathrm{NR}$ \\
\hline & & & & $03 / 03 / 88$ & & & NR & & NR \\
\hline FLUORID & PPB & 500 & 4000 & $03 / 10 / 88$ & & & NR & & $\mathrm{NR}$ \\
\hline & & & & $03 / 17 / 88$ & & & $N R$ & & NR \\
\hline & & . & & $03 / 25 / 88$ & & & $N R$ & & NR \\
\hline & & & & $03 / 31 / 88$ & & & NR & & NR \\
\hline & & & & $94 / 08 / 88$ & & & NR & & KR \\
\hline & & & & $04 / 15 / 88$ & & & NR & & NR \\
\hline & & & & $04 / 21 / 88$ & & & NR & & NR \\
\hline & & & & $04 / 28 / 88$ & & & NR & & NR \\
\hline & & & & $05 / 05 / 88$ & & & NR & & NR \\
\hline & & & & $05 / 12 / 88$ & & & NR & & NR \\
\hline & & & & $05 / 20 / 88$ & & & NR & & NR \\
\hline & & & & $05 / 26 / 88$ & & & NR & & NR \\
\hline & & & & $06 / 02 / 88$ & & & NR & & NR \\
\hline & & & & $06 / 08 / 88$ & & & NR & & NR \\
\hline & & & & $06 / 16 / 88$ & & & NR & & NR \\
\hline & & & & $06 / 23 / 88$ & & & NR & & NR \\
\hline FMAGNES & PPB & 50 & & $05 / 20 / 88$ & 4190 & $05 / 20 / 88$ & 6980 & $05 / 20 / 88$ & 4810 \\
\hline FMANGAN & PPB & 5 & & $05 / 20 / 88$ & & $05 / 20 / 88$ & 66 & $05 / 20 / 88$ & 23 \\
\hline FPOTASS & PPB & 100 & & $05 / 20 / 88$ & 1770 & $05 / 20 / 88$ & 6120 & $05 / 20 / 88$ & 10300 \\
\hline F SUU I UM & טיtr & ¿̇uิÛ & & $05 / 20 / 80$ & 10400 & $05 / 20 / 80$ & 51200 & $05 / 20 / 80$ & 65900 \\
\hline FSTRONT & PPB & 10 & & $05 / 20 / 88$ & 95 & $05 / 20 / 88$ & 110 & $05 / 20 / 88$ & 74 \\
\hline IRON & PPB & 50 & & $05 / 20 / 88$ & & $05 / 20 / 88$ & 159 & $05 / 20 / 88$ & 85 \\
\hline MAGNES & PPB & 0 & & $05 / 20 / 88$ & 4270 & $05 / 20 / 88$ & 6940 & $05 / 20 / 88$ & 4630 \\
\hline MANGESE & PPB & 5 & & $05 / 20 / 88$ & & $05 / 20 / 88$ & 66 & $05 / 20 / 88$ & 23 \\
\hline NITRATE & PPB & 500 & 45000 & $01 / 07 / 88$ & 2330 & $05 / 20 / 88$ & & $05 / 20 / 88$ & 581 \\
\hline & & & & $01 / 13 / 88$ & 2950 & & NR & & NR \\
\hline & & & & $01 / 20 / 88$ & 2790 & & $N R$ & & NR \\
\hline & & & & $01 / 28 / 88$ & 2930 & & NR & & NR \\
\hline & & & & $02 / 05 / 88$ & 2580 & & NR & & NR \\
\hline & & & & $02 / 11 / 88$ & 2370 & & NR & & $\mathrm{NR}$ \\
\hline & & & & $02 / 18 / 88$ & 2490 & & NR & & NR \\
\hline & & & & $02 / 26 / 88$ & 2320 & & NR & & $N R$ \\
\hline & & & & $03 / 03 / 88$ & 2160 & & NR & & NR \\
\hline & & & & $03 / 10 / 88$ & 2460 & & NR & & NR \\
\hline & & & & $03 / 17 / 88$ & & & NR & & NR \\
\hline & & & & $03 / 25 / 88$ & 1340 & & NR & & NR \\
\hline & & & & $03 / 31 / 88$ & 1450 & & $\mathrm{NR}$ & & NR \\
\hline & & & & $04 / 08 / 38$ & 1940 & & $N R$ & & NR \\
\hline
\end{tabular}


TABLE E. 5. (contd)

\begin{tabular}{|c|c|c|c|c|c|c|c|c|c|}
\hline \multirow{2}{*}{\multicolumn{2}{|c|}{ CONSTITUENT }} & \multirow{3}{*}{$\begin{array}{l}\text { CONTRACTUAL } \\
\text { DETECTION } \\
\text { LIMIT }\end{array}$} & \multirow{3}{*}{$\begin{array}{l}\text { DRINKING } \\
\text { WATER } \\
\text { STANDARD }\end{array}$} & \multirow[b]{2}{*}{ SAMPLE } & \multirow[b]{3}{*}{$3-1-17 \mathrm{~A}$} & \multirow{2}{*}{ SAMPLE } & \multirow[b]{3}{*}{$3-1-17 B$} & \multirow{3}{*}{$\begin{array}{l}\text { SAMPLE } \\
\text { DATE }\end{array}$} & \multirow[b]{3}{*}{$3+1-17 c$} \\
\hline & & & & & & & & & \\
\hline NAME & UNITS & & & DATE & & DATE & & & \\
\hline & & & & $04 / 15 / 88$ & 1740 & & NR & & NR \\
\hline & & & & $04 / 21 / 88$ & 1840 & & NR & & NR \\
\hline & & & & $04 / 28 / 88$ & 1050 & & NR & & NR \\
\hline & & & & $0.5 / 05 / 88$ & 1220 & & NR & & NR \\
\hline & & & & $05 / 12 / 88$ & 1860 & & NR & & NR \\
\hline & & & & $05 / 20 / 88$ & 1650 & & NR & & NR \\
\hline & & & & $05 / 26 / 88$ & 1740 & & NR & & NR \\
\hline & & & & $06 / 02 / 88$ & 2340 & & NR & & NR \\
\hline & & & & $06 / 08 / 88$ & 2180 & & NR & & NR \\
\hline & & & & $06 / 16 / 88$ & 1940 & & $\mathrm{NR}$ & & NR \\
\hline & & & & $06 / 23 / 88$ & 1870 & & NR & & NR \\
\hline PHFIELD & & 0.10 & & $01 / 07 / 88$ & 7.40 & $05 / 20 / 88$ & 7.90 & $05 / 20 / 88$ & 8.20 \\
\hline & & & & $01 / 13 / 88$ & 7.30 & & NR & & NR \\
\hline & & & & $01 / 20 / 88$ & 7.10 & & NR & & NR \\
\hline & & & & $01 / 28 / 88$ & 7.70 & & NR & & NR \\
\hline & & & & $02 / 05 / 88$ & 7.10 & & NR & & NR \\
\hline & & & & $02 / 11 / 88$ & 7.50 & & NR & & NR \\
\hline & & & & $02 / 18 / 88$ & 7.40 & & NR & & NR \\
\hline & & & & $02 / 26 / 88$ & 7.70 & & $\mathrm{NR}$ & & NR \\
\hline & & & & $03 / 03 / 88$ & 7.60 & & $\mathrm{NR}$ & & NR \\
\hline & & & & $03 / 10 / 88$ & 7.50 & & NR & & NR \\
\hline & & & & $03 / 17 / 88$ & 7.60 & & NR & & NR \\
\hline & & & & $03 / 25 / 88$ & 7.20 & & NR & & NR \\
\hline & & & & $03 / 31 / 88$ & 7.30 & & NR & & NR \\
\hline & & & & $04 / 08 / 88$ & 7 & & NR & & NR \\
\hline & & & & $04 / 15 / 88$ & 7.40 & & NR & & NR \\
\hline & & & & $04 / 21 / 88$ & 7.60 & & NR & & NR \\
\hline & & & & $04 / 28 / 88$ & 7.30 & & NR & & NR \\
\hline & & & & $05 / 05 / 88$ & 7.50 & & NR & & NR \\
\hline & & & & $05 / 12 / 88$ & 7 & & NR & & NR \\
\hline & & & & $05 / 20 / 88$ & 7.10 & & NR & & NR \\
\hline & & & & $05 / 26 / 88$ & 7.30 & & $M R$ & & NR \\
\hline & & & & $06 / 02 / 88$ & 7.10 & & NR & & NR \\
\hline & & & & $06 / 08 / 88$ & 7.90 & & NR & & NR \\
\hline & & & & $06 / 16 / 88$ & 7.10 & & NR & & NR \\
\hline & & & & $06 / 23 / 88$ & 7.40 & & $N R$ & & NR \\
\hline POTASUM & PPB & 100 & & $05 / 20 / 88$ & $1830^{\circ}$ & $05 / 20 / 88$ & 6150 & $05 / 20 / 88$ & 9960 \\
\hline SODIUM & PPB & 200 & & $05 / 20 / 88$ & 10800 & $05 / 20 / 88$ & 52000 & $05 / 20 / 88$ & 67100 \\
\hline SR 90 & $\mathrm{PCl} / \mathrm{L}$ & 5 & 8 & & NR & $02 / 03 / 88$ & & & NR \\
\hline & & & & & NR & $05 / 20 / 88$ & 5.28 & & $\mathrm{NR}$ \\
\hline & РPB & 10 & & $05 / 20 / 88$ & 96 & $05 / 20 / 88$ & $(108$ & $05 / 20 / 88$ & 72 \\
\hline SULFATE & PPB & 500 & & $01 / 07 / 88$ & 15200 & $05 / 20 / 88$ & 1110 & $05 / 20 / 88$ & \\
\hline & & & & $01 / 13 / 88$ & 15200 & & NR & & NR \\
\hline & & & & $01 / 20 / 88$ & 14200 & & $\mathrm{NR}$ & & $\mathrm{NR}$ \\
\hline
\end{tabular}


TABLE E. .5 . (contd)

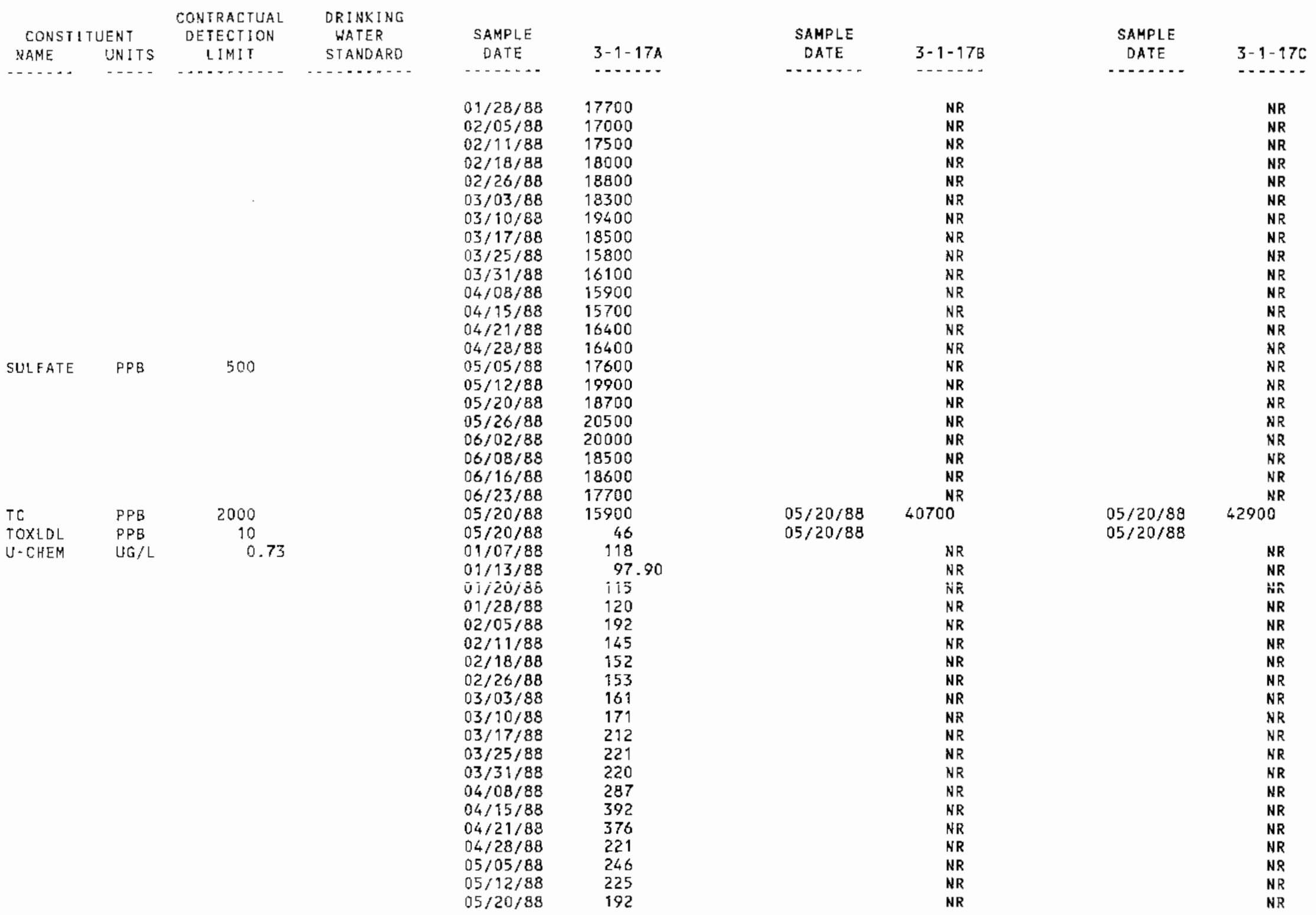


TABLE E.5. (contd)

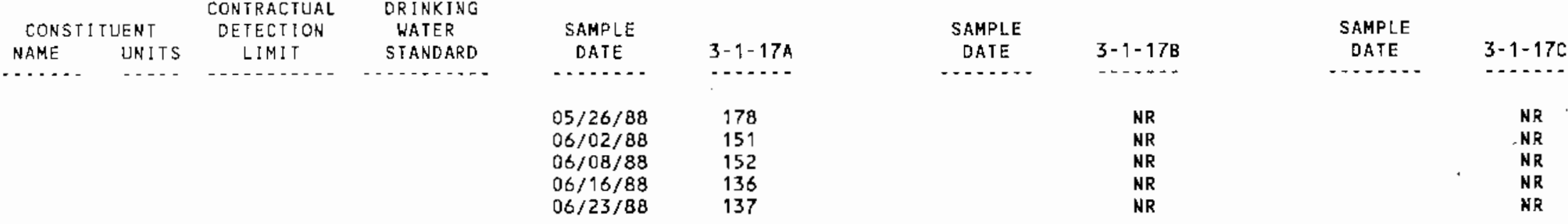


TABLE E.5. (contd)

\begin{tabular}{|c|c|c|}
\hline \multirow{2}{*}{\multicolumn{2}{|c|}{ CONST I TUENT }} & $\begin{array}{c}\text { CONTRACTUAL } \\
\text { DETECTION }\end{array}$ \\
\hline & & DETECTION \\
\hline NAME & UNITS & LIMIT \\
\hline - & $\cdots-$ & $-\cdots$ \\
\hline ALPHA & $P C I / L$ & 4 \\
\hline AMMONIU & PPE & 50 \\
\hline ARSENIC & $P P B$ & 5 \\
\hline BARIUM & PPB & 6 \\
\hline BETA & $\mathrm{PCI} / \mathrm{L}$ & 8 \\
\hline CALCIUM & PPB & 50 \\
\hline CHLORID & $\mathrm{PPB}$ & 500 \\
\hline CHROMUN & PPB & 10 \\
\hline CONDFLD & \#MHO & 1 \\
\hline FARSENI & PPB & 5 \\
\hline FBARIUM & PPB & 6 \\
\hline FCALCIU & PPB & 50 \\
\hline FIRON & PPB & 50 \\
\hline FLUORIO & PPB & 500 \\
\hline FMAGNES & $P P B$ & 50 \\
\hline F MANGAN & PPB & 5 \\
\hline FPOTASS & PPB & 100 \\
\hline FSODIUM & PPB & 200 \\
\hline FSTRONT & $\mathrm{PPQ}$ & 10 \\
\hline FVANADI & $\mathrm{PPB}$ & 5 \\
\hline$I R O N$ & PPB & 50 \\
\hline MAGNES & $P P B$ & 0 \\
\hline MANGESE & $\mathrm{PPB}$ & 5 \\
\hline N I CXKE! & PPR & 10 \\
\hline NITRATE & PPB & 500 \\
\hline PHF IELD & & 0.10 \\
\hline POTASUM & PPB & 100 \\
\hline RAO ILM & $\mathrm{PCI} / \mathrm{L}$ & 1 \\
\hline SOD I UM & PPB & 200 \\
\hline SR 90 & $\mathrm{PCI} / \mathrm{L}$ & 5 \\
\hline STRONUM & PPB & ס \\
\hline SULFATE & PPB & 500 \\
\hline TC & PPB & 2000 \\
\hline VANADUM & PPB & 5 \\
\hline
\end{tabular}

DRINKING

WATER SIANDARD

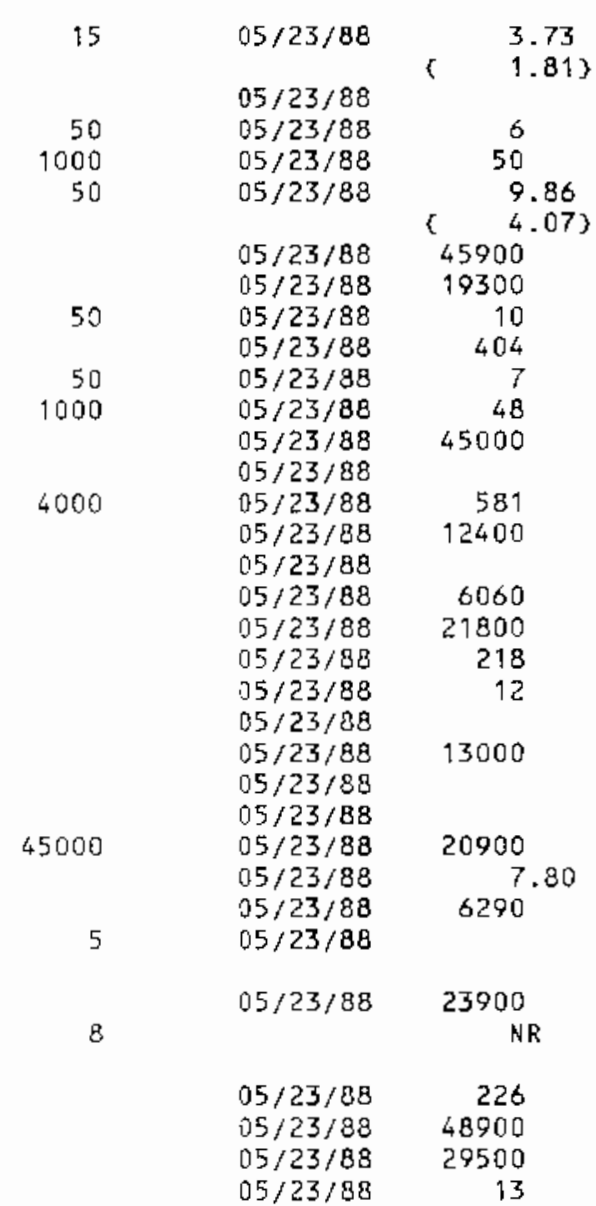

SAMPLE DATE $3-1-18 B$

$05 / 23 / 88$

$\begin{array}{lll}05 / 23 / 88 & & 90 \\ 05 / 23 / 88 & & \\ 05 / 23 / 88 & & 40 \\ 05 / 23 / 88 & & 9.40 \\ & \text { \& } & 3.973\end{array}$

05/23/88 ${ }_{11600} 3.97$

$05 / 23 / 88 \quad 11100$

$05 / 23 / 88$

$05 / 23 / 88$

$05 / 23 / 88$

$05 / 23 / 88$

$05 / 23 / 98$

$05 / 23 / 8 \mathrm{~B}$

$05 / 23 / 88$

$05 / 23 / 88$

$05 / 23 / 88$

$05 / 23 / 88$

$05 / 23 / 88$

$05 / 23 / 88$

$05 / 23 / 88$

$05 / 23 / 88$

$05 / 23 / 88$

$05 / 23 / 88$

$05 / 23 / 88$

$05 / 23 / 88$

$05 / 23 / 88$

$05 / 23 / 88$

$05 / 23 / 8 B$

$5 / 23 / 88$

$05 / 23 / 88$

$05 / 23 / 88$

$05 / 23 / 88$

$05 / 23 / 88$

$05 / 23 / 88$

$05 / 23 / 88$

6360

62000
77

222

5180

40

8.20

6080

65500

0.93

< $\quad 0.763$

42400
SAMPLE

DATE 3-1-18C

$05 / 23 / 88$

$05 / 23 / 88 \quad 115$

$05 / 23 / 88$

$05 / 23 / 88 \quad 8.81$

$05 / 23 / 88$ ( 3.85 \}

$\begin{array}{ll}05 / 23 / 88 & 12100 \\ 05 / 23 / 88 & 11000\end{array}$

$05 / 23 / 88 \quad 31$

$05 / 23 / 8 B \quad 359$

$05 / 23 / 88$
$05 / 23 / 88$

$05 / 23 / 88 \quad 13000$

$05 / 23 / 88 \quad 74$

$05 / 23 / 88 \quad 1790$

$05 / 23 / 88 \quad 5380$

$05 / 23 / 88 \quad 46$

$05 / 23 / 8 B \quad 6460$

$\begin{array}{rr}05 / 23 / 88 & 66800 \\ 05 / 23 / 88 & 80\end{array}$

$05 / 23 / 88$

$05 / 23 / 88$

$05 / 23 / 88$

$05 / 23 / 88$

$\overline{0} \overline{2} / \overline{\angle 3} / 86$

5310

48
13

$05 / 23 / 88$

$05 / 23 / 88$

8.10

$05 / 23 / 88$

NR

$05 / 23 / 88$

$05 / 23 / 88$

$05 / 23 / 88$

80 
TABLE E.5. (contd)

\begin{tabular}{|c|c|c|c|c|c|c|c|c|c|}
\hline \multicolumn{2}{|c|}{ CONSTI TUENT } & \multirow{2}{*}{$\begin{array}{l}\text { CONTRACTUAL } \\
\text { DETECTION } \\
\text { LIMIT }\end{array}$} & \multirow{2}{*}{$\begin{array}{l}\text { DRINKING } \\
\text { WATER } \\
\text { STANDARD }\end{array}$} & \multirow{2}{*}{$\begin{array}{l}\text { SAMPLE } \\
\text { DATE }\end{array}$} & \multirow[b]{2}{*}{$3-1-19$} & SAMPLE & \multirow[b]{2}{*}{$3-2-1$} & SAMPLE & \multirow[b]{2}{*}{$3-3-7$} \\
\hline NAME & UNITS & & & & & DATE & & DATE & \\
\hline$\cdots$ & $\cdots$ & - & - & $\cdots$ & $\cdots \cdots$ & $\ldots$ & $\cdots$ & $-\ldots+\infty$ & $\cdots$ \\
\hline ALPHA & $\mathrm{PCI} / \mathrm{L}$ & 4 & 15 & $05 / 17 / 88$ & $135+$ & $05 / 24 / 88$ & 7.93 & $05 / 27 / 88$ & 12 \\
\hline & & & & & $8.20\}$ & & 2.023 & & $2.82)$ \\
\hline AMMONIU & PPB & 50 & & $05 / 17 / 88$ & 60 & $05 / 24 / 8 B$ & & $05 / 27 / 88$ & \\
\hline BARIUM & PPB & 6 & 1000 & $05 / 17 / 88$ & 20 & $05 / 24 / 8 B$ & 43 & $05 / 27 / 88$ & 43 \\
\hline BETA & $\mathrm{PCI} / \mathrm{L}$ & 8 & 50 & $05 / 17 / 88$ & $\begin{array}{c}44.10 \\
\{\quad 6.39\}\end{array}$ & $05 / 24 / 88$ & $\begin{array}{ll} & 8.50 \\
\{\quad 3.96\}\end{array}$ & $05 / 27 / 88$ & $\begin{array}{r}12.40 \\
\{\quad 4.36\}\end{array}$ \\
\hline CALCIUM & PPB & 50 & & $05 / 17 / 88$ & 19900 & $05 / 24 / 88$ & 21300 & $05 / 27 / 88$ & 42400 \\
\hline CHLFORM & PPB & 10 & 100 & $01 / 07 / 88$ & 12 & $05 / 24 / 88$ & & $05 / 27 / 88$ & \\
\hline & & & & $01 / 13 / 88$ & 12 & & NR & & NR \\
\hline & & & & $01 / 20 / 88$ & 11 & & NR & & NR \\
\hline & & & & $01 / 28 / 88$ & 10 & & NR & & NR \\
\hline & & & & $02 / 05 / 88$ & 10 & & NR & & NR \\
\hline & & & & $02 / 18 / 88$ & 10 & & NR & & NR \\
\hline & & & & $02 / 26 / 88$ & 10 & & NR & & NR \\
\hline & & & & $03 / 03 / 88$ & 10 & & NR & & NR \\
\hline & & & & $03 / 10 / 88$ & 10 & & NR & & NR \\
\hline & & & & $03 / 17 / 88$ & 11 & & $N R$ & & NR \\
\hline & & & & $03 / 25 / 88$ & 11 & & NR & & NR \\
\hline & & & & $03 / 31 / 88$ & 11 & & NR & & NR \\
\hline & & & & $04 / 08 / 88$ & 10 & & NR & & NR \\
\hline & & & & $04 / 15 / 88$ & 10 & & NR & & NR \\
\hline & & & & $04 / 21 / 88$ & 12 & & NR & & NR. \\
\hline & & & & $04 / 28 / 88$ & 13 & & NR & & NR \\
\hline & & & & $05 / 05 / 88$ & 14 & & NR & & NR \\
\hline & & & & $05 / 12 / 88$ & 16 & & NR & & NR \\
\hline & & & & $05 / 17 / 88$ & 14 & & NR & & NR \\
\hline & & & & $05 / 26 / 88$ & 17 & & NR & & NR \\
\hline & & & & $06 / 02 / 88$ & 17 & & NR & & NR \\
\hline & & & & $06 / 08 / 88$ & 17 & & NR & & NR \\
\hline & & & & $06 / 16 / 88$ & 17 & & NR & & NR \\
\hline & & & & $06 / 23 / 88$ & 19 & & NR & & NR \\
\hline & & & & $06 / 29 / 88$ & 21 & & NR & & NR \\
\hline CHLORID & PPB & 500 & & $01 / 07 / 88$ & 33800 & $05 / 24 / 88$ & 11200 & $05 / 27 / 88$ & 17400 \\
\hline & & & & $01 / 13 / 88$ & 32700 & & NR & & NR \\
\hline & & & & $01 / 20 / 88$ & 22900 & & NR & & NR \\
\hline & & & & $01 / 28 / 88$ & 39000 & & NR & & NR \\
\hline & & & & $02 / 05 / 88$ & 25000 & & NR & & NR \\
\hline & & & & $02 / 18 / 88$ & 18300 & & NR & & NR \\
\hline & & & & $02 / 26 / 88$ & 26900 & & NR & & AR \\
\hline & & & & $03 / 03 / 88$ & 19600 & & NR & & NR \\
\hline & & & & $03 / 10 / 88$ & 19900 & & NR & & NR \\
\hline & & & & $03 / 17 / 88$ & 9580 & & NR & & NR \\
\hline & & & & $03 / 25 / 88$ & 15300 & & $N R$ & & NR \\
\hline & & & & $03 / 31 / 88$ & 11900 & & $\mathrm{NR}$ & & NR \\
\hline & & & & $04 / 08 / 8 B$ & 8950 & & NR & & NR \\
\hline
\end{tabular}


TABLE E.5. (contd)

\begin{tabular}{|c|c|c|c|c|c|c|c|c|c|}
\hline \multicolumn{2}{|c|}{ CONST I TUENT } & $\begin{array}{l}\text { CONTRACTUAL } \\
\text { DETECTION }\end{array}$ & $\begin{array}{l}\text { DRINKING } \\
\text { WATER }\end{array}$ & SAMPLE & & SAMPLE & & SAMPLE & \\
\hline \multirow{5}{*}{$\begin{array}{l}\text { NAME } \\
-\ldots .\end{array}$} & UNITS & LIMIT & STANDARD & DATE & $3-1-19$ & DATE & $3-2-1$ & DATE & $3-3-7$ \\
\hline & $\cdots$ & $\ldots$ & $\ldots \ldots$ & $\ldots .$. & $-\ldots$ & - - - - - - & $\cdots$ & - & $\cdots$ \\
\hline & & & & $04 / 15 / 88$ & 8170 & & NR & & NR \\
\hline & & & & $04 / 21 / 88$ & 13200 & & NR & & NR \\
\hline & & & & $04 / 28 / 88$ & 8680 & & NR & & NR \\
\hline \multirow[t]{9}{*}{ CHLORID } & $P P B$ & 500 & & $05 / 05 / 88$ & 18300 & & NR & & NR \\
\hline & & & & $05 / 12 / 88$ & 17500 & & NR & & NR \\
\hline & & & & $05 / 17 / 88$ & 12300 & & NR & & NR \\
\hline & & & & $05 / 26 / 8 B$ & 7960 & & NR & & NR \\
\hline & & & & $06 / 02 / 88$ & 7430 & & NR & & NR \\
\hline & & & & $06 / 08 / 88$ & 20500 & & $\mathrm{NR}$ & & NR \\
\hline & & & & $06 / 16 / 88$ & 26800 & & NR & & NR \\
\hline & & & & $06 / 23 / 88$ & 16600 & & $\mathrm{kR}$ & & NR \\
\hline & & & & $06 / 29 / 88$ & 28100 & & NR & & NR \\
\hline COLIFRM & MPN & 2 & 1 & $05 / 17 / 88$ & & $05 / 24 / 88$ & $5.10 *$ & $05 / 27 / 88$ & \\
\hline \multirow[t]{25}{*}{ CONDFLD } & DMHO & 1 & & $01 / 07 / 88$ & & $05 / 24 / 88$ & 168 & $05 / 27 / 88$ & 396 \\
\hline & & & & $01 / 13 / 88$ & 233 & & NR & & NR \\
\hline & & & & $01 / 20 / 88$ & 225 & & NR & & NR \\
\hline & & & & $01 / 28 / 88$ & 244 & & NR & & NR \\
\hline & & & & $02 / 05 / 88$ & 209 & & NR & & NR \\
\hline & & & & $02 / 18 / 88$ & 198 & & NR & & NR \\
\hline & & & & $02 / 26 / 88$ & 181 & & NR & & NR \\
\hline & & & & $03 / 03 / 88$ & 184 & & NR & & NR \\
\hline & & & & $03 / 10 / 88$ & 205 & & NR & & NR \\
\hline & & & & $03 / 17 / 88$ & 198 & & NR & & NR \\
\hline & & & & $03 / 25 / 88$ & 189 & & NR & & $\mathrm{NR}$ \\
\hline & & & & $03 / 31 / 88$ & 161 & & NR & & NR \\
\hline & & & & $04 / 08 / 88$ & 312 & & NR & & NR \\
\hline & & & & $04 / i 5 / 88$ & 160 & & NiR & & NQR \\
\hline & & & & $04 / 21 / 88$ & 267 & & NR & & NR \\
\hline & & & & $04 / 28 / 88$ & 212 & & NR & & $\mathrm{NR}$ \\
\hline & & & & $05 / 05 / 88$ & 229 & & $\mathrm{NR}$ & & NR \\
\hline & & & & $05 / 12 / 88$ & 259 & & NR & & NR \\
\hline & & & & $05 / 17 / 88$ & 174 & & NR & & NR \\
\hline & & & & $05 / 26 / 88$ & 160 & & NR & & NR \\
\hline & & & & $06 / 02 / 88$ & 169 & & NR & & NR \\
\hline & & & & $06 / 08 / 88$ & 193 & & NR & & NR \\
\hline & & & & $06 / 16 / 88$ & 210 & & $N R$ & & NR \\
\hline & & & & $06 / 23 / B B$ & 185 & & NR & & NR \\
\hline & & & & $06 / 29 / 8 B$ & 238 & & NR & & NR \\
\hline FBAR I UM & $P P B$ & 6 & 1000 & $05 / 17 / 88$ & 20 & $05 / 24 / 88$ & 46 & $05 / 27 / 88$ & 36 \\
\hline FCALCIU & $P P B$ & 50 & & $05 / 17 / 88$ & 20800 & $05 / 24 / 88$ & 22300 & $05 / 27 / 88$ & 40100 \\
\hline \multirow{5}{*}{ FLUORID } & PPB & 500 & 4000 & $01 / 07 / 88$ & & $05 / 24 / 88$ & 570 & $05 / 27 / 88$ & \\
\hline & & & & $01 / 13 / 8 B$ & 526 & & NR & & NR \\
\hline & & & & $01 / 20 / 88$ & & & NR & & $\mathrm{NR}$ \\
\hline & & & & $01 / 28 / 38$ & & & NR & & $N R$ \\
\hline & & & & $02 / 05 / 88$ & & & NR & & NR \\
\hline
\end{tabular}


TABLE E.5. (contd)

\begin{tabular}{|c|c|c|c|c|c|c|c|c|c|}
\hline \multicolumn{2}{|c|}{ CONSTIFUENT } & $\begin{array}{l}\text { CONTRACTUAL } \\
\text { DETECT!ON }\end{array}$ & $\begin{array}{l}\text { DR INKING } \\
\text { WATER }\end{array}$ & SAMPLE & & SAMPLE & & SAMPLE & \\
\hline NAME & UNITS & LIMIT & STANDARD & DATE & $3-1-19$ & DATE & $3-2-1$ & DATE & $3-3-7$ \\
\hline$\cdots \cdots$ & $\cdots-$ & - & - & $\cdots$ & $\cdots+$ & $\cdots$ & $-\cdots$ & $\cdots$ & $-\cdots$ \\
\hline & & & & $02 / 18 / 88$ & & & NR & & NR \\
\hline & & & & $02 / 26 / 88$ & & & NR & & NR \\
\hline & & & & $03 / 03 / 88$ & & & NR & & NR \\
\hline & & & & $03 / 10 / 88$ & & & NR & & NR \\
\hline & & & & $03 / 17 / 88$ & & & NR & & KR \\
\hline & & & & $03 / 25 / 88$ & & & NR & & NR \\
\hline & & & & $03 / 31 / 88$ & & & NR & & NR \\
\hline FLUOR ID & PPB & 500 & 4000 & $04 / 08 / 88$ & & & NR & & NR \\
\hline & & & & $04 / 15 / 88$ & & & NR & & NR \\
\hline & & & & $04 / 21 / 88$ & & & NR & & NR \\
\hline & & & & $04 / 28 / 88$ & & & NR & & MR \\
\hline & & & & $05 / 05 / 88$ & & & NR & & NR \\
\hline & & & & $05 / 12 / 88$ & & & NR & & NR \\
\hline & & & & $05 / 17 / 88$ & & & NR & & NR \\
\hline & & & & $05 / 26 / 88$ & & & NR & & NR \\
\hline & & & & $06 / 02 / 88$ & & & NR & & NR \\
\hline & & & & $06 / 08 / 88$ & & & NR & & NR \\
\hline & & & & $06 / 16 / 88$ & & & NR & & NR \\
\hline & & & & $06 / 23 / 88$ & & & NR & & NR \\
\hline & & & & $06 / 29 / 88$ & & & NR & & HR \\
\hline FHAGNES & PPB & 50 & & $05 / 17 / 88$ & 4620 & $05 / 24 / 88$ & 4850 & $05 / 27 / 88$ & 8480 \\
\hline FNICXEL & PPB & 10 & & $05 / 17 / 88$ & 10 & $05 / 24 / 88$ & & $05 / 27 / 88$ & \\
\hline FPOTASS & PPB & 100 & & $05 / 17 / 88$ & 2150 & $05 / 24 / 88$ & 3140 & $05 / 27 / 88$ & 4170 \\
\hline FSOD IUH & PPB & 200 & & $05 / 17 / 88$ & 13400 & $05 / 24 / 88$ & 12900 & $05 / 27 / 88$ & 17200 \\
\hline FSTRONT & PPB & 10 & & $05 / 17 / 88$ & 100 & $05 / 24 / 88$ & 99 & $05 / 27 / 88$ & . 157 \\
\hline FVANADI & PPB & 5 & & $05 / 17 / 88$ & & $05 / 24 / 88$ & & $05 / 27 / 88$ & 8 \\
\hline I RON & PPB & 50 & & $05 / 97 / 88$ & 198 & $05 / 24 / 88$ & & $05 / 27 / 88$ & 96 \\
\hline MAGNES & PPB & 0 & & $05 / 17 / 88$ & 4400 & $05 / 24 / 88$ & 4520 & $05 / 27 / 88$ & 9270 \\
\hline MANGESE & $P P B$ & 5 & & $05 / 17 / 88$ & 7 & $05 / 24 / 88$ & & $05 / 27 / 88$ & \\
\hline NITRATE & PPB & 500 & 45000 & $01 / 07 / 88$ & 2280 & $05 / 24 / 88$ & 6730 & $05 / 27 / 88$ & 14600 \\
\hline & & & & $01 / 13 / 88$ & 2780 & & NR & & $\mathrm{NR}$ \\
\hline & & & & $01 / 20 / 88$ & 2690 & & NR & & NR \\
\hline & & & & $01 / 28 / 88$ & 3120 & & NR & & NR \\
\hline & & & & $02 / 05 / 88$ & 2300 & & NR & & NR \\
\hline & & & & $02 / 18 / 88$ & 2230 & & NR & & NR \\
\hline & & & & $02 / 26 / 88$ & 2290 & & NR & & $\mathrm{NR}$ \\
\hline & & & & $03 / 03 / 88$ & 2250 & & NR & & NR \\
\hline & & & & $03 / 10 / 88$ & 2770 & & NR & & NR \\
\hline & & & & $03 / 17 / 88$ & 901 & & NR & & NR \\
\hline & & & & $03 / 25 / 88$ & 1380 & & NR & & NR \\
\hline & & & & $03 / 31 / 88$ & 1500 & & NR & & NR \\
\hline & & & & $04 / 08 / 88$ & 2120 & & NR & & NR \\
\hline & & & & $04 / 15 / 88$ & 1840 & & NR & & NR \\
\hline & & & & $04 / 21 / 88$ & 1890 & & $N R$ & & NR \\
\hline & & & & $04 / 28 / 88$ & 1170 & & NR & & NR \\
\hline
\end{tabular}


TABLE E.5. (contd)

\begin{tabular}{|c|c|c|c|c|c|c|c|c|c|}
\hline CONSTI & UENT & $\begin{array}{l}\text { CONTRACTUAL } \\
\text { DETECTION }\end{array}$ & $\begin{array}{l}\text { DRINKING } \\
\text { HATER }\end{array}$ & SAMPLE & & SAMPLE & & SAMPLE & \\
\hline NAME & UNITS & LIMIT & STANDARD & DATE & $3-1-19$ & DATE & $3-2-1$ & DATE & $3-3-7$ \\
\hline$\ldots$ & $\cdots$ & $\ldots \ldots$ & $\ldots \ldots$ & $\cdots \cdots$ & $\cdots$ & $\ldots$ & $\cdots$ & $\cdots$ & $\cdots$ \\
\hline & & & & $05 / 05 / 88$ & 1350 & & NR & & NR \\
\hline & & & & $05 / 12 / 88$ & 1740 & & NR & & NR \\
\hline & & & & $05 / 17 / 88$ & 1470 & & NR & & NR \\
\hline & & & & $05 / 26 / 88$ & 1710 & & NR & & NR \\
\hline & & & & $06 / 02 / 88$ & 2110 & & NR & & NR \\
\hline & & & & $06 / 08 / 88$ & 2100 & & NR & & NR \\
\hline & & & & $06 / 16 / 88$ & 3100 & & NR & & NR \\
\hline & & & & $06 / 23 / 88$ & 1920 & & NR & & NR \\
\hline & & & & $06 / 29 / 88$ & 1660 & & NR & & NR \\
\hline PHEIELD & & 0.10 & & $01 / 07 / 88$ & 7.90 & $05 / 24 / 88$ & 6.70 & $05 / 27 / 88$ & 7.70 \\
\hline & & & & $01 / 13 / 88$ & 8.20 & & NR & & NR \\
\hline PHFIELD & & 0.10 & & $01 / 20 / 88$ & 7.50 & & $N R$ & & NR \\
\hline & & & & $01 / 28 / 88$ & 7.10 & & NR & & NR \\
\hline & & & & $02 / 05 / 88$ & 7.20 & & NR & & NR \\
\hline & & & & $02 / 18 / 88$ & 7.40 & & NR & & $N R$ \\
\hline & & & & $02 / 26 / 88$ & 7.70 & & NR & & NR \\
\hline & & & & $03 / 03 / 88$ & 7.70 & & NR & & NR \\
\hline & & & & $03 / 10 / 88$ & 7.60 & & $N R$ & & NR \\
\hline & & & & $03 / 97 / 88$ & 7.80 & & NR & & NR \\
\hline & & & & $03 / 25 / 88$ & 7.30 & & $N R$ & & NR \\
\hline & & & & $03 / 31 / 88$ & 7.10 & & NR & & NR \\
\hline & & & & $04 / 08 / 88$ & 8.50 & & NR & & NR \\
\hline & & & & $04 / 15 / 88$ & 7.60 & & $N R$ & & NR \\
\hline & & & & $04 / 21 / 88$ & 7.40 & & NR & & NR \\
\hline & & & & $04 / 28 / 88$ & 7.20 & & $N R$ & & NR \\
\hline & & & & $05 / 05 / 88$ & 7.50 & & NR & & NR \\
\hline & & & & $05 / 12 / 88$ & 6.80 & & NR & & N R \\
\hline & & & & $05 / 17 / 88$ & 7.20 & & NR & & NR \\
\hline & & & & $05 / 26 / 88$ & 7.40 & & NR & & NR \\
\hline & & & & $06 / 02 / 8 B$ & 7.20 & & $\mathrm{NR}$ & & NR \\
\hline & & & & $06 / 08 / 88$ & 7.90 & & NR & & NR \\
\hline & & & & $06 / 16 / 88$ & 7.20 & & NR & & NR \\
\hline & & & & $06 / 23 / 88$ & 7.50 & & NR & & NR \\
\hline & & & & $06 / 29 / 88$ & 7 & & NR & & NR \\
\hline POTASUM & $\mathrm{PPB}$ & 100 & & $05 / 17 / 88$ & 1920 & $05 / 24 / 88$ & 2830 & $05 / 27 / 88$ & 5120 \\
\hline SODIUM & PPB & 200 & & $05 / 17 / 88$ & 12400 & $05 / 24 / 88$ & 12000 & $05 / 27 / 88$ & 21000 \\
\hline STRONUM & PPB & 10 & & $05 / 17 / 88$ & 95 & $05 / 24 / 88$ & 93 & $05 / 27 / 88$ & 176 \\
\hline SULFATE & PPB & 500 & & $01 / 07 / 88$ & 14800 & $05 / 24 / 88$ & 14000 & $05 / 27 / 88$ & 42600 \\
\hline & & & & $01 / 13 / 88$ & 15200 & & NR & & NR \\
\hline & & & & $01 / 20 / 8 \mathrm{~B}$ & 14200 & & NR & & NR \\
\hline & & & & $01 / 28 / 88$ & 17300 & & NR & & NR \\
\hline & & & & $02 / 05 / 88$ & 16700 & & $N R$ & & NR \\
\hline & & & & $02 / 18 / 88$ & 18100 & & NR & & NR \\
\hline & & & & $02 / 26 / 8 B$ & 19200 & & $N R$ & & NR \\
\hline & & & & $03 / 03 / 88$ & 18300 & & $N R$ & & NR \\
\hline
\end{tabular}


TABLE E.5. (contd)

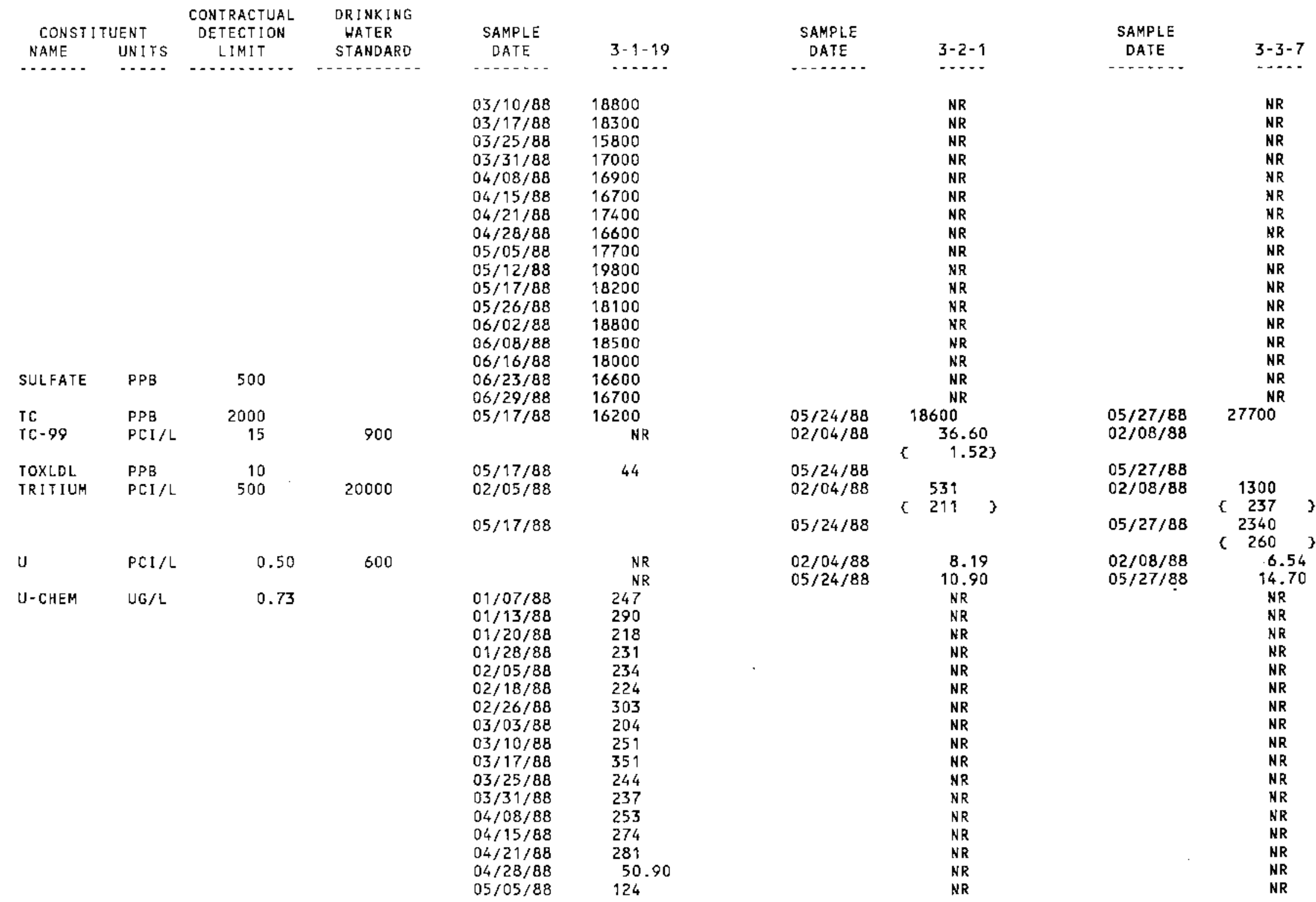


IABLE E.5. (contd)

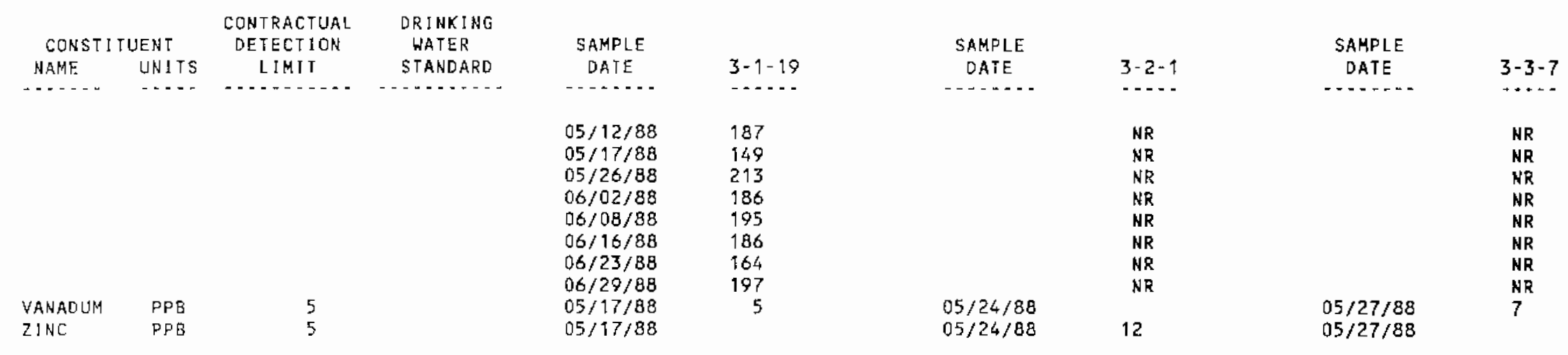


TABLE E.5. (contd)

\begin{tabular}{|c|c|c|c|c|c|c|c|c|c|}
\hline \multicolumn{2}{|c|}{ CONSTI TUENT } & $\begin{array}{l}\text { CONTRACTUAL } \\
\text { DETECTION }\end{array}$ & $\begin{array}{l}\text { DR I NKING } \\
\text { WATER }\end{array}$ & SAMPLE & & SAMPLE & & SAMP1E & \\
\hline NAME & UNITS & LIMIT & SIANDARD & DATE & $3-3-10$ & DATE & $3-4-1$ & DATE & $3 \cdot 4-7$ \\
\hline 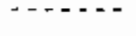 & $\cdots$ & - & - & $\ldots$ & $\cdots$ & $+\ldots$. & $\cdots$ & $\ldots . .$. & $\cdots$ \\
\hline ALPHA & $\mathrm{PCI} / \mathrm{L}$ & 4 & 15 & $05 / 09 / 88$ & $\begin{array}{l}14.60 \\
2.86\}\end{array}$ & $05 / 27 / 88$ & $\begin{array}{l}8.83 \\
2.323\end{array}$ & $05 / 16 / 88$ & $\begin{array}{r}22.20 \\
3.573\end{array}$ \\
\hline AMMONIU & PPB & 50 & & $05 / 09 / 88$ & 52 & $05 / 27 / 88$ & & $05 / 16 / 88$ & 38 \\
\hline BARIUM & PPB & 6 & 1000 & $05 / 09 / 88$ & 35 & $05 / 27 / 88$ & 34 & $05 / 16 / 88$ & 42 \\
\hline BETA & PCI/L & 8 & 50 & $05 / 09 / 88$ & $\begin{array}{r}11.80 \\
(\quad 4.21\}\end{array}$ & $05 / 27 / 88$ & $\begin{array}{l}8.29 \\
\mathrm{r} \quad 4.15\}\end{array}$ & $05 / 16 / 88$ & $\begin{array}{c}14.70 \\
C \quad 4.43\}\end{array}$ \\
\hline CALCIUM & PPB & 50 & & $05 / 09 / 88$ & 24000 & $05 / 27 / 88$ & 37300 & $05 / 16 / 88$ & 37900 \\
\hline CHLFORM & PPB & 10 & 100 & $05 / 09 / 88$ & 10 & $05 / 27 / 88$ & & $05 / 16 / 88$ & \\
\hline CHLORID & PPB & 500 & & $05 / 09 / 88$ & 9510 & $05 / 27 / 88$ & 9640 & $05 / 16 / 88$ & 11400 \\
\hline COLIFRH & MPN & 2 & 1 & $05 / 09 / 88$ & & $05 / 27 / 88$ & & $05 / 16 / 88$ & 9.20 \\
\hline CONDFLD & AMHO & 1 & & $05 / 09 / 88$ & 238 & $05 / 27 / 88$ & 317 & $05 / 16 / 88$ & 327 \\
\hline CYANIDE & PPB & 10 & & $05 / 09 / 88$ & & $05 / 27 / 88$ & 16 & $05 / 16 / 88$ & \\
\hline FBARIUM & PPB & 6 & 1000 & $05 / 09 / 88$ & 40 & $05 / 27 / 88$ & 34 & $05 / 16 / 88$ & 39 \\
\hline FCADMIU & $P P B$ & 2 & 10 & $05 / 09 / 88$ & & $05 / 27 / 88$ & 2 & $05 / 16 / 88$ & \\
\hline FCALCIU & $\mathrm{PPB}$ & 50 & & $05 / 09 / 88$ & 27800 & $05 / 27 / 88$ & 37600 & $05 / 16 / 88$ & 37000 \\
\hline FIRON & PPB & 50 & & $05 / 09 / 88$ & 95 & $05 / 27 / 88$ & & $05 / 16 / 88$ & \\
\hline FLUOR ID & PPB & 500 & 4000 & $05 / 09 / 88$ & 508 & $05 / 27 / 88$ & & $05 / 16 / 88$ & \\
\hline FMAGNES & PPB & 50 & & $05 / 09 / 88$ & 5890 & $05 / 27 / 88$ & 7570 & $05 / 16 / 88$ & 7250 \\
\hline FMANGAN & PPB & 5 & & $05 / 09 / 88$ & 10 & $05 / 27 / 88$ & & $05 / 16 / 88$ & \\
\hline FPOTASS & PPB & 100 & & $05 / 09 / 88$ & 3210 & $05 / 27 / 88$ & 4440 & $05 / 76 / 88$ & 4090 \\
\hline FSODIUM & PPB & 200 & & $05 / 09 / 88$ & 10600 & $05 / 27 / 88$ & 15700 & $05 / 16 / 88$ & 17100 \\
\hline FSTRONT & PPB & 10 & & $05 / 09 / 88$ & 137 & $05 / 27 / 88$ & 144 & $05 / 16 / 88$ & 160 \\
\hline FVANADI & PPB & 5 & & $05 / 09 / 88$ & & $05 / 27 / 88$ & 8 & $05 / 16 / 88$ & \\
\hline FZINC & PPB & 5 & & $05 / 09 / 88$ & 7 & $05 / 27 / 88$ & & $05 / 16 / 88$ & \\
\hline I RON & PPB & 50 & & $05 / 09 / 88$ & 225 & $05 / 27 / 88$ & 102 & $05 / 16 / 88$ & \\
\hline MAGNES & PPB & 0 & & $05 / 09 / 88$ & 5100 & $05 / 27 / 88$ & 7390 & $05 / 16 / 88$ & 7290 \\
\hline METHYCH & PPB & 5 & & $05 / 09 / 88$ & & $05 / 27 / 88$ & 13 & $05 / 16 / 88$ & \\
\hline NITRATE & PPB & 500 & 45000 & $05 / 09 / 88$ & 8630 & $05 / 27 / 88$ & 12200 & $05 / 16 / 88$ & 10900 \\
\hline PHFIELD & & 0.10 & & $05 / 09 / 88$ & 7.10 & $05 / 27 / 88$ & 7.60 & $05 / 16 / 88$ & 7.60 \\
\hline POTASUM & PPB & 100 & & $05 / 09 / 88$ & 2720 & $05 / 27 / 88$ & 4360 & $05 / 16 / 88$ & 4090 \\
\hline SODIUM & PPB & 200 & & $05 / 09 / 88$ & 9210 & $05 / 27 / 88$ & 15000 & $05 / 16 / 88$ & 17100 \\
\hline STRONUM & PPB & 10 & & $05 / 09 / 88$ & 120 & $05 / 27 / 88$ & 145 & $05 / 16 / 88$ & 160 \\
\hline SULFATE & $\mathrm{PPB}$ & 500 & & $05 / 09 / 88$ & 19500 & $05 / 27 / 88$ & 26900 & $05 / 16 / 88$ & 34600 \\
\hline TC & PPB & 2000 & & $05 / 09 / 88$ & 19200 & $05 / 27 / 88$ & 26800 & $05 / 16 / 88$ & 24900 \\
\hline TC- 99 & $\mathrm{PCI} / \mathrm{L}$ & 15 & 900 & & NR & & NR & $02 / 08 / 88$ & 9.27 \\
\hline & & & & & & & & & $1.23)$ \\
\hline TOXLDL & $P P B$ & 10 & & $05 / 09 / 88$ & 38 & $05 / 27 / 88$ & & $05 / 16 / 88$ & \\
\hline TRITIUM & $\mathrm{PCI} / \mathrm{L}$ & 500 & 20000 & $02 / 08 / 88$ & $\begin{array}{l}520 \\
212\end{array}$ & $02 / 11 / 88$ & 442 & $02 / 08 / 88$ & $\begin{array}{r}1490 \\
242\end{array}$ \\
\hline & & & & $05 / 09 / 88$ & 421 & $05 / 27 / 88$ & $\begin{array}{r}216 \\
537\end{array}$ & $05 / 16 / 88$ & $\left\{\begin{array}{r}242 \\
1550\end{array}\right.$ \\
\hline & & & & & $\{205\}$ & & 1207 & & C 201 \\
\hline$\downarrow$ & $\mathrm{PCI} / \mathrm{L}$ & 0.50 & 600 & $\begin{array}{l}02 / 08 / 88 \\
05 / 09 / 88\end{array}$ & $\begin{array}{l}27.80 \\
12.70\end{array}$ & $\begin{array}{l}02 / 11 / 88 \\
05 / 27 / 88\end{array}$ & $\begin{array}{r}9.13 \\
13.20\end{array}$ & $\begin{array}{l}02 / 08 / 88 \\
05 / 16 / 88\end{array}$ & $\begin{array}{l}33 \\
29.40\end{array}$ \\
\hline VANAD UM & PPB & 5 & & $05 / 09 / 88$ & & $05 / 27 / 88$ & 7 & $05 / 16 / 88$ & \\
\hline ZINC & PPE & 5 & & $05 / 09 / 88$ & 7 & $05 / 27 / 88$ & & $05 / 16 / 88$ & \\
\hline
\end{tabular}


TABLEE.5. (contd)

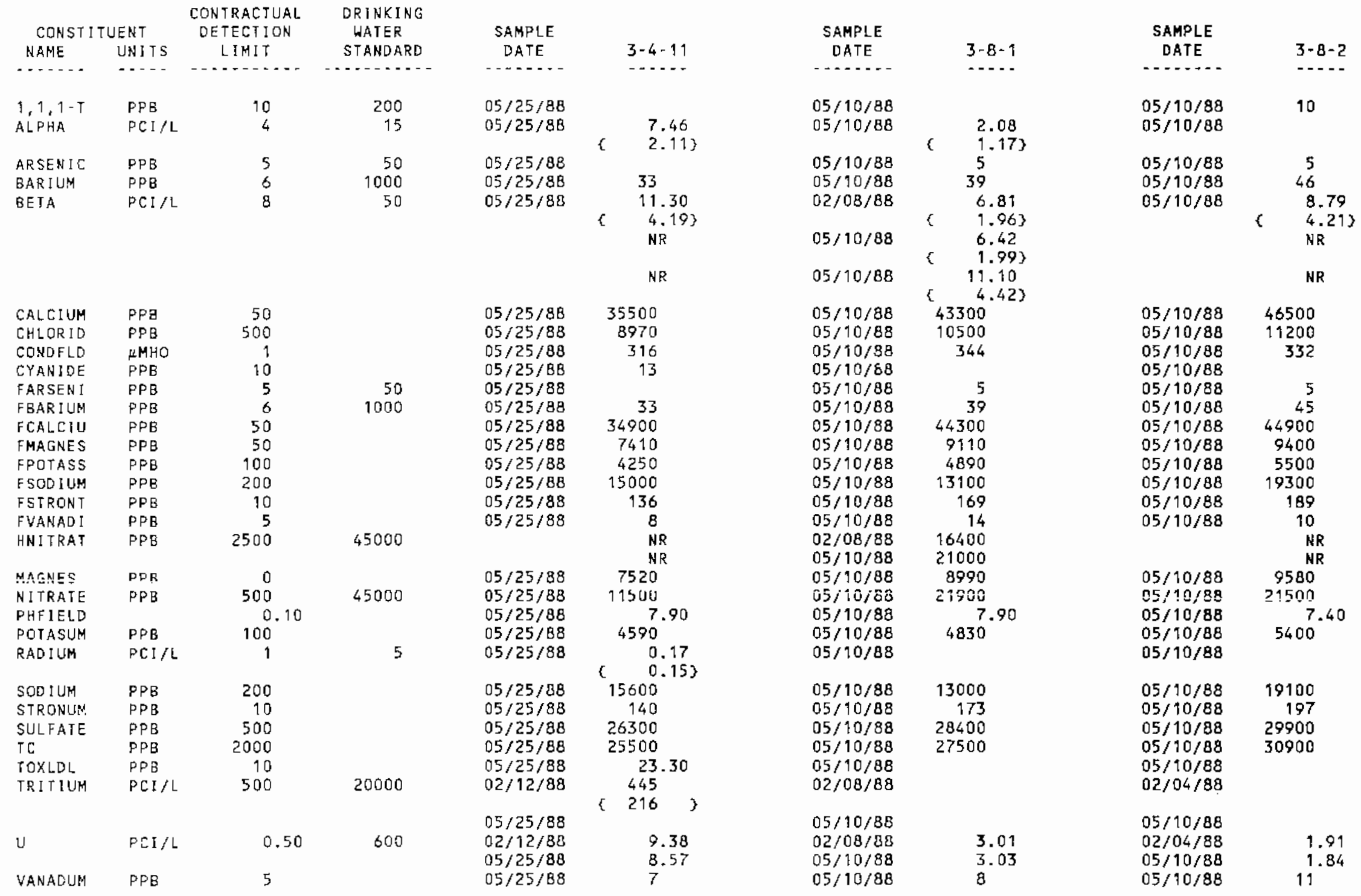


TABLE E.5. (contd)

\begin{tabular}{|c|c|c|c|c|c|c|c|c|c|}
\hline \multicolumn{2}{|c|}{ CONST I TUENT } & $\begin{array}{l}\text { CONTRACTUAL } \\
\text { DETECTION }\end{array}$ & $\begin{array}{l}\text { DRINKING } \\
\text { HATER }\end{array}$ & SAMPLE & & SAMPLE & & SAMPLE & \\
\hline NAME & UN I TS & LIMIT & STANDARD & DATE & $3-8-3$ & DATE & $6-530-E \uparrow 5 A$ & DATE & $6-S 19-E 13$ \\
\hline$\cdots \cdots$ & $\cdots$ & & & $\cdots$ & $\cdots$ & $\cdots$ & $\ldots$ & $\cdots$ & - n........ \\
\hline ALPHA & $\mathrm{PCI} / \mathrm{L}$ & 4 & 15 & $05 / 09 / 88$ & $\begin{array}{l}1.32 \\
1.03\}\end{array}$ & $05 / 05 / 88$ & & $05 / 27 / 88$ & $\begin{array}{l}3.80 \\
7.73\}\end{array}$ \\
\hline BARIUH & PPB & 6 & 1000 & $05 / 09 / 88$ & 38 & $05 / 05 / 88$ & 55 & $05 / 27 / 88$ & 49 \\
\hline BETA & $\mathrm{PCI} / \mathrm{L}$ & 8 & 50 & $\begin{array}{l}02 / 08 / 88 \\
05 / 09 / 88 \\
05 / 09 / 88\end{array}$ & 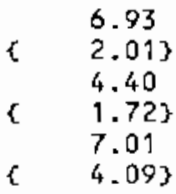 & $\begin{array}{l}02 / 12 / 88 \\
05 / 05 / 88\end{array}$ & $\begin{array}{l}5.99 \\
2.013 \\
5.19 \\
4.093 \\
\text { NR }\end{array}$ & $\begin{array}{l}02 / 17 / 88 \\
04 / 15 / 88 \\
05 / 27 / 88\end{array}$ & $\begin{array}{ll} & 7.33 \\
\{ & 2.12\} \\
& 8.01 \\
\{\quad 2.21\} \\
& 11.20 \\
\{\quad 4.27\}\end{array}$ \\
\hline CALCIUM & PPB & 50 & & $05 / 09 / 88$ & 39200 & $05 / 05 / 88$ & 59900 & $05 / 27 / 88$ & 44300 \\
\hline CHLORID & PPB & 500 & & $05 / 09 / 8 B$ & 10200 & $05 / 05 / 88$ & 5590 & $05 / 27 / 88$ & 18300 \\
\hline $50-60$ & PCI I L & 22.50 & 100 & $\begin{array}{l}02 / 08 / 88 \\
02 / 08 / 88 \\
05 / 09 / 88\end{array}$ & & & $\begin{array}{l}\text { NR } \\
\text { NR }\end{array}$ & $\begin{array}{l}02 / 17 / 88 \\
04 / 15 / 88\end{array}$ & $\begin{cases}6.08 \\
& 5.44 \\
N R\end{cases}$ \\
\hline COLIFRM & MPN & 2 & 1 & $05 / 09 / 88$ & & $05 / 05 / 88$ & & $05 / 27 / 88$ & $2.20 *$ \\
\hline CONDFLD & \#HO & 1 & & $05 / 09 / 88$ & 361 & $05 / 05 / 88$ & 609 & $05 / 27 / 88$ & 413 \\
\hline $\operatorname{cs}-137$ & $\mathrm{PCI} / \mathrm{L}$ & 20 & 200 & $\begin{array}{l}02 / 08 / 88 \\
02 / 08 / 88 \\
05 / 09 / 88\end{array}$ & & & $\begin{array}{l}\text { NR } \\
\text { NR } \\
\text { NR }\end{array}$ & $\begin{array}{l}02 / 17 / 88 \\
04 / 15 / 88\end{array}$ & 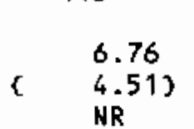 \\
\hline FARSEN! & PPB & 5 & 50 & $05 / 09 / 88$ & 5 & $05 / 05 / 88$ & & $05 / 27 / 88$ & 6 \\
\hline FBAR I UM & $P P B$ & 6 & 1000 & $05 / 09 / 88$ & 37 & $05 / 05 / 88$ & 57 & $05 / 27 / 88$ & 49 \\
\hline $\begin{array}{l}\text { FCALCIU } \\
\text { FIRON }\end{array}$ & $\begin{array}{l}\text { PPB } \\
\text { PPB }\end{array}$ & $\begin{array}{l}50 \\
50\end{array}$ & & $\begin{array}{l}05 / 09 / 88 \\
05 / 09 / 88\end{array}$ & 40200 & $\begin{array}{l}05 / 05 / 88 \\
05 / 05 / 88\end{array}$ & $\begin{array}{r}68600 \\
51\end{array}$ & $\begin{array}{l}05 / 27 / 88 \\
05 / 27 / 88\end{array}$ & 45100 \\
\hline FMAGNES & PPB & 50 & & $05 / 09 / 88$ & 8440 & $05 / 05 / 88$ & 12000 & $05 / 27 / 88$ & 13300 \\
\hline FPOTASS & PPB & 100 & & $05 / 09 / 88$ & 5130 & $05 / 05 / 88$ & 5870 & $05 / 27 / 88$ & 6310 \\
\hline FSODIUM & PPB & 200 & & $05 / 09 / 88$ & 19600 & $05 / 05 / 88$ & 12100 & $05 / 27 / 88$ & 22400 \\
\hline FSTRONT & PPB & 10 & & $05 / 09 / 88$ & 156 & $05 / 05 / 88$ & 247 & $05 / 27 / 88$ & 229 \\
\hline FVANAD I & PPB & 5 & & $05 / 09 / 88$ & 14 & $05 / 05 / 88$ & & $05 / 27 / 88$ & 17 \\
\hline FZINC & PPB & 5 & & $05 / 09 / 88$ & & $05 / 05 / 88$ & 106 & $05 / 27 / 88$ & \\
\hline HNITRAT & PPB & 2500 & 45000 & $\begin{array}{l}02 / 08 / 88 \\
05 / 09 / 88\end{array}$ & $\begin{array}{r}9730 \\
10400\end{array}$ & & $\begin{array}{l}\text { NR } \\
\text { NR }\end{array}$ & & $\begin{array}{l}\text { NR } \\
\text { NR }\end{array}$ \\
\hline I RON & PPB & 50 & & $05 / 09 / 88$ & 124 & $05 / 05 / 88$ & 468 & $05 / 27 / 88$ & 169 \\
\hline MAGNES & $\mathrm{PPB}$ & 0 & & $05 / 09 / 88$ & 8300 & $05 / 05 / 88$ & 11300 & $05 / 27 / 88$ & 12900 \\
\hline NITRATE & PPB & 500 & 45000 & $05 / 09 / 88$ & 10400 & $05 / 05 / 88$ & 14800 & $05 / 27 / 88$ & 21600 \\
\hline PHFIELD & & 0.10 & & $05 / 09 / 88$ & 7.80 & $05 / 05 / 88$ & 7.20 & $05 / 27 / 88$ & 7.70 \\
\hline POTASUM & PPB & 100 & & $05 / 09 / 88$ & $5010^{\circ}$ & $05 / 05 / 88$ & 5580 & $05 / 27 / 88$ & 6180 \\
\hline RADIUM & $\mathrm{PCI} / \mathrm{L}$ & 1 & 5 & $05 / 09 / 88$ & & $05 / 05 / 88$ & $\begin{array}{ll} & 0.23 \\
\{\quad 0.18\}\end{array}$ & $05 / 27 / 88$ & \\
\hline $500 I \cup M$ & PPB & 200 & & $05 / 09 / 88$ & 19400 & $05 / 05 / 88$ & 11600 & $05 / 27 / 88$ & 21300 \\
\hline STRONUM & PPB & 10 & & $05 / 09 / 88$ & 160 & $05 / 05 / 88$ & 242 & $05 / 27 / 88$ & 224 \\
\hline SULFATE & $P P B$ & 500 & & $05 / 09 / 88$ & 29600 & $05 / 05 / 88$ & 17500 & $05 / 27 / 88$ & 48700 \\
\hline TC & PPB & 2000 & & $05 / 09 / 88$ & 29600 & $05 / 05 / 88$ & 49700 & $05 / 27 / 88$ & 29400 \\
\hline
\end{tabular}


TABLE_E.5. (contd)

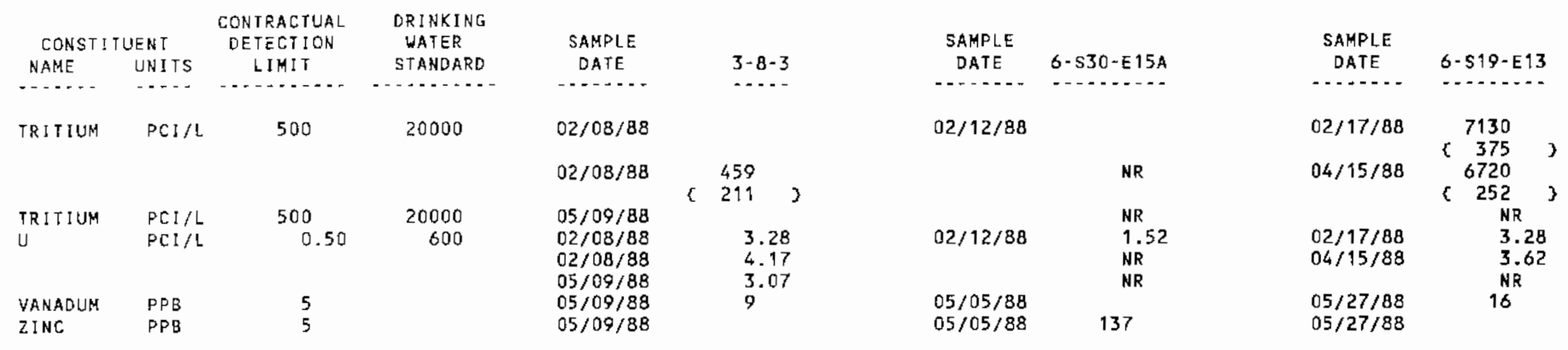


TABLE E.5. (contd)

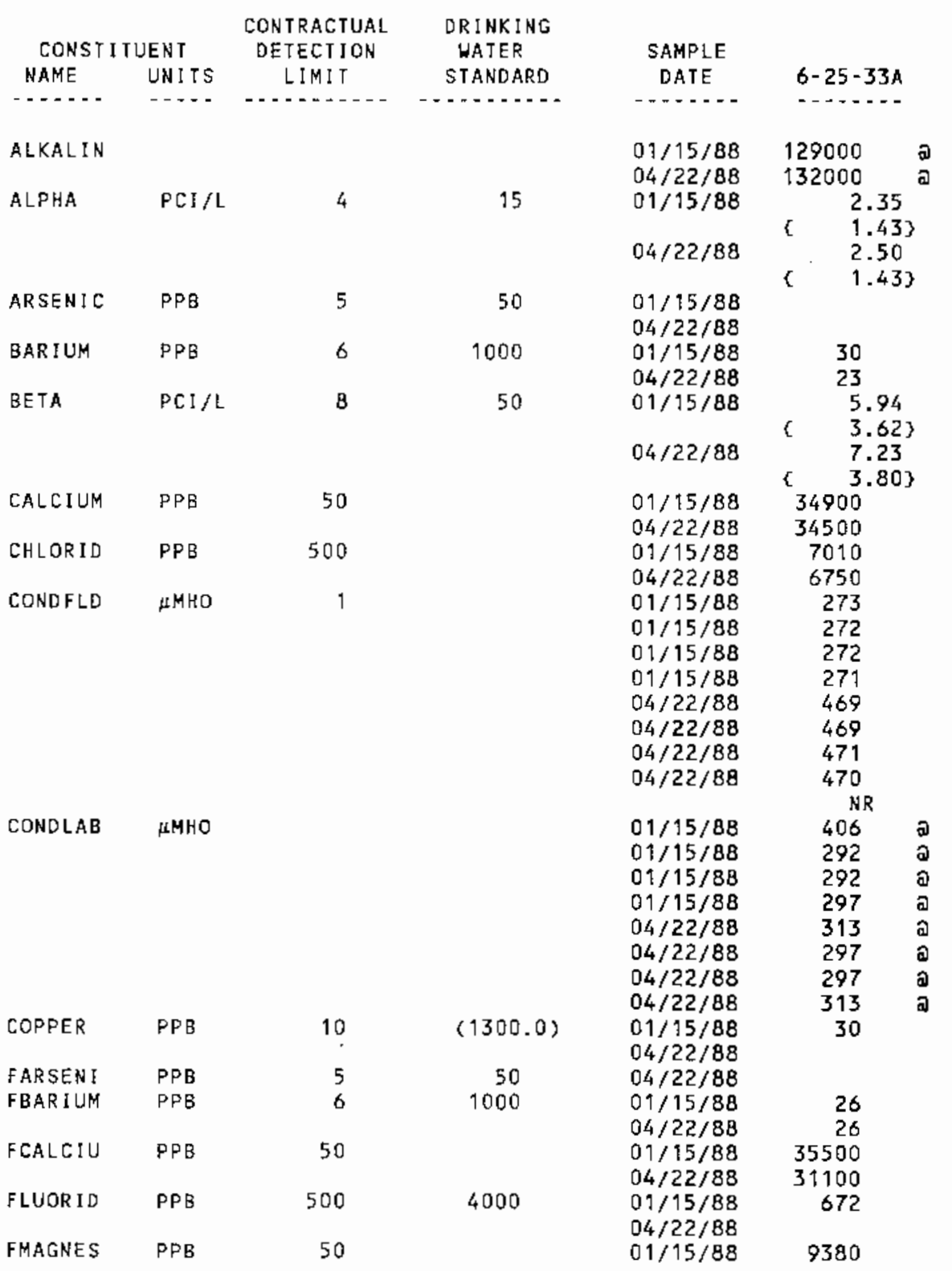

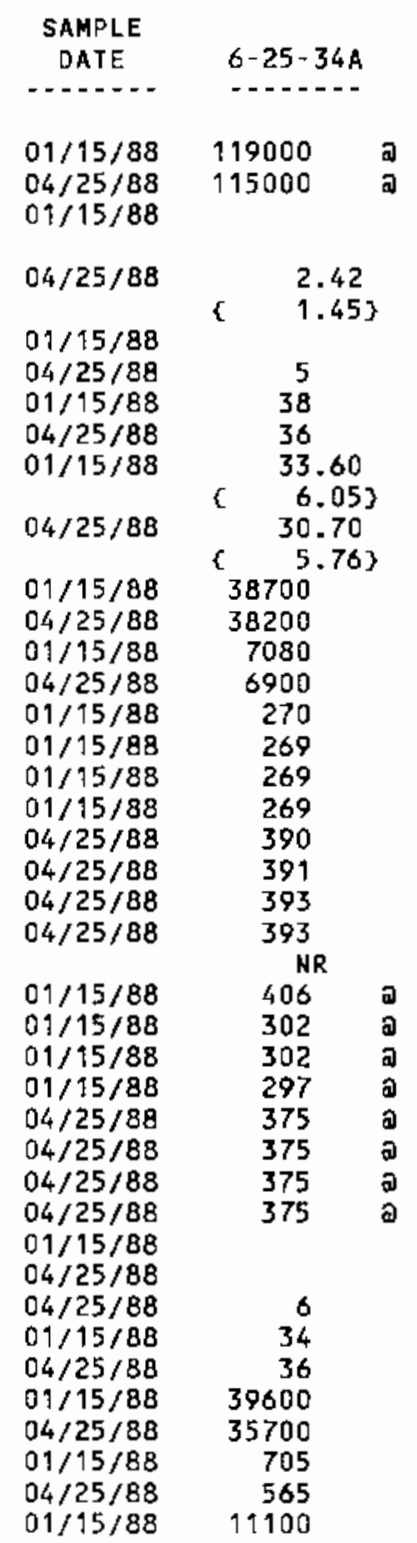

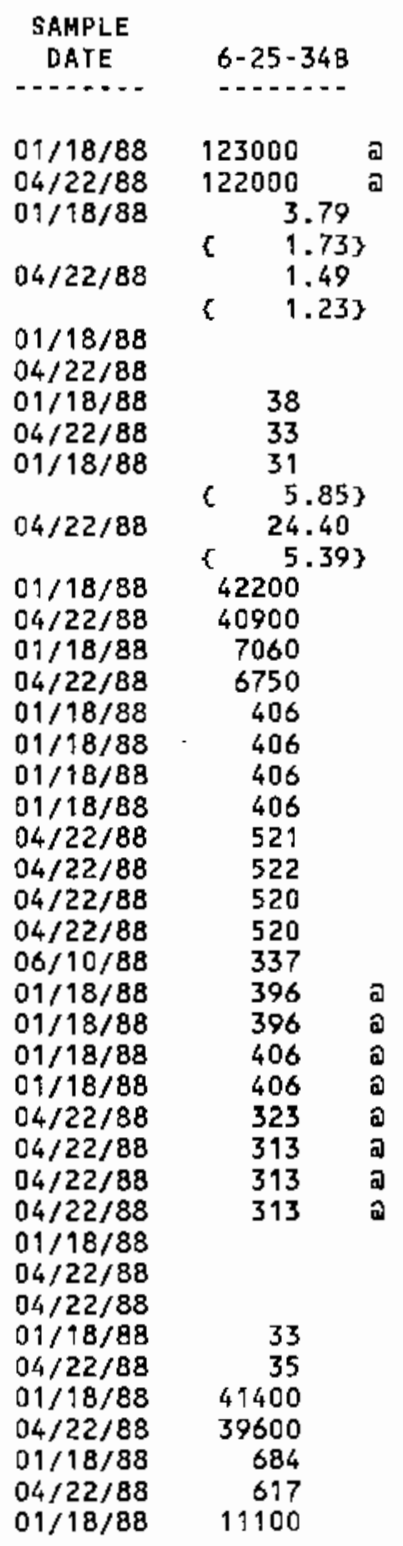


IABLE E.5. (contd)

\begin{tabular}{|c|c|c|c|c|c|c|c|c|c|}
\hline \multicolumn{2}{|c|}{ CONST I TUENT } & \multirow{2}{*}{$\begin{array}{c}\text { CONIRACTUAL } \\
\text { DETECTION } \\
\text { LIMIT }\end{array}$} & \multirow{2}{*}{$\begin{array}{l}\text { DRINKING } \\
\text { WATER } \\
\text { STANDARD }\end{array}$} & \multirow{2}{*}{$\begin{array}{l}\text { SAMPLE } \\
\text { DATE }\end{array}$} & \multirow[b]{2}{*}{$6-25-33 A$} & \multicolumn{2}{|l|}{ SAMPLE } & SAMPLE & \multirow[b]{2}{*}{$6-25-34 B$} \\
\hline NAME & UNI IS & & & & & DATE & $6-25-34 A$ & DATE & \\
\hline$\cdots$ & 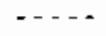 & & & 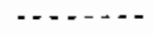 & $-\cdots$ & $\cdots$ & - & 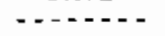 & $\ldots$ \\
\hline FMANGAN & PPB & 5 & & $\begin{array}{l}04 / 22 / 88 \\
01 / 15 / 88 \\
04 / 22 / 88\end{array}$ & $\begin{array}{r}8990 \\
7 \\
6\end{array}$ & $\begin{array}{l}04 / 25 / 88 \\
01 / 15 / 88 \\
04 / 25 / 88\end{array}$ & 10600 & $\begin{array}{l}04 / 22 / 88 \\
01 / 18 / 88 \\
04 / 22 / 88\end{array}$ & 10800 \\
\hline FPOTASS & PPB & 100 & & $\begin{array}{l}01 / 15 / 88 \\
04 / 22 / 88\end{array}$ & $\begin{array}{l}5360 \\
5320\end{array}$ & $\begin{array}{l}01 / 15 / 88 \\
04 / 25 / 88\end{array}$ & $\begin{array}{l}6010 \\
6100\end{array}$ & $\begin{array}{l}01 / 18 / 88 \\
04 / 22 / 88\end{array}$ & $\begin{array}{l}5720 \\
5210\end{array}$ \\
\hline FSODIUM & PPB & 200 & & $\begin{array}{l}01 / 15 / 88 \\
04 / 22 / 88\end{array}$ & $\begin{array}{l}30200 \\
26300\end{array}$ & $\begin{array}{l}01 / 15 / 88 \\
04 / 25 / 88\end{array}$ & $\begin{array}{l}22800 \\
20300\end{array}$ & $\begin{array}{l}01 / 18 / 88 \\
04 / 22 / 88\end{array}$ & $\begin{array}{l}21000 \\
20200\end{array}$ \\
\hline FSTRONT & PPB & 10 & & $\begin{array}{l}01 / 15 / 88 \\
04 / 22 / 88\end{array}$ & $\begin{array}{l}207 \\
197\end{array}$ & $\begin{array}{l}01 / 15 / 88 \\
04 / 25 / 88\end{array}$ & $\begin{array}{l}178 \\
171\end{array}$ & $\begin{array}{l}01 / 18 / 88 \\
04 / 22 / 88\end{array}$ & $\begin{array}{l}180 \\
173\end{array}$ \\
\hline FVANADI & $P P B$ & 5 & & $\begin{array}{l}01 / 15 / 88 \\
04 / 22 / 88\end{array}$ & $\begin{array}{r}10 \\
8\end{array}$ & $\begin{array}{l}01 / 15 / 88 \\
04 / 25 / 88\end{array}$ & $\begin{array}{l}25 \\
25\end{array}$ & $\begin{array}{l}01 / 18 / 88 \\
04 / 22 / 88\end{array}$ & $\begin{array}{l}24 \\
24\end{array}$ \\
\hline EZINC & $P P B$ & 5 & & $\begin{array}{l}01 / 15 / 88 \\
04 / 22 / 88\end{array}$ & 8 & $\begin{array}{l}01 / 15 / 88 \\
04 / 25 / 88\end{array}$ & & $\begin{array}{l}01 / 18 / 88 \\
04 / 22 / 88\end{array}$ & \\
\hline I RON & $\mathrm{PPB}$ & 50 & & $\begin{array}{l}01 / 15 / 88 \\
04 / 22 / 88\end{array}$ & $\begin{array}{r}132 \\
94\end{array}$ & $\begin{array}{l}01 / 15 / E 8 \\
04 / 25 / 83\end{array}$ & & $\begin{array}{l}01 / 18 / 88 \\
04 / 22 / 88\end{array}$ & 53 \\
\hline LEAOGF & PPG & 5 & 50 & $\begin{array}{l}01 / 15 / 88 \\
04 / 22 / 88\end{array}$ & 5 & $\begin{array}{l}01 / 15 / 88 \\
04 / 25 / 88\end{array}$ & & $\begin{array}{l}01 / 18 / 88 \\
04 / 22 / 88\end{array}$ & \\
\hline LFLUORO & PPB & 50 & 4000 & $\begin{array}{l}01 / 15 / 88 \\
04 / 22 / 88\end{array}$ & $\begin{array}{l}496 \\
395\end{array}$ & $\begin{array}{l}01 / 15 / 88 \\
04 / 25 / 88\end{array}$ & $\begin{array}{l}588 \\
476\end{array}$ & $\begin{array}{l}01 / 18 / 88 \\
04 / 22 / 88\end{array}$ & $\begin{array}{l}620 \\
482\end{array}$ \\
\hline MAGNES & PPB & 0 & & $\begin{array}{l}01 / 15 / 88 \\
04 / 22 / 88\end{array}$ & $\begin{array}{l}9420 \\
9350\end{array}$ & $\begin{array}{l}01 / 15 / 88 \\
04 / 25 / 88\end{array}$ & $\begin{array}{l}10900 \\
10700\end{array}$ & $\begin{array}{l}01 / 18 / 88 \\
04 / 22 / 88\end{array}$ & $\begin{array}{l}11200 \\
11300\end{array}$ \\
\hline MANGESE & $\mathrm{PPB}$ & 5 & & $\begin{array}{l}01 / 15 / 88 \\
04 / 22 / 88\end{array}$ & $\begin{array}{l}5 \\
6\end{array}$ & $\begin{array}{l}01 / 15 / 88 \\
04 / 25 / 88\end{array}$ & & $\begin{array}{l}01 / 18 / 88 \\
04 / 22 / 88\end{array}$ & \\
\hline NITRATE & PPB & 500 & 45000 & $\begin{array}{l}01 / 15 / 88 \\
04 / 22 / 88\end{array}$ & $\begin{array}{l}5830 \\
5580\end{array}$ & $\begin{array}{l}01 / 15 / 88 \\
04 / 25 / 88\end{array}$ & $\begin{array}{l}30900 \\
32900\end{array}$ & $\begin{array}{l}01 / 18 / 88 \\
04 / 22 / 88\end{array}$ & $\begin{array}{l}30700 \\
32700\end{array}$ \\
\hline$P H-I A B$ & & 0.01 & & $\begin{array}{l}01 / 15 / 88 \\
01 / 15 / 88 \\
01 / 15 / 88 \\
01 / 15 / 88 \\
04 / 22 / 88 \\
04 / 22 / 88 \\
04 / 22 / 88 \\
04 / 22 / 88\end{array}$ & $\begin{array}{l}7.99 \\
8.00 \\
8.04 \\
8.02 \\
8.20 \\
8.20 \\
8.20 \\
8.20\end{array}$ & $\begin{array}{l}01 / 15 / 88 \\
01 / 15 / 86 \\
01 / 15 / 88 \\
01 / 15 / 88 \\
04 / 25 / 88 \\
04 / 25 / 88 \\
04 / 25 / 88 \\
04 / 25 / 88\end{array}$ & $\begin{array}{l}7.78 \\
7.72 \\
7.90 \\
7.83 \\
8 \\
8 \\
8 \\
8\end{array}$ & $\begin{array}{l}01 / 18 / 88 \\
01 / 18 / 88 \\
01 / 18 / 88 \\
01 / 18 / 88 \\
04 / 22 / 88 \\
04 / 22 / 88 \\
04 / 22 / 88 \\
04 / 22 / 88\end{array}$ & $\begin{array}{l}7.63 \\
7.66 \\
7.70 \\
7.72 \\
8 \\
8.10 \\
8 \\
8\end{array}$ \\
\hline PHFIELD & & 0.10 & & $\begin{array}{l}01 / 15 / 88 \\
01 / 15 / 88 \\
01 / 15 / 88 \\
01 / 15 / 88 \\
04 / 22 / 88 \\
04 / 22 / 88 \\
04 / 22 / 88 \\
04 / 22 / 88\end{array}$ & $\begin{array}{l}8.20 \\
8.20 \\
8.20 \\
8.20 \\
7.80 \\
7.90 \\
7.90 \\
8\end{array}$ & $\begin{array}{l}01 / 15 / 88 \\
01 / 15 / 88 \\
01 / 15 / 88 \\
01 / 15 / 88 \\
04 / 25 / 88 \\
04 / 25 / 88 \\
04 / 25 / 88 \\
04 / 25 / 88\end{array}$ & $\begin{array}{l}7.90 \\
7.90 \\
8 \\
8 \\
8.40 \\
8.30 \\
8.40 \\
8.40\end{array}$ & $\begin{array}{l}01 / 18 / 88 \\
01 / 18 / 88 \\
01 / 18 / 88 \\
01 / 18 / 88 \\
04 / 22 / 88 \\
04 / 22 / 88 \\
04 / 22 / 88 \\
04 / 22 / 88\end{array}$ & $\begin{array}{l}5.80 \\
5.80 \\
5.80 \\
5.90 \\
7.50 \\
7.50 \\
7.60 \\
7.50\end{array}$ \\
\hline & & & & & $N R$ & & $\mathrm{NR}$ & $06 / 10 / 88$ & 7.80 \\
\hline POTASUM & $P P B$ & 100 & & $01 / 15 / 88$ & 5430 & $01 / 15 / 88$ & 5930 & $01 / 18 / 88$ & 5560 \\
\hline SOD IUM & РPB & 200 & & $04 / 15 / 88$ & 31800 & $\begin{array}{l}04 / 23 / 80 \\
01 / 15 / 88\end{array}$ & 22000 & $01 / 18 / 88$ & 20300 \\
\hline
\end{tabular}


TABLE E.5. (contd)

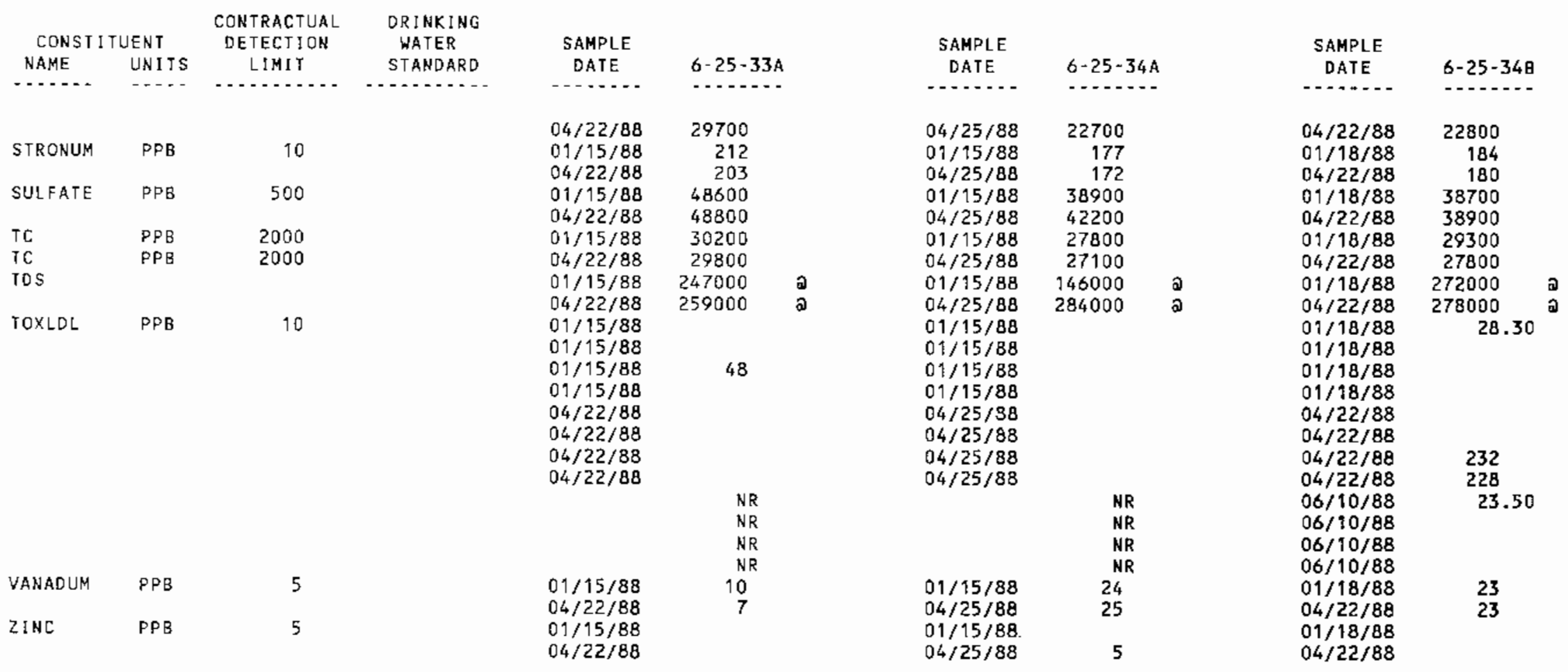


TABLE E.5. (contd)

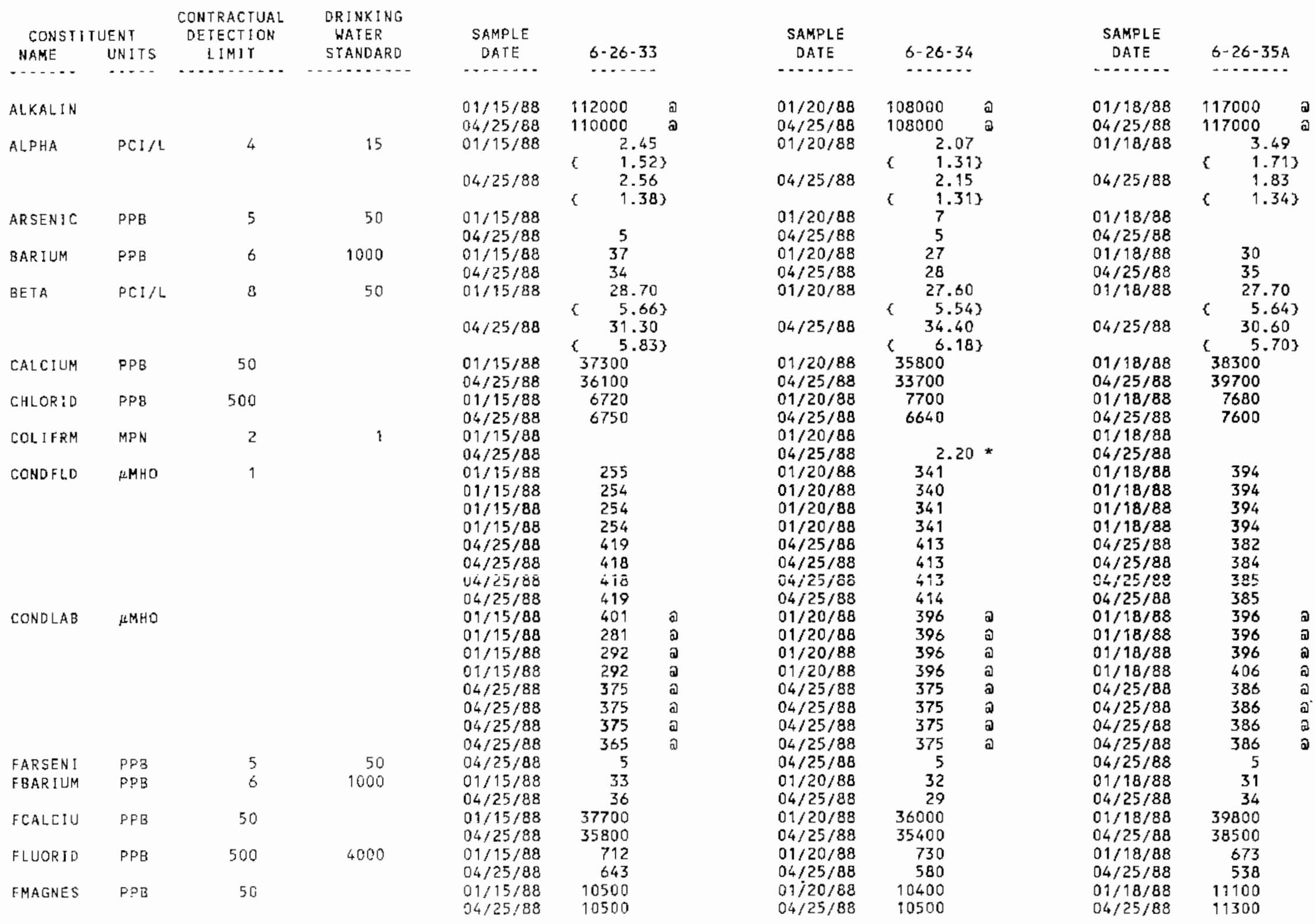


TABLE E.5. (contd)

\begin{tabular}{|c|c|c|c|c|c|}
\hline \multicolumn{2}{|c|}{ CONSTI ITUENT } & $\begin{array}{l}\text { CONTRACTUAL } \\
\text { DETECTION }\end{array}$ & $\begin{array}{l}\text { DRINKING } \\
\text { WATER }\end{array}$ & SAMPLE & \\
\hline NAME & UNITS & LIMII & STANDARD & DATE & $6-26-33$ \\
\hline 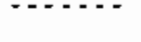 & $\cdots$ & - nen & $\cdots$ & 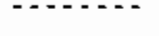 & $\cdots+-\cdots$ \\
\hline FPOTASS & РPB & 100 & & $\begin{array}{l}01 / 15 / 88 \\
04 / 25 / 88\end{array}$ & $\begin{array}{l}5780 \\
6100\end{array}$ \\
\hline FSODIUM & PPB & 200 & & $01 / 15 / 88$ & 22200 \\
\hline FSOD IUM & PPB & 200 & & $04 / 25 / 88$ & 20700 \\
\hline FSTRONT & PPB & 10 & & $01 / 15 / 88$ & 170 \\
\hline FVANAD I & PPB & 5 & & $\begin{array}{l}04 / 25 / 88 \\
01 / 15 / 88 \\
04 / 25 / 88\end{array}$ & $\begin{array}{r}170 \\
26 \\
26\end{array}$ \\
\hline IRON & PPB & 50 & & $\begin{array}{l}01 / 15 / 88 \\
04 / 25 / 88\end{array}$ & \\
\hline LFLUORD & PPB & 50 & 4000 & $\begin{array}{l}04 / 25 / 88 \\
01 / 15 / 88 \\
04 / 25 / 88\end{array}$ & $\begin{array}{l}614 \\
476\end{array}$ \\
\hline MAGNES & $\mathrm{PPB}$ & 0 & & $\begin{array}{l}01 / 15 / 88 \\
04 / 25 / 88\end{array}$ & $\begin{array}{l}10200 \\
10200\end{array}$ \\
\hline MANGESE & PPB & 5 & & $\begin{array}{l}01 / 15 / 88 \\
04 / 25 / 88\end{array}$ & \\
\hline NITRATE & PPB & 500 & 45000 & $\begin{array}{l}01 / 15 / 88 \\
04 / 25 / 88\end{array}$ & $\begin{array}{l}29100 \\
33100\end{array}$ \\
\hline$P H-L A B$ & & 0.01 & & $\begin{array}{l}01 / 15 / 88 \\
01 / 15 / 88 \\
01 / 15 / 88 \\
01 / 15 / 88 \\
04 / 25 / 88 \\
04 / 25 / 88 \\
04 / 25 / 88 \\
04 / 25 / 88\end{array}$ & $\begin{array}{l}7.92 \\
7.98 \\
7.95 \\
7.93 \\
8.10 \\
8.10 \\
8.10 \\
8.10\end{array}$ \\
\hline PHFIELD & & 0.10 & & $\begin{array}{l}01 / 15 / 88 \\
01 / 15 / 88 \\
01 / 15 / 88 \\
01 / 15 / 88 \\
04 / 25 / 88 \\
04 / 25 / 88 \\
04 / 25 / 88 \\
04 / 25 / 88\end{array}$ & $\begin{array}{l}8.10 \\
8.10 \\
8.10 \\
8.10 \\
8.20 \\
8.30 \\
8.50 \\
8.30\end{array}$ \\
\hline POTASUM & PPB & 100 & & $\begin{array}{l}01 / 15 / 88 \\
04 / 25 / 88\end{array}$ & $\begin{array}{l}5390 \\
5900\end{array}$ \\
\hline SODIUM & PPB & 200 & & $\begin{array}{l}01 / 15 / 88 \\
04 / 25 / 88\end{array}$ & $\begin{array}{l}20300 \\
22500\end{array}$ \\
\hline STRONUM & PPB & 10 & & $\begin{array}{l}01 / 15 / 88 \\
04 / 25 / 88\end{array}$ & $\begin{array}{l}169 \\
163\end{array}$ \\
\hline SULFATE & PPB & 500 & & $\begin{array}{l}01 / 15 / 88 \\
04 / 25 / 88\end{array}$ & $\begin{array}{l}37100 \\
40600\end{array}$ \\
\hline TC & PPB & 2000 & & $\begin{array}{l}01 / 15 / 88 \\
04 / 25 / 88 \\
01 / 15 / 88\end{array}$ & $\begin{array}{r}25900 \\
25800 \\
245000\end{array}$ \\
\hline
\end{tabular}

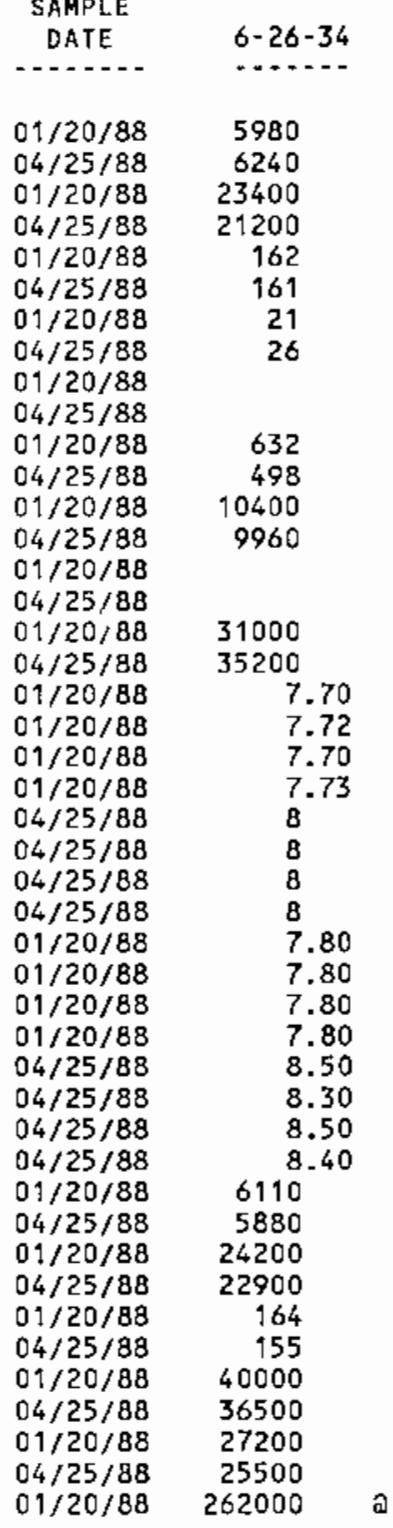

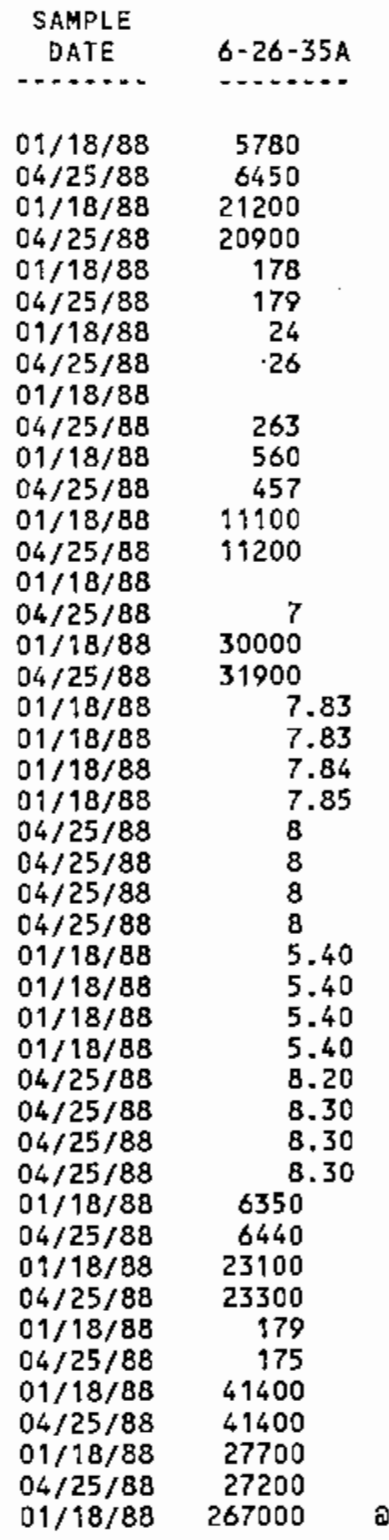


TABLE E.5. (contd)

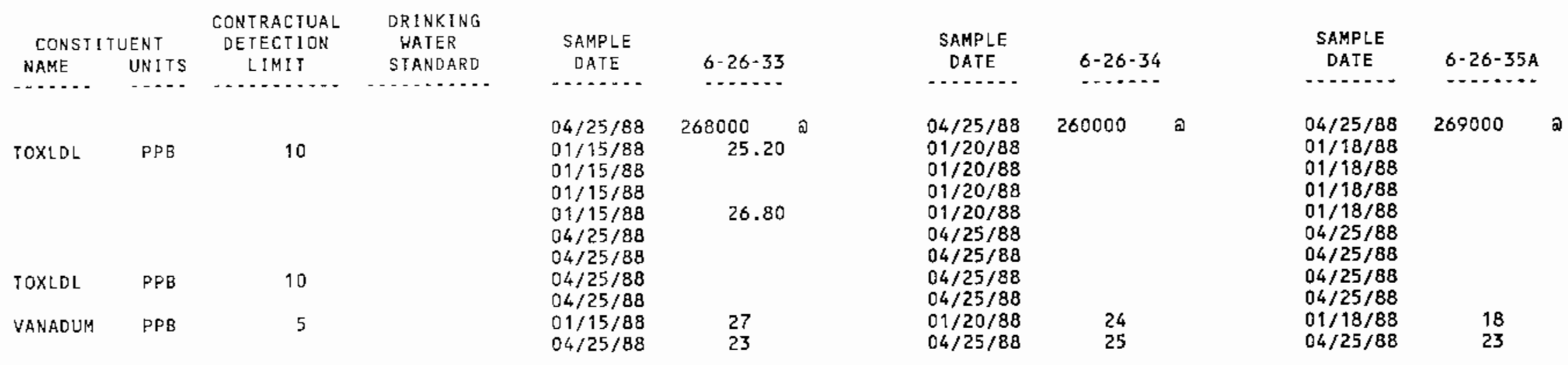


TABLE E.5. (contd)

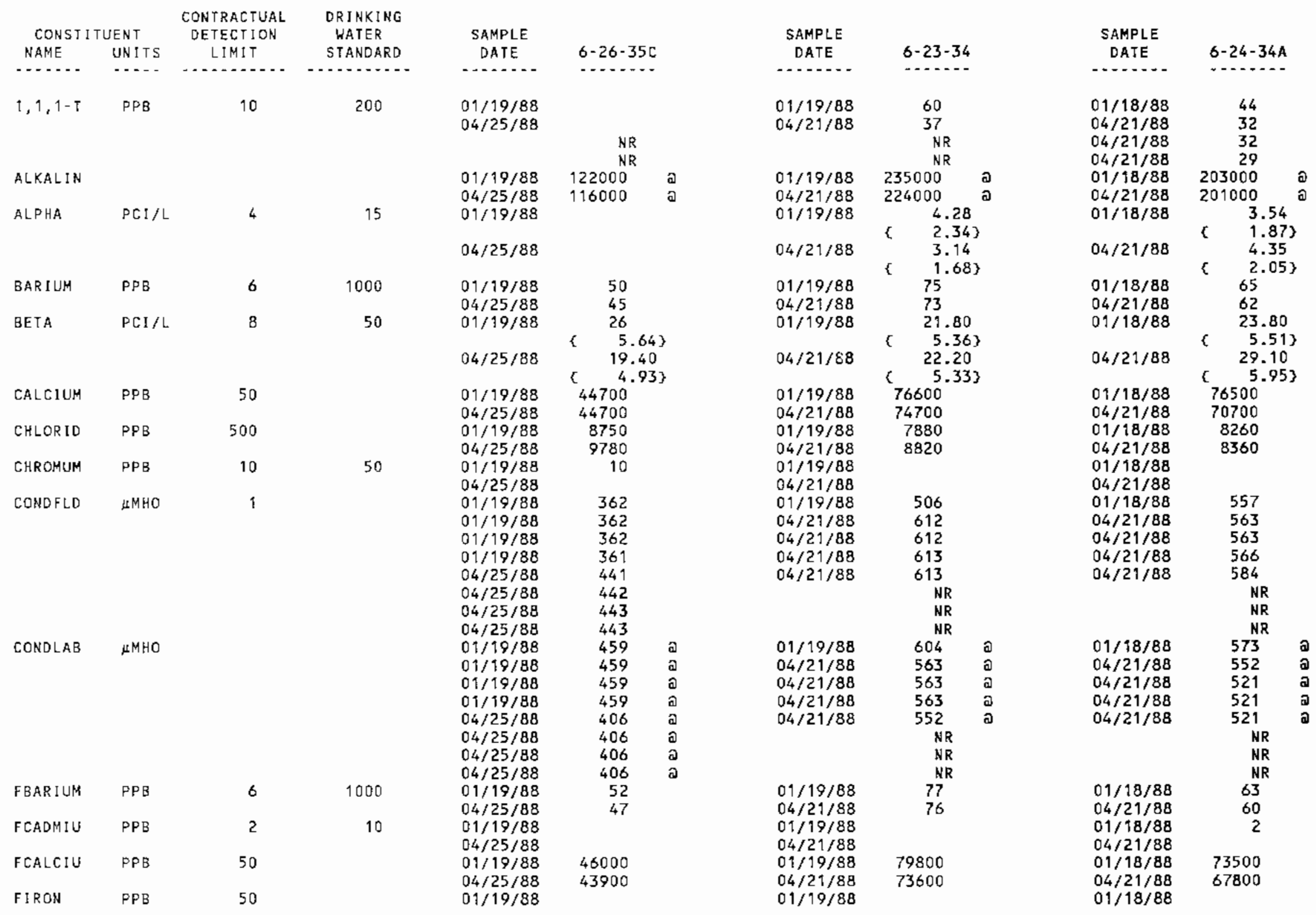


TABLE E.5. (contd)

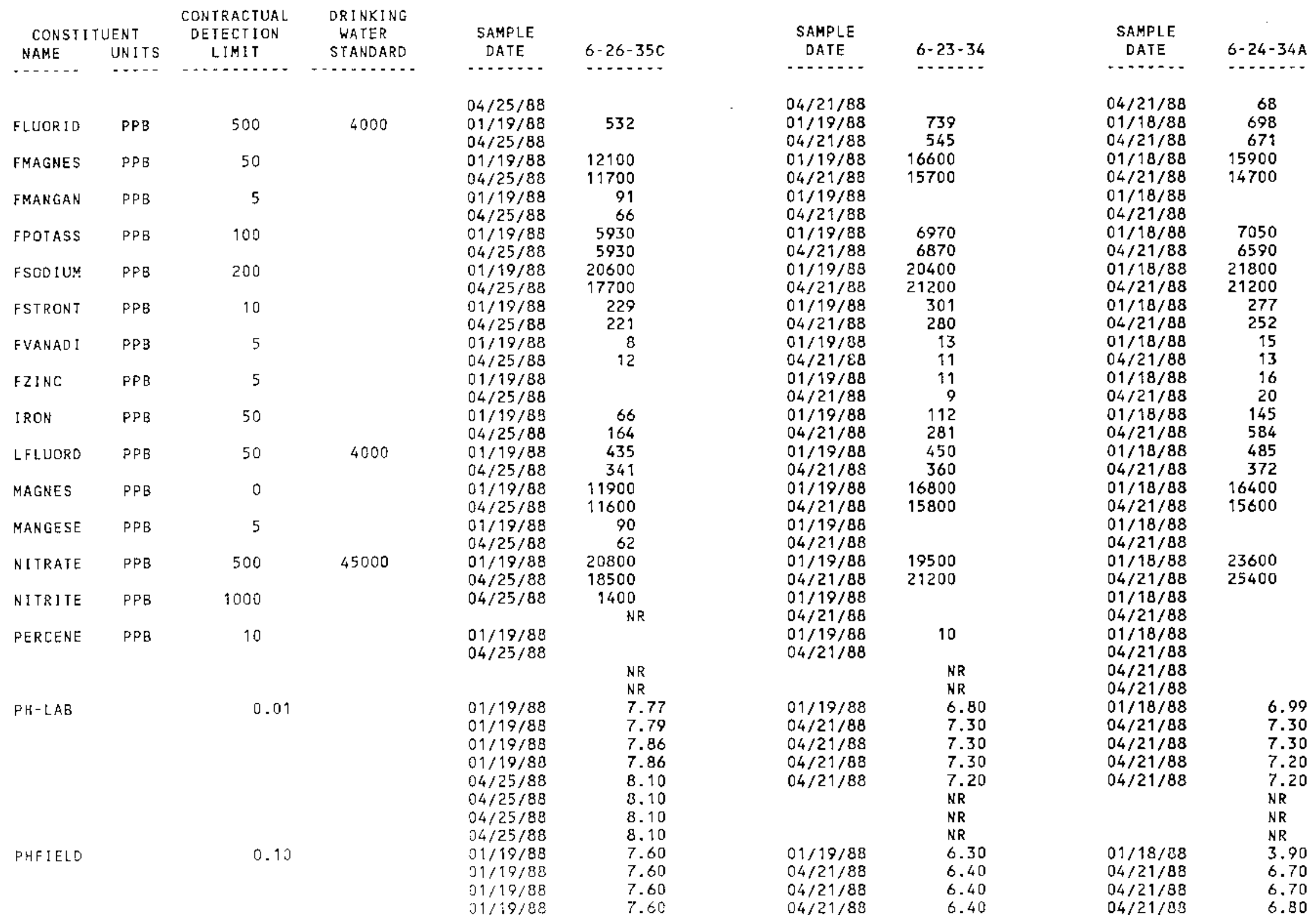


TABLE E. 5. (contd)

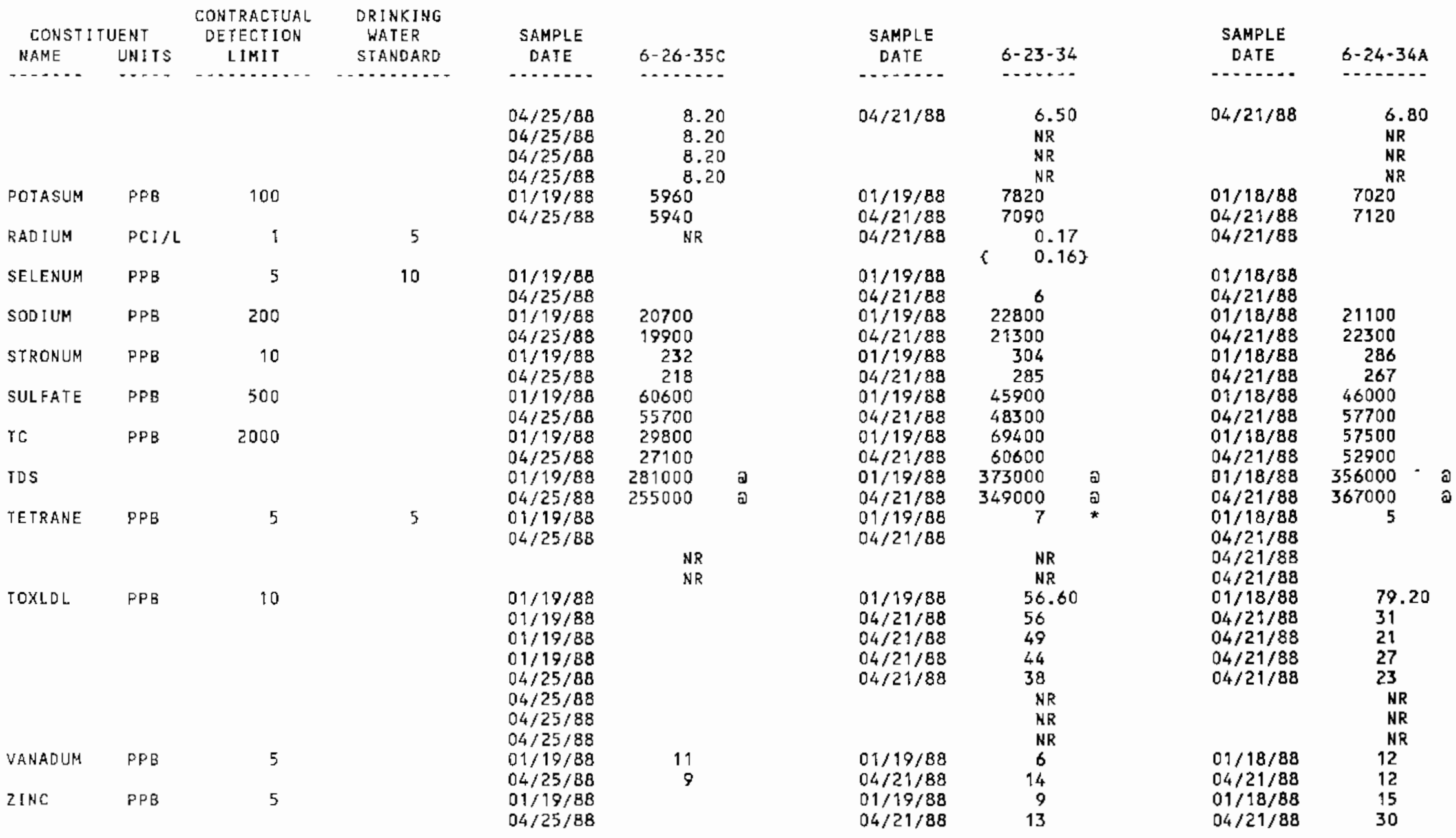


TABLE E.5. (contd)

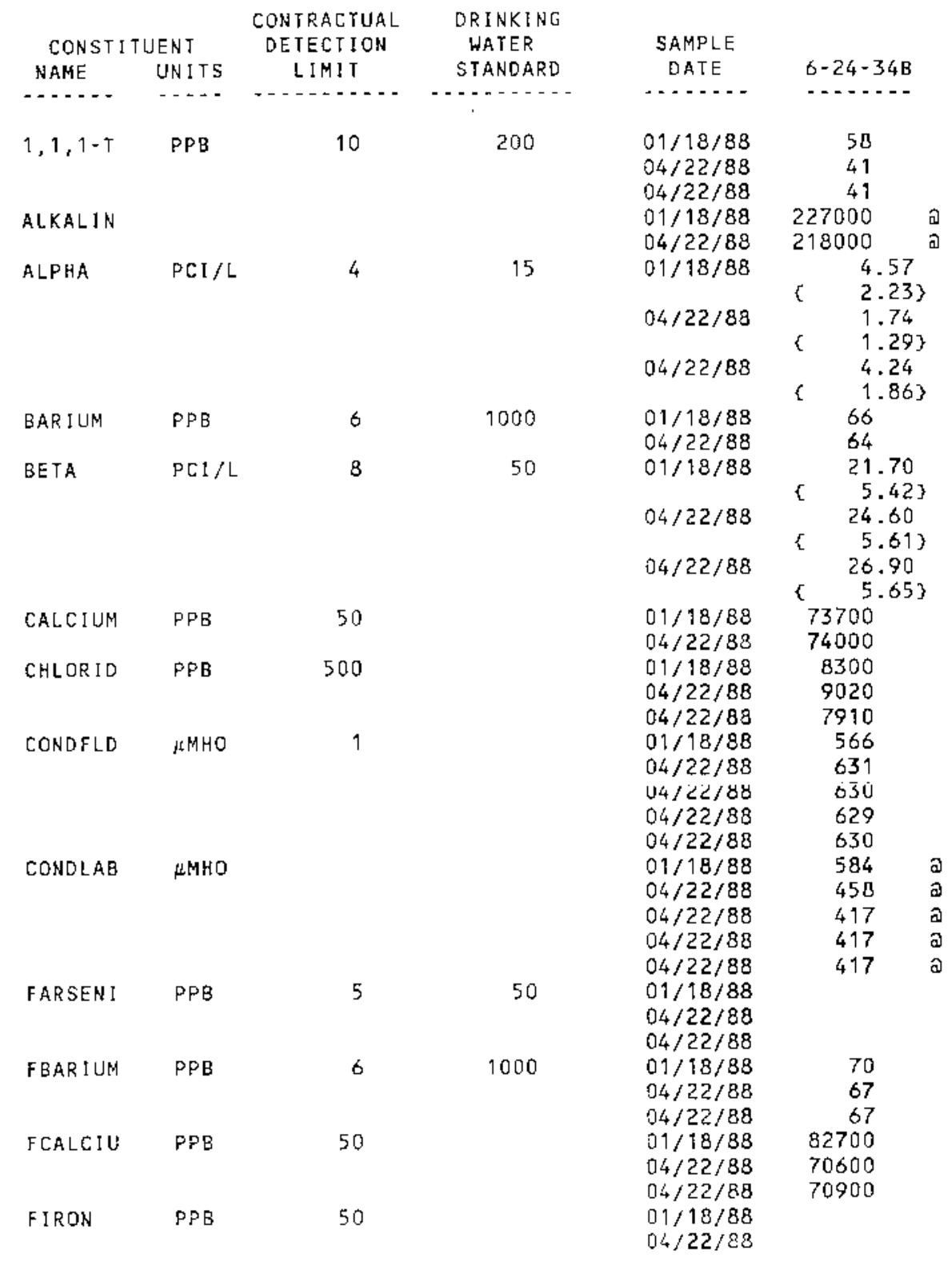

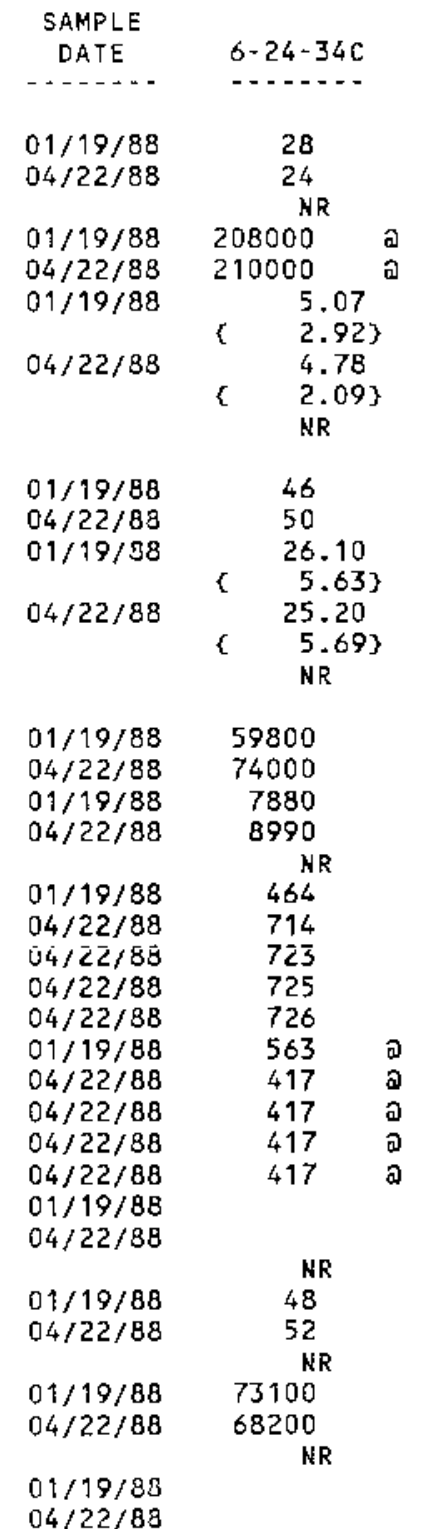

SAMPLE

DATE

$6-24-35$

81918

$01 / 19 / 88$

$04 / 21 / 88$

$01 / 19 / 88$

$04 / 21 / 88$

$01 / 19 / 88$

$04 / 21 / 88$

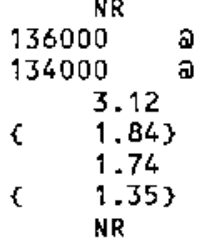

\begin{tabular}{|c|c|}
\hline $\begin{array}{l}01 / 19 / 88 \\
04 / 21 / 88 \\
01 / 19 / 88 \\
04 / 21 / 88\end{array}$ & $\begin{array}{r}42700 \\
45200 \\
7710 \\
8080\end{array}$ \\
\hline & NR \\
\hline $\begin{array}{l}01 / 19 / 88 \\
04 / 21 / 88 \\
04 / 2 \uparrow / 88 \\
04 / 2 \uparrow / 88 \\
04 / 21 / 88\end{array}$ & $\begin{array}{l}358 \\
485 \\
485 \\
483 \\
484\end{array}$ \\
\hline & $\begin{array}{l}434 \\
417\end{array}$ \\
\hline $04 / 21 / 88$ & $\begin{array}{l}417 \\
417\end{array}$ \\
\hline $04 / 21 / 88$ & 417 \\
\hline $\begin{array}{l}01 / 19 / 88 \\
04 / 21 / 88\end{array}$ & 5 \\
\hline & NR \\
\hline $\begin{array}{l}01 / 19 / 88 \\
04 / 21 / 88\end{array}$ & $\begin{array}{l}43 \\
44\end{array}$ \\
\hline & NR \\
\hline $\begin{array}{l}01 / 19 / 88 \\
04 / 21 / 88\end{array}$ & $\begin{array}{l}46500 \\
42800\end{array}$ \\
\hline
\end{tabular}

$\begin{array}{lll}01 / 19 / 88 & & 44 \\ 04 / 21 / 88 & & 45 \\ 01 / 19 / 88 & & 17.20 \\ & < & 4.773 \\ 04 / 21 / 88 & & 21.90 \\ & & 5.193 \\ & & \text { NR }\end{array}$

$\underset{\infty}{\omega}$
$01 / 19 / 88$

$04 / 21 / 88$ 
TABLE E.5. (contd)

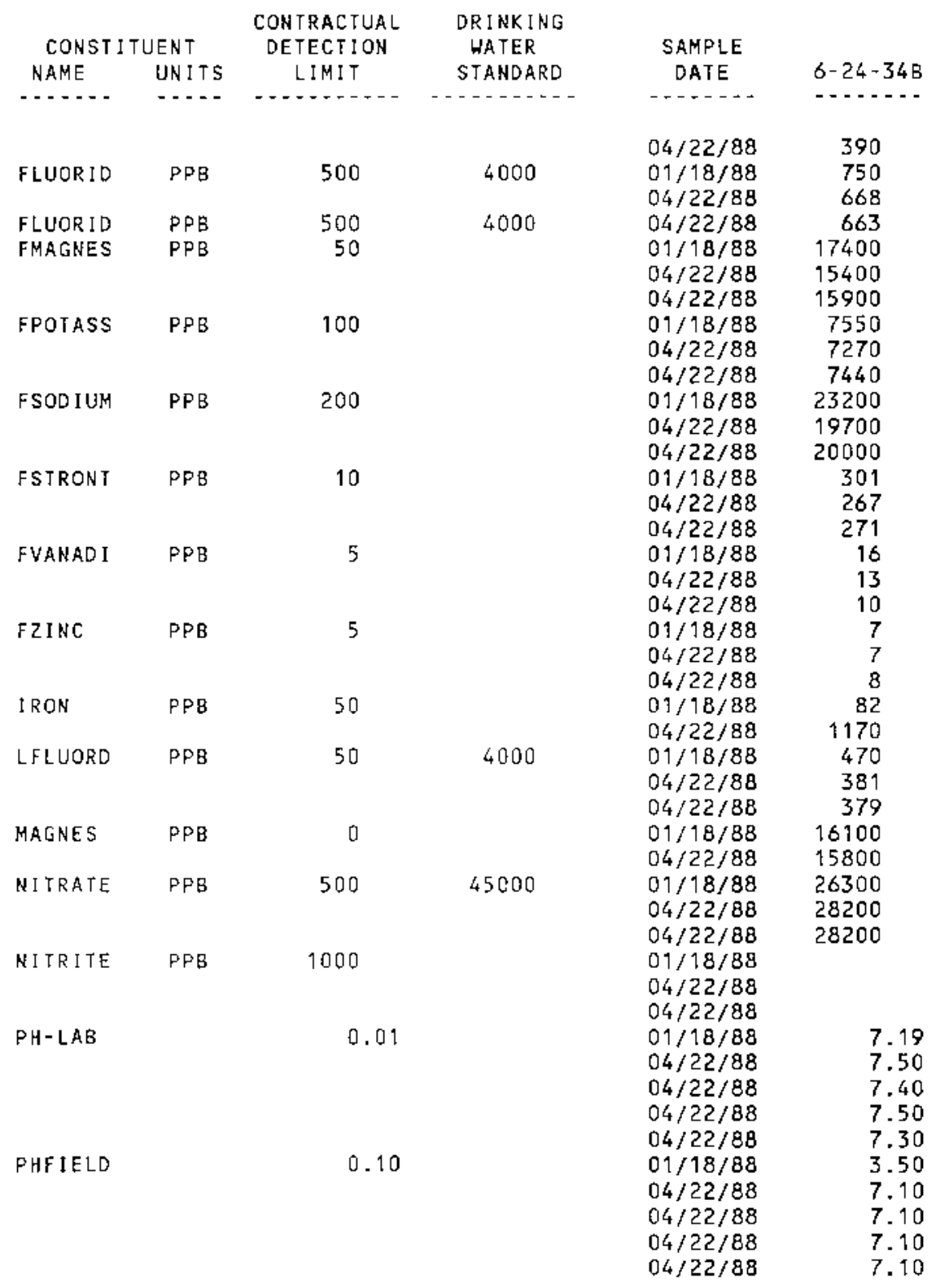

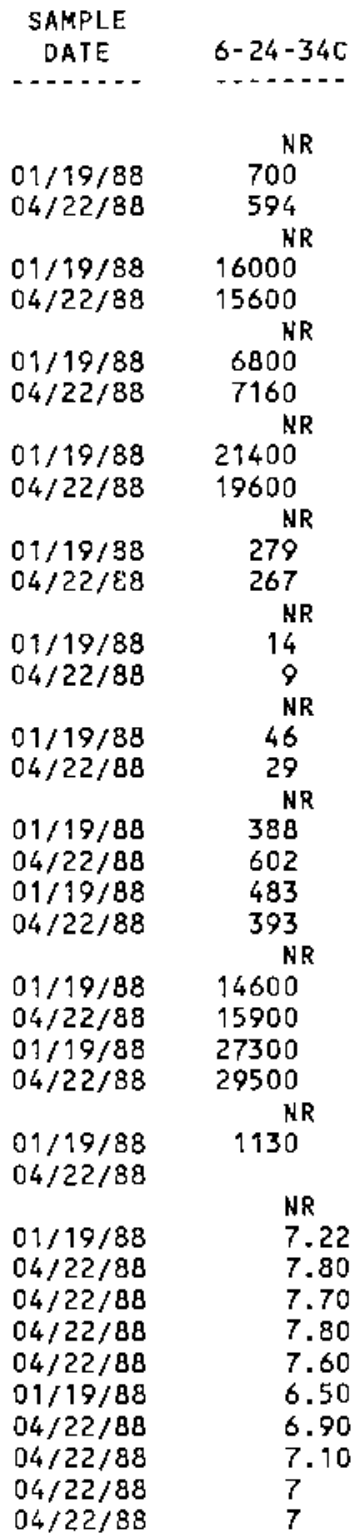

SAMPLE

DATE $6-24-35$

$\begin{array}{cc} & \mathrm{NR} \\ 01 / 19 / 88 & 676 \\ 04 / 21 / 88 & 644 \\ & \mathrm{NR} \\ 01 / 19 / 88 & 19400 \\ 04 / 21 / 88 & 10800 \\ & \mathrm{NR} \\ 01 / 19 / 88 & 6310 \\ 04 / 21 / 88 & 5940\end{array}$

$04 / 21 / 88$

$01 / 19 / 88$

$04 / 21 / 88$

NR

20400

$01 / 19 / 88 \quad 201$

$04 / 21 / 88 \quad 189$

$01 / 19 / 88$

$04 / 21 / 88$

$01 / 19 / 88$

$04 / 21 / 88$

$01 / 19 / 88$

$04 / 21 / 88$

$01 / 19 / 88$

$04 / 21 / 88$

$01 / 19 / 88$

$04 / 21 / 88$

$01 / 19 / 88$

$04 / 2 \uparrow / 88$

$01 / 19 / 88$

$04 / 21 / 88$

$01 / 19 / 88$

$04 / 21 / 88$

$04 / 21 / 88$

$04 / 21 / 88$

$04 / 21 / 88$

$01 / 19 / 88$

$04 / 21 / 88$

$04 / 21 / 88$

$04 / 21 / 88$

$04 / 21 / 88$ 
TABLE E.5. (contd)

\begin{tabular}{|c|c|c|c|c|c|c|}
\hline \multicolumn{2}{|c|}{ CONSTITUENT } & $\begin{array}{l}\text { CONTRACTUAL } \\
\text { OETECT ION }\end{array}$ & $\begin{array}{l}\text { OR I NKING } \\
\text { WATER }\end{array}$ & SAMPLE & & \\
\hline NAME & UNITS & LIMI T & STANDARD & DATE & $6-24-34 B$ & \\
\hline$\ldots \ldots$ & $\ldots$ & $\cdots-\cdots$ & $-\ldots, \ldots$ & $\ldots$ & $\cdots$ & \\
\hline POTASUM & PPB & 100 & & $\begin{array}{l}01 / 18 / 88 \\
04 / 22 / 88\end{array}$ & $\begin{array}{l}7500 \\
7010\end{array}$ & \\
\hline RAD I UIY & $\mathrm{PCI} / \mathrm{L}$ & 1 & 5 & $04 / 22 / 88$ & $\left\{\begin{array}{l}0.26 \\
0.18\end{array}\right\}$ & \\
\hline SODIUM & PPB & 200 & & $\begin{array}{l}01 / 18 / 88 \\
04 / 22 / 88\end{array}$ & $\begin{array}{l}22800 \\
21900\end{array}$ & \\
\hline STRONUM & PPB & 10 & & $01 / 18 / 88$ & 284 & \\
\hline STRONUM & PPB & 10 & & $04 / 22 / 88$ & 271 & \\
\hline SULFATE & PPB & 500 & & $\begin{array}{l}01 / 18 / 88 \\
04 / 22 / 88 \\
04 / 22 / 88\end{array}$ & $\begin{array}{l}43600 \\
45100 \\
44600\end{array}$ & \\
\hline TC & PPB & 2000 & & $\begin{array}{l}01 / 18 / 88 \\
04 / 22 / 88\end{array}$ & $\begin{array}{l}65400 \\
57600\end{array}$ & \\
\hline TOS & & & & $\begin{array}{l}01 / 18 / 88 \\
04 / 22 / 88\end{array}$ & $\begin{array}{l}383000 \\
360000\end{array}$ & a \\
\hline TEJRANE & PPB & 5 & 5 & $\begin{array}{l}01 / 18 / 88 \\
04 / 22 / 88 \\
04 / 22 / 88\end{array}$ & 6 & * \\
\hline TOXLDL & $\rho P B$ & 10 & & $\begin{array}{l}01 / 18 / 88 \\
04 / 22 / 88 \\
04 / 22 / 88 \\
04 / 22 / 88 \\
04 / 22 / 88\end{array}$ & $\begin{array}{l}57.20 \\
356 \\
373 \\
288 \\
356\end{array}$ & \\
\hline VANADU.Y & PPB & 5 & & $\begin{array}{l}01 / 18 / 88 \\
04 / 22 / 88\end{array}$ & $\begin{array}{r}8 \\
11\end{array}$ & \\
\hline $2 \mathrm{INC}$ & $P P B$ & 5 & & $\begin{array}{l}01 / 18 / 88 \\
04 / 26 / 88\end{array}$ & y & \\
\hline
\end{tabular}

$\begin{array}{lc}\text { SAMPLE } & \\ \text { DATE } & 6-24-34 \mathrm{C} \\ \cdots & \cdots \\ 01 / 19 / 88 & 6990 \\ 04 / 22 / 88 & 6900 \\ 04 / 22 / 88 & \\ 01 / 19 / 88 & 22400 \\ 04 / 22 / 88 & 21900 \\ 01 / 19 / 88 & 263 \\ 04 / 22 / 88 & 279 \\ 01 / 19 / 88 & 42000 \\ 04 / 22 / 88 & 44700 \\ 01 / 19 / 88 & 53900 \\ 04 / 22 / 88 & 52300 \\ 01 / 19 / 38 & 359000 \\ 04 / 22 / 88 & 365000 \\ 01 / 19 / 88 & \\ 04 / 22 / 88 & \\ & \\ 01 / 19 / 88 & \mathrm{NR} \\ 04 / 22 / 88 & 25 \\ 04 / 22 / 88 & 28 \\ 04 / 22 / 88 & 56 \\ 04 / 22 / 88 & 28 \\ 01 / 19 / 88 & 13 \\ 04 / 22 / 88 & 13 \\ 01 / 19 / 88 & 37 \\ 04 / 22 / 88 & 35 \\ & \end{array}$

SAMPLE

DATE

-.....

$01 / 19 / 88$

$04 / 21 / 88$

$04 / 21 / 88$

$01 / 19 / 88$

$04 / 21 / 88$

$01 / 19 / 88$

$04 / 21 / 88$

$01 / 19 / 88$

$04 / 21 / 88$

$01 / 19 / 88$

$04 / 21 / 88$

$01 / 19 / 88$

$04 / 21 / 88$

$01 / 19 / 88$

$04 / 21 / 88$

$01 / 19 / 88$

$04 / 21 / 88$

$04 / 21 / 88$

$04 / 21 / 88$

$04 / 21 / 88$

$01 / 19 / 88$

$04 / 21 / 88$

$01 / 19 / 88$

$04 / 21 / \overline{1} \overline{8}$
$6-24-35$

7090

6600

23200

21800

203

46500

46500

NR

34100

31900

276000

NR

20
20 
TABLE E.5. (contd)

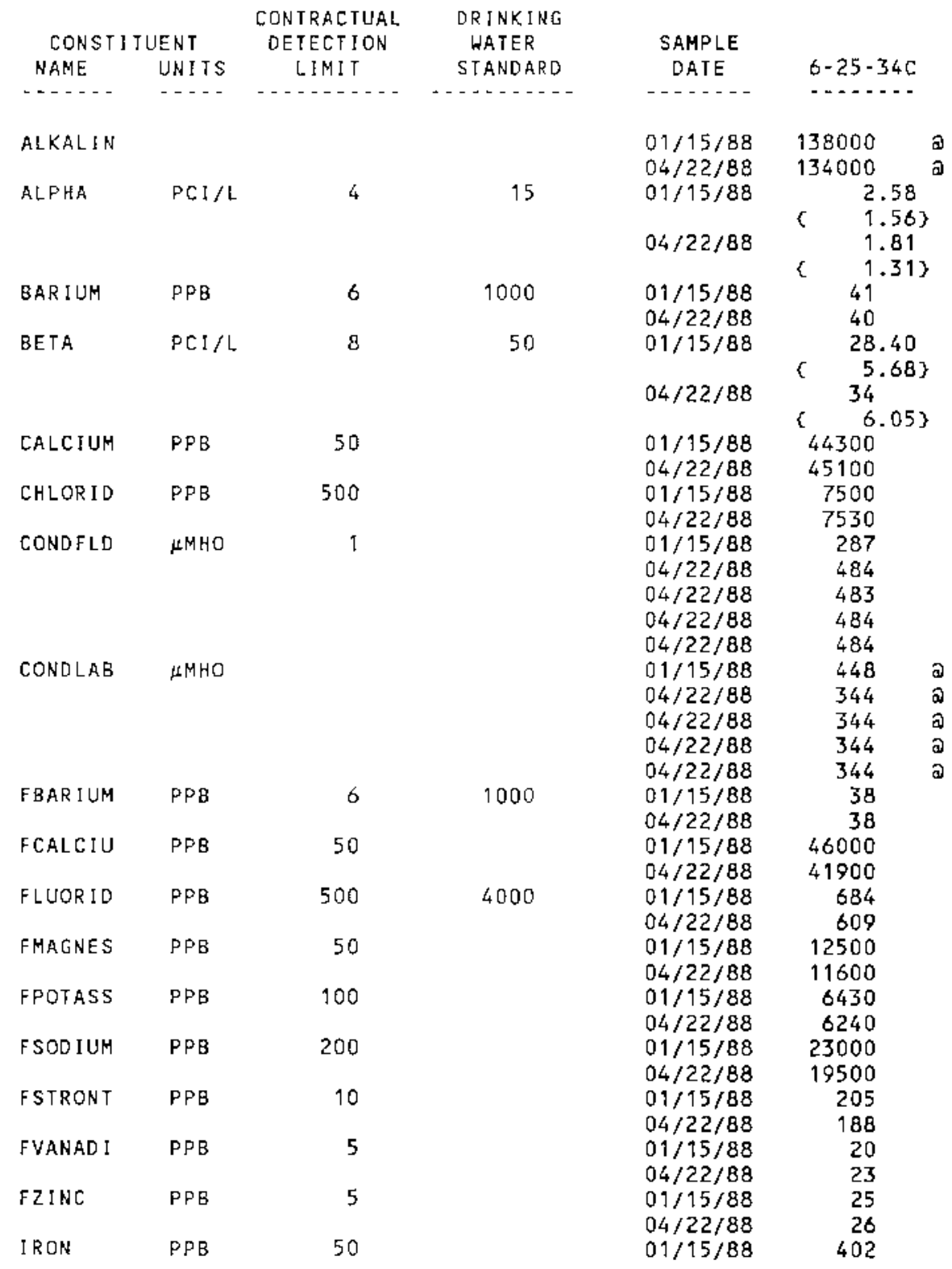


TABLE E.5. (contd)

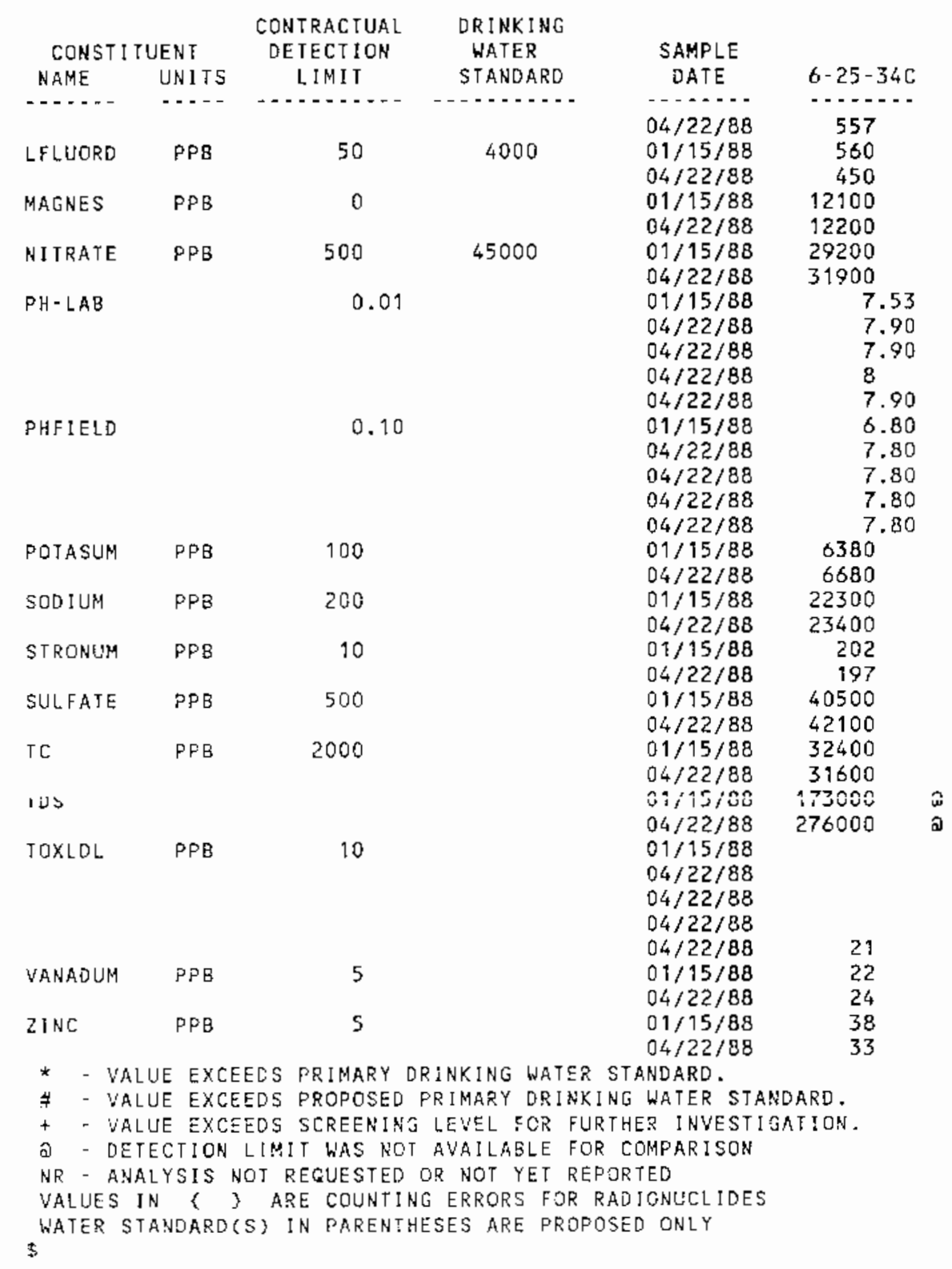


TABLE E.6. Constituents Analyzed for in Ground-Water Samples from Site-Wide Radiological (plus Nitrate) Monitoring Wells Outside the SiteWide Chemical or Compliance Monitoring Networks

$W$
$E$
$L$
$L$
$N$
$A$
$M$
$E$

$$
\begin{aligned}
& 1-B 3-2 P \\
& 1-B 3-2 Q \\
& 1-B 4-1 \\
& 1-B 4-2 \\
& 1-B 4-3 \\
& 1-B 4-4 \\
& 1-B 5-1 \\
& 1-B 9-1
\end{aligned}
$$

$1-\mathrm{D} 2-5$

$1-D 5-12$

$1-\mathrm{D} 8-3$

$1-\mathrm{F} 5-1$

$1-F 5-3$

$1-F 5-6$

$1-\mathrm{F} 8-1$

$1-F 8-2$

$1-K-11$

$1-K-19$

$1-K-20$

$1-K-22$

$1-K-27$

1 $-\mathrm{K}-28$

$1-K-29$

1-K-30

$1-N-5$

$1-N-6$

$1-\mathrm{N}-15$

$1-\mathrm{N}-16$

1 $-\mathrm{N}-18$

$1-N-19$

$1-\mathrm{N}-20$

$1-\mathrm{N}-21$

$1-\mathrm{N}-22$

I-N-23

$1-\mathrm{N}-24$

$1-\mathrm{N}-25$

$1-\mathrm{N}-30$

$1-\mathrm{N}-37$

l $-\mathrm{N}-45$

$$
\begin{array}{cccccccccccccc}
\mathrm{T} & \mathrm{H} & & & & & & & & \mathrm{I} & & & \\
\mathrm{R} & \mathrm{N} & & & & & & & & & & \mathrm{U} & & \mathrm{P} \\
\mathrm{I} & \mathrm{I} & \mathrm{A} & & \mathrm{G} & & \mathrm{N} & \mathrm{S} & \mathrm{T} & 1 & & & \mathrm{U} & \mathrm{U} \\
\mathrm{T} & \mathrm{T} & \mathrm{L} & \mathrm{B} & \mathrm{A} & \mathrm{C} & \mathrm{I} & \mathrm{R} & \mathrm{C} & 2 & & \mathrm{C} & & \\
\mathrm{I} & \mathrm{R} & \mathrm{P} & \mathrm{E} & \mathrm{M} & & & & & 9 & & \mathrm{H} & \mathrm{I} & \mathrm{I} \\
\mathrm{U} & \mathrm{A} & \mathrm{H} & \mathrm{T} & \mathrm{M} & 1 & 6 & 9 & 9 & 0 & & \mathrm{E} & \mathrm{S} & \mathrm{S} \\
\mathrm{M} & \mathrm{T} & \mathrm{A} & \mathrm{A} & \mathrm{A} & 4 & 3 & 0 & 9 & \text { W } & \mathrm{U} & \mathrm{M} & 0 & 0
\end{array}
$$$$
\begin{array}{lllll}
X & X & X & X & \\
X & X & X & X & \\
X & X & & X & X \\
X & X & & X & X \\
X & X & & X & X \\
X & X & & X & X \\
X & X & & X & \\
& X & & &
\end{array}
$$

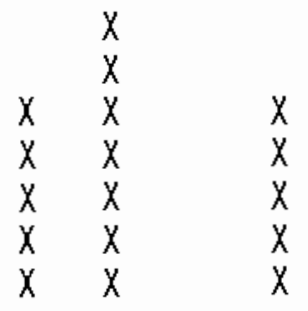$$
\begin{array}{ll}
X & X \\
X & X \\
X & X
\end{array}
$$$$
\begin{array}{llllll}
x & x & x & x \\
X & x & x & x & x & X \\
x & x & x & X
\end{array}
$$

$\begin{array}{ll}X & x \\ X & x \\ X & x \\ x & x \\ X & X\end{array}$

$$
\begin{array}{ll}
x & x \\
x & \\
x & x \\
x & x \\
x & x
\end{array}
$$

$\begin{array}{lll}X & X & X \\ X & x & x \\ X & x & x \\ X & x & X \\ X & x & X\end{array}$

$\begin{array}{ll}X & X \\ X & X \\ X & X \\ X & X \\ X & X \\ X & X \\ X & X \\ X & X\end{array}$

$\begin{array}{ll}X & X \\ X & X \\ X & X \\ X & X \\ X & X \\ X & X \\ X & X \\ X & X\end{array}$

$\begin{array}{lll}X & X & X \\ X & X & X \\ X & X & X \\ X & X & X \\ X & X & X \\ X & X & X \\ X & X & X \\ X & X & X\end{array}$

$\begin{array}{lllll}X & X & & X & X \\ X & X & & X & X \\ X & X & & X & X \\ X & X & & X & \\ X & X & & X & X \\ X & X & X & X & X \\ X & X & & X & X \\ X & X & & X & \\ X & X & & X & X \\ X & X & & X & X \\ X & X & & X & \\ X & X & & X & X \\ X & X & & X & X \\ X & X & & X & X \\ X & X & X & X & X\end{array}$

$\begin{array}{lll}X & X & X \\ X & X & X \\ X & X & X \\ X & X & X \\ X & X & X \\ X & X & X \\ X & X & X \\ X & X & X \\ X & & X \\ X & X & X \\ X & X & X \\ X & X & X \\ X & X & X \\ X & X & X \\ X & X & X\end{array}$


TABLE E.6. (contd)

$W$
$E$
$L$
L
A
$M$
E

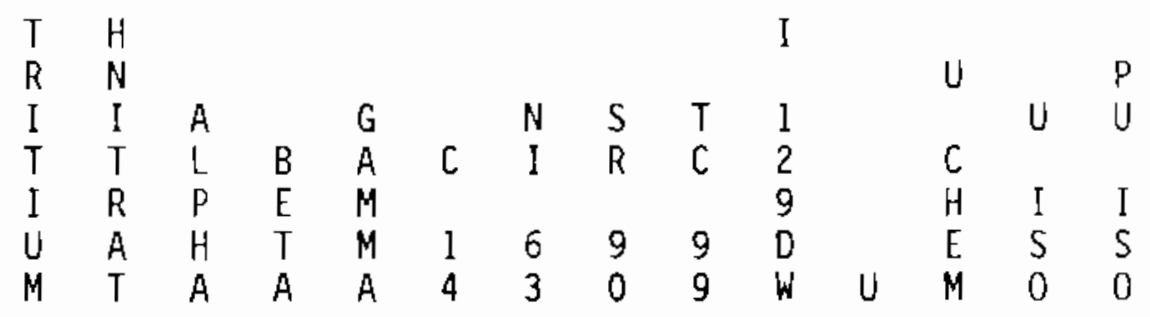

$1-N-50$
$1-N-51$

$\begin{array}{lll}x & x & x \\ x & x & x\end{array}$

$\begin{array}{lll}X & X & X \\ X & X & X\end{array}$

2-E13-8

$2-E 13-19$

$2-\mathrm{E} 17-2$

2-E17-8

$2-\mathrm{E} 17-13$

2-E23-1

$2-\mathrm{E} 24-1$

2-E24-4

2-E24-7

2-E24-8

2-E24-11

2-E24-12

2-E24-13

$2-E 25-2$

2-E25-3

2-E25-6

2-E25-9

2-E25-10

2-E25-11

$2-E 25-13$

$\begin{array}{llllll}X & X & & X & X & \\ X & X & & X & X & \\ X & X & x & X & X & \\ X & X & & X & X & X \\ X & X & x & X & X & \end{array}$

$\begin{array}{lll}X & X & X \\ X & X & X \\ X & & \end{array}$

$x$
$x$

$x$

2-E25-17

$2-E 26-1$

2-E26-2

2-E26-3

2-E26-4

$2-E 26-6$

2-E26-8

$2-E 27-1$

$2-E 27-5$

2-E27-7

2-E28-]

2-E28-5

2-E28-9

2-E28-12

$2-\mathrm{E} 28-16$

2-E28-17

2-E28-19

2-E28-24

2-E28-25

$2-E 33-7$

$x$

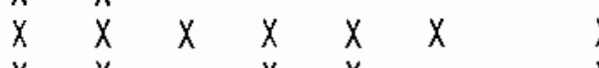

$x \quad x$

$\begin{array}{ll}x & x \\ x & \end{array}$

$\dot{x} x$

$x \quad x$

$x \quad x$

$x \quad x$

$x \quad x$

$x \quad x$

$x \quad x$

$\begin{array}{lllll}X & X & X & X\end{array}$

$\begin{array}{lllll}X & X & X & X\end{array}$

$x \quad \begin{array}{lll}X & X & X \\ X & X & X\end{array}$

$\begin{array}{ll}x & x \\ x & x\end{array}$

$\begin{array}{lll}x & x \\ x & x & x\end{array}$

$x \quad x \quad x \quad x \quad x$

$\begin{array}{ll}x & x \\ x & x\end{array}$

$x \quad x$

$\begin{array}{ll}x & x \\ x & x\end{array}$

$\begin{array}{lllll}x & x & & x & \\ x & x & x & x & x\end{array}$

$\begin{array}{llll}\hat{x} & \hat{x} & \hat{x} & \hat{x}\end{array}$

$\begin{array}{llll}x & x & x & \\ x & x & x & x\end{array}$

$x$
$x$

$x$
$x$
$x$

$x$

$\begin{array}{ll}x & x \\ x\end{array}$

$\begin{array}{llll}x & x & & x \\ x & x & x\end{array}$

$x \quad x \quad x$

$x$

$X$

$\begin{array}{lll}X & & \\ X & & \\ X & & \\ X & & \\ X & x & \\ X & & X \\ X & X & X\end{array}$

$x \quad x$

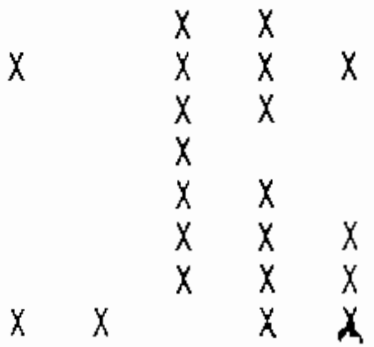

$x$

x

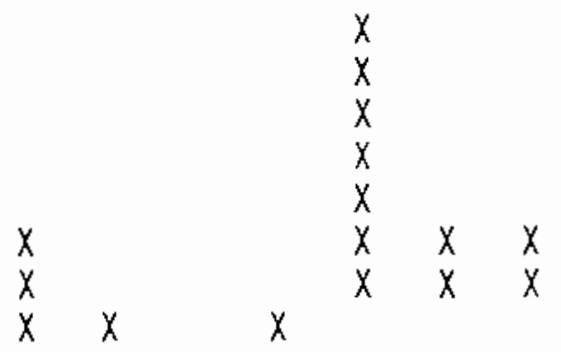

E. 314 
TABLE E.6. (contd)

$W$
$E$
$L$
$L$
$N$
$A$
$M$
$E$

$\begin{array}{cccccccccccccc}\mathrm{T} & \mathrm{H} & & & & & & & & \mathrm{I} & & & \\ \mathrm{R} & \mathrm{N} & & & & & & & & & & \mathrm{U} & & \mathrm{P} \\ \mathrm{I} & \mathrm{I} & \mathrm{A} & & \mathrm{G} & & \mathrm{N} & \mathrm{S} & \mathrm{T} & 1 & & & \mathrm{U} & \mathrm{U} \\ \mathrm{T} & \mathrm{T} & \mathrm{L} & \mathrm{B} & \mathrm{A} & \mathrm{C} & \mathrm{I} & \mathrm{R} & \mathrm{C} & 2 & & \mathrm{C} & & \\ \mathrm{I} & \mathrm{R} & \mathrm{P} & \mathrm{E} & \mathrm{M} & & & & & 9 & & \mathrm{H} & \mathrm{I} & \mathrm{I} \\ \mathrm{U} & \mathrm{A} & \mathrm{H} & \mathrm{T} & \mathrm{M} & 1 & 6 & 9 & 9 & \mathrm{D} & & \mathrm{E} & \mathrm{S} & \mathrm{S} \\ \mathrm{M} & \mathrm{T} & \mathrm{A} & \mathrm{A} & \mathrm{A} & 4 & 3 & 0 & 9 & \text { W } & \mathrm{U} & \mathrm{M} & 0 & 0\end{array}$

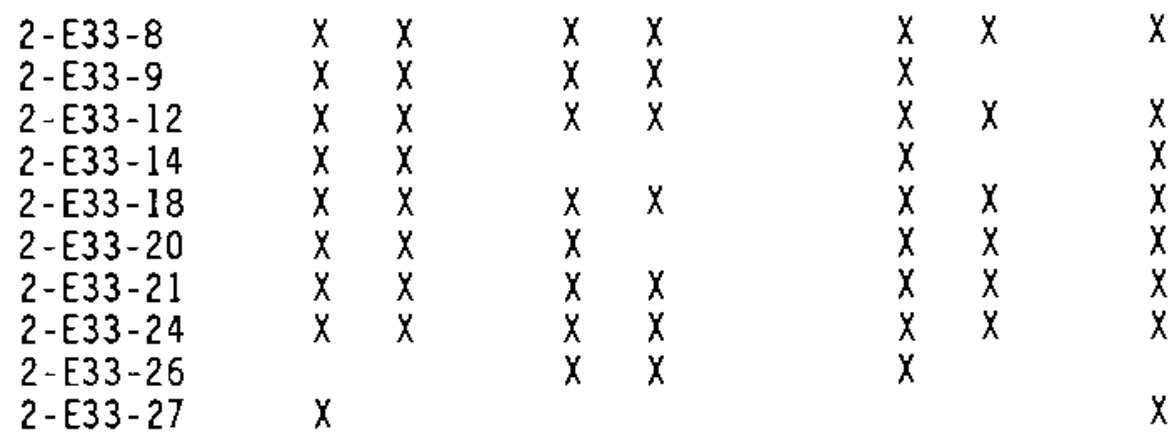

2-W6-1

2-W10-1

$2-W 10-3$

2-W10-5

$2-W 10-8$

2-W11-3

2-W11-9

2-W11-11

2-W11-15

$2-W 11-18$

2-W11-23

2-W11-24

2-W12-1

$2-W 15-2$

$2-W 15-3$

$2-W 15-6$

$2-W 18-3$

$2-W 18-7$

$2-W 18-20$

2-W19-1

2-W19-2

2-W19-5

$2-W 19-12$

2-W19-14

2-W19-17

2- $419-19$

2- $419-23$

$2-W 19-25$

2-W19-26

2-W19-27

2-W21-1

2-W22-2

$x \quad x$

$\begin{array}{ll}x & x \\ X & x\end{array}$

$X \quad X \quad x \quad x$

$x$
$x$

$\begin{array}{llll}X & X & & \\ X & X & X\end{array}$

$x$
$x$
$x$

$x \quad x$

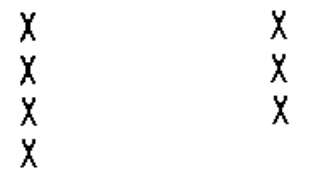

$\begin{array}{lll}x & x \\ x & \end{array}$

$\begin{array}{ll}x & x \\ x & \\ x & \end{array}$

$\begin{array}{lllll}x & x & x & x & \\ x & x & x & x\end{array}$

$x$
$x$
$x$
$x$
$x$
$x$
$x$ 
TABLE E.6. (contd)

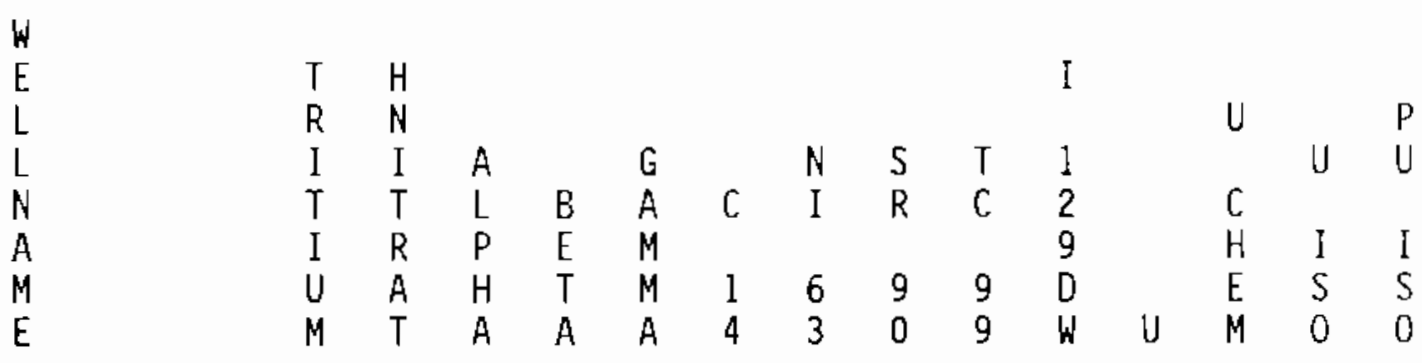

\begin{tabular}{|c|c|c|c|c|c|c|c|c|c|c|}
\hline $2-\omega 22-7$ & $x$ & $x$ & & & & & $x$ & & $x$ & \\
\hline $2-W 22-9$ & $x$ & $x$ & & & & & $x$ & & $x$ & \\
\hline $2-W 22-10$ & $x$ & & $x$ & $x$ & $x$ & & $x$ & & $x$ & \\
\hline $2-W 22-12$ & $x$ & $x$ & & $x$ & $x$ & & $x$ & & $x$ & \\
\hline $2-W 22-18$ & & & $x$ & $x$ & $x$ & & $x$ & $x$ & & \\
\hline $2-W 22-21$ & & $x$ & $x$ & $x$ & $x$ & $X$ & $x$ & & & \\
\hline $2-W 22-26$ & $x$ & $x$ & & $x$ & $x$ & & $x$ & & $X$ & \\
\hline $2-W 23-1$ & $x$ & $x$ & & $x$ & $x$ & & $x$ & & $x$ & \\
\hline $2-w 23-2$ & & $x$ & & $x$ & $x$ & & $x$ & $x$ & $x$ & \\
\hline $2-W 23-3$ & & $x$ & & $x$ & $x$ & & $x$ & & & \\
\hline $2-W 23-4$ & $x$ & $x$ & $x$ & $x$ & & & $x$ & & & $x$ \\
\hline $2-w 23-7$ & & & & $x$ & & & & $x$ & & \\
\hline $2-423-9$ & $x$ & $x$ & $x$ & $x$ & $x$ & & $x$ & $x$ & & $x$ \\
\hline $2-W 23-11$ & $x$ & $x$ & $x$ & $x$ & $x$ & & $x$ & $x$ & & $x$ \\
\hline $2-W 26-3$ & $x$ & $x$ & $X$ & $x$ & & & & & & \\
\hline $2-\omega 26-6$ & $x$ & $x$ & $x$ & $X$ & $x$ & & $x$ & & & \\
\hline
\end{tabular}

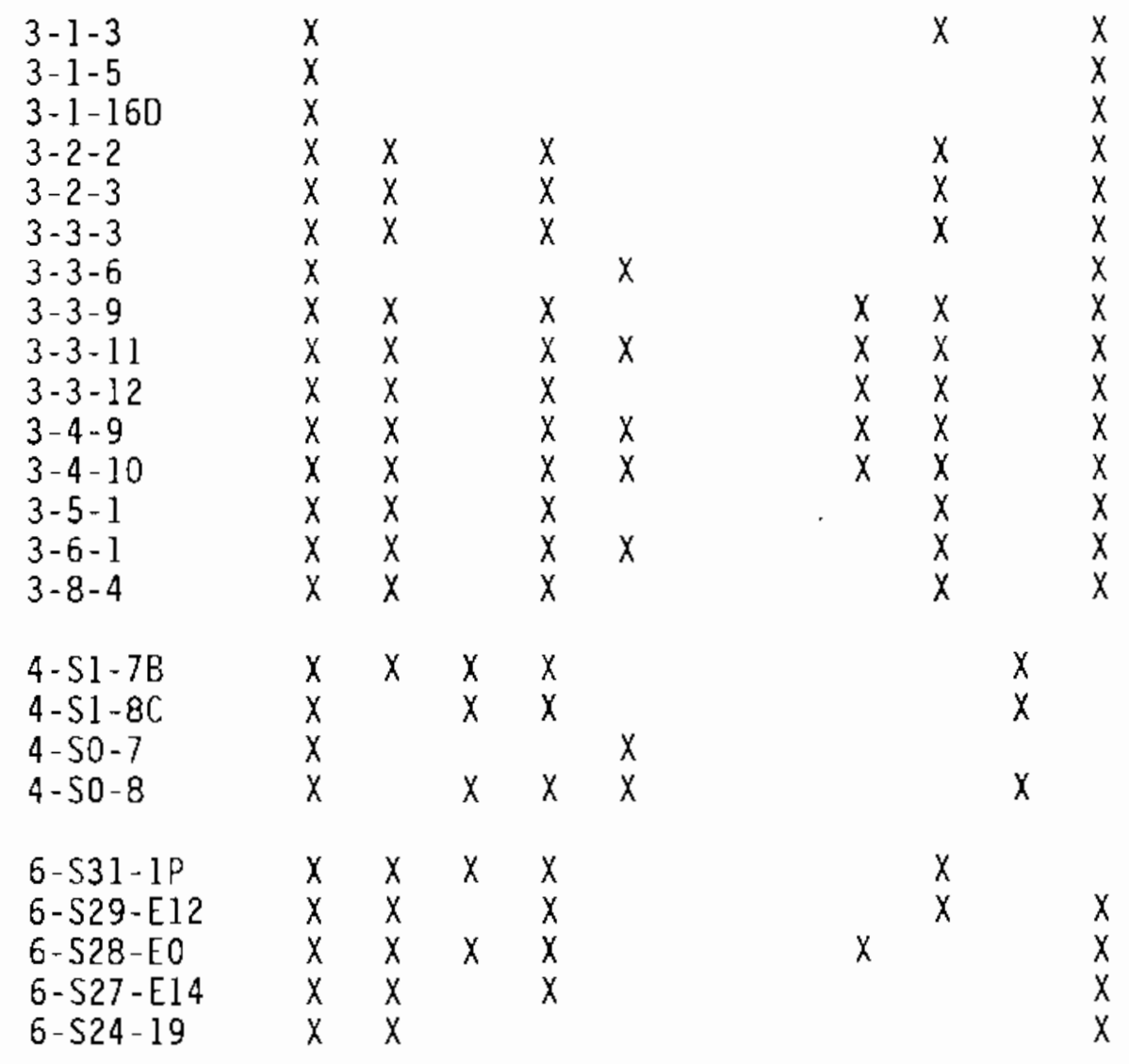


TABLE E.6. (contd)

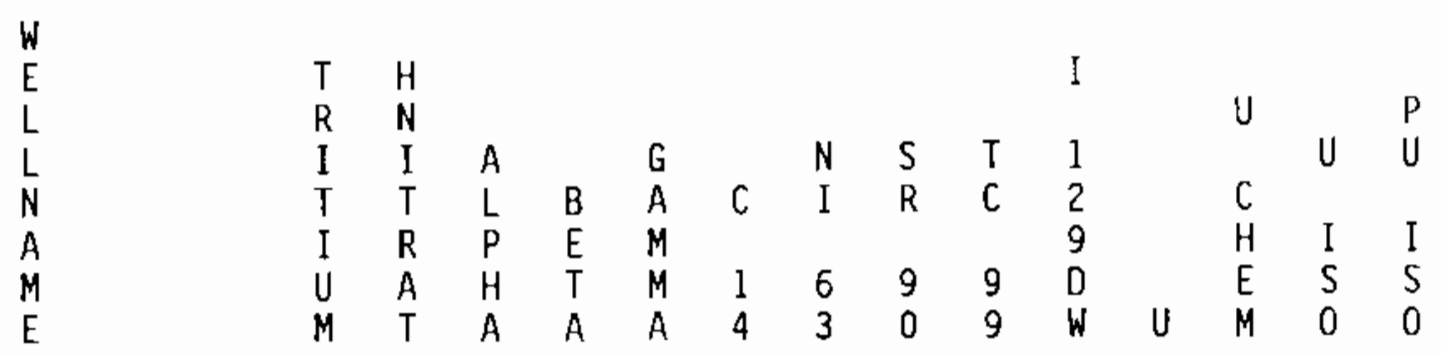

\begin{tabular}{|c|c|c|c|c|c|c|c|c|c|}
\hline $6-519-11$ & $\begin{array}{l}x \\
y\end{array}$ & $\begin{array}{l}X \\
y \\
y\end{array}$ & $\begin{array}{l}x \\
x\end{array}$ & $\begin{array}{l}x \\
x\end{array}$ & & $x$ & $\begin{array}{l}x \\
x\end{array}$ & & \\
\hline $\begin{array}{l}6-\$ 18-51 \\
6-S 14-20 A\end{array}$ & $\begin{array}{l}x \\
x\end{array}$ & $\begin{array}{l}x \\
x\end{array}$ & $x$ & & $x$ & & & & $x$ \\
\hline $6-512-3$ & $x$ & $x$ & $x$ & $x$ & $x$ & & & $x$ & $x$ \\
\hline $6-512-29$ & $x$ & $x$ & $x$ & $x$ & & & & & \\
\hline $6-\mathrm{S} 11-\mathrm{E} 12 \mathrm{~A}$ & $x$ & $x$ & & & & & & & \\
\hline 6-SII-El2AP & $x$ & $x$ & $x$ & $x$ & & & & & \\
\hline $6-58-19$ & $x$ & & $x$ & $x$ & & & & $x$ & \\
\hline $6-S 6-E 14 A$ & $x$ & $x$ & & $x$ & & & $x$ & & \\
\hline $6-56-E 4 B$ & $x$ & $x$ & & $x$ & $x$ & & & $x$ & $x$ \\
\hline $6-S 6-E 4 D$ & $x$ & $x$ & & $x$ & $x$ & & & $x$ & $x$ \\
\hline $6-53-25$ & $x$ & $x$ & $x$ & $x$ & & & & & \\
\hline $6-1-18$ & $x$ & $x$ & & & & & & & \\
\hline $6-2-3$ & $x$ & $x$ & $x$ & $x$ & & & & $x$ & \\
\hline $6-2-7$ & $x$ & $x$ & & & & & & & \\
\hline $6-2-33 A$ & $x$ & $x$ & $x$ & $x$ & & & & $x$ & \\
\hline $6-3-45$ & $x$ & $x$ & & $x$ & & $x$ & $x$ & & $x$ \\
\hline $6-8-17$ & $x$ & $x$ & $x$ & $x$ & & & & $x$ & \\
\hline $6-8-25$ & $x$ & $x$ & $x$ & $x$ & & & & $x$ & \\
\hline $6-8-32$ & $x$ & $x$ & $x$ & $x$ & & & & $x$ & \\
\hline $6-10-54 A$ & $x$ & $x$ & $x$ & $x$ & $x$ & & & & \\
\hline $6-13-64$ & $x$ & $x$ & $x$ & $x$ & & & & & \\
\hline $6-14-E 6 T$ & $x$ & $x$ & $x$ & $x$ & & & & & \\
\hline $6-14-47$ & $x$ & $x$ & $x$ & $\hat{x}$ & & & & & \\
\hline $6-15-15 B$ & $x$ & $x$ & $x$ & $\ddot{x}$ & & & & & \\
\hline $6-15-26$ & $x$ & $x$ & $x$ & $x$ & $x$ & & & $x$ & \\
\hline $6-17-5$ & $x$ & $\hat{x}$ & $x$ & $x$ & & & & & \\
\hline $6-17-47$ & $x$ & $x$ & & & & & & $x$ & \\
\hline $6-17-70$ & $x$ & $x$ & & & & & & & \\
\hline $6-19-58$ & & $x$ & & & & & & & \\
\hline $6-19-88$ & $x$ & $x$ & $x$ & $x$ & & & & & \\
\hline $6-20-E 12$ & $x$ & $x$ & & $x$ & & & & $x$ & $x$ \\
\hline $6-20-E 12 P$ & $\hat{x}$ & $\hat{x}$ & $x$ & $\hat{x}$ & & & $x$ & & \\
\hline $6-20-E 5 A$ & $x$ & $x$ & $x$ & $\hat{x}$ & & & & $x$ & \\
\hline $6-20-E 5 P$ & $x$ & $x$ & $x$ & $x$ & & & $x$ & & \\
\hline $6-20-E 5 Q$ & $x$ & $x$ & $x$ & $x$ & & & $x$ & & \\
\hline $6-20-E 5 R$ & $x$ & $x$ & $x$ & $x$ & & & $x$ & & \\
\hline $6-20-20$ & $x$ & $x$ & & $x$ & & & & $x$ & $x$ \\
\hline $6-20-82$ & $x$ & $x$ & $x$ & $x$ & & & & & \\
\hline $6-21-6$ & $x$ & $x$ & $x$ & $x$ & & & & $x$ & \\
\hline $6-22-70$ & $x$ & $x$ & $x$ & $x$ & & & & & \\
\hline $6-24-1 P$ & $x$ & $x$ & $x$ & $x$ & & & $x$ & & \\
\hline $6-24-10$ & $x$ & $x$ & $x$ & $x$ & & & $x$ & & \\
\hline & & & & & & & & & \\
\hline
\end{tabular}


TABLE E.6. (contd)

W
E
L
L
N
A
$M$
E

$\begin{array}{cccccccccccccc}\mathrm{T} & \mathrm{H} & & & & & & & & \mathrm{I} & & & & \\ R & \mathrm{~N} & & & & & & & & & & \mathrm{U} & & \mathrm{P} \\ \mathrm{I} & \mathrm{I} & \mathrm{A} & & \mathrm{G} & & \mathrm{N} & \mathrm{S} & \mathrm{T} & \mathrm{I} & & & \mathrm{U} & \mathrm{U} \\ \mathrm{T} & \mathrm{T} & \mathrm{L} & \mathrm{B} & \mathrm{A} & \mathrm{C} & \mathrm{I} & \mathrm{R} & \mathrm{C} & 2 & & \mathrm{C} & & \\ \mathrm{I} & \mathrm{R} & \mathrm{P} & \mathrm{E} & \mathrm{M} & & & & & 9 & & \mathrm{H} & \mathrm{I} & \mathrm{I} \\ \mathrm{U} & \mathrm{A} & \mathrm{H} & \mathrm{T} & \mathrm{M} & \mathrm{I} & 6 & 9 & 9 & \mathrm{D} & & \mathrm{E} & \mathrm{S} & \mathrm{S} \\ \mathrm{M} & \mathrm{T} & \mathrm{A} & \mathrm{A} & \mathrm{A} & 4 & 3 & 0 & 9 & \mathrm{~W} & \mathrm{U} & \mathrm{M} & 0 & 0\end{array}$

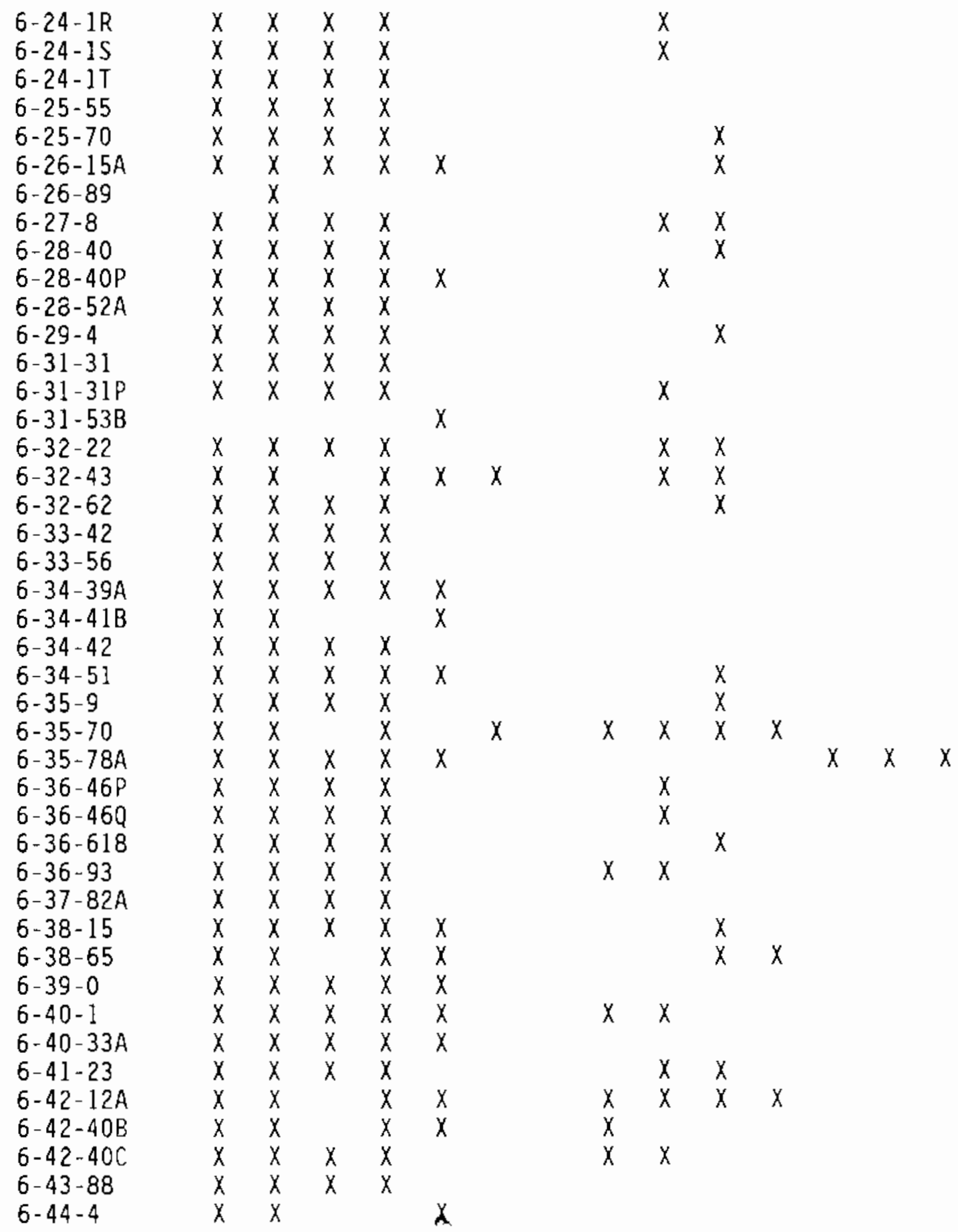


TABLEE.6. (contd)

$W$
$E$
$L$
$L$
$N$
$A$
$M$
$E$

$\begin{array}{cccccccccccccc}\mathrm{T} & \mathrm{H} & & & & & & & & \mathrm{I} & & & & \\ \mathrm{R} & \mathrm{N} & & & & & & & & & & \mathrm{U} & & \mathrm{P} \\ \mathrm{I} & \mathrm{I} & \mathrm{A} & & \mathrm{G} & & \mathrm{N} & \mathrm{S} & \mathrm{T} & 1 & & & \mathrm{U} & \mathrm{U} \\ \mathrm{T} & \mathrm{T} & \mathrm{L} & \mathrm{B} & \mathrm{A} & \mathrm{C} & \mathrm{I} & \mathrm{R} & \mathrm{C} & 2 & & \mathrm{C} & & \\ \mathrm{I} & \mathrm{R} & \mathrm{P} & \mathrm{E} & \mathrm{M} & & & & & 9 & & \mathrm{H} & \mathrm{I} & \mathrm{I} \\ \mathrm{U} & \mathrm{A} & \mathrm{H} & \mathrm{T} & \mathrm{M} & 1 & 6 & 9 & 9 & \mathrm{D} & & \mathrm{E} & \mathrm{S} & \mathrm{S} \\ \mathrm{M} & \mathrm{T} & \mathrm{A} & \mathrm{A} & \mathrm{A} & 4 & 3 & 0 & 9 & \mathrm{~W} & \mathrm{U} & \mathrm{M} & 0 & 0\end{array}$

$6-45-69 A$

$\begin{array}{llll}x & x & x & x\end{array}$

$6-46-21 B$

$6-47-35 A$

$6-48-7$

$6-48-18$

$6-48-71$

$6-49-13 \mathrm{E}$

$6-49-28$

$6-49-55 B$

6-49-100C

$6-50-30$

$6-50-42$

$6-50-45$

$6-50-48 B$

$6-51-46$

$6-51-63$

$6-51-75$

$6-52-19$

$6-52-46 A$

$6-52-48$

$6-53-47 B$

$6-53-48 A$

$6-53-50$

$6-53-55 A$

$6-53-103$

$6-54-34$

$6-54-45 A$

$6-54-48$

$6-54-49$

$6-54-57$

$6-55-40$

$6-55-44$

$6-55-50 A$

$6-55-50 D$

$6-55-70$

$6-55-89$

$6-56-43$

$6-56-53$

6-57-29A

$6-59-5 B$

$6-59-80 B$

$6-60-57$

$6-60-60$

$\begin{array}{llll}X & X & X & X\end{array}$

$\begin{array}{llll}X & X & X & X\end{array}$

$\begin{array}{llll}x & x & x & x\end{array}$

$\begin{array}{llll}X & X & X & x\end{array}$

$\begin{array}{llll}X & X & x & x\end{array}$

$\begin{array}{llll}x & x & x & x\end{array}$

$\begin{array}{llll}x & x & x & x \\ X & x & x & x\end{array}$

$\begin{array}{llll}X & x & X & x \\ X & x & x & x\end{array}$

$\begin{array}{llll}X & X & X & X \\ X & X & X & X\end{array}$

$\begin{array}{llll}X & X & X & X \\ X & X & X & X\end{array}$

$\begin{array}{llll}X & \hat{X} & \hat{X} & \hat{X}\end{array}$

$\begin{array}{llll}X & x & x & x\end{array}$

$\begin{array}{llll}x & X & X & x\end{array}$

$\begin{array}{lll}x & X & x\end{array}$

$x \quad x \quad x \quad x$

$\begin{array}{llll}x & X & X & x\end{array}$

$\begin{array}{llll}X & X & X & X\end{array}$

$x \quad x \quad x \quad x$

$x \quad x$

$x \quad x \quad x$
$x \quad x$

$x \quad x \quad x$

$\begin{array}{llll}X & X & X & X\end{array}$

$\begin{array}{llll}X & X & X & X\end{array}$

$\begin{array}{llll}X & X & X & X\end{array}$

$\begin{array}{lllll} & & x & x & x \\ & x & x & \\ x & x & x & x & \end{array}$

$x \quad x$

$x \quad x$

$\begin{array}{llll}x & x & x & x \\ X & x & x & \end{array}$

$\begin{array}{llll}X & X & X & X \\ X & x & & x\end{array}$

$X \quad X \quad X \quad X$

$x \quad x \quad x \quad x$

$\begin{array}{llll}x & x & x & x\end{array}$

$x \quad x \quad x$

$X X X$

$x$

$\begin{array}{ll}X & X \\ X & x\end{array}$

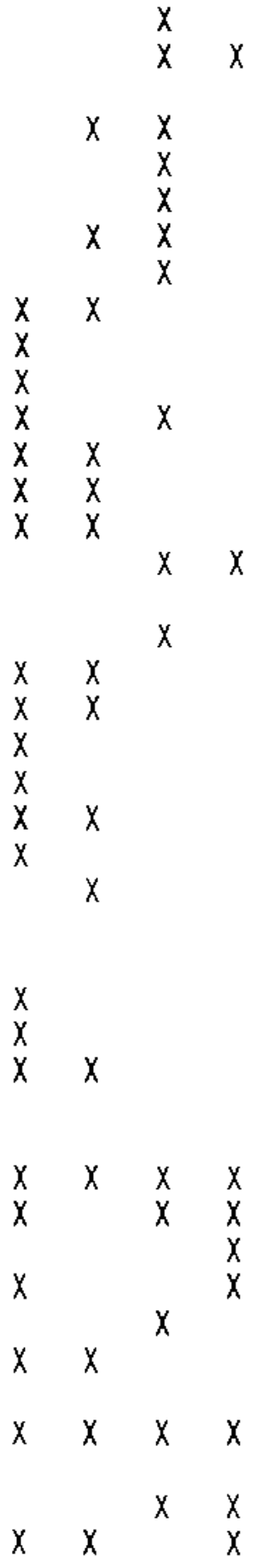


TABLE E.6. (contd)

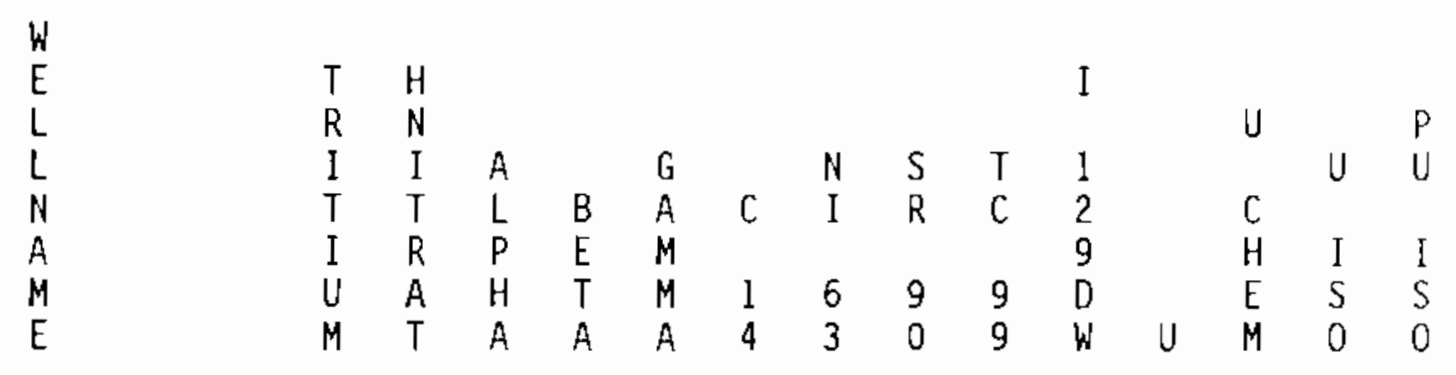

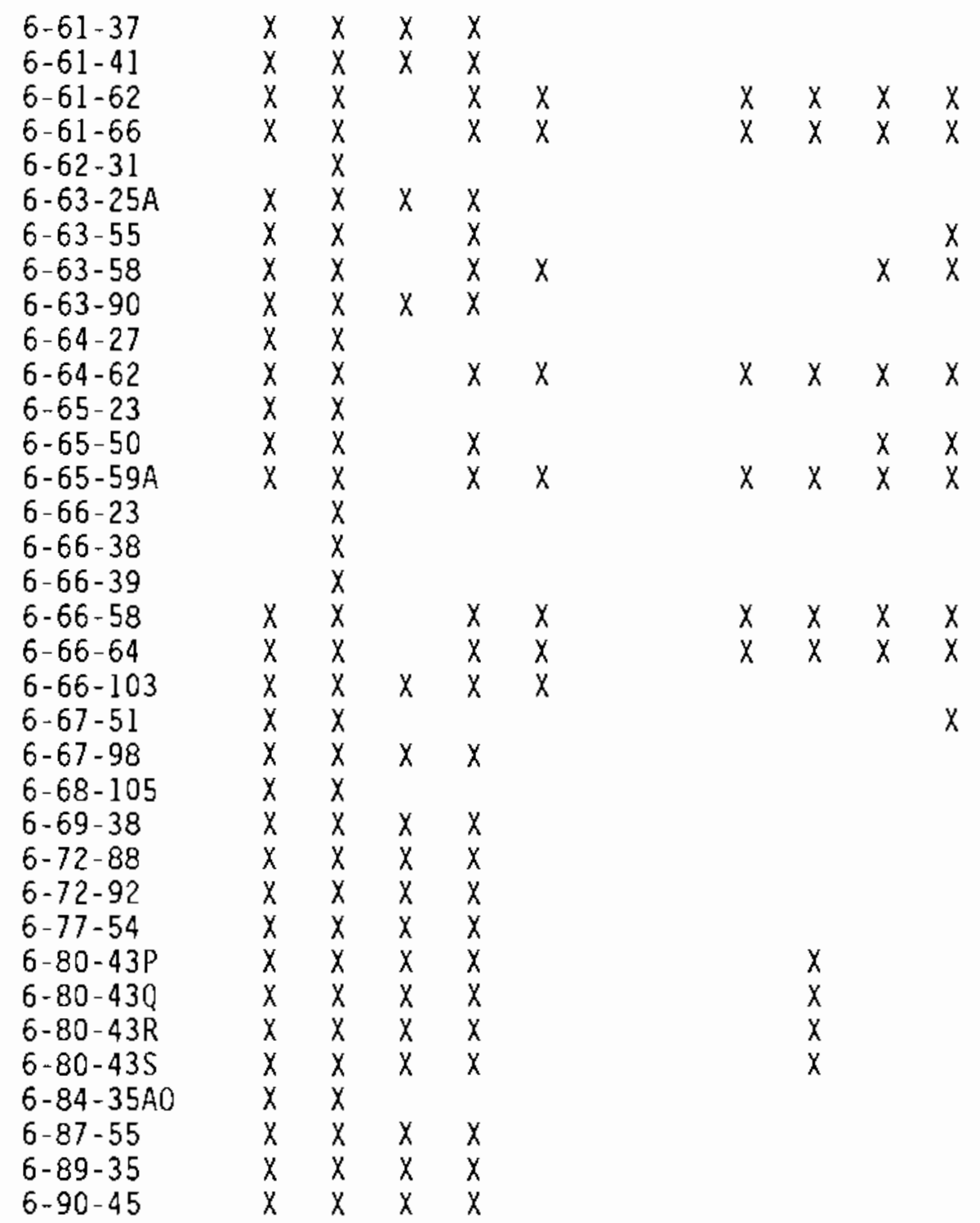


IABLE E.7. Results for Site-Wide Radiological (plus Nitrate) Monitoring Wells Outside the Site-Wide Chemical or Compliance Monitoring Networks

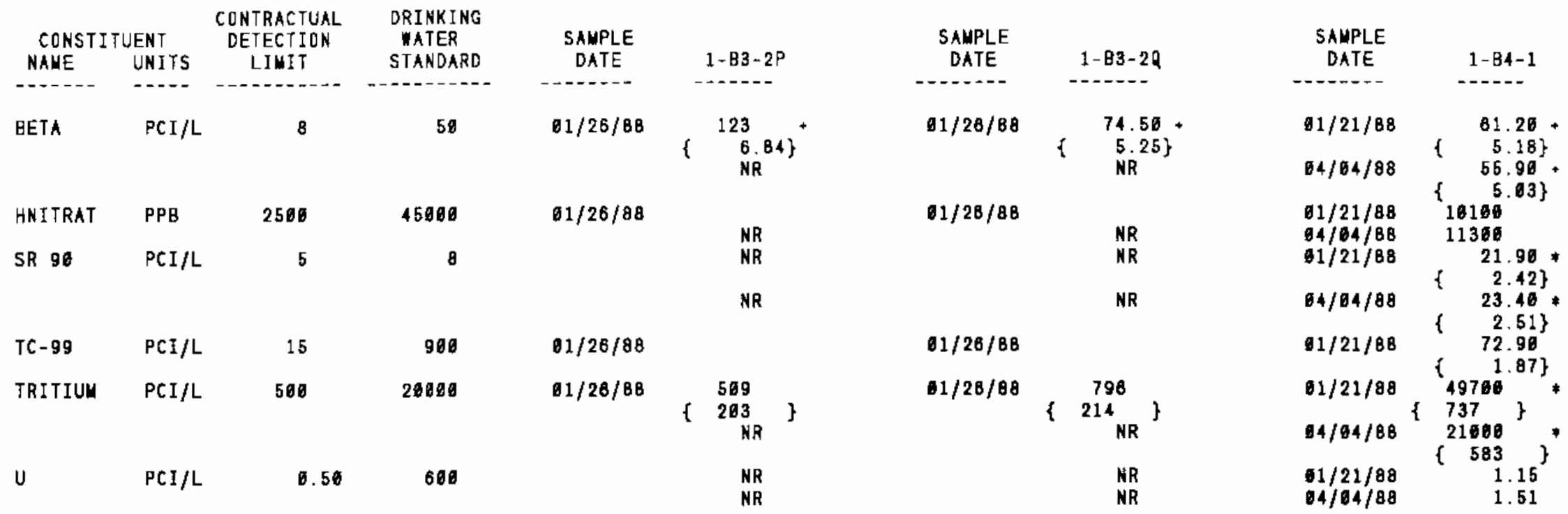


TABLE E.7. (contd)

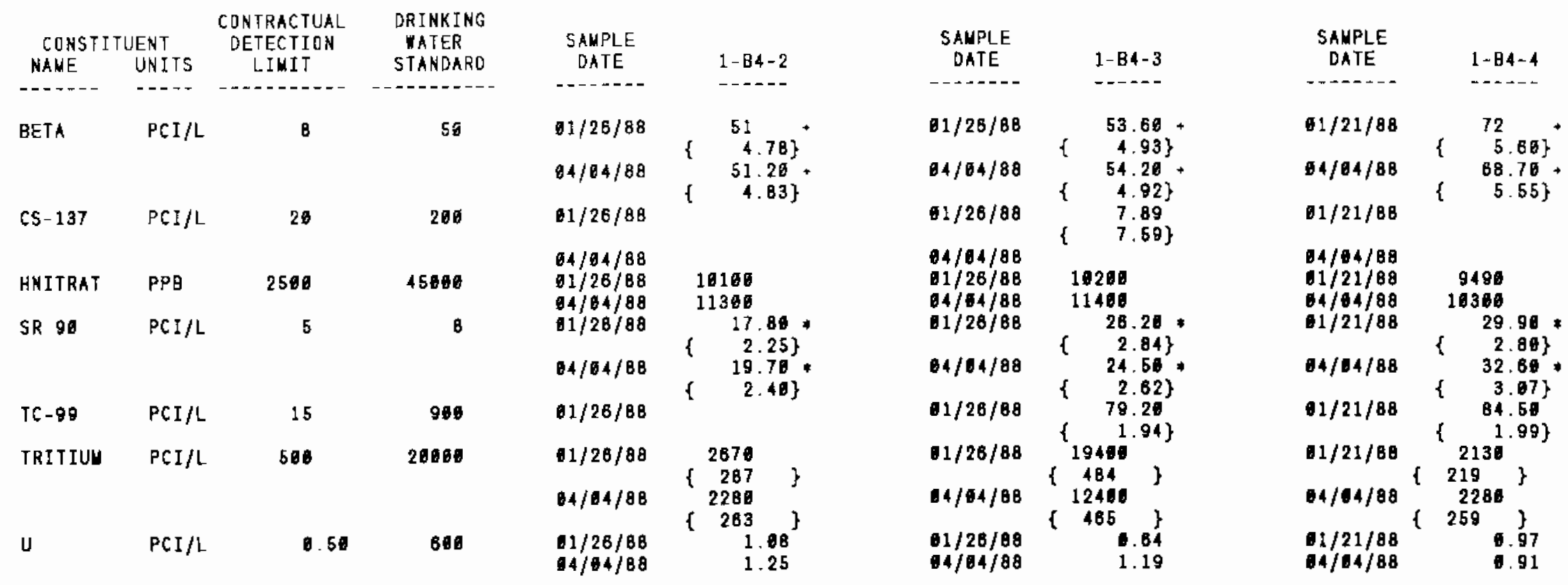


TABLE E.7. (contd)

\begin{tabular}{|c|c|c|c|c|c|c|c|c|c|}
\hline $\begin{array}{l}\text { CONSTI } \\
\text { NAME }\end{array}$ & $\begin{array}{l}\text { JENT } \\
\text { UNITS }\end{array}$ & $\begin{array}{l}\text { CONTRACTUAL } \\
\text { DETECTION } \\
\text { LIUIT }\end{array}$ & $\begin{array}{l}\text { DRINKING } \\
\text { TATER } \\
\text { STANDARD }\end{array}$ & $\begin{array}{l}\text { SAMPLE } \\
\text { DATE }\end{array}$ & $1-85-1$ & $\begin{array}{l}\text { SAMPLE } \\
\text { DATE }\end{array}$ & $1-89-1$ & $\begin{array}{l}\text { SAMPLE } \\
\text { DATE }\end{array}$ & $1-D 2-5$ \\
\hline BETA & PCI/L & $B$ & 59 & $\begin{array}{l}01 / 21 / 88 \\
04 / 84 / 88\end{array}$ & $\begin{array}{r}19.10 \\
\{\quad 3.84\} \\
19.20 \\
\{\quad 3.65\}\end{array}$ & & $\begin{array}{l}\text { NR } \\
\text { HR }\end{array}$ & $\begin{array}{l}61 / 11 / 88 \\
64 / 15 / 88\end{array}$ & $\begin{array}{ll} & 7.64 \\
\{ & 2.22\} \\
\{\quad 5.85 \\
\{\quad 2.28\end{array}$ \\
\hline HNITRAT & PPB & 2509 & 45000 & $\begin{array}{l}91 / 21 / 88 \\
04 / 94 / 88\end{array}$ & $\begin{array}{r}9720 \\
16900\end{array}$ & $\begin{array}{l}01 / 21 / 88 \\
04 / 04 / 88\end{array}$ & $\begin{array}{l}22100 \\
21900\end{array}$ & $\begin{array}{l}01 / 11 / 88 \\
04 / 15 / 88\end{array}$ & $\begin{array}{l}87500 \\
80300\end{array}$ \\
\hline SR 9 A & $\mathrm{PCI} / \mathrm{L}$ & 5 & 8 & $\begin{array}{l}01 / 21 / 88 \\
04 / 84 / 68\end{array}$ & $\begin{array}{ll}\{\quad & 2.18 \\
\{\quad 1.98\} \\
\{\quad 1.54 \\
\{.86\}\end{array}$ & & $\begin{array}{l}\text { NR } \\
\text { MR }\end{array}$ & $\begin{array}{l}01 / 11 / 88 \\
04 / 16 / 88\end{array}$ & \\
\hline TC-99 & $\mathrm{PCI} / \mathrm{L}$ & 15 & 966 & $01 / 21 / 88$ & $\begin{array}{l}119 \\
\{2.23\}\end{array}$ & & NR & $61 / 11 / 88$ & \\
\hline TRITIUY & $\mathrm{PCI} / \mathrm{L}$ & 500 & 26066 & $\begin{array}{l}81 / 21 / 68 \\
64 / 64 / 88\end{array}$ & $\left.\begin{array}{r}1476 \\
202 \\
1580\end{array}\right\}$ & & $\begin{array}{l}\text { MR } \\
\text { MR }\end{array}$ & $\begin{array}{l}61 / 11 / 88 \\
04 / 16 / 88\end{array}$ & $\left\{\begin{array}{c}32760 \\
692 \\
33560\end{array}\right\}$ \\
\hline u & $P C I / L$ & 0.58 & 880 & $\begin{array}{l}01 / 21 / 88 \\
04 / 84 / 86\end{array}$ & $\begin{array}{r}239.58^{8} \\
1.65\end{array}$ & & $\begin{array}{l}\text { NR } \\
\text { NR }\end{array}$ & $\begin{array}{l}01 / 11 / 88 \\
0 / / 15 / 8 \theta\end{array}$ & $\begin{array}{r}899 \\
2.48 \\
2.25\end{array}$ \\
\hline
\end{tabular}


IABLE E.7. (contd)

\begin{tabular}{|c|c|c|c|c|c|c|c|c|c|}
\hline $\begin{array}{l}\text { CONSTI } \\
\text { NAME }\end{array}$ & $\begin{array}{l}\text { UENT } \\
\text { UNITS }\end{array}$ & $\begin{array}{l}\text { CONTRACTUAL } \\
\text { OETECTION } \\
\text { LIUIT }\end{array}$ & $\begin{array}{l}\text { DRINKING } \\
\text { WATER } \\
\text { STANDARD }\end{array}$ & $\begin{array}{l}\text { SAMPLE } \\
\text { DATE }\end{array}$ & $1-05-12$ & $\begin{array}{l}\text { SALPLE } \\
\text { DATE }\end{array}$ & $1-D 8-3$ & $\begin{array}{l}\text { SALPLE } \\
\text { DATE }\end{array}$ & $1-F 5-1$ \\
\hline$---\infty$ & $\ldots-$ & -...- & $---1,-c--$ & $-\cdots-n$ & 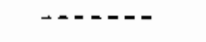 & 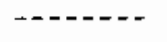 & ----- & $\cdots$ & ----- \\
\hline BETA & PCI/L & 8 & 56 & $\begin{array}{l}61 / 11 / 88 \\
04 / 08 / 88\end{array}$ & $\begin{array}{c}80.70 \\
6.42\} \\
79.60 \\
\{\quad 8.35\end{array}{ }^{+}{ }^{+}$ & $\begin{array}{l}01 / 11 / 88 \\
04 / 15 / 88\end{array}$ & $\left\{\begin{array}{l}9.61 \\
2.23\} \\
8.92 \\
\{\quad 2.23\}\end{array}\right.$ & $\begin{array}{l}01 / 11 / 88 \\
04 / 06 / 88\end{array}$ & $\begin{array}{c}35.30 \\
\{\quad 3.87\} \\
47.40 \\
\{\quad 4.59\}\end{array}$ \\
\hline HNITRAT & PPB & 2500 & 45060 & $\begin{array}{l}01 / 11 / 88 \\
04 / 68 / 88\end{array}$ & $\begin{array}{l}73800 \\
60400\end{array}$ & $\begin{array}{l}01 / 11 / 88 \\
04 / 15 / 88\end{array}$ & $\begin{array}{l}38700 \\
41500\end{array}$ & $\begin{array}{l}91 / 11 / 88 \\
04 / 08 / 88\end{array}$ & $\begin{array}{l}3290 \\
9428\end{array}$ \\
\hline$N I-B 3$ & $\mathrm{PCI} / \mathrm{L}$ & 10 & 50 & $\begin{array}{l}01 / 11 / 88 \\
04 / 98 / 88\end{array}$ & $\begin{array}{ll} & 2.46 \\
\{\quad 1.51\}\end{array}$ & & NR & 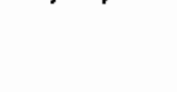 & NR \\
\hline$S R 90$ & $\mathrm{PCI} / \mathrm{L}$ & 5 & B & $04 / 68 / 88$ & $\left\{\begin{array}{c}39.76 \\
3.23 \\
36.4\}^{*} \\
\{\quad 3.15\}\end{array}\right.$ & $\begin{array}{l}01 / 11 / 88 \\
04 / 15 / 88\end{array}$ & $\begin{array}{ll} & 2.83 \\
\{\quad 1.07\} \\
2.83 \\
\{\quad 1.08\}\end{array}$ & $\begin{array}{l}01 / 11 / 88 \\
04 / 06 / 88\end{array}$ & $\begin{array}{c}20.30 \\
\{\quad 2.35\} \\
\{\quad 2.46 \\
2.53\end{array}$ \\
\hline TRITIUL & PCI /L & 500 & 20800 & $\begin{array}{l}01 / 11 / 88 \\
04 / 88 / 88\end{array}$ & 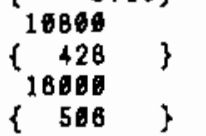 & $\begin{array}{l}61 / 11 / 88 \\
64 / 15 / 88\end{array}$ & $\left.\begin{array}{l}1440 \\
318 \\
3990 \\
212\end{array}\right\}$ & $\begin{array}{l}01 / 11 / 88 \\
04 / 66 / 88\end{array}$ & $\left.210^{581}\right\}$ \\
\hline $\mathrm{U}$ & $\mathrm{PCI} / \mathrm{L}$ & 0.50 & 600 & $\begin{array}{l}01 / 11 / 88 \\
04 / 08 / 88\end{array}$ & $\begin{array}{l}1.80^{\circ} \\
1.84\end{array}$ & $\begin{array}{l}01 / 11 / 88 \\
04 / 15 / 80\end{array}$ & $\begin{array}{r}.54 \\
.56\end{array}$ & $\begin{array}{l}01 / 11 / 88 \\
64 / 68 / 88\end{array}$ & $\begin{array}{r}0.52 \\
0.95\end{array}$ \\
\hline
\end{tabular}


TABLE E.7. (contd)

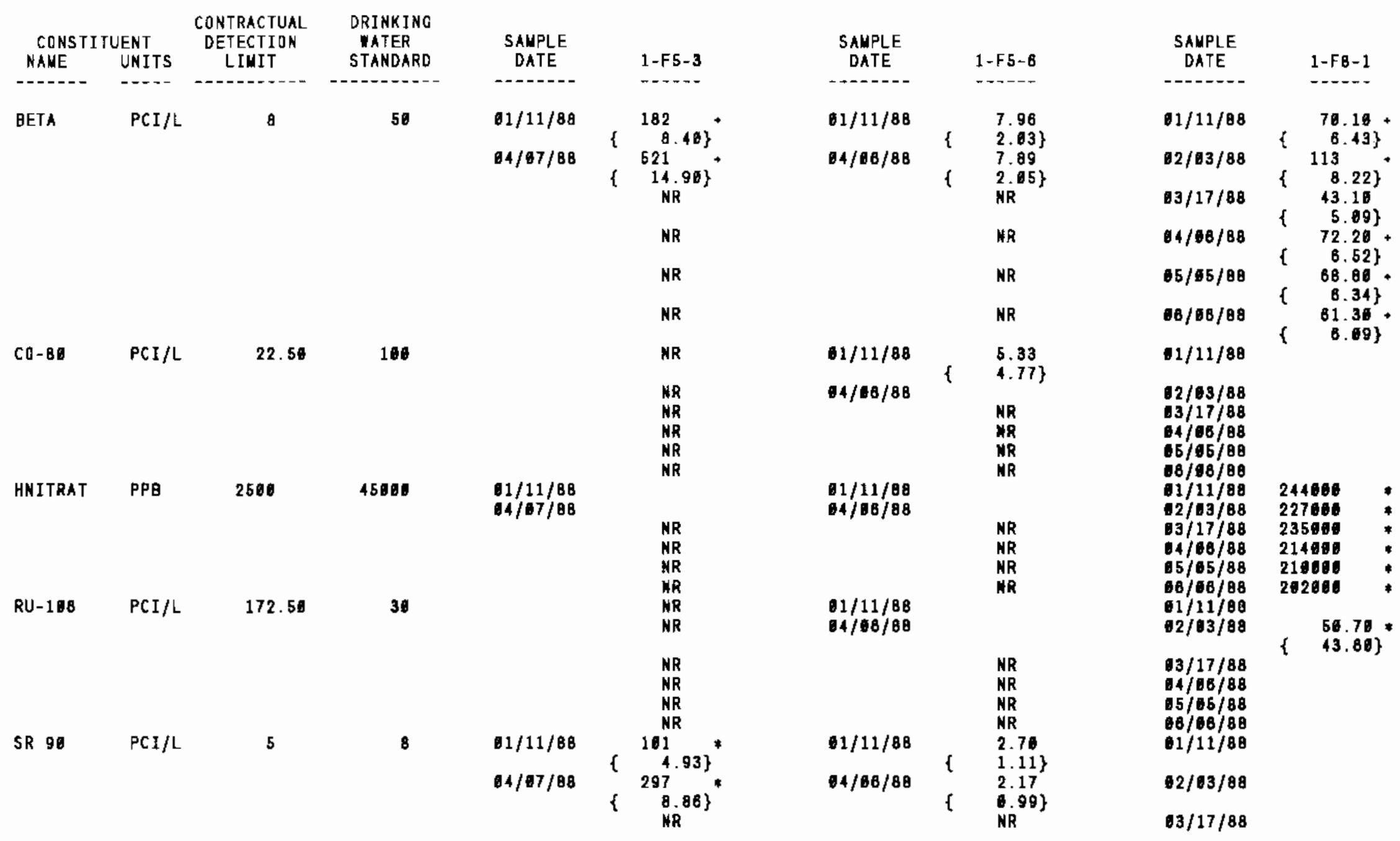


TABLE E.7. (contd)

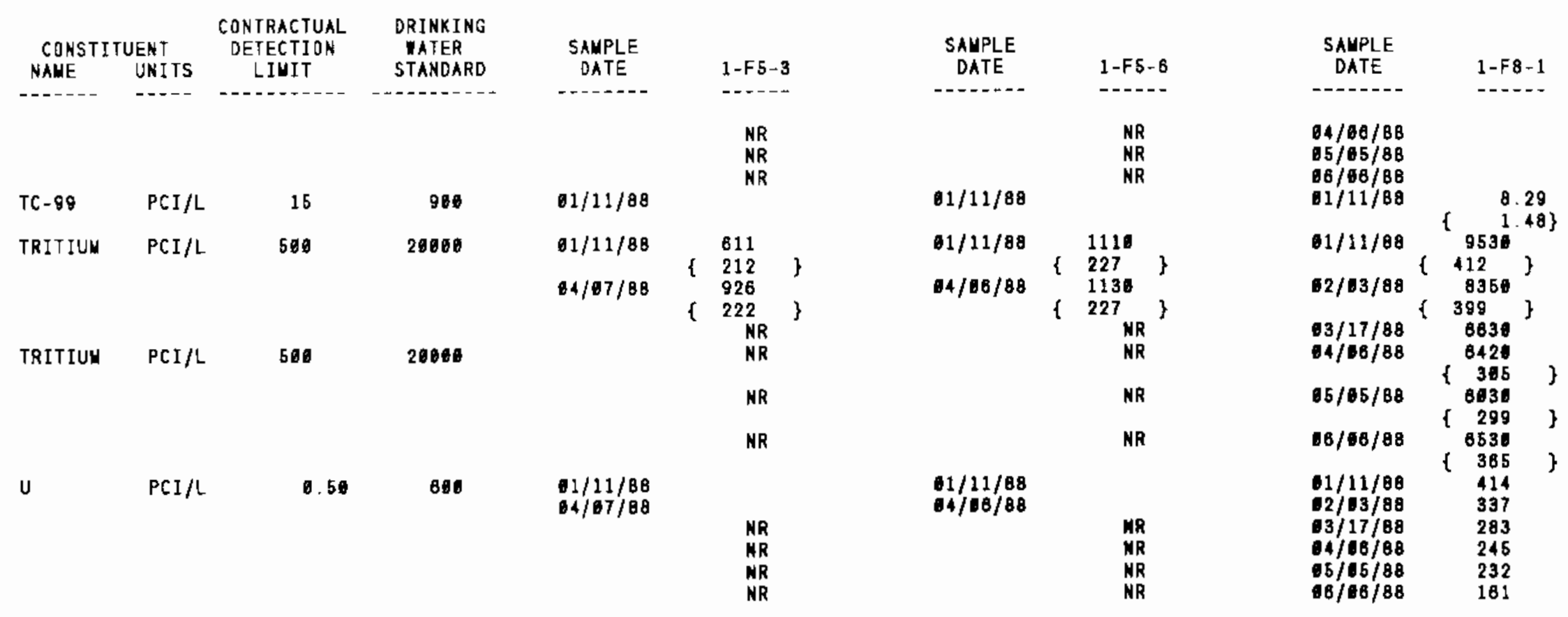


TABLE E.7. (contd)

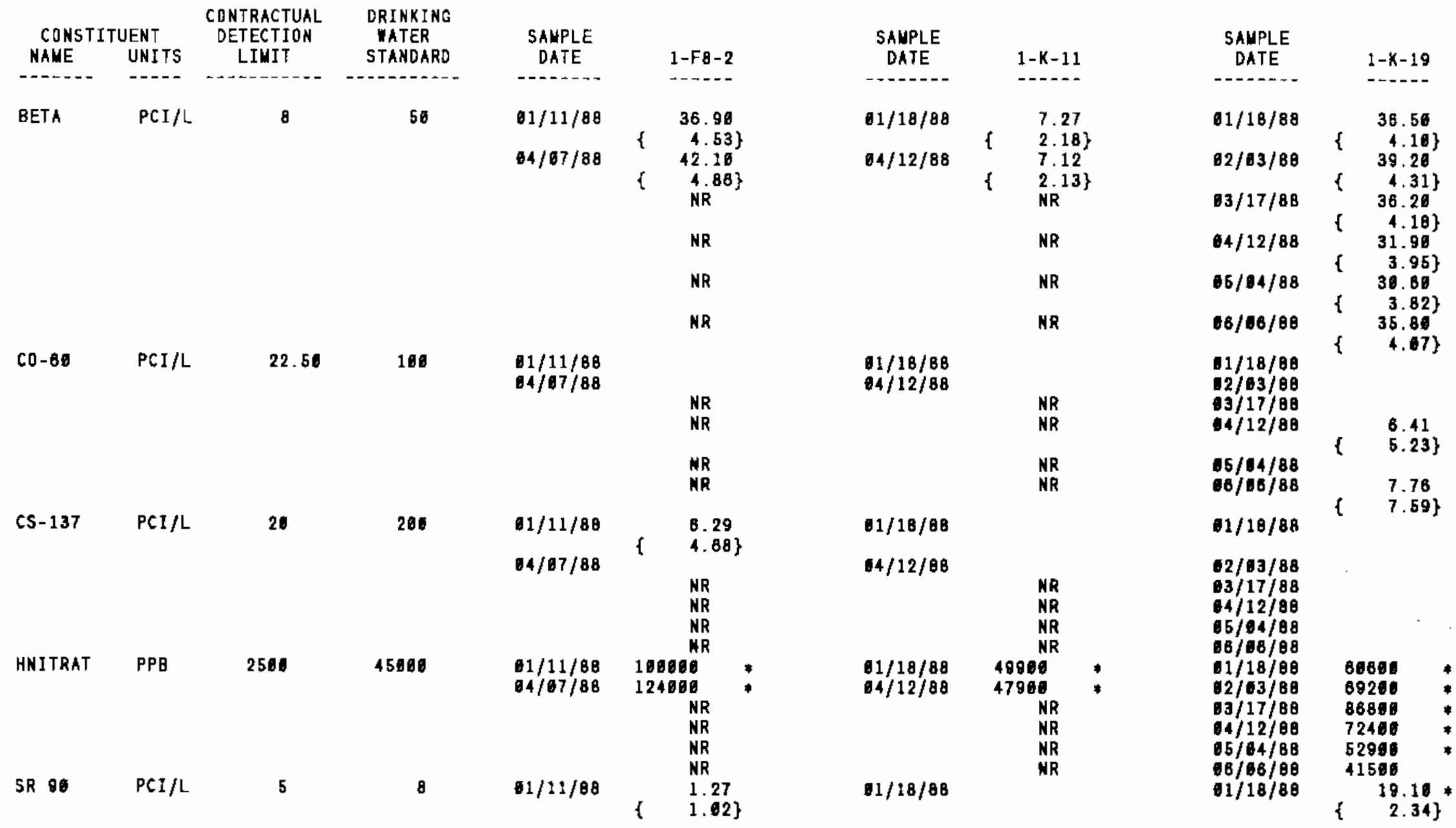


TABLE E.7. (contd)

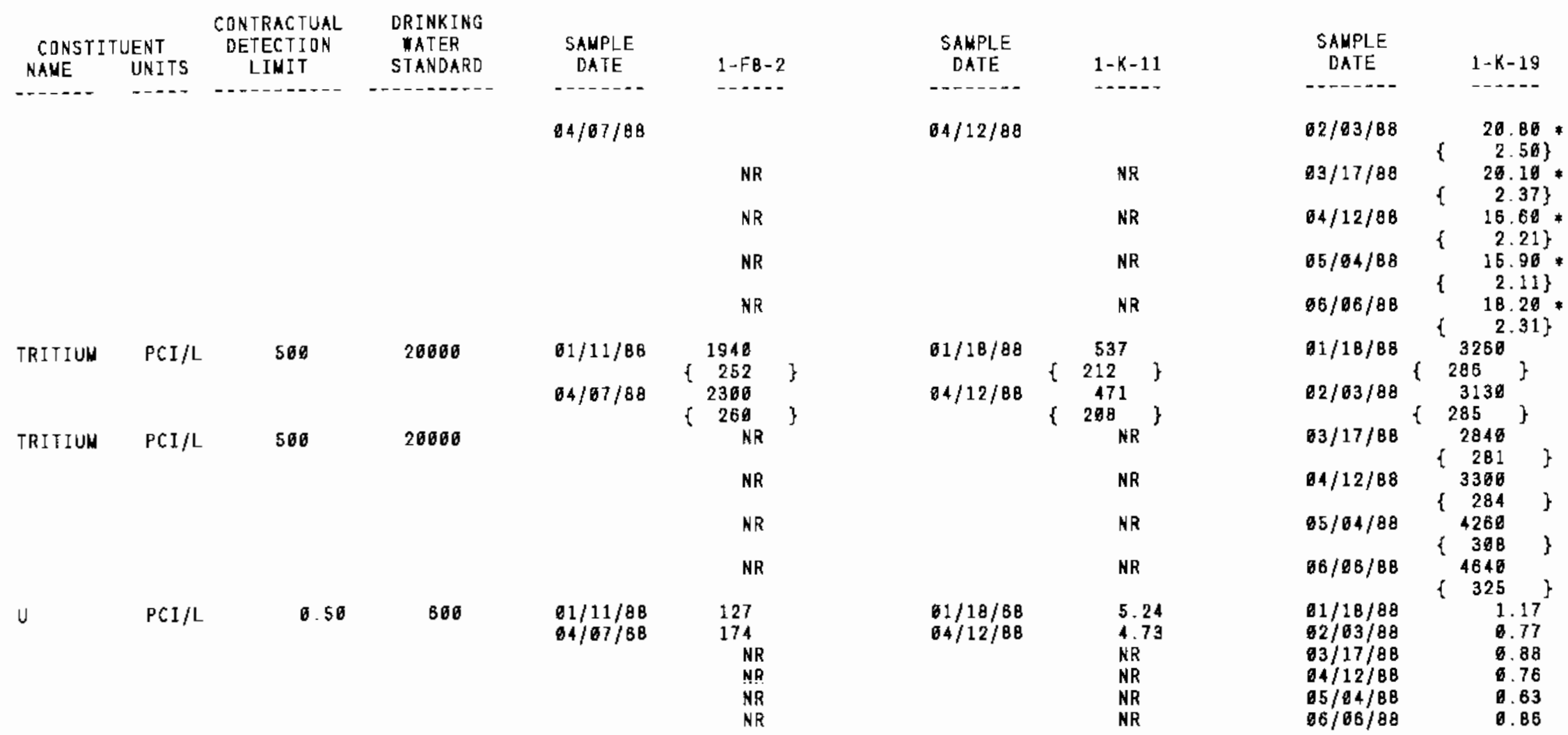


TABLE E.7. (contd)

\begin{tabular}{|c|c|c|c|c|c|c|c|c|c|}
\hline $\begin{array}{l}\text { CONSTI } \\
\text { NAME }\end{array}$ & $\begin{array}{l}\text { UENT } \\
\text { UNITS }\end{array}$ & $\begin{array}{c}\text { CONTRACTUAL } \\
\text { DETECTION } \\
\text { LIMIT }\end{array}$ & $\begin{array}{c}\text { DRINKING } \\
\text { WATER } \\
\text { STANDARD }\end{array}$ & $\begin{array}{l}\text { SAMPLE } \\
\text { DATE }\end{array}$ & $1-K-26$ & $\begin{array}{l}\text { SAMPLE } \\
\text { DATE }\end{array}$ & $1-K-22$ & $\begin{array}{l}\text { SAHPLE } \\
\text { DATE }\end{array}$ & $1-k-27$ \\
\hline------ & $-\cdots$ & $---\infty--$ & - - - - & ------ & $-\cdots$ & $-\cdots-1$ & $\cdots+$ & $\cdots-\cdots$ & $-\ldots$ \\
\hline BETA & $P C I / L$ & 8 & 50 & $\begin{array}{l}61 / 18 / 88 \\
64 / 12 / 88\end{array}$ & $\begin{array}{c}40.60 \\
\{\quad 4.25\} \\
35.10 \\
\{\quad 4.02\}\end{array}$ & $\begin{array}{l}61 / 18 / 88 \\
04 / 12 / 88\end{array}$ & 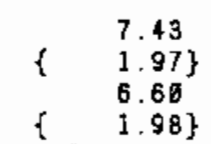 & $\begin{array}{l}61 / 18 / 88 \\
64 / 12 / 88\end{array}$ & $\begin{array}{cc} & 33.80 \\
\{\quad 3.91\} \\
\{\quad 30.90 \\
\{\quad 3.84\}\end{array}$ \\
\hline HNITRAT & PPB & 2568 & 45666 & $\begin{array}{l}01 / 18 / 88 \\
04 / 12 / 88\end{array}$ & $\begin{array}{l}22100 \\
22200\end{array}$ & $\begin{array}{l}01 / 18 / 88 \\
04 / 12 / 88\end{array}$ & $\begin{array}{l}2880 \\
2726\end{array}$ & $\begin{array}{l}61 / 18 / 88 \\
54 / 12 / 88\end{array}$ & $\begin{array}{r}11100 \\
7900\end{array}$ \\
\hline SR 90 & $P C I / L$ & 5 & 8 & $\begin{array}{l}01 / 18 / 88 \\
04 / 12 / 88\end{array}$ & $\begin{array}{ll}\left\{\quad \begin{array}{c}15.40 \\
2.85\end{array}\right\}^{*} \\
18.30^{*} \\
\{\quad 2.28 \\
\\
\text { NR }\end{array}$ & $\begin{array}{l}01 / 18 / 88 \\
01 / 18 / 88 \\
04 / 12 / 88\end{array}$ & $\begin{array}{ll}\{\quad 2.46 \\
1.05\} \\
\{\quad 2.99 \\
1.08\} \\
\{\quad 1.63 \\
\{\quad 1.03\}\end{array}$ & $\begin{array}{l}61 / 18 / 88 \\
64 / 12 / 88\end{array}$ & NR \\
\hline $\begin{array}{l}\text { TC-99 } \\
\text { TRITIUH }\end{array}$ & $\mathrm{PCI} / \mathrm{L}$ & 15 & 960 & $01 / 18 / 88$ & & $61 / 18 / 88$ & & $81 / 18 / 88$ & $\left\{\begin{array}{c}17.30 \\
\{\quad 1.35\}\end{array}\right.$ \\
\hline TRITIUM & PCI $/ L$ & 500 & 20600 & $\begin{array}{l}01 / 18 / 88 \\
04 / 12 / 88\end{array}$ & $\left.\begin{array}{r}1380 \\
238 \\
1086 \\
228 \\
\text { NR }\end{array}\right\}$ & $\begin{array}{l}61 / 18 / 88 \\
61 / 18 / 88 \\
64 / 12 / 88\end{array}$ & 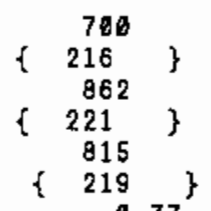 & $\begin{array}{l}01 / 18 / 88 \\
04 / 12 / 88\end{array}$ & $\begin{array}{c}\left.\begin{array}{c}2740 \\
274 \\
1850\end{array}\right\} \\
\left\{\begin{array}{c}249 \\
N R\end{array}\right\}\end{array}$ \\
\hline U & $\mathrm{PCI} / \mathrm{L}$ & 0.50 & 600 & $\begin{array}{l}61 / 18 / 88 \\
64 / 12 / 88\end{array}$ & $\begin{array}{l}1.76 \\
\text { J.94 } \\
\text { NR }\end{array}$ & $\begin{array}{l}01 / 18 / 88 \\
01 / 18 / 88 \\
04 / 12 / 88\end{array}$ & $\begin{array}{r}0.77^{5} \\
0.66 \\
0.77\end{array}$ & $\begin{array}{l}91 / 18 / 88 \\
84 / 12 / 88\end{array}$ & $\begin{array}{l}3.55 \\
3.37 \\
N R\end{array}$ \\
\hline
\end{tabular}


TABLE_E.7. (contd)

\begin{tabular}{|c|c|c|c|c|c|c|c|c|c|}
\hline $\begin{array}{l}\text { CONSTI } \\
\text { NAWE }\end{array}$ & $\begin{array}{l}\text { UENT } \\
\text { UNITS }\end{array}$ & $\begin{array}{l}\text { CONTRACTUAL } \\
\text { DETECTION } \\
\text { LIMIT }\end{array}$ & $\begin{array}{l}\text { DRINKING } \\
\text { WATER } \\
\text { STANDARD }\end{array}$ & $\begin{array}{l}\text { SAMPLE } \\
\text { DATE }\end{array}$ & $1-k-28$ & $\begin{array}{l}\text { SAMPLE } \\
\text { DATE }\end{array}$ & $1-k-29$ & $\begin{array}{c}\text { SAMPLE } \\
\text { DATE }\end{array}$ & $1-K-3 \theta$ \\
\hline$\ldots---$ & $\cdots-$ & - - - - & 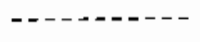 & 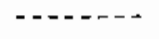 & ----- & $\cdots+\cdots$ & $-\infty---$ & $+\cdots--$ & ------ \\
\hline BETA & $\mathrm{PCI} / \mathrm{L}$ & 8 & 50 & $\begin{array}{l}01 / 18 / 88 \\
64 / 12 / 88\end{array}$ & $\left\{\begin{array}{l}9.17 \\
2.26\} \\
5.87 \\
1.99\}\end{array}\right.$ & $\begin{array}{l}01 / 18 / 88 \\
04 / 12 / 88\end{array}$ & $\begin{array}{ll} & 4.69 \\
1.65 & 1.96 \\
& 5.96 \\
\{\quad 1.87\}\end{array}$ & $\begin{array}{l}01 / 18 / 88 \\
64 / 12 / 88\end{array}$ & $\begin{array}{l}5.37 \\
\{\quad 1.82\} \\
5.66 \\
\{\quad 1.95\}\end{array}$ \\
\hline HNITRAT & PPB & 2509 & 45000 & $\begin{array}{l}91 / 18 / 88 \\
64 / 12 / 88\end{array}$ & $\begin{array}{l}18360 \\
26460\end{array}$ & $\begin{array}{l}01 / 18 / 88 \\
64 / 12 / 88\end{array}$ & $\begin{array}{r}8550 \\
9300\end{array}$ & $\begin{array}{l}01 / 18 / 88 \\
94 / 12 / 88\end{array}$ & $\begin{array}{r}68066 \\
76106\end{array}$ \\
\hline TRITIUN & PCI/L & 500 & 20000 & $\begin{array}{l}01 / 18 / 88 \\
04 / 12 / 88\end{array}$ & $\left\{\begin{array}{r}3050 \\
239 \\
3290 \\
288\end{array}\right\}$ & $\begin{array}{l}01 / 18 / 88 \\
64 / 12 / 88\end{array}$ & $\left\{\begin{array}{c}10800 \\
371 \\
17000 \\
521\end{array}\right\}$ & $\begin{array}{l}01 / 18 / 88 \\
64 / 12 / 88\end{array}$ & $\left.\begin{array}{c}1180000 \\
\left.\begin{array}{c}3500 \\
1220000\end{array}\right\} \\
2900\end{array}\right\}$ \\
\hline u & $P C I / L$ & 6.50 & 500 & $\begin{array}{l}01 / 18 / 88 \\
04 / 12 / 88\end{array}$ & $\begin{array}{l}3.77 \\
3.44\end{array}$ & $\begin{array}{l}91 / 18 / 88 \\
01 / 12 / 88\end{array}$ & $\begin{array}{l}1.78 \\
1.76\end{array}$ & $\begin{array}{l}91 / 18 / 88 \\
04 / 12 / 88\end{array}$ & $\begin{array}{l}1.89 \\
2\end{array}$ \\
\hline
\end{tabular}


IABLE E.7. (contd)

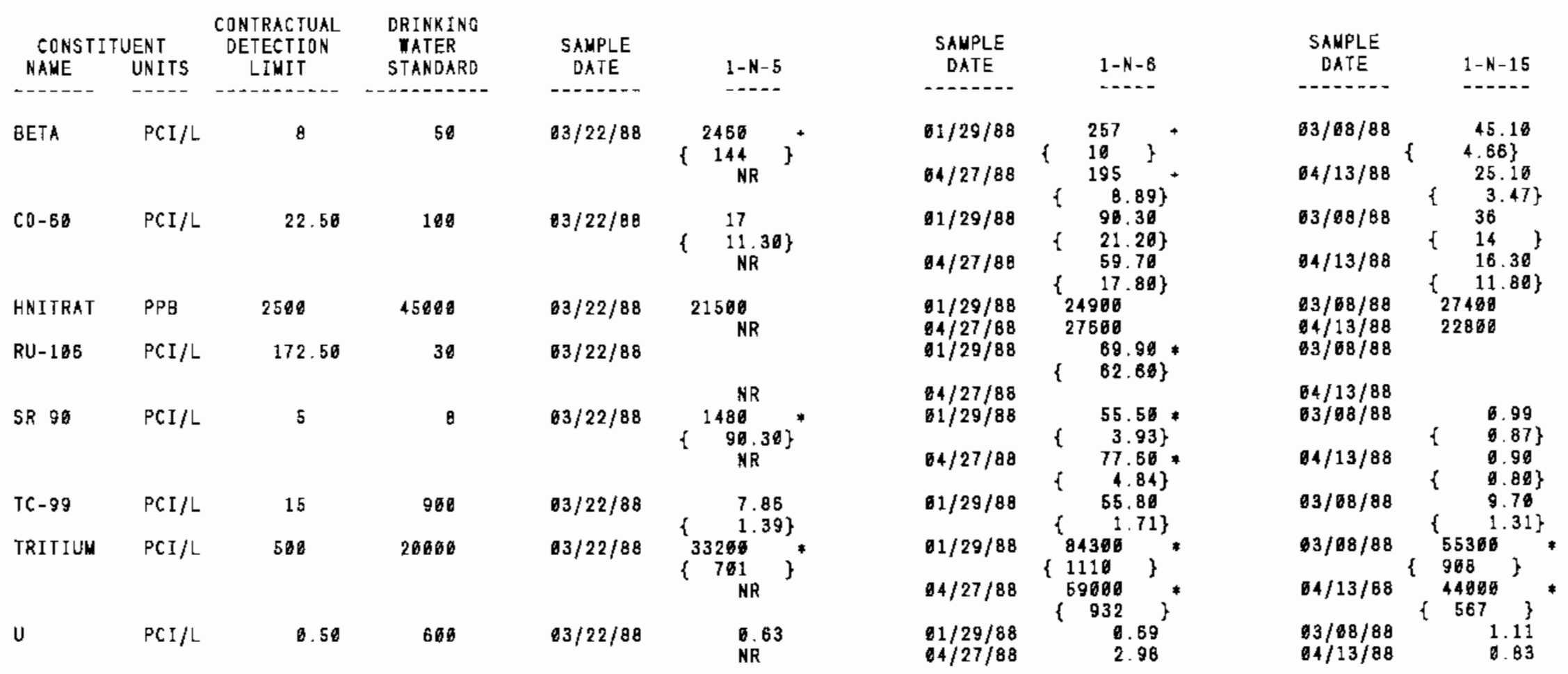


TABLE E.7. (contd)

\begin{tabular}{|c|c|c|c|c|c|c|c|c|c|}
\hline $\begin{array}{l}\text { CONSTI } \\
\text { NAKE }\end{array}$ & $\begin{array}{l}\text { UENT } \\
\text { UNITS }\end{array}$ & $\begin{array}{l}\text { CONTRACTUAL } \\
\text { DETECTION } \\
\text { LIMIT }\end{array}$ & $\begin{array}{l}\text { DRINKING } \\
\text { WATER } \\
\text { STANDARD }\end{array}$ & $\begin{array}{l}\text { SAMPLE } \\
\text { DATE }\end{array}$ & $1-N-16$ & $\begin{array}{l}\text { SAMPLE } \\
\text { DATE }\end{array}$ & $1-N-18$ & $\begin{array}{l}\text { SAMPLE } \\
\text { DATE }\end{array}$ & $1-N-19$ \\
\hline$\ldots$ & $\cdots-$ & 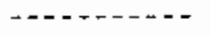 & - - & 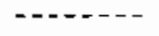 & $\cdots-$ & $+\cdots--\infty$ & $-\ldots--$ & $\cdots+-\cdots$ & ----- \\
\hline ALPHA & $\mathrm{PCI} / \mathrm{L}$ & 4 & 15 & & $\begin{array}{l}\text { NR } \\
\text { NR }\end{array}$ & & $\begin{array}{l}\text { NR } \\
\text { NR }\end{array}$ & $\begin{array}{l}04 / 28 / 88 \\
04 / 28 / 88\end{array}$ & 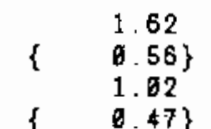 \\
\hline BETA & $\mathrm{PCI} / \mathrm{L}$ & 8 & 50 & $\begin{array}{l}03 / 22 / 88 \\
04 / 28 / 88\end{array}$ & $\begin{array}{l}12.10 \\
3.11\} \\
7.71 \\
2.35\} \\
N R\end{array}$ & $\begin{array}{l}03 / 21 / 88 \\
04 / 28 / 88\end{array}$ & $\left\{\begin{array}{r}1570 \\
118 \\
1420 \\
113 \\
N R\end{array}\right\}+$ & $\begin{array}{l}63 / 21 / 88 \\
64 / 28 / 88 \\
64 / 28 / 88\end{array}$ & 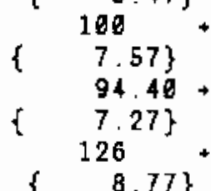 \\
\hline$c 0-60$ & $\mathrm{PCI} / \mathrm{L}$ & 22.50 & 100 & & $\begin{array}{l}\text { NR } \\
\text { NR } \\
\text { NR }\end{array}$ & $\begin{array}{l}03 / 21 / 88 \\
04 / 28 / 88\end{array}$ & $\left.\begin{array}{c}16.10 \\
9.66\} \\
18 \\
14 \\
\text { NR }\end{array}\right\}$ & $\begin{array}{l}03 / 21 / 88 \\
64 / 28 / 88 \\
04 / 28 / 88\end{array}$ & ( $50 .+1$ \\
\hline HNITRAT & PPB & 2500 & 45000 & $\begin{array}{l}83 / 22 / 8 B \\
04 / 2 B / 88\end{array}$ & & $\begin{array}{l}63 / 21 / 88 \\
64 / 28 / 88\end{array}$ & & $\begin{array}{l}03 / 21 / 88 \\
04 / 28 / 8 B\end{array}$ & $\begin{array}{l}4570 \\
4680\end{array}$ \\
\hline SR 90 & $\mathrm{PCI} / \mathrm{L}$ & 5 & 8 & $\begin{array}{l}63 / 22 / 88 \\
04 / 28 / 88\end{array}$ & NR & $\begin{array}{l}63 / 21 / 88 \\
64 / 28 / 88\end{array}$ & $\left\{\begin{array}{c}869 \\
58.79 \\
658 \\
59 \\
\text { NR }\end{array}\right\}^{*}$ & $\begin{array}{l}83 / 21 / 88 \\
64 / 28 / 88 \\
94 / 28 / 88\end{array}$ & $\left\{\begin{array}{c}85.70 * \\
4.57\}^{*} \\
78.90 * \\
4.62\} \\
78.86 \\
\{\quad 4.62\}\end{array}\right.$ \\
\hline TRI TIUM & $\mathrm{PCI} / \mathrm{L}$ & 500 & 20000 & $\begin{array}{l}03 / 22 / 88 \\
04 / 20 / 20\end{array}$ & NR & $\begin{array}{l}03 / 21 / 88 \\
64 / 28 / 88\end{array}$ & 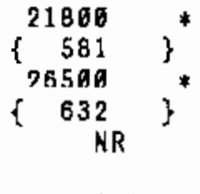 & $\begin{array}{l}83 / 21 / 88 \\
64 / 28 / 88 \\
04 / 28 / 88\end{array}$ & $\left\{\begin{array}{cc}3690 & \\
308 & \\
1696 & \\
251 & \\
1836 & \\
256 & \end{array}\right\}$ \\
\hline U & $\mathrm{PCI} / \mathrm{L}$ & 9.50 & 600 & $\begin{array}{l}03 / 22 / 88 \\
04 / 28 / 88\end{array}$ & $\begin{array}{l}3.88 \\
6.84 \\
\text { NR }\end{array}$ & $\begin{array}{l}93 / 21 / 88 \\
04 / 28 / 88\end{array}$ & $\begin{array}{l}1.14 \\
2.48 \\
\text { NR }\end{array}$ & $\begin{array}{l}93 / 21 / 88 \\
64 / 28 / 88 \\
64 / 28 / 88\end{array}$ & $\begin{array}{l}2.74 \\
1.89 \\
2.14\end{array}$ \\
\hline
\end{tabular}


TABLE E.7. (contd)

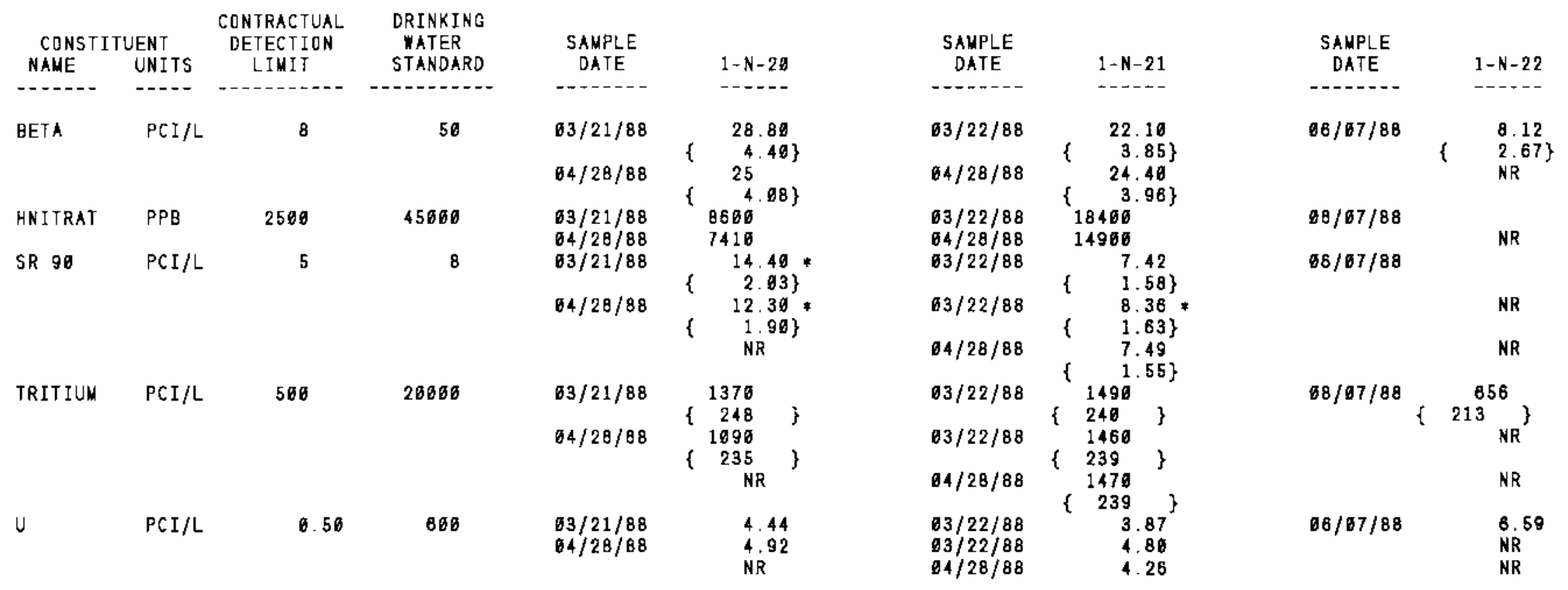


TABLE E.7. (contd)

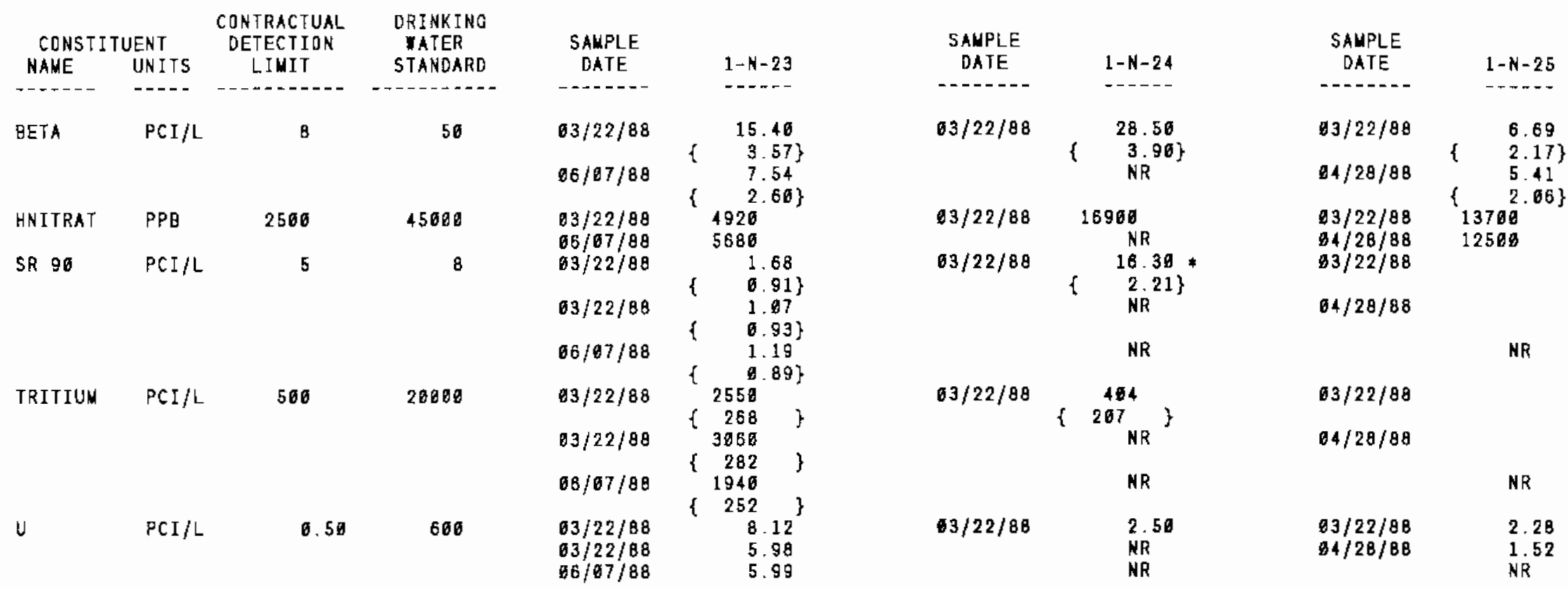


TABLE E.7. (contd)

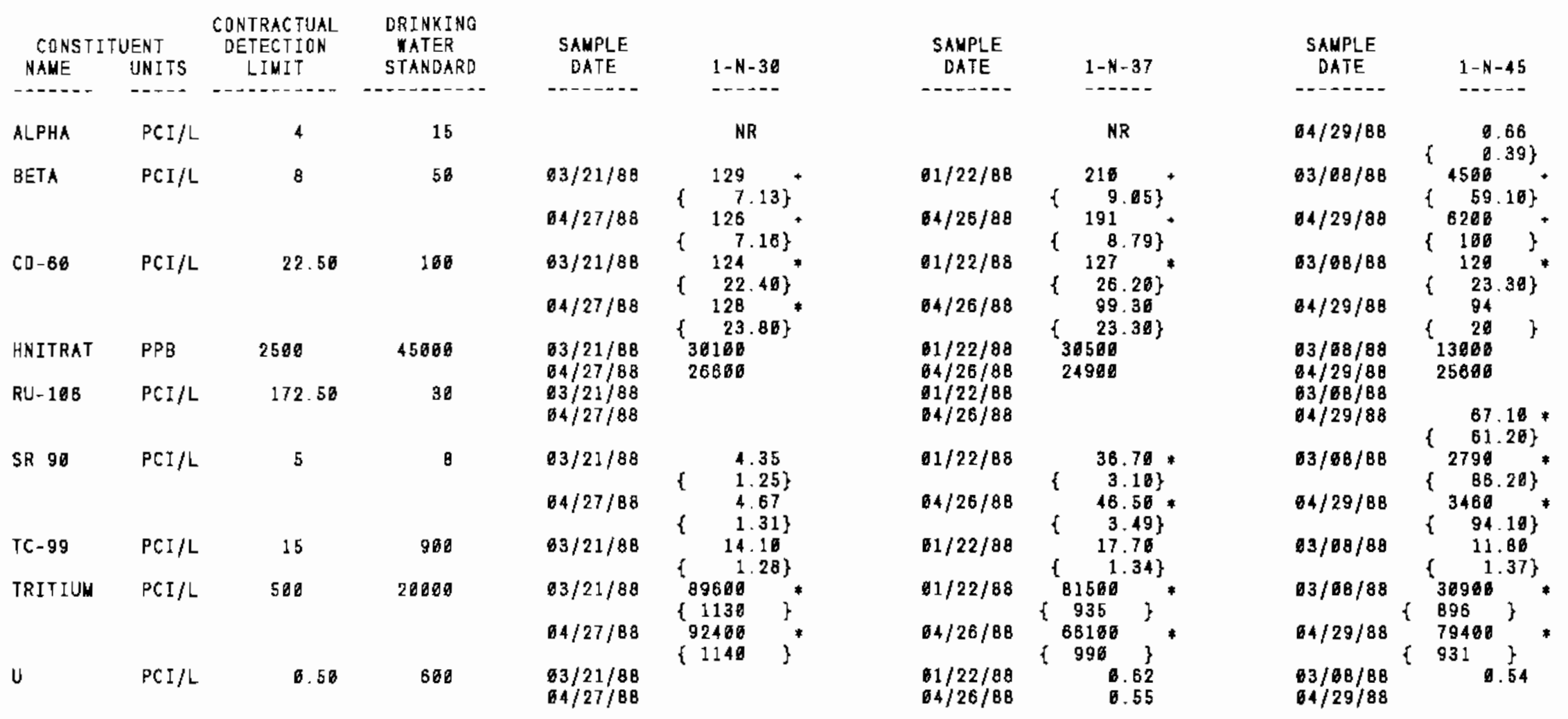


IABLE E.7. (contd)

\begin{tabular}{|c|c|c|c|c|c|c|c|c|c|}
\hline $\begin{array}{l}\text { CONSTI } \\
\text { NAME }\end{array}$ & $\begin{array}{l}\text { UENT } \\
\text { UNITS }\end{array}$ & $\begin{array}{l}\text { CONTRACTUAL } \\
\text { DETECTION } \\
\text { LIWIT }\end{array}$ & $\begin{array}{l}\text { DRINKING } \\
\text { WATER } \\
\text { STANOARD }\end{array}$ & $\begin{array}{l}\text { SANPLE } \\
\text { DATE }\end{array}$ & $1-N-50$ & $\begin{array}{l}\text { SAMPLEE } \\
\text { DATE }\end{array}$ & $1-N-51$ & $\begin{array}{l}\text { SAMPLE } \\
\text { DATE }\end{array}$ & $2-E 13-8$ \\
\hline------ & $\ldots \ldots$ & $\ldots \ldots---$ & $----n---$ & 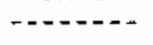 & ----- & 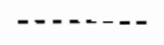 & ----- & -....- &.--+-- \\
\hline BETA & $\mathrm{PCI} / \mathrm{L}$ & 8 & 56 & $\begin{array}{l}61 / 29 / 88 \\
64 / 29 / 88\end{array}$ & $\left.\begin{array}{c}66.96 \\
\left\{\begin{array}{c}5.31 \\
68\end{array}\right. \\
\{\quad 5.44\end{array}\right\}^{+}$ & $\begin{array}{l}01 / 29 / 88 \\
64 / 26 / 88\end{array}$ & $\begin{array}{c}58.46 \\
4.64\} \\
41.19 \\
\{\quad 4.22\}\end{array}$ & $\begin{array}{l}62 / 01 / 88 \\
04 / 19 / 88\end{array}$ & $\begin{array}{l}7.99 \\
2.19\} \\
6.95 \\
2.09\}\end{array}$ \\
\hline HNITRAT & PPB & 2500 & 45666 & $\begin{array}{l}01 / 29 / 88 \\
64 / 29 / 88\end{array}$ & $\begin{array}{l}44809 \\
39560\end{array}$ & $\begin{array}{l}01 / 29 / 88 \\
04 / 26 / 88\end{array}$ & $\begin{array}{l}33900 \\
30900\end{array}$ & $62 / 01 / 88$ & 21390 \\
\hline TC -99 & $P C I / L$ & 15 & 968 & $61 / 29 / 88$ & $\begin{array}{ll} & 7.38 \\
\{\quad 1.21\}\end{array}$ & $61 / 29 / 88$ & $\begin{cases} & 8.65 \\
\{\quad 1.21\}\end{cases}$ & $2 / 81 / 88$ & \\
\hline TRITIUN & $\mathrm{PCI} / \mathrm{L}$ & 586 & 20000 & $\begin{array}{l}1 / 29 / 88 \\
04 / 29 / 88\end{array}$ & $\left.\begin{array}{l}133060 \\
\{1390 \\
124009 \\
11150\end{array}\right\}^{*}$ & $\begin{array}{l}91 / 29 / 88 \\
04 / 28 / 88\end{array}$ & $\left.\begin{array}{l}96680 \\
1190 \\
92908 \\
1150\end{array}\right\} *$ & $02 / 01 / 88$ & NR \\
\hline $\mathrm{U}$ & $\mathrm{PCI} / \mathrm{L}$ & a. 50 & 666 & $\begin{array}{l}01 / 29 / 88 \\
04 / 29 / 88\end{array}$ & ס. 68 & $\begin{array}{l}01 / 29 / 88 \\
04 / 26 / 88\end{array}$ & $\begin{array}{l}0.64 \\
0.54\end{array}$ & $62 / 01 / 88$ & 2.10 \\
\hline
\end{tabular}


IABLE E.7. (contd)

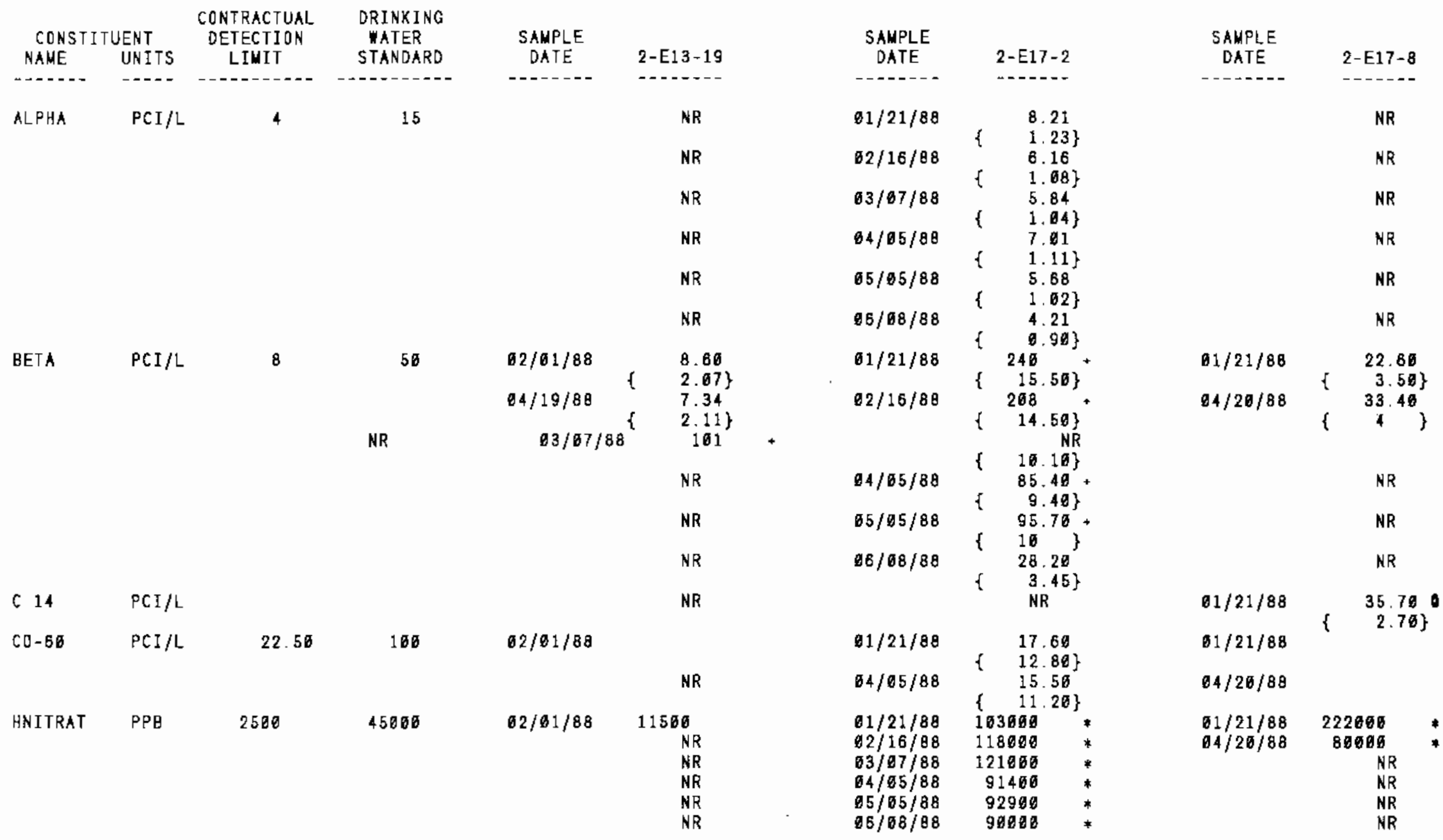


TABLE E.7. (contd)

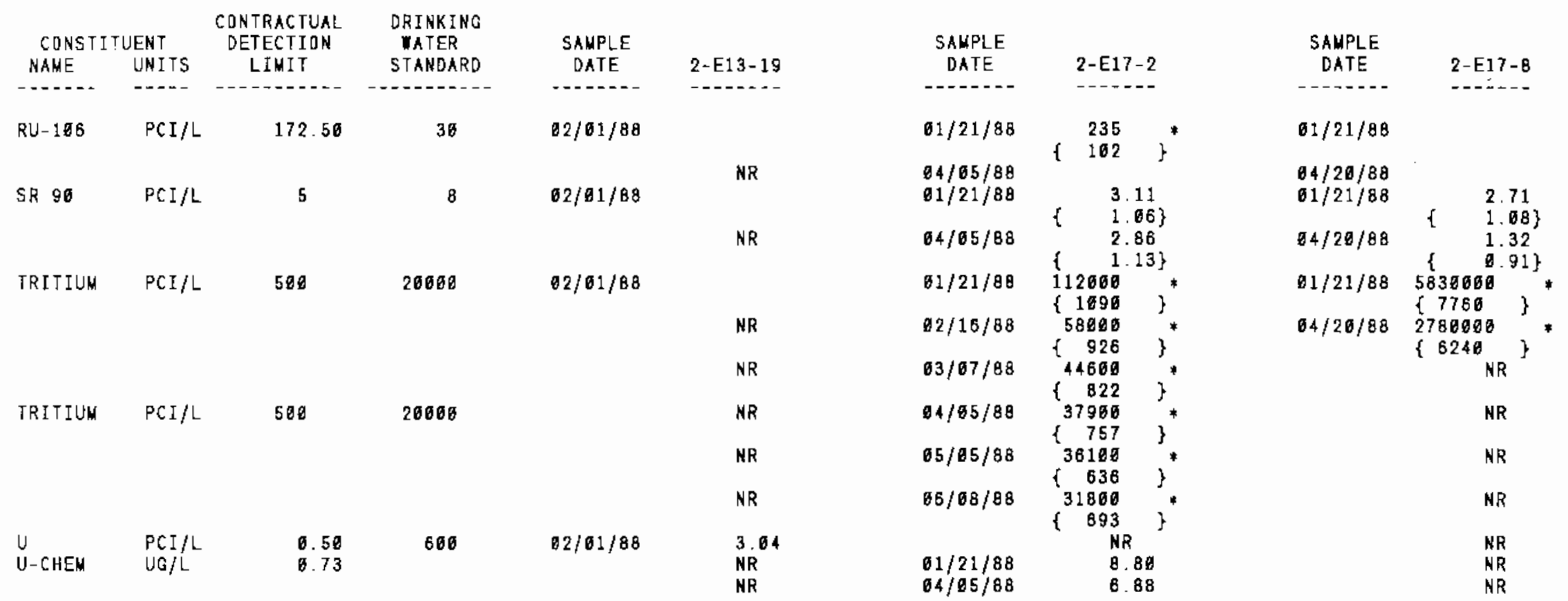


IABLE E.]. (contd)

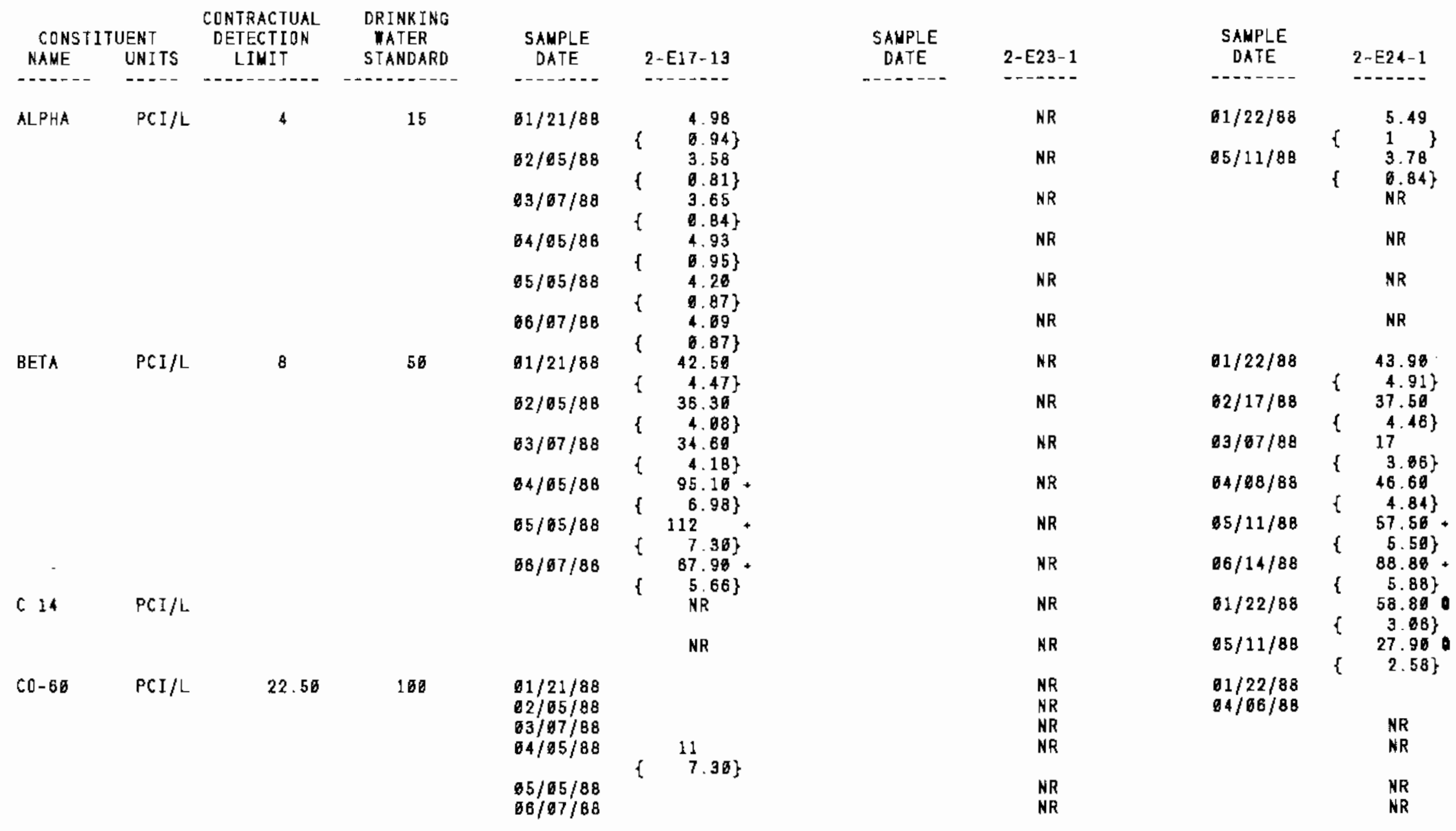


TABLEE.7. (contd)

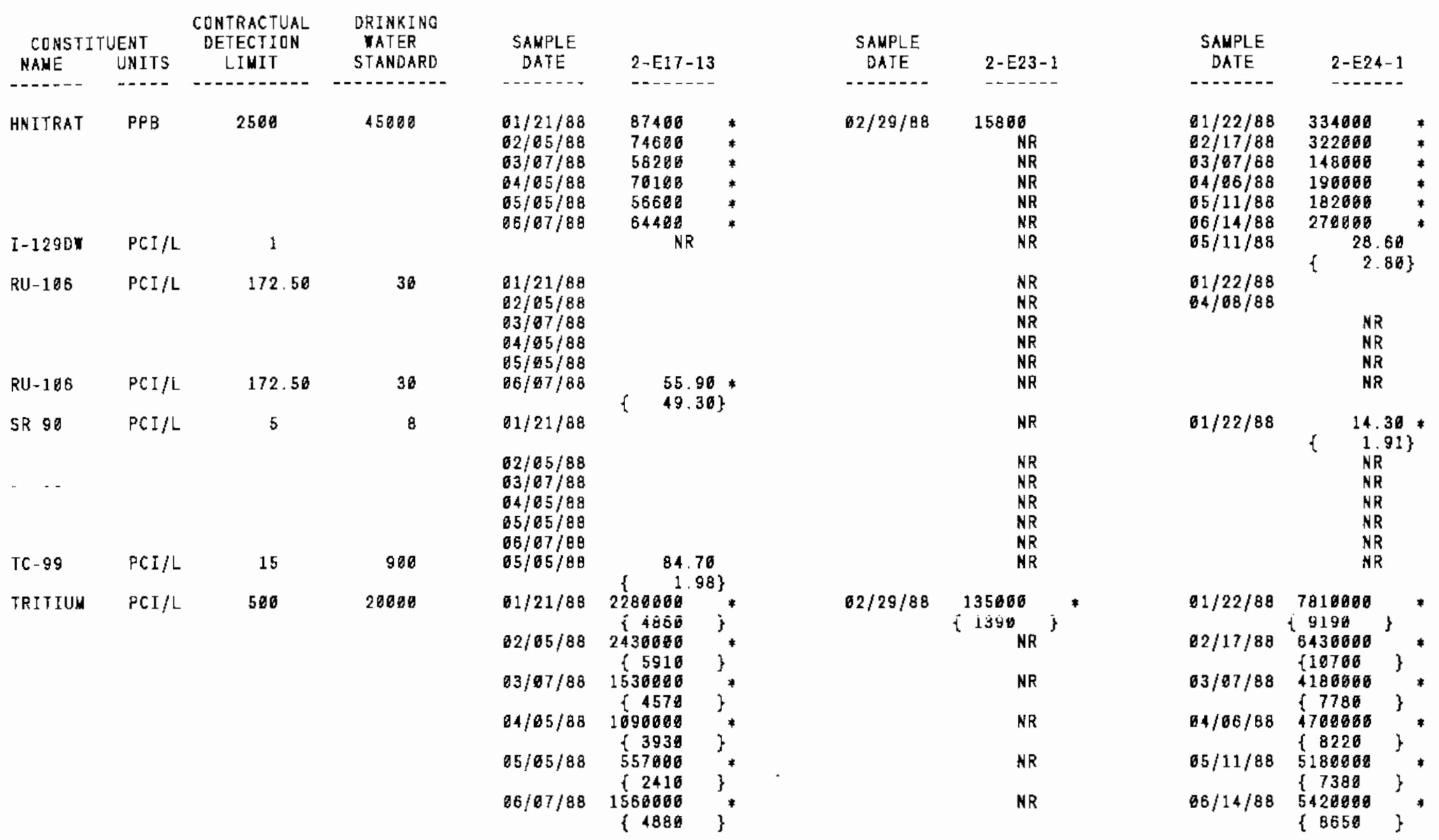


TABLE E.7. (contd)

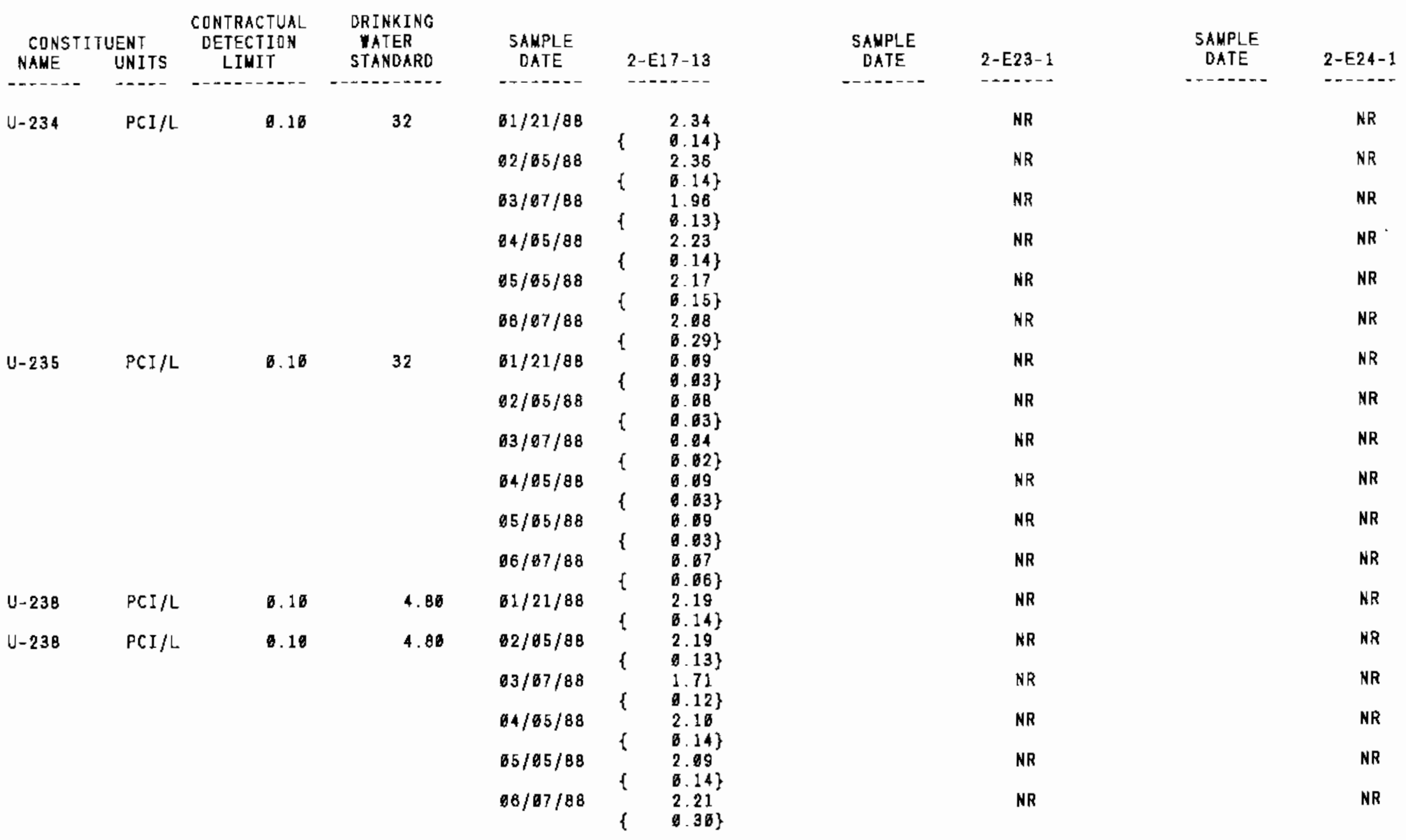


IABLE E. .7. (contd)

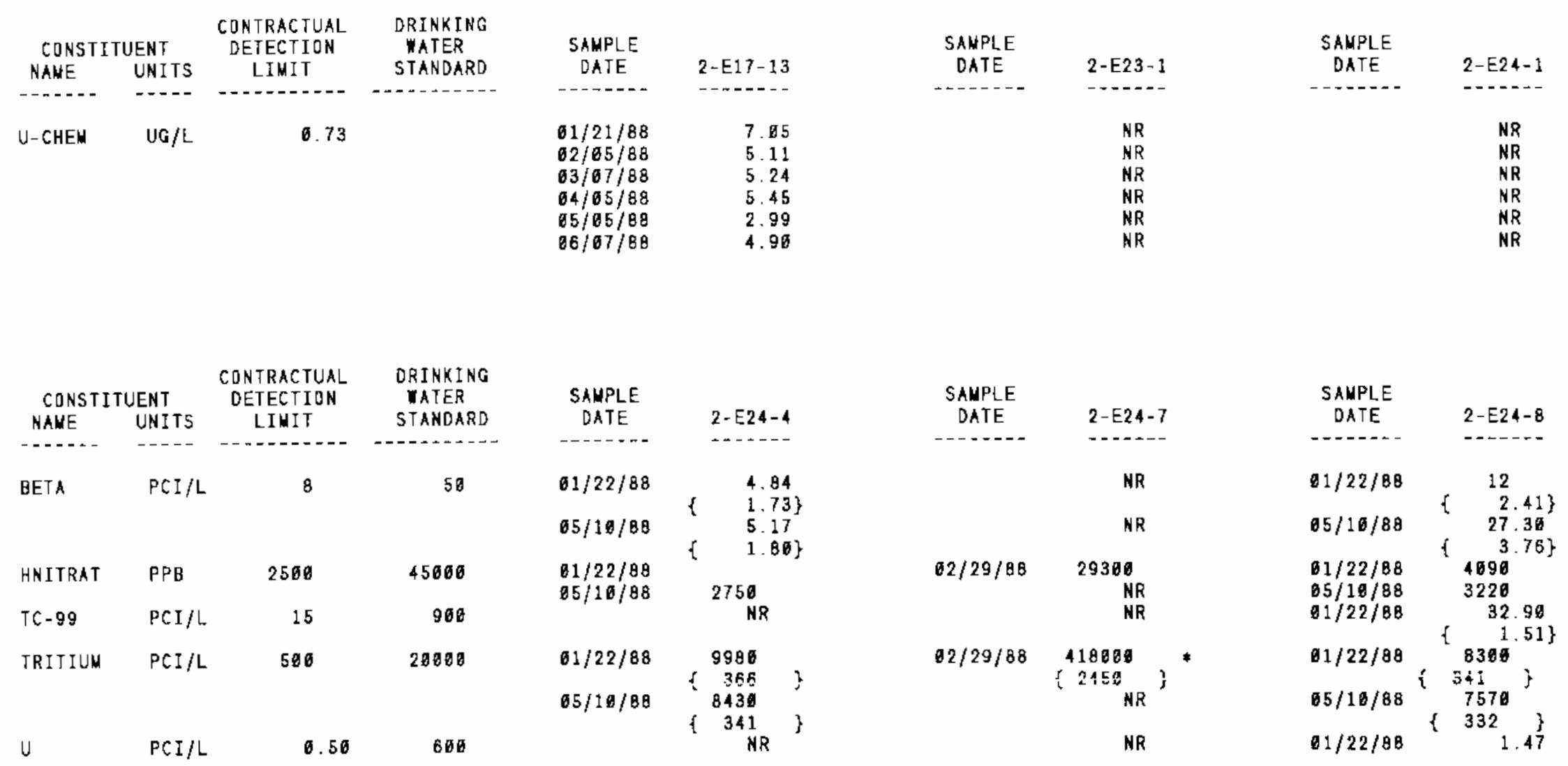


TABLE E.7. (contd)

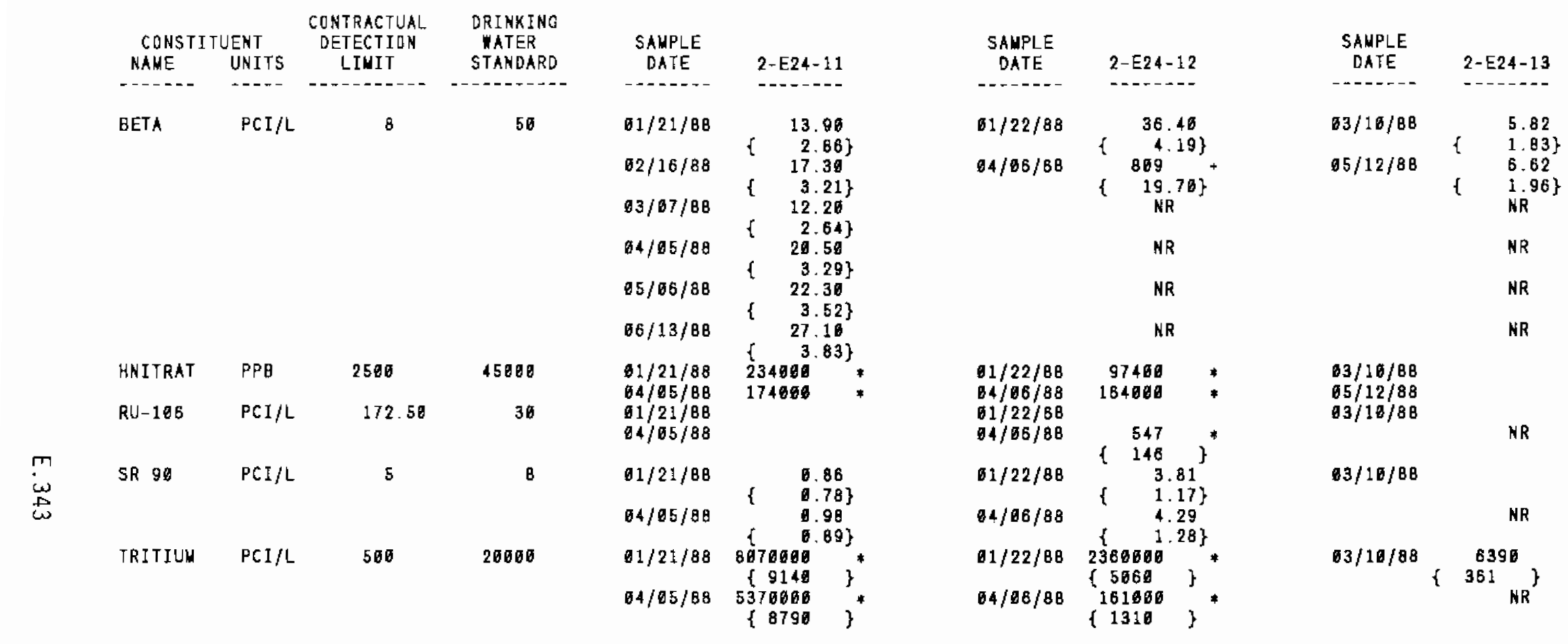


TABLE E.7. (contd)

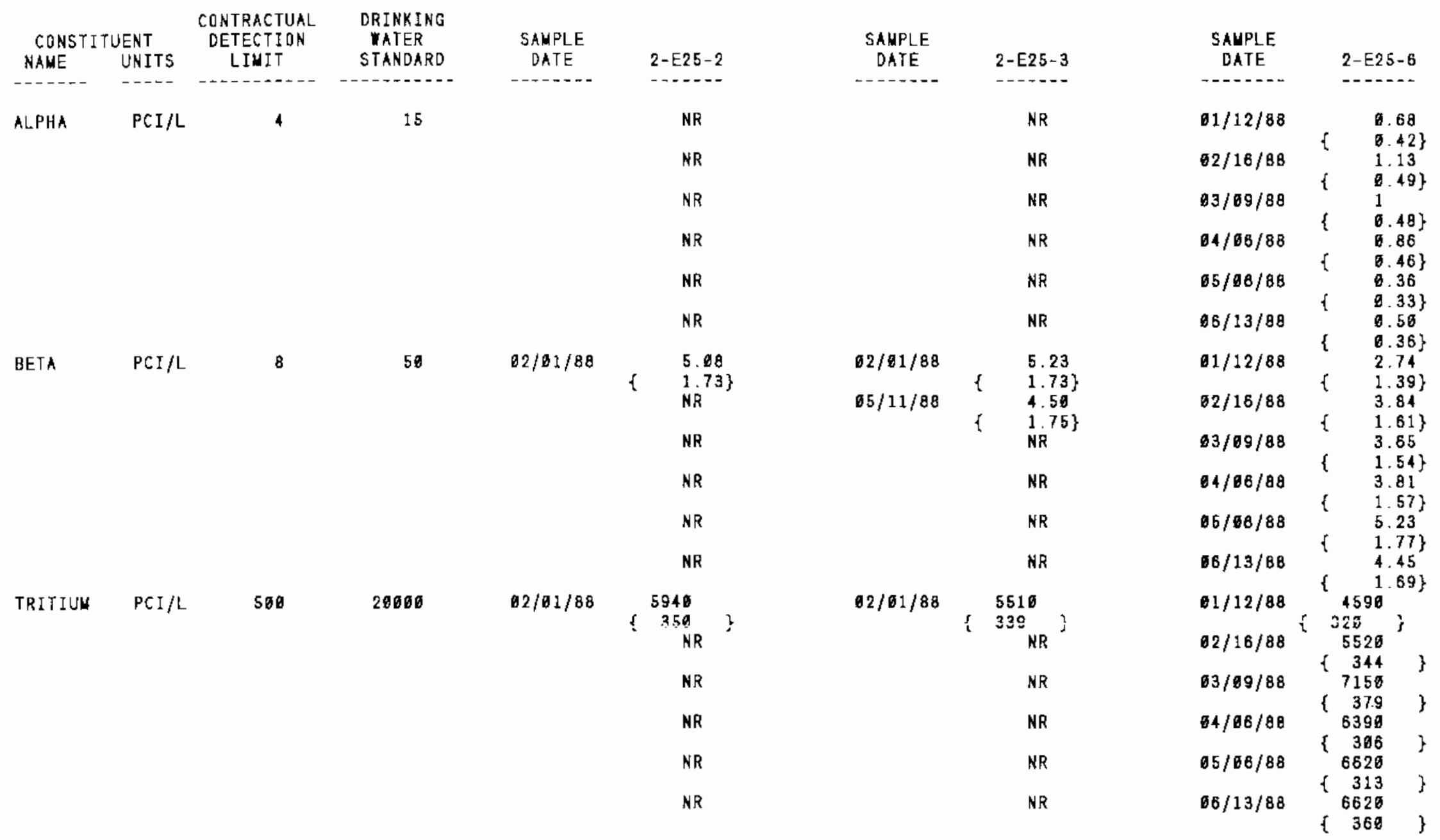


IABLEE.7. (contd)

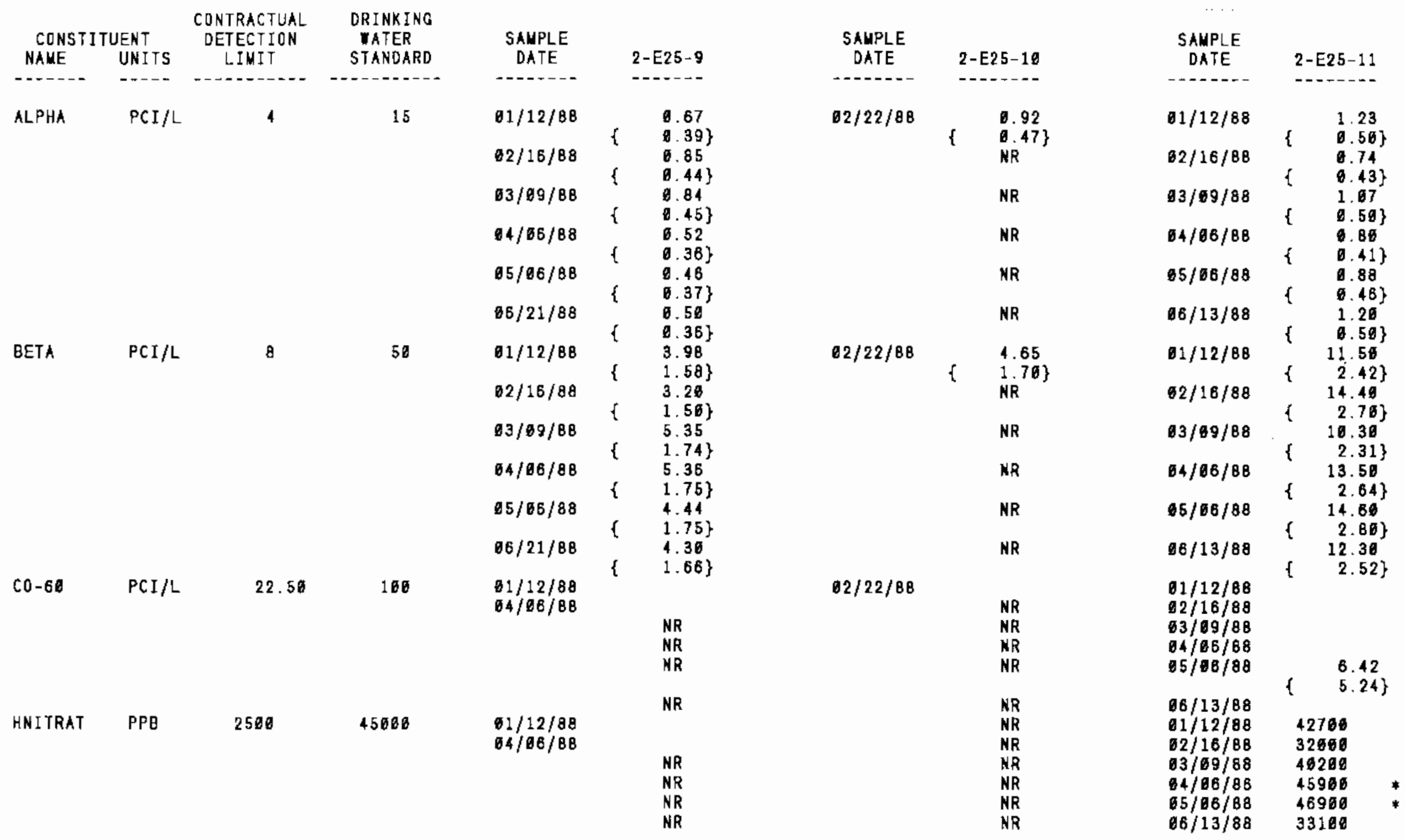


TABLE E.7. (contd)

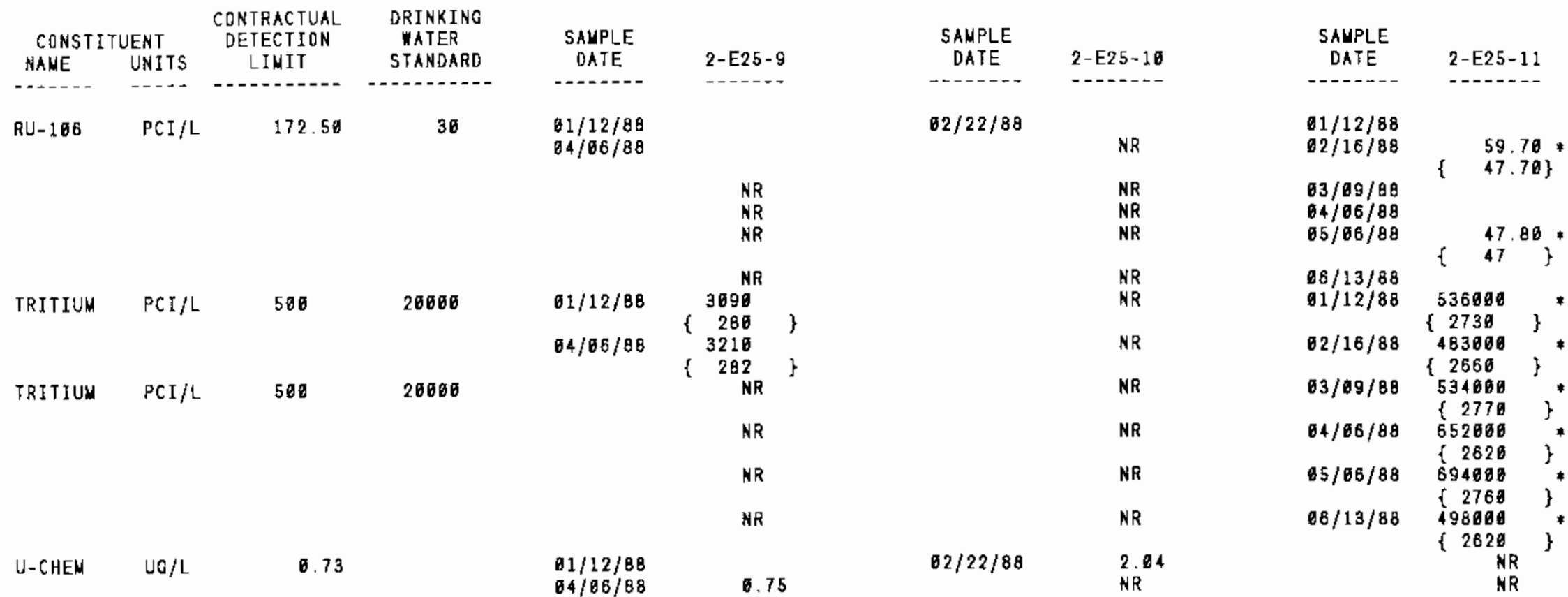


TABLE E.7. (contd)

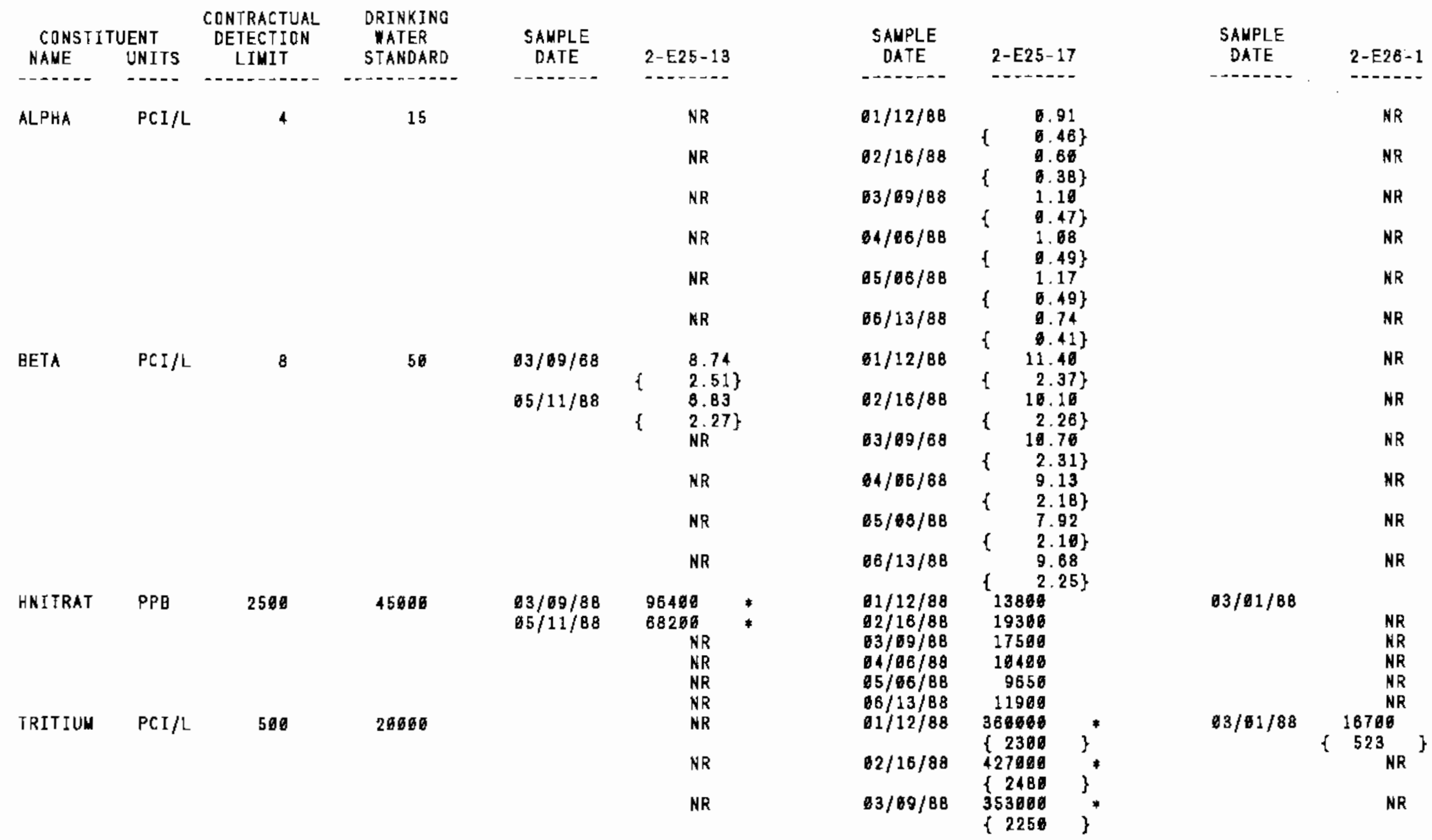


TABLE E.7. (contd)

\begin{tabular}{|c|c|c|c|c|c|c|c|c|c|}
\hline $\begin{array}{l}\text { CONSTI } \\
\text { NAME }\end{array}$ & $\begin{array}{l}\text { UENT } \\
\text { UNITS }\end{array}$ & $\begin{array}{c}\text { CONTRACTUAL } \\
\text { DETECTION } \\
\text { LIMIT }\end{array}$ & $\begin{array}{l}\text { DRINKING } \\
\text { SATER } \\
\text { STANDARD }\end{array}$ & $\begin{array}{l}\text { SANPLE } \\
\text { DATE }\end{array}$ & $2-E 25-13$ & $\begin{array}{l}\text { SAMPLE } \\
\text { DATE }\end{array}$ & $2-E 25-17$ & $\begin{array}{l}\text { SAMPLE } \\
\text { DATE }\end{array}$ & 2-E26-1 \\
\hline & & & & & $\begin{array}{l}\text { NR } \\
\text { NR } \\
\text { NR }\end{array}$ & $\begin{array}{l}04 / 66 / 88 \\
65 / 06 / 88 \\
66 / 13 / 88\end{array}$ & 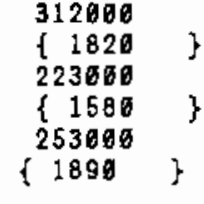 & & $\begin{array}{l}\text { NR } \\
\text { NR } \\
\text { NR }\end{array}$ \\
\hline $\begin{array}{l}\text { CONSTI } \\
\text { NAUE }\end{array}$ & $\begin{array}{l}\text { UENT } \\
\text { UNITS }\end{array}$ & $\begin{array}{c}\text { CONTRACTUA } \\
\text { DETECTION } \\
\text { LINIT }\end{array}$ & $\begin{array}{l}\text { DRINKING } \\
\text { IATER } \\
\text { STANDARD }\end{array}$ & $\begin{array}{l}\text { SAMPLE } \\
\text { DATE }\end{array}$ & $2-E 26-2$ & $\begin{array}{l}\text { SAMPLE } \\
\text { DATE }\end{array}$ & $2-E 26-3$ & $\begin{array}{l}\text { SAUPLE } \\
\text { DATE }\end{array}$ & $2-E 26-4$ \\
\hline BETA & PCI/L & 8 & 58 & $\begin{array}{l}62 / 01 / 88 \\
85 / 11 / 88\end{array}$ & $\begin{array}{l}4.58 \\
\{\quad 1.68\} \\
\{\quad 8.99 \\
\left\{\begin{array}{l}2.83 \\
\{\end{array}\right\}\end{array}$ & & $\begin{array}{l}\text { NR } \\
\text { NR }\end{array}$ & $\begin{array}{l}03 / 61 / 88 \\
05 / 11 / 88\end{array}$ & $\begin{array}{cc} & 19.66 \\
\{\quad 2.23\} \\
\{\quad 5.49 \\
\{\quad 1.82\}\end{array}$ \\
\hline TRITIUM & $\mathrm{PCI} / \mathrm{L}$ & 500 & 26860 & $\begin{array}{l}82 / 01 / 88 \\
95 / 11 / 88\end{array}$ & $\left.\begin{array}{r}2886 \\
284 \\
2389 \\
\left\{\begin{array}{r}284 \\
\{\end{array}\right\}\end{array}\right\}$ & $02 / 01 / 88$ & $\left\{\begin{array}{c}325 \\
{ }_{N R}\end{array}\right\}$ & $\begin{array}{l}83 / 81 / 88 \\
85 / 11 / 88\end{array}$ & $\left.\begin{array}{c}42866 \\
896 \\
39208 \\
589\end{array}\right\}$ \\
\hline
\end{tabular}


TABLE E.7. (contd)

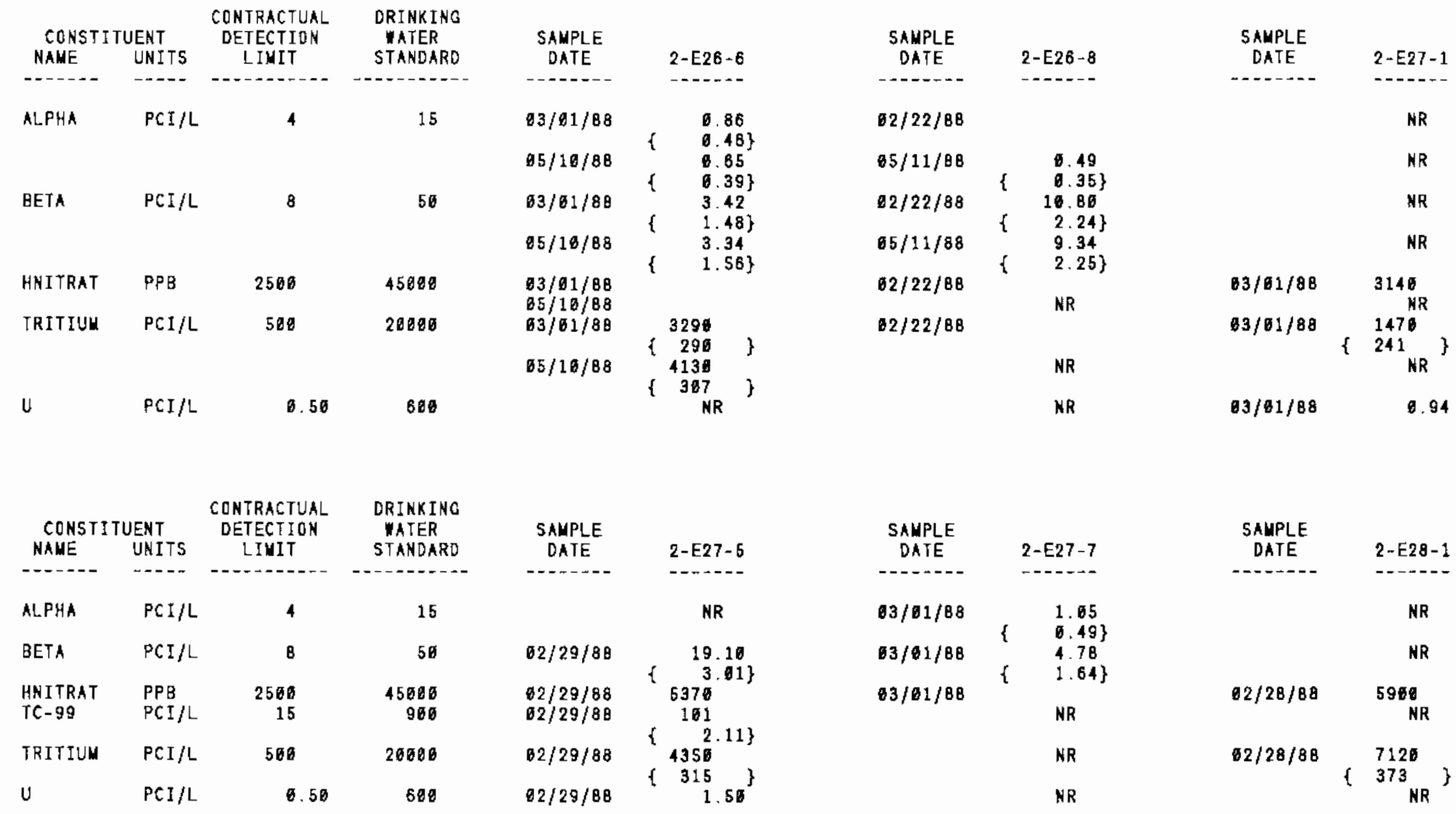


TABLE E.7. (contd)

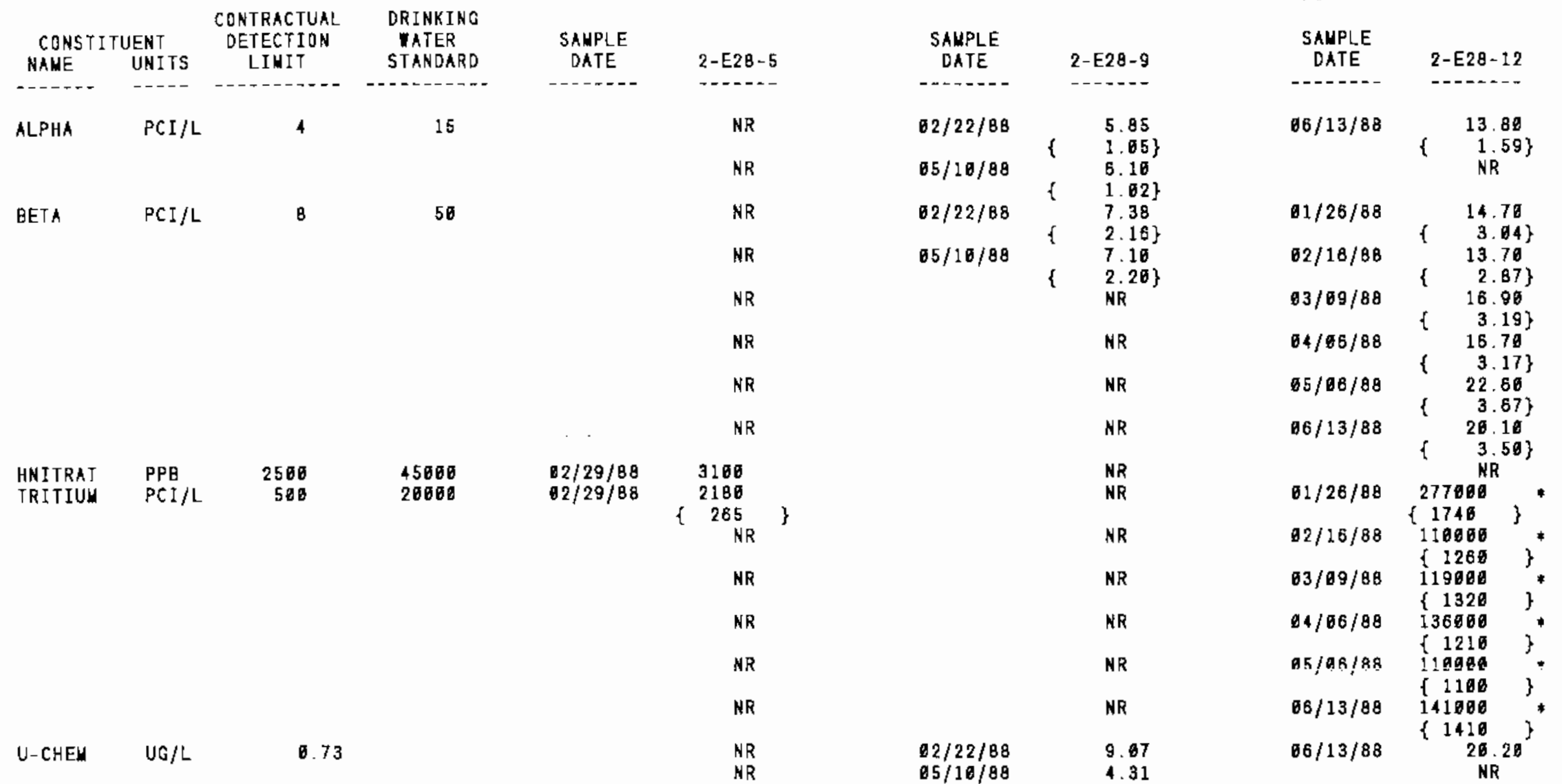


IABLE E.7. (contd)

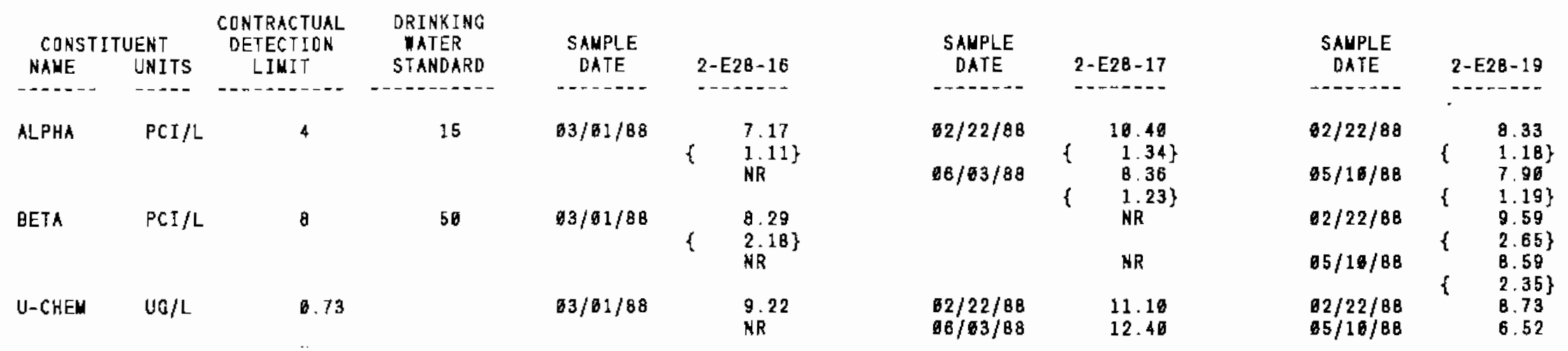


TABLE E.7. (contd)

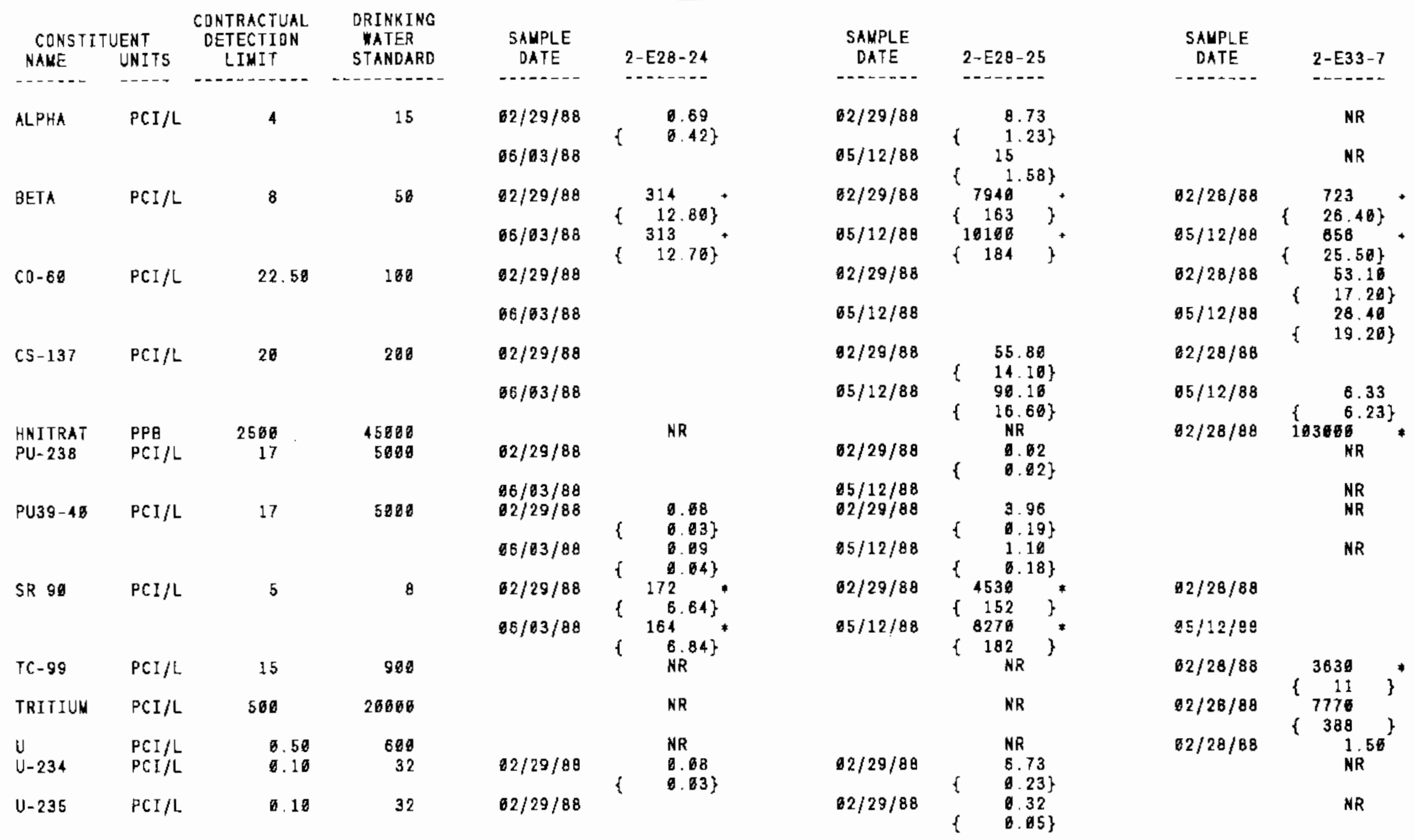


IABLE E.7. (contd)

\begin{tabular}{|c|c|c|c|c|c|c|c|c|c|}
\hline $\begin{array}{l}\text { CDNST } \\
\text { NAME }\end{array}$ & $\begin{array}{l}\text { UENT } \\
\text { UNITS }\end{array}$ & $\begin{array}{c}\text { CONTRACTUAL } \\
\text { DETECTION } \\
\text { LIMIT }\end{array}$ & $\begin{array}{l}\text { DRINKING } \\
\text { WATER } \\
\text { STANDARD }\end{array}$ & $\begin{array}{l}\text { SAMPLE } \\
\text { DATE }\end{array}$ & $2-E 28-24$ & $\begin{array}{l}\text { SAMPLE } \\
\text { DATE }\end{array}$ & $2-E 28-25$ & $\begin{array}{c}\text { SAUPLE } \\
\text { DATE }\end{array}$ & $2-E 33-7$ \\
\hline$U-238$ & $\mathrm{PCI} / \mathrm{L}$ & 0.19 & 4.89 & $82 / 29 / 88$ & 6.68 & $02 / 29 / 8 \theta$ & $6.69 *$ & & NR \\
\hline $\mathrm{U}$-CHEM & UG/L & 6.73 & & $\begin{array}{l}62 / 29 / 88 \\
66 / 03 / 88\end{array}$ & $0.63\}$ & $\begin{array}{l}82 / 29 / 88 \\
05 / 12 / 88\end{array}$ & & & $\begin{array}{l}\text { NR } \\
\text { NR }\end{array}$ \\
\hline
\end{tabular}

\begin{tabular}{|c|c|c|c|c|c|}
\hline \multicolumn{2}{|c|}{ CONSTITUENT } & $\begin{array}{c}\text { CONTRACTUAL } \\
\text { DETECTION } \\
\text { LIUIT }\end{array}$ & $\begin{array}{l}\text { DRINKING } \\
\text { WATER } \\
\text { STANDARD }\end{array}$ & $\begin{array}{c}\text { SAMPLE } \\
\text { DATE }\end{array}$ & $2-E 33-8$ \\
\hline------- & $-\cdots-$ & 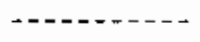 & $---n-\cdots$ & $--n-\infty$ & $\ldots+\cdots$ \\
\hline BETA & $\mathrm{PCI} / \mathrm{L}$ & 8 & $5 b$ & $92 / 28 / 88$ & $\begin{array}{r}55.86 \\
\quad 4.89\}\end{array}$ \\
\hline$C 0-60$ & $\mathrm{PCI} / \mathrm{L}$ & 22.50 & 100 & $82 / 28 / 88$ & \\
\hline CS-137 & $\mathrm{PCI} / \mathrm{L}$ & 26 & 260 & $02 / 28 / 8 B$ & \\
\hline $\begin{array}{l}\text { HNITRAT } \\
\text { TC-99 }\end{array}$ & $\begin{array}{l}\mathrm{PPB} \\
\mathrm{PCI} / \mathrm{L}\end{array}$ & $\begin{array}{r}2509 \\
15\end{array}$ & $\begin{array}{r}45000 \\
900\end{array}$ & $\begin{array}{l}82 / 28 / 88 \\
82 / 28 / 88\end{array}$ & $\begin{array}{r}7786 \\
287\end{array}$ \\
\hline TRITIUM & $\mathrm{PCI} / \mathrm{L}$ & 0.50 & 20660 & $\begin{array}{l}02 / 28 / 88 \\
62 / 28 / 88\end{array}$ & 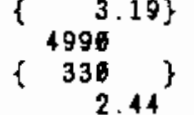 \\
\hline
\end{tabular}

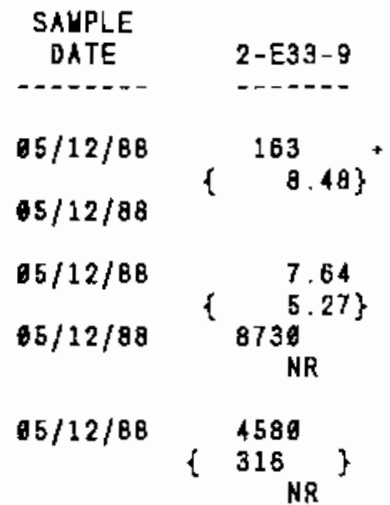

\begin{tabular}{|c|c|}
\hline $\begin{array}{l}\text { SAUPLE } \\
\text { DATE }\end{array}$ & $2-E 33-12$ \\
\hline 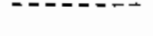 & 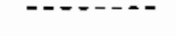 \\
\hline $62 / 28 / 88$ & 49.30 \\
\hline $92 / 28 / 68$ & $\begin{array}{l}4.25 \\
7.73\end{array}$ \\
\hline $02 / 28 / 88$ & $7.55\}$ \\
\hline $\begin{array}{l}92 / 28 / 88 \\
82 / 28 / 88\end{array}$ & \\
\hline $02 / 28 / 88$ & $\left.{ }_{214^{43}}\right\}$ \\
\hline
\end{tabular}


IABLE E.7. (contd)

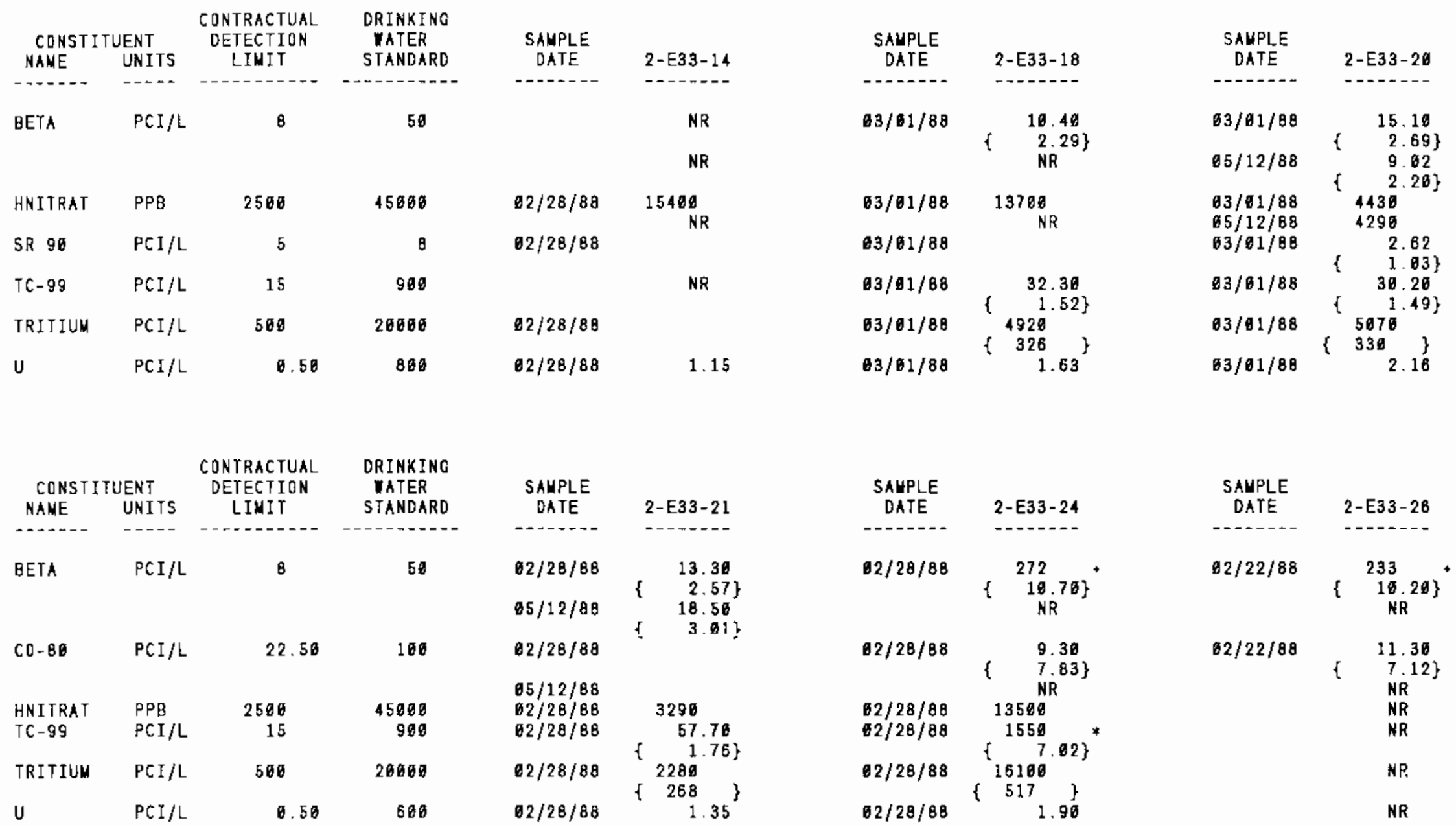


IABLE E.7. (contd)

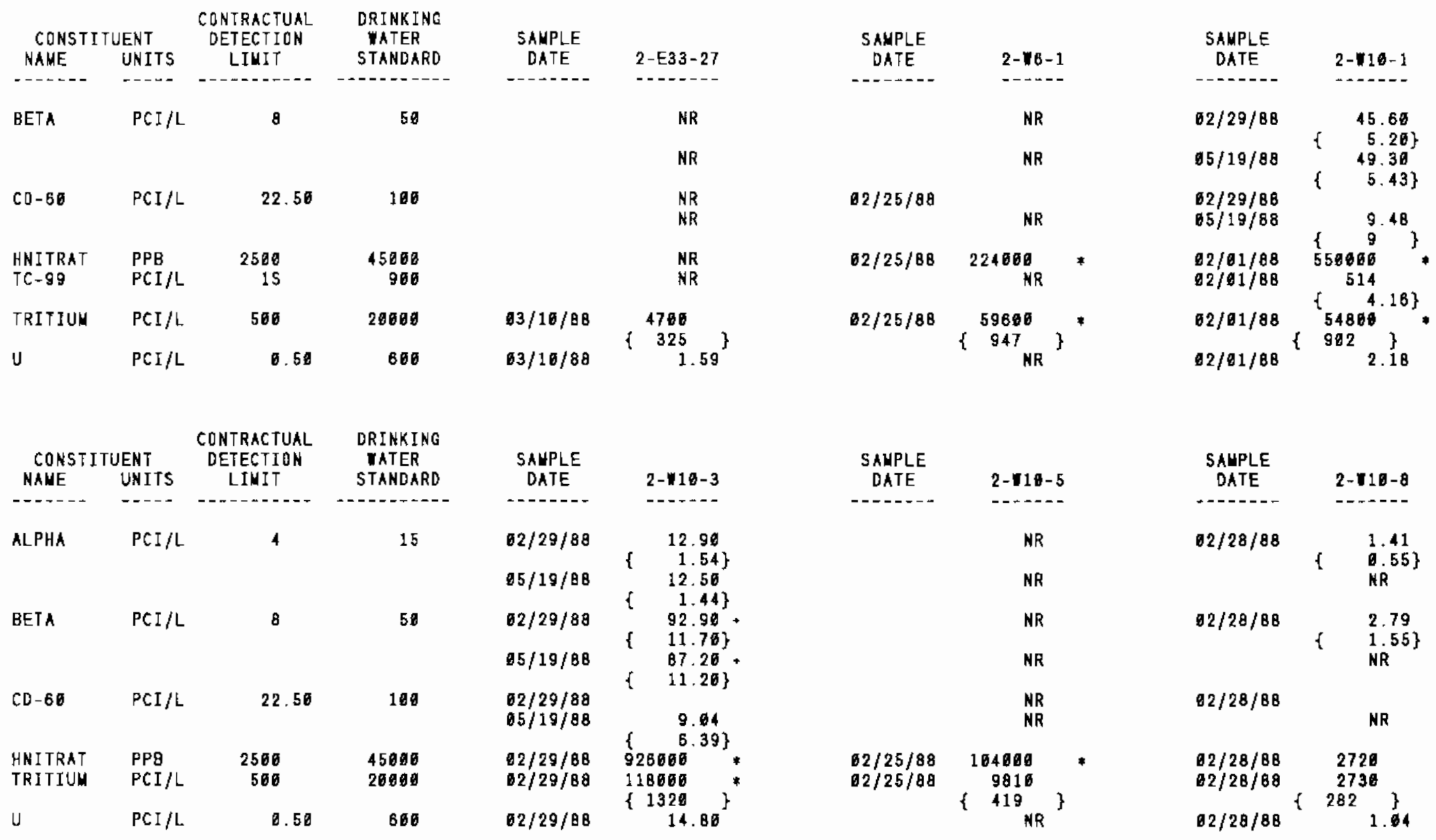


TABLEE.7. (contd)

\begin{tabular}{|c|c|c|c|c|c|c|c|c|c|}
\hline $\begin{array}{l}\text { CONSTI } \\
\text { NAME }\end{array}$ & $\begin{array}{l}\text { UENT } \\
\text { UNITS }\end{array}$ & $\begin{array}{l}\text { CONTRACTUAL } \\
\text { DETECTION } \\
\text { LIMIT }\end{array}$ & $\begin{array}{l}\text { DRINKING } \\
\text { WATER } \\
\text { STANDARD }\end{array}$ & $\begin{array}{l}\text { SAHPLE } \\
\text { DATE }\end{array}$ & 2-11-3 & $\begin{array}{c}\text { SANPLE } \\
\text { DATE }\end{array}$ & $2-11-9$ & $\begin{array}{c}\text { SAUPLE } \\
\text { DATE }\end{array}$ & $2-11-11$ \\
\hline----- & ---- & - & - & ----- & $---n-\infty$ & 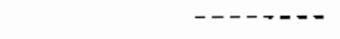 & - - - - & - & - - \\
\hline ALPHA & $\mathrm{PCI} / \mathrm{L}$ & 4 & 15 & & NR & & NR & $02 / 25 / 88$ & $\begin{array}{l}3.16 \\
0.79\}\end{array}$ \\
\hline BETA & $\mathrm{PCI} / \mathrm{L}$ & 8 & 56 & & NR & & NR & $62 / 25 / 88$ & $\begin{array}{c}54.40 \\
5.31\}\end{array}$ \\
\hline $\begin{array}{l}\text { HNITRAT } \\
\text { TRITIUN }\end{array}$ & $\begin{array}{l}\text { PPB } \\
\text { PCI } / L\end{array}$ & $\begin{array}{r}2500 \\
500\end{array}$ & $\begin{array}{l}45000 \\
20000\end{array}$ & $\begin{array}{l}82 / 25 / 88 \\
02 / 25 / 88\end{array}$ & 85900 & $\begin{array}{l}62 / 25 / 88 \\
62 / 25 / 88\end{array}$ & $\begin{array}{r}56400 \\
2740 \\
\{\quad 277\end{array}$ & & $\begin{array}{l}\text { NR } \\
\text { NR }\end{array}$ \\
\hline $\mathrm{u}$ & $\mathrm{PCI} / \mathrm{L}$ & 6. 5 & 609 & $02 / 25 / 88$ & 0.72 & $2 / 25 / 88$ & 1.08 & & NR \\
\hline
\end{tabular}

\begin{tabular}{|c|c|c|c|c|c|c|c|c|c|}
\hline $\begin{array}{l}\text { CONST I } \\
\text { NAUE }\end{array}$ & $\begin{array}{l}\text { UENI } \\
\text { UNITS }\end{array}$ & $\begin{array}{c}\text { CONTRACTUAL } \\
\text { DETECTION } \\
\text { LIMIT }\end{array}$ & $\begin{array}{l}\text { DRINKING } \\
\text { WATER } \\
\text { STANDARD }\end{array}$ & $\begin{array}{c}\text { SAMPLE } \\
\text { DATE }\end{array}$ & $2-\pi 11-15$ & $\begin{array}{l}\text { SAHPLE } \\
\text { DATE }\end{array}$ & $2-11-18$ & $\begin{array}{c}\text { SAWPLE } \\
\text { DATE }\end{array}$ & $2-11-23$ \\
\hline---- & --- & 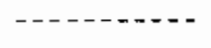 & $-\ldots$ & ----- & $\cdots$ & $-\cdots-1$ & $---\cdots$ & $---1--$ & $--\cdots--$ \\
\hline ALPHA & PCI/L & 4 & 15 & & NR & & $N R$ & $62 / 28 / 88$ & $\begin{array}{l}1.29 \\
b .53\}\end{array}$ \\
\hline BETA & $\mathrm{PCI} / \mathrm{L}$ & 8 & 50 & $\begin{array}{l}02 / 28 / 88 \\
65 / 16 / 88\end{array}$ & 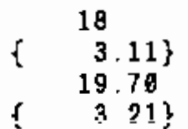 & $02 / 25 / 88$ & $\left\{\begin{array}{l}7 q .10 \\
6.11\} \\
N R\end{array}\right.$ & $82 / 28 / 88$ & $\begin{array}{ll} & 13.30 \\
\{\quad 3.26\} \\
\text { AR }\end{array}$ \\
\hline $\begin{array}{l}\text { HNITRAT } \\
\text { TC-99 }\end{array}$ & $\begin{array}{l}\text { PPB } \\
\text { PCI } / L\end{array}$ & $\begin{array}{r}2586 \\
15\end{array}$ & $\begin{array}{r}45666 \\
980\end{array}$ & & $\begin{array}{l}\text { NR } \\
\text { NR }\end{array}$ & $92 / 25 / 88$ & 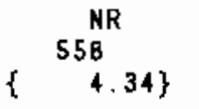 & $12 / 28 / 88$ & 113000 \\
\hline
\end{tabular}


IABLE E.7. (contd)

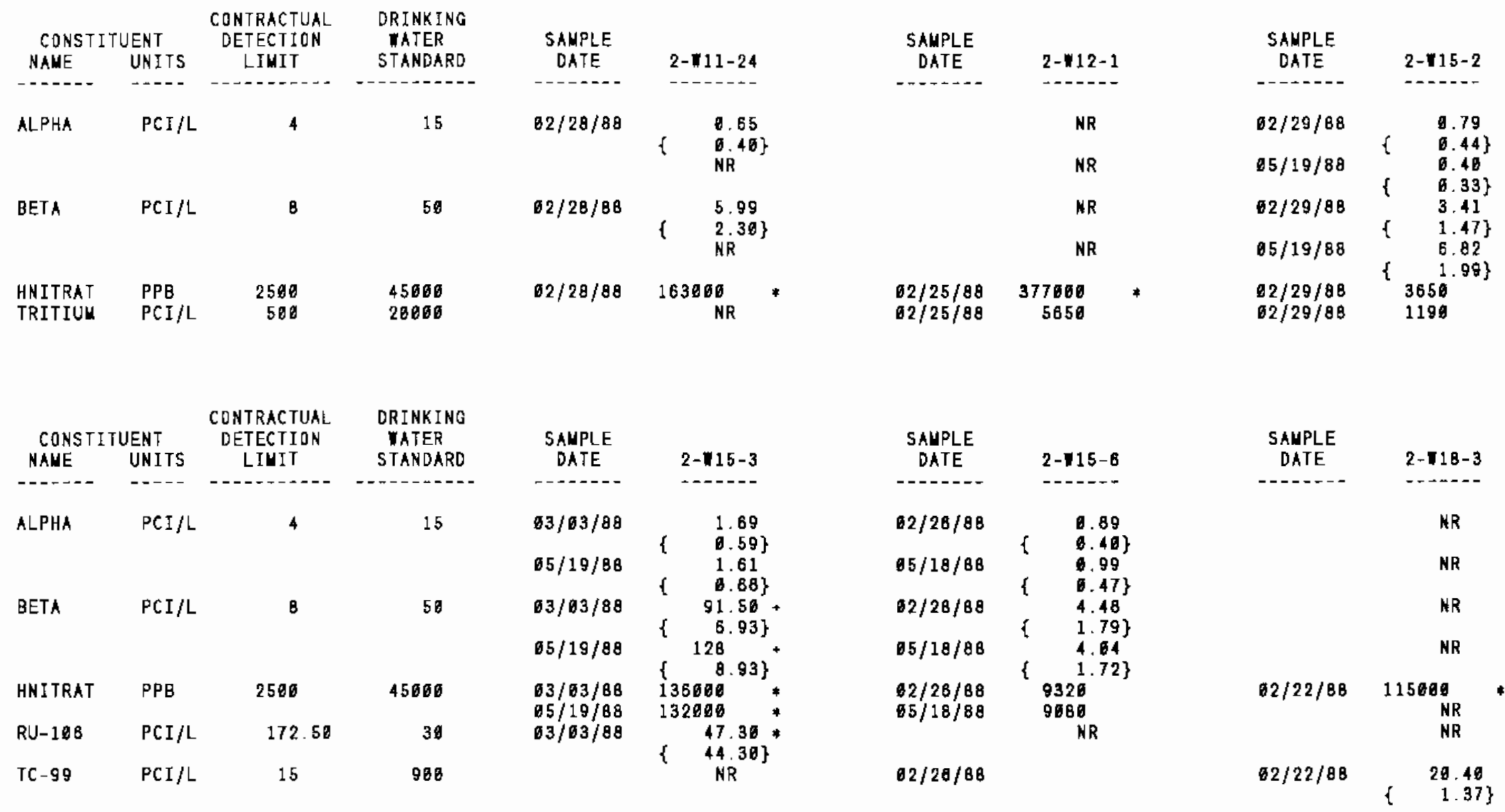


TABLE E.7. (contd)

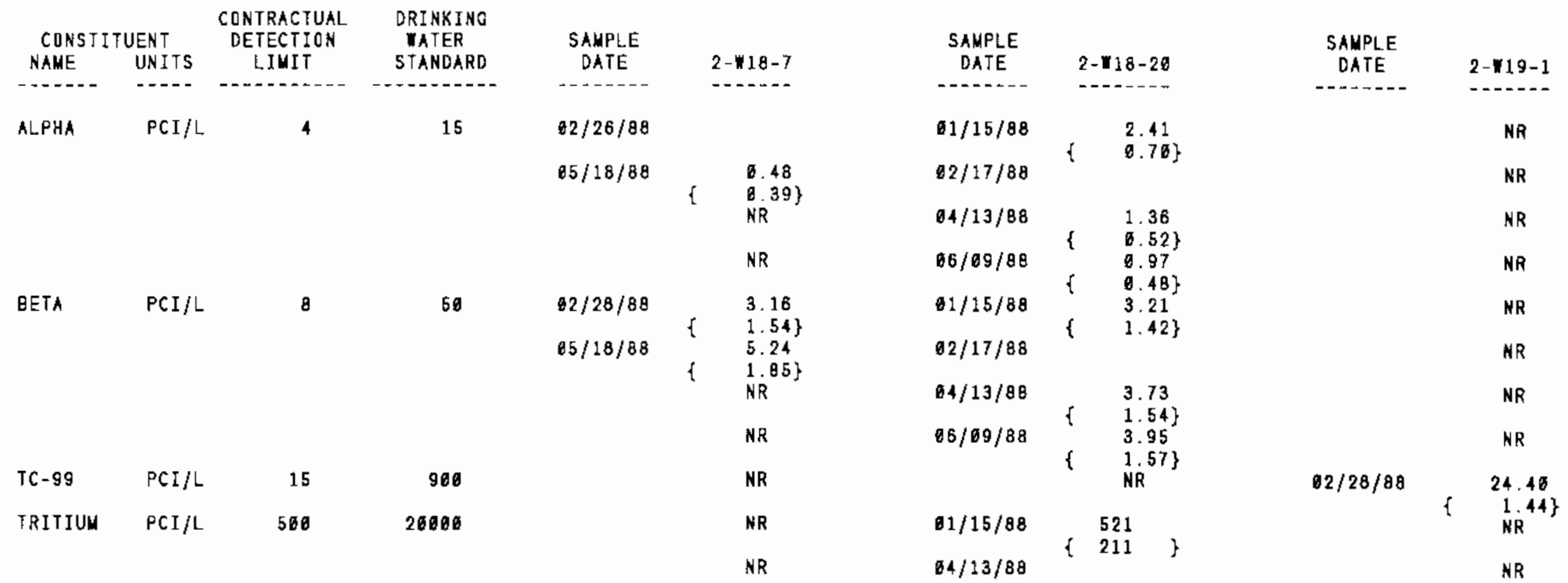


TABLE E. . . (contd)

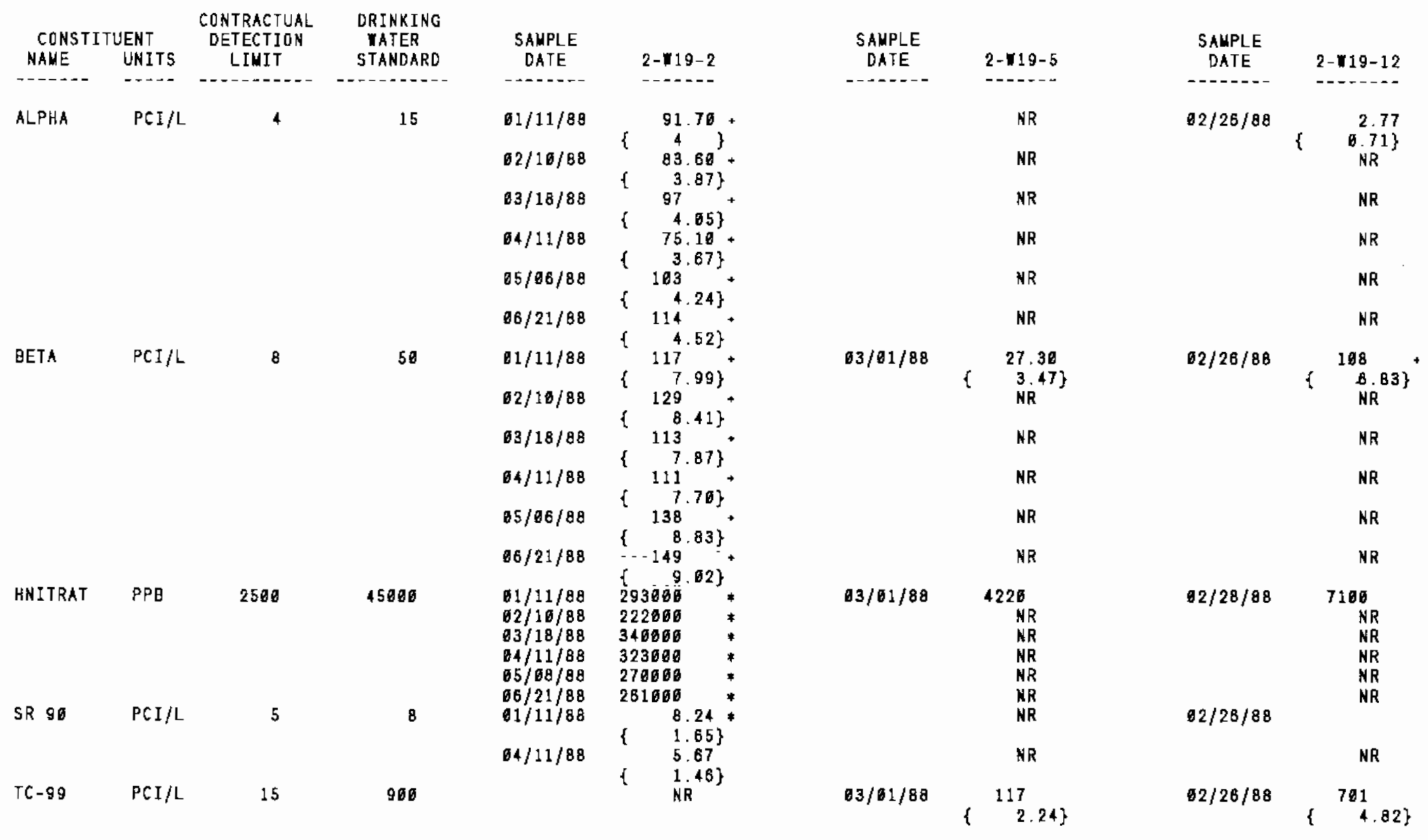


TABLE E.7. (contd)

\begin{tabular}{|c|c|c|c|c|c|c|c|c|c|}
\hline \multicolumn{2}{|c|}{ CONSTITUENT } & $\begin{array}{l}\text { CONTRACTUAL } \\
\text { DETECTION } \\
\text { LINIT }\end{array}$ & $\begin{array}{l}\text { DRINKING } \\
\text { WATER } \\
\text { STANDARD }\end{array}$ & $\begin{array}{l}\text { SAUPLE } \\
\text { DATE }\end{array}$ & $2-19-2$ & $\begin{array}{l}\text { SAMPLE } \\
\text { DATE }\end{array}$ & $2-19-5$ & $\begin{array}{l}\text { SAMPLE } \\
\text { DATE }\end{array}$ & $2-119-12$ \\
\hline$-\cdots---$ & --- & - & - & 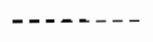 & ------ & $-\cdots---$ & ----- & $\ldots-\cdots$ & $\cdots-\cdots$ \\
\hline TRITIUM & $\mathrm{PCI} / \mathrm{L}$ & 500 & 20000 & $91 / 11 / 88$ & $\begin{array}{r}88300 \\
986\end{array}$ & $03 / 01 / 88$ & & $62 / 26 / 88$ & \\
\hline & & & & $04 / 11 / 88$ & 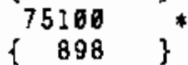 & & NR & & NR \\
\hline $\mathrm{U}$ & $\mathrm{PCI} / \mathrm{L}$ & 0.50 & 600 & & NR & $03 / 01 / 88$ & 6.70 & & NR \\
\hline U-CHEM & UG/L & 0.73 & & $\begin{array}{l}01 / 11 / 88 \\
02 / 10 / 88 \\
03 / 18 / 88 \\
04 / 11 / 88 \\
95 / 06 / 88 \\
06 / 21 / 88\end{array}$ & $\begin{array}{l}93.86 \\
183 \\
167 \\
86.36 \\
83.70 \\
138\end{array}$ & & $\begin{array}{l}\text { NR } \\
\text { NR } \\
\text { NR } \\
\text { NR } \\
\text { NR } \\
\text { NR }\end{array}$ & $92 / 26 / 88$ & $\begin{array}{l}3.33 \\
\text { NR } \\
\text { NR } \\
\text { NR } \\
\text { NR } \\
\text { NR }\end{array}$ \\
\hline
\end{tabular}


TABLE E.7. (contd)

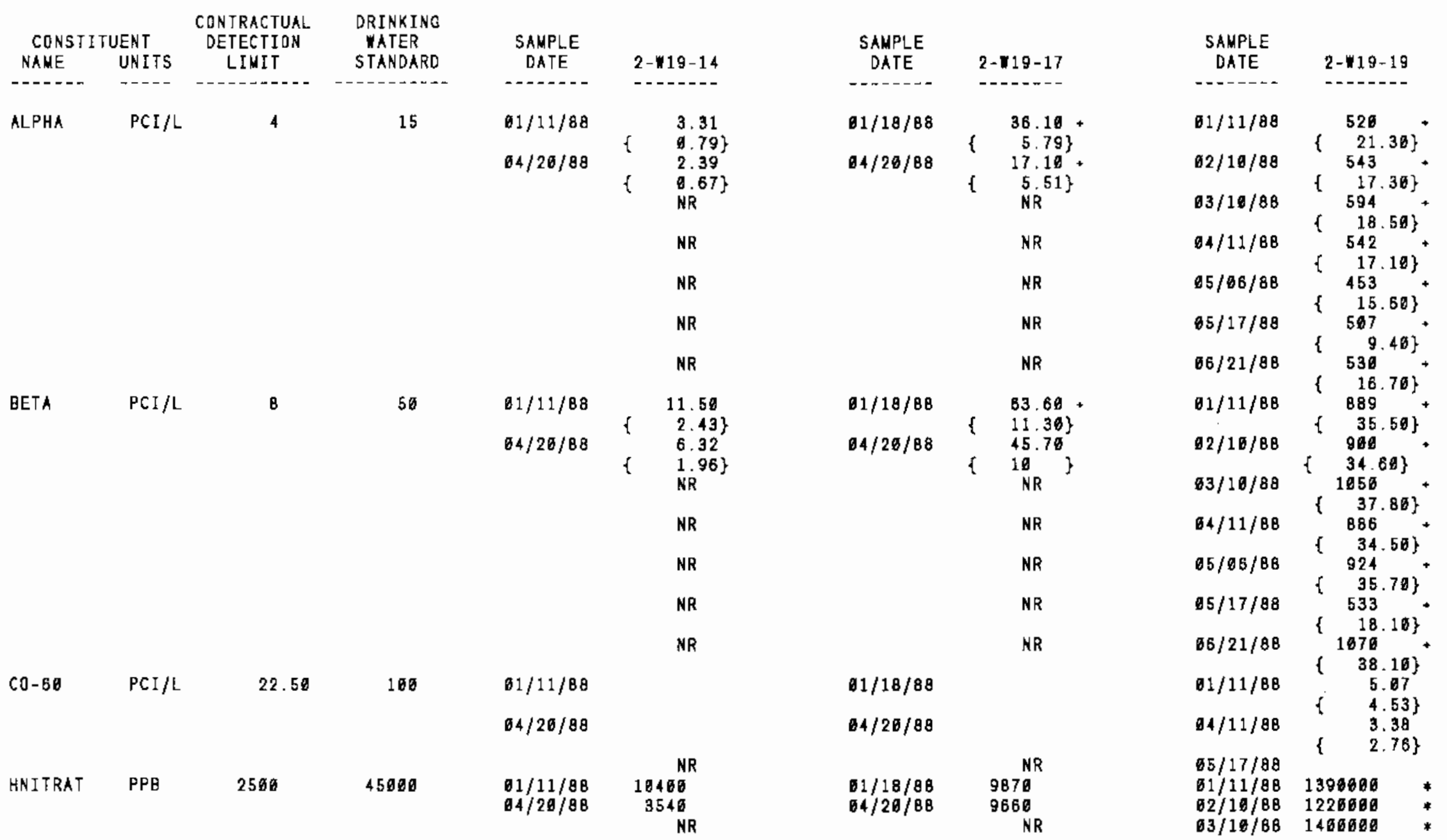


TABLE E.7. (contd)

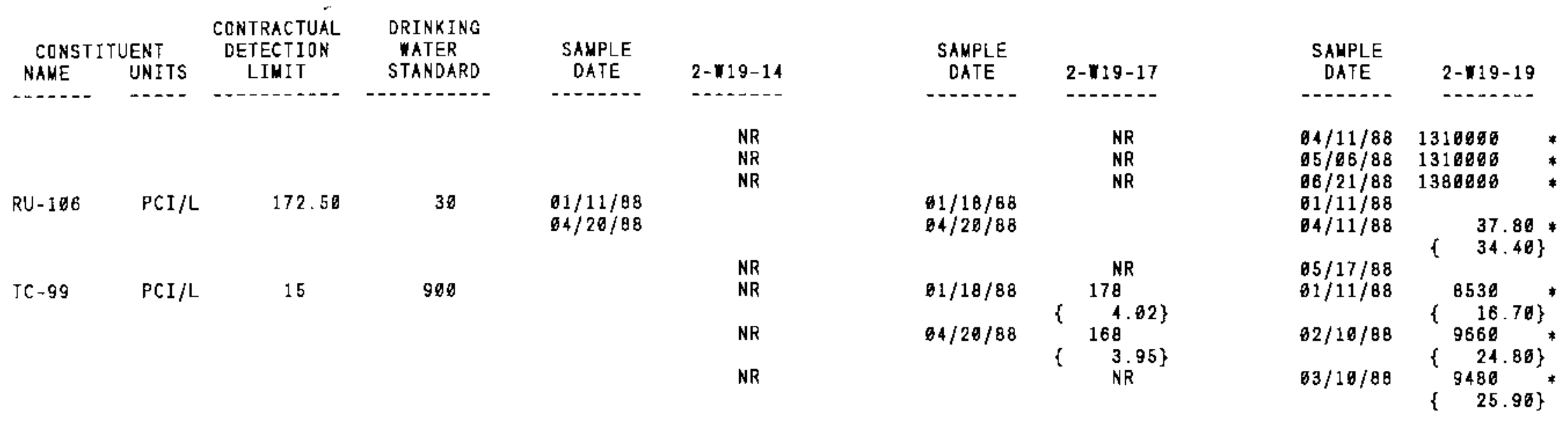


IABLE E.7. (contd)

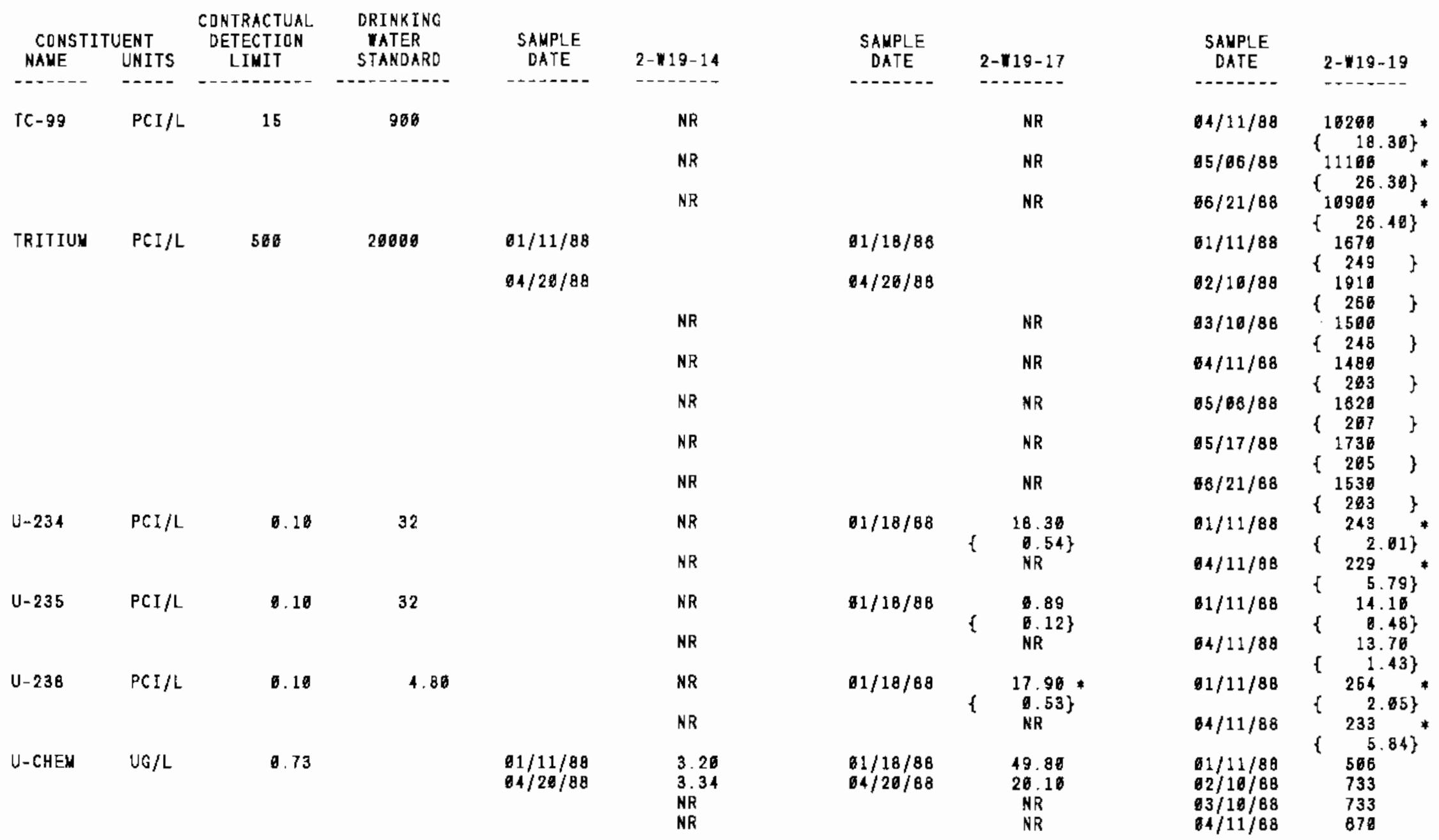


TABLE E.7. (contd)

CONTRACTUAL DRINKING

CONSTITUENT DETECTION WATER

UNITS DETECTION

SAMPLE LIMIT

STANDARD

SAMPLE

DATE

2-19-17

2-19-14

DATE-

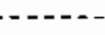

NR

NR

SAMPLE

DATE

2-19-19

$85 / 68 / 88$

$06 / 21 / 88$

657 
TABLE E.7. (contd)

\begin{tabular}{|c|c|c|c|c|c|}
\hline \multicolumn{2}{|c|}{ CDNSTITUENT } & $\begin{array}{l}\text { CONTRACTUAL } \\
\text { DETECTION } \\
\text { LINIT }\end{array}$ & $\begin{array}{l}\text { DRINKING } \\
\text { WATER } \\
\text { STANDARD }\end{array}$ & $\begin{array}{l}\text { SAMPLE } \\
\text { DATE }\end{array}$ & $2-19-23$ \\
\hline - - n. &.--- & -- & $-\cdots$ & $\cdots+\cdots$ & $\cdots$ \\
\hline ALPHA & $P C I / L$ & 4 & 15 & $\begin{array}{l}01 / 11 / 88 \\
02 / 18 / 88 \\
63 / 16 / 88 \\
64 / 11 / 88 \\
05 / 66 / 88 \\
66 / 15 / 8 B\end{array}$ & $\left\{\begin{array}{c}154 \\
11.09\} \\
156 \\
12 \\
172 \\
13.49 \\
142 \\
12 \\
132 \\
11 \\
126 \\
10.96\end{array}\right\}+$ \\
\hline BETA & $\mathrm{PCI} / \mathrm{L}$ & $B$ & 50 & $\begin{array}{l}01 / 11 / 88 \\
62 / 18 / 88 \\
03 / 16 / 88 \\
64 / 11 / 88 \\
05 / 66 / 88 \\
66 / 15 / 88\end{array}$ & 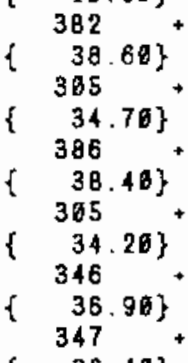 \\
\hline HNITRAT & PPB & 2566 & 45666 & $\begin{array}{l}61 / 11 / 88 \\
62 / 16 / 88 \\
03 / 16 / 88 \\
04 / 11 / 88 \\
65 / 66 / 88 \\
06 / 15 / 88\end{array}$ & $\begin{array}{l}436 . \\
487906 \\
389860 \\
426066 \\
419660 \\
437060 \\
453660\end{array}$ \\
\hline TC-99 & $\mathrm{PCI} / \mathrm{L}$ & 15 & 980 & $\begin{array}{l}61 / 11 / 88 \\
62 / 10 / 88 \\
83 / 10 / 88\end{array}$ & 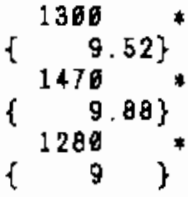 \\
\hline
\end{tabular}
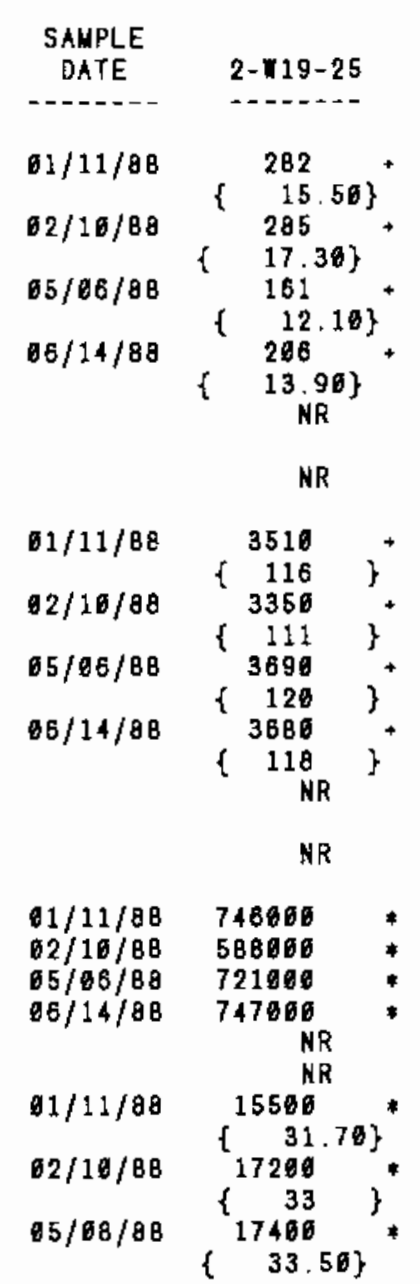

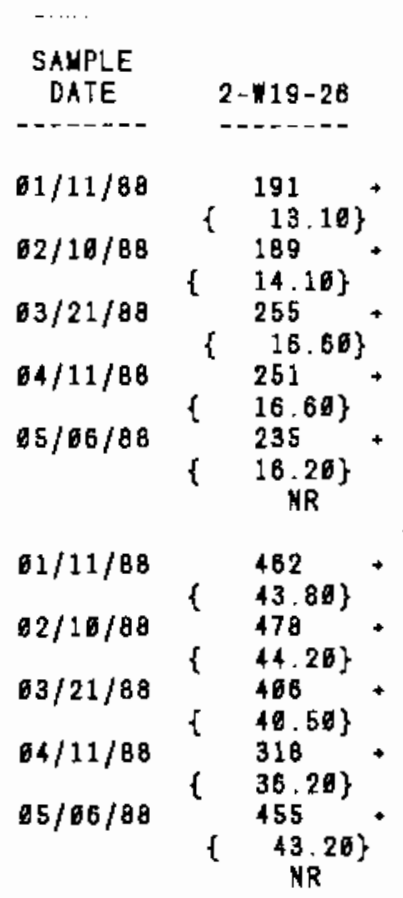

\begin{tabular}{|c|c|}
\hline $\begin{array}{l}61 / 11 / 88 \\
02 / 16 / 88 \\
03 / 21 / 88 \\
64 / 11 / 88 \\
05 / 86 / 88\end{array}$ & $\begin{array}{r}1646060 \\
850000 \\
1120000 \\
1676000 \\
1110000\end{array}$ \\
\hline $61 / 11 / 88$ & $\begin{array}{c}\text { NR } \\
1046 \\
5.82\}\end{array}$ \\
\hline $82 / 19 / 8 B$ & $\left\{\begin{array}{l}2800 \\
11.70\}\end{array}\right.$ \\
\hline $63 / 21 / 88$ & $\left\{\begin{array}{c}2100 \\
11.80\}\end{array}\right.$ \\
\hline
\end{tabular}


TABLE E.7. (contd)

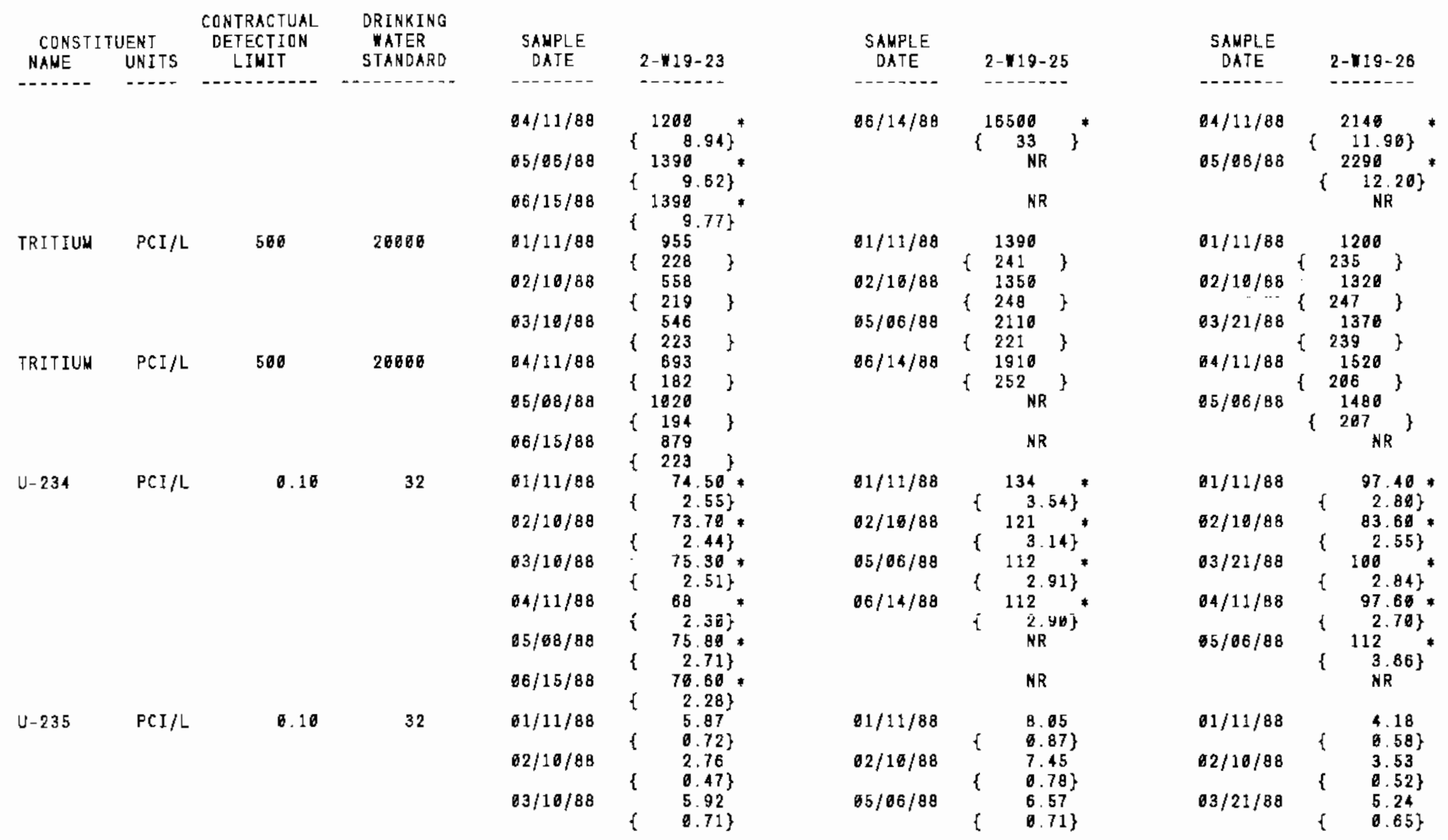


TABLE E.7. (contd)

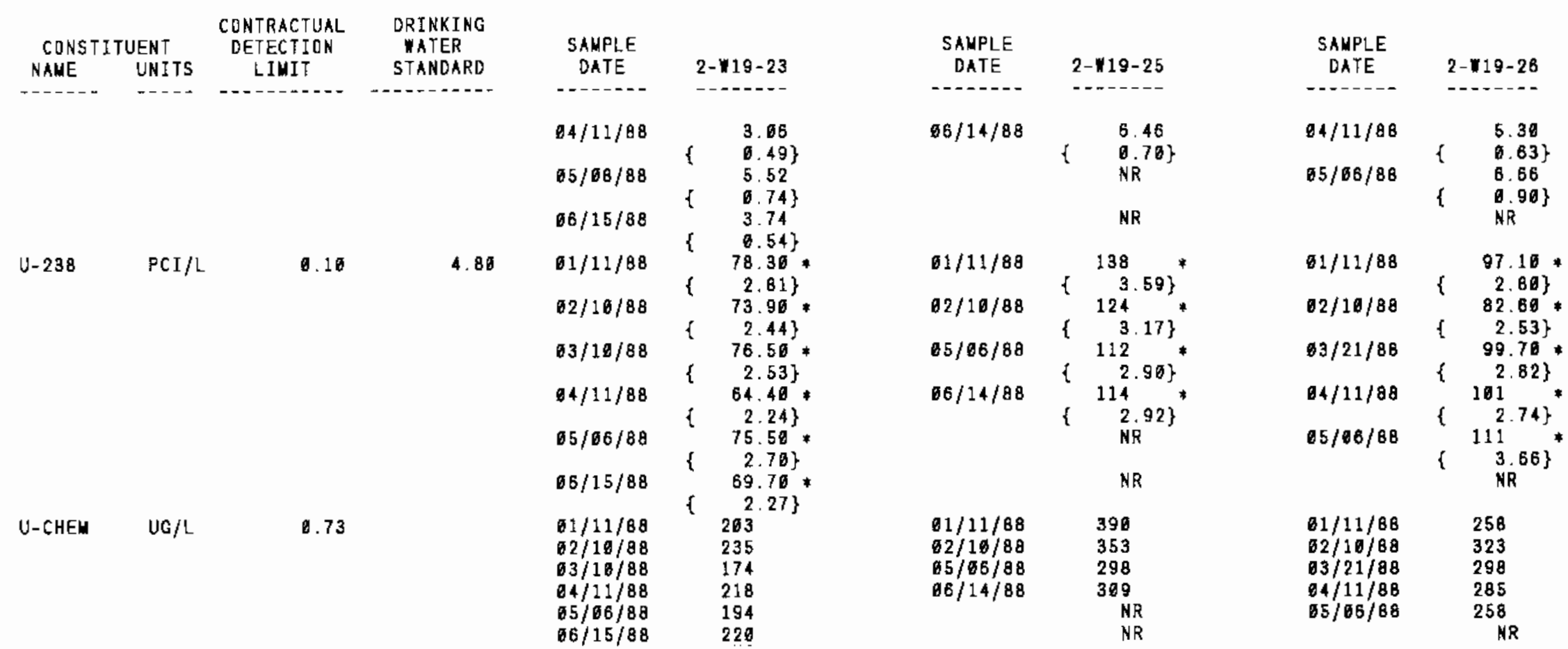


TABLE E.7. (contd)

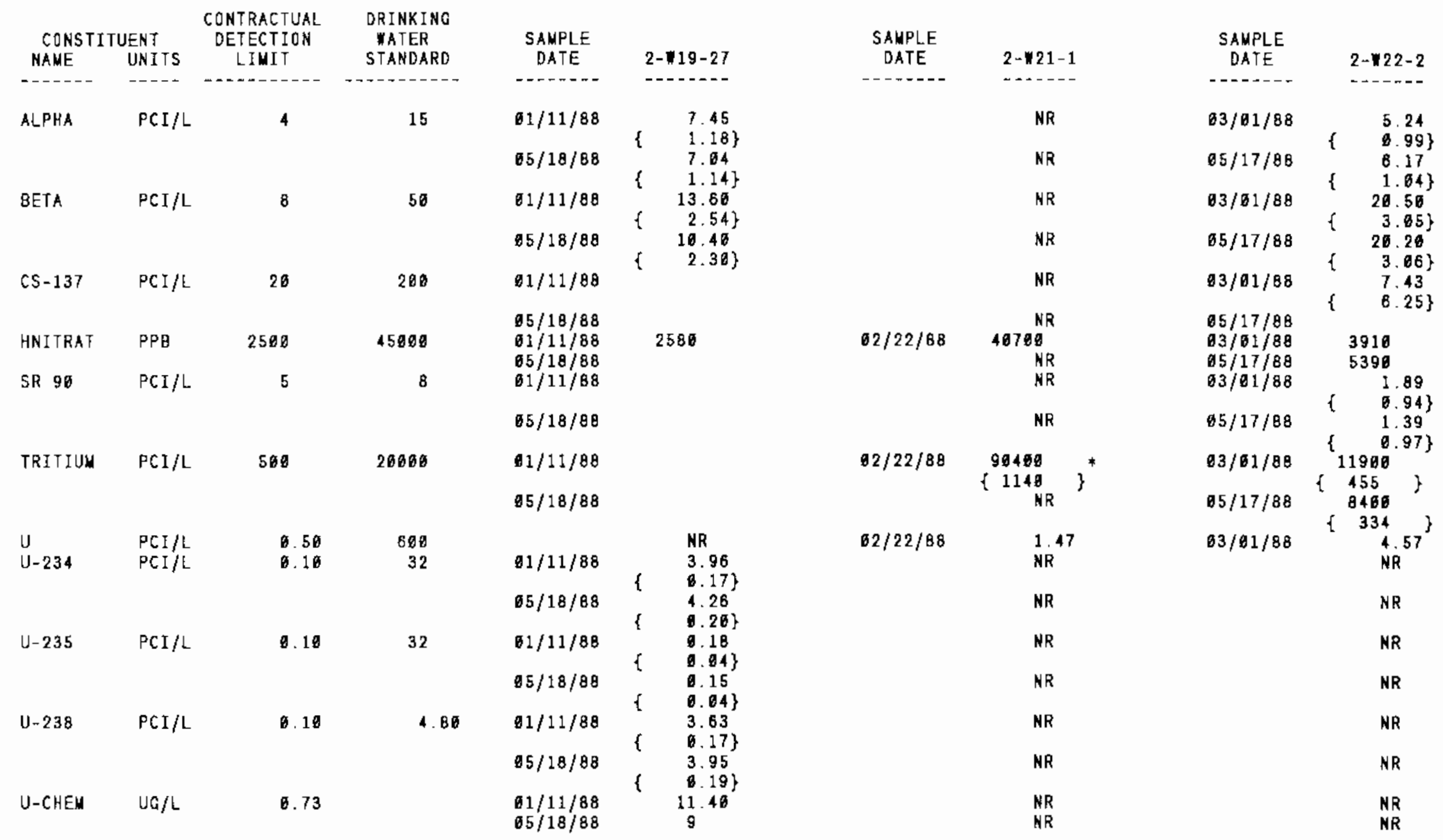


TABLE E.7. (contd)

\begin{tabular}{|c|c|c|c|c|c|c|c|c|c|}
\hline \multicolumn{2}{|c|}{ CONSTITUENT } & $\begin{array}{l}\text { CONTRACTUAL } \\
\text { DETECTION }\end{array}$ & $\begin{array}{l}\text { DRINKING } \\
\text { WATER } \\
\text { STANDARD }\end{array}$ & $\begin{array}{l}\text { SAMPLE } \\
\text { DATE }\end{array}$ & $2-122-7$ & $\begin{array}{l}\text { SAMPLE } \\
\text { DATE }\end{array}$ & $2-22-9$ & $\begin{array}{l}\text { SAMPLE } \\
\text { DATE }\end{array}$ & $2-\pi 22-10$ \\
\hline ALPHA & $\mathrm{PCI} / \mathrm{L}$ & 4 & 15 & & $\begin{array}{l}\text { NR } \\
\text { NR }\end{array}$ & & $\begin{array}{l}\text { NR } \\
\text { NR }\end{array}$ & $\begin{array}{l}02 / 28 / 88 \\
65 / 17 / 88\end{array}$ & 9.47 \\
\hline BETA & $\mathrm{PCI} / \mathrm{L}$ & 8 & 50 & & NR & & AR & $92 / 28 / 88$ & $\begin{array}{r}41.89 \\
\{\quad 4.12\}\end{array}$ \\
\hline $\begin{array}{l}\text { HNITRAT } \\
\text { SR } 9 g\end{array}$ & $\begin{array}{l}\text { PPB } \\
\text { PCI/L }\end{array}$ & $\begin{array}{r}2509 \\
5\end{array}$ & $\begin{array}{r}45060 \\
8\end{array}$ & $\begin{array}{l}62 / 22 / 88 \\
02 / 22 / 88\end{array}$ & NR & $\begin{array}{l}62 / 22 / 88 \\
92 / 22 / 88\end{array}$ & $\begin{array}{l}\text { NR } \\
5380\end{array}$ & $65 / 17 / 88$ & 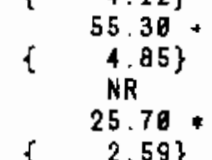 \\
\hline & & & & & NR & & NR & $65 / 17 / 88$ & $\begin{array}{r}26.46 \\
\{\quad 2.48\}\end{array}$ \\
\hline TRITIUM & $\begin{array}{l}\mathrm{PCI} / \mathrm{L} \\
\mathrm{PCI} / \mathrm{L}\end{array}$ & $\begin{array}{l}566 \\
0.59\end{array}$ & $\begin{array}{r}290090 \\
690\end{array}$ & $\begin{array}{l}92 / 22 / 88 \\
92 / 22 / 88\end{array}$ & $\left.\begin{array}{c}3640606 \\
\{2299\end{array}\right\}^{*}$ & $\begin{array}{l}62 / 22 / 88 \\
22 / 22 / 88\end{array}$ & $\left.\left.\begin{array}{l}7566686 \\
\{12666\end{array}\right\}\right\}_{4.19}$ & $\begin{array}{l}62 / 28 / 88 \\
62 / 28 / 88\end{array}$ & $\left.\begin{array}{l}1218 \delta 8 \\
1328\end{array}\right\}$ \\
\hline
\end{tabular}


TABLE E.7. (contd)

\begin{tabular}{|c|c|c|c|c|c|c|c|c|c|}
\hline $\begin{array}{l}\text { CDNSTI } \\
\text { NAME }\end{array}$ & $\begin{array}{l}\text { UENT } \\
\text { UNITS }\end{array}$ & $\begin{array}{c}\text { CONTRACTUAL } \\
\text { DETECTION } \\
\text { LIMIT }\end{array}$ & $\begin{array}{l}\text { DRINKING } \\
\text { WATER } \\
\text { STANDARD }\end{array}$ & $\begin{array}{l}\text { SANPLE } \\
\text { DATE }\end{array}$ & $2-122-12$ & $\begin{array}{l}\text { SALPLE } \\
\text { DATE }\end{array}$ & $2-122-18$ & $\begin{array}{l}\text { SANPLE } \\
\text { DATE }\end{array}$ & $2-22-21$ \\
\hline - - - & --- & - - & - n- & ------ & $---1---$ & $-\ldots-1$ & - - & $---\infty$ & $--\cdots$ \\
\hline ALPHA & $P C I / L$ & 4 & 15 & & $\begin{array}{l}\text { NR } \\
\text { NR }\end{array}$ & $\begin{array}{l}93 / 01 / 88 \\
95 / 17 / 88\end{array}$ & 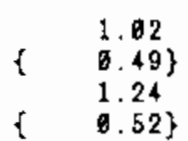 & $12 / 22 / 88$ & 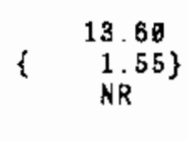 \\
\hline BETA & $P C I / L$ & 8 & 56 & $62 / 22 / 88$ & 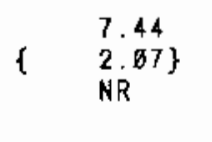 & $\begin{array}{l}63 / 01 / 88 \\
65 / 17 / 88\end{array}$ & 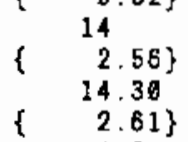 & $82 / 22 / 88$ & $\left\{\begin{array}{c}170 \\
8.56\} \\
N R\end{array}\right\}$ \\
\hline CS -137 & $\mathrm{PCI} / \mathrm{L}$ & 26 & 280 & $02 / 22 / 88$ & $\mathrm{NR}$ & $03 / 01 / 88$ & $\begin{array}{l}4.56 \\
\{\quad 4.19\}\end{array}$ & $02 / 22 / 88$ & \\
\hline $\begin{array}{l}\text { HNITRAT } \\
\text { TC-99 }\end{array}$ & $\begin{array}{l}\text { PPB } \\
P C I / L\end{array}$ & $\begin{array}{r}2596 \\
15\end{array}$ & $\begin{array}{r}45000 \\
900\end{array}$ & $92 / 22 / 88$ & $\begin{array}{c}\text { NR } \\
2778 \\
\text { NR }\end{array}$ & $\begin{array}{l}05 / 17 / 88 \\
03 / 01 / 88\end{array}$ & $\begin{array}{c}\text { NR } \\
69.46 \\
1.88\end{array}$ & $62 / 22 / 88$ & $\begin{array}{c}\text { NR } \\
35300^{N R}\end{array}$ \\
\hline TRITIUM & $\mathrm{PCI} / \mathrm{L}$ & 500 & 20000 & $62 / 22 / 88$ & $\left\{\begin{array}{r}25200 \\
631\end{array}\right\}^{*}$ & & NR & & NR \\
\hline$u$ & $P C I / L$ & 9.50 & 600 & $22 / 22 / 88$ & 1.64 & & NR & & NR \\
\hline
\end{tabular}


TABLE E.7. (contd)

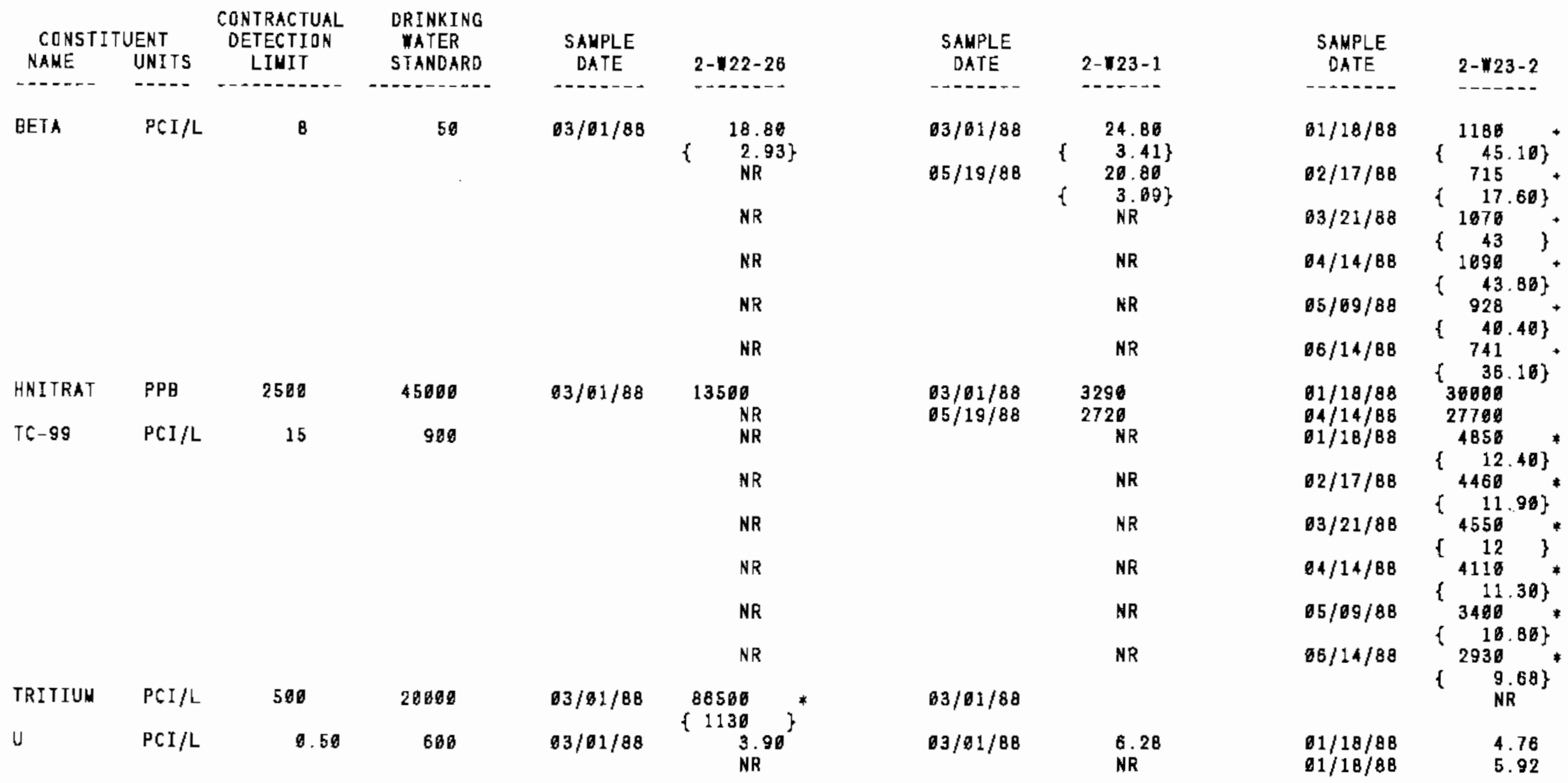


TABLE E.7. (contd)

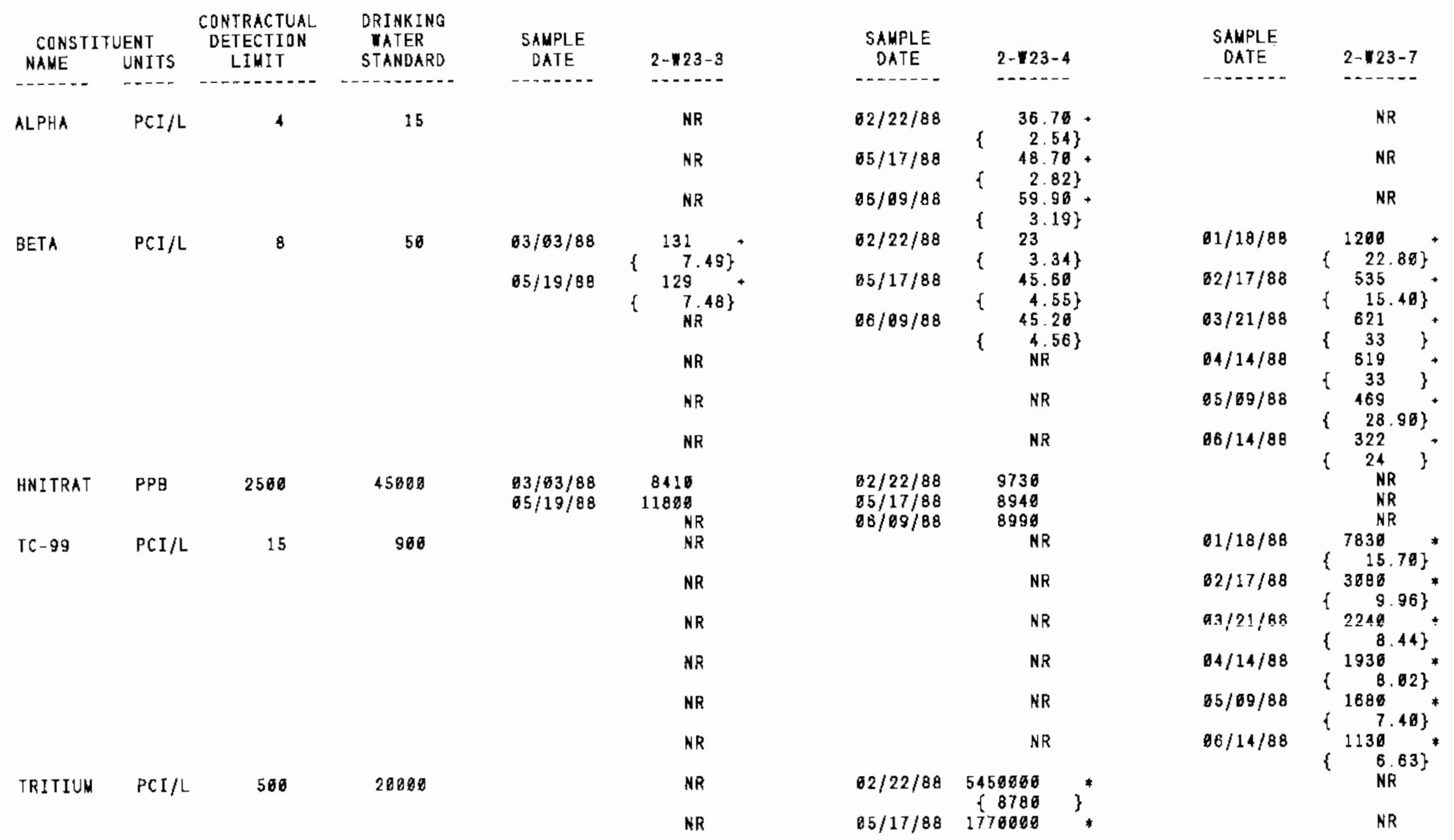


TABLE E.7. (contd)

\begin{tabular}{|c|c|c|c|c|c|c|c|c|c|}
\hline $\begin{array}{l}\text { CONST } \\
\text { NAME }\end{array}$ & $\begin{array}{l}\text { UENT } \\
\text { UNITS }\end{array}$ & $\begin{array}{l}\text { CONTRACTUAL } \\
\text { DETECTION } \\
\text { LIMIT }\end{array}$ & $\begin{array}{l}\text { DRINKING } \\
\text { MATER } \\
\text { STANDARD }\end{array}$ & $\begin{array}{c}\text { SAMPLE } \\
\text { OATE }\end{array}$ & $2-23-3$ & $\begin{array}{l}\text { SANPLE } \\
\text { DATE }\end{array}$ & $2-\pi 23-4$ & $\begin{array}{c}\text { SAMPLE } \\
\text { DATE }\end{array}$ & $2-23-7$ \\
\hline & & & & & NR & $08 / 69 / 88$ & $\left.\begin{array}{r}\{4290 \\
1550000 \\
\{4680\end{array}\right\}$ & & $\mathrm{NR}$ \\
\hline$U-234$ & $\mathrm{PC} 1 / \mathrm{L}$ & 6.10 & 32 & & NR & $92 / 22 / 8 B$ & $\begin{array}{r}21.76^{\circ} \\
\{\quad 6.42\}\end{array}$ & & NR \\
\hline$U-235$ & $P C I / L$ & 9.10 & 32 & & $N R$ & $62 / 22 / 88$ & $\begin{array}{ll}1.44 \\
\{\quad 11\}\end{array}$ & & NR \\
\hline$U-238$ & $\mathrm{PCI} / \mathrm{L}$ & 0.16 & 4.80 & & NR & $02 / 22 / 8 B$ & $\left\{\begin{array}{r}22.26{ }^{*} \\
\{\quad 0.43\}\end{array}\right.$ & & NR \\
\hline U-CHEM & UG $/ \mathrm{L}$ & 6.73 & & & $\begin{array}{l}\text { NR } \\
\text { NR } \\
\text { NR }\end{array}$ & $\begin{array}{l}62 / 22 / 88 \\
65 / 17 / 88 \\
96 / 89 / 88\end{array}$ & $\begin{array}{l}81.18 \\
66.76 \\
83.86\end{array}$ & & $\begin{array}{l}\mathrm{NR} \\
\mathrm{NR} \\
\mathrm{NR}\end{array}$ \\
\hline
\end{tabular}


TABLE E.7. (contd)

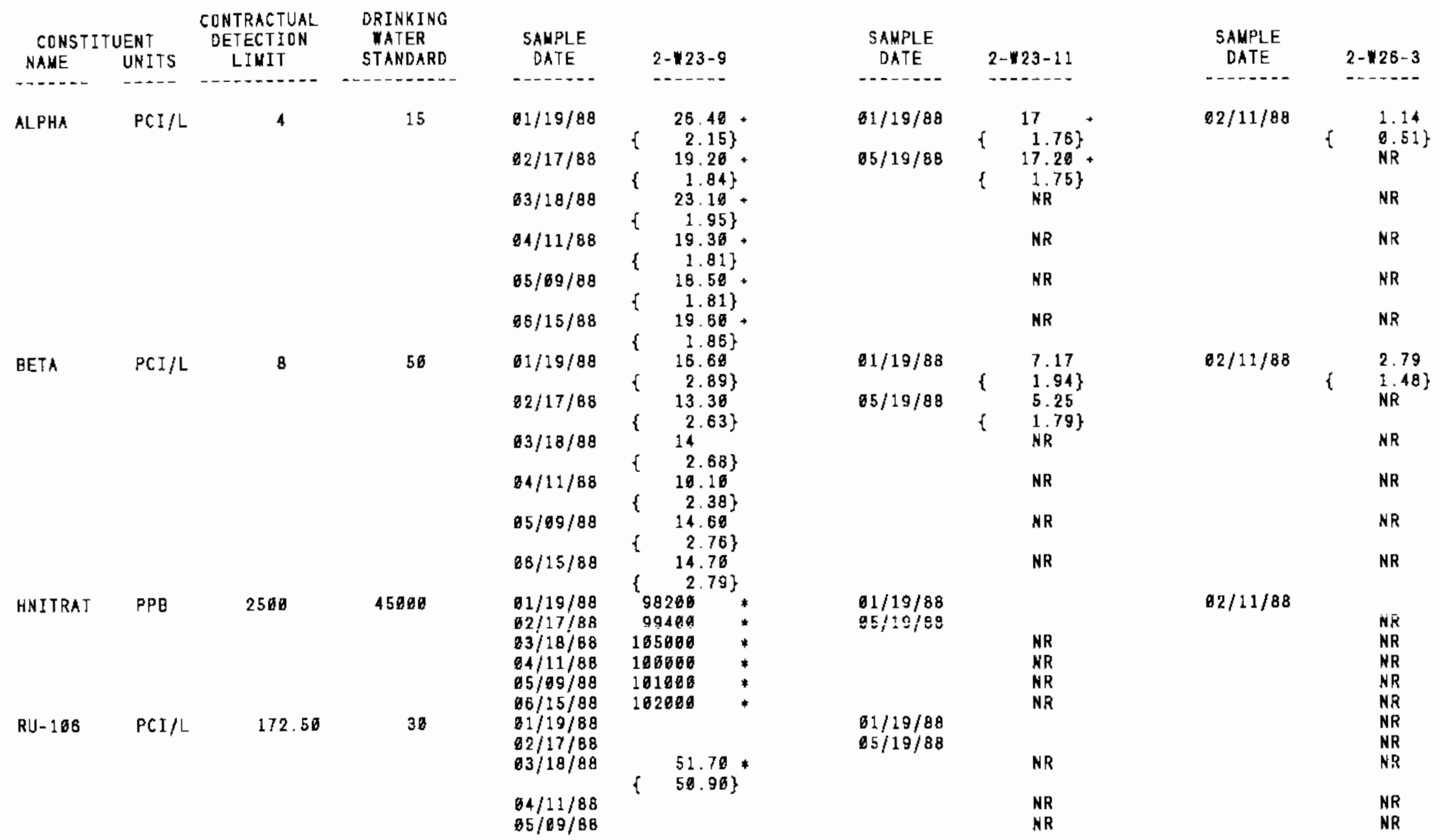


TABLE E.7. (contd)

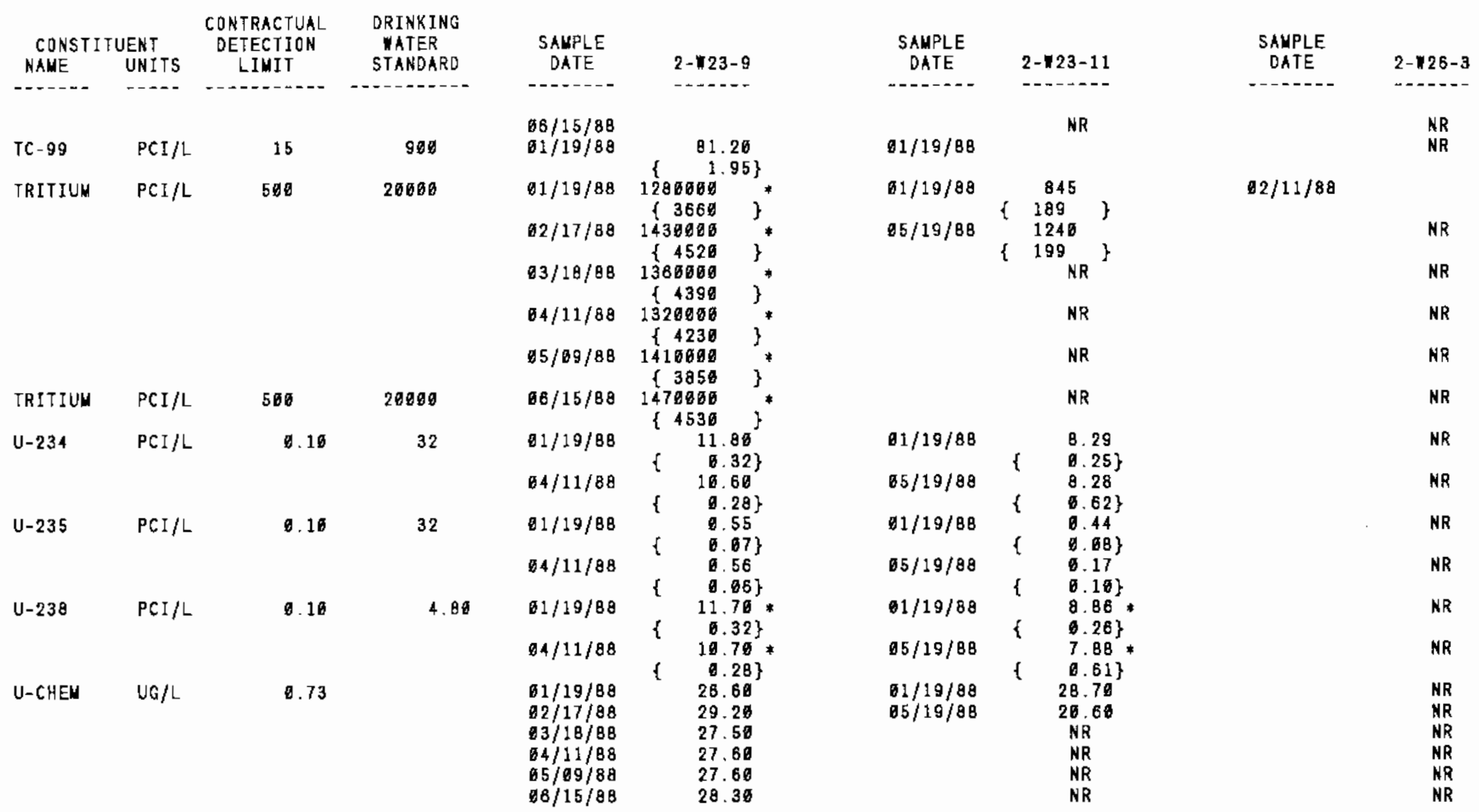


IABLE E.7. (contd)

\begin{tabular}{|c|c|c|c|}
\hline $\begin{array}{l}\text { CONSTI } \\
\text { NANE }\end{array}$ & $\begin{array}{l}\text { JENT } \\
\text { UNITS }\end{array}$ & $\begin{array}{l}\text { CONTRACTUAL } \\
\text { DETECTION } \\
\text { LIMIT }\end{array}$ & $\begin{array}{l}\text { DRINKING } \\
\text { WATER } \\
\text { STANDARD }\end{array}$ \\
\hline-+---- & $-\cdots$ & 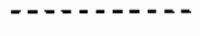 & \\
\hline ALPHA & $\mathrm{PCI} / \mathrm{L}$ & 4 & 15 \\
\hline BETA & PCI/L & 8 & 56 \\
\hline$T C-99$ & $\mathrm{PCI} / \mathrm{L}$ & 15 & 900 \\
\hline $\mathrm{U}$ & $\mathrm{PCI} / \mathrm{L}$ & 0.50 & 600 \\
\hline
\end{tabular}

$\begin{array}{ccc}\begin{array}{c}\text { SAMPLE } \\ \text { DATE }\end{array} & 2-126-6 \\ & & \\ 02 / 11 / 88 & & 1.25 \\ 62 / 11 / 88 & \{ & 6.51\} \\ & & 6.56 \\ & \left\{\begin{array}{l}1.95\} \\ \text { NR }\end{array}\right. \\ & & \text { NR } \\ & \text { NR }\end{array}$

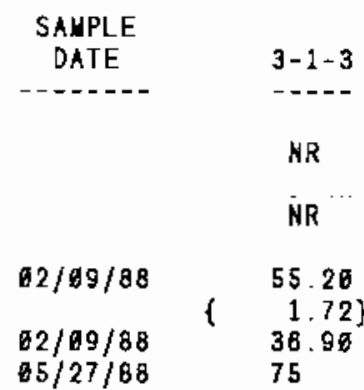

SAMPLE

DATE

3-1-5

NR

NR

NR

NR

$62 / 09 / 88-36.98$

$75.91 / 13 / 88$

$05 / 31 / 88$

48.26

36.26 
TABLE E.7. (contd)

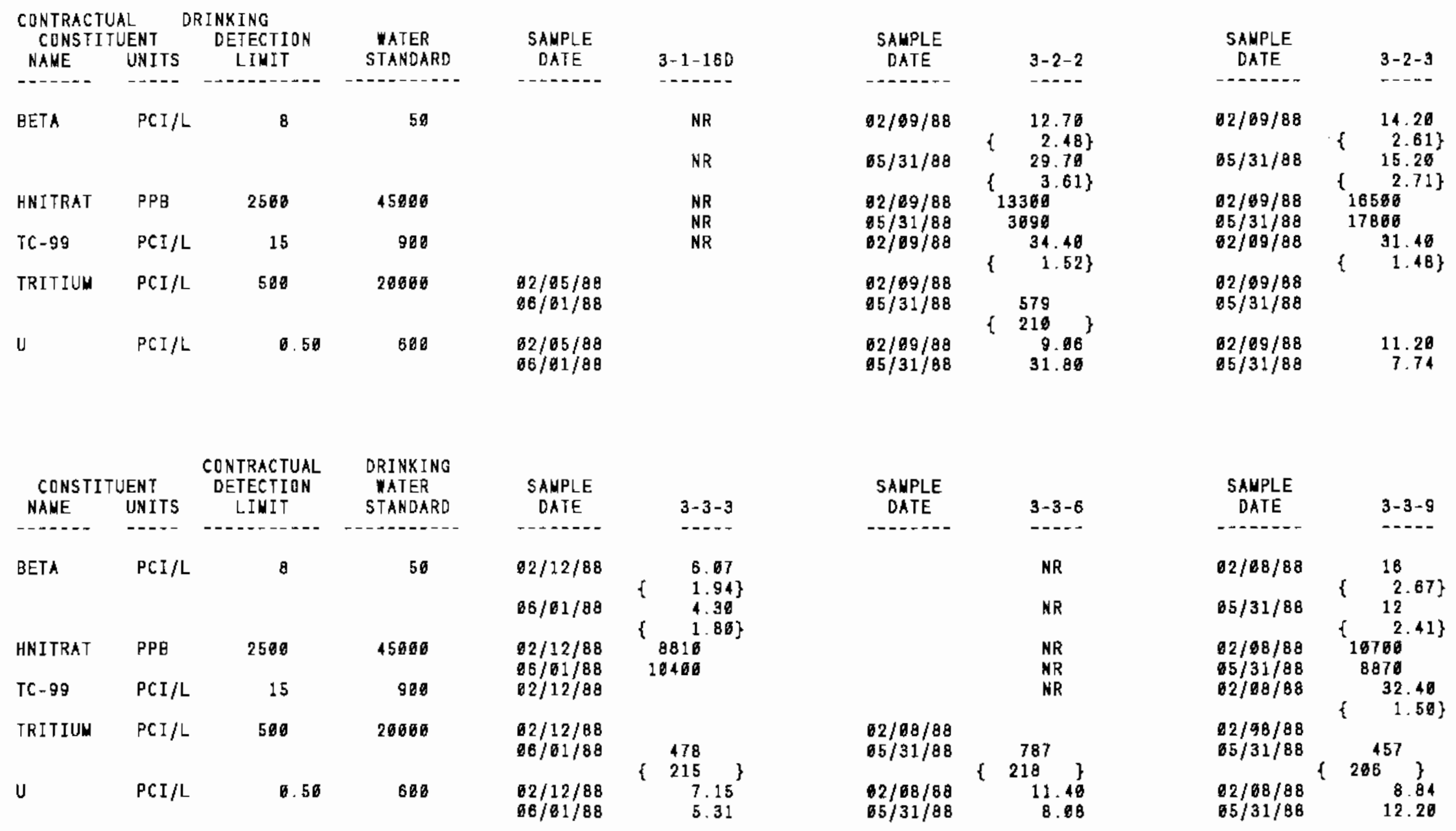


TABLE E.7. (contd)

\begin{tabular}{|c|c|c|c|c|c|c|c|c|c|}
\hline $\begin{array}{l}\text { CONSTI } \\
\text { NAHE }\end{array}$ & $\begin{array}{l}\text { JENT } \\
\text { UNITS }\end{array}$ & $\begin{array}{l}\text { CONTRACTUAL } \\
\text { DETECTION } \\
\text { LIMIT }\end{array}$ & $\begin{array}{l}\text { DRINKING } \\
\text { IATER } \\
\text { STANDARD }\end{array}$ & $\begin{array}{l}\text { SANPLE } \\
\text { DATE }\end{array}$ & $3-3-11$ & $\begin{array}{l}\text { SAUPLE } \\
\text { DATE }\end{array}$ & $3-3-12$ & $\begin{array}{c}\text { SAMPLE } \\
\text { DATE }\end{array}$ & $3-4-9$ \\
\hline$-\ldots$ & -... & $-\ldots$ & 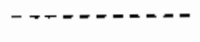 & $-\cdots+--$ & ---- & $-\cdots--$ & $--\cdots+$ & 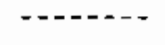 & $m--$ \\
\hline BETA & $P C I / L$ & 8 & 56 & $\begin{array}{l}82 / 12 / 88 \\
06 / 01 / 88\end{array}$ & $\begin{array}{r}21.90 \\
3.39\} \\
27.89 \\
\{\quad 3.72\}\end{array}$ & $\begin{array}{l}02 / 11 / 88 \\
68 / 01 / 88\end{array}$ & $\begin{array}{c}7.67 \\
\{\quad 2.15\} \\
11.48 \\
\{\quad 2.67\}\end{array}$ & $\begin{array}{l}02 / 88 / 88 \\
05 / 31 / 8 B\end{array}$ & $\begin{array}{c}13.60 \\
\{\quad 2.54\} \\
\{16.70 \\
\{\quad 2.90\}\end{array}$ \\
\hline HNITRAT & PPB & 2566 & 45990 & $\begin{array}{l}02 / 12 / 88 \\
B 8 / 1 / 88\end{array}$ & $\begin{array}{l}11300 \\
15400\end{array}$ & $\begin{array}{l}02 / 11 / 88 \\
66 / 61 / 88\end{array}$ & $\begin{array}{l}11600 \\
18460\end{array}$ & $\begin{array}{l}82 / 68 / 88 \\
05 / 31 / 88\end{array}$ & $\begin{array}{r}7800 \\
10200\end{array}$ \\
\hline SR 90 & PCI/L & 5 & 8 & $\begin{array}{l}02 / 12 / 88 \\
66 / 61 / 88\end{array}$ & $\begin{array}{l}6.43 \\
\{\quad 1.52\} \\
5.76 \\
\{\quad 1.38\}\end{array}$ & $\begin{array}{l}62 / 11 / B 8 \\
68 / 81 / 8 B\end{array}$ & & $\begin{array}{l}12 / 08 / 88 \\
65 / 31 / 88\end{array}$ & \\
\hline TC -99 & $\mathrm{PCI} / \mathrm{L}$ & 15 & 900 & $62 / 12 / 88$ & & $12 / 11 / 88$ & $\left\{\begin{array}{l}9.17 \\
1.21\}\end{array}\right.$ & $02 / 68 / 88$ & $\begin{array}{l}21.16 \\
1.37\}\end{array}$ \\
\hline TRITIUH & $\mathrm{PCI} / \mathrm{L}$ & 500 & 20000 & $\begin{array}{l}82 / 12 / 88 \\
66 / 1 / 8 B\end{array}$ & $\begin{array}{r}1850 \\
258 \\
2608 \\
\left\{\begin{array}{r}280 \\
\}\end{array}\right\}\end{array}$ & $\begin{array}{l}02 / 11 / 88 \\
06 / 01 / 88\end{array}$ & $\left.\begin{array}{r}2310 \\
\left\{\begin{array}{l}271 \\
2840 \\
282\end{array}\right\}\end{array}\right\}$ & $\begin{array}{l}62 / 08 / B 8 \\
05 / 31 / 88\end{array}$ & $\left\{\begin{array}{c}657 \\
217 \\
1140 \\
228\end{array}\right\}$ \\
\hline $\mathrm{U}$ & $\mathrm{PCI} / \mathrm{L}$ & 0.50 & 800 & $\begin{array}{l}02 / 12 / 88 \\
08 / 01 / 88\end{array}$ & $\begin{array}{l}22.40 \\
32.60\end{array}$ & $\begin{array}{l}02 / 11 / 88 \\
08 / 11 / 80\end{array}$ & $\begin{array}{l}18.39 \\
25.80\end{array}$ & $\begin{array}{l}12 / 88 / 88 \\
65 / 31 / 80\end{array}$ & $\begin{array}{l}22.80 \\
24.10\end{array}$ \\
\hline
\end{tabular}




\section{TABLE E.7. (contd)}

\begin{tabular}{|c|c|c|c|c|c|}
\hline \multicolumn{2}{|c|}{ CONSTITUENT } & $\begin{array}{c}\text { CONTRACTUAL } \\
\text { DETECTION } \\
\text { LIHIT }\end{array}$ & $\begin{array}{l}\text { DRINKING } \\
\text { VATER } \\
\text { STANDARD }\end{array}$ & $\begin{array}{c}\text { SAMPLE } \\
\text { DATE }\end{array}$ & $3-4-10$ \\
\hline------- & ---- & 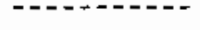 & $---1+-1--1$ & $----\infty-\infty$ & ----- \\
\hline BETA & $\mathrm{PCI} / \mathrm{L}$ & $B$ & 50 & $\begin{array}{l}82 / 08 / 88 \\
05 / 31 / 88\end{array}$ & $\begin{array}{r}18.40 \\
\{\quad 2.95\} \\
\{\quad 18 \\
\{\quad 2.99\}\end{array}$ \\
\hline HAITRAT & PPB & 2500 & 45000 & $\begin{array}{l}02 / 68 / 88 \\
65 / 31 / 88\end{array}$ & $\begin{array}{l}7590 \\
9810\end{array}$ \\
\hline$T C-99$ & $\mathrm{PCI} / \mathrm{L}$ & 15 & 960 & $02 / 88 / 88$ & $\{\quad 13$ \\
\hline TRITIUU & $\mathrm{PCI} / \mathrm{L}$ & 500 & 20060 & $\begin{array}{l}02 / 98 / 88 \\
05 / 31 / 88\end{array}$ & $\left.\begin{array}{c}854 \\
223 \\
1376 \\
235 \\
\text { NR }\end{array}\right\}$ \\
\hline U & $\mathrm{PCI} / \mathrm{L}$ & c. 50 & 806 & $\begin{array}{l}02 / 88 / 88 \\
95 / 31 / 88\end{array}$ & $\begin{array}{l}30.48 \\
28.78 \\
N R\end{array}$ \\
\hline
\end{tabular}

\begin{tabular}{|c|c|c|c|c|c|}
\hline \multicolumn{2}{|c|}{ CONSTITUENT } & $\begin{array}{c}\text { CONTRACTUAL } \\
\text { DETECTION } \\
\text { LIUIT }\end{array}$ & $\begin{array}{l}\text { DRINKING } \\
\text { WATER } \\
\text { STANDARD }\end{array}$ & $\begin{array}{l}\text { SAYPLE } \\
\text { DATE }\end{array}$ & $3-8-4$ \\
\hline$-\cdots--1$ & $-\cdots$ & 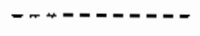 & $-\cdots-1-0$. & $\cdots-\cdots$ & --- \\
\hline BETA & $\mathrm{PCI} / \mathrm{L}$ & 8 & 50 & $\begin{array}{l}02 / 05 / 88 \\
05 / 31 / 88\end{array}$ & $\left\{\begin{array}{c}63.60 \\
5.41\} \\
7.35 \\
2.12\}\end{array}\right.$ \\
\hline $\begin{array}{l}\text { HHITRAT } \\
\text { TRITIUM }\end{array}$ & $\begin{array}{l}\text { PPB } \\
\text { PCI/L }\end{array}$ & $\begin{array}{r}2500 \\
500\end{array}$ & $\begin{array}{l}45000 \\
20000\end{array}$ & $\begin{array}{l}02 / 04 / 88 \\
05 / 31 / 88 \\
02 / 05 / 88\end{array}$ & $\begin{array}{l}19580 \\
21680\end{array}$ \\
\hline & & & & $05 / 31 / 88$ & \\
\hline U & $\mathrm{PCI} / \mathrm{L}$ & 9. 50 & 880 & $\begin{array}{l}02 / 05 / 88 \\
05 / 31 / 88\end{array}$ & $\begin{array}{l}2 \\
1.87\end{array}$ \\
\hline
\end{tabular}

\begin{tabular}{|c|c|}
\hline $\begin{array}{l}\text { SALPLE } \\
\text { DATE }\end{array}$ & $3-6-1$ \\
\hline n-con & ---- \\
\hline $02 / 11 / 8 B$ & 8 \\
\hline $05 / 31 / 88$ & $\begin{array}{ll}\{ & 2.19 \\
\{ & 7.83 \\
\{\quad 2.25\end{array}$ \\
\hline $\begin{array}{l}02 / 11 / 88 \\
05 / 31 / 88 \\
2 / 11 / 88\end{array}$ & $\begin{array}{l}29100 \\
32700\end{array}$ \\
\hline $02 / 11 / 88$ & \\
\hline $02 / 11 / 89$ & \\
\hline $\begin{array}{l}65 / 31 / 88 \\
22 / 11 / 88 \\
2 / 11 / 88 \\
65 / 31 / 88\end{array}$ & $\begin{array}{l}5.56 \\
6.25 \\
6.57\end{array}$ \\
\hline
\end{tabular}

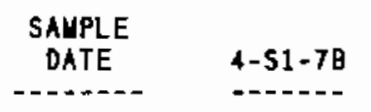

SAMPLE

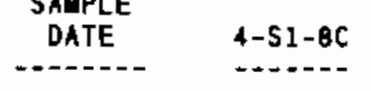

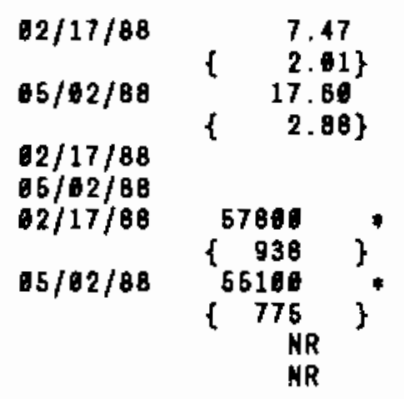

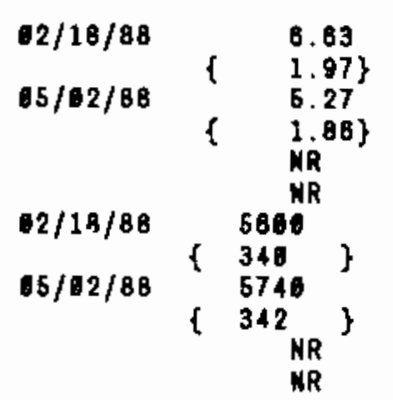


TABLE E.7. (contd)

\begin{tabular}{|c|c|c|c|c|c|c|c|c|c|}
\hline \multicolumn{2}{|c|}{$\begin{array}{l}\text { CONSTITUENT } \\
\text { NAME INITS }\end{array}$} & $\begin{array}{c}\text { CONTRACTUAL } \\
\text { DETECTION } \\
\text { LIUIT }\end{array}$ & $\begin{array}{l}\text { DRINKING } \\
\text { SATER } \\
\text { STANDARD }\end{array}$ & $\begin{array}{l}\text { SAUPLE } \\
\text { DATE }\end{array}$ & $4-50-7$ & $\begin{array}{l}\text { SAMPLE } \\
\text { DATE }\end{array}$ & $4-58-8$ & $\begin{array}{l}\text { SAUPLE } \\
\text { DATE }\end{array}$ & $6-531-1 P$ \\
\hline ALPHA & $\mathrm{PCI} / \mathrm{L}$ & 4 & 15 & & NR & $62 / 17 / 88$ & & $82 / 19 / 8 B$ & 1.22 \\
\hline & & & & & NR & $68 / 07 / 88$ & $\begin{array}{r}\quad 0.57 \\
\{\quad 0.41\}\end{array}$ & & NR \\
\hline BETA & $\mathrm{PCI} / \mathrm{L}$ & $B$ & 50 & & $\begin{array}{l}\text { NR } \\
\text { NR }\end{array}$ & $\begin{array}{l}02 / 17 / 88 \\
66 / 07 / 88\end{array}$ & 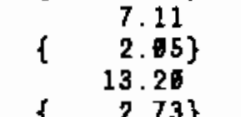 & $62 / 19 / 88$ & $\begin{array}{l}4.35 \\
1.67) \\
\text { NR }\end{array}$ \\
\hline $\begin{array}{l}\text { HNI TRAT } \\
\text { RU-106 }\end{array}$ & $\begin{array}{l}\mathrm{PPB} \\
\mathrm{PCI} / L\end{array}$ & $\begin{array}{l}2500 \\
172.50\end{array}$ & $\begin{array}{r}45006 \\
36\end{array}$ & $\begin{array}{l}02 / 17 / 88 \\
08 / 07 / 88\end{array}$ & 5008 & $\begin{array}{l}02 / 17 / 88 \\
08 / 67 / 88\end{array}$ & $\left.\begin{array}{ll}\{\quad & 2.73\} \\
& \text { NR } \\
& 59.76 * \\
\{\quad 54.18\end{array}\right\}$ & $02 / 19 / 88$ & $\begin{array}{r}3236 \\
\text { NR } \\
\text { NR }\end{array}$ \\
\hline TRITIUU & $\mathrm{PCI} / \mathrm{L}$ & 500 & 26800 & $\begin{array}{l}02 / 17 / 88 \\
06 / 67 / 88\end{array}$ & $\left.\begin{array}{r}53800 \\
\begin{array}{r}898 \\
34106\end{array} \\
718\end{array}\right\}$ & $\begin{array}{l}02 / 17 / 8 B \\
08 / 07 / 8 B\end{array}$ & $\left\{\begin{array}{c}4770 \\
327 \\
28268 \\
862\end{array}\right\}$ & $82 / 19 / 8 B$ & NR \\
\hline
\end{tabular}


IABLE E. . (contd)

\begin{tabular}{|c|c|c|c|c|c|c|c|c|c|}
\hline \multicolumn{2}{|c|}{ CQNSTITUENT } & $\begin{array}{c}\text { CONTRACTUAL } \\
\text { OETECTION } \\
\text { LIUIT }\end{array}$ & $\begin{array}{l}\text { DRINKING } \\
\text { WATER } \\
\text { STAMDARD }\end{array}$ & $\begin{array}{c}\text { SALPLE } \\
\text { DATE }\end{array}$ & $6-\$ 29-E 12$ & $\begin{array}{c}\text { SAMPLE } \\
\text { DATE }\end{array}$ & $B-S 28-E \theta$ & $\begin{array}{c}\text { SAHPLE } \\
\text { DATE }\end{array}$ & $6-527-E 14$ \\
\hline$m----$ & $-\cdots$ & 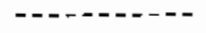 & $\cdots$ & $-\ldots \ldots$ & $-\ldots+\cdots$ & $\cdots$ & 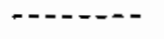 & 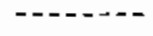 & $-\ldots+n-1$ \\
\hline ALPHA & PCI / & 4 & 15 & & $\begin{array}{l}\text { NR } \\
\text { NR }\end{array}$ & $\begin{array}{l}02 / 17 / 88 \\
04 / 15 / 88\end{array}$ & $\begin{array}{l}1.46 \\
0.55\} \\
1.50 \\
.55\end{array}$ & & $\begin{array}{l}\text { NR } \\
\text { NR }\end{array}$ \\
\hline BETA & $\mathrm{PCI} / \mathrm{L}$ & a & 50 & $\begin{array}{l}62 / 17 / 88 \\
68 / 17 / 88\end{array}$ & $\begin{array}{ll}\{ & 7.71 \\
& 2.12\} \\
& 8.61 \\
\{ & 2.31\} \\
& \text { NR }\end{array}$ & $\begin{array}{l}02 / 17 / 88 \\
04 / 15 / 88\end{array}$ & $\begin{array}{l}5.48 \\
1.88\} \\
4.23 \\
1.76\} \\
19\end{array}$ & $\begin{array}{l}01 / 67 / 88 \\
02 / 05 / 88 \\
03 / 03 / 88\end{array}$ & 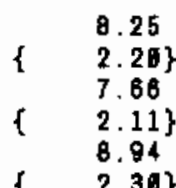 \\
\hline & & & & & NR & & NR & $04 / 06 / 88$ & 8.02 \\
\hline & & & & & NR & & NR & $65 / 04 / 88$ & $\begin{array}{l}2.20 \\
6.40 \\
2.063\end{array}$ \\
\hline & & & & & MR & & NR & $66 / 17 / 88$ & $\begin{array}{r}9.68 \\
+\quad 2.48\end{array}$ \\
\hline HNITRAT & PPB & 2500 & 46600 & $\begin{array}{l}02 / 17 / 88 \\
06 / 17 / 88\end{array}$ & $\begin{array}{c}18780 \\
28080 \\
\text { NR } \\
\text { NR } \\
\text { NR } \\
\text { NR } \\
27.38\end{array}$ & $\begin{array}{l}02 / 17 / 88 \\
04 / 15 / 88\end{array}$ & $\begin{array}{c}8998 \\
\text { OB96 } \\
\text { NR } \\
\text { NR } \\
\text { NR } \\
\text { NR } \\
\text { NR }\end{array}$ & $\begin{array}{l}01 / 07 / 88 \\
62 / 65 / 88 \\
03 / 03 / 80 \\
04 / 08 / 88 \\
66 / 64 / 88 \\
96 / 17 / 88\end{array}$ & $\begin{array}{l}22200 \\
21208 \\
22000 \\
19900 \\
19808 \\
25600\end{array}$ \\
\hline TC-99 & $\mathrm{PCI} / \mathrm{L}$ & 15 & 960 & $06 / 17 / 88$ & $\begin{array}{c}27.30 \\
\quad 1.45\}\end{array}$ & & NR & & NR \\
\hline U & $\mathrm{PCI} / \mathrm{L}$ & 0.50 & 868 & $\begin{array}{l}62 / 17 / 80 \\
08 / 17 / 88\end{array}$ & $\begin{array}{l}2.21 \\
1.97 \\
\text { NR } \\
\text { NR } \\
\text { NR } \\
\text { NR }\end{array}$ & $\begin{array}{l}02 / 17 / 88 \\
04 / 15 / 86\end{array}$ & $\begin{array}{l}1.39 \\
1.36 \\
\text { NR } \\
\text { NR } \\
\text { NR } \\
\text { NR }\end{array}$ & $\begin{array}{l}01 / 07 / 88 \\
62 / 65 / 88 \\
03 / 83 / 88 \\
64 / 68 / 88 \\
65 / 64 / 88 \\
06 / 17 / 88\end{array}$ & $\begin{array}{l}4.32 \\
3.45 \\
4.34 \\
4 . \\
3.32 \\
3.45\end{array}$ \\
\hline
\end{tabular}


IABLE E.7. (contd)

\begin{tabular}{|c|c|c|c|c|c|c|c|c|c|}
\hline $\begin{array}{l}\text { CONSTI } \\
\text { NAME }\end{array}$ & $\begin{array}{l}\text { UENT } \\
\text { UNITS }\end{array}$ & $\begin{array}{l}\text { CONTRACTUAL } \\
\text { DETECTION } \\
\text { LIUIT }\end{array}$ & $\begin{array}{l}\text { DRINKING } \\
\text { WATER } \\
\text { STANDARD }\end{array}$ & $\begin{array}{l}\text { SAMPLE } \\
\text { DATE }\end{array}$ & $6-524-19$ & $\begin{array}{l}\text { SAUPLE } \\
\text { DATE }\end{array}$ & $8-519-11$ & $\begin{array}{l}\text { SAHPLE } \\
\text { DATE }\end{array}$ & $6-S 18-51$ \\
\hline------ & $-\infty-$ & $-------\infty$ & --------- & 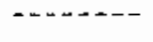 & ----- & -.-n-s- & $\cdots$ & 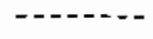 & - - \\
\hline ALPHA & $\mathrm{PCI} / \mathrm{L}$ & 4 & 15 & & NR & $02 / 17 / 88$ & $\begin{array}{ll} & 1.68 \\
\{.59\}\end{array}$ & $03 / 08 / 88$ & \\
\hline BETA & $\mathrm{PCI} / \mathrm{L}$ & 8 & 50 & & NR & $02 / 17 / 88$ & $\begin{array}{l}4.38 \\
\{\quad 1.68\}\end{array}$ & $63 / 08 / 88$ & $\begin{array}{l}2.87 \\
1.45\}\end{array}$ \\
\hline HNITRAT & PPB & 2500 & 45000 & $92 / 17 / 88$ & & $02 / 17 / 88$ & 8550 & $03 / 68 / 88$ & \\
\hline
\end{tabular}

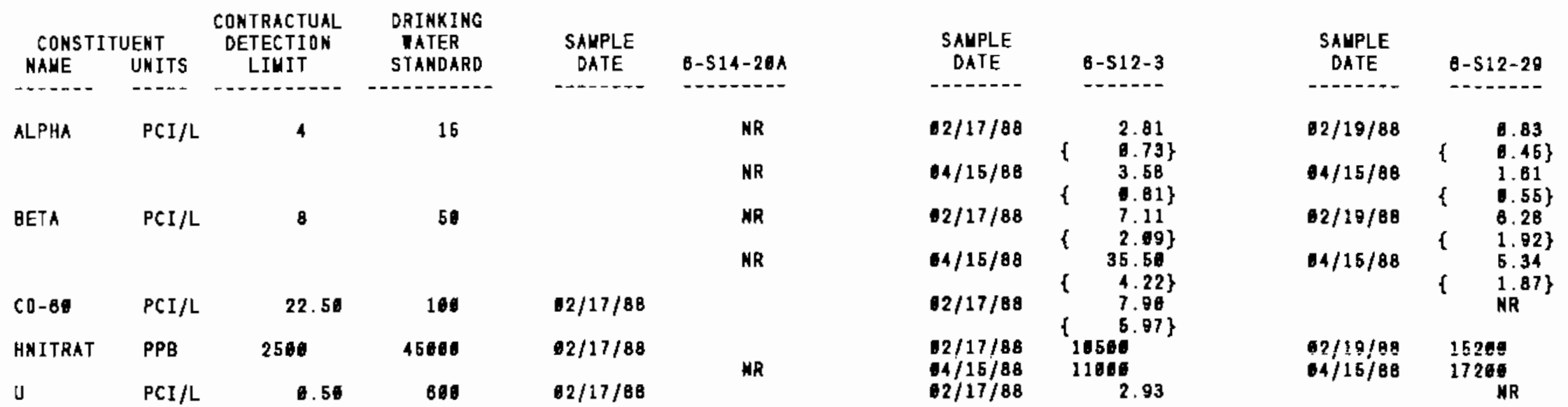


TABLE.E.7. (contd)

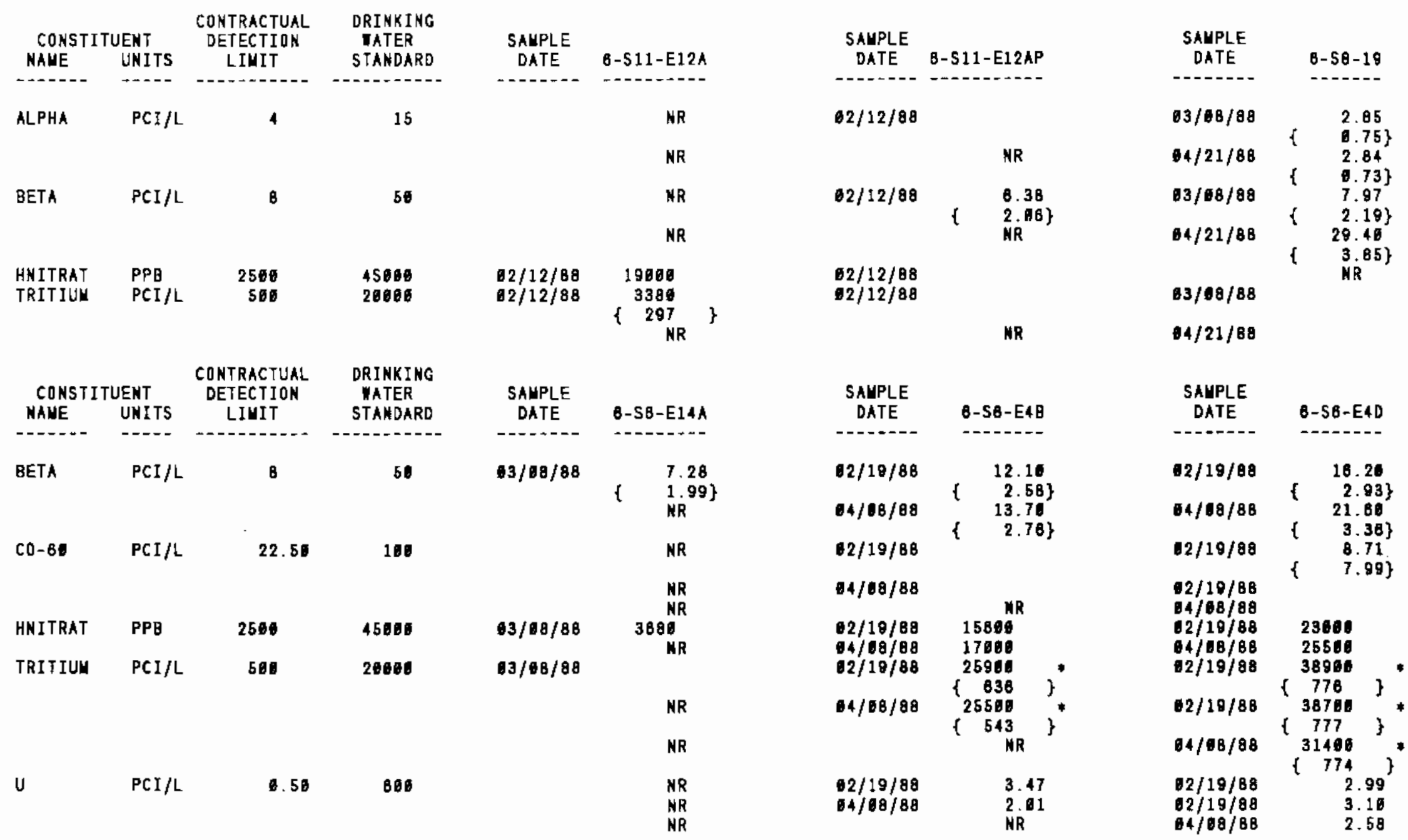


TABLE E.I. (contd)

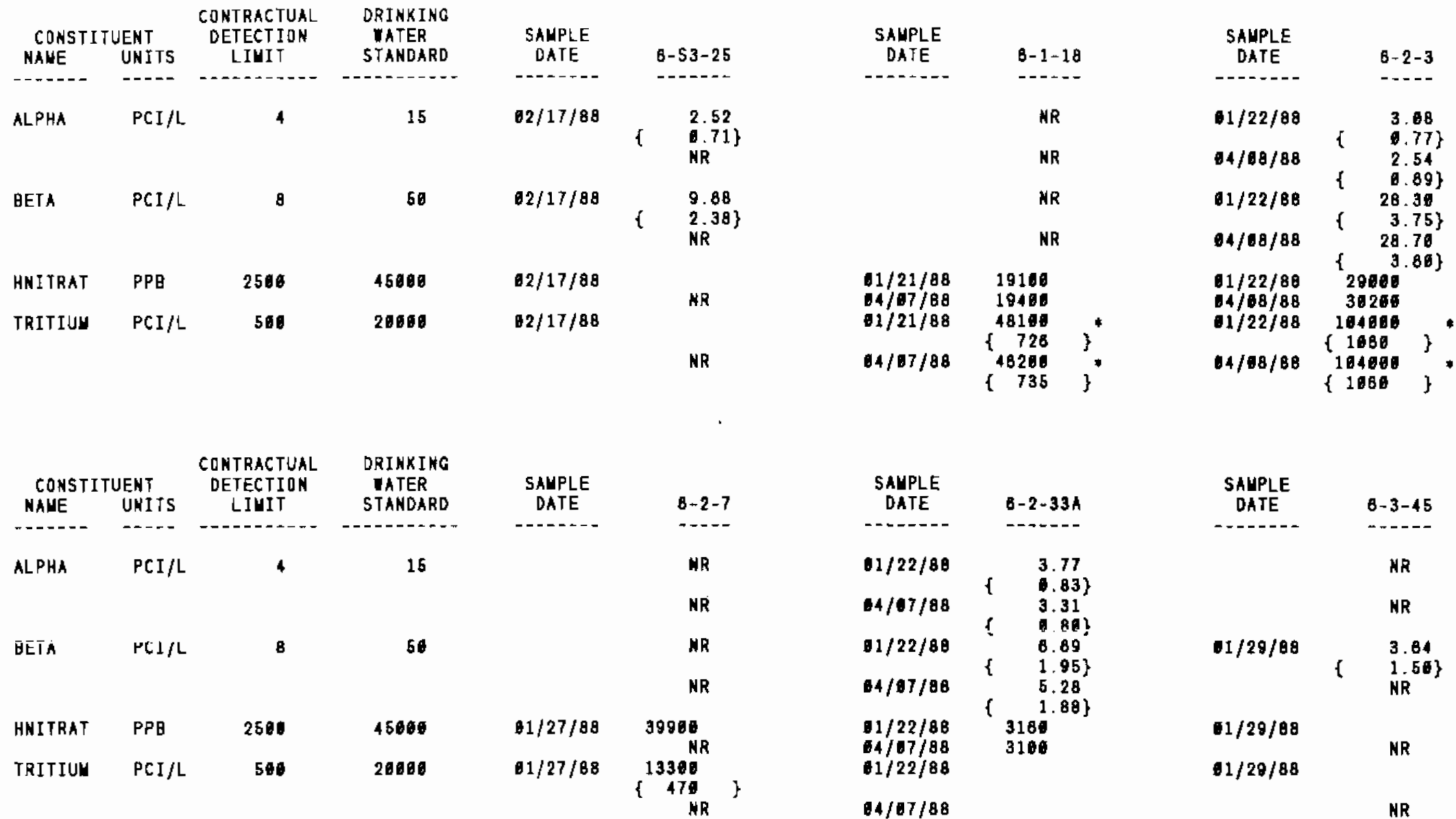




\section{TABLE E.7. (contd)}

\begin{tabular}{|c|c|c|c|c|c|}
\hline $\begin{array}{l}\text { CONSTI } \\
\text { NAME }\end{array}$ & $\begin{array}{l}\text { JENT } \\
\text { UNITS }\end{array}$ & $\begin{array}{l}\text { CONTRACTUAL } \\
\text { DETECTION } \\
\text { LINIT }\end{array}$ & $\begin{array}{l}\text { DRINXING } \\
\text { WATER } \\
\text { STANDARD }\end{array}$ & $\begin{array}{c}\text { SAMPLE } \\
\text { DATE }\end{array}$ & $8-8-17$ \\
\hline$+\cdots$ & $-\ldots+$ & - & $-\ldots-0-1,-1$ & $\cdots+\cdots$ & $\cdots--$ \\
\hline ALPHA & $\mathrm{PCI} / \mathrm{L}$ & 4 & 15 & $\begin{array}{l}01 / 27 / 88 \\
04 / 07 / 88\end{array}$ & $\begin{array}{l}3.58 \\
6.81\} \\
3.73 \\
.843\end{array}$ \\
\hline BETA & $\mathrm{PCI} / \mathrm{L}$ & 8 & 50 & $\begin{array}{l}01 / 27 / 88 \\
04 / 07 / 86\end{array}$ & 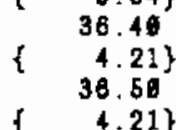 \\
\hline HNITRAT & PPQ & 2500 & 45600 & $\begin{array}{l}01 / 27 / 88 \\
04 / 67 / 88\end{array}$ & $\begin{array}{l}34260 \\
32700\end{array}$ \\
\hline$I-1290$ W & $\mathrm{PCI} / \mathrm{L}$ & 1 & & $04 / 07 / 88$ & $\begin{array}{r}\quad 0.53 \\
\{\quad 0.35\}\end{array}$ \\
\hline TRITIUH & $\mathrm{PCI} / \mathrm{L}$ & 500 & 20000 & $\begin{array}{l}01 / 27 / 88 \\
04 / 07 / 88\end{array}$ & $\left.\begin{array}{l}156000 \\
\{1320 \\
\{50000 \\
\{1280\end{array}\right\}$ \\
\hline
\end{tabular}

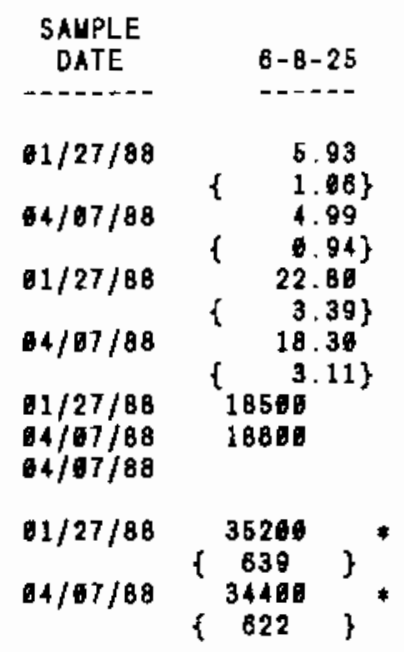

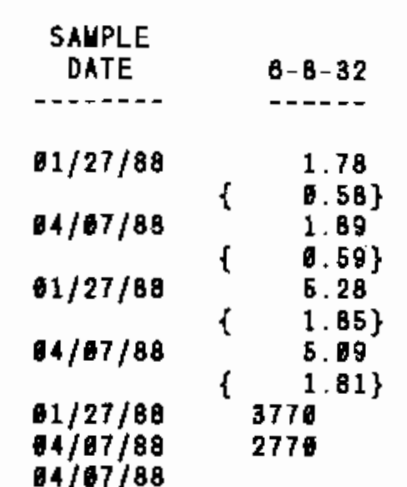

\begin{tabular}{|c|c|c|c|c|c|}
\hline \multicolumn{2}{|c|}{ CONST I TUENT } & $\begin{array}{c}\text { CONTRACTUAL } \\
\text { DETECTION } \\
\text { LIUIT }\end{array}$ & $\begin{array}{l}\text { DRINKING } \\
\text { WATER } \\
\text { STANDARD }\end{array}$ & $\begin{array}{l}\text { SANPLE } \\
\text { OATE }\end{array}$ & $8-16-54 A$ \\
\hline -....-- & $-\ldots+$ & $-\cdots-1, \ldots$. & 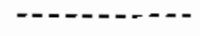 & 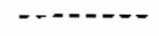 & $---\ldots$. \\
\hline ALPHA & $\mathrm{PCI} / \mathrm{L}$ & 4 & 15 & $\begin{array}{l}61 / 29 / 88 \\
65 / 24 / 88\end{array}$ & $\begin{array}{l}6.75 \\
\{\quad .41\} \\
1.89 \\
\{.83\}\end{array}$ \\
\hline BETA & $\mathrm{PCI} / \mathrm{L}$ & 8 & 50 & $\begin{array}{l}01 / 29 / 88 \\
65 / 24 / 88\end{array}$ & $\left\{\begin{array}{l}2.30 \\
1.41\} \\
3.73 \\
\{\quad 1.66\}\end{array}\right.$ \\
\hline $\begin{array}{l}\text { HNITRAT } \\
\text { TRITIUU }\end{array}$ & $\begin{array}{l}\mathrm{PPB} \\
\mathrm{PCI} / \mathrm{L}\end{array}$ & $\begin{array}{r}2500 \\
500\end{array}$ & $\begin{array}{l}45000 \\
20000\end{array}$ & $\begin{array}{l}01 / 29 / 88 \\
65 / 24 / 88 \\
81 / 29 / 88\end{array}$ & $\begin{array}{l}11406 \\
12000\end{array}$ \\
\hline & & & & $05 / 24 / 80$ & \\
\hline
\end{tabular}

\begin{tabular}{|c|c|}
\hline $\begin{array}{l}\text { SAHPLE } \\
\text { DATE }\end{array}$ & $6-13-84$ \\
\hline$\cdots$ & 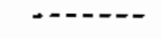 \\
\hline $01 / 29 / 88$ & 0.41 \\
\hline $85 / 22 / 88$ & $0.40^{\circ}$ \\
\hline & $0.33\}$ \\
\hline $1 / 28 / 88$ & 2.08 \\
\hline $05 / 22 / 8 B$ & 1.37\} \\
\hline $\begin{array}{l}61 / 29 / 88 \\
55 / 22 / 88 \\
01 / 29 / 88\end{array}$ & \\
\hline $05 / 22 / 88$ & \\
\hline
\end{tabular}

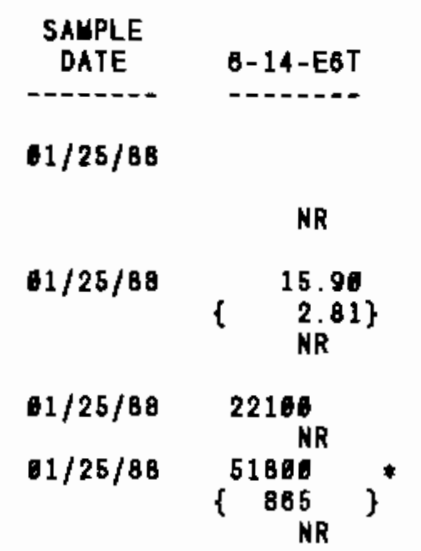


TABLE_E.7. (contd)

\begin{tabular}{|c|c|c|c|c|c|c|c|c|c|}
\hline \multicolumn{2}{|c|}{ CONSTITUENT } & $\begin{array}{l}\text { CONTRACTUAL } \\
\text { DETECTION } \\
\text { LINIT }\end{array}$ & $\begin{array}{l}\text { DRINKING } \\
\text { TATER } \\
\text { STANDARO }\end{array}$ & $\begin{array}{l}\text { SAUPLE } \\
\text { DATE }\end{array}$ & $8-14-17$ & $\begin{array}{l}\text { SAHPLE } \\
\text { DATE }\end{array}$ & $6-15-158$ & $\begin{array}{l}\text { SAUPLE } \\
\text { DATE }\end{array}$ & $6-15-26$ \\
\hline 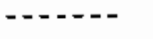 & $-\cdots-$ & 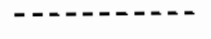 & 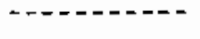 & 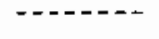 & $-\sim--\infty$ & $-\cdots+\cdots$ & $---n-\infty$ & $-\cdots----$ & $----\infty$ \\
\hline \multirow[t]{2}{*}{ ALPHA } & \multirow[t]{2}{*}{$\mathrm{PCI} / \mathrm{L}$} & \multirow[t]{2}{*}{4} & \multirow[t]{2}{*}{15} & \multirow[t]{2}{*}{$01 / 22 / 88$} & \multirow[t]{2}{*}{$\begin{array}{l}2.35 \\
\text { NR } \\
\text { SR }\end{array}$} & \multirow[t]{2}{*}{$1 / 21 / 88$} & \multirow[t]{2}{*}{$\begin{array}{l}8.03 \\
1.04\} \\
N R\end{array}$} & $\begin{array}{l}01 / 22 / 88 \\
01 / 22 / 88\end{array}$ & \multirow{2}{*}{ 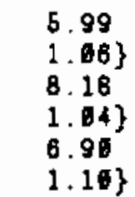 } \\
\hline & & & & & & & & $85 / 16 / 8 B$ & \\
\hline BETA & $\mathrm{PCI} / \mathrm{L}$ & 8 & 50 & \multirow[t]{3}{*}{$61 / 22 / 8 B$} & \multirow[t]{3}{*}{$\begin{array}{l}3.91 \\
1.57\} \\
M R\end{array}$} & \multirow[t]{2}{*}{$01 / 21 / 8 B$} & $\begin{array}{l}6.12 \\
1.93\} \\
\text { NR }\end{array}$ & $\begin{array}{l}01 / 22 / 88 \\
65 / 16 / 88\end{array}$ & $\begin{array}{r}21.30 \\
3.28\} \\
30 \\
3.92\}\end{array}$ \\
\hline \multirow[t]{2}{*}{$C O-80$} & \multirow[t]{2}{*}{$P C I / L$} & \multirow[t]{2}{*}{22.50} & \multirow[t]{2}{*}{106} & & & & NR & $01 / 22 / 88$ & $\begin{array}{l}7.68 \\
7.63\}\end{array}$ \\
\hline & & & & & & \multirow{3}{*}{$61 / 21 / 88$} & $\begin{array}{l}\text { NR } \\
\text { NR }\end{array}$ & $\begin{array}{l}01 / 22 / 88 \\
65 / 16 / 88\end{array}$ & \\
\hline HNYTRAT & PPB & 2500 & 45000 & \multirow[t]{2}{*}{$1 / 22 / 88$} & NR & & $\begin{array}{r}19300 \\
N R\end{array}$ & $\begin{array}{l}1 / 22 / 88 \\
5 / 16 / 88\end{array}$ & $\begin{array}{l}21501 \\
22800\end{array}$ \\
\hline I-1290" & $\mathrm{PCI} / \mathrm{L}$ & 1 & & & NR & & NR & $05 / 16 / 88$ & $\left\{\begin{array}{l}1.58 \\
\quad 0.38\}\end{array}\right.$ \\
\hline \multirow[t]{3}{*}{ TRITIUM } & \multirow[t]{3}{*}{$\mathrm{PCI} / \mathrm{L}$} & \multirow[t]{3}{*}{509} & \multirow[t]{3}{*}{26668} & \multirow[t]{3}{*}{$1 / 22 / 88$} & \multirow{3}{*}{\multicolumn{2}{|c|}{$01 / 21 / 88$}} & & $01 / 22 / 8 \theta$ & \multirow{3}{*}{ 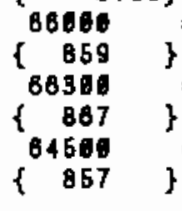 } \\
\hline & & & & & & & KR & $01 / 22 / 8 B$ & \\
\hline & & & & & & & NR & $15 / 18 / 88$ & \\
\hline \multirow{2}{*}{\multicolumn{2}{|c|}{$\begin{array}{l}\text { CONSTITUENT } \\
\text { NAHE } \\
\text { UNITS }\end{array}$}} & $\begin{array}{l}\text { CDNTRACTUAL } \\
\text { DETECTION } \\
\text { LIUIT }\end{array}$ & $\begin{array}{l}\text { DRINKING } \\
\text { TATER } \\
\text { STANDARD }\end{array}$ & $\begin{array}{l}\text { SAHPLE } \\
\text { DATE }\end{array}$ & $8-17-5$ & $\begin{array}{l}\text { SAUPLE } \\
\text { DATE }\end{array}$ & $6-17-47$ & $\begin{array}{l}\text { SAMPLE } \\
\text { DATE }\end{array}$ & $6-17-76$ \\
\hline & & 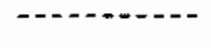 & $---1-20-1+$ & $---n-\infty$ & $-\cdots+-$ & $\cdots+-1$ & $\cdots+-1$ & 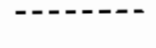 & $----n$ \\
\hline ALPHA & $\mathrm{PCI} / \mathrm{L}$ & 4 & 15 & $01 / 21 / 88$ & $\begin{array}{l}4.39 \\
.88\}\end{array}$ & & HR & & NR \\
\hline BETA & $\mathrm{PCI} / \mathrm{L}$ & $B$ & 59 & $61 / 21 / 80$ & $\begin{array}{l}6.82 \\
1.89\}\end{array}$ & & NR & & NR \\
\hline HNITRAT & PPB & 2500 & 45000 & $01 / 21 / 88$ & $\underset{N R}{62600}$ & $\begin{array}{l}01 / 22 / 88 \\
05 / 22 / 88\end{array}$ & & $\begin{array}{l}01 / 29 / 88 \\
04 / 29 / 88\end{array}$ & $\begin{array}{l}47460 \\
43800\end{array}$ \\
\hline
\end{tabular}


TABLE_E.7. (contd)

\begin{tabular}{|c|c|c|c|c|c|}
\hline \multicolumn{2}{|c|}{ CONSTI TUENT } & $\begin{array}{c}\text { CONTRACTUAL } \\
\text { DETECTION } \\
\text { LINIT }\end{array}$ & $\begin{array}{l}\text { DRINKING } \\
\text { IATER } \\
\text { STANDARD }\end{array}$ & $\begin{array}{l}\text { SAYPLE } \\
\text { DATE }\end{array}$ & $B-19-5 B$ \\
\hline ALPHA & PCI/L & 4 & 15 & & MR \\
\hline & & & & & NR \\
\hline BETA & $\mathrm{PCI} / \mathrm{h}$ & 8 & 58 & & NR \\
\hline & & & & & NR \\
\hline HNITRAT & PPB & 2506 & 45000 & $1 / 22 / 88$ & \\
\hline TRITIUY & $\mathrm{PCI} / \mathrm{L}$ & 506 & 20000 & & NR \\
\hline & & & & & NR \\
\hline$v$ & $\mathrm{PCI} / \mathrm{L}$ & 0.50 & 860 & & $\begin{array}{l}\mathrm{NR} \\
\mathrm{NR}\end{array}$ \\
\hline
\end{tabular}

\begin{tabular}{|c|c|}
\hline $\begin{array}{c}\text { SAYPLE } \\
\text { DATE }\end{array}$ & 6-19-88 \\
\hline $1 / 22 / 88$ & 0.58 \\
\hline $04 / 28 / 8 B$ & $\begin{array}{l}0.38 \mathrm{f} \\
1.18\end{array}$ \\
\hline $01 / 22 / 88$ & 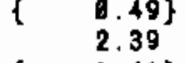 \\
\hline $04 / 29 / B B$ & $\begin{array}{l}1.41\} \\
1.87\end{array}$ \\
\hline $\begin{array}{l}01 / 22 / 88 \\
64 / 28 / 88 \\
61 / 22 / 88\end{array}$ & $1.43\}$ \\
\hline $04 / 29 / 88$ & \\
\hline & $\begin{array}{l}\text { NR } \\
\text { NR }\end{array}$ \\
\hline
\end{tabular}

\begin{tabular}{|c|c|}
\hline \multirow[t]{3}{*}{$\begin{array}{l}\text { SALPLE } \\
\text { DATE }\end{array}$} & $\theta-2 \theta-E 12$ \\
\hline & MR \\
\hline & NR \\
\hline $01 / 25 / 88$ & 4.35 \\
\hline$A B / 17 / 8 B$ & 5.83 \\
\hline $\begin{array}{l}01 / 25 / 88 \\
88 / 17 / 88 \\
1 / 25 / 88\end{array}$ & $\begin{array}{r}5670 \\
32809 \\
987\end{array}$ \\
\hline $68 / 17 / 88$ & $\left\{\begin{array}{r}189 \\
1390\end{array}\right.$ \\
\hline & $\{208\}$ \\
\hline $\begin{array}{l}1 / 25 / 8 B \\
60 / 17 / 88\end{array}$ & $\begin{array}{l}1.13 \\
1.34\end{array}$ \\
\hline
\end{tabular}

$\stackrel{!}{\omega_{\infty}}$

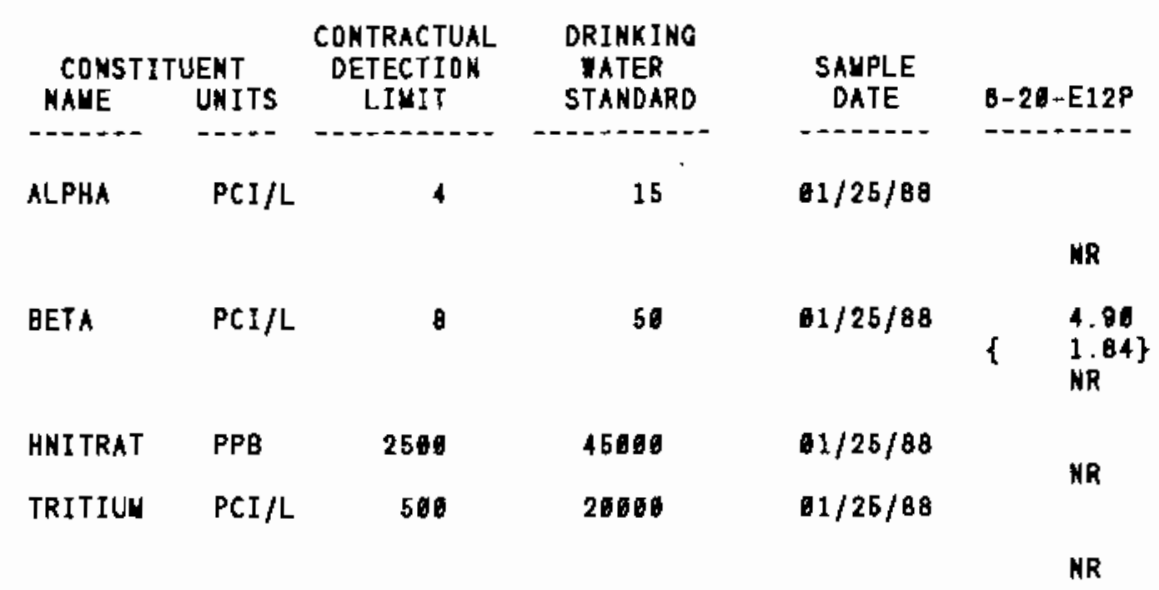

\begin{tabular}{|c|c|}
\hline $\begin{array}{l}\text { SAUPLEE } \\
\text { DATE }\end{array}$ & $8-20-E 5$ \\
\hline $11 / 25 / 88$ & 2.8 \\
\hline $65 / 20 / 88$ & $\begin{array}{l}8.73 \\
2.96 \\
6.76\end{array}$ \\
\hline $1 / 25 / 88$ & 21 \\
\hline $55 / 28 / 8 B$ & $\begin{array}{r}3.17 \\
22.48 \\
3.29\end{array}$ \\
\hline $\begin{array}{l}81 / 25 / 88 \\
65 / 2 \theta / 88 \\
81 / 25 / 88\end{array}$ & $\begin{array}{r}22208 \\
23386 \\
67460 \\
5 \quad 981\end{array}$ \\
\hline $95 / 26 / 8 B$ & 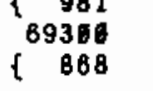 \\
\hline
\end{tabular}

\begin{tabular}{cc}
$\begin{array}{c}\text { SALPLE } \\
\text { DATE }\end{array}$ & $8-28-E S P$ \\
\hdashline $1 / 25 / 88$ & \\
& \\
$1 / 25 / 88$ & NR \\
& $\left\{\begin{array}{l}3.93 \\
1.78\} \\
N R\end{array}\right.$
\end{tabular}

$81 / 25 / 88$

$01 / 25 / 88$ 
TABLE E.7. (contd)

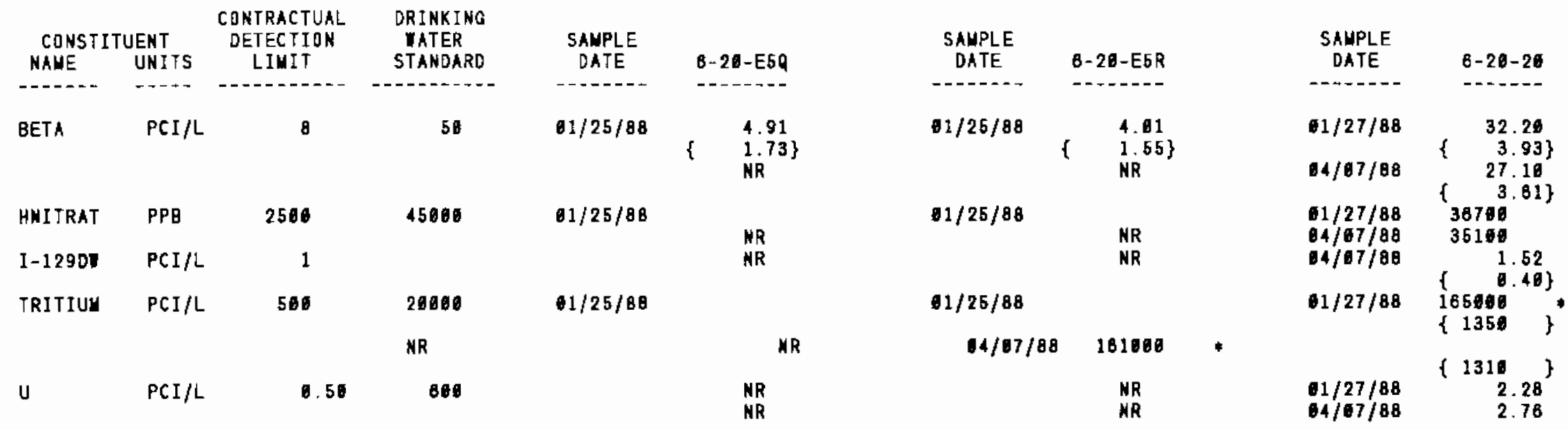

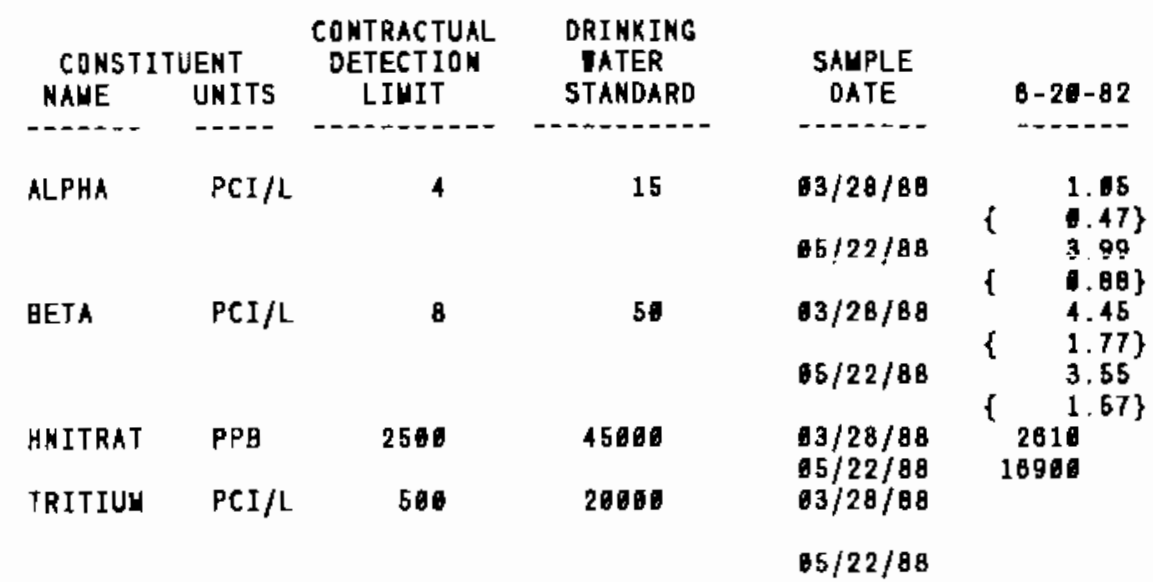

\begin{tabular}{|c|c|}
\hline $\begin{array}{l}\text { SAMPLE } \\
\text { DATE }\end{array}$ & $8-21-8$ \\
\hline $02 / 02 / 88$ & 2.16 \\
\hline $94 / 24 / 80$ & $\begin{array}{l}\begin{array}{l}8.65 \\
2.97\end{array} \\
\end{array}$ \\
\hline $02 / 62 / 88$ & $\begin{array}{r}0.78) \\
16.90 \\
0.913\end{array}$ \\
\hline $04 / 14 / 80$ & 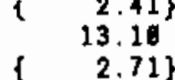 \\
\hline $\begin{array}{l}62 / 02 / 88 \\
4 / 14 / 88 \\
62 / 62 / 88\end{array}$ & $\begin{array}{r}39768 \\
35569 \\
51180 \\
\{\quad 869\end{array}$ \\
\hline $04 / 14 / 8 \theta$ & 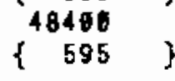 \\
\hline
\end{tabular}

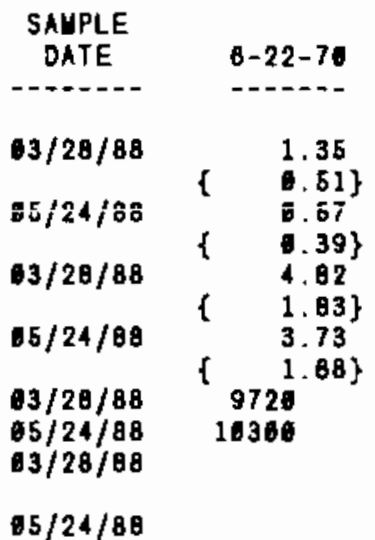


TABLE E.7. (contd)

\begin{tabular}{|c|c|c|c|c|c|}
\hline \multicolumn{2}{|c|}{ CONSTITUENT } & $\begin{array}{l}\text { CONTRACTUAL } \\
\text { DETECTION } \\
\text { LINIT }\end{array}$ & $\begin{array}{l}\text { DRINKING } \\
\text { DATER } \\
\text { STANDARD }\end{array}$ & $\begin{array}{l}\text { SAUPLE } \\
\text { DATE }\end{array}$ & $6-24-1 P$ \\
\hline$=---$ & $-\cdots+-$ & $--1-1-1-1$ & 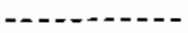 & $\cdots$ & $--\infty-$ \\
\hline SETA & $\mathrm{PCI} / \mathrm{L}$ & B & 50 & $01 / 25 / 88$ & $\begin{array}{l}5.8 B \\
1.90\}\end{array}$ \\
\hline TRITIUN & $\mathrm{PCI} / \mathrm{L}$ & 500 & 20000 & $1 / 25 / B B$ & $\begin{array}{l}389 \\
199\end{array}$ \\
\hline
\end{tabular}

$6-24-15$

HAUE

UNITS

CONTRACTUAL
DETECTIOA

$$
\begin{aligned}
& \text { DRINKINC } \\
& \text { WATER }
\end{aligned}
$$

STANDARD

ALPKA PCI/L

15

DATE

$61 / 25 / 88$

NR

50

BETA PCI/L

B

$1 / 25 / 98$

3.97

NR

HWITRAT PPB

2506

TRITIUN PCI/L
45000

580

20600

01/25/8B

$01 / 25 / 88$
NR

\begin{tabular}{|c|c|}
\hline $\begin{array}{l}\text { SAHPLE } \\
\text { DATE }\end{array}$ & $6-24-1 Q$ \\
\hline --nente- & --1 \\
\hline $1 / 25 / B B$ & $\begin{array}{l}3.58 \\
1.42\}\end{array}$ \\
\hline
\end{tabular}

NR
SAMPLE DATE

SAMPLE

DATE B-24-1R

$\begin{array}{ll}01 / 25 / 88 \quad & 3.94 \\ & 1.52\end{array}$

1/25/8B

SAMPLE

DATE

6-25-55

$1 / 25 / 88$

$62 / 61 / 8 B$

$05 / 24 / 88$

$62 / 01 / 88$

$85 / 24 / B 8$

$02 / 01 / 89$

$05 / 24 / 89$

$02 / 01 / 88$

$05 / 24 / 8 \mathrm{~B}$

\begin{tabular}{|c|c|}
\hline $1 / 25 / 88$ & \\
\hline & \\
\hline $01 / 25 / B B$ & $\left\{\begin{array}{r}14560 \\
484\end{array}\right.$ \\
\hline
\end{tabular}

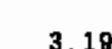

6.76

2.89
$\{\quad .75\}$

3.18

\{ 1.55$\}$

\{ 1.81\}

13860

13790

808:

\{ 354$\}$ 
TABLE E.7. (contd)

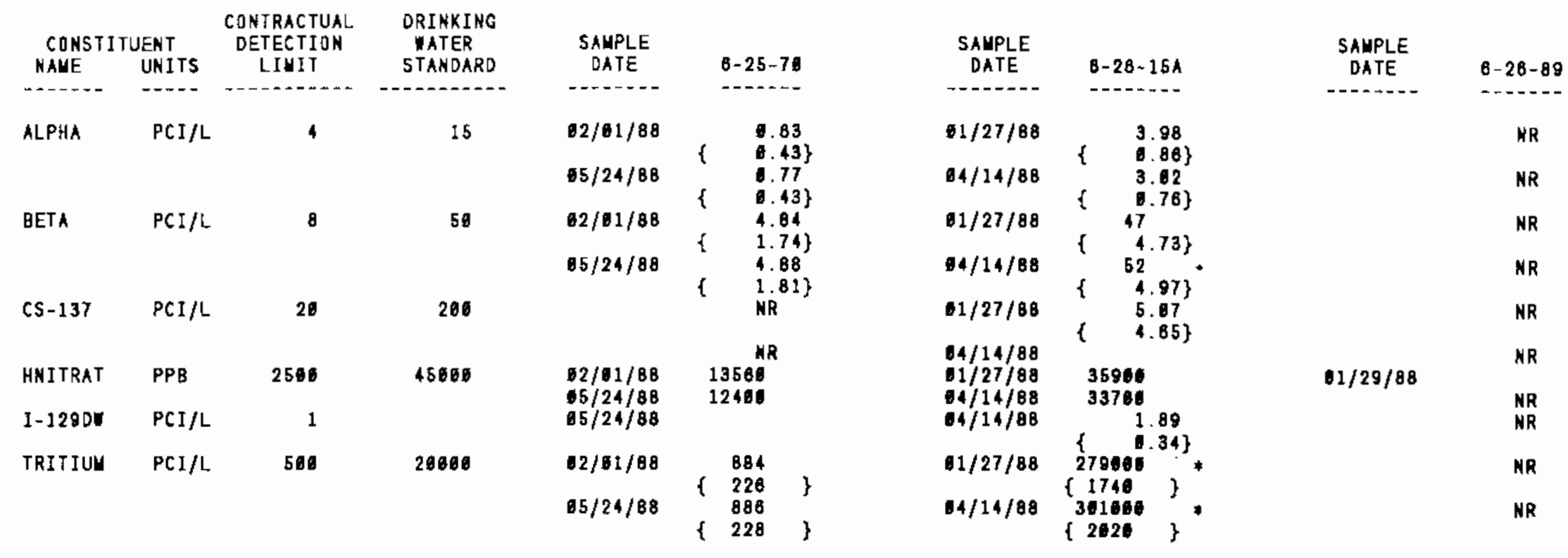


TABLEE.7. (contd)

\begin{tabular}{|c|c|c|c|c|c|c|c|c|c|c|}
\hline $\begin{array}{c}\text { CONSTI } \\
\text { NAHE }\end{array}$ & $\begin{array}{l}\text { UENT } \\
\text { UNITS }\end{array}$ & $\begin{array}{l}\text { CONTRACTUAL } \\
\text { DETECTIOA } \\
\text { LIMIT }\end{array}$ & $\begin{array}{l}\text { DRINKING } \\
\text { WATER } \\
\text { STANDARD }\end{array}$ & $\begin{array}{l}\text { SAMPLE } \\
\text { DATE }\end{array}$ & $6-27-8$ & $\begin{array}{l}\text { SAMPLE } \\
\text { DATE }\end{array}$ & $B-28-49$ & $\begin{array}{l}\text { SAMPLE } \\
\text { DATE }\end{array}$ & & $-2 B-4 B P$ \\
\hline$A L P H A$ & $\mathrm{PCI} / \mathrm{L}$ & 4 & 15 & $\begin{array}{l}81 / 27 / 88 \\
64 / 14 / 88\end{array}$ & $\left.\begin{array}{ll} & 3.12 \\
\{\quad & 0.77\} \\
\{ & 3.71 \\
\{ & 8.84\end{array}\right\}$ & $\begin{array}{l}01 / 28 / 88 \\
05 / 16 / 88\end{array}$ & $\left.\begin{array}{ll} & 3.32 \\
\{\quad & .78 \\
4 & 4.16 \\
\{\quad 6.84\end{array}\right\}$ & $91 / 28 / 8 B$ & & NR \\
\hline BETA & $\mathrm{PCI} / \mathrm{L}$ & $B$ & 50 & $\begin{array}{l}01 / 27 / 8 B \\
64 / 14 / 88\end{array}$ & $\begin{array}{c}45.98 \\
\{\quad 4.72\} \\
55.48 \\
\{\quad 5.17\}\end{array}$ & $\begin{array}{l}01 / 28 / 88 \\
55 / 16 / 88\end{array}$ & $\begin{array}{r}12.86 \\
\{\quad 2.85\} \\
\quad 11.28 \\
\{\quad 2.51\}\end{array}$ & $01 / 28 / 88$ & t & $\begin{array}{l}1.81 \\
1.21\} \\
\mathrm{NR}\end{array}$ \\
\hline $\begin{array}{l}\text { HNI TRAT } \\
\text { I-12SDT }\end{array}$ & $\begin{array}{l}\text { PPB } \\
\text { PCI } / L\end{array}$ & 2580 & 45000 & $\begin{array}{l}01 / 27 / 88 \\
04 / 14 / 88 \\
04 / 14 / 86\end{array}$ & $\begin{array}{l}37860 \\
338868 \\
2.27\end{array}$ & $\begin{array}{l}01 / 28 / 88 \\
05 / 18 / 88 \\
85 / 16 / 88\end{array}$ & $\begin{array}{l}13709 \\
14380\end{array}$ & $1 / 28 / B B$ & & $\begin{array}{l}\text { NR } \\
\text { NR }\end{array}$ \\
\hline TC- 99 & $\mathrm{PCI} / \mathrm{L}$ & 15 & 908 & & $\{331.45\}$ & & NR & $1 / 28 / 88$ & & \\
\hline TRITIUN & $\mathrm{PCI} / \mathrm{L}$ & 500 & 200100 & $\begin{array}{l}01 / 27 / 88 \\
04 / 14 / 88\end{array}$ & 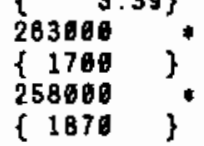 & $\begin{array}{l}01 / 28 / 88 \\
05 / 16 / 08\end{array}$ & $\left\{\begin{array}{c}18588 \\
544 \\
29488 \\
888\end{array}\right\}$ & $01 / 28 / 88$ & & NR \\
\hline
\end{tabular}


TABLE E.7. (contd)

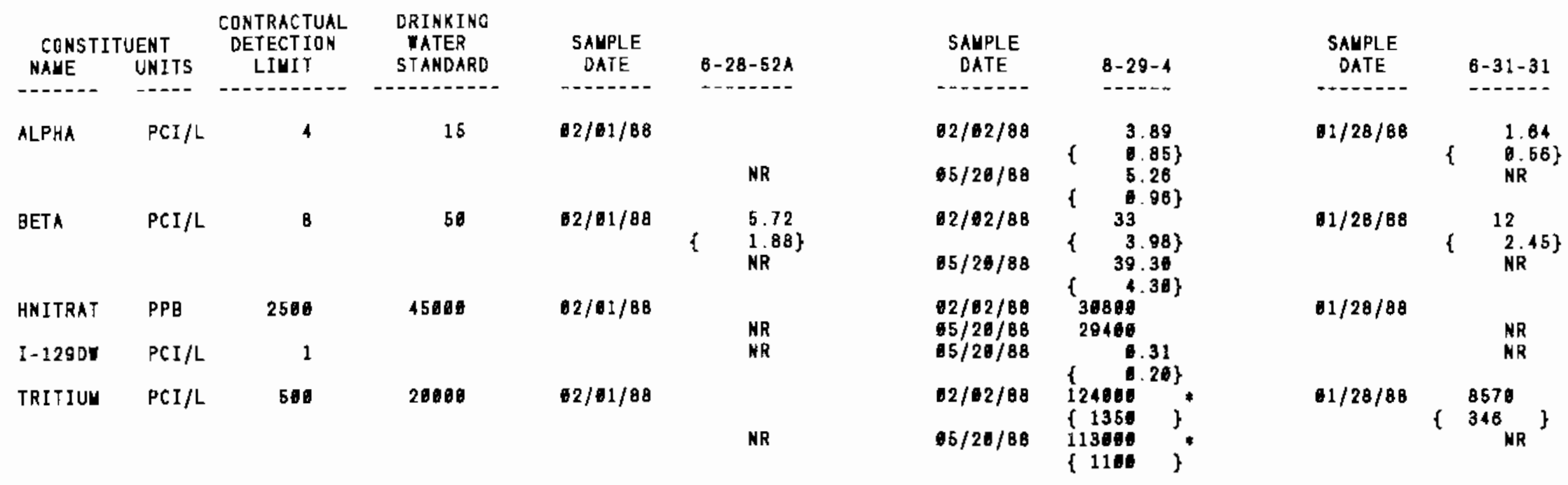


IABLE E. . (contd)

\begin{tabular}{|c|c|c|c|c|c|c|c|c|c|}
\hline $\begin{array}{c}\text { CONSTI } \\
\text { NAHE }\end{array}$ & $\begin{array}{l}\text { UENT } \\
\text { UNITS }\end{array}$ & $\begin{array}{c}\text { CONTRACTUAL } \\
\text { DETECTION } \\
\text { LIUIT }\end{array}$ & $\begin{array}{l}\text { DRINKING } \\
\text { IATER } \\
\text { STANDARD }\end{array}$ & $\begin{array}{l}\text { SAMPLEE } \\
\text { DATE }\end{array}$ & $8-31-31 P$ & $\begin{array}{l}\text { SAUPLE } \\
\text { DATE }\end{array}$ & $6-31-538$ & $\begin{array}{c}\text { SAYPLE } \\
\text { DATE }\end{array}$ & $6-32-22$ \\
\hline \multirow[t]{2}{*}{$A \perp P H A$} & $\mathrm{PCI} / \mathrm{L}$ & 4 & 15 & $01 / 28 / 88$ & & & NR & $83 / 16 / 88$ & 2.82 \\
\hline & & & & & NR & & NR & $08 / 01 / 88$ & $\begin{array}{l}2.49 \\
0.89\}\end{array}$ \\
\hline BETA & $\mathrm{PCI} / \mathrm{L}$ & 8 & 50 & $01 / 28 / 88$ & 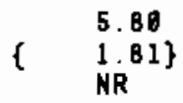 & & $\begin{array}{l}\text { NR } \\
\text { NR }\end{array}$ & $\begin{array}{l}03 / 10 / 88 \\
86 / 01 / 88\end{array}$ & $\begin{array}{c}48.98 \\
4.78\} \\
40\end{array}$ \\
\hline HNITRAT & PPG & 2500 & 45000 & $01 / 28 / \theta B$ & B258 & & $\begin{array}{l}\text { MR } \\
\text { MR }\end{array}$ & $\begin{array}{l}63 / 10 / 88 \\
06 / 01 / 88\end{array}$ & $\begin{array}{l}27286 \\
26260\end{array}$ \\
\hline I-1290X & $\mathrm{PCI} / \mathrm{L}$ & 1 & & & NR & & NR & $06 / 01 / 88$ & $\begin{array}{l}1.53 \\
\{\quad 0.28\}\end{array}$ \\
\hline TC-99 & $\mathrm{PCI} / \mathrm{L}$ & 15 & 960 & $01 / 28 / 88$ & & & $N R$ & $03 / 10 / 88$ & 244 \\
\hline \multirow[t]{2}{*}{ TRITIUN } & $\mathrm{PCI} / \mathrm{L}$ & 500 & 20080 & $81 / 28 / 88$ & & & NR & $83 / 10 / 88$ & 228080 \\
\hline & & & & & NR & & NR & $66 / 61 / 88$ & $\begin{array}{l}214868 \\
\{1778\end{array}$ \\
\hline
\end{tabular}


IABLE E.7. (contd)

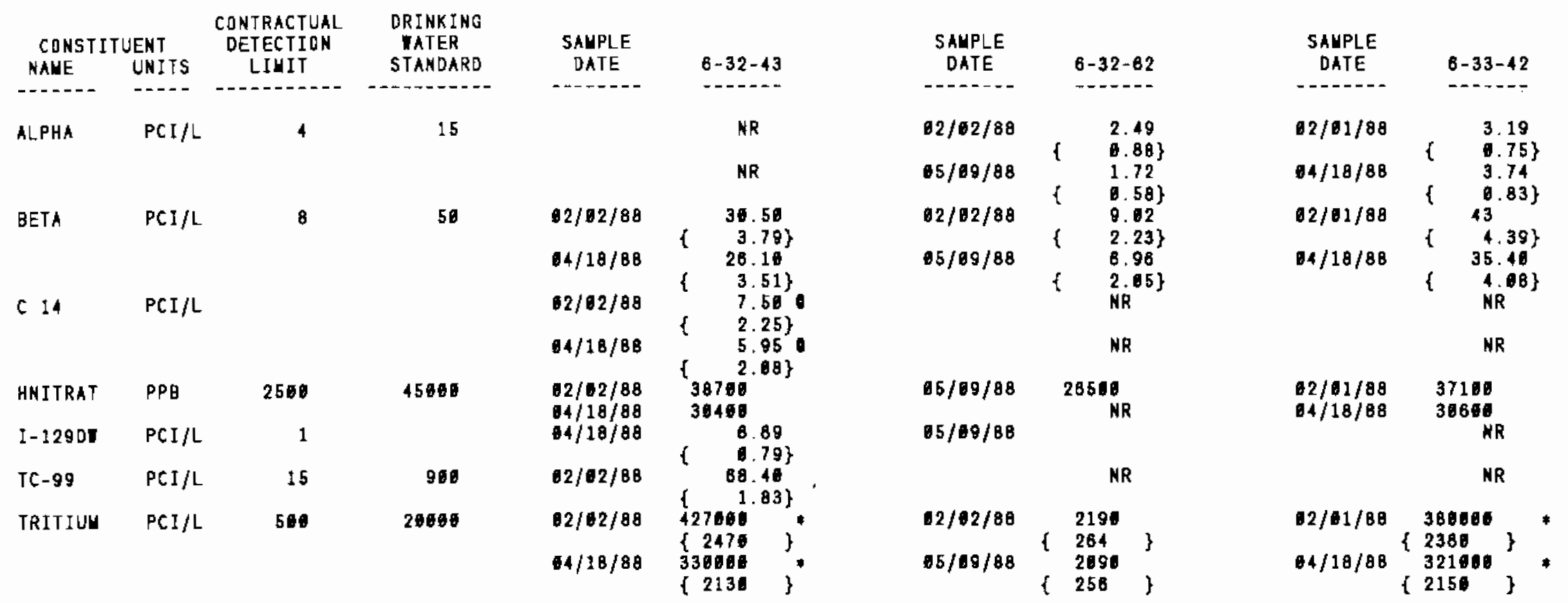


TABLE E.7. (contd)

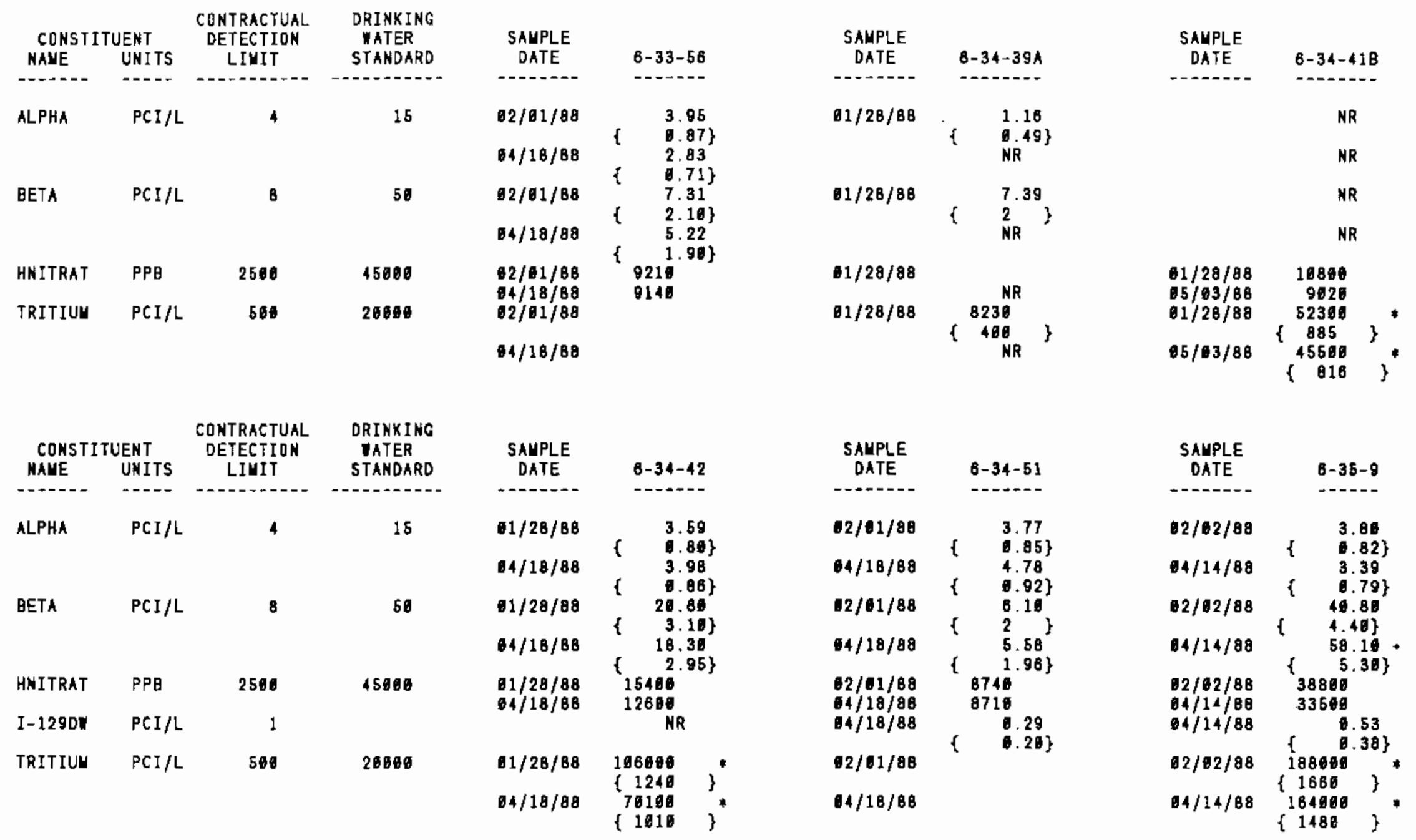


TABLE E.7. (contd)

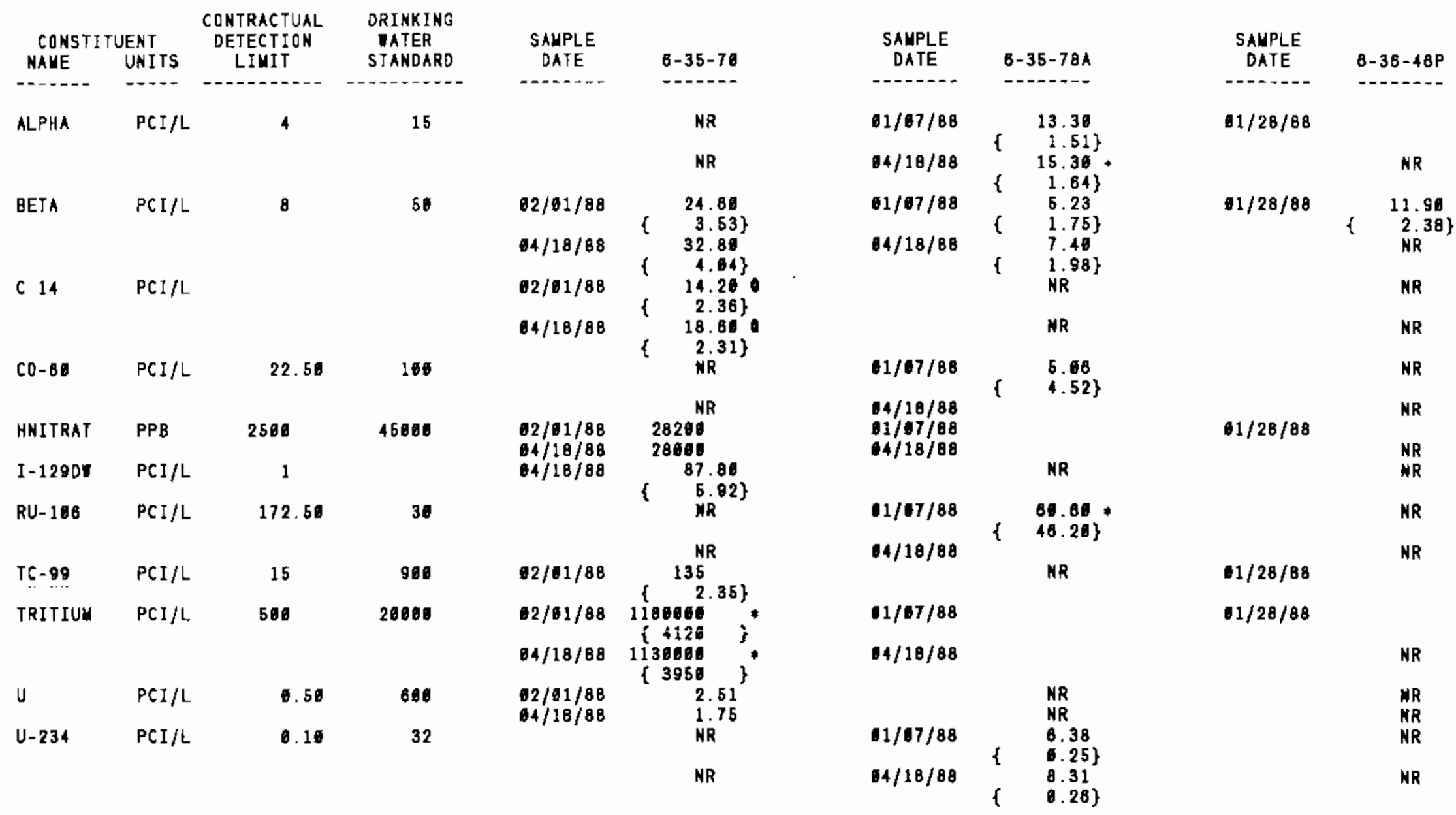


IABLEE.7. (contd)

\begin{tabular}{|c|c|c|c|c|c|}
\hline \multicolumn{2}{|c|}{ CONSTITUENT } & $\begin{array}{l}\text { CONTRACTUAL } \\
\text { DETECTION } \\
\text { THIT }\end{array}$ & $\begin{array}{l}\text { ORINKING } \\
\text { WATER }\end{array}$ & SAMPLE & $6-35-79$ \\
\hline - & - & - & 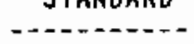 & - Dn & 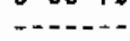 \\
\hline$U-235$ & $\mathrm{PCI} / \mathrm{L}$ & B. 10 & 32 & & NR \\
\hline & & & & & NR \\
\hline$U-238$ & $P C I / L$ & 8.18 & 4.80 & & NR \\
\hline & & & & & NR \\
\hline U-CHEV & UG/L & 0.73 & & & $\begin{array}{l}\text { NR } \\
\text { NR }\end{array}$ \\
\hline
\end{tabular}

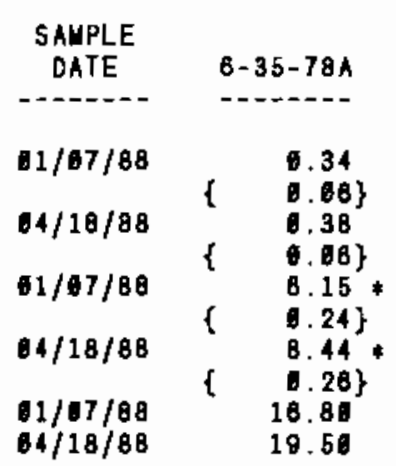

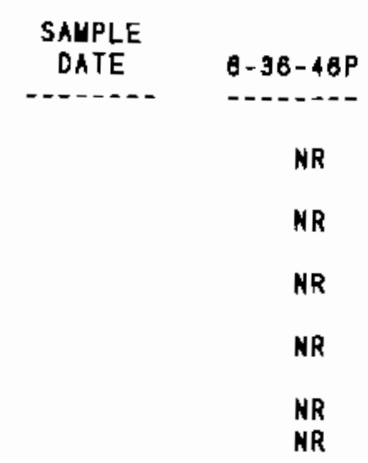

\begin{tabular}{|c|c|c|c|c|c|c|}
\hline \multirow{7}{*}{$\begin{array}{l}\pi \\
\omega \\
0\end{array}$} & \multicolumn{2}{|c|}{ CONSTITUENT } & $\begin{array}{c}\text { CONTRACTUAL } \\
\text { DETECTION } \\
\text { LINIT }\end{array}$ & $\begin{array}{l}\text { DRINKING } \\
\text { WATER } \\
\text { STANDARD }\end{array}$ & $\begin{array}{l}\text { SALPLE } \\
\text { DATE }\end{array}$ & $8-36-46 Q$ \\
\hline & ------ & --- & $-+-1,-1-1$ & $---n-1-1$ & ------ & $-\cdots \cdots$ \\
\hline & ALPHA & $\mathrm{PCI} / \mathrm{L}$ & 4 & 15 & $61 / 28 / 88$ & \\
\hline & & & & & & NR \\
\hline & BETA & $\mathrm{PCI} / \mathrm{L}$ & 8 & $5 B$ & $01 / 28 / 88$ & $\left\{\quad \frac{10}{2.24\}}\right.$ \\
\hline & HNI TRAT & PPB & 2500 & 45000 & $01 / 28 / 88$ & \\
\hline & TRITIUM & PCI/L & 500 & 20000 & $01 / 28 / 88$ & $\begin{array}{l}375 \\
\qquad \quad 281 \\
\text { NR }\end{array}$ \\
\hline
\end{tabular}

\begin{tabular}{|c|c|}
\hline $\begin{array}{l}\text { SALPLE } \\
\text { DATE }\end{array}$ & $8-36-618$ \\
\hline--- & 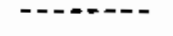 \\
\hline $02 / 03 / 88$ & 6.45 \\
\hline $05 / 25 / 80$ & 2.64 \\
\hline & 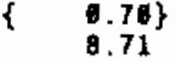 \\
\hline $65 / 25 / 88$ & $\begin{array}{l}2.23\} \\
7 \\
2.07\end{array}$ \\
\hline $\begin{array}{l}02 / 13 / 88 \\
65 / 25 / 88 \\
02 / 03 / 88\end{array}$ & $\begin{array}{r}3810 \\
26190 \\
1340\end{array}$ \\
\hline $65 / 25 / 88$ & $\begin{array}{r}236 \\
-2386 \\
\{\quad 260\end{array}$ \\
\hline
\end{tabular}

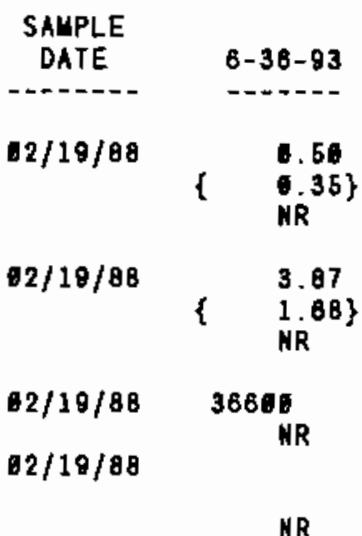


TABLE E.7. (contd)

\begin{tabular}{|c|c|c|c|c|c|c|c|c|c|}
\hline $\begin{array}{l}\text { CONSTI } \\
\text { NAME }\end{array}$ & $\begin{array}{l}\text { UENT } \\
\text { UNITS }\end{array}$ & $\begin{array}{l}\text { CONTRACTUAL } \\
\text { DETECTION } \\
\text { LIMIT }\end{array}$ & $\begin{array}{l}\text { ORINKING } \\
\text { WATER } \\
\text { STANDARD. }\end{array}$ & $\begin{array}{l}\text { SAMPLE } \\
\text { DATE }\end{array}$ & $8-37-82 A$ & $\begin{array}{l}\text { SAWPLE } \\
\text { DATE }\end{array}$ & $6-38-15$ & $\begin{array}{l}\text { SAMPLE } \\
\text { DATE }\end{array}$ & $6-38-85$ \\
\hline---- & ---- & 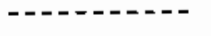 & - - - - - & 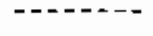 & $\cdots+-1$ & ------- & $-\infty=--$ & ------ & $-\cdots--$ \\
\hline ALPHA & $\mathrm{PCI} / \mathrm{L}$ & 4 & 15 & $\begin{array}{l}02 / 01 / 88 \\
05 / 24 / 88\end{array}$ & & $\begin{array}{l}03 / 10 / 88 \\
96 / 01 / 88\end{array}$ & $\begin{array}{l}2.86 \\
\{\quad .72\} \\
2.22 \\
\{.67\}\end{array}$ & & $\begin{array}{l}\text { NR } \\
\text { NR }\end{array}$ \\
\hline BETA & PCI/L & $B$ & 51 & $\begin{array}{l}02 / 01 / B B \\
65 / 24 / 88\end{array}$ & $\begin{array}{l}5.94 \\
\{.83\} \\
5.98 \\
1.83\}\end{array}$ & $03 / 10 / 88$ & $\begin{array}{c}89.90 \\
\left\{\begin{array}{c}8.46\} \\
80.89 \\
\{\quad 6.20\}\end{array}+\right.\end{array}$ & $03 / 28 / 88$ & $\begin{array}{r}18.10 \\
\{\quad 3.02\} \\
14.50 \\
\{\quad 2.88\}\end{array}$ \\
\hline$C D-8 B$ & $\mathrm{PCI} / \mathrm{L}$ & 22.50 & 100 & & $\begin{array}{l}\text { NR } \\
\text { NR }\end{array}$ & $\begin{array}{l}63 / 10 / 88 \\
06 / 61 / 88\end{array}$ & $\begin{array}{c}11.10 \\
9.28\} \\
13.58 \\
\{\quad 9.15\}\end{array}$ & $\begin{array}{l}63 / 28 / 88 \\
65 / 11 / 8 B\end{array}$ & \\
\hline HNITRAT & PPB & 2500 & 45600 & $\begin{array}{l}02 / 01 / 88 \\
05 / 24 / 88\end{array}$ & $\begin{array}{l}48700 \\
41200\end{array}$ & $\begin{array}{l}63 / 10 / 08 \\
60 / 01 / \mathrm{BB}\end{array}$ & $\begin{array}{l}55500 \\
51800\end{array}$ & $\begin{array}{l}63 / 28 / 88 \\
65 / 11 / 88\end{array}$ & $\begin{array}{l}153000 \\
159000\end{array}$ \\
\hline$I-12901$ & $\mathrm{PCI} / \mathrm{L}$ & 1 & & & NR & $06 / 01 / 88$ & $\left\{\begin{array}{l}\quad .43 \\
\quad .10\}\end{array}\right.$ & $55 / 11 / 88$ & $\left\{\begin{array}{l}3.51 \\
\{.55\}\end{array}\right.$ \\
\hline TRITIUU & $P C I / L$ & 500 & 20060 & $\begin{array}{l}62 / 01 / 88 \\
65 / 24 / 88\end{array}$ & & $\begin{array}{l}63 / 1 / / 88 \\
06 / 01 / 88\end{array}$ & $\left.\begin{array}{l}489600 \\
\{2630 \\
475000 \\
\{2586\end{array}\right\}$ & $\begin{array}{l}03 / 28 / 88 \\
05 / 11 / 88\end{array}$ & $\left.\begin{array}{c}397000 \\
2390 \\
397000 \\
2890\end{array}\right\}$ \\
\hline$U$ & $\mathrm{PCI} / \mathrm{L}$ & 0.50 & 806 & & $\begin{array}{l}\text { NR } \\
\text { NR }\end{array}$ & & $\begin{array}{l}\text { MR } \\
\text { NR }\end{array}$ & $\begin{array}{l}83 / 28 / 88 \\
65 / 11 / 88\end{array}$ & $\begin{array}{l}1.74 \\
1.54\end{array}$ \\
\hline
\end{tabular}


IABLE E.7. (contd)

\begin{tabular}{|c|c|c|c|c|c|c|c|c|c|}
\hline $\begin{array}{l}\text { CONSTI } \\
\text { NAHE }\end{array}$ & $\begin{array}{l}\text { UENT } \\
\text { UNITS }\end{array}$ & $\begin{array}{l}\text { CONTRACTUAL } \\
\text { DETECTION } \\
\text { LIMIT }\end{array}$ & $\begin{array}{l}\text { DRINKING } \\
\text { WAIER } \\
\text { STANDARD }\end{array}$ & $\begin{array}{l}\text { SALPLE } \\
\text { DATE }\end{array}$ & $6-39-6$ & $\begin{array}{l}\text { SAUPLE } \\
\text { OATE }\end{array}$ & $8-40-1$ & $\begin{array}{l}\text { SAMPLE } \\
\text { DATE }\end{array}$ & $6-46-33 A$ \\
\hline ALPHA & PCI I L & 4 & 16 & $02 / 18 / 8 B$ & $\begin{array}{l}\{.04 \\
\quad N R \\
N R\end{array}$ & $\begin{array}{l}82 / 16 / 88 \\
65 / 13 / 88\end{array}$ & $\begin{array}{l}\begin{array}{l}4.14 \\
0.87\} \\
3.29\end{array} \\
\{.792\end{array}$ & $02 / 82 / 88$ & $\begin{array}{l}1.65 \\
\text { NR } \\
\text { NR }\end{array}$ \\
\hline BETA & $\mathrm{PCI} / \mathrm{L}$ & 8 & 56 & $82 / 10 / 88$ & $\begin{array}{l}\begin{array}{c}72.49 \\
1.79\} \\
\mathrm{NR}\end{array} \\
\mathrm{NR}\end{array}$ & $\begin{array}{l}02 / 10 / 88 \\
05 / 13 / 88\end{array}$ & 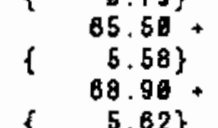 & $02 / 02 / 8 B$ & $\begin{array}{l}7.88 \\
2.87\} \\
\text { NR }\end{array}$ \\
\hline$C 0-50$ & $\mathrm{PCI} / \mathrm{L}$ & 22.58 & 100 & $2 / 10 / 88$ & & $62 / 10 / 88$ & $\begin{array}{r}11.4 \theta^{2} \\
\{\quad 9.95\}\end{array}$ & $02 / 02 / 8 B$ & \\
\hline $\begin{array}{l}\text { HNITRAT } \\
\text { TC- }-89\end{array}$ & $\begin{array}{l}\mathrm{PPB} \\
\mathrm{PCI} / \mathrm{L}\end{array}$ & $\begin{array}{r}2500 \\
15\end{array}$ & $\begin{array}{r}45008 \\
908\end{array}$ & $92 / 10 / 8 B$ & $\begin{array}{r}37460 \\
N R \\
N R\end{array}$ & $\begin{array}{l}02 / 10 / 88 \\
95 / 13 / 88 \\
-2 / 10 / 88\end{array}$ & $\begin{array}{r}37868 \\
40868 \\
350\end{array}$ & $02 / 02 / 88$ & $\begin{array}{l}N R \\
N R\end{array}$ \\
\hline TRITIUY & $\mathrm{PCI} / \mathrm{L}$ & 600 & 29060 & $82 / 18 / 8 B$ & $\underset{\text { NR }}{242686}\}^{1876}$ & $\begin{array}{l}62 / 10 / 80 \\
65 / 13 / 88\end{array}$ & $\left\{\begin{array}{c}237000 \\
243000 \\
\{1640\end{array}\right\} *$ & $02 / 62 / 88$ & NR \\
\hline
\end{tabular}



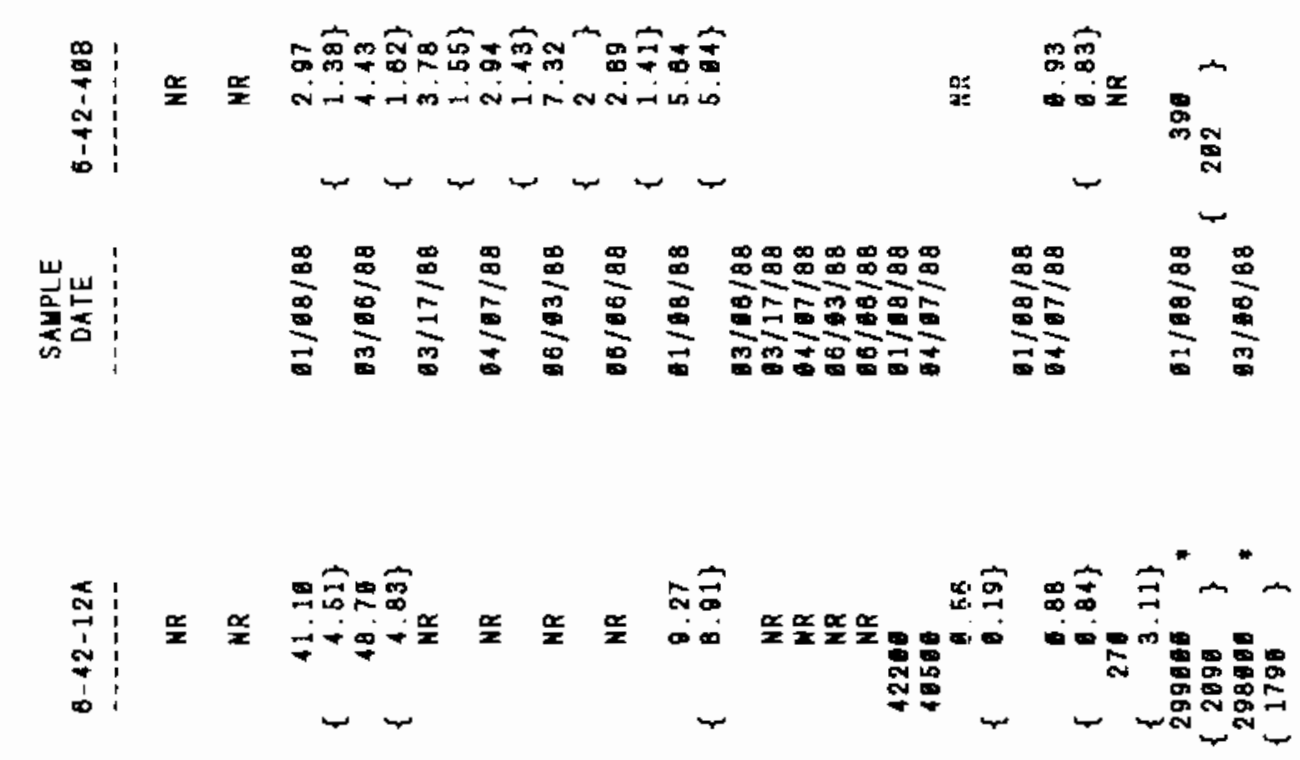

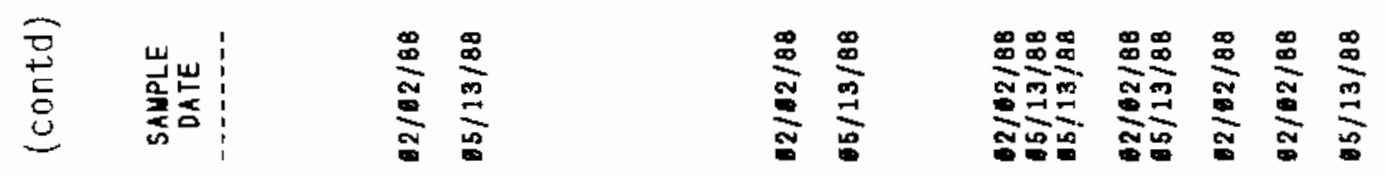

हो

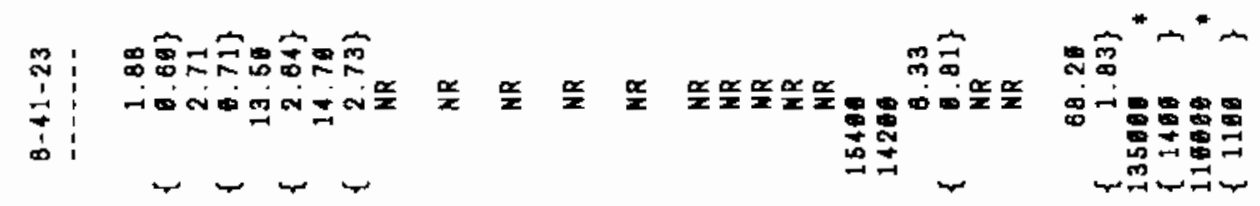

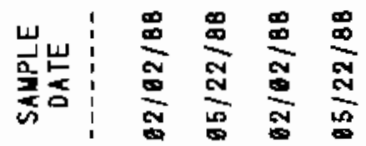

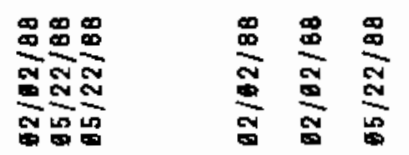
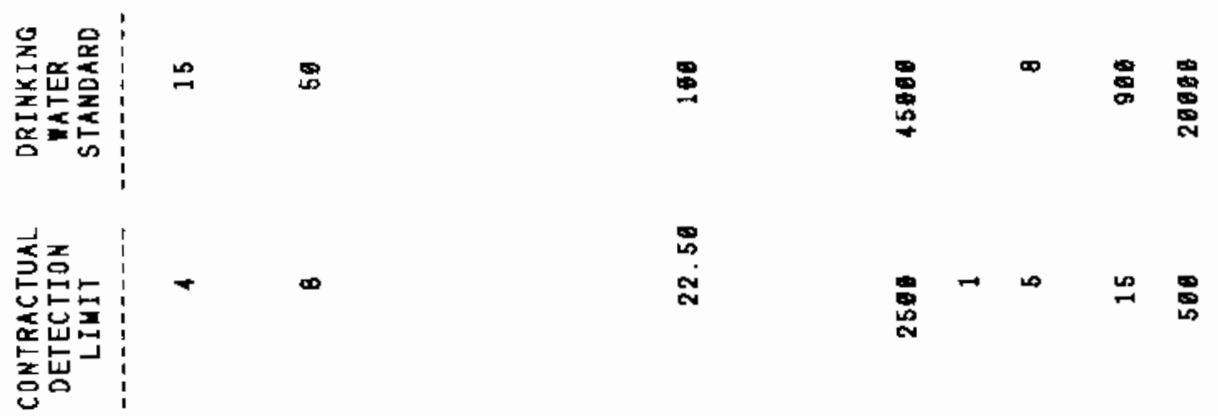
钽言言
产

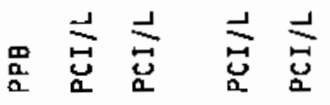
点:

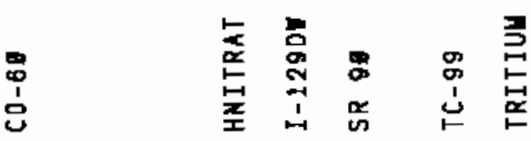


TABLE_E.7. (contd)

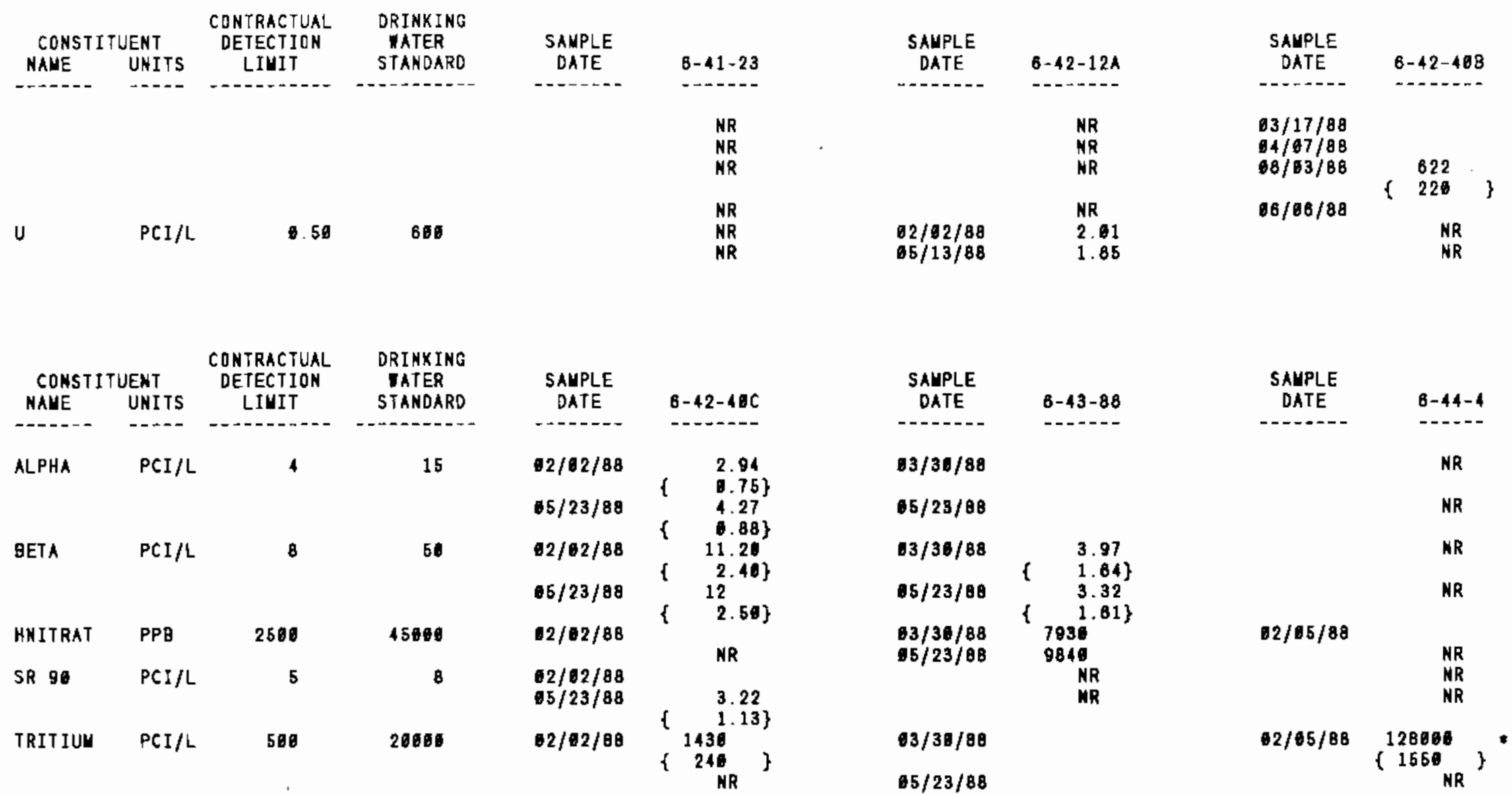


IABLE E.7. (contd)

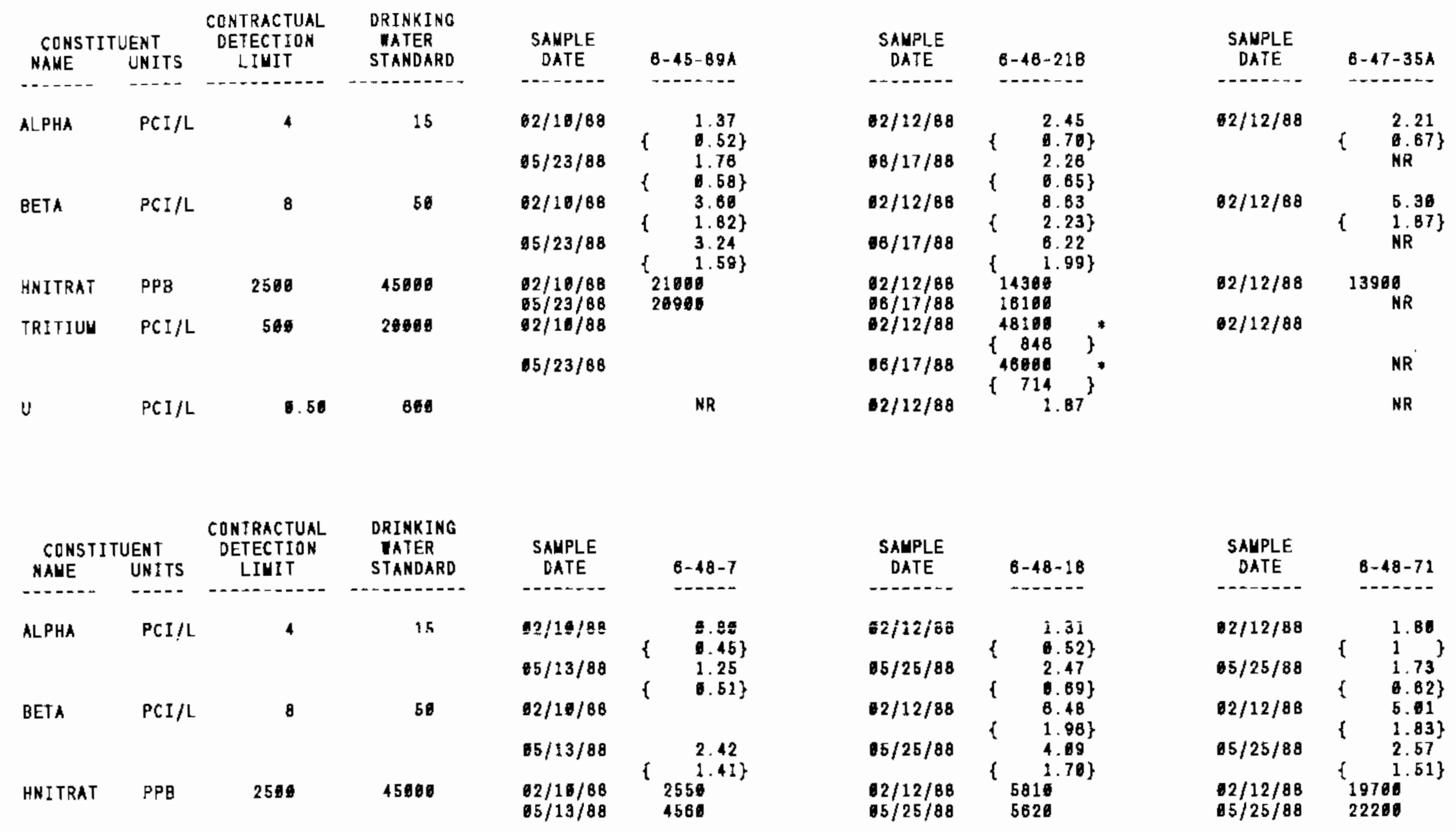


IABLE E.7. (contd)

\begin{tabular}{|c|c|c|c|c|c|}
\hline \multicolumn{2}{|c|}{ CONSTITUENT } & $\begin{array}{c}\text { CONTRACTUAL } \\
\text { DETECTION } \\
\text { LIUIT }\end{array}$ & $\begin{array}{l}\text { DRINKING } \\
\text { WATER } \\
\text { STANDARD }\end{array}$ & $\begin{array}{c}\text { SAMPLE } \\
\text { DATE }\end{array}$ & $6-49-13 E$ \\
\hline$-\cdots-\cdots$ & ---- & 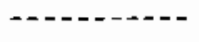 & $----\infty---$ & $----n-$ & 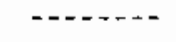 \\
\hline$A L P H A$ & $\mathrm{PCI} / \mathrm{L}$ & 4 & 15 & $\begin{array}{l}03 / 36 / 88 \\
06 / 81 / 88\end{array}$ & $\begin{array}{l}2.02 \\
\quad 6.82\} \\
2\end{array}$ \\
\hline GETA & $\mathrm{PCI} / \mathrm{L}$ & 8 & 50 & $\begin{array}{l}63 / 36 / 8 B \\
68 / 01 / 88\end{array}$ & $\begin{array}{l}3.56 \\
1.59\} \\
5.61 \\
1.89\}\end{array}$ \\
\hline $\begin{array}{l}\text { HNITRAT } \\
\text { TRITIUN }\end{array}$ & $\begin{array}{l}\mathrm{PPB} \\
\mathrm{PCI} / \mathrm{L}\end{array}$ & $\begin{array}{r}2580 \\
580\end{array}$ & $\begin{array}{l}45060 \\
20000\end{array}$ & $\begin{array}{l}03 / 30 / 88 \\
66 / 61 / 88 \\
63 / 30 / 88\end{array}$ & $\begin{array}{l}5170 \\
8050\end{array}$ \\
\hline & & & & $06 / 01 / 88$ & \\
\hline
\end{tabular}

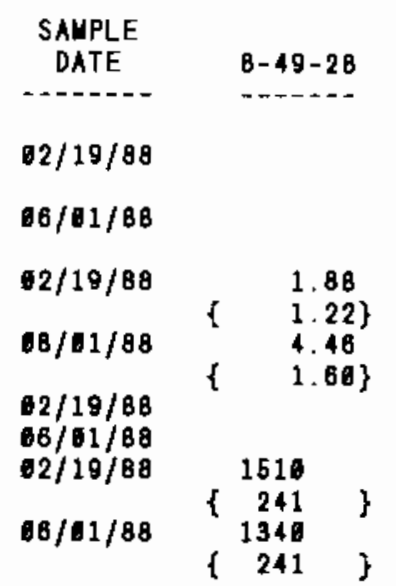

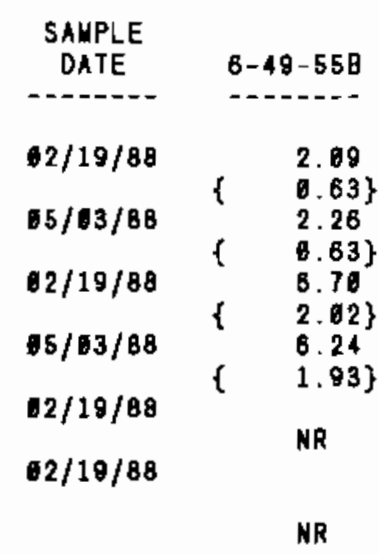

\begin{tabular}{|c|c|c|c|c|c|c|}
\hline \multirow{7}{*}{$\prod_{\omega}^{\infty}$} & $\begin{array}{l}\text { CONSTI } \\
\text { KALE }\end{array}$ & $\begin{array}{l}\text { UENT } \\
\text { UNITS }\end{array}$ & $\begin{array}{c}\text { CONTRACTUAL } \\
\text { DETECTION } \\
\text { LIUIT }\end{array}$ & $\begin{array}{l}\text { DRINKING } \\
\text { WATER } \\
\text { STANDARD }\end{array}$ & $\begin{array}{l}\text { 5AMPLE } \\
\text { DATE }\end{array}$ & $B+49-100 C$ \\
\hline & $--n-\infty$ & --- & $---1--n$ & 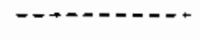 & $-\cdots-1$ & $-\cdots-1---$ \\
\hline & ALPHA & $\mathrm{PCI} / \mathrm{L}$ & 4 & 15 & $\begin{array}{l}62 / 12 / 88 \\
06 / 81 / 88\end{array}$ & $\begin{array}{ll} & 2.02 \\
\{\quad 0.81\}\end{array}$ \\
\hline & BETA & $\mathrm{PCI} / \mathrm{L}$ & 8 & 50 & $\begin{array}{l}62 / 12 / 88 \\
78 / 91 / 88\end{array}$ & $\begin{array}{c}6.57 \\
\{\quad 2.21\} \\
10.30 \\
\{\quad 2.37\}\end{array}$ \\
\hline & HNITRAT & PPB & 2580 & 45080 & $\begin{array}{l}02 / 12 / B 8 \\
06 / 01 / B 8\end{array}$ & 11868 \\
\hline & $I-1290$ & $\mathrm{PCI} / \mathrm{L}$ & 1 & & & NR \\
\hline & TRITIUM & $\mathrm{PCI} / \mathrm{L}$ & 506 & 20000 & $82 / 12 / B B$ & \\
\hline & & & & & $98 / 61 / 88$ & \\
\hline
\end{tabular}

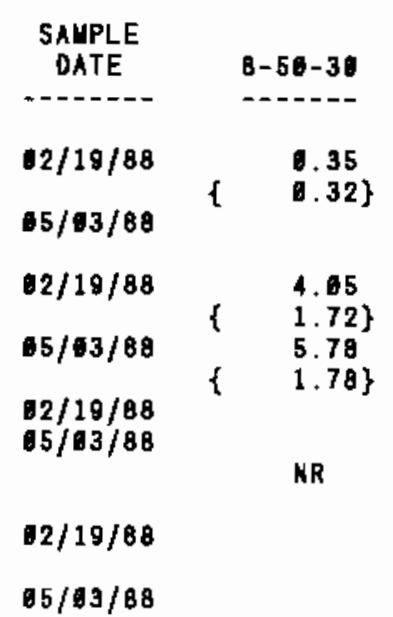

\begin{tabular}{|c|c|}
\hline $\begin{array}{c}\text { SAUPLE } \\
\text { DATE }\end{array}$ & $6-58-42$ \\
\hline - - & $---\ldots$. \\
\hline $12 / 02 / 88$ & 9. 93 \\
\hline $04 / 19 / 8 B$ & $\begin{array}{l}0.44\} \\
1.52\end{array}$ \\
\hline $02182 / 88$ & $\begin{array}{l}6.57) \\
6.48\end{array}$ \\
\hline $02 / 102 / 000$ & $\begin{array}{l}8.48 \\
1.92\}\end{array}$ \\
\hline $64 / 19 / 88$ & $\begin{array}{l}8.27 \\
2.17\}\end{array}$ \\
\hline $\begin{array}{l}02 / 62 / 88 \\
64 / 19 / 88\end{array}$ & $\begin{array}{l}2810 \\
4496\end{array}$ \\
\hline $04 / 19 / 8 B$ & $\begin{array}{r}\quad .32 \\
\{\quad 0.21\}\end{array}$ \\
\hline $62 / 02 / 88$ & $\left\{\begin{array}{r}4250 \\
312\end{array}\right.$ \\
\hline $04 / 19 / 88$ & $\begin{array}{r}4416 \\
\{\quad 315\end{array}$ \\
\hline
\end{tabular}




\section{APPENDIX REFERENCES}

EPA. 1982. Test Methods for Evaluating Solid Waste: Physical/Chemical Methods. 2nd ed., SW-846, U.S. Environmental Protection Agency, Washington, D.C.

Jaquish, R. E., and P. J. Mitche11, eds. 1988. Environmental Monitoring Hanford for 1987. PNL-6464, Pacific Northwest Laboratory, Richland, Washington.

Law, A. G., J. A. Serkowski, and A. L. Schatz. 1987. Results of the Separations Area Ground-Water Monitoring Network for 1986. RH0-RE-SR-87-24P, Rockwel] Hanford Operations, Richland, Washington. 


\section{DISTRIBUTION}

No. of

Copies

OFFSITE

10 DOE/Office of Scientific and Technical Information

\section{B. Blake}

133 lst Ave. North

Minneapolis, MN 55401

\section{ONSITE}

26 DOE Richland 0perations Office

M. J. Anthony

J. D. Goodenough

P. J. Krupin

R. J. Nevarez

M. W. Shupe

M. W. Tiernan (20)

$M$. Vargas

26 Westinghouse Hanford Company

M. R. Adams

L. C. Brown

G. D. Carpenter

M. A. Christie

M. L. Cramer

L. P. Diedicker

J. J. Dorian

K. R. Fecht

K. A. Gano

K. A. Gasper

E. M. Greagor

R. L. Jackson

M. J. Lauterbach

A. G. Law

D. L. Lund

A. C. McKarns

S. M. Mckinney

P. S. Peacock
No. of

Copies

K. M. Probasko

P. S. Schaus

J. A. Serkowski

L. L. Swanson

D. M. Tulburg

R. E. Wheeler

S. A. Wiegman

Public Reading Room

49 Pacific Northwest Laboratory

D. J. Bates

R. W. Bryce (5)

C. E. Elderkin

J. C. Evans (5)

F. W. Falco

J. S. Fruchter

R. M. Fruland

R. E. Gephart

R. H. Gray (2)

J. M. Hales

S. H. Haß1

M. S. Hanson (2)

P. C. Hays

W. W. Laity

P. E. Long

R. E. Lundgren

E. W. Lusty

S. P. Luttrel1

P. J. Mitchell

R. W. Nelson

J. T. Rieger

R. Schalla

D. R. Sherwood (5)

C. S. Simmons

R. L. Skaggs

R. M. Smith

J. A. Stottiemyre

G. P. Streile

R. E. Wildung

Publishing Coordination

Technical Report Files (5) 



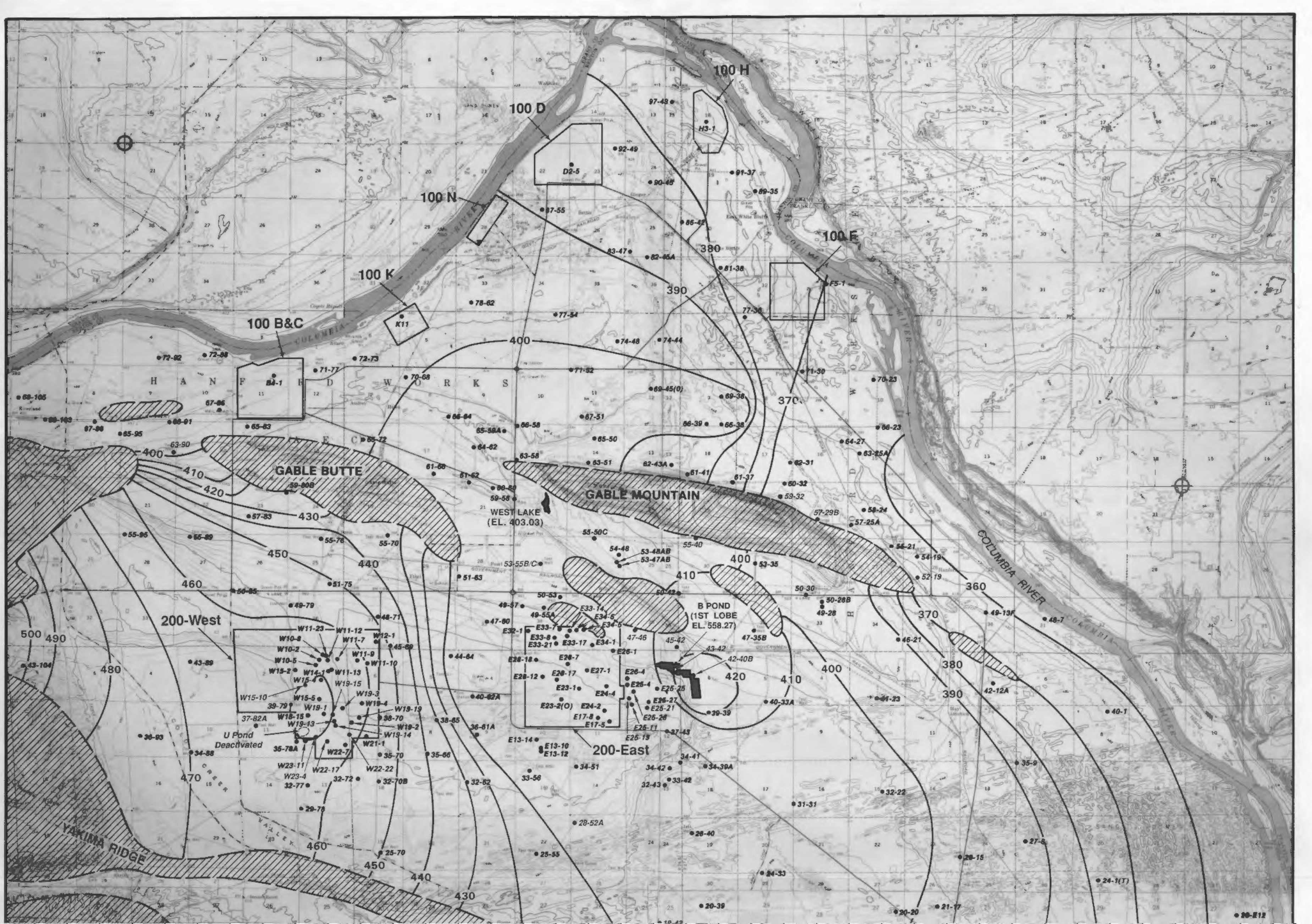


